\title{
On the rigid cohomology of certain Shimura varieties
}

\author{
Michael Harris ${ }^{1}$, Kai-Wen Lan ${ }^{2}$, Richard Taylor ${ }^{3^{*}}$ and Jack Thorne ${ }^{4}$ \\ In memory of Robert Coleman
}

\author{
${ }^{*}$ Correspondence: \\ rtaylor@ias.edu \\ ${ }^{3}$ School of Mathematics, IAS, \\ Princeton, NJ, USA \\ Full list of author information is \\ available at the end of the article
}

\begin{abstract}
We construct the compatible system of $I$-adic representations associated to a regular algebraic cuspidal automorphic representation of $G L_{n}$ over a CM (or totally real) field and check local-global compatibility for the /-adic representation away from / and a finite number of rational primes above which the CM field or the automorphic representation ramifies. The main innovation is that we impose no self-duality hypothesis on the automorphic representation.
\end{abstract}

\section{Contents}

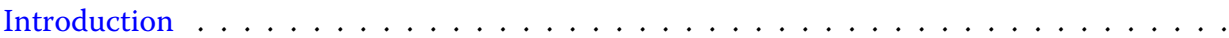

Notation

1 Some algebraic groups and automorphic forms $\ldots \ldots \ldots \ldots \ldots \ldots$

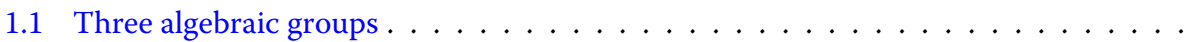

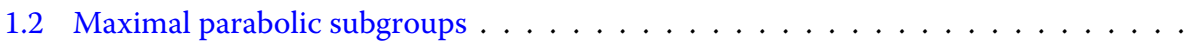

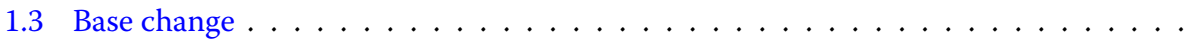

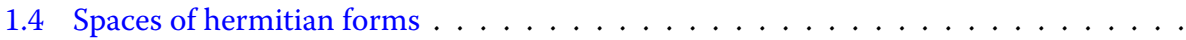

1.5 Locally symmetric spaces $\ldots \ldots \ldots \ldots \ldots \ldots$

2 Tori, torsors and torus embeddings $\ldots \ldots \ldots \ldots \ldots \ldots \ldots$

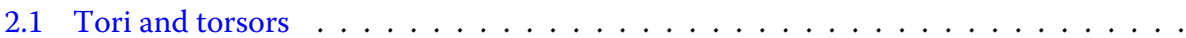

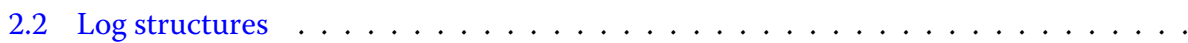

2.3 Torus embeddings $\ldots \ldots \ldots \ldots \ldots \ldots \ldots \ldots \ldots$

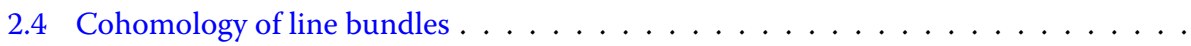

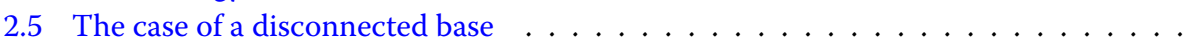

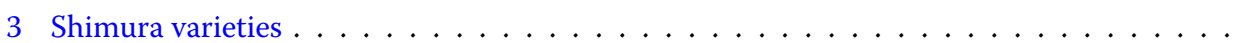

3.1 Some Shimura varieties . . . . . . . . . . . . . . . .

3.1 .1 Moduli problems . . . . . . . . . . . . . . . .

3.1 .2 Hecke actions . . . . . . . . . . . . . . . . . . .

3.1 .3 Representability .......................

3.2 Some Kuga-Sato varieties . . . . . . . . . . . . . . . . . . .

3.3 Some mixed Shimura varieties . . . . . . . . . . . . . . . . .

3.4 Vector bundles . . . . . . . . . . . . . . . . . . . .

3.4.1 Vector bundles on Shimura varieties in characteristic zero . . . . . . . .

3.4.2 Vector bundles on Kuga-Sato varieties in characteristic zero . . . . . . . .

3.4.3 Vector bundles on Shimura varieties in mixed characteristic . . . . . . . . .

3.4.4 Vector bundles on Kuga-Sato varieties in mixed characteristic

(c) The Author(s) 2016. This article is distributed under the terms of the Creative Commons Attribution 4.0 International License (http://creativecommons.org/licenses/by/4.0/), which permits unrestricted use, distribution, and reproduction in any medium, provided you give appropriate credit to the original author(s) and the source, provide a link to the Creative Commons license, and indicate if changes were made. 
3.4.5 Higher direct images from Kuga-Sato varieties to Shimura varieties, characteristic zero case . . . . . . . . . . . . . . .

3.4.6 Higher direct images from Kuga-Sato varieties to Shimura varieties, mixed

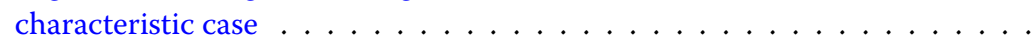

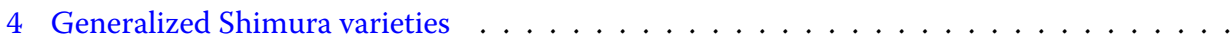

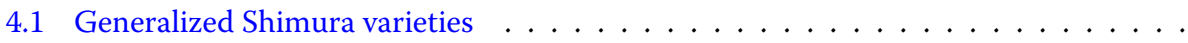

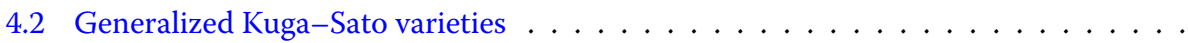

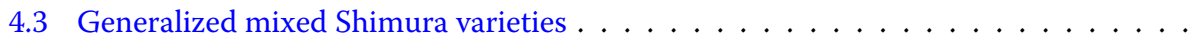

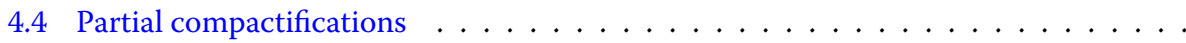

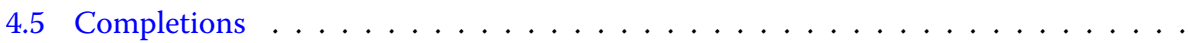

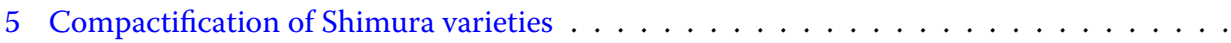

5.1 The minimal compactification $\ldots \ldots \ldots \ldots \ldots$

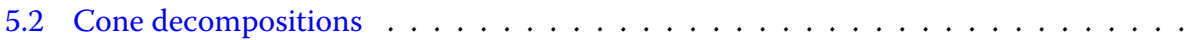

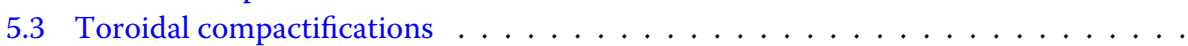

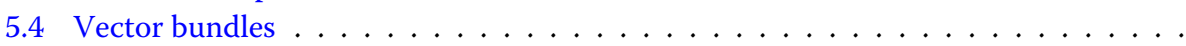

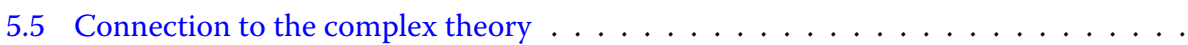

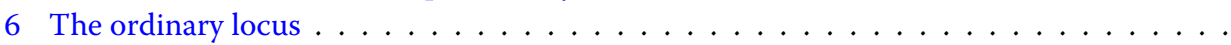

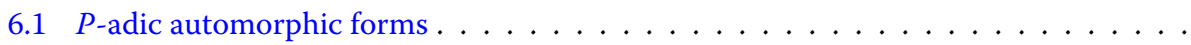

6.2 Interlude concerning linear algebra $\ldots \ldots \ldots \ldots \ldots \ldots \ldots \ldots$

6.3 The ordinary locus of a toroidal compactification as a dagger space . . . . . . . . .

6.3 .1 Review of dagger spaces . . . . . . . . . . . . . .

6.3 .2 The ordinary locus as a dagger space . . . . . . . . . . . .

6.3.3 The Frobenius lift $s_{p}$ and $\operatorname{tr}_{F} \ldots \ldots \ldots \ldots \ldots \ldots$

6.4 The ordinary locus of the minimal compactification as a dagger space . . . . . . .

6.4 .1 The ordinary locus as a dagger space . . . . . . . . . . . . . . . . . . . . . . . . . .

6.4 .2 The Frobenius lift $\varsigma_{p}$ and $\operatorname{tr}_{F} \ldots \ldots \ldots \ldots \ldots \ldots$

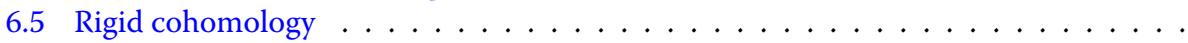

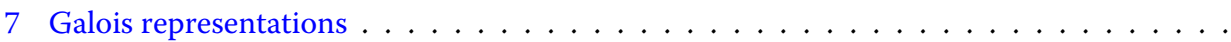

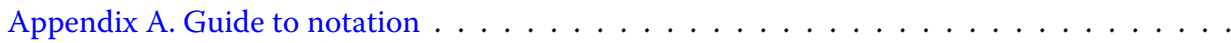

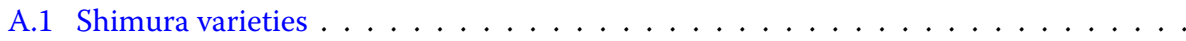

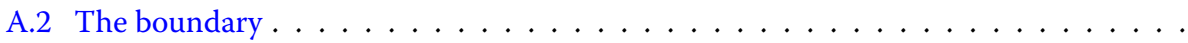

Appendix B. Comparison with $[44] \ldots \ldots \ldots \ldots \ldots \ldots$

B.1 Shimura varieties as PEL moduli in characteristic zero . . . . . . . . . . .

B.2 Filtrations ..........................

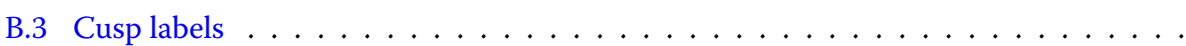

B.4 Minimal compactifications in characteristic zero . . . . . . . . . . . .

B.5 Toroidal compactifications of Shimura varieties in characteristic zero . . . . . . .

B.6 Kuga families in characteristic zero . . . . . . . . . . . . . . . . . .

B.7 Toroidal compactifications of Kuga families in characteristic zero . . . . . . . .

B.8 Automorphic bundles in characteristic zero . . . . . . . . . . . . . .

B.9 Total objects in mixed characteristics . . . . . . . . . . . . . .

B.10 Ordinary loci of Shimura varieties . . . . . . . . . . . . .

B.11 Ordinary cusp labels . . . . . . . . . . . . . . . . .

B.12 Partial minimal compactifications of ordinary PEL moduli . . . . . . . . . . . .

B.13 Partial toroidal compactifications of ordinary PEL moduli . . . . . . . . . . . .

B.14 Ordinary loci of Kuga families . . . . . . . . . . . . . . . .

B.15 Partial toroidal compactifications of ordinary loci of Kuga families . . . . . . . . .

B.16 Automorphic bundles in mixed characteristics . . . . . . . . . . . .

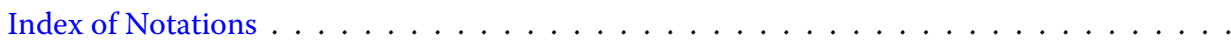

References

\section{Introduction}

Our main theorem is as follows (see Corollary 7.14). 
Theorem A Let $p$ denote a rational prime and let $\imath: \overline{\mathbb{Q}}_{p} \stackrel{\sim}{\rightarrow} \mathbb{C}$. Suppose that $E$ is a $C M$ (or totally real) field and that $\pi$ is a cuspidal automorphic representation of $G L_{n}\left(\mathbb{A}_{E}\right)$ such that $\pi_{\infty}$ has the same infinitesimal character as an irreducible algebraic representation $\rho_{\pi}$ of $\mathrm{RS}_{\mathbb{Q}}^{E} G L_{n}$. Then there is a unique continuous semi-simple representation

$$
r_{p, l}(\pi): G_{E} \longrightarrow G L_{n}\left(\overline{\mathbb{Q}}_{p}\right)
$$

such that, if $q \neq p$ is a rational prime above which $\pi$ is unramified and if $v \mid q$ is a prime of $E$, then $r_{p, l}(\pi)$ is unramified at $v$ and

$$
\left.r_{p, l}(\pi)\right|_{W_{E_{v}}} ^{\mathrm{SS}}=l^{-1} \operatorname{rec}_{E_{v}}\left(\pi_{\nu}|\operatorname{det}|_{\nu}^{(1-n) / 2}\right) .
$$

Here $\operatorname{rec}_{E_{v}}$ denotes the local Langlands correspondence for $E_{v}$. It may be possible to extend the local-global compatibility to other primes $v$. Ila Varma is considering this question.

The key point is that we make no self-duality assumption on $\pi$. In the presence of such a self-duality assumption ('polarizability', see [9]) the existence of $r_{p, l}(\pi)$ has been known for some years (see $[18,51])$. In almost all polarizable cases $r_{p, l}(\pi)$ is realized in the cohomology of a Shimura variety, and in all polarizable cases $r_{p, l}(\pi)^{\otimes 2}$ is realized in the cohomology of a Shimura variety (see [16]). In contrast, according to unpublished computations of one of us (M.H.) and of Laurent Clozel, in the non-polarizable case the representation $r_{p, l}(\pi)$ will never occur in the Betti or etale cohomology of a Shimura variety. Rather we construct it as a $p$-adic limit of representations which do occur in such cohomology groups.

We sketch our argument. We may easily reduce to the case of an imaginary CM field $F$ which contains an imaginary quadratic field in which $p$ splits. For all sufficiently large integers $N$, we construct a $2 n$-dimensional representation $R_{p}\left(l^{-1}\left(\pi\|\operatorname{det}\| I^{N}\right)^{\infty}\right)$ such that for good primes $v$ we have

$$
\begin{aligned}
& \left.R_{p}\left(l^{-1}\left(\pi|| \operatorname{det}||^{N}\right)^{\infty}\right)\right|_{W_{F_{v}}} ^{\mathrm{SS}} \\
& \quad \cong l^{-1} \operatorname{rec}_{F_{\nu}}\left(\pi_{v}|\operatorname{det}|_{\nu}^{N+(1-n) / 2}\right) \oplus \imath^{-1} \operatorname{rec}_{F_{\nu}}\left(\pi_{v}|\operatorname{det}|_{\nu}^{N+(1-n) / 2}\right)^{\vee, c} \epsilon_{p}^{1-2 n},
\end{aligned}
$$

as a $p$-adic limit of (presumably irreducible) $p$-adic representations associated to polarizable, regular algebraic cuspidal automorphic representations of $G L_{2 n}\left(\mathbb{A}_{F}\right)$. It is then elementary algebra to reconstruct $r_{p, l}(\pi)$.

We work on the quasi-split unitary similitude group $G_{n}$ associated to $F^{2 n}$. Note that $G_{n}$ has a maximal parabolic subgroup $P_{n,(n)}^{+}$with Levi component

$$
L_{n,(n)} \cong G L_{1} \times R S_{\mathbb{Q}}^{F} G L_{n} .
$$

(We will give all these groups integral structures.) We set

$$
\Pi(N)=\operatorname{Ind}_{P_{n,(n)}^{+}\left(\mathbb{A}^{\infty, p)}\right)}^{G_{n}\left(\mathbb{A}^{\infty, p}\right)}\left(1 \times i^{-1}\left(\pi\|\operatorname{det}\|^{N}\right)^{\infty, p}\right) .
$$

Then our strategy is to realize $\Pi(N)$, for sufficiently large $N$, in a space of overconvergent $p$-adic cusp forms for $G_{n}$ of finite slope. Once we have done this, we can use an argument of Katz (see [35]) to find congruences modulo arbitrarily high powers of $p$ to classical 
(holomorphic) cusp forms on $G_{n}$ (of other weights). (Alternatively it is presumably possible to construct an eigenvariety in this setting, but we have not carried this out.) One can attach Galois representations to these classical cusp forms by using the trace formula to lift them to polarizable, regular algebraic, discrete automorphic representations of $G L_{2 n}\left(\mathbb{A}_{F}\right)$ (see, e.g., [52]) and then applying the results of Chenevier and Harris [18], Shin [51].

We learnt the idea that one might try to realize $\Pi(N)$ in a space of overconvergent $p$-adic cusp forms for $G_{n}$ (of finite slope) from Chris Skinner. The key problem was how to achieve such a realization. To sketch our approach we must first establish some more notation.

To a neat open compact subgroup $U$ of $G_{n}$ we can associate a Shimura variety $X_{n, U} /$ Spec $\mathbb{Q}$. It is a moduli space for abelian $n[F: \mathbb{Q}]$-folds with an isogeny action of $F$ and certain additional structures. It is not proper. It has a canonical normal compactification $X_{n, U}^{\min }$ and, to certain auxiliary data $\Delta$, one can attach a smooth compactification $X_{n, U, \Delta}$ which naturally lies over $X_{n, U}^{\min }$ and whose boundary is a simple normal crossings divisor. To a representation $\rho$ of $L_{n,(n)}($ over $\mathbb{Q})$ one can attach a locally free sheaf $\mathcal{E}_{U, \rho} / X_{n, U}$ together with a canonical (locally free) extension $\mathcal{E}_{U, \Delta, \rho}$ to $X_{n, U, \Delta}$, whose global sections are holomorphic automorphic forms on $G_{n}$ 'of weight $\rho$ and level $U$ '. (The space of global sections does not depend on $\Delta$.) The product of $\mathcal{E}_{U, \Delta, \rho}$ with the ideal sheaf of the boundary of $X_{n, U, \Delta}$, which we denote $\mathcal{E}_{U, \Delta, \rho}^{\text {sub }}$, is again locally free, and its global sections are holomorphic cusp forms on $G_{n}$ 'of weight $\rho$ and level $U$ ' (and again the space of global sections does not depend on $\Delta$ ).

To the schemes $X_{n, U}, X_{n, U}^{\min }$ and $X_{n, U, \Delta}$ one can naturally attach dagger spaces $X_{n, U}^{\dagger}$, $X_{n, U}^{\min , \dagger}$ and $X_{n, U, \Delta}^{\dagger}$ in the sense of Grosse-Klönne [27]. These are like rigid analytic spaces except that one consistently works with overconvergent sections. If $U$ is the product of a neat open compact subgroup of $G_{n}\left(\mathbb{A}^{\infty, p}\right)$ and a suitable open compact subgroup of $G_{n}\left(\mathbb{Q}_{p}\right)$, then one can define admissible open subdagger spaces ('the ordinary loci')

$$
X_{n, U}^{\mathrm{ord}, \dagger} \subset X_{n, U}^{\dagger}
$$

and

$$
X_{n, U}^{\mathrm{ord}, \min , \dagger} \subset X_{n, U}^{\mathrm{min}, \dagger}
$$

and

$$
X_{n, U, \Delta}^{\mathrm{ord}, \dagger} \subset X_{n, U, \Delta}^{\dagger}
$$

By an overconvergent cusp form of weight $\rho$ and level $U$ one means a section of $\mathcal{E}_{U, \rho}^{\text {sub }}$ over $X_{n, U, \Delta}^{\mathrm{ord}, \dagger}$. (Again this definition does not depend on the choice of $\Delta$.)

We write $G_{n}^{(m)}$ for the semi-direct product of $G_{n}$ with the additive group with $\mathbb{Q}$-points $\operatorname{Hom}_{F}\left(F^{m}, F^{2 n}\right)$, and $P_{n,(n)}^{(m),+}$ for the pre-image of $P_{n,(n)}^{+}$in $G_{n}^{(m)}$. We also write $L_{n,(n)}^{(m)}$ for the semi-direct product of $L_{n,(n)}$ with the additive group with $\mathbb{Q}$-points $\operatorname{Hom}_{F}\left(F^{m}, F^{n}\right)$, which is naturally a quotient of $P_{n,(n)}^{(m),+}$. (Again we will give these groups integral structures.) To a neat open compact subgroup $U \subset G_{n}^{(m)}\left(\mathbb{A}^{\infty}\right)$ with projection $U^{\prime}$ in $G_{n}\left(\mathbb{A}^{\infty}\right)$ one can attach a (relatively smooth, projective) Kuga-Sato variety $A_{n, U}^{(m)} / X_{n, U^{\prime}}$. For a cofinal set of $U$ it is an abelian scheme isogenous to the $m$-fold self-product of the universal abelian 
scheme over $X_{n, U^{\prime}}$. To certain auxiliary data $\Sigma$ one can attach a smooth compactification $A_{n, U, \Sigma}^{(m)}$ of $A_{n, U}^{(m)}$ whose boundary is a simple normal crossings divisor; which lies over $X_{n, U}^{\min }$; and which, for suitable $\Sigma$ depending on $\Delta$, lies over $X_{n, U^{\prime}, \Delta}$. Thus

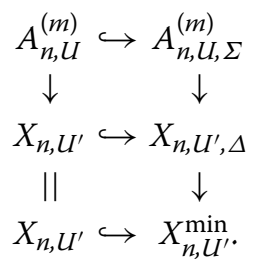

We define $A_{n, U}^{(m), \text { ord, } \dagger}$ and $A_{n, U, \Sigma}^{(m), \text { ord, } \dagger}$ to be the pre-image of $X_{n, U^{\prime}}^{\text {ord,min, } \dagger}$ in the dagger spaces associated to $A_{n, U}^{(m)}$ and $A_{n, U, \Sigma}^{(m)}$.

We will define

$$
H_{c-\partial}^{i}\left(\bar{A}_{n, U}^{(m), \text { ord }}, \overline{\mathbb{Q}}_{p}\right)
$$

to be the hypercohomology of the complex on $A_{n, U, \Sigma}^{(m), \text { ord, } \dagger}$ which is the tensor product of the de Rham complex with log poles towards the boundary, $A_{n, U, \Sigma}^{(m), \text { ord, } \dagger}-A_{n, U}^{(m), \text { ord, } \dagger}$, and the ideal sheaf defining the boundary. We believe it is a sort of rigid cohomology of the ordinary locus $\bar{A}_{n, U}^{(m), \text { ord }}$ in the special fibre of an integral model of $A_{n, U}^{(m)}$; more specifically, cohomology with compact support towards the toroidal boundary, but not towards the non-ordinary locus, hence our notation. However we have not bothered to verify that this group only depends on ordinary locus in the special fibre. The theory of Shimura varieties provides us with sufficiently canonical lifts that this will not matter to us. Our proof that for $N$ sufficiently large $\Pi(N)$ occurs in the space of overconvergent $p$-adic cusp forms for $G_{n}$ proceeds by evaluating $H_{c-\partial}^{i}\left(\bar{A}_{n, U}^{(m), \text { ord }}, \overline{\mathbb{Q}}_{p}\right)$ in two ways.

Firstly we use the usual Hodge spectral sequence. The higher direct images from $A_{n, U, \Sigma}^{(m)}$ to $X_{n, U^{\prime}, \Delta}$ of the tensor product of the ideal sheaf of the boundary and the sheaf of differentials of any degree with log poles along the boundary, are canonically filtered with graded pieces sheaves of the form $\mathcal{E}_{U^{\prime}, \Delta, \rho}^{\text {sub }}$. Thus $H_{c-\partial}^{i}\left(\bar{A}_{n, U}^{(m), \text { ord }}, \overline{\mathbb{Q}}_{p}\right)$ can be computed in terms of the groups

$$
H^{j}\left(X_{n, U, \Delta}^{\mathrm{ord}, \dagger}, \mathcal{E}_{U, \Delta, \rho}^{\mathrm{sub}}\right)
$$

A crucial observation for us is that for $j>0$ this group vanishes (see Theorem 5.4 and Proposition 6.12). This observation seems to have been made independently, at about the same time, by Andreatta, Iovita and Pilloni (see [1,2]). It seems quite surprising to us. It is false if one replaces $\mathcal{E}_{U, \Delta, \rho}^{\text {sub }}$ with $\mathcal{E}_{U, \Delta, \rho}^{\mathrm{can}}$. Its proof depends on a number of apparently unrelated facts, including:

- $X_{n, U}^{\text {ord,min, }} \dagger$ is affinoid.

- The stabilizer in $G L_{n}\left(\mathcal{O}_{F}\right)$ of a positive definite hermitian $n \times n$ matrix over $F$ is finite.

- Certain line bundles on self-products $A$ of the universal abelian scheme over $X_{n^{\prime}, U^{\prime}}$ (for $\left.n^{\prime}<n\right)$ are relatively ample for $A / X_{n^{\prime}, U^{\prime}}$

This observation implies that $H_{c-\partial}^{i}\left(\bar{A}_{n, U}^{(m), \text { ord }}, \overline{\mathbb{Q}}_{p}\right)$ can be computed by a complex whose terms are spaces of overconvergent cusp forms. Hence it suffices for us to show that, for $N$ sufficiently large, $\Pi(N)$ occurs in 


$$
H_{c-\partial}^{i}\left(\bar{A}_{n}^{(m), \text { ord }}, \overline{\mathbb{Q}}_{p}\right)=\lim _{\rightarrow U, \Sigma} H_{c-\partial}^{i}\left(\bar{A}_{n, U}^{(m), \text { ord }}, \overline{\mathbb{Q}}_{p}\right)
$$

for some $m$ and $i$ (depending on $N$ ).

To achieve this we use a second spectral sequence which computes the cohomology group $H_{c-\partial}^{i}\left(\bar{A}_{n, U}^{(m), \text { ord }}, \overline{\mathbb{Q}}_{p}\right)$ in terms of the rigid cohomology of $\bar{A}_{n, U, \Sigma}^{(m) \text {,ord }}$ and its various boundary strata. See Sect. 6.5. This is an analogue of the spectral sequence

$$
E_{1}^{i, j}=H^{j}\left(Y^{(i)}, \mathbb{C}\right) \Rightarrow H_{c}^{i+j}(Y-\partial Y, \mathbb{C})
$$

where $Y$ is a proper smooth variety over $\mathbb{C}$, where $\partial Y$ is a simple normal crossings divisor on $Y$, and where $Y^{(i)}$ is the disjoint union of the $i$-fold intersections of irreducible components of $\partial Y$. (So $Y^{(0)}=Y$.) Some of the terms in this spectral sequence seem a priori to be hard to control, e.g. $H_{\text {rig }}^{i}\left(\bar{A}_{n, U, \Sigma}^{(m) \text {,ord }}\right)$. However employing theorems about rigid cohomology due to Berthelot and Chiarellotto, we see that the eigenvalues of Frobenius on $H_{c-\partial}^{i}\left(\bar{A}_{n, U, \Sigma}^{(m), \text { ord }}, \overline{\mathbb{Q}}_{p}\right)$ are all Weil $p^{j}$-numbers for $j \geq 0$. Moreover the weight 0 part, $W_{0} H_{c-\partial}^{i}\left(\bar{A}_{n, U}^{(m), \text { ord }}, \overline{\mathbb{Q}}_{p}\right)$, equals the cohomology of a complex only involving the rigid cohomology in degree 0 of $\bar{A}_{n, U}^{(m) \text {,ord }}$ and its various boundary strata. (See Proposition 6.24.) This should have a purely combinatorial description. More precisely we define a simplicial complex $\mathcal{S}\left(\partial \bar{A}_{n, U, \Sigma}^{(m), \text { ord }}\right)$ whose vertices correspond to boundary components of $\bar{A}_{n, U, \Sigma}^{(m) \text {,ord }}$ and whose $j$-faces correspond to $j$-boundary components with non-trivial intersection. For $i>0$ we obtain an isomorphism

$$
H^{i}\left(\left|\mathcal{S}\left(\partial \bar{A}_{n, U, \Sigma}^{(m), \text { ord }}\right)\right|, \overline{\mathbb{Q}}_{p}\right) \cong W_{0} H_{c-\partial}^{i+1}\left(\bar{A}_{n, U}^{(m), \text { ord }}, \overline{\mathbb{Q}}_{p}\right)
$$

Thus it suffices to show that for $N$ sufficiently large $\Pi(N)$ occurs in

$$
H^{i}\left(\left|\mathcal{S}\left(\partial \bar{A}_{n}^{(m), \text { ord }}\right)\right|, \overline{\mathbb{Q}}_{p}\right)=\lim _{\rightarrow U, \Sigma} H^{i}\left(\left|\mathcal{S}\left(\partial \bar{A}_{n, U, \Sigma}^{(m), \text { ord }}\right)\right|, \overline{\mathbb{Q}}_{p}\right)
$$

for some $m$ and some $i>0$ (possibly depending on $N$ ).

The boundary of $\bar{A}_{n, U, \Sigma}^{(m), \text { ord }}$ comes in pieces indexed by the conjugacy classes of maximal parabolic subgroups of $G_{n}$. We shall be interested in the union of the irreducible components which are associated to $P_{n,(n)}^{+}$. These correspond to an open subset $\left|\mathcal{S}\left(\partial \bar{A}_{n, U, \Sigma}^{(m), \text { ord }}\right)\right|_{=n}$ of $\left|\mathcal{S}\left(\partial \bar{A}_{n, U, \Sigma}^{(m), \text { ord }}\right)\right|$. As $\left|\mathcal{S}\left(\partial \bar{A}_{n, U, \Sigma}^{(m), \text { ord }}\right)\right|$ is compact, the interior cohomology

$$
H_{\text {Int }}^{i}\left(\left|\mathcal{S}\left(\partial \bar{A}_{n}^{(m), \text { ord }}\right)\right|_{=n}, \overline{\mathbb{Q}}_{p}\right)=\lim _{\rightarrow U, \Sigma} H_{\text {Int }}^{i}\left(\left|\mathcal{S}\left(\partial \bar{A}_{n, U, \Sigma}^{(m), \text { ord }}\right)\right|_{=n}, \overline{\mathbb{Q}}_{p}\right)
$$

is naturally a subquotient of $H^{i}\left(\left|\mathcal{S}\left(\partial \bar{A}_{n}^{(m), \text { ord }}\right)\right|, \overline{\mathbb{Q}}_{p}\right)$. (By interior cohomology we mean the image of the cohomology with compact support in the cohomology. The interior cohomology of an open subset of an ambient compact Hausdorff space is naturally a subquotient of the cohomology of that ambient space.) Thus it even suffices to show that for $N$ sufficiently large $\Pi(N)$ occurs in

$$
H_{\text {Int }}^{i}\left(\left|\mathcal{S}\left(\partial \bar{A}_{n}^{(m), \text { ord }}\right)\right|_{=n}, \overline{\mathbb{Q}}_{p}\right)
$$

for some $m$ and some $i>0$ (possibly depending on $N$ ). 
However the data $\Sigma$ are a $G_{n}^{(m)}(\mathbb{Q})$-invariant (glued) collection of polyhedral cone decompositions and $\mathcal{S}\left(\partial \bar{A}_{n}^{(m) \text {,ord }}\right)$ is obtained from $\Sigma$ by replacing 1 -cones by vertices, 2 -cones by edges, etc. The cones corresponding to $\left|\mathcal{S}\left(\partial \bar{A}_{n}^{(m), \text { ord }}\right)\right|_{=n}$ are a disjoint union of polyhedral cones in the space of positive definite hermitian forms on $F^{n}$. From this one obtains an equality

$$
\left|\mathcal{S}\left(\partial \bar{A}_{n}^{(m), \text { ord }}\right)\right|_{=n}=\coprod_{h \in P_{n,(n)}^{+}\left(\mathbb{A}^{\infty, p}\right) \backslash G_{n}\left(\mathbb{A}^{\infty, p}\right) / U^{p}} \mathfrak{T}_{(n), h U h^{-1} \cap P_{n,(n)}^{(m),+}\left(\mathbb{A}^{\infty}\right)}^{(m)}
$$

where

$$
\mathfrak{T}_{(n), U^{\prime}}^{(m)}=L_{n,(n)}^{(m)}(\mathbb{Q}) \backslash L_{n,(n)}^{(m)}(\mathbb{A}) / U^{\prime}\left(\mathbb{R}_{>0}^{\times} \times\left(U(n)^{\left[F^{+}: \mathbb{Q}\right]} \mathbb{R}_{>0}^{\times}\right)\right),
$$

with $U(n)$ denoting the usual $n \times n$ compact unitary group. We deduce that

$$
H_{\text {Int }}^{i}\left(\left|\mathcal{S}\left(\partial \bar{A}_{n}^{(m), \text { ord }}\right)\right|_{=n}, \overline{\mathbb{Q}}_{p}\right)=\operatorname{Ind}_{P_{n,(n)}^{+}\left(\mathbb{A}^{\infty, p}\right)}^{G_{n}\left(\mathbb{A}^{\infty, p}\right)} H_{\text {Int }}^{i}\left(\mathfrak{T}_{(n)}^{(m)}, \overline{\mathbb{Q}}_{p}\right)^{\mathbb{Z}_{p}^{\times}}
$$

where

$$
H_{\text {Int }}^{i}\left(\mathfrak{T}_{(n)}^{(m)}, \overline{\mathbb{Q}}_{p}\right)=\lim _{\rightarrow U^{\prime}} H_{\text {Int }}^{i}\left(\mathfrak{T}_{(n), U^{\prime}}^{(m)}, \overline{\mathbb{Q}}_{p}\right)
$$

as $U^{\prime}$ runs over neat open compact subgroups of $L_{n,(n)}^{(m)}\left(\mathbb{A}^{\infty}\right)$. (The $\mathbb{Z}_{p}^{\times}$-invariants result from a restriction on the open compact subgroups of $G_{n}\left(\mathbb{A}^{\infty}\right)$ that we are considering.) Thus it suffices to show that for all sufficiently large $N$, the representation $1 \times\left(\left.\pi\|\operatorname{det}\|\right|^{N}\right)^{\infty, p}$ occurs in $H_{\text {Int }}^{i}\left(\mathfrak{T}_{(n)}^{(m)}, \mathbb{C}\right)$ for some $i>0$ and some $m$ (possibly depending on $N)$.

We will write simply $\mathfrak{T}_{(n), U^{\prime}}$ for $\mathfrak{T}_{(n), U^{\prime}}^{(0)}$, a locally symmetric space associated to $L_{n,(n)} \cong$ $G L_{1} \times \operatorname{RS}_{\mathbb{Q}}^{F} G L_{n}$. If $\rho$ is a representation of $L_{n,(n)}$ over $\mathbb{C}$, then it gives rise to a locally constant sheaf $\mathcal{L}_{\rho, U^{\prime}}$ over $\mathfrak{T}_{(n), U^{\prime}}$. We set

$$
H_{\text {Int }}^{i}\left(\mathfrak{T}_{(n)}, \mathcal{L}_{\rho}\right)=\lim _{\rightarrow U^{\prime}} H_{\text {Int }}^{i}\left(\mathfrak{T}_{(n), U^{\prime}}, \mathcal{L}_{\rho, U^{\prime}}\right)
$$

a smooth $L_{n,(n)}\left(\mathbb{A}^{\infty}\right)$-module. The space $\mathfrak{T}_{(n), U^{\prime}}^{(m)}$ is an $\left(S^{1}\right)^{n m[F: \mathbb{Q}]}$-bundle over the locally symmetric space $\mathfrak{T}_{(n), U^{\prime}}^{(0)}$ and if $\pi^{(m)}$ denotes the fibre map then

$$
R^{j} \pi_{*}^{(m)} \mathbb{C} \cong \mathcal{L}_{\wedge j \operatorname{Hom}_{F}\left(F^{m}, F^{n}\right)^{\vee} \otimes_{\mathbb{Q}} \mathbb{C}, U^{\prime}}
$$

where $L_{n,(n)}$ acts on $\operatorname{Hom}_{F}\left(F^{m}, F^{n}\right)$ via projection to $\mathrm{RS}_{\mathbb{Q}}^{F} G L_{n}$. Moreover the Leray spectral sequence

$$
E_{2}^{i, j}=H_{\text {Int }}^{i}\left(\mathfrak{T}_{(n)}, \mathcal{L}_{\wedge^{j} \operatorname{Hom}_{F}\left(F^{m}, F^{n}\right)^{\vee} \otimes_{\mathbb{Q}} \mathbb{C}}\right) \Rightarrow H_{\text {Int }}^{i+j}\left(\mathfrak{T}_{(n)}^{(m)}, \mathbb{C}\right)
$$

degenerates at the second page. (This can be seen by considering the action of the centre of $L_{n,(n)}\left(\mathbb{A}^{\infty}\right)$.) Thus it suffices to show that for all sufficiently large $N$, we can find nonnegative integers $j$ and $m$ and an irreducible constituent $\rho$ of $\wedge^{j} \operatorname{Hom}_{F}\left(F^{m}, F^{n}\right)^{\vee} \otimes_{\mathbb{Q}} \mathbb{C}$ such that the representation $1 \times\left(\pi \| \text { det } \|^{N}\right)^{\infty, p}$ occurs in $H_{\text {Int }}^{i}\left(\mathfrak{T}_{(n)}, \mathcal{L}_{\rho}\right)$ for some $i \in \mathbb{Z}_{>0}$. 
Clozel [20] checked that (for $n>1$ ) this will be the case as long as $1 \times\left(\pi\|\operatorname{det}\|{ }^{N}\right)_{\infty}$ has the same infinitesimal character as some irreducible constituent of $\wedge^{j} \operatorname{Hom}_{F}\left(F^{m}, F^{n}\right) \otimes_{\mathbb{Q}} \mathbb{C}$, i.e. if $\rho_{\pi} \otimes\left(\mathbf{N}_{F / \mathbb{Q}} \circ \operatorname{det}\right)^{N}$ occurs in $\wedge^{j} \operatorname{Hom}_{F}\left(F^{m}, F^{n}\right) \otimes_{\mathbb{Q}} \mathbb{C}$. From Weyl's construction of the irreducible representations of $G L_{n}$, for large enough $N$ this will indeed be the case for some $m$ and $j$.

We remark it is essential to work with $N$ sufficiently large. It is not an artefact of the fact that we are working with Kuga-Sato varieties rather than local systems on the Shimura variety. We can twist a local system on the Shimura variety by a power of the multiplier character of $G_{n}$. However the restriction of the multiplier factor of $G_{n}$ to $L_{n,(n)} \cong$ $G L_{1} \times \mathrm{RS}_{\mathbb{Q}}^{F} G L_{n}$ factors through the $G L_{1}$-factor and does not involve the $\mathrm{RS}_{\mathbb{Q}}^{F} G L_{n}$ factor.

We learnt from the series of papers [30-32] the key observation that $\left|\mathcal{S}\left(\partial A_{n, U, \Sigma}^{(m)}\right)\right|$ has a nice geometric interpretation involving the locally symmetric space for $L_{n,(n)}$ and that this could be used to calculate cohomology.

Although the central argument we have sketched above is not long, this paper has unfortunately become very long. If we had only wanted to construct $r_{p, l}(\pi)$ for all but finitely many primes $p$, then the argument would have been significantly shorter as we could have worked only with Shimura varieties $X_{n, U}$ which have good integral models at $p$. The fact that we want to construct $r_{p, l}(\pi)$ for all $p$ adds considerable technical complications and also requires appeal to the recent work [44]. (Otherwise we would only need to appeal to $[41,42]$.)

Another reason this paper has grown in length is the desire to use a language to describe toroidal compactifications of mixed Shimura varieties that is different from the language used in $[41,42,44]$. We do this because at least one of us (R.T.) finds this language clearer. In any case it would be necessary to establish a substantial amount of notation regarding toroidal compactifications of Shimura varieties, which would require significant space. We hope that the length of the paper, and the technicalities with which we have to deal, won't obscure the main line of the argument. On a first reading the reader might like to start with "Appendix A", which summarizes the extensive notation we use, and then turn to Sects. (5 and) 6 and 7. These sections will provide reference back to the key results from earlier sections. We have added "Appendix B" to help comparison between the notation of this paper and the notation of $[41,42,44]$, which we hope will make life easier for those readers that want to follow-up on our many references to these papers.

After we announced these results, but while we were writing up this paper, Scholze found another proof of Theorem A, relying on his theory of perfectoid spaces. His arguments seem to be in many ways more robust. For instance, he can handle torsion in the cohomology of the locally symmetric varieties associated to $G L_{n}$ over a CM field. Scholze's methods have some similarities with ours. Both methods first realize the Hecke eigenvalues of interest in the cohomology with compact support of the open Shimura variety by an analysis of the boundary and then show that they also occur in some space of $p$-adic cusp forms. We work with the ordinary locus of the Shimura variety, which for the minimal compactification is affinoid. Scholze works with the whole Shimura variety, but at infinite level. He (very surprisingly) shows that at infinite level, as a perfectoid space, (some compactification of) the Shimura variety has a Hecke invariant affinoid cover. 


\section{Notation}

If $G \rightarrow H$ is a surjective group homomorphism and if $U$ is a subgroup of $G$ we will sometimes use $U$ to also denote the image of $U$ in $H$.

Iff $: X \rightarrow Y$ and $f^{\prime}: Y \rightarrow Z$ then we will denote by $f^{\prime} \circ f: X \rightarrow Z$ the composite map $f$ followed by $f^{\prime}$. In this paper we will use both left and right actions. Suppose that $G$ is $a$ group acting on a set $X$ and that $g, h \in G$. If $G$ acts on $X$ on the left we will write gh for $g \circ h$. If $G$ acts on $X$ on the right we will write hg for $g \circ h$.

Iff is an automorphism of $\operatorname{Hom}(X, Y)$ we will sometimes use (of) to denote the map

$$
\begin{aligned}
\operatorname{Hom}(X, Y) & \longrightarrow \operatorname{Hom}(X, Y) \\
h & \longmapsto h \circ f .
\end{aligned}
$$

We will sometimes use / to denote a quotient, and sometimes we will use it to denote the fact that the object to the left lives 'over' the object to the right. Both these usages are standard, and we hope no confusion will arise.

If $G$ is a group (or group scheme) then $Z(G)$ will denote its centre.

We will write $S_{n}$ for the symmetric group on $n$ letters. We will write $U(n)$ for the group of $n \times n$ complex matrices $h$ with ${ }^{t} h\left({ }^{c} h\right)=1_{n}$.

If $G$ is an abelian group we will write $G[\infty]$ for the torsion subgroup of $G, G\left[\infty^{p}\right]$ for the subgroup of elements of order prime to $p$, and $G^{\mathrm{TF}}=G / G[\infty]$. We will write $T G=\lim _{\leftarrow N} G[N]$ and $T^{p} G=\lim _{\leftarrow p \not N} G[N]$. We will also write $V G=T G \otimes_{\mathbb{Z}} \mathbb{Q}$ and $V^{p} G=T^{p} G \otimes_{\mathbb{Z}} \mathbb{Q}$.

If $A$ is a ring, if $B$ is a locally free, finite $A$-algebra, and if $X / \operatorname{Spec} B$ is a quasi-projective scheme; then we will let $\mathrm{RS}_{A}^{B} X$ denote the restriction of scalars (or Weil restriction) of $X$ from $B$ to $A$. (See, for instance, section 7.6 of [12].)

By a $p$-adic formal scheme we mean a formal scheme such that $p$ generates an ideal of definition.

If $X$ is an $A$-module and $B$ is an $A$-algebra, we will sometimes write $X_{B}$ for $X \otimes_{A} B$. If $X$ is reflexive over $A$, then we will also use $X$ to denote the additive group scheme over $A$ defined by

$$
X(B)=X \otimes_{A} B=X_{B}
$$

for all $A$-algebras $B$.

If $X$ is a locally free $\mathcal{O}_{F}$-module we will write $G L\left(X / \mathcal{O}_{F}\right)$ for the group scheme over $\mathbb{Z}$ defined by

$$
G L\left(X / \mathcal{O}_{F}\right)(A)=\operatorname{Aut}\left(\left(X \otimes_{\mathbb{Z}} A\right) /\left(\mathcal{O}_{F} \otimes_{\mathbb{Z}} A\right)\right)
$$

If $Y$ is a scheme and if $G_{1}, G_{2} / Y$ are group schemes then we will let

$$
\mathcal{H o m}\left(G_{1}, G_{2}\right)
$$

denote the Zariski sheaf on $Y$ whose sections over an open $W$ are

$$
\operatorname{Hom}\left(\left.G_{1}\right|_{W},\left.G_{2}\right|_{W}\right)
$$


If in addition $R$ is a ring then we will let $\operatorname{Hom}\left(G_{1}, G_{2}\right)_{R}$ denote the tensor product of sheaves $\operatorname{Hom}\left(G_{1}, G_{2}\right) \otimes_{\mathbb{Z}} R$ and we will let $\operatorname{Hom}\left(G_{1}, G_{2}\right)_{R}$ denote the $R$-module of global sections of $\mathcal{H o m}\left(G_{1}, G_{2}\right)_{R}$. If $Y$ is noetherian this is the same as $\operatorname{Hom}\left(G_{1}, G_{2}\right) \otimes_{\mathbb{Z}} R$, but for a general base $Y$ it may differ.

If $\mathcal{S}$ is a simplicial complex we will write $|\mathcal{S}|$ for the corresponding topological space.

If $F$ is a field then $G_{F}$ will denote its absolute Galois group. If $F$ is a number field and $F_{0} \subset F$ is a subfield and $S$ is a finite set of primes of $F_{0}$, then we will denote by $G_{F}^{S}$ the maximal continuous quotient of $G_{F}$ in which all primes of $F$ not lying above an element of $S$ are unramified.

Suppose that $F$ is a number field and that $v$ is a place of $F$. If $v$ is finite we will write $\varpi_{v}$ for a uniformizer in $F_{v}$ and $k(v)$ for the residue field of $v$. We will write ||$_{\nu}$ for the absolute value on $F$ associated to $v$ and normalized as follows:

- if $v$ is finite then $\left|\varpi_{v}\right|_{v}=(\# k(v))^{-1}$;

- if $v$ is real then $|x|_{v}= \pm x$;

- if $v$ is complex then $|x|_{v}={ }^{c} x x$.

We write

$$
\|\|_{F}=\prod_{\nu}||_{v}: \mathbb{A}_{F}^{\times} \longrightarrow \mathbb{R}_{>0}^{\times} .
$$

We will write $\mathcal{D}_{F}^{-1}$ for the inverse different of $\mathcal{O}_{F}$.

If $w \in \mathbb{Z}$ and $p$ is a prime number then by a Weil $p^{w}$-number we mean an element $\alpha \in \overline{\mathbb{Q}}$ which is an integer away from $p$ and such that for each infinite place $v$ of $\overline{\mathbb{Q}}$ we have $|\alpha|_{v}=p^{w}$.

Suppose that $v$ is finite and that

$$
r: G_{F_{v}} \longrightarrow G L_{n}\left(\overline{\mathbb{Q}}_{l}\right)
$$

is a continuous representation, which in the case $v \mid l$ we assume to be de Rham. Then we will write $\mathrm{WD}(r)$ for the corresponding Weil-Deligne representation of the Weil group $W_{F_{v}}$ of $F_{v}$ (see, for instance, section 1 of [56]). If $\pi$ is an irreducible smooth representation of $G L_{n}\left(F_{v}\right)$ over $\mathbb{C}$ we will write $\operatorname{rec}_{F_{v}}(\pi)$ for the Weil-Deligne representation of $W_{F_{v}}$ corresponding to $\pi$ by the local Langlands conjecture (see, for instance, the introduction to [29]). If $\pi_{i}$ is an irreducible smooth representation of $G L_{n_{i}}\left(F_{v}\right)$ over $\mathbb{C}$ for $i=1,2$ then there is an irreducible smooth representation $\pi_{1} \boxplus \pi_{2}$ of $G L_{n_{1}+n_{2}}\left(F_{v}\right)$ over $\mathbb{C}$ satisfying

$$
\operatorname{rec}_{F_{v}}\left(\pi_{1} \boxplus \pi_{2}\right)=\operatorname{rec}_{F_{v}}\left(\pi_{1}\right) \oplus \operatorname{rec}_{F_{v}}\left(\pi_{2}\right) .
$$

Suppose that $G$ is a reductive group over $F_{v}$ and that $P$ is a parabolic subgroup of $G$ with unipotent radical $N$ and Levi component $L$. Suppose also that $\pi$ is a smooth representation of $L\left(F_{v}\right)$ on a vector space $W_{\pi}$ over a field $\Omega$ of characteristic 0 . We will define

$$
\operatorname{Ind}_{P\left(F_{v}\right)}^{G\left(F_{v}\right)} \pi
$$

to be the representation of $G\left(F_{v}\right)$ by right translation on the set of locally constant functions

$$
\varphi: G\left(F_{\nu}\right) \longrightarrow W_{\pi}
$$


such that

$$
\varphi(h g)=\pi(h) \varphi(g)
$$

for all $h \in P\left(F_{v}\right)$ and $g \in G\left(F_{v}\right)$. In the case $\Omega=\mathbb{C}$ we also define

$$
\mathrm{n}-\operatorname{Ind}_{P\left(F_{v}\right)}^{G\left(F_{v}\right)} \pi=\operatorname{Ind}_{P\left(F_{v}\right)}^{G\left(F_{v}\right)}\left(\pi \otimes \delta_{P}^{1 / 2}\right)
$$

where

$$
\delta_{P}(h)^{1 / 2}=\left|\operatorname{det}\left(\left.\operatorname{ad}(h)\right|_{\operatorname{Lie} N}\right)\right|_{v}^{1 / 2}
$$

If $G$ is a linear algebraic group over $F$ then the concept of a neat open compact subgroup of $G\left(\mathbb{A}_{F}^{\infty}\right)$ is defined, for instance, in section 0.6 of [49].

\section{Some algebraic groups and automorphic forms}

For the rest of this paper fix the following notation. Let $F^{+}$be a totally real field and $F_{0}$ an imaginary quadratic field, and set $F=F_{0} F^{+}$. Write $c$ for the non-trivial element of $\mathrm{Gal}\left(F / F^{+}\right)$. Also choose a rational prime $p$ which splits in $F_{0}$ and choose an element $\delta_{F} \in \mathcal{O}_{F,(p)}$ with $\operatorname{tr}_{F / F^{+}} \delta_{F}=1$ (which is possible as $p$ is unramified in $F / F^{+}$).

Fix an isomorphism $\imath: \overline{\mathbb{Q}}_{p} \stackrel{\sim}{\rightarrow} \mathbb{C}$. Fix a choice of $\sqrt{p} \in \overline{\mathbb{Q}}_{p}$ by $\imath \sqrt{p}>0$. If $v$ is a prime of $F$ and $\pi$ an irreducible admissible representation of $G L_{m}\left(F_{v}\right)$ over $\overline{\mathbb{Q}}_{p}$ define

$$
\operatorname{rec}_{F_{v}}(\pi)=l^{-1} \operatorname{rec}_{F_{v}}(\imath \pi)
$$

a Weil-Deligne representation of $W_{F_{v}}$ over $\overline{\mathbb{Q}}_{p}$.

Let $n$ be a non-negative integer. We will often attach $n$ as a subscript to other notation, when we need to record the particular choice of $n$ we are working with, but, at other times when the choice of $n$ is clear, we may drop it from the notation.

\subsection{Three algebraic groups}

Write $\Psi_{n}$ for the $n \times n$-matrix with 1's on the anti-diagonal and 0's elsewhere, and set

$$
J_{n}=\left(\begin{array}{cc}
0 & \Psi_{n} \\
-\Psi_{n} & 0
\end{array}\right) \in G L_{2 n}(\mathbb{Z}) .
$$

Let

$$
\Lambda_{n}=\left(\mathcal{D}_{F}^{-1}\right)^{n} \oplus \mathcal{O}_{F}^{n}
$$

and define a perfect pairing

$$
\langle,\rangle_{n}: \Lambda_{n} \times \Lambda_{n} \longrightarrow \mathbb{Z}
$$

by

$$
\langle x, y\rangle_{n}=\operatorname{tr}_{F / \mathbb{Q}}\left({ }^{t} x J_{n}^{c} y\right) .
$$


We will write $V_{n}$ for $\Lambda_{n} \otimes \mathbb{Q}$. Let $G_{n}$ denote the group scheme over $\mathbb{Z}$ defined by

$$
G_{n}(R)=\left\{(g, \mu) \in \operatorname{Aut}\left(\left(\Lambda_{n} \otimes_{\mathbb{Z}} R\right) /\left(\mathcal{O}_{F} \otimes_{\mathbb{Z}} R\right)\right) \times R^{\times}:{ }^{t} g J_{n}{ }^{c} g=\mu J_{n}\right\},
$$

for any ring $R$, and let $v: G_{n} \rightarrow G L_{1}$ denote the multiplier character which sends $(g, \mu)$ to $\mu$. Then $G_{n}$ is a quasi-split connected reductive group scheme over $\mathbb{Z}\left[1 / D_{F / \mathbb{Q}}\right]$ (where $D_{F / \mathbb{Q}}$ denotes the discriminant of $F / \mathbb{Q}$ ) and splits over $\mathcal{O}_{F^{\text {nc }}}\left[1 / D_{F / \mathbb{Q}}\right]$ (where $F^{\text {nc }}$ denotes the normal closure of $F / \mathbb{Q}$ ). In particular $G_{0}$ will denote $G L_{1}$ and $v: G_{0} \rightarrow G L_{1}$ is the identity map.

$$
\begin{aligned}
& \text { If } n>0 \text { set } \\
& \qquad C_{n}=\mathbb{G}_{m} \times \operatorname{ker}\left(N_{F / F^{+}}: \mathrm{RS}_{\mathbb{Z}}^{\mathcal{O}_{F}} \mathbb{G}_{m} \longrightarrow \mathrm{RS}_{\mathbb{Z}}^{\mathcal{O}_{F^{+}}} \mathbb{G}_{m}\right) .
\end{aligned}
$$

Then there is a natural map

$$
\begin{aligned}
G_{n} & \longrightarrow C_{n} \\
(g, \mu) & \longmapsto\left(\mu, \mu^{-n} \operatorname{det} g\right) .
\end{aligned}
$$

If $n=0$ we set $C_{0}=\mathbb{G}_{m}$ and let $G_{0} \longrightarrow C_{0}$ denote the map $v$. In either case this map identifies $C_{n}$ with $G_{n} /\left[G_{n}, G_{n}\right]$.

We will write $\Lambda_{n,(i)}$ for the submodule of $\Lambda_{n}$ consisting of elements whose last $2 n-i$ entries are 0 , and $V_{n,(i)}$ for $\Lambda_{n,(i)} \otimes \mathbb{Q}$. If $W$ is a submodule of $\Lambda_{n}$ we will write $W^{\perp}$ for its orthogonal complement with respect to $\langle,\rangle_{n}$. Thus $\Lambda_{n,(i)}^{\perp}$ is the submodule of $\Lambda_{n}$ consisting of vectors whose last $i$ entries are 0 . Also write

$$
\Lambda_{n}^{(m)}=\operatorname{Hom}\left(\mathcal{O}_{F}^{m}, \mathbb{Z}\right) \oplus \Lambda_{n}
$$

and set $V_{n}^{(m)}=\Lambda_{n}^{(m)} \otimes_{\mathbb{Z}} \mathbb{Q}$. Throughout this paper there will be various objects indexed by a superscript ${ }^{(m)}$. In the case $m=0$ we will sometimes simply drop it from the notation. For example, $\Lambda_{n}=\Lambda_{n}^{(0)}$.

Define an additive group scheme $\operatorname{Hom}_{n}^{(m)}$ over $\mathbb{Z}$ by

$$
\operatorname{Hom}_{n}^{(m)}(R)=\operatorname{Hom}_{\mathcal{O}_{F}}\left(\mathcal{O}_{F}^{m}, \Lambda_{n}\right) \otimes_{\mathbb{Z}} R
$$

Then $\operatorname{Hom}_{n}^{(m)}$ has an action of $G_{n} \times \mathrm{RS}_{\mathbb{Z}}^{\mathcal{O}_{F}} G L_{m}$ given by

$$
(g, h) f=g \circ f \circ h^{-1} .
$$

Also define a perfect pairing

$$
\langle,\rangle_{n}^{(m)}: \operatorname{Hom}_{n}^{(m)}(R) \times \operatorname{Hom}_{n}^{(m)}(R) \longrightarrow R
$$

by

$$
\left\langle f, f^{\prime}\right\rangle_{n}^{(m)}=\sum_{i=1}^{m}\left\langle f e_{i}, f^{\prime} e_{i}\right\rangle_{n},
$$

where $e_{1}, \ldots, e_{m}$ denotes the standard basis of $\mathcal{O}_{F}^{m}$. We have

$$
\left\langle(g, h) f, f^{\prime}\right\rangle_{n}^{(m)}=v(g)\left\langle f,\left(g^{-1, c, t} h\right) f^{\prime}\right\rangle_{n}^{(m)} .
$$


Moreover $G_{n}(R)$ is identified with the set of pairs

$$
(g, \mu) \in G L\left(\operatorname{Hom}_{\mathcal{O}_{F}}\left(\mathcal{O}_{F}^{m}, \Lambda_{n}\right) / \mathcal{O}_{F}\right)(R) \times R^{\times}
$$

such that $g$ commutes with the action of $G L_{m}\left(\mathcal{O}_{F} \otimes_{\mathbb{Z}} R\right)$ and such that

$$
\left\langle g f, g f^{\prime}\right\rangle_{n}^{(m)}=\mu\left\langle f, f^{\prime}\right\rangle_{n}^{(m)}
$$

for all $f, f^{\prime} \in \operatorname{Hom}_{\mathcal{O}_{F}}\left(\mathcal{O}_{F}^{m}, \Lambda_{n}\right)(R)$. We set

$$
G_{n}^{(m)}=G_{n} \ltimes \operatorname{Hom}_{n}^{(m)} .
$$

Then $G_{n}^{(m)}$ has an action of $\mathrm{RS}_{\mathbb{Z}}^{\mathcal{O}_{F}} G L_{m}$ by

$$
h(g, f)=(g,(1, h) f) .
$$

Moreover $G_{n}^{(m)}$ acts on $\Lambda_{n}^{(m)}$, by letting $f \in \operatorname{Hom}_{n}^{(m)}$ act by

$$
f:(h, x) \longmapsto\left(h+\langle x, f\rangle_{n}, x\right)
$$

and $g \in G_{n}$ act by

$$
g:(h, x) \longmapsto(h, g x) .
$$

Moreover $\mathrm{RS}_{\mathbb{Z}}^{\mathcal{O}_{F}} G L_{m}$ acts on $\Lambda_{n}^{(m)}$ by

$$
\gamma:(h, x) \longmapsto\left(h \circ \gamma^{-1}, x\right) .
$$

We have $\gamma \circ g=\gamma(g) \circ \gamma$.

If $m_{1} \geq m_{2}$ we embed $\mathcal{O}_{F}^{m_{2}} \hookrightarrow \mathcal{O}_{F}^{m_{1}}$ via

$$
i_{m_{2}, m_{1}}:\left(x_{1}, \ldots, x_{m_{2}}\right) \longmapsto\left(x_{1}, \ldots, x_{m_{2}}, 0, \ldots, 0\right) \text {. }
$$

This gives rise to maps

$$
i_{m_{2}, m_{1}}^{*}: \operatorname{Hom}_{n}^{\left(m_{1}\right)} \longrightarrow \operatorname{Hom}_{n}^{\left(m_{2}\right)}
$$

and

$$
i_{m_{2}, m_{1}}^{*}: G_{n}^{\left(m_{1}\right)} \longrightarrow G_{n}^{\left(m_{2}\right)} .
$$

It also gives rise to

$$
i_{m_{2}, m_{1}}^{*}: \Lambda_{n}^{\left(m_{1}\right)} \rightarrow \Lambda_{n}^{\left(m_{2}\right)} .
$$

Suppose that $R$ is a ring and that $X$ is an $\mathcal{O}_{F} \otimes_{\mathbb{Z}} R$-module. We will write Herm $X$ for the $R$-module of $R$-bilinear pairings

$$
(,): X \times X \longrightarrow R
$$

which satisfy 
(1) $(a x, y)=\left(x,{ }^{c} a y\right)$ for all $a \in \mathcal{O}_{F}$ and $x, y \in X$;

(2) $(x, y)=(y, x)$ for all $x, y \in X$.

If $z \in \operatorname{Herm}_{X}$ we will sometimes denote the corresponding pairing $(,)_{z}$. If $S$ is an $R$-algebra we have a natural map

$\operatorname{Herm}_{X} \otimes_{R} S \longrightarrow \operatorname{Herm}_{X \otimes_{R} S}$

If $X=\mathcal{O}_{F}^{m} \otimes_{\mathbb{Z}} R$ then we will write

$$
\operatorname{Herm}^{(m)}(R)=\operatorname{Herm}_{\mathcal{O}_{F}^{m}} \otimes_{\mathbb{Z}} R \longrightarrow \operatorname{Herm}_{\mathcal{O}_{F}^{m}} \otimes_{\mathbb{Z}} R .
$$

If $X \rightarrow Y$ then there is a natural map $\operatorname{Herm}_{Y} \rightarrow$ Herm $_{X}$. In particular if $m_{1} \geq m_{2}$, then there is a natural map

$$
\operatorname{Herm}^{\left(m_{1}\right)} \longrightarrow \operatorname{Herm}^{\left(m_{2}\right)}
$$

induced by the $\operatorname{map} \mathcal{O}_{F}^{\left(m_{2}\right)} \hookrightarrow \mathcal{O}_{F}^{\left(m_{1}\right)}$ described in the last paragraph. The group $G L\left(X / \mathcal{O}_{F}\right)$ acts on the left on $\operatorname{Herm}_{X}$ by

$$
(x, y)_{h z}=\left(h^{-1} x, h^{-1} y\right)_{z}
$$

There is a natural isomorphism

$$
\operatorname{Herm}_{X \oplus Y} \cong \operatorname{Herm}_{X} \oplus \operatorname{Hom}_{R}\left(X \otimes_{\mathcal{O}_{F} \otimes R, c \otimes 1} Y, R\right) \oplus \operatorname{Herm}_{Y}
$$

under which an element $(z, f, w)$ of the right hand side corresponds to

$$
\left((x, y),\left(x^{\prime}, y^{\prime}\right)\right)_{(z f, w)}=\left(x, x^{\prime}\right)_{z}+f\left(x \otimes y^{\prime}\right)+f\left(x^{\prime} \otimes y\right)+\left(y, y^{\prime}\right)_{w}
$$

If $X$ is an $\mathcal{O}_{F} \otimes_{\mathbb{Z}} R$-module, there is a natural pairing

$$
\begin{aligned}
\left(X \otimes_{\mathcal{O}_{F} \otimes R, c \otimes 1} X\right) \times \operatorname{Herm}_{X} & \longrightarrow R \\
(x \otimes y, z) & \longmapsto(x, y)_{z} .
\end{aligned}
$$

We further define

$$
\begin{aligned}
\mathrm{sw}:\left(X \otimes_{\mathcal{O}_{F} \otimes R, c \otimes 1} X\right) & \longrightarrow\left(X \otimes_{\mathcal{O}_{F} \otimes R, c \otimes 1} X\right) \\
x \otimes y & \longmapsto y \otimes x,
\end{aligned}
$$

and

$$
S(X)=\left(X \otimes_{\mathcal{O}_{F} \otimes R, c \otimes 1} X\right) /(\mathrm{sw}-1) .
$$

There is a natural map in the other direction

$$
\begin{aligned}
S(X) & \longrightarrow X \otimes_{\mathcal{O}_{F} \otimes R, c \otimes 1} X \\
w & \longmapsto w+\operatorname{sw}(w),
\end{aligned}
$$

such that the composite $S(X) \rightarrow X \otimes_{\mathcal{O}_{F} \otimes R, c \otimes 1} X \rightarrow S(X)$ is multiplication by 2 . Note that if $F / F^{+}$is ramified above 2 then $S\left(\mathcal{O}_{F}^{m}\right)$ can have 2-torsion, but that $S\left(\mathcal{O}_{F,(p)}^{m}\right)$ is torsion 
free. (Either $p>2$ or by assumption $F / F^{+}$is not ramified above 2.) There is a perfect duality

$$
S\left(\mathcal{O}_{F}^{m}\right)^{\mathrm{TF}} \times \operatorname{Herm}^{(m)}(\mathbb{Z}) \longrightarrow \mathbb{Z}
$$

We will write

$$
e=\sum_{i=1}^{m} e_{i} \otimes e_{i} \in \mathcal{O}_{F}^{m} \otimes_{\mathcal{O}_{F}, c} \mathcal{O}_{F}^{m},
$$

where $e_{1}, \ldots, e_{m}$ denotes the standard basis of $\mathcal{O}_{F}^{m}$.

Set $N_{n}^{(m)}(\mathbb{Z})$ to be the set of pairs

$$
(f, z) \in \operatorname{Hom}_{\mathcal{O}_{F}}\left(\mathcal{O}_{F}^{m}, \Lambda_{n}\right) \oplus\left(\frac{1}{2} \operatorname{Herm}^{(m)}(\mathbb{Z})\right)
$$

such that

$$
(x, y)_{z}-\frac{1}{2}\langle f x, f y\rangle_{n} \in \mathbb{Z}
$$

for all $x, y \in \mathcal{O}_{F}^{m}$. We define a group scheme $N_{n}^{(m)} / \operatorname{Spec} \mathbb{Z}$ by setting $N_{n}^{(m)}(R)$ to be the set of pairs

$$
(f, z) \in N_{n}^{(m)}(\mathbb{Z}) \otimes_{\mathbb{Z}} R
$$

with group law given by

$$
(f, z)\left(f^{\prime}, z^{\prime}\right)=\left(f+f^{\prime}, z+z^{\prime}+\frac{1}{2}\left(\left\langle f, f^{\prime}\right\rangle_{n}-\left\langle f^{\prime}, f\right\rangle_{n}\right)\right)
$$

where by $\left\langle f, f^{\prime}\right\rangle_{n}-\left\langle f^{\prime}, f\right\rangle_{n}$ we mean the hermitian form

$$
(x, y) \longmapsto\left\langle f(x), f^{\prime}(y)\right\rangle_{n}-\left\langle f^{\prime}(x), f(y)\right\rangle_{n} .
$$

Note that $(f, z)^{-1}=(-f,-z)$. Thus there is an exact sequence

$$
(0) \longrightarrow \operatorname{Herm}^{(m)} \longrightarrow N_{n}^{(m)} \longrightarrow \operatorname{Hom}_{n}^{(m)} \longrightarrow(0)
$$

In fact, $Z\left(N_{n}^{(m)}\right)=\operatorname{Herm}^{(m)}$. The commutator in $N_{n}^{(m)}$ induces an alternating map

$$
\operatorname{Hom}_{n}^{(m)}(R) \times \operatorname{Hom}_{n}^{(m)}(R) \longrightarrow \operatorname{Herm}^{(m)}(R)
$$

under which $\left(f, f^{\prime}\right)$ maps to the pairing

$$
(x, y) \longmapsto\left\langle f(x), f^{\prime}(y)\right\rangle_{n}-\left\langle f^{\prime}(x), f(y)\right\rangle_{n} .
$$

If $m_{1} \geq m_{2}$ there is a natural map

$$
N_{n}^{\left(m_{1}\right)} \longrightarrow N_{n}^{\left(m_{2}\right)}
$$


compatible with the previously described maps

$$
\operatorname{Hom}_{n}^{\left(m_{1}\right)} \rightarrow \operatorname{Hom}_{n}^{\left(m_{2}\right)}
$$

and

$$
\operatorname{Herm}^{\left(m_{1}\right)} \rightarrow \operatorname{Herm}^{\left(m_{2}\right)} .
$$

Note that $G_{n} \times \mathrm{RS}_{\mathbb{Z}}^{\mathcal{O}_{F}} G L_{m}$ acts on $N_{n}^{(m)}$ from the left by

$$
(g, h)(f, z)=\left(g \circ f \circ h^{-1}, v(g) h z\right) .
$$

If 2 is invertible in $R$ we see that

$$
\operatorname{Herm}^{(m)}(R)=\left\{g \in N_{n}^{(m)}(R):\left(-1_{m}\right)(g)=g\right\}
$$

and

$$
\operatorname{Hom}_{n}^{(m)}(R)=\left\{g \in N_{n}^{(m)}(R):\left(-1_{m}\right)(g)=g^{-1}\right\} .
$$

Set

$$
\widetilde{G}_{n}^{(m)}=G_{n} \ltimes N_{n}^{(m)},
$$

which has an $\mathrm{RS}_{\mathbb{Z}}^{\mathcal{O}_{F}} G L_{m}$-action via

$$
h(g, u)=(g, h(u)) .
$$

If $m_{1} \geq m_{2}$ then we get a natural map $\widetilde{G}_{n}^{\left(m_{1}\right)} \rightarrow \widetilde{G}_{n}^{\left(m_{2}\right)}$. Note that

$$
G_{n}^{(m)} \cong \widetilde{G}_{n}^{(m)} / \operatorname{Herm}^{(m)} .
$$

Let $B_{n}$ denote the subgroup of $G_{n}$ consisting of elements which preserve the chain $\Lambda_{n,(n)} \supset \Lambda_{n,(n-1)} \supset \cdots \supset \Lambda_{n,(1)} \supset \Lambda_{n,(0)}$ and let $N_{n}$ denote the normal subgroup of $B_{n}$ consisting of elements with $v=1$, which also act trivially on $\Lambda_{n,(i)} / \Lambda_{n,(i-1)}$ for all $i=1, \ldots, n$. Let $T_{n}$ denote the group consisting of the diagonal elements of $G_{n}$ and let $A_{n}$ denote the image of $\mathbb{G}_{m}$ in $G_{n}$ via the embedding that sends $t$ onto $t 1_{2 n}$. Over $\mathbb{Q}$ we see that $T_{n}$ is a maximal torus in a Borel subgroup $B_{n}$ of $G_{n}$ and that $N_{n}$ is the unipotent radical of $B_{n}$. Moreover $A_{n}$ is a maximal split torus in the centre of $G_{n}$.

If $\Omega$ is an algebraically closed field of characteristic 0 then set

$$
X^{*}\left(T_{n, / \Omega}\right)=\operatorname{Hom}\left(T_{n} \times \operatorname{Spec} \Omega, \mathbb{G}_{m} \times \operatorname{Spec} \Omega\right) .
$$

Also let $\Phi_{n} \subset X^{*}\left(T_{n, / \Omega}\right)$ denote the set of roots of $T_{n}$ on Lie $G_{n}$; let $\Phi_{n}^{+} \subset \Phi_{n}$ denote the set of positive roots with respect to $B_{n}$ and let $\Delta_{n} \subset \Phi_{n}^{+}$denote the set of simple positive roots. We will write $\varrho_{n}$ for half the sum of the elements of $\Phi_{n}^{+}$. If $R \subset \mathbb{R}$ is a subring then $X^{*}\left(T_{n, / \Omega}\right)_{R}^{+}$will denote the subset of $X^{*}\left(T_{n, / \Omega}\right)_{R}$ consisting of elements which pair non-negatively with the coroot $\check{\alpha} \in X_{*}\left(T_{n, / \Omega}\right)$ corresponding to each $\alpha \in \Delta_{n}$. We will write simply $X^{*}\left(T_{n, / \Omega}\right)^{+}$for $X^{*}\left(T_{n, / \Omega}\right)_{\mathbb{Z}}^{+}$. If $\lambda \in X^{*}\left(T_{n, / \Omega}\right)^{+}$we will let $\rho_{n, \lambda}$ (or simply $\rho_{\lambda}$ ) 
denote the irreducible representation of $G_{n}$ with highest weight $\lambda$. When $\rho_{\lambda}$ is used as a subscript we will sometimes replace it by just $\lambda$.

There is a natural identification

$$
G_{n} \times \operatorname{Spec} \Omega \cong\left\{\left(\mu, g_{\tau}\right) \in \mathbb{G}_{m} \times G L_{2 n}^{\operatorname{Hom}(F, \Omega)}: g_{\tau c}=\mu J_{n}{ }^{t} g_{\tau}^{-1} J_{n} \forall \tau\right\} .
$$

This gives rise to the further identification

$$
T_{n} \times \operatorname{Spec} \Omega \cong\left\{\left(t_{0},\left(t_{\tau, i}\right)\right) \in \mathbb{G}_{m} \times\left(\mathbb{G}_{m}^{2 n}\right)^{\operatorname{Hom}(F, \Omega)}: t_{\tau, i} t_{\tau c, 2 n+1-i}=t_{0} \forall \tau, i\right\} .
$$

We will use this to identify $X^{*}\left(T_{n, / \Omega}\right)$ with a quotient of

$$
X^{*}\left(\mathbb{G}_{m} \times\left(\mathbb{G}_{m}^{2 n}\right)^{\operatorname{Hom}(F, \Omega)}\right) \cong \mathbb{Z} \oplus\left(\mathbb{Z}^{2 n}\right)^{\operatorname{Hom}(F, \Omega)} .
$$

Under this identification $X^{*}\left(T_{n, / \Omega}\right)^{+}$is identified to the image of the set of

$$
\left(a_{0},\left(a_{\tau, i}\right)\right) \in \mathbb{Z} \oplus\left(\mathbb{Z}^{2 n}\right)^{\operatorname{Hom}(F, \Omega)}
$$

with

$$
a_{\tau, 1} \geq a_{\tau, 2} \geq \cdots \geq a_{\tau, 2 n}
$$

for all $\tau$.

If $R$ is a subring of $\mathbb{R}$ and $H$ an algebraic subgroup of $\widetilde{G}_{n}^{(m)}$ we will write $H(R)^{+}$for the subgroup of $H(R)$ consisting of elements with positive multiplier. Thus $G_{n}(\mathbb{R})^{+}$(resp. $G_{n}^{(m)}(\mathbb{R})^{+}$, resp. $\widetilde{G}_{n}^{(m)}(\mathbb{R})^{+}$) is the connected component of the identity in $G_{n}(\mathbb{R})$ (resp. $G_{n}^{(m)}(\mathbb{R})$, resp. $\left.\widetilde{G}_{n}^{(m)}(\mathbb{R})\right)$.

Let

$$
U_{n, \infty}=\left(U(n)^{2}\right)^{\operatorname{Hom}\left(F^{+}, \mathbb{R}\right)} \rtimes\{1, j\}
$$

with $j^{2}=1$ and $j\left(A_{\tau}, B_{\tau}\right) j=\left(B_{\tau}, A_{\tau}\right)$. Embed $U_{n, \infty}$ in $G_{n}(\mathbb{R})$ by sending $\left(A_{\tau}, B_{\tau}\right) \in$ $\left(U(n)^{2}\right)^{\operatorname{Hom}\left(F^{+}, \mathbb{R}\right)}$ to

$$
\begin{aligned}
& \left(1,\left(\left(\begin{array}{cc}
\left(A_{\tau}+B_{\tau}\right) / 2 & \left(A_{\tau}-B_{\tau}\right) \Psi_{n} / 2 i \\
\Psi_{n}\left(B_{\tau}-A_{\tau}\right) / 2 i & \Psi_{n}\left(A_{\tau}+B_{\tau}\right) \Psi_{n} / 2
\end{array}\right)\right)_{\tau}\right) \\
& \quad \in G_{n}(\mathbb{R}) \subset \mathbb{R}^{\times} \times \prod_{\tau \in \operatorname{Hom}\left(F^{+}, \mathbb{R}\right)} G L_{2 n}\left(F \otimes_{F^{+}, \tau} \mathbb{R}\right),
\end{aligned}
$$

and sending $j$ to

$$
\left(-1,\left(\left(\begin{array}{cc}
-1_{n} & 0 \\
0 & 1_{n}
\end{array}\right)\right)_{\tau}\right) .
$$

(This map depends on identifications $F \otimes_{F^{+}, \tau} \mathbb{R} \cong \mathbb{C}$, but the image of the map does not, and this image is all that will concern us.) Then $U_{n, \infty}$ is a maximal compact subgroup of $G_{n}(\mathbb{R})$ (and even of $\widetilde{G}_{n}^{(m)}(\mathbb{R})$ ). If $L \supset T_{n} \times$ Spec $\mathbb{R}$ is a Levi component of a parabolic subgroup $P \supset B_{n} \times \operatorname{Spec} \mathbb{R}$ then $U_{n, \infty} \cap L(\mathbb{R})$ is a maximal compact subgroup of $L(\mathbb{R})$. The connected component of the identity of $U_{n, \infty}$ is $U_{n, \infty}^{0}=U_{n, \infty} \cap G_{n}(\mathbb{R})^{+}$. 
We will write $\mathfrak{p}_{n}$ for the set of elements of Lie $G_{n}(\mathbb{R})$ of the form

$$
\left(0,\left(\left(\begin{array}{cc}
A_{\tau} & B_{\tau} \Psi_{n} \\
\Psi_{n} B_{\tau} & \Psi_{n} A_{\tau} \Psi_{n}
\end{array}\right)\right)_{\tau \in \operatorname{Hom}\left(F^{+}, \mathbb{R}\right)}\right),
$$

where ${ }^{c, t} A_{\tau}=A_{\tau}$ and ${ }^{c, t} B_{\tau}=B_{\tau}$ for all $\tau$. Then

$$
\text { Lie } G_{n}(\mathbb{R})=\mathfrak{p}_{n} \oplus \operatorname{Lie}\left(U_{n, \infty} A_{n}(\mathbb{R})\right) \text {. }
$$

We give the real vector space $\mathfrak{p}_{n}$ a complex structure by letting $i$ act by

$$
i_{0}:\left(A_{\tau}, B_{\tau}\right)_{\tau \in \operatorname{Hom}\left(F^{+}, \mathbb{R}\right)} \longmapsto\left(B_{\tau},-A_{\tau}\right)_{\tau \in \operatorname{Hom}\left(F^{+}, \mathbb{R}\right)} .
$$

We decompose

$$
\mathfrak{p}_{n} \otimes_{\mathbb{R}} \mathbb{C}=\mathfrak{p}_{n}^{+} \oplus \mathfrak{p}_{n}^{-}
$$

by setting

$$
\mathfrak{p}_{n}^{ \pm}=\left(\mathfrak{p}_{n} \otimes_{\mathbb{R}} \mathbb{C}\right)^{i_{0} \otimes 1= \pm 1 \otimes i}
$$

We also set

$$
\mathfrak{q}_{n}=\mathfrak{p}_{n}^{-} \oplus \operatorname{Lie}\left(U_{n, \infty} A_{n}(\mathbb{R})\right) \otimes_{\mathbb{R}} \mathbb{C} .
$$

It is a parabolic subalgebra of $\left(\right.$ Lie $\left.G_{n}(\mathbb{R})\right) \otimes_{\mathbb{R}} \mathbb{C}$ with unipotent radical $\mathfrak{p}_{n}^{-}$and Levi component Lie $\left(U_{n, \infty} A_{n}(\mathbb{R})\right) \otimes_{\mathbb{R}} \mathbb{C}$. We will write $\mathfrak{Q}_{n}$ for the parabolic subgroup of $G_{n} \times_{\mathbb{Q}} \mathbb{C}$ with Lie algebra $\mathfrak{q}_{n}$. Note that

$$
\mathfrak{Q}_{n}(\mathbb{C}) \cap G_{n}(\mathbb{R})=U_{n, \infty}^{0} A_{n}(\mathbb{R})^{0} .
$$

Let $\mathfrak{H}_{n}^{+}\left(\right.$resp. $\left.\mathfrak{H}_{n}^{ \pm}\right)$denote the set of $I$ in $G_{n}(\mathbb{R})$ with multiplier 1 such that $I^{2}=-1_{2 n}$ and such that the symmetric bilinear form $\langle I, \quad\rangle_{n}$ on $\Lambda_{n} \otimes_{\mathbb{Z}} \mathbb{R}$ is positive definite (respectively positive or negative definite). Then $G_{n}(\mathbb{R})$ (resp. $G_{n}(\mathbb{R})^{+}$) acts transitively on $\mathfrak{H}_{n}^{ \pm}$(resp. $\mathfrak{H}_{n}^{+}$) by conjugation. Moreover $J_{n} \in \mathfrak{H}_{n}^{+}$has stabilizer $U_{n, \infty}^{0} A_{n}(\mathbb{R})^{0}$ and so we get an identification of $\mathfrak{H}_{n}^{ \pm}\left(\right.$resp. $\left.\mathfrak{H}_{n}^{+}\right)$with $G_{n}(\mathbb{R}) / U_{n, \infty}^{0} A_{n}(\mathbb{R})^{0}\left(\right.$ resp. $\left.G_{n}(\mathbb{R})^{+} / U_{n, \infty}^{0} A_{n}(\mathbb{R})^{0}\right)$. The natural map

$$
\mathfrak{H}_{n}^{ \pm}=G_{n}(\mathbb{R}) / U_{n, \infty}^{0} A_{n}(\mathbb{R})^{0} \hookrightarrow G_{n}(\mathbb{C}) / \mathfrak{Q}_{n}(\mathbb{C})
$$

is an open embedding and gives $\mathfrak{H}_{n}^{ \pm}$the structure of a complex manifold. The action of $G_{n}(\mathbb{R})$ is holomorphic, and the complex structure induced on the tangent space $T_{J_{n}} \mathfrak{H}_{n}^{ \pm} \cong$ $\mathfrak{p}_{n}$ is the complex structure described in the previous paragraph.

If $\rho$ is a finite dimensional algebraic representation of $\mathfrak{Q}_{n}$ on a $\mathbb{C}$-vector space $W_{\rho}$, then there is a holomorphic vector bundle $\mathfrak{E}_{\rho} / \mathfrak{H}_{n}^{ \pm}$together with a holomorphic action of $G_{n}(\mathbb{R})$, defined as the pull-back to $\mathfrak{H}^{ \pm}$of $\left(G_{n}(\mathbb{C}) \times W_{\rho}\right) / \mathfrak{Q}_{n}(\mathbb{C})$, where

- $h \in \mathfrak{Q}_{n}(\mathbb{C})$ sends $(g, w)$ to $\left(g h, h^{-1} w\right)$,

- and where $h \in G_{n}(\mathbb{R})$ sends $[(g, w)]$ to $[(h g, w)]$. 
If $N_{2} \geq N_{1} \geq 0$ are integers we will write $U_{p}\left(N_{1}, N_{2}\right)_{n}$ for the subgroup of $G_{n}\left(\mathbb{Z}_{p}\right)$ consisting of elements whose reduction modulo $p^{N_{2}}$ preserves

$$
\Lambda_{n,(n)} \otimes_{\mathbb{Z}}\left(\mathbb{Z} / p^{N_{2}} \mathbb{Z}\right) \subset \Lambda_{n} \otimes_{\mathbb{Z}}\left(\mathbb{Z} / p^{N_{2}} \mathbb{Z}\right)
$$

and acts trivially on $\Lambda_{n} /\left(\Lambda_{n,(n)}+p^{N_{1}} \Lambda_{n}\right)$. If $N_{2} \geq N_{1} \geq N_{1}^{\prime} \geq 0$ then $U_{p}\left(N_{1}, N_{2}\right)_{n}$ is a normal subgroup of $U_{p}\left(N_{1}^{\prime}, N_{2}\right)_{n}$ and

$$
U_{p}\left(N_{1}^{\prime}, N_{2}\right)_{n} / U_{p}\left(N_{1}, N_{2}\right)_{n} \cong \operatorname{ker}\left(G L_{n}\left(\mathcal{O}_{F} / p^{N_{1}}\right) \rightarrow G L_{n}\left(\mathcal{O}_{F} / p^{N_{1}^{\prime}}\right)\right)
$$

We will also set

$$
\begin{aligned}
U_{p}\left(N_{1}, N_{2}\right)_{n}^{(m)} & =U_{p}\left(N_{1}, N_{2}\right)_{n} \ltimes \operatorname{Hom}_{\mathcal{O}_{F, p}}\left(\mathcal{O}_{F, p}^{m}, \Lambda_{n,(n)}+p^{N_{1}} \Lambda_{n}\right) \\
& \subset G_{n}^{(m)}\left(\mathbb{Z}_{p}\right)
\end{aligned}
$$

and set $\widetilde{U}_{p}\left(N_{1}, N_{2}\right)_{n}^{(m)}$ to be the pre-image of $U_{p}\left(N_{1}, N_{2}\right)_{n}^{(m)}$ in $\widetilde{G}_{n}^{(m)}\left(\mathbb{Z}_{p}\right)$. Pictorially we can think of $U_{p}\left(N_{1}, N_{2}\right)_{n}$ as

$$
\left(\begin{array}{c|c}
\mu 1_{n} \bmod p^{N_{1}} & * \\
\hline 0 \bmod p^{N_{2}} & 1_{n} \bmod p^{N_{1}}
\end{array}\right)
$$

and of $U_{p}\left(N_{1}, N_{2}\right)_{n}^{(m)}$ as

$$
\left(\begin{array}{c|c}
\mu 1_{n} \bmod p^{N_{1}} & * \\
\hline 0 \bmod p^{N_{2}} & 1_{n} \bmod p^{N_{1}}
\end{array}\right)\left(\begin{array}{c}
* \\
\hline 0 \bmod p^{N_{1}}
\end{array}\right)
$$

If $U^{p}$ is an open compact subgroup of $G_{n}\left(\mathbb{A}^{\infty, p}\right)\left(\right.$ resp. of $G_{n}^{(m)}\left(\mathbb{A}^{\infty, p}\right)$, resp. of $\left.\widetilde{G}_{n}^{(m)}\left(\mathbb{A}^{\infty, p}\right)\right)$ we will set $U^{p}\left(N_{1}, N_{2}\right)$ to be $U^{p} \times U_{p}\left(N_{1}, N_{2}\right)_{n}$ (resp. $U^{p} \times U_{p}\left(N_{1}, N_{2}\right)_{n}^{(m)}$, resp. $U^{p} \times$ $\left.\widetilde{U}_{p}\left(N_{1}, N_{2}\right)_{n}^{(m)}\right)$, a compact open subgroup of $G_{n}\left(\mathbb{A}^{\infty}\right)\left(\right.$ resp. $G_{n}^{(m)}\left(\mathbb{A}^{\infty}\right)$, resp. $\left.\widetilde{G}_{n}^{(m)}\left(\mathbb{A}^{\infty}\right)\right)$.

\subsection{Maximal parabolic subgroups}

We will write $P_{n,(i)}^{+}$(resp. $P_{n,(i)}^{(m),+}$, resp. $\widetilde{P}_{n,(i)}^{(m),+}$ ) for the subgroup of $G_{n}$ (resp. $G_{n}^{(m)}$, resp. $\widetilde{G}_{n}^{(m)}$ ) consisting of elements which (after projection to $G_{n}$ ) take $\Lambda_{n,(i)}$ to itself. We will also write $N_{n,(i)}^{+}\left(\right.$resp. $N_{n,(i)}^{(m),+}$, resp. $\widetilde{N}_{n,(i)}^{(m),+}$ ) for the subgroups of $P_{n,(i)}^{+}$(resp. $P_{n,(i)}^{(m),+}$, resp. $\widetilde{P}_{n,(i)}^{(m),+}$ ) consisting of elements which act trivially on $\Lambda_{n,(i)}$ and $\Lambda_{n,(i)}^{\perp} / \Lambda_{n,(i)}$ and $\Lambda_{n} / \Lambda_{n,(i)}^{\perp}$. Over $\mathbb{Q}$ the groups $P_{n,(i)}^{+}$(resp. $P_{n,(i)}^{(m),+}$, resp. $\widetilde{P}_{n,(i)}^{(m),+}$ ) are maximal parabolic subgroups of $G_{n}\left(\right.$ resp. $G_{n}^{(m)}$, resp. $\left.\widetilde{G}_{n}^{(m)}\right)$ containing the pre-image of $B_{n}$. The groups $N_{n,(i)}^{+}\left(\operatorname{resp} . N_{n,(i)}^{(m),+}\right.$, resp. $\widetilde{N}_{n,(i)}^{(m),+}$ ) are their unipotent radicals.

In some instances it will be useful to replace these groups by their 'hermitian part'. We will write $P_{n,(i)}$ for the normal subgroup of $P_{n,(i)}^{+}$consisting of elements which act trivially on $\Lambda_{n} / \Lambda_{n,(i)}^{\perp}$. We will also write $P_{n,(i)}^{(m)}$ for the normal subgroup

$$
P_{n,(i)} \ltimes \operatorname{Hom}_{\mathcal{O}_{F}}\left(\mathcal{O}_{F}^{m}, \Lambda_{n,(i)}^{\perp}\right)
$$

of $P_{n,(i)}^{(m),+}$, and $\widetilde{P}_{n,(i)}^{(m)}$ for the pre-image of $P_{n,(i)}^{(m)}$ in $\widetilde{P}_{n,(i)}^{(m),+}$. We will let

$$
N_{n,(i)}=N_{n,(i)}^{+}
$$


and

$$
N_{n,(i)}^{(m)}=N_{n,(i)}^{(m),+} \cap P_{n,(i)}^{(m)}
$$

and

$$
\widetilde{N}_{n,(i)}^{(m)}=\widetilde{N}_{n,(i)}^{(m),+} \cap \widetilde{P}_{n,(i)}^{(m)}
$$

Over $\mathbb{Q}$ these are the unipotent radicals of $P_{n,(i)}\left(\operatorname{resp} . P_{n,(i)}^{(m)}\right.$, resp. $\left.\widetilde{P}_{n,(i)}^{(m)}\right)$.

Pictorially one can think of $P_{n,(i)}^{+}$and $P_{n,(i)}$ as matrices of the following shapes

$$
\left(\begin{array}{l|l|l}
* & * & * \\
\hline 0 & * & * \\
\hline 0 & 0 & *
\end{array}\right)
$$

and

$$
\left(\begin{array}{c|c|c}
v(g) 1_{i} & * & * \\
\hline 0 & g & * \\
\hline 0 & 0 & 1_{i}
\end{array}\right)
$$

respectively. If we picture an element of $G_{n}^{(m)}$ as a pair of matrices

$$
()()
$$

(the first $2 n \times 2 n$ and the second $2 n \times m$ ) then we can picture $P_{n,(i)}^{(m),+}$ and $P_{n,(i)}^{(m)}$ as consisting of matrices of the shape

$$
\left(\begin{array}{c|c|c}
* & * & * \\
\hline 0 & * & * \\
\hline 0 & 0 & *
\end{array}\right)\left(\begin{array}{c}
* \\
\hline * \\
\hline *
\end{array}\right)
$$

and

$$
\left(\begin{array}{c|c|c}
v(g) 1_{i} & * & * \\
\hline 0 & g & * \\
\hline 0 & 0 & 1_{i}
\end{array}\right)\left(\begin{array}{c}
* \\
\hline * \\
\hline 0
\end{array}\right)
$$

respectively.

We have an isomorphism

$$
P_{n,(i)} \cong \widetilde{G}_{n-i}^{(i)}
$$

To describe it let $\Lambda_{n,(i)}^{\prime}$ denote the subspace of $\Lambda_{n}$ consisting of vectors with their first $2 n-i$ entries 0 , so that

$$
\Lambda_{n,(i)}^{\prime} \cong \mathcal{O}_{F}^{i}
$$

and

$$
\Lambda_{n-i} \cong \Lambda_{n,(i)}^{\perp} \cap\left(\Lambda_{n,(i)}^{\prime}\right)^{\perp} \stackrel{\sim}{\longrightarrow} \Lambda_{n,(i)}^{\perp} / \Lambda_{n,(i)} .
$$


We define

$$
G_{n-i} \hookrightarrow P_{n,(i)}
$$

by letting $g \in G_{n-i}$ act as $v(g)$ on $\Lambda_{n,(i)}$, as $g$ on $\Lambda_{n-i} \cong \Lambda_{n,(i)}^{\perp} \cap\left(\Lambda_{n,(i)}^{\prime}\right)^{\perp}$ and as 1 on $\Lambda_{n,(i)}^{\prime}$, i.e.

$$
g \longmapsto\left(\begin{array}{ccc}
v(g) 1_{i} & 0 & 0 \\
0 & g & 0 \\
0 & 0 & 1_{i}
\end{array}\right) \in P_{n,(i)}
$$

We define

$$
N_{n,(i)} \longrightarrow \operatorname{Hom}_{n-i}^{(i)}
$$

by sending $h$ to the map

$$
\mathcal{O}_{F}^{i} \cong \Lambda_{n,(i)}^{\prime} \stackrel{h-1_{2 n}}{\longrightarrow} \Lambda_{n,(i)}^{\perp} \rightarrow \Lambda_{n-i} .
$$

We also define

$$
Z\left(N_{n,(i)}\right) \stackrel{\sim}{\longrightarrow} \operatorname{Herm}_{\Lambda_{n,(i)}^{\prime}} \cong \operatorname{Herm}^{(i)}
$$

by sending $z$ to the pairing

$$
(x, y)_{z}=\left\langle\left(z-1_{2 n}\right) x, y\right\rangle_{n}
$$

on $\Lambda_{n,(i)}^{\prime}$. In the other direction $(f, z) \in N_{n-i}^{(i)}$ is mapped to

$$
\left(\begin{array}{ccc}
1_{i} & \Psi_{i}^{c, t} f J_{n-i} & \Psi_{i}^{t}\left(z-\frac{1}{2}^{t} f J_{n-i}{ }^{c} f\right) \\
0 & 1_{2(n-i)} & f \\
0 & 0 & 1_{i}
\end{array}\right) \in N_{n,(i)},
$$

where we think of $f \in M_{2(n-i) \times i}(F)$ with first $n-i$ rows in $\left(\mathcal{D}_{F}^{-1}\right)^{i}$ and second $(n-i)$ rows in $\mathcal{O}_{F}^{i}$, and we think of $z \in M_{i \times i}(F)^{t=c}$.

We also have isomorphisms

$$
P_{n,(i)}^{(m)} \cong \widetilde{G}_{n-i}^{(i+m)} / \operatorname{Herm}^{(m)}
$$

and

$$
\widetilde{P}_{n,(i)}^{(m)} \cong \widetilde{G}_{n-i}^{(i+m)}
$$

We will describe the second of these isomorphisms. Suppose $f \in \operatorname{Hom}_{n-i}^{(i)}$ and $g \in$ $\operatorname{Hom}_{n-i}^{(m)}$. Also suppose that $z \in \frac{1}{2} \operatorname{Herm}^{(i)}$ and $w \in \frac{1}{2} \operatorname{Herm}^{(m)}$ and

$$
u \in \frac{1}{2} \operatorname{Hom}\left(\mathcal{O}_{F}^{i} \otimes_{\mathcal{O}_{F}, c} \mathcal{O}_{F}^{m}, \mathbb{Z}\right),
$$


so that

$$
((f, g),(z, u, w)) \in N_{n-i}^{(i+m)}
$$

Let $h(f, z)$ denote the element of $P_{n,(i)}$ corresponding to $(f, z) \in N_{n,(i)}$. Think of $g$ as a map

$$
g: \mathcal{O}_{F}^{m} \longrightarrow \Lambda_{n-i} \cong \Lambda_{n,(i)}^{\perp} \cap\left(\Lambda_{n,(i)}^{\prime}\right)^{\perp} \subset \Lambda_{n} .
$$

Define $j(f, g, u) \in \operatorname{Hom}\left(\mathcal{O}_{F}^{m}, \Lambda_{n,(i)}\right)$ by

$$
\langle y, j(f, g, u)(x)\rangle_{n}=1 / 2\langle f(y), g(x)\rangle_{n-i}-u(y \otimes x)
$$

for all $x \in \mathcal{O}_{F}^{m}$ and $y \in \Lambda_{n,(i)}^{\prime} \cong \mathcal{O}_{F}^{i}$. Then

$$
((f, g),(z, u, w)) \longmapsto h(f, z)(g+j(f, g, u), w) \in N_{n,(i)} \ltimes \tilde{N}_{n}^{(m)} .
$$

\section{Note that}

$$
Z\left(\widetilde{N}_{n,(i)}^{(m)}\right) \cong \operatorname{Herm}^{(i+m)}
$$

and that

$$
Z\left(N_{n,(i)}^{(m)}\right) \cong \operatorname{Herm}^{(i+m)} / \operatorname{Herm}^{(m)}
$$

Write $L_{n,(i) \text { lin }}$ for the subgroup of $P_{n,(i)}^{+}$consisting of elements with $v=1$ which preserve $\Lambda_{n,(i)}^{\prime} \subset \Lambda_{n}$ and act trivially on $\Lambda_{n,(i)}^{\perp} / \Lambda_{n,(i)}$. We set $N\left(L_{n,(i), \text { lin }}^{(m)}\right)$ to be the additive group scheme over $\mathbb{Z}$ associated to

$$
\operatorname{Hom}_{\mathcal{O}_{F}}\left(\mathcal{O}_{F}^{m}, \Lambda_{n,(i)}^{\prime}\right)
$$

and write $L_{n,(i), \text { lin }}^{(m)}$ for

$$
L_{n,(i), \text { lin }} \ltimes N\left(L_{n,(i), \text { lin }}^{(m)}\right) \subset P_{n,(i)}^{(m),+}
$$

and $\widetilde{L}_{n,(i), \text { lin }}^{(m)}$ for

$$
L_{n,(i), \operatorname{lin}} \ltimes N\left(L_{n,(i), \operatorname{lin}}^{(m)}\right) \subset \widetilde{P}_{n,(i)}^{(m),+} .
$$

Note that

$$
P_{n,(i)}^{+}=L_{n,(i), \text { lin }} \ltimes P_{n,(i)}
$$

and

$$
P_{n,(i)}^{(m),+}=L_{n,(i), \operatorname{lin}}^{(m)} \ltimes P_{n,(i)}^{(m)}
$$

and

$$
\widetilde{P}_{n,(i)}^{(m),+}=\widetilde{L}_{n,(i), \operatorname{lin}}^{(m)} \ltimes \widetilde{P}_{n,(i)}^{(m)} .
$$


Also note that

$$
L_{n,(i), \text { lin }} \cong \mathrm{RS}_{\mathbb{Z}}^{\mathcal{O}_{F}} G L_{i}
$$

via its action on $\Lambda_{n,(i)}^{\prime} \cong \mathcal{O}_{F}^{i}$, and that

$$
\widetilde{L}_{n,(i), \text { lin }}^{(m)} \stackrel{\sim}{\longrightarrow} L_{n,(i), \text { lin }}^{(m)} \cong\left(\mathrm{RS}_{\mathbb{Z}}^{\mathcal{O}_{F}} G L_{i}\right) \ltimes \operatorname{Hom}_{\mathcal{O}_{F}}\left(\mathcal{O}_{F}^{m}, \mathcal{O}_{F}^{i}\right) .
$$

Pictorially we can think of $L_{n,(i), \text { lin }}$ as consisting of matrices of the form

$$
\left(\begin{array}{c|c|c}
\Psi_{i}^{c, t} h^{-1} \Psi_{n} & 0 & 0 \\
\hline 0 & 1_{2(n-i)} & 0 \\
\hline 0 & 0 & h
\end{array}\right)
$$

and $L_{n,(i), \text { lin }}^{(m)}$ as consisting of matrices of the form

$$
\left(\begin{array}{c|c|c}
\Psi_{i}^{c, t} h^{-1} \Psi_{n} & 0 & 0 \\
\hline 0 & 1_{2(n-i)} & 0 \\
\hline 0 & 0 & h
\end{array}\right)\left(\begin{array}{c}
0 \\
\hline 0 \\
\hline *
\end{array}\right) .
$$

We let $L_{n,(i) \text { herm }}$ denote the subgroup of $P_{n,(i)}$ consisting of elements which preserve $\Lambda_{n,(i)}^{\prime}$. Thus

$$
L_{n,(i), \text { herm }} \cong G_{n-i} .
$$

In particular

$$
v: L_{n,(n), \text { herm }} \stackrel{\sim}{\longrightarrow} \mathbb{G}_{m} .
$$

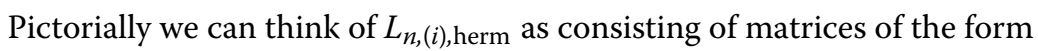

$$
\left(\begin{array}{c|c|c}
v(g) 1_{i} & 0 & 0 \\
\hline 0 & g & 0 \\
\hline 0 & 0 & 1_{i}
\end{array}\right)
$$

Over $\mathbb{Q}$ it is a Levi component for $P_{n,(i)}$ and $P_{n,(i)}^{(m)}$ and $\widetilde{P}_{n,(i)}^{(m)}$, so in particular

$$
P_{n,(i)}=L_{n,(i), \text { herm }} \ltimes N_{n,(i)}
$$

and

$$
P_{n,(i)}^{(m)}=L_{n,(i), \text { herm }} \ltimes N_{n,(i)}^{(m)}
$$

and

$$
\widetilde{P}_{n,(i)}^{(m)}=L_{n,(i), \text { herm }} \ltimes \widetilde{N}_{n,(i)}^{(m)} .
$$

We also set

$$
L_{n,(i)}=L_{n,(i), \text { herm }} \times L_{n,(i), \text { lin }}
$$


and

$$
L_{n,(i)}^{(m)}=L_{n,(i), \text { herm }} \times L_{n,(i), \text { lin }}^{(m)}
$$

and

$$
\widetilde{L}_{n,(i)}^{(m)}=L_{n,(i), \text { herm }} \times \widetilde{L}_{n,(i), \text { lin }}^{(m)} .
$$

Over $\mathbb{Q}$ we see that $L_{n,(i)}$ is a Levi component for each of $P_{n,(i)}^{+}$and $P_{n,(i)}^{(m),+}$ and $\widetilde{P}_{n,(i)}^{(m),+}$. Moreover

$$
P_{n,(i)}^{+}=L_{n,(i)} \ltimes N_{n,(i)}
$$

and

$$
P_{n,(i)}^{(m)++}=L_{n,(i)}^{(m)} \ltimes N_{n,(i)}^{(m)}
$$

and

$$
\widetilde{P}_{n,(i)}^{(m),+}=\widetilde{L}_{n,(i)}^{(m)} \ltimes \widetilde{N}_{n,(i)}^{(m)}
$$

We will occasionally write $P_{n,(i)}^{(m),-}\left(\right.$ resp. $L_{n,(i), \text { herm }}^{-}$) for the kernel of the map $P_{n,(i)}^{(m)} \rightarrow C_{n-i}$

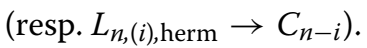

We will write $R_{n,(n),(i)}$ for the subgroup of $L_{n,(n)}$ mapping $\Lambda_{n,(i)}$ to itself. We will write $N\left(R_{n,(n),(i)}\right)$ for the subgroup of $R_{n,(n),(i)}$ which acts trivially on $\Lambda_{n,(i)}$ and $\Lambda_{n,(i)}^{\perp} / \Lambda_{n,(i)}$ and $\Lambda_{n} / \Lambda_{n,(i)}^{\perp}$.

We will also write $R_{n,(n)}^{(m)}$ for the semi-direct product

$$
L_{n,(n)} \ltimes \operatorname{Hom}_{\mathcal{O}_{F}}\left(\mathcal{O}_{F}^{m}, \Lambda_{n,(n)}\right) .
$$

If $m^{\prime} \leq m$ we will fix $\mathbb{Z}^{m} \rightarrow \mathbb{Z}^{m-m^{\prime}}$ to be projection onto the last $m-m^{\prime}$ coordinates and define $Q_{m,\left(m^{\prime}\right)}$ for the subgroup of $G L_{m}$ consisting of elements preserving the kernel of this map. We also define $Q_{m,\left(m^{\prime}\right)}^{\prime}$ to be the subgroup of $Q_{m,\left(m^{\prime}\right)}$ consisting of elements which induce $1_{\mathbb{Z}^{m-m^{\prime}}}$ on $\mathbb{Z}^{m-m^{\prime}}$. Thus there is an exact sequence

$$
(0) \longrightarrow \operatorname{Hom}\left(\mathbb{Z}^{m-m^{\prime}}, \mathbb{Z}^{m^{\prime}}\right) \longrightarrow Q_{m,\left(m^{\prime}\right)}^{\prime} \longrightarrow G L_{m^{\prime}} \longrightarrow\{1\}
$$

\section{Moreover}

$$
\widetilde{L}_{n,(i), \text { lin }}^{(m)} \cong L_{n,(i), \text { lin }}^{(m)} \cong \mathrm{RS}_{\mathbb{Z}}^{\mathcal{O}_{F}} Q_{m+i,(i)}^{\prime}
$$

We will also write $A_{n,(i), \text { lin }}$ (resp. $A_{n,(i) \text {,herm }}$ ) for the image of the map from $\mathbb{G}_{m}$ to $L_{n,(i) \text {,lin }}$ (resp. $L_{n,(i), \text { herm }}$ ) sending $t$ to $t 1_{i}$ (resp. $\left.\left(t^{2}, t 1_{2(n-i)}\right)\right)$. Moreover write $A_{n,(i)}$ for $A_{n,(i), \text { lin }} \times$ $A_{n,(i), \text { herm }}$. Over $\mathbb{Q}$ the group $A_{n,(i)}\left(\right.$ resp. $A_{n,(i), \text { lin }}$, resp. $\left.A_{n,(i), \text { herm }}\right)$ is the maximal split torus

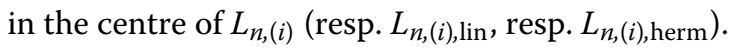

Again suppose that $\Omega$ is an algebraically closed field of characteristic 0 . Let $\Phi_{(n)} \subset \Phi_{n}$ denote the set of roots of $T_{n}$ on Lie $L_{n,(n)}$, and set $\Phi_{(n)}^{+}=\Phi_{n}^{+} \cap \Phi_{(n)}$ and $\Delta_{(n)}=\Delta_{n} \cap \Phi_{(n)}$. We will write $\varrho_{n,(n)}$ for half the sum of the elements of $\Phi_{(n)}^{+}$. If $R \subset \mathbb{R}$ then $X^{*}\left(T_{n, / \Omega}\right)_{(n), R}^{+}$ 
will denote the subset of $X^{*}\left(T_{n, / \Omega}\right)_{R}$ consisting of elements which pair non-negatively with the coroot $\check{\alpha} \in X_{*}\left(T_{n, / \Omega}\right)$ corresponding to each $\alpha \in \Delta_{(n)}$. We write $X^{*}\left(T_{n, / \Omega}\right)_{(n)}^{+}$for $X^{*}\left(T_{n, / \Omega}\right)_{(n), \mathbb{Z}^{*}}^{+}$If $\lambda \in X^{*}\left(T_{n, / \Omega}\right)_{(n)}^{+}$we will let $\rho_{(n), \lambda}$ denote the irreducible representation of $L_{n,(n)}$ with highest weight $\lambda$. When $\rho_{(n), \lambda}$ is used as a subscript we will sometimes replace it by just (n), $\lambda$.

Note that Lie $P_{n,(n)}(\mathbb{C})$ and $\mathfrak{q}_{n}$ are conjugate under $G_{n}(\mathbb{C})$ and hence we obtain an identification ('Cayley transform') of $\left(\operatorname{Lie} U_{n, \infty} A_{n}(\mathbb{R})\right) \otimes_{\mathbb{R}} \mathbb{C}$ and Lie $L_{n,(n)}(\mathbb{C})$, which is well defined up to conjugation by $L_{n,(n)}(\mathbb{C})$. Similarly $\mathfrak{Q}_{n}$ and $P_{n,(n)}(\mathbb{C})$ are conjugate in $G_{n} \times \mathbb{Q} \mathbb{C}$. Thus $L_{n,(n)}(\mathbb{C})$ can be identified with $\mathfrak{Q}_{n}$ modulo its unipotent radical, canonically up to $L_{n,(n)}(\mathbb{C})$-conjugation. Thus if $\rho$ is a finite dimensional algebraic representation of $L_{n,(n)}$ over $\mathbb{C}$, we can associate to it a representation of $\mathfrak{Q}_{n}$ and of $\mathfrak{q}_{n}$, and hence a holomorphic vector bundle $\mathfrak{E}_{\rho} / \mathfrak{H}_{n}^{ \pm}$with $G_{n}(\mathbb{R})$-action.

The isomorphism $L_{n,(n)} \cong G L_{1} \times \mathrm{RS}_{\mathbb{Z}}^{\mathcal{O}_{F}} G L_{n}$ gives rise to a natural identification

$$
L_{n,(n)} \times \operatorname{Spec} \Omega \cong G L_{1} \times G L_{n}^{\operatorname{Hom}(F, \Omega)},
$$

and hence to identifications

$$
T_{n} \times \operatorname{Spec} \Omega \cong G L_{1} \times\left(G L_{1}^{n}\right)^{\operatorname{Hom}(F, \Omega)}
$$

and

$$
X^{*}\left(T_{n, / \Omega}\right) \cong \mathbb{Z} \oplus\left(\mathbb{Z}^{n}\right)^{\operatorname{Hom}(F, \Omega)}
$$

Under this identification $X^{*}\left(T_{n, / \Omega}\right)_{(n)}^{+}$is identified to the set of

$$
\left(b_{0},\left(b_{\tau, i}\right)\right) \in \mathbb{Z} \oplus\left(\mathbb{Z}^{n}\right)^{\operatorname{Hom}(F, \Omega)}
$$

with

$$
b_{\tau, 1} \geq b_{\tau, 2} \geq \cdots \geq b_{\tau, n}
$$

for all $\tau$.

To compare this parametrization of $X^{*}\left(T_{n, / \Omega}\right)$ with the one introduced in Sect. 1.1 note that the map

$$
G L_{1} \times G L_{n}^{\operatorname{Hom}(F, \Omega)} \hookrightarrow\left\{\left(\mu, g_{\tau}\right) \in \mathbb{G}_{m} \times G L_{2 n}^{\operatorname{Hom}(F, \Omega)}: g_{\tau c}=\mu J_{n}{ }^{t} g_{\tau}^{-1} J_{n} \forall \tau\right\}
$$

coming from $L_{n,(n)} \hookrightarrow G_{n}$ sends

$$
\left(\mu,\left(g_{\tau}\right)_{\tau \in \operatorname{Hom}(F, \Omega)}\right) \longmapsto\left(\mu,\left(\left(\begin{array}{cc}
\mu \Psi_{n}^{t} g_{\tau c}^{-1} \Psi_{n} & 0 \\
0 & g_{\tau}
\end{array}\right)\right)_{\tau \in \operatorname{Hom}(F, \Omega)}\right)
$$

Thus the map

$$
\mathbb{Z} \oplus\left(\mathbb{Z}^{2 n}\right)^{\operatorname{Hom}(F, \Omega)} \rightarrow X^{*}\left(T_{n, / \Omega}\right) \cong \mathbb{Z} \oplus\left(\mathbb{Z}^{n}\right)^{\operatorname{Hom}(F, \Omega)}
$$


sends

$$
\left(a_{0},\left(a_{\tau, i}\right)_{\tau \in \operatorname{Hom}(F, \Omega) ; i=1, \ldots, 2 n}\right) \longmapsto\left(a_{0}+\sum_{\tau} \sum_{j=1}^{n} a_{\tau, j},\left(a_{\tau, n+i}-a_{\tau c, n+1-i}\right)_{\tau, i}\right) .
$$

A section is provided by the map

$$
\left(b_{0},\left(b_{\tau, i}\right)\right) \longmapsto\left(b_{0},\left(0, \ldots, 0, b_{\tau, 1}, \ldots, b_{\tau, n}\right)_{\tau}\right) .
$$

In particular we see that $X^{*}\left(T_{n, / \Omega}\right)^{+} \subset X^{*}\left(T_{n, / \Omega}\right)_{(n)}^{+}$is identified with the set of

$$
\left(b_{0},\left(b_{\tau, i}\right)\right) \in \mathbb{Z} \oplus\left(\mathbb{Z}^{n}\right)^{\operatorname{Hom}(F, \Omega)}
$$

with

$$
b_{\tau, 1} \geq b_{\tau, 2} \geq \cdots \geq b_{\tau, n}
$$

and

$$
b_{\tau, 1}+b_{\tau c, 1} \leq 0
$$

for all $\tau$.

Note that

$$
2\left(\varrho_{n}-\varrho_{n,(n)}\right)=\left(n^{2}\left[F^{+}: \mathbb{Q}\right],(-n)_{\tau, i}\right) .
$$

We write Std for the representation of $L_{n,(n)}$ on $\Lambda_{n} / \Lambda_{n,(n)}$ over $\mathbb{Z}$, and if $\tau: F \hookrightarrow \overline{\mathbb{Q}}$ we write $\operatorname{Std}_{\tau}$ for the representation of $L_{n,(n)}$ on $\left(\Lambda_{n} / \Lambda_{n,(n)}\right) \otimes_{\mathcal{O}_{F}, \tau} \mathcal{O}_{\overline{\mathbb{Q}}}$. If $\Omega$ is an algebraically closed field of characteristic 0 and if $\tau: F \hookrightarrow \Omega$ we will sometimes write $\operatorname{Std}_{\tau}$ for the representation of $L_{n,(n)}$ on $\left(\Lambda_{n} / \Lambda_{n,(n)}\right) \otimes_{\mathcal{O}_{F}, \tau} \Omega$. We hope that context will make clear the distinction between these two slightly different meaning of $\operatorname{Std}_{\tau}$. We also let KS denote the representation

$$
S\left(\operatorname{Std}^{\vee}\right) \otimes v
$$

of $L_{n,(n)}$ over $\mathbb{Z}$. (See Sect. 1.1.) Note that over $\overline{\mathbb{Q}}$ the representation $\operatorname{Std}_{\tau}^{\vee}$ is irreducible and in our normalizations has highest weight $\left(0, b_{\tau^{\prime}}\right)$ where

$$
b_{\tau}=(0, \ldots, 0,-1)
$$

but $b_{\tau^{\prime}}=0$ for $\tau^{\prime} \neq \tau$. Similarly the representation $\wedge^{n[F: \mathbb{Q}]} \operatorname{Std}^{\vee}$ is irreducible with highest weight

$$
\left(0,(-1, \ldots,-1)_{\tau}\right) .
$$

Finally KS is the direct sum of the $\left[F^{+}: \mathbb{Q}\right]$ irreducible representations indexed by $\tau \in$ $\operatorname{Hom}\left(F^{+}, \overline{\mathbb{Q}}\right)$ with highest weights $\left(1, b_{\tau^{\prime}}\right)$, where

$$
b_{\tau^{\prime}}=(0, \ldots, 0,-1)
$$


if $\tau^{\prime}$ extends $\tau$, and $b_{\tau^{\prime}}=0$ otherwise.

We will let $\varsigma_{p} \in L_{n,(n), \text { herm }}\left(\mathbb{Q}_{p}\right) \cong \mathbb{Q}_{p}^{\times}$denote the unique element with multiplier $p^{-1}$. Set

$$
U_{p}(N)_{n,(i)}=\operatorname{ker}\left(L_{n,(i), \operatorname{lin}}\left(\mathbb{Z}_{p}\right) \rightarrow L_{n,(i), \operatorname{lin}}\left(\mathbb{Z} / p^{N} \mathbb{Z}\right)\right)
$$

and

$$
U_{p}(N)_{n,(i)}^{(m)}=\operatorname{ker}\left(L_{n,(i), \operatorname{lin}}^{(m)}\left(\mathbb{Z}_{p}\right) \rightarrow L_{n,(i), \operatorname{lin}}^{(m)}\left(\mathbb{Z} / p^{N} \mathbb{Z}\right)\right) .
$$

Also set

$$
U_{p}\left(N_{1}, N_{2}\right)_{n,(i)}^{(m)}=U_{p}\left(N_{1}, N_{2}\right)_{n-i} \times U_{p}\left(N_{1}\right)_{n,(i)}^{(m)} \subset L_{n,(i)}^{(m)}\left(\mathbb{Z}_{p}\right)
$$

and

$$
\widetilde{U}_{p}\left(N_{1}, N_{2}\right)_{n,(i)}^{(m),+}=U_{p}\left(N_{1}\right)_{n,(i)}^{(m)} \ltimes \widetilde{U}_{p}\left(N_{1}, N_{2}\right)_{n-i}^{(m+i)} \subset \widetilde{P}_{n,(i)}^{(m),+}\left(\mathbb{Z}_{p}\right)
$$

and

$$
U_{p}\left(N_{1}, N_{2}\right)_{n,(i)}^{(m),+}=\widetilde{U}_{p}\left(N_{1}, N_{2}\right)_{n,(i)}^{(m),+} / \operatorname{Herm}_{\mathcal{O}_{F, p}^{m}} \subset P_{n,(i)}^{(m),+}\left(\mathbb{Z}_{p}\right) .
$$

Let $U^{p}$ be an open compact subgroup of $L_{n,(i)}\left(\mathbb{A}^{\infty, p}\right)\left(\operatorname{resp} \cdot L_{n,(i), \text { lin }}^{(m)}\left(\mathbb{A}^{\infty, p}\right)\right.$, resp. $L_{n,(i)}^{(m)}\left(\mathbb{A}^{\infty, p}\right)$, resp. $\left(P_{n,(i)}^{(m),+} / Z\left(N_{n,(i)}^{(m)}\right)\right)\left(\mathbb{A}^{\infty, p}\right)$, resp. $P_{n,(i)}^{(m),+}\left(\mathbb{A}^{\infty, p}\right)$, resp. $\left.\widetilde{P}_{n,(i)}^{(m),+}\left(\mathbb{A}^{\infty, p}\right)\right)$. Then set

$$
U^{p}\left(N_{1}, N_{2}\right)=U^{p} \times U_{p}\left(N_{1}, N_{2}\right)_{n,(i)} \subset L_{n,(i)}\left(\mathbb{A}^{\infty}\right)
$$

(resp.

$$
U^{p}(N)=U^{p} \times U_{p}(N)_{n,(i)}
$$

resp.

$$
U^{p}\left(N_{1}, N_{2}\right)=U^{p} \times U_{p}\left(N_{1}, N_{2}\right)_{n,(i)}^{(m)} \subset L_{n,(i)}^{(m)}\left(\mathbb{A}^{\infty}\right),
$$

resp.

$$
U^{p}\left(N_{1}, N_{2}\right)=U^{p} \times\left(U_{p}\left(N_{1}, N_{2}\right)_{n,(i)}^{(m),+} / Z\left(N_{n,(i)}^{(m)}\right)\left(\mathbb{Z}_{p}\right)\right) \subset P_{n,(i)}^{(m),+} / Z\left(N_{n,(i)}^{(m)}\right)\left(\mathbb{A}^{\infty}\right),
$$

resp.

$$
U^{p}\left(N_{1}, N_{2}\right)=U^{p} \times U_{p}\left(N_{1}, N_{2}\right)_{n,(i)}^{(m),+} \subset P_{n,(i)}^{(m),+}\left(\mathbb{A}^{\infty}\right),
$$

resp.

$$
\left.U^{p}\left(N_{1}, N_{2}\right)=U^{p} \times \widetilde{U}_{p}\left(N_{1}, N_{2}\right)_{n,(i)}^{(m),+} \subset \widetilde{P}_{n,(i)}^{(m),+}\left(\mathbb{A}^{\infty}\right)\right) .
$$

In the case $i=n$ these groups do not depend on $N_{2}$, so we will write simply $U^{p}\left(N_{1}\right)$. 
For the study of the ordinary locus we will need a variant of $G_{n}\left(\mathbb{A}^{\infty}\right)$ and $G_{n}^{(m)}\left(\mathbb{A}^{\infty}\right)$ and $\widetilde{G}_{n}^{(m)}\left(\mathbb{A}^{\infty}\right)$. More specifically define a semigroup

$$
\widetilde{G}_{n}^{(m)}\left(\mathbb{A}^{\infty}\right)^{\text {ord }}=\widetilde{G}_{n}^{(m)}\left(\mathbb{A}^{\infty, p}\right) \times\left(\varsigma_{p}^{\mathbb{Z}_{\geq 0}} \widetilde{P}_{n,(n)}^{(m),+}\left(\mathbb{Z}_{p}\right)\right)
$$

Its maximal sub-semigroup that is also a group is

$$
\widetilde{G}_{n}^{(m)}\left(\mathbb{A}^{\infty}\right)^{\mathrm{ord}, \times}=\widetilde{G}_{n}^{(m)}\left(\mathbb{A}^{\infty, p}\right) \times \widetilde{P}_{n,(n)}^{(m),+}\left(\mathbb{Z}_{p}\right)
$$

If $H$ is an algebraic subgroup of $\widetilde{G}_{n}^{(m)}$ (over Spec $\mathbb{Q}$ ) we set

$$
H\left(\mathbb{A}^{\infty}\right)^{\text {ord }}=H\left(\mathbb{A}^{\infty}\right) \cap \widetilde{G}_{n}^{(m)}\left(\mathbb{A}^{\infty}\right)^{\text {ord }}
$$

Its maximal sub-semigroup that is also a group is

$$
H\left(\mathbb{A}^{\infty}\right)^{\text {ord }, \times}=H\left(\mathbb{A}^{\infty}\right) \cap \widetilde{G}_{n}^{(m)}\left(\mathbb{A}^{\infty}\right)^{\text {ord }, \times} .
$$

Thus

$$
G_{n}\left(\mathbb{A}^{\infty}\right)^{\text {ord }, \times}=G_{n}\left(\mathbb{A}^{\infty, p}\right) \times P_{n,(n)}^{+}\left(\mathbb{Z}_{p}\right)
$$

and

$$
G_{n}^{(m)}\left(\mathbb{A}^{\infty}\right)^{\text {ord }, \times}=G_{n}^{(m)}\left(\mathbb{A}^{\infty, p}\right) \times P_{n,(n)}^{(m),+}\left(\mathbb{Z}_{p}\right)
$$

If $U^{p}$ is an open compact subgroup of $H\left(\mathbb{A}^{\infty, p}\right)$, we set

$$
U^{p}(N)=H\left(\mathbb{A}^{\infty}\right)^{\mathrm{ord}, \times} \cap\left(U^{p} \times \widetilde{U}_{p}\left(N, N^{\prime}\right)_{n,(n)}^{(m),+}\right)
$$

for any $N^{\prime} \geq N$. The group does not depend on the choice of $N^{\prime}$.

\subsection{Base change}

We will write $B_{G L_{m}}$ for the subgroup of upper triangular elements of $G L_{m}$ and $T_{G L_{m}}$ for the subgroup of diagonal elements of $B_{G L_{m}}$.

We will also let $G_{n}^{1}$ denote the group scheme over $\mathcal{O}_{F^{+}}$defined by

$$
G_{n}^{1}(R)=\left\{g \in \operatorname{Aut}\left(\left(\Lambda_{n} \otimes \mathcal{O}_{F^{+}} R\right) /\left(\mathcal{O}_{F} \otimes_{\mathcal{O}_{F^{+}}} R\right)\right):{ }^{t} g J_{n}{ }^{c} g=J_{n}\right\}
$$

Thus

$$
\text { ker } v \cong \mathrm{RS}_{\mathbb{Z}}^{\mathcal{O}^{+}} G_{n}^{1}
$$

We will write $B_{n}^{1}$ for the subgroup of $G_{n}^{1}$ consisting of upper triangular matrices and $T_{n}^{1}$ for the subgroup of $B_{n}^{1}$ consisting of diagonal matrices. There is a natural projection $B_{n}^{1} \rightarrow T_{n}^{1}$ obtained by setting the off diagonal entries of an element of $B_{n}^{1}$ to 0 . 
Suppose that $q$ is a rational prime. Let $u_{1}, \ldots, u_{r}$ denote the primes of $F^{+}$above $q$ which split $u_{i}=w_{i}{ }^{c} w_{i}$ in $F$ and let $v_{1}, \ldots, v_{s}$ denote the primes of $F^{+}$above $q$ which do not split in $F$. Then

$$
G_{n}\left(\mathbb{Q}_{q}\right) \cong \prod_{i=1}^{r} G L_{2 n}\left(F_{w_{i}}\right) \times H
$$

where

$$
H=\left\{\left(\mu, g_{i}\right) \in \mathbb{Q}_{q}^{\times} \times \prod_{i=1}^{s} G L_{2 n}\left(F_{v_{i}}\right):{ }^{t} g_{i} J_{n}{ }^{c} g_{i}=\mu J_{n} \forall i\right\} \supset \prod_{i=1}^{s} G_{n}^{1}\left(F_{v_{i}}^{+}\right) .
$$

If $\Pi$ is an irreducible smooth representation of $G_{n}\left(\mathbb{Q}_{q}\right)$ then

$$
\Pi=\left(\bigotimes_{i=1}^{r} \Pi_{w_{i}}\right) \otimes \Pi_{H}
$$

We define $\mathrm{BC}(\Pi)_{w_{i}}=\Pi_{w_{i}}$ and $\mathrm{BC}(\Pi)_{c w_{i}}=\Pi_{w_{i}}^{\vee, c}$. Note that this does not depend on the choice of primes $w_{i} \mid u_{i}$. We will say that $\Pi$ is unramified at $w_{i}$ if $\mathrm{BC}(\Pi)_{w_{i}}^{G L_{2 n}\left(\mathcal{O}_{F, w_{i}}\right)} \neq(0)$. We will say that $\Pi$ is unramified at $v_{i}$ if $v_{i}$ is unramified in $F$ and

$$
\Pi^{G_{n}^{1}\left(\mathcal{O}_{F^{+}, v_{i}}\right)} \neq(0)
$$

We will say that $\Pi$ is unramified at $q$ if $\Pi$ is unramified at all primes above $q$ and either $q$ splits in $F_{0}$ or $q$ is unramified in $F$.

Suppose that $\Pi$ is unramified at $v_{i}$. Then there is a character $\chi$ of the quotient $T_{n}^{1}\left(F_{v_{i}}^{+}\right) / T_{n}^{1}\left(\mathcal{O}_{F^{+}, v_{i}}\right)$ such that $\left.\Pi\right|_{G_{n}^{1}\left(F_{v_{i}}^{+}\right)}$and n-Ind ${ }_{B_{n}^{1}\left(F_{v_{i}}^{+}\right)}^{G_{1}^{1}\left(F^{+}\right)} \chi$ share an irreducible subquotient with a $G_{n}^{1}\left(\mathcal{O}_{F^{+}, v_{i}}\right)$-fixed vector. Moreover this character $\chi$ is unique modulo the action of the normalizer $N_{G_{n}^{1}\left(F_{v_{i}}^{+}\right)}\left(T_{n}^{1}\left(F_{v_{i}}^{+}\right)\right) / T_{n}^{1}\left(F_{v_{i}}^{+}\right)$. (If $\pi$ and $\pi^{\prime}$ are two irreducible subquotients of $\left.\Pi_{H}\right|_{G_{n}^{1}\left(F_{v_{i}}^{+}\right)}$then we must have $\pi^{\prime} \cong \pi^{S_{v_{i}}^{-1}}$ where

$$
\varsigma_{v_{i}}=\left(\begin{array}{cc}
\varpi_{v_{i}}^{-1} 1_{n} & 0 \\
0 & 1_{n}
\end{array}\right) \in G L_{2 n}\left(F_{v_{i}}\right)
$$

However

$$
\left.\left(\mathrm{n}-\operatorname{Ind}_{B_{n}^{1}\left(F_{v_{i}}^{+}\right)}^{G_{1}^{1}\left(F_{\nu^{+}}^{+}\right)} \chi\right)^{\zeta_{v}^{-1}} \cong \mathrm{n}-\operatorname{Ind}_{B_{n}^{1}\left(F_{v_{i}}^{+}\right)}^{G_{1}^{1}\left(F_{F^{+}}^{+}\right)} \chi \cdot\right)
$$

Let

$$
\begin{aligned}
\mathbf{N}: T_{G L_{2 n}}\left(F_{v_{i}}\right) & \longrightarrow T_{n}^{1}\left(F_{v_{i}}^{+}\right) \\
\operatorname{diag}\left(t_{1}, \ldots, t_{2 n}\right) & \longmapsto \operatorname{diag}\left(t_{1} /{ }^{c} t_{2 n}, \ldots, t_{2 n} /{ }^{c} t_{1}\right) .
\end{aligned}
$$

Then we define $\mathrm{BC}(\Pi)_{v_{i}}$ to be the unique subquotient of

$$
\mathrm{n}-\operatorname{Ind}_{B_{G L_{2 n}}\left(F_{v_{i}}\right)}^{G L_{2 n}\left(F_{v_{i}}\right)}(\chi \circ \mathbf{N})
$$

with a $G L_{2 n}\left(\mathcal{O}_{F, v_{i}}\right)$-fixed vector. The next lemma is easy to prove. 
Lemma 1.1 Suppose that $\psi \otimes \pi$ is an irreducible smooth representation of

$$
L_{n,(n)}\left(\mathbb{Q}_{q}\right) \cong L_{n,(n), \operatorname{herm}}\left(\mathbb{Q}_{q}\right) \times L_{n,(n), \operatorname{lin}}\left(\mathbb{Q}_{q}\right)=\mathbb{Q}_{q}^{\times} \times G L_{n}\left(F_{q}\right) .
$$

(1) If $v$ is unramified over $F^{+}$and $\pi_{v}$ is unramified then $\mathrm{n}-\operatorname{Ind}_{P_{n,(n)}\left(\mathbb{Q}_{q}\right)}^{G_{n}\left(\mathbb{Q}_{q}\right)}(\psi \otimes \pi)$ has a subquotient $\Pi$ which is unramified at $v$. Moreover $\mathrm{BC}(\Pi)_{v}$ is the unramified irreducible subquotient of $\mathrm{n}$-Ind $\operatorname{In}_{Q_{2 n}(n)}^{G L_{2 n}\left(F_{v}\right)}\left(F_{v}\right)\left(\pi_{v}^{\vee, c} \otimes \pi_{v}\right)$.

(2) If $v$ is split over $F^{+}$and $\Pi$ is an irreducible subquotient of the normalized induction $\mathrm{n}-\operatorname{Ind}_{P_{n,(n)}\left(\mathbb{Q}_{q}\right)}^{G_{n}\left(\mathbb{Q}_{2}\right)}(\psi \otimes \pi)$, then $\mathrm{BC}(\Pi)_{v}$ is an irreducible subquotient of $\mathrm{n}-\operatorname{Ind}_{Q_{2 n,(n)}\left(F_{v}\right)}^{G L_{2 n}\left(F_{v}\right)}\left(\left(\pi c_{v}\right)^{\vee, c} \otimes \pi_{v}\right)$.

Note that in both cases $\mathrm{BC}\left(\Pi_{v}\right)$ does not depend on $\psi$.

In this paragraph let $K$ be a number field, $m \in \mathbb{Z}_{>0}$, and write $U_{K, \infty}$ for a maximal compact subgroup of $G L_{m}\left(K_{\infty}\right)$. We shall (slightly abusively) refer to an admissible

$$
G_{n}\left(\mathbb{A}^{\infty}\right) \times\left(\left(\operatorname{Lie} G_{n}(\mathbb{R})\right)_{\mathbb{C}}, U_{n, \infty}\right)
$$

(resp.

$$
L_{n,(i)}\left(\mathbb{A}^{\infty}\right) \times\left(\left(\operatorname{Lie} L_{n,(i)}(\mathbb{R})\right)_{\mathbb{C}}, U_{n, \infty} \cap L_{n,(i)}(\mathbb{R})\right)
$$

resp.

$$
\left.G L_{m}\left(\mathbb{A}_{K}^{\infty}\right) \times\left(\left(\operatorname{Lie} G L_{m}\left(K_{\infty}\right)\right)_{\mathbb{C}}, U_{K, \infty}\right)\right)
$$

module as an admissible $G_{n}(\mathbb{A})$-module (resp. $L_{n,(i)}(\mathbb{A})$-module, resp. $G L_{m}\left(\mathbb{A}_{K}\right)$-module). By a square integrable automorphic representation of $G_{n}(\mathbb{A})$ (resp. $L_{n,(i)}(\mathbb{A})$, resp. $\left.G L_{m}\left(\mathbb{A}_{K}\right)\right)$ we shall mean the twist by a character of an irreducible admissible $G_{n}(\mathbb{A})$ module (resp. $L_{n,(i)}(\mathbb{A})$-module, resp. $G L_{m}\left(\mathbb{A}_{K}\right)$-module) that occurs discretely in the space of square integrable automorphic forms on the double coset space $G_{n}(\mathbb{Q}) \backslash G_{n}(\mathbb{A}) / A_{n}(\mathbb{R})^{0}$ (respectively $L_{n,(i)}(\mathbb{Q}) \backslash L_{n,(i)}(\mathbb{A}) / A_{n,(i)}(\mathbb{R})^{0}$ or $\left.G L_{m}(K) \backslash G L_{m}\left(\mathbb{A}_{K}\right) / \mathbb{R}_{>0}^{\times}\right)$. By a cuspidal automorphic representation of $G_{n}(\mathbb{A})$ (resp. $L_{n,(i)}(\mathbb{A})$, resp. $G L_{m}\left(\mathbb{A}_{K}\right)$ ) we shall mean an irreducible admissible $G_{n}(\mathbb{A})$-submodule (resp. $L_{n,(i)}(\mathbb{A})$-submodule, resp. $G L_{m}\left(\mathbb{A}_{K}\right)$ submodule) of the space of cuspidal automorphic forms on $G_{n}(\mathbb{A})\left(\right.$ resp. $L_{n,(i)}(\mathbb{A})$, resp. $\left.G L_{m}\left(\mathbb{A}_{K}\right)\right)$.

Proposition 1.2 Suppose that $\Pi$ is a square integrable automorphic representation of $G_{n}(\mathbb{A})$ and that $\Pi_{\infty}$ is cohomological. Then there is an expression

$$
2 n=m_{1} n_{1}+\cdots+m_{r} n_{r}
$$

with $m_{i}, n_{i} \in \mathbb{Z}_{>0}$ and cuspidal automorphic representations $\widetilde{\Pi}_{i}$ of $G L_{m_{i}}\left(\mathbb{A}_{F}\right)$ such that

- $\widetilde{\Pi}_{i}^{\vee} \cong \widetilde{\Pi}_{i}^{c}$;

- $\widetilde{\Pi}_{i}\|\operatorname{det}\|{ }^{\left(m_{i}+n_{i}-1\right) / 2}$ is cohomological;

- if $v$ is a prime of $F$ above a rational prime $q$ such that 
- either $q$ splits in $F_{0}$

- or $F$ and $\Pi$ are unramified above $q$,

then

$$
\mathrm{BC}\left(\Pi_{q}\right)_{v}=\boxplus_{i=1}^{r} \boxplus_{j=0}^{n_{i}-1} \widetilde{\Pi}_{i, v}|\operatorname{det}|_{v}^{\left(n_{i}-1\right) / 2-j} .
$$

Proof This follows from the main theorem of [52] and the classification of square integrable automorphic representations of $G L_{m}\left(\mathbb{A}_{F}\right)$ in [48]. (Here we are using our assumption that $F$ contains an imaginary quadratic field.)

Corollary 1.3 Keep the assumptions of the proposition. Then there is a continuous, semisimple, algebraic (i.e. unramified almost everywhere and de Rham above p) representation

$$
r_{p, l}(\Pi): G_{F} \longrightarrow G L_{2 n}\left(\overline{\mathbb{Q}}_{p}\right)
$$

with the following property: If $v$ is a prime of $F$ above a rational prime $q \neq p$ such that

- either $q$ splits in $F_{0}$,

- or $F$ and $\Pi$ are unramified above q,

then

$$
\imath \mathrm{WD}\left(\left.r_{p, l}(\Pi)\right|_{G_{F_{v}}}\right)^{\mathrm{ss}} \cong \operatorname{rec}_{F_{v}}\left(\mathrm{BC}\left(\Pi_{q}\right)_{v}|\operatorname{det}|_{v}^{(1-2 n) / 2}\right) .
$$

Proof Combine the proposition with, for instance, theorem 1.2 of [11] and theorem A of [10]. (These results are due to many people and we simply choose these particular references for convenience.)

\subsection{Spaces of hermitian forms}

If $R \subset \mathbb{R}$ then we will denote by $\operatorname{Herm}_{X}^{>0}$ (resp. $\operatorname{Herm}_{X}^{\geq 0}$ ) the set of pairings ( , ) in $\operatorname{Herm}_{X}$ such that

$$
(x, x)>0
$$

(resp. $\geq 0$ ) for all $x \in X-\{0\}$. We will denote by $S\left(F^{m}\right)^{>0}$ (resp. $S\left(F^{m}\right)^{\geq 0}$ ) the set of elements $a \in S\left(F^{m}\right)$ such that for each $\tau: F \hookrightarrow \mathbb{C}$ the image of $a$ under the map

$$
\begin{aligned}
S\left(F^{m}\right) & \longrightarrow M_{m}(F)^{t=c} \\
x \otimes y & \longmapsto x^{c, t} y+y^{c, t} x
\end{aligned}
$$

is positive definite (resp. positive semi-definite), i.e. all the roots of its characteristic polynomial are strictly positive (resp. non-negative) real numbers. Then $S\left(F^{m}\right)^{>0}$ is the set of elements of $S\left(F^{m}\right)$ whose pairing with every nonzero element of $\operatorname{Herm}_{\bar{F}^{m}} \geq 0$ is strictly positive, and $\mathrm{Herm}_{F^{m}}^{>0}$ is the set of elements of $\mathrm{Herm}_{F^{m}}$ whose pairing with every nonzero element of $S\left(F^{m}\right)^{\geq 0}$ is strictly positive. We will also write

$$
S\left(\mathcal{O}_{F,(p)}^{m}\right)^{>0}=S\left(\mathcal{O}_{F,(p)}^{m}\right) \cap S\left(F^{m}\right)^{>0} .
$$


We will next turn to the study of certain spaces which play a key role in the definition of the auxiliary data controlling toroidal compactifications.

Suppose that $W \subset V_{n}$ is an isotropic $F$-direct summand. We set

$$
\mathfrak{C}^{(m)}(W)=\left(\operatorname{Herm}_{V_{n} / W^{\perp}} \oplus \operatorname{Hom}_{F}\left(F^{m}, W\right)\right) \otimes_{\mathbb{Q}} \mathbb{R}
$$

If $m=0$ we will drop it from the notation. Note that we have a natural identification

$$
\mathfrak{C}^{(m)}\left(V_{n,(i)}\right) \cong Z\left(N_{n,(i)}^{(m)}\right)(\mathbb{R}) .
$$

There is also a natural map

$$
\mathfrak{C}^{(m)}(W) \longrightarrow \mathfrak{C}(W)
$$

Note that if $f \in \operatorname{Hom}_{F}\left(F^{m}, W\right)$ we can define $f^{\prime} \in \operatorname{Hom}\left(F^{m} \otimes_{F, c}\left(V_{n} / W^{\perp}\right), \mathbb{Q}\right)$ by

$$
f^{\prime}(x \otimes y)=\langle f(x), y\rangle_{n} .
$$

This establishes an isomorphism

$$
\operatorname{Hom}_{F}\left(F^{m}, W\right) \stackrel{\sim}{\longrightarrow} \operatorname{Hom}\left(F^{m} \otimes_{F, c}\left(V_{n} / W^{\perp}\right), \mathbb{Q}\right)
$$

and hence an isomorphism

$$
\mathfrak{C}^{(m)}(W) \stackrel{\sim}{\longrightarrow}\left(\operatorname{Herm}_{V_{n} / W^{\perp} \oplus F^{m}} / \operatorname{Herm}_{F^{m}}\right) \otimes_{\mathbb{Q}} \mathbb{R}
$$

Thus

$$
\mathfrak{C}^{(m)}\left(V_{n,(i)}\right) \cong Z\left(N_{n,(i)}^{(m)}\right)(\mathbb{R}) .
$$

If $g \in G_{n}(\mathbb{Q})$ we define

$$
\begin{aligned}
g: \mathfrak{C}^{(m)}(W) & \longrightarrow \mathfrak{C}^{(m)}(g W) \\
(z, f) & \longmapsto(g z, g \circ f),
\end{aligned}
$$

where

$$
(x, y)_{g z}=|v(g)|\left(g^{-1} x, g^{-1} y\right)_{z} .
$$

We extend this to an action of $G_{n}^{(m)}(\mathbb{Q})$ as follows: If $g \in \operatorname{Hom}_{F}\left(F^{m}, V_{n}\right)$ then we set

$$
g(z, f)=\left(z, f-\theta_{z} \circ g\right)
$$

where $\theta_{z}: V_{n} \rightarrow W$ satisfies

$$
\left(x \bmod W^{\perp}, y \bmod W^{\perp}\right)_{z}=\left\langle\theta_{z}(x), y\right\rangle_{n}
$$

for all $x, y \in V_{n}$. If $W^{\prime} \subset W$ there is a natural embedding

$$
\mathfrak{C}^{(m)}\left(W^{\prime}\right) \hookrightarrow \mathfrak{C}^{(m)}(W) .
$$


We will write $\mathfrak{C}^{>0}(W)=\operatorname{Herm}_{V_{n} / W^{\perp} \otimes_{\mathbb{Q}} \mathbb{R}}^{>0}$ and $\mathfrak{C}^{\geq 0}(W)=\operatorname{Herm}_{V_{n} / W^{\perp} \otimes_{\mathbb{Q}} \mathbb{R}}^{\geq 0}$. We will also write $\mathfrak{C}^{(m),>0}(W)\left(\right.$ resp. $\left.\mathfrak{C}^{(m), \geq 0}(W)\right)$ for the pre-image of $\mathfrak{C}^{>0}(W)\left(\right.$ resp. $\left.\mathfrak{C}^{\geq 0}(W)\right)$ in $\mathfrak{C}^{(m)}(W)$. Moreover we will set

$$
\mathfrak{C}^{(m), \succ 0}(W)=\bigcup_{W^{\prime} \subset W} \mathfrak{C}^{(m),>0}\left(W^{\prime}\right)
$$

and $\mathfrak{C}^{\succ 0}(W)=\mathfrak{C}^{(0), \succ 0}(W)$. Thus

$$
\mathfrak{C}^{(m),>0}(W) \subset \mathfrak{C}^{(m),>0}(W) \subset \mathfrak{C}^{(m), \geq 0}(W) .
$$

Note that the natural map $\mathfrak{C}^{(m)}(W) \rightarrow \mathfrak{C}(W)$ gives rise to a surjection

$$
\mathfrak{C}^{(m), \succ 0}(W) \rightarrow \mathfrak{C}^{\succ 0}(W)
$$

and that the pre-image of a point in $\mathfrak{C}^{>0}\left(W^{\prime}\right)$ is $\operatorname{Hom}_{F}\left(F^{m}, W^{\prime}\right.$ ) (and in particular the pre-image of (0) is (0)). Also note that if $W^{\prime} \subset W$ then there is a closed embedding

$$
\mathfrak{C}^{(m), \succ 0}\left(W^{\prime}\right) \hookrightarrow \mathfrak{C}^{(m), \succ 0}(W) .
$$

Finally note that the action of $G_{n}^{(m)}(\mathbb{Q})$ takes $\mathfrak{C}^{(m), \succ 0}(W)$ (resp. $\mathfrak{C}^{(m),>0}(W)$, resp. $\left.\mathfrak{C}^{(m), \geq 0}(W)\right)$ to $\mathfrak{C}^{(m), \succ 0}(g W)\left(\right.$ resp. $\mathfrak{C}^{(m),>0}(g W)$, resp. $\left.\mathfrak{C}^{(m), \geq 0}(g W)\right)$.

Note that $L_{n,(i)}^{(m)}(\mathbb{R})$ acts on

$$
\pi_{0}\left(L_{n,(i), \text { herm }}(\mathbb{R})\right) \times \mathfrak{C}^{(m)}\left(V_{n,(i)}\right)
$$

and preserves

$$
\pi_{0}\left(L_{n,(i), \operatorname{herm}}(\mathbb{R})\right) \times \mathfrak{C}^{(m),>0}\left(V_{n,(i)}\right) .
$$

Moreover $L_{n,(i)}^{(m)}(\mathbb{Q})$ preserves

$$
\pi_{0}\left(L_{n,(i), \operatorname{herm}}(\mathbb{R})\right) \times \mathfrak{C}^{(m), \succ 0}\left(V_{n,(i)}\right) .
$$

In fact, $L_{n,(i)}^{(m)}(\mathbb{R})$ acts transitively on $\pi_{0}\left(L_{n,(i), \text { herm }}(\mathbb{R})\right) \times \mathfrak{C}^{(m),>0}\left(V_{n,(i)}\right)$. For this paragraph let $(,)_{0} \in \mathfrak{C}^{>0}\left(V_{n,(i)}\right)$ denote the pairing on $\left(V_{n} / V_{n,(i)}^{\perp} \otimes \mathbb{Q} \mathbb{R}\right)^{2}$ induced by $\left.J_{n},\right\rangle_{n}$. Then the stabilizer of $1 \times\left((,)_{0}, 0\right)$ in $L_{n,(i)}^{(m)}(\mathbb{R})$ is

$$
L_{n,(i) \text {,herm }}(\mathbb{R})^{\nu=1}\left(U_{n, \infty} \cap L_{n,(i) \text {,lin }}^{(m)}(\mathbb{R})\right) A_{n}(\mathbb{R})^{0} .
$$

Thus we get an $L_{n,(i)}^{(m)}(\mathbb{R})$-equivariant identification

$$
\begin{aligned}
& \pi_{0}\left(L_{n,(i), \text { herm }}(\mathbb{R})\right) \times \mathfrak{C}^{(m),>0}\left(V_{n,(i)}\right) / \mathbb{R}_{>0}^{\times} \\
& \cong L_{n,(i)}^{(m)}(\mathbb{R}) / L_{n,(i), \text { herm }}(\mathbb{R})^{+}\left(U_{n, \infty} \cap L_{n,(i), \text { lin }}^{(m)}(\mathbb{R})\right)^{0} A_{n,(i)}(\mathbb{R})^{0} .
\end{aligned}
$$

We define $\mathfrak{C}^{(m)}$ to be the topological space

$$
\left(\bigcup_{W} \mathfrak{C}^{(m), \succ 0}(W)\right) / \sim,
$$


where $\sim$ is the equivalence relation generated by the identification of $\mathfrak{C}^{(m), \succ 0}\left(W^{\prime}\right)$ with its image in $\mathfrak{C}^{(m), \succ 0}(W)$ whenever $W^{\prime} \subset W$. (This is sometimes referred to as the 'conical complex'.) Thus as a set

$$
\mathfrak{C}^{(m)}=\coprod_{W} \mathfrak{C}^{(m),>0}(W) .
$$

We will let $\mathfrak{C}_{=i}^{(m)}$ denote

$$
\coprod_{\operatorname{dim}_{F} W=i} \mathfrak{C}^{(m),>0}(W)
$$

Note that $\mathfrak{C}_{=n}^{(m)}$ is a dense open subset of $\mathfrak{C}^{(m)}$. If $m=0$ we drop it from the notation.

The space $\mathfrak{C}^{(m)}$ has a natural, continuous, left action of $G_{n}^{(m)}(\mathbb{Q}) \times \mathbb{R}_{>0}^{\times}$. (The second factor acts on each $\mathfrak{C}^{(m), \succ 0}(W)$ by scalar multiplication.)

We have homeomorphisms

$$
\begin{aligned}
G_{n}^{(m)}(\mathbb{Q}) \backslash\left(G_{n}^{(m)}\left(\mathbb{A}^{\infty}\right) / U \times \pi_{0}\left(G_{n}(\mathbb{R})\right) \times \mathfrak{C}_{=i}^{(m)} / \mathbb{R}_{>0}^{\times}\right) \\
\cong \\
\cong P_{n,(i)}^{(m),+}(\mathbb{Q}) \backslash\left(G_{n}^{(m)}\left(\mathbb{A}^{\infty}\right) / U \times \pi_{0}\left(G_{n}(\mathbb{R})\right) \times\left(\mathfrak{C}^{(m),>0}\left(V_{n,(i)}\right) / \mathbb{R}_{>0}^{\times}\right)\right) \\
\cong \coprod_{h \in P_{n,(i)}^{(m),}\left(\mathbb{A}^{\infty}\right) \backslash G_{n}^{(m)}\left(\mathbb{A}^{\infty}\right) / U} L_{n,(i)}^{(m)}(\mathbb{Q}) \backslash L_{n,(i)}^{(m)}(\mathbb{A}) /\left(h U h^{-1} \cap P_{n,(i)}^{(m),+}\left(\mathbb{A}^{\infty}\right)\right) \\
\quad L_{n,(i), \text { herm }}(\mathbb{R})^{+}\left(L_{n,(i), \operatorname{lin}}^{(m)}(\mathbb{R}) \cap U_{n, \infty}^{0}\right) A_{n,(i)}(\mathbb{R})^{0} .
\end{aligned}
$$

(Use the fact, strong approximation for unipotent groups, that

$$
N_{n,(i)}^{(m),+}\left(\mathbb{A}^{\infty}\right)=V+N_{n,(i)}^{(m),+}(\mathbb{Q})
$$

for any open compact subgroup $V$ of $N_{n,(i)}^{(m),+}\left(\mathbb{A}^{\infty}\right)$.) If $g \in G_{n}^{(m)}\left(\mathbb{A}^{\infty}\right)$ and if $g^{-1} U g \subset U^{\prime}$ then the right translation map

$$
\begin{aligned}
& g: G_{n}^{(m)}(\mathbb{Q}) \backslash\left(G_{n}^{(m)}\left(\mathbb{A}^{\infty}\right) / U \times \pi_{0}\left(G_{n}(\mathbb{R})\right) \times \mathfrak{C}_{=i}^{(m)} / \mathbb{R}_{>0}^{\times}\right) . \\
& \quad \longrightarrow G_{n}^{(m)}(\mathbb{Q}) \backslash\left(G_{n}^{(m)}\left(\mathbb{A}^{\infty}\right) / U^{\prime} \times \pi_{0}\left(G_{n}(\mathbb{R})\right) \times \mathfrak{C}_{=i}^{(m)} / \mathbb{R}_{>0}^{\times}\right) .
\end{aligned}
$$

corresponds to the coproduct of the right translation maps

$$
\begin{aligned}
g^{\prime}: & L_{n,(i)}^{(m)}(\mathbb{Q}) \backslash L_{n,(i)}^{(m)}(\mathbb{A}) /\left(h U h^{-1} \cap P_{n,(i)}^{(m),+}\left(\mathbb{A}^{\infty}\right)\right) L_{n,(i), \text { herm }}(\mathbb{R})^{+} \\
& \left(L_{n,(i), \operatorname{lin}}^{(m)}(\mathbb{R}) \cap U_{n, \infty}^{0}\right) A_{n,(i)}(\mathbb{R})^{0} \\
& \longrightarrow L_{n,(i)}^{(m)}(\mathbb{Q}) \backslash L_{n,(i)}^{(m)}(\mathbb{A}) /\left(h^{\prime} U^{\prime}\left(h^{\prime}\right)^{-1} \cap P_{n,(i)}^{(m),+}\left(\mathbb{A}^{\infty}\right)\right) L_{n,(i), \text { herm }}(\mathbb{R})^{+} \\
& \left(L_{n,(i), \operatorname{lin}}^{(m)}(\mathbb{R}) \cap U_{n, \infty}^{0}\right) A_{n,(i)}(\mathbb{R})^{0}
\end{aligned}
$$

where $h g=g^{\prime} h^{\prime} u^{\prime}$ with $g^{\prime} \in P_{n,(i)}^{(m),+}\left(\mathbb{A}^{\infty}\right)$ and $u^{\prime} \in U^{\prime}$.

When considering compactifications of just the ordinary locus we will need a variant of the above discussion.

We set

$$
\left(G_{n}^{(m)}\left(\mathbb{A}^{\infty}\right) \times \pi_{0}\left(G_{n}(\mathbb{R})\right) \times \mathfrak{C}^{(m)}\right)^{\text {ord }}
$$


to be the subset of $G_{n}^{(m)}\left(\mathbb{A}^{\infty}\right) \times \pi_{0}\left(G_{n}(\mathbb{R})\right) \times \mathfrak{C}^{(m)}$ consisting of elements $(g, \delta, x)$ such that for some $W$ we have

$$
x \in \mathfrak{C}^{(m), \succ 0}(W)
$$

and

$$
W \otimes_{\mathbb{Q}} \mathbb{Q}_{p}=g_{p}\left(V_{n,(n)} \otimes_{\mathbb{Q}} \mathbb{Q}_{p}\right)
$$

It has a left action of $G_{n}^{(m)}(\mathbb{Q})$ and a right action of $G_{n}^{(m)}\left(\mathbb{A}^{\infty}\right)^{\text {ord }} \times \mathbb{R}_{>0}^{\times}$. We define

$$
\left(G_{n}^{(m)}\left(\mathbb{A}^{\infty}\right) \times \pi_{0}\left(G_{n}(\mathbb{R})\right) \times \mathfrak{C}_{=i}^{(m)}\right)^{\text {ord }}
$$

similarly. We also set

$$
G_{n}^{(m)}(\mathbb{Q}) \backslash\left(G_{n}^{(m)}\left(\mathbb{A}^{\infty}\right) / U^{p}\left(N_{1}, N_{2}\right) \times \pi_{0}\left(G_{n}(\mathbb{R})\right) \times \mathfrak{C}^{(m)}\right)^{\text {ord }}
$$

(resp.

$$
\left.G_{n}^{(m)}(\mathbb{Q}) \backslash\left(G_{n}^{(m)}\left(\mathbb{A}^{\infty}\right) / U^{p}\left(N_{1}, N_{2}\right) \times \pi_{0}\left(G_{n}(\mathbb{R})\right) \times \mathfrak{C}_{=i}^{(m)}\right)^{\text {ord }}\right)
$$

to be the image of $\left(G_{n}^{(m)}\left(\mathbb{A}^{\infty}\right) \times \pi_{0}\left(G_{n}(\mathbb{R})\right) \times \mathfrak{C}^{(m)}\right)^{\text {ord }}$ in

$$
G_{n}^{(m)}(\mathbb{Q}) \backslash\left(G_{n}^{(m)}\left(\mathbb{A}^{\infty}\right) / U^{p}\left(N_{1}, N_{2}\right) \times \pi_{0}\left(G_{n}(\mathbb{R})\right) \times \mathfrak{C}^{(m)}\right)
$$

(resp.

$$
\left.G_{n}^{(m)}(\mathbb{Q}) \backslash\left(G_{n}^{(m)}\left(\mathbb{A}^{\infty}\right) / U^{p}\left(N_{1}, N_{2}\right) \times \pi_{0}\left(G_{n}(\mathbb{R})\right) \times \mathfrak{C}_{=i}^{(m)}\right)\right) .
$$

Then as a set

$$
\begin{aligned}
& G_{n}^{(m)}(\mathbb{Q}) \backslash\left(G_{n}^{(m)}\left(\mathbb{A}^{\infty}\right) / U^{p}\left(N_{1}, N_{2}\right) \times \pi_{0}\left(G_{n}(\mathbb{R})\right) \times \mathfrak{C}^{(m)}\right)^{\text {ord }} \\
& \quad=\coprod_{i} G_{n}^{(m)}(\mathbb{Q}) \backslash\left(G_{n}^{(m)}\left(\mathbb{A}^{\infty}\right) / U^{p}\left(N_{1}, N_{2}\right) \times \pi_{0}\left(G_{n}(\mathbb{R})\right) \times \mathfrak{C}_{=i}^{(m)}\right)^{\text {ord }} .
\end{aligned}
$$

In the case $i=n$ we have a simpler description of

$$
G_{n}^{(m)}(\mathbb{Q}) \backslash\left(G_{n}^{(m)}\left(\mathbb{A}^{\infty}\right) / U^{p}\left(N_{1}, N_{2}\right) \times \pi_{0}\left(G_{n}(\mathbb{R})\right) \times \mathfrak{C}_{=i}^{(m)}\right)^{\text {ord }} .
$$

\section{Lemma 1.4}

$$
\begin{aligned}
& G_{n}^{(m)}(\mathbb{Q}) \backslash\left(G_{n}^{(m)}\left(\mathbb{A}^{\infty}\right) \times \pi_{0}\left(G_{n}(\mathbb{R})\right) \times \mathfrak{C}_{=n}^{(m)}\right)^{\text {ord }} / U^{p}\left(N_{1}\right) \\
& \quad \stackrel{\sim}{\longrightarrow} G_{n}^{(m)}(\mathbb{Q}) \backslash\left(G_{n}^{(m)}\left(\mathbb{A}^{\infty}\right) / U^{p}\left(N_{1}, N_{2}\right) \times \pi_{0}\left(G_{n}(\mathbb{R})\right) \times \mathfrak{C}_{=n}^{(m)}\right)^{\text {ord }} .
\end{aligned}
$$


Proof There is a natural surjection. We must check that it is also injective. The right hand side equals

$$
\begin{aligned}
& P_{n,(n)}^{(m),+}(\mathbb{Q}) \backslash\left(G_{n}^{(m)}\left(\mathbb{A}^{\infty, p}\right) / U^{p} \times\left(P_{n,(n)}^{(m),+}\left(\mathbb{Q}_{p}\right) U_{p}\left(N_{1}, N_{2}\right)_{n}^{(m)}\right) / U_{p}\left(N_{1}, N_{2}\right)_{n}^{(m)}\right. \\
& \left.\quad \times \pi_{0}\left(G_{n}(\mathbb{R})\right) \times \mathfrak{C}^{(m),>0}\left(V_{n,(n)}\right)\right) \\
& \quad \cong P_{n,(n)}^{(m),+}(\mathbb{Q}) \backslash\left(G_{n}^{(m)}\left(\mathbb{A}^{\infty}\right)^{\text {ord }} / U^{p}\left(N_{1}\right) \times \pi_{0}\left(G_{n}(\mathbb{R})\right) \times \mathfrak{C}^{(m),>0}\left(V_{n,(n)}\right)\right),
\end{aligned}
$$

which is clearly isomorphic to the left hand side.

There does not seem to be such a simple description of

$$
G_{n}^{(m)}(\mathbb{Q}) \backslash\left(G_{n}^{(m)}\left(\mathbb{A}^{\infty}\right) / U^{p}\left(N_{1}, N_{2}\right) \times \pi_{0}\left(G_{n}(\mathbb{R})\right) \times \mathfrak{C}_{=i}^{(m)}\right)^{\text {ord }}
$$

for $i \neq n$. However the interested reader can see the end of this section for a partial result, with a very unpleasant proof.

We set

$$
\mathfrak{T}_{U^{p}\left(N_{1}\right),=n}^{(m) \text { ord }}=G_{n}^{(m)}(\mathbb{Q}) \backslash\left(G_{n}^{(m)}\left(\mathbb{A}^{\infty}\right) / U^{p}\left(N_{1}, N_{2}\right) \times \pi_{0}\left(G_{n}(\mathbb{R})\right) \times \mathfrak{C}_{=n}^{(m)}\right)^{\text {ord }} / \mathbb{R}_{>0}^{\times} .
$$

If $U$ is a neat open compact subgroup of $L_{n,(i)}^{(m)}\left(\mathbb{A}^{\infty}\right)$, set

$$
\mathfrak{T}_{(i), U}^{(m)}=L_{n,(i)}^{(m)}(\mathbb{Q}) \backslash L_{n,(i)}^{(m)}(\mathbb{A}) / U L_{n,(i), \text { herm }}(\mathbb{R})^{0}\left(L_{n,(i), \text { lin }}^{(m)}(\mathbb{R}) \cap U_{n, \infty}^{0}\right) A_{n,(n)}(\mathbb{R})^{0} .
$$

\section{Corollary 1.5}

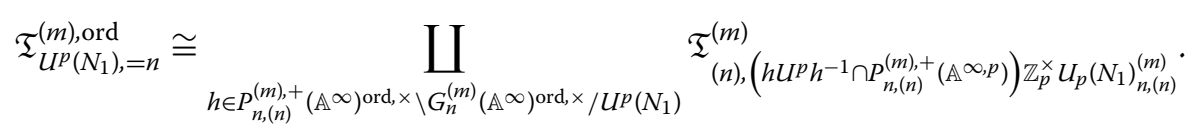

If $Y$ is a locally compact, Hausdorff topological space then we write $H_{\text {Int }}^{i}(Y, \mathbb{C})$ for the image of

$$
H_{c}^{i}(Y, \mathbb{C}) \longrightarrow H^{i}(Y, \mathbb{C})
$$

We define

$$
H_{\text {Int }}^{i}\left(\mathfrak{T}_{=n}^{(m), \text { ord }}, \overline{\mathbb{Q}}_{p}\right)=\lim _{\rightarrow U^{p}, N} H_{\text {Int }}^{i}\left(\mathfrak{T}_{U^{p}(N),=n}^{(m), \text { ord }}, \overline{\mathbb{Q}}_{p}\right)
$$

a smooth $G_{n}^{(m)}\left(\mathbb{A}^{\infty}\right)^{\text {ord }}$-module, and

$$
H_{\text {Int }}^{i}\left(\mathfrak{T}_{(n)}^{(m)}, \overline{\mathbb{Q}}_{p}\right)=\lim _{\rightarrow U} H_{\text {Int }}^{i}\left(\mathfrak{T}_{(n), U}^{(m)}, \overline{\mathbb{Q}}_{p}\right)
$$

a smooth $L_{n,(n)}^{(m)}\left(\mathbb{A}^{\infty}\right)$-module. Note that

$$
H_{\text {Int }}^{i}\left(\mathfrak{T}_{(n)}^{(m)}, \overline{\mathbb{Q}}_{p}\right)^{\mathbb{Z}_{p}^{\times}}=\lim _{\rightarrow U^{p}, N} H_{\text {Int }}^{i}\left(\mathfrak{T}_{(n), U^{p} U_{p}(N)_{n,(n)}^{(m)}}^{(m)} \mathbb{Z}_{p}^{\times}, \overline{\mathbb{Q}}_{p}\right)
$$

as $N$ runs over positive integers and $U^{p}$ runs over neat open compact subgroups of $L_{n,(n)}^{(m)}\left(\mathbb{A}^{\infty, p}\right)$. With these definitions we have the following corollary. 
Corollary 1.6 There is a $G_{n}^{(m)}\left(\mathbb{A}^{\infty}\right)^{\text {ord }}$-equivariant isomorphism

$$
\operatorname{Ind}_{P_{n,(n)}^{(m),+}\left(\mathbb{A}^{\infty, p}\right)}^{G_{\text {Int }}^{(m)}\left(\mathbb{T}_{(n)}^{\infty, p}\right)} H_{p}^{i}\left(\mathfrak{\mathbb { Q }}_{p}\right)^{\mathbb{Z}_{p}^{\times}} \cong H_{\text {Int }}^{i}\left(\mathfrak{T}_{=n}^{(m), \text { ord }}, \overline{\mathbb{Q}}_{p}\right) .
$$

Interior cohomology has the following property which will be key for us.

Lemma 1.7 Suppose that $G$ is a locally compact, totally disconnected topological group. Suppose that for any sufficiently small open compact subgroup $U \subset G$ we are given a compact Hausdorff space $Z_{U}$ and an open subset $Y_{U} \subset Z_{U}$. Suppose moreover that whenever $U, U^{\prime}$ are sufficiently small open compact subgroups of $G$ and $g \in G$ with $g^{-1} U g \subset U U^{\prime}$, then there is a proper continuous map

$$
g: Z_{U} \longrightarrow Z_{U^{\prime}}
$$

with $g Y_{U} \subset Y_{U^{\prime}}$. Also suppose that $g \circ h=h g$ whenever these maps are all defined and that if $g \in U$ then the map $g: Z_{U} \rightarrow Z_{U}$ is the identity.

If $\Omega$ is a field, set

$$
H^{i}(Z, \Omega)=\lim _{\rightarrow U} H^{i}\left(Z_{U}, \Omega\right)
$$

and

$$
H_{\mathrm{Int}}^{i}(Y, \Omega)=\lim _{\rightarrow U} H_{\mathrm{Int}}^{i}\left(Y_{U}, \Omega\right)
$$

These are both smooth G-modules. Moreover $H_{\mathrm{Int}}^{i}(Y, \Omega)$ is a subquotient of $H^{i}(Z, \Omega)$ as G-modules.

Proof Note that the diagram

$$
\begin{aligned}
H_{c}^{i}\left(Y_{U}, \Omega\right) & \longrightarrow H^{i}\left(Y_{U}, \Omega\right) \\
\downarrow & \uparrow \\
H_{c}^{i}\left(Z_{U}, \Omega\right) & =H^{i}\left(Z_{U}, \Omega\right)
\end{aligned}
$$

is commutative. Set

$$
A=\lim _{\rightarrow U} \operatorname{Im}\left(H_{c}^{i}\left(Y_{U}, \Omega\right) \longrightarrow H_{c}^{i}\left(Z_{U}, \Omega\right)=H^{i}\left(Z_{U}, \Omega\right)\right)
$$

and

$$
B=\lim _{\rightarrow U} \operatorname{Im}\left(\operatorname{ker}\left(H_{c}^{i}\left(Y_{U}, \Omega\right) \longrightarrow H^{i}\left(Y_{U}, \Omega\right)\right) \longrightarrow H^{i}\left(Z_{U}, \Omega\right)\right)
$$

Then

$$
B \subset A \subset H^{i}(Z, \Omega)
$$

are $G$-invariant subspaces with

$$
A / B \stackrel{\sim}{\longrightarrow} H_{\text {Int }}^{i}(Y, \Omega) .
$$


We finish this section with our promised generalization of Lemma 1.4. This generalization is not needed for the proofs of the main results of this paper, but we include it for completeness sake. The reader may wish to skip the proof.

Lemma 1.8 There is a natural homeomorphism

$$
\begin{gathered}
G_{n}^{(m)}(\mathbb{Q}) \backslash\left(G_{n}^{(m)}\left(\mathbb{A}^{\infty}\right) / U^{p}\left(N_{1}, N_{2}\right) \times \pi_{0}\left(G_{n}(\mathbb{R})\right) \times \mathfrak{C}_{=i}^{(m)}\right)^{\text {ord }} \\
\cong \amalg_{h \in P_{n,(i)}^{(m)+}\left(\mathbb{A}^{\infty}\right)_{\text {ord, } \times} \backslash G_{n}^{(m)}\left(\mathbb{A}^{\infty}\right)^{\text {ord } \times} \times U^{p}\left(N_{1}\right)} L_{n,(i)}^{(m)}(\mathbb{Q}) \\
\backslash L_{n,(i)}^{(m)}(\mathbb{A}) /\left(\left(h U^{p}\left(N_{1}\right) h^{-1} \cap P_{n,(i)}^{(m)+}\left(\mathbb{A}^{\infty}\right)^{\text {ord }, \times}\right)\right. \\
\left.L_{n,(i), \text { herm }}^{-}\left(\mathbb{Z}_{p}\right) L_{n,(i), \text { herm }}(\mathbb{R})^{0}\left(L_{n,(i), \text { lin }}^{(m)}(\mathbb{R}) \cap U_{n, \infty}^{0}\right)\right)
\end{gathered}
$$

where $U^{p}\left(N_{1}\right) \subset G_{n}^{(m)}\left(\mathbb{A}^{\infty}\right)^{\text {ord }, \times}$.

In particular

$$
G_{n}^{(m)}(\mathbb{Q}) \backslash\left(G_{n}^{(m)}\left(\mathbb{A}^{\infty}\right) / U^{p}\left(N_{1}, N_{2}\right) \times \pi_{0}\left(G_{n}(\mathbb{R})\right) \times \mathfrak{C}_{=i}^{(m)}\right)^{\text {ord }}
$$

and

$$
G_{n}^{(m)}(\mathbb{Q}) \backslash\left(G_{n}^{(m)}\left(\mathbb{A}^{\infty}\right) / U^{p}\left(N_{1}, N_{2}\right) \times \pi_{0}\left(G_{n}(\mathbb{R})\right) \times \mathfrak{C}^{(m)}\right)^{\text {ord }}
$$

are independent of $N_{2} \geq N_{1}$.

Proof Firstly we have that

$$
\begin{aligned}
G_{n}^{(m)} & (\mathbb{Q}) \backslash\left(G_{n}^{(m)}\left(\mathbb{A}^{\infty}\right) / U^{p}\left(N_{1}, N_{2}\right) \times \pi_{0}\left(G_{n}(\mathbb{R})\right) \times \mathfrak{C}_{=i}^{(m)}\right)^{\text {ord }} \\
\cong & P_{n,(i)}^{(m)+}(\mathbb{Q}) \backslash\left(G_{n}^{(m)}\left(\mathbb{A}^{\infty, p}\right) / U^{p}\right. \\
& \times\left(P_{n,(i)}^{(m),+}(\mathbb{Q}) P_{n,(n)}^{(m),+}\left(\mathbb{Q}_{p}\right) U_{p}\left(N_{1}, N_{2}\right)_{n}^{(m)}\right) / U_{p}\left(N_{1}, N_{2}\right)_{n}^{(m)} \\
& \left.\times \pi_{0}\left(G_{n}(\mathbb{R})\right) \times \mathfrak{C}^{(m),>0}\left(V_{n,(i)}\right)\right) .
\end{aligned}
$$

We can replace the second $P_{n,(i)}^{(m),+}(\mathbb{Q})$ by $P_{n,(i)}^{(m),+}\left(\mathbb{Q}_{p}\right)$, and then, using in particular the Iwasawa decomposition for $L_{n,(n)}\left(\mathbb{Q}_{p}\right)$, replace $P_{n,(n)}^{(m),+}\left(\mathbb{Q}_{p}\right)$ by $P_{n,(n)}^{(m),+}\left(\mathbb{Z}_{p}\right)$. Next we can replace $P_{n,(i)}^{(m),+}\left(\mathbb{Q}_{p}\right)$ by $P_{n,(i)}^{(m),+}\left(\mathbb{Z}_{p}\right)$ as long as we also replace $P_{n,(i)}^{(m),+}(\mathbb{Q})$ by $P_{n,(i)}^{(m),+}\left(\mathbb{Z}_{(p)}\right)$. This gives

$$
\begin{aligned}
G_{n}^{(m)} & (\mathbb{Q}) \backslash\left(G_{n}^{(m)}\left(\mathbb{A}^{\infty}\right) / U^{p}\left(N_{1}, N_{2}\right) \times \pi_{0}\left(G_{n}(\mathbb{R})\right) \times \mathfrak{C}_{=i}^{(m)}\right)^{\text {ord }} \\
\cong & P_{n,(i)}^{(m)+}\left(\mathbb{Z}_{(p)}\right) \backslash\left(G_{n}^{(m)}\left(\mathbb{A}^{\infty, p}\right) / U^{p}\right. \\
& \times\left(P_{n,(i)}^{(m),+}\left(\mathbb{Z}_{p}\right) P_{n,(n)}^{(m),+}\left(\mathbb{Z}_{p}\right) U_{p}\left(N_{1}, N_{2}\right)_{n}^{(m)}\right) / U_{p}\left(N_{1}, N_{2}\right)_{n}^{(m)} \\
& \left.\times \pi_{0}\left(G_{n}(\mathbb{R})\right) \times \mathfrak{C}^{(m),>0}\left(V_{n,(i)}\right)\right) .
\end{aligned}
$$

Note that

$$
P_{n-i,(n-i)}^{+}\left(\mathbb{Z}_{p}\right) \rightarrow C_{n-i}\left(\mathbb{Z}_{p}\right)
$$


[This follows from the fact that primes above $p$ of $F^{+}$are unramified in $F$, which implies that

$$
\left.\operatorname{ker}\left(\mathbf{N}_{F / F^{+}}: \mathcal{O}_{F, p}^{\times} \rightarrow \mathcal{O}_{F^{+}, p}^{\times}\right)=\left\{{ }^{c} x x^{-1}: x \in \mathcal{O}_{F, p}^{\times}\right\} \cdot\right]
$$

Thus

$$
L_{n,(i), \text { herm }}^{-}\left(\mathbb{Z}_{p}\right) P_{n-i,(n-i)}^{+}\left(\mathbb{Z}_{p}\right)=L_{n,(i), \text { herm }}\left(\mathbb{Z}_{p}\right)
$$

and

$$
P_{n,(i)}^{(m),+}\left(\mathbb{Z}_{p}\right) P_{n,(n)}^{(m),+}\left(\mathbb{Z}_{p}\right)=P_{n,(i)}^{(m),-}\left(\mathbb{Z}_{p}\right) P_{n,(n)}^{(m),+}\left(\mathbb{Z}_{p}\right)
$$

Moreover, by strong approximation, $P_{n,(i)}^{(m),-}\left(\mathbb{Z}_{(p)}\right)$ (resp. $\left.L_{n,(i), \text { herm }}^{-}\left(\mathbb{Z}_{(p)}\right)\right)$ is dense in $P_{n,(i)}^{(m),-}\left(\mathbb{A}^{\infty, p} \times \mathbb{Z}_{p}\right)\left(\right.$ resp. $\left.L_{n,(i) \text { herm }}^{-}\left(\mathbb{A}^{\infty, p} \times \mathbb{Z}_{p}\right)\right)$. Thus

$$
\begin{aligned}
G_{n}^{(m)} & (\mathbb{Q}) \backslash\left(G_{n}^{(m)}\left(\mathbb{A}^{\infty}\right) / U^{p}\left(N_{1}, N_{2}\right) \times \pi_{0}\left(G_{n}(\mathbb{R})\right) \times \mathfrak{C}_{=i}^{(m)}\right)^{\text {ord }} \\
\cong & P_{n,(i)}^{(m),}\left(\mathbb{Z}_{(p)}\right) \backslash\left(G_{n}^{(m)}\left(\mathbb{A}^{\infty, p}\right) / U^{p}\right. \\
& \times\left(P_{n,(i)}^{(m),-}\left(\mathbb{Z}_{p}\right) P_{n,(n)}^{(m),+}\left(\mathbb{Z}_{p}\right) U_{p}\left(N_{1}, N_{2}\right)_{n}^{(m)}\right) / U_{p}\left(N_{1}, N_{2}\right)_{n}^{(m)} \\
& \left.\times \pi_{0}\left(G_{n}(\mathbb{R})\right) \times \mathfrak{C}^{(m),>0}\left(V_{n,(i)}\right)\right) \\
\cong & L_{n,(i)}^{(m)}\left(\mathbb{Z}_{(p)}\right) \backslash\left(\left(P_{n,(i)}^{(m),-}\left(\mathbb{A}^{\infty, p}\right) \backslash G_{n}^{(m)}\left(\mathbb{A}^{\infty, p}\right) / U^{p}\right)\right. \\
& \times\left(P_{n,(i)}^{(m),-}\left(\mathbb{Z}_{p}\right) \backslash\left(P_{n,(i)}^{(m),}\left(\mathbb{Z}_{p}\right) P_{n,(n)}^{(m),+}\left(\mathbb{Z}_{p}\right) U_{p}\left(N_{1}, N_{2}\right)_{n}^{(m)}\right) / U_{p}\left(N_{1}, N_{2}\right)_{n}^{(m)}\right) \\
& \left.\times \pi_{0}\left(G_{n}(\mathbb{R})\right) \times \mathfrak{C}^{(m),>0}\left(V_{n,(i)}\right)\right) .
\end{aligned}
$$

Next we claim that the natural map

$$
\begin{aligned}
& \left(P_{n,(i)}^{(m),-} \cap P_{n,(n)}^{(m),+}\right)\left(\mathbb{Z}_{p}\right) \backslash P_{n,(n)}^{(m),+}\left(\mathbb{Z}_{p}\right) /\left(U_{p}\left(N_{1}\right)_{n,(n)}^{(m)} \mathbb{Z}_{p}^{\times} N_{n,(n)}^{(m)}\left(\mathbb{Z}_{p}\right)\right) \\
& \longrightarrow P_{n,(i)}^{(m),-}\left(\mathbb{Z}_{p}\right) \backslash\left(P_{n,(i)}^{(m),-}\left(\mathbb{Z}_{p}\right) P_{n,(n)}^{(m),+}\left(\mathbb{Z}_{p}\right) U_{p}\left(N_{1}, N_{2}\right)_{n}^{(m)}\right) / U_{p}\left(N_{1}, N_{2}\right)_{n}^{(m)}
\end{aligned}
$$

is an isomorphism. It suffices to check this modulo $p^{N_{2}}$, where the map becomes

$$
\begin{aligned}
& \left(P_{n,(i)}^{(m),} \cap P_{n,(n)}^{(m),+}\right)\left(\mathbb{Z} / p^{N_{2}} \mathbb{Z}\right) \backslash P_{n,(n)}^{(m),+}\left(\mathbb{Z} / p^{N_{2}} \mathbb{Z}\right) /\left(U_{p}\left(N_{1}\right)_{n,(n)}^{(m)} \mathbb{Z}_{p}^{\times} N_{n,(n)}^{(m)}\left(\mathbb{Z}_{p}\right)\right) \\
& \quad \longrightarrow P_{n,(i)}^{(m),-}\left(\mathbb{Z} / p^{N_{2}} \mathbb{Z}\right) \backslash\left(P_{n,(i)}^{(m),-}\left(\mathbb{Z} / p^{N_{2}} \mathbb{Z}\right) P_{n,(n)}^{(m),+}\left(\mathbb{Z} / p^{N_{2}} \mathbb{Z}\right) /\left(U_{p}\left(N_{1}\right)_{n,(n)}^{(m)} \mathbb{Z}_{p}^{\times} N_{n,(n)}^{(m)}\left(\mathbb{Z}_{p}\right)\right),\right.
\end{aligned}
$$

which is clearly an isomorphism. Thus we have

$$
\begin{aligned}
G_{n}^{(m)} & (\mathbb{Q}) \backslash\left(G_{n}^{(m)}\left(\mathbb{A}^{\infty}\right) / U^{p}\left(N_{1}, N_{2}\right) \times \pi_{0}\left(G_{n}(\mathbb{R})\right) \times \mathfrak{C}_{=i}^{(m)}\right)^{\text {ord }} \\
\cong & L_{n,(i)}^{(m)}\left(\mathbb{Z}_{(p)}\right) \backslash\left(\left(P_{n,(i)}^{(m),}\left(\mathbb{A}^{\infty, p}\right) \backslash G_{n}^{(m)}\left(\mathbb{A}^{\infty, p}\right) / U^{p}\right)\right. \\
& \times\left(\left(P_{n,(i)}^{(m),} \cap P_{n,(n)}^{(m),+}\right)\left(\mathbb{Z}_{p}\right) \backslash P_{n,(n)}^{(m),+}\left(\mathbb{Z}_{p}\right) /\left(U_{p}\left(N_{1}\right)_{n,(n)}^{(m)} \mathbb{Z}_{p}^{\times} N_{n,(n)}^{(m)}\left(\mathbb{Z}_{p}\right)\right)\right) \\
& \left.\times \pi_{0}\left(G_{n}(\mathbb{R})\right) \times \mathfrak{C}^{(m),>0}\left(V_{n,(i)}\right)\right),
\end{aligned}
$$

where $\gamma \in L_{n,(i)}^{(m)}\left(\mathbb{Z}_{(p)}\right)$ acts on $\left(P_{n,(i)}^{(m),-} \cap P_{n,(n)}^{(m),+}\right)\left(\mathbb{Z}_{p}\right) \backslash P_{n,(n)}^{(m),+}\left(\mathbb{Z}_{p}\right)$ via an element of $P_{n-i,(n-i)}^{+}\left(\mathbb{Z}_{p}\right) \times L_{n,(i), \text { lin }}\left(\mathbb{Z}_{p}\right)$ with the same image in $C_{n-i}\left(\mathbb{Z}_{p}\right) \times L_{n,(i), \text { lin }}\left(\mathbb{Z}_{p}\right)$. 
Note that

$$
\begin{gathered}
P_{n,(i)}^{(m),-}\left(\mathbb{A}^{\infty, p}\right) \backslash G_{n}^{(m)}\left(\mathbb{A}^{\infty, p}\right) / U^{p}=\coprod_{h \in P_{n,(i)}^{(m),+}\left(\mathbb{A}^{\infty, p}\right) \backslash G_{n}^{(m)}\left(\mathbb{A}^{\infty, p}\right) / U^{p}} \\
L_{n,(i), \mathrm{herm}}^{(m),-}\left(\mathbb{A}^{\infty, p}\right) \backslash L_{n,(i)}^{(m)}\left(\mathbb{A}^{\infty, p}\right) /\left(h U^{p} h^{-1} \cap P_{n,(i)}^{(m),+}\left(\mathbb{A}^{\infty, p}\right)\right) .
\end{gathered}
$$

Also note that, if we set $U_{p}=\left(U_{p}\left(N_{1}\right)_{n,(n)}^{(m)} \mathbb{Z}_{p}^{\times} N_{n,(n)}^{(m)}\left(\mathbb{Z}_{p}\right)\right)$, then

$$
\begin{gathered}
\left(P_{n,(i)}^{(m),-} \cap P_{n,(n)}^{(m),+}\right)\left(\mathbb{Z}_{p}\right) \backslash P_{n,(n)}^{(m),+}\left(\mathbb{Z}_{p}\right) / U_{p}=\coprod_{h \in\left(P_{n,(i)}^{(m),} \cap P_{n,(n)}^{(m),+}\right)\left(\mathbb{Z}_{p}\right) \backslash P_{n,(n)}^{(m),+}\left(\mathbb{Z}_{p}\right) / U_{p}} \\
\quad\left(L_{n,(i), \operatorname{lin}}^{(m)}\left(\mathbb{Z}_{p}\right) \times \operatorname{Im}\left(P_{n-i,(n-i)}\left(\mathbb{Z}_{p}\right) \rightarrow C_{n-i}\left(\mathbb{Z}_{p}\right)\right)\right)^{\prime} /\left(h U_{p} h^{-1} \cap P_{n,(i)}^{(m),+}\left(\mathbb{Z}_{p}\right)\right) .
\end{gathered}
$$

However as the primes above $p$ split in $F^{+}$split in $F$ we see that

$$
\operatorname{Im}\left(P_{n-i,(n-i)}\left(\mathbb{Z}_{p}\right) \rightarrow C_{n-i}\left(\mathbb{Z}_{p}\right)\right)=L_{n,(i), \text { herm }}\left(\mathbb{Z}_{p}\right) / L_{n,(i), \text { herm }}^{-}\left(\mathbb{Z}_{p}\right)
$$

and so

$$
\begin{aligned}
& \left(P_{n,(i)}^{(m),-} \cap P_{n,(n)}^{(m),+}\right)\left(\mathbb{Z}_{p}\right) \backslash P_{n,(n)}^{(m),+}\left(\mathbb{Z}_{p}\right) / U_{p}=\coprod_{h \in\left(P_{n,(i)}^{(m),} \cap P_{n,(n)}^{(m),+}\right)\left(\mathbb{Z}_{p}\right) \backslash P_{n,(n)}^{(m),+}\left(\mathbb{Z}_{p}\right) / U_{p}} \\
& \quad L_{n,(i)}^{(m)}\left(\mathbb{Z}_{p}\right) / L_{n,(i), \text { herm }}^{-}\left(\mathbb{Z}_{p}\right)\left(h U_{p} h^{-1} \cap P_{n,(i)}^{(m),+}\left(\mathbb{Z}_{p}\right)\right) .
\end{aligned}
$$

Thus we see that

$$
\begin{aligned}
& G_{n}^{(m)}(\mathbb{Q}) \backslash\left(G_{n}^{(m)}\left(\mathbb{A}^{\infty}\right) / U^{p}\left(N_{1}, N_{2}\right) \times \pi_{0}\left(G_{n}(\mathbb{R})\right) \times \mathfrak{C}_{=i}^{(m)}\right)^{\text {ord }} \\
& \cong \bigsqcup_{h \in P_{n,(i)}^{(m),+}\left(\mathbb{A}^{\infty}\right)^{\text {ord }, \times} \backslash G_{n}^{(m)}\left(\mathbb{A}^{\infty}\right)^{\text {ord }, \times} / U^{p}\left(N_{1}\right)} L_{n,(i)}^{(m)}\left(\mathbb{Z}_{(p)}\right) \\
& \backslash\left(L_{n,(i)}^{(m)}\left(\mathbb{A}^{\infty, p} \times \mathbb{Z}_{p}\right) / L_{n,(i), \text { herm }}^{-}\left(\mathbb{A}^{\infty, p} \times \mathbb{Z}_{p}\right)\right. \\
&\left.\left(h U^{p}\left(N_{1}\right) h^{-1} \cap P_{n,(i)}^{(m),+}\left(\mathbb{A}^{\infty}\right)^{\text {ord }, \times}\right) \times \pi_{0}\left(G_{n}^{(m)}(\mathbb{R})\right) \times \mathfrak{C}^{(m),>0}\left(V_{n,(i)}\right)\right) .
\end{aligned}
$$

As $L_{n,(i), \text { herm }}^{-}\left(\mathbb{Z}_{(p)}\right)$ acts trivially on

$$
\left(L_{n,(i)}^{(m)}\left(\mathbb{Z}_{p}\right) / L_{n,(i), \text { herm }}^{-}\left(\mathbb{Z}_{p}\right)\right) \times \pi_{0}\left(G_{n}^{(m)}(\mathbb{R})\right) \times \mathfrak{C}^{(m),>0}\left(V_{n,(i)}\right)
$$

and is dense in $L_{n,(i) \text {,herm }}^{-}\left(\mathbb{A}^{\infty, p}\right)$, we further see that

$$
\begin{aligned}
& G_{n}^{(m)}(\mathbb{Q}) \backslash\left(G_{n}^{(m)}\left(\mathbb{A}^{\infty}\right) / U^{p}\left(N_{1}, N_{2}\right) \times \pi_{0}\left(G_{n}(\mathbb{R})\right) \times \mathfrak{C}_{=i}^{(m)}\right)^{\text {ord }} \\
& \cong \coprod_{h \in P_{n,(i)}^{(m),+}\left(\mathbb{A}^{\infty}\right)^{\text {ord }, \times} \backslash G_{n}^{(m)}\left(\mathbb{A}^{\infty}\right)^{\text {ord }, \times} / U^{p}\left(N_{1}\right)} \\
& L_{n,(i)}^{(m)}\left(\mathbb{Z}_{(p)}\right) \backslash\left(L_{n,(i)}^{(m)}\left(\mathbb{A}^{\infty, p} \times \mathbb{Z}_{p}\right) / L_{n,(i), \text { herm }}^{-}\left(\mathbb{Z}_{p}\right)\right. \\
& \left(h U^{p}\left(N_{1}\right) h^{-1} \cap P_{n,(i)}^{(m),+}\left(\mathbb{A}^{\infty}\right)^{\text {ord }, \times}\right) \\
& \left.\times \pi_{0}\left(G_{n}^{(m)}(\mathbb{R})\right) \times \mathfrak{C}^{(m),>0}\left(V_{n,(i)}\right)\right) \\
& \cong \coprod_{h \in P_{n,(i)}^{(m),+}\left(\mathbb{A}^{\infty}\right)^{\text {ord }, \times} \backslash G_{n}^{(m)}\left(\mathbb{A}^{\infty}\right)^{\text {ord }, \times} / U^{p}\left(N_{1}\right)} \\
& L_{n,(i)}^{(m)}\left(\mathbb{Z}_{(p)}\right) \backslash L_{n,(i)}^{(m)}\left(\mathbb{A}^{p} \times \mathbb{Z}_{p}\right) /\left(\left(h U^{p}\left(N_{1}\right) h^{-1} \cap P_{n,(i)}^{(m),+}\left(\mathbb{A}^{\infty}\right)^{\text {ord }, \times}\right)\right. \\
& \left.L_{n,(i), \operatorname{herm}}^{-}\left(\mathbb{Z}_{p}\right) L_{n,(i), \operatorname{herm}}(\mathbb{R})^{0}\left(L_{n,(i), \operatorname{lin}}^{(m)}(\mathbb{R}) \cap U_{n, \infty}^{0}\right)\right), \\
& \cong \coprod_{h \in P_{n,(i)}^{(m),+}\left(\mathbb{A}^{\infty}\right) \text { ord } \times \backslash G_{n}^{(m)}\left(\mathbb{A}^{\infty}\right) \text { ord } \times / U^{p}\left(N_{1}\right)} \\
& L_{n,(i)}^{(m)}(\mathbb{Q}) \backslash L_{n,(i)}^{(m)}(\mathbb{A}) /\left(\left(h U^{p}\left(N_{1}\right) h^{-1} \cap P_{n,(i)}^{(m),+}\left(\mathbb{A}^{\infty}\right)^{\text {ord }, \times}\right)\right. \\
& \left.L_{n,(i), \text { herm }}^{-}\left(\mathbb{Z}_{p}\right) L_{n,(i), \text { herm }}(\mathbb{R})^{0}\left(L_{n,(i), \text { lin }}^{(m)}(\mathbb{R}) \cap U_{n, \infty}^{0}\right)\right),
\end{aligned}
$$

as desired. 
Abusing notation slightly, we will write

$$
G_{n}^{(m)}(\mathbb{Q}) \backslash\left(G_{n}^{(m)}\left(\mathbb{A}^{\infty}\right) / U^{p}\left(N_{1}\right) \times \pi_{0}\left(G_{n}(\mathbb{R})\right) \times \mathfrak{C}_{=i}^{(m)}\right)^{\text {ord }}
$$

for

$$
G_{n}^{(m)}(\mathbb{Q}) \backslash\left(G_{n}^{(m)}\left(\mathbb{A}^{\infty}\right) / U^{p}\left(N_{1}, N_{2}\right) \times \pi_{0}\left(G_{n}(\mathbb{R})\right) \times \mathfrak{C}_{=i}^{(m)}\right)^{\text {ord }},
$$

and

$$
G_{n}^{(m)}(\mathbb{Q}) \backslash\left(G_{n}^{(m)}\left(\mathbb{A}^{\infty}\right) / U^{p}\left(N_{1}\right) \times \pi_{0}\left(G_{n}(\mathbb{R})\right) \times \mathfrak{C}^{(m)}\right)^{\text {ord }}
$$

for

$$
G_{n}^{(m)}(\mathbb{Q}) \backslash\left(G_{n}^{(m)}\left(\mathbb{A}^{\infty}\right) / U^{p}\left(N_{1}, N_{2}\right) \times \pi_{0}\left(G_{n}(\mathbb{R})\right) \times \mathfrak{C}^{(m)}\right)^{\text {ord }} .
$$

\subsection{Locally symmetric spaces}

In this section we will calculate $H_{\text {Int }}^{i}\left(\mathfrak{T}_{(n)}^{(m)}, \overline{\mathbb{Q}}_{p}\right)$ in terms of automorphic forms on $L_{n,(n)}(\mathbb{A})$. Our main result will be the following, which will be an immediate consequence of Corollary 1.12 and Lemma 1.13 below.

Corollary 1.9 Suppose that $n>1$ and that $\rho$ is an irreducible algebraic representation of $L_{n,(n), \text { lin }}$ on a finite dimensional $\mathbb{C}$-vector space. Suppose also that $\pi$ is a cuspidal automorphic representation of $L_{n,(n), \text { lin }}(\mathbb{A})$ such that $\pi_{\infty}$ has the same infinitesimal character as $\rho^{\vee}$ and that $\psi$ is a continuous character of $\mathbb{Q}^{\times} \backslash \mathbb{A}^{\times} / \mathbb{R}_{>0}^{\times}$. Then for all sufficiently large integers $N$ there are integers $m(N) \in \mathbb{Z}_{\geq 0}$ and $i(N) \in \mathbb{Z}_{>0}$, and an $L_{n,(n)}\left(\mathbb{A}^{\infty}\right)$-equivariant embedding

$$
\left(\pi^{\infty}\|\operatorname{det}\|^{N}\right) \times \psi^{\infty} \hookrightarrow H_{\text {Int }}^{i(N)}\left(\mathfrak{T}_{(n)}^{(m(N))}, \mathbb{C}\right) .
$$

If $m=0$ we will write $\mathfrak{T}_{(n)}$ for $\mathfrak{T}_{(n)}^{(0)}$. Let $\Omega$ denote an algebraically closed field of characteristic 0 . If $\rho$ is a finite dimensional algebraic representation of $L_{n,(n)}$ on a $\Omega$-vector space $W_{\rho}$ then we define a locally constant sheaf $\mathcal{L}_{\rho, U} / \mathfrak{T}_{(n), U}$ as

$$
\begin{gathered}
L_{n,(n)}(\mathbb{Q}) \backslash\left(W_{\rho} \times L_{n,(n)}(\mathbb{A}) / U\left(L_{n,(n)}(\mathbb{R}) \cap U_{n, \infty}^{0}\right) A_{n,(n)}(\mathbb{R})^{0}\right) \\
\quad \downarrow \\
L_{n,(n)}(\mathbb{Q}) \backslash L_{n,(n)}(\mathbb{A}) / U\left(L_{n,(n)}(\mathbb{R}) \cap U_{n, \infty}^{0}\right) A_{n,(n)}(\mathbb{R})^{0} .
\end{gathered}
$$

The system of sheaves $\mathcal{L}_{\rho, U}$ has a right action of $L_{n,(n)}\left(\mathbb{A}^{\infty}\right)$. We define

$$
H_{\text {Int }}^{i}\left(\mathfrak{T}_{(n)}, \mathcal{L}_{\rho}\right)=\lim _{\rightarrow U} H_{\text {Int }}^{i}\left(\mathfrak{T}_{(n), U}, \mathcal{L}_{\rho, U}\right),
$$

smooth $L_{n,(n)}\left(\mathbb{A}^{\infty}\right)$-module. Note that if $\rho$ has a central character $\chi_{\rho}$ then,

$$
\alpha \in Z\left(L_{n,(n)}\right)(\mathbb{Q})^{+} \subset L_{n,(n)}\left(\mathbb{A}^{\infty}\right)
$$

acts on $H_{\text {Int }}^{i}\left(\mathfrak{T}_{(n)}, \mathcal{L}_{\rho}\right)$ via $\chi_{\rho}(\alpha)^{-1}$. (Use the fact that $Z\left(L_{n,(n)}\right)(\mathbb{Q})^{+} \subset\left(L_{n,(n)}(\mathbb{R}) \cap\right.$ $\left.U_{n, \infty}^{0}\right) A_{n,(n)}(\mathbb{R})^{0}$.) 
The natural map $L_{n,(n)}^{(m)} \rightarrow L_{n,(n)}$ gives rise to continuous maps

$$
\pi^{(m)}: \mathfrak{T}_{(n), U}^{(m)} \longrightarrow \mathfrak{T}_{(n), U}
$$

compatible with the action of $L_{n,(n)}^{(m)}\left(\mathbb{A}^{\infty}\right)$.

Lemma 1.10 (1) The maps $\pi^{(m)}$ are real-torus bundles (i.e. $\left(S^{1}\right)^{r}$-bundles for some $r$ ), and in particular are proper maps.

(2) There are $L_{n,(n)}^{(m)}\left(\mathbb{A}^{\infty}\right)$-equivariant identifications

$$
R^{i} \pi_{*}^{(m)} \Omega \cong \mathcal{L}_{\wedge i}\left(\bigoplus_{\tau: F \hookrightarrow \Omega} \operatorname{Std}_{\tau}^{\oplus m}\right)^{\vee}
$$

In particular the action of $L_{n,(n)}^{(m)}\left(\mathbb{A}^{\infty}\right)$ on the relative cohomology sheaf $R^{i} \pi_{*}^{(m)} \Omega$ factors through $L_{n,(n)}\left(\mathbb{A}^{\infty}\right)$.

Proof Recall that

$$
N\left(L_{n,(n), \operatorname{lin}}^{(m)}\right)=\operatorname{ker}\left(L_{n,(n)}^{(m)} \rightarrow L_{n,(n)}\right)
$$

Suppose that $U$ is a neat open compact subgroup of $L_{n,(n)}^{(m)}\left(\mathbb{A}^{\infty}\right)$ with image $U^{\prime}$ in $L_{n,(n)}\left(\mathbb{A}^{\infty}\right)$. Then $L_{n,(n)}(\mathbb{Q}) \times U^{\prime}$ acts freely on

$$
L_{n,(n)}(\mathbb{A}) /\left(L_{n,(n)}(\mathbb{R}) \cap U_{n, \infty}^{0}\right) A_{n,(n)}(\mathbb{R})^{0}
$$

Thus it suffices to prove that the map $\widetilde{\pi}^{(m)}$

$$
\begin{gathered}
N\left(L_{n,(n), \text { lin }}^{(m)}\right)(\mathbb{Q}) \backslash L_{n,(n)}^{(m)}(\mathbb{A}) /\left(U \cap N\left(L_{n,(n), \text { lin }}^{(m)}\right)\left(\mathbb{A}^{\infty}\right)\right)\left(L_{n,(n)}(\mathbb{R}) \cap U_{n, \infty}^{0}\right) A_{n,(n)}(\mathbb{R})^{0} \\
\downarrow \\
L_{n,(n)}(\mathbb{A}) /\left(L_{n,(n)}(\mathbb{R}) \cap U_{n, \infty}^{0}\right) A_{n,(n)}(\mathbb{R})^{0}
\end{gathered}
$$

is a real-torus bundle and that there are $L_{n,(n)}(\mathbb{Q}) \times L_{n,(n)}^{(m)}\left(\mathbb{A}^{\infty}\right)$-equivariant isomorphisms

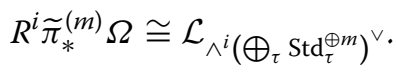

Using the identification of spaces (but not of groups) that comes from the group product

$$
L_{n,(n)}^{(m)}(\mathbb{A})=N\left(L_{n,(n), \operatorname{lin}}^{(m)}\right)(\mathbb{A}) \times L_{n,(n)}(\mathbb{A}),
$$

we see that $\tilde{\pi}^{(m)}$ can be identified with the map

$$
\begin{gathered}
\left(N\left(L_{n,(n), \operatorname{lin}}^{(m)}\right)(\mathbb{Q}) \backslash N\left(L_{n,(n), \operatorname{lin}}^{(m)}\right)(\mathbb{A}) /\left(U \cap N\left(L_{n,(n), \text { lin }}^{(m)}\right)\left(\mathbb{A}^{\infty}\right)\right)\right) \\
\times\left(L_{n,(n)}(\mathbb{A}) /\left(L_{n,(n)}(\mathbb{R}) \cap U_{n, \infty}^{0}\right) A_{n,(n)}(\mathbb{R})^{0}\right) \\
\downarrow \\
L_{n,(n)}(\mathbb{A}) /\left(L_{n,(n)}(\mathbb{R}) \cap U_{n, \infty}^{0}\right) A_{n,(n)}(\mathbb{R})^{0}
\end{gathered}
$$


or, using the equality $N\left(L_{n,(n), \text { lin }}^{(m)}\right)\left(\mathbb{A}^{\infty}\right)=N\left(L_{n,(n), \text { lin }}^{(m)}\right)(\mathbb{Q})\left(U \cap N\left(L_{n,(n), \text { lin }}^{(m)}\right)\left(\mathbb{A}^{\infty}\right)\right)$, even with

$$
\begin{gathered}
\left(\left(N\left(L_{n,(n), \operatorname{lin}}^{(m)}\right)(\mathbb{Q}) \cap U\right) \backslash N\left(L_{n,(n), \operatorname{lin}}^{(m)}\right)(\mathbb{R})\right) \times\left(L_{n,(n)}(\mathbb{A}) /\left(L_{n,(n)}(\mathbb{R}) \cap U_{n, \infty}^{0}\right) A_{n,(n)}(\mathbb{R})^{0}\right) \\
\downarrow \\
L_{n,(n)}(\mathbb{A}) /\left(L_{n,(n)}(\mathbb{R}) \cap U_{n, \infty}^{0}\right) A_{n,(n)}(\mathbb{R})^{0},
\end{gathered}
$$

The right $L_{n,(n)}^{(m)}\left(\mathbb{A}^{\infty}\right)$-action is by right translation on the second factor. The left action of $L_{n,(n)}(\mathbb{Q})$ is via conjugation on the first factor and left translation on the second.

The first part of the lemma follows, and we see that

$$
R^{i} \tilde{\pi}_{*}^{(m)} \Omega
$$

is $L_{n,(n)}(\mathbb{Q}) \times L_{n,(n)}^{(m)}\left(\mathbb{A}^{\infty}\right)$ equivariantly identified with the locally constant sheaf

$$
\begin{gathered}
\left(\wedge^{i} N\left(L_{n,(n), \text { lin }}^{(m)}\right)(\Omega)^{\vee}\right) \times\left(L_{n,(n)}(\mathbb{A}) /\left(L_{n,(n)}(\mathbb{R}) \cap U_{n, \infty}^{0}\right) A_{n,(n)}(\mathbb{R})^{0}\right) \\
\downarrow \\
L_{n,(n)}(\mathbb{A}) /\left(L_{n,(n)}(\mathbb{R}) \cap U_{n, \infty}^{0}\right) A_{n,(n)}(\mathbb{R})^{0} .
\end{gathered}
$$

The lemma follows.

Lemma 1.11 There is an $L_{n,(n)}^{(m)}\left(\mathbb{A}^{\infty}\right)$-equivariant isomorphism

$$
H_{\mathrm{Int}}^{k}\left(\mathfrak{T}_{(n)}^{(m)}, \Omega\right) \cong \bigoplus_{i+j=k} H_{\mathrm{Int}}^{i}\left(\mathfrak{T}_{(n)}, \mathcal{L}_{\wedge^{j}}\left(\oplus_{\tau} \operatorname{Std}_{\tau}^{\oplus m}\right)^{\vee}\right)
$$

Proof There is an $L_{n,(n)}^{(m)}\left(\mathbb{A}^{\infty}\right)$-equivariant spectral sequence

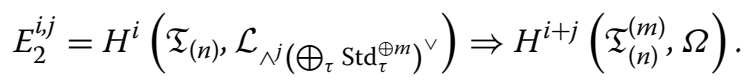

If $\alpha \in \mathbb{Q}_{>0}^{\times} \subset Z\left(L_{n,(n), \text { lin }}\right)\left(\mathbb{A}^{\infty}\right)$, then $\alpha$ acts on $E_{2}^{i, j}$ via $\alpha^{j}$. We deduce that all the differentials (on the second and any later page) vanish, and so the spectral sequence degenerates on the second page. Moreover the $\alpha \mapsto \alpha^{j}$ eigenspace in $H^{i+j}\left(\mathfrak{T}_{(n)}^{(m)}, \Omega\right)$ is naturally identified with

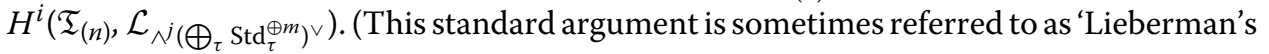
trick'.)

As the maps $\pi^{(m)}$ are proper, there is also an $L_{n,(n)}^{(m)}\left(\mathbb{A}^{\infty}\right)$-equivariant spectral sequence

$$
E_{c, 2}^{i, j}=H_{c}^{i}\left(\mathfrak{T}_{(n)}, \mathcal{L}_{\wedge^{j}}\left(\oplus_{\tau} \operatorname{Std}_{\tau}^{\oplus m}\right)^{\vee}\right) \Rightarrow H_{c}^{i+j}\left(\mathfrak{T}_{(n)}^{(m)}, \Omega\right)
$$

and $\alpha \in \mathbb{Q}_{>0}^{\times} \subset Z\left(L_{n,(n), \text { lin }}\right)\left(\mathbb{A}^{\infty}\right)$ acts on $E_{c, 2}^{i, j}$ via $\alpha^{j}$. Again we see that the spectral sequence degenerates on the second page and that the $\alpha \mapsto \alpha^{j}$ eigenspace in $H_{c}^{i+j}\left(\mathfrak{T}_{(n)}^{(m)}, \Omega\right)$ is naturally identified with $H_{c}^{i}\left(\mathfrak{T}_{(n)}, \mathcal{L}_{\wedge^{j}}\left(\oplus_{\tau} \operatorname{Std}_{\tau}^{\oplus m}\right)^{\vee}\right)$.

The lemma follows.

Corollary 1.12 Suppose that $\rho$ is an irreducible representation of $L_{n,(n), \text { lin }}$ over $\Omega$, which we extend to a representation of $L_{n,(n)}$ by letting it be trivial on $L_{n,(n), \text { herm. }}$ Let $d=N_{F / \mathbb{Q}} \circ$ det : 
$L_{n,(n), \text { lin }} \rightarrow \mathbb{G}_{m}$. Then for all $N$ sufficiently large there are $j(N), m(N) \in \mathbb{Z}_{\geq 0}$ such that, for all $i$,

$$
H_{\text {Int }}^{i}\left(\mathfrak{T}_{(n)}, \mathcal{L}_{\rho \otimes d^{-N}}\right)
$$

is an $L_{n,(n)}\left(\mathbb{A}^{\infty}\right)$-direct summand of

$$
H_{\mathrm{Int}}^{i+j(N)}\left(\mathfrak{T}_{(n)}^{(m(N))}, \Omega\right)
$$

Proof It follows from Weyl's construction of the irreducible representations of $G L_{n}$ that, for $N$ sufficiently large, $\rho \otimes d^{-N}$ is a direct summand of

$$
\bigotimes_{\tau}\left(\operatorname{Std}_{\tau}^{\vee}\right)^{\otimes m_{\tau}(N)}
$$

for certain non-negative integers $m_{\tau}(N)$. Hence for $N$ sufficiently large and $m(N)=$ $\max \left\{m_{\tau}(N)\right\}$ the representation $\rho \otimes d^{-N}$ is also a direct summand of

$$
\wedge^{\sum_{\tau} m_{\tau}(N)}\left(\bigoplus_{\tau} \operatorname{Std}_{\tau}^{\oplus m(N)}\right)^{\vee} .
$$

Lemma 1.13 Suppose that $\rho$ is an irreducible algebraic representation of $L_{n,(n)}$ on a finite dimensional $\mathbb{C}$-vector space.

(1) Then

$$
\bigoplus_{\Pi} \Pi^{\infty} \otimes H^{i}\left(\operatorname{Lie} L_{n,(n)},\left(L_{n,(n)}(\mathbb{R}) \cap U_{n, \infty}^{0}\right) A_{n,(n)}(\mathbb{R})^{0}, \Pi_{\infty} \otimes \rho\right) \hookrightarrow H_{\text {Int }}^{i}\left(\mathfrak{T}_{(n)}, \mathcal{L}_{\rho}\right),
$$

where $\Pi$ runs over cuspidal automorphic representations of $L_{n,(n)}(\mathbb{A})$.

(2) If $n>1$ and if $\Pi$ is a cuspidal automorphic representation of $L_{n,(n)}(\mathbb{A})$ such that $\Pi_{\infty}$ has the same infinitesimal character as $\rho^{\vee}$, then

$$
H^{i}\left(\operatorname{Lie} L_{n,(n)},\left(L_{n,(n)}(\mathbb{R}) \cap U_{n, \infty}^{0}\right) A_{n,(n)}(\mathbb{R})^{0}, \Pi_{\infty} \otimes \rho\right) \neq(0)
$$

for some $i>0$.

Proof The first part results from [13], more precisely from combining theorem 5.2, the discussion in section 5.4 and corollary 5.5 of that paper. The second part results from [20], see the proof of theorem 3.13, and in particular lemma 3.14, of that paper.

We are now in a position to deduce Corollary 1.9, which we stated at the start of this section. 


\section{Tori, torsors and torus embeddings}

The main aim of this section is to recall some basic facts about relative torus embeddings of tori torsors, which will provide local models for the boundary of toroidal compactifications of Shimura and Kuga-Sato varieties. Much of this material is in some sense standard, but we need to work with infinite cone decompositions, which are not treated in much of the literature. It will also be convenient to use a notation which emphasizes the boundary of the torus embedding and the completion of the torus embedding along the boundary. These seem to be more naturally parameterized by certain partial fans rather than fans. In Sect. 2.4 we compute certain cohomology groups. For finite fans (or partial fans) such results are fairly standard, but we found it quite tricky to formulate and prove the results we need in the presence of infinitely many cones. Maybe this is just our incompetence.

Throughout this section let $R_{0}$ denote an irreducible noetherian ring (i.e. a noetherian ring with a unique minimal prime ideal). In the applications of this section elsewhere in this paper it will be either $\mathbb{Q}$ or $\mathbb{Z}_{(p)}$ or $\mathbb{Z} / p^{r} \mathbb{Z}$ for some $r$. We will consider $R_{0}$ endowed with the discrete topology so that $\operatorname{Spf} R_{0} \cong \operatorname{Spec} R_{0}$.

\subsection{Tori and torsors}

If $S / Y$ is a torus (i.e. a group scheme etale locally on $Y$ isomorphic to $\mathbb{G}_{m}^{N}$ for some $N$ ) then we can define its sheaf of characters $X^{*}(S)=\operatorname{Hom}\left(S, \mathbb{G}_{m}\right)$ and its sheaf of cocharacters $X_{*}(S)=\operatorname{Hom}\left(\mathbb{G}_{m}, S\right)$. These are locally constant sheaves of free $\mathbb{Z}$-modules in the etale topology on $Y$. They are naturally $\mathbb{Z}$-dual to each other. More generally if $S_{1} / Y$ and $S_{2} / Y$ are two tori then $\operatorname{Hom}\left(S_{1}, S_{2}\right)$ is a locally constant sheaf of free $\mathbb{Z}$-modules in the etale topology on $Y$. In fact,

$$
\operatorname{Hom}\left(S_{1}, S_{2}\right)=\operatorname{Hom}\left(X_{*}\left(S_{1}\right), X_{*}\left(S_{2}\right)\right)=\operatorname{Hom}\left(X^{*}\left(S_{2}\right), X^{*}\left(S_{1}\right)\right) .
$$

By a quasi-isogeny (resp. isogeny) from $S_{1}$ to $S_{2}$ we shall mean a global section of the sheaf $\operatorname{Hom}\left(S_{1}, S_{2}\right)_{\mathbb{Q}}\left(\right.$ resp. $\left.\operatorname{Hom}\left(S_{1}, S_{2}\right)\right)$ with an inverse in $\operatorname{Hom}\left(S_{2}, S_{1}\right)_{\mathbb{Q}}$. We will write $[S]_{\text {isog }}$ for the category whose objects are tori over $Y$ quasi-isogenous to $S$ and whose morphisms are isogenies. The sheaves $X_{*}(S)_{\mathbb{Q}}$ and $X^{*}(S)_{\mathbb{Q}}$ only depend on the quasi-isogeny class of $S$ so we will write $X_{*}\left([S]_{\text {isog }}\right)_{\mathbb{Q}}$ and $X^{*}\left([S]_{\text {isog }}\right)_{\mathbb{Q}}$.

If $\bar{y}$ is a geometric point of $Y$ then we define

$$
T S_{\bar{y}}=\lim _{N} S[N](k(\bar{y}))
$$

and

$$
T^{p} S_{\bar{y}}={\underset{p}{p \chi_{N}}}_{\lim } S[N](k(\bar{y}))
$$

with the transition map from $M N$ to $N$ being multiplication by $M$. (The Tate modules of S.) Also define

$$
V S_{\bar{y}}=T S_{\bar{y}} \otimes_{\mathbb{Z}} \mathbb{Q}
$$

and

$$
V^{p} S_{\bar{y}}=T^{p} S_{\bar{y}} \otimes_{\mathbb{Z}} \mathbb{Q}
$$


If $Y$ is a scheme over Spec $\mathbb{Q}$ then

$$
T S_{\bar{y}} \cong X_{*}(S)_{\bar{y}} \otimes_{\mathbb{Z}} \widehat{\mathbb{Z}}(1) .
$$

If $Y$ is a scheme over Spec $\mathbb{Z}_{(p)}$ then

$$
T^{p} S_{\bar{y}} \cong X_{*}(S)_{\bar{y}} \otimes_{\mathbb{Z}} \widehat{\mathbb{Z}}^{p}(1)
$$

Now suppose that $S$ is split, i.e. isomorphic to $\mathbb{G}_{m}^{N}$ for some $N$. By an $S$-torsor $T / Y$ we mean a scheme $T / Y$ with an action of $S$, which locally in the Zariski topology on $Y$ is isomorphic to $S$. By a rigidification of $T$ along $e: Y^{\prime} \rightarrow Y$ we mean an isomorphism of $S$-torsors $e^{*} T \cong S$ over $Y^{\prime}$. If $U$ is a connected open subset of $Y$ then

$$
\left.T\right|_{U}=\text { Spec } \bigoplus_{\chi \in X^{*}(S)(U)} \mathcal{L}_{T}(\chi)
$$

where $\mathcal{L}_{T}(\chi)$ is a line bundle on $U$. If $Z$ is any open subset of $Y$ and if $\chi \in X^{*}(S)(Z)$ then there is a unique line bundle $\mathcal{L}_{T}(\chi)$ on $Z$ whose restriction to any connected open subset $U \subset Z$ is $\mathcal{L}_{T}\left(\left.\chi\right|_{U}\right)$. Multiplication gives isomorphisms

$$
\mathcal{L}_{T}\left(\chi_{1}\right) \otimes \mathcal{L}_{T}\left(\chi_{2}\right) \stackrel{\sim}{\longrightarrow} \mathcal{L}_{T}\left(\chi_{1}+\chi_{2}\right) .
$$

The map

$$
T \longmapsto \mathcal{L}_{T, 1}^{\vee}
$$

gives a bijection between isomorphism classes of $\mathbb{G}_{m}$-torsors and isomorphism classes of line bundles on $Y$. The inverse map sends $\mathcal{L}$ to

$$
\text { Spec } \bigoplus_{N \in \mathbb{Z}} \mathcal{L}^{\vee, \otimes N}
$$

If $\alpha: S \rightarrow S^{\prime}$ is a morphism of split tori and if $T / Y$ is an $S$-torsor we can form a push-out $\alpha_{*} T$, an $S^{\prime}$ torsor on $Y$ defined as the quotient

$$
\left(S^{\prime} \times_{Y} T\right) / S
$$

where $S$ acts by

$$
s:\left(s^{\prime}, t\right) \longmapsto\left(s^{\prime} s, s^{-1} t\right) .
$$

There is a natural map $T \rightarrow \alpha_{*} T$ compatible with $\alpha: S \rightarrow S^{\prime}$. If $\alpha$ is an isogeny then

$$
\alpha_{*} T=(\operatorname{ker} \alpha) \backslash T .
$$

If $T_{1}$ and $T_{2}$ are $S$-torsors over $Y$ we define

$$
\left(T_{1} \otimes_{S} T_{2}\right) / Y
$$


to be the $S$-torsor

$$
\left(T_{1} \times_{Y} T_{2}\right) / S
$$

where $S$ acts by

$$
s:\left(t_{1}, t_{2}\right) \longmapsto\left(s t_{1}, s^{-1} t_{2}\right) .
$$

If $T$ is an $S$-torsor on $Y$ we define an $S$-torsor $T^{\vee} / Y$ by taking $T^{\vee}=T$ as schemes but defining an $S$ action. on $T^{\vee}$ by

$$
\text { s.t }=s^{-1} t \text {, }
$$

i.e. $T^{\vee}=[-1]_{S, *} T$. Then

$$
T^{\vee} \otimes_{S} T \cong S
$$

via the map that sends $\left(t_{1}, t_{2}\right)$ to the unique section $s$ of $S$ with $s t_{1}=t_{2}$.

\subsection{Log structures}

We will call a formal scheme

$$
\mathfrak{X} \longrightarrow \operatorname{Spf} R_{0}
$$

suitable if it has a cover by affine opens $\mathfrak{U}_{i}=\operatorname{Spf}\left(A_{i}\right)_{I_{i}}^{\wedge}$, where $A_{i}$ is a finitely generated $R_{0}$-algebra and $I_{i}$ is an ideal of $A_{i}$ whose inverse image in $R_{0}$ is (0).

By a log structure on a scheme $X$ (resp. formal scheme $\mathfrak{X}$ ) we mean a sheaf of monoids $\mathcal{M}$ on $X$ (resp. $\mathfrak{X})$ together with a morphism

$$
\alpha: \mathcal{M} \longrightarrow\left(\mathcal{O}_{X}, \times\right)
$$

(resp.

$$
\left.\alpha: \mathcal{M} \longrightarrow\left(\mathcal{O}_{\mathfrak{X}}, \times\right)\right)
$$

such that the induced map

$$
\alpha^{-1} \mathcal{O}_{X}^{\times} \longrightarrow \mathcal{O}_{X}^{\times}
$$

(resp.

$$
\left.\alpha^{-1} \mathcal{O}_{\mathfrak{X}}^{\times} \longrightarrow \mathcal{O}_{\mathfrak{X}}^{\times}\right)
$$

is an isomorphism. We will refer to a scheme (resp. formal scheme) endowed with a log structure as a log scheme (resp. log formal scheme). By a morphism of log schemes (resp. morphism of log formal schemes)

$$
(\phi, \psi):(X, \mathcal{M}, \alpha) \longrightarrow(Y, \mathcal{N}, \beta)
$$


(resp.

$$
(\phi, \psi):(\mathfrak{X}, \mathcal{M}, \alpha) \longrightarrow(\mathfrak{Y}, \mathcal{N}, \beta))
$$

we shall mean a morphism $\phi: X \rightarrow Y($ resp. $\phi: \mathfrak{X} \rightarrow \mathfrak{Y})$ and a map

$$
\psi: \phi^{-1} \mathcal{N} \longrightarrow \mathcal{M}
$$

such that $\phi^{*} \circ \phi^{-1}(\beta)=\alpha \circ \psi$. We will consider $R_{0}$ endowed with the trivial log structure $\left(\mathcal{O}_{\text {Spec } R_{0}}^{\times}, 1\right)$ (resp. $\left.\left(\mathcal{O}_{\text {Spf } R_{0}}^{\times}, 1\right)\right)$. We will call a log formal scheme $(\mathfrak{X}, \mathcal{M}, \alpha) /$ Spf $R_{0}$ suitable if $\mathfrak{X} / \operatorname{Spf} R_{0}$ is suitable and if, locally in the Zariski topology, $\mathcal{M} / \alpha^{-1} \mathcal{O}_{\mathfrak{X}}^{\times}$is finitely generated. (In the case of schemes these definitions are well known: See, for example, [34]. We have not attempted to optimize the definition in the case of formal schemes. We are simply making a definition which works for the limited purposes of this article. The reader might like to compare our definitions with those in [5].)

If $D$ is a closed subscheme of $X$ we define a $\log$ structure $\mathcal{M}(D)$ on $X$ by setting

$$
\mathcal{M}(D)(U)=\mathcal{O}_{X}(U) \cap \mathcal{O}_{X}(U-D)^{\times} .
$$

If $X / \operatorname{Spec} R_{0}$ is a scheme of finite type and if $Z \subset X$ is a closed subscheme which is flat over Spec $R_{0}$, then the formal completion $X_{Z}^{\wedge}$ is a suitable formal scheme. Let $i^{\wedge}$ denote the map of ringed spaces $X_{Z}^{\wedge} \rightarrow X$. If $(\mathcal{M}, \alpha)$ is a log structure on $X$, then we get a map

$$
\left(i^{\wedge}\right)^{-1}(\alpha):\left(i^{\wedge}\right)^{-1} \mathcal{M} \longrightarrow \mathcal{O}_{X_{\tilde{Z}}}
$$

It induces a $\log$ structure $\left(\mathcal{M}^{\wedge}, \alpha^{\wedge}\right)$ on $X_{Z}^{\wedge}$, where $\mathcal{M}^{\wedge}$ denotes the push-out

$$
\begin{aligned}
& \begin{aligned}
\left(\left(i^{\wedge}\right)^{-1}(\alpha)\right)^{-1} \mathcal{O}_{X_{Z}^{\wedge}}^{\times} & \hookrightarrow \\
\downarrow & \left(i^{\wedge}\right)^{-1} \mathcal{M} \\
\downarrow & \downarrow
\end{aligned} \\
& \mathcal{O}_{X_{Z}^{\wedge}}^{\times} \longrightarrow \mathcal{M}^{\wedge} \text {. }
\end{aligned}
$$

If

$$
(\phi, \psi):(X, \mathcal{M}, \alpha) \longrightarrow(Y, \mathcal{N}, \beta)
$$

is a morphism of schemes with $\log$ structures over $\operatorname{Spec} R_{0}$ then there is a right exact sequence

$$
\phi^{*} \Omega_{Y}^{1}(\log \mathcal{N}) \longrightarrow \Omega_{X}^{1}(\log \mathcal{M}) \longrightarrow \Omega_{X / Y}^{1}(\log \mathcal{M} / \mathcal{N}) \longrightarrow(0)
$$

of sheaves of $\log$ differentials. If the map $(\phi, \psi)$ is log smooth then this sequence is also left exact and the sheaf $\Omega_{X / Y}^{1}(\log \mathcal{M} / \mathcal{N})$ is locally free. (See, e.g., [34].) As usual, we write $\Omega_{X}^{i}(\log \mathcal{M})=\wedge^{i} \Omega_{X}^{1}(\log \mathcal{M})$ and $\Omega_{X / Y}^{i}(\log \mathcal{M} / \mathcal{N})=\wedge^{i} \Omega_{X / Y}^{1}(\log \mathcal{M} / \mathcal{N})$.

By a coherent sheaf of differentials on a formal scheme $\mathfrak{X} / \mathrm{Spf} R_{0}$ we will mean a coherent sheaf $\Omega / \mathfrak{X}$ together with a differential $d: \mathcal{O}_{\mathfrak{X}} \rightarrow \Omega$ which vanishes on $R_{0}$. By a coherent sheaf of log differentials on a $\log$ formal scheme $(\mathfrak{X}, \mathcal{M}, \alpha) / \operatorname{Spf} R_{0}$ we shall mean a coherent sheaf $\Omega / \mathfrak{X}$ together with a differential, which vanishes on $R_{0}$,

$$
d: \mathcal{O}_{\mathfrak{X}} \longrightarrow \Omega \text {, }
$$


and a homomorphism

$$
\operatorname{dlog}: \mathcal{M} \longrightarrow \Omega
$$

such that

$$
\alpha(m) \operatorname{dlog} m=d(\alpha(m))
$$

By a universal coherent sheaf of differentials (resp. universal coherent sheaf of log differentials) we shall mean a coherent sheaf of differentials $(\Omega, d)$ (resp. a coherent sheaf of $\log$ differentials $\left(\Omega, d\right.$, dlog)) such that for any other coherent sheaf of differentials $\left(\Omega^{\prime}, d^{\prime}\right)$ (resp. a coherent sheaf of $\log$ differentials $\left(\Omega^{\prime}, d^{\prime}, \operatorname{dlog}\right)$ ) there is a unique map $f: \Omega \rightarrow \Omega^{\prime}$ such that $f \circ d=d^{\prime}$ (resp. $f \circ d=d^{\prime}$ and $f \circ \operatorname{dlog}=\operatorname{dlog}{ }^{\prime}$ ).

Note that if a universal coherent sheaf of differentials (resp. universal coherent sheaf of $\log$ differentials) exists, it is unique up to unique isomorphism.

Lemma 2.1 Suppose that $R_{0}$ is a discrete, noetherian topological ring.

(1) A universal sheaf of coherent differentials $\Omega_{\mathfrak{X} / \mathrm{Spf} R_{0}}^{1}$ exists for any suitable formal scheme $\mathfrak{X} / \operatorname{Spf} R_{0}$.

(2) If $X / \operatorname{Spec} R_{0}$ is a scheme of finite type and if $Z \subset X$ is flat over $R_{0}$ then

$$
\Omega_{X_{Z}^{\wedge} / \operatorname{Spf} R_{0}}^{1} \cong\left(\Omega_{X / \operatorname{Spec} R_{0}}^{1}\right)^{\wedge}
$$

(3) A universal sheaf of coherent log differentials $\Omega_{\mathfrak{X} / \mathrm{Spf} R_{0}}^{1}(\log \mathcal{M})$ exists for any suitable log formal scheme $(\mathfrak{X}, \mathcal{M}, \alpha) / \operatorname{Spf} R_{0}$.

(4) Suppose that $X / \operatorname{Spec} R_{0}$ is a scheme of finite type, that $Z \subset X$ is flat over $R_{0}$ and that $(\mathcal{M}, \alpha)$ is a log structure on $X$ such that Zariski locally $\mathcal{M} / \alpha^{-1} \mathcal{O}_{X}^{\times}$is finitely generated. Then

$$
\Omega_{X_{\bar{Z}} / \operatorname{Spf} R_{0}}^{1}\left(\log \mathcal{M}^{\wedge}\right) \cong\left(\Omega_{X / \operatorname{Spec} R_{0}}^{1}(\log \mathcal{M})\right)^{\wedge}
$$

Proof Consider the first part. Suppose that $\mathfrak{U}=\operatorname{Spf} A_{I}^{\wedge}$ is an affine open in $\mathfrak{X}$, where $A$ is a finitely generated $R_{0}$-algebra and $I$ is an ideal of $A$ with inverse image (0) in $R_{0}$. Then there exists a universal finite module of differentials $\Omega_{\mathfrak{U}}^{1}$ for $\mathfrak{U}$, namely the coherent sheaf of $\mathcal{O}_{\mathfrak{U}}$-modules associated to $\left(\Omega_{A / R_{0}}^{1}\right)_{I}^{\wedge}$. (See sections 11.5 and 12.6 of [40].) We must show that if $\mathfrak{U}^{\prime} \subset \mathfrak{U}$ is open then $\left.\Omega_{\mathfrak{U}}^{1}\right|_{\mathfrak{U}^{\prime}}$ is a universal finite module of differentials for $\mathfrak{U}^{\prime}$. For then uniqueness will allow us to glue the coherent sheaves $\Omega_{\mathfrak{U}}^{1}$ to form $\Omega_{\mathfrak{X}}^{1}$.

So suppose that $\left(\Omega^{\prime}, d^{\prime}\right)$ is a finite module of differentials for $\mathfrak{U}^{\prime}$. We must show that there is a unique map of $\mathcal{O}_{\mathfrak{U}^{\prime}}$-modules

$$
f:\left.\Omega_{\mathfrak{U}}^{1}\right|_{\mathfrak{U}^{\prime}} \longrightarrow \Omega^{\prime}
$$

such that $d^{\prime}=f \circ d$. We may cover $\mathfrak{U}^{\prime}$ by affine opens of the form $\operatorname{Spf}\left(A_{g}\right)_{I}^{\wedge}$ and it will suffice to find, for each $g$, a unique

$$
f_{g}:\left.\Omega_{\mathfrak{U}}^{1}\right|_{\operatorname{Spf}\left(A_{g}\right)_{I}^{\wedge}} \longrightarrow \Omega^{\prime} \mid \operatorname{Spf}\left(A_{g}\right)_{I}^{\wedge}
$$


with $d^{\prime}=f_{g} \circ d$. Thus we may assume that $\mathfrak{U}^{\prime}=\operatorname{Spf}\left(A_{g}\right)_{I}^{\wedge}$. But in this case we know $\Omega_{\mathfrak{U}^{\prime}}^{1}$ exists, and is the coherent sheaf associated to

$$
\left(\Omega_{A_{g} / R_{0}}^{1}\right)_{I}^{\wedge} \cong\left(\Omega_{A / R_{0}}^{1} \otimes_{A} A_{g}\right)_{I}^{\wedge} .
$$

On the other hand $\left.\Omega_{\mathfrak{U}}^{1}\right|_{\mathfrak{U}^{\prime}}$ is the coherent sheaf associated to

$$
\left(\Omega_{A / R_{0}}^{1}\right)_{I}^{\wedge} \otimes_{A_{I}^{\wedge}}\left(A_{g}\right)_{I}^{\wedge}
$$

Thus

$$
\left.\Omega_{\mathfrak{U}^{\prime}}^{1} \stackrel{\sim}{\longrightarrow} \Omega_{\mathfrak{U}}^{1}\right|_{\mathfrak{U}^{\prime}}
$$

and the first part follows. The second part also follows from the proof of the first part.

For the third part, because of uniqueness, it suffices to work locally. Thus we may assume that there are finitely many sections $m_{1}, \ldots, m_{r} \in \mathcal{M}(\mathfrak{X})$, which together with $\alpha^{-1} \mathcal{O}_{\mathfrak{X}}^{\times}$ generate $\mathcal{M}$. Then we define $\Omega_{(\mathfrak{X}, \mathcal{M}, \alpha)}^{1}$ to be the cokernel of the map

$$
\begin{aligned}
\mathcal{O}_{\mathfrak{X}}^{\oplus r} & \longrightarrow \Omega_{\mathfrak{X}}^{1} \oplus \mathcal{O}_{\mathfrak{X}}^{\oplus r} \\
\left(f_{i}\right)_{i} & \longmapsto\left(-\sum_{i} f_{i} d \alpha\left(m_{i}\right),\left(f_{i} \alpha\left(m_{i}\right)\right)_{i}\right) .
\end{aligned}
$$

It is elementary to check that this has the desired universal property. The fourth part is also elementary to check.

If

$$
(\phi, \psi):(\mathfrak{X}, \mathcal{M}, \alpha) \longrightarrow(\mathfrak{Y}, \mathcal{N}, \beta)
$$

is a map of suitable $\log$ formal schemes over $\operatorname{Spf} R_{0}$ then we set

$$
\Omega_{\mathfrak{X} / \mathfrak{Y}}^{1}(\log \mathcal{M} / \mathcal{N})=\Omega_{\mathfrak{X} / \operatorname{Spf} R_{0}}^{1}(\log \mathcal{M}) / \phi^{*} \Omega_{\mathfrak{Y} / \operatorname{Spf} R_{0}}^{1}(\log \mathcal{N}) .
$$

We also set

$$
\Omega_{\mathfrak{X} / \mathrm{Spf} R_{0}}^{i}=\wedge^{i} \Omega_{\mathfrak{X} / \mathrm{Spf} R_{0}}^{1}
$$

and

$$
\Omega_{\mathfrak{X} / \operatorname{Spf} R_{0}}^{i}(\log \mathcal{M})=\wedge^{i} \Omega_{\mathfrak{X} / \operatorname{Spf} R_{0}}^{1}(\log \mathcal{M})
$$

and

$$
\Omega_{\mathfrak{X} / \mathfrak{Y}}^{i}(\log \mathcal{M} / \mathcal{N})=\wedge^{i} \Omega_{\mathfrak{X} / \mathfrak{Y}}^{1}(\log \mathcal{M} / \mathcal{N}) .
$$

Corollary 2.2 Suppose that $R_{0}$ is a discrete, noetherian topological ring; that

$$
(X, \mathcal{M}, \alpha) \rightarrow(Y, \mathcal{N}, \beta)
$$

is a map of log schemes over Spec $R_{0}$; and that $Z \subset X$ and $W \subset Y$ are closed subschemes flat over Spec $R_{0}$ which map to each other under $X \rightarrow Y$. Suppose moreover that $X$ and $Y$ have finite type over $\operatorname{Spec} R_{0}$ and that $\mathcal{M} / \alpha^{-1} \mathcal{O}_{\mathfrak{X}}^{\times}$and $\mathcal{N} / \beta^{-1} \mathcal{O}_{\mathfrak{Y}}^{\times}$are locally (in the Zariski topology) finitely generated. Then

$$
\Omega_{\left(X_{Z}^{\wedge}, \mathcal{M}^{\wedge}, \alpha^{\wedge}\right) /\left(Y_{\hat{W}}^{\wedge}, \mathcal{N}^{\wedge}, \beta^{\wedge}\right)}^{1} \cong\left(\Omega_{X / Y}^{1}(\log \mathcal{M} / \mathcal{N})\right)_{Z}^{\hat{Z}}
$$


Proof This follows from the lemma and from the exactness of completion.

If $Y$ is a scheme we will let

$$
\operatorname{Aff}_{Y}^{n}=\underline{\operatorname{Spec}} \mathcal{O}_{Y}\left[T_{1}, \ldots, T_{n}\right]
$$

denote affine $n$-space over $Y$ and

$$
\operatorname{Coord}_{Y}^{n}=\underline{\operatorname{Spec}} \mathcal{O}_{Y}\left[T_{1}, \ldots, T_{n}\right] /\left(T_{1} \ldots T_{n}\right) \subset \operatorname{Aff}_{Y}^{n}
$$

denote the union of the coordinate hyperplanes in $\operatorname{Aff}_{Y}^{n}$. Now suppose that $X \rightarrow Y$ is a smooth map of schemes of relative dimension $n$. By a simple normal crossings divisor in $X$ relative to $Y$ we shall mean a closed subscheme $D \subset X$ such that $X$ has an affine Zariski-open cover $\left\{U_{i}\right\}$ such that each $U_{i}$ admits an etale map $f_{i}: U_{i} \rightarrow \operatorname{Aff}_{Y}^{n}$ so that $\left.D\right|_{U_{i}}$ is the (scheme-theoretic) pre-image of $\operatorname{Coord}_{Y}^{n}$. In the case that $Y$ is just the spectrum of a field we will refer simply to a simple normal crossings divisor in $X$.

Suppose that $Y$ is locally noetherian and separated and that the connected components of $Y$ are irreducible. If $S$ is a finite set of irreducible components of $D$ we will set

$$
D_{S}=\bigcap_{E \in S} E
$$

It is smooth over $Y$. We will also set

$$
D^{(s)}=\coprod_{\# S=s} D_{S}
$$

If $E$ is an irreducible component of $D^{(s)}$ then the set $S(E)$ of irreducible components of $D$ containing $E$ has cardinality $s$. If $\geq$ is a total order on the set of irreducible components of $D$, we can define a delta set $\mathcal{S}(D, \geq$ ), or simply $\mathcal{S}(D)$, as follows. (For the definition of 'delta set', see, for instance, [25]. We can, if we prefer to be more abstract, replace $\mathcal{S}(D, \geq)$ by the associated simplicial set.) The $n$ cells consist of all irreducible components of $D^{(n+1)}$. If $E$ is such an irreducible component and if $i \in\{0, \ldots, n\}$ then the image of $E$ under the face map $d_{i}$ is the unique irreducible component of

$$
\bigcap_{F \in S(E)_{i}} F
$$

which contains $E$. Here $S(E)_{i}$ equals $S(E)$ with its $(i+1)$ th smallest element removed. The topological realization $|\mathcal{S}(D, \geq)|$ does not depend on the total order $\geq$, so we will often write $|\mathcal{S}(D)|$.

We record a general observation about log de Rham complexes and divisors with simple normal crossings, which is probably well known. We include a proof because it is of crucial importance for our argument.

Lemma 2.3 Suppose that $Y$ is a smooth scheme of finite type over a field $k$ and that $Z \subset Y$ is a divisor with simple normal crossings. Let $Z_{1}, \ldots, Z_{m}$ denote the distinct irreducible components of $Z$ and set

$$
Z_{S}=\bigcap_{j \in S} Z_{j} \subset Y
$$


(in particular $Z_{\emptyset}=Y$ ), and

$$
Z^{(s)}=\coprod_{\# S=s} Z_{S}
$$

Let is $\left(\right.$ resp. $\left.i^{(s)}\right)$ denote the natural maps $Z_{S} \rightarrow Y\left(\right.$ resp. $Z^{(s)} \rightarrow Y$ ). Also let $\mathcal{I}_{Z}$ denote the ideal of definition of $Z$.

There is a double complex

$$
i_{*}^{(s)} \Omega_{Z^{(s)}}^{r}
$$

with maps

$$
d: i_{*}^{(s)} \Omega_{Z^{(s)}}^{r} \longrightarrow i_{*}^{(s)} \Omega_{Z^{(s)}}^{r+1}
$$

and

$$
i_{*}^{(s)} \Omega_{Z^{(s)}}^{r} \longrightarrow i_{*}^{(s+1)} \Omega_{Z^{(s+1)}}^{r}
$$

being the sum of the maps

$$
i_{S, *} \Omega_{Z_{S}}^{r} \longrightarrow i_{S^{\prime}, *} \Omega_{Z_{S^{\prime}}}^{r}
$$

which are

- 0 if $S \not \subset S^{\prime}$,

- and $(-1)^{\#\{i \in S: i<j\}}$ times the natural pull-back if $S \cup\{j\}=S^{\prime}$.

The natural inclusions

$$
\Omega_{Y}^{r}(\log \mathcal{M}(Z)) \otimes \mathcal{I}_{Z} \longrightarrow \Omega_{Y}^{r}
$$

give rise to a map of complexes

$$
\Omega_{Y}^{\bullet}(\log \mathcal{M}(Z)) \otimes \mathcal{I}_{Z} \longrightarrow \Omega_{Y}^{\bullet}=i_{*}^{(0)} \Omega_{Z^{(0)}}^{\bullet}
$$

For fixed $r$ the simple complexes

$$
(0) \longrightarrow \Omega_{Y}^{r}(\log \mathcal{M}(Z)) \otimes \mathcal{I}_{Z} \longrightarrow i_{*}^{(0)} \Omega_{Z^{(0)}}^{r} \longrightarrow i_{*}^{(1)} \Omega_{Z^{(1)}}^{r} \longrightarrow \cdots
$$

are exact.

Proof Only the last assertion is not immediate. So consider the last assertion. We can work Zariski locally, so we may assume that the complex is pulled back from the corresponding complex for the case $Y=\operatorname{Spec} k\left[X_{1}, \ldots, X_{d}\right]$ and $Z$ is given by $X_{1} X_{2} \ldots X_{m}=0$. In this case we take $Z_{j}$ to be the scheme $X_{j}=0$, for $j=1, \ldots, m$. In this case

$$
\Omega_{Y}^{r}(\log \mathcal{M}(Z)) \otimes \mathcal{I}_{Z}=\bigoplus_{T} k\left[X_{1}, \ldots, X_{d}\right]\left(\prod_{j=1, j \notin T}^{m} X_{j}\right) \bigwedge_{j \in T} d X_{j}
$$


where $T$ runs over $r$ element subsets of $\{1, \ldots, d\}$. On the other hand

$$
i_{S, *} \Omega_{Z_{S}}^{r}=\bigoplus_{T} k\left[X_{1}, \ldots, X_{d}\right] /\left(X_{j}\right)_{j \in S} \bigwedge_{j \in T} d X_{j}
$$

where $T$ runs over $r$ element subsets of $\{1, \ldots, d\}-S$. Thus it suffices to show that, for each subset $T \subset\{1, \ldots, d\}$ the sequence

$$
\begin{gathered}
(0) \longrightarrow\left(\prod_{j=1, j \notin T}^{m} X_{j}\right) k\left[X_{1}, \ldots, X_{d}\right] \longrightarrow k\left[X_{1}, \ldots, X_{d}\right] \longrightarrow \cdots \\
\quad \ldots \longrightarrow \bigoplus_{\# S=s, S \cap T=\emptyset}^{\longrightarrow} k\left[X_{1}, \ldots, X_{d}\right] /\left(X_{j}\right)_{j \in S} \longrightarrow \cdots
\end{gathered}
$$

is exact, where $S \subset\{1, \ldots, m\}$. The sequence for $T \subset\{1, \ldots, d\}$ is obtained from the sequence for $\emptyset \subset\{1, \ldots, m\}-T$ by tensoring over $k$ with $k\left[X_{j}\right]_{j \in T \cup\{m+1, \ldots, d\}}$, and so we only need treat the case $m=d$ and $T=\emptyset$.

If $\mu$ is a monomial in the variables $X_{1}, \ldots, X_{m}$, let $R(\mu)$ denote the subset of $\{1, \ldots, m\}$ consisting of the indices $j$ for which $X_{j}$ does not occur in $\mu$. Then our complex is the direct sum over $\mu$ of the complexes

$$
(0) \longrightarrow A_{\mu} \longrightarrow k \longrightarrow \cdots \longrightarrow \bigoplus_{S \subset R(\mu), \# S=s} k \longrightarrow \cdots
$$

where $A_{\mu}=k$ if $R(\mu)=\emptyset$ and $=(0)$ otherwise. So it suffices to prove this latter complex exact for all $\mu$. If $R(\mu)=\emptyset$ then it becomes

$$
(0) \longrightarrow k \longrightarrow k \longrightarrow(0) \longrightarrow(0) \longrightarrow \cdots,
$$

which is clearly exact. If $R(\mu) \neq \emptyset$, our complex becomes

$$
(0) \longrightarrow k \longrightarrow \bigoplus_{S \subset R(\mu), \# S=1} k \longrightarrow \cdots \longrightarrow \bigoplus_{S \subset R(\mu), \# S=s} k \longrightarrow \cdots
$$

If we suppress the first $k$, this is the complex that computes the simplicial cohomology with $k$-coefficients of the simplex with $\# R(\mu)$ vertices. Thus it is exact everywhere except $\bigoplus_{S \subset R(\mu), \# S=1} k$ and the kernel of

$$
\bigoplus_{S \subset R(\mu), \# S=1} k \rightarrow \bigoplus_{S \subset R(\mu), \# S=2} k
$$

is just $k$. The desired exactness follows.

\subsection{Torus embeddings}

We will now discuss relative torus embeddings, crucially in the context of infinite fans.

If $W$ is a real vector space with dual $W^{\vee}$ and if $A \subset W$ is a subspace we set

$$
A^{\vee}=\left\{\chi \in W^{\vee}: \chi(A) \subset \mathbb{R}_{\geq 0}\right\}
$$

and

$$
A^{\vee, 0}=\left\{\chi \in W^{\vee}: \chi(A-\{0\}) \subset \mathbb{R}_{>0}\right\}
$$


and

$$
A^{\perp}=\left\{\chi \in W^{\vee}: \chi(A)=\{0\}\right\}
$$

We will suppose that $Y / \operatorname{Spec} R_{0}$ is flat and locally of finite type. To simplify the notation, for now we will restrict to the case of a split torus $S / Y$ with $Y$ connected. We will record the (trivial) generalization to the case of a disconnected base below. Thus we can think of $X^{*}(S)$ and $X_{*}(S)$ as abelian groups, rather than as locally constant sheaves on $Y$, i.e. we replace the sheaf by its global sections over $Y$. We will let $T / Y$ denote an $S$-torsor.

By a rational polyhedral cone $\sigma \subset X_{*}(S)_{\mathbb{R}}$ we mean a non-empty subset consisting of all $\mathbb{R}_{\geq 0}$-linear combinations of a finite set of elements of $X_{*}(S)$, but which contains no complete line through 0 . (We include the case $\sigma=\{0\}$. The notion we define here is sometimes called a 'non-degenerate rational polyhedral cone'.) By the interior $\sigma^{0}$ of $\sigma$ we shall mean the complement in $\sigma$ of all its proper faces. (We consider $\sigma$ as a face of $\sigma$, but not a proper face.) We call $\sigma$ smooth if it consists of all $\mathbb{R}_{\geq 0}$-linear combinations of a subset of a $\mathbb{Z}$-basis of $X_{*}(S)$. Note that any face of a smooth cone is smooth. Moreover we set

$$
T_{\sigma}=\text { Spec } \bigoplus_{\chi \in X^{*}(S) \cap \sigma^{\vee}} \mathcal{L}_{T}(\chi)
$$

Then $T_{\sigma}$ is a scheme over $Y$ with an action of $S$ and there is a natural $S$-equivariant dense open embedding $T \hookrightarrow T_{\sigma}$. If $\sigma^{\prime} \subset \sigma$ there is a natural map $T_{\sigma^{\prime}} \rightarrow T_{\sigma}$ compatible with the embeddings of $T$. If $f: Y^{\prime} \rightarrow Y$ then $T_{\sigma} / Y$ pulls back under $f$ to $\left(f^{*} T\right)_{\sigma} / Y^{\prime}$ compatibly with the maps $T_{\sigma^{\prime}} \hookrightarrow T_{\sigma}$ for $\sigma^{\prime} \subset \sigma$.

Suppose that $\Sigma_{0}$ is a set of faces of $\sigma$ such that

- $\{0\} \notin \Sigma_{0}$,

- and, if $\tau^{\prime} \supset \tau \in \Sigma_{0}$, then $\tau^{\prime} \in \Sigma_{0}$.

In this case define

$$
\left|\Sigma_{0}\right|^{0}=\sigma-\bigcup_{\tau \notin \Sigma_{0}} \tau
$$

Thus

$$
\left|\Sigma_{0}\right|^{0, \vee, 0} \cap \sigma^{\vee}=\sigma^{\vee}-\bigcup_{\tau \in \Sigma_{0}} \tau^{\perp}
$$

Then we define $\partial_{\Sigma_{0}} T_{\sigma} \subset T_{\sigma}$ to be the closed subscheme defined by the sheaf of ideals

$$
\bigoplus_{\chi \in X^{*}(S) \cap\left|\Sigma_{0}\right|^{0, \vee, 0} \cap \sigma^{\vee}} \mathcal{L}_{T}(\chi) \subset \bigoplus_{\chi \in X^{*}(S) \cap \sigma^{\vee}} \mathcal{L}_{T}(\chi) .
$$

If $\Sigma_{0}$ contains all the faces of $\sigma$ other than $\{0\}$ we will write $\partial T_{\sigma}$ for $\partial_{\Sigma_{0}} T_{\sigma}$. Note that $\partial_{\emptyset} T_{\sigma}=\emptyset$. If $\sigma^{\prime}$ is a face of $\sigma$ then under the open embedding

$$
T_{\sigma^{\prime}} \hookrightarrow T_{\sigma}
$$


$\partial_{\Sigma_{0}} T_{\sigma}$ pulls back to $\partial_{\left\{\tau \in \Sigma_{0}: \tau \subset \sigma^{\prime}\right\}} T_{\sigma^{\prime}}$.

By a fan in $X_{*}(S)_{\mathbb{R}}$ we shall mean a non-empty collection $\Sigma$ of rational polyhedral cones $\sigma \subset X_{*}(S)_{\mathbb{R}}$ which satisfy

- if $\sigma \in \Sigma$, so is each face of $\sigma$,

- if $\sigma, \sigma^{\prime} \in \Sigma$ then $\sigma \cap \sigma^{\prime}$ is a face of $\sigma$ and of $\sigma^{\prime}$.

Note that unless otherwise stated we will not assume that $\Sigma$ is finite. We call $\Sigma$ smooth if each $\sigma \in \Sigma$ is smooth. We will call $\Sigma$ full if every element of $\Sigma$ is contained in an element of $\Sigma$ with the same dimension as $X_{*}(S)_{\mathbb{R}}$. Define

$$
|\Sigma|=\bigcup_{\sigma \in \Sigma} \sigma .
$$

We call $\Sigma^{\prime}$ a refinement of $\Sigma$ if each $\sigma^{\prime} \in \Sigma^{\prime}$ is a subset of some element of $\Sigma$ and each element $\sigma \in \Sigma$ is a finite union of elements of $\Sigma^{\prime}$.

Lemma 2.4 (1) If $\Sigma$ is a fan and $\Sigma^{\prime} \subset \Sigma$ is a finite cardinality subfan then there is a refinement $\widetilde{\Sigma}$ of $\Sigma$ with the following properties:

- any element of $\Sigma$ which is smooth also lies in $\widetilde{\Sigma}$;

- any element of $\widetilde{\Sigma}$ contained in an element of $\Sigma^{\prime}$ is smooth;

- and if $\sigma^{\prime} \in \Sigma-\widetilde{\Sigma}$ then $\sigma^{\prime}$ has a non-smooth face lying in $\Sigma^{\prime}$.

(2) Any fan $\Sigma$ has a smooth refinement $\Sigma^{\prime}$ such that every smooth cone $\sigma \in \Sigma$ also lies in $\Sigma^{\prime}$.

Proof The first part is proved just as for finite fans by making a finite series of elementary subdivisions by 1 cones that lie in some element $\sigma^{\prime} \in \Sigma^{\prime}$ but not in any of its smooth faces. See, for instance, section 2.6 of [26].

For the second part, consider the set $\mathcal{S}$ of pairs $(\widetilde{\Sigma}, \Delta)$ where $\widetilde{\Sigma}$ is a refinement of $\Sigma$ and $\Delta$ is a subfan of $\Sigma$ such that

- every smooth element of $\Sigma$ lies in $\widetilde{\Sigma}$;

- and if $\sigma \in \widetilde{\Sigma}$ is contained in an element of $\Delta$ then $\sigma$ is smooth.

It suffices to show that $\mathcal{S}$ contains an element $(\widetilde{\Sigma}, \Delta)$ with $\Delta=\Sigma$.

If $(\widetilde{\Sigma}, \Delta) \in \mathcal{S}$ and $\sigma \in \Sigma$ we define $\widetilde{\Sigma}(\sigma)$ to be the set of elements of $\widetilde{\Sigma}$ contained in $\sigma$. We define a partial order on $\mathcal{S}$ by decreeing that $(\widetilde{\Sigma}, \Delta) \geq\left(\widetilde{\Sigma}^{\prime}, \Delta^{\prime}\right)$ if and only if the following conditions are satisfied:

- $\widetilde{\Sigma}$ refines $\widetilde{\Sigma}^{\prime}$;

- $\Delta \supset \Delta^{\prime}$

- $\tilde{\Sigma}^{\prime}(\sigma)=\tilde{\Sigma}(\sigma)$ unless $\sigma$ has a face that is contained in an element of $\Delta$ but in no element of $\Delta^{\prime}$.

Suppose that $\mathcal{S}^{\prime} \subset \mathcal{S}$ is totally ordered. Set

$$
\Delta=\bigcup_{\left(\widetilde{\Sigma}^{\prime}, \Delta^{\prime}\right) \in \mathcal{S}^{\prime}} \Delta^{\prime}
$$


and let $\widetilde{\Sigma}$ denote the set of cones $\sigma^{\prime}$ which lie in $\widetilde{\Sigma}^{\prime}$ for all sufficiently large elements of $\left(\widetilde{\Sigma}^{\prime}, \Delta^{\prime}\right) \in \mathcal{S}^{\prime}$. If $\sigma \in \Sigma$ then we can choose $\left(\widetilde{\Sigma}^{\prime}, \Delta^{\prime}\right) \in \mathcal{S}^{\prime}$ so that the number of faces of $\sigma$ in $\Delta^{\prime}$ is maximal. If $\left(\widetilde{\Sigma}^{\prime}, \Delta^{\prime}\right) \leq\left(\widetilde{\Sigma}^{\prime \prime}, \Delta^{\prime \prime}\right) \in \mathcal{S}^{\prime}$ then $\widetilde{\Sigma}^{\prime}(\sigma)=\widetilde{\Sigma}^{\prime \prime}(\sigma)$. Thus $\widetilde{\Sigma}(\sigma)=\widetilde{\Sigma}^{\prime}(\sigma)$. We conclude that $\widetilde{\Sigma}$ is a refinement of $\Sigma$. Thus $(\widetilde{\Sigma}, \Delta) \in \mathcal{S}$ and it is an upper bound for $\mathcal{S}^{\prime}$.

By Zorn's lemma $\mathcal{S}$ has a maximal element $(\widetilde{\Sigma}, \Delta)$. We will show that $\Delta=\Sigma$, which will complete the proof of the lemma. Suppose not. Choose $\sigma \in \Sigma-\Delta$. Set $\Delta^{\prime}$ to be the union of $\Delta$ and the faces of $\sigma$. Let $\widetilde{\Sigma}^{\prime}$ be a refinement of $\widetilde{\Sigma}$ such that

- any element of $\widetilde{\Sigma}$ which is smooth also lies in $\widetilde{\Sigma}^{\prime}$;

- any element of $\widetilde{\Sigma}^{\prime}$ contained in $\sigma$ is smooth;

- and if $\sigma^{\prime} \in \widetilde{\Sigma}-\widetilde{\Sigma}^{\prime}$ then $\sigma^{\prime}$ has a non-smooth face contained in $\sigma$.

Then $\left(\widetilde{\Sigma}^{\prime}, \Delta^{\prime}\right) \in \mathcal{S}$ and $\left(\widetilde{\Sigma}^{\prime}, \Delta^{\prime}\right)>(\widetilde{\Sigma}, \Delta)$, a contradiction.

To a fan $\Sigma$ one can attach a connected scheme $T_{\Sigma}$ that is separated, locally (on $T_{\Sigma}$ ) of finite type and flat over $Y$ of relative dimension $\operatorname{dim}_{\mathbb{R}} X_{*}(S)_{\mathbb{R}}$, together with an action of $S$ and an $S$-equivariant dense open embedding $T \hookrightarrow T_{\Sigma}$ over $Y$. The scheme $T_{\Sigma}$ has an open cover by the $T_{\sigma}$ for $\sigma \in \Sigma$ such that $T_{\sigma^{\prime}} \subset T_{\sigma}$ if and only if $\sigma^{\prime} \subset \sigma$. We write $\mathcal{O}_{T_{\Sigma}}$ for the structure sheaf of $T_{\Sigma}$. If $\Sigma$ is smooth then $T_{\Sigma} / Y$ is smooth. If $\Sigma$ is finite and $|\Sigma|=X_{*}(S)_{\mathbb{R}}$, then $T_{\Sigma} / Y$ is proper. If $\Sigma^{\prime} \subset \Sigma$ then $T_{\Sigma^{\prime}}$ can be identified with an open subscheme of $T_{\Sigma}$. If $\Sigma^{\prime}$ refines $\Sigma$ then there is an $S$-equivariant proper map

$$
T_{\Sigma^{\prime}} \rightarrow T_{\Sigma}
$$

which restricts to the identity on $T$ : Its restriction to $T_{\sigma^{\prime}}$ equals the map

$$
T_{\sigma^{\prime}} \longrightarrow T_{\sigma} \hookrightarrow T_{\Sigma}
$$

where $\sigma^{\prime} \subset \sigma \in \Sigma$.

By boundary data for $\Sigma$ we shall mean a proper subset $\Sigma_{0} \subset \Sigma$ such that $\Sigma-\Sigma_{0}$ is a fan. (Note that $\Sigma_{0}$ may not be closed under taking faces.) If $\Sigma_{0}$ is boundary data we define $\partial_{\Sigma_{0}} T_{\Sigma}$ to be the closed subscheme of $T_{\Sigma}$ with

$$
\left(\partial_{\Sigma_{0}} T_{\Sigma}\right) \cap T_{\sigma}=\partial_{\left\{\tau \in \Sigma_{0}: \tau \subset \sigma\right\}} T_{\sigma} .
$$

Note that

$$
\partial_{\Sigma_{0}} T_{\Sigma} \subset \bigcup_{\sigma \in \Sigma_{0}} T_{\sigma}
$$

Thus $\partial_{\Sigma_{0}} T_{\Sigma}$ has an open cover by the sets

$$
\left(\partial_{\Sigma_{0}} T_{\Sigma}\right)_{\sigma}=T_{\sigma} \cap \partial_{\Sigma_{0}} T_{\Sigma}
$$

as $\sigma$ runs over $\Sigma_{0}$. We write $\mathcal{I}_{\partial_{\Sigma_{0}} T_{\Sigma}}$ for the ideal sheaf in $\mathcal{O}_{T_{\Sigma}}$ defining $\partial_{\Sigma_{0}} T_{\Sigma}$. If $\Sigma_{0} \subset$ $\Sigma^{\prime} \subset \Sigma$ then

$$
\partial_{\Sigma_{0}} T_{\Sigma^{\prime}} \stackrel{\sim}{\longrightarrow} \partial_{\Sigma_{0}} T_{\Sigma}
$$


Note that $\left.\mathcal{I}_{\partial_{\Sigma_{0}} T_{\Sigma}}\right|_{T_{\sigma}}$ corresponds to the ideal

$$
\bigoplus_{\chi \in \mathfrak{X}_{\Sigma_{0}, \sigma, 1}} \mathcal{L}_{T}(\chi)
$$

of

$$
\bigoplus_{\chi \in X^{*}(S) \cap \sigma^{\vee}} \mathcal{L}_{T}(\chi)
$$

where

$$
\mathfrak{X}_{\Sigma_{0}, \sigma, 1}=X^{*}(S) \cap \sigma^{\vee}-\bigcup_{\tau \in \Sigma_{0}, \tau \subset \sigma} \tau^{\perp}
$$

and $\tau^{\perp}$ denotes the annihilator of $\tau$ in $X^{*}(S)_{\mathbb{R}}$. If we let $\mathfrak{X}_{\Sigma_{0}, \sigma, m}$ denote the set of sums of $m$ elements of $\mathfrak{X}_{\Sigma_{0}, \sigma, 1}$, then $\left.\mathcal{I}_{\partial_{\Sigma_{0}} T_{\Sigma}}^{m}\right|_{T_{\sigma}}$ corresponds to the ideal

$$
\bigoplus_{\chi \in \mathfrak{X}_{\Sigma_{0}, \sigma, m}} \mathcal{L}_{T}(\chi)
$$

If $\sigma \notin \Sigma_{0}$ then

$$
\mathfrak{X}_{\Sigma_{0}, \sigma, m}=X^{*}(S) \cap \sigma^{\vee}
$$

for all $m$. If on the other hand $\sigma \in \Sigma_{0}$ then

$$
\bigcap_{m} \mathfrak{X}_{\Sigma_{0}, \sigma, m}=\emptyset .
$$

(For if $\chi \in \sigma^{0} \cap X_{*}(S)$ then $\chi \geq m$ on $\mathfrak{X}_{\Sigma_{0}, \sigma, m}$.)

In the special case $\Sigma_{0}=\Sigma-\{\{0\}\}$ we will write $\partial T_{\Sigma}$ for $\partial_{\Sigma_{0}} T_{\Sigma}$ and $\mathcal{I}_{\partial T_{\Sigma}}$ for $\mathcal{I}_{\partial_{\Sigma_{0}} T_{\Sigma}}$. Then

$$
T=T_{\Sigma}-\partial T_{\Sigma}
$$

We will write $\mathcal{M}_{\Sigma} \rightarrow \mathcal{O}_{T_{\Sigma}}$ for the log structure corresponding to the closed embedding $\partial T_{\Sigma} \hookrightarrow T_{\Sigma}$. We will write $\Omega_{T_{\Sigma} / \operatorname{Spec} R_{0}}^{1}(\log \infty)$ for the log differentials previously denoted $\Omega_{T_{\Sigma} / \text { Spec } R_{0}}^{1}\left(\log \mathcal{M}_{\Sigma}\right)$.

If $\Sigma$ is smooth then $\partial T_{\Sigma}$ is a simple normal crossings divisor on $T_{\Sigma}$ relative to $Y$.

If $\Sigma_{0}$ is boundary data for $\Sigma$ we will set

$$
\left|\Sigma_{0}\right|=\{0\} \cup \bigcup_{\sigma \in \Sigma_{0}} \sigma .
$$

and

$$
\left|\Sigma_{0}\right|^{0}=\left|\Sigma_{0}\right|-\bigcup_{\sigma \in \Sigma-\Sigma_{0}} \sigma .
$$

We will call $\Sigma_{0}$ 
- open if $\left|\Sigma_{0}\right|^{0}$ is open in $X_{*}(S)_{\mathbb{R}}$

- finite if it has finite cardinality;

- locally finite if for every rational polyhedral cone $\tau \subset\left|\Sigma_{0}\right|$ (not necessarily in $\Sigma_{0}$ ) the intersection $\tau \cap\left|\Sigma_{0}\right|^{0}$ meets only finitely many elements of $\Sigma_{0}$. (We remark that although this condition may be intuitive in the case $\left|\Sigma_{0}\right|^{0}=\left|\Sigma_{0}\right|$, in other cases it may be less so.)

Let $\Sigma$ continue to denote a fan and $\Sigma_{0}$ boundary data for $\Sigma$. If $\sigma \in \Sigma$ we write

$$
\Sigma(\sigma)=\{\tau \in \Sigma: \tau \supset \sigma\} .
$$

If $\sigma \neq\{0\}$, then this is an example of boundary data for $\Sigma$. If $\sigma \in \Sigma_{0}$ then

$$
\Sigma(\sigma)=\left\{\tau \in \Sigma_{0}: \tau \supset \sigma\right\}
$$

and we will sometimes denote it $\Sigma_{0}(\sigma)$. If $\Sigma_{0}$ is locally finite then $\Sigma_{0}(\sigma)$ is finite for all $\sigma \in \Sigma_{0}$. If $\{0\} \neq \sigma \in \Sigma$ we write

$$
\partial_{\sigma} T_{\Sigma}=\partial_{\Sigma(\sigma)} T_{\Sigma}
$$

and

$$
\partial_{\sigma}^{0} T_{\Sigma}=\partial_{\sigma} T_{\Sigma}-\bigcup_{\sigma^{\prime} \supsetneq \sigma} \partial_{\sigma^{\prime}} T_{\Sigma}
$$

Sometimes we also write

$$
\partial_{\{0\}}^{0} T_{\Sigma}=T
$$

If $\Sigma_{0}$ is locally finite then the $\partial_{\sigma} T_{\Sigma}$ for $\sigma \in \Sigma_{0}$ form a locally finite closed cover of $\partial_{\Sigma_{0}} T_{\Sigma}$. Set theoretically we have

$$
\partial_{\sigma} T_{\Sigma}=\coprod_{\sigma^{\prime} \in \Sigma(\sigma)} \partial_{\sigma^{\prime}}^{0} T_{\Sigma}
$$

and

$$
\left(\partial_{\Sigma_{0}} T_{\Sigma}\right)_{\sigma}=\coprod_{\substack{\sigma^{\prime} \in \Sigma_{0} \\ \sigma^{\prime} \subset \sigma}} \partial_{\sigma^{\prime}}^{0} T_{\Sigma}
$$

and

$$
T_{\sigma}=\coprod_{\sigma^{\prime} \subset \sigma} \partial_{\sigma^{\prime}}^{0} T_{\Sigma}
$$

and

$$
\partial_{\Sigma_{0}} T_{\Sigma}=\coprod_{\sigma^{\prime} \in \Sigma_{0}} \partial_{\sigma^{\prime}}^{0} T_{\Sigma}
$$

If $\operatorname{dim} \sigma=1$ then $\partial_{\sigma}^{0} T_{\Sigma}=\partial T_{\sigma}$. 
Keep the notation of the previous paragraph. We define $S(\sigma)$ to be the split torus with cocharacter group $X_{*}(S)$ divided by the subgroup generated by $\sigma \cap X_{*}(S)$, and $T(\sigma)$ to be the push-out of $T$ to $S(\sigma)$. We also define $\bar{\Sigma}(\sigma)$ to be the set of images in $X_{*}(S(\sigma))_{\mathbb{R}}$ of elements of $\Sigma(\sigma)$. It is a fan for $X_{*}(S)_{\mathbb{R}} /\langle\sigma\rangle_{\mathbb{R}}$. [The main point to check is that if $\tau, \tau^{\prime} \in \Sigma(\sigma)$ then $\left(\tau \cap \tau^{\prime}\right)+\langle\sigma\rangle_{\mathbb{R}}=\left(\tau+\langle\sigma\rangle_{\mathbb{R}}\right) \cap\left(\tau^{\prime}+\langle\sigma\rangle_{\mathbb{R}}\right)$. To verify this suppose that $x \in \tau$ and $y \in \tau^{\prime}$ with $x-y \in\langle\sigma\rangle_{\mathbb{R}}$. Then $x-y=z-w$ with $z, w \in \sigma$. Thus $x+w=y+z \in \tau \cap \tau^{\prime}$ and $x+\langle\sigma\rangle_{\mathbb{R}}=(x+w)+\langle\sigma\rangle_{\mathbb{R}}$.] If $\sigma \in \Sigma_{0}$ we will sometimes write $\bar{\Sigma}_{0}(\sigma)$ for $\bar{\Sigma}(\sigma)$, as it depends only on $\Sigma_{0}$ and not on $\Sigma$. Then

$$
\partial_{\sigma}^{0} T_{\Sigma} \cong T(\sigma) \subset T(\sigma)_{\bar{\Sigma}(\sigma)} \cong \partial_{\sigma} T_{\Sigma}
$$

Thus $\partial_{\sigma} T_{\Sigma}$ is separated, locally (on the source) of finite type and flat over $Y$. The closed subscheme $\partial_{\sigma} T_{\Sigma}$ has codimension in $T_{\Sigma}$ equal to the dimension of $\sigma$. If $\Sigma(\sigma)$ is smooth then $\partial_{\sigma} T_{\Sigma}$ is smooth over $Y$.

If $\Sigma(\sigma)$ is open then $\partial_{\sigma} T_{\Sigma}$ satisfies the valuative criterion of properness over $Y$. If in addition $\Sigma(\sigma)$ is finite then $\partial_{\sigma} T_{\Sigma}$ is proper over $Y$. If $\Sigma_{0}$ is open, then $\partial_{\Sigma_{0}} T_{\Sigma}$ satisfies the valuative criterion of properness over $Y$. If in addition $\Sigma_{0}$ is finite then $\partial_{\Sigma_{0}} T_{\Sigma}$ is proper over $Y$.

The schemes $\partial_{\sigma_{1}} T_{\Sigma}, \ldots, \partial_{\sigma_{s}} T_{\Sigma}$ intersect if and only if $\sigma_{1}, \ldots, \sigma_{s}$ are all contained in some $\sigma \in \Sigma$. In this case the intersection equals $\partial_{\sigma} T_{\Sigma}$ for the smallest such $\sigma$. We set

$$
\partial_{i} T_{\Sigma}=\coprod_{\operatorname{dim} \sigma=i} \partial_{\sigma} T_{\Sigma}
$$

If $Y$ is irreducible then $T_{\Sigma}$ and each $\partial_{\sigma} T_{\Sigma}$ is irreducible. Moreover the irreducible components of $\partial T_{\Sigma}$ are the $\partial_{\sigma} T_{\Sigma}$ as $\sigma$ runs over one-dimensional elements of $\Sigma$. If $\Sigma$ is smooth then we see that $\mathcal{S}\left(\partial T_{\Sigma}\right)$ is the delta set with cells in bijection with the elements of $\Sigma-\{\{0\}\}$ and with the same 'face relations'. In particular it is in fact a simplicial complex and

$$
\left|\mathcal{S}\left(\partial T_{\Sigma}\right)\right|=(|\Sigma|-\{0\}) / \mathbb{R}_{>0}^{\times} .
$$

We say that $\left(\Sigma^{\prime}, \Sigma_{0}^{\prime}\right)$ refines $\left(\Sigma, \Sigma_{0}\right)$ if $\Sigma^{\prime}$ refines $\Sigma$ and $\Sigma^{\prime}-\Sigma_{0}^{\prime}$ is the set of elements of $\Sigma^{\prime}$ contained in some element of $\Sigma-\Sigma_{0}$. In this case $\partial_{\Sigma_{0}^{\prime}} T_{\Sigma^{\prime}}$ maps to $\partial_{\Sigma_{0}} T_{\Sigma}$, and in fact set theoretically $\partial_{\Sigma_{0}^{\prime}} T_{\Sigma^{\prime}}$ is the pre-image of $\partial_{\Sigma_{0}} T_{\Sigma}$ in $T_{\Sigma^{\prime}}$.

If $\Sigma$ is a fan, then by line bundle data for $\Sigma$ we mean a continuous function $\psi:|\Sigma| \rightarrow \mathbb{R}$, such that for each cone $\sigma \in \Sigma$, the restriction $\left.\psi\right|_{\sigma}$ equals some $\psi_{\sigma} \in X^{*}(S)$. To $\psi$ we can attach a line bundle $\mathcal{L}_{\psi}$ on $T_{\Sigma}$ : On $T_{\sigma}$ (with $\sigma \in \Sigma$ ) it corresponds to the $\bigoplus_{\chi \in \sigma^{\vee} \cap X^{*}(S)} \mathcal{L}_{T}(\chi)$-module

$$
\bigoplus_{\substack{\chi \in X^{*}(S) \\ \chi-\psi \geq 0 \text { on } \sigma}} \mathcal{L}_{T}(\chi) .
$$

Note that there are natural isomorphisms

$$
\mathcal{L}_{\psi} \otimes \mathcal{L}_{\psi^{\prime}} \cong \mathcal{L}_{\psi+\psi^{\prime}}
$$


and that

$$
\mathcal{L}_{\psi}^{\otimes-1} \cong \mathcal{L}_{-\psi}
$$

We have the following examples of line bundle data.

(1) $\mathcal{O}_{T_{\Sigma}}$ is the line bundle associated to $\psi \equiv 0$.

(2) If $\Sigma$ is smooth then $\mathcal{I}_{\partial T_{\Sigma}}$ is the line bundle associated to the unique such function $\psi_{\Sigma}$ which for every one-dimensional cone $\sigma \in \Sigma$ satisfies

$$
\psi_{\Sigma}\left(X_{*}(S) \cap \sigma\right)=\mathbb{Z}_{\geq 0}
$$

Suppose that $\alpha: S \rightarrow S^{\prime}$ is a surjective map of split tori over $Y$. Then $X^{*}(\alpha): X^{*}\left(S^{\prime}\right) \hookrightarrow$ $X^{*}(S)$ and $X_{*}(\alpha): X_{*}(S) \rightarrow X_{*}\left(S^{\prime}\right)$, the latter with finite cokernel. We call fans $\Sigma$ for $X_{*}(S)$ and $\Sigma^{\prime}$ for $X_{*}\left(S^{\prime}\right)$ compatible if for all $\sigma \in \Sigma$ the image $X_{*}(\alpha) \sigma$ is contained in some element of $\Sigma^{\prime}$. In this case the map $\alpha: T \rightarrow \alpha_{*} T$ extends to an $S$-equivariant map

$$
\alpha: T_{\Sigma} \longrightarrow\left(\alpha_{*} T\right)_{\Sigma^{\prime}}
$$

We will write

$$
\Omega_{T_{\Sigma} /\left(\alpha_{*} T\right)_{\Sigma^{\prime}}}^{1}(\log \infty)=\Omega_{T_{\Sigma} /\left(\alpha_{*} T\right)_{\Sigma^{\prime}}}^{1}\left(\log \mathcal{M}_{\Sigma} / \mathcal{M}_{\Sigma^{\prime}}\right) .
$$

If for all $\sigma^{\prime} \in \Sigma^{\prime}$ the pre-image $X_{*}(\alpha)^{-1}\left(\sigma^{\prime}\right)$ is a finite union of elements of $\Sigma$, then $\alpha: T_{\Sigma} \rightarrow\left(\alpha_{*} T\right)_{\Sigma^{\prime}}$ is proper.

If $\alpha$ is an isogeny, if $\Sigma$ and $\Sigma^{\prime}$ are compatible, and if every element of $\Sigma^{\prime}$ is a finite union of elements of $\Sigma$, then we call $\Sigma$ a quasi-refinement of $\Sigma^{\prime}$. In that case the map $\alpha: T_{\Sigma} \rightarrow\left(\alpha_{*} T\right)_{\Sigma^{\prime}}$ is proper.

Lemma 2.5 If $\alpha$ is surjective and \# coker $X_{*}(\alpha)$ is invertible on $Y$ then

$$
\alpha:\left(T_{\Sigma}, \mathcal{M}_{\Sigma}\right) \rightarrow\left(\left(\alpha_{*} T\right)_{\Sigma^{\prime}}, \mathcal{M}_{\Sigma^{\prime}}\right)
$$

is log smooth, and there is a natural isomorphism

$$
\left(X^{*}(S) / X^{*}(\alpha) X^{*}\left(S^{\prime}\right)\right) \otimes_{\mathbb{Z}} \mathcal{O}_{T_{\Sigma}} \stackrel{\sim}{\longrightarrow} \Omega_{T_{\Sigma} /\left(\alpha_{*} T\right)_{\Sigma^{\prime}}}^{1}(\log \infty) .
$$

Proof We can work Zariski locally on $T_{\Sigma}$. Thus we may replace $T_{\Sigma}$ by $T_{\sigma}$ and $\left(\alpha_{*} T\right)_{\Sigma^{\prime}}$ by $\left(\alpha_{*} T\right)_{\sigma^{\prime}}$ for cones $\sigma$ and $\sigma^{\prime}$ with $X_{*}(\alpha) \sigma \subset \sigma^{\prime}$. We may also replace $Y$ by an affine open subset $U$ such that $\left.T\right|_{U}$ is trivial, i.e. each $\mathcal{L}_{T}(\chi) \cong \mathcal{O}_{Y}$ compatibly with $\mathcal{L}_{T}(\chi) \otimes \mathcal{L}_{T}\left(\chi^{\prime}\right) \stackrel{\sim}{\rightarrow}$ $\mathcal{L}_{T}\left(\chi+\chi^{\prime}\right)$. Then the log structure on $T_{\sigma}$ has a chart $\mathbb{Z}\left[\sigma^{\vee} \cap X^{*}(S)\right] \rightarrow \mathcal{O}_{T_{\sigma}}$ sending $\chi$ to

$$
1 \in \mathcal{O}_{Y}(Y) \cong \mathcal{L}_{T}(\chi)
$$

Similarly the log structure on $\left(\alpha_{*} T\right)_{\sigma^{\prime}}$ has a chart $\mathbb{Z}\left[\left(\sigma^{\prime}\right)^{\vee} \cap X^{*}\left(S^{\prime}\right)\right] \rightarrow \mathcal{O}_{\left(\alpha_{*} T\right)_{\sigma^{\prime}}}$ sending $\chi$ to

$$
1 \in \mathcal{O}_{Y}(Y) \cong \mathcal{L}_{\alpha_{*} T}(\chi) .
$$


The lemma follows because

$$
X^{*}(\alpha): X^{*}\left(S^{\prime}\right) \longrightarrow X^{*}(S)
$$

is injective and the torsion subgroup of the cokernel is finite with order invertible on $Y$.

We will call pairs $\left(\Sigma, \Sigma_{0}\right)$ and $\left(\Sigma^{\prime}, \Sigma_{0}^{\prime}\right)$ of fans and boundary data for $S$ and $S^{\prime}$, respectively, compatible if $\Sigma$ and $\Sigma^{\prime}$ are compatible and if no cone of $\Sigma_{0}$ maps into any cone of $\Sigma^{\prime}-\Sigma_{0}^{\prime}$. In this case

$$
\partial_{\Sigma_{0}} T_{\Sigma} \longrightarrow \partial_{\Sigma_{0}^{\prime}}\left(\alpha_{*} T\right)_{\Sigma^{\prime}}
$$

We will call them strictly compatible if they are compatible and $\Sigma-\Sigma_{0}$ is the set of cones in $\Sigma$ mapping into some element of $\Sigma^{\prime}-\Sigma_{0}^{\prime}$.

Lemma 2.6 Suppose that $\alpha: S \rightarrow S^{\prime}$ is a surjective map of split tori, that $T / Y$ is an $S$-torsor and that $\left(\Sigma, \Sigma_{0}\right)$ and $\left(\Sigma^{\prime}, \Sigma_{0}^{\prime}\right)$ are strictly compatible fans with boundary data for $S$ and $S^{\prime}$, respectively. Then locally on $T_{\Sigma}$ there is a strictly positive integer $m$ such that

$$
\alpha^{*} \mathcal{I}_{\partial_{\Sigma_{0}^{\prime}}\left(\alpha_{*} T\right)_{\Sigma^{\prime}}} \supset \mathcal{I}_{\partial_{\Sigma_{0}} T_{\Sigma}}^{m}
$$

and

$$
\mathcal{I}_{\partial_{\Sigma_{0}} T_{\Sigma}} \supset \alpha^{*} \mathcal{I}_{\partial_{\Sigma_{0}^{\prime}}\left(\alpha_{*} T\right)_{\Sigma^{\prime}}}
$$

Proof We may work locally on $Y$ and so we may suppose that $Y=\operatorname{Spec} A$ is affine and that each $\mathcal{L}_{T}(\chi)$ is trivial. It also suffices to check the lemma locally on $T_{\Sigma}$. Thus we may suppose that $\Sigma$ consists of a cone $\sigma$ and all its faces. Let $\sigma^{\prime}$ denote the smallest element of $\Sigma^{\prime}$ containing the image of $\sigma$. Then we may further suppose that $\Sigma^{\prime}$ consists of $\sigma^{\prime}$ and all its faces. We may further suppose that $\sigma \in \Sigma_{0}$ and $\sigma^{\prime} \in \Sigma_{0}^{\prime}$, else there is nothing to prove.

Then

$$
T_{\Sigma}=\operatorname{Spec} \bigoplus_{\chi \in X^{*}(S) \cap \sigma^{\vee}} \mathcal{L}_{T}(\chi)
$$

and $\partial_{\Sigma_{0}} T_{\Sigma}$ is defined by

$$
\bigoplus_{\chi \in X^{*}(S) \cap\left|\Sigma_{0}\right|^{0, v, 0}} \mathcal{L}_{T}(\chi)
$$

Moreover $T_{\Sigma} \times_{\left(\alpha_{*} T\right)_{\Sigma^{\prime}}} \partial_{\Sigma_{0}^{\prime}}\left(\alpha_{*} T\right)_{\Sigma^{\prime}}$ is defined by

$$
\bigoplus_{\substack{\chi_{1} \in X^{*}\left(S^{\prime}\right) \cap\left|\Sigma_{0}^{\prime}\right| 0, v, 0 \\ \chi_{2} \in X^{*}(S) \cap \sigma^{\vee}}} \mathcal{L}_{T}\left(X^{*}(\alpha) \chi_{1}+\chi_{2}\right) .
$$

Thus it suffices to show that for some positive integer $m$ we have

$$
\begin{aligned}
X^{*}(S) \cap\left|\Sigma_{0}\right|^{0, \vee, 0} & \supset X^{*}(\alpha)\left(X^{*}\left(S^{\prime}\right) \cap\left|\Sigma_{0}^{\prime}\right|^{0, \vee, 0}\right)+\left(X^{*}(S) \cap \sigma^{\vee}\right) \\
& \supset m\left(X^{*}(S) \cap\left|\Sigma_{0}\right|^{0, \vee, 0}\right) .
\end{aligned}
$$


This is equivalent to

$$
\left|\Sigma_{0}\right|^{0, \vee, 0}=X^{*}(\alpha)\left|\Sigma_{0}^{\prime}\right|^{0, \vee, 0}+\sigma^{\vee}
$$

Suppose that $\chi_{1} \in\left|\Sigma_{0}^{\prime}\right|^{0, \vee, 0}$ and $\chi_{2} \in \sigma^{\vee}$. Then

$$
X^{*}(\alpha)\left(\chi_{1}\right)\left(\sigma-\left|\Sigma-\Sigma_{0}\right|\right)=\chi_{1}\left(X_{*}(\alpha)\left(\sigma-\left|\Sigma-\Sigma_{0}\right|\right)\right) \subset \chi_{1}\left(\sigma^{\prime}-\left|\Sigma^{\prime}-\Sigma_{0}^{\prime}\right|\right) \subset \mathbb{R}_{>0}
$$

and so

$$
\left(X^{*}(\alpha)\left(\chi_{1}\right)+\chi_{2}\right)\left(\sigma-\left|\Sigma-\Sigma_{0}\right|\right) \subset \mathbb{R}_{>0}
$$

Thus

$$
\left|\Sigma_{0}\right|^{0, \vee, 0} \supset X^{*}(\alpha)\left|\Sigma_{0}^{\prime}\right|^{0, \vee, 0}+\sigma^{\vee}
$$

Conversely suppose that $\chi \in\left|\Sigma_{0}\right|^{0, \vee, 0}$. Let $\tau$ denote the face of $\sigma$, where $\chi=0$. Then $\tau \in \Sigma-\Sigma_{0}$. Let $\tau^{\prime}$ denote the smallest face of $\sigma^{\prime}$ containing $X_{*}(\alpha) \tau$. Then $\tau^{\prime} \in \Sigma^{\prime}-\Sigma_{0}^{\prime}$. We can find $\chi_{1} \in\left|\Sigma_{0}^{\prime}\right|^{0, \vee, 0}$ with $\chi_{1}\left(\tau^{\prime}\right)=\{0\}$. Note that if $a \in \sigma$ and $\chi(a)=0$ then $\left(X^{*}(\alpha)\left(\chi_{1}\right)\right)(a)=0$. Thus we can find $\epsilon>0$ such that

$$
\chi-X^{*}(\alpha)\left(\epsilon \chi_{1}\right) \in \sigma^{\vee}
$$

It follows that

$$
\left|\Sigma_{0}\right|^{0, \vee, 0} \subset X^{*}(\alpha)\left|\Sigma_{0}^{\prime}\right|^{0, \vee, 0}+\sigma^{\vee}
$$

The lemma follows.

Suppose that $\left(\Sigma, \Sigma_{0}\right)$ and $\left(\Sigma^{\prime}, \Sigma_{0}^{\prime}\right)$ are strictly compatible. We will say that

- $\Sigma_{0}$ is open over $\Sigma_{0}^{\prime}$ if $\left|\Sigma_{0}\right|^{0}$ is open in $X_{*}(\alpha)^{-1}\left|\Sigma_{0}^{\prime}\right|^{0}$;

- and that $\Sigma_{0}$ is finite over $\Sigma_{0}^{\prime}$ if only finitely many elements of $\Sigma_{0}$ map into any element of $\Sigma_{0}^{\prime}$.

If $\alpha$ is an isogeny, if $\Sigma$ is a quasi-refinement of $\Sigma^{\prime}$ and if $\left(\Sigma, \Sigma_{0}\right)$ and $\left(\Sigma^{\prime}, \Sigma_{0}^{\prime}\right)$ are strictly compatible, then we call $\left(\Sigma, \Sigma_{0}\right)$ a quasi-refinement of $\left(\Sigma^{\prime}, \Sigma_{0}^{\prime}\right)$. In this case $\Sigma_{0}$ is open and finite over $\Sigma_{0}^{\prime}$.

Lemma 2.7 Suppose that $\alpha: S \rightarrow S^{\prime}$ is a surjective map of split tori, that $T / Y$ is an $S$-torsor, and that $\left(\Sigma, \Sigma_{0}\right)$ and $\left(\Sigma^{\prime}, \Sigma_{0}^{\prime}\right)$ are strictly compatible fans with boundary data for $S$ and $S^{\prime}$, respectively. If $\Sigma_{0}$ is locally finite and $\Sigma_{0}$ is open over $\Sigma_{0}^{\prime}$ then

$$
\partial_{\Sigma_{0}} T_{\Sigma} \longrightarrow \partial_{\Sigma_{0}^{\prime}}\left(\alpha_{*} T\right)_{\Sigma^{\prime}}
$$

satisfies the valuative criterion of properness. If in addition $\Sigma_{0}$ is finite over $\Sigma_{0}^{\prime}$ then this morphism is proper. 
Proof It suffices to show that if $\sigma \in \Sigma_{0}$ and if $\sigma^{\prime}$ is the smallest element of $\Sigma_{0}^{\prime}$ containing $X_{*}(\alpha) \sigma$, then

$$
\partial_{\sigma} T_{\Sigma} \longrightarrow \partial_{\sigma^{\prime}}\left(\alpha_{*} T\right)_{\Sigma^{\prime}}
$$

satisfies the valuative criterion of properness. However this is the map of toric varieties

$$
T(\sigma)_{\bar{\Sigma}_{0}(\sigma)} \longrightarrow\left(\alpha_{*} T\right)\left(\sigma^{\prime}\right)_{\bar{\Sigma}_{0}^{\prime}\left(\sigma^{\prime}\right)}
$$

As $\bar{\Sigma}_{0}(\sigma)$ is finite, it suffices to check that

$$
\bigcup_{\substack{\tau^{\prime} \supset \sigma^{\prime} \\ \tau^{\prime} \in \Sigma_{0}^{\prime}}} X_{*}(\alpha)^{-1}\left(\left(\tau^{\prime}\right)^{0}+\left\langle\sigma^{\prime}\right\rangle_{\mathbb{R}}\right)=\bigcup_{\substack{\tau \supset \sigma \\ \tau \in \Sigma_{0}}}\left(\tau^{0}+\langle\sigma\rangle_{\mathbb{R}}\right) .
$$

Choose a point $P \in \sigma^{0}$ such that

$$
X_{*}(\alpha) P \in\left(X_{*}(\alpha) \sigma\right)^{0} \subset\left(\sigma^{\prime}\right)^{0} .
$$

Then

$$
\left\langle\sigma^{\prime}\right\rangle_{\mathbb{R}}=\sigma^{\prime}+\mathbb{R} X_{*}(\alpha)(P)
$$

[To see this choose nonzero vectors $v_{i}$ in each one-dimensional face of $\sigma^{\prime}$. Then we can write $X_{*}(\alpha)(P)=\sum_{i} \mu_{i} v_{i}$ with each $\mu_{i}>0$. If $\lambda_{i} \in \mathbb{R}$, then for $\lambda$ sufficiently large $\lambda_{i}+\lambda \mu_{i} \in \mathbb{R}_{>0}$ for all $i$, and so

$$
\left.\sum_{i} \lambda_{i} v_{i}=\sum_{i}\left(\lambda_{i}+\lambda \mu_{i}\right) v_{i}-\lambda X_{*}(\alpha)(P) \in \sigma^{\prime}+\mathbb{R} X_{*}(\alpha)(P) .\right]
$$

Thus

$$
\left\langle\sigma^{\prime}\right\rangle_{\mathbb{R}}=\sigma^{\prime}+X_{*}(\alpha)\langle\sigma\rangle_{\mathbb{R}}
$$

Hence for all $\tau^{\prime} \in \Sigma_{0}^{\prime}$ with $\tau^{\prime} \supset \sigma^{\prime}$, we have

$$
\left(\tau^{\prime}\right)^{0}+\left\langle\sigma^{\prime}\right\rangle_{\mathbb{R}}=\left(\tau^{\prime}\right)^{0}+X_{*}(\alpha)\langle\sigma\rangle_{\mathbb{R}}
$$

and so

$$
X_{*}(\alpha)^{-1}\left(\left(\tau^{\prime}\right)^{0}+\left\langle\sigma^{\prime}\right\rangle_{\mathbb{R}}\right)=\langle\sigma\rangle_{\mathbb{R}}+X_{*}(\alpha)^{-1}\left(\tau^{\prime}\right)^{0} .
$$

We deduce that it suffices to check that

$$
\langle\sigma\rangle_{\mathbb{R}}+\bigcup_{\substack{\tau^{\prime} \supset \sigma^{\prime} \\ \tau^{\prime} \in \Sigma_{0}^{\prime}}} X_{*}(\alpha)^{-1}\left(\tau^{\prime}\right)^{0}=\langle\sigma\rangle_{\mathbb{R}}+\bigcup_{\substack{\tau \supset \sigma \\ \tau \in \Sigma_{0}}} \tau^{0}
$$


The left hand side certainly contains the right hand side, so it suffices to prove that for all $\tau^{\prime} \in \Sigma_{0}^{\prime}$ with $\tau^{\prime} \supset X_{*}(\alpha) \sigma$ we have

$$
\langle\sigma\rangle_{\mathbb{R}}+X_{*}(\alpha)^{-1} \tau^{\prime} \subset\langle\sigma\rangle_{\mathbb{R}}+\bigcup_{\substack{\tau \supset \sigma \\ \tau \in \Sigma_{0}}} \tau^{0} .
$$

Let $\pi$ denote the map

$$
\pi: X_{*}(S)_{\mathbb{R}} \rightarrow X_{*}(S)_{\mathbb{R}} /\langle\sigma\rangle_{\mathbb{R}}
$$

Because $X_{*}(\alpha)^{-1} \tau^{\prime}$ and $\bigcup_{\substack{\tau \supset \sigma \\ \tau \in \Sigma_{0}}} \tau^{0}$ are invariant under the action of $\mathbb{R}_{>0}^{\times}$it suffices to find an open set $U \subset X_{*}(S)_{\mathbb{R}}$ containing $P$ such that

$$
(\pi U) \cap \pi X_{*}(\alpha)^{-1} \tau^{\prime} \subset \pi \bigcup_{\substack{\tau \supset \sigma \\ \tau \in \Sigma_{0}}} \tau^{0},
$$

or equivalently such that

$$
U \cap\left(\langle\sigma\rangle_{\mathbb{R}}+X_{*}(\alpha)^{-1} \tau^{\prime}\right) \subset\langle\sigma\rangle_{\mathbb{R}}+\bigcup_{\substack{\tau \supset \sigma \\ \tau \in \Sigma_{0}}} \tau^{0} .
$$

Thus it suffices to find an open set $U \subset X_{*}(S)_{\mathbb{R}}$ containing $P$ such that

(1) $U \cap X_{*}(\alpha)^{-1}\left|\Sigma_{0}^{\prime}\right|^{0} \subset \bigcup_{\substack{\tau \supset \sigma \\ \tau \in \Sigma_{0}}} \tau^{0}$;

(2) $U \cap X_{*}(\alpha)^{-1} \tau^{\prime} \subset X_{*}(\alpha)^{-1}\left|\Sigma_{0}^{\prime}\right|^{0}$;

(3) and for all open $U^{\prime} \subset U$ containing $P$ we have $U^{\prime} \cap\left(\langle\sigma\rangle_{\mathbb{R}}+X_{*}(\alpha)^{-1} \tau^{\prime}\right)=U^{\prime} \cap$ $X_{*}(\alpha)^{-1} \tau^{\prime}$.

Moreover in order to find such a $U \ni P$ it suffices to find one satisfying each property independently and take their intersection.

One can find an open set $U \ni P$ satisfying the first property because

$$
\bigcup_{\substack{\tau \supset \sigma \\ \tau \in \Sigma_{0}}} \tau^{0} \subset\left|\Sigma_{0}\right|^{0} \subset X^{*}(\alpha)^{-1}\left|\Sigma_{0}^{\prime}\right|^{0}
$$

are both open inclusions.

To find $U \ni P$ satisfying the second condition we just need to avoid the faces of $X_{*}(\alpha)^{-1} \tau^{\prime}$ which do not contain $P$.

It remains to check that we can find an open $U \ni P$ satisfying the last condition. Suppose that $X_{*}(\alpha)^{-1} \tau^{\prime}$ is defined by inequalities $\chi_{i} \geq 0$ for $i=1, \ldots, r$ with $\chi_{i} \in X^{*}(S)_{\mathbb{R}}$. Suppose that $\chi_{i}=0$ on $\sigma$ for $i=1, \ldots, s$, but that $\chi_{i}(P)>0$ for $i=s+1, \ldots, r$. It suffices to choose $U$ so that $\chi_{i}>0$ on $U$ for $i=s+1, \ldots, r$. For then if $x \in X_{*}(\alpha)^{-1} \tau^{\prime}$ and $y \in\langle\sigma\rangle_{\mathbb{R}}$ with $x+y \in U$ we see that

$$
\chi_{i}(x+y)=\chi_{i}(x) \geq 0
$$

for $i=1, \ldots, s$, while $\chi_{i}(x+y)>0$ for $i=s+1, \ldots, r$. Thus for $U^{\prime} \subset U$ we have

$$
U^{\prime} \cap\left(\langle\sigma\rangle_{\mathbb{R}}+X_{*}(\alpha)^{-1} \tau^{\prime}\right)=U^{\prime} \cap X_{*}(\alpha)^{-1} \tau^{\prime},
$$

as desired. 
By a partial fan we will mean a collection $\Sigma_{0}$ of rational polyhedral cones satisfying

- $(0) \notin \Sigma_{0}$;

- if $\sigma_{1}, \sigma_{2} \in \Sigma_{0}$, then $\sigma_{1} \cap \sigma_{2}$ is a face of $\sigma_{1}$ and of $\sigma_{2}$;

- if $\sigma_{1}, \sigma_{2} \in \Sigma_{0}$, and if $\sigma \supset \sigma_{2}$ is a face of $\sigma_{1}$, then $\sigma \in \Sigma_{0}$.

(Again note that $\Sigma_{0}$ may not be closed under taking faces.) In this case we will let $\widetilde{\Sigma}_{0}$ denote the set of faces of elements of $\Sigma_{0}$ together with $\{0\}$. Then $\widetilde{\Sigma}_{0}$ and $\widetilde{\Sigma}_{0}-\Sigma_{0}$ are fans, and $\Sigma_{0}$ is boundary data for $\widetilde{\Sigma}_{0}$. [To see this suppose that $\tau_{i}$ is a face of $\sigma_{i} \in \Sigma_{0}$ for $i=1$, 2. Then $\sigma_{1} \cap \sigma_{2}$ is a face of $\sigma_{1}$ and so $\tau_{1} \cap \sigma_{2}=\tau_{1} \cap\left(\sigma_{1} \cap \sigma_{2}\right)$ is a face of $\sigma_{1} \cap \sigma_{2}$ and hence of $\sigma_{2}$. Thus $\tau_{1} \cap \tau_{2}=\tau_{2} \cap\left(\tau_{1} \cap \sigma_{2}\right)$ is a face of $\tau_{2}$.] If $\Sigma$ is a fan and $\Sigma_{0}$ is boundary data for $\Sigma$, then $\Sigma_{0}$ is a partial fan, and $\Sigma \supset \widetilde{\Sigma}_{0}$. Thus

$$
\partial_{\Sigma_{0}} T_{\Sigma} \cong \partial_{\Sigma_{0}} T_{\widetilde{\Sigma}_{0}}
$$

If $\Sigma_{0}$ and $\Sigma_{0}^{\prime}$ are partial fans we will say that $\Sigma_{0}$ refines $\Sigma_{0}^{\prime}$ if every element of $\Sigma_{0}$ is contained in an element of $\Sigma_{0}^{\prime}$ and if every element of $\Sigma_{0}^{\prime}$ is a finite union of elements of $\Sigma_{0}$. In this case $\widetilde{\Sigma}_{0}$ also refines $\widetilde{\Sigma}_{0}^{\prime}$.

If $\Sigma_{0}$ is a partial fan we will set

$$
\left|\Sigma_{0}\right|=\{0\} \cup \bigcup_{\sigma \in \Sigma_{0}} \sigma=\left|\widetilde{\Sigma}_{0}\right|
$$

and

$$
\left|\Sigma_{0}\right|^{0}=\left|\Sigma_{0}\right|-\bigcup_{\sigma \in \widetilde{\Sigma}_{0}-\Sigma_{0}} \sigma .
$$

We will call $\Sigma_{0}$

- smooth if each $\sigma \in \Sigma_{0}$ is smooth;

- full if every element of $\Sigma_{0}$ which is not a face of any other element of $\Sigma_{0}$, has the same dimension as $S$;

- open if $\left|\Sigma_{0}\right|^{0}$ is open in $X_{*}(S)_{\mathbb{R}}$;

- finite if it has finite cardinality;

- locally finite if for every rational polyhedral cone $\tau \subset\left|\Sigma_{0}\right|$ (not necessarily in $\Sigma_{0}$ ) the intersection $\tau \cap\left|\Sigma_{0}\right|^{0}$ meets only finitely many elements of $\Sigma_{0}$.

If $\Sigma_{0}$ is smooth, so is $\widetilde{\Sigma}_{0}$.

Suppose that $\Sigma_{0}$ is a partial fan. If $\Sigma \supset \widetilde{\Sigma}_{0}$ is a fan then the natural maps

$$
\partial_{\Sigma_{0}} T_{\widetilde{\Sigma}_{0}} \longrightarrow \partial_{\Sigma_{0}} T_{\Sigma}
$$

and

$$
\left(T_{\widetilde{\Sigma}_{0}}\right)_{\partial_{\Sigma_{0}} T}^{\wedge} \longrightarrow\left(T_{\Sigma}\right)_{\partial_{\Sigma_{0}} T}^{\wedge}
$$

are isomorphisms, and we will denote these schemes/formal schemes $\partial_{\Sigma_{0}} T$ and $T_{\Sigma_{0}}^{\wedge}$, respectively. Moreover the $\log$ structures induced on $T_{\Sigma_{0}}^{\wedge}$ by $\mathcal{M}_{\widetilde{\Sigma}_{0}}$ and by $\mathcal{M}_{\Sigma}$ are the same and we will denote them $\mathcal{M}_{\Sigma_{0}}^{\wedge}$. If $\Sigma_{0}^{\prime} \subset \Sigma_{0}$ is also a partial fan, then $T_{\Sigma_{0}^{\prime}}^{\wedge}$ can be identified with the completion of $T_{\Sigma_{0}}^{\wedge}$ along $\partial_{\Sigma_{0}^{\prime}} T$, and $\mathcal{M}_{\Sigma_{0}}^{\wedge}$ induces $\mathcal{M}_{\Sigma_{0}^{\prime}}^{\wedge}$. If $\sigma \in \widetilde{\Sigma}_{0}$ then we will let 


$$
\left(T_{\Sigma_{0}}^{\wedge}\right)_{\sigma}
$$

denote the restriction of $T_{\Sigma_{0}}^{\wedge}$ to the topological space $\left(\partial_{\Sigma_{0}} T_{\widetilde{\Sigma}_{0}}\right)_{\sigma}$. Thus the $\left(T_{\Sigma_{0}}^{\wedge}\right)_{\sigma}$ for $\sigma \in \Sigma_{0}$ form an affine open cover of $T_{\Sigma_{0}}^{\wedge}$. We have

$$
\left(T_{\Sigma_{0}}^{\wedge}\right)_{\{0\}}=\emptyset
$$

and

$$
\left(T_{\Sigma_{0}}^{\wedge}\right)_{\sigma_{1}} \cap\left(T_{\Sigma_{0}}^{\wedge}\right)_{\sigma_{2}}=\left(T_{\Sigma_{0}}^{\wedge}\right)_{\sigma_{1} \cap \sigma_{2}}
$$

If $\Sigma_{0}^{\prime}$ refines $\Sigma_{0}$ then there is an induced map

$$
T_{\Sigma_{0}^{\prime}}^{\wedge} \longrightarrow T_{\Sigma_{0}}^{\wedge}
$$

Continue to suppose that $\Sigma_{0}$ is a partial fan. We will call $\Sigma_{1} \subset \Sigma_{0}$ boundary data if, whenever $\sigma \in \Sigma_{0}$ contains $\sigma^{\prime} \in \Sigma_{1}$, then $\sigma \in \Sigma_{1}$. In this case $\Sigma_{1}$ is a partial fan and $T_{\Sigma_{1}}^{\wedge}$ is canonically identified with the completion of $T_{\Sigma_{0}}^{\wedge}$ along $\partial_{\Sigma_{1}} T_{\Sigma_{0}}$.

We will also use the following notation.

- $\mathcal{O}_{T_{\Sigma_{0}}}$ will denote the structure sheaf of $T_{\Sigma_{0}}^{\wedge}$.

- $\mathcal{I}_{T_{\Sigma_{0}}}$ will denote the completion of $\mathcal{I}_{\partial_{\Sigma_{0}}} T_{\widetilde{\Sigma}_{0}}$, an ideal of definition for $T_{\Sigma_{0}}$.

- $\mathcal{I}_{\partial, \Sigma_{0}}^{\wedge}$ will denote the completion of $\mathcal{I}_{\partial T_{\widetilde{\Sigma}_{0}}}$. Thus $\mathcal{I}_{T_{\Sigma_{0}}} \supset \mathcal{I}_{\partial, \Sigma_{0}}^{\wedge}$.

- $\Omega_{T_{\Sigma_{0}} / \operatorname{Spf} R_{0}}^{1}(\log \infty)$ will denote $\Omega_{T_{\Sigma_{0}} / \operatorname{Spf} R_{0}}^{1}\left(\log \mathcal{M}_{\Sigma}^{\wedge}\right)$, which is isomorphic to the completion of $\Omega_{T_{\widetilde{\Sigma}_{0}} / \operatorname{Spec} R_{0}}^{1}(\log \infty)$.

For $\sigma \in \widetilde{\Sigma}_{0}$ recall that $\left.\mathcal{I}_{\partial \Sigma_{0}}^{m} T_{\widetilde{\Sigma}_{0}}\right|_{T_{\sigma}}$ corresponds to the ideal

$$
\bigoplus_{\chi \in \mathfrak{X}_{\Sigma_{0}, \sigma, m}} \mathcal{L}_{T}(\chi)
$$

of

$$
\bigoplus_{\chi \in \sigma^{\vee} \cap X^{*}(S)} \mathcal{L}_{T}(\chi)
$$

Also recall that if $\sigma \notin \Sigma_{0}$ then

$$
\mathfrak{X}_{\Sigma_{0}, \sigma, m}=\sigma^{\vee} \cap X^{*}(S)
$$

for all $m$, while if $\sigma \in \Sigma_{0}$ then

$$
\bigcap_{m} \mathfrak{X}_{\Sigma_{0}, \sigma, m}=\emptyset
$$

By line bundle data for $\Sigma_{0}$ we mean a continuous function $\psi:\left|\Sigma_{0}\right| \rightarrow \mathbb{R}$, such that for each cone $\sigma \in \widetilde{\Sigma}_{0}$, the restriction $\left.\psi\right|_{\sigma}$ equals some $\psi_{\sigma} \in X^{*}(S)$. This is the same as line bundle data for the fan $\widetilde{\Sigma}_{0}$, and we will write $\mathcal{L}_{\psi}^{\wedge}$ for the line bundle on $T_{\Sigma_{0}}$, which is the completion of $\mathcal{L}_{\psi} / T_{\widetilde{\Sigma}_{0}}$. Note that 


$$
\mathcal{L}_{\psi}^{\wedge} \otimes \mathcal{L}_{\psi^{\prime}}^{\wedge}=\mathcal{L}_{\psi+\psi^{\prime}}^{\wedge}
$$

and that

$$
\left(\mathcal{L}_{\psi}^{\wedge}\right)^{\otimes-1}=\mathcal{L}_{-\psi}^{\wedge}
$$

We have the following examples of line bundle data.

(1) $\mathcal{O}_{T_{\Sigma_{0}}}$ is the line bundle associated to $\psi \equiv 0$.

(2) If $\Sigma_{0}$ is smooth then $\mathcal{I}_{\partial, \Sigma_{0}}^{\wedge}$ is the line bundle associated to the unique such function $\psi \widetilde{\Sigma}_{0}$ which for every one-dimensional cone $\sigma \in \widetilde{\Sigma}_{0}$ satisfies

$$
\psi_{\widetilde{\Sigma}_{0}}\left(X_{*}(S) \cap \sigma\right)=\mathbb{Z}_{\geq 0}
$$

Suppose that $\alpha: S \rightarrow S^{\prime}$ is a surjective map of tori, and that $\Sigma_{0}$ (resp. $\Sigma_{0}^{\prime}$ ) is a partial fan for $S$ (resp. $S^{\prime}$ ). We call $\Sigma_{0}$ and $\Sigma_{0}^{\prime}$ compatible if for every $\sigma \in \Sigma_{0}$ the image $X_{*}(\alpha) \sigma$ is contained in some element of $\Sigma_{0}^{\prime}$ but in no element of $\widetilde{\Sigma}_{0}^{\prime}-\Sigma_{0}^{\prime}$. In this case $\left(\widetilde{\Sigma}_{0}, \Sigma_{0}\right)$ and $\left(\widetilde{\Sigma}_{0}^{\prime}, \Sigma_{0}^{\prime}\right)$ are compatible, and there is a natural morphism

$$
\alpha:\left(T_{\Sigma_{0}}^{\wedge}, \mathcal{M}_{\Sigma_{0}}^{\wedge}\right) \longrightarrow\left(\left(\alpha_{*} T\right)_{\Sigma_{0}^{\prime}}^{\wedge}, \mathcal{M}_{\Sigma_{0}^{\prime}}^{\wedge}\right)
$$

We will write

$$
\Omega_{T_{\Sigma_{0}}^{\wedge} /\left(\alpha_{*} T\right)_{\Sigma_{0}^{\prime}}^{\wedge}}^{1}(\log \infty)=\Omega_{T_{\Sigma_{0}}^{\wedge} /\left(\alpha_{*} T\right)_{\Sigma_{0}^{\prime}}^{\wedge}}^{1}\left(\log \mathcal{M}_{\Sigma_{0}}^{\wedge} / \mathcal{M}_{\Sigma_{0}^{\prime}}^{\wedge}\right)
$$

The following lemma follows immediately from Lemma 2.5.

Lemma 2.8 If $\alpha$ is surjective and \#coker $X_{*}(\alpha)$ is invertible on $Y$ then there is a natural isomorphism

$$
\left(X^{*}(S) / X^{*}(\alpha) X^{*}\left(S^{\prime}\right)\right) \otimes_{\mathbb{Z}} \mathcal{O}_{T_{\Sigma_{0}}^{\wedge}} \stackrel{\sim}{\longrightarrow} \Omega_{T_{\Sigma_{0}}^{\wedge} /\left(\alpha_{*} T\right)_{\Sigma_{0}^{\prime}}^{\wedge}}^{1}(\log \infty)
$$

We will call $\Sigma_{0}$ and $\Sigma_{0}^{\prime}$ strictly compatible if they are compatible and if an element of $\widetilde{\Sigma}_{0}$ lies in $\Sigma_{0}$ if and only if it maps to no element of $\widetilde{\Sigma}_{0}^{\prime}-\Sigma_{0}^{\prime}$. In this case $\left(\widetilde{\Sigma}_{0}, \Sigma_{0}\right)$ and $\left(\widetilde{\Sigma}_{0}^{\prime}, \Sigma_{0}^{\prime}\right)$ are strictly compatible. We will say that

- $\Sigma_{0}$ is open over $\Sigma_{0}^{\prime}$ if $\left|\Sigma_{0}\right|^{0}$ is open in $X_{*}(\alpha)^{-1}\left|\Sigma_{0}^{\prime}\right|^{0}$;

- and that $\Sigma_{0}$ is finite over $\Sigma_{0}^{\prime}$ if only finitely many elements of $\Sigma_{0}$ map into any element of $\Sigma_{0}^{\prime}$.

If $\alpha$ is an isogeny, if $\Sigma_{0}$ and $\Sigma_{0}^{\prime}$ are strictly compatible and if every element of $\Sigma_{0}^{\prime}$ is a finite union of elements of $\Sigma_{0}$, then we call $\Sigma_{0}$ a quasi-refinement of $\Sigma_{0}^{\prime}$. In this case $\Sigma_{0}$ is open and finite over $\Sigma_{0}^{\prime}$. The next lemma follows immediately from Lemmas 2.6 and 2.7.

Lemma 2.9 Suppose that $\Sigma_{0}^{\prime}$ and $\Sigma_{0}$ are strictly compatible.

(1) $T_{\Sigma_{0}}^{\wedge}$ is the formal completion of $T_{\widetilde{\Sigma}_{0}}$ along $\partial_{\Sigma_{0}^{\prime}}\left(\alpha_{*} T\right)$, and $T_{\Sigma_{0}}^{\wedge}$ is locally (on the source) topologically of finite type over $\left(\alpha_{*} T\right)_{\Sigma_{0}^{\prime}}^{\wedge}$

(2) If $\Sigma_{0}$ is locally finite and if it is open and finite over $\Sigma_{0}^{\prime}$ then $T_{\Sigma_{0}}^{\wedge}$ is proper over $\left(\alpha_{*} T\right)_{\Sigma_{0}^{\prime}}^{\wedge}$ 
Corollary 2.10 If $\alpha$ is an isogeny, if $\Sigma_{0}$ is locally finite and if $\Sigma_{0}$ is a quasi-refinement of $\Sigma_{0}^{\prime}$ then $T_{\Sigma_{0}}^{\wedge}$ is proper over $\left(\alpha_{*} T\right)_{\Sigma_{0}^{\prime}}^{\wedge}$

If $\Sigma_{0}$ and $\Sigma_{0}^{\prime}$ are compatible partial fans and if $\Sigma_{1}^{\prime} \subset \Sigma_{0}^{\prime}$ is boundary data then $\Sigma_{0}\left(\Sigma_{1}^{\prime}\right)$ will denote the set of elements $\sigma \in \Sigma_{0}$ such that $X_{*}(\alpha) \sigma$ is contained in no element of $\Sigma_{0}^{\prime}-\Sigma_{1}^{\prime}$. It is boundary data for $\Sigma_{0}$. Moreover the formal completion of $T_{\Sigma_{0}}^{\wedge}$ along the reduced subscheme of $\left(\alpha_{*} T\right)_{\Sigma_{1}^{\prime}}^{\wedge}$ is canonically identified with $T_{\Sigma_{0}\left(\Sigma_{1}^{\prime}\right)}^{\wedge}$. If $\Sigma_{1}^{\prime}=\left\{\sigma^{\prime}\right\}$ is a singleton we will write $\Sigma_{0}\left(\sigma^{\prime}\right)$ for $\Sigma_{0}\left(\left\{\sigma^{\prime}\right\}\right)$.

\subsection{Cohomology of line bundles}

In this section we will compute the cohomology of line bundles on formal completions of torus embeddings. We will work throughout over a base scheme $Y$ which is connected, separated and flat and locally of finite type over $\operatorname{Spec} R_{0}$.

We start with some definitions. We continue to assume that $S / Y$ is a split torus, that $T / Y$ is an $S$-torsor, that $\Sigma_{0}$ is a partial fan and that $\psi$ is line bundle data for $\Sigma_{0}$. If $\sigma \in \widetilde{\Sigma}_{0}$ then we set

$$
\mathfrak{X}_{\Sigma_{0}, \psi, \sigma, 0}=\left\{\chi \in X^{*}(S) \cap \sigma^{\vee}: \chi \geq \psi \text { on } \sigma\right\} .
$$

For $m>0$ we define $\mathfrak{X}_{\Sigma_{0}, \psi, \sigma, m}$ to be the set of sums of an element of $\mathfrak{X}_{\Sigma_{0}, \psi, \sigma, 0}$ and an element of $\mathfrak{X}_{\Sigma_{0}, \sigma, m}$. If $\sigma \notin \Sigma_{0}$ then

$$
\mathfrak{X}_{\Sigma_{0}, \psi, \sigma, m}=\mathfrak{X}_{\Sigma_{0}, \psi, \sigma, 0}
$$

for all $m$, while if $\sigma \in \Sigma_{0}$

$$
\bigcap_{m} \mathfrak{X}_{\Sigma_{0}, \psi, \sigma, m}=\emptyset
$$

Further suppose that $\chi \in X^{*}(S)$.

- Set $Y_{\psi}(\chi)=\left\{x \in X^{*}(S)_{\mathbb{R}}:(\psi-\chi)(x)>0\right\}$.

- If $U \subset Y$ is open let $H_{\Sigma_{0}, \psi, m}^{j}(\chi)(U)$ denote the $j$ th cohomology of the Cech complex with $i$ th term

$$
\prod_{\substack{\left(\sigma_{0}, \ldots, \sigma_{i}\right) \in \Sigma_{0}^{i+1} \\ \chi \in \mathcal{X}_{\Sigma_{0}, \psi, \sigma_{0}} \cap \cdots \cap \sigma_{i}, 0 \\ \chi \notin \mathcal{X} \Sigma_{0}, \psi, \sigma_{0} \cap \cdots \cap \sigma_{i}, m}} \mathcal{L}_{T}(\chi)(U) .
$$

Note the examples:

(1) $Y_{0}(\chi) \cap\left|\Sigma_{0}\right|^{0}=\emptyset$ if and only if $\chi \in\left|\Sigma_{0}\right|^{\vee}$.

(2) $Y_{\psi_{\tilde{\Sigma}_{0}}}(\chi) \cap\left|\Sigma_{0}\right|^{0}=\emptyset$ if and only if $\chi \in\left|\Sigma_{0}\right|^{\vee, 0}$.

Also note that if $\Sigma_{0}$ is finite then, for $m$ large enough, $H_{\Sigma_{0}, \psi, m}^{j}(\chi)(U)$ does not depend on $m$. We will denote it simply $H_{\Sigma_{0}, \psi}^{j}(\chi)(U)$. It equals the cohomology of the Cech complex 


$$
\prod_{\substack{\left(\sigma_{0}, \ldots, \sigma_{i}\right) \in \Sigma_{0}^{i+1} \\ \sigma_{0} \cap \cdots \cap \sigma_{i} \in \Sigma_{0}}} \mathcal{L}_{T}(\chi)(U)
$$

\section{Lemma 2.11 If $U$ is connected then}

$$
H_{\Sigma_{0}, \psi}^{i}(\chi)(U)=H_{\left|\Sigma_{0}\right|^{0}-Y_{\psi}(\chi)}^{i}\left(\left|\Sigma_{0}\right|^{0}, \mathcal{L}_{T}(\chi)(U)\right)
$$

Proof Write $M$ for $\mathcal{L}_{T}(\chi)(U)$. We follow the argument of section 3.5 of [26]. As $\sigma_{0} \cap \cdots \cap$ $\sigma_{i} \cap\left|\Sigma_{0}\right|^{0}$ and $\sigma_{0} \cap \cdots \cap \sigma_{i} \cap\left|\Sigma_{0}\right|^{0} \cap Y_{\psi}(\chi)$ are convex, we see that

$$
\begin{aligned}
& H_{\left(\sigma_{0} \cap \cdots \cap \sigma_{i} \cap\left|\Sigma_{0}\right|^{0}\right)-Y_{\psi}(\chi)}^{j}\left(\sigma_{0} \cap \cdots \cap \sigma_{i} \cap\left|\Sigma_{0}\right|^{0}, M\right) \\
& \quad= \begin{cases}M & \text { if } j=0 \text { and }\left(\sigma_{0} \cap \cdots \cap \sigma_{i} \cap\left|\Sigma_{0}\right|^{0}\right) \cap Y_{\psi}(\chi)=\emptyset \\
(0) & \text { otherwise. }\end{cases}
\end{aligned}
$$

(See the first paragraph of section 3.5 of [26].) Thus the $i$ th term of our Cech complex becomes

$$
\prod_{\left(\sigma_{0}, \ldots, \sigma_{i}\right) \in \Sigma_{0}^{i+1}} H_{\left(\sigma_{0} \cap \cdots \cap \sigma_{i} \cap\left|\Sigma_{0}\right|^{0}\right)-Y_{\psi}(\chi)}^{0}\left(\sigma_{0} \cap \cdots \cap \sigma_{i} \cap\left|\Sigma_{0}\right|^{0}, M\right)
$$

Thus it suffices to show that the Cech complex with $i$ th term

$$
\prod_{\left(\sigma_{0}, \ldots, \sigma_{i}\right) \in \Sigma_{0}^{i+1}} H_{\left(\sigma_{0} \cap \cdots \cap \sigma_{i} \cap\left|\Sigma_{0}\right|^{0}\right)-Y_{\psi}(\chi)}^{0}\left(\sigma_{0} \cap \cdots \cap \sigma_{i} \cap\left|\Sigma_{0}\right|^{0}, M\right)
$$

computes

$$
H_{\left|\Sigma_{0}\right|^{0}-Y_{\psi}(\chi)}^{i}\left(\left|\Sigma_{0}\right|^{0}, M\right)
$$

To this end choose an injective resolution

$$
M \longrightarrow \mathcal{I}^{0} \longrightarrow \mathcal{I}^{1} \longrightarrow \cdots
$$

as sheaves of abelian groups on $\left|\Sigma_{0}\right|^{0}$, and consider the double complex

$$
\prod_{\left(\sigma_{0}, \ldots, \sigma_{i}\right) \in \Sigma_{0}^{i+1}} H_{\left(\sigma_{0} \cap \cdots \cap \sigma_{i} \cap\left|\Sigma_{0}\right|^{0}\right)-Y_{\psi}(\chi)}^{0}\left(\sigma_{0} \cap \cdots \cap \sigma_{i} \cap\left|\Sigma_{0}\right|^{0}, \mathcal{I}^{j}\right) .
$$

We compute the cohomology of the corresponding total complex in two ways. Firstly the $j$ th cohomology of the complex

$$
\begin{aligned}
& H_{\left(\sigma_{0} \cap \cdots \cap \sigma_{i} \cap\left|\Sigma_{0}\right|^{0}\right)-Y_{\psi}(\chi)}^{0}\left(\sigma_{0} \cap \cdots \cap \sigma_{i} \cap\left|\Sigma_{0}\right|^{0}, \mathcal{I}^{0}\right) \\
& \downarrow \\
& H_{\left(\sigma_{0} \cap \cdots \cap \sigma_{i} \cap\left|\Sigma_{0}\right|^{0}\right)-Y_{\psi}(\chi)}^{0}\left(\sigma_{0} \cap \cdots \cap \sigma_{i} \cap\left|\Sigma_{0}\right|^{0}, \mathcal{I}^{1}\right)
\end{aligned}
$$


equals

$$
H_{\left(\sigma_{0} \cap \cdots \cap \sigma_{i} \cap\left|\Sigma_{0}\right|^{0}\right)-Y_{\psi}(\chi)}^{j}\left(\sigma_{0} \cap \cdots \cap \sigma_{i} \cap\left|\Sigma_{0}\right|^{0}, M\right) .
$$

(See theorem 4.1, proposition 5.3 and theorem 5.5 of chapter II of [14].) This vanishes for $j>0$, and so the cohomology of our total complex is the same as the cohomology of the Cech complex with $i$ th term

$$
\prod_{\left(\sigma_{0}, \ldots, \sigma_{i}\right) \in \Sigma_{0}^{i+1}} H_{\left(\sigma_{0} \cap \cdots \cap \sigma_{i} \cap\left|\Sigma_{0}\right|^{0}\right)-Y_{\psi}(\chi)}^{0}\left(\sigma_{0} \cap \cdots \cap \sigma_{i} \cap\left|\Sigma_{0}\right|^{0}, M\right) .
$$

Thus it suffices to identify the cohomology of our double complex with

$$
H_{\left|\Sigma_{0}\right|^{0}-Y_{\psi}(\chi)}^{i}\left(\left|\Sigma_{0}\right|^{0}, M\right)
$$

For this it suffices to show that

$$
\begin{aligned}
& (0) \longrightarrow H_{\left|\Sigma_{0}\right|^{0}-Y_{\psi}(\chi)}^{0}\left(\left|\Sigma_{0}\right|^{0}, \mathcal{I}^{j}\right) \longrightarrow \prod_{\sigma_{0} \in \Sigma_{0}} H_{\sigma_{0} \cap\left|\Sigma_{0}\right|^{0}-Y_{\psi}(\chi)}^{0}\left(\sigma_{0} \cap\left|\Sigma_{0}\right|^{0}, \mathcal{I}^{j}\right) \\
& \quad \longrightarrow \prod_{\left(\sigma_{0}, \sigma_{1}\right) \in \Sigma_{0}^{2}} H_{\left(\sigma_{0} \cap \sigma_{1} \cap\left|\Sigma_{0}\right|^{0}\right)-Y_{\psi}(\chi)}^{0}\left(\sigma_{0} \cap \sigma_{1} \cap\left|\Sigma_{0}\right|^{0}, \mathcal{I}^{j}\right) \longrightarrow \cdots
\end{aligned}
$$

is exact for all $j$. Let $\widetilde{\mathcal{I}}^{j}$ denote the sheaf of discontinuous sections of $\mathcal{I}^{j}$, i.e. $\widetilde{\mathcal{I}}^{j}(V)$ denotes the set of functions which assign to each point of $x \in V$ an element of the stalk $\mathcal{I}_{x}^{j}$ of $\mathcal{I}^{j}$ at $x$. Then $\mathcal{I}^{j}$ is a direct summand of $\widetilde{\mathcal{I}}^{j}$ so it suffices to show that

$$
\begin{aligned}
& (0) \longrightarrow H_{\left|\Sigma_{0}\right|^{0}-Y_{\psi}(\chi)}^{0}\left(\left|\Sigma_{0}\right|^{0}, \widetilde{\mathcal{I}}^{j}\right) \longrightarrow \prod_{\sigma_{0} \in \Sigma_{0}} H_{\sigma_{0} \cap\left|\Sigma_{0}\right|^{0}-Y_{\psi}(\chi)}^{0}\left(\sigma_{0} \cap\left|\Sigma_{0}\right|^{0}, \widetilde{\mathcal{I}}^{j}\right) \\
& \quad \longrightarrow \prod_{\left(\sigma_{0}, \sigma_{1}\right) \in \Sigma_{0}^{2}} H_{\left(\sigma_{0} \cap \sigma_{1} \cap\left|\Sigma_{0}\right|^{0}\right)-Y_{\psi}(\chi)}^{0}\left(\sigma_{0} \cap \sigma_{1} \cap\left|\Sigma_{0}\right|^{0}, \widetilde{\mathcal{I}}^{j}\right) \longrightarrow \cdots
\end{aligned}
$$

is exact for all $j$. However $\widetilde{\mathcal{I}}^{j}$ is the direct product over $x$ in $\left|\Sigma_{0}\right|^{0}$ of the sky-scraper $\overline{\mathcal{I}}_{x}^{j}$ sheaf at $x$ with stalk $\mathcal{I}_{x}^{j}$. Thus it suffices to show that

$$
\begin{aligned}
& (0) \longrightarrow H_{\left|\Sigma_{0}\right|^{0}-Y_{\psi}(\chi)}^{0}\left(\left|\Sigma_{0}\right|^{0}, \overline{\mathcal{I}}_{x}^{j}\right) \longrightarrow \prod_{\sigma_{0} \in \Sigma_{0}} H_{\sigma_{0} \cap\left|\Sigma_{0}\right|^{0}-Y_{\psi}(\chi)}^{0}\left(\sigma_{0} \cap\left|\Sigma_{0}\right|^{0}, \overline{\mathcal{I}}_{x}^{j}\right) \\
& \quad \longrightarrow \prod_{\left(\sigma_{0}, \sigma_{1}\right) \in \Sigma_{0}^{2}} H_{\left(\sigma_{0} \cap \sigma_{1} \cap\left|\Sigma_{0}\right|^{0}\right)-Y_{\psi}(\chi)}^{0}\left(\sigma_{0} \cap \sigma_{1} \cap\left|\Sigma_{0}\right|^{0}, \overline{\mathcal{I}}_{x}^{j}\right) \longrightarrow \cdots
\end{aligned}
$$

is exact for all $x \in\left|\Sigma_{0}\right|^{0}$ and for all $j$. If $x \in Y_{\psi}(\chi) \cap\left|\Sigma_{0}\right|^{0}$ all the terms in this sequence are 0 , so the sequence is certainly exact. If $x \in\left|\Sigma_{0}\right|^{0}-Y_{\psi}(\chi)$, this sequence equals

$$
(0) \longrightarrow \mathcal{I}_{x}^{j} \longrightarrow \prod_{\substack{\sigma_{0} \in \Sigma_{0} \\ x \in \sigma}} \mathcal{I}_{x}^{j} \longrightarrow \prod_{\substack{\left(\sigma_{0}, \sigma_{1}\right) \in \Sigma_{0}^{2} \\ x \in\left(\sigma_{0} \cap \sigma_{1}\right)}} \mathcal{I}_{x}^{j} \longrightarrow \cdots
$$

A standard argument shows that this is indeed exact: Choose $\sigma \in \Sigma_{0}$ with $x \in \sigma$. Suppose

$$
\left(a\left(\sigma_{0}, \ldots, \sigma_{i}\right)\right) \in \operatorname{ker}\left(\prod_{\substack{\left(\sigma_{0}, \ldots, \sigma_{i}\right) \in \Sigma_{0}^{i+1} \\
x \in \sigma_{0} \cap \cdots \cap \sigma_{i}}} \mathcal{I}_{x}^{j} \longrightarrow \prod_{\begin{array}{c}
\left(\sigma_{0}, \ldots, \sigma_{i+1}\right) \in \Sigma_{0}^{i+2} \\
x \in \sigma_{0} \cap \cdots \cap \sigma_{i+1}
\end{array}} \mathcal{I}_{x}^{j}\right) .
$$


Define

$$
\left(a^{\prime}\left(\sigma_{0}, \ldots, \sigma_{i-1}\right)\right) \in \prod_{\substack{\left(\sigma_{0}, \ldots, \sigma_{i-1}\right) \in \Sigma_{0}^{i} \\ x \in \sigma_{0} \cap \cdots \cap \sigma_{i-1}}} \mathcal{I}_{x}^{j}
$$

by

$$
a^{\prime}\left(\sigma_{0}, \ldots, \sigma_{i-1}\right)=a\left(\sigma_{0}, \ldots, \sigma_{i-1}, \sigma\right) .
$$

If $\partial a^{\prime}$ denotes the image of $a^{\prime}$ in

$$
\prod_{\substack{\left(\sigma_{0}, \ldots, \sigma_{i}\right) \in \Sigma_{0}^{i+1} \\ x \in \sigma_{0} \cap \cdots \cap \sigma_{i}}} \mathcal{I}_{x}^{j}
$$

then

$$
\left(\partial a^{\prime}\right)\left(\sigma_{0}, \ldots, \sigma_{i}\right)=\sum_{k=0}^{i}(-1)^{k} a\left(\sigma_{0}, \ldots, \widehat{\sigma}_{k}, \ldots, \sigma_{i}, \sigma\right)=(-1)^{i} a\left(\sigma_{0}, \ldots, \sigma_{i}\right),
$$

i.e. $a=(-1)^{i} \partial a^{\prime}$.

In general we will let $H_{\left|\Sigma_{0}\right|^{0}-Y_{\psi}(\chi)}^{i}\left(\left|\Sigma_{0}\right|^{0}, \mathcal{L}_{T}(\chi)\right)$ denote the sheaf of $\mathcal{O}_{Y}$-modules on $Y$ associated to the pre-sheaf

$$
U \longmapsto H_{\left|\Sigma_{0}\right|^{0}-Y_{\psi}(\chi)}^{i}\left(\left|\Sigma_{0}\right|^{0}, \mathcal{L}_{T}(\chi)(U)\right) .
$$

Lemma 2.12 Let $Y$ be a connected, separated scheme which is flat and locally of finite type over an irreducible noetherian ring $R_{0}$, let $S / Y$ be a split torus, let $T / Y$ be an $S$-torsor, let $\Sigma_{0}$ be a partial fan for $S$, let $\psi$ be line bundle data for $\Sigma_{0}$, and let $\pi_{\Sigma_{0}}$ denote the map $T_{\Sigma_{0}}^{\wedge} \rightarrow Y$. Suppose that $\Sigma_{0}$ is finite, non-empty and open. Then

$$
R^{i} \pi_{\Sigma_{0}, *}^{\wedge} \mathcal{L}_{\psi}^{\wedge}=\prod_{\chi \in X^{*}(S)} H_{\left|\Sigma_{0}\right|^{0}-Y_{\psi}(\chi)}^{i}\left(\left|\Sigma_{0}\right|^{0}, \mathcal{L}_{T}(\chi)\right) .
$$

(Note that $R^{i} \pi_{\Sigma_{0, *}}^{\wedge} \mathcal{L}_{\psi}^{\wedge}$ may not be quasi-coherent on $Y$. Infinite products of quasi-coherent sheaves may not be quasi-coherent.)

Proof The left hand side is the sheaf associated to the pre-sheaf

$$
U \longmapsto H^{i}\left(\left.T_{\Sigma_{0}}^{\wedge}\right|_{U}, \mathcal{L}_{\psi}^{\wedge}\right)
$$

and the right hand side is the sheaf associated to the pre-sheaf

$$
U \longmapsto \prod_{\chi \in X^{*}(S)(U)} H_{\left|\Sigma_{0}\right|^{0}-Y_{\psi}(\chi)}^{i}\left(\left|\Sigma_{0}\right|^{0}, \mathcal{L}_{T}(\chi)(U)\right) .
$$

Thus it suffices to establish isomorphisms

$$
H^{i}\left(\left.T_{\Sigma_{0}}\right|_{U}, \mathcal{L}_{\psi}^{\wedge}\right) \cong \prod_{\chi \in X^{*}(S)(U)} H_{\left|\Sigma_{0}\right|^{0}-Y_{\psi}(\chi)}^{i}\left(\left|\Sigma_{0}\right|^{0}, \mathcal{L}_{T}(\chi)(U)\right),
$$

compatibly with restriction, for $U=\operatorname{Spec} A$, with $A$ noetherian and $\operatorname{Spec} A$ connected. 
Write $\partial_{\Sigma_{0}, m} T_{\widetilde{\Sigma}_{0}}$ for the closed subscheme of $T_{\widetilde{\Sigma}_{0}}$ defined by $\mathcal{I}_{\partial_{\Sigma_{0}}}^{m} T_{\widetilde{\Sigma}_{0}}$. It has the same underlying topological space as $\partial_{\Sigma_{0}} T_{\widetilde{\Sigma}_{0}}$. We will first compute

$$
H^{i}\left(\left.\partial_{\Sigma_{0}, m} T_{\widetilde{\Sigma}_{0}}\right|_{U}, \mathcal{L}_{\psi} / \mathcal{I}_{\partial_{\Sigma_{0}} T_{\widetilde{\Sigma}_{0}}^{m}} \mathcal{L}_{\psi}\right)
$$

using the affine cover of $\partial_{\Sigma_{0}, m} T_{\widetilde{\Sigma}_{0}}$ by the open sets $T_{\sigma}$ for $\sigma \in \Sigma_{0}$. This gives rise to a Cech complex with terms

$$
\prod_{\left(\sigma_{0}, \ldots, \sigma_{i}\right) \in \Sigma_{0}^{i+1}} \bigoplus_{\substack{\chi \in X^{*}(S) \\ \chi \in \mathfrak{X}_{\Sigma_{0}, \psi, \sigma_{0} \cap \cdots \cap \sigma_{i}, 0} \\ \chi \notin \mathcal{X}_{\Sigma_{0}, \psi, \sigma_{0} \cap \cdots \cap \sigma_{i}, m}}} \mathcal{L}_{T}(\chi)(U) .
$$

As $\Sigma_{0}$ is finite, we see that

$$
H^{i}\left(\left.\partial_{\Sigma_{0}, m} T_{\widetilde{\Sigma}_{0}}\right|_{U}, \mathcal{L}_{\psi} / \mathcal{I}_{\partial_{\Sigma_{0}} T_{\widetilde{\Sigma}_{0}}} \mathcal{L}_{\psi}\right)=\bigoplus_{\chi \in X^{*}(S)} H_{\Sigma_{0}, \psi, m}^{i}(\chi)(U)
$$

Because $A$ is noetherian, because $\partial_{\Sigma_{0}, m} T_{\widetilde{\Sigma}_{0}}$ is proper over Spec $A$ and because $\mathcal{L}_{\psi} / \mathcal{I}_{\partial_{\Sigma_{0}} T_{\widetilde{\Sigma}_{0}}^{m}} \mathcal{L}_{\psi}$ is a coherent sheaf on $\partial_{\Sigma_{0}, m} T_{\widetilde{\Sigma}_{0}}$, we see that the cohomology group $H^{i}\left(\partial_{\Sigma_{0}, m} T_{\widetilde{\Sigma}_{0}} \mid U, \mathcal{L}_{\psi} / \mathcal{I}_{\partial_{\Sigma_{0}}}^{m} T_{\widetilde{\Sigma}_{0}} \mathcal{L}_{\psi}\right)$ is a finitely generated $A$-module, and hence, for fixed $m$ and $i$, we see that the groups $H_{\Sigma_{0}, \psi, m}^{i}(\chi)(U)=(0)$ for all but finitely many $\chi$. In particular

$$
H^{i}\left(\left.\partial_{\Sigma_{0}, m} T_{\widetilde{\Sigma}_{0}}\right|_{U}, \mathcal{L}_{\psi} / \mathcal{I}_{\partial_{\Sigma_{0}} T_{\widetilde{\Sigma}_{0}}} \mathcal{L}_{\psi}\right)=\prod_{\chi \in X^{*}(S)} H_{\Sigma_{0}, \psi, m}^{i}(\chi)(U)
$$

Moreover, combining this observation with the fact that $\left\{H_{\Sigma_{0}, \psi, m}^{i}(\chi)(U)\right\}$ satisfies the Mittag-Leffler condition, we see that the system

$$
\left\{H^{i}\left(\left.\partial_{\Sigma_{0}, m} T_{\widetilde{\Sigma}_{0}}\right|_{U}, \mathcal{L}_{\psi} / \mathcal{I}_{\partial_{\Sigma_{0}}}^{m} T_{\widetilde{\Sigma}_{0}} \mathcal{L}_{\psi}\right)\right\}
$$

satisfies the Mittag-Leffler condition. Hence from proposition 0.13.3.1 of [23] we see that

$$
\begin{aligned}
H^{i}\left(\left.T_{\Sigma_{0}}^{\wedge}\right|_{U}, \mathcal{L}_{\psi}^{\wedge}\right) & \cong \lim _{\leftarrow m} H^{i}\left(\partial_{\Sigma_{0}, m} T_{\widetilde{\Sigma}_{0}} \mid U, \mathcal{L}_{\psi} / \mathcal{I}_{\partial_{\Sigma_{0}} T_{\widetilde{\Sigma}_{0}}} \mathcal{L}_{\psi}\right) \\
& \cong \prod_{\chi \in X^{*}(S)} \lim _{\leftarrow m} H_{\Sigma_{0}, \psi, m}^{i}(\chi)(U),
\end{aligned}
$$

and the present lemma follows from Lemma 2.11.

Lemma 2.13 Let $Y$ be a connected, separated scheme which is flat and locally of finite type over an irreducible noetherian ring $R_{0}, S / Y$ be a split torus, let $T / Y$ be an $S$-torsor, let $\Sigma_{\infty}$ be a partial fan for $S$, let

$$
\Sigma_{1} \subset \Sigma_{2} \subset \cdots
$$

be a nested sequence of partial fans with $\Sigma_{\infty}=\bigcup_{i} \Sigma_{i}$ and let $\psi$ be line bundle data for $\Sigma_{\infty}$. For $i=1,2,3, \ldots, \infty$ let $\pi_{\Sigma_{i}}^{\wedge}$ denote the map $T_{\Sigma_{i}}^{\wedge} \rightarrow Y$. 
Suppose that for $i \in \mathbb{Z}_{>0}$ the partial fan $\Sigma_{i}$ is finite, non-empty and open. Suppose also that for all $i \in \mathbb{Z}_{\geq 0}$ and all connected, noetherian, affine open sets $U \subset Y$, the inverse system

$$
\left\{H_{\left|\Sigma_{j}\right|^{0}-Y_{\psi}(\chi)}^{i}\left(\left|\Sigma_{j}\right|^{0}, \mathcal{O}_{Y}(U)\right)\right\}_{j}
$$

satisfies the Mittag-Leffler condition. Then

$$
R^{i} \pi_{\Sigma_{\infty}, *} \mathcal{L}_{\psi}^{\wedge} \cong \prod_{\chi \in X^{*}(S)} \lim _{\leftarrow j} H_{\left|\Sigma_{j}\right|^{0}-Y_{\psi}(\chi)}^{i}\left(\left|\Sigma_{j}\right|^{0}, \mathcal{L}_{T}(\chi)\right) .
$$

Proof The left hand side is the sheaf associated to the pre-sheaf

$$
U \longmapsto H^{i}\left(\left.T_{\Sigma_{\infty}}^{\wedge}\right|_{U}, \mathcal{L}_{\psi}^{\wedge}\right)
$$

and the right hand side is the sheaf associated to the pre-sheaf

$$
U \longmapsto \prod_{\chi \in X^{*}(S)} \lim _{\leftarrow j} H_{\left|\Sigma_{j}\right|^{0}-Y_{\psi}(\chi)}\left(\left|\Sigma_{j}\right|^{0}, \mathcal{O}_{Y}(U)\right) \otimes \mathcal{L}_{T}(\chi)(U)
$$

Thus it suffices to establish isomorphisms

$$
H^{i}\left(\left.T_{\Sigma_{\infty}}^{\wedge}\right|_{U}, \mathcal{L}_{\psi}^{\wedge}\right) \cong \prod_{\chi \in X^{*}(S)(Y)} \lim _{\leftarrow j} H_{\left|\Sigma_{j}\right|^{0}-Y_{\psi}(\chi)}^{i}\left(\left|\Sigma_{j}\right|^{0}, \mathcal{O}_{Y}(U)\right) \otimes \mathcal{L}_{T}(\chi)(U),
$$

compatibly with restriction, for $U=\operatorname{Spec} A$, with $A$ noetherian and $\operatorname{Spec} A$ connected.

We can compute $H^{i}\left(T_{\Sigma_{\infty}}^{\wedge} \mid U, \mathcal{L}_{\psi}^{\wedge}\right)$ as the cohomology of the Cech complex with $i$ th term

$$
\prod_{\left(\sigma_{0}, \ldots, \sigma_{i}\right) \in \Sigma_{\infty}^{i+1}} \mathcal{L}_{\psi}^{\wedge}\left(\left(T_{\Sigma_{\infty}}^{\wedge}\right)_{\left(\sigma_{0} \cap \cdots \cap \sigma_{i}\right)} \mid U\right)
$$

and we can compute $H^{i}\left(T_{\Sigma_{j}}^{\wedge} \mid u, \mathcal{L}_{\psi}^{\wedge}\right)$ as the cohomology of the Cech complex with $i$ th term

$$
\prod_{\left(\sigma_{0}, \ldots, \sigma_{i}\right) \in \Sigma_{j}^{i+1}} \mathcal{L}_{\psi}^{\wedge}\left(\left.\left(T_{\Sigma_{j}}^{\wedge}\right)_{\left(\sigma_{0} \cap \cdots \cap \sigma_{i}\right)}\right|_{U}\right)
$$

Note that as soon as the faces of $\sigma$ in $\Sigma_{j}$ equals the faces of $\sigma$ in $\Sigma_{\infty}$ then $\left(T_{\Sigma_{\infty}}^{\wedge}\right)_{\sigma}=\left(T_{\Sigma_{j}}^{\wedge}\right)_{\sigma}$. Thus

$$
\lim _{\leftarrow j} \prod_{\left(\sigma_{0}, \ldots, \sigma_{i}\right) \in \Sigma_{j}^{i+1}} \mathcal{L}_{\psi}^{\wedge}\left(\left.\left(T_{\Sigma_{j}}^{\wedge}\right)_{\left(\sigma_{0} \cap \cdots \cap \sigma_{i}\right)}\right|_{U}\right) \cong \prod_{\left(\sigma_{0}, \ldots, \sigma_{i}\right) \in \Sigma_{\infty}^{i+1}} \mathcal{L}_{\psi}^{\wedge}\left(\left.\left(T_{\Sigma_{\infty}}^{\wedge}\right)_{\left(\sigma_{0} \cap \cdots \cap \sigma_{i}\right)}\right|_{U}\right),
$$

and

$$
\left\{\prod_{\left(\sigma_{0}, \ldots, \sigma_{i}\right) \in \Sigma_{j}^{i+1}} \mathcal{L}_{\psi}^{\wedge}\left(\left(T_{\Sigma_{j}}^{\wedge}\right)_{\left(\sigma_{0} \cap \cdots \cap \sigma_{i}\right)(U)}\right)\right\}
$$

satisfies the Mittag-Leffler condition (with $j$ varying but $i$ fixed). 
From theorem 3.5.8 of [57] we see that there is a short exact sequence

$(0) \longrightarrow \lim _{\leftarrow j}^{1} H^{i-1}\left(\left.T_{\Sigma_{j}}^{\wedge}\right|_{U}, \mathcal{L}_{\psi}^{\wedge}\right) \longrightarrow H^{i}\left(\left.T_{\Sigma_{\infty}}^{\wedge}\right|_{U}, \mathcal{L}_{\psi}^{\wedge}\right) \longrightarrow \lim _{\leftarrow j} H^{i}\left(\left.T_{\Sigma_{j}}^{\wedge}\right|_{U}, \mathcal{L}_{\psi}^{\wedge}\right) \longrightarrow(0)$.

Applying Lemma 2.12 and the fact that $\lim _{\leftarrow}$ and $\lim _{\leftarrow}{ }^{1}$ in the category of abelian groups commute with arbitrary products, the present lemma follows. (It follows easily from definition 3.5.1 of [57] and the exactness of infinite products in the category of abelian groups that $\lim _{\leftarrow}$ and $\lim _{\leftarrow}{ }^{1}$ commute with arbitrary products in the category of abelian groups.)

We now turn to two specific line bundles: $\mathcal{O}_{T_{\Sigma_{0}}^{\wedge}}$ and, in the case that $\Sigma_{0}$ is smooth, $\mathcal{I}_{\partial, \Sigma_{0}}^{\wedge}$.

Lemma 2.14 Let $Y$ be a connected, separated scheme which is flat and locally of finite type over an irreducible noetherian ring $R_{0}$, let $S / Y$ be a split torus, let $T / Y$ be an $S$-torsor, let $\Sigma_{0}$ be a partial fan for $S$, and let $\pi_{\Sigma_{0}}^{\wedge}$ denote the map $T_{\Sigma_{0}}^{\wedge} \rightarrow Y$. Suppose that $\Sigma_{0}$ is non-empty, finite and open and that $\left|\Sigma_{0}\right|^{0}$ is convex.

(1) Then

$$
R^{i} \pi_{\Sigma_{0}, *}^{\wedge} \mathcal{O}_{T_{\Sigma_{0}}^{\wedge}}= \begin{cases}\prod_{\chi \in\left|\Sigma_{0}\right|^{\vee}} \mathcal{L}(\chi) & \text { if } i=0 \\ (0) & \text { otherwise }\end{cases}
$$

(2) If in addition $\Sigma_{0}$ is smooth then

$$
R^{i} \pi_{\Sigma_{0}, *}^{\wedge} \mathcal{I}_{\partial, \Sigma_{0}}^{\wedge}= \begin{cases}\prod_{\chi \in\left|\Sigma_{0}\right|^{\vee, 0}} \mathcal{L}(\chi) & \text { if } i=0 \\ (0) & \text { otherwise. }\end{cases}
$$

Proof The first part follows from Lemma 2.12 because $Y_{0}(\chi) \cap\left|\Sigma_{0}\right|^{0}$ is empty if $\chi \in\left|\Sigma_{0}\right|^{\vee}$ and otherwise, being the intersection of two convex sets, it is convex.

For the second part we have that $Y_{\psi_{\Sigma_{0}}}(\chi) \cap\left|\Sigma_{0}\right|^{0}=\emptyset$ if and only if $\chi \in\left|\Sigma_{0}\right|^{\vee, 0}$. Thus it suffices to show that each $Y_{\psi_{\tilde{\Sigma}_{0}}}(\chi) \cap\left|\Sigma_{0}\right|^{0}$ is empty or contractible.

To this end, consider the sets

$$
Y^{\prime}(\chi)=\bigcup_{\substack{\sigma \in \Sigma_{0} \\ \chi \leq 0 \text { on } \sigma}} \sigma
$$

and

$$
Y^{\prime \prime}(\chi)=\bigcup_{\substack{\sigma \in \Sigma_{0} \\ \chi \ngtr 0 \text { on } \sigma-\{0\}}} \sigma .
$$

If $\chi>0$ on $\sigma-\{0\}$ then $\chi \geq \psi_{\widetilde{\Sigma}_{0}}$ on $\sigma$ so that $\sigma \cap Y_{\psi_{\widetilde{\Sigma}_{0}}}(\chi)=\emptyset$. Thus

$$
Y^{\prime \prime}(\chi) \supset Y_{\psi_{\widetilde{\Sigma}_{0}}}(\chi) \cap\left|\Sigma_{0}\right|^{0} \supset Y^{\prime}(\chi) \cap\left|\Sigma_{0}\right|^{0}
$$

and

$$
Y^{\prime \prime}(\chi) \supset\left\{x \in\left|\Sigma_{0}\right|^{0}: \chi(x) \leq 0\right\} \supset Y^{\prime}(\chi) \cap\left|\Sigma_{0}\right|^{0}
$$


We will describe a deformation retraction

$$
H: Y^{\prime \prime}(\chi) \times[0,1] \longrightarrow Y^{\prime \prime}(\chi)
$$

from $Y^{\prime \prime}(\chi)$ to $Y^{\prime}(\chi)$, which restricts to deformation retractions

$$
\left(Y_{\psi_{\Sigma_{0}}}(\chi) \cap\left|\Sigma_{0}\right|^{0}\right) \times[0,1] \longrightarrow Y_{\psi_{\Sigma_{0}}} \cap\left|\Sigma_{0}\right|^{0}(\chi)
$$

from $Y_{\psi_{\tilde{\Sigma}_{0}}}(\chi) \cap\left|\Sigma_{0}\right|^{0}$ to $Y^{\prime}(\chi) \cap\left|\Sigma_{0}\right|^{0}$, and

$$
\left\{x \in\left|\Sigma_{0}\right|^{0}: \chi(x) \leq 0\right\} \times[0,1] \longrightarrow\left\{x \in\left|\Sigma_{0}\right|^{0}: \chi(x) \leq 0\right\}
$$

from $\left\{x \in\left|\Sigma_{0}\right|^{0}: \chi(x) \leq 0\right\}$ to $Y^{\prime}(\chi) \cap\left|\Sigma_{0}\right|^{0}$. (Recall that in particular $\left.H\right|_{Y^{\prime}(\chi) \times[0,1]}$ is just projection to the first factor.) As $\left\{x \in\left|\Sigma_{0}\right|^{0}: \chi(x) \leq 0\right\}$ is empty or convex, it would follow that $Y_{\psi_{\widetilde{\Sigma}_{0}}}(\chi) \cap\left|\Sigma_{0}\right|^{0}$ is empty or contractible and the second part of the corollary would follow.

To define $H$ it suffices to define, for each $\sigma \in \widetilde{\Sigma}_{0}$ with $\sigma \subset Y^{\prime \prime}(\chi)$, a deformation retraction

$$
H_{\sigma}: \sigma \times[0,1] \longrightarrow \sigma
$$

from $\sigma$ to $\sigma \cap Y^{\prime}(\chi)$ with the following properties:

- If $\sigma^{\prime} \subset \sigma$ then $\left.H_{\sigma}\right|_{\sigma^{\prime} \times[0,1]}=H_{\sigma^{\prime}}$.

- $\left.H_{\sigma}\right|_{\left(\sigma \cap Y_{\psi_{\Sigma_{0}}}(\chi) \cap\left|\Sigma_{0}\right|^{0}\right) \times[0,1]}$ is a deformation retraction from $\sigma \cap Y_{\psi_{\Sigma_{0}}}(\chi) \cap\left|\Sigma_{0}\right|^{0}$ to $\sigma \cap$ $Y^{\prime}(\chi) \cap\left|\Sigma_{0}\right|^{0}$.

- $\left.H_{\sigma}\right|_{\left(\sigma \cap\left\{x \in\left|\Sigma_{0}\right|^{0}: \chi(x) \leq 0\right\}\right) \times[0,1]}$ is a deformation retraction from $\sigma \cap\left\{x \in\left|\Sigma_{0}\right|^{0}: \chi(x) \leq 0\right\}$ to $\sigma \cap Y^{\prime}(\chi) \cap\left|\Sigma_{0}\right|^{0}$.

To define $H_{\sigma}$, let $v_{1}, \ldots, v_{r}, w_{1}, \ldots, w_{s}$ denote the shortest nonzero vectors in $X_{*}(S)$ on each of the one-dimensional faces of $\sigma$ (so that $r+s=\operatorname{dim} \sigma$ ), with the notation chosen such that $\chi\left(v_{i}\right) \leq 0$ for all $i$ and $\chi\left(w_{j}\right)>0$ for all $j$. Note that $1-\chi\left(v_{i}\right)>0$ for all $i$ and $1-\chi\left(w_{j}\right) \leq 0$ for all $j$. We set

$$
H_{\sigma}\left(\sum_{i} a_{i} v_{i}+\sum_{j} b_{i} w_{j}, t\right)=\sum_{i} a_{i} v_{i}+(1-t) \sum_{j} b_{i} w_{j} .
$$

Because

$$
\begin{aligned}
\sigma \cap Y_{\psi_{\tilde{\Sigma}_{0}}}(\chi) \cap\left|\Sigma_{0}\right|^{0}= & \left\{\sum_{i} a_{i} v_{i}+\sum_{j} b_{j} w_{j}: a_{i}, b_{j} \in \mathbb{R}_{\geq 0}\right. \text { and } \\
& \left.\sum_{i} a_{i}\left(1-\chi\left(v_{i}\right)\right)+\sum_{j} b_{j}\left(1-\chi\left(w_{j}\right)\right)>0\right\} \cap\left|\Sigma_{0}\right|^{0}
\end{aligned}
$$

and

$$
\begin{aligned}
\sigma & \cap\left\{x \in\left|\Sigma_{0}\right|^{0}: \chi(x) \leq 0\right\} \\
& =\left\{\sum_{i} a_{i} v_{i}+\sum_{j} b_{j} w_{j}: a_{i}, b_{j} \in \mathbb{R}_{\geq 0} \text { and } \sum_{i} a_{i} \chi\left(v_{i}\right)+\sum_{j} b_{j} \chi\left(w_{j}\right) \leq 0\right\} \cap\left|\Sigma_{0}\right|^{0}
\end{aligned}
$$


are convex sets, and because

$$
\sigma \cap Y^{\prime}(\chi)=\left\{\sum_{i} a_{i} v_{i}: a_{i} \in \mathbb{R}_{\geq 0}\right\},
$$

it is easy to check that it has the desired properties and the proof of the lemma is complete.

Lemma 2.15 Let $Y$ be a connected, separated scheme which is flat and locally of finite type over an irreducible noetherian ring $R_{0}$, let $\alpha: S \rightarrow S^{\prime}$ be an isogeny of split tori over $Y$, and let $\Sigma_{0}^{\prime}\left(\right.$ resp. $\Sigma_{0}$ ) be a locally finite partial fan for $S^{\prime}$ (resp. S). Suppose that $\Sigma_{0}^{\prime}$ is full. Also suppose that $\Sigma_{0}$ is a quasi-refinement of $\Sigma_{0}^{\prime}$, and let $\pi^{\wedge}$ denote the map $T_{\Sigma_{0}}^{\wedge} \rightarrow\left(\alpha_{*} T\right)_{\Sigma_{0}^{\prime}}^{\wedge}$.

Then for $i>0$ we have

$$
R^{i} \pi_{*}^{\wedge} \mathcal{O}_{T_{\Sigma_{0}}^{\wedge}}=(0)
$$

while

$$
\mathcal{O}_{\left(\alpha_{*} T\right)_{\Sigma_{0}^{\prime}}^{\wedge}} \stackrel{\sim}{\longrightarrow}\left(\pi_{*}^{\wedge} \mathcal{O}_{T_{\Sigma_{0}}}\right)^{\mathrm{ker} \alpha} .
$$

If moreover $\Sigma_{0}$ and $\Sigma_{0}^{\prime}$ are smooth then, for $i>0$ we have

$$
R^{i} \pi_{*}^{\wedge} \mathcal{I}_{\partial, \Sigma_{0}}^{\wedge}=(0)
$$

while

$$
\mathcal{I}_{\partial, \Sigma_{0}^{\prime}}^{\wedge} \stackrel{\sim}{\longrightarrow}\left(\pi_{*}^{\wedge} \mathcal{I}_{\partial, \Sigma_{0}}^{\wedge}\right)^{\operatorname{ker} \alpha} .
$$

Proof We may reduce to the case that $Y=\operatorname{Spec} A$ is affine. The map $\pi^{\wedge}$ is proper and hence

$$
R^{i} \pi_{*}^{\wedge} \mathcal{O}_{T_{\Sigma_{0}}^{\wedge}}
$$

and

$$
R^{i} \pi_{*}^{\wedge} \mathcal{I}_{\partial, \Sigma_{0}}^{\wedge}
$$

and

$$
\operatorname{coker}\left(\mathcal{O}_{\left(\alpha_{*} T\right)_{\Sigma_{0}^{\prime}}^{\wedge}} \longrightarrow\left(\pi_{*}^{\wedge} \mathcal{O}_{T_{\Sigma_{0}}}\right)^{\operatorname{ker} \alpha}\right)
$$

and

$$
\operatorname{coker}\left(\mathcal{I}_{\partial, \Sigma_{0}^{\prime}}^{\wedge} \longrightarrow\left(\pi_{*}^{\wedge} \mathcal{I}_{\partial, \Sigma_{0}}^{\wedge}\right)^{\operatorname{ker} \alpha}\right)
$$

are coherent sheaves. Thus they have closed support. Their support is also $S$-invariant. Thus it suffices to show that for each maximal element $\sigma^{\prime} \in \Sigma_{0}^{\prime}$ the space $\partial_{\sigma^{\prime}}\left(\alpha_{*} T\right)_{\widetilde{\Sigma}_{0}^{\prime}}$ does not lie in the support of these sheaves. Let $\Sigma_{0}\left(\sigma^{\prime}\right)$ denote the subset of elements $\sigma \in \Sigma_{0}$ which lie in $\sigma^{\prime}$, but in no face of $\sigma^{\prime}$. Then $\Sigma_{0}\left(\sigma^{\prime}\right)$ is a partial fan and $T_{\Sigma_{0}\left(\sigma^{\prime}\right)}^{\wedge}$ equals the 
formal completion of $T_{\Sigma_{0}}^{\wedge}$ along $\partial_{\sigma^{\prime}} T_{\widetilde{\Sigma}_{0}^{\prime}}$. Thus the formal completion of the above four sheaves along $\partial_{\sigma^{\prime}} T_{\widetilde{\Sigma}_{0}^{\prime}}$ equal the corresponding sheaf for the pair $\Sigma_{0}\left(\sigma^{\prime}\right)$ and $\left\{\sigma^{\prime}\right\}$, so that we are reduced to the case that $\Sigma_{0}^{\prime}=\left\{\sigma^{\prime}\right\}$ is a singleton.

In the case that $\Sigma_{0}^{\prime}=\left\{\sigma^{\prime}\right\}$ then $\left(\alpha_{*} T\right)_{\Sigma_{0}^{\prime}}$ and $Y$ have the same underlying topological space. Let $\pi_{1}^{\wedge}$ denote the map of ringed spaces $T_{\Sigma_{0}}^{\wedge} \rightarrow Y$. Then it suffices to show that for $i>0$ we have

$$
R^{i} \pi_{1, *}^{\wedge} \mathcal{O}_{T_{\Sigma_{0}}}=(0)
$$

and

$$
R^{i} \pi_{1, *}^{\wedge} \mathcal{I}_{\partial, \Sigma_{0}}^{\wedge}=(0)
$$

and that we have

$$
\mathcal{O}_{\left(\alpha_{*} T\right)_{\Sigma_{0}^{\prime}}^{\wedge}} \stackrel{\sim}{\longrightarrow}\left(\pi_{1, *}^{\wedge} \mathcal{O}_{T_{\Sigma_{0}}}\right)^{\operatorname{ker} \alpha}
$$

and

$$
\mathcal{I}_{\partial, \Sigma_{0}^{\prime}}^{\wedge} \stackrel{\sim}{\longrightarrow}\left(\pi_{1, *}^{\wedge} \mathcal{I}_{\partial, \Sigma_{0}}^{\wedge}\right)^{\operatorname{ker} \alpha} .
$$

This follows from Lemma 2.14. (Note that

$$
\prod_{\chi \in\left|\Sigma_{0}\right|^{\vee} \cap X^{*}(S)} \mathcal{L}(\chi)=\bigoplus_{\xi \in(\operatorname{ker} \alpha)^{\vee}} \prod_{\substack{\chi \in\left|\Sigma_{0}\right|^{\vee} \cap X^{*}(S) \\ \chi \mid \operatorname{ker} \alpha=\xi}} \mathcal{L}(\chi),
$$

where $\operatorname{ker} \alpha$ acts on the $\xi$ term via $\xi$; and that

$$
\left\{\chi \in\left|\Sigma_{0}\right|^{\vee} \cap X^{*}(S):\left.\chi\right|_{\operatorname{ker} \alpha}=1\right\}=\left|\Sigma_{0}\right|^{\vee} \cap X^{*}\left(S^{\prime}\right)=\left|\left\{\sigma^{\prime}\right\}\right|^{\vee} \cap X^{*}\left(S^{\prime}\right) .
$$

These assertions remain true with $\left|\Sigma_{0}\right|^{\vee, 0}$ replacing $\left|\Sigma_{0}\right|^{\vee}$ and $\left|\left\{\sigma^{\prime}\right\}\right|^{\vee, 0}$ replacing $\left|\left\{\sigma^{\prime}\right\}\right|^{\vee}$.)

Lemma 2.16 Let $Y$ be a connected, separated scheme which is flat and locally of finite type over an irreducible noetherian ring $R_{0}$, let $S / Y$ be a split torus, let $T / Y$ be an $S$-torsor, let $\Sigma_{0}$ be a partial fan for $S$, and let $\pi_{\Sigma_{0}}$ denote the map of ringed spaces $T_{\Sigma_{0}}^{\wedge} \rightarrow Y$. Suppose that $\Sigma_{0}$ is non-empty, full, locally finite and open, and that $\left|\Sigma_{0}\right|^{0}$ is convex.

(1) Then

$$
R^{i} \pi_{\Sigma_{0}, *}^{\wedge} \mathcal{O}_{T_{\Sigma_{0}}}= \begin{cases}\prod_{\chi \in\left|\Sigma_{0}\right|^{\vee}} \mathcal{L}(\chi) & \text { if } i=0 \\ (0) & \text { otherwise. }\end{cases}
$$

(2) If in addition $\Sigma_{0}$ is smooth then

$$
R^{i} \pi_{\Sigma_{0}, *} \mathcal{I}_{\partial, \Sigma_{0}}^{\wedge}= \begin{cases}\prod_{\chi \in\left|\Sigma_{0}\right|^{\vee, 0}} \mathcal{L}(\chi) & \text { if } i=0 \\ (0) & \text { otherwise }\end{cases}
$$


Proof Let $\sigma_{1}, \sigma_{2}, \ldots$ be an enumeration of the 1 cones in $\widetilde{\Sigma}_{0}$. Let $\Delta^{(i)} \subset|\Sigma|$ denote the convex hull of $\sigma_{1}, \ldots, \sigma_{i}$. It is a rational polyhedral cone contained in $\left|\Sigma_{0}\right|$, and there exists $i_{0}$ such that for $i \geq i_{0}$ the cone $\Delta^{(i)}$ will have the same dimension as $X_{*}(S)_{\mathbb{R}}$. Let $\partial \Delta^{(i)}$ denote the union of the proper faces of $\Delta^{(i)}$; and let $\Delta^{(i), c}$ denote the closure of $\left|\Sigma_{0}\right|-\Delta^{(i)}$ in $\left|\Sigma_{0}\right|$.

Define recursively fans $\Sigma^{(i)}$ and boundary data $\Sigma_{0}^{(i)}$ as follows. We set $\Sigma^{\left(i_{0}-1\right)}=\widetilde{\Sigma}_{0}$ and $\Sigma_{0}^{\left(i_{0}-1\right)}=\Sigma_{0}$. For $i \geq i_{0}$ set

$$
\Sigma^{(i)}=\left\{\sigma \cap \Delta^{(i)}, \sigma \cap \partial \Delta^{(i)}, \sigma \cap \Delta^{(i), c}: \sigma \in \Sigma^{(i-1)}\right\} .
$$

Then $\Sigma^{(i)}$ refines $\Sigma^{(i-1)}$ and we choose $\Sigma_{0}^{(i)}$ to be the unique subset of $\Sigma^{(i)}$ such that $\left(\Sigma^{(i)}, \Sigma_{0}^{(i)}\right)$ refines $\left(\Sigma^{(i-1)}, \Sigma_{0}^{(i-1)}\right)$. Then $\widetilde{\Sigma_{0}^{(i)}}=\Sigma^{(i)}$. We also check by induction on $i$ that - $\Sigma^{(i)} \cup \Sigma^{(i-1)}-\left(\Sigma^{(i)} \cap \Sigma^{(i-1)}\right)$ is finite;

- and $\Sigma_{0}^{(i)}$ is locally finite.

(The point being that the local finiteness of $\Sigma_{0}^{(i-1)}$ implies that only finitely many elements of $\Sigma_{0}^{(i-1)}$, and hence of $\Sigma^{(i-1)}$, meet both $\Delta^{(i)}-\partial \Delta^{(i)}$ and $X_{*}(S)_{\mathbb{R}}-\Delta^{(i)}$.)

Now define $\Sigma^{(\infty)}\left(\right.$ resp. $\left.\Sigma_{0}^{(\infty)}\right)$ to be the set of cones that occur in $\Sigma^{(i)}\left(\right.$ resp. $\left.\Sigma_{0}^{(i)}\right)$ for infinitely many $i$. Alternatively

$$
\Sigma^{(\infty)}=\bigcup_{i}\left\{\sigma \in \Sigma^{(i)}: \sigma \subset \Delta^{(i)}\right\}
$$

Also let $\Sigma^{(\infty), \text { sm }}$ denote a smooth refinement of $\Sigma^{(\infty)}$ and let $\Sigma_{0}^{(\infty), s m}$ denote the elements of $\sigma \in \Sigma^{(\infty), s m}$ for which there exists $\tau \in \Sigma_{0}$ such that $\sigma \subset \tau$ and $\sigma \cap \tau^{0} \neq \emptyset$. (See Lemma 2.4.) Then $\Sigma^{(\infty), s m}$ is a fan, $\Sigma_{0}^{(\infty), s m}$ provides locally finite boundary data for $\Sigma^{(\infty), s m}$, we have $\Sigma_{0}^{\widetilde{(\infty), \mathrm{sm}}}=\Sigma^{(\infty), \mathrm{sm}}$, and $\left(\Sigma^{(\infty), \mathrm{sm}}, \Sigma_{0}^{(\infty), \mathrm{sm}}\right)$ refines $\left(\widetilde{\Sigma}_{0}, \Sigma_{0}\right)$. Moreover $\Sigma_{0}^{(\infty), \mathrm{sm}}$ is open. We also define $\Sigma_{i}^{(\infty) \text {,sm }}$ to be the set of $\sigma \in \Sigma_{0}^{(\infty), \text { sm }}$ such that $\sigma \subset \Delta^{(i)}$ but $\sigma \not \subset \partial \Delta^{(i)}$. Note that:

- $\Sigma_{i}^{(\infty), s m}$ is finite and open;

- $\Sigma_{i}^{(\infty), \mathrm{sm}} \supset \Sigma_{i-1}^{(\infty), \mathrm{sm}}$;

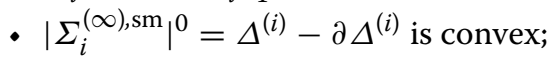

- and $\Sigma_{0}^{(\infty), \mathrm{sm}}=\bigcup_{i>0} \Sigma_{i}^{(\infty), \mathrm{sm}}$.

(For the last of these properties use the fact that $\Sigma_{0}^{(\infty), s m}$ is open.)

By Lemma 2.15 it suffices to prove this lemma after replacing the pair $\Sigma_{0}$ by $\Sigma_{0}^{(\infty), s m}$. This lemma then follows from Lemmas 2.13 and 2.14.

\subsection{The case of a disconnected base}

Throughout this section we will continue to assume that $Y$ is a separated scheme, flat and locally of finite type over $\operatorname{Spec} R_{0}$. We prove nothing new in this section, we simply explain how to re-express the last two sections in a way that makes sense over a disconnected base, so that the results we have already established immediately extend.

Let $S$ be a split torus over $Y$ and let $T / Y$ be an $S$-torsor. By a rational polyhedral cone $\sigma$ in $X_{*}(S)_{\mathbb{R}}$ we shall mean a locally constant sheaf of subsets $\sigma \subset X_{*}(S)_{\mathbb{R}}$, such that 
- for each connected open $U \subset Y$ the set $\sigma(U) \subset X_{*}(S)_{\mathbb{R}}(U)$ is either empty or a rational polyhedral cone,

- and the locus where $\sigma \neq \emptyset$ is non-empty and connected. We call this locus the support of $\sigma$.

We call $\sigma^{\prime}$ a face of $\sigma$ if for each open connected $U$ either $\sigma(U)=\sigma^{\prime}(U)=\emptyset$ or the cone $\sigma^{\prime}(U)$ is a face of $\sigma(U)$. We call $\sigma$ smooth if each $\sigma(U)$ is smooth. By a fan $\Sigma$ in $X_{*}(S)_{\mathbb{R}}$ we mean a set of rational polyhedral cones in $X_{*}(S)_{\mathbb{R}}$, such that

- if $\sigma \in \Sigma$ then so is any face $\sigma^{\prime}$ of $\sigma$;

- if $\sigma, \sigma^{\prime} \in \Sigma$ then $\sigma \cap \sigma^{\prime}$ is either empty or a face of $\sigma$ and $\sigma^{\prime}$,

- any connected component of $Y$ arises as the support of some element of $\Sigma$.

Thus to give a fan in $X_{*}(S)_{\mathbb{R}}$ is the same as giving a fan in $X_{*}(S)_{\mathbb{R}}(Z)$ for each connected component $Z$ of $Y$. If $U$ is a non-empty connected open in $Y$ then we set

$$
\Sigma(U)=\{\sigma(U): \sigma \in \Sigma\}-\{\emptyset\}
$$

It is a fan for $X_{*}(S)_{\mathbb{R}}(U)$.

We call $\Sigma$ smooth (resp. full, resp. finite) if each $\Sigma(U)$ is. We define a locally constant sheaf $|\Sigma|$ of subsets of $X_{*}(S)_{\mathbb{R}}$ by setting

$$
|\Sigma|(U)=\bigcup_{\sigma \in \Sigma} \sigma(U)
$$

(resp.

$$
\left.|\Sigma|^{*}(U)=\bigcup_{\sigma \in \Sigma}(\sigma(U)-\{0\})\right)
$$

for $U$ any connected open subset of $Y$. We will call $|\Sigma|\left(\right.$ resp. $\left.|\Sigma|^{*}\right)$ convex if $|\Sigma|(U)$ (resp. $\left.|\Sigma|^{*}(U)\right)$ is for each connected open $U \subset Y$. We also define locally constant sheaves of subsets $|\Sigma|^{\vee}$ and $|\Sigma|^{\vee, 0}$ of $X^{*}(S)_{\mathbb{R}}$ by setting

$$
|\Sigma|^{\vee}(U)=\left\{\chi \in X^{*}(S)_{\mathbb{R}}(U): \chi(|\Sigma|(U)) \subset \mathbb{R}_{\geq 0}\right\}=\bigcap_{\sigma \in \Sigma} \sigma(U)^{\vee}
$$

and

$$
|\Sigma|^{\vee, 0}(U)=\left\{\chi \in X^{*}(S)_{\mathbb{R}}(U): \chi\left(|\Sigma|^{*}(U)\right) \subset \mathbb{R}_{>0}\right\}=\bigcap_{\sigma \in \Sigma} \sigma(U)^{\vee, 0}
$$

We call $\Sigma^{\prime}$ a refinement of $\Sigma$ if each $\Sigma^{\prime}(U)$ is a refinement of $\Sigma(U)$ for each open, connected $U$. Any fan $\Sigma$ has a smooth refinement $\Sigma^{\prime}$ such that every smooth cone $\sigma \in \Sigma$ also lies in $\Sigma^{\prime}$.

To a fan $\Sigma$ one can attach a scheme $T_{\Sigma}$ flat and separated over $Y$ and locally (on $T_{\Sigma}$ ) of finite type over $Y$, together with an action of $S$ and an $S$-equivariant embedding $T \hookrightarrow T_{\Sigma}$. It has an open cover $\left\{T_{\sigma}\right\}_{\sigma \in \Sigma}$, with each $T_{\sigma}$ relatively affine over $Y$. Over a connected open $U \subset Y$ this restricts to the corresponding picture for $\Sigma(U)$. We write $\mathcal{O}_{T_{\Sigma}}$ for the 
structure sheaf of $T_{\Sigma}$. If $\Sigma$ is smooth then $T_{\Sigma} / Y$ is smooth. If $\Sigma$ is finite and $|\Sigma|=X_{*}(S)_{\mathbb{R}}$, then $T_{\Sigma}$ is proper over $Y$. If $\Sigma^{\prime}$ refines $\Sigma$ then there is an $S$-equivariant proper map

$$
T_{\Sigma^{\prime}} \rightarrow T_{\Sigma}
$$

which restricts to the identity on $T$.

By boundary data we shall mean a proper subset $\Sigma_{0} \subset \Sigma$ such that $\Sigma-\Sigma_{0}$ is a fan. If $U \subset Y$ is a connected open we set

$$
\Sigma_{0}(U)=\left\{\sigma(U): \sigma \in \Sigma_{0}\right\}-\{\emptyset\}
$$

If $\Sigma_{0}$ is boundary data, then we can associate to it a closed subscheme $\partial_{\Sigma_{0}} T_{\Sigma} \subset T_{\Sigma}$, which over a connected open $U \subset Y$ restricts to $\partial_{\Sigma_{0}(U)}\left(\left.T\right|_{U}\right)_{\Sigma(U)} \subset\left(\left.T\right|_{U}\right)_{\Sigma(U)}$.

In the case that $\Sigma_{0}$ is the set of elements of $\Sigma$ of dimension bigger than 0 we shall simply write $\partial T_{\Sigma}$ for $\partial_{\Sigma_{0}} T_{\Sigma}$. Thus $T=T_{\Sigma}-\partial T_{\Sigma}$. We will write $\mathcal{I}_{\partial T_{\Sigma}}$ for the ideal of definition of $\partial T_{\Sigma}$ in $\mathcal{O}_{T_{\Sigma}}$. We will also write $\mathcal{M}_{\Sigma} \rightarrow \mathcal{O}_{T_{\Sigma}}$ for the associated log structure and $\Omega_{T_{\Sigma} / \operatorname{Spec} R_{0}}^{1}(\log \infty)$ for the log differentials $\Omega_{T_{\Sigma} / \operatorname{Spec} R_{0}}^{1}\left(\log \mathcal{M}_{\Sigma}\right)$.

If $\Sigma$ is smooth then $\partial T_{\Sigma}$ is a simple normal crossings divisor on $T_{\Sigma}$ relative to $Y$.

If $\sigma \in \Sigma$ has positive dimension and if $\Sigma_{0}$ denotes the set of elements of $\Sigma$ which have $\sigma$ for a face, then we will write $\partial_{\sigma} T_{\Sigma}$ for $\partial_{\Sigma_{0}} T_{\Sigma}$. It is connected and flat over $Y$, and, locally on $Y$, it has codimension in $T_{\Sigma}$ equal to the dimension of $\sigma$. If $\Sigma$ is smooth then each $\partial_{\sigma} T_{\Sigma}$ is smooth over $Y$. The schemes $\partial_{\sigma_{1}} T_{\Sigma}, \ldots, \partial_{\sigma_{s}} T_{\Sigma}$ intersect if and only if $\sigma_{1}, \ldots, \sigma_{s}$ are all contained in some $\sigma \in \Sigma$. In this case the intersection equals $\partial_{\sigma} T_{\Sigma}$ for the smallest such $\sigma$. We set

$$
\partial_{i} T_{\Sigma}=\coprod_{\operatorname{dim} \sigma=i} \partial_{\sigma} T_{\Sigma}
$$

If the connected components of $Y$ are irreducible then each $\partial_{\sigma} T_{\Sigma}$ is irreducible. Moreover the irreducible components of $\partial T_{\Sigma}$ are the $\partial_{\sigma} T_{\Sigma}$ as $\sigma$ runs over one-dimensional elements of $\Sigma$. If $\Sigma$ is smooth then we see that $\mathcal{S}\left(\partial T_{\Sigma}\right)$ is the delta set with cells in bijection with the elements of $\Sigma$ with dimension bigger than 0 and with the same 'face relations'. In particular it is in fact a simplicial complex and

$$
\left|\mathcal{S}\left(\partial T_{\Sigma}\right)\right|=\coprod_{Z \in \pi_{0}(Y)}|\Sigma|^{*}(Z) / \mathbb{R}_{>0}^{\times}
$$

We will call $\Sigma_{0}$ open (resp. finite, resp. locally finite) if $\Sigma_{0}(U)$ is for each open connected $U \subset Y$. If $\Sigma_{0}$ is finite and open, then $\partial_{\Sigma_{0}} T_{\Sigma}$ is proper over $Y$.

By a partialfan in $X_{*}(S)$ we mean a collection $\Sigma_{0}$ of rational polyhedral cones in $X_{*}(S)$ such that

- $\Sigma_{0}$ does not contain $(0) \subset X_{*}(S)(U)_{\mathbb{R}}$ for any open connected $U$;

- if $\sigma_{1}, \sigma_{2} \in \Sigma_{0}$ then $\sigma_{1} \cap \sigma_{2}$ is either empty or a face of $\sigma_{1}$ and of $\sigma_{2}$;

- if $\sigma_{1}, \sigma_{2} \in \Sigma_{0}$ and if $\sigma \supset \sigma_{2}$ is a face of $\sigma_{1}$, then $\sigma \in \Sigma_{0}$.

In this case we will let $\widetilde{\Sigma}_{0}$ denote the set of faces of elements of $\Sigma_{0}$ together with $\{0\}$ supported on any connected component of $Y$. It is a fan, and $\Sigma_{0}$ is boundary data for $\widetilde{\Sigma}_{0}$. 
By boundary data $\Sigma_{1}$ for $\Sigma_{0}$ we shall mean a subset $\Sigma_{1} \subset \Sigma_{0}$ such that if $\sigma \in \Sigma_{0}$ contains $\sigma_{1} \in \Sigma_{1}$, then $\sigma \in \Sigma_{1}$. In this case $\Sigma_{1}$ is again a partial fan and boundary data for $\widetilde{\Sigma}_{0}$. We say that a partial fan $\Sigma_{0}$ for $X_{*}(S)$ refines a partial fan $\Sigma_{0}^{\prime}$ for $X_{*}(S)$ if every element of $\Sigma_{0}$ lies in an element of $\Sigma_{0}^{\prime}$ and if every element of $\Sigma_{0}^{\prime}$ is a finite union of elements of $\Sigma_{0}$.

If $\Sigma_{0}$ is a partial fan we define locally constant sheaves of subsets $\left|\Sigma_{0}\right|,\left|\Sigma_{0}\right|^{*},\left|\Sigma_{0}\right|^{\vee}$ and $\left|\Sigma_{0}\right|^{\vee, 0}$ of $X_{*}(S)_{\mathbb{R}}$ or $X^{*}(S)_{\mathbb{R}}$ to be $\left|\widetilde{\Sigma}_{0}\right|,\left|\widetilde{\Sigma}_{0}\right|^{*},\left|\widetilde{\Sigma}_{0}\right|^{\vee}$ and $\left|\widetilde{\Sigma}_{0}\right|^{\vee, 0}$, respectively. We also define a sheaf of subsets $\left|\Sigma_{0}\right|^{0}$ by

$$
\left|\Sigma_{0}\right|^{0}(U)=\left|\widetilde{\Sigma}_{0}\right|(U)-\bigcup_{\sigma \in \widetilde{\Sigma}_{0}(U)-\Sigma_{0}(U)} \sigma
$$

for any connected open set $U \subset Y$. We will call $\left|\Sigma_{0}\right|$ (resp. $\left.\left|\Sigma_{0}\right|^{0}\right)$ convex if $\left|\Sigma_{0}\right|(U)$ (resp. $\left.\left|\Sigma_{0}\right|^{0}(U)\right)$ is convex for all open connected subsets $U \subset Y$.

We will call $\Sigma_{0}$ smooth (resp. full, resp. open, resp. finite, resp. locally finite) if $\Sigma_{0}(U)$ is for each $U \subset Y$ open and connected. We will call $\Sigma_{0}$ non-degenerate if for each non-empty connected open subset $U \subset Y$ the set $\Sigma_{0}(U)$ is non-empty.

If $\Sigma_{0}$ is a partial fan we will write

$$
\partial_{\Sigma_{0}} T
$$

for $\partial_{\Sigma_{0}} T_{\widetilde{\Sigma}_{0}}$

$$
T_{\Sigma_{0}}^{\wedge}
$$

for the completion of $T_{\widetilde{\Sigma}_{0}}$ along $\partial_{\Sigma_{0}} T_{\widetilde{\Sigma}_{0}} ;$ and

$$
\mathcal{M}_{\Sigma_{0}}^{\wedge} \longrightarrow \mathcal{O}_{T_{\Sigma_{0}}}
$$

for the $\log$ structure induced by $\mathcal{M}_{\widetilde{\Sigma}_{0}}$. We make the following definitions.

- $\mathcal{I}_{T_{\Sigma_{0}}}$ will denote the completion of $\mathcal{I}_{\partial_{\Sigma_{0}}} T_{\widetilde{\Sigma}_{0}}$, the sheaf of ideals defining $\partial_{\Sigma_{0}} T_{\widetilde{\Sigma}_{0}}$. It is an ideal of definition for $T_{\Sigma_{0}}^{\wedge}$.

- $\mathcal{I}_{\partial, \Sigma_{0}}^{\wedge}$ will denote the completion of $\mathcal{I}_{\partial T_{\widetilde{\Sigma}_{0}}}$, the sheaf of ideals defining $\partial_{\Sigma_{0}} T_{\widetilde{\Sigma}_{0}}$. Thus $\mathcal{I}_{\partial, \Sigma_{0}}^{\wedge} \subset \mathcal{I}_{T_{\Sigma_{0}}}$

- $\Omega_{T_{\Sigma_{0}} / \operatorname{Spf} R_{0}}^{1}(\log \infty)$ will denote $\Omega_{T_{\Sigma_{0}} / \operatorname{Spf} R_{0}}^{1}\left(\log \mathcal{M}_{\Sigma}^{\wedge}\right)$.

We will write

$$
\prod_{\chi \in\left|\Sigma_{0}\right|^{\vee}} \mathcal{L}_{T}(\chi)
$$

(resp.

$$
\left.\prod_{\chi \in\left|\Sigma_{0}\right| \vee, 0} \mathcal{L}_{T}(\chi)\right)
$$

for the sheaf (of abelian groups) on $Y$ such that for any connected open subset $U \subset Y$ we have

$$
\left.\left(\prod_{\chi \in\left|\Sigma_{0}\right|^{\vee}} \mathcal{L}_{T}(\chi)\right)\right|_{U}=\prod_{\chi \in\left|\Sigma_{0}\right|^{\vee}(U) \cap X^{*}(S)(U)} \mathcal{L}_{T}(\chi)
$$


(resp.

$$
\left.\left.\left(\prod_{\chi \in\left|\Sigma_{0}\right|^{\vee, 0}} \mathcal{L}_{T}(\chi)\right)\right|_{U}=\prod_{\chi \in\left|\Sigma_{0}\right|^{\vee, 0}(U) \cap X^{*}(S)(U)} \mathcal{L}_{T}(\chi)\right) .
$$

Suppose that $\alpha: S \rightarrow S^{\prime}$ is a surjective map of split tori over $Y$. Then $X^{*}(\alpha): X^{*}\left(S^{\prime}\right) \hookrightarrow$ $X^{*}(S)$ and $X_{*}(\alpha): X_{*}(S)_{\mathbb{R}} \rightarrow X_{*}\left(S^{\prime}\right)_{\mathbb{R}}$. We call fans $\Sigma$ for $X_{*}(S)$ and $\Sigma^{\prime}$ for $X_{*}\left(S^{\prime}\right)$ compatible if for all $\sigma \in \Sigma$ the image $X_{*}(\alpha) \sigma$ is contained in some element of $\Sigma^{\prime}$. In this case the map $\alpha: T \rightarrow \alpha_{*} T$ extends to an $S$-equivariant map

$$
\alpha: T_{\Sigma} \longrightarrow\left(\alpha_{*} T\right)_{\Sigma^{\prime}}
$$

We will write

$$
\Omega_{T_{\Sigma} /\left(\alpha_{*} T\right)_{\Sigma^{\prime}}}^{1}(\log \infty)=\Omega_{T_{\Sigma} /\left(\alpha_{*} T\right)_{\Sigma^{\prime}}}^{1}\left(\log \mathcal{M}_{\Sigma} / \mathcal{M}_{\Sigma^{\prime}}\right) .
$$

The following lemma is an immediate consequence of Lemma 2.5.

Lemma 2.17 If $\alpha$ is surjective and \#coker $X_{*}(\alpha)$ is invertible on $Y$ then $\alpha:\left(T_{\Sigma}, \mathcal{M}_{\Sigma}\right) \rightarrow$ $\left(\left(\alpha_{*} T\right)_{\Sigma^{\prime}}, \mathcal{M}_{\Sigma^{\prime}}\right)$ is log smooth, and there is a natural isomorphism

$$
\left(X^{*}(S) / X^{*}(\alpha) X^{*}\left(S^{\prime}\right)\right) \otimes_{\mathbb{Z}} \mathcal{O}_{T_{\Sigma}} \stackrel{\sim}{\longrightarrow} \Omega_{T_{\Sigma} /\left(\alpha_{*} T\right)_{\Sigma^{\prime}}}^{1}(\log \infty) .
$$

If $\alpha$ is an isogeny, if $\Sigma$ and $\Sigma^{\prime}$ are compatible, and if every element of $\Sigma^{\prime}$ is a finite union of elements of $\Sigma$, then we call $\Sigma$ a quasi-refinement of $\Sigma^{\prime}$. In that case the map $\alpha: T_{\Sigma} \rightarrow\left(\alpha_{*} T\right)_{\Sigma^{\prime}}$ is proper.

Suppose that $\alpha: S \rightarrow S^{\prime}$ is a surjective map of tori, and that $\Sigma_{0}$ (resp. $\Sigma_{0}^{\prime}$ ) is a partial fan for $S$ (resp. $S^{\prime}$ ). We call $\Sigma_{0}$ and $\Sigma_{0}^{\prime}$ compatible if for every $\sigma \in \Sigma_{0}$ the image $X_{*}(\alpha) \sigma$ is contained in some element of $\Sigma_{0}^{\prime}$ but in no element of $\widetilde{\Sigma}_{0}^{\prime}-\Sigma_{0}^{\prime}$. In this case there is a natural morphism

$$
\alpha:\left(T_{\Sigma_{0}}^{\wedge}, \mathcal{M}_{\Sigma_{0}}^{\wedge}\right) \longrightarrow\left(\left(\alpha_{*} T\right)_{\Sigma_{0}^{\prime}}^{\wedge}, \mathcal{M}_{\Sigma_{0}^{\prime}}^{\wedge}\right) .
$$

We will write

$$
\Omega_{T_{\Sigma_{0}}^{\wedge} /\left(\alpha_{*} T\right)_{\Sigma_{0}^{\prime}}^{\wedge}}^{1}(\log \infty)=\Omega_{T_{\Sigma_{0}}^{\wedge} /\left(\alpha_{*} T\right)_{\Sigma_{0}^{\prime}}^{\wedge}}^{1}\left(\log \mathcal{M}_{\Sigma_{0}}^{\wedge} / \mathcal{M}_{\Sigma_{0}^{\prime}}^{\wedge}\right)
$$

The following lemma follows immediately from Lemma 2.8 .

Lemma 2.18 If $\alpha$ is surjective and \# $\operatorname{coker} X_{*}(\alpha)$ is invertible on $Y$ then there is a natural isomorphism

$$
\left(X^{*}(S) / X^{*}(\alpha) X^{*}\left(S^{\prime}\right)\right) \otimes_{\mathbb{Z}} \mathcal{O}_{T_{\Sigma_{0}}} \stackrel{\sim}{\longrightarrow} \Omega_{T_{\Sigma_{0}} /\left(\alpha_{*} T\right)_{\Sigma_{0}^{\prime}}^{\wedge}}^{1}(\log \infty) .
$$

We will call $\Sigma_{0}$ and $\Sigma_{0}^{\prime}$ strictly compatible if they are compatible and if an element of $\widetilde{\Sigma}_{0}$ lies in $\Sigma_{0}$ if and only if it maps to no element of $\widetilde{\Sigma}_{0}^{\prime}-\Sigma_{0}^{\prime}$. We will say that

- $\Sigma_{0}$ is open over $\Sigma_{0}^{\prime}$ if $\left|\Sigma_{0}\right|^{0}(U)$ is open in $X_{*}(\alpha)^{-1}\left|\Sigma_{0}^{\prime}\right|^{0}(U)$ for all connected opens $U \subset Y$; 
- and that $\Sigma_{0}$ is finite over $\Sigma_{0}^{\prime}$ if only finitely many elements of $\Sigma_{0}$ map into any element of $\Sigma_{0}^{\prime}$.

If $\alpha$ is an isogeny, if $\Sigma_{0}$ and $\Sigma_{0}^{\prime}$ are strictly compatible and if every element of $\Sigma_{0}^{\prime}$ is a finite union of elements of $\Sigma_{0}$, then we call $\Sigma_{0}$ a quasi-refinement of $\Sigma_{0}^{\prime}$. In this case $\Sigma_{0}$ is open and finite over $\Sigma_{0}^{\prime}$. The next lemma follows immediately from Lemma 2.9.

Lemma 2.19 Suppose that $\Sigma_{0}^{\prime}$ and $\Sigma_{0}$ are strictly compatible.

(1) $T_{\Sigma_{0}}^{\wedge}$ is the formal completion of $T_{\widetilde{\Sigma}_{0}}$ along $\partial_{\Sigma_{0}^{\prime}}\left(\alpha_{*} T\right)$, and $T_{\Sigma_{0}}^{\wedge}$ is locally (on the source) topologically of finite type over $\left(\alpha_{*} T\right)_{\Sigma_{0}^{\prime}}^{\wedge}$

(2) If $\Sigma_{0}$ is locally finite and if it is open and finite over $\Sigma_{0}^{\prime}$ then $T_{\Sigma_{0}}^{\wedge}$ is proper over $\left(\alpha_{*} T\right)_{\Sigma_{0}^{\prime}}^{\wedge}$

Corollary 2.20 If $\alpha$ is an isogeny, if $\Sigma_{0}$ is locally finite and if $\Sigma_{0}$ is a quasi-refinement of $\Sigma_{0}^{\prime}$ then $T_{\Sigma_{0}}^{\wedge}$ is proper over $\left(\alpha_{*} T\right)_{\Sigma_{0}^{\prime}}^{\wedge}$

If $\Sigma_{0}$ and $\Sigma_{0}^{\prime}$ are compatible partial fans and if $\Sigma_{1}^{\prime} \subset \Sigma_{0}^{\prime}$ is boundary data then $\Sigma_{0}\left(\Sigma_{1}^{\prime}\right)$ will denote the set of elements $\sigma \in \Sigma_{0}$ such that $X_{*}(\alpha) \sigma$ is contained in no element of $\Sigma_{0}^{\prime}-\Sigma_{1}^{\prime}$. It is boundary data for $\Sigma_{0}$. Moreover the formal completion of $T_{\Sigma_{0}}^{\wedge}$ along the reduced subscheme of $\left(\alpha_{*} T\right)_{\Sigma_{1}^{\prime}}^{\wedge}$ is canonically identified with $T_{\Sigma_{0}\left(\Sigma_{1}^{\prime}\right)}^{\wedge}$. If $\Sigma_{1}^{\prime}=\left\{\sigma^{\prime}\right\}$ is a singleton we will write $\Sigma_{0}\left(\sigma^{\prime}\right)$ for $\Sigma_{0}\left(\left\{\sigma^{\prime}\right\}\right)$.

The next two lemmas follow immediately from Lemmas 2.15 and 2.16, respectively.

Lemma 2.21 Let $Y$ be a separated scheme which is flat and locally of finite type over an irreducible noetherian ring $R_{0}$, let $\alpha: S \rightarrow S^{\prime}$ be an isogeny of split tori over $Y$, and let $\Sigma_{0}^{\prime}$ (resp. $\Sigma_{0}$ ) be a locally finite partial fan for $S^{\prime}$ (resp. S). Suppose that $Y$ is separated and locally noetherian, that $\Sigma_{0}^{\prime}$ is full and that $\Sigma_{0}$ is locally finite. Also suppose that $\Sigma_{0}$ is a quasi-refinement of $\Sigma_{0}^{\prime}$. Let $\pi^{\wedge}$ denote the map $T_{\sigma_{0}}^{\wedge} \rightarrow\left(\alpha_{*} T\right)_{\sigma_{0}^{\prime}}^{\wedge}$

Then for $i>0$ we have

$$
R^{i} \pi_{*}^{\wedge} \mathcal{O}_{T_{\Sigma_{0}}}=(0)
$$

while

$$
\mathcal{O}_{\left(\alpha_{*} T\right)_{\Sigma_{0}^{\prime}}^{\wedge}} \stackrel{\sim}{\longrightarrow}\left(\pi_{*}^{\wedge} \mathcal{O}_{T_{\Sigma_{0}}}\right)^{\text {ker } \alpha} .
$$

If moreover $\Sigma_{0}$ and $\Sigma_{0}^{\prime}$ are smooth then, for $i>0$ we have

$$
R^{i} \pi_{*}^{\wedge} \mathcal{I}_{\partial, \Sigma_{0}}^{\wedge}=(0)
$$

while

$$
\mathcal{I}_{\partial, \Sigma_{0}^{\prime}}^{\wedge} \stackrel{\sim}{\longrightarrow}\left(\pi_{*}^{\wedge} \mathcal{I}_{\partial, \Sigma_{0}}^{\wedge}\right)^{\mathrm{ker} \alpha} .
$$

Lemma 2.22 Let $Y$ be a separated scheme which is flat and locally of finite type over an irreducible noetherian ring $R_{0}$, let $S / Y$ be a split torus, let $T / Y$ is an $S$-torsor, let $\Sigma_{0}$ be a partial fan for $S$, and let $\pi_{\Sigma_{0}}^{\wedge}$ denote the map $T_{\Sigma_{0}}^{\wedge} \rightarrow Y$. Suppose that $Y$ is separated and locally noetherian, that $\Sigma_{0}$ is non-degenerate, full, locally finite and open and that $\left|\Sigma_{0}\right|^{0}$ is convex. 
(1) Then

$$
R^{i} \pi_{\Sigma_{0, *}}^{\wedge} \mathcal{O}_{T_{\Sigma_{0}}}= \begin{cases}\prod_{\chi \in\left|\Sigma_{0}\right|^{\vee}} \mathcal{L}(\chi) & \text { if } i=0 \\ (0) & \text { otherwise. }\end{cases}
$$

(2) If in addition $\Sigma_{0}$ is smooth then

$$
R^{i} \pi_{\Sigma_{0}, *}^{\wedge} \mathcal{I}_{\partial, \Sigma_{0}}^{\wedge}= \begin{cases}\prod_{\chi \in\left|\Sigma_{0}\right|^{\vee, 0}} \mathcal{L}(\chi) & \text { if } i=0 \\ (0) & \text { otherwise. }\end{cases}
$$

\section{Shimura varieties}

In this section we will describe the Shimura varieties associated to $G_{n}$ and the mixed Shimura varieties associated to $G_{n}^{(m)}$ and $\widetilde{G}_{n}^{(m)}$. We assume that all schemes discussed in this section are locally noetherian.

\subsection{Some Shimura varieties}

\subsubsection{Moduli problems}

By a $G_{n}$-abelian scheme over a scheme $Y / \mathbb{Q}$ we shall mean an abelian scheme $A / Y$ of relative dimension $n[F: \mathbb{Q}]$ together with an embedding

$$
i: F \hookrightarrow \operatorname{End}(A / Y)_{\mathbb{Q}}
$$

such that Lie $A$ is a locally free right $\mathcal{O}_{Y} \otimes_{\mathbb{Q}} F$-module of rank $n$. By a morphism (resp. quasi-isogeny) of $G_{n}$-abelian schemes we mean a morphism (resp. quasi-isogeny) of abelian schemes which commutes with the $F$-action. If $(A, i)$ is a $G_{n}$-abelian scheme then we give $A^{\vee}$ the structure $\left(A^{\vee}, i^{\vee}\right)$ of a $G_{n}$-abelian scheme by setting $i^{\vee}(a)=i\left({ }^{c} a\right)^{\vee}$. By a quasipolarization of $a G_{n}$-abelian scheme $(A, i) / Y$ we shall mean a quasi-isogeny $\lambda: A \rightarrow A^{\vee}$ of $G_{n}$-abelian schemes, some $\mathbb{Q}^{\times}$-multiple of which is a polarization. (Note that according to this convention, if $\lambda$ is a polarization, then $-\lambda$ is a quasi-polarization.) If $Y=\operatorname{Spec} k$ with $k$ a field, we will let $\langle,\rangle_{\lambda}$ denote the Weil pairing induced on the adelic Tate module $V A$ (see section 23 of [47]).

Lemma 3.1 If $k$ is a field of characteristic 0 and if $(A, i, \lambda) / k$ is a quasi-polarized $G_{n^{-}}$ abelian scheme, then $V_{p}(A \times \bar{k})$ is a free $F_{p}$-module of rank $2 n$.

Proof We may suppose that $k$ is a finitely generated field extension of $\mathbb{Q}$, which we may embed into $\mathbb{C}$. Then

$$
\left(V_{p}(A \times \bar{k}) \otimes_{\mathbb{Q}_{p}, l} \mathbb{C}\right) \cong\left(\operatorname{Lie} A_{\bar{y}} \otimes_{k} \mathbb{C}\right) \oplus\left(\operatorname{Lie} A_{\bar{y}} \otimes_{k, c} \mathbb{C}\right)
$$

so that $V_{p}(A \times \bar{k}) \otimes_{\mathbb{Q}_{p}, l} \mathbb{C}$ is a free $F \otimes_{\mathbb{Q}} \mathbb{C}$-module. As $F \otimes_{\mathbb{Q}} \mathbb{C}=F_{p} \otimes_{\mathbb{Q}_{p}, l} \mathbb{C}$ we deduce that $V_{p}(A \times \bar{k})$ is a free $F_{p}$-module, as desired.

By an ordinary $G_{n}$-abelian scheme over a $\mathbb{Z}_{(p)}$ - scheme $Y$ we shall mean an abelian scheme $A / Y$ of relative dimension $n[F: \mathbb{Q}]$, such that for each geometric point $\bar{y}$ of $Y$ we have $\# A[p](k(\bar{y})) \geq p^{n[F: \mathbb{Q}]}$, together with an embedding

$$
i: \mathcal{O}_{F,(p)} \hookrightarrow \operatorname{End}(A / Y)_{\mathbb{Z}_{(p)}}
$$


such that Lie $A$ is a locally free right $\mathcal{O}_{Y} \otimes_{\mathbb{Z}_{(p)}} \mathcal{O}_{F,(p)}$-module of rank $n$. By a morphism of ordinary $G_{n}$-abelian schemes we mean a morphism of abelian schemes which commutes with the $\mathcal{O}_{F,(p)}$-action. If $(A, i)$ is an ordinary $G_{n}$-abelian scheme then we give $A^{\vee}$ the structure, $\left(A^{\vee}, i^{\vee}\right)$, of a $G_{n}$-abelian scheme by setting $i^{\vee}(a)=i\left({ }^{c} a\right)^{\vee}$. By a prime-to- $p$ quasi-polarization of an ordinary $G_{n}$-abelian scheme $(A, i) / Y$ we shall mean a prime-to- $p$ quasi-isogeny $\lambda: A \rightarrow A^{\vee}$ of ordinary $G_{n^{-}}$-abelian schemes, some $\mathbb{Z}_{(p)}^{\times}$-multiple of which is a prime-to- $p$ polarization.

If $U$ is an open compact subgroup of $G_{n}\left(\mathbb{A}^{\infty}\right)$ then by a $U$-level structure on a quasipolarized $G_{n}$-abelian scheme $(A, i, \lambda)$ over a connected scheme $Y /$ Spec $\mathbb{Q}$ with a geometric point $\bar{y}$, we mean a $\pi_{1}(Y, \bar{y})$-invariant $U$-orbit $[\eta]$ of pairs $\left(\eta_{0}, \eta_{1}\right)$ of $\mathbb{A}^{\infty}$-linear isomorphisms

$$
\eta_{0}: \mathbb{A}_{\bar{y}}^{\infty} \stackrel{\sim}{\longrightarrow} \mathbb{A}^{\infty}(1)_{\bar{y}}=V \mathbb{G}_{m, \bar{y}}
$$

and

$$
\eta_{1}: V_{n} \otimes_{\mathbb{Q}} \mathbb{A}^{\infty} \stackrel{\sim}{\longrightarrow} V A_{\bar{y}}
$$

such that

$$
\eta_{1}(a x)=i(a) \eta_{1}(x)
$$

for all $a \in F$ and $x \in V_{n} \otimes_{\mathbb{Q}} \mathbb{A}^{\infty}$, and such that

$$
\left\langle\eta_{1} x, \eta_{1} y\right\rangle_{\lambda}=\eta_{0}\langle x, y\rangle_{n}
$$

for all $x, y \in V_{n} \otimes_{\mathbb{Q}} \mathbb{A}^{\infty}$. This definition is independent of the choice of geometric point $\bar{y}$ of $Y$. By a $U$-level structure on a quasi-polarized $G_{n}$-abelian scheme $(A, i, \lambda)$ over a general (locally noetherian) scheme $Y / \operatorname{Spec} \mathbb{Q}$, we mean the collection of a $U$-level structure over each connected component of $Y$. If $\left[\left(\eta_{0}, \eta_{1}\right)\right]$ is a level structure we define $\left\|\eta_{0}\right\| \in \mathbb{Q}_{>0}^{\times}$by

$$
\left\|\eta_{0}\right\| \eta_{0} \widehat{\mathbb{Z}}=\widehat{\mathbb{Z}}(1)
$$

Now suppose that $U^{p}$ is an open compact subgroup of $G_{n}\left(\mathbb{A}^{\infty, p}\right)$ and that $N_{1} \leq N_{2}$ are non-negative integers. By a $U^{p}\left(N_{1}, N_{2}\right)$-level structure on an ordinary, prime-to- $p$ quasi-polarized, $G_{n}$-abelian scheme $(A, i, \lambda)$ over a connected scheme $Y / \operatorname{Spec} \mathbb{Z}_{(p)}$ with a geometric point $\bar{y}$, we mean a $\pi_{1}(Y, \bar{y})$-invariant $U^{p}$-orbit $[\eta]$ of four-tuples $\left(\eta_{0}^{p}, \eta_{1}^{p}, C, \eta_{p}\right)$ consisting of

- an $\mathbb{A}^{\infty, p}$-linear isomorphism $\eta_{0}^{p}: \mathbb{A}_{\bar{y}}^{\infty, p} \stackrel{\sim}{\longrightarrow} \mathbb{A}^{\infty, p}(1)_{\bar{y}}=V^{p} \mathbb{G}_{m, \bar{y}}$;

- an $\mathbb{A}_{F}^{\infty, p}$-linear isomorphism

$$
\eta_{1}^{p}: V_{n} \otimes_{\mathbb{Q}} \mathbb{A}^{\infty, p} \stackrel{\sim}{\longrightarrow} V^{p} A_{\bar{y}}
$$

such that

$$
\left\langle\eta_{1}^{p} x, \eta_{1}^{p} y\right\rangle_{\lambda}=\eta_{0}\langle x, y\rangle_{n}
$$

for all $x, y \in V_{n} \otimes_{\mathbb{Q}} \mathbb{A}^{\infty, p}$ 
- a locally free sub- $\mathcal{O}_{F,(p)}$-module scheme $C \subset A\left[p^{N_{2}}\right]$, such that for every geometric point $\tilde{y}$ of $Y$ there is an $\mathcal{O}_{F,(p)}$-invariant sub-Barsotti-Tate group $\widetilde{C}_{\tilde{y}} \subset A_{\tilde{y}}\left[p^{\infty}\right]$ with the following properties

- $C_{\tilde{y}}=\widetilde{C}_{\tilde{y}}\left[p^{N_{2}}\right]$,

- for all $N$ the subgroup scheme $\widetilde{C}_{\tilde{y}}\left[p^{N}\right]$ is isotropic in $A\left[p^{N}\right]_{\tilde{y}}$ for the $\lambda$-Weil pairing,

- $A_{\tilde{y}}\left[p^{\infty}\right] / \widetilde{C}_{\tilde{y}}$ is ind-etale,

- the Tate module $T\left(A_{\tilde{y}}\left[p^{\infty}\right] / \widetilde{C}_{\tilde{y}}\right)$ is free over $\mathcal{O}_{F, p}$ of rank $n$;

- and an isomorphism

$$
\eta_{p}: p^{-N_{1}} \Lambda_{n} /\left(p^{-N_{1}} \Lambda_{n,(n)}+\Lambda_{n}\right) \stackrel{\sim}{\longrightarrow} A\left[p^{N_{1}}\right] /\left(A\left[p^{N_{1}}\right] \cap C\right)
$$

such that

$$
\eta_{p}(a x)=i(a) \eta_{p}(x)
$$

for all $a \in \mathcal{O}_{F,(p)}$ and $x \in p^{-N_{1}} \Lambda_{n} /\left(p^{-N_{1}} \Lambda_{n,(n)}+\Lambda_{n}\right)$.

This definition is independent of the choice of the geometric point $\bar{y} \in Y$. By a $U^{p}\left(N_{1}, N_{2}\right)$ level structure on an ordinary, prime-to- $p$ quasi-polarized, $G_{n}$-abelian scheme $(A, i, \lambda)$ over a general (locally noetherian) scheme $Y / \operatorname{Spec} \mathbb{Z}_{(p)}$, we mean the collection of a $U^{p}\left(N_{1}, N_{2}\right)$ level structure over each connected component of $Y$. If $\left[\left(\eta_{0}^{p}, \eta_{1}^{p}, C, \eta_{p}\right)\right]$ is a level structure we define $\left\|\eta_{0}^{p}\right\| \in \mathbb{Z}_{(p),>0}^{\times}$by

$$
\left\|\eta_{0}^{p}\right\| \eta_{0}^{p} \widehat{\mathbb{Z}}^{p}=\widehat{\mathbb{Z}}^{p}(1)
$$

By a quasi-isogeny (resp. prime-to- $p$ quasi-isogeny) between quasi-polarized, $G_{n}$ abelian schemes with $U$-level structures (resp. ordinary, prime-to- $p$ quasi-polarized, $G_{n^{-}}$ abelian schemes with $U^{p}\left(N_{1}, N_{2}\right)$-level structures)

$$
(\beta, \delta):(A, i, \lambda,[\eta]) \longrightarrow\left(A^{\prime}, i^{\prime}, \lambda^{\prime},\left[\eta^{\prime}\right]\right)
$$

we mean a quasi-isogeny (resp. prime-to- $p$ quasi-isogeny) of abelian schemes $\beta \in$ $\operatorname{Hom}\left(A, A^{\prime}\right)_{\mathbb{Q}}\left(\right.$ resp. $\left.\beta \in \operatorname{Hom}\left(A, A^{\prime}\right)_{\mathbb{Z}_{(p)}}\right)$ and $\delta \in \mathbb{Q}^{\times}$(resp. $\left.\delta \in \mathbb{Z}_{(p)}^{\times}\right)$such that

- $\beta \circ i(a)=i^{\prime}(a) \circ \beta$ for all $a \in F\left(\operatorname{resp} . \mathcal{O}_{F,(p)}\right)$;

- $\delta \lambda=\beta^{\vee} \circ \lambda^{\prime} \circ \beta$;

- $\left[\left(\delta \eta_{0},(V \beta) \circ \eta_{1}\right)\right]=\left[\eta^{\prime}\right]\left(\operatorname{resp} .\left[\left(\delta \eta_{0}^{p},\left(V^{p} \beta\right) \circ \eta_{1}^{p}, \beta C, \beta \circ \eta_{p}\right)\right]=\left[\eta^{\prime}\right]\right)$.

Lemma 3.2 Suppose that $T$ is an $\mathcal{O}_{F, p}$-module, which is free over $\mathcal{O}_{F, p}$ of rank $2 n$, with a perfect alternating pairing

$$
\langle,\rangle: T \times T \longrightarrow \mathbb{Z}_{p}
$$

such that

$$
\langle a x, y\rangle=\left\langle x,{ }^{c} a y\right\rangle
$$


for all $x, y \in T$ and $a \in \mathcal{O}_{F, p}$. Also suppose that $\widetilde{T} \subset T$ is a sub- $\mathcal{O}_{F, p}$-module which is isotropic for $\langle$,$\rangle and such that T / \widetilde{T}$ is free of rank $n$ over $\mathcal{O}_{F, p}$. Finally suppose that

$$
\eta_{p}: p^{-N_{1}} \Lambda_{n} /\left(p^{-N_{1}} \Lambda_{n,(n)}+\Lambda_{n}\right) \stackrel{\sim}{\longrightarrow} p^{-N_{1}} T /\left(p^{-N_{1}} \widetilde{T}+T\right)
$$

is an $\mathcal{O}_{F, p}$-module isomorphism.

Consider the set $[\eta]$ of isomorphisms

$$
\eta: \Lambda_{n} \otimes \mathbb{Z}_{p} \stackrel{\sim}{\longrightarrow} T
$$

such that

- $\eta(a x)=a \eta(x)$ for all $a \in \mathcal{O}_{F,(p)}$;

- there exists $\delta \in \mathbb{Z}_{p}^{\times}$such that

$$
\langle\eta x, \eta y\rangle=\delta\langle x, y\rangle_{n}
$$

for all $x, y \in \Lambda_{n} \otimes \mathbb{Z}_{p}$

- $\eta\left(\left(p^{-N_{2}} \Lambda_{n,(n)}\right) \otimes \mathbb{Z}_{p}+\Lambda_{n} \otimes \mathbb{Z}_{p}\right)=p^{-N_{2}} \widetilde{T}+T$;

- the map

$$
p^{-N_{1}} \Lambda_{n} /\left(p^{-N_{1}} \Lambda_{n,(n)}+\Lambda_{n}\right) \stackrel{\sim}{\longrightarrow} p^{-N_{1}} T /\left(p^{-N_{1}} \widetilde{T}+T\right)
$$

induced by $\eta$ equals $\eta_{p}$.

Then $[\eta]$ is non-empty and a single $U_{p}\left(N_{1}, N_{2}\right)$-orbit.

Proof Let $e_{1}, \ldots, e_{n}$ denote a $\mathcal{O}_{F, p}$-basis of $T / \widetilde{T}$. Note that $\langle$,$\rangle induces a perfect pairing$ between $\widetilde{T}$ and $T / \widetilde{T}$. We recursively lift the $e_{i}$ to elements $\widetilde{e}_{i} \in T$ with $\widetilde{e}_{i}$ orthogonal to the $\mathcal{O}_{F, p}$ span of the $\widetilde{e}_{j}$ for $j<i$. Suppose that $\widetilde{e}_{1}, \ldots, \widetilde{e}_{i-1}$ have already been chosen. Choose some lift $e_{i}^{\prime}$ of $e_{i}$. Then choose $t \in \widetilde{T}$ such that

- $\langle t, x\rangle=\left\langle e_{i}^{\prime}, x\right\rangle$ for all $x \in \bigoplus_{j=1}^{i-1} \mathcal{O}_{F, p} \widetilde{e}_{j}$,

- and $\left\langle t, \alpha e_{i}^{\prime}\right\rangle=\left\langle e_{i}^{\prime} / 2, \alpha e_{i}^{\prime}\right\rangle$ for all $\alpha \in \mathcal{O}_{F, p}^{c=-1}$.

(If $p=2$ some explanation is required as to why we can do this. The map

$$
\begin{aligned}
\mathcal{O}_{F, p} & \longrightarrow \mathbb{Z}_{p} \\
\alpha & \longmapsto\left\langle e_{i}^{\prime}, \alpha e_{i}^{\prime}\right\rangle
\end{aligned}
$$

is of the form

$$
\alpha \longmapsto \operatorname{tr}_{F / \mathbb{Q}}(\beta \alpha)
$$

for some $\beta \in\left(\mathcal{D}_{F, p}^{-1}\right)^{c=-1}$. Because $p=2$ is unramified in $F / F^{+}$, we can write $\beta=\gamma-{ }^{c} \gamma$ for some $\gamma \in \mathcal{D}_{F, p}^{-1}$. Thus the second condition can be replaced by the condition

$$
\left\langle t, \alpha e_{i}^{\prime}\right\rangle=\operatorname{tr}_{F / \mathbb{Q}}(\gamma \alpha)
$$

for all $\alpha \in \mathcal{O}_{F, p}^{c=-1}$. Now it is clear that the required element $t$ exists.) Then take $\widetilde{e}_{i}=e_{i}^{\prime}-t$. Then $\widetilde{e}_{i}$ is orthogonal to $\bigoplus_{j=1}^{i-1} \mathcal{O}_{F, p} \widetilde{e}_{j}$. Moreover for $\alpha \in \mathcal{O}_{F, p}$ we have 


$$
\begin{aligned}
\left\langle\widetilde{e}_{i}, \alpha \widetilde{e}_{i}\right\rangle & =\left\langle e_{i}^{\prime}, \alpha e_{i}^{\prime}\right\rangle-\left\langle t,\left(\alpha-{ }^{c} \alpha\right) e_{i}^{\prime}\right\rangle \\
& =\left\langle e_{i}^{\prime}, \alpha e_{i}^{\prime}\right\rangle-\left\langle e_{i}^{\prime} / 2,\left(\alpha-{ }^{c} \alpha\right) e_{i}^{\prime}\right\rangle \\
& =\left(\left\langle e_{i}^{\prime}, \alpha e_{i}^{\prime}\right\rangle+\left\langle e_{i}^{\prime},{ }^{c} \alpha e_{i}^{\prime}\right\rangle\right) / 2 \\
& =0 .
\end{aligned}
$$

Thus we can write

$$
T=\widetilde{T} \oplus \widetilde{T}^{\prime}
$$

with $\widetilde{T}^{\prime}$ an isotropic $\mathcal{O}_{F, p}$-subspace of $T$, which is free over $\mathcal{O}_{F, p}$ of rank $n$. We see that

$$
\widetilde{T}^{\prime} \cong \operatorname{Hom}_{\mathbb{Z}_{p}}\left(\widetilde{T}, \mathbb{Z}_{p}\right)
$$

The lemma now follows without difficulty.

Corollary 3.3 If $Y$ is a $\mathbb{Q}$-scheme with geometric point $\bar{y}$, if $(A, i, \lambda) / Y$ is an ordinary $G_{n^{-}}$ abelian scheme and if $\left[\left(\eta_{0}^{p}, \eta_{1}^{p}, C, \eta_{p}\right)\right]$ is a $U^{p}\left(N_{1}, N_{2}\right)$-level structure on $(A, i, \lambda)$, then there is a unique $U_{p}\left(N_{1}, N_{2}\right)$-orbit of pairs of isomorphisms

$$
\eta_{0, p}: \mathbb{Z}_{p, \bar{y}} \stackrel{\sim}{\longrightarrow} \mathbb{Z}_{p}(1)_{\bar{y}}
$$

and

$$
\eta_{1, p}: \Lambda_{n} \otimes \mathbb{Z}_{p} \stackrel{\sim}{\longrightarrow} T_{p} A_{\bar{y}}
$$

such that

- $\eta_{1, p}(a x)=a \eta_{1, p}(x)$ for all $a \in \mathcal{O}_{F,(p)}$,

- $\left\langle\eta_{1, p} x, \eta_{1, p} y\right\rangle_{\lambda}=\eta_{0, p}\langle x, y\rangle_{n}$ for all $x, y \in \Lambda_{n} \otimes \mathbb{Z}_{p}$,

- $\eta_{1, p} p^{-N_{2}} \Lambda_{n,(n)} / \Lambda_{n,(n)}=C$,

- $\eta_{1, p}$ induces $\eta_{p}$.

Proof This follows on combining Lemmas 3.1 and 3.2.

Corollary 3.4 Suppose that $Y$ is a scheme over $\operatorname{Spec} \mathbb{Q}$. There is a natural bijection between prime-to-p quasi-isogeny classes of ordinary, prime-to-p quasi-polarized $G_{n^{-}}$ abelian schemes with $U^{p}\left(N_{1}, N_{2}\right)$-level structure and quasi-isogeny classes of quasipolarized $G_{n}$-abelian schemes with $U^{p}\left(N_{1}, N_{2}\right)$-level structure.

Proof We may assume that $Y$ is connected with geometric point $\bar{y}$. We will show both sets are in natural bijection with the set of prime-to-p quasi-isogeny classes of four-tuples $(A, i, \lambda,[\eta])$, where $(A, i)$ is a $G_{n}$-abelian scheme, $\lambda$ is a prime-to- $p$ quasi-polarization of $(A, i)$, and $[\eta]$ is a $\pi_{1}(Y, \bar{y})$-invariant $U^{p}\left(N_{1}, N_{2}\right)$-orbit of pairs $\left(\eta_{0}, \eta_{1}\right)$, where

- $\eta_{0}: \mathbb{A}^{\infty, p} \times \mathbb{Z}_{p} \stackrel{\sim}{\rightarrow} \mathbb{A}^{\infty, p}(1) \times \mathbb{Z}_{p}(1)$,

- and $\eta_{1}: \Lambda_{n} \otimes\left(\mathbb{A}^{\infty, p} \times \mathbb{Z}_{p}\right) \stackrel{\sim}{\rightarrow} V^{p} A_{\bar{y}} \times T_{p} A_{\bar{y}}$ satisfies

$$
\eta_{0}\langle x, y\rangle_{n}=\left\langle\eta_{1} x, \eta_{1} y\right\rangle_{\lambda}
$$


There is a natural map from this set to the set of quasi-isogeny classes of quasi-polarized $G_{n}$-abelian schemes with $U^{p}\left(N_{1}, N_{2}\right)$-level structure, which is easily checked to be a bijection. The bijection between this set and the set of prime-to- $p$ quasi-isogeny classes of ordinary, prime-to- $p$ quasi-polarized $G_{n}$-abelian schemes with a $U^{p}\left(N_{1}, N_{2}\right)$-level structure, follows by the usual arguments (see, for instance, section III.1 of [29]) from Corollary 3.3.

\subsubsection{Hecke actions}

If $(A, i, \lambda,[\eta]) / Y$ is a quasi-polarized, $G_{n}$-abelian scheme with $U$-level structure and if $g \in G_{n}\left(\mathbb{A}^{\infty}\right)$ with $U^{\prime} \supset g^{-1} U g$, then we can define a quasi-polarized, $G_{n}$-abelian scheme with $U^{\prime}$-level structure $(A, i, \lambda,[\eta]) g / Y$ by

$$
\left(A, i, \lambda,\left[\left(\eta_{0}, \eta_{1}\right)\right]\right) g=\left(A, i, \lambda,\left[\left(v(g) \eta_{0}, \eta_{1} \circ g\right]\right) .\right.
$$

This action takes one quasi-isogeny class to another.

If $(A, i, \lambda,[\eta]) / Y$ is an ordinary, prime-to- $p$ quasi-polarized, $G_{n}$-abelian scheme with $U^{p}\left(N_{1}, N_{2}\right)$-level structure and if $g \in G_{n}\left(\mathbb{A}^{\infty}\right)^{\text {ord, } \times}$ with

$$
\left(U^{\prime}\right)^{p}\left(N_{1}^{\prime}, N_{2}^{\prime}\right) \supset g^{-1} U^{p}\left(N_{1}, N_{2}\right) g
$$

(so that in particular $N_{i} \geq N_{i}^{\prime}$ for $i=1,2$ ), then we can define an ordinary, prime-to- $p$ quasi-polarized, $G_{n}$-abelian scheme with $\left(U^{\prime}\right)^{p}\left(N_{1}^{\prime}, N_{2}^{\prime}\right)$-level structure $(A, i, \lambda,[\eta]) g / Y$ by

$$
\left(A, i, \lambda,\left[\left(\eta_{0}^{p}, \eta_{1}^{p}, C, \eta_{p}\right)\right]\right) g=\left(A, i, \lambda,\left[\left(v\left(g^{p}\right) \eta_{0}^{p}, \eta_{1}^{p} \circ g^{p}, C\left[p^{N_{2}^{\prime}}\right], \eta_{p} \circ g_{p}\right)\right]\right) .
$$

Recall the definition of $\varsigma_{p}$ towards the end of Sect. 1.2. If $\left(U^{\prime}\right)^{p}\left(N_{1}^{\prime}, N_{2}^{\prime}\right) \supset$ $\varsigma_{p}^{-1} U^{p}\left(N_{1}, N_{2}\right) \varsigma_{p}$ (so that in particular $N_{1} \geq N_{1}^{\prime}$ and $N_{2}>N_{2}^{\prime}$ ), then we can define an ordinary, prime-to- $p$ quasi-polarized, $G_{n}$-abelian scheme with $\left(U^{\prime}\right)^{p}\left(N_{1}^{\prime}, N_{2}^{\prime}\right)$-level structure $(A, i, \lambda,[\eta]) \varsigma_{p} / Y$ by

$$
\left(A, i, \lambda,\left[\left(\eta_{0}^{p}, \eta_{1}^{p}, C, \eta_{p}\right)\right]\right) \varsigma_{p}=\left(A / C[p], i, F(\lambda),\left[\left(p \eta_{0}^{p}, F\left(\eta_{1}^{p}\right), C\left[p^{1+N_{2}^{\prime}}\right] / C[p], F\left(\eta_{p}\right)\right)\right]\right)
$$

where

$$
F(\lambda): A / C[p] \stackrel{\lambda}{\longrightarrow} A^{\vee} / \lambda C[p]=A^{\vee} / C[p]^{\perp} \stackrel{\sim}{\longrightarrow}(A / C[p])^{\vee}
$$

with the last isomorphism being induced by the dual of the map $A / C[p] \rightarrow A$ induced by multiplication by $p$ on $A$; where $F\left(\eta_{1}^{p}\right)$ is the composition of $\eta_{1}^{p}$ with the natural map $V^{p} A \stackrel{\sim}{\rightarrow} V^{p}(A / C[p])$; and where $F\left(\eta_{p}\right)$ is the composition of $\eta_{p}$ with the natural identification

$$
A\left[p^{N_{1}^{\prime}}\right] /\left(C \cap A\left[p^{N_{1}^{\prime}}\right]\right)=(A / C[p])\left[p^{N_{1}^{\prime}}\right] /\left(C\left[p^{1+N_{2}^{\prime}}\right] / C[p] \cap(A / C[p])\left[p^{N_{1}^{\prime}}\right]\right) .
$$

If $Y$ is an $\mathbb{F}_{p}$-scheme then $\varsigma_{p}$ is the composite of pull-back by absolute Frobenius followed by forgetting some of the structure.

Together these two definitions give an action of $G_{n}\left(\mathbb{A}^{\infty}\right)^{\text {ord }}$. This action takes one prime-to- $p$ quasi-isogeny class to another.

With these definitions the correspondence of Corollary 3.4 is $G_{n}\left(\mathbb{A}^{\infty}\right)^{\text {ord }}$-equivariant. 


\subsubsection{Representability}

If $U$ is a neat open compact subgroup of $G_{n}\left(\mathbb{A}^{\infty}\right)$ then the functor that sends a (locally noetherian) scheme $S / \mathbb{Q}$ to the set of quasi-isogeny classes of polarized $G_{n}$-abelian schemes with $U$-level structures is represented by a quasi-projective scheme $X_{n, U}$ which is smooth of relative dimension $n^{2}\left[F^{+}: \mathbb{Q}\right]$ over $\mathbb{Q}$. Let

$$
\left[\left(A^{\text {univ }}, i^{\text {univ }}, \lambda^{\text {univ }},\left[\eta^{\text {univ }}\right]\right)\right] / X_{n, U}
$$

denote the universal equivalence class of polarized $G_{n}$-abelian schemes with $U$-level structure. If $U^{\prime} \supset g^{-1} U g$ then there is a map $g: X_{n, U} \rightarrow X_{n, U^{\prime}}$ arising from $\left(A^{\text {univ }}, i^{\text {univ }}, \lambda^{\text {univ }},\left[\eta^{\text {univ }}\right]\right) g / X_{n, U}$ and the universal property of $X_{n, U^{\prime}}$. This makes $\left\{X_{n, U}\right\}$ an inverse system of schemes with right $G_{n}\left(\mathbb{A}^{\infty}\right)$-action. The maps $g$ are finite etale. If $U_{1} \subset U_{2}$ is a normal subgroup then $X_{n, U_{1}} / X_{n, U_{2}}$ is Galois with group $U_{2} / U_{1}$.

There are identifications of topological spaces:

$$
X_{n, U}(\mathbb{C}) \cong G_{n}(\mathbb{Q})^{+} \backslash\left(G_{n}\left(\mathbb{A}^{\infty}\right) / U \times \mathfrak{H}_{n}^{+}\right) \cong G_{n}(\mathbb{Q}) \backslash\left(G_{n}\left(\mathbb{A}^{\infty}\right) / U \times \mathfrak{H}_{n}^{ \pm}\right)
$$

compatible with the right action of $G_{n}\left(\mathbb{A}^{\infty}\right)$. (See sections 7 and 8 of [39]. Note that $\operatorname{ker}^{1}\left(\mathbb{Q}, G_{n}\right)=(0)$, as is explained in section 7 of [39].) More precisely we associate to $(g, I) \in G_{n}\left(\mathbb{A}^{\infty}\right) / U \times \mathfrak{H}_{n}^{+}$the torus $\left(\Lambda_{n} \otimes_{\mathbb{Z}} \mathbb{R}\right) / \Lambda_{n}$ with complex structure coming from $I$; with polarization corresponding to the Riemann form given by $\langle$,$\rangle ; and with level$ structure coming from

$$
\eta_{1}: \Lambda_{n} \otimes \mathbb{A}^{\infty} \stackrel{g}{\longrightarrow} \Lambda_{n} \otimes \mathbb{A}^{\infty}=V\left(\left(\Lambda_{n} \otimes_{\mathbb{Z}} \mathbb{R}\right) / \Lambda_{n}\right)
$$

and

$$
\begin{aligned}
\eta_{0}: \mathbb{A}^{\infty} & \stackrel{\sim}{\longrightarrow} \mathbb{A}^{\infty}(1) \\
x & \longmapsto-v(g) x \zeta,
\end{aligned}
$$

where $\zeta=\lim _{\leftarrow N} e^{2 \pi i / N} \in \widehat{\mathbb{Z}}(1)$. We deduce that

$$
\begin{aligned}
\pi_{0}\left(X_{n, U} \times \operatorname{Spec} \overline{\mathbb{Q}}\right) & \cong G_{n}(\mathbb{Q}) \backslash G_{n}(\mathbb{A}) /\left(U G_{n}(\mathbb{R})^{+}\right) \\
& \cong G_{n}(\mathbb{Q}) \backslash\left(G_{n}\left(\mathbb{A}^{\infty}\right) / U \times \pi_{0}\left(G_{n}(\mathbb{R})\right)\right) \\
& \cong C_{n}(\mathbb{Q}) \backslash C_{n}(\mathbb{A}) / U C_{n}(\mathbb{R})^{0} .
\end{aligned}
$$

If $U^{p}$ is neat then the functor that sends a scheme $Y / \mathbb{Z}_{(p)}$ to the set of prime-to- $p$ quasi-isogeny classes of ordinary, prime-to- $p$ quasi-polarized, $G_{n}$-abelian schemes with $U^{p}\left(N_{1}, N_{2}\right)$-level structure is represented by a scheme $\mathcal{X}_{n, U^{p}\left(N_{1}, N_{2}\right)}^{\text {ord }}$ quasi-projective over $\mathbb{Z}_{(p)}$. (See theorems 3.4.1.9 and 3.4.2.5 in [44]. Note that, by theorem 3.4.1.9 in [44], the naive moduli problem there is already smooth, and hence the submoduli problem with the right Lie algebra condition agrees with the normalization in theorem 3.4.2.5 in [44].) Let

$$
\left[\left(\mathcal{A}^{\text {univ }}, i^{\text {univ }}, \lambda^{\text {univ }},\left[\eta^{\text {univ }}\right]\right)\right] / \mathcal{X}_{n, U^{p}\left(N_{1}, N_{2}\right)}^{\text {ord }}
$$

denote the universal equivalence class. If $g \in G_{n}\left(\mathbb{A}^{\infty}\right)^{\text {ord }}$ and $\left(U^{p}\right)^{\prime}\left(N_{1}^{\prime}, N_{2}^{\prime}\right) \supset$ $g^{-1} U^{p}\left(N_{1}, N_{2}\right) g$, then there is a quasi-finite map

$$
g: \mathcal{X}_{n, U^{p}\left(N_{1}, N_{2}\right)}^{\text {ord }} \longrightarrow \mathcal{X}_{\left(U^{p}\right)^{\prime}\left(N_{1}^{\prime}, N_{2}^{\prime}\right)}^{\text {ord }}
$$


arising from ( $\left.\mathcal{A}^{\text {univ }}, i^{\text {univ }}, \lambda^{\text {univ }},\left[\eta^{\text {univ }}\right]\right) g / \mathcal{X}_{n, U^{p}\left(N_{1}, N_{2}\right)}^{\text {ord }}$ and the universal property of $\mathcal{X}_{n,\left(U^{p}\right)^{\prime}\left(N_{1}^{\prime}, N_{2}^{\prime}\right)}^{\text {ord }}$. If $g \in G_{n}\left(\mathbb{A}^{\infty}\right)^{\text {ord, } \times}$ then the map $g$ is etale, and, if further $N_{2}=$ $N_{2}^{\prime}$, then it is finite etale. If $U^{p}\left(N_{1}, N_{2}\right)$ is a normal subgroup of $\left(U^{p}\right)^{\prime}\left(N_{1}^{\prime}, N_{2}\right)$ then $\mathcal{X}_{n, U^{p}\left(N_{1}, N_{2}\right)}^{\text {ord }} / \mathcal{X}_{n,\left(U^{p}\right)^{\prime}\left(N_{1}^{\prime}, N_{2}\right)}^{\text {ord }}$ is Galois with group $\left(U^{p}\right)^{\prime}\left(N_{1}^{\prime}\right) / U^{p}\left(N_{1}\right)$. There are $G_{n}\left(\mathbb{A}^{\infty}\right)^{\text {ord }}$ equivariant identifications

$$
\mathcal{X}_{n, U^{p}\left(N_{1}, N_{2}\right)}^{\text {ord }} \times \operatorname{Spec} \mathbb{Q} \cong X_{n, U^{p}\left(N_{1}, N_{2}\right)}
$$

The scheme $\mathcal{X}_{n, U^{p}\left(N_{1}, N_{2}\right)}^{\text {ord }}$ is smooth over $\mathbb{Z}_{(p)}$ of relative dimension $n^{2}\left[F^{+}: \mathbb{Q}\right]$. (By the Serre-Tate theorem (see [36]) the formal completion of $\mathcal{X}_{n, U^{p}\left(N_{1}, N_{2}\right)}^{\text {ord }}$ at a point $x$ in the special fibre is isomorphic to

$$
\operatorname{Hom}_{\mathbb{Z}_{p}}\left(S\left(T_{p} \mathcal{A}_{x}^{\text {univ }}\right), \widehat{\mathbb{G}}_{m}\right)
$$

This is formally smooth as long as $S\left(T_{p} \mathcal{A}_{x}^{\text {univ }}\right) \cong S\left(\mathcal{O}_{F, p}^{n}\right)$ is torsion free. This module is torsion free because in the case $p=2$ we are assuming that $p=2$ is unramified in $F / F^{+}$.) Suppose that $g \in G_{n}\left(\mathbb{A}^{\infty}\right)^{\text {ord }}$ and $\left(U^{p}\right)^{\prime}\left(N_{1}^{\prime}, N_{2}^{\prime}\right) \supset g^{-1} U^{p}\left(N_{1}, N_{2}\right) g$, then the quasi-finite map

$$
g: \mathcal{X}_{n, U^{p}\left(N_{1}, N_{2}\right)}^{\text {ord }} \longrightarrow \mathcal{X}_{n,\left(U^{p}\right)^{\prime}\left(N_{1}^{\prime}, N_{2}^{\prime}\right)}^{\text {ord }}
$$

is in fact flat, because it is a quasi-finite map between locally noetherian regular schemes which are equidimensional of the same dimension. (See pages 507 and 508 of [37].)

On $\mathbb{F}_{p}$-fibres the map

$$
\varsigma_{p}: \mathcal{X}_{n, U^{p}\left(N_{1}, N_{2}+1\right)}^{\text {ord }} \times \operatorname{Spec} \mathbb{F}_{p} \longrightarrow \mathcal{X}_{n, U^{p}\left(N_{1}, N_{2}\right)}^{\text {ord }} \times \operatorname{Spec} \mathbb{F}_{p}
$$

is the absolute Frobenius map composed with the forgetful map $1: \mathcal{X}_{n, U^{p}\left(N_{1}, N_{2}+1\right)}^{\text {ord }} \rightarrow$ $\mathcal{X}_{n, U^{p}\left(N_{1}, N_{2}\right)}^{\text {ord }}$ (for any $\left.N_{2} \geq N_{1} \geq 0\right)$. Thus if $N_{2}>0$, then the quasi-finite, flat map

$$
\varsigma_{p}: \mathcal{X}_{n, U^{p}\left(N_{1}, N_{2}+1\right)}^{\text {ord }} \longrightarrow \mathcal{X}_{n, U^{p}\left(N_{1}, N_{2}\right)}^{\text {ord }}
$$

has all its fibres of degree $p^{n^{2}\left[F^{+}: \mathbb{Q}\right]}$ and hence is finite flat of this degree. (A flat, quasi-finite morphism $f: X \rightarrow Y$ between noetherian schemes with constant fibre degree is proper and hence, by theorem 8.11.1 of [24], finite. We give the argument for properness. By the valuative criterion we may reduce to the case $Y=\operatorname{Spec} B$ for a DVR $B$ with fraction field $L$. By theorem 8.12.6 of [24] $X$ is a dense open subscheme of $\operatorname{Spec} A$, for $A$ a finite $B$ algebra. Let $I$ denote the ideal of $A$ consisting of all $\mathfrak{m}_{B}$-torsion elements. If $f \in A$ and Spec $A_{f} \subset X$, then by flatness the map $A \rightarrow A_{f}$ factors through $A / I$. Thus $X \subset \operatorname{Spec} A / I$ and in fact $I=(0)$, so that $A / B$ is finite flat. Because an open subscheme is determined by its points, we conclude that we must have $X=\operatorname{Spec} A^{\prime}$ for some $A \subset A^{\prime} \subset A \otimes_{B} L$. By the constancy of the fibre degree we conclude that $A^{\prime}$ is finite over $B$.) We deduce that for any $g \in G_{n}\left(\mathbb{A}^{\infty}\right)^{\text {ord }}$, if $N_{2}^{\prime}>0$ and $p^{N_{2}-N_{2}^{\prime}} v\left(g_{p}\right) \in \mathbb{Z}_{p}^{\times}$, then the map

$$
g: \mathcal{X}_{n, U^{p}\left(N_{1}, N_{2}\right)}^{\text {ord }} \longrightarrow \mathcal{X}_{\left(U^{p}\right)^{\prime}\left(N_{1}^{\prime}, N_{2}^{\prime}\right)}^{\text {ord }}
$$

is finite. 
Lemma 3.5 Write $\mathcal{X}_{n, U^{p}\left(N_{1}, N_{2}\right)}^{\mathrm{ord} \wedge}$ for the completion of $\mathcal{X}_{U^{p}\left(N_{1}, N_{2}\right)}^{\text {ord }}$ along its $\mathbb{F}_{p}$-fibre. If $N_{2}^{\prime}>$ $N_{2} \geq N_{1}$ then the map

$$
1: \mathcal{X}_{n, U^{p}\left(N_{1}, N_{2}^{\prime}\right)}^{\mathrm{ord} \wedge} \longrightarrow \mathcal{X}_{n, U^{p}\left(N_{1}, N_{2}\right)}^{\mathrm{ord} \wedge}
$$

is an isomorphism.

Proof The map has an inverse which sends a tuple $\left[\left(\mathcal{A}^{\text {univ }}, i^{\text {univ }}, \lambda^{\text {univ }},\left[\eta^{\text {univ }}\right]\right)\right]$ over $\mathcal{X}_{n, U^{p}\left(N_{1}, N_{2}\right)}^{\text {ord } \wedge}$ to

$$
\left[\left(\mathcal{A}^{\text {univ }}, i^{\text {univ }}, \lambda^{\text {univ }},\left[\left(\eta_{0}^{\text {univ }, p}, \eta_{1}^{\text {univ }, p}, \mathcal{A}^{\text {univ }}\left[p^{N_{2}^{\prime}}\right]^{0}, \eta_{p}^{\text {univ }}\right)\right]\right)\right]
$$

over $\mathcal{X}_{n, U^{p}\left(N_{1}, N_{2}^{\prime}\right)}^{\text {ord } \wedge}$

Thus we will denote $\mathcal{X}_{n, U^{p}\left(N_{1}, N_{2}\right)}^{\text {ord } \wedge}$ simply

$$
\mathfrak{X}_{n, U^{p}\left(N_{1}\right)}^{\text {ord }}
$$

Then $\left\{\mathfrak{X}_{n, U^{p}(N)}^{\text {ord }}\right\}$ is a system of $p$-adic formal schemes with right $G_{n}\left(\mathbb{A}^{\infty}\right)^{\text {ord }}$-action. We will write $\bar{X}_{n, U^{p}(N)}^{\text {ord }}$ for the reduced subscheme of $\mathfrak{X}_{n, U^{p}(N)}^{\text {ord }}$.

Throughout the paper we will use usual Roman letters, such as $X$, for 'Shimura-like' varieties of finite type over $\mathbb{Q}$, cursive letters, such as $\mathcal{X}$, for models of them of finite type over $\mathbb{Z}_{(p)}$, over-lined usual Roman letters, such as $\bar{X}$, for their $\mathbb{F}_{p}$-fibre, and Gothic letters, such as $\mathfrak{X}$, for their formal completion along this special fibre.

\subsection{Some Kuga-Sato varieties}

Recall that a semi-abelian scheme is a smooth separated commutative group scheme such that each geometric fibre is the extension of an abelian scheme by a torus. To a semi-abelian scheme $G / Y$ one can associate an etale constructible sheaf of abelian groups $X^{*}(G)$, the 'character group of the toric part of $G$ '. See theorem I.2.10 of [17]. If $X^{*}(G)$ is locally constant then $G$ is an extension

$$
(0) \longrightarrow S_{G} \longrightarrow G \longrightarrow A_{G} \longrightarrow(0)
$$

of a uniquely determined abelian scheme $A_{G}$ by a uniquely determined split torus $S_{G}$ with character group $X^{*}(G)$. By an isogeny (resp. prime-to-p isogeny) of semi-abelian schemes we mean a morphism which is quasi-finite and surjective (resp. quasi-finite and surjective and whose geometric fibres have orders relatively prime to $p$ ). If $Y$ is locally noetherian, then by a quasi-isogeny (resp. prime-to-p quasi-isogeny) $\alpha: G \rightarrow G^{\prime}$ we mean an element of $\operatorname{Hom}\left(G, G^{\prime}\right)_{\mathbb{Q}}\left(\operatorname{resp}\right.$. $\left.\operatorname{Hom}\left(G, G^{\prime}\right)_{\mathbb{Z}_{(p)}}\right)$ with an inverse in $\operatorname{Hom}\left(G^{\prime}, G\right)_{\mathbb{Q}}$ (resp. $\left.\operatorname{Hom}\left(G^{\prime}, G\right)_{\mathbb{Z}_{(p)}}\right)$.

Suppose that $Y / \operatorname{Spec} \mathbb{Q}$ is a locally noetherian scheme. By a $G_{n}^{(m)}$-semi-abelian scheme $G$ over $Y$ we mean a triple $(G, i, j)$ where

- $G / Y$ is a semi-abelian scheme,

- $i: F \hookrightarrow \operatorname{End}(G)_{\mathbb{Q}}$,

- and $j: F^{m} \stackrel{\sim}{\rightarrow} X^{*}(G)_{\mathbb{Q}}$ is an $F$-linear isomorphism; 
- such that Lie $A_{G}$ is a free $\mathcal{O}_{Y} \otimes_{\mathbb{Q}} F$ module of $\operatorname{rank} n[F: \mathbb{Q}]$.

Then $A_{G}$ is a $G_{n}$-abelian scheme. By a quasi-isogeny of $G_{n}^{(m)}$-semi-abelian schemes we mean a quasi-isogeny of semi-abelian schemes

$$
\beta: G \rightarrow G^{\prime}
$$

such that

$$
i^{\prime}(a) \circ \beta=\beta \circ i(a)
$$

for all $a \in F$, and

$$
j=X^{*}(\beta) \circ j^{\prime}
$$

Note that, if $\bar{y}$ is a geometric point of $Y$, then $j$ induces a map

$$
j^{*}: V S_{G, \bar{y}} \stackrel{\sim}{\longrightarrow} \operatorname{Hom}_{\mathbb{Q}}\left(F^{m}, V \mathbb{G}_{m, \bar{y}}\right) .
$$

By a quasi-polarization of $(G, i, j)$ we mean a quasi-polarization of $A_{G}$.

If $Y$ is connected and $\bar{y}$ is a geometric point of $Y$ and if $U \subset G_{n}^{(m)}\left(\mathbb{A}^{\infty}\right)$ is a neat open compact subgroup then by a $U$ level structure on a quasi-polarized $G_{n}^{(m)}$-semi-abelian scheme $(G, i, j, \lambda)$ we mean a $\pi_{1}(Y, \bar{y})$-invariant $U$-orbit of pairs $\left(\eta_{0}, \eta_{1}\right)$ where

$$
\eta_{0}: \mathbb{A}^{\infty} \stackrel{\sim}{\longrightarrow} V \mathbb{G}_{m, \bar{y}}
$$

is an $\mathbb{A}^{\infty}$-linear map, and where

$$
\eta_{1}: \Lambda_{n}^{(m)} \otimes_{\mathbb{Z}} \mathbb{A}^{\infty} \longrightarrow V G_{\bar{y}}
$$

is an $\mathbb{A}_{F}^{\infty}$-linear map such that

$$
\left.\eta_{1}\right|_{\operatorname{Hom}_{\mathbb{Z}}\left(\mathcal{O}_{F}^{m}, \mathbb{A}^{\infty}\right)}=\left(j^{*}\right)^{-1} \circ \operatorname{Hom}\left(1_{F^{m}}, \eta_{0}\right)
$$

and

$$
\left[\left(\eta_{0}, \eta_{1} \bmod V S_{G, \bar{y}}\right)\right]
$$

is a $U$-level structure on $A_{G}$. This is canonically independent of $\bar{y}$. We define a $U$ level structure on a $G_{n}^{(m)}$-semi-abelian scheme over a general locally noetherian scheme $Y$ to be such a level structure over each connected component of $Y$. By a quasi-isogeny between two quasi-polarized, $G_{n}^{(m)}$-semi-abelian schemes with $U$-level structure

$$
(\beta, \delta):\left(G, i, j, \lambda,\left[\left(\eta_{0}, \eta_{1}\right)\right]\right) \longrightarrow\left(G^{\prime}, i^{\prime}, j^{\prime}, \lambda^{\prime},\left[\left(\eta_{0}^{\prime}, \eta_{1}^{\prime}\right)\right]\right)
$$

we mean a quasi-isogeny

$$
\beta:(G, i, j) \longrightarrow\left(G^{\prime}, i^{\prime}, j^{\prime}\right)
$$


and an element $\delta \in \mathbb{Q}^{\times}$such that

$$
\delta \lambda=\beta^{\vee} \circ \lambda^{\prime} \circ \beta
$$

and

$$
\left[\left(\eta_{0}^{\prime}, \eta_{1}^{\prime}\right)\right]=\left[\left(\delta \eta_{0}, V(\beta) \circ \eta_{1}\right)\right]
$$

If $\left(G, i, j, \lambda,\left[\left(\eta_{0}, \eta_{1}\right)\right]\right)$ is a quasi-polarized, $G_{n}^{(m)}$-semi-abelian scheme with $U$-level structure, if $g \in G_{n}^{(m)}\left(\mathbb{A}^{\infty}\right)$ and if $U^{\prime} \supset g^{-1} U g$ then we define a quasi-polarized, $G_{n}^{(m)}$-semiabelian scheme with $U^{\prime}$-level structure

$$
\left(G, i, j, \lambda,\left[\left(\eta_{0}, \eta_{1}\right)\right]\right) g=\left(G, i, j, \lambda,\left[\left(v(g) \eta_{0}, \eta_{1} \circ g\right)\right]\right)
$$

The quasi-isogeny class of $\left(G, i, j, \lambda,\left[\left(\eta_{0}, \eta_{1}\right)\right]\right) g$ only depends on the quasi-isogeny class of $\left(G, i, j, \lambda,\left[\left(\eta_{0}, \eta_{1}\right)\right]\right)$. If $\left(G, i, j, \lambda,\left[\left(\eta_{0}, \eta_{1}\right)\right]\right)$ is a quasi-polarized, $G_{n}^{(m)}$-semi-abelian scheme with $U$-level structure, if $\gamma \in G L_{m}(F)$ and $U^{\prime} \supset \gamma U$ then we define a quasi-polarized, $G_{n}^{(m)}$-semi-abelian scheme with $U^{\prime}$-level structure

$$
\gamma\left(G, i, j, \lambda,\left[\left(\eta_{0}, \eta_{1}\right)\right]\right)=\left(G, i, j \circ \gamma^{-1}, \lambda,\left[\left(\eta_{0}, \eta_{1} \circ \gamma^{-1}\right)\right]\right) .
$$

The quasi-isogeny class of $\gamma\left(G, i, j, \lambda,\left[\left(\eta_{0}, \eta_{1}\right)\right]\right)$ only depends on the quasi-isogeny class of $\left(G, i, j, \lambda,\left[\left(\eta_{0}, \eta_{1}\right)\right]\right)$. We have $\gamma \circ g=\gamma(g) \circ \gamma$. If $\left(G, i, j, \lambda,\left[\left(\eta_{0}, \eta_{1}\right)\right]\right)$ is a quasi-polarized, $G_{n}^{(m)}$-semi-abelian scheme with $U$-level structure, if $m^{\prime} \leq m$ and if $U^{\prime} \supset i_{m^{\prime}, m}^{*} U$, then we define a quasi-polarized, $G_{n}^{\left(m^{\prime}\right)}$-semi-abelian scheme with $U^{\prime}$-level structure

$$
\pi_{m, m^{\prime}}\left(G, i, j, \lambda,\left[\left(\eta_{0}, \eta_{1}\right)\right]\right)=\left(G / S, i, j \circ i_{m^{\prime}, m}, \lambda,\left[\left(\eta_{0}, \eta_{1}^{\prime}\right)\right]\right)
$$

where $S \subset S_{G}$ is the subtorus with

$$
X^{*}(S)=X^{*}\left(S_{G}\right) /\left(X^{*}\left(S_{G}\right) \cap j \circ i_{m^{\prime}, m} F^{m^{\prime}}\right)
$$

and where

$$
\eta_{1}^{\prime} \circ i_{m^{\prime}, m}^{*}=\eta_{1} \bmod V S
$$

The quasi-isogeny class of $\pi_{m, m^{\prime}}\left(G, i, j, \lambda,\left[\left(\eta_{0}, \eta_{1}\right)\right]\right)$ only depends on the quasi-isogeny class of $\left(G, i, j, \lambda,\left[\left(\eta_{0}, \eta_{1}\right)\right]\right)$. If $\gamma \in Q_{m, m^{\prime}}(F)$ then $\pi_{m, m^{\prime}} \circ \gamma=\bar{\gamma} \circ \pi_{m, m^{\prime}}$, where $\bar{\gamma}$ denotes the image of $\gamma$ in $G L_{m^{\prime}}(F)$. If $g \in G_{n}^{(m)}\left(\mathbb{A}^{\infty}\right)$ then $\pi_{m, m^{\prime}} \circ g=i_{m^{\prime}, m}^{*}(g) \circ \pi_{m, m^{\prime}}$.

If $U$ is a neat open compact subgroup of $G_{n}^{(m)}\left(\mathbb{A}^{\infty}\right)$ then the functor which sends a locally noetherian scheme $Y / \mathbb{Q}$ to the set of quasi-isogeny classes of quasi-polarized $G_{n}^{(m)}$-semiabelian schemes with $U$-level structure is represented by a quasi-projective scheme $A_{n, U}^{(m)}$, which is smooth of dimension $n(n+2 m)\left[F^{+}: \mathbb{Q}\right]$. (See proposition 1.3.2.14 of [44].) We remark that according to our notational conventions we have

$$
A_{n, U}^{(0)}=X_{n, U}
$$


Let

$$
\left[\left(G^{\text {univ }}, i^{\text {univ }}, j^{\text {univ }}, \lambda^{\text {univ }},\left[\eta^{\text {univ }}\right]\right)\right] / A_{n, U}^{(m)}
$$

denote the universal quasi-isogeny class of quasi-polarized $G_{n}^{(m)}$-semi-abelian schemes with $U$-level structure. If $g \in G_{n}^{(m)}\left(\mathbb{A}^{\infty}\right)$ and $U_{1}, U_{2}$ are neat open compact subgroups of $G_{n}^{(m)}\left(\mathbb{A}^{\infty}\right)$ with $U_{2} \supset g^{-1} U_{1} g$ then there is a map

$$
g: A_{n, U_{1}}^{(m)} \longrightarrow A_{n, U_{2}}^{(m)}
$$

arising from $\left(G^{\text {univ }}, i^{\text {univ }}, j^{\text {univ }}, \lambda^{\text {univ }},\left[\eta^{\text {univ }}\right]\right) g / A_{n, U_{1}}^{(m)}$ and from the universal property of $A_{n, U_{2}}^{(m)}$. Similarly if $\gamma \in G L_{m}(F)$ and $U_{1}, U_{2}$ are neat open compact subgroups of $G_{n}^{(m)}\left(\mathbb{A}^{\infty}\right)$ with $U_{2} \supset \gamma U_{1}$ then there is a map

$$
\gamma: A_{n, U_{1}}^{(m)} \longrightarrow A_{n, U_{2}}^{(m)}
$$

arising from $\gamma\left(G^{\text {univ }}, i^{\text {univ }}, j^{\text {univ }}, \lambda^{\text {univ }},\left[\eta^{\text {univ }}\right]\right) / A_{n, U_{1}}^{(m)}$ and from the universal property of $A_{n, U_{2}}^{(m)}$. Moreover if $m^{\prime} \leq m$, if $U \subset G_{n}^{(m)}\left(\mathbb{A}^{\infty}\right)$ and if $U^{\prime}$ denotes the image of $U$ in $G_{n}^{\left(m^{\prime}\right)}\left(\mathbb{A}^{\infty}\right)$, then there is a smooth projective map

$$
\pi_{A_{n}^{(m)} / A_{n}^{\left(m^{\prime}\right)}}: A_{n, U}^{(m)} \longrightarrow A_{n, U^{\prime}}^{\left(m^{\prime}\right)}
$$

arising from $\pi_{m, m^{\prime}}\left(G^{\text {univ }}, i^{\text {univ }}, j^{\text {univ }}, \lambda^{\text {univ }},\left[\eta^{\text {univ }}\right]\right) / A_{n, U}^{(m)}$ and the universal property of $A_{n, U^{\prime}}^{\left(m^{\prime}\right)}$. (We will sometimes write $\pi_{A_{n}^{(m)} / X_{n}}$ for $\pi_{A_{n}^{(m)} / A_{n}^{(0)}}$.) We see that these actions have the following properties.

- $g_{1} \circ g_{2}=g_{2} g_{1}$ (i.e. this is a right action) and $\gamma_{1} \circ \gamma_{2}=\gamma_{1} \gamma_{2}$ (i.e. this is a left action) and $\gamma \circ g=\gamma(g) \circ \gamma$.

- If $\gamma \in Q_{m, m^{\prime}}(F)$ then $\pi_{A_{n}^{(m)} / A_{n}^{\left(m^{\prime}\right)}} \circ \gamma=\bar{\gamma} \circ \pi_{A_{n}^{(m)} / A_{n}^{\left(m^{\prime}\right)}}$, where $\bar{\gamma}$ denotes the image of $\gamma$ in $G L_{m^{\prime}}(F)$.

- $\pi_{A_{n}^{(m)} / A_{n}^{\left(m^{\prime}\right)}} \circ g=g^{\prime} \circ \pi_{A_{n}^{(m)} / A_{n}^{\left(m^{\prime}\right)}}$, where $g^{\prime}$ denotes the image of $g$ in $G_{n}^{\left(m^{\prime}\right)}\left(\mathbb{A}^{\infty}\right)$.

Moreover we have the following properties.

- The maps $g$ and $\gamma$ are finite etale. The maps $\pi_{m, m^{\prime}}$ are smooth and projective.

- If $U_{1} \subset U_{2}$ is an open normal subgroup of a neat open compact subgroup then $A_{n, U_{1}}^{(m)} / A_{n, U_{2}}^{(m)}$ is Galois with group $U_{2} / U_{1}$.

- If $U=U^{\prime} \ltimes M$ with $U^{\prime} \subset G_{n}\left(\mathbb{A}^{\infty}\right)$ and $M \subset \operatorname{Hom}_{n}^{(m)}\left(\mathbb{A}^{\infty}\right)$ then $A_{n, U}^{(m)} / X_{n, U^{\prime}}$ is an abelian scheme of relative dimension $m n[F: \mathbb{Q}]$.

- In general $A_{n, U}^{(m)}$ is a principal homogenous space for $A_{n, U^{\prime} \ltimes\left(U \cap \operatorname{Hom}_{n}^{(m)}\left(\mathbb{A}^{\infty}\right)\right)}^{(m)}$ over $X_{n, U^{\prime}}$, where $U^{\prime}$ denotes the image of $U$ in $G_{n}\left(\mathbb{A}^{\infty}\right)$.

- There are $G_{n}^{(m)}\left(\mathbb{A}^{\infty}\right)$ and $G L_{m}(F)$ equivariant homeomorphisms

$$
A_{n, U}^{(m)}(\mathbb{C}) \cong G_{n}^{(m)}(\mathbb{Q}) \backslash G_{n}^{(m)}(\mathbb{A}) /\left(U \times U_{n, \infty}^{0} A_{n}(\mathbb{R})^{0}\right) .
$$

Moreover in the case $U=U^{\prime} \ltimes M$, if $G^{\text {univ }} / A_{n, U}^{(m)}$ and $A^{\text {univ }} / X_{n, U^{\prime}}$ are chosen so that $\pi_{A_{n}^{(m)} / X_{n}}^{*} A^{\text {univ }} \cong A_{G}$ univ , then there is a $\mathbb{Q}$-linear map 


$$
i_{A^{\text {univ }}}^{(m)}: F^{m} \longrightarrow \operatorname{Hom}_{/ X_{n, U^{\prime}}}\left(A_{n, U}^{(m)},\left(A^{\text {univ }}\right)^{\vee}\right)_{\mathbb{Q}}
$$

with the following properties.

- If $a \in F$ then

$$
i_{A^{\text {univ }}}^{(m)}(a x)=i^{\text {univ }, \vee}\left({ }^{c} a\right) \circ i_{A^{\text {univ }}}^{(m)}(x)
$$

- If $(\beta, \delta)$ is a quasi-isogeny

$$
\left(G^{\text {univ }}, i^{\text {univ }}, j^{\text {univ }}, \lambda^{\text {univ }},\left[\eta^{\text {univ }}\right]\right) \longrightarrow\left(G^{\text {univ }, \prime}, i^{\text {univ }, \prime}, j^{\text {univ }, \prime}, \lambda^{\text {univ }, \prime},\left[\eta^{\text {univ }, \prime}\right]\right)
$$

then

$$
\beta^{\vee} \circ i_{\left(A^{\text {univ }}\right)^{\prime}}^{(m)}(x)=i_{A^{\text {univ }}}^{(m)}(x) .
$$

In particular $i_{A^{\text {univ }}}^{(m)}$ depends only on $A^{\text {univ }}$ and not on $G^{\text {univ }}$.

- If $g \in G_{n}^{(m)}\left(\mathbb{A}^{\infty}\right)$ and $\gamma \in G L_{m}(F)$ then

$$
i_{A^{\text {univ }}}^{(m)}(x) \circ g=i_{g^{*} A^{\text {univ }}}^{(m)}(x)
$$

and

$$
i_{A^{\text {univ }}}^{(m)}(x) \circ \gamma=i_{\gamma^{*} A^{\text {univ }}}^{(m)}\left(\gamma^{-1} x\right)
$$

- If $e_{1}, \ldots, e_{m}$ denotes the standard basis of $F^{m}$ then

$$
i_{A^{\text {univ }}}=\left\|\eta_{0}^{\text {univ }}\right\|^{-1}\left(\left(\lambda^{\text {univ }}\right)^{-1} \circ i_{A^{\text {univ }}}^{(m)}\left(e_{1}\right), \ldots,\left(\lambda^{\text {univ }}\right)^{-1} \circ i_{A^{\text {univ }}}^{(m)}\left(e_{m}\right)\right): A_{n, U}^{(m)} \longrightarrow\left(A^{\text {univ }}\right)^{m}
$$

is a quasi-isogeny. If $(\beta, \delta)$ is a quasi-isogeny

$$
\left(G^{\text {univ }}, i^{\text {univ }}, j^{\text {univ }}, \lambda^{\text {univ }},\left[\eta^{\text {univ }}\right]\right) \longrightarrow\left(G^{\text {univ }, \prime}, i^{\text {univ }, \prime}, j^{\text {univ }, \prime}, \lambda^{\text {univ }, \prime},\left[\eta^{\text {univ }, \prime}\right]\right),
$$

then

$$
\beta^{\oplus m} \circ i_{A^{\text {univ }}}=i_{\left(A^{\text {univ }}\right)^{\prime}}
$$

- The map

$$
\begin{aligned}
\eta_{n, U}^{(m)}: \operatorname{Hom}_{F}\left(F^{m}, V_{n}\right) \otimes_{\mathbb{Q}} \mathbb{A}^{\infty} \stackrel{\sim}{\rightarrow} V\left(A^{\text {univ }}\right)^{m} & \stackrel{\sim}{\rightarrow} V A_{n, U}^{(m)} \\
f & \mapsto\left(\eta_{1}^{\text {univ }}\left(f\left(e_{1}\right)\right), \ldots, \eta_{1}^{\text {univ }}\left(f\left(e_{m}\right)\right)\right) \\
x & \mapsto V\left(i_{A^{\text {univ }}}\right)^{-1} x
\end{aligned}
$$

is an isomorphism, which does not depend on the choice of $G^{\text {univ }}$. It satisfies

$$
\eta_{n, U}^{(m)} M=T A_{n, U}^{(m)}
$$


(See lemmas 1.3.2.7 and 1.3.2.50, propositions 1.3.2.14, 1.3.2.24 and 1.3.2.55, theorem 1.3.3.15, and remark 1.3.3.33 of [44]; and section 3.5 of [43].)

Note that

$$
i_{A^{\text {univ }}} \circ g=i_{g^{*} A^{\text {univ }}}
$$

and

$$
i_{A^{\text {univ }}} \circ \gamma={ }^{t} \gamma^{-1} \circ i_{\gamma^{*} A^{\text {univ }}} .
$$

Define

$$
i_{\lambda}^{(m)}: F^{m} \otimes_{F, c} F^{m} \longrightarrow \operatorname{Hom}_{/ X_{n, U^{\prime}}}\left(A_{n, U}^{(m)}, A_{n, U}^{(m), v}\right)_{\mathbb{Q}}
$$

by

$$
i_{\lambda}^{(m)}(x \otimes y)=\left\|\eta_{0}^{\text {univ }}\right\|^{-1} i_{A^{\text {univ }}}^{(m)}(x)^{\vee} \circ \lambda^{\text {univ },-1} \circ i_{A^{\text {univ }}}^{(m)}(y) .
$$

This does not depend on the choice of $A^{\text {univ }}$. We have

$$
i_{\lambda}^{(m)}(x \otimes y)^{\vee}=i_{\lambda}^{(m)}(y \otimes x) .
$$

Moreover

$$
\left(i_{A^{\text {univ }}}^{-1}\right)^{\vee} \circ i_{\lambda}^{(m)}(x \otimes y) \circ i_{A^{\text {univ }}}^{-1}=\left(\lambda^{\text {univ }}\right)^{\oplus m} \circ i^{\text {univ }}(c, t \text { xy }) .
$$

If $a \in\left(F^{m} \otimes_{F, c} F^{m}\right)^{\mathrm{sw}=1}$ has image in $S\left(F^{m}\right)$ lying in $S\left(F^{m}\right)^{>0}$ then

$$
\left(i_{A^{\text {univ }}}^{-1}\right)^{\vee} \circ i_{\lambda}^{(m)}(a) \circ i_{A^{\text {univ }}}^{-1}=\left(\lambda^{\text {univ }}\right)^{\oplus m} \circ i^{\text {univ }}\left(a^{\prime}\right)
$$

for some matrix $a^{\prime} \in M_{m \times m}(F)^{t=c}$ all whose eigenvalues are positive real numbers. (See Sect. 1.1 for the definition of sw.) Thus $i_{\lambda}^{(m)}(a)$ is a quasi-polarization. (See the end of section 21 of [47].)

Now suppose that $Y / \operatorname{Spec} \mathbb{Z}_{(p)}$ is a locally noetherian scheme. By an ordinary $G_{n}^{(m)}$-emiabelian scheme $G$ over $Y$ we mean a triple $(G, i, j)$ where

- $G / Y$ is a semi-abelian scheme such that $X^{*}(G)$ is locally constant over $Y$, and such that $\# G[p](k(\bar{y})) \geq p^{(n+m)[F: \mathbb{Q}]}$ for each geometric point $\bar{y}$ of $Y$,

- $i: \mathcal{O}_{F,(p)} \hookrightarrow \operatorname{End}(G)_{\mathbb{Z}_{(p)}}$ such that Lie $A_{G}$ is a free $\mathcal{O}_{Y} \otimes_{\mathbb{Z}_{(p)}} \mathcal{O}_{F,(p)}$ module of rank $n[F: \mathbb{Q}]$,

- and $j: \mathcal{O}_{F,(p)}^{m} \stackrel{\sim}{\rightarrow} X^{*}(G)_{\mathbb{Z}_{(p)}}$ is a $\mathcal{O}_{F,(p)}$-linear isomorphism.

Then $A_{G}$ is an ordinary $G_{n}$-abelian scheme. By a prime-to-p quasi-isogeny of ordinary $G_{n}^{(m)}$-semi-abelian schemes we mean a prime-to-p quasi-isogeny of semi-abelian schemes

$$
\beta: G \rightarrow G^{\prime}
$$

such that

$$
i^{\prime}(a) \circ \beta=\beta \circ i(a)
$$


for all $a \in \mathcal{O}_{F,(p)}$, and

$$
j=X^{*}(\beta) \circ j^{\prime}
$$

Note that, if $\bar{y}$ is a geometric point of $Y$, then $j$ induces a map

$$
j^{*}: V^{p} S_{G, \bar{y}} \stackrel{\sim}{\longrightarrow} \operatorname{Hom}_{\mathbb{Z}_{(p)}}\left(\mathcal{O}_{F,(p)}^{m}, V^{p} \mathbb{G}_{m, \bar{y}}\right) .
$$

By a prime-to- $p$ quasi-polarization of $(G, i, j)$ we shall mean a prime-to- $p$ quasipolarization of $A_{G}$.

If $Y$ is connected and $\bar{y}$ is a geometric point of $Y$, if $U^{p} \subset G_{n}^{(m)}\left(\mathbb{A}^{\infty, p}\right)$ is a neat open compact subgroup and if $N_{2} \geq N_{1} \geq 0$ then by a $U^{p}\left(N_{1}, N_{2}\right)$ level structure on a prime-to- $p$ quasi-polarized ordinary $G_{n}^{(m)}$-semi-abelian scheme $(G, i, j, \lambda)$ we mean a $\pi_{1}(Y, \bar{y})$-invariant $U^{p}$-orbit $[\eta]$ of five-tuples $\left(\eta_{0}^{p}, \eta_{1}^{p}, C, D, \eta_{p}\right)$ consisting of

- an $\mathbb{A}^{\infty, p}$-linear isomorphism $\eta_{0}^{p}: \mathbb{A}^{\infty, p} \stackrel{\sim}{\longrightarrow} \mathbb{A}^{\infty, p}(1)_{\bar{y}}=V^{p} \mathbb{G}_{m, \bar{y}}$;

- an $\mathbb{A}_{F}^{\infty, p}$-linear isomorphism

$$
\eta_{1}^{p}: \Lambda_{n}^{(m)} \otimes_{\mathbb{Z}} \mathbb{A}^{\infty, p} \stackrel{\sim}{\longrightarrow} V^{p} G_{\bar{y}}
$$

such that $\left.\eta_{1}^{p}\right|_{\operatorname{Hom}_{\mathbb{Z}}\left(\mathcal{O}_{F}^{m}, \mathbb{A} \infty, p\right)}=\left(j^{*}\right)^{-1} \circ \operatorname{Hom}\left(1_{\mathcal{O}_{F}^{m}}, \eta_{0}^{p}\right)$;

- a locally free sub- $\mathcal{O}_{F,(p)}$-module scheme $C \subset G\left[p^{N_{2}}\right]$, such that for every geometric

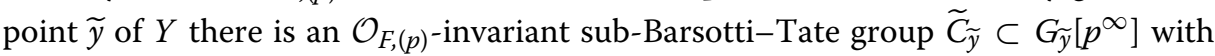
the following properties

- $C_{\tilde{y}}=\widetilde{C}_{\tilde{y}}\left[p^{N_{2}}\right]$,

- $\widetilde{C}_{\tilde{y}} \supset S_{G, \tilde{y}}\left[p^{\infty}\right]$,

- for all $N$ the subgroup scheme $\widetilde{C}_{\widetilde{y}}\left[p^{N}\right] / S_{G, \tilde{y}}\left[p^{N}\right]$ is isotropic in $A_{G}\left[p^{N}\right]_{\tilde{y}}$ for the $\lambda$-Weil pairing,

- $G_{\tilde{y}}\left[p^{\infty}\right] / \widetilde{C}_{\tilde{y}}$ is ind-etale,

- the Tate module $T\left(G_{\tilde{y}}\left[p^{\infty}\right] / \widetilde{C}_{\tilde{y}}\right)$ is free over $\mathcal{O}_{F, p}$ of rank $n$;

- a locally free sub- $\mathcal{O}_{F,(p)}$-module scheme $D \subset C\left[p^{N_{1}}\right]$ such that

$$
D \stackrel{\sim}{\rightarrow} C\left[p^{N_{1}}\right] / S_{G}\left[p^{N_{1}}\right] ;
$$

- and an isomorphism

$$
\eta_{p}: p^{-N_{1}} \Lambda_{n} /\left(p^{-N_{1}} \Lambda_{n,(n)}+\Lambda_{n}\right) \stackrel{\sim}{\longrightarrow} G\left[p^{N_{1}}\right] / C\left[p^{N_{1}}\right]
$$

such that

$$
\eta_{p}(a x)=i(a) \eta_{p}(x)
$$

for all $a \in \mathcal{O}_{F,(p)}$ and $x \in p^{-N_{1}} \Lambda_{n} /\left(p^{-N_{1}} \Lambda_{n,(n)}+\Lambda_{n}\right)$;

such that

$$
\left[\left(\eta_{0}^{p}, \eta_{1}^{p} \bmod V^{p} S_{G}, C / S_{G}\left[p^{N_{2}}\right], \eta_{p}\right)\right]
$$


is a $U^{p}\left(N_{1}, N_{2}\right)$-level structure for $\left(A_{G}, i, \lambda\right)$. This definition is independent of the choice of geometric point $\bar{y}$ of $Y$. By a $U^{p}\left(N_{1}, N_{2}\right)$-level structure on an ordinary, prime-to- $p$ quasi-polarized, $G_{n}^{(m)}$-semi-abelian scheme $(G, i, j, \lambda)$ over a general (locally noetherian) scheme $Y / \operatorname{Spec} \mathbb{Z}_{(p)}$, we mean the collection of a $U^{p}\left(N_{1}, N_{2}\right)$-level structure over each connected component of $Y$.

By a prime-to- $p$ quasi-isogeny between two quasi-polarized, ordinary $G_{n}^{(m)}$-semi-abelian schemes with $U^{p}\left(N_{1}, N_{2}\right)$-level structure

$$
(\beta, \delta):\left(G, i, j, \lambda,\left[\left(\eta_{0}, \eta_{1}\right)\right]\right) \longrightarrow\left(G^{\prime}, i^{\prime}, j^{\prime}, \lambda^{\prime},\left[\left(\eta_{0}^{\prime}, \eta_{1}^{\prime}\right)\right]\right)
$$

we mean a prime-to- $p$ quasi-isogeny

$$
\beta:(G, i, j) \longrightarrow\left(G^{\prime}, i^{\prime}, j^{\prime}\right)
$$

and an element $\delta \in \mathbb{Z}_{(p)}^{\times}$such that

$$
\delta \lambda=\beta^{\vee} \circ \lambda^{\prime} \circ \beta
$$

and

$$
\left[\left(\left(\eta_{0}^{p}\right)^{\prime},\left(\eta_{1}^{p}\right)^{\prime}, C^{\prime}, D^{\prime}, \eta_{p}^{\prime}\right)\right]=\left[\left(\delta \eta_{0}^{p}, V^{p}(\beta) \circ \eta_{1}^{p}, \beta C, \beta D, \beta \circ \eta_{p}\right)\right] .
$$

If $\left(G, i, j, \lambda,\left[\left(\eta_{0}^{p}, \eta_{1}^{p}, C, D, \eta_{p}\right)\right]\right)$ is a prime-to- $p$ quasi-polarized, ordinary $G_{n}^{(m)}$-semiabelian scheme with $U^{p}\left(N_{1}, N_{2}\right)$-level structure, if $g \in G_{n}^{(m)}\left(\mathbb{A}^{\infty}\right)^{\text {ord, } \times}$ and if

$$
\left(U^{p}\right)^{\prime}\left(N_{1}^{\prime}, N_{2}^{\prime}\right) \supset g^{-1} U^{p}\left(N_{1}, N_{2}\right) g
$$

then we define a prime-to- $p$ quasi-polarized, ordinary $G_{n}^{(m)}$-semi-abelian scheme with $\left(U^{p}\right)^{\prime}\left(N_{1}^{\prime}, N_{2}^{\prime}\right)$-level structure

$$
\left(G, i, j, \lambda,\left[\left(\eta_{0}^{p}, \eta_{1}^{p}, C, D, \eta_{p}\right)\right]\right) g=\left(G, i, j, \lambda,\left[\left(v(g) \eta_{0}^{p}, \eta_{1}^{p} \circ g^{p}, C, D, \eta_{p} \circ g_{p}\right)\right]\right) .
$$

The prime-to- $p$ quasi-isogeny class of $\left(G, i, j, \lambda,\left[\left(\eta_{0}^{p}, \eta_{1}^{p}, C, D, \eta_{p}\right)\right]\right) g$ only depends on the prime-to- $p$ quasi-isogeny class of $\left(G, i, j, \lambda,\left[\left(\eta_{0}^{p}, \eta_{1}^{p}, C, D, \eta_{p}\right)\right]\right)$. Similarly, if

$$
\left(G, i, j, \lambda,\left[\left(\eta_{0}^{p}, \eta_{1}^{p}, C, D, \eta_{p}\right)\right]\right)
$$

is a prime-to- $p$ quasi-polarized, ordinary $G_{n}^{(m)}$-semi-abelian scheme with $U^{p}\left(N_{1}, N_{2}\right)$-level structure and if

$$
\left(U^{p}\right)^{\prime}\left(N_{1}^{\prime}, N_{2}^{\prime}\right) \supset \varsigma_{p}^{-1} U^{p}\left(N_{1}, N_{2}\right) \varsigma_{p}
$$

then we define a prime-to- $p$ quasi-polarized, ordinary $G_{n}^{(m)}$-semi-abelian scheme with $\left(U^{p}\right)^{\prime}\left(N_{1}^{\prime}, N_{2}^{\prime}\right)$-level structure

$$
\begin{aligned}
& \left(G, i, j, \lambda,\left[\left(\eta_{0}^{p}, \eta_{1}^{p}, C, D, \eta_{p}\right)\right]\right) \varsigma_{p} \\
& \quad=\left(G / C[p], i, p j, F(\lambda),\left[\left(p \eta_{0}^{p}, F\left(\eta_{1}^{p}\right), C\left[p^{1+N_{2}^{\prime}}\right] / C[p],\left(D^{\prime} / C[p]\right)\left[p^{N_{1}^{\prime}}\right], F\left(\eta_{p}\right)\right)\right]\right)
\end{aligned}
$$


where

$$
F(\lambda): A_{G} / C[p] \stackrel{\lambda}{\longrightarrow} A_{G}^{\vee} / \lambda C[p]=A_{G}^{\vee} / C[p]^{\perp} \stackrel{\sim}{\longrightarrow}\left(A_{G} / C[p]\right)^{\vee}
$$

with the latter isomorphism being induced by the dual of the map $A_{G} / C[p] \rightarrow A_{G}$ induced by multiplication by $p$ on $A_{G}$; where $F\left(\eta_{1}^{p}\right)$ is the composition of $\eta_{1}^{p}$ with the natural map $V^{p} G \stackrel{\sim}{\rightarrow} V^{p}(G / C[p])$; where $D^{\prime}$ denotes the pre-image of $D$ under the multiplication by $p$ map $C \rightarrow C$; and where $F\left(\eta_{p}\right)$ is the composition of $\eta_{p}$ with the natural identification

$$
G\left[p^{N_{1}^{\prime}}\right] /\left(C \cap G\left[p^{N_{1}^{\prime}}\right]\right)=(G / C[p])\left[p^{N_{1}^{\prime}}\right] /\left(C\left[p^{1+N_{2}^{\prime}}\right] / C[p] \cap(G / C[p])\left[p^{N_{1}^{\prime}}\right]\right) .
$$

Together these two definitions give an action of $G_{n}\left(\mathbb{A}^{\infty}\right)^{\text {ord }}$.

If $\left(G, i, j, \lambda,\left[\left(\eta_{0}^{p}, \eta_{1}^{p}, C, D, \eta_{p}\right)\right]\right)$ is a prime-to- $p$ quasi-polarized, ordinary $G_{n}^{(m)}$-semiabelian scheme with $U^{p}\left(N_{1}, N_{2}\right)$-level structure, if $\gamma \in G L_{m}\left(\mathcal{O}_{F,(p)}\right)$ and if

$$
\left(U^{p}\right)^{\prime}\left(N_{1}^{\prime}, N_{2}^{\prime}\right) \supset \gamma U^{p}\left(N_{1}, N_{2}\right)
$$

then we define a prime-to- $p$ quasi-polarized, ordinary $G_{n}^{(m)}$-semi-abelian scheme with $\left(U^{p}\right)^{\prime}\left(N_{1}^{\prime}, N_{2}^{\prime}\right)$-level structure

$$
\gamma\left(G, i, j, \lambda,\left[\left(\eta_{0}^{p}, \eta_{1}^{p}, C, D, \eta_{p}\right)\right]\right)=\left(G, i, j \circ \gamma^{-1}, \lambda,\left[\left(\eta_{0}^{p}, \eta_{1}^{p} \circ \gamma^{-1}, C, D, \eta_{p}\right)\right]\right) .
$$

The prime-to- $p$ quasi-isogeny class of $\gamma\left(G, i, j, \lambda,\left[\left(\eta_{0}^{p}, \eta_{1}^{p}, C, D, \eta_{p}\right)\right]\right)$ only depends on the quasi-isogeny class of $\left(G, i, j, \lambda,\left[\left(\eta_{0}^{p}, \eta_{1}^{p}, C, D, \eta_{p}\right)\right]\right)$. We have $\gamma \circ g=\gamma(g) \circ \gamma$. If $\left(G, i, j, \lambda,\left[\left(\eta_{0}^{p}, \eta_{1}^{p}, C, D, \eta_{p}\right)\right]\right)$ is a prime-to- $p$ quasi-polarized, ordinary $G_{n}^{(m)}$-semiabelian scheme with $U^{p}\left(N_{1}, N_{2}\right)$-level structure, if $m^{\prime} \leq m$ and if $\left(U^{p}\right)^{\prime}\left(N_{1}^{\prime}, N_{2}^{\prime}\right) \supset$ $i_{m^{\prime}, m}^{*} U^{p}\left(N_{1}, N_{2}\right)$, then we define a quasi-polarized, ordinary $G_{n}^{\left(m^{\prime}\right)}$-semi-abelian scheme with $\left(U^{p}\right)^{\prime}\left(N_{1}^{\prime}, N_{2}^{\prime}\right)$-level structure

$$
\pi_{m, m^{\prime}}\left(G, i, j, \lambda,\left[\left(\eta_{0}^{p}, \eta_{1}^{p}, C, D, \eta_{p}\right)\right]\right)=\left(G / S, i, j \circ i_{m^{\prime}, m}, \lambda,\left[\left(\eta_{0}^{p},\left(\eta_{1}^{p}\right)^{\prime}, C^{\prime}, D^{\prime}, \eta_{p}\right)\right]\right),
$$

where $S \subset S_{G}$ is the subtorus with

$$
X^{*}(S)=X^{*}\left(S_{G}\right) /\left(X^{*}\left(S_{G}\right) \cap j \circ i_{m^{\prime}, m} \mathcal{O}_{F,(p)}^{m^{\prime}}\right)
$$

and where

$$
\left(\eta_{1}^{p}\right)^{\prime} \circ i_{m^{\prime}, m}^{*}=\eta_{1}^{p} \bmod V^{p} S
$$

and $C^{\prime}$ (resp. $D^{\prime}$ ) denotes the image of $C$ (resp. D). The prime-to-p quasi-isogeny class of $\pi_{m, m^{\prime}}\left(G, i, j, \lambda,\left[\left(\eta_{0}^{p}, \eta_{1}^{p}, C, D, \eta_{p}\right)\right]\right)$ only depends on the quasi-isogeny class of $\left(G, i, j, \lambda,\left[\left(\eta_{0}^{p}, \eta_{1}^{p}, C, D, \eta_{p}\right)\right]\right)$. If $\gamma \in Q_{m, m^{\prime}}\left(\mathcal{O}_{F,(p)}\right)$ then $\pi_{m, m^{\prime}} \circ \gamma=\bar{\gamma} \circ \pi_{m, m^{\prime}}$, where $\bar{\gamma}$ denotes the image of $\gamma$ in $G L_{m^{\prime}}\left(\mathcal{O}_{F,(p)}\right)$. If $g \in G_{n}^{(m)}\left(\mathbb{A}^{\infty}\right)$ then $\pi_{m, m^{\prime}} \circ g=i_{m^{\prime}, m}^{*}(g) \circ \pi_{m, m^{\prime}}$.

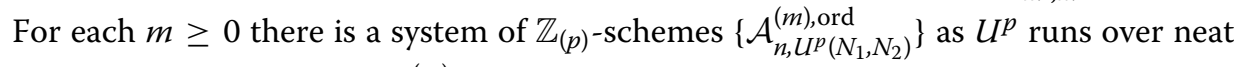
open compact subgroups of $G_{n}^{(m)}\left(\mathbb{A}^{\infty, p}\right)$ and $N_{1}, N_{2}$ run over integers with $N_{2} \geq N_{1} \geq 0$, together with the following extra structures: 
- If $g \in G_{n}^{(m)}\left(\mathbb{A}^{\infty}\right)^{\text {ord }}$ and $U_{2}^{p}\left(N_{21}, N_{22}\right) \supset g^{-1} U_{1}^{p}\left(N_{11}, N_{12}\right) g$ then there is a quasi-finite, flat map

$$
g: \mathcal{A}_{n, U_{1}^{p}\left(N_{11}, N_{12}\right)}^{(m), \text { ord }} \longrightarrow \mathcal{A}_{n, U_{2}^{p}\left(N_{21}, N_{22}\right)}^{(m) \text { ord }}
$$

- If $m^{\prime} \leq m$ and if $\left(U^{p}\right)^{\prime}$ denotes the image of $U^{p}$ in $G_{n}^{\left(m^{\prime}\right)}\left(\mathbb{A}^{\infty, p}\right)$, then there is a smooth projective map with geometrically connected fibres

$$
\pi_{\mathcal{A}_{n}^{(m), \text { ord }} / \mathcal{A}_{n}^{\left(m^{\prime}\right), \text { ord }}}: \mathcal{A}_{n, U^{p}\left(N_{1}, N_{2}\right)}^{(m), \text { ord }} \longrightarrow \mathcal{A}_{n,\left(U^{p}\right)^{\prime}\left(N_{1}, N_{2}\right)^{*}}^{\left(m^{\prime}\right) \text { ord }}
$$

- If $\gamma \in G L_{m}\left(\mathcal{O}_{F,(p)}\right)$ and $U_{2}^{p} \supset \gamma U_{1}^{p}$ then there is a finite etale map

$$
\gamma: \mathcal{A}_{n, U_{1}^{p}\left(N_{1}, N_{2}\right)}^{(m) \text { ord }} \longrightarrow \mathcal{A}_{n, U_{2}^{p}\left(N_{1}, N_{2}\right)^{\circ}}^{(m) \text { ord }}
$$

Moreover there is a canonical prime-to- $p$ quasi-isogeny class of ordinary $G_{n}^{(m)}$-semiabelian schemes with $U^{p}\left(N_{1}, N_{2}\right)$ level structure

$$
\left(\mathcal{G}^{\text {univ }}, i^{\text {univ }}, j^{\text {univ }}, \lambda^{\text {univ }},\left[\eta^{\text {univ }}\right]\right) / \mathcal{A}_{n, U^{p}\left(N_{1}, N_{2}\right)}^{(m) \text { ord }}
$$

These enjoy the following properties:

- $\mathcal{A}_{n, U^{p}\left(N_{1}, N_{2}\right)}^{(0) \text {,rd }}=\mathcal{X}_{n, U^{p}\left(N_{1}, N_{2}\right)}^{\text {ord }}$. (We will sometimes write $\pi_{\mathcal{A}_{n}^{(m), \text { ord }} / \mathcal{X}_{n}^{\text {ord }}}$ instead of

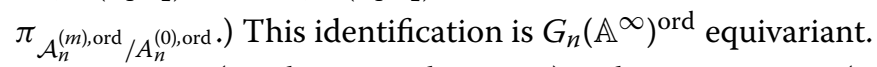

- $g_{1} \circ g_{2}=g_{2} g_{1}$ (i.e. this is a right action) and $\gamma_{1} \circ \gamma_{2}=\gamma_{1} \gamma_{2}$ (i.e. this is a left action) and $\gamma \circ g=\gamma(g) \circ \gamma$.

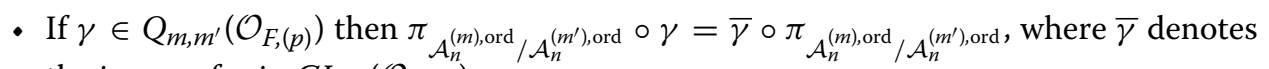
the image of $\gamma$ in $G L_{m^{\prime}}\left(\mathcal{O}_{F,(p)}\right)$.

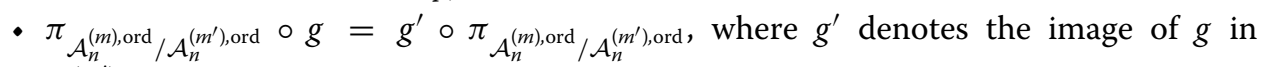
$G_{n}^{\left(m^{\prime}\right)}\left(\mathbb{A}^{\infty}\right)^{\text {ord }}$.

- If $g \in G_{n}^{(m)}\left(\mathbb{A}^{\infty}\right)^{\text {ord }}$, then the induced map

$$
g: \mathcal{A}_{n, U_{1}^{p}\left(N_{11}, N_{12}\right)}^{(m), \text { ord }} \longrightarrow g^{*} \mathcal{A}_{n, U_{2}^{p}\left(N_{21}, N_{22}\right)}^{(m) \text { ord }}
$$

over $\mathcal{X}_{n, U_{1}^{p}\left(N_{11}, N_{12}\right)}^{\text {ord }}$ is finite flat of degree $p^{n m[F: \mathbb{Q}]}$. If $g \in G_{n}^{(m)}\left(\mathbb{A}^{\infty}\right)^{\text {ord, } \times}$, then this map is also etale.

- If $U_{1}^{p} \subset U_{2}^{p}$ is an open normal subgroup of a neat open compact of $G_{n}^{(m)}\left(\mathbb{A}^{\infty, p}\right)$ and if $N_{11} \geq N_{21}$, then $\mathcal{A}_{n, U_{1}^{p}\left(N_{11}, N_{2}\right)}^{(m) \text { ord }} / \mathcal{A}_{n, U_{2}^{p}\left(N_{21}, N_{2}\right)}^{(m), \text { ord }}$ is Galois with Galois group $U_{2}^{p}\left(N_{21}\right) / U_{1}^{p}\left(N_{11}\right)$.

- On $\mathbb{F}_{p}$-fibres the map

$$
\varsigma_{p}: \mathcal{A}_{n, U^{p}\left(N_{1}, N_{2}+1\right)}^{(m), \text { ord }} \times \operatorname{Spec} \mathbb{F}_{p} \longrightarrow \mathcal{A}_{n, U^{p}\left(N_{1}, N_{2}\right)}^{(m), \text { ord }} \times \operatorname{Spec} \mathbb{F}_{p}
$$

equals the composition of the absolute Frobenius map with the forgetful map (for any $N_{2} \geq N_{1} \geq 0$ ). 
- If $g \in G_{n}^{(m)}\left(\mathbb{A}^{\infty}\right)^{\text {ord }}$ and $U_{2}^{p}\left(N_{21}, N_{22}\right) \supset g^{-1} U_{1}^{p}\left(N_{11}, N_{12}\right) g$ then the pull-back

$$
g^{*}\left(\mathcal{G}_{2}^{\text {univ }}, i_{2}^{\text {univ }}, j_{2}^{\text {univ }}, \lambda_{2}^{\text {univ }},\left[\eta_{2}^{\text {univ }}\right]\right)
$$

is prime-to- $p$ quasi-isogenous to the tuple $\left(\mathcal{G}_{1}^{\text {univ }}, i_{1}^{\text {univ }}, j_{1}^{\text {univ }}, \lambda_{1}^{\text {univ }},\left[\eta_{1}^{\text {univ }}\right]\right) g$.

- If $\gamma \in G L_{m}\left(\mathcal{O}_{F,(p)}\right)$ and $U_{2}^{p}\left(N_{21}, N_{22}\right) \supset \gamma U_{1}^{p}\left(N_{11}, N_{12}\right)$ then the pull-back

$$
\gamma^{*}\left(\mathcal{G}_{2}^{\text {univ }}, i_{2}^{\text {univ }}, j_{2}^{\text {univ }}, \lambda_{2}^{\text {univ }},\left[\eta_{2}^{\text {univ }}\right]\right)
$$

is prime-to- $p$ quasi-isogenous to the tuple $\gamma\left(\mathcal{G}_{1}^{\text {univ }}, i_{1}^{\text {univ }}, j_{1}^{\text {univ }}, \lambda_{1}^{\text {univ }},\left[\eta_{1}^{\text {univ }}\right]\right)$.

- If $m^{\prime} \leq m$ and if $U_{2}^{p}\left(N_{21}, N_{22}\right) \supset i_{m^{\prime}, m}^{*} U_{1}^{p}\left(N_{11}, N_{12}\right)$ then the pull-back $\pi_{\mathcal{A}_{n}^{(m)} / \mathcal{A}_{n}^{\left(m^{\prime}\right)}}^{*}\left(\mathcal{G}_{2}^{\text {univ }}, i_{2}^{\text {univ }}, j_{2}^{\text {univ }}, \lambda_{2}^{\text {univ }},\left[\eta_{2}^{\text {univ }}\right]\right)$ is prime-to- $p$ quasi-isogenous to the tuple $\pi_{m, m^{\prime}}\left(\mathcal{G}_{1}^{\text {univ }}, i_{1}^{\text {univ }}, j_{1}^{\text {univ }}, \lambda_{1}^{\text {univ }},\left[\eta_{1}^{\text {univ }}\right]\right)$.

- If $U^{p}=\left(U^{p}\right)^{\prime} \ltimes M^{p}$ with $\left(U^{p}\right)^{\prime} \subset G_{n}\left(\mathbb{A}^{\infty, p}\right)$ and $M^{p} \subset \operatorname{Hom}_{n}^{(m)}\left(\mathbb{A}^{\infty, p}\right)$ then

$$
\mathcal{A}_{n, U^{p}\left(N_{1}, N_{2}\right)}^{(m) \text {,ord }} / \mathcal{X}_{n,\left(U^{p}\right)^{\prime}\left(N_{1}, N_{2}\right)}^{\text {ord }}
$$

is an abelian scheme of relative dimension $m n[F: \mathbb{Q}]$.

- In general $\mathcal{A}_{n, U^{p}\left(N_{1}, N_{2}\right)}^{(m) \text {,ord }}$ is a principal homogenous space for the abelian scheme $\mathcal{A}_{n,\left(\left(U^{p}\right)^{\prime} \ltimes M^{p}\right)\left(N_{1}, N_{2}\right)}^{(m) \text { ord }}$ over $\mathcal{X}_{n,\left(U^{p}\right)^{\prime}\left(N_{1}, N_{2}\right)}^{\text {ord }}$, where $\left(U^{p}\right)^{\prime}$ denotes the image of $U^{p}$ in $G_{n}\left(\mathbb{A}^{\infty, p}\right)$ and $M^{p}=U^{p} \cap \operatorname{Hom}_{n}^{(m)}\left(\mathbb{A}^{\infty, p}\right)$.

- There are natural identifications

$$
\mathcal{A}_{n, U^{p}\left(N_{1}, N_{2}\right)}^{(m) \text { ord }} \times \operatorname{Spec} \mathbb{Q} \cong A_{n, U^{p}\left(N_{1}, N_{2}\right)}^{(m)} .
$$

These identifications are compatible with the identifications

$$
\mathcal{X}_{n,\left(U^{p}\right)^{\prime}\left(N_{1}, N_{2}\right)}^{\text {ord }} \times \operatorname{Spec} \mathbb{Q} \cong X_{n,\left(U^{p}\right)^{\prime}\left(N_{1}, N_{2}\right)}
$$

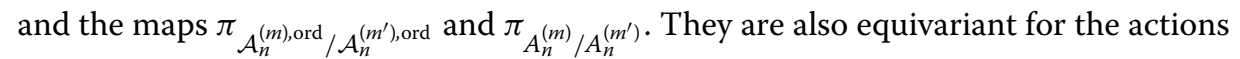
of the semi-group $G_{n}^{(m)}\left(\mathbb{A}^{\infty}\right)^{\text {ord }}$ and the group $G L_{m}\left(\mathcal{O}_{F,(p)}\right)$.

Moreover in the case $U^{p}=\left(U^{p}\right)^{\prime} \ltimes M^{p}$, if $\mathcal{G}^{\text {univ }} / \mathcal{A}_{n, U^{p}\left(N_{1}, N_{2}\right)}^{(m) \text { ord }}$ and $\mathcal{A}^{\text {univ }} / \mathcal{X}_{n, U^{p}\left(N_{1}, N_{2}\right)}^{\text {ord }}$ are chosen so that $\pi_{\mathcal{A}_{n}^{(m), \text { ord }} / \mathcal{X}_{n}^{\text {ord }}}^{*} \mathcal{A}^{\text {univ }} \cong A_{\mathcal{G}}$ univ , then there is a $\mathbb{Z}_{(p) \text {-linear map }}$

$$
i_{\mathcal{A}^{\text {univ }}}^{(m)}: \mathcal{O}_{F,(p)}^{m} \longrightarrow \operatorname{Hom}_{/ \mathcal{X}_{n,\left(U^{p}\right)^{\prime}\left(N_{1}, N_{2}\right)}^{\text {ord }}}\left(\mathcal{A}_{n, U^{p}\left(N_{1}, N_{2}\right)^{\prime}}^{(m), \text { ord }}\left(\mathcal{A}^{\text {univ }} / C^{\text {univ }}\left[p^{N_{1}}\right]\right)^{\vee}\right)_{\mathbb{Z}_{(p)}}
$$

with the following properties.

- If $a \in \mathcal{O}_{F,(p)}$ then

$$
i_{\mathcal{A}^{\text {univ }}}^{(m)}(a x)=i^{\text {univ, },}\left({ }^{c} a\right) \circ i_{\mathcal{A}^{\text {univ }}}^{(m)}(x) .
$$

- If $(\beta, \delta)$ is a prime-to- $p$ quasi-isogeny

$$
\left(\mathcal{G}^{\text {univ }}, i^{\text {univ }}, j^{\text {univ }}, \lambda^{\text {univ }},\left[\eta^{\text {univ }}\right]\right) \longrightarrow\left(\mathcal{G}^{\text {univ }, \prime}, i^{\text {univ }, \prime}, j^{\text {univ }, \prime}, \lambda^{\text {univ }, \prime},\left[\eta^{\text {univ }, \prime}\right]\right)
$$


then

$$
\beta^{\vee} \circ i_{\left(\mathcal{A}^{\text {univ }}\right)^{\prime}}^{(m)}(x)=i_{\mathcal{A}^{\text {univ }}}^{(m)}(x) .
$$

In particular $i_{\mathcal{A}^{\text {univ }}}^{(m)}$ depends only on $\mathcal{A}^{\text {univ }}$ and not on $\mathcal{G}^{\text {univ }}$.

- If $g \in G_{n}^{(m)}\left(\mathbb{A}^{\infty}\right)^{\text {ord }}$ and $\gamma \in G L_{m}\left(\mathcal{O}_{F,(p)}\right)$ then

$$
i_{\mathcal{A}^{\text {univ }}}^{(m)}(x) \circ g=i_{g^{*} \mathcal{A}^{\text {univ }}}^{(m)}(x)
$$

and

$$
i_{\mathcal{A}^{\text {univ }}}^{(m)}(x) \circ \gamma=i_{\gamma^{*} \mathcal{A}^{\text {univ }}}^{(m)}\left(\gamma^{-1} x\right)
$$

- If $e_{1}, \ldots, e_{m}$ denotes the standard basis of $\mathcal{O}_{F,(p)}^{m}$ then

$$
i_{\mathcal{A}^{\text {univ }}}=\left\|\eta_{0}^{p \text { univ }}\right\|^{-1}\left(\left(\lambda\left(N_{1}\right)^{\text {univ }}\right)^{-1} \circ i_{\mathcal{A}^{\text {univ }}}^{(m)}\left(e_{1}\right), \ldots,\left(\lambda\left(N_{1}\right)^{\text {univ }}\right)^{-1} \circ i_{\mathcal{A}^{\text {univ }}}^{(m)}\left(e_{m}\right)\right)
$$

is a prime-to- $p$ quasi-isogeny

$$
\mathcal{A}_{n, U^{p}\left(N_{1}, N_{2}\right)}^{(m) \text { ord }} \longrightarrow\left(\mathcal{A}^{\text {univ }} / C^{\text {univ }}\left[p^{N_{1}}\right]\right)^{m} \text {. }
$$

Here $\lambda\left(N_{1}\right)^{\text {univ }}$ refers to the prime-to-p quasi-polarization $\mathcal{A}^{\text {univ }} / C\left[p^{N_{1}}\right] \rightarrow\left(\mathcal{A}^{\text {univ }} / C\left[p^{N_{1}}\right]\right)^{\vee}$ for which the composite

$$
\mathcal{A}^{\text {univ }} \longrightarrow \mathcal{A}^{\text {univ }} / C\left[p^{N_{1}}\right] \stackrel{\lambda\left(N_{1}\right)^{\text {univ }}}{\longrightarrow}\left(\mathcal{A}^{\text {univ }} / C\left[p^{N_{1}}\right]\right)^{\vee} \longrightarrow \mathcal{A}^{\text {univ }, \vee}
$$

equals $p^{N_{1}} \lambda^{\text {univ }}$.

We have

$$
\beta^{\oplus m} \circ i_{\mathcal{A}^{\text {univ }}}=i_{\left(\mathcal{A}^{\text {univ })^{\prime}}\right.}
$$

The composite map

$$
\begin{aligned}
\eta_{n, U^{p}\left(N_{1}, N_{2}\right)}^{(m)}: \operatorname{Hom}_{\mathcal{O}_{F}}\left(\mathcal{O}_{F}^{m}, \Lambda_{n}\right) \otimes_{\mathbb{Z}} \mathbb{A}^{\infty, p} & \longrightarrow V^{p}\left(\mathcal{A}^{\text {univ }}\right)^{m} \\
& \stackrel{p^{-N_{1}}}{\longrightarrow} V^{p}\left(\mathcal{A}^{\text {univ }} / C^{\text {univ }}\left[p^{N_{1}}\right]\right)^{m} \\
& \longrightarrow V^{p} \mathcal{A}_{n, U^{p}\left(N_{1}, N_{2}\right)}^{(m), \text { ord }}
\end{aligned}
$$

where the first maps sends

$$
f \longmapsto\left(\eta_{1}^{p \text {,univ }}\left(f\left(e_{1}\right)\right), \ldots, \eta_{1}^{p \text {,univ }}\left(f\left(e_{m}\right)\right)\right)
$$

and the third map sends

$$
x \longmapsto V^{p}\left(i_{\mathcal{A}^{\text {univ }}}\right)^{-1} x,
$$

is an isomorphism, which does not depend on the choice of $\mathcal{G}^{\text {univ }}$. It satisfies

$$
\eta_{n, U^{p}\left(N_{1}, N_{2}\right)}^{(m)} M^{p}=T^{p} \mathcal{A}_{n, U^{p}\left(N_{1}, N_{2}\right)}^{(m), \text { ord }}
$$


(See lemmas 5.2.4.7 and 7.1.2.1, propositions 5.2.4.13, 5.2.4.25 and 7.1.2.5, remarks 7.1.2.38 and 7.1.4.27, and theorem 7.1.4.1 of [44].)

We deduce the following additional properties:

- If $g \in G_{n}^{(m)}\left(\mathbb{A}^{\infty}\right)^{\text {ord, } \times}$ then the map $g: \mathcal{A}_{n, U_{1}^{p}\left(N_{11}, N_{12}\right)}^{(m) \text { ord }} \rightarrow \mathcal{A}_{n, U_{2}^{p}\left(N_{21}, N_{22}\right)}^{(m) \text { ord }}$ is etale. If further $N_{12}=N_{22}$, then it is finite etale.

- If $g \in G_{n}^{(m)}\left(\mathbb{A}^{\infty}\right)^{\text {ord }}$, if $N_{22}>0$ and if $p^{N_{12}-N_{22}} v\left(g_{p}\right) \in \mathbb{Z}_{p}^{\times}$then

$$
g: \mathcal{A}_{n, U_{1}^{p}\left(N_{11}, N_{12}\right)}^{(m) \text { ord }} \longrightarrow \mathcal{A}_{n, U_{2}^{p}\left(N_{21}, N_{22}\right)}^{(m), \text { ord }}
$$

is finite. If $N_{2}>0$ then the finite flat map

$$
\varsigma_{p}: \mathcal{A}_{n, U^{p}\left(N_{1}, N_{2}+1\right)}^{(m) \text { ord }} \longrightarrow \mathcal{A}_{n, U^{p}\left(N_{1}, N_{2}\right)}^{(m), \text { ord }}
$$

has degree $p^{n(n+2 m)\left[F^{+}: \mathbb{Q}\right]}$.

$$
i_{\mathcal{A}^{\text {univ }}} \circ g=i_{g^{*} \mathcal{A}^{\text {univ }}}
$$

and

$$
i_{\mathcal{A}^{\text {univ }}} \circ \gamma={ }^{t} \gamma^{-1} \circ i_{\gamma^{*} \mathcal{A}^{\text {univ }}}
$$

Also in this case define

$$
i_{\lambda}^{(m)}: \mathcal{O}_{F,(p)}^{m} \otimes_{\mathcal{O}_{F,(p), c}} \mathcal{O}_{F,(p)}^{m} \longrightarrow \operatorname{Hom}_{/ \mathcal{X}_{n,\left(U^{p}\right)^{\prime}\left(N_{1}, N_{2}\right)}^{\text {ord }}}\left(\mathcal{A}_{n, U^{p}\left(N_{1}, N_{2}\right)}^{(m), \text { ord }}, A_{n, U^{p}\left(N_{1}, N_{2}\right)}^{(m) \text { ord } \vee}\right)_{\mathbb{Z}_{(p)}}
$$

by

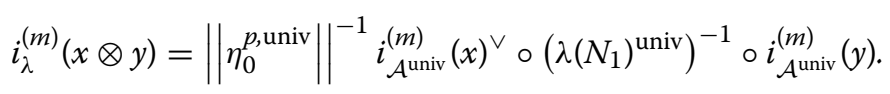

This does not depend on the choice of $\mathcal{A}^{\text {univ }}$. We have

$$
i_{\lambda}^{(m)}(x \otimes y)^{\vee}=i_{\lambda}^{(m)}(y \otimes x) .
$$

Moreover

$$
\left(i_{\mathcal{A}^{\text {univ }}}^{-1}\right)^{\vee} \circ i_{\lambda}^{(m)}(x \otimes y) \circ i_{\mathcal{A}^{\text {univ }}}^{-1}=\left(\lambda\left(N_{1}\right)^{\text {univ }}\right)^{\oplus m} \circ i^{\text {univ }}(c, t x y) .
$$

If $a \in\left(\mathcal{O}_{F,(p)}^{m} \otimes_{\mathcal{O}_{F,(p), c}} \mathcal{O}_{F,(p)}^{m}\right)^{\mathrm{sw}=1}$ has image in $S\left(\mathcal{O}_{F,(p)}^{m}\right)$ lying in $S\left(\mathcal{O}_{F,(p)}^{m}\right)^{>0}$ then

$$
\left(i_{\mathcal{A}^{\text {univ }}}^{-1}\right)^{\vee} \circ i_{\lambda}^{(m)}(a) \circ i_{\mathcal{A}^{\text {univ }}}^{-1}=\left(\lambda\left(N_{1}\right)^{\text {univ }}\right)^{\oplus m} \circ i^{\text {univ }}\left(a^{\prime}\right)
$$

for some matrix $a^{\prime} \in M_{m \times m}\left(\mathcal{O}_{F,(p)}\right)^{t=c}$ all whose eigenvalues are positive real numbers. Thus $i_{\lambda}^{(m)}(a)$ is a quasi-polarization. (See the end of section 21 of [47].)

The completion of $\mathcal{A}_{U^{p}\left(N_{1}, N_{2}\right)}^{(m), \text { ord }}$ along its $\mathbb{F}_{p}$-fibre does not depend on $N_{2}$, so we will denote it

$$
\mathfrak{A}_{U^{p}\left(N_{1}\right)^{\circ}}^{(m) \text { ord }}
$$


(See theorem 7.1.4.1 of [44].) Then $\left\{\mathfrak{A}_{U^{p}(N)}^{(m), \text { ord }}\right\}$ is a system of $p$-adic formal schemes with a right $G_{n}^{(m)}\left(\mathbb{A}^{\infty}\right)^{\text {ord }}$-action and a left $G L_{m}\left(\mathcal{O}_{F,(p)}\right)$-action. There is an equivariant map

$$
\left\{\mathfrak{A}_{n, U^{p}(N)}^{(m) \text { ord }}\right\} \rightarrow\left\{\mathfrak{X}_{n,\left(U^{\prime}\right)^{p}(N)}^{\text {ord }}\right\}
$$

We will write $\bar{A}_{n, U^{p}(N)}^{(m) \text {,ord }}$ for the reduced subscheme of $\mathfrak{A}_{n, U^{p}(N)}^{(m) \text {,ord }}$.

\subsection{Some mixed Shimura varieties}

If $\widetilde{U}\left(\right.$ resp. $\left.\widetilde{U}^{p}\right)$ is a neat open compact subgroup of $\widetilde{G}_{n}^{(m)}\left(\mathbb{A}^{\infty}\right)\left(\right.$ resp. $\left.\widetilde{G}_{n}^{(m)}\left(\mathbb{A}^{\infty, p}\right)\right)$ we will denote by $S_{n, \widetilde{U}}^{(m)}\left(\right.$ resp. $\mathcal{S}_{n, \widetilde{U}^{p}}^{(m), \text { ord }}$ ) the split torus over Spec $\mathbb{Q}\left(\right.$ resp. Spec $\left.\mathbb{Z}_{(p)}\right)$ with

$$
X_{*}\left(S_{n, \widetilde{U}}^{(m)}\right)=Z\left(N_{n}^{(m)}\right)(\mathbb{Q}) \cap \widetilde{U} \subset \operatorname{Herm}^{(m)}(\mathbb{Q})
$$

(resp.

$$
\left.X_{*}\left(\mathcal{S}_{n, \widetilde{U}^{p}}^{(m), \text { ord }}\right)=Z\left(N_{n}^{(m)}\right)\left(\mathbb{Z}_{(p)}\right) \cap \widetilde{U}^{p} \subset \operatorname{Herm}^{(m)}\left(\mathbb{Z}_{(p)}\right)\right) .
$$

If $g \in \widetilde{G}_{n}^{(m)}\left(\mathbb{A}^{\infty}\right)$ (resp. $\left.\widetilde{G}_{n}^{(m)}\left(\mathbb{A}^{\infty}\right)^{\text {ord }}\right)$ and $\widetilde{U}_{2} \supset g^{-1} \widetilde{U}_{1} g$ (resp. $\widetilde{U}_{2}^{p} \supset g^{-1} \widetilde{U}_{1}^{p} g$ ) we get a map

$$
g: S_{n, \widetilde{U}_{1}}^{(m)} \longrightarrow S_{n, \widetilde{U}_{2}}^{(m)}
$$

(resp.

$$
\left.g: \mathcal{S}_{n, \widetilde{U}_{1}^{p}}^{(m), \text { ord }} \longrightarrow \mathcal{S}_{n, \widetilde{U}_{2}^{p}}^{(m), \text { ord }}\right)
$$

corresponding to

$$
\|v(g)\|: X_{*}\left(S_{n, \widetilde{U}_{1}}^{(m)}\right) \longrightarrow X_{*}\left(S_{n, \widetilde{U}_{2}}^{(m)}\right)
$$

(resp.

$$
\left.\|v(g)\|: X_{*}\left(\mathcal{S}_{n, \widetilde{U}_{1}^{p}}^{(m), \text { ord }}\right) \longrightarrow X_{*}\left(\mathcal{S}_{n, \widetilde{U}_{2}^{p}}^{(m), \text { ord }}\right)\right)
$$

where we think of the domain and codomain both as subspaces of Herm ${ }^{(m)}$. If $\gamma \in G L_{m}(\mathbb{Q})$ (resp. $\left.G L_{m}\left(\mathbb{Z}_{(p)}\right)\right)$ and $\widetilde{U}_{2} \supset \gamma \widetilde{U}_{1}$ (resp. $\widetilde{U}_{2}^{p} \supset \gamma \widetilde{U}_{1}^{p}$ ) we get a map

$$
\gamma: S_{n, \widetilde{U}_{1}}^{(m)} \longrightarrow S_{n, \widetilde{U}_{2}}^{(m)}
$$

(resp.

$$
\left.\gamma: \mathcal{S}_{n, \widetilde{U}_{1}^{p}}^{(m), \text { ord }} \longrightarrow \mathcal{S}_{n, \widetilde{U}_{2}^{p}}^{(m), \text { ord }}\right)
$$

corresponding to

$$
\gamma: X_{*}\left(S_{n, \widetilde{U}_{1}}^{(m)}\right) \longrightarrow X_{*}\left(S_{n, \widetilde{U}_{2}}^{(m)}\right)
$$


(resp.

$$
\left.\gamma: X_{*}\left(\mathcal{S}_{n, \widetilde{U}_{1}^{p}}^{(m), \text { ord }}\right) \longrightarrow X_{*}\left(\mathcal{S}_{n, \widetilde{U}_{2}^{p}}^{(m), \text { ord }}\right)\right)
$$

where again we think of the domain and codomain both as subspaces of Herm ${ }^{(m)}$. If $m_{1} \geq$ $m_{2}$ and if $\widetilde{U}_{2}$ (resp. $\left.\widetilde{U}_{2}^{p}\right)$ is the image of $\widetilde{U}_{1}\left(\right.$ resp. $\left.\widetilde{U}_{1}^{p}\right)$ in $\widetilde{G}_{n}^{\left(m_{2}\right)}\left(\mathbb{A}^{\infty}\right)\left(\right.$ resp. $\left.\widetilde{G}_{n}^{\left(m_{2}\right)}\left(\mathbb{A}^{\infty, p}\right)\right)$, then our chosen map $\operatorname{Herm}^{\left(m_{1}\right)} \rightarrow \operatorname{Herm}^{\left(m_{2}\right)}$ induces a map

$$
S_{n, \widetilde{U}_{1}}^{\left(m_{1}\right)} \longrightarrow S_{n, \widetilde{U}_{2}}^{\left(m_{2}\right)}
$$

(resp.

$$
\left.\mathcal{S}_{n, \widetilde{U}_{1}^{p}}^{\left(m_{1}\right) \text {,ord }} \longrightarrow \mathcal{S}_{n, \widetilde{U}_{2}^{p}}^{\left(m_{2}\right), \text { ord }}\right)
$$

As $\widetilde{U}$ runs over neat open compact subgroups of $\widetilde{G}_{n}^{(m)}\left(\mathbb{A}^{\infty}\right)$, there is a system of $S_{n, \widetilde{U}^{-}}^{(m)}$. torsors

$$
T_{n, \widetilde{U}}^{(m)}=\underline{\text { Spec }} \bigoplus_{\chi \in X^{*}\left(S_{n, \widetilde{U}}^{(m)}\right)} \mathcal{L}_{n, \widetilde{U}}^{(m)}(\chi)
$$

over $A_{n, \widetilde{U}}^{(m)}$ together with the following extra structures:

- If $g \in \widetilde{G}_{n}^{(m)}\left(\mathbb{A}^{\infty}\right)$ and $\widetilde{U}_{1}, \widetilde{U}_{2}$ are neat open compact subgroups of $\widetilde{G}_{n}^{(m)}\left(\mathbb{A}^{\infty}\right)$ with $\widetilde{U}_{2} \supset g^{-1} \widetilde{U}_{1} g$ then there is a finite etale map

$$
g: T_{n, \widetilde{U}_{1}}^{(m)} \longrightarrow T_{n, \widetilde{U}_{2}}^{(m)}
$$

compatible with the maps $g: A_{n, \widetilde{U}_{1}}^{(m)} \longrightarrow A_{n, \widetilde{U}_{2}}^{(m)}$ and $g: S_{n, \widetilde{U}_{1}}^{(m)} \longrightarrow S_{n, \widetilde{U}_{2}}^{(m)}$.

- If $\gamma \in G L_{m}(F)$ and $\widetilde{U}_{1}, \widetilde{U}_{2}$ are neat open compact subgroups of $\widetilde{G}_{n}^{(m)}\left(\mathbb{A}^{\infty}\right)$ with $\widetilde{U}_{2} \supset$ $\gamma \widetilde{U}_{1}$ then there is a finite etale map

$$
\gamma: T_{n, \widetilde{U}_{1}}^{(m)} \longrightarrow T_{n, \widetilde{U}_{2}}^{(m)}
$$

compatible with the maps $\gamma: A_{n, \widetilde{U}_{1}}^{(m)} \longrightarrow A_{n, \widetilde{U}_{2}}^{(m)}$ and $\gamma: S_{n, \widetilde{U}_{1}}^{(m)} \longrightarrow S_{n, \widetilde{U}_{2}}^{(m)}$.

- If $m_{1} \geq m_{2}$ and $\widetilde{U}_{2}$ is the image of $\widetilde{U}_{1}$ in $\widetilde{G}^{\left(m_{2}\right)}\left(\mathbb{A}^{\infty}\right)$, then there is a map

$$
T_{n, \widetilde{U}_{1}}^{\left(m_{1}\right)} \longrightarrow T_{n, \widetilde{U}_{2}}^{\left(m_{2}\right)}
$$

compatible with the maps $S_{n, \widetilde{U}_{1}}^{\left(m_{1}\right)} \longrightarrow S_{n, \widetilde{U}_{2}}^{\left(m_{2}\right)}$ and $A_{n, \widetilde{U}_{1}}^{\left(m_{1}\right)} \longrightarrow A_{n, \widetilde{U}_{2}}^{\left(m_{2}\right)}$.

These enjoy the following properties:

- $g_{1} \circ g_{2}=g_{2} g_{1}$ (i.e. this is a right action) and $\gamma_{1} \circ \gamma_{2}=\gamma_{1} \gamma_{2}$ (i.e. this is a left action) and $\gamma \circ g=\gamma(g) \circ \gamma$.

- If $\widetilde{U}_{1} \subset \widetilde{U}_{2}$ is an open normal subgroup of a neat open compact subgroup of $\widetilde{G}_{n}^{(m)}\left(\mathbb{A}^{\infty}\right)$, then $T_{n, \widetilde{U}_{1}}^{(m)} / T_{n, \widetilde{U}_{2}}^{(m)}$ is Galois with group $\widetilde{U}_{2} / \widetilde{U}_{1}$. 
- The maps $T_{n, \widetilde{U}_{1}}^{\left(m_{1}\right)} \longrightarrow T_{n, \widetilde{U}_{2}}^{\left(m_{2}\right)}$ are compatible with the actions of $\widetilde{G}_{n}^{\left(m_{1}\right)}\left(\mathbb{A}^{\infty}\right)$ and $\widetilde{G}_{n}^{\left(m_{2}\right)}\left(\mathbb{A}^{\infty}\right)$ and the map $\widetilde{G}_{n}^{\left(m_{1}\right)}\left(\mathbb{A}^{\infty}\right) \rightarrow \widetilde{G}_{n}^{\left(m_{2}\right)}\left(\mathbb{A}^{\infty}\right)$, and also with the action of $Q_{m_{1}, m_{2}}(F)$.

- Suppose that $\widetilde{U}=U^{\prime} \ltimes M$ with $U^{\prime} \subset G_{n}\left(\mathbb{A}^{\infty}\right)$ and $M \subset N_{n}^{(m)}\left(\mathbb{A}^{\infty}\right)$. Also suppose that

$$
\chi \in X^{*}\left(S_{n, \widetilde{U}}^{(m)}\right) \subset S\left(F^{m}\right)
$$

is sufficiently divisible. Then we can find $a \in F^{m} \otimes_{F, c} F^{m}$ lifting $\chi$ such that

$$
i_{\lambda}^{(m)}(a): A_{n, \widetilde{U}}^{(m)} \longrightarrow\left(A_{n, \widetilde{U}}^{(m)}\right)^{\vee}
$$

is a homomorphism. For any such $a$

$$
\mathcal{L}_{n, \widetilde{U}}^{(m)}(\chi)=\left(1, i_{\lambda}^{(m)}(a)\right)^{*} \mathcal{P}_{A_{n, \widetilde{U}}^{(m)}}
$$

- If $\chi \in X^{*}\left(S_{n, \widetilde{U}}^{(m)}\right) \cap S\left(F^{m}\right)^{>0}$ then $\mathcal{L}_{n, \widetilde{U}}^{(m)}(\chi)$ is relatively ample for $A_{n, \widetilde{U}}^{(m)} / X_{n, \widetilde{U}}$.

- There are $\widetilde{G}_{n}^{(m)}\left(\mathbb{A}^{\infty}\right)$ and $G L_{m}(F)$ equivariant homeomorphisms

$$
T_{n, \widetilde{U}}^{(m)}(\mathbb{C}) \cong \widetilde{G}_{n}^{(m)}(\mathbb{Q}) \backslash \widetilde{G}_{n}^{(m)}(\mathbb{A}) \operatorname{Herm}^{(m)}(\mathbb{C}) /\left(\widetilde{U} \times U_{n, \infty}^{0} A_{n}(\mathbb{R})^{0}\right)
$$

(See lemmas 1.3.2.25 and 1.3.2.72, and propositions 1.3.2.31, 1.3.2.45 and 1.3.2.90 of [44]; section 3.6 of [43]; and the second paragraph of Sect. 3.2 above.)

Similarly as $\widetilde{U}^{p}$ runs over neat open compact subgroups of $\widetilde{G}_{n}^{(m)}(\mathbb{A} \infty, p)$ and $N_{1}, N_{2}$ run over integers with $N_{2} \geq N_{1} \geq 0$, there is a system of $\mathcal{S}_{n, \widetilde{U}^{p}}^{(m) \text {,ord }}$-torsors

$$
\mathcal{T}_{n, \widetilde{U}^{p}\left(N_{1}, N_{2}\right)}^{(m) \text { ord }}=\underline{\text { Spec }} \underset{\chi \in X^{*}\left(\mathcal{S}_{n, \widetilde{U}^{(m)}\left(N_{1}, N_{2}\right)}\right)}{ } \mathcal{L}_{n, \widetilde{U}^{p}\left(N_{1}, N_{2}\right)}^{(m), \text { ord }}(\chi)
$$

over $\mathcal{A}_{n, \widetilde{U}^{p}\left(N_{1}, N_{2}\right)}^{(m) \text { ord }}$ together with the following extra structures:

- If $g \in \widetilde{G}_{n}^{(m)}\left(\mathbb{A}^{\infty}\right)^{\text {ord }}$ and $\widetilde{U}_{2}^{p}\left(N_{21}, N_{22}\right) \supset g^{-1} \widetilde{U}_{1}^{p}\left(N_{11}, N_{12}\right) g$ then there is a quasi-finite, flat map

$$
g: \mathcal{T}_{n, \widetilde{U}_{1}^{p}\left(N_{11}, N_{12}\right)}^{(m), \text { ord }} \longrightarrow \mathcal{T}_{n, \widetilde{U}_{2}^{p}\left(N_{21}, N_{22}\right)}^{(m) \text { ord }}
$$

compatible with the map $g: \mathcal{A}_{n, \widetilde{U}_{1}^{p}\left(N_{11}, N_{12}\right)}^{(m), \text { ord }} \longrightarrow \mathcal{A}_{n, \widetilde{U}_{2}^{p}\left(N_{21}, N_{22}\right)}^{(m) \text { ord }}$ and the map $g$ : $\mathcal{S}_{n, \widetilde{U}_{1}^{p}}^{(m) \text { ord }} \longrightarrow \mathcal{S}_{n, \widetilde{U}_{2}^{p}}^{(m) \text {,ord }}$

- If $\gamma \in G L_{m}\left(\mathcal{O}_{F,(p)}\right)$ and $\widetilde{U}_{2}^{p} \supset \gamma \widetilde{U}_{1}^{p}$ then there is a finite etale map

$$
\gamma: \mathcal{T}_{n, \widetilde{U}_{1}^{p}\left(N_{1}, N_{2}\right)}^{(m) \text { ord }} \longrightarrow \mathcal{T}_{n, \widetilde{U}_{2}^{p}\left(N_{1}, N_{2}\right)}^{(m) \text {,ord }}
$$

compatible with the maps

$$
\gamma: \mathcal{A}_{n, \widetilde{U}_{1}^{p}\left(N_{1}, N_{2}\right)}^{(m), \text { ord }} \longrightarrow \mathcal{A}_{n, \widetilde{U}_{2}^{p}\left(N_{1}, N_{2}\right)}^{(m)}
$$

and

$$
\gamma: \mathcal{S}_{n, \widetilde{U}_{1}^{p}}^{(m), \text { ord }} \longrightarrow \mathcal{S}_{n, \widetilde{U}_{2}^{p}}^{(m) \text { ord }}
$$


- If $m_{1} \geq m_{2}$ and $\widetilde{U}_{2}^{p}$ is the image of $\widetilde{U}_{1}^{p}$ in $\widetilde{G}^{\left(m_{2}\right)}\left(\mathbb{A}^{\infty, p}\right)$, then there is a map

$$
\mathcal{T}_{n, \widetilde{U}_{1}^{p}\left(N_{1}, N_{2}\right)}^{\left(m_{1}\right) \text { ord }} \longrightarrow \mathcal{T}_{n, \widetilde{U}_{2}^{p}\left(N_{1}, N_{2}\right)}^{\left(m_{2}\right) \text { ord }}
$$

compatible with the map $\mathcal{S}_{n, \widetilde{U}_{1}^{p}}^{\left(m_{1}\right) \text {,ord }} \longrightarrow \mathcal{S}_{n, \widetilde{U}_{2}^{p}}^{\left(m_{2}\right) \text {,ord }}$ and the map $\mathcal{A}_{n, \widetilde{U}_{1}^{p}\left(N_{1}, N_{2}\right)}^{\left(m_{1}\right) \text { ord }} \longrightarrow$ $\mathcal{A}_{n, \widetilde{U}_{2}^{p}\left(N_{1}, N_{2}\right)}^{\left(m_{2}\right) \text { ord }}$

These enjoy the following properties:

- $g_{1} \circ g_{2}=g_{2} g_{1}$ (i.e. this is a right action) and $\gamma_{1} \circ \gamma_{2}=\gamma_{1} \gamma_{2}$ (i.e. this is a left action) and $\gamma \circ g=\gamma(g) \circ \gamma$.

- If $g \in \widetilde{G}_{n}^{(m)}\left(\mathbb{A}^{\infty}\right)^{\text {ord, } \times}$ then the map $g: \mathcal{T}_{n, \widetilde{U}_{1}^{p}\left(N_{11}, N_{12}\right)}^{(m), \text { ord }} \rightarrow \mathcal{T}_{n, \widetilde{U}_{2}^{p}\left(N_{21}, N_{22}\right)}^{(m) \text { ord }}$ is etale. If further $N_{12}=N_{22}$, then it is finite etale.

- The maps $\mathcal{T}_{n, \widetilde{U}_{1}^{p}\left(N_{1}, N_{2}\right)}^{\left(m_{1}\right), \text { ord }} \longrightarrow \mathcal{T}_{n, \widetilde{U}_{2}^{p}\left(N_{1}, N_{2}\right)}^{\left(m_{2}\right) \text {,ord }}$ are compatible with the actions of $\widetilde{G}_{n}^{\left(m_{1}\right)}\left(\mathbb{A}^{\infty}\right)^{\text {ord }}$ and $\widetilde{G}_{n}^{\left(m_{2}\right)}\left(\mathbb{A}^{\infty}\right)^{\text {ord }}$ and the map $\widetilde{G}_{n}^{\left(m_{1}\right)}\left(\mathbb{A}^{\infty}\right) \rightarrow \widetilde{G}_{n}^{\left(m_{2}\right)}\left(\mathbb{A}^{\infty}\right)$, and with the action of $Q_{m_{1}, m_{2}}\left(\mathcal{O}_{F,(p)}\right)$.

- If $\widetilde{U}_{1}^{p} \subset \widetilde{U}_{2}^{p}$ is an open normal subgroup of a neat open compact of $\widetilde{G}_{n}^{(m)}\left(\mathbb{A}^{\infty, p}\right)$, and if $N_{11} \geq N_{21}$ then $\mathcal{T}_{n, \widetilde{U}_{1}^{p}\left(N_{11}, N_{2}\right)}^{(m) \text {,ord }} / \mathcal{T}_{n, \widetilde{U}_{2}^{p}\left(N_{21}, N_{2}\right)}^{(m) \text {,ord }}$ is Galois with Galois group $\widetilde{U}_{2}^{p}\left(N_{21}\right) / \widetilde{U}_{1}^{p}\left(N_{11}\right)$.

- If $g \in G_{n}^{(m)}\left(\mathbb{A}^{\infty}\right)^{\text {ord }}$, if $N_{22}>0$ and if $p^{N_{12}-N_{22}} v\left(g_{p}\right) \in \mathbb{Z}_{p}^{\times}$, then the map $g$ : $\mathcal{T}_{n, \widetilde{U}_{1}^{p}\left(N_{11}, N_{12}\right)}^{(m) \text {,ord }} \rightarrow \mathcal{T}_{n, \widetilde{U}_{2}^{p}\left(N_{21}, N_{22}\right)}^{(m) \text {,ord }}$ is finite. If $N_{2}>0$ then the finite flat map

$$
\varsigma_{p}: \mathcal{T}_{n, \widetilde{U}_{1}^{p}\left(N_{1}, N_{2}+1\right)}^{(m), \text { ord }} \rightarrow \mathcal{T}_{n, \widetilde{U}_{2}^{p}\left(N_{1}, N_{2}\right)}^{(m) \text { ord }}
$$

has degree $p^{(n+m)^{2}\left[F^{+}: \mathbb{Q}\right]}$.

- On the $\mathbb{F}_{p}$-fibre

$$
\varsigma_{p}: \mathcal{T}_{n, \widetilde{U}^{p}\left(N_{1}, N_{2}+1\right)}^{(m) \text { ord }} \times \operatorname{Spec} \mathbb{F}_{p} \longrightarrow \mathcal{T}_{n, \widetilde{U}^{p}\left(N_{1}, N_{2}\right)}^{(m), \text { ord }} \times \operatorname{Spec} \mathbb{F}_{p}
$$

equals the composition of the absolute Frobenius map with the forgetful map (for any $N_{2} \geq N_{1} \geq 0$ ).

- Suppose that $\widetilde{U}^{p}=\left(U^{p}\right)^{\prime} \ltimes M^{p}$ with $\left(U^{p}\right)^{\prime} \subset G_{n}\left(\mathbb{A}^{\infty, p}\right)$ and $M^{p} \subset N_{n}^{(m)}\left(\mathbb{A}^{\infty, p}\right)$. Also suppose that

$$
\chi \in X^{*}\left(\mathcal{S}_{n, \widetilde{U}^{p}}^{(m), \text { ord }}\right) \subset S\left(\mathcal{O}_{F,(p)}^{m}\right)
$$

is sufficiently divisible. Then we can find $a \in \mathcal{O}_{F,(p)}^{m} \otimes_{\mathcal{O}_{F,(p)}} \mathcal{O}_{F,(p)}^{m}$ lifting $\chi$ such that

$$
i_{\lambda}^{(m)}(a): \mathcal{A}_{n, \widetilde{U}^{p}\left(N_{1}, N_{2}\right)}^{(m), \text { ord }} \longrightarrow\left(\mathcal{A}_{n, \widetilde{U}^{p}\left(N_{1}, N_{2}\right)}^{(m), \text { ord }}\right)^{\vee}
$$

is a homomorphism. For any such $a$

$$
\mathcal{L}_{n, \widetilde{U}^{p}\left(N_{1}, N_{2}\right)}^{(m), \text { ord }}(\chi)=\left(1, i_{\lambda}^{(m)}(a)\right)^{*} \mathcal{P}_{\mathcal{A}_{n, U^{p}\left(N_{1}, N_{2}\right)}^{(m), \text { ord }}}
$$


- If $\chi \in X^{*}\left(\mathcal{S}_{n, \widetilde{U}^{p}}^{(m)}\right) \cap S\left(\mathcal{O}_{F,(p)}^{m}\right)^{>0}$ then $\mathcal{L}_{n, \widetilde{U}^{p}\left(N_{1}, N_{2}\right)}^{(m), \text { ord }}(\chi)$ is relatively ample for

$$
\mathcal{A}_{n, \widetilde{U}^{p}\left(N_{1}, N_{2}\right)}^{(m) \text { ord }} / \mathcal{X}_{n, \widetilde{U}^{p}\left(N_{1}, N_{2}\right)}^{\text {ord }}
$$

- There are natural identifications

$$
\mathcal{T}_{n, \widetilde{U}^{p}\left(N_{1}, N_{2}\right)}^{(m), \text { ord }} \times \operatorname{Spec} \mathbb{Q} \cong T_{n, \widetilde{U}^{p}\left(N_{1}, N_{2}\right)}^{(m)} .
$$

These identifications are compatible with the identifications

$$
\mathcal{A}_{n, \widetilde{U}^{p}\left(N_{1}, N_{2}\right)}^{(m), \text { ord }} \times \operatorname{Spec} \mathbb{Q} \cong A_{n, \widetilde{U}^{p}\left(N_{1}, N_{2}\right)}^{(m)}
$$

and the maps

$$
\mathcal{T}_{n, \widetilde{U}^{p}\left(N_{1}, N_{2}\right)}^{(m) \text { ord }} \longrightarrow \mathcal{A}_{n, \widetilde{U}^{p}\left(N_{1}, N_{2}\right)}^{(m) \text { ord }}
$$

and

$$
T_{n, \widetilde{U}^{p}\left(N_{1}, N_{2}\right)}^{(m)} \longrightarrow A_{n, \widetilde{U}^{p}\left(N_{1}, N_{2}\right)}^{(m)}
$$

The identifications are also equivariant for the actions of the semi-group $\widetilde{G}_{n}^{(m)}\left(\mathbb{A}^{\infty}\right)^{\text {ord }}$ and the group $G L_{m}\left(\mathcal{O}_{F,(p)}\right)$.

(See lemmas 5.2.4.26 and 7.1.2.22, propositions 5.2.4.30, 5.2.4.41 and 7.1.2.36, and remark 7.1.2.38 of [44].)

\subsection{Vector bundles}

\subsubsection{Vector bundles on Shimura varieties in characteristic zero}

Suppose that $U$ is a neat open compact subgroup of $G_{n}\left(\mathbb{A}^{\infty}\right)$. We will let $\Omega_{n, U}$ denote the pull-back by the identity section of the sheaf of relative differentials $\Omega_{A^{\text {univ }} / X_{n, U}}^{1}$. This is a locally free sheaf of $\operatorname{rank} n[F: \mathbb{Q}]$. Up to unique isomorphism its definition does not depend on the choice of $A^{\text {univ }}$. (Because, by the neatness of $U$, there is a unique quasi-isogeny between any two universal four-tuples ( $\left.A^{\text {univ }}, i^{\text {univ }}, \lambda^{\text {univ }},\left[\eta^{\text {univ }}\right]\right)$ ). The system of sheaves $\left\{\Omega_{n, U}\right\}$ has an action of $G_{n}\left(\mathbb{A}^{\infty}\right)$. There is a natural isomorphism between $\Omega_{A^{\text {univ }} / X_{n, U}}$ and the pull-back of $\Omega_{n, U}$ from $X_{n, U}$ to $A^{\text {univ }}$. We will write

$$
\omega_{U}=\omega_{n, U}=\wedge^{n[F: \mathbb{Q}]} \Omega_{n, U} .
$$

Similarly, if $\pi: A^{\text {univ }} \rightarrow X_{n, U}$ is the structural map, then the sheaf

$$
R^{i} \pi_{*} \Omega_{A^{\text {univ }} / X_{n, U}}^{j} \cong\left(\wedge^{j} \Omega_{n, U}\right) \otimes R^{i} \pi_{*} \mathcal{O}_{A^{\text {univ }}}
$$

is locally free and canonically independent of the choice of $A^{\text {univ }}$. These sheaves again have an action of $G_{n}\left(\mathbb{A}^{\infty}\right)$.

We will also write $\Xi_{n, U}=\mathcal{O}_{X_{n, U}}(\|\nu\|)$ for the sheaf $\mathcal{O}_{X_{n, U}}$ but with the $G_{n}\left(\mathbb{A}^{\infty}\right)$-action multiplied by $\|v\|$. 
For any $m \in \mathbb{Z}$ such that $m \lambda^{\text {univ }}$ is a true isogeny we get a class

$$
\begin{aligned}
{\left[\left(1,[m] \lambda^{\text {univ }}\right)^{*} \mathcal{P}_{A^{\text {univ }}}\right] } & \in H^{1}\left(A^{\text {univ }}, \mathcal{O}_{A^{\text {univ }}}^{\times}\right) \\
& \longrightarrow H^{0}\left(X_{n, U}, R^{1} \pi_{*} \mathcal{O}_{A^{\text {univ }}}^{\times}\right) \\
& \stackrel{d \log }{\longrightarrow} H^{0}\left(X_{n, U}, R^{1} \pi_{*} \Omega_{A^{\text {univ }} / X_{n, U}}^{1}\right)
\end{aligned}
$$

The class

$$
\left[\left(1, \lambda^{\text {univ }}\right)^{*} \mathcal{P}_{A^{\text {univ }}}\right]=\left[\left(1,[m] \lambda^{\text {univ }}\right)^{*} \mathcal{P}_{A^{\text {univ }}}\right] / m \in H^{0}\left(X_{n, U}, R^{1} \pi_{*} \Omega_{A^{\text {univ }} / X_{n, U}}^{1}\right)
$$

is well defined independently of $m$. We obtain an embedding

$$
\Xi_{n, U} \hookrightarrow R^{1} \pi_{*} \Omega_{A^{\text {univ }} / X_{n, U}}^{1}
$$

sending 1 to $\left\|\eta^{\text {univ }}\right\|\left[\left(1, \lambda^{\text {univ }}\right) * \mathcal{P}_{A^{\text {univ }}}\right]$. (See Sect. 3.1 for the definition of $\left\|\eta^{\text {univ }}\right\|$.) These maps are compatible with the isomorphisms

$$
R^{1} \pi_{*} \Omega_{A^{\text {univ }} / X_{n, U}}^{1} \stackrel{\sim}{\longrightarrow} R^{1} \pi_{*} \Omega_{A^{\text {univ },} / X_{n, U}}^{1}
$$

induced by the unique quasi-isogeny between two universal four-tuples. They are also $G_{n}\left(\mathbb{A}^{\infty}\right)$-equivariant.

The composites of induced maps

$$
\begin{aligned}
\operatorname{Hom}\left(\Omega_{n, U}, \Xi_{n, U}\right) & \hookrightarrow \operatorname{Hom}\left(\Omega_{n, U}, R^{1} \pi_{*} \Omega_{A^{\text {univ }} / X_{n, U}}^{1}\right) \\
& \stackrel{\sim}{\sim} \operatorname{Hom}\left(\Omega_{n, U}, \Omega_{n, U} \otimes R^{1} \pi_{*} \mathcal{O}_{A^{\text {univ }}}\right) \\
& \stackrel{\operatorname{tr}}{\longrightarrow} R^{1} \pi_{*} \mathcal{O}_{A^{\text {univ }}}
\end{aligned}
$$

are $G_{n}\left(\mathbb{A}^{\infty}\right)$-equivariant isomorphisms, independent of the choice of $A^{\text {univ }}$. Moreover the short exact sequence

$$
(0) \longrightarrow \Omega_{X_{n, U}}^{1} \otimes \mathcal{O}_{A^{\text {univ }}} \longrightarrow \Omega_{A^{\text {univ }}}^{1} \longrightarrow \Omega_{n, U} \otimes \mathcal{O}_{A^{\text {univ }}} \longrightarrow(0)
$$

gives rise to a map

$$
\begin{aligned}
\Omega_{n, U} & \longrightarrow \Omega_{X_{n, U}}^{1} \otimes R^{1} \pi_{*} \mathcal{O}_{A_{\text {univ }}} \\
& \stackrel{\sim}{\longleftarrow} \Omega_{X_{n, U}}^{1} \otimes \operatorname{Hom}\left(\Omega_{n, U}, \Xi_{n, U}\right)
\end{aligned}
$$

and hence to a map

$$
\Omega_{n, U}^{\otimes 2} \longrightarrow \Omega_{X_{n, U}}^{1} \otimes \Xi_{n, U}
$$

These maps do not depend on the choice of $A^{\text {univ }}$ and are $G_{n}\left(\mathbb{A}^{\infty}\right)$-equivariant. They further induce $G_{n}\left(\mathbb{A}^{\infty}\right)$-equivariant isomorphisms

$$
S\left(\Omega_{n, U}\right) \stackrel{\sim}{\longrightarrow} \Omega_{X_{n, U}}^{1} \otimes \Xi_{n, U}
$$

which again do not depend on the choice of $A^{\text {univ }}$. (See, for instance, propositions 2.1.7.3 and 2.3.5.2 of [41]. This is referred to as the 'Kodaira-Spencer isomorphism'.) 
Let $\mathcal{E}_{U}$ denote the principal $L_{n,(n)}$-bundle on $X_{n, U}$ in the Zariski topology defined by setting, for $W \subset X_{n, U}$ a Zariski open, $\mathcal{E}_{U}(W)$ to be the set of pairs $\left(\xi_{0}, \xi_{1}\right)$, where

$$
\xi_{0}:\left.\Xi_{n, U}\right|_{W} \stackrel{\sim}{\longrightarrow} \mathcal{O}_{W}
$$

and

$$
\xi_{1}: \Omega_{n, U} \stackrel{\sim}{\longrightarrow} \operatorname{Hom}_{\mathbb{Q}}\left(V_{n} / V_{n,(n)}, \mathcal{O}_{W}\right) .
$$

We define the $L_{n,(n)}$-action on $\mathcal{E}_{U}$ by

$$
h\left(\xi_{0}, \xi_{1}\right)=\left(v(h)^{-1} \xi_{0},\left(\circ h^{-1}\right) \circ \xi_{1}\right) .
$$

The inverse system $\left\{\mathcal{E}_{U}\right\}$ has an action of $G_{n}\left(\mathbb{A}^{\infty}\right)$.

Suppose that $R_{0}$ is a $\mathbb{Q}$-algebra and that $\rho$ is a representation of $L_{n,(n)}$ on a finite, locally free $R_{0}$-module $W_{\rho}$. We define a locally free sheaf $\mathcal{E}_{U, \rho}$ over $X_{n, U} \times \operatorname{Spec} R_{0}$ by setting $\mathcal{E}_{U, \rho}(W)$ to be the set of $L_{n,(n)}\left(\mathcal{O}_{W}\right)$-equivariant maps of Zariski sheaves of sets

$$
\left.\mathcal{E}_{U}\right|_{W} \rightarrow W_{\rho} \otimes_{R_{0}} \mathcal{O}_{W}
$$

Then $\left\{\mathcal{E}_{U, \rho}\right\}$ is a system of locally free sheaves with $G_{n}\left(\mathbb{A}^{\infty}\right)$-action over the system of schemes $\left\{X_{n, U} \times \operatorname{Spec} R_{0}\right\}$. If $g \in G_{n}\left(\mathbb{A}^{\infty}\right)$, then the natural map

$$
g^{*} \mathcal{E}_{U, \rho} \longrightarrow \mathcal{E}_{U^{\prime}, \rho}
$$

is an isomorphism.

In the case $R_{0}=\mathbb{C}$, the holomorphic vector bundle on $X_{n, U}(\mathbb{C})$ associated to $\mathcal{E}_{U, \rho}$ is

$$
\mathfrak{E}_{U, \rho}=G_{n}(\mathbb{Q}) \backslash\left(G_{n}\left(\mathbb{A}^{\infty}\right) / U \times \mathfrak{E}_{\rho}\right)
$$

over

$$
X_{n, U}(\mathbb{C})=G_{n}(\mathbb{Q}) \backslash\left(G_{n}\left(\mathbb{A}^{\infty}\right) / U \times \mathfrak{H}_{n}^{ \pm}\right) .
$$

(See Sect. 1.1 for the definition of the holomorphic vector bundle $\mathfrak{E}_{\rho} / \mathfrak{H}_{n}^{ \pm}$.)

Note that

$$
\mathcal{E}_{U, \mathrm{Std}^{\vee}} \cong \Omega_{n, U}
$$

and

$$
\mathcal{E}_{U, v^{-1}} \cong \Xi_{n, U}
$$

and

$$
\mathcal{E}_{U, \wedge\left[{ }^{n[: \mathbb{Q}]} \operatorname{Std}^{\vee}\right.} \cong \omega_{U}
$$

and

$$
\mathcal{E}_{U, \mathrm{KS}} \cong \Omega_{X_{n, U}}^{1} .
$$

(See Sect. 1.2 for the definition of the representation KS.) 


\subsubsection{Vector bundles on Kuga-Sato varieties in characteristic zero}

Suppose now that $U$ is a neat open compact subgroup of $G_{n}^{(m)}\left(\mathbb{A}^{\infty}\right)$ with image $U^{\prime}$ in $G_{n}\left(\mathbb{A}^{\infty}\right)$. We will let $\Omega_{n, U}^{(m)}$ denote the pull-back by the identity section of the sheaf of relative differentials $\Omega_{G^{\text {univ }} / A_{n, U}^{(m)}}^{1}$. This is a locally free sheaf of rank $(n+m)[F: \mathbb{Q}]$. Up to unique isomorphism its definition does not depend on the choice of $G^{\text {univ }}$. The system of sheaves $\left\{\Omega_{n, U}^{(m)}\right\}$ has actions of $G_{n}^{(m)}\left(\mathbb{A}^{\infty}\right)$ and of $G L_{m}(F)$. Moreover there is an exact sequence

$$
(0) \longrightarrow \pi_{A_{n}^{(m)} / X_{n}}^{*} \Omega_{n, U^{\prime}} \longrightarrow \Omega_{n, U}^{(m)} \longrightarrow F^{m} \otimes_{\mathbb{Q}} \mathcal{O}_{A_{n, U}^{(m)}} \longrightarrow(0)
$$

which is equivariant for the actions of $G_{n}^{(m)}\left(\mathbb{A}^{\infty}\right)$ and $G L_{m}(F)$.

Let $\mathcal{E}_{U}^{(m)}$ denote the principal $R_{n,(n)}^{(m)}$-bundle on $A_{n, U}^{(m)}$ in the Zariski topology defined by setting, for $W \subset A_{n, U}^{(m)}$ a Zariski open, $\mathcal{E}_{U}^{(m)}(W)$ to be the set of pairs $\left(\xi_{0}, \xi_{1}\right)$, where

$$
\xi_{0}:\left.\Xi_{n, U}\right|_{W} \stackrel{\sim}{\longrightarrow} \mathcal{O}_{W}
$$

and

$$
\xi_{1}: \Omega_{n, U}^{(m)} \stackrel{\sim}{\longrightarrow} \operatorname{Hom}_{\mathbb{Q}}\left(V_{n} / V_{n,(n)} \oplus \operatorname{Hom}_{\mathbb{Q}}\left(F^{m}, \mathbb{Q}\right), \mathcal{O}_{W}\right)
$$

satisfies

$$
\xi_{1}: \Omega_{n, U} \stackrel{\sim}{\longrightarrow} \operatorname{Hom}_{\mathbb{Q}}\left(V_{n} / V_{n,(n)}, \mathcal{O}_{W}\right)
$$

and induces the canonical isomorphism

$$
F^{m} \otimes_{\mathbb{Q}} \mathcal{O}_{W} \longrightarrow \operatorname{Hom}_{\mathbb{Q}}\left(\operatorname{Hom}_{\mathbb{Q}}\left(F^{m}, \mathbb{Q}\right), \mathcal{O}_{W}\right)
$$

We define the $R_{n,(n)}^{(m)}$-action on $\mathcal{E}_{U}^{(m)}$ by

$$
h\left(\xi_{0}, \xi_{1}\right)=\left(v(h)^{-1} \xi_{0},\left(\circ h^{-1}\right) \circ \xi_{1}\right) .
$$

The inverse system $\left\{\mathcal{E}_{U}^{(m)}\right\}$ has an action of $G_{n}^{(m)}\left(\mathbb{A}^{\infty}\right)$ and of $G L_{m}(F)$.

Suppose that $R_{0}$ is a $\mathbb{Q}$-algebra and that $\rho$ is a representation of $R_{n,(n)}^{(m)}$ on a finite, locally free $R_{0}$-module $W_{\rho}$. We define a locally free sheaf $\mathcal{E}_{U, \rho}^{(m)}$ over $A_{n, U}^{(m)} \times \operatorname{Spec} R_{0}$ by setting $\mathcal{E}_{U, \rho}^{(m)}(W)$ to be the set of $R_{n,(n)}^{(m)}\left(\mathcal{O}_{W}\right)$-equivariant maps of Zariski sheaves of sets

$$
\left.\mathcal{E}_{U}^{(m)}\right|_{W} \longrightarrow W_{\rho} \otimes_{R_{0}} \mathcal{O}_{W}
$$

Then $\left\{\mathcal{E}_{U, \rho}^{(m)}\right\}$ is a system of locally free sheaves with both $G_{n}^{(m)}\left(\mathbb{A}^{\infty}\right)$-action and $G L_{m}(F)$ action over the system of schemes $\left\{A_{n, U}^{(m)} \times \operatorname{Spec} R_{0}\right\}$. If $g \in G_{n}^{(m)}\left(\mathbb{A}^{\infty}\right)$ and $\gamma \in G L_{m}(F)$, then the natural maps

$$
g^{*} \mathcal{E}_{U, \rho}^{(m)} \longrightarrow \mathcal{E}_{U^{\prime}, \rho}^{(m)}
$$

and

$$
\gamma^{*} \mathcal{E}_{U, \rho}^{(m)} \longrightarrow \mathcal{E}_{U^{\prime}, \rho}^{(m)}
$$


are isomorphisms. If $\rho$ factors through $R_{n,(n)}^{(m)} \rightarrow L_{n,(n)}$ then $\mathcal{E}_{U, \rho}^{(m)}$ is canonically isomorphic to the pull-back of $\mathcal{E}_{U, \rho}$ from $X_{n, U}$. In general $W_{\rho}$ has a filtration by $R_{n,(n)}^{(m)}$-invariant direct summands such that the action of $R_{n,(n)}^{(m)}$ on each graded piece factors through $L_{n,(n)}$. (To see this apply proposition 4.7.3 of exposé I of [53] to the action of $L_{n,(n) \text {,herm }}$ on $W_{\rho}$.) Thus $\mathcal{E}_{U, \rho}^{(m)}$ has a $G_{n}^{(m)}\left(\mathbb{A}^{\infty}\right)$ and $G L_{m}(F)$ invariant filtration by local direct summands such that each graded piece is the pull-back of some $\mathcal{E}_{U, \rho^{\prime}}$ from $X_{n, U}$.

\subsubsection{Vector bundles on Shimura varieties in mixed characteristic}

Similarly suppose that $U^{p}$ is a neat open compact subgroup of $G_{n}\left(\mathbb{A}^{\infty, p}\right)$, and that $N_{2} \geq N_{1} \geq 0$ are integers. We will let $\Omega_{n, U^{p}\left(N_{1}, N_{2}\right)}^{\text {ord }}$ denote the pull-back by the identity section of $\Omega_{\mathcal{A}^{\text {univ }} / \mathcal{X}_{n, U^{p}\left(N_{1}, N_{2}\right)}^{\text {ord }}}^{1}$. This is a locally free sheaf of rank $n[F: \mathbb{Q}]$. Up to unique isomorphism its definition does not depend on the choice of $\mathcal{A}^{\text {univ }}$. (Because, by the neatness of $U^{p}$, there is a unique prime-to- $p$ quasi-isogeny between any two universal fourtuples $\left(\mathcal{A}^{\text {univ }}, i^{\text {univ }}, \lambda^{\text {univ }},\left[\eta^{\text {univ }}\right]\right)$.) The system of sheaves $\left\{\Omega_{n, U^{p}\left(N_{1}, N_{2}\right)}^{\text {ord }}\right\}$ has an action of $G_{n}\left(\mathbb{A}^{\infty}\right)^{\text {ord }}$. There is a natural isomorphism between $\Omega_{\mathcal{A}^{\text {univ }} / \mathcal{X}_{n, U} \text { ord }_{\left(N_{1}, N_{2}\right)}}^{1}$ and the pull-back of $\Omega_{n, U^{p}\left(N_{1}, N_{2}\right)}^{\text {ord }}$. We will write

$$
\omega_{U^{p}\left(N_{1}, N_{2}\right)}=\omega_{n, U^{p}\left(N_{1}, N_{2}\right)}=\wedge^{n[F: \mathbb{Q}]} \Omega_{n, U^{p}\left(N_{1}, N_{2}\right)}^{\text {ord }}
$$

We will also write $\Xi_{n, U^{p}\left(N_{1}, N_{2}\right)}=\mathcal{O}_{\mathcal{X}_{n, U^{p}\left(N_{1}, N_{2}\right)}^{\text {ord }}}(\|\nu\|)$ for the sheaf $\mathcal{O}_{\mathcal{X}_{n, U}^{\text {ord }}\left(N_{1}, N_{2}\right)}$ but with the $G_{n}\left(\mathbb{A}^{\infty}\right)^{\text {ord }}$-action multiplied by $\|\nu\|$.

For any $m \in \mathbb{Z}$ such that $p \nmid m$ and $m \lambda^{\text {univ }}$ is a true isogeny we get a class

$$
\begin{aligned}
{\left[\left(1,[m] \lambda^{\text {univ }}\right)^{*} \mathcal{P}_{\mathcal{A}^{\text {univ }}}\right] } & \in H^{1}\left(\mathcal{A}^{\text {univ }}, \mathcal{O}_{\mathcal{A}^{\text {univ }}}^{\times}\right) \\
& \longrightarrow H^{0}\left(\mathcal{X}_{n, U^{p}\left(N_{1}, N_{2}\right)}^{\text {ord }}, R^{1} \pi_{*} \mathcal{O}_{\mathcal{A}^{\text {univ }}}^{\times}\right) \\
& \stackrel{d \log }{\longrightarrow} H^{0}\left(\mathcal{X}_{n, U^{p}\left(N_{1}, N_{2}\right)}^{\text {ord }}, R^{1} \pi_{*} \Omega_{\mathcal{A}^{\text {univ }} / \mathcal{X}_{n, U^{p}\left(N_{1}, N_{2}\right)}^{\text {ord }}}^{1}\right)
\end{aligned}
$$

The class

$$
\begin{aligned}
{\left[\left(1, \lambda^{\text {univ }}\right)^{*} \mathcal{P}_{\mathcal{A}^{\text {univ }}}\right] } & =\left[\left(1,[m] \lambda^{\text {univ }}\right)^{*} \mathcal{P}_{\mathcal{A}^{\text {univ }}}\right] / m \\
& \in H^{0}\left(\mathcal{X}_{n, U^{p}\left(N_{1}, N_{2}\right)}^{\text {ord }}, R^{1} \pi_{*} \Omega_{\mathcal{A}^{\text {univ }} / \mathcal{X}_{n, U^{p}\left(N_{1}, N_{2}\right)}^{\text {ord }}}^{1}\right)
\end{aligned}
$$

is well defined independently of $m$. We obtain an embedding

$$
\Xi_{n, U^{p}\left(N_{1}, N_{2}\right)}^{\text {ord }} \hookrightarrow R^{1} \pi_{*} \Omega_{\mathcal{A}^{\text {univ }} / \mathcal{X}_{n, U^{p}\left(N_{1}, N_{2}\right)}^{\text {ord }}}^{1}
$$

sending 1 to $\left\|\eta^{\text {univ }}\right\|\left[\left(1, \lambda^{\text {univ }}\right)^{*} \mathcal{P}_{\mathcal{A}^{\text {univ }}}\right]$. These maps are compatible with the isomorphisms

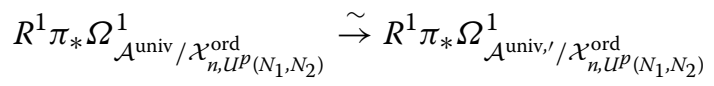

induced by the unique prime-to- $p$ quasi-isogeny between two universal four-tuples. They are also $G_{n}\left(\mathbb{A}^{\infty}\right)^{\text {ord }}$-equivariant. 
The composites of induced maps

$$
\begin{aligned}
& \operatorname{Hom}\left(\Omega_{n, U^{p}\left(N_{1}, N_{2}\right)}^{\text {ord }}, \Xi_{n, U^{p}\left(N_{1}, N_{2}\right)}^{\text {ord }}\right) \\
& \hookrightarrow \operatorname{Hom}\left(\Omega_{n, U^{p}\left(N_{1}, N_{2}\right)}^{\text {ord }}, R^{1} \pi_{*} \Omega_{\mathcal{A}^{\text {univ }} / \mathcal{X}_{n, U^{p}\left(N_{1}, N_{2}\right)}^{\text {ord }}}^{1}\right) \\
& \stackrel{\sim}{\longleftarrow} \operatorname{Hom}\left(\Omega_{n, U^{p}\left(N_{1}, N_{2}\right)}^{\text {ord }}, \Omega_{n, U^{p}\left(N_{1}, N_{2}\right)}^{\text {ord }} \otimes R^{1} \pi_{*} \mathcal{O}_{\mathcal{A}^{\text {univ }}}\right) \\
& \stackrel{\operatorname{tr}}{\longrightarrow} R^{1} \pi_{*} \mathcal{O}_{\mathcal{A}^{\text {univ }}}
\end{aligned}
$$

are $G_{n}\left(\mathbb{A}^{\infty}\right)^{\text {ord }}$-equivariant isomorphisms, independent of the choice of $\mathcal{A}^{\text {univ }}$. Moreover the short exact sequence

$$
(0) \longrightarrow \Omega_{\mathcal{X}_{n, U^{p}\left(N_{1}, N_{2}\right)}^{\text {ord }}}^{1} \otimes \mathcal{O}_{\mathcal{A}^{\text {univ }}} \longrightarrow \Omega_{\mathcal{A}^{\text {univ }}}^{1} \longrightarrow \Omega_{n, U^{p}\left(N_{1}, N_{2}\right)}^{\text {ord }} \otimes \mathcal{O}_{\mathcal{A}^{\text {univ }}} \longrightarrow(0)
$$

gives rise to a map

$$
\begin{aligned}
\Omega_{n, U^{p}\left(N_{1}, N_{2}\right)}^{\text {ord }} & \longrightarrow \Omega_{\mathcal{X}_{n, U^{p}\left(N_{1}, N_{2}\right)}^{\text {ord }}}^{1} \otimes R^{1} \pi_{*} \mathcal{O}_{\mathcal{A}^{\text {univ }}} \\
& \longleftarrow \Omega_{\mathcal{X}_{n, U^{p}\left(N_{1}, N_{2}\right)}^{\text {ord }}}^{1} \otimes \operatorname{Hom}\left(\Omega_{n, U^{p}\left(N_{1}, N_{2}\right)}^{\text {ord }}, \Xi_{n, U^{p}\left(N_{1}, N_{2}\right)}^{\text {ord }}\right)
\end{aligned}
$$

and hence to a map

$$
\left(\Omega_{n, U^{p}\left(N_{1}, N_{2}\right)}^{\text {ord }}\right)^{\otimes 2} \longrightarrow \Omega_{\mathcal{X}_{n, U^{p}\left(N_{1}, N_{2}\right)}^{\text {ord }}}^{1} \otimes \Xi_{n, U^{p}\left(N_{1}, N_{2}\right)}^{\text {ord }}
$$

These maps do not depend on the choice of $\mathcal{A}^{\text {univ }}$ and are $G_{n}\left(\mathbb{A}^{\infty}\right)^{\text {ord }}$-equivariant. They further induce $G_{n}\left(\mathbb{A}^{\infty}\right)^{\text {ord }}$ isomorphisms

$$
S\left(\Omega_{n, U^{p}\left(N_{1}, N_{2}\right)}^{\text {ord }}\right) \stackrel{\sim}{\longrightarrow} \Omega_{\mathcal{X}_{n, U^{p}\left(N_{1}, N_{2}\right)}^{\text {ord }}}^{1} \otimes \Xi_{n, U^{p}\left(N_{1}, N_{2}\right)}^{\text {ord }}
$$

which again do not depend on the choice of $\mathcal{A}^{\text {univ }}$. (See, for instance, proposition 3.4.3.3 of [44].)

Let $\mathcal{E}_{U^{p}\left(N_{1}, N_{2}\right)}^{\text {ord }}$ denote the principal $L_{n,(n)}$-bundle on $\mathcal{X}_{n, U^{p}\left(N_{1}, N_{2}\right)}^{\text {ord }}$ in the Zariski topology defined by setting, for $W \subset \mathcal{X}_{n, U^{p}\left(N_{1}, N_{2}\right)}^{\text {ord }}$ a Zariski open, $\mathcal{E}_{U^{p}\left(N_{1}, N_{2}\right)}^{\text {ord }}(W)$ to be the set of pairs $\left(\xi_{0}, \xi_{1}\right)$, where

$$
\xi_{0}:\left.\Xi_{n, U^{p}\left(N_{1}, N_{2}\right)}^{\text {ord }}\right|_{W} \stackrel{\sim}{\longrightarrow} \mathcal{O}_{W}
$$

and

$$
\xi_{1}: \Omega_{n, U^{p}\left(N_{1}, N_{2}\right)}^{\text {ord }} \stackrel{\sim}{\longrightarrow} \operatorname{Hom}_{\mathbb{Z}}\left(\Lambda_{n} / \Lambda_{n,(n)}, \mathcal{O}_{W}\right)
$$

We define the $L_{n,(n)}$-action on $\mathcal{E}_{U^{p}\left(N_{1}, N_{2}\right)}^{\text {ord }}$ by

$$
h\left(\xi_{0}, \xi_{1}\right)=\left(v(h)^{-1} \xi_{0},\left(\circ h^{-1}\right) \circ \xi_{1}\right) .
$$

The inverse system $\left\{\mathcal{E}_{U^{p}\left(N_{1}, N_{2}\right)}^{\text {ord }}\right\}$ has an action of $G_{n}\left(\mathbb{A}^{\infty}\right)^{\text {ord, }} \times$.

Suppose that $R_{0}$ is a $\mathbb{Z}_{(p)}$-algebra and that $\rho$ is a representation of the algebraic group $L_{n,(n)}$ on a finite, locally free $R_{0}$-module $W_{\rho}$. We define a locally free sheaf $\mathcal{E}_{U^{p}\left(N_{1}, N_{2}\right), \rho}^{\text {ord }}$ over 
$\mathcal{X}_{n, U^{p}\left(N_{1}, N_{2}\right)}^{\text {ord }} \times \operatorname{Spec} R_{0}$ by setting $\mathcal{E}_{U^{p}\left(N_{1}, N_{2}\right), \rho}^{\text {ord }}(W)$ to be the set of $L_{n,(n)}\left(\mathcal{O}_{W}\right)$-equivariant maps of Zariski sheaves of sets

$$
\left.\mathcal{E}_{U^{p}\left(N_{1}, N_{2}\right)}^{\text {ord }}\right|_{W} \rightarrow W_{\rho} \otimes_{R_{0}} \mathcal{O}_{W}
$$

Then $\left\{\mathcal{E}_{U^{p}\left(N_{1}, N_{2}\right), \rho}^{\text {ord }}\right\}$ is a system of locally free sheaves with $G_{n}\left(\mathbb{A}^{\infty}\right)^{\text {ord, } \times}$-action over the system of schemes $\left\{\mathcal{X}_{n, U^{p}\left(N_{1}, N_{2}\right)}^{\text {ord }} \times \operatorname{Spec} R_{0}\right\}$. The maps

$$
g^{*} \mathcal{E}_{U^{p}\left(N_{1}, N_{2}\right), \rho} \longrightarrow \mathcal{E}_{\left(U^{p}\right)^{\prime}\left(N_{1}^{\prime}, N_{2}^{\prime}\right), \rho}
$$

are isomorphisms. The pull-back of $\mathcal{E}_{U^{p}\left(N_{1}, N_{2}\right), \rho}^{\text {ord }}$ to

$$
\mathcal{X}_{n, U^{p}\left(N_{1}, N_{2}\right)}^{\text {ord }} \times \operatorname{Spec} R_{0}[1 / p]
$$

is canonically identified with the sheaf $\mathcal{E}_{U^{p}\left(N_{1}, N_{2}\right), \rho \otimes_{R_{0}} R_{0}[1 / p]}$. This identification is $G_{n}\left(\mathbb{A}^{\infty}\right)^{\text {ord, } \times}$-equivariant.

Note that

$$
\mathcal{E}_{U^{p}\left(N_{1}, N_{2}\right), \operatorname{Std}^{\vee}}^{\text {ord }} \cong \Omega_{n, U^{p}\left(N_{1}, N_{2}\right)}^{\text {ord }}
$$

and

$$
\mathcal{E}_{U^{p}\left(N_{1}, N_{2}\right), v^{-1}}^{\text {ord }} \cong \Xi_{n, U^{p}\left(N_{1}, N_{2}\right)}^{\text {ord }}
$$

and

$$
\mathcal{E}_{U^{p}\left(N_{1}, N_{2}\right), \wedge^{n[F: \mathbb{Q}]} \operatorname{Std}^{\vee}}^{\text {ord }} \cong \omega_{U^{p}\left(N_{1}, N_{2}\right)}^{\text {ord }}
$$

and

$$
\mathcal{E}_{U^{p}\left(N_{1}, N_{2}\right), \mathrm{KS}}^{\mathrm{ord}} \cong \Omega_{\mathcal{X}_{n, U^{p}\left(N_{1}, N_{2}\right)}^{\text {ord }}}^{1}
$$

\subsubsection{Vector bundles on Kuga-Sato varieties in mixed characteristic}

Suppose now that $U^{p}$ is a neat open compact subgroup of $G_{n}^{(m)}\left(\mathbb{A}^{\infty, p}\right)$ with image $\left(U^{p}\right)^{\prime}$ in $G_{n}\left(\mathbb{A}^{\infty, p}\right)$. We will let $\Omega_{n, U^{p}\left(N_{1}, N_{2}\right)}^{(m) \text { ord }}$ denote the pull-back by the identity section of the sheaf of relative differentials $\Omega_{\mathcal{G}^{\text {univ }} / \mathcal{A}_{n, U^{p}\left(N_{1}, N_{2}\right)}^{(m), \text { ord }}}^{1}$. This is a locally free sheaf of rank $(n+m)[F: \mathbb{Q}]$. Up to unique isomorphism its definition does not depend on the choice of $\mathcal{G}^{\text {univ }}$. The system of sheaves $\left\{\Omega_{n, U^{p}\left(N_{1}, N_{2}\right)}^{(m) \text {,ord }}\right\}$ has actions of $G_{n}^{(m)}\left(\mathbb{A}^{\infty}\right)^{\text {ord }}$ and of $G L_{m}\left(\mathcal{O}_{F,(p)}\right)$. Moreover there is an exact sequence

$$
(0) \rightarrow \pi_{\mathcal{A}_{n}^{(m), \text { ord }} / \mathcal{X}_{n}^{\text {ord }}}^{*} \Omega_{n,\left(U^{p}\right)^{\prime}\left(N_{1}, N_{2}\right)}^{\text {ord }} \rightarrow \Omega_{n, U^{p}\left(N_{1}, N_{2}\right)}^{(m)} \rightarrow \mathcal{O}_{F,(p)}^{m} \otimes_{\mathbb{Q}} \mathcal{O}_{\mathcal{A}_{n, U^{p}\left(N_{1}, N_{2}\right)}^{(m)}} \rightarrow(0)
$$

which is equivariant for the actions of $G_{n}^{(m)}\left(\mathbb{A}^{\infty}\right)^{\text {ord }}$ and $G L_{m}\left(\mathcal{O}_{F,(p)}\right)$.

Let $\mathcal{E}_{U^{p}\left(N_{1}, N_{2}\right)}^{(m) \text { ord }}$ denote the principal $R_{n,(n)}^{(m)}$-bundle on $\mathcal{A}_{n, U^{p}\left(N_{1}, N_{2}\right)}^{(m) \text {,ord }}$ in the Zariski topology defined by setting, for $W \subset \mathcal{A}_{n, U^{p}\left(N_{1}, N_{2}\right)}^{(m) \text {,ord }}$ a Zariski open, $\mathcal{E}_{U^{p}\left(N_{1}, N_{2}\right)}^{(m) \text {,ord }}(W)$ to be the set of pairs $\left(\xi_{0}, \xi_{1}\right)$, where

$$
\xi_{0}:\left.\Xi_{n, U^{p}\left(N_{1}, N_{2}\right)}^{\text {ord }}\right|_{W} \stackrel{\sim}{\longrightarrow} \mathcal{O}_{W}
$$


and

$$
\xi_{1}: \Omega_{n, U^{p}\left(N_{1}, N_{2}\right)}^{(m) \text { ord }} \stackrel{\sim}{\longrightarrow} \operatorname{Hom}\left(\Lambda_{n} / \Lambda_{n,(n)} \oplus \operatorname{Hom}\left(\mathcal{O}_{F}^{m}, \mathbb{Z}\right), \mathcal{O}_{W}\right)
$$

satisfies

$$
\xi_{1}: \Omega_{n, U^{p}\left(N_{1}, N_{2}\right)}^{\text {ord }} \stackrel{\sim}{\longrightarrow} \operatorname{Hom}\left(\Lambda_{n} / \Lambda_{n,(n)}, \mathcal{O}_{W}\right)
$$

and induces the canonical isomorphism

$$
\mathcal{O}_{F,(p)}^{m} \otimes_{\mathbb{Z}_{(p)}} \mathcal{O}_{W} \longrightarrow \operatorname{Hom}\left(\operatorname{Hom}\left(\mathcal{O}_{F}^{m}, \mathbb{Z}\right), \mathcal{O}_{W}\right)
$$

We define the $R_{n,(n)}^{(m)}$-action on $\mathcal{E}_{U^{p}\left(N_{1}, N_{2}\right)}^{(m) \text {,ord }}$ by

$$
h\left(\xi_{0}, \xi_{1}\right)=\left(v(h)^{-1} \xi_{0},\left(\circ h^{-1}\right) \circ \xi_{1}\right) .
$$

The inverse system $\left\{\mathcal{E}_{U^{p}\left(N_{1}, N_{2}\right)}^{(m), \text { ord }}\right\}$ has an action of $G_{n}^{(m)}\left(\mathbb{A}^{\infty}\right)^{\text {ord, } \times}$ and of $G L_{m}\left(\mathcal{O}_{F,(p)}\right)$.

Suppose that $R_{0}$ is a $\mathbb{Z}_{(p)}$-algebra and that $\rho$ is a representation of $R_{n,(n)}^{(m)}$ on a finite, locally free $R_{0}$-module $W_{\rho}$. We define a locally free sheaf $\mathcal{E}_{U^{p}\left(N_{1}, N_{2}\right), \rho}^{(m) \text { ord }}$ over $\mathcal{A}_{n, U^{p}\left(N_{1}, N_{2}\right)}^{(m) \text {,ord }} \times$ Spec $R_{0}$ by setting $\mathcal{E}_{U, \rho}^{(m)}(W)$ to be the set of $R_{n,(n)}^{(m)}\left(\mathcal{O}_{W}\right)$-equivariant maps of Zariski sheaves of sets

$$
\left.\mathcal{E}_{U^{p}\left(N_{1}, N_{2}\right)}^{(m), \text { ord }}\right|_{W} \longrightarrow W_{\rho} \otimes_{R_{0}} \mathcal{O}_{W}
$$

Then $\left\{\mathcal{E}_{U^{p}\left(N_{1}, N_{2}\right), \rho}^{(m), \text { ord }}\right\}$ is a system of locally free sheaves with $G_{n}^{(m)}\left(\mathbb{A}^{\infty}\right)^{\text {ord, } \times}$-action and $G L_{m}\left(\mathcal{O}_{F,(p)}\right)$-action over the system of schemes $\left\{\mathcal{A}_{n, U^{p}\left(N_{1}, N_{2}\right)}^{(m) \text {,ord }} \times \operatorname{Spec} R_{0}\right\}$. If $g \in$ $G_{n}^{(m)}\left(\mathbb{A}^{\infty}\right)^{\text {ord, } \times}$ and $\gamma \in G L_{m}\left(\mathcal{O}_{F,(p)}\right)$, then the natural maps

$$
g^{*} \mathcal{E}_{U^{p}\left(N_{1}, N_{2}\right), \rho}^{(m), \text { ord }} \longrightarrow \mathcal{E}_{\left(U^{p}\right)^{\prime}\left(N_{1}^{\prime}, N_{2}^{\prime}\right), \rho}^{(m), \text { ord }}
$$

and

$$
\gamma^{*} \mathcal{E}_{U^{p}\left(N_{1}, N_{2}\right), \rho}^{(m), \text { ord }} \longrightarrow \mathcal{E}_{\left(U^{p}\right)^{\prime}\left(N_{1}^{\prime}, N_{2}^{\prime}\right), \rho}^{(m), \text { ord }}
$$

are isomorphisms. If $\rho$ factors through $R_{n,(n)}^{(m)} \rightarrow L_{n,(n)}$ then $\mathcal{E}_{U^{p}\left(N_{1}, N_{2}\right), \rho}^{(m), \text { isd }}$ canonically isomorphic to the pull-back of $\mathcal{E}_{U^{p}\left(N_{1}, N_{2}\right), \rho}^{\text {ord }}$ from $\mathcal{X}_{n, U^{p}\left(N_{1}, N_{2}\right)}^{\text {ord }}$. In general $W_{\rho}$ has a filtration by $R_{n,(n)}^{(m)}$-invariant local direct summands such that the action of $R_{n,(n)}^{(m)}$ on each graded piece factors through $L_{n,(n)}$. (To see this apply proposition 4.7.3 of exposé I of [53] to the action of $L_{n,(n), \text { herm }}$ on $W_{\rho}$.) Thus $\mathcal{E}_{U^{p}\left(N_{1}, N_{2}\right), \rho}^{(m), \text { ord }}$ has a $G_{n}^{(m)}\left(\mathbb{A}^{\infty}\right)$ and $G L_{m}\left(\mathcal{O}_{F,(p)}\right)$ invariant filtration by local direct summands such that each graded piece is the pull-back of some $\mathcal{E}_{U^{p}\left(N_{1}, N_{2}\right), \rho^{\prime}}^{\text {ord }}$ from $\mathcal{X}_{n, U^{p}\left(N_{1}, N_{2}\right)}^{\text {ord }}$. 


\subsubsection{Higher direct images from Kuga-Sato varieties to Shimura varieties, characteristic zero}

case

If $m \geq m^{\prime}$ and if $U$ is a neat open compact subgroup of $G_{n}^{(m)}\left(\mathbb{A}^{\infty}\right)$ with image $U^{\prime}$ in $G_{n}^{\left(m^{\prime}\right)}\left(\mathbb{A}^{\infty}\right)$ then the sheaf

$$
R^{j} \pi_{n}^{(m)} / A_{n}^{\left(m^{\prime}\right)}, *{ }_{A_{n, U}^{(m)} / A_{n, U^{\prime}}^{\left(m^{\prime}\right)}}^{i}
$$

depends only on $U^{\prime}$ and not on $U$. We will denote it

$$
\left(R^{j} \pi_{*} \Omega_{A_{n}^{(m)} / A_{n}^{\left(m^{\prime}\right)}}^{i}\right)_{U^{\prime}}
$$

If $g \in G_{n}^{(m)}\left(\mathbb{A}^{\infty}\right)$ and $g^{-1} U_{1} g \subset U_{2}$ then there is a natural isomorphism

$$
g:\left(g^{\prime}\right)^{*}\left(R^{j} \pi_{*} \Omega_{A_{n}^{(m)} / A_{n}^{\left(m^{\prime}\right)}}^{i}\right)_{U_{2}^{\prime}} \stackrel{\sim}{\longrightarrow}\left(R^{j} \pi_{*} \Omega_{A_{n}^{(m)} / A_{n}^{\left(m^{\prime}\right)}}^{i}\right)_{U_{1}^{\prime}},
$$

where $g^{\prime}$ (resp. $U_{1}^{\prime}$, resp. $U_{2}^{\prime}$ ) denotes the image of $g$ (resp. $U_{1}$, resp. $\left.U_{2}\right)$ in $G_{n}^{\left(m^{\prime}\right)}\left(\mathbb{A}^{\infty}\right)$. This isomorphism only depends on $g^{\prime}, U_{1}^{\prime}$ and $U_{2}^{\prime}$ and not on $g, U_{1}$ and $U_{2}$. This gives the system of sheaves $\left\{\left(R^{j} \pi_{*} \Omega_{A_{n}^{(m)} / A_{n}^{\left(m^{\prime}\right)}}^{i}\right)_{U^{\prime}}\right\}$ a left action of $G_{n}^{\left(m^{\prime}\right)}\left(\mathbb{A}^{\infty}\right)$. Also if $\gamma \in Q_{m, m^{\prime}}(F)$ then $\gamma: A_{n, U}^{(m)} \rightarrow A_{n, \gamma U}^{(m)}$ gives a natural isomorphism

$$
\gamma:\left(R^{j} \pi_{*} \Omega_{A_{n}^{(m)} / A_{n}^{\left(m^{\prime}\right)}}^{i}\right)_{U^{\prime}} \stackrel{\sim}{\longrightarrow}\left(R^{j} \pi_{*} \Omega_{A_{n}^{(m)} / A_{n}^{\left(m^{\prime}\right)}}^{i}\right)_{U^{\prime}},
$$

which depends only on $U^{\prime}$ and not on $U$. This gives the system of sheaves

$$
\left\{\left(R^{j} \pi_{*} \Omega_{A_{n}^{(m)} / A_{n}^{\left(m^{\prime}\right)}}^{i}\right)_{U^{\prime}}\right\}
$$

a right action of $Q_{m, m^{\prime}}(F)$. We have $\gamma \circ g=\gamma(g) \circ \gamma$.

If $U_{1}^{\prime} \supset U_{2}^{\prime}$ and $g^{\prime} \in U_{2}^{\prime}$ normalizes $U_{1}^{\prime}$ then on

$$
\left(R^{j} \pi_{*} \Omega_{A_{n}^{(m)} / A_{n}^{\left(m^{\prime}\right)}}^{i}\right)_{U_{2}^{\prime}} \cong\left(R^{j} \pi_{*} \Omega_{A_{n}^{(m)} / A_{n}^{\left(m^{\prime}\right)}}^{i}\right)_{U_{1}^{\prime}} \otimes_{\mathcal{O}_{A_{n, U_{1}^{\prime}}^{\left(m^{\prime}\right)}}} \mathcal{O}_{A_{n, U_{2}^{\prime}}^{\left(m^{\prime}\right)}}
$$

the actions of $g$ and $1 \otimes g$ agree. Moreover if $U$ is a neat open compact subgroup of $G_{n}^{(m)}\left(\mathbb{A}^{\infty}\right)$ with image $U^{\prime}$ in $G_{n}^{\left(m^{\prime}\right)}\left(\mathbb{A}^{\infty}\right)$ then the natural map

$$
\pi_{A_{n}^{(m)} / A_{n}^{\left(m^{\prime}\right)}}^{*}\left(\pi_{*} \Omega_{A_{n}^{(m)} / A_{n}^{\left(m^{\prime}\right)}}^{1}\right)_{U^{\prime}} \longrightarrow \Omega_{A_{n, U}^{(m)} / A_{n, U^{\prime}}^{\left(m^{\prime}\right)}}^{1}
$$

is an isomorphism. These isomorphisms are equivariant for the actions of the groups $G_{n}^{(m)}\left(\mathbb{A}^{\infty}\right)$ and $Q_{m, m^{\prime}}(F)$.

The natural maps

$$
\wedge^{i}\left(\pi_{*} \Omega_{A_{n}^{(m)} / A_{n}^{\left(m^{\prime}\right)}}^{1}\right)_{U^{\prime}} \otimes \wedge^{j}\left(R^{1} \pi_{*} \mathcal{O}_{A_{n}^{(m)}}\right)_{U^{\prime}} \longrightarrow\left(R^{j} \pi_{*} \Omega_{A_{n}^{(m)} / A_{n}^{\left(m^{\prime}\right)}}^{i}\right)_{U^{\prime}}
$$

are $G_{n}^{\left(m^{\prime}\right)}\left(\mathbb{A}^{\infty}\right)$ and $Q_{m, m^{\prime}}(F)$ equivariant isomorphisms. 
Suppose that $U$ is a neat open compact subgroup of $G_{n}^{(m)}\left(\mathbb{A}^{\infty}\right)$ with image $U^{\prime}$ in $G_{n}^{\left(m^{\prime}\right)}\left(\mathbb{A}^{\infty}\right)$ and $U^{\prime \prime}$ in $G_{n}\left(\mathbb{A}^{\infty}\right)$. If $U$ is of the form $U^{\prime} \ltimes M$, then the quasi-isogeny $i_{A^{\text {univ }}}: A_{n, U}^{(m)} \rightarrow\left(A^{\text {univ }}\right)^{m-m^{\prime}}$ over $A_{n, U^{\prime}}^{\left(m^{\prime}\right)}$ gives rise to an isomorphism

$$
\operatorname{Hom}_{F}\left(F^{m-m^{\prime}}, \Omega_{n, U^{\prime \prime}}\right) \otimes \mathcal{O}_{A_{n, U}^{(m)}} \cong \Omega_{A_{n, U}^{(m)} / A_{n, U^{\prime}}^{\left(m^{\prime}\right)}}^{1}
$$

and a canonical embedding

$$
\Xi_{n, U^{\prime \prime}} \otimes \mathcal{O}_{A_{n, U^{\prime}}^{\left(m^{\prime}\right)}} \hookrightarrow \Xi_{n, U^{\prime \prime}}^{\oplus\left(m-m^{\prime}\right)} \otimes \mathcal{O}_{A_{n, U^{\prime}}^{\left(m^{\prime}\right)}} \hookrightarrow\left(R^{1} \pi_{*} \Omega_{A^{(m)} / A^{\left(m^{\prime}\right)}}^{1}\right)_{U^{\prime}},
$$

where the first map denotes the diagonal embedding. These maps do not depend on the choice of $A^{\text {univ }}$. They are $G_{n}^{(m)}\left(\mathbb{A}^{\infty}\right)$-equivariant. The first map is also $Q_{m, m^{\prime}}(F)$ equivariant, where an element $\gamma \in Q_{m, m^{\prime}}(F)$ acts on the left hand sides by composition with the inverse of the projection of $\gamma$ to $G L_{m-m^{\prime}}(F)$. This remains true if we do not assume that $U$ has the form $U^{\prime} \ltimes M$.

This gives rise to canonical $G_{n}^{\left(m^{\prime}\right)}\left(\mathbb{A}^{\infty}\right)$-equivariant isomorphisms

$$
\operatorname{Hom}_{F}\left(F^{m-m^{\prime}}, \Omega_{n, U^{\prime \prime}}\right) \otimes \mathcal{O}_{A_{n, U^{\prime}}^{\left(m^{\prime}\right)}} \cong\left(\pi_{*} \Omega_{A_{n}^{(m)} / A_{n}^{\left(m^{\prime}\right)}}^{1}\right)_{U^{\prime}}
$$

Moreover the composite maps

$$
\begin{aligned}
& \operatorname{Hom}\left(\left(\pi_{*} \Omega_{A_{n}^{(m)} / A_{n}^{\left(m^{\prime}\right)}}^{1}\right)_{U^{\prime}}, \Xi_{n, U^{\prime \prime}} \otimes \mathcal{O}_{A_{n, U^{\prime}}^{\left(m^{\prime}\right)}}\right) \\
& \hookrightarrow \operatorname{Hom}\left(\left(\pi_{*} \Omega_{A_{n}^{(m)} / A_{n}^{\left(m^{\prime}\right)}}^{1}\right)_{U^{\prime}},\left(R^{1} \pi_{*} \Omega_{A_{n}^{(m)} / A_{n}^{\left(m^{\prime}\right)}}^{1}\right)_{U^{\prime}}\right) \\
& \quad \stackrel{\operatorname{Hom}}{ }\left(\left(\pi_{*} \Omega_{A_{n}^{(m)} / A_{n}^{\left(m^{\prime}\right)}}^{1}\right)_{U^{\prime}},\left(\pi_{*} \Omega_{A_{n}^{(m)} / A_{n}^{\left(m^{\prime}\right)}}^{1}\right)_{U^{\prime}} \otimes\left(R^{1} \pi_{*} \mathcal{O}_{A_{n}^{(m)}}\right)_{U^{\prime}}\right) \\
& \stackrel{\operatorname{tr}}{\longrightarrow}\left(R^{1} \pi_{*} \mathcal{O}_{A_{n}^{(m)}}\right)_{U^{\prime}}
\end{aligned}
$$

are $G_{n}^{\left(m^{\prime}\right)}\left(\mathbb{A}^{\infty}\right)$-equivariant isomorphisms.

\subsubsection{Higher direct images from Kuga-Sato varieties to Shimura varieties, mixed} characteristic case

If $m \geq m^{\prime}$ and if $U^{p}$ is a neat open compact subgroup of $G_{n}^{(m)}\left(\mathbb{A}^{\infty, p}\right)$ with image $\left(U^{p}\right)^{\prime}$ in $G_{n}^{\left(m^{\prime}\right)}\left(\mathbb{A}^{\infty, p}\right)$, and if $0 \leq N_{1} \leq N_{2}$ are integers, then the sheaf

$$
R^{j} \pi_{\mathcal{A}_{n}^{(m), \text { ord }} / \mathcal{A}_{n}^{\left(m^{\prime}\right) \text {,ord }}, *} \Omega^{i}{ }_{\mathcal{A}_{n, U^{p}\left(N_{1}, N_{2}\right)}^{(m), \text { ord }}} / \mathcal{A}_{n,\left(U^{p}\right)^{\prime}\left(N_{1}, N_{2}\right)}^{\left(m^{\prime}\right) \text { ord }}
$$

depends only on $\left(U^{p}\right)^{\prime}$ and not on $U^{p}$. We will denote it

$$
\left(R^{j} \pi_{*} \Omega_{\mathcal{A}_{n}^{(m), \text { ord }} / \mathcal{A}_{n}^{\left(m^{\prime}\right), \text { ord }}}^{i}\right)_{\left(U^{p}\right)^{\prime}\left(N_{1}, N_{2}\right)}
$$

If $g \in G_{n}^{(m)}\left(\mathbb{A}^{\infty}\right)^{\text {ord }}$ and $g^{-1} U_{1}^{p}\left(N_{11}, N_{12}\right) g \subset U_{2}^{p}\left(N_{21}, N_{22}\right)$, then there is a natural map

$$
g:\left(g^{\prime}\right)^{*}\left(R^{j} \pi_{*} \Omega_{\mathcal{A}_{n}^{(m), \text { ord }} / \mathcal{A}_{n}^{\left(m^{\prime}\right) \text {,ord }}}^{i}\right)_{\left(U_{2}^{p}\right)^{\prime}\left(N_{21}, N_{22}\right)} \rightarrow\left(R^{j} \pi_{*} \Omega_{\mathcal{A}_{n}^{(m), \text { ord }} / \mathcal{A}_{n}^{\left(m^{\prime}\right), \text { ord }}}^{i}\right)_{\left(U_{1}^{p}\right)^{\prime}\left(N_{11}, N_{12}\right)},
$$


where $\left(U_{i}^{p}\right)^{\prime}$ denotes the image of $U_{i}^{p}$ in $G_{n}^{\left(m^{\prime}\right)}\left(\mathbb{A}^{\infty, p}\right)$ and $g^{\prime}$ denotes the image of $g$ in $G_{n}^{\left(m^{\prime}\right)}\left(\mathbb{A}^{\infty}\right)^{\text {ord }}$. If $g \in G_{n}^{(m)}\left(\mathbb{A}^{\infty}\right)^{\text {ord, } \times}$ then it is an isomorphism. Moreover this map only depends on $g^{\prime},\left(U_{1}^{p}\right)^{\prime}\left(N_{11}, N_{12}\right)$ and $\left(U_{2}^{p}\right)^{\prime}\left(N_{21}, N_{22}\right)$ and not on $g, U_{1}^{p}\left(N_{11}, N_{12}\right)$ and $U_{2}^{p}\left(N_{21}, N_{22}\right)$. This gives the system of sheaves

$$
\left\{\left(R^{j} \pi_{*} \Omega_{\mathcal{A}_{n}^{(m), \text { ord }} / \mathcal{A}_{n}^{\left(m^{\prime}\right), \text { ord }}}^{i}\right)_{\left(U^{p}\right)^{\prime}\left(N_{1}, N_{2}\right)}\right\}
$$

a left action of $G_{n}^{\left(m^{\prime}\right)}\left(\mathbb{A}^{\infty}\right)^{\text {ord }}$.

If $\gamma \in Q_{m, m^{\prime}}\left(\mathcal{O}_{F,(p)}\right)$ then $\gamma: A_{n, U^{p}\left(N_{1}, N_{2}\right)}^{(m)} \rightarrow A_{n, \gamma U^{p}\left(N_{1}, N_{2}\right)}^{(m)}$ gives a natural isomorphism

$$
\gamma:\left(R^{j} \pi_{*} \Omega_{\mathcal{A}_{n}^{(m), \text { ord }} / \mathcal{A}_{n}^{\left(m^{\prime}\right), \text { ord }}}^{i}\right)_{\left(U^{p}\right)^{\prime}\left(N_{1}, N_{2}\right)} \stackrel{\sim}{\longrightarrow}\left(R^{j} \pi_{*} \Omega_{\mathcal{A}_{n}^{(m), \text { ord }} / \mathcal{A}_{n}^{\left(m^{\prime}\right), \text { ord }}}^{i}\right)_{\left(U^{p}\right)^{\prime}\left(N_{1}, N_{2}\right)},
$$

which depends only on $\left(U^{p}\right)^{\prime}\left(N_{1}, N_{2}\right)$ and not on $U^{p}\left(N_{1}, N_{2}\right)$. This gives the system of sheaves

$$
\left\{\left(R^{j} \pi_{*} \Omega_{\mathcal{A}_{n}^{(m), \text { ord }} / \mathcal{A}_{n}^{\left(m^{\prime}\right), \text { ord }}}\right)_{\left(U^{p}\right)^{\prime}\left(N_{1}, N_{2}\right)}\right\}
$$

a right action of $Q_{m, m^{\prime}}\left(\mathcal{O}_{F,(p)}\right)$. We have $\gamma \circ g=\gamma(g) \circ \gamma$.

If $\left(U_{1}^{p}\right)^{\prime}\left(N_{11}, N_{12}\right) \supset\left(U_{2}^{p}\right)^{\prime}\left(N_{21}, N_{22}\right)$ and $g \in\left(U_{1}^{p}\right)^{\prime}\left(N_{11}, N_{12}\right)$ normalizes the subgroup $\left(U_{2}^{p}\right)^{\prime}\left(N_{21}, N_{22}\right)$, then on

$$
\begin{aligned}
& \left(R^{j} \pi_{*} \Omega_{\mathcal{A}_{n}^{(m), \text { ord }} / \mathcal{A}_{n}^{\left(m^{\prime}\right), \text { ord }}}^{i}\right)_{\left(U_{2}^{p}\right)^{\prime}\left(N_{21}, N_{22}\right)}
\end{aligned}
$$

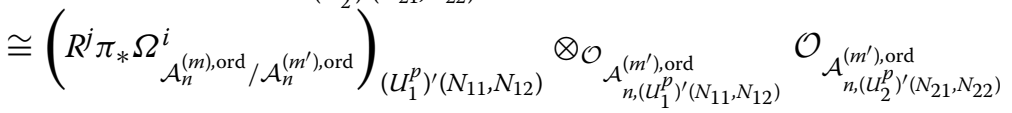

the actions of $g$ and $1 \otimes g$ agree. Moreover if $U^{p}$ is a neat open compact subgroup of $G_{n}^{(m)}\left(\mathbb{A}^{\infty, p}\right)$ with image $\left(U^{p}\right)^{\prime}$ in $G_{n}^{\left(m^{\prime}\right)}\left(\mathbb{A}^{\infty, p}\right)$, and if $0 \leq N_{1} \leq N_{2}$, then the natural map

$$
\pi_{\mathcal{A}_{n}^{(m), \text { ord }} / \mathcal{A}_{n}^{\left(m^{\prime}\right), \text { ord }}}^{*}\left(\pi_{*} \Omega_{\mathcal{A}_{n}^{(m), \text { ord }} / \mathcal{A}_{n}^{\left(m^{\prime}\right) \text {,ord }}}^{1}\right)_{\left(U^{p}\right)^{\prime}\left(N_{1}, N_{2}\right)} \longrightarrow \Omega_{\mathcal{A}_{n, U^{p}\left(N_{1}, N_{2}\right)}^{(m)} / \mathcal{A}_{n,\left(U^{p}\right)^{\prime}\left(N_{1}, N_{2}\right)}^{\left(m^{\prime},\right. \text { ord }}}^{1}
$$

is an isomorphism. These isomorphisms are $G_{n}^{(m)}\left(\mathbb{A}^{\infty}\right)^{\text {ord }}$ and $Q_{m, m^{\prime}}\left(\mathcal{O}_{F,(p)}\right)$ equivariant.

The natural maps

$$
\begin{aligned}
& \wedge^{i}\left(\pi_{*} \Omega_{\mathcal{A}_{n}^{(m), \text { ord }} / \mathcal{A}_{n}^{\left(m^{\prime}\right), \text { ord }}}\right)_{\left(U^{p}\right)^{\prime}\left(N_{1}, N_{2}\right)} \otimes \wedge^{j}\left(R^{1} \pi_{*} \mathcal{O}_{\mathcal{A}_{n}^{(m), \text { ord }}}\right)_{\left(U^{p}\right)^{\prime}\left(N_{1}, N_{2}\right)} \\
& \quad \longrightarrow\left(R^{j} \pi_{*} \Omega_{\mathcal{A}_{n}^{(m), \text { ord }} / \mathcal{A}_{n}^{\left(m^{\prime}\right) \text {,ord }}}^{i}\right)_{\left(U^{p}\right)^{\prime}\left(N_{1}, N_{2}\right)}
\end{aligned}
$$

are $G_{n}^{\left(m^{\prime}\right)}\left(\mathbb{A}^{\infty}\right)^{\text {ord }}$ and $Q_{m, m^{\prime}}\left(\mathcal{O}_{F,(p)}\right)$ equivariant isomorphisms.

Under the identification

$$
\mathcal{X}_{n,\left(U^{p}\right)^{\prime}\left(N_{1}, N_{2}\right)}^{\text {ord }} \times \operatorname{Spec} \mathbb{Q} \cong X_{n,\left(U^{p}\right)^{\prime}\left(N_{1}, N_{2}\right)}
$$


the sheaves $\Omega_{n,\left(U^{p}\right)^{\prime}\left(N_{1}, N_{2}\right)}^{\text {ord }}$ (resp. $\left.\Xi_{n,\left(U^{p}\right)^{\prime}\left(N_{1}, N_{2}\right)}^{\text {ord }}\right)$ are naturally identified with the sheaves $\Omega_{n,\left(U^{p}\right)^{\prime}\left(N_{1}, N_{2}\right)}$ (resp. $\left.\Xi_{n,\left(U^{p}\right)^{\prime}\left(N_{1}, N_{2}\right)}\right)$. Moreover, under the identification

$$
\mathcal{A}_{n, U^{p}\left(N_{1}, N_{2}\right)}^{\left(m^{\prime}\right) \text { ord }} \times \operatorname{Spec} \mathbb{Q} \cong A_{n, U^{p}\left(N_{1}, N_{2}\right)}^{\left(m^{\prime}\right)}
$$

the sheaf $\left(R^{j} \pi_{*} \Omega_{\mathcal{A}^{(m), \text { ord }} / \mathcal{A}_{n}^{\left(m^{\prime}\right) \text {,ord }}}^{i}\right)_{\left(U^{p}\right)^{\prime}\left(N_{1}, N_{2}\right)}$ is naturally identified with the sheaf $\left(R^{j} \pi_{*} \Omega_{A_{n}^{(m)} / A_{n}^{\left(m^{\prime}\right)}}^{i}\right)_{\left(U^{p}\right)^{\prime}\left(N_{1}, N_{2}\right)}$. These identifications are equivariant for the actions of $G_{n}\left(\mathbb{A}^{\infty}\right)^{\text {ord }}$ and $Q_{m, m^{\prime}}\left(\mathcal{O}_{F,(p)}\right)$.

\section{Generalized Shimura varieties}

We will introduce certain disjoint unions of mixed Shimura varieties, which are associated to $L_{n,(i) \text {,lin }}$ and $L_{n,(i)}$ and $P_{n,(i)}^{+} / Z\left(N_{n,(i)}\right)$ and $P_{n,(i)}^{+}$; to $L_{n,(i), \text { lin }}^{(m)}$ and $L_{n,(i)}^{(m)}$ and $P_{n,(i)}^{(m),+} / Z\left(N_{n,(i)}^{(m)}\right)$ and $P_{n,(i)}^{(m),+}$; and to $\widetilde{P}_{n,(i)}^{(m),+}$. The differences with the last section are purely book keeping. We then describe certain torus embeddings for these generalized Shimura varieties and discuss their completion along the boundary. These completions will serve as formal local models near the boundary of the toroidal compactifications of the $X_{n, U}$ and the $A_{n, \widetilde{U}}^{(m)}$ to be discussed in the next section.

We remind the reader of our convention that, if $U$ is a subgroup of $G$ and $H$ is a quotient of $G$, then we will sometimes use $U$ to denote its image in $H$. We hope that this causes no confusion as we will only do this when the context makes clear we are referring to a subgroup of $H$.

\subsection{Generalized Shimura varieties}

If $U$ is a neat open compact subgroup of $L_{n,(i), \text { lin }}^{(m)}\left(\mathbb{A}^{\infty}\right)$ we set

$$
Y_{n,(i), U}^{(m),+}=\coprod_{L_{n,(i), \text { lin }}^{(m)}\left(\mathbb{A}^{\infty}\right) / U} \operatorname{Spec} \mathbb{Q}
$$

In the case $m=0$ we will write simply $Y_{n,(i), U}^{+}$. Then $\left\{Y_{n,(i), U}^{(m),+}\right\}$ is a system of schemes (locally of finite type over Spec $\mathbb{Q}$ ) with right $L_{n,(i), \text { lin }}^{(m)}\left(\mathbb{A}^{\infty}\right)$-action. Each $Y_{n,(i), U}^{(m),+}$ also has a left action of $L_{n,(i), \text { lin }}^{(m)}(\mathbb{Q})$, and the inverse system has a right action of $L_{n,(i), \text { lin }}^{(m)}\left(\mathbb{A}^{\infty}\right)$. If $\delta \in G L_{m}(F)$ we get a map

$$
\delta: Y_{n,(i), U}^{(m)} \longrightarrow Y_{n,(i), \delta(U)}^{(m)}
$$

which sends $(\operatorname{Spec} \mathbb{Q})_{h U} \rightarrow(\operatorname{Spec} \mathbb{Q})_{\delta(h) \delta(U)}$ via the identity. This gives a left action of $G L_{m}(F)$ on the inverse system of the $Y_{n,(i), U}^{(m)}$. If $\delta \in G L_{m}(F)$ and $\gamma \in L_{n,(i), \text { lin }}^{(m)}(\mathbb{Q})$ and $g \in L_{n,(i), \text { lin }}^{(m)}\left(\mathbb{A}^{\infty}\right)$ then $\delta \circ \gamma=\delta(\gamma) \circ \delta$ and $\delta \circ g=\delta(g) \circ \delta$. If $U^{\prime}$ denotes the image of $U$ in $L_{n,(i), \text { lin }}\left(\mathbb{A}^{\infty}\right)$ then there is a natural map

$$
Y_{n,(i), U}^{(m),+} \rightarrow Y_{n,(i), U^{\prime}}^{+}
$$

These maps are equivariant for

$$
L_{n,(i), \operatorname{lin}}^{(m)}(\mathbb{Q}) \times L_{n,(i), \operatorname{lin}}^{(m)}\left(\mathbb{A}^{\infty}\right) \longrightarrow L_{n,(i), \operatorname{lin}}(\mathbb{Q}) \times L_{n,(i), \operatorname{lin}}\left(\mathbb{A}^{\infty}\right) .
$$


The naive quotient

$$
L_{n,(i), \operatorname{lin}}^{(m)}(\mathbb{Q}) \backslash Y_{n,(i), U}^{(m),+}
$$

makes sense. We will denote this space

$$
Y_{n,(i), U}^{(m), \downarrow}
$$

and drop the $(m)$ if $m=0$. The inverse system of these spaces has a right action of $L_{n,(i), \text { lin }}^{(m)}\left(\mathbb{A}^{\infty}\right)$, and a left action of $G L_{m}(F)$. The induced map

$$
Y_{n,(i), U}^{(m), 4} \longrightarrow Y_{n,(i), U}^{\natural}
$$

is an isomorphism, and $G L_{m}(F)$ acts trivially on these spaces. (Use the fact that

$$
\left.\left(U \cap\left(\operatorname{Hom}_{F}\left(F^{m}, F^{i}\right) \otimes_{\mathbb{Q}} \mathbb{A}^{\infty}\right)\right)+\operatorname{Hom}_{F}\left(F^{m}, F^{i}\right)=\operatorname{Hom}_{F}\left(F^{m}, F^{i}\right) \otimes_{\mathbb{Q}} \mathbb{A}^{\infty} .\right)
$$

Similarly if $U^{p}$ is a neat open compact subgroup of $L_{n,(i), \operatorname{lin}}^{(m)}\left(\mathbb{A}^{\infty, p}\right)$ and if $N \in \mathbb{Z}_{\geq 0}$ we set

$$
\mathcal{Y}_{n,(i), U^{p}(N)}^{(m), \text { ord },+}=\coprod_{L_{n,(i), \text { lin }}^{(m)}\left(\mathbb{A}^{\infty}\right)^{\text {ord }, \times} / U^{p}(N)} \operatorname{Spec} \mathbb{Z}_{(p)}
$$

In the case $m=0$ we drop it from the notation. Each $\mathcal{Y}_{n,(i), U^{p}(N)}^{(m), \text { ord }+}$ has a left action of $L_{n,(i), \text { lin }}^{(m)}\left(\mathbb{Z}_{(p)}\right)$ and the inverse system of the $\mathcal{Y}_{n,(i), U^{p}(N)}^{(m), \text { ord }+}$ has a commuting right action of $L_{n,(i) \text { lin }}^{(m)}\left(\mathbb{A}^{\infty}\right)^{\text {ord }}$. It also has a left action of $G L_{m}\left(\mathcal{O}_{F,(p)}\right)$. If $\delta \in G L_{m}\left(\mathcal{O}_{F,(p)}\right)$ and $\gamma \in L_{n,(i), \text { lin }}^{(m)}\left(\mathbb{Z}_{(p)}\right)$ and $g \in L_{n,(i), \text { lin }}^{(m)}\left(\mathbb{A}^{\infty}\right)^{\text {ord }}$ then $\delta \circ \gamma=\delta(\gamma) \circ \delta$ and $\delta \circ g=\delta(g) \circ \delta$. There are equivariant maps

$$
\mathcal{Y}_{n,(i), U^{p}(N)}^{(m), \text { ord }+} \longrightarrow \mathcal{Y}_{n,(i), U^{p}(N)}^{\text {ord, }}
$$

We set

$$
\mathcal{Y}_{n,(i), U^{p}(N)}^{\mathrm{ord}, \curvearrowleft}=L_{n,(i), \operatorname{lin}}\left(\mathbb{Z}_{(p)}\right) \backslash \mathcal{Y}_{n,(i), U^{p}(N)}^{\mathrm{ord},+}=L_{n,(i), \operatorname{lin}}^{(m)}\left(\mathbb{Z}_{(p)}\right) \backslash \mathcal{Y}_{n,(i), U^{p}(N)}^{(m), \text { ord },+}
$$

There are maps

$$
\mathcal{Y}_{n,(i), U^{p}(N)}^{(m), \text { ord }+} \times \operatorname{Spec} \mathbb{Q} \hookrightarrow Y_{n,(i), U^{p}(N)}^{(m),+}
$$

which are equivariant for the actions of the groups $L_{n,(i), \text { lin }}^{(m)}\left(\mathbb{Z}_{(p)}\right)$ and $L_{n,(i), \text { lin }}^{(m)}\left(\mathbb{A}^{\infty}\right)^{\text {ord }}$ and $G L_{m}\left(\mathcal{O}_{F,(p)}\right)$. Moreover the maps $\mathcal{Y}_{n,(i), U^{p}(N)}^{(m), \text { ord },+} \rightarrow \mathcal{Y}_{n,(i), U^{p}(N)}^{\text {ord, }}$ and $Y_{n,(i), U^{p}(N)}^{(m),+} \rightarrow Y_{n,(i), U^{p}(N)}^{+}$ are compatible. The induced maps

$$
\mathcal{Y}_{n,(i), U^{p}(N)}^{(m), \text { ord },} \times \operatorname{Spec} \mathbb{Q} \stackrel{\sim}{\longrightarrow} Y_{n,(i), U^{p}(N)}^{(m), \natural}
$$

are isomorphisms. 
Suppose now that $U$ is a neat open compact subgroup of $L_{n,(i)}^{(m)}\left(\mathbb{A}^{\infty}\right)$. We set

$$
X_{n,(i), U}^{(m),+}=\left(X_{n-i, U \cap G_{n-i}\left(\mathbb{A}^{\infty}\right)} \times Y_{n,(i), U \cap L_{n,(i), \operatorname{lin}}^{(m)}\left(\mathbb{A}^{\infty}\right)}^{(m),+}\right) / U
$$

In the case $m=0$ we will write simply $X_{n,(i), U}^{+}$. Then $\left\{X_{n,(i), U}^{(m),+}\right\}$ is a system of schemes (locally of finite type over Spec $\mathbb{Q}$ ) with right $L_{n,(i)}^{(m)}\left(\mathbb{A}^{\infty}\right)$-action via finite etale maps. Each $X_{n,(i), U}^{(m),+}$ has a left action of $L_{n,(i), \text { lin }}^{(m)}(\mathbb{Q})$, which commutes with the right $L_{n,(i)}^{(m)}\left(\mathbb{A}^{\infty}\right)$-action. The system also has a left action of $G L_{m}(F)$. If $\delta \in G L_{m}(F)$ and $\gamma \in L_{n,(i), \text { lin }}^{(m)}(\mathbb{Q})$ and $g \in L_{n,(i)}^{(m)}\left(\mathbb{A}^{\infty}\right)$ then $\delta \circ \gamma=\delta(\gamma) \circ \delta$ and $\delta \circ g=\delta(g) \circ \delta$. If $U^{\prime}$ is an open normal subgroup of $U$ then $X_{n,(i), U}^{(m),+}$ is identified with $X_{n,(i), U^{\prime}}^{(m),+} / U$. Projection to the second factor gives $L_{n,(i), \text { lin }}^{(m)}(\mathbb{Q}) \times L_{n,(i)}^{(m)}\left(\mathbb{A}^{\infty}\right)$ and $G L_{m}(F)$ equivariant maps

$$
X_{n,(i), U}^{(m),+} \longrightarrow Y_{n,(i), U}^{(m),+}
$$

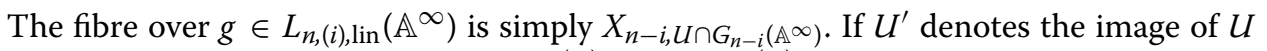
in $L_{n,(i)}\left(\mathbb{A}^{\infty}\right)$ then there is a natural, $L_{n,(i), \operatorname{lin}}^{(m)}(\mathbb{Q}) \times L_{n,(i)}^{(m)}\left(\mathbb{A}^{\infty}\right)$-equivariant, commutative diagram

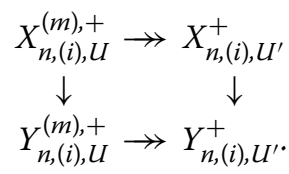

We have

$$
X_{n,(i), U}^{(m),+}(\mathbb{C})=L_{n,(i), \operatorname{herm}}(\mathbb{Q}) \backslash\left(L_{n,(i)}^{(m)}\left(\mathbb{A}^{\infty}\right) / U \times \mathfrak{H}_{n-i}^{ \pm}\right)
$$

and

$$
\pi_{0}\left(X_{n,(i), U}^{(m),+} \times \operatorname{Spec} \overline{\mathbb{Q}}\right) \cong\left(L_{n,(i), \operatorname{lin}}^{(m)}\left(\mathbb{A}^{\infty}\right) \times\left(C_{n-i}(\mathbb{Q}) \backslash C_{n-i}(\mathbb{A}) / C_{n-i}(\mathbb{R})^{0}\right)\right) / U
$$

The naive quotient

$$
X_{n,(i), U}^{(m), \natural}=L_{n,(i), \operatorname{lin}}^{(m)}(\mathbb{Q}) \backslash X_{n,(i), U}^{(m),+}
$$

makes sense and fibres over $Y_{n,(i), U}^{(m), \text { }}$, the fibre over $g$ being $X_{n-i, U_{1}}$, where $U_{1}$ denotes the projection to $G_{n-i}\left(\mathbb{A}^{\infty}\right)$ of the subgroup $U_{2} \subset U$ consisting of elements whose projection to $L_{n,(i), \text { lin }}^{(m)}\left(\mathbb{A}^{\infty}\right)$ lies in $g^{-1} L_{n,(i), \text { lin }}^{(m)}(\mathbb{Q}) g$. We sometimes write $X_{n,(i), U}^{\natural}$ for $X_{n,(i), U}^{(0), \natural}$. If $U^{\prime}$ denotes the projection of $U$ to $L_{n,(i)}\left(\mathbb{A}^{\infty}\right)$, then the induced map

$$
X_{n,(i), U}^{(m), \downarrow} \stackrel{\sim}{\longrightarrow} X_{n,(i), U^{\prime}}^{\natural}
$$

is an isomorphism. The action of $L_{n,(i)}^{(m)}\left(\mathbb{A}^{\infty}\right)$ is by finite etale maps and if $U^{\prime}$ is an open normal subgroup of $U$ then $X_{n,(i), U}^{(m), \natural}$ is identified with $X_{n,(i), U^{\prime}}^{(m), \natural} / U$. We have

$$
\pi_{0}\left(X_{n,(i), U}^{(m), 4} \times \operatorname{Spec} \overline{\mathbb{Q}}\right) \cong\left(F^{\times} \times C_{n-i}(\mathbb{Q})\right) \backslash\left(\mathbb{A}_{F}^{\times} \times C_{n-i}(\mathbb{A})\right) / U\left(F_{\infty}^{\times} \times C_{n-i}(\mathbb{R})^{0}\right) .
$$


We define sheaves $\Omega_{n,(i), U}^{+}$and $\Xi_{n,(i), U}^{+}$over $X_{n,(i), U}^{+}$as the quotients of

$$
\Omega_{n-i, U \cap G_{n-i}\left(\mathbb{A}^{\infty}\right)} / X_{n-i, U \cap G_{n-i}\left(\mathbb{A}^{\infty}\right)} \times Y_{n,(i), U \cap L_{n,(i), \operatorname{lin}}\left(\mathbb{A}^{\infty}\right)}
$$

and

$$
\Xi_{n-i, U \cap G_{n-i}\left(\mathbb{A}^{\infty}\right)} / X_{n-i, U \cap G_{n-i}\left(\mathbb{A}^{\infty}\right)} \times Y_{n,(i), U \cap L_{n,(i), \operatorname{lin}}\left(\mathbb{A}^{\infty}\right)}^{+}
$$

by $U$. Then $\left\{\Omega_{n,(i), U}^{+}\right\}$and $\left\{\Xi_{n,(i), U}^{+}\right\}$are systems of locally free sheaves on $X_{n,(i), U}^{+}$with left $L_{n,(i)}\left(\mathbb{A}^{\infty}\right)$-action and commuting right $L_{n,(i), \text { lin }}(\mathbb{Q})$-action.

Let $\mathcal{E}_{(i), U}^{+}$denote the principal $R_{n,(n),(i)} / N\left(R_{n,(n),(i)}\right)$-bundle on $X_{n,(i), U}^{+}$in the Zariski topology defined by setting, for $W \subset X_{n,(i), U}^{+}$a Zariski open, $\mathcal{E}_{(i), U}^{+}(W)$ to be the set of triples $\left(\xi_{0}, \xi_{11}, \xi_{12}\right)$, where

$$
\xi_{0}:\left.\Xi_{n,(i), U}^{+}\right|_{W} \stackrel{\sim}{\longrightarrow} \mathcal{O}_{W}
$$

and

$$
\xi_{11}: \Omega_{n,(i), U}^{+} \stackrel{\sim}{\longrightarrow} \operatorname{Hom}_{\mathbb{Q}}\left(V_{n-i} / V_{n-i,(n-i)}, \mathcal{O}_{W}\right)
$$

and

$$
\xi_{12}: F^{i} \otimes_{\mathbb{Q}} \mathcal{O}_{W} \stackrel{\sim}{\longrightarrow} \operatorname{Hom}\left(V_{n} / V_{n,(i)}^{\perp}, \mathcal{O}_{W}\right) .
$$

We define the $R_{n,(n),(i)} / N\left(R_{n,(n),(i)}\right)$-action on $\mathcal{E}_{(i), U}^{+}$by

$$
h\left(\xi_{0}, \xi_{11}, \xi_{12}\right)=\left(v(h)^{-1} \xi_{0},\left(\circ h^{-1}\right) \circ \xi_{11},\left(\circ h^{-1}\right) \circ \xi_{12}\right) .
$$

The inverse system $\left\{\mathcal{E}_{(i), U}^{+}\right\}$has an action of $L_{n,(i)}\left(\mathbb{A}^{\infty}\right)$ and of $L_{n,(i), \text { lin }}(\mathbb{Q})$.

Suppose that $R_{0}$ is a $\mathbb{Q}$-algebra and that $\rho$ is a representation of the algebraic group $R_{n,(n),(i)} / N\left(R_{n,(n),(i)}\right)$ on a finite, locally free $R_{0}$-module $W_{\rho}$. We define a locally free sheaf $\mathcal{E}_{(i), U, \rho}^{+}$over $X_{n,(i), U}^{+} \times \operatorname{Spec} R_{0}$ by setting $\mathcal{E}_{(i), U, \rho}^{+}(W)$ to be the set of $\left(R_{n,(n),(i)} / N\left(R_{n,(n),(i)}\right)\right)\left(\mathcal{O}_{W}\right)$-equivariant maps of Zariski sheaves of sets

$$
\mathcal{E}_{(i), U}^{+} \mid W \longrightarrow W_{\rho} \otimes_{R_{0}} \mathcal{O}_{W}
$$

Then $\left\{\mathcal{E}_{(i), U, \rho}^{+}\right\}$is a system of locally free sheaves with an $L_{n,(i)}\left(\mathbb{A}^{\infty}\right)$-action and an $L_{n,(i) \text {,lin }}(\mathbb{Q})$-action over the system of schemes $\left\{X_{n,(i), U}^{+} \times \operatorname{Spec} R_{0}\right\}$. The restriction of $\mathcal{E}_{(i), U, \rho}^{+}$to $X_{n-i, h U h^{-1} \cap G_{n-i}\left(\mathbb{A}^{\infty}\right)}$ can be identified with $\mathcal{E}_{h U h^{-1} \cap G_{n-i}\left(\mathbb{A}^{\infty}\right),\left.\rho\right|_{L_{n-i,(n-i)}}}$. However the description of the actions of $L_{n,(i)}\left(\mathbb{A}^{\infty}\right)$ and $L_{n,(i), \operatorname{lin}}(\mathbb{Q})$ involve $\rho$ and not just $\left.\rho\right|_{L_{n-i,(n-i)}}$. If $g \in L_{n,(i)}\left(\mathbb{A}^{\infty}\right)$ and $\gamma \in L_{n,(i), \text { lin }}(\mathbb{Q})$, then the natural maps

$$
g^{*} \mathcal{E}_{(i), U, \rho}^{+} \longrightarrow \mathcal{E}_{(i), U^{\prime}, \rho}^{+}
$$

and

$$
\gamma^{*} \mathcal{E}_{(i), U, \rho}^{+} \longrightarrow \mathcal{E}_{(i), U^{\prime}, \rho}^{+}
$$

are isomorphisms. 
We will also write

$$
\Omega_{n,(i), U}^{\natural}=L_{n,(i), \operatorname{lin}}(\mathbb{Q}) \backslash \Omega_{n,(i), U}^{+}
$$

and

$$
\Xi_{n,(i), U}^{\natural}=L_{n,(i), \operatorname{lin}}(\mathbb{Q}) \backslash \Xi_{n,(i), U}^{+}
$$

locally free sheaves on $X_{n,(i), U}^{\natural}$. (If $\rho$ is trivial on $L_{n,(i), \text { lin }}$ then one can also form the quotient of $\mathcal{E}_{(i), U, \rho}^{+}$by $L_{n,(i), \text { lin }}(\mathbb{Q})$, but in general this quotient does not make sense.)

If $U^{p}$ is a neat open compact subgroup of $L_{n,(i)}^{(m)}\left(\mathbb{A}^{\infty, p}\right)$ and $N_{2} \geq N_{1} \geq 0$ we set

$$
\mathcal{X}_{n,(i), U^{p}\left(N_{1}, N_{2}\right)}^{(m), \text { ord, }}=\left(\mathcal{X}_{n-i,\left(U^{p} \cap G_{n-i}\left(\mathbb{A}^{\infty, p}\right)\right)\left(N_{1}, N_{2}\right)}^{\text {ord }} \times \mathcal{Y}_{n,(i),\left(U^{p} \cap L_{n,(i), \operatorname{lin}}^{(m)}\left(\mathbb{A}^{\infty, p}\right)\right)\left(N_{1}\right)}^{(m), d}\right) / U^{p} .
$$

In the case $m=0$ we drop it from the notation. Each $\mathcal{X}_{n,(i), U^{p}\left(N_{1}, N_{2}\right)}^{(m), \text { ord, }}$ has a left action of $L_{n,(i) \text {,lin }}^{(m)}\left(\mathbb{Z}_{(p)}\right)$ and the inverse system has a commuting right action of $L_{n,(i)}^{(m)}\left(\mathbb{A}^{\infty}\right)^{\text {ord }}$. There is also a left action of $G L_{m}\left(\mathcal{O}_{F,(p)}\right)$. If $\delta \in G L_{m}\left(\mathcal{O}_{F,(p)}\right)$ and $\gamma \in L_{n,(i), \text { lin }}^{(m)}\left(\mathbb{Z}_{(p)}\right)$ and $g \in L_{n,(i) \text { lin }}^{(m)}\left(\mathbb{A}^{\infty}\right)^{\text {ord }}$ then $\delta \circ \gamma=\delta(\gamma) \circ \delta$ and $\delta \circ g=\delta(g) \circ \delta$. If $g \in L_{n,(i)}^{(m)}\left(\mathbb{A}^{\infty}\right)^{\text {ord }}$ and if

$$
g: \mathcal{X}_{n,(i), U^{p}\left(N_{1}, N_{2}\right)}^{(m), \text { ord }+} \longrightarrow \mathcal{X}_{n,(i),\left(U^{p}\right)^{\prime}\left(N_{1}^{\prime}, N_{2}^{\prime}\right)^{\prime}}^{(m), \text { ord },}
$$

then this map is quasi-finite and flat. If $g \in L_{n,(i)}^{(m)}\left(\mathbb{A}^{\infty}\right)^{\text {ord, } \times}$ then it is etale, and, if further $N_{2}=N_{2}^{\prime}$, then it is finite etale. If $N_{2}^{\prime}>0$ and $p^{N_{2}-N_{2}^{\prime}} v\left(g_{p}\right) \in \mathbb{Z}_{p}^{\times}$then the map is finite. On $\mathbb{F}_{p}$-fibres the map $\varsigma_{p}$ is absolute Frobenius composed with the forgetful map. If $\left(U^{p}\right)^{\prime}$ is an open normal subgroup of $U^{p}$ and if $N_{1} \leq N_{1}^{\prime} \leq N_{2}$ then

$$
\mathcal{X}_{n,(i),\left(U^{p}\right)^{\prime}\left(N_{1}^{\prime}, N_{2}\right)}^{(m), \text { ord }+} / U^{p}\left(N_{1}, N_{2}\right) \stackrel{\sim}{\longrightarrow} \mathcal{X}_{n,(i), U^{p}\left(N_{1}, N_{2}\right)}^{(m), o r d,}
$$

There are commutative diagrams

$$
\begin{array}{cc}
\mathcal{X}_{n,(i), U^{p}\left(N_{1}, N_{2}\right)}^{(m), \text { ord, }} & \rightarrow \mathcal{X}_{n,(i), U^{p}\left(N_{1}, N_{2}\right)}^{\text {ord },} \\
\downarrow & \downarrow \downarrow \\
\mathcal{Y}_{n,(i), U^{p}\left(N_{1}, N_{2}\right)}^{(m), \text { ord, }} & \rightarrow \mathcal{Y}_{n,(i), U^{p}\left(N_{1}, N_{2}\right)}^{\text {ord, }}
\end{array}
$$

We set

$$
\mathcal{X}_{n,(i), U^{p}\left(N_{1}, N_{2}\right)}^{(m), \text { ord }}=L_{n,(i), \text { lin }}^{(m)}\left(\mathbb{Z}_{(p)}\right) \backslash \mathcal{X}_{n,(i), U^{p}\left(N_{1}, N_{2}\right)}^{(m), o r d,}
$$

and write $\mathcal{X}_{n,(i), U^{p}\left(N_{1}, N_{2}\right)}^{\text {ord, }}$ for $\mathcal{X}_{n,(i), U^{p}\left(N_{1}, N_{2}\right)}^{(0), \text { ord },}$. The system of these spaces has a right action of $L_{n,(i)}^{(m)}\left(\mathbb{A}^{\infty}\right)^{\text {ord }}$ and a left action of $G L_{m}\left(\mathcal{O}_{F,(p)}\right)$. If $\delta \in G L_{m}\left(\mathcal{O}_{F,(p)}\right)$ and $g \in L_{n,(i) \text { lin }}^{(m)}\left(\mathbb{A}^{\infty}\right)^{\text {ord }}$ then $\delta \circ g=\delta(g) \circ \delta$. If $g \in L_{n,(i)}^{(m)}\left(\mathbb{A}^{\infty}\right)^{\text {ord }}$ and if

$$
g: \mathcal{X}_{n,(i), U^{p}\left(N_{1}, N_{2}\right)}^{(m), \text { ord }, \sharp} \longrightarrow \mathcal{X}_{n,(i),\left(U^{p}\right)^{\prime}\left(N_{1}^{\prime}, N_{2}^{\prime}\right)^{\prime}}^{(m), \text { ord },}
$$

then this map is quasi-finite and flat. If $g \in L_{n,(i)}^{(m)}\left(\mathbb{A}^{\infty}\right)^{\text {ord, } \times}$ then it is etale, and, if further $N_{2}=N_{2}^{\prime}$, then it is finite etale. If $N_{2}^{\prime}>0$ and $p^{N_{2}-N_{2}^{\prime}} v\left(g_{p}\right) \in \mathbb{Z}_{p}^{\times}$then the map is finite. 
On $\mathbb{F}_{p}$-fibres the map $\varsigma_{p}$ is absolute Frobenius composed with the forgetful map. If $\left(U^{p}\right)^{\prime}$ is an open normal subgroup of $U^{p}$ and if $N_{1} \leq N_{1}^{\prime} \leq N_{2}$ then

$$
\mathcal{X}_{n,(i),\left(U^{p}\right)^{\prime}\left(N_{1}^{\prime}, N_{2}\right)}^{(m), \text { ord }} / U^{p}\left(N_{1}, N_{2}\right) \stackrel{\sim}{\longrightarrow} \mathcal{X}_{n,(i), U^{p}\left(N_{1}, N_{2}\right)}^{(m), \text { ord } \boxminus}
$$

The natural maps

$$
\mathcal{X}_{n,(i), U^{p}\left(N_{1}, N_{2}\right)}^{(m), \text { ord } \boxminus} \longrightarrow \mathcal{X}_{n,(i), U^{p}\left(N_{1}, N_{2}\right)}^{\text {ord }, \natural}
$$

are isomorphisms.

We define sheaves $\Omega_{n,(i), U^{p}\left(N_{1}, N_{2}\right)}^{\text {ord, }}$ and $\Xi_{n,(i), U^{p}\left(N_{1}, N_{2}\right)}^{\text {ord, }}$ over $\mathcal{X}_{n,(i), U^{p}\left(N_{1}, N_{2}\right)}^{\text {ord }+}$ as the quotients of

$$
\Omega_{n-i,\left(U^{p} \cap G_{n-i}\left(\mathbb{A}^{\infty, p}\right)\right)\left(N_{1}, N_{2}\right)}^{\text {ord }} / \mathcal{X}_{n-i,\left(U^{p} \cap G_{n-i}\left(\mathbb{A}^{\infty, p}\right)\right)\left(N_{1}, N_{2}\right)}^{\text {ord }} \times \mathcal{Y}_{n,(i),\left(U^{p} \cap L_{n,(i), \operatorname{lin}}\left(\mathbb{A}^{\infty, p}\right)\right)\left(N_{1}\right)}^{\text {ord }++}
$$

and

$$
\Xi_{n-i,\left(U^{p} \cap G_{n-i}\left(\mathbb{A}^{\infty, p}\right)\right)\left(N_{1}, N_{2}\right)}^{\text {ord }} / \mathcal{X}_{n-i,\left(U^{p} \cap G_{n-i}\left(\mathbb{A}^{\infty, p}\right)\right)\left(N_{1}, N_{2}\right)}^{\text {ord }} \times \mathcal{Y}_{n,(i),\left(U^{p} \cap L_{n,(i), \operatorname{lin}}\left(\mathbb{A}^{\infty, p}\right)\right)\left(N_{1}\right)}^{\mathrm{ord},+}
$$

by $U^{p}$. Then the systems of sheaves $\Omega_{n,(i), U^{p}\left(N_{1}, N_{2}\right)}^{\text {ord, }}$ and $\Xi_{n,(i), U^{p}\left(N_{1}, N_{2}\right)}^{\text {ord, }+}$ have commuting actions of $L_{n,(i) \text {,in }}\left(\mathbb{Z}_{(p)}\right)$ and $L_{n,(i)}\left(\mathbb{A}^{\infty}\right)^{\text {ord }}$.

Let $\mathcal{E}_{(i), U^{p}\left(N_{1}, N_{2}\right)}^{\text {ord, }}$ denote the principal $R_{n,(n),(i)} / N\left(R_{n,(n),(i)}\right)$-bundle for the Zariski topology on $\mathcal{X}_{n,(i), U^{p}\left(N_{1}, N_{2}\right)}^{\text {ord, }}$ defined by setting, for $W \subset \mathcal{X}_{n,(i), U^{p}\left(N_{1}, N_{2}\right)}^{\text {ord, }}$ a Zariski open, $\mathcal{E}_{(i), U^{p}\left(N_{1}, N_{2}\right)}^{\text {ord, }}(W)$ to be the set of triples $\left(\xi_{0}, \xi_{11}, \xi_{12}\right)$, where

$$
\xi_{0}: \Xi_{n,(i), U^{p}\left(N_{1}, N_{2}\right)}^{\text {ord, }} \mid W \stackrel{\sim}{\longrightarrow} \mathcal{O}_{W}
$$

and

$$
\xi_{11}: \Omega_{n,(i), U^{p}\left(N_{1}, N_{2}\right)}^{\mathrm{ord},+} \stackrel{\sim}{\longrightarrow} \operatorname{Hom}\left(\Lambda_{n-i} / \Lambda_{n-i,(n-i)}, \mathcal{O}_{W}\right)
$$

and

$$
\xi_{12}: \mathcal{O}_{F}^{i} \otimes_{\mathbb{Z}} \mathcal{O}_{W} \stackrel{\sim}{\longrightarrow} \operatorname{Hom}\left(\Lambda_{n} / \Lambda_{n,(i)}^{\perp}, \mathcal{O}_{W}\right)
$$

We define the $R_{n,(n),(i)} / N\left(R_{n,(n),(i)}\right)$-action on $\mathcal{E}_{(i), U^{p}\left(N_{1}, N_{2}\right)}^{\text {ord }+}$ by

$$
h\left(\xi_{0}, \xi_{11}, \xi_{12}\right)=\left(v(h)^{-1} \xi_{0},\left(\circ h^{-1}\right) \circ \xi_{11},\left(\circ h^{-1}\right) \circ \xi_{12}\right) .
$$

The inverse system $\left\{\mathcal{E}_{(i), U^{p}\left(N_{1}, N_{2}\right)}^{+}\right\}$has an action of $L_{n,(i)}\left(\mathbb{A}^{\infty}\right)^{\text {ord, } \times}$ and an action of $L_{n,(i), \operatorname{lin}}\left(\mathbb{Z}_{(p)}\right)$.

Suppose that $R_{0}$ is a $\mathbb{Z}_{(p)}$-algebra and that $\rho$ is a representation of the algebraic group $R_{n,(n),(i)} / N\left(R_{n,(n),(i)}\right)$ on a finite, locally free $R_{0}$-module $W_{\rho}$. We define a locally free sheaf $\mathcal{E}_{(i), U^{p}\left(N_{1}, N_{2}\right), \rho}^{\text {ord, }}$ over $\mathcal{X}_{n,(i), U^{p}\left(N_{1}, N_{2}\right)}^{\text {ord, }+} \times \operatorname{Spec} R_{0}$ by setting $\mathcal{E}_{(i), U^{p}\left(N_{1}, N_{2}\right), \rho}^{\text {ord, }}(W)$ to be the set of $\left(R_{n,(n),(i)} / N\left(R_{n,(n),(i)}\right)\right)\left(\mathcal{O}_{W}\right)$-equivariant maps of Zariski sheaves of sets

$$
\left.\mathcal{E}_{(i), U^{p}\left(N_{1}, N_{2}\right)}^{\mathrm{ord},}\right|_{W} \longrightarrow W_{\rho} \otimes_{R_{0}} \mathcal{O}_{W}
$$


Then $\left\{\mathcal{E}_{(i), U^{p}\left(N_{1}, N_{2}\right), \rho}^{\text {ord },}\right\}$ is a system of locally free sheaves with $L_{n,(i)}\left(\mathbb{A}^{\infty}\right)^{\text {ord, } \times}$-action and $L_{n,(i), \text { lin }}\left(\mathbb{Z}_{(p)}\right)$-action over the system of schemes $\left\{\mathcal{X}_{n,(i), U^{p}\left(N_{1}, N_{2}\right)}^{\text {ord }+} \times \operatorname{Spec} R_{0}\right\}$. The restriction of $\mathcal{E}_{(i), U^{p}\left(N_{1}, N_{2}\right), \rho}^{\text {ord, }}$ to $\mathcal{X}_{n-i,\left(h U^{p} h^{-1} \cap G_{n-i}\left(\mathbb{A}^{\infty, p}\right)\left(N_{1}, N_{2}\right)\right)}^{\text {ord }}$ can be identified with $\mathcal{E}_{\left(h U^{p} h^{-1} \cap G_{n-i}\left(\mathbb{A}^{\infty, p}\right)\right)\left(N_{1}, N_{2}\right),\left.\rho\right|_{L_{n-i}(n-i)}}^{\text {ord }}$. However the description of the actions of the groups $L_{n,(i)}\left(\mathbb{A}^{\infty}\right)^{\text {ord }, \times}$ and $L_{n,(i), \text { lin }}\left(\mathbb{Z}_{(p)}\right)$ involves $\rho$ and not just $\left.\rho\right|_{L_{n-i,(n-i)}}$. If $g \in L_{n,(i)}\left(\mathbb{A}^{\infty}\right)^{\text {ord, } \times}$ and $\gamma \in L_{n,(i), \text { lin }}\left(\mathbb{Z}_{(p)}\right)$, then the natural maps

$$
g^{*} \mathcal{E}_{(i), U^{p}\left(N_{1}, N_{2}\right), \rho}^{\mathrm{ord},+} \longrightarrow \mathcal{E}_{(i),\left(U^{p}\right)^{\prime}\left(N_{1}^{\prime}, N_{2}^{\prime}\right), \rho}^{\mathrm{ord},+}
$$

and

$$
\gamma^{*} \mathcal{E}_{(i), U^{p}\left(N_{1}, N_{2}\right), \rho}^{\mathrm{ord},+} \longrightarrow \mathcal{E}_{(i),\left(U^{p}\right)^{\prime}\left(N_{1}^{\prime}, N_{2}^{\prime}\right), \rho}^{\mathrm{ord},+}
$$

are isomorphisms.

We will also write

$$
\Omega_{n,(i), U^{p}\left(N_{1}, N_{2}\right)}^{\mathrm{ord}, \sharp}=L_{n,(i), \operatorname{lin}}\left(\mathbb{Z}_{(p)}\right) \backslash \Omega_{n,(i), U^{p}\left(N_{1}, N_{2}\right)}^{\mathrm{ord},+}
$$

and

$$
\Xi_{n,(i), U^{p}\left(N_{1}, N_{2}\right)}^{\mathrm{ord},}=L_{n,(i), \operatorname{lin}}\left(\mathbb{Z}_{(p)}\right) \backslash \Xi_{n,(i), U^{p}\left(N_{1}, N_{2}\right)}^{\mathrm{ord},+}
$$

locally free sheaves on $\mathcal{X}_{n,(i), U^{p}\left(N_{1}, N_{2}\right)}^{\text {ord, }}$.

There are maps

$$
\mathcal{X}_{n,(i), U^{p}\left(N_{1}, N_{2}\right)}^{(m), \text { ord },+} \times \operatorname{Spec} \mathbb{Q} \hookrightarrow X_{n,(i), U^{p}\left(N_{1}, N_{2}\right)}^{(m),+}
$$

which are equivariant for the actions of the groups $L_{n,(i)}^{(m)}\left(\mathbb{A}^{\infty}\right)^{\text {ord }}$ and $L_{n,(i), \text { lin }}^{(m)}\left(\mathbb{Z}_{(p)}\right)$ and $G L_{m}\left(\mathcal{O}_{F,(p)}\right)$. Under these maps the sheaves $\Omega_{n,(i), U^{p}\left(N_{1}, N_{2}\right)}^{\text {ord }}$ (resp. $\Xi_{n,(i), U^{p}\left(N_{1}, N_{2}\right)}^{\text {ord, }}$, resp. $\left.\mathcal{E}_{(i), U^{p}\left(N_{1}, N_{2}\right), \rho}^{\text {ord },}\right)$ correspond to $\Omega_{n,(i), U^{p}\left(N_{1}, N_{2}\right)}^{+}$(resp. to $\Xi_{n,(i), U^{p}\left(N_{1}, N_{2}\right)}^{+}$, resp. to $\left.\mathcal{E}_{(i), U^{p}\left(N_{1}, N_{2}\right), \rho \otimes \mathbb{Q}}^{\text {ord }}\right)$. The induced maps

$$
\mathcal{X}_{n,(i), U^{p}\left(N_{1}, N_{2}\right)}^{(m), \mathrm{rad}} \times \operatorname{Spec} \mathbb{Q} \longrightarrow X_{n,(i), U^{p}\left(N_{1}, N_{2}\right)}^{(m),}
$$

are isomorphisms.

\subsection{Generalized Kuga-Sato varieties}

Now suppose that $U$ is a neat open compact subgroup of

$$
\left(P_{n,(i)}^{(m),+} / Z\left(N_{n,(i)}^{(m)}\right)\right)\left(\mathbb{A}^{\infty}\right)=\left(\widetilde{P}_{n,(i)}^{(m),+} / Z\left(\widetilde{N}_{n,(i)}^{(m)}\right)\right)\left(\mathbb{A}^{\infty}\right) .
$$

We set

$$
A_{n,(i), U}^{(m),+}=\coprod_{h \in L_{n,(i), \operatorname{lin}}^{(m)}\left(\mathbb{A}^{\infty}\right) / U} A_{n-i, h U h^{-1} \cap G_{n-i}^{(i+m)}\left(\mathbb{A}^{\infty}\right)}^{(i+m)}
$$

In the case $m=0$ we will write $\operatorname{simply} A_{n,(i), U}^{+}$. 
If $g \in\left(P_{n,(i)}^{(m),+} / Z\left(N_{n,(i)}^{(m)}\right)\right)\left(\mathbb{A}^{\infty}\right)$ and $g^{-1} U g \subset U^{\prime}$, then we define a finite etale map

$$
g: A_{n,(i), U}^{(m),+} \longrightarrow A_{n,(i), U^{\prime}}^{(m),+}
$$

to be the coproduct of the maps

$$
g^{\prime}: A_{n-i, h U h^{-1} \cap G_{n-i}^{(i+m)}\left(\mathbb{A}^{\infty}\right)}^{(i+m)} \longrightarrow A_{n-i, h^{\prime} U^{\prime}\left(h^{\prime}\right)^{-1} \cap G_{n-i}^{(i+m)}\left(\mathbb{A}^{\infty}\right)^{\prime}}^{(i+m)}
$$

where $h, h^{\prime} \in L_{n,(i), \text { lin }}^{(m)}\left(\mathbb{A}^{\infty}\right)$ and $g^{\prime} \in G_{n-i}^{(i+m)}\left(\mathbb{A}^{\infty}\right)$ satisfy $h g=g^{\prime} h^{\prime}$. This makes $\left\{A_{n,(i), U}^{(m),+}\right\}$ a system of schemes (locally of finite type over Spec $\mathbb{Q}$ ) with right action of the group $\left(P_{n,(i)}^{(m),+} / Z\left(N_{n,(i)}^{(m)}\right)\right)\left(\mathbb{A}^{\infty}\right)$. If $U^{\prime}$ is an open normal subgroup of $U$ then $A_{n,(i), U}^{(m),+}$ is identified with $A_{n,(i), U^{\prime}}^{(m),+} / U$.

If $\gamma \in L_{n,(i), \text { lin }}^{(m)}(\mathbb{Q})$, then we define

$$
\gamma: A_{n,(i), U}^{(m),+} \longrightarrow A_{n,(i), U}^{(m),+}
$$

to be the coproduct of the maps

$$
\gamma: A_{n-i, h U h^{-1} \cap G_{n-i}^{(i+m)}\left(\mathbb{A}^{\infty}\right)}^{(i+m)} \longrightarrow A_{n-i,(\gamma h) U(\gamma h)^{-1} \cap G_{n-i}^{(i+m)}\left(\mathbb{A}^{\infty}\right)^{\prime}}^{(i+m)}
$$

This gives a left action of $L_{n,(i), \text { lin }}^{(m)}(\mathbb{Q})$ on each $A_{n,(i), U}^{(m),+}$, which commutes with the action of $\left(P_{n,(i)}^{(m),+} / Z\left(N_{n,(i)}^{(m)}\right)\right)\left(\mathbb{A}^{\infty}\right)$.

If $\delta \in G L_{m}(F)$ define a map

$$
\delta: A_{n,(i), U}^{(m),+} \longrightarrow A_{n,(i), \delta(U)}^{(m),+}
$$

as the coproduct of the maps

$$
\delta: A_{n-i, h U h^{-1} \cap G_{n-i}^{(i+m)}\left(\mathbb{A}^{\infty}\right)}^{(i+m)} \longrightarrow A_{n-i, \delta\left(h U h^{-1}\right) \cap G_{n-i}^{(i+m)}\left(\mathbb{A}^{\infty}\right)^{(i+m)}}
$$

This gives a left $G L_{m}(F)$-action on the system of the $A_{n,(i), U}^{(m),+}$. If $\delta \in G L_{m}(F)$ and $\gamma \in$ $L_{n,(i), \text { lin }}^{(m)}(\mathbb{Q})$ and $g \in\left(P_{n,(i)}^{(m),+} / Z\left(N_{n,(i)}^{(m)}\right)\right)\left(\mathbb{A}^{\infty}\right)$ then $\delta \circ \gamma=\delta(\gamma) \circ \delta$ and $\delta \circ g=\delta(g) \circ \delta$.

There are natural maps

$$
A_{n,(i), U}^{(m),+} \longrightarrow X_{n,(i), U}^{(m),+}
$$

which are equivariant for the actions of $\left(P_{n,(i)}^{(m),+} / Z\left(N_{n,(i)}^{(m)}\right)\right)\left(\mathbb{A}^{\infty}\right)$ and $L_{n,(i), \text { lin }}^{(m)}(\mathbb{Q})$ and $G L_{m}(F)$. If $U^{\prime}$ denotes the image of $U$ in $\left(P_{n,(i)}^{+} / Z\left(N_{n,(i)}\right)\right)\left(\mathbb{A}^{\infty}\right)$ then there is a natural commutative diagram:

$$
\begin{array}{ccc}
A_{n,(i), U}^{(m),+} & \rightarrow & A_{n,(i), U^{\prime}}^{+} \\
\downarrow & & \downarrow \\
X_{n,(i), U}^{(m),+} & \rightarrow & X_{n,(i), U^{\prime}}^{+} \\
\downarrow & & \downarrow \\
Y_{n,(i), U}^{(m),+} & \rightarrow & Y_{n,(i), U^{\prime}}^{+}
\end{array}
$$


which is $L_{n,(i), \text { lin }}^{(m)}(\mathbb{Q})$ and $\left(P_{n,(i)}^{(m),+} / Z\left(N_{n,(i)}^{(m)}\right)\right)\left(\mathbb{A}^{\infty}\right)$ equivariant.

We have

$$
A_{n,(i), U}^{(m),+}(\mathbb{C})=\left(P_{n,(i)}^{(m)} / Z\left(N_{n,(i)}^{(m)}\right)\right)(\mathbb{Q}) \backslash\left(P_{n,(i)}^{(m),+} / Z\left(N_{n,(i)}^{(m)}\right)\right)(\mathbb{A}) /\left(U U_{n-i, \infty}^{0} A_{n-i}(\mathbb{R})^{0}\right) .
$$

Note that it does not make sense to divide $A_{n,(i), U}^{(m),+}$ by $L_{n,(i), \operatorname{lin}}^{(m)}(\mathbb{Q})$, so we don't do so.

We define a semi-abelian scheme $\widetilde{G}^{\text {univ }} / A_{n,(i), U}^{+}$by requiring that over the open and closed subscheme $A_{n-i, h U h^{-1} \cap G_{n-i}^{(i)}\left(\mathbb{A}^{\infty}\right)}$ it restricts to $G^{\text {univ }}$. It is unique up to unique quasiisogeny. We also define a sheaf $\widetilde{\Omega}_{n,(i), U}^{+}\left(\right.$resp. $\left.\widetilde{\Xi}_{n,(i), U}^{+}\right)$over $A_{n,(i), U}^{+}$to be the unique sheaf which, for each $h$, restricts to $\Omega_{n-i, h U h^{-1} \cap G_{n-i}^{(i)}\left(\mathbb{A}^{\infty}\right)}^{(i)}\left(\right.$ resp. $\left.\Xi_{n-i, h U h^{-1} \cap G_{n-i}^{(i)}\left(\mathbb{A}^{\infty}\right)}\right)$ on $A_{n-i, h U h^{-1} \cap G_{n-i}^{(i)}\left(\mathbb{A}^{\infty}\right)}^{(i)}$. Thus $\widetilde{\Omega}_{n,(i), U}^{+}$is the pull-back by the identity section of $\Omega_{\widetilde{G}^{\text {univ }} / A_{n,(i), U}^{+}}^{1}$. Then $\left\{\widetilde{\Omega}_{n,(i), U}^{+}\right\}$(resp. $\left\{\widetilde{\Xi}_{n,(i), U}^{+}\right\}$) is a system of locally free sheaves on $A_{n,(i), U}^{+}$with a left $\left(P_{n,(i)}^{+} / Z\left(N_{n,(i)}\right)\right)\left(\mathbb{A}^{\infty}\right)$-action and a commuting right $L_{n,(i), \text { lin }}(\mathbb{Q})$-action. There are equivariant exact sequences

$$
(0) \longrightarrow \pi^{*} \Omega_{n,(i), U}^{+} \longrightarrow \widetilde{\Omega}_{n,(i), U}^{+} \longrightarrow F^{i} \otimes_{\mathbb{Q}} \mathcal{O}_{A_{n,(i), U}^{+}} \longrightarrow(0),
$$

where $\pi$ denotes the map $A_{n,(i), U}^{+} \rightarrow X_{n,(i), U}^{+}$.

Let $\widetilde{\mathcal{E}}_{(i), U}^{+}$denote the principal $R_{n,(n),(i)}$-bundle on $A_{n,(i), U}^{+}$in the Zariski topology defined by setting, for $W \subset A_{n,(i), U}^{+}$a Zariski open, $\widetilde{\mathcal{E}}_{(i), U}^{+}(W)$ to be the set of pairs $\left(\xi_{0}, \xi_{1}\right)$, where

$$
\xi_{0}:\left.\Xi_{n,(i), U}^{+}\right|_{W} \stackrel{\sim}{\longrightarrow} \mathcal{O}_{W}
$$

and

$$
\xi_{1}: \widetilde{\Omega}_{n,(i), U}^{+} \stackrel{\sim}{\longrightarrow} \operatorname{Hom}_{\mathbb{Q}}\left(V_{n-i} / V_{n-i,(n-i)} \oplus \operatorname{Hom}_{\mathbb{Q}}\left(F^{i}, \mathbb{Q}\right), \mathcal{O}_{W}\right)
$$

satisfies

$$
\xi_{1}: \Omega_{n,(i), U}^{+} \stackrel{\sim}{\longrightarrow} \operatorname{Hom}_{\mathbb{Q}}\left(V_{n-i} / V_{n-i,(n-i)}, \mathcal{O}_{W}\right)
$$

We define the $R_{n,(n),(i)}$-action on $\mathcal{E}_{(i), U}^{+}$by

$$
h\left(\xi_{0}, \xi_{1}\right)=\left(v(h)^{-1} \xi_{0},\left(\circ h^{-1}\right) \circ \xi_{1}\right) .
$$

The inverse system $\left\{\widetilde{\mathcal{E}}_{(i), U}^{+}\right\}$has an action of $P_{n,(i)}^{+}\left(\mathbb{A}^{\infty}\right)$ and of $L_{n,(i), \text { lin }}(\mathbb{Q})$.

Suppose that $R_{0}$ is a $\mathbb{Q}$-algebra and that $\rho$ is a representation of $R_{n,(n),(i)}$ on a finite, locally free $R_{0}$-module $W_{\rho}$. We define a locally free sheaf $\mathcal{E}_{(i), U, \rho}^{+}$over $A_{n,(i), U}^{+} \times \operatorname{Spec} R_{0}$ by setting $\mathcal{E}_{(i), U, \rho}^{+}(W)$ to be the set of $R_{n,(n),(i)}\left(\mathcal{O}_{W}\right)$-equivariant maps of Zariski sheaves of sets

$$
\widetilde{\mathcal{E}}_{(i), U}^{+} \mid W \longrightarrow W_{\rho} \otimes_{R_{0}} \mathcal{O}_{W}
$$

Then $\left\{\mathcal{E}_{(i), U, \rho}^{+}\right\}$is a system of locally free sheaves with both a $P_{n,(i)}^{+}\left(\mathbb{A}^{\infty}\right)$-action and an $L_{n,(i), \text { lin }}(\mathbb{Q})$-action over the system of schemes $\left\{A_{n,(i), U}^{+} \times \operatorname{Spec} R_{0}\right\}$. The restriction of $\mathcal{E}_{(i), U, \rho}^{+}$to $A_{n-i, h U h^{-1} \cap G_{n-i}^{(i)}\left(\mathbb{A}^{\infty}\right)}^{(i)}$ can be identified with $\mathcal{E}_{h U h^{-1} \cap G_{n-i}^{(i)}\left(\mathbb{A}^{\infty}\right),\left.\rho\right|_{R_{n-i,(n-i)}^{(i)}}}$. However 
the description of the actions of $P_{n,(i)}^{+}\left(\mathbb{A}^{\infty}\right)$ and $L_{n,(i), \text { lin }}(\mathbb{Q})$ involve $\rho$ and not just $\left.\rho\right|_{R_{n-i,(n-i)}^{(i)}}$. If $g \in P_{n,(i)}^{+}\left(\mathbb{A}^{\infty}\right)$ and $\gamma \in L_{n,(i) \text {,in }}(\mathbb{Q})$, then the natural maps

$$
g^{*} \mathcal{E}_{(i), U, \rho}^{+} \longrightarrow \mathcal{E}_{(i), U^{\prime}, \rho}^{+}
$$

and

$$
\gamma^{*} \mathcal{E}_{(i), U, \rho}^{+} \longrightarrow \mathcal{E}_{(i), U^{\prime}, \rho}^{+}
$$

are isomorphisms. If $\rho$ factors through $R_{n,(n),(i)} / N\left(R_{n,(n),(i)}\right)$ then $\mathcal{E}_{(i), U, \rho}^{+}$is canonically isomorphic to the pull-back of $\mathcal{E}_{(i), U, \rho}^{+}$from $X_{n,(i), U}^{+}$. In general $W_{\rho}$ has a filtration by $R_{n,(n),(i)}$-invariant local direct summands such that the action of $R_{n,(n),(i)}$ on each graded piece factors through $R_{n,(n),(i)} / N\left(R_{n,(n),(i)}\right)$. (To see this apply proposition 4.7.3 of exposé I of [53] to the action of $A_{n,(i), \text { lin }}$ on $W_{\rho}$.) Thus $\mathcal{E}_{(i), U, \rho}^{+}$has a $P_{n,(i)}^{+}\left(\mathbb{A}^{\infty}\right)$ and $L_{n,(i), \text { lin }}(\mathbb{Q})$ invariant filtration by local direct summands such that each graded piece is the pull-back of some $\mathcal{E}_{(i), U, \rho^{\prime}}^{+}$from $X_{n,(i), U}^{+}$.

Similarly if $U^{p}$ is a neat open compact subgroup of $\left(P_{n,(i)}^{(m),+} / Z\left(N_{n,(i)}^{(m)}\right)\right)\left(\mathbb{A}^{\infty, p}\right)=$ $\left(\widetilde{P}_{n,(i)}^{(m)+} / Z\left(\widetilde{N}_{n,(i)}^{(m)}\right)\right)\left(\mathbb{A}^{\infty, p}\right)$ we set

$$
\mathcal{A}_{n,(i), U^{p}\left(N_{1}, N_{2}\right)}^{(m), \text { ord }+}=\coprod_{h \in L_{n,(i), \text { lin }}^{(m)}\left(\mathbb{A}^{\infty}\right)^{\text {ord } \times} / U^{p}\left(N_{1}\right)} \mathcal{A}_{n-i,\left(h U^{p} h^{-1} \cap G_{n-i}^{(i+m)}\left(\mathbb{A}^{\infty, p}\right)\right)\left(N_{1}, N_{2}\right)}^{(i+m), \text { ord }}
$$

In the case $m=0$ we will write simply $\mathcal{A}_{n,(i), U^{p}\left(N_{1}, N_{2}\right)}^{\text {ord, }}$. The inverse system of the $\mathcal{A}_{n,(i), U^{p}\left(N_{1}, N_{2}\right)}^{(m) \text {,ord, }}$ has a right action of $\left(P_{n,(i)}^{(m),+} / Z\left(N_{n,(i)}^{(m)}\right)\right)\left(\mathbb{A}^{\infty}\right)^{\text {ord }}$ and a commuting left action of $L_{n,(i), \text { lin }}^{(m)}\left(\mathbb{Z}_{(p)}\right)$. If $g \in\left(P_{n,(i)}^{(m),+} / Z\left(N_{n,(i)}^{(m)}\right)\right)\left(\mathbb{A}^{\infty}\right)^{\text {ord }}$ then the map

$$
g: \mathcal{A}_{n,(i), U^{p}\left(N_{1}, N_{2}\right)}^{(m), \text { ord }+} \longrightarrow \mathcal{A}_{n,(i),\left(U^{p}\right)^{\prime}\left(N_{1}^{\prime}, N_{2}^{\prime}\right)}^{(m), \text { ord },}
$$

is quasi-finite and flat. If $g \in\left(P_{n,(i)}^{(m),+} / Z\left(N_{n,(i)}^{(m)}\right)\right)\left(\mathbb{A}^{\infty}\right)^{\text {ord, } \times}$ then it is etale, and, if further $N_{2}=N_{2}^{\prime}$, then it is finite etale. If $N_{2}^{\prime}>0$ and $p^{N_{2}-N_{2}^{\prime}} v\left(g_{p}\right) \in \mathbb{Z}_{p}^{\times}$then the map is finite. On $\mathbb{F}_{p}$-fibres the map $\varsigma_{p}$ is absolute Frobenius composed with the forgetful map. If $\left(U^{p}\right)^{\prime}$ is an open normal subgroup of $U^{p}$ and if $N_{1} \leq N_{1}^{\prime} \leq N_{2}$ then $\mathcal{A}_{n,(i),\left(U^{p}\right)^{\prime}\left(N_{1}^{\prime}, N_{2}\right)}^{(m), \text { ord, }} / U^{p}\left(N_{1}, N_{2}\right)$

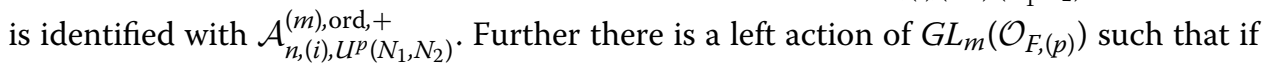
$\delta \in G L_{m}\left(\mathcal{O}_{F,(p)}\right)$ and $\gamma \in L_{n,(i), \text { lin }}^{(m)}\left(\mathbb{Z}_{(p)}\right)$ and $g \in\left(P_{n,(i)}^{(m),+} / Z\left(N_{n,(i)}^{(m)}\right)\right)\left(\mathbb{A}^{\infty}\right)^{\text {ord }}, \delta \circ \gamma=\delta(\gamma) \circ \delta$ and $\delta \circ g=\delta(g) \circ \delta$. There are natural equivariant maps

$$
\mathcal{A}_{n,(i), U^{p}\left(N_{1}, N_{2}\right)}^{(m), \text { ord }+} \longrightarrow \mathcal{X}_{n,(i), U^{p}\left(N_{1}, N_{2}\right)}^{(m), o r d,+}
$$

If $\left(U^{p}\right)^{\prime}$ denotes the image of $U^{p}$ in $\left(P_{n,(i)}^{+} / Z\left(N_{n,(i)}\right)\right)\left(\mathbb{A}^{\infty, p}\right)$ then there is a natural equivariant, commutative diagram:

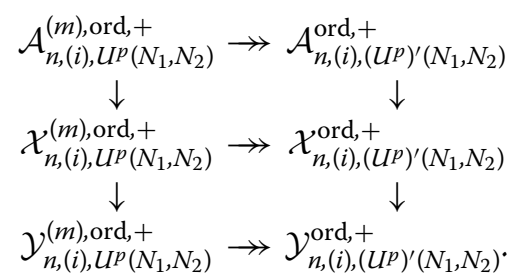


There are equivariant embeddings

$$
\mathcal{A}_{n,(i), U^{p}\left(N_{1}, N_{2}\right)}^{(m), \text { ord }+} \times \operatorname{Spec} \mathbb{Q} \hookrightarrow A_{n,(i), U^{p}\left(N_{1}, N_{2}\right)}^{(m),+}
$$

We define a semi-abelian scheme $\widetilde{\mathcal{G}}^{\text {univ }} / \mathcal{A}_{n,(i), U^{p}\left(N_{1}, N_{2}\right)}^{\text {ord, }}$ over $\mathcal{A}_{n,(i), U^{p}\left(N_{1}, N_{2}\right)}^{\text {ord, }}$ by requiring that over $\mathcal{A}_{n-i,\left(h U^{p} h^{-1} \cap G_{n-i}^{(i)}\left(\mathbb{A}^{\infty, p}\right)\right)\left(N_{1}, N_{2}\right)}$ it restricts to $\mathcal{G}^{\text {univ }}$. It is unique up to unique primeto- $p$ quasi-isogeny. We define a locally free sheaf $\widetilde{\Omega}_{n,(i), U^{p}\left(N_{1}, N_{2}\right)}^{\text {ord })}$ (resp. $\widetilde{\Xi}_{n,(i), U^{p}\left(N_{1}, N_{2}\right)}^{\text {ord, }}$ ) over the scheme $\mathcal{A}_{h,(i), U^{p}\left(N_{1}, N_{2}\right)}^{\text {ord }}$ to be the sheaf which, for each $h$, restricts to the sheaf

$$
\Omega_{n-i,\left(h U^{p} h^{-1} \cap G_{n-i}^{(i)}\left(\mathbb{A}^{\infty, p}\right)\right)\left(N_{1}, N_{2}\right)}^{(i) \text { ord }}
$$

(resp. $\left.\Xi_{n-i,\left(h U^{p} h^{-1} \cap G_{n-i}^{(i)}\left(\mathbb{A}^{\infty, p}\right)\right)\left(N_{1}, N_{2}\right)}^{(i) \text { ord }}\right)$ on the subscheme $\mathcal{A}_{n-i,\left(h U^{p} h^{-1} \cap G_{n-i}^{(i)}\left(\mathbb{A}^{\infty, p}\right)\right)\left(N_{1}, N_{2}\right)}^{(i) \text { ord }}$. Then $\widetilde{\Omega}_{n,(i), U^{p}\left(N_{1}, N_{2}\right)}^{\text {ord, }}$ is the pull-back by the identity section of $\Omega_{\widetilde{\mathcal{G}}^{\text {univ }} / \mathcal{A}_{n, i), U^{p}\left(N_{1}, N_{2}\right)}^{\text {ord, }}}^{1}$. The collection $\left\{\widetilde{\Omega}_{n,(i), U^{p}\left(N_{1}, N_{2}\right)}^{\text {ord, }}\right\}$ (resp. $\left\{\widetilde{\Xi}_{n,(i), U^{p}\left(N_{1}, N_{2}\right)}^{\text {ord, }}\right\}$ ) is a system of locally free sheaves on $\mathcal{A}_{n,(i), U^{p}\left(N_{1}, N_{2}\right)}^{\text {ord, }}$ with a left $\left(P_{n,(i)}^{+} / Z\left(N_{n,(i)}\right)\right)\left(\mathbb{A}^{\infty}\right)^{\text {ord }}$-action and a commuting right $L_{n,(i), \text { lin }}\left(\mathbb{Z}_{(p)}\right)$-action. Also there are equivariant exact sequences

$$
(0) \longrightarrow \pi^{*} \Omega_{n,(i), U^{p}\left(N_{1}, N_{2}\right)}^{\mathrm{ord},+} \longrightarrow \widetilde{\Omega}_{n,(i), U^{p}\left(N_{1}, N_{2}\right)}^{\mathrm{ord},+} \longrightarrow \mathcal{O}_{F}^{i} \otimes_{\mathbb{Z}} \mathcal{O}_{\mathcal{A}_{n,(i), U^{p}\left(N_{1}, N_{2}\right)}^{\text {ord }+}} \longrightarrow(0)
$$

where $\pi$ denotes the map $\mathcal{A}_{n,(i), U}^{\text {ord, }+} \rightarrow \mathcal{X}_{n,(i), U}^{\text {ord, }+}$.

Let $\widetilde{\mathcal{E}}_{(i), U^{p}\left(N_{1}, N_{2}\right)}^{\text {ord, }}$ denote the principal $R_{n,(n),(i)}$-bundle on the scheme $\mathcal{A}_{n,(i), U^{p}\left(N_{1}, N_{2}\right)}^{\text {ord, }}$ in the Zariski topology defined by setting, for $W \subset \mathcal{A}_{n,(i), U^{p}\left(N_{1}, N_{2}\right)}^{\text {ord, }}$ a Zariski open, $\widetilde{\mathcal{E}}_{(i), U^{p}\left(N_{1}, N_{2}\right)}^{\text {ord }+}(W)$ to be the set of pairs $\left(\xi_{0}, \xi_{1}\right)$, where

$$
\xi_{0}:\left.\Xi_{n,(i), U^{p}\left(N_{1}, N_{2}\right)}^{\text {ord },+}\right|_{W} \stackrel{\sim}{\longrightarrow} \mathcal{O}_{W}
$$

and

$$
\xi_{1}: \widetilde{\Omega}_{n,(i), U^{p}\left(N_{1}, N_{2}\right)}^{\mathrm{ord},} \stackrel{\sim}{\longrightarrow} \operatorname{Hom}_{\mathbb{Z}}\left(\Lambda_{n-i} / \Lambda_{n-i,(n-i)} \oplus \operatorname{Hom}_{\mathbb{Z}}\left(\mathcal{O}_{F}^{i}, \mathbb{Z}\right), \mathcal{O}_{W}\right)
$$

satisfies

$$
\xi_{1}: \Omega_{n,(i), U^{p}\left(N_{1}, N_{2}\right)}^{\mathrm{ord},+} \stackrel{\sim}{\longrightarrow} \operatorname{Hom}_{\mathbb{Z}}\left(\Lambda_{n-i} / \Lambda_{n-i,(n-i)}, \mathcal{O}_{W}\right)
$$

We define the $R_{n,(n),(i) \text {-action on }} \widetilde{\mathcal{E}}_{(i), U^{p}\left(N_{1}, N_{2}\right)}^{\text {ord }+}$ by

$$
h\left(\xi_{0}, \xi_{1}\right)=\left(v(h)^{-1} \xi_{0},\left(\circ h^{-1}\right) \circ \xi_{1}\right)
$$

The inverse system $\left\{\widetilde{\mathcal{E}}_{(i), U^{p}\left(N_{1}, N_{2}\right)}^{\text {ord, }}\right\}$ has an action both of the groups $P_{n,(i)}^{+}\left(\mathbb{A}^{\infty}\right)^{\text {ord, } \times}$ and of $L_{n,(i), \operatorname{lin}}\left(\mathbb{Z}_{(p)}\right)$.

Suppose that $R_{0}$ is a $\mathbb{Q}$-algebra and that $\rho$ is a representation of $R_{n,(n),(i)}$ on a finite, locally free $R_{0}$-module $W_{\rho}$. We define a locally free sheaf $\mathcal{E}_{(i), U^{p}\left(N_{1}, N_{2}\right), \rho}^{\text {ord },}$ over $\mathcal{A}_{n,(i), U^{p}\left(N_{1}, N_{2}\right)}^{\text {ord, }} \times$ 
Spec $R_{0}$ by setting $\mathcal{E}_{(i), U^{p}\left(N_{1}, N_{2}\right), \rho}^{\text {ord }+}(W)$ to be the set of $R_{n,(n),(i)}\left(\mathcal{O}_{W}\right)$-equivariant maps of Zariski sheaves of sets

$$
\left.\widetilde{\mathcal{E}}_{(i), U^{p}\left(N_{1}, N_{2}\right)}^{\text {ord }+}\right|_{W} \longrightarrow W_{\rho} \otimes_{R_{0}} \mathcal{O}_{W}
$$

Then $\left\{\mathcal{E}_{(i), U^{p}\left(N_{1}, N_{2}\right), \rho}^{\text {ord },}\right\}$ is a system of locally free sheaves with $P_{n,(i)}^{+}\left(\mathbb{A}^{\infty}\right)^{\text {ord, } \times}$-action and $L_{n,(i), \text { lin }}\left(\mathbb{Z}_{(p)}\right)$-action over the system of schemes $\left\{\mathcal{A}_{n,(i), U^{p}\left(N_{1}, N_{2}\right)}^{\text {ord, }} \times \operatorname{Spec} R_{0}\right\}$. The restriction of $\mathcal{E}_{(i), U^{p}\left(N_{1}, N_{2}\right), \rho}^{\text {ord, }}$ to $\mathcal{A}_{n-i,\left(h U^{p} h^{-1} \cap G_{n-i}^{(i)}\left(\mathbb{A}^{\infty, p}\right)\right)\left(N_{1}, N_{2}\right)}^{(\text {ord }}$ can be identified with

$$
\mathcal{E}_{\left(h U^{p} h^{-1} \cap G_{n-i}^{(i)}\left(\mathbb{A}^{\infty, p}\right)\right)\left(N_{1}, N_{2}\right),\left.\rho\right|_{R_{n-i,(n-i)}^{(i)}}} .
$$

However the description of the actions of the groups $P_{n,(i)}^{+}\left(\mathbb{A}^{\infty}\right)^{\text {ord, } \times}$ and $L_{n,(i), \text { lin }}\left(\mathbb{Z}_{(p)}\right)$ involves $\rho$ and not just $\left.\rho\right|_{R_{n-i,(n-i)}^{(i)}}$. If $g \in P_{n,(i)}^{+}\left(\mathbb{A}^{\infty}\right)^{\text {ord, } \times}$ and $\gamma \in L_{n,(i), \text { lin }}\left(\mathbb{Z}_{(p)}\right)$, then the natural maps

$$
g^{*} \mathcal{E}_{(i), U^{p}\left(N_{1}, N_{2}\right), \rho}^{\mathrm{ord},+} \longrightarrow \mathcal{E}_{(i),\left(U^{p}\right)^{\prime}\left(N_{1}^{\prime}, N_{2}^{\prime}\right), \rho}^{\mathrm{ord},+}
$$

and

$$
\gamma^{*} \mathcal{E}_{(i), U^{p}\left(N_{1}, N_{2}\right), \rho}^{\mathrm{ord},+} \longrightarrow \mathcal{E}_{(i),\left(U^{p}\right)^{\prime}\left(N_{1}^{\prime}, N_{2}^{\prime}\right), \rho}^{\mathrm{ord},}
$$

are isomorphisms. If $\rho$ factors through $R_{n,(n),(i)} / N\left(R_{n,(n),(i)}\right)$ then $\mathcal{E}_{(i), U^{p}\left(N_{1}, N_{2}\right), \rho}^{\text {ord },}$ is canonically isomorphic to the pull-back of $\mathcal{E}_{(i), U^{p}\left(N_{1}, N_{2}\right), \rho}^{\text {ord },}$ from $\mathcal{X}_{n,(i), U^{p}\left(N_{1}, N_{2}\right)}^{\text {ord, }}$. In general $W_{\rho}$ has a filtration by $R_{n,(n),(i)}$-invariant local direct summands such that the action of $R_{n,(n),(i)}$ on each graded piece factors through $R_{n,(n),(i)} / N\left(R_{n,(n),(i)}\right)$. (To see this apply proposition 4.7.3 of exposé I of [53] to the action of $A_{n,(i), \text { lin }}$ on $W_{\rho}$.) Thus $\mathcal{E}_{(i), U^{p}\left(N_{1}, N_{2}\right), \rho}^{\text {ord, }}$ has a $P_{n,(i)}^{+}\left(\mathbb{A}^{\infty}\right)^{\text {ord, } \times}$ and $L_{n,(i), \text { lin }}\left(\mathbb{Z}_{(p)}\right)$ invariant filtration by local direct summands such that each graded piece is the pull-back of some $\mathcal{E}_{(i), U^{p}\left(N_{1}, N_{2}\right), \rho^{\prime}}^{\text {ord, }}$ from $\mathcal{X}_{n,(i), U^{p}\left(N_{1}, N_{2}\right)}^{\text {ord, }}$.

The next lemma follows from the discussion in Sect. 3.4.

Lemma 4.1 If $U^{\prime}$ is the image of $U\left(\right.$ resp. $\left.U^{p}\right)$ and if $\pi$ denotes the map $A_{n,(i), U}^{(m),+} \rightarrow A_{n,(i), U^{\prime}}^{+}$ then there are $L_{n,(i), \text { lin }}(\mathbb{Q})$-equivariant, $\left(P_{n,(i)}^{+} / Z\left(N_{n,(i)}\right)\right)\left(\mathbb{A}^{\infty}\right)$-equivariant and $G L_{m}(F)$ equivariant isomorphisms

$$
\begin{aligned}
& R^{j} \pi_{*} \Omega_{A_{n,(i), U}^{(m),+} / A_{n,(i), U^{\prime}}^{+}}^{k} \\
& \quad \cong\left(\wedge^{k}\left(F^{m} \otimes_{F} \Omega_{n,(i), U^{\prime}}^{+}\right)\right) \otimes\left(\wedge^{j}\left(F^{m} \otimes_{F} \operatorname{Hom}\left(\Omega_{n,(i), U^{\prime}}^{+}, \Xi_{n,(i), U^{\prime}}^{+}\right)\right)\right) .
\end{aligned}
$$

\subsection{Generalized mixed Shimura varieties}

Next suppose $\widetilde{U}$ is an open compact subgroup of $\widetilde{P}_{n,(i)}^{(m),+}\left(\mathbb{A}^{\infty}\right)$. We define a split torus $\widetilde{S}_{n,(i), \widetilde{U}}^{(m),+} / Y_{n,(i), \widetilde{U}}^{(m),+}$ as

$$
\coprod_{h \in L_{n,(i), \operatorname{lin}}^{(m)}\left(\mathbb{A}^{\infty}\right) / \widetilde{U}} S_{n-i, h \widetilde{U} h^{-1} \cap \widetilde{G}_{n-i}^{(m+i)}\left(\mathbb{A}^{\infty}\right)^{\cdot}}^{(m+i)}
$$


Thus $X_{*}\left(\widetilde{S}_{n,(i), \widetilde{U}}^{(m),+}\right)_{\mathbb{Q}}$ is a constant sheaf:

$$
X_{*}\left(\widetilde{S}_{n,(i), \widetilde{U}}^{(m),+}\right)_{\mathbb{Q}} \cong \operatorname{Herm}_{\mathbb{Q}}^{(m+i)} \cong Z\left(N_{n,(i)}^{(m)}\right)(\mathbb{Q}) .
$$

If $\widetilde{g} \in \widetilde{P}_{n,(i)}^{(m),+}\left(\mathbb{A}^{\infty}\right)$ and $\widetilde{g}^{-1} \widetilde{U} \widetilde{g} \subset \widetilde{U}^{\prime}$, then we define

$$
\widetilde{g}: \widetilde{S}_{n,(i), \widetilde{U}}^{(m),+} \longrightarrow \widetilde{S}_{n,(i), \widetilde{U}^{\prime}}^{(m),+}
$$

to be the coproduct of the maps

$$
\widetilde{g}^{\prime}: S_{n-i, h \widetilde{U} h^{-1} \cap \widetilde{G}_{n-i}^{(m+i)}\left(\mathbb{A}^{\infty}\right)}^{(m+i)} \longrightarrow S_{n-i, h^{\prime} \widetilde{U}^{\prime}\left(h^{\prime}\right)^{-1} \cap \widetilde{G}_{n-i}^{(m+i)}\left(\mathbb{A}^{\infty}\right)^{\prime}}^{(m+i)}
$$

where $h, h^{\prime} \in L_{n,(i), \text { lin }}^{(m)}\left(\mathbb{A}^{\infty}\right)$ and $\widetilde{g}^{\prime} \in \widetilde{G}_{n-i}^{(m+i)}\left(\mathbb{A}^{\infty}\right)$ satisfy $h \widetilde{g}=\widetilde{g}^{\prime} h^{\prime}$. This makes $\left\{\widetilde{S}_{n,(i), \widetilde{U}}^{(m),+}\right\}$ a system of relative tori with right $\widetilde{P}_{n,(i)}^{(m),+}\left(\mathbb{A}^{\infty}\right)$-action. If $\gamma \in L_{n,(i), \text { lin }}^{(m)}(\mathbb{Q})$, then we define

$$
\gamma: \widetilde{S}_{n,(i), \widetilde{U}}^{(m)+} \longrightarrow \widetilde{S}_{n,(i), \widetilde{U}}^{(m)+}
$$

to be the coproduct of the maps

$$
\gamma: S_{n-i, h \widetilde{U} h^{-1} \cap \widetilde{G}_{n-i}^{(m+i)}\left(\mathbb{A}^{\infty}\right)}^{(m+i)} \longrightarrow S_{n-i,(\gamma h) \widetilde{U}(\gamma h)^{-1} \cap \widetilde{G}_{n-i}^{(m+i)}\left(\mathbb{A}^{\infty}\right)^{\cdot}}^{(m+i)}
$$

This gives a left action of $L_{n,(i), \text { lin }}^{(m)}(\mathbb{Q})$ on each $\widetilde{S}_{n,(i), \widetilde{U}}^{(m),+}$, which commutes with the action of $\widetilde{P}_{n,(i)}^{(m),+}\left(\mathbb{A}^{\infty}\right)$.

Similarly suppose $\widetilde{U}^{p}$ is an open compact subgroup of $\widetilde{P}_{n,(i)}^{(m),+}\left(\mathbb{A}^{\infty, p}\right)$ and that $N$ is a non-negative integer. We define a split torus $\widetilde{\mathcal{S}}_{n,(i), \widetilde{U}^{p}(N)}^{(m), \text { ord, }+} / \mathcal{Y}_{n,(i), \widetilde{U}^{p}(N)}^{(m), \text { ord, }}$ as

$$
\coprod_{h \in L_{n,(i), \operatorname{lin}}^{(m)}\left(\mathbb{A}^{\infty}\right)^{\mathrm{ord}, \times} / \widetilde{U}^{p}(N)} \mathcal{S}_{n-i,\left(h \widetilde{U}^{p} h^{-1} \cap \widetilde{G}_{n-i}^{(m+i)}\left(\mathbb{A}^{\infty, p}\right)\right)}^{(m+i)}
$$

for any $N^{\prime} \geq N$. Thus $X_{*}\left(\widetilde{S}_{n,(i), \widetilde{U}^{p}(N)}^{(m), \text { ord }+}\right)_{\mathbb{Z}_{(p)}}$ is a constant sheaf:

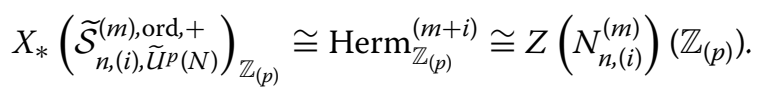

If $\widetilde{g} \in \widetilde{P}_{n,(i)}^{(m),+}\left(\mathbb{A}^{\infty}\right)^{\text {ord }}$ and $\widetilde{g}^{-1} \widetilde{U}^{p}(N) \widetilde{g} \subset\left(\widetilde{U}^{p}\right)^{\prime}\left(N^{\prime}\right)$, then we define

$$
\widetilde{g}: \widetilde{\mathcal{S}}_{n,(i), \widetilde{U}^{p}(N)}^{(m), \text { rd }+} \longrightarrow \widetilde{\mathcal{S}}_{n,(i),\left(\widetilde{U}^{p}\right)^{\prime}\left(N^{\prime}\right)}^{(m), \text { ord }+}
$$

to be the coproduct of the maps

$$
\tilde{g}^{\prime}: \mathcal{S}_{n-i,\left(h \widetilde{U}^{p} h^{-1} \cap \widetilde{G}_{n-i}^{(m+i)}\left(\mathbb{A}^{\infty, p}\right)\right)}^{(m+i)} \longrightarrow \mathcal{S}_{n-i,\left(h^{\prime}\left(\widetilde{U}^{p}\right)^{\prime}\left(h^{\prime}\right)^{-1} \cap \widetilde{G}_{n-i}^{(m+i)}\left(\mathbb{A}^{\infty, p}\right)\right)^{\prime}},
$$


where $h, h^{\prime} \in L_{n,(i) \text { lin }}^{(m)}\left(\mathbb{A}^{\infty}\right)^{\text {ord }}$ and $\widetilde{g}^{\prime} \in \widetilde{G}_{n-i}^{(m+i)}\left(\mathbb{A}^{\infty}\right)^{\text {ord }}$ satisfy $h \widetilde{g}=\widetilde{g}^{\prime} h^{\prime}$. This makes $\left\{\widetilde{\mathcal{S}}_{n,(i), \widetilde{U}^{p}(N)}^{(m),+}\right\}$ a system of relative tori with right $\widetilde{P}_{n,(i)}^{(m),+}\left(\mathbb{A}^{\infty}\right)^{\text {ord }}$-action. If $\gamma$ is an element of $L_{n,(i), \text { lin }}^{(m)}\left(\mathbb{Z}_{(p)}\right)$, then we define

$$
\gamma: \widetilde{\mathcal{S}}_{n,(i), \widetilde{U}^{p}(N)}^{(m),+} \longrightarrow \widetilde{\mathcal{S}}_{n,(i), \widetilde{U}^{p}(N)}^{(m),+}
$$

to be the coproduct of the maps

$$
\gamma: \mathcal{S}_{n-i,\left(h \widetilde{U}^{p} h^{-1} \cap \widetilde{G}_{n-i}^{(m+i)}\left(\mathbb{A}^{\infty, p}\right)\right)}^{(m+i)} \longrightarrow \mathcal{S}_{n-i,\left((\gamma h) \widetilde{U}^{p}(\gamma h)^{-1} \cap \widetilde{G}_{n-i}^{(m+i)}\left(\mathbb{A}^{\infty, p}\right)\right)}^{(m+i)}
$$

This gives a left action of $L_{n,(i), \text { lin }}^{(m)}\left(\mathbb{Z}_{(p)}\right)$ on each $\widetilde{\mathcal{S}}_{n,(i), \widetilde{U}^{p}(N)}^{(m)}$, which commutes with the action of $\widetilde{P}_{n,(i)}^{(m),+}\left(\mathbb{A}^{\infty}\right)^{\text {ord }}$.

The sheaves $X^{*}\left(\widetilde{S}_{n,(i), \widetilde{U}}^{(m),+}\right)$ and $X_{*}\left(\widetilde{S}_{n,(i), \widetilde{U}}^{(m),+}\right)$ have actions of $L_{n,(i), \text { lin }}^{(m)}(\mathbb{Q})$. The sheaves $X^{*}\left(\widetilde{\mathcal{S}}_{n,(i), \widetilde{U}^{p}(N)}^{(m), \text { rdd }+}\right)$ and $X_{*}\left(\widetilde{\mathcal{S}}_{n,(i), \widetilde{U}^{p}(N)}^{(m), \text { rdd }+}\right)$ have actions of the group $L_{n,(i), \text { lin }}^{(m)}\left(\mathbb{Z}_{(p)}\right)$. The systems of sheaves $\left\{X^{*}\left(\widetilde{S}_{n,, i), \widetilde{U}}^{(m),+}\right)\right\}$ and $\left\{X_{*}\left(\widetilde{S}_{n,(i), \widetilde{U}}^{(m),+}\right)\right\}$ (resp. $\left\{X^{*}\left(\widetilde{\mathcal{S}}_{n,(i), \widetilde{U}^{p}(N)}^{(m), \text { ord }+}\right)\right\}$ and $\left.\left\{X_{*}\left(\widetilde{\mathcal{S}}_{n,(i), \widetilde{U}^{p}(N)}^{(m), \text { ord }+}\right)\right\}\right)$ have actions of $\widetilde{P}_{n,(i)}^{(m),+}\left(\mathbb{A}^{\infty}\right)\left(\operatorname{resp} . \widetilde{P}_{n,(i)}^{(m),+}\left(\mathbb{A}^{\infty}\right)^{\text {ord }}\right)$.

The sheaf

$$
\left(X_{*}\left(\widetilde{S}_{n,(i), \widetilde{U}}^{(m),+}\right) \cap \operatorname{Herm}_{F^{m}}\right)=\coprod_{h \in L_{n,(i), \operatorname{lin}}^{(m)}\left(\mathbb{A}^{\infty}\right) / \widetilde{U}}\left(X_{*}\left(\widetilde{S}_{n-i, h \tilde{U} h^{-1} \cap \widetilde{G}_{n-i}^{(m+i)}\left(\mathbb{A}^{\infty}\right)}^{(m+i)}\right) \cap \operatorname{Herm}_{F^{m}}\right)
$$

is a subsheaf of $X_{*}\left(\widetilde{S}_{n,(i), \widetilde{U}}^{(m),+}\right)$. (Recall the embedding

$$
\left.\operatorname{Herm}^{(m)} \cong \operatorname{ker}\left(Z\left(\widetilde{N}_{n,(i)}^{(m)}\right) \rightarrow Z\left(N_{n,(i)}^{(m)}\right)\right) \subset \operatorname{Herm}^{(i+m)} .\right)
$$

It is invariant by the actions of the groups $L_{n,(i), \text { lin }}^{(m)}(\mathbb{Q})$ and $\widetilde{P}_{n,(i)}^{(m),+}\left(\mathbb{A}^{\infty}\right)$. We define a split torus

$$
\widehat{S}_{n,(i), \widetilde{U}}^{(m),+} / Y_{n,(i), \widetilde{U}}^{(m),+}
$$

by

$$
X_{*}\left(\widehat{S}_{n,(i), \widetilde{U}}^{(m),+}\right)=X_{*}\left(\widetilde{S}_{n,(i), \widetilde{U}}^{(m),+}\right) \cap \operatorname{Herm}_{F^{m}}
$$

If $U$ denote the image of $\widetilde{U}$ in $P_{n,(i)}^{(m),+}\left(\mathbb{A}^{\infty}\right)$, then we will write

$$
S_{n,(i), U}^{(m),+}=\widetilde{S}_{n,(i), \widetilde{U}}^{(m),+} / \widehat{S}_{n,(i), \widetilde{U}}^{(m),+}
$$

It depends only on $U$ and not on the choice of $\widetilde{U}$ mapping onto $U$. The sheaf $X_{*}\left(S_{n,(i), U}^{(m),+}\right)_{\mathbb{Q}}$ is constant:

$$
X_{*}\left(S_{n,(i), U}^{(m),+}\right)_{\mathbb{Q}} \cong Z\left(N_{n,(i)}^{(m)}\right)(\mathbb{Q}) .
$$

In the case $m=0$ we will write simply $S_{n,(i), U}^{+}$. The tori $\widetilde{S}_{n,(i), \widetilde{U}}^{(m),+}$ and $S_{n,(i), \widetilde{U}}^{(m),+}$ inherit a left action of $L_{n,(i), \text { lin }}^{(m)}(\mathbb{Q})$ and a right action of $\widetilde{P}_{n,(i)}^{(m),+}\left(\mathbb{A}^{\infty}\right)$. In the case of $S_{n,(i), \widetilde{U}}^{(m),+}$ the latter factors 
through $P_{n,(i)}^{(m),+}\left(\mathbb{A}^{\infty}\right)$. If $\widetilde{U}$ is a neat open compact subgroup of $\widetilde{P}_{n,(i)}^{(m),+}\left(\mathbb{A}^{\infty}\right)$ with image $U$ in $P_{n,(i)}^{(m),+}\left(\mathbb{A}^{\infty}\right)$ and image $U^{\prime}$ in $P_{n,(i)}^{+}\left(\mathbb{A}^{\infty}\right)$, then there is a natural, $L_{n,(i), \text { lin }}^{(m)}(\mathbb{Q})$-equivariant and $\widetilde{P}_{n,(i)}^{(m),+}\left(\mathbb{A}^{\infty}\right)$-equivariant, commutative diagram:

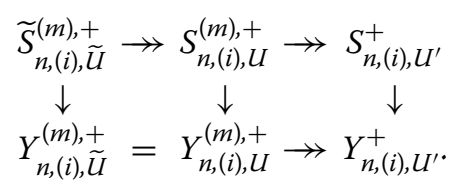

Similarly the sheaf

$$
\begin{aligned}
& \left(X_{*}\left(\widetilde{\mathcal{S}}_{n,(i), \widetilde{U}^{p}(N)}^{(m), \text { ord }+}\right) \cap \operatorname{Herm}_{F^{m}}\right) \\
& \quad=\coprod_{h \in L_{n,(i), \operatorname{lin}}^{(m)}\left(\mathbb{A}^{\infty}\right)^{\text {ord }, \times} / \widetilde{U}^{p}(N)}\left(X_{*}\left(\begin{array}{c}
\widetilde{\mathcal{S}}_{n-i,\left(h \widetilde{U}^{p} h^{-1} \cap \widetilde{G}_{n-i}^{(m+i)}\left(\mathbb{A}^{\infty, p}\right)\right)}^{(m+i) \text { ord }} \\
n
\end{array}\right) \cap \operatorname{Herm}_{F^{m}}\right)
\end{aligned}
$$

is a subsheaf of $X_{*}\left(\widetilde{\mathcal{S}}_{n,(i), \widetilde{U}^{p}(N)}^{(m), \text { ord, }}\right)$. It is invariant by the actions of $L_{n,(i) \text {,lin }}^{(m)}\left(\mathbb{Z}_{(p)}\right)$ and $\widetilde{P}_{n,(i)}^{(m),+}\left(\mathbb{A}^{\infty}\right)^{\text {ord }}$. We define a split torus

$$
\widehat{\mathcal{S}}_{n,(i), \widetilde{U}^{p}(N)}^{(m), \text { ord }+} / \mathcal{Y}_{n,(i), \widetilde{U}^{p}(N)}^{(m), \text { ord }+}
$$

by

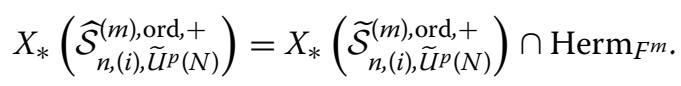

If $U^{p}$ denotes the image of $\widetilde{U}^{p}$ in $P_{n,(i)}^{(m)}\left(\mathbb{A}^{\infty, p}\right)$, then we will write

$$
\mathcal{S}_{n,(i), U^{p}(N)}^{(m), \text { ord, }}=\widetilde{\mathcal{S}}_{n,(i), \widetilde{U}^{p}(N)}^{(m), \text { ord }+} / \widehat{\mathcal{S}}_{n,(i), \widetilde{U}^{p}(N)}^{(m), \text { ord }+}
$$

It depends only on $U^{p}$ and not the $\widetilde{U}^{p}$ mapping to $U^{p}$. The sheaf $X_{*}\left(\mathcal{S}_{n,(i), U^{p}(N)}^{(m), \text { ord, }+}\right)_{\mathbb{Z}_{(p)}}$ is constant:

$$
X_{*}\left(\mathcal{S}_{n,(i), U^{p}(N)}^{(m), \text { ord },+}\right)_{\mathbb{Z}_{(p)}} \cong Z\left(N_{n,(i)}^{(m)}\right)\left(\mathbb{Z}_{(p)}\right) .
$$

In the case $m=0$ we will write simply $\mathcal{S}_{n,(i), U^{p}(N)}^{+}$. The tori $\widehat{\mathcal{S}}_{n,(i), \widetilde{U}^{p}(N)}^{(m), \text { ord, }}$ and $\mathcal{S}_{n,(i), U^{p}(N)}^{(m), \text { ord, }}$ inherit a left action of $L_{n,(i), \text { lin }}^{(m)}\left(\mathbb{Z}_{(p)}\right)$ and a right action of $\widetilde{P}_{n,(i)}^{(m),+}\left(\mathbb{A}^{\infty}\right)^{\text {ord }}$. In the case of $\mathcal{S}_{n,(i), U^{p}(N)}^{(m), \text { ord }+}$ the latter factors through $P_{n,(i)}^{(m),+}\left(\mathbb{A}^{\infty}\right)^{\text {ord }}$. If $\widetilde{U}^{p}$ is a neat open compact subgroup of $\widetilde{P}_{n,(i)}^{(m),+}\left(\mathbb{A}^{\infty, p}\right)$ with image $U^{p}$ in $P_{n,(i)}^{(m),+}\left(\mathbb{A}^{\infty, p}\right)$ and image $\left(U^{p}\right)^{\prime}$ in $P_{n,(i)}^{+}\left(\mathbb{A}^{\infty, p}\right)$, then there is a natural, $L_{n,(i), \text { lin }}^{(m)}\left(\mathbb{Z}_{(p)}\right)$-equivariant and $\widetilde{P}_{n,(i)}^{(m),+}\left(\mathbb{A}^{\infty}\right)^{\text {ord }}$-equivariant, commutative diagram:

$$
\begin{aligned}
& \underset{n,(i), \widetilde{U}^{p}(N)}{\widetilde{\mathcal{S}}_{(m), \text { ord }+}^{(m)}} \rightarrow \mathcal{S}_{n,(i), U^{p}(N)}^{(m), \text { ord }+} \rightarrow \mathcal{S}_{n,(i),\left(U^{p}\right)^{\prime}(N)}^{\mathrm{ord},+} \\
& \downarrow \quad \downarrow \quad \downarrow \\
& \mathcal{Y}_{n,(i), \widetilde{U}^{p}(N)}^{(m), \text { ord, }+}=\mathcal{Y}_{n,(i), U^{p}(N)}^{(m), \text { ord }+} \rightarrow \mathcal{Y}_{n,(i),\left(U^{p}\right)^{\prime}(N)}^{\text {ord }+}
\end{aligned}
$$

There are natural equivariant embeddings

$$
\widetilde{\mathcal{S}}_{n,(i), \widetilde{U}^{p}(N)}^{(m), \text { Spec }} \mathbb{Q} \hookrightarrow \widetilde{S}_{n,(i), \widetilde{U}^{p}(N)}^{(m),+}
$$


and

$$
\mathcal{S}_{n,(i), U^{p}(N)}^{(m), \text { ord }+} \times \operatorname{Spec} \mathbb{Q} \hookrightarrow S_{n,(i), U^{p}(N)}^{(m),+}
$$

and

$$
\widehat{\mathcal{S}}_{n,(i),\left(U^{p}\right)^{\prime}(N)}^{(m), \text { ord }+} \times \operatorname{Spec} \mathbb{Q} \hookrightarrow \widehat{S}_{n,(i),\left(U^{p}\right)^{\prime}(N)}^{(m),}
$$

We write $X_{*}\left(S_{n,(i), U}^{(m),+}\right)_{\mathbb{R}}^{\succ 0}$ (resp. $X_{*}\left(S_{n,(i), U}^{(m),+}\right)_{\mathbb{R}}^{>0}$, resp. $X_{*}\left(S_{n,(i), U}^{(m),+}\right)_{\mathbb{R}}^{\geq 0}$ ) for the subsheaves (of monoids) of $X_{*}\left(S_{n,(i), U}^{(m),+}\right)_{\mathbb{R}}$ corresponding to the subset $\mathfrak{C}^{(m), \succ 0}\left(V_{n,(i)}\right) \subset Z\left(N_{n,(i)}^{(m)}\right)(\mathbb{R})$ (resp. to the subset $\mathfrak{C}^{(m),>0}\left(V_{n,(i)}\right) \subset Z\left(N_{n,(i)}^{(m)}\right)(\mathbb{R})$, resp. to the subset $\mathfrak{C}^{(m), \geq 0}\left(V_{n,(i)}\right) \subset$ $\left.Z\left(N_{n,(i)}^{(m)}\right)(\mathbb{R})\right)$.

We will also write $X^{*}\left(S_{n,(i), U}^{(m),+}\right)_{\mathbb{R}}^{\geq 0}$ (resp. $X^{*}\left(S_{n,(i), U}^{(m),+}\right)_{\mathbb{R}}^{>0}$, resp. $X^{*}\left(S_{n,(i), U}^{(m),+}\right) \geq 0$, resp. $\left.X^{*}\left(S_{n,(i), U}^{(m),+}\right)^{>0}\right)$ for the subsheaves (of monoids) of $X^{*}\left(S_{n,(i), U}^{(m),+}\right)_{\mathbb{R}}\left(\right.$ resp. $X^{*}\left(S_{n,(i), U}^{(m),+}\right)_{\mathbb{R}}$, resp. $X^{*}\left(S_{n,(i), U}^{(m),+}\right)$, resp. $\left.X^{*}\left(S_{n,(i), U}^{(m),+}\right)\right)$ consisting of sections that have non-negative (resp. strictly positive, resp. non-negative, resp. strictly positive) pairing with each nonzero section of $X_{*}\left(S_{n,(i), U}^{(m),+}\right)_{\mathbb{R}}^{\geq 0}$. All these sheaves have (compatible) actions of $L_{n,(i), \text { lin }}^{(m)}(\mathbb{Q})$. The system of sheaves $\left\{X_{*}\left(S_{n,(i), U}^{(m),+}\right)\right\}$ has an action of $P_{n,(i)}^{(m),+}\left(\mathbb{A}^{\infty}\right)$, and the same is true for all the other systems of sheaves we are considering in this paragraph.

We may take the quotients of the sheaves $X^{*}\left(S_{n,(i), U}^{(m),+}\right)$ (resp. $X^{*}\left(S_{n,(i), U}^{(m),+}\right)^{>0}$, resp. $\left.X^{*}\left(S_{n,(i), U}^{(m),+}\right)^{\geq 0}\right)$ by $L_{n,(i), \text { lin }}^{(m)}(\mathbb{Q})$ to give sheaves of sets on $Y_{n,(i), U}^{(m), 4}$, which we will denote $X^{*}\left(S_{n,(i), U}^{(m),+}\right)^{\natural}\left(\operatorname{resp} . X^{*}\left(S_{n,(i), U}^{(m),+}\right)^{>0, \natural}\right.$, resp. $\left.X^{*}\left(S_{n,(i), U}^{(m),+}\right)^{\geq 0, \natural}\right)$. If $y=h U$ lies in $Y_{n,(i), U}^{(m),+}$ above

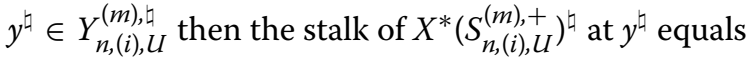

$$
\left\{\gamma \in L_{n,(i), \operatorname{lin}}^{(m)}(\mathbb{Q}): \gamma y=y\right\} \backslash X^{*}\left(\begin{array}{l}
S_{n-i, h U h^{-1} \cap \widetilde{G}_{n-i}^{(m+i)}\left(\mathbb{A}^{\infty}\right)}^{(m+i)} \\
n .
\end{array}\right.
$$

We will write $X_{*}\left(\mathcal{S}_{n,(i), U^{p}(N)}^{(m), \text { ord, }}\right)_{\mathbb{R}}^{\geq 0}$ (resp. $X_{*}\left(\mathcal{S}_{n,(i), U^{p}(N)}^{(m), \text { ord, }+}\right)_{\mathbb{R}}^{>0}$, resp. $\left.X_{*}\left(\mathcal{S}_{n,(i), U^{p}(N)}^{(m), \text { ord }+}\right)_{\mathbb{R}}^{\geq 0}\right)$ for the subsheaves (of monoids) of $X_{*}\left(\mathcal{S}_{n,(i), U^{p}(N)}^{(m), \text { ord }+}\right)_{\mathbb{R}}$ corresponding to

$$
\mathfrak{C}^{(m), \succ 0}\left(V_{n,(i)}\right) \subset Z\left(N_{n,(i)}^{(m)}\right)(\mathbb{R})
$$

(resp.

$$
\mathfrak{C}^{(m),>0}\left(V_{n,(i)}\right) \subset Z\left(N_{n,(i)}^{(m)}\right)(\mathbb{R})
$$

resp.

$$
\left.\mathfrak{C}^{(m), \geq 0}\left(V_{n,(i)}\right) \subset Z\left(N_{n,(i)}^{(m)}\right)(\mathbb{R})\right) .
$$

Again we will write $X^{*}\left(\mathcal{S}_{n,(i), U^{p}(N)}^{(m), \text { ord, }}\right)_{\mathbb{R}}^{\geq 0}$ (resp. $X^{*}\left(\mathcal{S}_{n,(i), U^{p}(N)}^{(m), \text { ord, }+}\right)^{\geq 0}$ ) for the subsheaves (of monoids) of $X^{*}\left(\mathcal{S}_{n,(i), U^{p}(N)}^{(m), \text { ord, }+}\right)_{\mathbb{R}}\left(\right.$ resp. $\left.X^{*}\left(\mathcal{S}_{n,(i), U^{p}(N)}^{(m), \text { ord }+}\right)\right)$ consisting of sections that have nonnegative pairing with each section of $X_{*}\left(\mathcal{S}_{n,(i), U^{p}(N)}^{(m), \text { ord }+}\right)_{\mathbb{R}}^{\geq 0}$. We will also write $X^{*}\left(\mathcal{S}_{n,(i), U^{p}(N)}^{(m), \text { ord, }}\right)_{\mathbb{R}}^{>0}$ (resp. $X^{*}\left(\mathcal{S}_{n,(i), U^{p}(N)}^{(m), \text { ord }+}\right)^{>0}$ ) for the subsheaves (of monoids) of the sheaves $X^{*}\left(\mathcal{S}_{n,(i), U^{p}(N)}^{(m), \text { ord, }}\right)_{\mathbb{R}}$ $\left(\right.$ resp. $\left.X^{*}\left(\mathcal{S}_{n,(i), U^{p}(N)}^{(m), \text { ord, }}\right)\right)$ consisting of sections that have strictly positive pairing with each 
nonzero section of $\left.X_{*}\left(\mathcal{S}_{n,(i), U^{p}(N)}^{(m), \text { ord, }}\right)\right)_{\mathbb{R}}^{\geq 0}$. All these sheaves have actions of $L_{n,(i), \text { lin }}^{(m)}\left(\mathbb{Z}_{(p)}\right)$. The system of sheaves $\left\{X_{*}\left(\mathcal{S}_{n,(i), U^{p}(N)}^{(m), \text { ord }+}\right)\right\}$ has an action of $P_{n,(i)}^{(m),+}\left(\mathbb{A}^{\infty}\right)^{\text {ord }}$, and the same is true for all the other systems of sheaves we are considering in this paragraph.

We may take the quotients of the sheaves $X^{*}\left(\mathcal{S}_{n,(i), U^{p}(N)}^{(m), \text { ord }+}\right)$ and $X^{*}\left(\mathcal{S}_{n,(i), U^{p}(N)}^{(m), \text { ord, }}\right)>0$ and

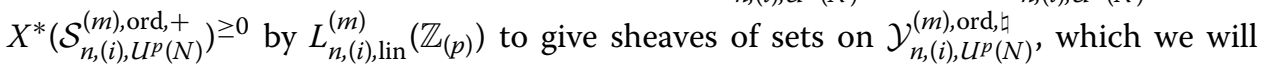
denote $X^{*}\left(\mathcal{S}_{n,(i), U^{p}(N)}^{(m), \text { ord }+}\right)^{\natural}$ and $X^{*}\left(\mathcal{S}_{n,(i), U^{p}(N)}^{(m), \text { ord, }+}\right)^{>0, \natural}$ and $X^{*}\left(\mathcal{S}_{n,(i), U^{p}(N)}^{(m), \text { ord, }+}\right) \geq 0, \natural$.

Suppose again that $\widetilde{U}$ is a neat open compact subgroup of $\widetilde{P}_{n,(i)}^{(m),+}\left(\mathbb{A}^{\infty}\right)$ and set

$$
\widetilde{T}_{n,(i), \widetilde{U}}^{(m),+}=\coprod_{h \in L_{n,(i), \operatorname{lin}}^{(m)}\left(\mathbb{A}^{\infty}\right) / \widetilde{U}} T_{n-i, h \widetilde{U} h^{-1} \cap \widetilde{G}_{n-i}^{(m+i)}\left(\mathbb{A}^{\infty}\right)}^{(m+i)}
$$

It is an $\widetilde{S}_{n,(i), \widetilde{U}}^{(m),+}$-torsor over $A_{n,(i), \widetilde{U}}^{(m),+}$. If $U$ denotes the image of $\widetilde{U}$ in $P_{n,(i)}^{(m)++}\left(\mathbb{A}^{\infty}\right)$ then the push-out of $\widetilde{T}_{n,(i), \widetilde{U}}^{(m),+}$ under $\widetilde{S}_{n,(i), \widetilde{U}}^{(m),+} \rightarrow S_{n,(i), U}^{(m),+}$ is an $S_{n,(i), U}^{(m),+}$-torsor over $A_{n,(i), U}^{(m),+}=A_{n,(i), \widetilde{U}}^{(m),+}$, which only depends on $U$ (and not $\widetilde{U}$ ), and which we will denote $T_{n,(i), U}^{(m),+}$. In the case $m=0$ we will write simply $T_{n,(i), U}^{+}$. Note that $\widetilde{T}_{n,(i), \widetilde{U}}^{(m),+}$ is an $\widehat{S}_{n,(i), \widetilde{U}}^{(m),+}$-torsor over $T_{n,(i), \widetilde{U}}^{(m),+}$.

$$
\text { If } \widetilde{g} \in \widetilde{P}_{n,(i)}^{(m),+}\left(\mathbb{A}^{\infty}\right) \text { and } \widetilde{g}^{-1} \widetilde{U} \widetilde{g} \subset \widetilde{U}^{\prime} \text {, then we define }
$$

$$
\widetilde{g}: \widetilde{T}_{n,(i), \widetilde{U}}^{(m),+} \longrightarrow \widetilde{T}_{n,(i), \widetilde{U}^{\prime}}^{(m),}
$$

to be the coproduct of the maps

$$
\widetilde{g}^{\prime}: \widetilde{T}_{n-i, h \widetilde{U} h^{-1} \cap \widetilde{G}_{n-i}^{(m+i)}\left(\mathbb{A}^{\infty}\right)}^{(m+i)} \longrightarrow \widetilde{T}_{n-i, h^{\prime} \widetilde{U}^{\prime}\left(h^{\prime}\right)^{-1} \cap \widetilde{G}_{n-i}^{(m+i)}\left(\mathbb{A}^{\infty}\right)^{\prime}}^{(m+i)}
$$

where $h, h^{\prime} \in L_{n,(i), \text { lin }}^{(m)}\left(\mathbb{A}^{\infty}\right)$ and $\widetilde{g}^{\prime} \in \widetilde{G}_{n-i}^{(m+i)}\left(\mathbb{A}^{\infty}\right)$ satisfy $h \widetilde{g}=\widetilde{g}^{\prime} h^{\prime}$. This makes $\left\{\widetilde{T}_{n,(i), \widetilde{U}}^{(m),+}\right\}$ a system of $\left\{\widetilde{S}_{n,(i), \widetilde{U}}^{(m),}\right\}$-torsors over $\left\{A_{n,(i), \widetilde{U}}^{(m),+}\right\}$ with right $\widetilde{P}_{n,(i)}^{(m),+}\left(\mathbb{A}^{\infty}\right)$-action. It also induces an action of $P_{n,(i)}^{(m),+}\left(\mathbb{A}^{\infty}\right)$ on $\left\{T_{n,(i), U}^{(m),+}\right\}$, which makes $\left\{T_{n,(i), U}^{(m),+}\right\}$ a system of $\left\{S_{n,(i), U}^{(m),+}\right\}$-torsors over $\left\{A_{n,(i), U}^{(m),+}\right\}$ with right $P_{n,(i)}^{(m),+}\left(\mathbb{A}^{\infty}\right)$-action. If $\gamma \in L_{n,(i), \text { lin }}^{(m)}(\mathbb{Q})$, then we define

$$
\gamma: \widetilde{T}_{n,(i), \widetilde{U}}^{(m)+} \longrightarrow \widetilde{T}_{n,(i), \widetilde{U}}^{(m),+}
$$

to be the coproduct of the maps

$$
\gamma: T_{n-i, h \widetilde{U} h^{-1} \cap \widetilde{G}_{n-i}^{(m+i)}\left(\mathbb{A}^{\infty}\right)}^{(m+i)} \longrightarrow T_{n-i,(\gamma h) \widetilde{U}(\gamma h)^{-1} \cap \widetilde{G}_{n-i}^{(m+i)}\left(\mathbb{A}^{\infty}\right)}^{(m+i)}
$$

This gives a left action of $L_{n,(i) \text {,lin }}^{(m)}(\mathbb{Q})$ on each $\widetilde{T}_{n,(i), \widetilde{U}}^{(m),+}$, which commutes with the action of $\widetilde{P}_{n,(i)}^{(m),+}\left(\mathbb{A}^{\infty}\right)$. It induces a left action of $L_{n,(i), \text { lin }}^{(m)}(\mathbb{Q})$ on each $T_{n,(i), U}^{(m),+}$, which commutes with the action of $P_{n,(i)}^{(m),+}\left(\mathbb{A}^{\infty}\right)$. Suppose that $\widetilde{U}$ is a neat open compact subgroup of $\widetilde{P}_{n,(i)}^{(m),+}\left(\mathbb{A}^{\infty}\right)$ with image $U$ in $P_{n,(i)}^{(m),+}\left(\mathbb{A}^{\infty}\right)$ and image $U^{\prime}$ in $P_{n,(i)}^{+}\left(\mathbb{A}^{\infty}\right)$. Then there is a commutative diagram 


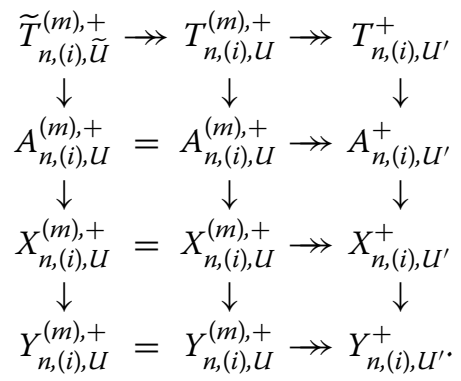

This diagram is $L_{n,(i), \text { lin }}^{(m)}(\mathbb{Q})$-equivariant and $\widetilde{P}_{n,(i)}^{(m),+}\left(\mathbb{A}^{\infty}\right)$-equivariant. We have

$$
T_{n,(i), U}^{(m),+}(\mathbb{C})=P_{n,(i)}^{(m)}(\mathbb{Q}) \backslash\left(P_{n,(i)}^{(m),+}(\mathbb{A}) Z\left(N_{n,(i)}^{(m)}\right)(\mathbb{C})\right) /\left(U U_{n-i, \infty}^{0} A_{n-i}(\mathbb{R})^{0}\right) .
$$

Similarly if $\widetilde{U}^{p}$ is a neat open compact subgroup of $\widetilde{P}_{n,(i)}^{(m),+}\left(\mathbb{A}^{\infty, p}\right)$ and $0 \leq N_{1} \leq N_{2}$ we set

$$
\widetilde{\mathcal{T}}_{n,(i), \widetilde{U}^{p}\left(N_{1}, N_{2}\right)}^{(m) \text { ord }+}=\coprod_{h \in L_{n,(i), \operatorname{lin}}^{(m)}\left(\mathbb{A}^{\infty}\right)^{\text {ord, } \times} / \widetilde{U}^{p}\left(N_{1}, N_{2}\right)} \mathcal{T}_{n-i,\left(h \widetilde{U}^{p} h^{-1} \cap \widetilde{G}_{n-i}^{(m+i)}\left(\mathbb{A}^{\infty, p}\right)\right)\left(N_{1}, N_{2}\right)}^{(m+i) \text { ord }}
$$

It is an $\widetilde{\mathcal{S}}_{n,(i), \widetilde{U}^{p}\left(N_{1}\right)}^{(m), \text { torsor }+}$ over $\mathcal{A}_{n,(i), \widetilde{U}^{p}\left(N_{1}, N_{2}\right)}^{(m), \text { If }}$. If $U^{p}$ denotes the image of $\widetilde{U}^{p}$ in $P_{n,(i)}^{(m),+}\left(\mathbb{A}^{\infty, p}\right)$ then the push-out of $\widetilde{\mathcal{T}}_{n,(i), \widetilde{U}^{p}\left(N_{1}, N_{2}\right)}^{(m), \text { ord, }}$ under $\widetilde{\mathcal{S}}_{n,(i), \widetilde{U}^{p}\left(N_{1}\right)}^{(m), \text { ord },} \rightarrow \mathcal{S}_{n,(i), U^{p}\left(N_{1}\right)}^{(m), \text { ord },+}$ is an $\mathcal{S}_{n,(i), U^{p}\left(N_{1}\right)}^{(m), \text { ord, }}$-torsor over $\mathcal{A}_{n,(i), U^{p}\left(N_{1}, N_{2}\right)}^{(m), \text { ord, }}$, which only depends on $U^{p}$ (and not $\widetilde{U}^{p}$ ) and

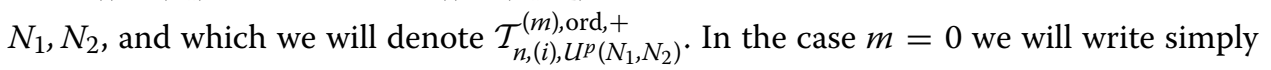
$\mathcal{T}_{n,(i), U^{p}\left(N_{1}, N_{2}\right)}^{\text {ord, Note that }} \widetilde{\mathcal{T}}_{n,(i), \widetilde{U}^{p}\left(N_{1}, N_{2}\right)}^{(m), \text { ord }+}$ is a $\widehat{\mathcal{S}}_{n,(i), \widetilde{U}^{p}\left(N_{1}\right)}^{(m) \text {,ord }+}$-torsor over $\mathcal{T}_{n,(i), \widetilde{U}^{p}\left(N_{1}, N_{2}\right)}^{(m) \text { ord, }}$.

As above the system $\left\{\widetilde{\mathcal{T}}_{n,(i), \widetilde{U}^{p}\left(N_{1}, N_{2}\right)}^{(m), \text { ord },}\right\}$ has a right action of $\widetilde{P}_{n,(i)}^{(m),+}\left(\mathbb{A}^{\infty}\right)^{\text {ord }}$ and a commuting left action of $L_{n,(i), \text { lin }}^{(m)}\left(\mathbb{Z}_{(p)}\right)$. If $g \in \widetilde{P}_{n,(i)}^{(m),+}\left(\mathbb{A}^{\infty}\right)^{\text {ord, } \times}$ then the map $g$ is finite etale. The map

$$
\varsigma_{p}: \widetilde{\mathcal{T}}_{n,(i), \widetilde{U}^{p}\left(N_{1}, N_{2}\right)}^{(m), \text { Spd, }} \times \operatorname{Spec} \mathbb{F}_{p} \longrightarrow \widetilde{\mathcal{T}}_{n,(i), \widetilde{U}^{p}\left(N_{1}, N_{2}-1\right)}^{(m), \text { rd },} \times \operatorname{Spec} \mathbb{F}_{p}
$$

equals absolute Frobenius composed with the forgetful map. If $N_{2}>1$ then the map

$$
\varsigma_{p}: \widetilde{\mathcal{T}}_{n,(i), \widetilde{U}^{p}\left(N_{1}, N_{2}\right)}^{(m), r_{d},+} \longrightarrow \widetilde{\mathcal{T}}_{n,(i), \widetilde{U}^{p}\left(N_{1}, N_{2}-1\right)}^{(m), \text { ord }+}
$$

is finite flat. Further there is a left action of $G L_{m}\left(\mathcal{O}_{F,(p)}\right)$ such that if $\delta \in G L_{m}\left(\mathcal{O}_{F,(p)}\right)$ and $\gamma \in L_{n,(i) \text { lin }}^{(m)}\left(\mathbb{Z}_{(p)}\right)$ and $g \in \widetilde{P}_{n,(i)}^{(m),+}\left(\mathbb{A}^{\infty}\right)^{\text {ord }}$, then $\gamma$ followed by $\delta$ equals $\delta$ followed by $\delta \gamma \delta^{-1}$, and $g$ followed by $\delta$ equals $\delta$ followed by $\delta g \delta^{-1}$. These actions are also all compatible with the actions on $\left\{\widetilde{\mathcal{S}}_{n,(i), \widetilde{U}^{p}\left(N_{1}\right)}^{(m), \text { ord, }}\right\}$. There are induced actions of the groups $G L_{m}\left(\mathcal{O}_{F,(p)}\right)$ and $L_{n,(i), \text { lin }}^{(m)}\left(\mathbb{Z}_{(p)}\right)$ and $P_{n,(i)}^{(m),+}\left(\mathbb{A}^{\infty}\right)^{\text {ord }}$ on $\left\{\mathcal{T}_{n,(i), U^{p}\left(N_{1}, N_{2}\right)}^{(m), \text {,rd, }}\right\}$ which are compatible with the actions on $\left\{\mathcal{S}_{n,(i), U^{p}\left(N_{1}\right)}^{(m), \text { odd }+}\right\}$. There is an equivariant commutative diagram 


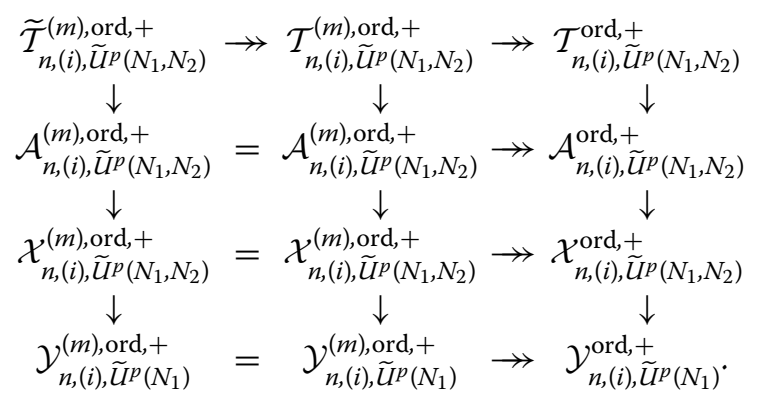

There are natural equivariant embeddings

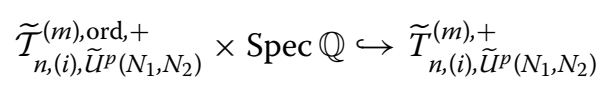

and

$$
\mathcal{T}_{n,(i), U^{p}\left(N_{1}, N_{2}\right)}^{(m), \text { rdd }+} \times \operatorname{Spec} \mathbb{Q} \hookrightarrow T_{n,(i), U^{p}\left(N_{1}, N_{2}\right)}^{(m),+}
$$

If $a$ is a section of $X^{*}\left(S_{n,(i), U}^{(m),+}\right)$ over $W \subset Y_{n,(i), U}^{(m)}$ then we can associate to it a line bundle

$$
\mathcal{L}_{U}^{+}(a)
$$

$\left.\operatorname{over} A_{n,(i), U}^{(m),+}\right|_{W}$ as in Sect. 2.1. There are natural isomorphisms

$$
\mathcal{L}_{U}^{+}(a) \otimes \mathcal{L}_{U}^{+}\left(a^{\prime}\right) \cong \mathcal{L}_{U}^{+}\left(a+a^{\prime}\right)
$$

Suppose that $R_{0}$ is a noetherian $\mathbb{Q}$-algebra. Suppose also that $U$ is a neat open compact subgroup of $P_{n,(i)}^{+}\left(\mathbb{A}^{\infty}\right)$. If $a$ is a section in $X^{*}\left(S_{n,(i), U}^{+}\right)^{>0}(W)$ then $\mathcal{L}_{U}^{+}(a)$ is relatively ample for $\left.A_{n,(i), U}^{+}\right|_{W} /\left.X_{n,(i), U}^{+}\right|_{W}$. If $\pi^{+}$denotes the map

$$
\left.A_{n,(i), U}^{+}\right|_{W} \times\left.\operatorname{Spec} R_{0} \longrightarrow X_{n,(i), U}^{+}\right|_{W} \times \operatorname{Spec} R_{0}
$$

then we see that

$$
R^{i} \pi_{*}^{+} \mathcal{L}_{U}^{+}(a)=(0)
$$

for $i>0$. (Because $A_{n,(i), U}^{+}\left|W / X_{n,(i), U}^{+}\right| W$ is a torsor for an abelian scheme and $\mathcal{L}_{U}^{+}(a)$ is relatively ample for this morphism.) We will denote by $\left(\pi_{A^{+} / X^{+}, *} \mathcal{L}\right)_{U}^{+}(a)$ the image $\pi_{*}^{+} \mathcal{L}_{U}^{+}(a)$. Suppose further that $\mathcal{F}$ is a locally free sheaf on $X_{n,(i), U}^{+} \times \operatorname{Spec} R_{0}$ with $L_{n,(i), \text { lin }}(\mathbb{Q})$-action. If $a^{\natural}$ is a section of $X^{*}\left(S_{n,(i), U}^{+}\right)^{>0, \natural}$ we will define

$$
\left(\pi_{A^{+} / X^{\natural}, *} \mathcal{L} \otimes \mathcal{F}\right)_{U}^{+}\left(a^{\natural}\right)
$$

as follows: Over a point $y^{\natural}$ of $Y_{n,(i), U}^{\natural}$ we take the sheaf

$$
\prod_{y, a}\left(\pi_{A^{+} / X^{+}, *} \mathcal{L}\right)_{U}^{+}(a)_{y} \otimes \mathcal{F}_{y}
$$

over $X_{n,(i), U, y^{\natural}}^{\natural} \times \operatorname{Spec} R_{0}$, where $y$ runs over points of $Y_{n,(i), U}^{+}$above $y^{\natural}$ and $a$ runs over sections of $X^{*}\left(S_{n,(i), U}^{+}\right)_{y}$ above $a^{\natural}$. It is a sheaf with an action of $L_{n,(i), \text { lin }}(\mathbb{Q})$. 
Lemma 4.2 Keep the notation and assumptions of the previous paragraph.

(1)

$$
\left(\pi_{A^{+} / X^{\natural}, *} \mathcal{L} \otimes \mathcal{F}\right)_{U}^{+}\left(a^{\natural}\right) \cong \operatorname{Ind}_{\{1\}}^{L_{n,(i), \operatorname{lin}}(\mathbb{Q})}\left(\left(\pi_{A^{+} / X^{\natural}, *} \mathcal{L} \otimes \mathcal{F}\right)_{U}^{+}\left(a^{\natural}\right)^{L_{n,(i), \operatorname{lin}}(\mathbb{Q})}\right)
$$

as a sheaf on $X_{n,(i), U}^{\natural} \times \operatorname{Spec} R_{0}$ with $L_{n,(i), \operatorname{lin}}(\mathbb{Q})$-action.

(2) If

$$
\pi: A_{n,(i), U}^{+} \times \operatorname{Spec} R_{0} \longrightarrow X_{n,(i), U}^{\natural} \times \operatorname{Spec} R_{0}
$$

then

$$
\begin{aligned}
& R^{i} \pi_{*} \prod_{a \in X^{*}\left(S_{n, i(i),}^{+}\right)^{>0}\left(\mathcal{L}_{U}^{+}(a) \otimes \mathcal{F}\right)} \\
& \quad \cong \begin{cases}\prod_{a^{\natural} \in X^{*}\left(S_{n,(i), U}^{+}\right.}^{+>0, \sharp}\left(\pi_{A^{+} / X^{\natural}, *} \mathcal{L} \otimes \mathcal{F}\right)_{U}^{+}\left(a^{\natural}\right) & \text { if } i=0 \\
(0) & \text { otherwise. }\end{cases}
\end{aligned}
$$

Proof For the first part note that if $y$ in $Y_{n,(i), U}^{+}$and if $a \in X^{*}\left(S_{n,(i), U}^{+}\right)_{y}^{>0}$ then the stabilizer of $a$ in $\left\{\gamma \in L_{n,(i)}(\mathbb{Q}): \gamma y=y\right\}$ is finite, and that if $U$ is neat then it is trivial. The second part follows from the observations of the previous paragraph together with proposition 0.13.3.1 of [23].

Similarly if $a$ is a section of $X^{*}\left(\mathcal{S}_{n,(i), U^{p}\left(N_{1}\right)}^{(m), \text { ord, }}\right)$ over $W \subset \mathcal{Y}_{n,(i), U^{p}\left(N_{1}\right)}^{(m), \text { ord, }}$ then we can associate to it a line bundle

$$
\mathcal{L}_{U^{p}\left(N_{1}, N_{2}\right)}^{+}(a)
$$

over $\mathcal{A}_{n,(i), U^{p}\left(N_{1}, N_{2}\right)}^{(m), \text { Wrd },} \mid$ There are natural isomorphisms

$$
\mathcal{L}_{U^{p}\left(N_{1}, N_{2}\right)}^{+}(a) \otimes \mathcal{L}_{U^{p}\left(N_{1}, N_{2}\right)}^{+}\left(a^{\prime}\right) \cong \mathcal{L}_{U^{p}\left(N_{1}, N_{2}\right)}^{+}\left(a+a^{\prime}\right)
$$

Suppose that $R_{0}$ is a noetherian $\mathbb{Z}_{(p)}$-algebra. Suppose that $U^{p}$ is a neat open compact subgroup of $P_{n,(i)}^{+}\left(\mathbb{A}^{\infty, p}\right)$ and that $0 \leq N_{1} \leq N_{2}$. If $a$ is a section in $X^{*}\left(\mathcal{S}_{n,(i), U^{p}\left(N_{1}\right)}^{+}\right)^{>0}(W)$

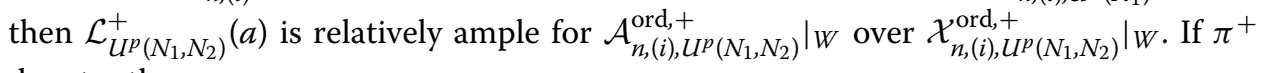
denotes the map

$$
\left.\mathcal{A}_{n,(i), U^{p}\left(N_{1}, N_{2}\right)}^{\mathrm{ord},+}\right|_{W} \times\left.\operatorname{Spec} R_{0} \longrightarrow \mathcal{X}_{n,(i), U^{p}\left(N_{1}, N_{2}\right)}^{\mathrm{ord},+}\right|_{W} \times \operatorname{Spec} R_{0}
$$

then we see that

$$
R^{i} \pi_{*}^{+} \mathcal{L}_{U^{p}\left(N_{1}, N_{2}\right)}^{+}(a)=(0)
$$

for $i>0$. (Again because $\mathcal{A}_{n,(i), U^{p}\left(N_{1}, N_{2}\right)}^{\text {ord, }}\left|W / \mathcal{X}_{n,(i), U^{p}\left(N_{1}, N_{2}\right)}^{\mathrm{ord}}\right| W$ is a torsor for an abelian scheme and $\mathcal{L}_{U^{p}\left(N_{1}, N_{2}\right)}^{+}(a)$ is relatively ample for this morphism.) We will denote by $\left(\pi_{\mathcal{A}^{\text {ord },+} / \mathcal{X}^{\text {ord },+},{ }_{*}} \mathcal{L}\right)_{U^{p}\left(N_{1}, N_{2}\right)}^{+}(a)$ the image $\pi_{*}^{+} \mathcal{L}^{+}(a)$. Suppose further that $\mathcal{F}$ is a locally 
free sheaf on $\mathcal{X}_{n,(i), U^{p}\left(N_{1}, N_{2}\right)}^{\text {ord, }} \times \operatorname{Spec} R_{0}$ with $L_{n,(i), \text { lin }}\left(\mathbb{Z}_{(p)}\right)$-action. If $a^{\natural}$ is a section of $X^{*}\left(\mathcal{S}_{n,(i), U^{p}\left(N_{1}\right)}^{\text {ord, }}\right)^{>0, \llbracket}$ we define a sheaf

$$
\left(\pi_{\mathcal{A}^{\mathrm{ord},+} / \mathcal{X}^{\mathrm{ord}, \sharp, *}} \mathcal{L} \otimes \mathcal{F}\right)_{U^{p}\left(N_{1}, N_{2}\right)}^{+}\left(a^{\natural}\right)
$$

as follows: Over a point $y^{\natural}$ of $\mathcal{Y}_{n,(i), U^{p}\left(N_{1}, N_{2}\right)}^{\text {ord, we take the sheaf }}$

$$
\prod_{y, a}\left(\pi_{\mathcal{A}^{\mathrm{ord},+} / \mathcal{X}^{\mathrm{ord},+, *}} \mathcal{L}\right)_{U^{p}\left(N_{1}, N_{2}\right)}^{+}(a)_{y} \otimes \mathcal{F}_{y}
$$

over $\mathcal{X}_{n,(i), U^{p}\left(N_{1}, N_{2}\right), y^{\natural}}^{\text {ord },} \times$ Spec $R_{0}$, where $y$ runs over points of $\mathcal{Y}_{n,(i), U^{p}\left(N_{1}, N_{2}\right)}^{\text {ord, }}$ above $y^{\natural}$ and $a$ runs over sections of $X^{*}\left(\mathcal{S}_{n,(i), U^{p}\left(N_{1}\right)}^{\text {ord, }}\right)_{y}$ above $a^{\natural}$. It is a sheaf with an action of $L_{n,(i), \operatorname{lin}}\left(\mathbb{Z}_{(p)}\right)$. As above we have the following lemma.

Lemma 4.3 Keep the notation and assumptions of the previous paragraph.

(1)

(2) If

$$
\begin{aligned}
& \left(\pi_{\mathcal{A}^{\text {ord },+} / \mathcal{X}^{\text {ord }, \natural, *}} \mathcal{L} \otimes \mathcal{F}\right)_{U^{p}\left(N_{1}, N_{2}\right)}^{+}\left(a^{\natural}\right) \\
& \cong \operatorname{Ind}_{\{1\}}^{L_{n,(i), \operatorname{lin}}\left(\mathbb{Z}_{(p)}\right)}\left(\left(\pi_{\mathcal{A}^{\text {ord },+} / \mathcal{X}^{\text {ord },+, *}} \mathcal{L} \otimes \mathcal{F}\right)_{U^{p}\left(N_{1}, N_{2}\right)}^{+}\left(a^{\natural}\right)^{L_{n,(i), \operatorname{lin}}\left(\mathbb{Z}_{(p)}\right)}\right)
\end{aligned}
$$

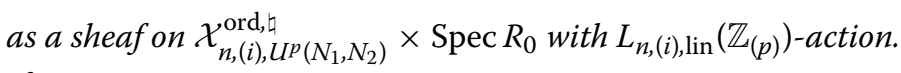

If

$$
\pi: \mathcal{A}_{n,(i), U^{p}\left(N_{1}, N_{2}\right)}^{\mathrm{ord},+} \times \operatorname{Spec} R_{0} \longrightarrow \mathcal{X}_{n,(i), U^{p}\left(N_{1}, N_{2}\right)}^{\mathrm{ord}, \natural} \times \operatorname{Spec} R_{0}
$$

then

$$
R^{i} \pi_{*} \prod_{a \in X^{*}\left(\mathcal{S}_{n,(i), U^{p}\left(N_{1}\right)}^{\mathrm{ord},+}\right)}^{>0}\left(\mathcal{L}_{U^{p}\left(N_{1}, N_{2}\right)}^{+}(a) \otimes \mathcal{F}\right)
$$

is isomorphic to

$$
\begin{aligned}
& \prod_{a^{\natural} \in X^{*}}\left(\mathcal{S}_{n,(i), U^{p}\left(N_{1}\right)}^{\mathrm{ord},+}\right)^{>0, \natural} \\
& \text { if } i=0 \text {, and otherwise is }(0) .
\end{aligned}
$$

\subsection{Partial compactifications}

We will now turn to the partial compactification of the generalized Shimura varieties, $T_{n,(i), U}^{(m)}$, we discussed in the last section. These will serve as models for the full compactification of the $A_{n, U}^{(m)}$, which near the boundary can be formally modelled on the partial compactifications of the $T_{n,(i), U}^{(m)}$.

Suppose that $U$ (resp. $U^{p}$ ) is a neat open compact subgroup of $L_{n,(i), \operatorname{lin}}^{(m)}\left(\mathbb{A}^{\infty}\right)$ (resp. $\left.L_{n,(i), \text { lin }}^{(m)}\left(\mathbb{A}^{\infty, p}\right)\right)$ and that $N$ is a non-negative integer. By an admissible cone decomposition 
$\Sigma_{0}$ for $X_{*}\left(S_{n,(i), U}^{(m),+}\right)_{\mathbb{R}}^{\succ 0}\left(\operatorname{resp} . X_{*}\left(\mathcal{S}_{n,(i), U^{p}(N)}^{(m), \text { ord }+}\right)_{\mathbb{R}}^{\succ 0}\right)$ we shall mean a partial fan $\Sigma_{0}$ in $X_{*}\left(S_{n,(i), U}^{(m),+}\right)_{\mathbb{R}}$ (resp. $\left.X_{*}\left(\mathcal{S}_{n,(i), U^{p}(N)}^{(m), \text { ord }+}\right) \mathbb{R}\right)$ such that

- $\left|\Sigma_{0}\right|=X_{*}\left(S_{n,(i), U}^{(m),+}\right)_{\mathbb{R}}^{\succ 0}\left(\operatorname{resp} . X_{*}\left(\mathcal{S}_{n,(i), U^{p}(N)}^{(m), \text { ord }+}\right)_{\mathbb{R}}^{\succ 0}\right)$;

- $\left|\Sigma_{0}\right|^{0}=X_{*}\left(S_{n,(i), U}^{(m),+}\right)_{\mathbb{R}}^{>0}\left(\right.$ resp. $\left.X_{*}\left(\mathcal{S}_{n,(i), U^{p}(N)}^{(m), \text { ord }+}\right)_{\mathbb{R}}^{>0}\right)$;

- $\Sigma_{0}$ is invariant under the left action of $L_{n,(i), \text { lin }}^{(m)}(\mathbb{Q})$ (resp. $\left.L_{n,(i), \text { lin }}^{(m)}\left(\mathbb{Z}_{(p)}\right)\right)$;

- $L_{n,(i), \text { lin }}^{(m)}(\mathbb{Q}) \backslash \Sigma_{0}$ (resp. $\left.L_{n,(i), \text { lin }}^{(m)}\left(\mathbb{Z}_{(p)}\right) \backslash \Sigma_{0}\right)$ is a finite set;

- if $\sigma \in \Sigma_{0}$ and $1 \neq \gamma \in L_{n,(i), \text { lin }}^{(m)}(\mathbb{Q})\left(\operatorname{resp} . L_{n,(i), \text { lin }}^{(m)}\left(\mathbb{Z}_{(p)}\right)\right)$ then

$$
\sigma \cap \gamma \sigma \notin \Sigma_{0}
$$

(Many authors would not include the last condition in the definition of an 'admissible cone decomposition'.) In concrete terms $\Sigma_{0}$ consists of a partial fan $\Sigma_{g, 0}$ in $Z\left(N_{n,(i)}^{(m)}\right)(\mathbb{R})$ for each $g \in L_{n,(i), \text { lin }}^{(m)}\left(\mathbb{A}^{\infty}\right)\left(\operatorname{resp} . L_{n,(i), \text { lin }}^{(m)}\left(\mathbb{A}^{\infty}\right)^{\text {ord } \times}\right)$, such that

- $\Sigma_{\gamma g u, 0}=\gamma \Sigma_{g, 0}$ for all $\gamma \in L_{n,(i), \text { lin }}^{(m)}(\mathbb{Q})\left(\operatorname{resp} . L_{n,(i), \text { lin }}^{(m)}\left(\mathbb{Z}_{(p)}\right)\right)$ and $u \in U\left(\right.$ resp. $\left.U^{p}(N)\right)$;

- $\left|\Sigma_{g, 0}\right|=\mathfrak{C}^{(m), \succ 0}\left(V_{n,(i)}\right)$ and $\left|\Sigma_{g, 0}\right|^{0}=\mathfrak{C}^{(m),>0}\left(V_{n,(i)}\right)$ for each $g$;

- $\left(L_{n,(i), \text { lin }}^{(m)}(\mathbb{Q}) \cap g U g^{-1}\right) \backslash \Sigma_{g, 0}\left(\operatorname{resp} .\left(L_{n,(i), \text { lin }}^{(m)}\left(\mathbb{Z}_{(p)}\right) \cap g U^{p}(N) g^{-1}\right) \backslash \Sigma_{g}\right)$ is finite for all $g$;

- for each $g$ and each $\sigma \in \Sigma_{g, 0}$, if $1 \neq \gamma \in\left(L_{n,(i), \operatorname{lin}}^{(m)}(\mathbb{Q}) \cap g U g^{-1}\right)\left(\operatorname{resp} .\left(L_{n,(i), \operatorname{lin}}^{(m)}\left(\mathbb{Z}_{(p)}\right) \cap\right.\right.$ $\left.\left.g U^{p}(N) g^{-1}\right)\right)$ then

$$
\sigma \cap \gamma \sigma \notin \Sigma_{g, 0}
$$

Note that an admissible cone decomposition $\Sigma_{0}$ for $X_{*}\left(S_{n,(i), U^{p}(N)}^{(m),+}\right)_{\mathbb{R}}^{\succ 0}$ induces (by restriction) one, which we will denote $\Sigma_{0}^{\text {ord }}$, for $X_{*}\left(\mathcal{S}_{n,(i), U^{p}(N)}^{(m),{ }^{\circ}}\right)_{\mathbb{R}}^{\succ 0}$. This sets up a bijection between admissible cone decompositions for $X_{*}\left(S_{n,(i), U^{p}(N)}^{(m),}\right)_{\mathbb{R}}^{\succ 0}$ and for $X_{*}\left(\mathcal{S}_{n,(i), U^{p}(N)}^{(m), \text { ord },+}\right)_{\mathbb{R}}^{\succ 0}$.

Lemma 4.4 Suppose that $U$ (resp. $U^{p}$ ) is a neat open compact subgroup of the group $L_{n,(i), \text { lin }}^{(m)}\left(\mathbb{A}^{\infty}\right)$ (resp. $\left.L_{n,(i), \text { lin }}^{(m)}\left(\mathbb{A}^{\infty}\right)\right)$ and that $N$ is a non-negative integer. Suppose also that $\Sigma_{0}$ is an admissible cone decomposition for $X_{*}\left(S_{n,(i), U}^{(m),+}\right)_{\mathbb{R}}^{\succ 0}\left(\right.$ resp. for $\left.X_{*}\left(\mathcal{S}_{n,(i), U^{p}(N)}^{(m), \text { ord },+}\right)_{\mathbb{R}}^{\succ 0}\right)$. Also suppose that $\tau \subset\left|\Sigma_{0}\right|$ is a rational polyhedral cone. Then the set

$$
\left\{\gamma \in L_{n,(i), \operatorname{lin}}^{(m)}(\mathbb{Q}): \gamma \tau \cap \tau \cap\left|\Sigma_{0}\right|^{0} \neq \emptyset\right\}
$$

(resp.

$$
\left.\left\{\gamma \in L_{n,(i), \operatorname{lin}}^{(m)}\left(\mathbb{Z}_{(p)}\right): \gamma \tau \cap \tau \cap\left|\Sigma_{0}\right|^{0} \neq \emptyset\right\}\right)
$$

is finite.

Proof We treat the case of $X_{*}\left(S_{n,(i), U}^{(m),+}\right)_{\mathbb{R}}^{\succ 0}$, the other being exactly similar. Suppose that $\tau$ has support $y=h U$ and set $\Gamma=L_{n,(i), \operatorname{lin}}^{(m)}(\mathbb{Q}) \cap h U h^{-1}$ a discrete subgroup of $L_{n,(i), \operatorname{lin}}^{(m)}(\mathbb{Q})$. We certainly have

$$
\left\{\gamma \in L_{n,(i), \operatorname{lin}}^{(m)}(\mathbb{Q}): \gamma \tau \cap \tau \cap\left|\Sigma_{0}\right|^{0} \neq \emptyset\right\}=\left\{\gamma \in \Gamma: \gamma \tau \cap \tau \cap\left|\Sigma_{0}\right|^{0}(y) \neq \emptyset\right\} .
$$

That this set is finite follows from theorem II.4.6 and the remark (ii) at the end of section II.4.1 of [3]. 
Corollary 4.5 If $\Sigma_{0}$ is an admissible cone decomposition for $X_{*}\left(S_{n,(i), U}^{(m),+}\right)_{\mathbb{R}}^{\succ 0}$ or for $X_{*}\left(\mathcal{S}_{n,(i), U^{p}(N)}^{(m), \text { ord }+}\right)_{\mathbb{R}}^{\succ 0}$, then $\Sigma_{0}$ is locally finite.

Proof Let $\tau \subset\left|\Sigma_{0}\right|$ be a rational polyhedral cone. Let $\sigma_{1}, \ldots, \sigma_{r}$ be representatives for $L_{n,(i) \text {,lin }}^{(m)}\left(\mathbb{A}^{\infty}\right) \backslash \Sigma_{0}$ (resp. $\left.L_{n,(i), \text { lin }}^{(m)}\left(\mathbb{Z}_{(p)}\right) \backslash \Sigma_{0}\right)$; and suppose they are chosen with the same support as $\tau$ whenever possible. Let $\tau^{\prime}$ be the rational polyhedral cone spanned by $\tau$ and those $\sigma_{i}$ with the same support as $\tau$. If $\gamma \in L_{n,(i), \text { lin }}^{(m)}\left(\mathbb{A}^{\infty}\right)\left(\operatorname{resp} . L_{n,(i), \text { lin }}^{(m)}\left(\mathbb{Z}_{(p)}\right)\right)$ and

$$
\gamma \sigma_{i} \cap \tau \cap\left|\Sigma_{0}\right|^{0} \neq \emptyset
$$

then

$$
\gamma \tau^{\prime} \cap \tau^{\prime} \cap\left|\Sigma_{0}\right|^{0} \neq \varnothing
$$

and so by the previous lemma $\gamma$ lies in a finite set. The corollary follows.

If $g \in P_{n,(i)}^{(m),+}\left(\mathbb{A}^{\infty}\right)$, if $U^{\prime} \supset g^{-1} U g$ are neat open compact subgroups of the group $P_{n,(i)}^{(m),+}\left(\mathbb{A}^{\infty}\right)$ and if $\Sigma_{0}^{\prime}$ is a $U^{\prime}$-admissible cone decomposition for $X_{*}\left(S_{n,(i), U^{\prime}}^{(m),+}\right)_{\mathbb{R}}^{\succ 0}$, then $\Sigma_{0}^{\prime} g^{-1}$ is a $U$-admissible cone decomposition for $X_{*}\left(S_{n,(i), U}^{(m),+}\right)_{\mathbb{R}}^{\succ 0}$. We will call a $U$-admissible cone decomposition $\Sigma_{0}$ for $X_{*}\left(S_{n,(i), U}^{(m),+}\right)_{\mathbb{R}}^{\succ 0}$ compatible with $\Sigma_{0}^{\prime}$ with respect to $g$ if $\Sigma_{0}$ refines $\Sigma_{0}^{\prime} g^{-1}$. Similarly if $g \in P_{n,(i)}^{(m),+}\left(\mathbb{A}^{\infty}\right)^{\text {ord }}$, if $\left(U^{p}\right)^{\prime}\left(N^{\prime}\right) \supset\left(g^{-1} U^{p} g\right)(N)$ and if $\Sigma_{0}^{\prime}$ is a $\left(U^{p}\right)^{\prime}\left(N^{\prime}\right)$-admissible cone decomposition for $X_{*}\left(\mathcal{S}_{n,(i),\left(U^{p}\right)^{\prime}\left(N^{\prime}\right)}^{(m)}\right)_{\mathbb{R}}^{\succ 0}$, then $\left(\Sigma^{\prime} g^{-1}, \Sigma_{0}^{\prime} g^{-1}\right)$ is an admissible cone decomposition for $X_{*}\left(\mathcal{S}_{n,(i), U^{p}(N)}^{(m), \text { ord, }}\right)_{\mathbb{R}}^{\succ 0}$. We will call a $U^{p}(N)$-admissible cone decomposition $\Sigma_{0}$ for $X_{*}\left(\mathcal{S}_{n,(i), U^{p}(N)}^{\left.(m),{ }^{\prime}\right)}\right)_{\mathbb{R}}^{\succ 0}$ compatible with $\Sigma_{0}^{\prime}$ with respect to $g$ if $\Sigma_{0}$ refines $\Sigma_{0}^{\prime} g^{-1}$.

If $U^{\prime}$ is a neat open compact subgroup of $P_{n,(i)}^{+}\left(\mathbb{A}^{\infty}\right)$ which contains the image of $U$, we will call an admissible cone decomposition $\Sigma_{0}$ of $X_{*}\left(S_{n,(i), U}^{(m),+}\right)_{\mathbb{R}}^{\succ 0}$ and an admissible cone decomposition $\Delta_{0}$ of $X_{*}\left(S_{n,(i), U^{\prime}}^{+}\right)_{\mathbb{R}}^{\succ 0}$ compatible if, under the natural map

$$
X_{*}\left(S_{n,(i), U}^{(m),+}\right)_{\mathbb{R}}^{\succ 0} \rightarrow X_{*}\left(S_{n,(i), U^{\prime}}^{+}\right)_{\mathbb{R}}^{\succ 0}
$$

the image of each $\sigma \in \Sigma_{0}$ is contained in some element of $\Delta_{0}$. Similarly if $\left(U^{p}\right)^{\prime}$ is a neat open compact subgroup of $P_{n,(i)}^{+}\left(\mathbb{A}^{\infty, p}\right)$ which contains the image of $U^{p}$ and if $N^{\prime} \geq N$, we will call an admissible cone decomposition $\Sigma_{0}$ of $X_{*}\left(\mathcal{S}_{n,(i), U^{p}(N)}^{(m),+}\right)_{\mathbb{R}}^{\succ 0}$ and an admissible cone decomposition $\Delta_{0}$ of $X_{*}\left(\mathcal{S}_{n,(i),\left(U^{p}\right)^{\prime}\left(N^{\prime}\right)}^{\text {ord, }}\right)_{\mathbb{R}}^{\succ 0}$ compatible if, under the natural map

$$
X_{*}\left(\mathcal{S}_{n,(i), U^{p}(N)}^{(m), \text { ord }+}\right)_{\mathbb{R}}^{\succ 0} \rightarrow X_{*}\left(\mathcal{S}_{n,(i),\left(U^{p}\right)^{\prime}\left(N^{\prime}\right)}^{\text {ord }+}\right)_{\mathbb{R}}^{\succ 0}
$$

the image of each $\sigma \in \Sigma_{0}$ is contained in some element of $\Delta_{0}$.

If $\Sigma_{0}$ is a smooth admissible cone decomposition for $X_{*}\left(S_{n,(i), U}^{(m),+}\right)_{\mathbb{R}}^{\succ 0}$ (resp. for $\left.X_{*}\left(\mathcal{S}_{\left.n,(i), U^{p}\left(N_{1}, N_{2}\right)\right)}^{(m), \text { ord, }}\right)_{\mathbb{R}}^{\succ 0}\right)$, then the log smooth, log scheme

$$
\left(T_{n,(i), U, \widetilde{\Sigma}_{0}}^{(m),}, \mathcal{M}_{\widetilde{\Sigma}_{0}}\right)
$$


(resp. the log smooth, log scheme

$$
\left.\left(\mathcal{T}_{n,(i), U^{p}\left(N_{1}, N_{2}\right), \widetilde{\Sigma}_{0}}^{(m), \mathcal{M}_{\widetilde{\Sigma}_{0}}}\right)\right)
$$

has a left action of $L_{n,(i), \text { lin }}^{(m)}(\mathbb{Q})$ (resp. $L_{n,(i), \text { lin }}^{(m)}\left(\mathbb{Z}_{(p)}\right)$ ) extending that on $T_{n,(i), U}^{(m),+}$ (resp. $\mathcal{T}_{n,(i), U^{p}\left(N_{1}, N_{2}\right)}^{(m) \text {,ord }+}$ (Recall the definition of $\widetilde{\Sigma}_{0}$ from Sect. 2.5.) If $g \in P_{n,(i)}^{(m),+}\left(\mathbb{A}^{\infty}\right)$ (resp. $\left.g \in P_{n,(i)}^{(m),+}\left(\mathbb{A}^{\infty}\right)^{\text {ord }}\right)$ and if $\Sigma_{0}$ is compatible with $\Sigma_{0}^{\prime}$ with respect to $g$ then the map

$$
g: T_{n,(i), U}^{(m),+} \longrightarrow T_{n,(i), U^{\prime}}^{(m),+}
$$

(resp.

$$
\left.g: \mathcal{T}_{n,(i), U^{p}\left(N_{1}, N_{2}\right)}^{(m), \text { ord }+} \longrightarrow \mathcal{T}_{n,(i),\left(U^{p}\right)^{\prime}\left(N_{1}^{\prime}, N_{2}^{\prime}\right)}^{(m), \text { ord }+}\right)
$$

uniquely extends to an $L_{n,(i), \text { lin }}^{(m)}(\mathbb{Q})$-equivariant (resp. $L_{n,(i), \text { lin }}^{(m)}\left(\mathbb{Z}_{(p)}\right)$-equivariant) log etale map

$$
g:\left(T_{n,(i), U, \widetilde{\Sigma}_{0}}^{(m),+}, \mathcal{M}_{\widetilde{\Sigma}_{0}}\right) \longrightarrow\left(T_{n,(i), U^{\prime}, \widetilde{\Sigma}_{0}^{\prime}}^{(m)}, \mathcal{M}_{\widetilde{\Sigma}_{0}^{\prime}}\right)
$$

(resp.

$$
\left.g:\left(\mathcal{T}_{n,(i), U^{p}\left(N_{1}, N_{2}\right), \widetilde{\Sigma}_{0}}^{(m), \text { ord }+}, \mathcal{M}_{\widetilde{\Sigma}_{0}}\right) \longrightarrow\left(\mathcal{T}_{n,(i),\left(U^{p}\right)^{\prime}\left(N_{1}^{\prime}, N_{2}^{\prime}\right), \widetilde{\Sigma}_{0}^{\prime}}^{(m), \text { ord }+} \mathcal{M}_{\widetilde{\Sigma}_{0}^{\prime}}\right)\right)
$$

This makes $\left\{\left(T_{n,(i), U, \widetilde{\Sigma}_{0}}^{(m),+}, \mathcal{M}_{\widetilde{\Sigma}_{0}}\right)\right\}$ (resp. $\left.\left\{\left(\mathcal{T}_{n,(i), U^{p}\left(N_{1}, N_{2}\right), \widetilde{\Sigma}_{0}}^{(m), \text { ord }+}, \mathcal{M}_{\widetilde{\Sigma}_{0}}\right)\right\}\right)$ a system of log schemes with $P_{n,(i)}^{(m),+}\left(\mathbb{A}^{\infty}\right)$-action (resp. $P_{n,(i)}^{(m),+}\left(\mathbb{A}^{\infty}\right)^{\text {ord }}$-action). There are equivariant embeddings

$$
\left(\mathcal{T}_{n,(i), U^{p}\left(N_{1}, N_{2}\right), \widetilde{\Sigma}_{0}^{\text {ord }}}^{(m), \text { ord }+} \times \operatorname{Spec} \mathbb{Q}, \mathcal{M}_{\widetilde{\Sigma}_{0}^{\text {ord }}}\right) \hookrightarrow\left(T_{n,(i), U^{p}\left(N_{1}, N_{2}\right), \widetilde{\Sigma}_{0}}^{(m),+}, \mathcal{M}_{\widetilde{\Sigma}_{0}}\right)
$$

We have

$$
\begin{aligned}
& \left|\mathcal{S}\left(\partial T_{n,(i), U, \widetilde{\Sigma}_{0}}^{(m),+}\right)\right|-\left|\mathcal{S}\left(\partial T_{n,(i), U, \widetilde{\Sigma}_{0}-\Sigma_{0}}^{(m),+}\right)\right| \\
& \quad=\left(L_{n,(i), \operatorname{lin}}^{(m)}\left(\mathbb{A}^{\infty}\right) \times\left(C_{n-i}(\mathbb{Q}) \backslash C_{n-i}(\mathbb{A}) / C_{n-i}(\mathbb{R})^{0}\right)\right) / U \times\left(\mathfrak{C}_{(i)}^{(m),>0} / \mathbb{R}_{>0}^{\times}\right) .
\end{aligned}
$$

If $U^{\prime}$ (resp. $\left.\left(U^{\prime}\right)^{p}\right)$ is a neat open compact subgroup of the group $P_{n,(i)}^{+}\left(\mathbb{A}^{\infty}\right)$ (resp. $\left.P_{n,(i)}^{+}\left(\mathbb{A}^{\infty, p}\right)\right)$ which contains the image of $U$ (resp. $\left.U^{p}\right)$, if $\Delta_{0}$ is a smooth admissible cone decomposition of $X_{*}\left(S_{n,(i), U^{\prime}}^{+}\right)_{\mathbb{R}}^{\succ 0}\left(\operatorname{resp} . X_{*}\left(\mathcal{S}_{n,(i),\left(U^{\prime}\right) p\left(N_{1}\right)}^{\text {ord, }}\right)_{\mathbb{R}}^{\succ 0}\right)$ and if $\Sigma_{0}$ is a compatible smooth admissible cone decomposition of $X_{*}\left(S_{n,(i), U}^{(m),+}\right)_{\mathbb{R}}^{\succ 0}\left(\operatorname{resp} . X_{*}\left(\mathcal{S}_{n,(i), U^{p}\left(N_{1}\right)}^{(m), \text { ord, }}\right)_{\mathbb{R}}^{\succ 0}\right)$, then the map

$$
T_{n,(i), U}^{(m),+} \longrightarrow T_{n,(i), U^{\prime}}^{+}
$$

(resp.

$$
\left.\mathcal{T}_{n,(i), U^{p}\left(N_{1}, N_{2}\right)}^{(m), \text { ord }+} \longrightarrow \mathcal{T}_{n,(i),\left(U^{\prime}\right)^{p}\left(N_{1}, N_{2}\right)}^{\text {ord, }}\right)
$$


extends to an $L_{n,(i), \text { lin }}^{(m)}(\mathbb{Q})$-equivariant (resp. $L_{n,(i), \text { lin }}^{(m)}\left(\mathbb{Z}_{(p)}\right)$-equivariant) log smooth map

$$
\left(T_{n,(i), U, \widetilde{\Sigma}_{0}}^{(m),}, \mathcal{M}_{\widetilde{\Sigma}_{0}}\right) \longrightarrow\left(T_{n,(i), U^{\prime}, \tilde{\Delta}_{0}}^{+}, \mathcal{M}_{\tilde{\Delta}_{0}}\right)
$$

(resp.

$$
\left.\left(\mathcal{T}_{n,(i), U^{p}\left(N_{1}, N_{2}\right), \widetilde{\Sigma}_{0}}^{(m), \text { ord }+}, \mathcal{M}_{\widetilde{\Sigma}_{0}}\right) \longrightarrow\left(\mathcal{T}_{n,(i),\left(U^{\prime}\right)^{p}\left(N_{1}, N_{2}\right), \widetilde{\Delta}_{0}}^{\text {ord }+}, \mathcal{M}_{\widetilde{\Delta}_{0}}\right)\right)
$$

This gives rise to a $P_{n,(i)}^{(m),+}\left(\mathbb{A}^{\infty}\right)$-equivariant (resp. $P_{n,(i)}^{(m),+}\left(\mathbb{A}^{\infty}\right)^{\text {ord }}$-equivariant) map of systems of $\log$ schemes

$$
\left\{\left(T_{n,(i), U, \widetilde{\Sigma}_{0}}^{(m),+}, \mathcal{M}_{\widetilde{\Sigma}_{0}}\right)\right\} \longrightarrow\left\{\left(T_{n,(i), U^{\prime}, \widetilde{\Delta}_{0}}^{+}, \mathcal{M}_{\widetilde{\Delta}_{0}}\right)\right\}
$$

(resp.

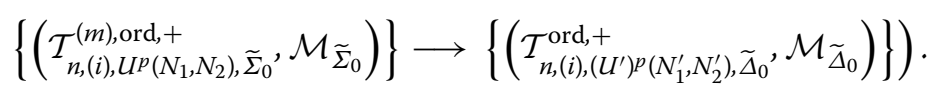

These maps are compatible with the embeddings

$$
\left(\mathcal{T}_{n,(i), U^{p}\left(N_{1}, N_{2}\right), \widetilde{\Sigma}_{0}}^{(m), \text { ord }+} \times \operatorname{Spec} \mathbb{Q}, \mathcal{M}_{\widetilde{\Sigma}_{0}}\right) \hookrightarrow\left(T_{n,(i), U^{p}\left(N_{1}, N_{2}\right), \widetilde{\Sigma}_{0}}^{(m),+}, \mathcal{M}_{\widetilde{\Sigma}_{0}}\right)
$$

and

$$
\left(\mathcal{T}_{n,(i), U^{p}\left(N_{1}, N_{2}\right), \tilde{\Delta}_{0}}^{\text {ord }+} \times \operatorname{Spec} \mathbb{Q}, \mathcal{M}_{\widetilde{\Delta}_{0}}\right) \hookrightarrow\left(T_{n,(i), U^{p}\left(N_{1}, N_{2}\right), \widetilde{\Delta}_{0}}^{+}, \mathcal{M}_{\widetilde{\Delta}_{0}}\right)
$$

\subsection{Completions}

If $\Sigma_{0}$ denotes a smooth admissible cone decomposition of $X_{*}\left(S_{n,(i), U}^{(m),+}\right)_{\mathbb{R}}^{\succ 0}$ (resp. of

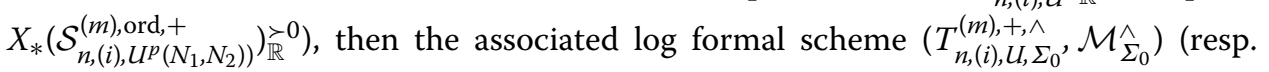
$\left.\left(\mathcal{T}_{n,(i), U^{p}\left(N_{1}, N_{2}\right), \Sigma_{0}}^{(m), \text { ord }+, \wedge}, \mathcal{M}_{\Sigma_{0}}^{\wedge}\right)\right)$ inherits a left action of $L_{n,(i), \text { lin }}^{(m)}(\mathbb{Q})\left(\right.$ resp. $\left.L_{n,(i), \text { lin }}^{(m)}\left(\mathbb{Z}_{(p)}\right)\right)$. If $g \in$ $P_{n,(i)}^{(m),+}\left(\mathbb{A}^{\infty}\right)$ (resp. $\left.P_{n,(i)}^{(m),+}\left(\mathbb{A}^{\infty}\right)^{\text {ord }}\right)$ and if $\Sigma_{0}$ is compatible with $\Sigma_{0}^{\prime}$ with respect to $g$, then there is an induced $L_{n,(i), \text { lin }}^{(m)}(\mathbb{Q})$-equivariant (resp. $L_{n,(i), \text { lin }}^{(m)}\left(\mathbb{Z}_{(p)}\right)$-equivariant) map

$$
g:\left(T_{n,(i), U, \Sigma_{0}}^{(m),+, \wedge}, \mathcal{M}_{\Sigma_{0}}^{\wedge}\right) \longrightarrow\left(T_{n,(i), U^{\prime}, \Sigma_{0}^{\prime}}^{(m),+\mathcal{M}_{\Sigma_{0}^{\prime}}}\right)
$$

(resp.

$$
\left.g:\left(\mathcal{T}_{n,(i), U^{p}\left(N_{1}, N_{2}\right), \Sigma_{0}}^{(m), \text { ord }+, \wedge}, \mathcal{M}_{\Sigma_{0}}^{\wedge}\right) \longrightarrow\left(\mathcal{T}_{n,(i),\left(U^{p}\right)^{\prime}\left(N_{1}^{\prime}, N_{2}^{\prime}\right), \Sigma_{0}^{\prime}}^{(m), \mathcal{M}_{\Sigma_{0}^{\prime}}^{\prime}}\right)\right)
$$

This makes $\left\{\left(T_{n,(i), U, \Sigma_{0}}^{(m),+, \wedge}, \mathcal{M}_{\Sigma_{0}}^{\wedge}\right)\right\}$ (resp. $\left.\left\{\left(\mathcal{T}_{n,(i), U^{p}\left(N_{1}, N_{2}\right), \Sigma_{0}}^{(m), \text { ord }+, \wedge}, \mathcal{M}_{\Sigma_{0}}^{\wedge}\right)\right\}\right)$ a system of log formal schemes with $P_{n,(i)}^{(m),+}\left(\mathbb{A}^{\infty}\right)$-action (resp. $P_{n,(i)}^{(m),+}\left(\mathbb{A}^{\infty}\right)^{\text {ord }}$-action).

Similarly the schemes $\partial_{\Sigma_{0}} T_{n,(i), U}^{(m),+}\left(\operatorname{resp} . \partial_{\Sigma_{0}} \mathcal{T}_{n,(i), U^{p}\left(N_{1}, N_{2}\right)}^{(m), \text { ord, }}\right)$ inherit a left action of the group $L_{n,(i), \text { lin }}^{(m)}(\mathbb{Q})\left(\right.$ resp. $\left.L_{n,(i), \text { lin }}^{(m)}\left(\mathbb{Z}_{(p)}\right)\right)$. If $g \in P_{n,(i)}^{(m),+}\left(\mathbb{A}^{\infty}\right)\left(\right.$ resp. $\left.P_{n,(i)}^{(m),+}\left(\mathbb{A}^{\infty}\right)^{\text {ord }}\right)$ and if $\Sigma_{0}$ is compatible with $\Sigma_{0}^{\prime}$ with respect to $g$, then there is an induced $L_{n,(i), \text { lin }}^{(m)}(\mathbb{Q})$-equivariant (resp. $L_{n,(i), \text { lin }}^{(m)}\left(\mathbb{Z}_{(p)}\right)$-equivariant) map

$$
g: \partial_{\Sigma_{0}} T_{n,(i), U}^{(m),+} \longrightarrow \partial_{\Sigma_{0}^{\prime}} T_{n,(i), U^{\prime}}^{(m),+}
$$


(resp.

$$
\left.g: \partial_{\Sigma_{0}} \mathcal{T}_{n,(i), U^{p}\left(N_{1}, N_{2}\right)}^{(m), \mathrm{ord}+} \longrightarrow \partial_{\Sigma_{0}^{\prime}} \mathcal{T}_{n,(i),\left(U^{p}\right)^{\prime}\left(N_{1^{\prime}}^{\prime}, N_{2}^{\prime}\right)}^{(m), \mathrm{rrd},}\right)
$$

This makes $\left\{\partial_{\Sigma_{0}} T_{n,(i), U}^{(m),+}\right\}$ (resp. $\left.\left\{\partial_{\Sigma_{0}} \mathcal{T}_{n,(i), U^{p}\left(N_{1}, N_{2}\right)}^{(m), \text { ord },}\right\}\right)$ a system of log formal schemes with $P_{n,(i)}^{(m),+}\left(\mathbb{A}^{\infty}\right)$-action (resp. $P_{n,(i)}^{(m),+}\left(\mathbb{A}^{\infty}\right)^{\text {ord }}$-action).

If $U^{\prime}$ (resp. $\left.\left(U^{p}\right)^{\prime}\right)$ is a neat open compact subgroup of the group $P_{n,(i)}^{+}\left(\mathbb{A}^{\infty}\right)$ (resp. $\left.P_{n,(i)}^{+}\left(\mathbb{A}^{\infty, p}\right)\right)$ which contains the image of $U$ (resp. $\left.U^{p}\right)$, if $\Delta_{0}$ is a smooth admissible cone decomposition of $X_{*}\left(S_{n,(i), U^{\prime}}^{+}\right)_{\mathbb{R}}^{\succ 0}$ (resp. $\left.X_{*}\left(\mathcal{S}_{n,(i),\left(U^{p}\right)^{\prime}\left(N_{1}\right)}^{\text {ord, }}\right)_{\mathbb{R}}^{\succ 0}\right)$ and if $\Sigma_{0}$ is a compatible smooth admissible cone decomposition of $X_{*}\left(S_{n,(i), U}^{(m),+}\right)_{\mathbb{R}}^{\succ 0}\left(\right.$ resp. $\left.X_{*}\left(\mathcal{S}_{n,(i), U^{p}\left(N_{1}\right)}^{(m) \text { ord, }}\right)_{\mathbb{R}}^{\succ 0}\right)$, then there are induced maps

$$
\left(T_{n,(i), U, \Sigma_{0}}^{(m),+, \wedge}, \mathcal{M}_{\Sigma_{0}}^{\wedge}\right) \longrightarrow\left(T_{n,(i), U^{\prime}, \Delta_{0}}^{+, \wedge}, \mathcal{M}_{\Delta_{0}}^{\wedge}\right)
$$

and

$$
\partial_{\Sigma_{0}} T_{n,(i), U}^{(m),+} \longrightarrow \partial_{\Delta_{0}} T_{n,(i), U^{\prime}}^{+}
$$

(resp.

$$
\left(\mathcal{T}_{n,(i), U^{p}\left(N_{1}, N_{2}\right), \Sigma_{0}}^{(m), \text { ord },+\wedge}, \mathcal{M}_{\Sigma_{0}}^{\wedge}\right) \longrightarrow\left(\mathcal{T}_{n,(i),\left(U^{p}\right)^{\prime}\left(N_{1}, N_{2}\right), \Delta_{0}}^{\mathrm{ord},+, \wedge}, \mathcal{M}_{\Delta_{0}}^{\wedge}\right)
$$

and

$$
\left.\partial_{\Sigma_{0}} \mathcal{T}_{n,(i), U^{p}\left(N_{1}, N_{2}\right)}^{(m), \mathrm{rd},+} \longrightarrow \partial_{\Delta_{0}} \mathcal{T}_{n,(i),\left(U^{p}\right)^{\prime}\left(N_{1}, N_{2}\right)}^{\mathrm{ord},+}\right)
$$

which are $L_{n,(i) \text {,lin }}^{(m)}(\mathbb{Q})$-equivariant (resp. $L_{n,(i) \text {,lin }}^{(m)}\left(\mathbb{Z}_{(p)}\right)$-equivariant). This gives rise to $P_{n,(i)}^{(m),+}\left(\mathbb{A}^{\infty}\right)$-equivariant (resp. $P_{n,(i)}^{(m),+}\left(\mathbb{A}^{\infty}\right)^{\text {ord }}$-equivariant) maps of systems of log formal schemes

$$
\left\{\left(T_{n,(i), U, \Sigma_{0}}^{(m),+, \wedge}, \mathcal{M}_{\Sigma_{0}}^{\wedge}\right)\right\} \rightarrow\left\{\left(T_{n,(i), U^{\prime}, \Delta_{0}}^{+, \wedge}, \mathcal{M}_{\Delta_{0}}^{\wedge}\right)\right\}
$$

and of systems of schemes

$$
\left\{\partial_{\Sigma_{0}} T_{n,(i), U}^{(m),+}\right\} \longrightarrow\left\{\partial_{\Delta_{0}} T_{n,(i), U^{\prime}}^{+}\right\}
$$

(resp.

$$
\left\{\left(\mathcal{T}_{n,(i), U^{p}\left(N_{1}, N_{2}\right), \Sigma_{0}}^{(m), \mathrm{ord}+, \wedge}, \mathcal{M}_{\Sigma_{0}}^{\wedge}\right)\right\} \rightarrow\left\{\left(\mathcal{T}_{n,(i),\left(U^{\prime}\right) p\left(N_{1}^{\prime}, N_{2}^{\prime}\right), \Delta_{0}}^{\mathrm{ord},+\wedge}, \mathcal{M}_{\Delta_{0}}^{\wedge}\right)\right\}
$$

and

$$
\left.\left\{\partial_{\Sigma_{0}} \mathcal{T}_{n,(i), U^{p}\left(N_{1}, N_{2}\right)}^{(m), \text { ord }+}\right\} \rightarrow\left\{\partial_{\Delta_{0}} \mathcal{T}_{n,(i),\left(U^{p}\right)^{\prime}\left(N_{1}, N_{2}\right)}^{\mathrm{ord},+}\right\}\right)
$$

If $\sigma \in \Sigma_{0}$ and if $1 \neq \gamma \in L_{n,(i) \text { lin }}^{(m)}(\mathbb{Q})\left(\operatorname{resp} . L_{n,(i) \text { lin }}^{(m)}\left(\mathbb{Z}_{(p)}\right)\right)$ then $\sigma \cap \gamma \sigma \notin \Sigma_{0}$. Thus

$$
\left(T_{n,(i), U, \Sigma_{0}}^{(m),+, \wedge}\right)_{\sigma} \cap\left(T_{n,(i), U, \Sigma_{0}}^{(m),+\wedge}\right)_{\gamma \sigma}=\emptyset
$$


and

$$
\left(\partial_{\Sigma_{0}} T_{n,(i), U}^{(m),+}\right)_{\sigma} \cap\left(\partial_{\Sigma_{0}} T_{n,(i), U}^{(m),+}\right)_{\gamma \sigma}=\emptyset
$$

(resp.

$$
\left(\mathcal{T}_{n,(i), U^{p}\left(N_{1}, N_{2}\right), \Sigma_{0}}^{(m), \text { ord },+\wedge}\right)_{\sigma} \cap\left(\mathcal{T}_{n,(i), U^{p}\left(N_{1}, N_{2}\right), \Sigma_{0}}^{(m), \text { ord }+, \wedge}\right)_{\gamma \sigma}=\emptyset
$$

and

$$
\left.\left(\partial_{\Sigma_{0}} \mathcal{T}_{n,(i), U^{p}\left(N_{1}, N_{2}\right)}^{(m), \text { ord, }}\right)_{\sigma} \cap\left(\partial_{\Sigma_{0}} \mathcal{T}_{n,(i), U^{p}\left(N_{1}, N_{2}\right)}^{(m), \text { ord },+}\right)_{\gamma \sigma}=\emptyset\right) .
$$

It follows we can form log formal schemes

$$
\left(T_{n,(i), U, \Sigma_{0}}^{(m), \downarrow}, \mathcal{M}_{\Sigma_{0}}^{\wedge}\right)=L_{n,(i), \operatorname{lin}}^{(m)}(\mathbb{Q}) \backslash\left(T_{n,(i), U, \Sigma_{0}}^{(m),+, \wedge}, \mathcal{M}_{\Sigma_{0}}^{\wedge}\right)
$$

(resp.

$$
\left.\left(\mathcal{T}_{n,(i), U^{p}\left(N_{1}, N_{2}\right), \Sigma_{0}}^{(m), \text { ord }, \wedge}, \mathcal{M}_{\Sigma_{0}}^{\wedge}\right)=L_{n,(i), \text { lin }}^{(m)}\left(\mathbb{Z}_{(p)}\right) \backslash\left(\mathcal{T}_{n,(i), U^{p}\left(N_{1}, N_{2}\right), \Sigma_{0}}^{(m), \text { ord },+\wedge}, \mathcal{M}_{\Sigma_{0}}^{\wedge}\right)\right)
$$

and

$$
\left(T_{n,(i), U, \Sigma_{0}}^{(m), \downarrow+, \wedge}, \mathcal{M}_{\Sigma_{0}}^{\wedge}\right)=\operatorname{Hom}_{F}\left(F^{m}, F^{i}\right) \backslash\left(T_{n,(i), U, \Sigma_{0}}^{(m),+, \wedge}, \mathcal{M}_{\Sigma_{0}}^{\wedge}\right)
$$

(resp.

$$
\left.\left(\mathcal{T}_{n,(i), U^{p}\left(N_{1}, N_{2}\right), \Sigma_{0}}^{(m), \text { ord },+, \wedge}, \mathcal{M}_{\Sigma_{0}}^{\wedge}\right)=\operatorname{Hom}_{\mathcal{O}_{F,(p)}}\left(\mathcal{O}_{F,(p)}^{m}, \mathcal{O}_{F,(p)}^{i}\right) \backslash\left(\mathcal{T}_{n,(i), U^{p}\left(N_{1}, N_{2}\right), \Sigma_{0}}^{(m), \text { ord },+\wedge}, \mathcal{M}_{\Sigma_{0}}^{\wedge}\right)\right) .
$$

We can also form schemes

$$
\partial_{\Sigma_{0}} T_{n,(i), U}^{(m), \natural}=L_{n,(i), \operatorname{lin}}^{(m)}(\mathbb{Q}) \backslash \partial_{\Sigma_{0}} T_{n,(i), U}^{(m),+}
$$

(resp.

$$
\left.\partial_{\Sigma_{0}} \mathcal{T}_{n,(i), U^{p}\left(N_{1}, N_{2}\right)}^{(m), \text { ord },}=L_{n,(i), \operatorname{lin}}^{(m)}\left(\mathbb{Z}_{(p)}\right) \backslash \partial_{\Sigma_{0}} \mathcal{T}_{n,(i), U^{p}\left(N_{1}, N_{2}\right)}^{(m), \text { ord },}\right) .
$$

The quotient maps

$$
\left(T_{n,(i), U, \Sigma_{0}}^{(m),+, \wedge}, \mathcal{M}_{\Sigma_{0}}^{\wedge}\right) \rightarrow\left(T_{n,(i), U, \Sigma_{0}}^{(m), \natural+, \wedge}, \mathcal{M}_{\Sigma_{0}}^{\wedge}\right) \rightarrow\left(T_{n,(i), U, \Sigma_{0}}^{(m), \downarrow, \wedge}, \mathcal{M}_{\Sigma_{0}}^{\wedge}\right)
$$

and

$$
\partial_{\Sigma_{0}} T_{n,(i), U}^{(m),+} \rightarrow \partial_{\Sigma_{0}} T_{n,(i), U}^{(m), \natural}
$$

(resp.

$$
\left(\mathcal{T}_{n,(i), U^{p}\left(N_{1}, N_{2}\right), \Sigma_{0}}^{(m), \text { ord },+\wedge}, \mathcal{M}_{\Sigma_{0}}^{\wedge}\right) \rightarrow\left(\mathcal{T}_{n,(i), U^{p}\left(N_{1}, N_{2}\right), \Sigma_{0}}^{(m), \text { ord },++,}, \mathcal{M}_{\Sigma_{0}}^{\wedge}\right) \rightarrow\left(\mathcal{T}_{n,(i), U^{p}\left(N_{1}, N_{2}\right), \Sigma_{0}}^{(m), \text { ord }, \wedge}, \mathcal{M}_{\Sigma_{0}}^{\wedge}\right)
$$


and

$$
\left.\partial_{\Sigma_{0}} \mathcal{T}_{n,(i), U^{p}\left(N_{1}, N_{2}\right)}^{(m), \text { ord },+} \rightarrow \partial_{\Sigma_{0}} \mathcal{T}_{n,(i), U^{p}\left(N_{1}, N_{2}\right)}^{(m), \text { ord },}\right)
$$

are Zariski locally isomorphisms. The log formal scheme $\left(T_{n,(i), U, \Sigma_{0}}^{(m), \underline{4}+, \wedge}, \mathcal{M}_{\Sigma_{0}}^{\wedge}\right.$ ) (resp. $\left.\left(\mathcal{T}_{n,(i), U^{p}\left(N_{1}, N_{2}\right), \Sigma_{0}}^{(m), \text { or },+, \wedge} \mathcal{M}_{\Sigma_{0}}^{\wedge}\right)\right)$ inherits an action of $L_{n,(i), \operatorname{lin}}(\mathbb{Q})\left(\right.$ resp. $\left.L_{n,(i), \operatorname{lin}}\left(\mathbb{Z}_{(p)}\right)\right)$.

If $g \in P_{n,(i)}^{(m),+}\left(\mathbb{A}^{\infty}\right)\left(\operatorname{resp} . P_{n,(i)}^{(m),+}\left(\mathbb{A}^{\infty}\right)^{\text {ord }}\right)$ and if $\Sigma_{0}$ is compatible with $\Sigma_{0}^{\prime}$ with respect to $g$ then there are induced maps

$$
g:\left(T_{n,(i), U, \Sigma_{0}}^{(m), \mathfrak{M}^{\prime} \wedge} \mathcal{M}_{\Sigma_{0}}^{\wedge}\right) \longrightarrow\left(T_{n,(i), U^{\prime}, \Sigma_{0}^{\prime}}^{(m), \mathcal{M}_{\Sigma_{0}^{\prime}}}\right)
$$

(resp.

$$
\left.g:\left(\mathcal{T}_{n,(i), U^{p}\left(N_{1}, N_{2}\right), \Sigma_{0}}^{(m), \text { ord }, \wedge}, \mathcal{M}_{\Sigma_{0}}^{\wedge}\right) \longrightarrow\left(\mathcal{T}_{n,(i),\left(U^{p}\right)^{\prime}\left(N_{1}^{\prime}, N_{2}^{\prime}\right), \Sigma_{0}^{\prime}}^{(m), \mathcal{M}_{\Sigma_{0}^{\prime}}}\right)\right)
$$

and

$$
g:\left(T_{n,(i), U, \Sigma_{0}}^{(m), 4+, \wedge}, \mathcal{M}_{\Sigma_{0}}^{\wedge}\right) \longrightarrow\left(T_{n,(i), U^{\prime}, \Sigma_{0}^{\prime}}^{(m),+, \wedge} \mathcal{M}_{\Sigma_{0}^{\prime}}^{\wedge}\right)
$$

(resp.

$$
\left.g:\left(\mathcal{T}_{n,(i), U^{p}\left(N_{1}, N_{2}\right), \Sigma_{0}}^{(m), \text { ord },+, \wedge}, \mathcal{M}_{\Sigma_{0}}^{\wedge}\right) \longrightarrow\left(\mathcal{T}_{n,(i),\left(U^{p}\right)^{\prime}\left(N_{1}^{\prime}, N_{2}^{\prime}\right), \Sigma_{0}^{\prime}}^{(m), \mathcal{M}_{\Sigma_{0}^{\prime}}^{\prime}}\right)\right)
$$

and

$$
g: \partial_{\Sigma_{0}} T_{n,(i), U}^{(m), \sharp} \longrightarrow \partial_{\Sigma_{0}^{\prime}} T_{n,(i), U^{\prime}}^{(m),}
$$

(resp.

$$
\left.g: \partial_{\Sigma_{0}} \mathcal{T}_{n,(i), U^{p}\left(N_{1}, N_{2}\right)}^{(m), \text { ord } \sharp} \longrightarrow \partial_{\Sigma_{0}^{\prime}} \mathcal{T}_{n,(i),\left(U^{p}\right)^{\prime}\left(N_{1}^{\prime}, N_{2}^{\prime}\right)}^{(m), \text { ord })}\right)
$$

This makes the collections $\left\{\left(T_{n,(i), U, \Sigma_{0}}^{(m), t, \wedge}, \mathcal{M}_{\Sigma_{0}}^{\wedge}\right)\right\}$ (resp. $\left.\left\{\left(\mathcal{T}_{n,(i), U U^{p}\left(N_{1}, N_{2}\right), \Sigma_{0}}^{(m), \text { ord }, \wedge}, \mathcal{M}_{\Sigma_{0}}^{\wedge}\right)\right\}\right)$ and

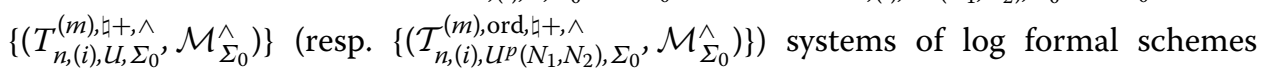
with $P_{n,(i)}^{(m),+}\left(\mathbb{A}^{\infty}\right)$-action (resp. $P_{n,(i)}^{(m),+}\left(\mathbb{A}^{\infty}\right)^{\text {ord }}$-action). It also makes the collections $\left\{\partial_{\Sigma_{0}} T_{n,(i), U}^{(m),}\right\}\left(\right.$ resp. $\left.\left\{\partial_{\Sigma_{0}} \mathcal{T}_{n,(i), U^{p}\left(N_{1}, N_{2}\right)}^{(m), \text { ord },}\right\}\right)$ systems of schemes with $P_{n,(i)}^{(m),+}\left(\mathbb{A}^{\infty}\right)$-action (resp. $P_{n,(i)}^{(m),+}\left(\mathbb{A}^{\infty}\right)^{\text {ord }}$-action $)$.

If $U^{\prime}$ (resp. $\left.\left(U^{p}\right)^{\prime}\right)$ is a neat open compact subgroup of the group $P_{n,(i)}^{+}\left(\mathbb{A}^{\infty}\right)$ (resp. $\left.P_{n,(i)}^{+}\left(\mathbb{A}^{\infty, p}\right)\right)$ which contains the image of $U$ (resp. $\left.U^{p}\right)$, if $\Delta_{0}$ is a smooth admissible cone decomposition of $X_{*}\left(S_{n,(i), U^{\prime}}^{+}\right)_{\mathbb{R}}^{\succ 0}\left(\right.$ resp. $\left.X_{*}\left(\mathcal{S}_{n,(i),\left(U^{p}\right)^{\prime}\left(N_{1}\right)}^{\text {ord }}\right)_{\mathbb{R}}^{\succ 0}\right)$ and if $\Sigma_{0}$ is a compatible smooth admissible cone decomposition of $X_{*}\left(S_{n,(i), U^{(m),+}}^{\succ_{\mathbb{R}}}\left(\right.\right.$ resp. $\left.X_{*}\left(\mathcal{S}_{n,(i), U^{p}\left(N_{1}\right)}^{(m), \text { ord }+}\right)_{\mathbb{R}}^{\succ 0}\right)$, then there are induced maps

$$
\left(T_{n,(i), U, \Sigma_{0}}^{(m), 4, \wedge}, \mathcal{M}_{\Sigma_{0}}^{\wedge}\right) \longrightarrow\left(T_{n,(i), U^{\prime}, \Delta_{0}}^{\natural, \wedge}, \mathcal{M}_{\Delta_{0}}^{\wedge}\right)
$$

(resp.

$$
\left.\left(\mathcal{T}_{n,(i), U^{p}\left(N_{1}, N_{2}\right), \Sigma_{0}}^{(m), \text { ord }, \wedge}, \mathcal{M}_{\Sigma_{0}}^{\wedge}\right) \longrightarrow\left(\mathcal{T}_{n,(i),\left(U^{p}\right)^{\prime}\left(N_{1}, N_{2}\right), \Delta_{0}}^{\text {ord, }, \mathcal{M}_{\Delta_{0}}}\right)\right)
$$


and

$$
\left(T_{n,(i), U, \Sigma_{0}}^{(m), 4+, \wedge}, \mathcal{M}_{\Sigma_{0}}^{\wedge}\right) \longrightarrow\left(T_{n,(i), U^{\prime}, \Delta_{0}}^{+, \wedge}, \mathcal{M}_{\Delta_{0}}^{\wedge}\right)
$$

(resp.

$$
\left.\left(\mathcal{T}_{n,(i), U^{p}\left(N_{1}, N_{2}\right), \Sigma_{0}}^{(m), \text { ord },+, \wedge}, \mathcal{M}_{\Sigma_{0}}^{\wedge}\right) \longrightarrow\left(\mathcal{T}_{n,(i),\left(U^{p}\right)^{\prime}\left(N_{1}, N_{2}\right), \Delta_{0}}^{\text {ord },+, \wedge} \mathcal{M}_{\Delta_{0}}^{\wedge}\right)\right)
$$

and

$$
\partial_{\Sigma_{0}} T_{n,(i), U}^{(m), \natural} \longrightarrow \partial_{\Delta_{0}} T_{n,(i), U^{\prime}}^{\natural}
$$

(resp.

$$
\left.\partial_{\Sigma_{0}} \mathcal{T}_{n,(i), U^{p}\left(N_{1}, N_{2}\right)}^{(m), \mathrm{rd}, \natural} \longrightarrow \partial_{\Delta_{0}} \mathcal{T}_{n,(i),\left(U^{p}\right)^{\prime}\left(N_{1}, N_{2}\right)}^{\mathrm{ord}, \mathrm{q}}\right)
$$

These give rise to $P_{n,(i)}^{(m),+}\left(\mathbb{A}^{\infty}\right)$-equivariant (resp. $P_{n,(i)}^{(m),+}\left(\mathbb{A}^{\infty}\right)^{\text {ord }}$-equivariant) maps of systems of $\log$ formal schemes

$$
\left\{\left(T_{n,(i), U, \Sigma_{0}}^{(m), \natural_{1} \wedge}, \mathcal{M}_{\Sigma_{0}}^{\wedge}\right)\right\} \longrightarrow\left\{\left(T_{n,(i), U^{\prime}, \Delta_{0}}^{\natural, \wedge}, \mathcal{M}_{\Delta_{0}}^{\wedge}\right)\right\}
$$

(resp.

$$
\left.\left\{\left(\mathcal{T}_{n,(i), U^{p}\left(N_{1}, N_{2}\right), \Sigma_{0}}^{(m), \text { ord }, \wedge}, \mathcal{M}_{\Sigma_{0}}^{\wedge}\right)\right\} \rightarrow\left\{\left(\mathcal{T}_{n,(i),\left(U^{p}\right)^{\prime}\left(N_{1}^{\prime}, N_{2}^{\prime}\right), \Delta_{0}}^{\text {ord, }, \wedge} \mathcal{M}_{\Delta_{0}}^{\wedge}\right)\right\}\right)
$$

and

$$
\left\{\left(T_{n,(i), U, \Sigma_{0}}^{(m),++\wedge}, \mathcal{M}_{\Sigma_{0}}^{\wedge}\right)\right\} \rightarrow\left\{\left(T_{n,(i), U^{\prime}, \Delta_{0}}^{+, \wedge}, \mathcal{M}_{\Delta_{0}}^{\wedge}\right)\right\}
$$

(resp.

$$
\left.\left\{\left(\mathcal{T}_{n,(i), U^{p}\left(N_{1}, N_{2}\right), \Sigma_{0}}^{(m), \text { ord },++, \mathcal{M}_{\Sigma_{0}}}\right)\right\} \rightarrow\left\{\left(\mathcal{T}_{n,(i),\left(U^{p}\right)^{\prime}\left(N_{1}^{\prime}, N_{2}^{\prime}\right), \Delta_{0}}^{\text {ord },+\wedge} \mathcal{M}_{\Delta_{0}}^{\wedge}\right)\right\}\right)
$$

They also give rise to a $P_{n,(i)}^{(m)}\left(\mathbb{A}^{\infty}\right)$-equivariant (resp. $P_{n,(i)}^{(m)}\left(\mathbb{A}^{\infty}\right)^{\text {ord }}$-equivariant) map of systems of schemes

$$
\left\{\partial_{\Sigma_{0}} T_{n,(i), U}^{(m), \natural}\right\} \longrightarrow\left\{\partial_{\Delta_{0}} T_{n,(i), U^{\prime}}^{\natural}\right\}
$$

(resp.

$$
\left.\left\{\partial_{\Sigma_{0}} \mathcal{T}_{n,(i), U^{p}\left(N_{1}, N_{2}\right)}^{(m), \text { ord },}\right\} \rightarrow\left\{\partial_{\Delta_{0}} \mathcal{T}_{n,(i),\left(U^{p}\right)^{\prime}\left(N_{1}, N_{2}\right)}^{\mathrm{ord}, \natural}\right\}\right) .
$$

We will write

$$
\partial_{\Sigma_{0}} \bar{T}_{n,(i), U^{p}(N)}^{(m), \text { ord },}=\partial_{\Sigma_{0}} \mathcal{T}_{n,(i), U^{p}\left(N_{1}, N_{2}\right)}^{(m), \text { Sr, }} \times \operatorname{Spec} \mathbb{F}_{p} .
$$

It is independent of $N_{2}$. 
We also get a commutative diagram

$$
\begin{array}{ccc}
T_{n,(i), U, \Sigma_{0}}^{(m),+, \wedge} & & \\
\downarrow & & \\
T_{n,(i), U, \Sigma_{0}}^{(m),+, \wedge} & & T_{n,(i), U^{\prime}, \Delta_{0}}^{+, \wedge} \\
\downarrow & & \downarrow \\
T_{n,(i), U, \Sigma_{0}}^{(m),\lrcorner} & & T_{n,(i), U^{\prime}, \Delta_{0}}^{\natural, \wedge} \\
\downarrow & & \downarrow \\
X_{n,(i), U}^{(m), \sharp} & = & X_{n,(i), U^{\prime}}^{\natural} \\
\downarrow & & \downarrow \\
Y_{n,(i), U}^{(m), \sharp} & = & Y_{n,(i), U^{\prime}}^{\natural}
\end{array}
$$

(resp.

$$
\begin{aligned}
& \mathcal{T}^{(m), \text { ord, }+ \text {, }}
\end{aligned}
$$

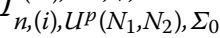

$$
\begin{aligned}
& \downarrow \\
& \mathcal{T}_{n,(i), U^{p}\left(N_{1}, N_{2}\right), \Sigma_{0}}^{(m), \text { ord },+, \wedge} \longrightarrow \mathcal{T}_{n,(i),\left(U^{p}\right)^{\prime}\left(N_{1}, N_{2}\right), \Delta_{0}}^{\mathrm{ord},+, \wedge} \\
& \downarrow \\
& \mathcal{T}_{n,(i), U^{p}\left(N_{1}, N_{2}\right), \Sigma_{0}}^{(m), \operatorname{ord}, \wedge} \longrightarrow \mathcal{T}_{n,(i),}^{\natural, \wedge} \\
& U^{\gamma^{\prime}}\left(N_{1}, N_{2}\right), \Delta_{0} \\
& \downarrow \\
& \mathcal{X}_{n,(i), U^{p}\left(N_{1}, N_{2}\right)}^{(m) \text { ord }}=\mathcal{X}_{n,(i),(U,)}^{\text {ord }, ~} \\
& \downarrow \downarrow \downarrow \\
& \left.\mathcal{Y}_{n,(i), U^{p}\left(N_{1}, N_{2}\right)}^{(m), \text { ord },}=\mathcal{Y}_{n,(i),\left(U^{\prime}\right)^{p}\left(N_{1}, N_{2}\right)}^{\text {ord, }}\right) .
\end{aligned}
$$

We will let $\mathcal{I}_{\partial, n,(i), U, \Sigma_{0}}^{(m),+, \wedge}$ denote the formal completion of the ideal sheaf defining

$$
\partial T_{n,(i), U, \widetilde{\Sigma}_{0}}^{(m),+} \subset T_{n,(i), U, \widetilde{\Sigma}_{0}}^{(m),+}
$$

We will let $\mathcal{I}_{\partial, n,(i), U, \Sigma_{0}}^{(m), \downarrow+, \wedge}$ denote its quotient by $\operatorname{Hom}_{F}\left(F^{m}, F^{i}\right)$ and $\mathcal{I}_{\partial, n,(i), U, \Sigma_{0}}^{(m), \downarrow}$ denote its quotient by $L_{n,(i), \text { lin }}^{(m)}(\mathbb{Q})$. Similarly we will let $\mathcal{I}_{\partial, n,(i), U^{p}\left(N_{1}, N_{2}\right), \Sigma_{0}}^{(m), \text { ord },+\wedge}$ denote the formal completion of the ideal sheaf defining

$$
\partial \mathcal{T}_{n,(i), U^{p}\left(N_{1}, N_{2}\right), \widetilde{\Sigma}_{0}}^{(m), \text { rd },} \subset \mathcal{T}_{n,(i), U^{p}\left(N_{1}, N_{2}\right), \widetilde{\Sigma}_{0}}^{(m), \text { ord },+}
$$

We will let $\mathcal{I}_{\partial, n,(i), U^{p}\left(N_{1}, N_{2}\right), \Sigma_{0}}^{(m), \text { ord },+, \wedge}$ denote its quotient by $\operatorname{Hom}_{\mathcal{O}_{F,(p)}}\left(\mathcal{O}_{F,(p)}^{m}, \mathcal{O}_{F,(p)}^{i}\right)$ and

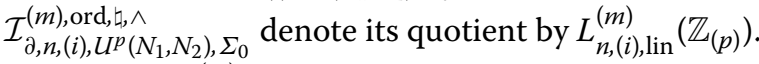

There are $P_{n,(i)}^{(m),+}\left(\mathbb{A}^{\infty}\right)^{\text {ord }}$ and $L_{n,(i), \text { lin }}\left(\mathbb{Z}_{(p)}\right)$ equivariant maps

$$
\mathcal{T}_{n,(i), U^{p}\left(N_{1}, N_{2}\right), \Sigma_{0}^{\text {ord }}}^{(m), \text { ord }+, \wedge} \times \operatorname{Spf} \mathbb{Q} \hookrightarrow T_{n,(i), U^{p}\left(N_{1}, N_{2}\right), \Sigma_{0}}^{(m),+, \wedge}
$$

if $\Sigma_{0}^{\text {ord }}$ and $\Sigma_{0}$ correspond under the bijection of Sect. 4.4. These embeddings are compatible with the maps

$$
\mathcal{T}_{n,(i), U^{p}\left(N_{1}, N_{2}\right), \Sigma_{0}^{\text {ord }}}^{(m), \text { ord },+\wedge} \longrightarrow \mathcal{T}_{n,(i), U^{p}\left(N_{1}, N_{2}\right), \Delta_{0}^{\text {ord }}}^{\text {ord }+, \wedge}
$$


and

$$
T_{n,(i), U^{p}\left(N_{1}, N_{2}\right), \Sigma_{0}}^{(m),+, \wedge} \longrightarrow T_{n,(i), U^{p}\left(N_{1}, N_{2}\right), \Delta_{0}}^{+, \wedge}
$$

Moreover they are also compatible with the $\log$ structures and with the sheaves $\mathcal{I}_{\partial, n,(i), U^{p}\left(N_{1}, N_{2}\right), \Sigma_{0}^{\text {ord }}}^{(m), \text { ord },+\wedge}$ and $\mathcal{I}_{\partial, n,(i), U^{p}\left(N_{1}, N_{2}\right), \Sigma_{0}}^{(m),+,}$. They induce isomorphisms

$$
\mathcal{T}_{n,(i), U^{p}\left(N_{1}, N_{2}\right), \Sigma_{0}^{\text {ord }}}^{(m), \text { ord },, \wedge} \times \operatorname{Spf} \mathbb{Q} \stackrel{\sim}{\longrightarrow} T_{n,(i), U^{p}\left(N_{1}, N_{2}\right), \Sigma_{0}}^{(m), \sharp}
$$

Lemma 4.6 Suppose that $R_{0}$ is an irreducible noetherian $\mathbb{Q}$-algebra (resp. $\mathbb{Z}_{(p)}$-algebra) with the discrete topology. Suppose also that $U \supset U^{\prime}$ (resp. $\left.U^{p} \supset\left(U^{p}\right)^{\prime}\right)$ are neat open compact subgroups of $P_{n,(i)}^{(m),+}\left(\mathbb{A}^{\infty}\right)\left(\right.$ resp. $\left.P_{n,(i)}^{(m),+}\left(\mathbb{A}^{\infty, p}\right)\right)$, that $N_{2}^{\prime} \geq N_{1}^{\prime} \geq 0$ and $N_{2} \geq N_{1} \geq 0$ are integers with $N_{2}^{\prime} \geq N_{2}$ and $N_{1}^{\prime} \geq N_{1}$ and that $\Sigma_{0}$ and $\Sigma_{0}^{\prime}$ are compatible smooth admissible cone decompositions for $X_{*}\left(S_{n,(i), U}^{(m),+}\right)_{\mathbb{R}}^{\succ 0}$ and $X_{*}\left(S_{n,(i), U^{\prime}}^{(m),+}\right)_{\mathbb{R}}^{\succ 0}$ (resp. for $X_{*}\left(\mathcal{S}_{n,(i), U^{p}\left(N_{1}, N_{2}\right)}^{(m)}\right)_{\mathbb{R}}^{\succ 0}$ and $\left.X_{*}\left(\mathcal{S}_{n,(i),\left(U^{p}\right)^{\prime}\left(N_{1}^{\prime}, N_{2}^{\prime}\right)}^{\left.(m),{ }^{\prime}\right)}\right)_{\mathbb{R}}^{\succ 0}\right)$. Let $\pi_{\left(U^{\prime}, \Sigma_{0}^{\prime}\right),\left(U, \Sigma_{0}\right)}\left(\right.$ resp. $\left.\pi_{\left(\left(U^{p}\right)^{\prime}\left(N_{1}^{\prime}, N_{2}^{\prime}\right), \Sigma_{0}^{\prime}\right),\left(U^{p}\left(N_{1}, N_{2}\right), \Sigma_{0}\right)}\right)$ denote the map

$$
1_{*}: T_{n,(i), U^{\prime}, \Sigma_{0}^{\prime}}^{(m), \natural_{n}} \rightarrow T_{n,(i), U, \Sigma_{0}}^{(m), \forall, \wedge}
$$

(resp.

$$
\left.1_{*}: \mathcal{T}_{n,(i),\left(U^{p}\right)^{\prime}\left(N_{1}^{\prime}, N_{2}^{\prime}\right), \Sigma_{0}^{\prime}}^{(m), \text { ord }, \wedge} \rightarrow \mathcal{T}_{n,(i), U^{p}\left(N_{1}, N_{2}\right), \Sigma_{0}}^{(m), \text { ord }, \wedge}\right)
$$

(1) If $i>0$ then

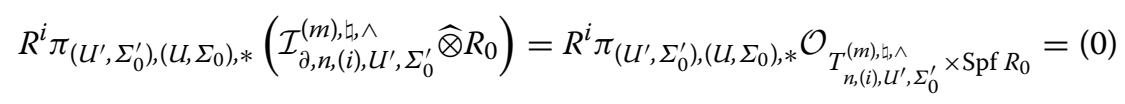

(resp.

$$
R^{i} \pi_{\left(\left(U^{p}\right)^{\prime}\left(N_{1}^{\prime}, N_{2}^{\prime}\right), \Sigma_{0}^{\prime}\right),\left(U^{p}\left(N_{1}, N_{2}\right), \Sigma_{0}\right), *}\left(\mathcal{I}_{\partial, n,(i),\left(U^{p}\right)^{\prime}\left(N_{1}^{\prime}, N_{2}^{\prime}\right), \Sigma_{0}^{\prime}}^{(m), \text { ord }{ }^{\prime}, \wedge} R_{0}\right)=(0)
$$

and

$$
\left.R^{i} \pi_{\left(\left(U^{p}\right)^{\prime}\left(N_{1}^{\prime}, N_{2}^{\prime}\right), \Sigma_{0}^{\prime}\right),\left(U^{p}\left(N_{1}, N_{2}\right), \Sigma_{0}\right), *} \mathcal{O}_{\mathcal{T}_{\left.m,(i),(U)^{\prime}\right)^{\prime}\left(N_{1}^{\prime}, N_{2}^{\prime}\right), \Sigma_{0}^{\prime}}^{(m)} \times \operatorname{Spf} R_{0}}=(0)\right) .
$$

(2) Suppose further that $U^{\prime}\left(\right.$ resp. $\left.\left(U^{p}\right)^{\prime}\right)$ is a normal subgroup of $U$ (resp. $\left.U^{p}\right)$ and that $\Sigma_{0}^{\prime}$ is $U$-invariant (resp. $U^{p}\left(N_{1}, N_{2}\right)$-invariant). Then the natural maps

$$
\mathcal{O}_{T_{n,(i), U, \Sigma_{0}}^{(m), \wedge} \times \operatorname{Spf} R_{0}} \longrightarrow\left(\pi_{\left(U^{\prime}, \Sigma_{0}^{\prime}\right),\left(U, \Sigma_{0}\right), *} \mathcal{O}_{T_{n,(i), U^{\prime}, \Sigma_{0}^{\prime}}^{(m), 4} \times \operatorname{Spf} R_{0}}\right)^{U}
$$

(resp.

$$
\begin{aligned}
& \mathcal{O}_{\mathcal{T}_{n,(i), U p}^{(m), \text { ord }, \uplus_{1} \wedge}\left(N_{1}, N_{2}\right), \Sigma_{0}} \times \operatorname{Spf} R_{0} \\
& \left.\longrightarrow\left(\pi_{\left(\left(\left(U^{p}\right)^{\prime}\left(N_{2}, N_{2}\right), \Sigma_{0}^{\prime}\right),\left(U^{p}\left(N_{1}, N_{2}\right), \Sigma_{0}\right), *\right.} \mathcal{O}_{\mathcal{T}_{n,(i),\left(U^{p}\right)^{\prime}\left(N_{2}, N_{2}\right), \Sigma_{0}^{\prime}}^{(m), \operatorname{Spd}^{\prime}, \wedge}}\right)^{U^{p}\left(N_{1}, N_{2}\right)}\right)
\end{aligned}
$$


and

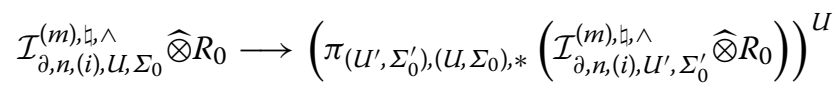

(resp.

$$
\begin{aligned}
& \mathcal{I}_{\partial, n,(i), U^{p}\left(N_{1}, N_{2}\right), \Sigma_{0}}^{(m), \text { ord } \sharp \wedge R_{0}} \\
& \left.\quad \longrightarrow\left(\pi_{\left(\left(U^{p}\right)^{\prime}\left(N_{2}, N_{2}\right), \Sigma_{0}^{\prime}\right),\left(U^{p}\left(N_{1}, N_{2}\right), \Sigma_{0}\right), *}\left(\mathcal{I}_{\partial, n,(i),\left(U^{p}\right)^{\prime}\left(N_{2}, N_{2}\right), \Sigma_{0}^{\prime}}^{(m), \text { ord }, \text { }, \wedge} R_{0}\right)\right)^{U^{p}\left(N_{1}, N_{2}\right)}\right)
\end{aligned}
$$

are isomorphisms.

The same statements are true with $\downarrow$ replaced by + or by $\downarrow+$.

Proof It suffices to treat the case of + . We treat the case of $T_{n,(i), U^{\prime}, \Sigma_{0}^{\prime}}^{(m),+, \wedge} \times \operatorname{Spf} R_{0}$, the case of $\mathcal{T}_{n,(i),\left(U^{p}\right)^{\prime}\left(N_{1}, N_{2}\right), \Sigma_{0}^{\prime}}^{(m), \text { ord },+\wedge} \times \operatorname{Spf} R_{0}$ being exactly similar.

Let $U^{\prime \prime}$ denote the open compact subgroup of $P_{n,(i)}^{(m),+}\left(\mathbb{A}^{\infty}\right)$ generated by $U^{\prime}$ and $U \cap$ $Z\left(N_{n,(i)}^{(m)}\right)\left(\mathbb{A}^{\infty}\right)$. Then $\Sigma_{0}$ is a $U^{\prime \prime}$ admissible smooth cone decomposition of $X_{*}\left(S_{n,(i), U^{\prime \prime}}^{(m),}\right)_{\mathbb{R}}^{\succ 0}$. Moreover

$$
T_{n,(i), U^{\prime \prime}, \widetilde{\Sigma}_{0}}^{(m),+, \wedge} \times \operatorname{Spf} R_{0} \longrightarrow T_{n,(i), U, \widetilde{\Sigma}_{0}}^{(m),+, \wedge} \times \operatorname{Spf} R_{0}
$$

is finite etale, and if $U^{\prime}$ is normal in $U$ then it is Galois with group $U / U^{\prime \prime}$. Thus we may replace $U$ by $U^{\prime \prime}$ and reduce to the case that $U$ and $U^{\prime}$ have the same projection to $\left(P_{n,(i)}^{(m),+} / Z\left(N_{n,(i)}^{(m)}\right)\right)\left(\mathbb{A}^{\infty}\right)$. In this case the result follows from Lemma 2.15 .

Define $\widetilde{\Omega}_{n,(i), U, \Delta_{0}}^{\natural}$ on $T_{n,(i), U, \Delta_{0}}^{\natural, \wedge}$ as the quotient by $L_{n,(i), \text { lin }}(\mathbb{Q})$ of the pull-back of $\widetilde{\Omega}_{n,(i), U}^{+}$ from $A_{n,(i), U}^{+}$to $T_{n,(i), U, \Delta_{0}}^{+, \wedge}$. Also define $\widetilde{\Omega}_{n,(i), U^{p}\left(N_{1}, N_{2}\right), \Delta_{0}}^{\text {ord, } \natural}$ on $\mathcal{T}_{n,(i), U^{p}\left(N_{1}, N_{2}\right), \Delta_{0}}^{\text {ord } \natural, \wedge}$ as the quotient by $L_{n,(i), \text { lin }}\left(\mathbb{Z}_{(p)}\right)$ of the pull-back of the sheaf $\widetilde{\Omega}_{n,(i), U^{p}\left(N_{1}, N_{2}\right)}^{\text {ord, }}$ from $\mathcal{A}_{n,(i), U^{p}\left(N_{1}, N_{2}\right)}^{\text {ord, }}$ to $\mathcal{T}_{n,(i), U^{p}\left(N_{1}, N_{2}\right), \Delta_{0}}^{\text {ord }+, \wedge}$

Suppose that $R_{0}$ is a $\mathbb{Q}$-algebra and that $\rho$ is a representation of $R_{n,(n),(i)}$ on a finite, locally free $R_{0}$-module $W_{\rho}$. Then we define a locally free sheaf $\mathcal{E}_{(i), U, \Delta_{0}, \rho}^{\natural}$ on $T_{n,(i), U, \Delta_{0}}^{\natural, \wedge}$ as the quotient by $L_{n,(i), \operatorname{lin}}(\mathbb{Q})$ of the pull-back of $\mathcal{E}_{(i), U, \rho}^{+}$from $A_{n,(i), U}^{+}$to $T_{n,(i), U, \Delta_{0}}^{+, \wedge}$. Then the system of sheaves $\left\{\mathcal{E}_{(i), U, \Delta_{0}, \rho}^{\natural}\right\}$ over $\left\{T_{n,(i), U, \Delta_{0}}^{\natural, \wedge}\right\}$ has an action of $P_{n,(i)}^{+}\left(\mathbb{A}^{\infty}\right)$. If $g \in P_{n,(i)}^{+}\left(\mathbb{A}^{\infty}\right)$, then the natural map

$$
g^{*} \mathcal{E}_{(i), U, \Delta_{0}, \rho}^{\natural} \longrightarrow \mathcal{E}_{(i), U^{\prime}, \Delta_{0}^{\prime}, \rho}^{\natural}
$$

is an isomorphism. The sheaves $\mathcal{E}_{(i), U, \Delta_{0}, \rho}^{\natural}$ have $P_{n,(i)}^{+}\left(\mathbb{A}^{\infty}\right)$-invariant filtrations by local direct summands whose graded pieces pull-backed to $T_{n,(i), U, \Delta_{0}}^{+, \wedge}$ are equivariantly isomorphic to the pull-backs of sheaves of the form $\mathcal{E}_{(i), U, \rho^{\prime}}^{+}$on $X_{n,(i), U}^{+}$.

Similarly in the case of mixed characteristic suppose that $R_{0}$ is a $\mathbb{Z}_{(p)}$-algebra and that $\rho$ is a representation of $R_{n,(n),(i)}$ on a finite, locally free $R_{0}$-module $W_{\rho}$. Then we define a locally free sheaf $\mathcal{E}_{(i), U^{p}\left(N_{1}, N_{2}\right), \Delta_{0}, \rho}^{\text {ord, }}$ on $\mathcal{T}_{n,(i), U^{p}\left(N_{1}, N_{2}\right), \Delta_{0}}^{\text {ord, }, \wedge}$ as the quotient by $L_{n,(i), \operatorname{lin}}\left(\mathbb{Z}_{(p)}\right)$ of the pull-back of $\mathcal{E}_{(i), U^{p}\left(N_{1}, N_{2}\right), \rho}^{\text {ord, }}$ from $\mathcal{A}_{n,(i), U^{p}\left(N_{1}, N_{2}\right)}^{\text {ord, }}$ to $\mathcal{T}_{n,(i), U^{p}\left(N_{1}, N_{2}\right), \Delta_{0}}^{\text {ord, }}$. Then the collection $\left\{\mathcal{E}_{(i), U^{p}\left(N_{1}, N_{2}\right), \Delta_{0}, \rho}^{\text {ord },}\right\}$ is a system of sheaves over $\left\{\mathcal{T}_{n,(i), U^{p}\left(N_{1}, N_{2}\right), \Delta_{0}}^{\text {ord }, \downarrow \wedge}\right\}$ with an action of $P_{n,(i)}^{+}\left(\mathbb{A}^{\infty}\right)^{\text {ord }, \times}$. If $g \in P_{n,(i)}^{+}\left(\mathbb{A}^{\infty}\right)^{\text {ord, } \times}$, then the natural map 


$$
g^{*} \mathcal{E}_{(i), U U^{p}\left(N_{1}, N_{2}\right), \Delta_{0}, \rho}^{\mathrm{ord}, \natural} \mathcal{E}_{(i),\left(U^{p}\right)^{\prime}\left(N_{1}, N_{2}\right), \Delta_{0}^{\prime}, \rho}^{\mathrm{ord}, \natural}
$$

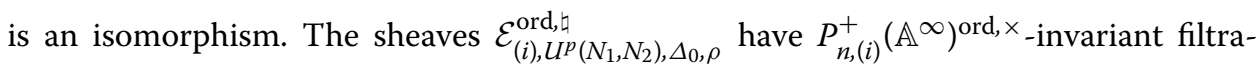
tions by local direct summands whose graded pieces pull-backed to the formal scheme $\mathcal{T}_{n,(i), U^{p}\left(N_{1}, N_{2}\right), \Delta_{0}}^{\text {ord, }}$ are equivariantly isomorphic to the pull-backs of sheaves of the form

$\mathcal{E}_{(i), U^{p}\left(N_{1}, N_{2}\right), \rho^{\prime}}^{\text {ord, }}$ on $\mathcal{X}_{n,(i), U^{p}\left(N_{1}, N_{2}\right)}^{\text {ord, }}$.

Corollary 4.7 Suppose that $R_{0}$ is an irreducible noetherian $\mathbb{Q}$-algebra (resp. $\mathbb{Z}_{(p) \text {-algebra) }}$ with the discrete topology. Let $\rho$ be a representation of $R_{n,(n),(i)}$ on a finite, locally free $R_{0}$-module $W_{\rho}$. Suppose also that $U \supset U^{\prime}$ (resp. $\left.U^{p} \supset\left(U^{p}\right)^{\prime}\right)$ are neat open compact subgroups of $P_{n,(i)}^{(m),+}\left(\mathbb{A}^{\infty}\right)$ (resp. $\left.P_{n,(i)}^{(m),+}\left(\mathbb{A}^{\infty, p}\right)\right)$, that $N_{2}^{\prime} \geq N_{1}^{\prime} \geq 0$ and $N_{2} \geq N_{1} \geq 0$ are integers with $N_{2}^{\prime} \geq N_{2}$ and $N_{1}^{\prime} \geq N_{1}$ and that $\Sigma_{0}$ and $\Sigma_{0}^{\prime}$ are compatible smooth admissible cone decompositions for $X_{*}\left(S_{n,(i), U}^{(m),+}\right)_{\mathbb{R}}^{\succ 0}$ and $X_{*}\left(S_{n,(i), U^{\prime}}^{(m),+}\right)_{\mathbb{R}}^{\succ 0}\left(\right.$ resp. $X_{*}\left(\mathcal{S}_{n,(i), U^{p}\left(N_{1}, N_{2}\right)}^{(m), \text { ord, }}\right)_{\mathbb{R}}^{\succ 0}$ and $\left.X_{*}\left(\mathcal{S}_{n,(i),\left(U^{p}\right)^{\prime}\left(N_{1}^{\prime}, N_{2}^{\prime}\right)}^{(m), \text { ord }+}\right)_{\mathbb{R}}^{\succ 0}\right)$. Let $\pi_{\left(U^{\prime}, \Sigma_{0}^{\prime}\right),\left(U, \Sigma_{0}\right)}\left(\right.$ resp. $\left.\pi_{\left(\left(U^{p}\right)^{\prime}\left(N_{1}^{\prime}, N_{2}^{\prime}\right), \Sigma_{0}^{\prime}\right),\left(U^{p}\left(N_{1}, N_{2}\right), \Sigma_{0}\right)}\right)$ denote the map

$$
1_{*}: T_{n,(i), U^{\prime}, \Sigma_{0}^{\prime}}^{(m), 4, \wedge} \rightarrow T_{n,(i), U, \Sigma_{0}}^{(m), \natural, \wedge}
$$

(resp.

$$
\left.1_{*}: \mathcal{T}_{n,(i),\left(U^{p}\right)^{\prime}\left(N_{1}^{\prime}, N_{2}^{\prime}\right), \Sigma_{0}^{\prime}}^{(m), \text { rd }, \wedge} \rightarrow \mathcal{T}_{n,(i), U^{p}\left(N_{1}, N_{2}\right), \Sigma_{0}}^{(m), \text { ord },, \wedge}\right)
$$

(1) If $i>0$ then

$$
R^{i} \pi_{\left(U^{\prime}, \Sigma_{0}^{\prime}\right),\left(U, \Sigma_{0}\right), *}\left(\mathcal{I}_{\partial, n,(i), U^{\prime}, \Sigma_{0}^{\prime}}^{(m), \natural} \widehat{\mathcal{E}}_{(i), U^{\prime}, \Sigma_{0}^{\prime}, \rho}^{\natural}\right)=R^{i} \pi_{\left(U^{\prime}, \Sigma_{0}^{\prime}\right),\left(U, \Sigma_{0}\right), *} \mathcal{E}_{(i), U^{\prime}, \Sigma_{0}^{\prime}, \rho}^{\natural}=(0)
$$

(resp.

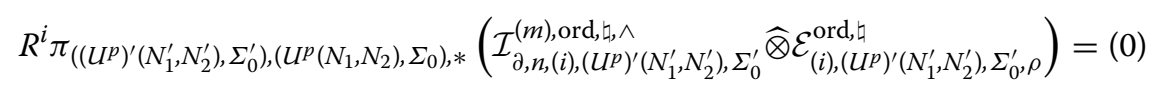

and

$$
\left.R^{i} \pi_{\left(\left(U^{p}\right)^{\prime}\left(N_{1}^{\prime}, N_{2}^{\prime}\right), \Sigma_{0}^{\prime}\right),\left(U^{p}\left(N_{1}, N_{2}\right), \Sigma_{0}\right), *} \mathcal{E}_{(i),\left(U^{p}\right)^{\prime}\left(N_{1}^{\prime}, N_{2}^{\prime}\right), \Sigma_{0}^{\prime}, \rho}^{\mathrm{ord}, \natural}=(0)\right)
$$

(2) Suppose further that $U^{\prime}$ (resp. $\left.\left(U^{p}\right)^{\prime}\right)$ is a normal subgroup of $U$ (resp. $U^{p}$ ) and that $\Sigma_{0}^{\prime}$ is $U$-invariant (resp. $U^{p}\left(N_{1}, N_{2}\right)$-invariant). Then the natural maps

$$
\mathcal{E}_{(i), U, \Sigma_{0}, \rho}^{\natural} \longrightarrow\left(\pi_{\left(U^{\prime}, \Sigma_{0}^{\prime}\right),\left(U, \Sigma_{0}\right), *} \mathcal{E}_{(i), U^{\prime}, \Sigma_{0}^{\prime}, \rho}^{\natural}\right)^{U}
$$

(resp.

$$
\begin{aligned}
& \mathcal{E}_{(i), U^{p}\left(N_{1}, N_{2}\right), \Sigma_{0}, \rho}^{\text {ord },} \\
& \left.\quad \longrightarrow\left(\pi_{\left(\left(\left(U^{p}\right)^{\prime}\left(N_{2}, N_{2}\right), \Sigma_{0}^{\prime}\right),\left(U^{p}\left(N_{1}, N_{2}\right), \Sigma_{0}\right), *\right.} \mathcal{E}_{(i),\left(U^{p}\right)^{\prime}\left(N_{2}, N_{2}\right), \Sigma_{0}^{\prime}, \rho}^{\text {ord }, \natural}\right)^{U^{p}\left(N_{1}, N_{2}\right)}\right)
\end{aligned}
$$

and

$$
\mathcal{I}_{\partial, n,(i), U, \Sigma_{0}}^{(m), \natural} \widehat{\otimes} \mathcal{E}_{(i), U, \Sigma_{0}, \rho}^{\natural} \longrightarrow\left(\pi_{\left(U^{\prime}, \Sigma_{0}^{\prime}\right),\left(U, \Sigma_{0}\right), *}\left(\mathcal{I}_{\partial, n,(i), U^{\prime}, \Sigma_{0}^{\prime}}^{(m), \widehat{\mathcal{E}}_{(i), U^{\prime}, \Sigma_{0}^{\prime}, \rho}^{\natural}}\right)\right)^{U}
$$


(resp.

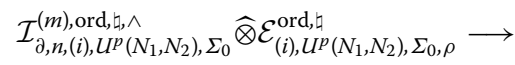

$$
\begin{aligned}
& \left(\pi_{\left(\left(U^{p}\right)^{\prime}\left(N_{2}, N_{2}\right), \Sigma^{\prime}\right),\left(U^{p}\left(N_{1}, N_{2}\right), \Sigma\right), *}\left(\mathcal{I}_{\partial, n, m,(i),\left(U^{p}\right)^{\prime}\left(N_{2}, N_{2}\right), \Sigma_{0}^{\prime}}^{(m), \mathcal{E}_{(i),\left(U^{p}\right)^{\prime}\left(N_{2}, N_{2}\right), \Sigma_{0}^{\prime}, \rho}}\right)^{U^{p}\left(N_{1}, N_{2}\right)}\right)
\end{aligned}
$$

are isomorphisms.

Lemma 4.8 Suppose that $U$ is a neat open compact subgroup of $P_{n,(i)}^{(m),+}\left(\mathbb{A}^{\infty}\right)$ and let $U^{\prime}$ denote the image of $U$ in $P_{n,(i)}^{+}\left(\mathbb{A}^{\infty}\right)$. Let $\Delta_{0}$ be a smooth admissible cone decomposition for $X_{*}\left(S_{n,(i), U^{\prime}}^{+}\right)$and let $\Sigma_{0}$ be a compatible smooth admissible cone decomposition for $X_{*}\left(S_{n,(i), U}^{(m),+}\right)$. Let $\pi^{+}=\pi_{\left(U, \Sigma_{0}\right),\left(U^{\prime}, \Delta_{0}\right)}^{+}$denote the map

$$
T_{n,(i), U, \Sigma_{0}}^{(m), \natural+\wedge} \longrightarrow T_{n,(i), U^{\prime}, \Delta_{0}}^{+, \wedge}
$$

and let $\pi^{\natural}=\pi_{\left(U, \Sigma_{0}\right),\left(U^{\prime}, \Delta_{0}\right)}^{\natural}$ denote the map

$$
T_{n,(i), U, \Sigma_{0}}^{(m), \sharp, \wedge} \longrightarrow T_{n,(i), U^{\prime}, \Delta_{0}}^{\natural, \wedge}
$$

(1) The maps $\pi_{\left(U, \Sigma_{0}\right),\left(U^{\prime}, \Delta_{0}\right)}^{+}$and $\pi_{\left(U, \Sigma_{0}\right),\left(U^{\prime}, \Delta_{0}\right)}^{\natural}$ are proper.

(2) The natural maps

$$
\mathcal{O}_{T_{n,(i), U^{\prime}, \Delta_{0}}^{+, \wedge}} \longrightarrow \pi_{\left(U, \Sigma_{0}\right),\left(U^{\prime}, \Delta_{0}\right), *}^{+} \mathcal{O}_{T_{n,(i), U, \Sigma_{0}}^{(m),+,}}
$$

and

$$
\mathcal{I}_{\partial, n,(i), U^{\prime}, \Delta_{0}}^{+, \wedge} \longrightarrow \pi_{\left(U, \Sigma_{0}\right),\left(U^{\prime}, \Delta_{0}\right), *}^{+} \mathcal{I}_{\partial, n,(i), U, \Sigma_{0}}^{(m), \natural+, \wedge}
$$

and

$$
\mathcal{O}_{T_{n,(i), U^{\prime}, \Delta_{0}}^{\natural, \wedge}} \longrightarrow \pi_{\left(U, \Sigma_{0}\right),\left(U^{\prime}, \Delta_{0}\right), *}^{\natural} \mathcal{O}_{T_{n,(i), U, \Sigma_{0}}^{(m), \downarrow \wedge}}
$$

and

$$
\mathcal{I}_{\partial, n,(i), U^{\prime}, \Delta_{0}}^{\natural, \wedge} \longrightarrow \pi_{\left(U, \Sigma_{0}\right),\left(U^{\prime}, \Delta_{0}\right), *}^{\natural} \mathcal{I}_{\partial, n,(i), U, \Sigma_{0}}^{(m), \Sigma_{0}, \wedge}
$$

are isomorphisms.

(3) The natural maps

$$
\mathcal{I}_{\partial, n,(i), U^{\prime}, \Delta_{0}}^{+, \wedge} \otimes R^{j} \pi_{\left(U, \Sigma_{0}\right),\left(U^{\prime}, \Delta_{0}\right), *}^{+} \mathcal{O}_{T_{n,(i), U, \Sigma_{0}}^{(m),+, \wedge}} \longrightarrow R^{j} \pi_{\left(U, \Sigma_{0}\right),\left(U^{\prime}, \Delta_{0}\right), *}^{+} \mathcal{I}_{\partial, n,(i), U, \Sigma_{0}}^{(m), \natural+, \wedge}
$$

and

$$
\mathcal{I}_{\partial, n,(i), U^{\prime}, \Delta_{0}}^{\natural, \wedge} \otimes R^{j} \pi_{\left(U, \Sigma_{0}\right),\left(U^{\prime}, \Delta_{0}\right), *}^{\natural} \mathcal{O}_{T_{n, i), U, \Sigma_{0}}^{(m), \wedge}} \longrightarrow R^{j} \pi_{\left(U, \Sigma_{0}\right),\left(U^{\prime}, \Delta_{0}\right), *}^{\natural} \mathcal{I}_{\partial, n,(i), U, \Sigma_{0}}^{(m), \downarrow}
$$

are isomorphisms. 
Proof It suffices to treat the + case.

The first part follows from Lemma 2.19. We deduce that all the sheaves mentioned in the remaining parts are coherent.

Thus, by theorem 4.1.5 of [23] ('the theorem on formal functions'), it suffices to prove the remaining assertions after completing at a point of $T_{n,(i), U^{\prime}, \Delta_{0}}^{+, \wedge}$. The set points where the assertions are true after completing at that point is open. (Again because the sheaves involved are all coherent.) This open set is $S_{n,(i), U^{\prime}}^{+}$-invariant. (The sheaves in question do not all have $S_{n,(i), U^{\prime}}^{+}$-actions. However locally on $T_{n,(i), U^{\prime}, \Delta_{0}}^{+, \wedge}$ they do.) Thus it will do to prove the lemma after completion at $\partial_{\sigma} T_{n,(i), U^{\prime}, \widetilde{\Delta}_{0}}^{+}$, for $\sigma \in \Delta_{0}$ maximal. We will add a subscript $\sigma$ to denote completion along $\partial_{\sigma} T_{n,(i), U^{\prime}, \tilde{\Delta}_{0}}^{+}$.

We write $\tilde{\pi}$ for the map

$$
T_{n,(i), U, \Sigma_{0}}^{(m),+, \wedge} \longrightarrow T_{n,(i), U^{\prime}, \Delta_{0}}^{+, \wedge}
$$

and factor $\tilde{\pi}=\pi_{2} \circ \pi_{1}$, where

$$
\pi_{1}: T_{n,(i), U, \Sigma_{0}}^{(m),+\wedge} \longrightarrow T_{n,(i), U^{\prime}, \Delta_{0}}^{+, \wedge} \times_{A_{n,(i), U^{\prime}}^{+}} A_{n,(i), U}^{(m),+}
$$

and

$$
\pi_{2}: T_{n,(i), U^{\prime}, \Delta_{0}}^{+, \wedge} \times_{A_{n,(i), U^{\prime}}^{+}} A_{n,(i), U}^{(m),+} \longrightarrow T_{n,(i), U^{\prime}, \Delta_{0}}^{+, \wedge}
$$

Also write $\pi_{3}$ for the other projection

$$
\pi_{3}: T_{n,(i), U^{\prime}, \Delta_{0}}^{+, \wedge} \times_{A_{n,(i), U^{\prime}}^{+}} A_{n,(i), U}^{(m),+} \longrightarrow A_{n,(i), U}^{(m),+}
$$

We will first show that

$$
R^{j} \pi_{1, \sigma, *} \mathcal{O}_{T_{n,(i), U, \Sigma_{0}, \sigma}^{(m),+\wedge}}= \begin{cases}(0) & \text { if } j>0 \\ \mathcal{O}_{T_{n,(i), U^{\prime}, \Delta_{0}, \sigma}^{+, \wedge} \times_{A_{n,(i), U^{\prime}}^{+}} A_{n,(i), U}^{(m),+}} & \text { if } j=0\end{cases}
$$

and

$$
R^{j} \pi_{1, \sigma, *} \mathcal{I}_{\partial, n,(i), U, \Sigma_{0}, \sigma}^{(m),+, \wedge}= \begin{cases}(0) & \text { if } j>0 \\ \pi_{2, \sigma}^{*} \mathcal{I}_{\partial, n,(i), U^{\prime}, \Delta_{0}, \sigma}^{+, \wedge} & \text { if } j=0\end{cases}
$$

As $T_{n,(i), U^{\prime}, \Delta_{0}, \sigma}^{+, \wedge} \times_{A_{n,(i), U^{\prime}}^{+}} A_{n,(i), U}^{(m),+}$ has the same underlying topological space as $A_{n,(i), U}^{(m),+}$, i.e. $\pi_{3, \sigma}$ is a homeomorphism on the underlying topological space, it suffices to show that

$$
R^{j}\left(\pi_{3} \circ \pi_{1}\right)_{\sigma, *} \mathcal{O}_{T_{n,(i), U, \Sigma_{0}, \sigma}^{(m),+, \wedge}}= \begin{cases}(0) & \text { if } j>0 \\ \pi_{3, \sigma, *} \mathcal{O}_{T_{n,(i), U^{\prime}, \Delta_{0}, \sigma}^{+, \wedge} \times_{A_{n,(i), U^{\prime}}^{+}} A_{n,(i), U}^{(m),+}} & \text { if } j=0\end{cases}
$$

and

$$
R^{j} \pi_{1, \sigma, *} \mathcal{I}_{\partial, n,(i), U, \Sigma_{0}, \sigma}^{(m),+, \wedge}= \begin{cases}(0) & \text { if } j>0 \\ \pi_{3, \sigma, *} \pi_{2, \sigma}^{*} \mathcal{I}_{\partial, n,(i), U^{\prime}, \Delta_{0}, \sigma}^{+, \wedge} & \text { if } j=0\end{cases}
$$


This would follow from Lemma 2.22 as long as we can show that, for all $y \in Y_{n,(i), U}^{(m),+}$ with image $y^{\prime}$ in $Y_{n,(i), U^{\prime}}^{+}$, we have $\left|\Sigma_{0}\right|^{\vee}(y)=\left|\Delta_{0}\right|^{\vee}\left(y^{\prime}\right)$ and $\left|\Sigma_{0}\right|^{\vee, 0}(y)=\left|\Delta_{0}\right|^{\vee, 0}\left(y^{\prime}\right)$. Concretely these required equalities are

$$
\begin{aligned}
\{\chi & \left.\in \mathfrak{C}\left(V_{n,(i)}\right)^{\vee}: \chi \geq 0 \text { on } \mathfrak{C}^{\succ 0}\left(V_{n,(i)}\right)\right\} \\
& \stackrel{\sim}{\longrightarrow}\left\{\chi \in \mathfrak{C}^{(m)}\left(V_{n,(i)}\right)^{\vee}: \chi \geq 0 \text { on } \mathfrak{C}^{(m), \succ 0}\left(V_{n,(i)}\right)\right\}
\end{aligned}
$$

and

$$
\begin{aligned}
\{\chi & \left.\in \mathfrak{C}\left(V_{n,(i)}\right)^{\vee}: \chi>0 \text { on } \mathfrak{C}^{\succ 0}\left(V_{n,(i)}\right)-\{0\}\right\} \\
& \stackrel{\sim}{\longrightarrow}\left\{\chi \in \mathfrak{C}^{(m)}\left(V_{n,(i)}\right)^{\vee}: \chi>0 \text { on } \mathfrak{C}^{(m), \succ 0}\left(V_{n,(i)}\right)-\{0\}\right\}
\end{aligned}
$$

If $\chi$ lies in one of the right hand sides then $\chi(z, f) \geq 0$ for all $z \in \mathfrak{C}^{>0}\left(V_{n,(i)}\right)$ and all $f \in \operatorname{Hom}\left(F^{m}, V_{n,(i)}\right) \otimes_{\mathbb{Q}} \mathbb{R}$. Taking the limit as $z \rightarrow 0$ we see that $\chi \geq 0$ on the vector space $\operatorname{Hom}\left(F^{m}, V_{n,(i)}\right) \otimes_{\mathbb{Q}} \mathbb{R}$ and so $\chi=0$ on this space. Thus the right hand sides are the set of $\chi \in \mathfrak{C}\left(V_{n,(i)}\right)^{\vee}$ such that $\chi \geq 0$ (resp. $\chi>0$ ) on the images of

$$
\mathfrak{C}^{(m), \succ 0}\left(V_{n,(i)}\right) \longrightarrow \mathfrak{C}\left(V_{n,(i)}\right)
$$

(resp.

$$
\left.\mathfrak{C}^{(m), \succ 0}\left(V_{n,(i)}\right)-\{0\} \longrightarrow \mathfrak{C}\left(V_{n,(i)}\right)\right) .
$$

But these images are $\mathfrak{C}^{\succ 0}\left(V_{n,(i)}\right)$ (resp. $\left.\mathfrak{C}^{\succ 0}\left(V_{n,(i)}\right)-\{0\}\right)$ and so the required equalities hold.

We deduce that

$$
R^{j} \widetilde{\pi}_{\sigma, *} \mathcal{O}_{T_{n,(i), U, \Sigma_{0}, \sigma}^{(m),+\wedge}}=\left(\wedge^{j} \operatorname{Hom}_{F}\left(\Omega_{n,(i), U^{\prime}}^{+}, F^{m} \otimes_{\mathbb{Q}} \Xi_{n,(i), U^{\prime}}^{+}\right)\right) \otimes_{\mathcal{O}_{X_{n,(i), U^{\prime}}^{+}}} \mathcal{O}_{T_{n,(i), U^{\prime}, \Delta_{0}, \sigma}^{+, \wedge}}
$$

and

$$
R^{j} \tilde{\pi}_{\sigma, *} \mathcal{I}_{\partial, n,(i), U, \Sigma_{0}, \sigma}^{(m),+, \wedge}=\left(\wedge^{j} \operatorname{Hom}_{F}\left(\Omega_{n,(i), U^{\prime}}^{+}, F^{m} \otimes_{\mathbb{Q}} \Xi_{n,(i), U^{\prime}}^{+}\right)\right) \otimes_{\mathcal{O}_{X_{n,(i), U^{\prime}}^{+}}} \mathcal{I}_{\partial, n,(i), U^{\prime}, \Delta_{0}, \sigma^{*}}^{+, \wedge}
$$

As $T_{n,(i), U, \Sigma_{0}, \sigma}^{(m), 4+, \wedge}$ is the quotient of $T_{n,(i), U, \Sigma_{0}, \sigma}^{(m),+\wedge}$ by $\operatorname{Hom}_{F}\left(F^{m}, F^{i}\right)$, we obtain spectral sequences

$$
\begin{aligned}
& H^{j_{1}}\left(\operatorname{Hom}_{F}\left(F^{m}, F^{i}\right),\left(\wedge^{j_{2}} \operatorname{Hom}_{F}\left(\Omega_{n,(i), U^{\prime}}^{+}, F^{m} \otimes_{\mathbb{Q}} \Xi_{n,(i), U^{\prime}}^{+}\right)\right)\right) \otimes_{\mathcal{O}_{X_{n,(i), U^{\prime}}^{+}}} \mathcal{O}_{T_{n,(i), U^{\prime}, \Delta_{0}, \sigma}^{+, \wedge}} \\
& \quad \Rightarrow R^{j_{1}+j_{2}} \pi_{*}^{+} \mathcal{O}_{T_{n,(i), U, \Sigma_{0}, \sigma}^{(m), \natural+, \wedge}}
\end{aligned}
$$

and

$$
\begin{aligned}
& H^{j_{1}}\left(\operatorname{Hom}_{F}\left(F^{m}, F^{i}\right),\left(\wedge^{j_{2}} \operatorname{Hom}_{F}\left(\Omega_{n,(i), U^{\prime}}^{+}, F^{m} \otimes_{\mathbb{Q}} \Xi_{n,(i), U^{\prime}}^{+}\right)\right)\right) \otimes_{\mathcal{O}_{X_{n,(i), U^{\prime}}^{+}}} \mathcal{I}_{\partial, n,(i), U^{\prime}, \Delta_{0}, \sigma}^{+, \wedge} \\
& \quad \Rightarrow R^{j_{1}+j_{2}} \pi_{*}^{+} \mathcal{I}_{\partial, n,(i), U, \Sigma_{0}, \sigma^{\circ}}^{(m), \natural+, \wedge}
\end{aligned}
$$

These can also be written

$$
\begin{aligned}
& \operatorname{Hom}\left(\wedge^{j_{1}} \operatorname{Hom}_{F}\left(F^{m}, F^{i}\right), \wedge^{j_{2}} \operatorname{Hom}_{F}\left(\Omega_{n,(i), U^{\prime}}^{+} F^{m} \otimes_{\mathbb{Q}} \Xi_{n,(i), U^{\prime}}^{+}\right)\right) \otimes_{\mathcal{O}_{X_{n,(i), U^{\prime}}^{+}}} \mathcal{O}_{n,(i), U^{\prime}, \Delta_{0}, \sigma}^{+, \wedge} \\
& \Rightarrow R^{j_{1}+j_{2}} \pi_{*}^{+} \mathcal{O}_{T_{n,(i), U, \Sigma_{0}, \sigma}^{(m), \ell+\wedge}}
\end{aligned}
$$


and

$$
\begin{aligned}
& \operatorname{Hom}\left(\wedge^{j_{1}} \operatorname{Hom}_{F}\left(F^{m}, F^{i}\right), \wedge^{j_{2}} \operatorname{Hom}_{F}\left(\Omega_{n,(i), U^{\prime}}^{+}, F^{m} \otimes_{\mathbb{Q}} \Xi_{n,(i), U^{\prime}}^{+}\right)\right) \otimes_{\mathcal{O}_{X_{n,(i), U^{\prime}}^{+}, n,(i), U^{\prime}, \Delta_{0}, \sigma}} \mathcal{I}^{+, \wedge} \\
& \quad \Rightarrow R^{j_{1}+j_{2}} \pi_{*}^{+} \mathcal{I}_{\partial, n,(i), U, \Sigma_{0}, \sigma^{\circ}}^{(m), \natural+, \wedge}
\end{aligned}
$$

The lemma follows (as $\mathcal{I}_{\partial, n,(i), U^{\prime}, \Delta_{0}, \sigma}^{+, \wedge}$ is flat over $\left.\mathcal{O}_{T_{n,(i), U^{\prime}, \Delta_{0}, \sigma}^{+, \wedge}}\right)$.

The following lemma is equation (1.3.2.86) in lemma 1.3.2.79 of [44].

Lemma 4.9 Suppose that $U$ is a neat open compact subgroup of $P_{n,(i)}^{(m),+}\left(\mathbb{A}^{\infty}\right)$ and let $U^{\prime}$ denote the image of $U$ in $P_{n,(i)}^{+}\left(\mathbb{A}^{\infty}\right)$. Let $\Delta_{0}$ be a smooth admissible cone decomposition for $X_{*}\left(S_{n,(i), U^{\prime}}^{+}\right)$and let $\Sigma_{0}$ be a compatible smooth admissible cone decomposition for $X_{*}\left(S_{n,(i), U}^{(m),+}\right)$. There are canonical equivariant isomorphisms

$$
\operatorname{Hom}_{F}\left(F^{m}, \widetilde{\Omega}_{n,(i), U^{\prime}}^{+}\right) \otimes_{\mathcal{O}_{A_{n,(i), U^{\prime}}^{+}}} \mathcal{O}_{T_{n,(i), U, \Sigma_{0}}^{(m),+, \wedge}} \stackrel{\sim}{\longrightarrow} \Omega_{T_{n,(i), U, \Sigma_{0}}^{(m),+, \wedge} / T_{n,(i), U^{\prime}, \Delta_{0}}^{+, \wedge}}^{1}(\log \infty)
$$

We deduce the following lemmas.

Lemma 4.10 Suppose that $U$ is a neat open compact subgroup of $P_{n,(i)}^{(m),+}\left(\mathbb{A}^{\infty}\right)$ and let $U^{\prime}$ denote the image of $U$ in $P_{n,(i)}^{+}\left(\mathbb{A}^{\infty}\right)$. Let $\Delta_{0}$ be a smooth admissible cone decomposition for $X_{*}\left(S_{n,(i), U^{\prime}}^{+}\right)$and let $\Sigma_{0}$ be a compatible smooth admissible cone decomposition for $X_{*}\left(S_{n,(i), U}^{(m),+}\right)$. Let $\pi^{+}=\pi_{\left(U, \Sigma_{0}\right),\left(U^{\prime}, \Delta_{0}\right)}^{+}$denote the map

$$
T_{n,(i), U, \Sigma_{0}}^{(m), \downarrow+, \wedge} \longrightarrow T_{n,(i), U^{\prime}, \Delta_{0}}^{+, \wedge}
$$

and let $\pi^{\natural}=\pi_{\left(U, \Sigma_{0}\right),\left(U^{\prime}, \Delta_{0}\right)}^{\natural}$ denote the map

$$
T_{n,(i), U, \Sigma_{0}}^{(m), \downarrow, \wedge} \longrightarrow T_{n,(i), U^{\prime}, \Delta_{0}}^{\natural,}
$$

(1) $\pi_{*}^{\natural} \Omega_{T_{n,(i), U, \Sigma_{0}}^{(m), \natural} / T_{n,(i), U^{\prime}, \Delta_{0}}^{\natural, \wedge}}^{1}(\log \infty) \cong \operatorname{Hom}_{F}\left(F^{m}, \widetilde{\Omega}_{n,(i), U^{\prime}}^{\natural}\right)$ is locally free of finite rank.

(2) The natural map

$$
\begin{gathered}
\pi_{\left(U, \Sigma_{0}\right),\left(U^{\prime}, \Delta_{0}\right)}^{\natural, *} \pi_{\left(U, \Sigma_{0}\right),\left(U^{\prime}, \Delta_{0}\right), *}^{\natural} \Omega_{T_{n,(i), U, \Sigma_{0}}^{(m), \downarrow, \wedge}}^{1} / T_{n,(i), U^{\prime}, \Delta_{0}}^{\natural, \wedge}(\log \infty) \\
\longrightarrow \Omega_{T_{n,(i), U, \Sigma_{0}}^{(m), \downarrow, \wedge}}^{1} / T_{n,(i), U^{\prime}, \Delta_{0}}^{\natural, \wedge}(\log \infty)
\end{gathered}
$$

is an isomorphism.

(3) The natural maps

$$
\begin{aligned}
& \left(R^{j_{1}} \pi_{*}^{\natural} \mathcal{O}_{T_{n,(i), U, \Sigma_{0}}^{(m), \sharp \wedge}}\right) \otimes\left(\wedge^{j_{2}} \pi_{*}^{\natural} \Omega_{T_{n,(i), U, \Sigma_{0}}^{(m), \sharp, \wedge} / T_{n,(i), U^{\prime}, \Delta_{0}}^{\natural, \wedge}}^{1}(\log \infty)\right) \\
& \longrightarrow R^{j_{1}} \pi_{*}^{\natural} \Omega_{T_{n,(i), U, \Sigma_{0}}^{(m), \Sigma_{2}} / T_{n,(i), U^{\prime}, \Delta_{0}}^{\natural, \wedge}}^{j_{2}}(\log \infty)
\end{aligned}
$$


and

$$
\begin{aligned}
& \left(R^{j_{1}} \pi_{*}^{\natural} \mathcal{O}_{T_{n, i(i), U, \Sigma_{0}}^{(m), \wedge}}\right) \otimes\left(\wedge^{j_{2}} \pi_{*}^{\natural} \Omega_{T_{n,(i), U, \Sigma_{0}}^{(m), \wedge} / T_{n,(i), U^{\prime}, \Delta_{0}}^{\natural, \wedge}}^{1}(\log \infty)\right) \otimes \mathcal{I}_{\partial, n,(i), U^{\prime}, \Delta_{0}}^{\natural, \wedge} \\
& \longrightarrow R^{j_{1}} \pi_{*}^{\natural}\left(\Omega_{T_{n, i}(i), \Sigma_{0}, \Sigma_{0}}^{j_{2}} / T_{n, i, U^{\prime}, \Delta_{0}}^{\natural, \wedge}(\log \infty) \otimes \mathcal{I}_{\partial, n,(i), U, \sigma_{0}}^{(m), \downarrow}\right)
\end{aligned}
$$

are isomorphisms.

Lemma 4.11 Suppose that $U \supset U^{\prime}$ are neat open compact subgroups of the group $P_{n,(i)}^{(m),+}\left(\mathbb{A}^{\infty}\right)$ and let $V$ and $V^{\prime}$ denote the images of $U$ and $U^{\prime}$ in $P_{n,(i)}^{+}\left(\mathbb{A}^{\infty}\right)$. Let $\Delta_{0}$ (resp. $\left.\Delta_{0}^{\prime}\right)$ be a smooth admissible cone decomposition for $X_{*}\left(S_{n,(i), V}^{+}\right)\left(\right.$resp. $\left.X_{*}\left(S_{n,(i), V^{\prime}}^{+}\right)\right)$and let $\Sigma_{0}\left(\right.$ resp. $\left.\Sigma_{0}^{\prime}\right)$ be a compatible smooth admissible cone decomposition for $X_{*}\left(S_{n,(i), U}^{(m),+}\right)$ (resp. $X_{*}\left(S_{n,(i), U^{\prime}}^{(m)}\right)$ ). Further suppose that $\Sigma_{0}$ and $\Sigma_{0}^{\prime}$ are compatible and that $\Delta_{0}$ and $\Delta_{0}^{\prime}$ are compatible.

(1) The natural map

$$
\pi_{\left(U^{\prime}, \Sigma_{0}^{\prime}\right),\left(U, \Sigma_{0}\right)}^{*} \Omega_{T_{n, i}^{(i), U, \Sigma_{0}}}^{1(m), \wedge} / T_{n,(i), V, \Delta_{0}}^{\natural, \wedge}(\log \infty) \longrightarrow \Omega_{T_{n, i}^{(i), U^{\prime}, \Sigma_{0}^{\prime}}}^{1(m), T_{n,(i), V^{\prime}, \Delta_{0}^{\prime}}^{\natural, \wedge}}(\log \infty)
$$

is an isomorphism.

(2) The natural map

$$
\begin{gathered}
\pi_{\left(V^{\prime}, \Delta_{0}^{\prime}\right),\left(V, \Delta_{0}\right)}^{*} \pi_{\left(U, \Sigma_{0}\right),\left(V, \Delta_{0}\right), *} \Omega_{T_{n,(i), U, \Sigma_{0}}^{(m),} / T_{n,(i), V, \Delta_{0}}^{\natural, \wedge}}^{1}(\log \infty) \\
\longrightarrow \pi_{\left(U^{\prime}, \Sigma_{0}^{\prime}\right),\left(V^{\prime}, \Delta_{0}^{\prime}\right), *} \Omega_{T_{n,(i), U^{\prime}, \Sigma_{0}^{\prime}}^{(m), T_{n,(i), V^{\prime}, \Delta_{0}^{\prime}}^{\natural, \wedge}}}^{1}(\log \infty)
\end{gathered}
$$

is an isomorphism.

Similarly we have the following lemma.

Lemma 4.12 Suppose that $U^{p}$ is a neat open compact subgroup of $P_{n,(i)}^{(m),+}\left(\mathbb{A}^{\infty, p}\right)$ and let $\left(U^{p}\right)^{\prime}$ denote the image of $U^{p}$ in $P_{n,(i)}^{+}\left(\mathbb{A}^{\infty, p}\right)$. Suppose that $N_{2} \geq N_{1} \geq 0$ are integers. Let $\Delta_{0}$ be a smooth admissible cone decomposition for $X_{*}\left(\mathcal{S}_{n,(i),\left(U^{p}\right)^{\prime}\left(N_{1}, N_{2}\right)}^{\mathrm{ord}}\right)$ and let $\Sigma_{0}$ be a compatible smooth admissible cone decomposition for $X_{*}\left(\mathcal{S}_{n,(i), U^{p}\left(N_{1}, N_{2}\right)}^{(m), \text { ord, }}\right)$ Let $\pi^{\natural}=$ $\pi_{\left(U^{p}\left(N_{1}, N_{2}\right), \Sigma_{0}\right),\left(\left(U^{p}\right)^{\prime}\left(N_{1}, N_{2}\right), \Delta_{0}\right)}^{\natural}$ denote the map

$$
\mathcal{T}_{n,(i), U^{p}\left(N_{1}, N_{2}\right), \Sigma_{0}}^{(m), \text { ord }, \wedge} \longrightarrow \mathcal{T}_{n,(i),\left(U^{p}\right)^{\prime}\left(N_{1}, N_{2}\right), \Delta_{0}}^{\mathrm{ord}, \iota^{\circ}}
$$

(1) The map $\pi_{\left(U^{p}\left(N_{1}, N_{2}\right), \Sigma_{0}\right),\left(\left(U^{p}\right)^{\prime}\left(N_{1}, N_{2}\right), \Delta_{0}\right)}^{\natural \text { is proper. }}$

(2) The natural maps

$$
\mathcal{O}_{\mathcal{T}_{n,(i),\left(U^{p}\right)^{\prime}\left(N_{1}, N_{2}\right), \Delta_{0}}^{\text {ord } h_{1} \wedge}} \longrightarrow \pi_{\left(U^{p}\left(N_{1}, N_{2}\right), \Sigma_{0}\right),\left(\left(U^{p}\right)^{\prime}\left(N_{1}, N_{2}\right), \Delta_{0}\right), *}^{\natural} \mathcal{O}_{\mathcal{T}_{n,(i), U p\left(N_{1}, N_{2}\right), \Sigma_{0}}^{(m), \text { ord }, \wedge}}
$$

and

$$
\mathcal{I}_{\partial, n,(i),\left(U^{p}\right)^{\prime}\left(N_{1}, N_{2}\right), \Delta_{0}}^{\mathrm{ord}, \downarrow} \longrightarrow \pi_{\left(U^{p}\left(N_{1}, N_{2}\right), \Sigma_{0}\right),\left(\left(U^{p}\right)^{\prime}\left(N_{1}, N_{2}\right), \Delta_{0}\right), *}^{\natural} \mathcal{I}_{\partial, n,(i), U^{p}\left(N_{1}, N_{2}\right), \Sigma_{0}}^{(m), \text { ord, } \natural^{\wedge}}
$$

are isomorphisms. 
(3) The natural map

$$
\mathcal{I}_{\partial, n,(i),\left(U^{p}\right)^{\prime}\left(N_{1}, N_{2}\right), \Delta_{0}}^{\mathrm{ord}, \sharp, \wedge} \otimes R^{j} \pi_{*}^{\natural} \mathcal{O}_{\mathcal{T}_{n,(i), U^{p}\left(N_{1}, N_{2}\right), \Sigma_{0}}^{(m), \text { ord }, \wedge}}^{\longrightarrow} \longrightarrow R^{j} \pi_{*}^{\natural} \mathcal{I}_{\partial, n,(i), U^{p}\left(N_{1}, N_{2}\right), \Sigma_{0}}^{(m), \text { ord, }, \wedge}
$$

is an isomorphism.

We finish this section with an important vanishing result.

Lemma 4.13 Suppose that $R_{0}$ is an irreducible, noetherian $\mathbb{Q}$-algebra (resp. $\mathbb{Z}_{(p) \text {-algebra) }}$ with the discrete topology. Suppose also that $U$ (resp. $U^{p}$ ) is a neat open compact subgroup of $P_{n,(i)}^{+}\left(\mathbb{A}^{\infty}\right)$ (resp. $\left.P_{n,(i)}^{+}\left(\mathbb{A}^{\infty, p}\right)\right)$, that $N_{2} \geq N_{1} \geq 0$ are integers and that $\Delta_{0}$ is a smooth admissible cone decomposition for $X_{*}\left(S_{n,(i), U}^{+}\right)_{\mathbb{R}}^{\succ 0}$ (resp. $\left.X_{*}\left(\mathcal{S}_{n,(i), U}^{\text {ord },+}\right)_{\mathbb{R}}^{\succ 0}\right)$. Let $\pi$ denote the map

$$
\pi: T_{n,(i), U, \Delta_{0}}^{\natural, \wedge} \longrightarrow X_{n,(i), U}^{\natural}
$$

(resp.

$$
\left.\pi: \mathcal{T}_{n,(i), U^{p}\left(N_{1}, N_{2}\right), \Delta_{0}}^{\mathrm{ord}, \mathfrak{A}} \longrightarrow \mathcal{X}_{n,(i), U^{p}\left(N_{1}, N_{2}\right)}^{\mathrm{ord}, \mathfrak{l}}\right)
$$

Further suppose that $\mathcal{E}$ is a coherent sheaf on the formal scheme $T_{n,(i), U, \Delta_{0}}^{\natural, \wedge} \times \operatorname{Spf} R_{0}$ (resp. $\left.\mathcal{T}_{n,(i), U^{p}\left(N_{1}, N_{2}\right), \Delta_{0}}^{\text {ord } \natural_{,}} \times \operatorname{Spf} R_{0}\right)$ with an exhaustive separated filtration, such that the pull-back to $T_{n,(i), U, \Delta_{0}}^{+, \wedge} \times \operatorname{Spf} R_{0}\left(\operatorname{resp} . \mathcal{T}_{n,(i), U^{p}\left(N_{1}, N_{2}\right), \Delta_{0}}^{\mathrm{ord},+\wedge} \times \operatorname{Spf} R_{0}\right)$ of each

$$
\operatorname{gr}^{i} \mathcal{E}
$$

is $L_{n,(i), \operatorname{lin}}(\mathbb{Q})$-equivariantly (resp. $L_{n,(i), \operatorname{lin}}\left(\mathbb{Z}_{(p)}\right)$-equivariantly) isomorphic to the pullback to $T_{n,(i), U, \Delta_{0}}^{+, \wedge} \times \operatorname{Spf} R_{0}$ (resp. $\left.\mathcal{T}_{n,(i), U^{p}\left(N_{1}, N_{2}\right), \Delta_{0}}^{\text {ord }+, \wedge} \times \operatorname{Spf} R_{0}\right)$ of a locally free sheaf $\mathcal{F}_{i}$ with $L_{n,(i), \operatorname{lin}}(\mathbb{Q})$-action (resp. $L_{n,(i), \operatorname{lin}}\left(\mathbb{Z}_{(p)}\right)$-action) over $X_{n,(i), U}^{+} \times \operatorname{Spec} R_{0}\left(\right.$ resp. $\mathcal{X}_{n,(i), U}^{\mathrm{ord},+} \times$ Spec $\left.R_{0}\right)$.

Then for $i>0$

$$
R^{i} \pi_{*}\left(\mathcal{E} \otimes \mathcal{I}_{\partial, n,(i), U, \Delta_{0}}^{\natural, \wedge}\right)=(0)
$$

(resp.

$$
R^{i} \pi_{*}\left(\mathcal{E} \otimes \mathcal{I}_{\partial, n,(i), U^{p}\left(N_{1}, N_{2}\right), \Delta_{0}}^{\text {ord }, \llbracket \wedge}=(0)\right)
$$

Proof We will treat the case of $T_{n,(i), U, \Delta_{0}}^{\natural, \wedge} \times \operatorname{Spf} R_{0}$, the other case being exactly similar. We can immediately reduce to the case that the pull-back to $T_{n,(i), U, \Delta_{0}}^{+, \wedge} \times \operatorname{Spf} R_{0}$ of $\mathcal{E}$ is $L_{n,(i) \text {,lin }}(\mathbb{Q})$-equivariantly isomorphic to the pull-back to $T_{n,(i), U, \Delta_{0}}^{+, \wedge} \operatorname{Spf} R_{0}$ of a locally free sheaf $\mathcal{F}$ with $L_{n,(i), \text { lin }}(\mathbb{Q})$-action over $X_{n,(i), U}^{+} \times \operatorname{Spec} R_{0}$.

Let $\pi^{+}$denote the map

$$
\pi^{+}: T_{n,(i), U, \Delta_{0}}^{+, \wedge} \times \operatorname{Spf} R_{0} \longrightarrow X_{n,(i), U}^{\natural} \times \operatorname{Spec} R_{0}
$$

Also write $\pi^{+}=\pi_{1}^{+} \circ \pi_{2}^{+}$, where

$$
\pi_{1}^{+}: A_{n,(i), U}^{+} \times \operatorname{Spec} R_{0} \longrightarrow X_{n,(i), U}^{\natural} \times \operatorname{Spec} R_{0}
$$


and

$$
\pi_{2}^{+}: T_{n,(i), U, \Delta_{0}}^{+, \wedge} \times \operatorname{Spec} R_{0} \longrightarrow A_{n,(i), U}^{+} \times \operatorname{Spec} R_{0}
$$

By Lemma 2.22 we have that

$$
R^{i} \pi_{2, *}^{+}\left(\mathcal{F} \otimes \mathcal{I}_{\partial, n,(i), U, \Delta_{0}}^{\wedge}\right)= \begin{cases}\mathcal{F} \otimes \prod_{a \in X^{*}\left(S_{n,(i), U}^{+}\right)>0} \mathcal{L}_{U}^{+}(a) & \text { if } i=0 \\ (0) & \text { otherwise. }\end{cases}
$$

Then by Lemma 4.2 (or in the case of $\mathcal{T}_{n,(i), U^{p}\left(N_{1}, N_{2}\right), \Delta_{0}}^{\text {ord, }, \wedge} \times \operatorname{Spf} R_{0}$ Lemma 4.3) we deduce that

$$
\begin{aligned}
& R^{i} \pi_{*}^{+}\left(\mathcal{F} \otimes \mathcal{I}_{\partial, n,(i), U, \Delta_{0}}^{\wedge}\right) \\
& = \begin{cases}\operatorname{Ind}_{\{1\}}^{\left.L_{n, i}\right) \text { lin }(\mathbb{Q})}\left(\prod_{a^{\natural} \in X^{*}\left(S_{n,(i), U}^{+}\right)>0, \sharp}\left(\pi_{A^{+} / X^{\natural}, *} \mathcal{L} \otimes \mathcal{F}\right)_{U}^{+}\left(a^{\natural}\right)^{L_{n,(i), l i n}(\mathbb{Q})}\right) & \text { if } i=0 \\
(0) & \text { otherwise }\end{cases}
\end{aligned}
$$

Finally there is a spectral sequence

$$
H^{i}\left(L_{n,(i), \operatorname{lin}}(\mathbb{Q}), R^{j} \pi_{*}^{+}\left(\mathcal{F} \otimes \mathcal{I}_{\partial, n,(i), U, \Delta_{0}}^{\wedge}\right)\right) \Rightarrow R^{i+j} \pi_{*}\left(\mathcal{F} \otimes \mathcal{I}_{\partial, n,(i), U, \Delta_{0}}^{\wedge}\right),
$$

and so the present lemma follows on applying Shapiro's lemma.

Corollary 4.14 Suppose that $U$ (resp. $\left.U^{p}\right)$ is a neat open compact subgroup of $P_{n,(i)}^{+}\left(\mathbb{A}^{\infty}\right)$ (resp. $\left.P_{n,(i)}^{+}\left(\mathbb{A}^{\infty, p}\right)\right)$, that $N_{2} \geq N_{1} \geq 0$ are integers, and that $\Delta_{0}$ is a smooth admissible cone decomposition for $X_{*}\left(S_{n,(i), U}^{+}\right)_{\mathbb{R}}^{\succ 0}\left(\right.$ resp. $\left.X_{*}\left(\mathcal{S}_{n,(i), U}^{\text {ord },+}\right)_{\mathbb{R}}^{\succ 0}\right)$. Let $\pi$ denote the map

$$
\pi: T_{n,(i), U, \Delta_{0}}^{\natural, \wedge} \longrightarrow X_{n,(i), U}^{\natural}
$$

(resp.

$$
\pi: \mathcal{T}_{n,(i), U^{p}\left(N_{1}, N_{2}\right), \Delta_{0}}^{\left.\mathrm{ord}, \sharp \mathcal{X}_{n,(i), U^{p}\left(N_{1}, N_{2}\right)}^{\mathrm{ord}, \boldsymbol{B}}\right)}
$$

Also suppose that $R_{0}$ is an irreducible noetherian $\mathbb{Q}$-algebra (resp. $\mathbb{Z}_{(p)}$-algebra) with the discrete topology and that $\rho$ is a representation of $R_{n,(n),(i)}$ on a finite locally free $R_{0}$-module.

Then for $i>0$

$$
R^{i} \pi_{*}\left(\mathcal{E}_{n,(i), U, \Delta_{0}, \rho}^{\natural} \otimes \mathcal{I}_{\partial, n,(i), U, \Delta_{0}}^{\natural, \wedge}\right)=(0)
$$

(resp.

$$
\left.R^{i} \pi_{*}\left(\mathcal{E}_{n,(i), U^{p}\left(N_{1}, N_{2}\right), \Delta_{0}, \rho}^{\mathrm{ord},} \otimes \mathcal{I}_{\partial, n,(i), U^{p}\left(N_{1}, N_{2}\right), \Delta_{0}}^{\mathrm{ord}, \downarrow \wedge}\right)=(0)\right) .
$$




\section{Compactification of Shimura varieties}

We now turn to the compactification of the $X_{n, U}$ and the $A_{n, U}^{(m)}$.

\subsection{The minimal compactification}

There is a canonically defined system of normal projective schemes with $G_{n}\left(\mathbb{A}^{\infty}\right)$-action, $\left\{X_{n, U}^{\min } / \operatorname{Spec} \mathbb{Q}\right\}$ (for $U \subset G_{n}\left(\mathbb{A}^{\infty}\right)$ a neat open compact subgroup), together with compatible, $G_{n}\left(\mathbb{A}^{\infty}\right)$-equivariant, dense open embeddings

$$
j_{U}^{\min }: X_{n, U} \hookrightarrow X_{n, U}^{\min }
$$

These schemes are referred to as the minimal (or sometimes 'Baily-Borel') compactifications. (The introduction to [49] asserts that the scheme $X_{n, U}^{\min }$ is the minimal normal compactification of $X_{n, U}$, although we won't need this fact.) For $g \in G_{n}\left(\mathbb{A}^{\infty}\right)$ and $g^{-1} U g \subset U^{\prime}$ the maps

$$
g: X_{n, U}^{\min } \longrightarrow X_{n, U^{\prime}}^{\min }
$$

are finite.

Write

$$
\partial X_{n, U}^{\min }=X_{n, U}^{\min }-X_{n, U} .
$$

There is a family of closed subschemes

$$
\partial_{0} X_{n, U}^{\min }=X_{n, U}^{\min } \supset \partial_{1} X_{n, U}^{\min }=\partial X_{n, U}^{\min } \supset \partial_{2} X_{n, U}^{\min } \supset \cdots \supset \partial_{n} X_{n, U}^{\min } \supset \partial_{n+1} X_{n, U}^{\min }=\emptyset
$$

such that each

$$
\partial_{i}^{0} X_{n, U}^{\min }=\partial_{i} X_{n, U}^{\min }-\partial_{i+1} X_{n, U}^{\min }
$$

is smooth of dimension $(n-i)^{2}\left[F^{+}: \mathbb{Q}\right]$. The families $\left\{\partial_{i} X_{n, U}^{\min }\right\}$ and $\left\{\partial_{i}^{0} X_{n, U}^{\min }\right\}$ are families of schemes with $G_{n}\left(\mathbb{A}^{\infty}\right)$-action. Moreover we have a decomposition

$$
\partial_{i}^{0} X_{n, U}^{\min }=\coprod_{h \in P_{n, i}^{+}\left(\mathbb{A}^{\infty}\right) \backslash G_{n}\left(\mathbb{A}^{\infty}\right) / U} X_{n,(i), h U h^{-1} \cap P_{n, i}^{+}\left(\mathbb{A}^{\infty}\right)}^{\natural}
$$

If $g \in G_{n}\left(\mathbb{A}^{\infty}\right)$ and if $g^{-1} U g \subset U^{\prime}$ then the map

$$
g: \partial_{i}^{0} X_{n, U}^{\min } \longrightarrow \partial_{i}^{0} X_{n, U^{\prime}}^{\min }
$$

is the coproduct of the maps

$$
g^{\prime}: X_{n,(i), h U h^{-1} \cap P_{n,(i)}^{+}\left(\mathbb{A}^{\infty}\right)}^{\natural} \longrightarrow X_{n,(i), h^{\prime} U^{\prime}\left(h^{\prime}\right)^{-1} \cap P_{n,(i)}^{+}\left(\mathbb{A}^{\infty}\right)}^{\natural}
$$

where $h g=g^{\prime} h^{\prime}$ with $g^{\prime} \in P_{n,(i)}^{+}\left(\mathbb{A}^{\infty}\right)$. We will write $X_{n, U, i}^{\min , \wedge}$ for the completion of $X_{n, U}^{\min }$ along $\partial_{i}^{0} X_{n, U}^{\min }$. (See theorem 1.3.1.5 and proposition 1.3.1.14 of [44].)

There is also a canonically defined system of normal quasi-projective schemes with $G_{n}\left(\mathbb{A}^{\infty}\right)^{\text {ord }}$-action, $\left\{\mathcal{X}_{n, U^{p}\left(N_{1}, N_{2}\right)}^{\text {ord min }} / \operatorname{Spec} \mathbb{Z}_{(p)}\right\}$, together with compatible, dense open embeddings

$$
j_{U^{p}\left(N_{1}, N_{2}\right)}^{\min }: \mathcal{X}_{n, U^{p}\left(N_{1}, N_{2}\right)}^{\text {ord }} \hookrightarrow \mathcal{X}_{n, U^{p}\left(N_{1}, N_{2}\right)}^{\text {ord, min }}
$$


which are $G_{n}\left(\mathbb{A}^{\infty}\right)^{\text {ord }}$-equivariant. Suppose that $g \in G_{n}\left(\mathbb{A}^{\infty}\right)^{\text {ord }}$ and that

$$
g^{-1} U^{p}\left(N_{1}, N_{2}\right) g \subset\left(U^{p}\right)^{\prime}\left(N_{1}^{\prime}, N_{2}^{\prime}\right),
$$

then

$$
g: \mathcal{X}_{n, U^{p}\left(N_{1}, N_{2}\right)}^{\mathrm{ord} \min } \longrightarrow \mathcal{X}_{n,\left(U^{p}\right)^{\prime}\left(N_{1}^{\prime}, N_{2}^{\prime}\right)}^{\mathrm{ord}, \min }
$$

is quasi-finite. If $p^{N_{2}-N_{2}^{\prime}} v(g) \in \mathbb{Z}_{p}^{\times}$and either $N_{2}^{\prime}=N_{2}$ or $N_{2}^{\prime}>0$, then it is also finite. On $\mathbb{F}_{p}$-fibres $\varsigma_{p}$ acts as absolute Frobenius composed with the forgetful map. (See theorem 6.2.1.1, proposition 6.2.2.1 and corollary 6.2.2.9 of [44]. We remark that we are, perhaps unfortunately, following a different convention from [44]. According to our convention

$$
\mathcal{X}_{n, U^{p}\left(N_{1}, N_{2}\right)}^{\mathrm{ord} \min } \times \operatorname{Spec} \mathbb{Q} \cong X_{n, U^{p}\left(N_{1}, N_{2}\right)}^{\min }
$$

In contrast Lan [44] works with an open subscheme which only contains the 'ordinary' part of the boundary. Our $\mathcal{X}_{n, U^{p}\left(N_{1}, N_{2}\right)}^{\text {ord, }}$ is the union of this open subscheme with $X_{n, U^{p}\left(N_{1}, N_{2}\right)}^{\min }$.)

Write

$$
\partial \mathcal{X}_{n, U^{p}\left(N_{1}, N_{2}\right)}^{\mathrm{ord}, \min }=\mathcal{X}_{n, U^{p}\left(N_{1}, N_{2}\right)}^{\mathrm{ord} \min }-\mathcal{X}_{n, U^{p}\left(N_{1}, N_{2}\right)}^{\text {ord }}
$$

There is a family of closed subschemes

$$
\begin{aligned}
\partial_{0} \mathcal{X}_{n, U^{p}\left(N_{1}, N_{2}\right)}^{\text {ord,min }}= & \mathcal{X}_{n, U^{p}\left(N_{1}, N_{2}\right)}^{\text {ord,min }} \supset \partial_{1} \mathcal{X}_{n, U^{p}\left(N_{1}, N_{2}\right)}^{\text {ord,min }} \\
= & \partial \mathcal{X}_{n, U^{p}\left(N_{1}, N_{2}\right)}^{\text {ord,min }} \supset \partial_{2} \mathcal{X}_{n, U^{p}\left(N_{1}, N_{2}\right)}^{\text {ord,min }} \\
& \supset \cdots \supset \partial_{n} \mathcal{X}_{n, U^{p}\left(N_{1}, N_{2}\right)}^{\text {ord, }} \supset \partial_{n+1} \mathcal{X}_{n, U^{p}\left(N_{1}, N_{2}\right)}^{\text {ord,min }}=\emptyset
\end{aligned}
$$

such that each

$$
\partial_{i}^{0} \mathcal{X}_{n, U^{p}\left(N_{1}, N_{2}\right)}^{\text {ordmin }}=\partial_{i} \mathcal{X}_{n, U^{p}\left(N_{1}, N_{2}\right)}^{\text {ord,min }}-\partial_{i+1} \mathcal{X}_{n, U^{p}\left(N_{1}, N_{2}\right)}^{\text {ord,min }}
$$

is smooth over $\mathbb{Z}_{(p)}$ of relative dimension $(n-i)^{2}\left[F^{+}: \mathbb{Q}\right]$. Then

$$
\left\{\partial_{i} \mathcal{X}_{n, U^{p}\left(N_{1}, N_{2}\right)}^{\text {ord,min }}\right\}
$$

and

$$
\left\{\partial_{i}^{0} \mathcal{X}_{n, U^{p}\left(N_{1}, N_{2}\right)}^{\text {ordmin }}\right\}
$$

are families of schemes with $G_{n}\left(\mathbb{A}^{\infty}\right)^{\text {ord }}$-action. We will write $\mathcal{X}_{n, U^{p}\left(N_{1}, N_{2}\right), i}^{\text {ord, } i n}, \wedge$ for the completion of $\mathcal{X}_{n, U^{p}\left(N_{1}, N_{2}\right)}^{\text {ordmin }}$ along $\partial_{i}^{0} \mathcal{X}_{n, U^{p}\left(N_{1}, N_{2}\right)}^{\text {ord,min }}$. We have a decomposition

$$
\begin{aligned}
& \partial_{i}^{0} \mathcal{X}_{n, U^{p}\left(N_{1}, N_{2}\right)}^{\text {ordmin }}=\coprod_{h \in P_{n,(i)}^{+}\left(\mathbb{A}^{\infty}\right)^{\text {ord }, \times} \backslash G_{n}\left(\mathbb{A}^{\infty}\right)^{\text {ord }, \times} / U^{p}\left(N_{1}\right)} \mathcal{X}_{n,(i),\left(h U^{p} h^{-1} \cap P_{n,(i)}^{+}\right.}^{\text {ord } \left.\left(\mathbb{A}^{\infty, p}\right)\right)\left(N_{1}, N_{2}\right)} \\
& \amalg \coprod_{h} X_{n,(i), h U^{p}\left(N_{1}, N_{2}\right) h^{-1} \cap P_{n, i}^{+}\left(\mathbb{A}^{\infty}\right)^{\prime}}
\end{aligned}
$$

where the second coproduct runs over

$$
h \in\left(P_{n, i)}^{+}\left(\mathbb{A}^{\infty}\right) \backslash G_{n}\left(\mathbb{A}^{\infty}\right) / U^{p}\left(N_{1}, N_{2}\right)\right)-\left(P_{n,(i)}^{+}\left(\mathbb{A}^{\infty}\right)^{\text {ord }, \times} \backslash G_{n}\left(\mathbb{A}^{\infty}\right)^{\text {ord }, \times} / U^{p}\left(N_{1}\right)\right) .
$$


(Again see theorems 6.2.1.1 and proposition 6.2.2.1 of [44].)

[We explain why the map

$$
P_{n,(i)}^{+}\left(\mathbb{A}^{\infty}\right)^{\text {ord }, \times} \backslash G_{n}\left(\mathbb{A}^{\infty}\right)^{\text {ord }, \times} / U^{p}\left(N_{1}\right) \longrightarrow P_{n,(i)}^{+}\left(\mathbb{A}^{\infty}\right) \backslash G_{n}\left(\mathbb{A}^{\infty}\right) / U^{p}\left(N_{1}, N_{2}\right)
$$

is injective. It suffices to check that

$$
\begin{aligned}
& \left(P_{n,(i)}^{+} \cap P_{n,(n)}^{+}\right)\left(\mathbb{Z}_{p}\right) \backslash P_{n,(n)}^{+}\left(\mathbb{Z}_{p}\right) / U_{p}\left(N_{1}, N_{1}\right)_{n,(n)}^{+} \\
& \quad \hookrightarrow P_{n,(i)}^{+}\left(\mathbb{Q}_{p}\right) \backslash G_{n}\left(\mathbb{Q}_{p}\right) / U_{p}\left(N_{1}, N_{2}\right)_{n} \\
& \quad=P_{n,(i)}^{+}\left(\mathbb{Z}_{p}\right) \backslash G_{n}\left(\mathbb{Z}_{p}\right) / U_{p}\left(N_{1}, N_{2}\right)_{n},
\end{aligned}
$$

or even that

$$
\begin{aligned}
& \left(P_{n,(i)}^{+} \cap P_{n,(n)}^{+}\right)\left(\mathbb{Z} / p^{N_{2}} \mathbb{Z}\right) \backslash P_{n,(n)}^{+}\left(\mathbb{Z} / p^{N_{2}} \mathbb{Z}\right) / V \\
& \hookrightarrow P_{n,(i)}^{+}\left(\mathbb{Z} / p^{N_{2}} \mathbb{Z}\right) \backslash G_{n}\left(\mathbb{Z} / p^{N_{2}} \mathbb{Z}\right) / V
\end{aligned}
$$

where

$$
V=\operatorname{ker}\left(P_{n,(n)}^{+}\left(\mathbb{Z} / p^{N_{2}} \mathbb{Z}\right) \rightarrow L_{n,(n), \operatorname{lin}}\left(\mathbb{Z} / p^{N_{1}} \mathbb{Z}\right)\right)
$$

This is clear.]

If $g \in G_{n}\left(\mathbb{A}^{\infty}\right)^{\text {ord }}$ and if $g^{-1} U^{p}\left(N_{1}, N_{2}\right) g \subset\left(U^{p}\right)^{\prime}\left(N_{1}^{\prime}, N_{2}^{\prime}\right)$ then the map

$$
g: \partial_{i}^{0} \mathcal{X}_{n, U^{p}\left(N_{1}, N_{2}\right)}^{\mathrm{ord} \min } \longrightarrow \partial_{i}^{0} \mathcal{X}_{n,\left(U^{p}\right)^{\prime}\left(N_{1}^{\prime}, N_{2}^{\prime}\right)}^{\text {ord,min }}
$$

is the coproduct of the maps

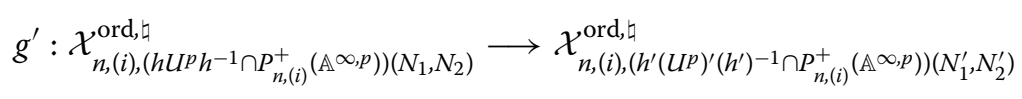

where $h g=g^{\prime} h^{\prime}$ with $g^{\prime} \in P_{n,(i)}^{+}\left(\mathbb{A}^{\infty}\right)^{\text {ord }}$, and of the maps

$$
g^{\prime}: X_{n,(i), h U^{p}\left(N_{1}, N_{2}\right) h^{-1} \cap P_{n,(i)}^{+}\left(\mathbb{A}^{\infty}\right)}^{\natural} X_{n,(i), h^{\prime}\left(U^{p}\right)^{\prime}\left(N_{1}^{\prime}, N_{2}^{\prime}\right)\left(h^{\prime}\right)^{-1} \cap P_{n,(i)}^{+}\left(\mathbb{A}^{\infty}\right)}^{\natural}
$$

where $h g=g^{\prime} h^{\prime}$ with $g^{\prime} \in P_{n,(i)}^{+}\left(\mathbb{A}^{\infty}\right)$. (Again see theorems 6.2.1.1 and proposition 6.2.2.1 of [44].)

$$
\text { If } N_{2}^{\prime} \geq N_{2} \geq N_{1} \text { then the natural map }
$$

$$
\mathcal{X}_{n, U^{p}\left(N_{1}, N_{2}^{\prime}\right)}^{\text {ord,min }} \longrightarrow \mathcal{X}_{n, U^{p}\left(N_{1}, N_{2}\right)}^{\text {ord,min }}
$$

is etale in a Zariski neighbourhood of the $\mathbb{F}_{p}$-fibre, and the natural map

$$
\mathfrak{X}_{n, U^{p}\left(N_{1}, N_{2}^{\prime}\right)}^{\text {ord,min }} \longrightarrow \mathfrak{X}_{n, U^{p}\left(N_{1}, N_{2}\right)}^{\text {ord,min }}
$$

between formal completions along the $\mathbb{F}_{p}$-fibres is an isomorphism. (See corollary 6.2.2.8 and example 3.4.4.5 of [44].) We will denote this $p$-adic formal scheme

$$
\mathfrak{X}_{n, U^{p}\left(N_{1}\right)}^{\text {ord,min }}
$$


and will denote its reduced subscheme

$$
\bar{X}_{n, U^{p}\left(N_{1}\right)}^{\text {ord } \min }
$$

We will also write

$$
\partial \bar{X}_{n, U^{p}\left(N_{1}\right)}^{\text {ord min }}=\bar{X}_{n, U^{p}\left(N_{1}\right)}^{\text {ord,min }}-\bar{X}_{n, U^{p}\left(N_{1}\right)}^{\text {ord }}
$$

The families $\left\{\mathfrak{X}_{n, U^{p}(N)}^{\text {ord,min }}\right\}$ and $\left\{\bar{X}_{n, U^{p}(N)}^{\text {ord, } m}\right\}$ and $\left\{\partial \bar{X}_{n, U^{p}(N)}^{\text {ord, min }}\right\}$ have $G_{n}\left(\mathbb{A}^{\infty}\right)^{\text {ord }}$-actions. There is a family of closed subschemes

$$
\begin{aligned}
\partial_{0} \bar{X}_{n, U^{p}(N)}^{\text {ord,min }}= & \bar{X}_{n, U^{p}(N)}^{\text {ord,min }} \supset \partial_{1} \bar{X}_{n, U^{p}(N)}^{\text {ord,min }} \\
= & \partial \bar{X}_{n, U^{p}(N)}^{\text {ord,min }} \supset \partial_{2} \bar{X}_{n, U^{p}(N)}^{\text {ord,min }} \\
& \supset \cdots \supset \partial_{n} \bar{X}_{n, U^{p}(N)}^{\text {ord,min }} \supset \partial_{n+1} \bar{X}_{n, U^{p}(N)}^{\text {ord,min }}=\emptyset
\end{aligned}
$$

such that each

$$
\partial_{i}^{0} \bar{X}_{n, U^{p}(N)}^{\text {ord,min }}=\partial_{i} \bar{X}_{n, U^{p}(N)}^{\text {ord,min }}-\partial_{i+1} \bar{X}_{n, U^{p}(N)}^{\text {ord,min }}
$$

is smooth of dimension $(n-i)^{2}\left[F^{+}: \mathbb{Q}\right]$. Then $\left\{\partial_{i} \bar{X}_{n, U^{p}(N)}^{\min }\right\}$ and $\left\{\partial_{i}^{0} \bar{X}_{n, U^{p}(N)}^{\min }\right\}$ are families of schemes with $G_{n}\left(\mathbb{A}^{\infty}\right)^{\text {ord }}$-action. Moreover we have a decomposition

$$
\partial_{i}^{0} \bar{X}_{n, U^{p}(N)}^{\text {ord,min }}=\coprod_{h \in P_{n,(i)}^{+}\left(\mathbb{A}^{\infty}\right)^{\text {ord }, \times} \backslash G_{n}\left(\mathbb{A}^{\infty}\right)^{\text {ord }, \times} / U^{p}(N)} \bar{X}_{n,(i),\left(h U^{p} h^{-1} \cap P_{n,(i)}^{+}\left(\mathbb{A}^{\infty, p}\right)\right)(N)^{*}}^{\text {ord } \curvearrowleft}
$$

If $g \in G_{n}\left(\mathbb{A}^{\infty}\right)^{\text {ord }}$ and if $g^{-1} U^{p}(N) g \subset\left(U^{p}\right)^{\prime}\left(N^{\prime}\right)$ then the map

$$
g: \partial_{i}^{0} \bar{X}_{n, U^{p}(N)}^{\text {ord,min }} \longrightarrow \partial_{i}^{0} \bar{X}_{n,\left(U^{p}\right)^{\prime}\left(N^{\prime}\right)}^{\text {ord, min }}
$$

is the coproduct of the maps

$$
g^{\prime}: \bar{X}_{n,(i),\left(h U^{p} h^{-1} \cap P_{n,(i)}^{+}\left(\mathbb{A}^{\infty, p}\right)\right)(N)}^{\mathrm{ord}, \natural} \bar{X}_{n,(i),\left(h^{\prime}\left(U^{p}\right)^{\prime}\left(h^{\prime}\right)^{-1} \cap P_{n,(i)}^{+}\left(\mathbb{A}^{\infty, p}\right)\right)\left(N^{\prime}\right)}^{\mathrm{ord}, \natural}
$$

where $h g=g^{\prime} h^{\prime}$ with $g^{\prime} \in P_{n,(i)}^{+}\left(\mathbb{A}^{\infty}\right)^{\text {ord }}$. In particular $\varsigma_{p}$ acts as absolute Frobenius.

The schemes $\mathcal{X}_{n, U^{p}\left(N_{1}, N_{2}\right)}^{\text {ord, } i n}$ are not proper. There are proper integral models of the schemes $X_{n, U}^{\min }$, but we have less control over them.

More specifically suppose that $U \subset G_{n}\left(\mathbb{A}^{\infty, p} \times \mathbb{Z}_{p}\right)$ is an open compact subgroup whose projection to $G_{n}\left(\mathbb{A}^{\infty, p}\right)$ is neat. Then there is a normal, projective, flat $\mathbb{Z}_{(p)}$-scheme $\mathcal{X}_{n, U}^{\min }$ with generic fibre $X_{n, U}^{\min }$. If $g \in G_{n}\left(\mathbb{A}^{\infty, p} \times \mathbb{Z}_{p}\right)$ and if

$$
g^{-1} U g \subset U^{\prime}
$$

then there is a map

$$
g: \mathcal{X}_{n, U}^{\min } \longrightarrow \mathcal{X}_{n, U^{\prime}}^{\min }
$$

extending the map $g: X_{n, U}^{\min } \rightarrow X_{n, U^{\prime}}^{\min }$ This gives the system $\left\{\mathcal{X}_{n, U}^{\min }\right\}$ an action of $G_{n}\left(\mathbb{A}^{\infty, p} \times\right.$ $\left.\mathbb{Z}_{p}\right)$. We set

$$
\bar{X}_{n, U}^{\min }=\mathcal{X}_{n, U}^{\min } \times_{\mathbb{Z}_{(p)}} \mathbb{F}_{p} .
$$


On $\mathcal{X}_{n, U}^{\min }$ there is an ample line bundle $\omega_{U}$, and the system of line bundles $\left\{\omega_{U}\right\}$ over $\left\{\mathcal{X}_{n, U}^{\min }\right\}$ has an action of $G_{n}\left(\mathbb{A}^{\infty, p} \times \mathbb{Z}_{p}\right)$. The pull-back of $\omega_{U}$ to $X_{n, U}$ is $G_{n}\left(\mathbb{A}^{\infty, p} \times \mathbb{Z}_{p}\right)$-equivariantly identified with $\wedge^{n[F: \mathbb{Q}]} \Omega_{n, U}$. (See propositions 2.2.1.2 and 2.2.3.1 of [44]. The rough idea is to take $\mathcal{X}_{n, U}^{\min }$ as the normalization of $X_{n, U}^{\min }$ over the minimal compactification of some Siegel moduli of genus $n[F: \mathbb{Q}]$ and a suitable neat level away from $p$, and take $\omega_{U}$ to be the pull-back of the corresponding Hodge invertible sheaf there. It is not easy to describe such normalizations in as much detail as in $[17,41]$, but we can still verify the assertions in this paragraph using the corresponding assertions for the minimal compactifications of Siegel moduli.)

Moreover there are canonical sections

$$
\operatorname{Hasse}_{U} \in H^{0}\left(\bar{X}_{U}^{\min }, \omega_{U}^{\otimes(p-1)}\right)
$$

such that

$$
g^{*} \operatorname{Hasse}_{U^{\prime}}=\operatorname{Hasse}_{U}
$$

whenever $g \in G_{n}\left(\mathbb{A}^{\infty, p} \times \mathbb{Z}_{p}\right)$ and $U^{\prime} \supset g^{-1} U g$. We will write $\bar{X}_{n, U}^{\text {min,n-ord }}$ for the zero locus in $\bar{X}_{n, U}^{\min }$ of Hasse $U$. (See corollaries 6.3.1.7 and 6.3.1.8 of [44]. The rough idea is to take Hasse H $_{U}$ to be the pull-back of the corresponding section over the minimal compactifications of some Siegel moduli.) Then $\bar{X}_{n, U}^{\min }-\bar{X}_{n, U}^{\min , \mathrm{n} \text {-ord }}$ is relatively affine over $\bar{X}_{n, U}^{\min }$ associated to the sheaf of algebras

$$
\left(\bigoplus_{i=0}^{\infty} \omega^{\otimes(p-1) a i}\right) /\left(\operatorname{Hasse}_{U}^{a}-1\right)
$$

for any $a \in \mathbb{Z}_{>0}$. It is also affine over $\mathbb{F}_{p}$ associated to the algebra

$$
\left(\bigoplus_{i=0}^{\infty} H^{0}\left(\bar{X}_{n, U}^{\min }, \omega^{\otimes(p-1) a i}\right)\right) /\left(\operatorname{Hasse}_{U}^{a}-1\right)
$$

for any $a \in \mathbb{Z}_{>0}$.

There are $G_{n}\left(\mathbb{A}^{\infty}\right)^{\text {ord, } \times}$-equivariant open embeddings

$$
\mathcal{X}_{n, U^{p}\left(N_{1}, N_{2}\right)}^{\text {ord,min }} \hookrightarrow \mathcal{X}_{n, U^{p}\left(N_{1}, N_{2}\right)}^{\min }
$$

These induce maps

$$
\bar{X}_{n, U^{p}\left(N_{1}, N_{2}\right)}^{\text {ord } \min } \hookrightarrow \bar{X}_{n, U^{p}\left(N_{1}, N_{2}\right)}^{\min }-\bar{X}_{n, U^{p}\left(N_{1}, N_{2}\right)}^{\mathrm{min}, \mathrm{ord}}
$$

on $\mathbb{F}_{p}$-fibres which are open and closed embeddings. (See proposition 6.3.2.2 of [44].) In the case $N_{1}=N_{2}=0$ this map is in fact an isomorphism. (See lemmas 6.3.2.7 and 6.3.2.9 of [44].) We remark that for $N_{2}>0$ this map is not an isomorphism: The definition of $\bar{X}_{n, U^{p}\left(N_{1}, N_{2}\right)}^{\text {ord }}$ requires not only that the universal abelian scheme is ordinary, the condition that defines $\bar{X}_{n, U^{p}\left(N_{1}, N_{2}\right)}-\bar{X}_{n, U^{p}\left(N_{1}, N_{2}\right)}^{\text {min, }}$, but also that the universal subgroup $C^{\text {univ }} \subset \mathcal{A}^{\text {univ }}\left[p^{N_{2}}\right]$ is connected above each geometric point.

Also the pull-back of $\omega_{U^{p}\left(N_{1}, N_{2}\right)}$ to $\mathcal{X}_{n, U^{p}\left(N_{1}, N_{2}\right)}^{\text {ord }}$ is $G_{n}\left(\mathbb{A}^{\infty}\right)^{\text {ord, } \times}$-equivariantly identified with the sheaf $\wedge^{n[F: \mathbb{Q}]} \Omega_{n, U^{p}\left(N_{1}, N_{2}\right)}^{\text {ord }}$. If $g \in G_{n}\left(\mathbb{A}^{\infty}\right)^{\text {ord, } \times}$ and

$$
g^{-1}\left(U^{p}\right)^{\prime}\left(N_{1}^{\prime}, N_{2}\right) g \subset U^{p}\left(N_{1}, N_{2}\right),
$$


then the commutative square

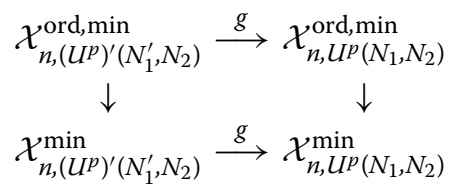

is a pull-back square. (See theorem 6.2.1.1 and proposition 6.2.2.1 of [44].)

At the referee's suggestion we include a few remarks about the construction of $\mathcal{X}_{U^{p}\left(N_{1}, N_{2}\right)}^{\text {ord, min }}$ and $\mathcal{X}_{U}^{\min }$ in [44]. If $p$ is unramified in $F$ then one has good control of certain integral toroidal compactifications $\mathcal{X}_{U^{p}(0,0), \Delta}$ and of the integral minimal compactification $\mathcal{X}_{U^{p}(0,0)}^{\min }$. Moreover over $\mathcal{X}_{U^{p}(0,0), \Delta}$ there is a 'universal' semi-abelian scheme and 'ordinarity' and $U^{p}\left(N_{1}, N_{2}\right)$-level structure can be defined for this semi-abelian scheme. For $U=U^{p} U_{p}$ with $U_{p} \subset G_{n}\left(\mathbb{Z}_{p}\right)$ one can then define $\mathcal{X}_{U}^{\text {min }}$ as a normalization of $\mathcal{X}_{U^{p}(0,0)}^{\min }$ in $X_{U}^{\min }$. One can then define $\mathcal{X}_{U, \Delta^{\prime}}$ as a suitable normalized blow-up of $\mathcal{X}_{U}^{\min }$. We don't have very much control of $\mathcal{X}_{U, \Delta^{\prime}}$ or $\mathcal{X}_{U}^{\text {min }}$. One can also define $\mathcal{X}_{U^{p}\left(N_{1}, N_{2}\right), \Delta}^{\text {ord }}$, and it solves a relative moduli problem over $\mathcal{X}_{U^{p}(0,0), \Delta}^{\text {ord }} \subset \mathcal{X}_{U^{p}(0,0), \Delta}$. There are maps

$$
\mathcal{X}_{U^{p}\left(N_{1}, N_{2}\right), \Delta}^{\mathrm{ord}} \longrightarrow \mathcal{X}_{U^{p}\left(N_{1}, N_{2}\right), \Delta} \longrightarrow \mathcal{X}_{U^{p}\left(N_{1}, N_{2}\right)}^{\min }
$$

The key point is to show that this map has open image, which we define to be $\mathcal{X}_{U^{p}\left(N_{1}, N_{2}\right)}^{\text {ord, min }}$ and that $\mathcal{X}_{U^{p}\left(N_{1}, N_{2}\right), \Delta}^{\text {ord }}$ is the pre-image of this open set in $\mathcal{X}_{U^{p}\left(N_{1}, N_{2}\right), \Delta}$.

In the case that $p$ ramifies in $F$ things are a bit harder, because we don't even have a good candidate for $\mathcal{X}_{U^{p}(0,0)}^{\min }$. In this case one first constructs the corresponding spaces in the Siegel case, which is analogous to the good case discussed in the previous paragraph. One then has a proper map $\mathcal{X}_{U^{p}\left(N_{1}, N_{2}\right), \Delta}^{\text {ord }} \rightarrow \mathcal{Z}_{V^{p}\left(N_{1}, N_{2}\right)}^{\text {ord, }}$, where $\mathcal{Z}_{V^{p}\left(N_{1}, N_{2}\right)}^{\text {ord, in }}$ is the integral minimal partial compactification of the ordinary locus of a Siegel variety. One applies Stein factorization to this map and uses this to define $\mathcal{X}_{U^{p}\left(N_{1}, N_{2}\right)}^{\text {ord, }}$ :

$$
\mathcal{X}_{U^{p}\left(N_{1}, N_{2}\right), \Delta}^{\text {ord }} \longrightarrow \mathcal{X}_{U^{p}\left(N_{1}, N_{2}\right)}^{\text {ord, min }} \longrightarrow \mathcal{Z}_{V^{p}\left(N_{1}, N_{2}\right)}^{\text {ord, }{ }^{\prime}}
$$

Then $\mathcal{X}_{U^{p}\left(N_{1}, N_{2}\right)}^{\text {ord,min }}$ admits a quasi-finite map to $\mathcal{X}_{U^{p}\left(N_{1}, N_{2}\right)}^{\min }$, which is shown to be an open immersion using Zariski's main theorem.

\subsection{Cone decompositions}

Let $U \subset G^{(m)}\left(\mathbb{A}^{\infty}\right)$ be an open compact subgroup. By a $U$-admissible cone decomposition $\Sigma$ of $G_{n}^{(m)}\left(\mathbb{A}^{\infty}\right) \times \pi_{0}\left(G_{n}(\mathbb{R})\right) \times \mathfrak{C}^{(m)}$ we shall mean a set of closed subsets $\sigma \subset G_{n}^{(m)}\left(\mathbb{A}^{\infty}\right) \times$ $\pi_{0}\left(G_{n}(\mathbb{R})\right) \times \mathfrak{C}^{(m)}$ such that

(1) each $\sigma$ is contained in $\{(g, \delta)\} \times \mathfrak{C}^{(m), \succ 0}(W)$ for some isotropic subspace $W \subset V_{n}$ and some $(g, \delta) \in G_{n}^{(m)}\left(\mathbb{A}^{\infty}\right) \times \pi_{0}\left(G_{n}(\mathbb{R})\right)$ and is the set of $\mathbb{R}_{\geq 0}$-linear combinations of a finite set of elements of Herm $_{V / W^{\perp}} \times W^{m}$;

(2) no $\sigma \in \Sigma$ contains a complete line through the origin in any $(g, \delta) \times \mathfrak{C}^{(m)}(W)$;

(3) if $\sigma \in \Sigma$ then any face of $\sigma$ also lies in $\Sigma$;

(4) if $\sigma, \sigma^{\prime} \in \Sigma$ then either $\sigma \cap \sigma^{\prime}=\emptyset$ or $\sigma \cap \sigma^{\prime}$ is a face of $\sigma$ and $\sigma^{\prime}$;

(5) $G_{n}^{(m)}\left(\mathbb{A}^{\infty}\right) \times \pi_{0}\left(G_{n}(\mathbb{R})\right) \times \mathfrak{C}^{(m)}=\bigcup_{\sigma \in \Sigma} \sigma$;

(6) $\Sigma$ is invariant by the diagonal action of $G_{n}^{(m)}(\mathbb{Q})$ on $G_{n}^{(m)}\left(\mathbb{A}^{\infty}\right) \times \pi_{0}\left(G_{n}(\mathbb{R})\right) \times \mathfrak{C}^{(m)}$; 
(7) $\Sigma$ is invariant by the right action of $U$ on $G_{n}^{(m)}\left(\mathbb{A}^{\infty}\right) \times \pi_{0}\left(G_{n}(\mathbb{R})\right) \times \mathfrak{C}^{(m)}$ (acting only on the first factor);

(8) $G_{n}^{(m)}(\mathbb{Q}) \backslash \Sigma / U$ is a finite set;

(9) if $\sigma \in \Sigma$ lies in $G_{n}^{(m)}\left(\mathbb{A}^{\infty}\right) \times \pi_{0}\left(G_{n}(\mathbb{R})\right) \times \mathfrak{C}^{(m), \succ 0}\left(V_{n,(i)}\right)$ and if $h \in P_{n,(i)}^{(m)}(\mathbb{A})$, then $h \sigma \in \Sigma$;

(10) if $\sigma \in \Sigma$ lies in $G_{n}^{(m)}\left(\mathbb{A}^{\infty}\right) \times \pi_{0}\left(G_{n}(\mathbb{R})\right) \times \mathfrak{C}^{(m), \succ 0}\left(V_{n,(i)}\right)$, if $\gamma \in G_{n}^{(m)}(\mathbb{Q})$, if $u \in U$ and if $h \in P_{n,(i)}^{(m)}(\mathbb{A})$ satisfy

$$
\sigma \cap \gamma h \sigma u \cap\left(G_{n}^{(m)}\left(\mathbb{A}^{\infty}\right) \times \pi_{0}\left(G_{n}(\mathbb{R})\right) \times \mathfrak{C}^{(m),>0}\left(V_{n,(i)}\right)\right) \neq \emptyset
$$

then $\gamma \in P_{n,(i)}^{(m)}(\mathbb{Q})$.

(Here we let $G_{n}^{(m)}(\mathbb{A})$ act on $G_{n}^{(m)}\left(\mathbb{A}^{\infty}\right) \times \pi_{0}\left(G_{n}(\mathbb{R})\right) \times \mathfrak{C}^{(m)}$ via multiplication on the first two factors. The restriction of this action to $G_{n}^{(m)}(\mathbb{Q})$ does not coincide with the standard action of $G_{n}^{(m)}(\mathbb{Q})$, which we are using.) Note that if $U^{\prime} \subset U$ and if $\Sigma$ is a $U$-admissible cone decomposition of $G_{n}^{(m)}\left(\mathbb{A}^{\infty}\right) \times \pi_{0}\left(G_{n}(\mathbb{R})\right) \times \mathfrak{C}^{(m)}$ then $\Sigma$ is also $U^{\prime}$-admissible. We will call a set $\Sigma$ of closed subsets of $G_{n}^{(m)}\left(\mathbb{A}^{\infty}\right) \times \pi_{0}\left(G_{n}(\mathbb{R})\right) \times \mathfrak{C}^{(m)}$ an admissible cone decomposition of $G_{n}^{(m)}\left(\mathbb{A}^{\infty}\right) \times \pi_{0}\left(G_{n}(\mathbb{R})\right) \times \mathfrak{C}^{(m)}$ if it is $U$-admissible for some open compact subgroup $U$.

We remark that different authors use the term ' $U$-admissible cone decomposition' in somewhat different ways.

We call $\Sigma^{\prime}$ a refinement of $\Sigma$ if every element of $\Sigma$ is a union of elements of $\Sigma^{\prime}$. We define a partial order on the set of pairs $(U, \Sigma)$, where $U \subset G_{n}^{(m)}\left(\mathbb{A}^{\infty}\right)$ is an open compact subgroup and $\Sigma$ is a $U$-admissible cone decomposition of $G_{n}^{(m)}\left(\mathbb{A}^{\infty}\right) \times \pi_{0}\left(G_{n}(\mathbb{R})\right) \times \mathfrak{C}^{(m)}$, as follows: We set

$$
\left(U^{\prime}, \Sigma^{\prime}\right) \geq(U, \Sigma)
$$

if and only if $U^{\prime} \subset U$ and $\Sigma^{\prime}$ is a refinement of $\Sigma$. If $g \in G_{n}^{(m)}\left(\mathbb{A}^{\infty}\right)$ and $\Sigma$ is a $U$-admissible cone decomposition of $G_{n}^{(m)}\left(\mathbb{A}^{\infty}\right) \times \pi_{0}\left(G_{n}(\mathbb{R})\right) \times \mathfrak{C}^{(m)}$, then

$$
\Sigma g=\{\sigma(g \times 1): \sigma \in \Sigma\}
$$

is a $g^{-1} U g$-admissible cone decomposition of $G_{n}^{(m)}\left(\mathbb{A}^{\infty}\right) \times \pi_{0}\left(G_{n}(\mathbb{R})\right) \times \mathfrak{C}^{(m)}$. The action of $G_{n}^{(m)}\left(\mathbb{A}^{\infty}\right)$ preserves $\geq$.

There is a natural projection

$$
G_{n}^{(m)}\left(\mathbb{A}^{\infty}\right) \times \pi_{0}\left(G_{n}(\mathbb{R})\right) \times \mathfrak{C}^{(m)} \rightarrow G_{n}\left(\mathbb{A}^{\infty}\right) \times \pi_{0}\left(G_{n}(\mathbb{R})\right) \times \mathfrak{C} .
$$

We will call admissible cone decompositions $\Sigma$ of $G_{n}^{(m)}\left(\mathbb{A}^{\infty}\right) \times \pi_{0}\left(G_{n}(\mathbb{R})\right) \times \mathfrak{C}^{(m)}$ and $\Delta$ of $G_{n}\left(\mathbb{A}^{\infty}\right) \times \pi_{0}\left(G_{n}(\mathbb{R})\right) \times \mathfrak{C}$ compatible if the image of every $\sigma \in \Sigma$ is contained in an element of $\Delta$. If in addition $\Sigma$ is $U$-admissible, $\Delta$ is $U^{\prime}$-admissible and $U^{\prime}$ contains the image of $U$ in $G_{n}\left(\mathbb{A}^{\infty}\right)$ we will say that $(U, \Sigma)$ and $\left(U^{\prime}, \Delta\right)$ are compatible and write

$$
(U, \Sigma) \geq\left(U^{\prime}, \Delta^{\prime}\right) .
$$

Now let $U^{p} \subset G^{(m)}\left(\mathbb{A}^{\infty, p}\right)$ be an open compact subgroup and let $N \geq 0$ be an integer and consider $U^{p}(N) \subset G_{n}^{(m)}\left(\mathbb{A}^{\infty}\right)^{\text {ord, } \times}$. By a $U^{p}(N)$-admissible cone decomposition $\Sigma$ of 
$\left(G_{n}^{(m)}\left(\mathbb{A}^{\infty}\right) \times \pi_{0}\left(G_{n}(\mathbb{R})\right) \times \mathfrak{C}^{(m)}\right)^{\text {ord }}$ we shall mean a set of closed subsets $\sigma \subset\left(G_{n}^{(m)}\left(\mathbb{A}^{\infty}\right) \times\right.$ $\left.\pi_{0}\left(G_{n}(\mathbb{R})\right) \times \mathfrak{C}^{(m)}\right)^{\text {ord }}$ such that

(1) each $\sigma$ is contained in $\{(g, \delta)\} \times \mathfrak{C}^{(m), \succ 0}(W)$ for some isotropic subspace $W \subset V_{n}$ and some $(g, \delta) \in G_{n}^{(m)}\left(\mathbb{A}^{\infty}\right) \times \pi_{0}\left(G_{n}(\mathbb{R})\right)$ and is the set of $\mathbb{R}_{\geq 0}$-linear combinations of a finite set of elements of $\operatorname{Herm}_{V / W^{\perp}} \times W^{m}$;

(2) no $\sigma \in \Sigma$ contains a complete line through the origin in any $(g, \delta) \times \mathfrak{C}^{(m)}(W)$;

(3) if $\sigma \in \Sigma$ then any face of $\sigma$ also lies in $\Sigma$;

(4) if $\sigma, \sigma^{\prime} \in \Sigma$ then either $\sigma \cap \sigma^{\prime}=\emptyset$ or $\sigma \cap \sigma^{\prime}$ is a face of $\sigma$ and $\sigma^{\prime}$;

(5) $\left(G_{n}^{(m)}\left(\mathbb{A}^{\infty}\right) \times \pi_{0}\left(G_{n}(\mathbb{R})\right) \times \mathfrak{C}^{(m)}\right)^{\text {ord }}=\bigcup_{\sigma \in \Sigma} \sigma$;

(6) if $\sigma \in \Sigma$, if $\gamma \in G_{n}^{(m)}(\mathbb{Q})$ and if $u \in U^{p}(N, N)$ are such that $\gamma \sigma u \subset\left(G_{n}^{(m)}\left(\mathbb{A}^{\infty}\right) \times\right.$ $\left.\pi_{0}\left(G_{n}(\mathbb{R})\right) \times \mathfrak{C}^{(m)}\right)^{\text {ord }}$, then $\gamma \sigma u \in \Sigma$;

(7) there is a finite subset of $\Sigma$ such that any element of $\Sigma$ has the form $\gamma \sigma u$ with $\gamma \in G_{n}^{(m)}(\mathbb{Q})$ and $u \in U^{p}(N, N)$ and $\sigma$ in the given finite subset;

(8) if $\sigma \in \Sigma$ lies in $G_{n}^{(m)}\left(\mathbb{A}^{\infty}\right) \times \pi_{0}\left(G_{n}(\mathbb{R})\right) \times \mathfrak{C}^{(m), \succ 0}\left(V_{n,(i)}\right)$ and meets $G_{n}^{(m)}\left(\mathbb{A}^{\infty}\right) \times$ $\pi_{0}\left(G_{n}(\mathbb{R})\right) \times \mathfrak{C}^{(m),>0}\left(V_{n,(i)}\right)$, and if $h \in P_{n,(i)}^{(m)}\left(\mathbb{A}^{\infty}\right)^{\text {ord, } \times} \times P_{n,(i)}^{(m)}(\mathbb{R})$, then $h \sigma \in \Sigma ;$

(9) if $\sigma \in \Sigma$ lies in $G_{n}^{(m)}\left(\mathbb{A}^{\infty}\right) \times \pi_{0}\left(G_{n}(\mathbb{R})\right) \times \mathfrak{C}^{(m), \succ 0}\left(V_{n,(i)}\right)$, if $\gamma \in G_{n}^{(m)}(\mathbb{Q})$, if $u \in$ $U^{p}(N, N)$ and if $h \in P_{n,(i)}^{(m)}\left(\mathbb{A}^{\infty}\right)^{\text {ord }, \times} \times P_{n,(i)}^{(m)}(\mathbb{R})$ satisfy $\gamma h \sigma u \in \Sigma$ and

$$
\sigma \cap \gamma h \sigma u \cap\left(G_{n}^{(m)}\left(\mathbb{A}^{\infty}\right) \times \pi_{0}\left(G_{n}(\mathbb{R})\right) \times \mathfrak{C}^{(m),>0}\left(V_{n,(i)}\right)\right) \neq \emptyset
$$

then $\gamma \in P_{n,(i)}^{(m)}(\mathbb{Q})$.

Note that if $\left(U^{p}\right)^{\prime}\left(N^{\prime}\right) \subset U^{p}(N)$ and if $\Sigma$ is a $U^{p}(N)$-admissible cone decomposition of $\left(G_{n}^{(m)}\left(\mathbb{A}^{\infty}\right) \times \pi_{0}\left(G_{n}(\mathbb{R})\right) \times \mathfrak{C}^{(m)}\right)^{\text {ord }}$ then $\Sigma$ is also $\left(U^{p}\right)^{\prime}\left(N^{\prime}\right)$-admissible. We will call a set $\Sigma$ of closed subsets of $\left(G_{n}^{(m)}\left(\mathbb{A}^{\infty}\right) \times \pi_{0}\left(G_{n}(\mathbb{R})\right) \times \mathfrak{C}^{(m)}\right)^{\text {ord }}$ an admissible cone decomposition of $\left(G_{n}^{(m)}\left(\mathbb{A}^{\infty}\right) \times \pi_{0}\left(G_{n}(\mathbb{R})\right) \times \mathfrak{C}^{(m)}\right)^{\text {ord }}$ if it is $U^{p}(N)$-admissible for some open compact subgroup $U^{p}$ and for some $N$.

If $\Sigma$ is a $U^{p}\left(N_{1}, N_{2}\right)$-admissible cone decomposition of $G_{n}^{(m)}\left(\mathbb{A}^{\infty}\right) \times \pi_{0}\left(G_{n}(\mathbb{R})\right) \times \mathfrak{C}^{(m)}$ then

$$
\Sigma^{\text {ord }}=\left\{\sigma \in \Sigma: \sigma \subset\left(G_{n}^{(m)}\left(\mathbb{A}^{\infty}\right) \times \pi_{0}\left(G_{n}(\mathbb{R})\right) \times \mathfrak{C}^{(m)}\right)^{\text {ord }}\right\}
$$

is a $U^{p}\left(N_{1}\right)$-admissible cone decomposition for $\left(G_{n}^{(m)}\left(\mathbb{A}^{\infty}\right) \times \pi_{0}\left(G_{n}(\mathbb{R})\right) \times \mathfrak{C}^{(m)}\right)^{\text {ord }}$.

We call $\Sigma^{\prime}$ a refinement of $\Sigma$ if every element of $\Sigma$ is a union of elements of $\Sigma^{\prime}$. We define a partial order on the set of pairs $\left(U^{p}(N), \Sigma\right)$, where $U^{p} \subset G_{n}^{(m)}\left(\mathbb{A}^{\infty, p}\right)$ is an open compact subgroup, $N \in \mathbb{Z}_{\geq 0}$ and $\Sigma$ is a $U^{p}(N)$-admissible cone decomposition of $\left(G_{n}^{(m)}\left(\mathbb{A}^{\infty}\right) \times \pi_{0}\left(G_{n}(\mathbb{R})\right) \times \mathfrak{C}^{(m)}\right)^{\text {ord }}$, as follows: We set

$$
\left(\left(U^{p}\right)^{\prime}\left(N^{\prime}\right), \Sigma^{\prime}\right) \geq\left(U^{p}(N), \Sigma\right)
$$

if and only if $\left(U^{p}\right)^{\prime}\left(N^{\prime}\right) \subset U^{p}(N)$ and $\Sigma^{\prime}$ is a refinement of $\Sigma$. If $g \in G_{n}^{(m)}\left(\mathbb{A}^{\infty}\right)^{\text {ord }}$ and $\Sigma$ is a $U^{p}(N)$-admissible cone decomposition of $\left(G_{n}^{(m)}\left(\mathbb{A}^{\infty}\right) \times \pi_{0}\left(G_{n}(\mathbb{R})\right) \times \mathfrak{C}^{(m)}\right)^{\text {ord }}$, then

$$
\Sigma g=\{\sigma(g \times 1): \sigma \in \Sigma\}
$$

is a $g^{-1} U^{p}(N) g$-admissible cone decomposition of

$$
\left(G_{n}^{(m)}\left(\mathbb{A}^{\infty}\right) \times \pi_{0}\left(G_{n}(\mathbb{R})\right) \times \mathfrak{C}^{(m)}\right)^{\text {ord }}
$$


The action of $G_{n}^{(m)}\left(\mathbb{A}^{\infty}\right)^{\text {ord }}$ preserves $\geq$.

There is a natural projection

$$
\left(G_{n}^{(m)}\left(\mathbb{A}^{\infty}\right) \times \pi_{0}\left(G_{n}(\mathbb{R})\right) \times \mathfrak{C}^{(m)}\right)^{\text {ord }} \rightarrow\left(G_{n}\left(\mathbb{A}^{\infty}\right) \times \pi_{0}\left(G_{n}(\mathbb{R})\right) \times \mathfrak{C}\right)^{\text {ord }} .
$$

We will call admissible cone decompositions $\Sigma$ of $\left(G_{n}^{(m)}\left(\mathbb{A}^{\infty}\right) \times \pi_{0}\left(G_{n}(\mathbb{R})\right) \times \mathfrak{C}^{(m)}\right)^{\text {ord }}$ and $\Delta$ of $\left(G_{n}\left(\mathbb{A}^{\infty}\right) \times \pi_{0}\left(G_{n}(\mathbb{R})\right) \times \mathfrak{C}\right)^{\text {ord }}$ compatible if the image of every $\sigma \in \Sigma$ is contained in an element of $\Delta$. If in addition $\Sigma$ is $U^{p}(N)$-admissible, $\Delta$ is $\left(U^{p}\right)^{\prime}\left(N^{\prime}\right)$-admissible and $\left(U^{p}\right)^{\prime}\left(N^{\prime}\right)$ contains the image of $U^{p}(N)$ in $G_{n}\left(\mathbb{A}^{\infty}\right)^{\text {ord }}$ we will say that $\left(U^{p}(N), \Sigma\right)$ and $\left(\left(U^{p}\right)^{\prime}\left(N^{\prime}\right), \Delta\right)$ are compatible and write

$$
\left(U^{p}(N), \Sigma\right) \geq\left(\left(U^{p}\right)^{\prime}\left(N^{\prime}\right), \Delta^{\prime}\right)
$$

If $\Sigma$ is a $U$-admissible cone decomposition of $G_{n}^{(m)}\left(\mathbb{A}^{\infty}\right) \times \pi_{0}\left(G_{n}(\mathbb{R})\right) \times \mathfrak{C}^{(m)}$ and if $h \in G_{n}^{(m)}\left(\mathbb{A}^{\infty}\right)$ then we define an admissible cone decomposition $\Sigma(h)_{0}$ for

$$
X_{*}\left(S_{n,(i), h U h^{-1} \cap P_{n, i)}^{(m),+}\left(\mathbb{A}^{\infty}\right)}^{(m),+}\right)_{\mathbb{R}}^{\succ 0}
$$

as follows: The cones in $\Sigma(h)_{0}$ over an element

$$
y=\left[h^{\prime}\left(\left(h U h^{-1} \cap P_{n,(i)}^{(m),+}\left(\mathbb{A}^{\infty}\right)\right) /\left(h U h^{-1} \cap P_{n,(i)}^{(m)}\left(\mathbb{A}^{\infty}\right)\right)\right)\right] \in Y_{n,(i), h U h^{-1} \cap P_{n,(i)}^{(m),+}\left(\mathbb{A}^{\infty}\right)}^{(m),+}
$$

are the cones

$$
\sigma \subset \mathfrak{C}^{(m), \succ 0}\left(V_{n,(i)}\right) \cong X_{*}\left(S_{n,(i), h U h^{-1} \cap P_{n,(i)}^{(m),+}\left(\mathbb{A}^{\infty}\right)}^{(m),}\right)_{\mathbb{R}, y}^{\succ 0}
$$

which meet $\mathfrak{C}^{(m),>0}\left(V_{n,(i)}\right)$ and satisfy

$$
\left\{\left(h^{\prime} h, 1\right)\right\} \times \sigma \in \Sigma .
$$

This does not depend on the representative $h^{\prime}$ we choose for $y$. It also only depends on

$$
h \in P_{n,(i)}^{(m)}\left(\mathbb{A}^{\infty}\right) \backslash G_{n}^{(m)}\left(\mathbb{A}^{\infty}\right) / U
$$

If $h_{1} \in L_{n,(i), \text { lin }}^{(m)}\left(\mathbb{A}^{\infty}\right)$ then under the natural isomorphism

$$
h_{1}: Y_{n,(i), h U h^{-1} \cap P_{n, i}^{(m),+}\left(\mathbb{A}^{\infty}\right)}^{(m),+} \stackrel{\sim}{\longrightarrow} Y_{n,(i), h_{1} h U\left(h_{1} h\right)^{-1} \cap P_{n,(i)}^{(m),+}\left(\mathbb{A}^{\infty}\right)}^{(m),+}
$$

we see that $\Sigma(h)_{0}$ and $\Sigma\left(h_{1} h\right)_{0}$ correspond.

Similarly if $\Sigma$ is a $U^{p}(N)$-admissible cone decomposition of

$$
\left(G_{n}^{(m)}\left(\mathbb{A}^{\infty}\right) \times \pi_{0}\left(G_{n}(\mathbb{R})\right) \times \mathfrak{C}^{(m)}\right)^{\text {ord }}
$$

and if $h \in G_{h}^{(m)}\left(\mathbb{A}^{\infty}\right)^{\text {ord, } \times}$ then we define an admissible cone decomposition $\Sigma(h)_{0}$ for

$$
X_{*}\left(\mathcal{S}_{n,(i), h U^{p}(N) h^{-1} \cap P_{n, i)}^{(m),+}\left(\mathbb{A}^{\infty}\right) \text { ord, },}^{(m)}\right)_{\mathbb{R}}^{\succ 0}
$$


as follows: The cones in $\Sigma(h)_{0}$ over an element $y$ given as

$$
\begin{aligned}
& {\left[h^{\prime}\left(h U^{p}(N) h^{-1} \cap P_{n,(i)}^{(m),+}\left(\mathbb{A}^{\infty}\right)^{\text {ord }, \times}\right) /\left(h U^{p}(N) h^{-1} \cap P_{h,(i)}^{(m),+}\left(\mathbb{A}^{\infty}\right)^{\text {ord }, \times}\right)\right]} \\
& \in \mathcal{Y}_{n,(i), h U^{p}(N) h^{-1} \cap P_{n,(i)}^{(m),+}\left(\mathbb{A}^{\infty}\right) \text { ord, } \times}^{(m)}
\end{aligned}
$$

are the cones

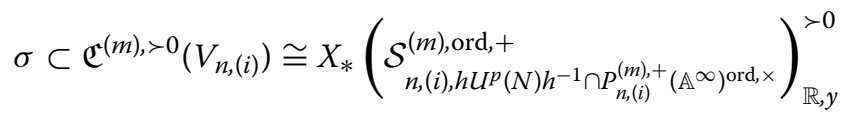

which meet $\mathfrak{C}^{(m),>0}\left(V_{n,(i)}\right)$ and satisfy

$$
\left\{\left(h^{\prime} h, 1\right)\right\} \times \sigma \in \Sigma .
$$

This does not depend on the representative $h^{\prime}$ we choose for $y$. It also only depends on

$$
h \in P_{n,(i)}^{(m)}\left(\mathbb{A}^{\infty}\right)^{\text {ord }, \times} \backslash G_{n}^{(m)}\left(\mathbb{A}^{\infty}\right)^{\text {ord }, \times} / U^{p}(N) .
$$

If $h_{1} \in L_{n,(i), \text { lin }}^{(m)}\left(\mathbb{A}^{\infty}\right)^{\text {ord, } \times}$ then under the natural isomorphism

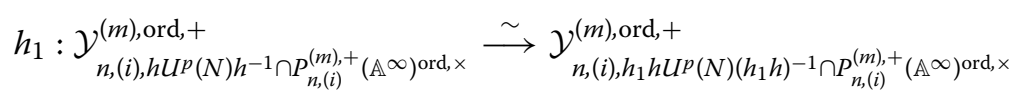

we see that $\Sigma(h)_{0}$ and $\Sigma\left(h_{1} h\right)_{0}$ correspond.

There are sets $\mathcal{J}_{n}^{(m), \text { tor }}$ (resp. $\mathcal{J}_{n}^{(m) \text {,tor,ord }}$ ) of pairs $(U, \Sigma)\left(\right.$ resp. $\left.\left(U^{p}(N), \Sigma\right)\right)$ where $U \subset$ $G_{n}^{(m)}\left(\mathbb{A}^{\infty}\right)$ is a neat open compact subgroup (resp. $U^{p} \subset G_{n}^{(m)}\left(\mathbb{A}^{\infty, p}\right)$ is a neat open compact subgroup and $N \in \mathbb{Z}_{\geq 0}$ ) and $\Sigma$ is a $U$-admissible (resp. $U^{p}(N)$-admissible) cone decomposition of $G_{n}^{(m)}\left(\mathbb{A}^{\infty}\right) \times \pi_{0}\left(G_{n}(\mathbb{R})\right) \times \mathfrak{C}^{(m)}\left(\operatorname{resp} .\left(G_{n}^{(m)}\left(\mathbb{A}^{\infty}\right) \times \pi_{0}\left(G_{n}(\mathbb{R})\right) \times \mathfrak{C}^{(m)}\right)^{\text {ord }}\right)$, with a number of properties which will be listed in this section and the next section. (See [44].)

Firstly we have the following properties:

(1) The sets $\mathcal{J}_{n}^{(m) \text {,tor }}$ (resp. $\mathcal{J}_{n}^{(m) \text {,tor,ord }}$ ) are invariant under the action of $G_{n}^{(m)}\left(\mathbb{A}^{\infty}\right)$ (resp. $\left.G_{n}^{(m)}\left(\mathbb{A}^{\infty}\right)^{\text {ord } \times}\right)$.

(2) If $U$ is any neat open compact subgroup of $G_{n}^{(m)}\left(\mathbb{A}^{\infty}\right)$, then there is some $\Sigma$ with $(U, \Sigma) \in \mathcal{J}_{n}^{(m), \text { tor }}$.

(3) If $U^{p}$ is any neat open compact subgroup of $G_{n}^{(m)}\left(\mathbb{A}^{\infty, p}\right)$ and if $N \in \mathbb{Z}_{\geq 0}$, then there is some $\Sigma$ with $\left(U^{p}(N), \Sigma\right) \in \mathcal{J}_{n}^{(m), \text { tor,ord }}$.

(4) If $(U, \Sigma) \in \mathcal{J}_{n}^{(m), \text { tor }}$ and if $U^{\prime} \subset U$ then there exists $\left(U^{\prime}, \Sigma^{\prime}\right) \in \mathcal{J}_{n}^{(m), \text { tor }}$ with $\left(U^{\prime}, \Sigma^{\prime}\right) \geq(U, \Sigma)$.

(5) If $\left(U^{p}(N), \Sigma^{\prime}\right) \in \mathcal{J}_{n}^{(m), \text { tor,ord }}$, if $N^{\prime} \geq N$ and if $\left(U^{p}\right)^{\prime}\left(N^{\prime}\right) \subset U^{p}(N)$ then there exists an element $\left(\left(U^{p}\right)^{\prime}\left(N^{\prime}\right), \Sigma^{\prime}\right) \in \mathcal{J}_{n}^{(m), \text { tor,ord }}$ with $\left(\left(U^{p}\right)^{\prime}\left(N^{\prime}\right), \Sigma^{\prime}\right) \geq\left(U^{p}(N), \Sigma\right)$.

(6) If $\left(U^{\prime}, \Sigma^{\prime}\right) \geq(U, \Sigma)$ are elements of $\mathcal{J}_{n}^{(m) \text {,tor }}$ and if moreover $U^{\prime}$ is a normal subgroup of $U$, then we may choose $\left(U^{\prime}, \Sigma^{\prime \prime}\right) \in \mathcal{J}_{n}^{(m) \text {,tor }}$ such that $\Sigma^{\prime \prime}$ is $U$-invariant and such that $\left(U^{\prime}, \Sigma^{\prime \prime}\right) \geq\left(U^{\prime}, \Sigma^{\prime}\right)$.

(7) If $\left(\left(U^{p}\right)^{\prime}\left(N^{\prime}\right), \Sigma^{\prime}\right) \geq\left(U^{p}(N), \Sigma\right)$ are elements of $\mathcal{J}_{n}^{(m), \text { tor,ord }}$ with $\left(U^{p}\right)^{\prime}$ a normal subgroup of $U^{p}$, then there is an element $\left(\left(U^{p}\right)^{\prime}\left(N^{\prime}\right), \Sigma^{\prime \prime}\right) \in \mathcal{J}_{n}^{(m) \text {,tor }}$ such that $\Sigma^{\prime \prime}$ is $U^{p}(N)$-invariant and such that $\left(\left(U^{p}\right)^{\prime}\left(N^{\prime}\right), \Sigma^{\prime \prime}\right) \geq\left(\left(U^{p}\right)^{\prime}\left(N^{\prime}\right), \Sigma^{\prime}\right)$. 
(8) If $(U, \Sigma)$ and $\left(U, \Sigma^{\prime}\right) \in \mathcal{J}_{n}^{(m), \text { tor }}$ (resp. if $\left(U^{p}(N), \Sigma\right)$ and $\left(U^{p}(N), \Sigma^{\prime}\right) \in \mathcal{J}_{n}^{(m), \text { tor.ord }}$ ) then there exists $\left(U, \Sigma^{\prime \prime}\right) \in \mathcal{J}_{n}^{(m) \text {,tor }}$ (resp. there exists $\left(U^{p}(N), \Sigma^{\prime \prime}\right)$ in $\mathcal{J}_{n}^{(m) \text {,tor,ord }}$ ) with $\left(U, \Sigma^{\prime \prime}\right) \geq(U, \Sigma)$ and $\left(U, \Sigma^{\prime \prime}\right) \geq\left(U, \Sigma^{\prime}\right)\left(\operatorname{resp}\right.$. with $\left(U^{p}(N), \Sigma^{\prime \prime}\right) \geq\left(U^{p}(N), \Sigma\right)$ and $\left.\left(U^{p}(N), \Sigma^{\prime \prime}\right) \geq\left(U^{p}(N), \Sigma^{\prime}\right)\right)$.

(9) If $\left(U^{\prime}, \Delta\right) \in \mathcal{J}_{n}^{\text {tor }}$ (resp. $\left.\left(\left(U^{p}\right)^{\prime}\left(N^{\prime}\right), \Delta\right) \in \mathcal{J}_{n}^{\text {tor,ord }}\right)$ and if $U$ is a neat open compact subgroup of $G_{n}^{(m)}\left(\mathbb{A}^{\infty}\right)$ mapping into $U^{\prime}$ (resp. $U^{p}$ is a neat open compact subgroup of $G_{n}^{(m)}\left(\mathbb{A}^{\infty, p}\right)$ mapping into $\left(U^{p}\right)^{\prime}$ and $\left.N \geq N^{\prime}\right)$, then there exists $(U, \Sigma) \in$ $\mathcal{J}_{n}^{(m) \text {,tor }}$ (resp. $\left(U^{p}(N), \Sigma\right) \in \mathcal{J}_{n}^{(m) \text {,tor,ord }}$ ) compatible with $\left(U^{\prime}, \Delta\right)$ (resp. $\left(\left(U^{p}\right)^{\prime}\left(N^{\prime}\right)\right.$, $\Delta))$.

(10) If $\left(U^{p}\left(N_{1}, N_{2}\right), \Sigma\right) \in \mathcal{J}_{n}^{(m), \text { tor }}$ then $\left(U^{p}\left(N_{1}\right), \Sigma^{\text {ord }}\right) \in \mathcal{J}_{n}^{(m) \text {,tor,ord }}$.

(11) If $\left(U^{p}(N), \Sigma^{\prime}\right) \in \mathcal{J}_{n}^{(m), \text { tor,ord }}$ and if $N^{\prime} \geq N$, then there exists $\left(U^{p}\left(N, N^{\prime}\right), \Sigma\right) \in$ $\mathcal{J}_{n}^{(m) \text {,tor }}$ with $\Sigma^{\text {ord }}=\Sigma^{\prime}$.

(12) If $\left(U^{p}\left(N_{1}, N_{2}\right), \Sigma\right)$ and $\left(U^{p}\left(N_{1}, N_{2}\right), \Sigma^{\prime}\right) \in \mathcal{J}_{n}^{(m) \text {,tor }}$ with $\Sigma^{\text {ord }}=\left(\Sigma^{\prime}\right)^{\text {ord }}$, then there is an element $\left(U^{p}\left(N_{1}, N_{2}\right), \Sigma^{\prime \prime}\right) \in \mathcal{J}_{n}^{(m) \text {,tor }}$ with $\left(\Sigma^{\prime \prime}\right)^{\text {ord }}=\Sigma^{\text {ord }}=\left(\Sigma^{\prime}\right)^{\text {ord }}$ and with $\left(U^{p}\left(N_{1}, N_{2}\right), \Sigma^{\prime \prime}\right) \geq\left(U^{p}\left(N_{1}, N_{2}\right), \Sigma\right)$ and with $\left(U^{p}\left(N_{1}, N_{2}\right), \Sigma^{\prime \prime}\right) \geq\left(U^{p}\left(N_{1}, N_{2}\right)\right.$, $\left.\Sigma^{\prime}\right)$.

(13) If $\left(U^{p}\left(N_{1}, N_{2}\right), \Sigma\right)$ and $\left(\left(U^{p}\right)^{\prime}\left(N_{1}^{\prime}, N_{2}^{\prime}\right), \Sigma^{\prime}\right) \in \mathcal{J}_{n}^{(m) \text {,tor }}$ with $\left(U^{p}\right)^{\prime}\left(N_{1}^{\prime}, N_{2}^{\prime}\right) \subset$ $U^{p}\left(N_{1}, N_{2}\right)$ and with $\left(\Sigma^{\prime}\right)^{\text {ord }}$ refining $\Sigma^{\text {ord }}$, then there also exists another pair $\left(\left(U^{p}\right)^{\prime}\left(N_{1}^{\prime}, N_{2}^{\prime}\right), \Sigma^{\prime \prime}\right) \in \mathcal{J}_{n}^{(m), \text { tor }}$ with $\Sigma^{\prime \prime}$ refining both $\Sigma$ and $\Sigma^{\prime}$ and with $\left(\Sigma^{\prime \prime}\right)^{\text {ord }}=$ $\left(\Sigma^{\prime}\right)^{\text {ord }}$.

(14) If $\left(U^{p}\left(N_{1}, N_{2}\right), \Delta\right) \in \mathcal{J}_{n}^{\text {tor }}$ and $\left(\left(U^{p}\right)^{\prime}\left(N_{1}^{\prime}, N_{2}^{\prime}\right), \Sigma^{\prime}\right) \in \mathcal{J}_{n}^{(m) \text {,tor }}$ are such that $\left(U^{p}\right)^{\prime}\left(N_{1}^{\prime}, N_{2}^{\prime}\right) \subset U^{p}\left(N_{1}, N_{2}\right)$ and $\left(\Sigma^{\prime}\right)^{\text {ord }}$ is compatible with $\Delta^{\text {ord }}$, then there exists $\left(\left(U^{p}\right)^{\prime}\left(N_{1}^{\prime}, N_{2}^{\prime}\right), \Sigma^{\prime \prime}\right) \in \mathcal{J}_{n}^{(m), \text { tor }}$ with $\Sigma^{\prime \prime}$ refining $\Sigma^{\prime}$ and compatible with $\Delta$ and with $\left(\Sigma^{\prime \prime}\right)^{\text {ord }}=\left(\Sigma^{\prime}\right)^{\text {ord }}$.

(15) If $\left(U^{p}\left(N_{1}, N_{2}\right), \Sigma\right) \in \mathcal{J}_{n}^{(m) \text {,tor }}$ and if $N_{2}^{\prime} \geq N_{2}$ then there exists a new pair $\left(U^{p}\left(N_{1}, N_{2}^{\prime}\right), \Sigma^{\prime}\right) \in \mathcal{J}_{n}^{(m), \text { tor }}$ with $\left(\Sigma^{\prime}\right)^{\text {ord }}=\Sigma^{\text {ord }}$.

(See propositions 1.2.4.52 and 7.1.1.21 of [44].)

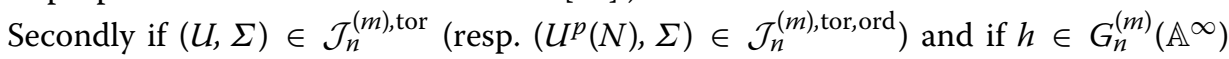
(resp. $h \in G_{n}^{(m)}\left(\mathbb{A}^{\infty}\right)^{\text {ord, } \times}$ ) then $\Sigma(h)_{0}$ is smooth.

Thirdly if $(U, \Sigma) \in \mathcal{J}_{n}^{(m) \text {,tor }}$, then there is a simplicial complex $\mathcal{S}(U, \Sigma)$ whose simplices are in bijection with the cones in

$$
G_{n}^{(m)}(\mathbb{Q}) \backslash \Sigma / U
$$

which have dimension bigger than 0 , and have the same face relations. We will write $\mathcal{S}(U, \Sigma)_{\leq i}$ for the subcomplex of $\mathcal{S}(U, \Sigma)$ consisting of simplices associated to the orbits of cones $(g, \delta) \times \sigma \in \Sigma$ with $\sigma \subset \mathfrak{C}^{(m)}, \succ 0(W)$ for some $W$ with $\operatorname{dim}_{F} W \leq i$. We will also set

$$
|\mathcal{S}(U, \Sigma)|_{=i}=\left|\mathcal{S}(U, \Sigma)_{\leq i}\right|-\left|\mathcal{S}(U, \Sigma)_{\leq i-1}\right|
$$

an open subset of $\left|\mathcal{S}(U, \Sigma)_{\leq i}\right|$. Then one sees that

$$
|\mathcal{S}(U, \Sigma)| \cong G_{n}^{(m)}(\mathbb{Q}) \backslash\left(\left(G_{n}^{(m)}\left(\mathbb{A}^{\infty}\right) / U\right) \times \pi_{0}\left(G_{n}(\mathbb{R})\right) \times\left(\left(\mathfrak{C}^{(m)}-\mathfrak{C}_{=0}^{(m)}\right) / \mathbb{R}_{>0}^{\times}\right)\right)
$$


and

$$
\begin{aligned}
|\mathcal{S}(U, \Sigma)|_{=i} & \\
\cong & G_{n}^{(m)}(\mathbb{Q}) \backslash\left(\left(G_{n}^{(m)}\left(\mathbb{A}^{\infty}\right) / U\right) \times \pi_{0}\left(G_{n}(\mathbb{R})\right) \times\left(\left(\mathfrak{C}_{=i}^{(m)}\right) / \mathbb{R}_{>0}^{\times}\right)\right) \\
\cong & \bigsqcup_{h \in P_{n,(i)}^{(m),+}\left(\mathbb{A}^{\infty}\right) \backslash G_{n}^{(m)}\left(\mathbb{A}^{\infty}\right) / U} L_{n,(i)}^{(m)}(\mathbb{Q}) \backslash L_{n,(i)}^{(m)}(\mathbb{A}) /\left(h U h^{-1} \cap P_{n,(i)}^{(m),+}\left(\mathbb{A}^{\infty}\right)\right) \\
& L_{n,(i), \operatorname{herm}}(\mathbb{R})^{+}\left(L_{n,(i), \operatorname{lin}}^{(m)}(\mathbb{R}) \cap U_{n, \infty}^{0}\right) A_{n,(i)}(\mathbb{R})^{0} .
\end{aligned}
$$

(See Sect. 1.4.)

If $\left(U^{p}(N), \Sigma\right) \in \mathcal{J}_{n}^{(m), \text { tor,ord }}$ then there is a simplicial complex $\mathcal{S}\left(U^{p}(N), \Sigma\right)^{\text {ord }}$ whose simplices are in bijection with equivalence classes of cones of dimension greater than 0 in $\Sigma$, where $\sigma$ and $\sigma^{\prime}$ are considered equivalent if $\sigma^{\prime}=\gamma \sigma u$ for some $\gamma \in G_{n}^{(m)}(\mathbb{Q})$ and some $u \in U^{p}(N, N)$. We will write $\mathcal{S}\left(U^{p}(N), \Sigma\right)_{\leq i}^{\text {ord }}$ for the subcomplex of $\mathcal{S}\left(U^{p}(N), \Sigma\right)^{\text {ord }}$ consisting of simplices associated to the orbits of cones $(g, \delta) \times \sigma \in \Sigma$ with $\sigma \subset \mathfrak{C}^{(m)}, \succ 0(W)$ for some $W$ with $\operatorname{dim}_{F} W \leq i$. We will also set

$$
\left|\mathcal{S}\left(U^{p}(N), \Sigma\right)^{\text {ord }}\right|_{=i}=\left|\mathcal{S}\left(U^{p}(N), \Sigma\right)_{\leq i}^{\text {ord }}\right|-\left|\mathcal{S}\left(U^{p}(N), \Sigma\right)_{\leq i-1}^{\text {ord }}\right|
$$

an open subset of $\left|\mathcal{S}\left(U^{p}, \Sigma\right)_{\leq i}^{\text {ord }}\right|$. Then we see that

$$
\begin{aligned}
& \left|\mathcal{S}\left(U^{p}(N), \Sigma\right)^{\text {ord }}\right| \\
& \left.\quad \cong G_{n}^{(m)}(\mathbb{Q}) \backslash\left(\left(G_{n}^{(m)}\left(\mathbb{A}^{\infty}\right) / U^{p}(N)\right) \times \pi_{0}\left(G_{n}(\mathbb{R})\right) \times\left(\mathfrak{C}^{(m)}-\mathfrak{C}_{=0}^{(m)}\right) / \mathbb{R}_{>0}^{\times}\right)\right)^{\text {ord }},
\end{aligned}
$$

where

$$
\left.\left(\left(G_{n}^{(m)}\left(\mathbb{A}^{\infty}\right) / U^{p}(N)\right) \times \pi_{0}\left(G_{n}(\mathbb{R})\right) \times\left(\mathfrak{C}^{(m)}-\mathfrak{C}_{=0}^{(m)}\right) / \mathbb{R}_{>0}^{\times}\right)\right)^{\text {ord }}
$$

denotes the image of

$$
\left(G_{n}^{(m)}\left(\mathbb{A}^{\infty}\right) \times \pi_{0}\left(G_{n}(\mathbb{R})\right) \times \mathfrak{C}^{(m)}\right)^{\text {ord }}-\left(G_{n}^{(m)}\left(\mathbb{A}^{\infty}\right) \times \pi_{0}\left(G_{n}(\mathbb{R})\right) \times \mathfrak{C}_{=0}^{(m)}\right)^{\text {ord }}
$$

in

$$
\left.G_{n}^{(m)}(\mathbb{Q}) \backslash\left(\left(G_{n}^{(m)}\left(\mathbb{A}^{\infty}\right) / U^{p}(N, N)\right) \times \pi_{0}\left(G_{n}(\mathbb{R})\right) \times\left(\mathfrak{C}^{(m)}-\mathfrak{C}_{=0}^{(m)}\right) / \mathbb{R}_{>0}^{\times}\right)\right) .
$$

Moreover

$$
\begin{aligned}
&\left|\mathcal{S}\left(U^{p}(N), \Sigma\right)^{\text {ord }}\right|_{=i} \\
& \cong G_{n}^{(m)}(\mathbb{Q}) \backslash\left(\left(G_{n}^{(m)}\left(\mathbb{A}^{\infty}\right) / U^{p}(N, N)\right) \times \pi_{0}\left(G_{n}(\mathbb{R})\right) \times\left(\left(\mathfrak{C}_{=i}^{(m)}\right) / \mathbb{R}_{>0}^{\times}\right)\right)^{\text {ord }} \\
& \cong \bigsqcup_{h \in P_{n,(i)}^{(m),}\left(\mathbb{A}^{\infty}\right)^{\text {ord }, \times} \backslash G_{n}^{(m)}\left(\mathbb{A}^{\infty}\right)^{\text {ord } \times} / U^{p}(N)} L_{n,(i)}^{(m)}(\mathbb{Q}) \backslash L_{n,(i)}^{(m)}(\mathbb{A}) \\
& /\left(h U^{p}(N) h^{-1} \cap P_{n,(i)}^{(m),+}\left(\mathbb{A}^{\infty}\right)^{\text {ord }}\right) \\
& L_{n,(i), \text { herm }}^{-}\left(\mathbb{Z}_{p}\right) L_{n,(i) \text {,herm }}(\mathbb{R})^{+}\left(L_{n,(i), \text { lin }}^{(m)}(\mathbb{R}) \cap U_{n, \infty}^{0}\right) A_{n,(i)}(\mathbb{R})^{0} .
\end{aligned}
$$

(Use the same argument as in the proof of Lemma 1.8.) In particular

$$
\left|\mathcal{S}\left(U^{p}(N), \Sigma\right)^{\text {ord }}\right|_{=n} \cong \mathfrak{T}_{U^{p}(N),=n}^{(m), \text { ord }} .
$$




\subsection{Toroidal compactifications}

If $(U, \Sigma) \in \mathcal{J}_{n}^{(m), \text { tor }}$, then there is a smooth projective scheme $A_{n, U, \Sigma}^{(m)}$ and a divisor with simple normal crossings

$$
\partial A_{n, U, \Sigma}^{(m)} \subset A_{n, U, \Sigma}^{(m)}
$$

together with an isomorphism

$$
j_{U, \Sigma}^{(m)}: A_{n, U}^{(m)} \stackrel{\sim}{\longrightarrow} A_{n, U, \Sigma}^{(m)}-\partial A_{n, U, \Sigma}^{(m)}
$$

and a projection

$$
\pi_{A^{(m), \text { tor } / X^{\min }}}: A_{n, U, \Sigma}^{(m)} \longrightarrow X_{n, U}^{\min }
$$

such that

$$
\begin{array}{cccc}
A_{n, U}^{(m)} & \hookrightarrow & A_{n, U, \Sigma}^{(m)} \\
\downarrow & & \downarrow \\
X_{n, U} & \hookrightarrow & X_{n, U}^{\min }
\end{array}
$$

is a commutative pull-back square. (The set $\mathcal{J}_{n}^{(m) \text {,tor }}$ was chosen so that inter alia these properties are true.) The divisor $\partial A_{n, U, \Sigma}^{(m)}$ induces a $\log$ structure $\mathcal{M}_{\Sigma}$ on $A_{n, U, \Sigma}^{(m)}$.

If $(U, \Sigma) \in \mathcal{J}_{n}^{(m), \text { tor }}$ and $\left(U^{\prime}, \Delta\right) \in \mathcal{J}_{n}^{\text {tor }}$ with $(U, \Sigma) \geq\left(U^{\prime}, \Delta\right)$ then there is a log smooth map

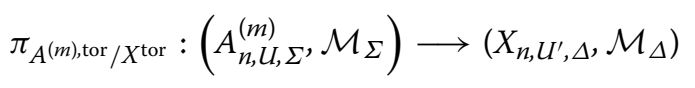

over $X_{n, U^{\prime}}^{\min }$ extending the map

$$
\pi_{A^{(m)} / X}: A_{n, U}^{(m)} \longrightarrow X_{n, U^{\prime}}
$$

If $\left(U^{\prime}, \Sigma^{\prime}\right)$ and $(U, \Sigma) \in \mathcal{J}_{n}^{(m) \text {,tor }}$; if $g \in G_{n}^{(m)}\left(\mathbb{A}^{\infty}\right)$; if $U^{\prime} \supset g^{-1} U g$; and if $\Sigma g$ is a refinement of $\Sigma^{\prime}$ then the map $g: A_{n, U}^{(m)} \rightarrow A_{n, U^{\prime}}^{(m)}$ extends to a log etale morphism

$$
g:\left(A_{n, U, \Sigma}^{(m)}, \mathcal{M}_{\Sigma}\right) \longrightarrow\left(A_{n, U^{\prime}, \Sigma^{\prime}}^{(m)}, \mathcal{M}_{\Sigma^{\prime}}\right)
$$

The collection $\left\{A_{n, U, \Sigma}^{(m)}\right\}$ becomes a system of schemes with right $G_{n}^{(m)}\left(\mathbb{A}^{\infty}\right)$-action, indexed by $\mathcal{J}_{n}^{(m) \text {,tor }}$. The maps $j_{U, \Sigma}^{(m)}$ and $\pi_{A^{(m), \text { tor }} / X^{\text {min }}}$ and $\pi_{A^{(m) \text {,tor }} / X^{\text {tor }}}$ are all $G_{n}^{(m)}\left(\mathbb{A}^{\infty}\right)$-equivariant. If $(U, \Sigma) \geq\left(U^{\prime}, \Sigma^{\prime}\right)$ we will write $\pi_{(U, \Sigma),\left(U^{\prime}, \Sigma^{\prime}\right)}$ for the map $1: A_{n, U, \Sigma}^{(m)} \rightarrow A_{n, U^{\prime}, \Sigma^{\prime}}^{(m)}$. (See theorem 1.3.3.15 of [44] for the assertions of the last three paragraphs.)

Any of the (canonically quasi-isogenous) universal abelian schemes $A^{\text {univ }} / X_{n, U}$ extend uniquely to semi-abelian schemes $A_{\Delta}^{\text {univ }} / X_{n, U, \Delta}$. The quasi-isogenies between the $A^{\text {univ }}$ extend uniquely to quasi-isogenies between the $A_{\Delta}^{\text {univ }}$. If $g \in G_{n}\left(\mathbb{A}^{\infty}\right)$ and $(U, \Delta) \geq$ $\left(U^{\prime}, \Delta^{\prime}\right) g$ then $g^{*} A_{\Delta^{\prime}}^{\text {univ }}$ is one of the $A_{\Delta}^{\text {univ }}$. (See remarks 1.1.2.1 and 1.3.1.4 of [44].)

We will write $\partial_{i} A_{n, U, \Sigma}^{(m)}$ for the pre-image under $\left.\pi_{A^{(m), t o r} / X^{\min }}\right|_{\partial A_{n, U, \Sigma}^{(m)}}$ of $\partial_{i} X_{U}^{\min }$. We also set

$$
\partial_{i}^{0} A_{n, U, \Sigma}^{(m)}=\partial_{i} A_{n, U, \Sigma}^{(m)}-\partial_{i+1} A_{n, U, \Sigma}^{(m)} .
$$


We will also write $A_{n, U, \Sigma, i}^{(m), \wedge}$ for the formal completion of $A_{n, U, \Sigma}^{(m)}$ along $\partial_{i}^{0} A_{n, U, \Sigma}^{(m)}$ and $\mathcal{M}_{\Sigma, i}^{\wedge}$ for the $\log$ structure induced on $A_{n, U, \Sigma, i}^{(m), \wedge}$ by $\mathcal{M}_{\Sigma}$. There are isomorphisms

$$
\left(A_{n, U, \Sigma, i}^{(m), \wedge}, \mathcal{M}_{\Sigma, i}^{\wedge}\right) \cong \coprod_{h \in P_{n, i(i)}^{(m)+}\left(\mathbb{A}^{\infty}\right) \backslash G_{n}^{(m)}\left(\mathbb{A}^{\infty}\right) / U}\left(T_{n,(i), h U h^{-1} \cap P_{n, i)}^{(m)+}\left(\mathbb{A}^{\infty}\right), \Sigma(h)_{0}}^{(m), \mathcal{M}_{\Sigma(h)_{0}}}\right) .
$$

Suppose that $g^{-1} U g \subset U^{\prime}$ and that $\Sigma g$ is a refinement of $\Sigma^{\prime}$. Suppose also that $h, h^{\prime} \in$ $G_{n}^{(m)}\left(\mathbb{A}^{\infty}\right)$ with

$$
h g\left(h^{\prime}\right)^{-1} \in P_{n,(i)}^{(m),+}\left(\mathbb{A}^{\infty}\right) .
$$

Then the diagram

$$
\begin{aligned}
& T_{n,(i), h U h^{-1} \cap P_{n, i}^{(m),+}\left(\mathbb{A}^{\infty}\right), \Sigma(h)_{0}}^{(m), \downarrow \wedge} \stackrel{h g\left(h^{\prime}\right)^{-1}}{\longrightarrow} T_{n,(i), h^{\prime} U^{\prime}\left(h^{\prime}\right)^{-1} \cap P_{n,(i)}^{(m),+}\left(\mathbb{A}^{\infty}\right), \Sigma^{\prime}\left(h^{\prime}\right)_{0}}^{(m), \wedge} \\
& \downarrow \\
& A_{n, U, \Sigma, i}^{(m), \wedge} \quad \stackrel{g}{\longrightarrow} \quad A_{n, U^{\prime}, \Sigma^{\prime}, i}^{(m) \wedge}
\end{aligned}
$$

commutes, and is compatible with the log structures on each of these formal schemes. (See theorem 1.3.3.15 of [44].)

If $U^{\prime}$ is a neat subgroup of $G_{n}\left(\mathbb{A}^{\infty}\right)$ containing the image of $U$; if $\left(U^{\prime}, \Delta\right) \in \mathcal{J}_{n}^{\text {tor }}$; and if $\Sigma$ and $\Delta$ are compatible, then for all $h \in P_{n,(i)}^{(m),+}\left(\mathbb{A}^{\infty}\right)$ with image $h^{\prime} \in P_{n,(i)}^{+}\left(\mathbb{A}^{\infty}\right)$ the cone decompositions $\Sigma(h)_{0}$ and $\Delta\left(h^{\prime}\right)_{0}$ are compatible and we have a diagram

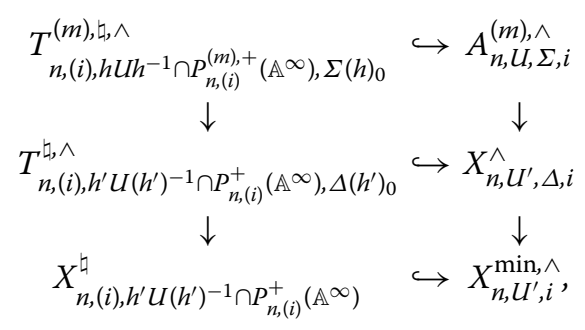

which is commutative as a diagram of topological spaces (but not as a diagram of locally ringed spaces). The top square is commutative as a diagram of formal schemes and is compatible with the log structures. (Again see theorem 1.3.3.15 of [44].)

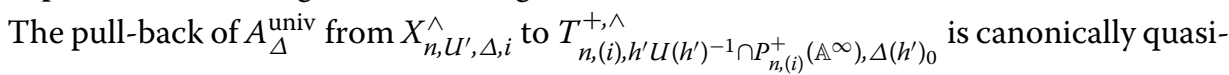
isogenous to the pull-back of $\widetilde{G}^{\text {univ }}$ from $A_{n,(i), h^{\prime} U\left(h^{\prime}\right)^{-1} \cap P_{n, i(}^{+}\left(\mathbb{A}^{\infty}\right), \Delta\left(h^{\prime}\right)_{0}}$.

We will write

$$
\left|\mathcal{S}\left(\partial A_{n, U, \Sigma}^{(m)}\right)\right|_{=i}=\left|\mathcal{S}\left(\partial A_{n, U, \Sigma}^{(m)}-\partial_{i+1} A_{n, U, \Sigma}^{(m)}\right)\right|-\left|\mathcal{S}\left(\partial A_{n, U, \Sigma}^{(m)}-\partial_{i} A_{n, U, \Sigma}^{(m)}\right)\right| .
$$

Then there are compatible identifications

$$
\mathcal{S}\left(\partial A_{n, U, \Sigma}^{(m)}\right) \cong \mathcal{S}(U, \Sigma)
$$

and

$$
\mathcal{S}\left(\partial A_{n, U, \Sigma}^{(m)}-\partial_{i+1} A_{n, U, \Sigma}^{(m)}\right) \cong \mathcal{S}(U, \Sigma)_{\leq i}
$$


and

$$
\left|\mathcal{S}\left(\partial A_{n, U, \Sigma}^{(m)}\right)\right|_{=i} \cong|\mathcal{S}(U, \Sigma)|_{=i} ;
$$

and the latter is compatible with the identifications

$$
\begin{aligned}
& \left|\mathcal{S}\left(\partial A_{n, U, \Sigma}^{(m)}\right)\right|_{=i} \\
& \cong \coprod_{h \in P_{n,(i)}^{(m),}\left(\mathbb{A}^{\infty}\right) \backslash G_{n}^{(m)}\left(\mathbb{A}^{\infty}\right) / U} L_{n,(i), \operatorname{lin}}^{(m)}(\mathbb{Q}) \backslash\left(\left|\mathcal{S}\left(\partial T_{n,(i), h U h^{-1} \cap P_{n,(i)}^{(m),+}\left(\mathbb{A}^{\infty}\right), \widetilde{\Sigma(h)_{0}}}^{(m),+}\right)\right|\right. \\
& \left.-\left|\mathcal{S}\left(\partial T_{\left.n,(i), h U h^{-1} \cap P_{n,(i)}^{(m),}\left(\mathbb{A}^{\infty}\right), \widetilde{\Sigma(h)}\right)_{0}-\Sigma(h)}^{(m),+}\right)\right|\right) \\
& \cong \coprod_{h \in P_{n,(i)}^{(m),+}\left(\mathbb{A}^{\infty}\right) \backslash G_{n}^{(m)}\left(\mathbb{A}^{\infty}\right) / U} L_{n,(i)}^{(m)}(\mathbb{Q}) \backslash L_{n,(i)}^{(m)}(\mathbb{A}) /\left(h U h^{-1} \cap P_{n,(i)}^{(m),+}\left(\mathbb{A}^{\infty}\right)\right) \\
& L_{n,(i), \text { herm }}(\mathbb{R})^{+}\left(L_{n,(i), \text { lin }}^{(m)}(\mathbb{R}) \cap U_{n, \infty}^{0}\right) A_{n,(i)}(\mathbb{R})^{0} \\
& \cong|\mathcal{S}(U, \Sigma)|_{=i} \text {. }
\end{aligned}
$$

(See theorem 1.3.3.15 of [44].) If $[\sigma] \in \mathcal{S}(U, \Sigma)$ we will write

$$
\partial_{[\sigma]} A_{n, U, \Sigma}^{(m)}
$$

for the corresponding closed boundary stratum of $A_{n, U, \Sigma}^{(m)}$.

Similarly if $\left(U^{p}\left(N_{1}, N_{2}\right), \Sigma\right) \in \mathcal{J}_{n}^{(m)}$, tor , then there is a smooth quasi-projective scheme $\mathcal{A}_{n, U^{p}\left(N_{1}, N_{2}\right), \Sigma}^{(m), \text { ond }}$ and a divisor with simple normal crossings

$$
\partial \mathcal{A}_{n, U^{p}\left(N_{1}, N_{2}\right), \Sigma}^{(m), \text { rd }} \subset \mathcal{A}_{n, U^{p}\left(N_{1}, N_{2}\right), \Sigma}^{(m), \text { ord }}
$$

together with an isomorphism

$$
j_{U^{p}\left(N_{1}, N_{2}\right), \Sigma}^{(m), \text { ord }}: \mathcal{A}_{n, U^{p}\left(N_{1}, N_{2}\right)}^{(m), \text { ord }} \stackrel{\sim}{\longrightarrow} \mathcal{A}_{n, U^{p}\left(N_{1}, N_{2}\right), \Sigma}^{(m), \text { ord }}-\partial \mathcal{A}_{n, U^{p}\left(N_{1}, N_{2}\right), \Sigma}^{(m), \text { ord }}
$$

and a projection

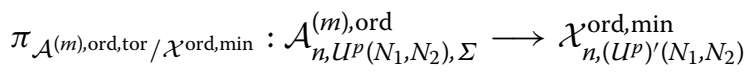

such that

$$
\begin{array}{ccc}
\mathcal{A}_{n, U^{p}\left(N_{1}, N_{2}\right)}^{(m), \text { ord }} & \hookrightarrow \mathcal{A}_{n, U^{p}\left(N_{1}, N_{2}\right), \Sigma}^{(m) \text { ord }} \\
\downarrow & \downarrow \\
\mathcal{X}_{n,\left(U^{p}\right)^{\prime}\left(N_{1}, N_{2}\right)}^{\text {ord }} & \hookrightarrow \mathcal{X}_{n,\left(U^{p}\right)^{\prime}\left(N_{1}, N_{2}\right)}^{\text {ord,min }}
\end{array}
$$

is a commutative pull-back square. The divisor $\partial \mathcal{A}_{n, U^{p}\left(N_{1}, N_{2}\right), \Sigma}^{(m), \text { ind }}$ induces a log structure $\mathcal{M}_{\Sigma}$ on $\mathcal{A}_{n, U^{p}\left(N_{1}, N_{2}\right), \Sigma}^{(m) \text { ord }}$

$$
\text { If }\left(U^{p}\left(N_{1}, N_{2}\right), \Sigma\right) \in \mathcal{J}_{n}^{(m), \text { tor }} \text { and }\left(\left(U^{p}\right)^{\prime}\left(N_{1}, N_{2}\right), \Delta\right) \in \mathcal{J}_{n}^{\text {tor }} \text { satisfy }
$$

$$
\left(U^{p}\left(N_{1}, N_{2}\right), \Sigma\right) \geq\left(\left(U^{p}\right)^{\prime}\left(N_{1}, N_{2}\right), \Delta\right)
$$

then there is a $\log$ smooth map

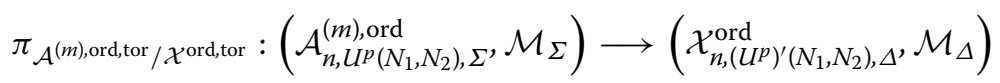


over $\mathcal{X}_{n,\left(U^{p}\right)^{\prime}\left(N_{1}, N_{2}\right)}^{\text {ord,min }}$ extending the map

$$
\pi_{\mathcal{A}^{(m), \text { ord }} / X^{\text {ord }}}: \mathcal{A}_{n, U^{p}\left(N_{1}, N_{2}\right)}^{(m), \text { ord }} \longrightarrow \mathcal{X}_{n,\left(U^{p}\right)^{\prime}\left(N_{1}, N_{2}\right)}^{\text {ord }}
$$

If $\left(\left(U^{p}\right)^{\prime}\left(N_{1}, N_{2}\right), \Sigma^{\prime}\right)$ and $\left.\left(U^{p}\right)^{\prime}\left(N_{1}, N_{2}\right), \Sigma\right) \in \mathcal{J}_{n}^{(m) \text {,tor }}$; if $g \in G_{n}^{(m)}\left(\mathbb{A}^{\infty}\right)^{\text {ord }}$; if $\left(U^{p}\right)^{\prime}\left(N_{1}^{\prime}, N_{2}^{\prime}\right) \supset g^{-1} U^{p}\left(N_{1}, N_{2}\right) g$; and if $\Sigma g$ is a refinement of $\Sigma^{\prime}$ then the map $g: \mathcal{A}_{n, U^{p}\left(N_{1}, N_{2}\right)}^{(m), \text { ord }} \rightarrow \mathcal{A}_{n,\left(U^{p}\right)^{\prime}\left(N_{1}, N_{2}\right)}^{(m), \text { oxt }}$ exds to a log etale morphism

$$
g:\left(\mathcal{A}_{n, U^{p}\left(N_{1}, N_{2}\right), \Sigma}^{(m), \text { ord }}, \mathcal{M}_{\Sigma}\right) \longrightarrow\left(\mathcal{A}_{n,\left(U^{p}\right)^{\prime}\left(N_{1}, N_{2}\right), \Sigma^{\prime}}^{(m), \text { ord }}, \mathcal{M}_{\Sigma^{\prime}}\right)
$$

Then $\left\{\mathcal{A}_{n, U^{p}\left(N_{1}, N_{2}\right), \Sigma}^{(m), \text { ord }}\right\}$ is a system of schemes with right $G_{n}^{(m)}\left(\mathbb{A}^{\infty}\right)^{\text {ord }}$-action, indexed by the subset of $\mathcal{J}_{n}^{(m) \text {,tor }}$ consisting of elements of the form $\left(U^{p}\left(N_{1}, N_{2}\right), \Sigma\right)$. The

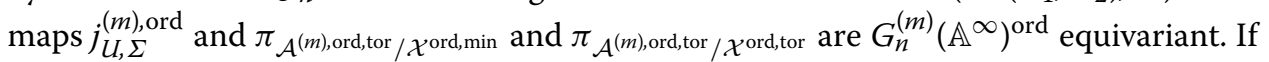
$\left(U^{p}\left(N_{1}, N_{2}\right), \Sigma\right) \geq\left(\left(U^{p}\right)^{\prime}\left(N_{1}^{\prime}, N_{2}^{\prime}\right), \Sigma^{\prime}\right)$, then we will denote the map $1: \mathcal{A}_{n, U^{p}\left(N_{1}, N_{2}\right), \Sigma}^{(m) \text { ord }} \rightarrow$

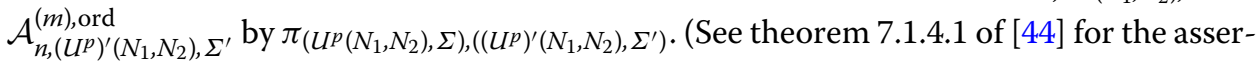
tions of the last three paragraphs.)

Any of the (canonically prime-to- $p$ quasi-isogenous) universal abelian schemes $\mathcal{A}^{\text {univ }} / \mathcal{X}_{n, U^{p}\left(N_{1}, N_{2}\right)}^{\text {ord }}$ extend uniquely to semi-abelian schemes $\mathcal{A}_{\Delta}^{\text {univ }} / \mathcal{X}_{n, U^{p}\left(N_{1}, N_{2}\right), \Delta}^{\text {ord }}$. The prime-to- $p$ quasi-isogenies between the $\mathcal{A}^{\text {univ }}$ extend uniquely to prime-to- $p$ quasiisogenies between the $\mathcal{A}_{\Delta}^{\text {univ }}$. If $g \in G_{n}\left(\mathbb{A}^{\infty}\right)^{\text {ord, } \times}$ and

$$
\left(U^{p}\left(N_{1}, N_{2}\right), \Delta\right) \geq\left(\left(U^{p}\right)^{\prime}\left(N_{1}, N_{2}\right), \Delta^{\prime}\right) g
$$

then $g^{*} \mathcal{A}_{\Delta^{\prime}}^{\text {univ }}$ is one of the $\mathcal{A}_{\Delta}^{\text {univ }}$. (See remarks 3.4.2.8 and 5.2.1.5 of [44].)

We will write $\partial_{i} \mathcal{A}_{n, U^{p}\left(N_{1}, N_{2}\right), \Sigma}^{(m) \text {,ord }}$ for the pre-image of $\partial_{i} \mathcal{X}_{n, U^{p}\left(N_{1}, N_{2}\right)}^{\text {ord,min }}$ under the map

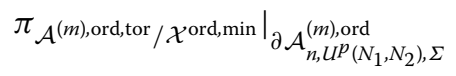

and set

$$
\partial_{i}^{0} \mathcal{A}_{n, U^{p}\left(N_{1}, N_{2}\right), \Sigma}^{(m), \text { ord }}=\partial_{i} \mathcal{A}_{n, U^{p}\left(N_{1}, N_{2}\right), \Sigma}^{(m), \text { ord }}-\partial_{i+1} \mathcal{A}_{n, U^{p}\left(N_{1}, N_{2}\right), \Sigma}^{(m), \text { ord }}
$$

We will also write $\mathcal{A}_{n, U^{p}\left(N_{1}, N_{2}\right), \Sigma, i}^{(m), \text { ord } \wedge}$ for the formal completion of $\mathcal{A}_{n, U^{p}\left(N_{1}, N_{2}\right), \Sigma}^{(m) \text { ard }}$ along $\partial_{i}^{0} \mathcal{A}_{n, U^{p}\left(N_{1}, N_{2}\right), \Sigma}^{(m) \text {,ord }}$, and $\mathcal{M}_{\Sigma, i}^{\wedge}$ for the log structure induced on $\mathcal{A}_{n, U^{p}\left(N_{1}, N_{2}\right), \Sigma, i}^{(m) \text {,ord, } \wedge}$ by $\mathcal{M}_{\Sigma}$. There are isomorphisms

$$
\begin{aligned}
& \left(\mathcal{A}_{n, U^{p}\left(N_{1}, N_{2}\right), \Sigma, i}^{(m), \text { ord } \wedge}, \mathcal{M}_{\Sigma, i}^{\wedge}\right) \cong \coprod_{h \in P_{n,(i)}^{(m),+}\left(\mathbb{A}^{\infty}\right)^{\text {ord }, \times} \backslash G_{n}^{(m)}\left(\mathbb{A}^{\infty}\right)^{\text {ord } \times} \times U^{p}\left(N_{1}\right)} \\
& \left(\mathcal{T}_{n,(i),\left(h U^{p} h^{-1} \cap P_{n,(i)}^{(m),+}\left(\mathbb{A}^{\infty, p}\right)\right)\left(N_{1}, N_{2}\right), \Sigma^{\operatorname{ord}}(h)_{0}}^{(m), \mathcal{M}^{\text {ord }}(h)_{0}}\right)
\end{aligned}
$$

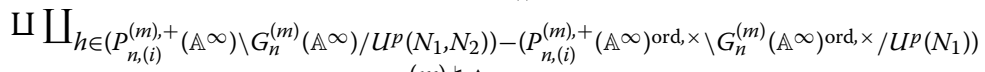

$$
\begin{aligned}
& \left(T_{n,(i), h U^{p}\left(N_{1}, N_{2}\right) h^{-1} \cap P_{n,(i)}^{(m),+}\left(\mathbb{A}^{\infty}\right), \Sigma(h)_{0}}^{(m), \emptyset}, \mathcal{M}_{\Sigma(h)_{0}}^{\wedge}\right) .
\end{aligned}
$$

Suppose that $g \in G_{n}^{(m)}\left(\mathbb{A}^{\infty}\right)^{\text {ord }}$ and $g^{-1} U^{p}\left(N_{1}, N_{2}\right) g \subset\left(U^{p}\right)^{\prime}\left(N_{1}^{\prime}, N_{2}^{\prime}\right)$ and that $\Sigma g$ is a refinement of $\Sigma^{\prime}$. Suppose also that $h, h^{\prime} \in G_{n}^{(m)}\left(\mathbb{A}^{\infty}\right)^{\text {ord, } \times}$ with

$$
h g\left(h^{\prime}\right)^{-1} \in P_{n,(i)}^{(m),+}\left(\mathbb{A}^{\infty}\right)^{\text {ord }}
$$


Then the diagram

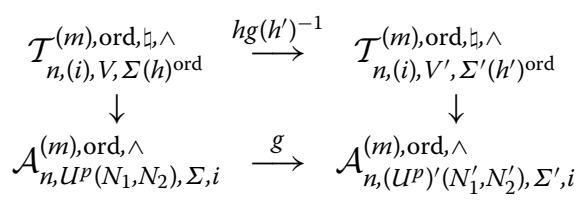

commutes, where

$$
V=\left(h U^{p} h^{-1} \cap P_{n,(i)}^{(m)+}\left(\mathbb{A}^{\infty, p}\right)\right)\left(N_{1}, N_{2}\right)
$$

and

$$
V^{\prime}=\left(h^{\prime}\left(U^{p}\right)^{\prime}\left(h^{\prime}\right)^{-1} \cap P_{n,(i)}^{(m),+}\left(\mathbb{A}^{\infty, p}\right)\right)\left(N_{1}^{\prime}, N_{2}^{\prime}\right)
$$

Moreover this is compatible with the log structures defined on each of the four formal schemes. (See theorem 7.1.4.1 of [44].)

If $[\sigma] \in \mathcal{S}\left(U^{p}\left(N_{1}, N_{2}\right), \Sigma\right)$ we will write

$$
\partial_{[\sigma]} \mathcal{A}_{n, U^{p}\left(N_{1}, N_{2}\right), \Sigma}^{(m), \text { ord }}
$$

for the closure of $\partial_{[\sigma]} A_{n, U^{p}\left(N_{1}, N_{2}\right), \Sigma}^{(m)}$ in $\mathcal{A}_{n, U^{p}\left(N_{1}, N_{2}\right), \Sigma}^{(m) \text { ord }}$. The special fibre

$$
\left(\partial_{[\sigma]} \mathcal{A}_{n, U^{p}\left(N_{1}, N_{2}\right), \Sigma}^{(m) \text { ord }}\right) \times \operatorname{Spec} \mathbb{F}_{p}
$$

is non-empty if and only if $[\sigma] \in \mathcal{S}\left(U^{p}\left(N_{1}\right), \Sigma^{\text {ord }}\right)^{\text {ord }}$. (We remind the reader that the first superscript ${ }^{\text {ord }}$ associates the 'ordinary' cone decomposition $\Sigma^{\text {ord }}$ to the cone decomposition $\Sigma$, while the second superscript ${ }^{\text {ord }}$ is the notation we are using for the simplicial complex associated to an 'ordinary' cone decomposition.) We will write

$$
\left(\mathcal{A}_{n, U^{p}\left(N_{1}, N_{2}\right), \Sigma}^{(m), \text { ord }}\right)^{0}=\mathcal{A}_{n, U^{p}\left(N_{1}, N_{2}\right), \Sigma}^{(m), \text { ord }}-\bigcup_{[\sigma] \in \mathcal{S}\left(U^{p}\left(N_{1}, N_{2}\right), \Sigma\right)-\mathcal{S}\left(U^{p}\left(N_{1}\right), \Sigma^{\text {ord }}\right)^{\text {ord }}} \partial_{[\sigma]} \mathcal{A}_{n, U^{p}\left(N_{1}, N_{2}\right), \Sigma}^{(m), \text { ord }}
$$

This only depends on $\Sigma^{\text {ord }}$.

If $\left(U^{p}\right)^{\prime}$ is a neat subgroup of $G_{n}\left(\mathbb{A}^{\infty, p}\right)$ containing the image of $U^{p}$; if the pair $\left(\left(U^{p}\right)^{\prime}\left(N_{1}, N_{2}\right), \Delta\right) \in \mathcal{J}_{n}^{\text {tor }}$; and if $\Sigma$ and $\Delta$ are compatible, then for all $h \in P_{n,(i)}^{(m),+}\left(\mathbb{A}^{\infty}\right)^{\text {ord }, \times}$ with image $h^{\prime} \in P_{n,(i)}^{+}\left(\mathbb{A}^{\infty}\right)^{\text {ord, } \times}$ the cone decompositions $\Sigma^{\text {ord }}(h)_{0}$ and $\Delta^{\text {ord }}\left(h^{\prime}\right)_{0}$ are compatible and we have a diagram

$$
\begin{aligned}
& \mathcal{T}_{n,(i),\left(h U^{p} h^{-1} \cap P_{n,(i)}^{(m),+}\left(\mathbb{A}^{\infty, p}\right)\right)\left(N_{1}, N_{2}\right), \Sigma^{\mathrm{ord}}(h)_{0}}^{(m), \text { ord },, \wedge} \hookrightarrow \mathcal{A}_{n, U^{p}\left(N_{1}, N_{2}\right), \Sigma, i}^{(m), \text { ord } \wedge}
\end{aligned}
$$

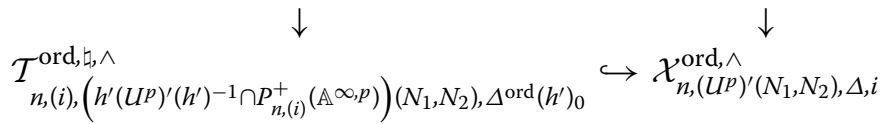

$$
\begin{aligned}
& \mathcal{X}^{\text {ord, }, ~} \quad \downarrow \quad \hookrightarrow \mathcal{X}_{n,\left(U^{p}\right)^{\prime}\left(N_{1}, N_{2}\right)}^{\text {ord,min }, \wedge}
\end{aligned}
$$

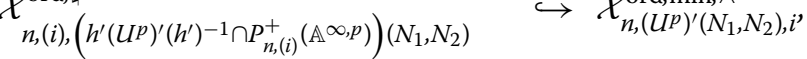


which is commutative as a diagram of topological spaces (but not as a diagram of locally ringed spaces). The top square is commutative as a diagram of formal schemes and is compatible with the log structures. (See theorem 7.1.4.1 of [44].)

The pull-back of $\mathcal{A}_{\Delta}^{\text {univ }}$ to $\mathcal{T}_{n,(i),\left(h^{\prime}\left(U^{p}\right)^{\prime}\left(h^{\prime}\right)^{-1} \cap P_{n,(i)}^{+}\left(\mathbb{A}^{\infty, p}\right)\right)\left(N_{1}, N_{2}\right), \Delta^{\circ r d}\left(h^{\prime}\right)_{0}}^{\text {ord }+, \wedge}$ is canonically quasiisogenous to the pull-back of $\widetilde{\mathcal{G}}^{\text {univ }}$ from

$$
\mathcal{A}_{n,(i),\left(h^{\prime}\left(U^{p}\right)^{\prime}\left(h^{\prime}\right)^{-1} \cap P_{n,(i)}^{+}\left(\mathbb{A}^{\infty, p}\right)\right)\left(N_{1}, N_{2}\right), \Delta^{\text {ord }}\left(h^{\prime}\right)_{0}}
$$

All this is compatible with passage to the generic fibre and our previous discussion. (Again see theorem 7.1.4.1 of [44].)

If $N_{2}^{\prime} \geq N_{2} \geq N_{1}$, if $\Sigma^{\prime}$ is a refinement of $\Sigma$ and if $\Sigma^{\text {ord }}=\left(\Sigma^{\prime}\right)^{\text {ord }}$ then the natural map

$$
\mathcal{A}_{n, U^{p}\left(N_{1}, N_{2}^{\prime}\right), \Sigma^{\prime}}^{(m) \text { ord }} \longrightarrow \mathcal{A}_{n, U^{p}\left(N_{1}, N_{2}\right), \Sigma}^{(m) \text { ord }}
$$

is etale in a neighbourhood of the $\mathbb{F}_{p}$-fibre of $\mathcal{A}_{n, U^{p}\left(N_{1}, N_{2}^{\prime}\right), \Sigma^{\prime}}^{(m) \text { ord }}$ and induces an isomorphism between the formal completions of these schemes along their $\mathbb{F}_{p}$-fibres. (See theorem 7.1.4.1(4) of [44].) We will denote this $p$-adic formal scheme

$$
\mathfrak{A}_{n, U^{p}\left(N_{1}\right), \Sigma^{\text {ord }}}^{(m), \text { ord }}
$$

and will denote its reduced subscheme

$$
\bar{A}_{n, U^{p}\left(N_{1}\right), \Sigma^{\text {ord }}}^{(m) \text { ord }}
$$

(In the case $m=0$ we could also write $\mathfrak{X}_{n, U^{p}\left(N_{1}\right), \Sigma^{\text {ord }}}^{\text {ord }}$ and $\bar{x}_{n, U^{p}\left(N_{1}\right), \Sigma^{\text {ord }}}^{\text {ord }}$. We will also write

$$
\partial \bar{A}_{n, U^{p}\left(N_{1}\right), \Sigma^{\text {ord }}}^{(m), \text { ord }}=\bar{A}_{n, U^{p}\left(N_{1}\right), \Sigma^{\text {ord }}}^{(m) \text { ord }}-\bar{A}_{n, U^{p}\left(N_{1}\right)}^{(m) \text { ord }} .
$$

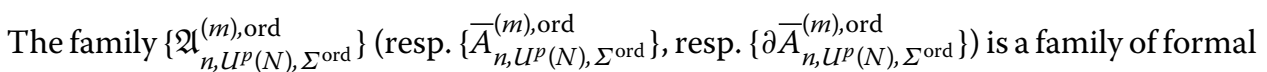
schemes (resp. schemes, resp. schemes) indexed by $\mathcal{J}_{n}^{(m) \text {,tor,ord }}$ with $G_{n}\left(\mathbb{A}^{\infty}\right)^{\text {ord }}$ action. Let

$$
\partial_{i} \bar{A}_{n, U^{p}(N), \Sigma^{\text {ord }}}^{(m), \text { ord }}
$$

denote the pre-image of $\partial_{i} \bar{X}_{n, U^{p}(N)}^{\mathrm{ord}, \min }$ in $\partial \bar{A}_{n, U^{p}(N), \Sigma^{\text {ord }}}^{(m) \text {, ord }}$, and set

$$
\partial_{i}^{0} \bar{A}_{n, U^{p}(N), \Sigma^{\text {ord }}}^{(m), \text { ord }}=\partial_{i} \bar{A}_{n, U^{p}(N), \Sigma^{\text {ord }}}^{(m), \text { ord }}-\partial_{i+1} \bar{A}_{n, U^{p}(N), \Sigma^{\text {ord }}}^{(m), \text { ord }} .
$$

The families $\left\{\partial_{i} \bar{A}_{n, U^{p}(N), \Sigma^{\text {ord }}}^{(m) \text { ord }}\right\}$ and $\left\{\partial_{i}^{0} \bar{A}_{n, U^{p}(N), \Sigma^{\text {ord }}}^{(m) \text { ord }}\right\}$ have actions of $G_{n}\left(\mathbb{A}^{\infty}\right)^{\text {ord }}$. Moreover we have a decomposition

$$
\begin{aligned}
& \partial_{i}^{0} \bar{A}_{n, U^{p}(N), \Sigma^{\text {ord }}}^{(m) \text { ord }} \\
& \quad=\coprod_{h \in P_{n,(i)}^{+}\left(\mathbb{A}^{\infty}\right)^{\text {ord }} \backslash G_{n}\left(\mathbb{A}^{\infty}\right)^{\text {ord }} / U^{p}(N)} \partial_{\Sigma^{\text {ord }}(h) 0} \bar{T}_{n,(i),\left(h U^{p} h^{-1} \cap P_{n,(i)}^{+}\left(\mathbb{A}^{\infty}, p\right)\right)(N)}^{(m),} .
\end{aligned}
$$

If $g \in G_{n}^{(m)}\left(\mathbb{A}^{\infty}\right)^{\text {ord }}$, if $g^{-1} U^{p}(N) g \subset\left(U^{p}\right)^{\prime}\left(N^{\prime}\right)$ and if $\Sigma^{\text {ord }} g$ is a refinement of $\left(\Sigma^{\prime}\right)^{\text {ord }}$, then the map

$$
g: \partial_{i}^{0} \bar{A}_{n, U^{p}(N), \Sigma^{\text {ord }}}^{(m), \text { ord }} \longrightarrow \partial_{i}^{0} \bar{A}_{n,\left(U^{p}\right)^{\prime}\left(N^{\prime}\right),\left(\Sigma^{\prime}\right) \text { ord }}^{(m) \text { ord }}
$$


is the coproduct of the maps

$$
\begin{aligned}
& g^{\prime}: \partial_{\Sigma^{\operatorname{ord}}(h)_{0}} \bar{T}_{n,(i),\left(h U^{p} h^{-1} \cap P_{n,(i)}^{+}\left(\mathbb{A}^{\infty, p}\right)\right)(N)}^{(m), \text { ord } \curvearrowleft} \\
& \longrightarrow \partial_{\Sigma^{\mathrm{ord}}(h)_{0}} \bar{T}_{n,(i),\left(h^{\prime}\left(U^{\prime}\right)^{p}\left(h^{\prime}\right)^{-1} \cap P_{n,(i)}^{+}\left(\mathbb{A}^{\infty, p}\right)\right)\left(N^{\prime}\right)}^{- \text {ord },}
\end{aligned}
$$

where $h g=g^{\prime} h^{\prime}$ with $g^{\prime} \in P_{n,(i)}^{+}\left(\mathbb{A}^{\infty}\right)^{\text {ord }}$.

The map

$$
\varsigma_{p}: \mathfrak{A}_{n, U^{p}(N), \Sigma^{\text {ord }}}^{(m) \text { ord }} \longrightarrow \mathfrak{A}_{n, U^{p}(N), \Sigma^{\text {ord }}}^{(m), \text { ord }}
$$

is finite flat of degree $p^{(2 m+n) n\left[F^{+}: \mathbb{Q}\right]}$ and on $\mathbb{F}_{p}$-fibres it is identified with absolute Frobenius.

If $N_{2}^{\prime} \geq N_{2} \geq N_{1}$, if $\Sigma^{\prime}$ is a refinement of $\Sigma$ and if $\sigma \in \Sigma^{\text {ord }}=\left(\Sigma^{\prime}\right)^{\text {ord }}$ then the natural map

$$
\partial_{[\sigma]} \mathcal{A}_{n, U^{p}\left(N_{1}, N_{2}^{\prime}\right), \Sigma^{\prime}}^{(m), \text { ord }} \longrightarrow \partial_{[\sigma]} \mathcal{A}_{n, U^{p}\left(N_{1}, N_{2}\right), \Sigma}^{(m), \text { ord }}
$$

is etale in a neighbourhood of the $\mathbb{F}_{p}$-fibre of $\partial_{[\sigma]} \mathcal{A}_{n, U^{p}\left(N_{1}, N_{2}^{\prime}\right), \Sigma^{\prime}}^{(m) \text {,ord }}$ and so induces an isomorphism of the formal completions of these schemes along their $\mathbb{F}_{p}$-fibres. We will denote this $p$-adic formal scheme

$$
\partial_{[\sigma]} \mathfrak{A}_{n, U^{p}\left(N_{1}\right), \Sigma^{\text {ord }}}^{(m) \text { ord }}
$$

and will denote its reduced subscheme

$$
\partial_{[\sigma]} \bar{A}_{n, U^{p}\left(N_{1}\right), \Sigma^{\text {ord }}}^{(m) \text { ord }}
$$

For $s>0$ we will write

$$
\partial^{(s)} \mathfrak{A}_{n, U^{p}\left(N_{1}\right), \Sigma^{\text {ord }}}^{(m) \text {,ord }}=\coprod_{\begin{array}{c}
{[\sigma] \in \mathcal{S}\left(U^{p}\left(N_{1}\right), \Sigma^{\text {ord }}\right)^{\text {ord }}} \\
\operatorname{dim} \sigma=s
\end{array}} \partial_{[\sigma]} \mathfrak{A}_{n, U^{p}\left(N_{1}\right), \Sigma^{\text {ord }}}^{(m), \text { ord }}
$$

and

$$
\partial^{(s)} \bar{A}_{n, U^{p}\left(N_{1}\right), \Sigma^{\text {ord }}}^{(m) \text { ord }}=\coprod_{\begin{array}{c}
{[\sigma] \in \mathcal{S}\left(U^{p}\left(N_{1}\right), \Sigma^{\text {ord }}\right)^{\text {ord }}} \\
\operatorname{dim} \sigma=s
\end{array}} \partial_{[\sigma]} \bar{A}_{n, U^{p}\left(N_{1}\right), \Sigma^{\text {ord }}}^{(m), \text { ord }}
$$

The maps

$$
\varsigma_{p}: \partial^{(s)} \mathfrak{A}_{n, U^{p}\left(N_{1}\right), \Sigma^{\text {ord }}}^{(m), \text { ord }} \longrightarrow \partial^{(s)} \mathfrak{A}_{n, U^{p}\left(N_{1}\right), \Sigma^{\text {ord }}}^{(m) \text { ord }}
$$

are finite flat of degree $p^{(2 m+n) n\left[F^{+}: \mathbb{Q}\right]-s}$.

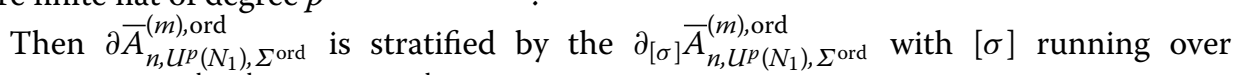
$\mathcal{S}\left(U^{p}\left(N_{1}\right), \Sigma^{\text {ord }}\right)^{\text {ord }}$. If $\sigma \in \Sigma^{\text {ord }}$ but $\sigma$ is not contained in

$$
\bigcup_{i<n}\left(G_{n}^{(m)}\left(\mathbb{A}^{\infty}\right) \times \pi_{0}\left(G_{n}^{(m)}(\mathbb{R})\right) \times \mathfrak{C}_{=i}^{(m)}\right)^{\text {ord }}
$$


then $\partial_{[\sigma]} \bar{A}_{n, U^{p}\left(N_{1}\right), \Sigma^{\text {ord }}}^{(m) \text {,ord }}$ is irreducible. (Because $\partial_{[\sigma]} \bar{A}_{n, U^{p}\left(N_{1}\right), \Sigma^{\text {ord }}}^{(m) \text {,ord }}$ is a toric variety over $\mathbb{F}_{p}$.

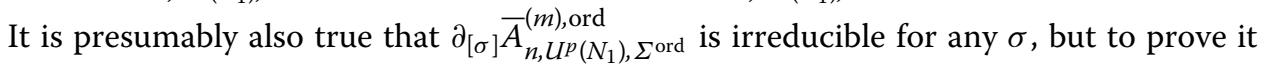
one would need an irreducibility statement about the special fibre of a Shimura variety. In many cases such a theorem has been proved by Hida [33], but not in the full generality in which we are working here.)

We will write

$$
\begin{aligned}
& \left|\mathcal{S}\left(\partial \bar{A}_{n, U^{p}(N), \Sigma^{\text {ord }}}^{(m), \text { ord }}\right)\right|_{=i} \\
& =\left|\mathcal{S}\left(\partial \bar{A}_{n, U^{p}(N), \Sigma^{\text {ord }}}^{(m), \text { ord }}-\partial_{i+1} \bar{A}_{n, U^{p}(N), \Sigma^{\text {ord }}}^{(m) \text { ord }}\right)\right|-\left|\mathcal{S}\left(\partial \bar{A}_{n, U^{p}(N), \Sigma^{\text {ord }}}^{(m), \text { ord }}-\partial_{i} \bar{A}_{n, U^{p}(N), \Sigma^{\text {ord }}}^{(m), \text { ord }}\right)\right|
\end{aligned}
$$

an open subset of $\left|\mathcal{S}\left(\partial \bar{A}_{n, U^{p}(N), \Sigma^{\text {ord }}}^{(m), \text { ord }}-\partial_{i+1} \bar{A}_{n, U^{p}(N), \Sigma^{\text {ord }}}^{(m) \text {,ord }}\right)\right|$. Then there are natural surjections

$$
\mathcal{S}\left(\partial \bar{A}_{n, U^{p}(N), \Sigma^{\text {ord }}}^{(m), \text { ord }}\right) \rightarrow \mathcal{S}\left(U^{p}(N), \Sigma^{\text {ord }}\right)^{\text {ord }}
$$

which restrict to surjections

$$
\mathcal{S}\left(\partial \bar{A}_{n, U^{p}(N), \Sigma^{\text {ord }}}^{(m) \text { ord }}-\partial_{i+1} \bar{A}_{n, U^{p}(N), \Sigma^{\text {ord }}}^{(m) \text { ord }}\right) \rightarrow \mathcal{S}\left(U^{p}(N), \Sigma^{\text {ord }}\right)_{\leq i}^{\text {ord }}
$$

This gives rise to surjections

$$
\left|\mathcal{S}\left(\partial \bar{A}_{n, U^{p}(N), \Sigma^{\text {ord }}}^{(m) \text { ord }}\right)\right|_{=i} \rightarrow\left|\mathcal{S}\left(U^{p}(N), \Sigma^{\text {ord }}\right)^{\text {ord }}\right|_{=i} .
$$

In the case $n=i$ this is actually a homeomorphism

$$
\left|\mathcal{S}\left(\partial \bar{A}_{n, U^{p}(N), \Sigma^{\text {ord }}}^{(m) \text { ord }}\right)\right|_{=n} \cong\left|\mathcal{S}\left(U^{p}(N), \Sigma^{\text {ord }}\right)^{\text {ord }}\right|_{=n} \cong \mathfrak{T}_{U^{p}(N),=n^{*}}^{(m) \text {,ord }}
$$

This is compatible with the identifications

$$
\begin{aligned}
& \left|\mathcal{S}\left(\partial \bar{A}_{n, U^{p}(N), \Sigma \text { ord }}^{(m), \text { ord }}\right)\right|=n \\
& \cong \coprod_{h \in P_{n,(n)}^{(m),+}\left(\mathbb{A}^{\infty}\right)^{\text {ord }, \times} \backslash G_{n}^{(m)}\left(\mathbb{A}^{\infty}\right)^{\text {ord }, \times} / U^{p}(N)} L_{n,(n), \operatorname{lin}}^{(m)}\left(\mathbb{Z}_{(p)}\right) \backslash \\
& \left(\left|\mathcal{S}\left(\partial \bar{T}_{n,(n), h U^{p}(N) h^{-1} \cap P_{n,(n)}^{(m),+}\left(\mathbb{A}^{\infty}\right)^{\text {ord }}, \Sigma^{\text {ord }}(h)_{0}}\right)\right|-\right. \\
& \left.\left|\mathcal{S}\left(\partial \bar{T}_{n,(n), h U^{p}(N) h^{-1} \cap P_{n,(n)}^{(m),+}\left(\mathbb{A}^{\infty}\right){ }^{\text {ord }}, \Sigma^{\text {ord }}(h)_{0}-\Sigma^{\text {ord }}(h)_{0}}\right)\right|\right) \\
& \cong \coprod_{h \in P_{n,(i)}^{(m),+}\left(\mathbb{A}^{\infty}\right)^{\text {ord }, \times} \backslash G_{n}^{(m)}\left(\mathbb{A}^{\infty}\right)^{\text {ord } \times} \times U^{p}(N)} L_{n,(n)}^{(m)}(\mathbb{Q}) \backslash L_{n,(n)}^{(m)}(\mathbb{A}) / \\
& \left(h U^{p}(N) h^{-1} \cap P_{n,(n)}^{(m),+}\left(\mathbb{A}^{\infty}\right)\right) L_{n,(n), \operatorname{herm}}(\mathbb{R})^{+}\left(L_{n,(n), \operatorname{lin}}^{(m)}(\mathbb{R}) \cap U_{n, \infty}^{0}\right) A_{n,(n)}(\mathbb{R})^{0} \\
& \cong\left|\mathcal{S}\left(U^{p}(N), \Sigma^{\text {ord }}\right)^{\text {ord }}\right|_{=n} \text {. }
\end{aligned}
$$

\subsection{Vector bundles}

We will write $\mathcal{I}_{\partial X_{n, U} \min }\left(\operatorname{resp} . \mathcal{I}_{\partial X_{n, U, \Delta}}\right.$, resp. $\left.\mathcal{I}_{\partial A_{U, \Sigma}^{(m)}}\right)$ for the ideal sheaf in $\mathcal{O}_{X_{n, U}^{\min }}\left(\operatorname{resp} . \mathcal{O}_{X_{n, U, \Delta}}\right.$, resp. $\left.\mathcal{O}_{A_{U, \Sigma}^{(m)}}\right)$ defining the boundary $\partial X_{n, U}^{\min }\left(\right.$ resp. $\partial X_{n, U, \Delta}$, resp. $\left.\partial A_{U, \Sigma}^{(m)}\right)$. More generally we will write $\mathcal{I}_{\partial X_{n, U}^{\min } \times \operatorname{Spec} R_{0}}\left(\operatorname{resp} . \mathcal{I}_{\partial X_{n, U, \Delta} \times \operatorname{Spec} R_{0}}, \operatorname{resp} . \mathcal{I}_{\partial A_{U, \Sigma}^{(m)} \times \operatorname{Spec} R_{0}}\right)$ for the correspondingly defined sheaves on $X_{n, U}^{\min } \times \operatorname{Spec} R_{0}\left(\right.$ resp. $\left.X_{n, U, \Delta} \times \operatorname{Spec} R_{0}, \operatorname{resp} . A_{U, \Sigma}^{(m)} \times \operatorname{Spec} R_{0}\right)$.

Lemma 5.1 Suppose that $R_{0}$ is an irreducible, noetherian $\mathbb{Q}$-algebra. 
(1) If $i>0$ then

$$
R^{i} \pi_{(U, \Sigma),\left(U^{\prime}, \Sigma^{\prime}\right), *} \mathcal{O}_{A_{n, U, \Sigma}^{(m)} \times \operatorname{Spec} R_{0}}=(0)
$$

and

$$
R^{i} \pi_{(U, \Sigma),\left(U^{\prime}, \Sigma^{\prime}\right), *} \mathcal{I}_{\partial A_{n, U, \Sigma}^{(m)} \times \operatorname{Spec} R_{0}}=(0) .
$$

(2) If $(U, \Sigma) \geq\left(U^{\prime}, \Sigma^{\prime}\right)$ and $U$ is a normal subgroup of $U^{\prime}$, then the natural maps

$$
\mathcal{O}_{A_{U^{\prime}, \Sigma^{\prime}}^{(m)} \times \operatorname{Spec} R_{0}} \longrightarrow\left(\pi(U, \Sigma),\left(U^{\prime}, \Sigma^{\prime}\right), * \mathcal{O}_{A_{U, \Sigma}^{(m)} \times \operatorname{Spec} R_{0}}\right)^{U^{\prime}}
$$

and

$$
\mathcal{I}_{\partial A_{U^{\prime}, \Sigma^{\prime}}^{(m)} \times \operatorname{Spec} R_{0}} \longrightarrow\left(\pi(U, \Sigma),\left(U^{\prime}, \Sigma^{\prime}\right), * \mathcal{I}_{\partial A_{U, \Sigma}^{(m)} \times \operatorname{Spec} R_{0}}\right)^{U^{\prime}}
$$

are isomorphisms.

(3) If $U^{\prime}$ is the image in $G_{n}\left(\mathbb{A}^{\infty}\right)$ of $U \subset G_{n}^{(m)}\left(\mathbb{A}^{\infty}\right)$ and if $\Sigma$ and $\Delta$ are compatible, then

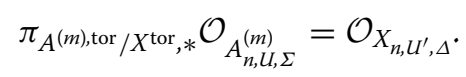

Proof If $\Sigma$ is $U^{\prime}$-invariant the first two parts follow from Lemma 4.6. In the general case we choose $\left(U, \Sigma^{\prime \prime}\right) \geq(U, \Sigma)$ with $\Sigma^{\prime \prime}$ being $U^{\prime}$-invariant, and apply the cases of the lemma already proved to the pairs $\left(\left(U, \Sigma^{\prime \prime}\right),\left(U^{\prime}, \Sigma^{\prime}\right)\right)$ and $\left(\left(U, \Sigma^{\prime \prime}\right),(U, \Sigma)\right)$.

The third part follows from Lemma 4.8.

Similarly we will write $\mathcal{I}_{\partial \mathcal{X}_{n, U^{p}\left(N_{1}, N_{2}\right)}^{\text {ordmin }}}$ (resp. $\mathcal{I}_{\partial \mathcal{X}_{n, U^{p}\left(N_{1}, N_{2}\right), 4}^{\text {ord }}}$, resp. $\mathcal{I}_{\partial \mathcal{A}_{n, U^{\prime}\left(N_{1}, N_{2}\right), \Sigma}^{(m) \text { ord }}}$ ) for the ideal sheaf in $\mathcal{O}_{\mathcal{X}_{n, U^{p}\left(N_{1}, N_{2}\right)}^{\text {ordmin }}}\left(\right.$ resp. $\mathcal{O}_{\mathcal{X}_{n, U^{p}\left(N_{1}, N_{2}\right), \Delta}^{\text {ord }}}$, resp. $\left.\mathcal{O}_{\mathcal{A}_{n, U^{p}\left(N_{1}, N_{2}\right), \Sigma}^{(m), \text { ord }}}\right)$ defining the boundary $\partial \mathcal{X}_{n, U^{p}\left(N_{1}, N_{2}\right)}^{\text {ord,min }}\left(\right.$ resp. $\partial \mathcal{X}_{n, U^{p}\left(N_{1}, N_{2}\right), \Delta}^{\text {ord }}$, resp. $\partial \mathcal{A}_{n, U^{p}\left(N_{1}, N_{2}\right), \Sigma}^{(m), \text { ord }}$. More generally we will also

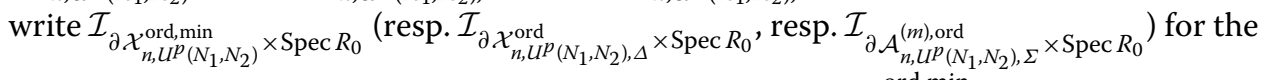
sheaf defined in the corresponding manner on the scheme $\mathcal{X}_{n, U^{p}\left(N_{1}, N_{2}\right)}^{\text {ordmin }} \times \operatorname{Spec} R_{0}$ (resp. $\mathcal{X}_{n, U^{p}\left(N_{1}, N_{2}\right), \Delta}^{\text {ord }} \times \operatorname{Spec} R_{0}$, resp. $\left.\mathcal{A}_{n, U^{p}\left(N_{1}, N_{2}\right), \Sigma}^{(m) \text {,rd }} \times \operatorname{Spec} R_{0}\right)$. The next lemma follows from Lemmas 4.6 and 4.12 .

Lemma 5.2 Suppose that $R_{0}$ is an irreducible, noetherian $\mathbb{Z}_{(p)}$-algebra.

(1) If $i>0$ then

$$
R^{i} \pi_{\left(U^{p}\left(N_{1}, N_{2}\right), \Sigma\right),\left(\left(U^{p}\right)^{\prime}\left(N_{1}^{\prime}, N_{2}^{\prime}\right), \Sigma^{\prime}\right), *} \mathcal{O}_{\mathcal{A}_{n, U P\left(N_{1}, N_{2}\right), \Sigma}^{(m), \text { ord }} \times \operatorname{Spec} R_{0}}=(0)
$$

and

$$
R^{i} \pi_{\left(U^{p}\left(N_{1}, N_{2}\right), \Sigma\right),\left(\left(U^{p}\right)^{\prime}\left(N_{1}^{\prime}, N_{2}^{\prime}\right), \Sigma^{\prime}\right), * \mathcal{I}_{\partial \mathcal{A}_{n, U p}^{(m), 0 \text { ord }}\left(N_{1}, N_{2}\right), \Sigma} \times \operatorname{Spec} R_{0}}=(0) .
$$


(2) If $\left(U^{p}\left(N_{1}, N_{2}\right), \Sigma\right) \geq\left(\left(U^{p}\right)^{\prime}\left(N_{1}^{\prime}, N_{2}\right), \Sigma^{\prime}\right)$ and $U^{p}$ is a normal subgroup of $\left(U^{p}\right)^{\prime}$, then the natural maps

$$
\mathcal{O}_{\mathcal{A}_{\left.n,(U)^{\prime}\right)^{\prime}\left(N_{1}^{\prime}, N_{2}^{\prime}\right), \Sigma^{\prime}}^{(m) \text { oSpec } R_{0}}} \rightarrow\left(\pi_{\left(U^{p}\left(N_{1}, N_{2}\right), \Sigma\right),\left(\left(U^{p}\right)^{\prime}\left(N_{1}^{\prime}, N_{2}^{\prime}\right), \Sigma^{\prime}\right), *} \mathcal{O}_{\mathcal{A}_{n, U^{p}\left(N_{1}, N_{2}\right), \Sigma}^{(m), \text { ord }} \times \operatorname{Spec} R_{0}}\right)^{\left(U^{p}\right)^{\prime}\left(N_{1}^{\prime}\right)}
$$

and

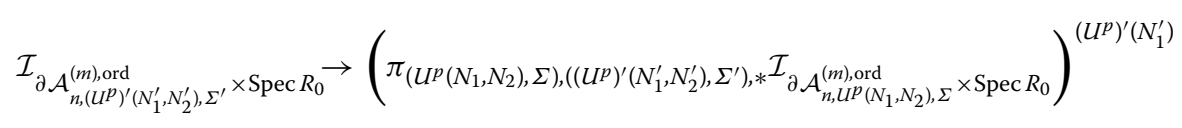

are isomorphisms.

(3) If $\left(U^{p}\right)^{\prime}$ is the image in $G_{n}\left(\mathbb{A}^{\infty, p}\right)$ of $U^{p} \subset G_{n}^{(m)}\left(\mathbb{A}^{\infty, p}\right)$ and if $\Sigma$ and $\Delta$ are compatible, then

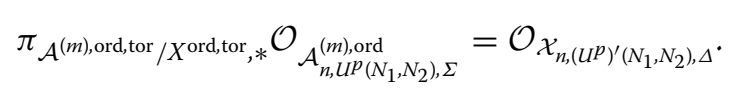

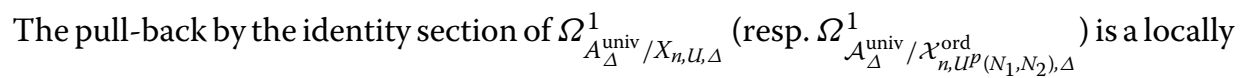
free sheaf, which is canonically independent of the choice of $A^{\text {univ }}$ (resp. $\mathcal{A}^{\text {univ }}$ ). We will denote it $\Omega_{n, U, \Delta}$ (resp. $\Omega_{n, U^{p}\left(N_{1}, N_{2}\right), \Delta}^{\text {ord }}$. If $g \in G_{n}\left(\mathbb{A}^{\infty}\right)$ (resp. $g \in G_{n}\left(\mathbb{A}^{\infty}\right)^{\text {ord, } \times}$ ) and $(U, \Delta) g \geq\left(U^{\prime}, \Delta^{\prime}\right)\left(\operatorname{resp} .\left(U^{p}\left(N_{1}, N_{2}\right), \Delta\right) g \geq\left(\left(U^{p}\right)^{\prime}\left(N_{1}^{\prime}, N_{2}^{\prime}\right), \Delta^{\prime}\right)\right)$ then there is a natural isomorphism

$$
g^{*} \Omega_{n, U^{\prime}, \Delta^{\prime}} \longrightarrow \Omega_{n, U, \Delta}
$$

(resp.

$$
\left.g^{*} \Omega_{n,\left(U^{p}\right)^{\prime}\left(N_{1}, N_{2}\right), \Delta^{\prime}}^{\mathrm{ord}} \longrightarrow \Omega_{n, U^{p}\left(N_{1}, N_{2}\right), \Delta}^{\mathrm{ord}}\right) .
$$

This gives the inverse system $\left\{\Omega_{n, U, \Delta}\right\}$ (resp. $\left.\left\{\Omega_{n, U^{p}\left(N_{1}, N_{2}\right), \Delta}^{\text {ord }}\right\}\right)$ an action of $G_{n}\left(\mathbb{A}^{\infty}\right)$ (resp. $\left.G_{n}\left(\mathbb{A}^{\infty}\right)^{\text {ord } \times}\right)$. There is also a natural map

$$
\varsigma_{p}: \varsigma_{p}^{*} \Omega_{n, U^{p}\left(N_{1}, N_{2}-1\right), \Delta}^{\mathrm{ord}} \longrightarrow \Omega_{n, U^{p}\left(N_{1}, N_{2}\right), \Delta}^{\mathrm{ord}}
$$

There is a canonical identification

$$
\left.\Omega_{n, U, \Delta}\right|_{n, U} \cong \Omega_{n, U}
$$

(resp.

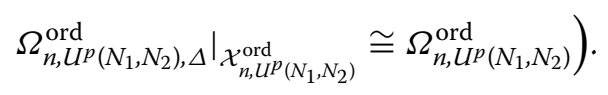

We will write

$$
\omega_{U, \Delta}=\wedge^{n[F: \mathbb{Q}]} \Omega_{n, U, \Delta}
$$

(resp.

$$
\left.\omega_{U^{p}\left(N_{1}, N_{2}\right), \Delta}^{\mathrm{ord}}=\wedge^{n[F: \mathbb{Q}]} \Omega_{n, U^{p}\left(N_{1}, N_{2}\right), \Delta}^{\mathrm{ord}}\right) .
$$


The pull-back of $\Omega_{n, U, \Delta}$ to $T_{n,(i), h U h^{-1} \cap P_{n,(i)}^{+}\left(\mathbb{A}^{\infty}\right), \Delta(h)_{0}}$ is canonically and equivariantly identified with the sheaf $\widetilde{\Omega}_{\left.n,(i), h U h^{-1} \cap P_{n,(i)}^{+}\left(\mathbb{A}^{\infty}\right), \Delta(h)\right)_{0}}^{\natural}$. Similarly the pull-back of $\Omega_{n, U^{p}\left(N_{1}, N_{2}\right), \Delta}^{\text {ord }}$ to $\mathcal{T}_{n,(i),\left(h U^{p} h^{-1} \cap P_{n,(i)}^{+}\right.}^{\left.\left.\text {ord }, \wedge \mathbb{A}^{\infty, p}\right)\right)\left(N_{1}, N_{2}\right), \Delta^{\text {ord }}(h)_{0}}$ is canonically and equivariantly identified with the sheaf $\widetilde{\Omega}_{n,(i),\left(h U^{p} h^{-1} \cap P_{n,(i)}^{+}\left(\mathbb{A}^{\infty, p}\right)\right)\left(N_{1}, N_{2}\right), \Delta^{\text {ord }}(h)_{0}}^{\text {ord }}$. (See lemmas 1.3.2.41 and 5.2.4.38 of [44].)

We will write

$$
\Xi_{n, U, \Delta}=\mathcal{O}_{X_{n, U, \Delta}}(\|v\|)
$$

(resp.

$$
\left.\Xi_{n, U^{p}\left(N_{1}, N_{2}\right), \Delta}^{\text {ord }}=\mathcal{O}_{\mathcal{X}_{n, U^{p}\left(N_{1}, N_{2}\right), \Delta}^{\text {ord }}}(\|v\|)\right)
$$

for the structure sheaf of $X_{n, U, \Delta}\left(\operatorname{resp} . \mathcal{X}_{n, U^{p}\left(N_{1}, N_{2}\right), \Delta}^{\text {ord }}\right)$ with the $G_{n}\left(\mathbb{A}^{\infty}\right)\left(\right.$ resp. $\left.G_{n}\left(\mathbb{A}^{\infty}\right)^{\text {ord }}\right)$ action twisted by $\|v\|$. If $g \in G_{n}\left(\mathbb{A}^{\infty}\right)$ (resp. $g \in G_{n}\left(\mathbb{A}^{\infty}\right)^{\text {ord, } \times}$ ) then the maps

$$
g^{*} \Xi_{n, U, \Delta} \longrightarrow \Xi_{n, U^{\prime}, \Delta^{\prime}}
$$

(resp.

$$
\left.g^{*} \Xi_{n, U^{p}\left(N_{1}, N_{2}\right), \Delta}^{\text {ord }} \longrightarrow \Xi_{n,\left(U^{p}\right)^{\prime}\left(N_{1}^{\prime}, N_{2}^{\prime}\right), \Delta^{\prime}}^{\text {ord }}\right)
$$

are isomorphisms.

The pull-back of $\Xi_{n, U, \Delta}$ to the formal scheme $T_{n,(i), h U h^{-1} \cap P_{n,(i)}^{+}\left(\mathbb{A}^{\infty}\right), \Delta(h)_{0}}$ equals the pull-back of the sheaf $\Xi_{n,(i), h U h^{-1} \cap P_{n,(i)}^{+}\left(\mathbb{A}^{\infty}\right)}^{\natural}$ from $X_{n,(i), h U h^{-1} \cap P_{n,(i)}^{+}\left(\mathbb{A}^{\infty}\right)}^{\natural}$. Similarly the pull-back of $\Xi_{n, U^{p}\left(N_{1}, N_{2}\right), \Delta}^{\text {ord }}$ to $\mathcal{T}_{n,(i),\left(h U^{p} h^{-1} \cap P_{n,(i)}^{+}\left(\mathbb{A}^{\infty, p)}\right)\left(N_{1}, N_{2}\right), \Delta^{\text {ord }}(h)_{0}\right.}^{\text {ord }, \wedge}$ is naturally isomor-

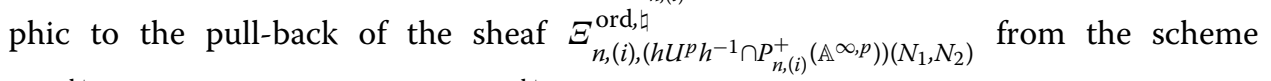
$\mathcal{X}_{n,(i),\left(h U^{p} h^{-1} \cap P_{n,(i)}^{+}\left(\mathbb{A}^{\infty, p}\right)\right)\left(N_{1}, N_{2}\right)}^{\text {ord }, \natural}$ to $\mathcal{T}_{\left.n,(i),\left(h U^{p} h^{-1} \cap P_{n,(i)}^{+} \text {ord },\left(\mathbb{A}^{\infty, p}\right)\right)\left(N_{1}, N_{2}\right), \Delta^{\text {ord }}(h)\right)_{0}}$.

Let $\mathcal{E}_{U, \Delta}^{\text {can }}$ (resp. $\mathcal{E}_{U^{p}\left(N_{1}, N_{2}\right), \Delta}^{\text {ord,can }}$ ) denote the principal $L_{n,(n)}$-bundle on $X_{n, U, \Delta}$ (resp. on $\mathcal{X}_{n, U^{p}\left(N_{1}, N_{2}\right), \Delta}^{\text {ord }}$ ) in the Zariski topology defined by setting, for $W \subset X_{n, U, \Delta}$ (resp. $\left.\mathcal{X}_{n, U^{p}\left(N_{1}, N_{2}\right), \Delta}^{\text {ord }}\right)$ a Zariski open, $\mathcal{E}_{U, \Delta}^{\text {can }}(W)\left(\right.$ resp. $\left.\mathcal{E}_{U^{p}\left(N_{1}, N_{2}\right), \Delta}^{\text {ord,can }}(W)\right)$ to be the set of pairs $\left(\xi_{0}, \xi_{1}\right)$, where

$$
\xi_{0}:\left.\Xi_{n, U, \Delta}\right|_{W} \stackrel{\sim}{\longrightarrow} \mathcal{O}_{W}
$$

(resp.

$$
\left.\xi_{0}:\left.\Xi_{h, U^{p}\left(N_{1}, N_{2}\right), \Delta}^{\text {ord }}\right|_{W} \stackrel{\sim}{\longrightarrow} \mathcal{O}_{W}\right)
$$

and

$$
\xi_{1}: \Omega_{n, U, \Delta} \stackrel{\sim}{\longrightarrow} \operatorname{Hom}_{\mathbb{Q}}\left(V_{n} / V_{n,(n)}, \mathcal{O}_{W}\right)
$$

(resp.

$$
\left.\xi_{1}: \Omega_{n, U^{p}\left(N_{1}, N_{2}\right), \Delta}^{\mathrm{ord}} \stackrel{\sim}{\longrightarrow} \operatorname{Hom}_{\mathbb{Z}}\left(\Lambda_{n} / \Lambda_{n,(n)}, \mathcal{O}_{W}\right)\right) .
$$


We define the $L_{n,(n)}$-action on $\mathcal{E}_{U, \Delta}^{\mathrm{can}}\left(\right.$ resp. $\left.\mathcal{E}_{U^{p}\left(N_{1}, N_{2}\right), \Delta}^{\text {ord,can }}\right)$ by

$$
h\left(\xi_{0}, \xi_{1}\right)=\left(v(h)^{-1} \xi_{0},\left(\circ h^{-1}\right) \circ \xi_{1}\right)
$$

The inverse system $\left\{\mathcal{E}_{U, \Delta}^{\text {can }}\right\}$ (resp. $\left\{\mathcal{E}_{U^{p}\left(N_{1}, N_{2}\right), \Delta}^{\text {ord,can }}\right\}$ ) has an action of $G_{n}\left(\mathbb{A}^{\infty}\right.$ ) (resp. $\left.G_{n}\left(\mathbb{A}^{\infty}\right)^{\text {ord }, \times}\right)$.

Suppose that $R_{0}$ is a $\mathbb{Q}$-algebra (resp. $\mathbb{Z}_{(p)}$-algebra) and that $\rho$ is a representation of $L_{n,(n)}$ on a finite, locally free $R_{0}$-module $W_{\rho}$. We define a locally free sheaf $\mathcal{E}_{U, \Delta, \rho}^{\text {can }}$ (resp. $\mathcal{E}_{U^{p}\left(N_{1}, N_{2}\right), \Delta, \rho}^{\text {ord,can }}$ ) over $X_{n, U, \Delta} \times \operatorname{Spec} R_{0}$ (resp. $\left.\mathcal{X}_{n, U^{p}\left(N_{1}, N_{2}\right), \Delta}^{\text {ord }} \times \operatorname{Spec} R_{0}\right)$ by setting $\mathcal{E}_{U, \Delta, \rho}^{\text {can }}(W)$ (resp. $\left.\mathcal{E}_{U^{p}\left(N_{1}, N_{2}\right), \Delta, \rho}^{\text {ord,can }}(W)\right)$ to be the set of $L_{n,(n)}\left(\mathcal{O}_{W}\right)$-equivariant maps of Zariski sheaves of sets

$$
\left.\mathcal{E}_{U, \Delta}^{\mathrm{can}}\right|_{W} \rightarrow W_{\rho} \otimes_{R_{0}} \mathcal{O}_{W}
$$

(resp.

$$
\left.\mathcal{E}_{U^{p}\left(N_{1}, N_{2}\right), \Delta}^{\text {ord,can }} \mid W \rightarrow W_{\rho} \otimes_{R_{0}} \mathcal{O}_{W}\right)
$$

Then $\left\{\mathcal{E}_{U, \Delta, \rho}^{\text {can }}\right\}$ (resp. $\left\{\mathcal{E}_{U^{p}\left(N_{1}, N_{2}\right), \Delta, \rho}^{\text {ord,can }}\right\}$ ) is a system of locally free sheaves with $G_{n}\left(\mathbb{A}^{\infty}\right)$ action (resp. $G_{n}\left(\mathbb{A}^{\infty}\right)^{\text {ord, } \times}$-action) over the system of schemes $\left\{X_{n, U, \Delta} \times \operatorname{Spec} R_{0}\right\}$ (resp. $\left.\left\{\mathcal{X}_{n, U^{p}\left(N_{1}, N_{2}\right), \Delta}^{\text {ord }} \times \operatorname{Spec} R_{0}\right\}\right)$.

Note that

$$
\mathcal{E}_{U, \Delta, \mathrm{Std}^{\mathrm{can}}}^{\mathrm{can}} \cong \Omega_{n, U, \Delta}
$$

and

$$
\mathcal{E}_{U, \Delta, v^{-1}}^{\mathrm{can}} \cong \Xi_{n, U, \Delta}
$$

and

$$
\mathcal{E}_{U, \Delta, \wedge}^{\mathrm{can}}{ }^{n[F: \mathbb{Q}]} \operatorname{Std}^{\vee} \cong \omega_{U, \Delta}
$$

Similarly

$$
\mathcal{E}_{U^{p}\left(N_{1}, N_{2}\right), \Delta, \operatorname{Std}}^{\text {ord,can }} \cong \Omega_{n, U^{p}\left(N_{1}, N_{2}\right), \Delta}^{\text {ord }}
$$

and

$$
\mathcal{E}_{U^{p}\left(N_{1}, N_{2}\right), \Delta, v^{-1}}^{\text {ord,can }} \cong \Xi_{n, U^{p}\left(N_{1}, N_{2}\right), \Delta}^{\text {ord }}
$$

and

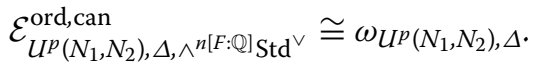

Also note that the pull-back of $\mathcal{E}_{U, \Delta, \rho}^{\mathrm{can}}$ (resp. $\mathcal{E}_{U^{p}\left(N_{1}, N_{2}\right), \Delta, \rho}^{\text {ord,can }}$ ) to $X_{n, U} \times \operatorname{Spec} R_{0}$ (resp. $\left.\mathcal{X}_{n, U^{p}\left(N_{1}, N_{2}\right)}^{\text {ord }} \times \operatorname{Spec} R_{0}\right)$ is canonically identified with $\mathcal{E}_{U, \rho}$ (resp. $\left.\mathcal{E}_{U^{p}\left(N_{1}, N_{2}\right), \rho}^{\text {ord }}\right)$. These identifications are $G_{n}\left(\mathbb{A}^{\infty}\right)$ (resp. $\left.G_{n}\left(\mathbb{A}^{\infty}\right)^{\text {ord } \times} \times\right)$ equivariant. 
Moreover note that the pull-back of $\mathcal{E}_{U, \Delta, \rho}^{\mathrm{can}}$ to $T_{n,(i), h U h^{-1} \cap P_{n, i()}^{\dagger}\left(\mathbb{A}^{\infty}\right), \Delta(h)_{0}}$ is canonically and

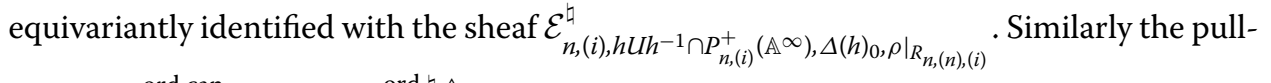
back of $\mathcal{E}_{U^{p}\left(N_{1}, N_{2}\right), \Delta, \rho}^{\text {ord,can }}$ to $\mathcal{T}_{n,(i),\left(h U^{p} h^{-1} \cap P_{n,(i)}^{+} \text {ord }, \mathbb{A}^{\infty, p}, \wedge\right)\left(N_{1}, N_{2}\right), \Delta^{\text {ord }}(h) 0}$ is canonically and equivariantly identified with

$$
\mathcal{E}_{n,(i),\left(h U^{p} h^{-1} \cap P_{n,(i)}^{+}\left(\mathbb{A}^{\infty, p}\right)\right)\left(N_{1}, N_{2}\right), \Delta^{\operatorname{ord}}(h)_{0},\left.\rho\right|_{R_{n,(n),(i)}}} .
$$

Set

$$
\mathcal{E}_{U, \Delta, \rho}^{\mathrm{sub}}=\mathcal{I}_{\partial X_{n, U, \Delta}} \mathcal{E}_{U, \Delta, \rho} \cong \mathcal{I}_{\partial X_{n, U, \Delta}} \otimes \mathcal{E}_{U, \Delta, \rho}
$$

and

$$
\mathcal{E}_{U^{p}\left(N_{1}, N_{2}\right), \Delta, \rho}^{\text {ord,sub }}=\mathcal{I}_{\partial \mathcal{X}_{n, U^{p}\left(N_{1}, N_{2}\right), \Delta}^{\text {ord }}} \mathcal{E}_{U^{p}\left(N_{1}, N_{2}\right), \Delta, \rho}^{\text {ord }} \cong \mathcal{I}_{\partial \mathcal{X}_{n, U^{p}\left(N_{1}, N_{2}\right), \Delta}^{\text {ord }}} \otimes \mathcal{E}_{U^{p}\left(N_{1}, N_{2}\right), \Delta, \rho}^{\text {ord }}
$$

Then $\left\{\mathcal{E}_{U, \Delta, \rho}^{\text {sub }}\right\}$ (resp. $\left.\left\{\mathcal{E}_{U^{p}\left(N_{1}, N_{2}\right), \Delta, \rho}^{\text {ord sub }}\right\}\right)$ is also a system of locally free sheaves with $G_{n}\left(\mathbb{A}^{\infty}\right)$ action (resp. $G_{n}\left(\mathbb{A}^{\infty}\right)^{\text {ord, } \times}$-action) over the systems of schemes $\left\{X_{n, U, \Delta} \times \operatorname{Spec} R_{0}\right\}$ (resp. $\left.\left\{\mathcal{X}_{n, U^{p}\left(N_{1}, N_{2}\right), \Delta}^{\text {ord }} \times \operatorname{Spec} R_{0}\right\}\right)$.

Lemma 5.3 (1) If $g \in G_{n}\left(\mathbb{A}^{\infty}\right)$ (resp. $\left.G_{n}\left(\mathbb{A}^{\infty}\right)^{\text {ord } \times} \times\right)$ and $g: X_{n, U, \Delta} \rightarrow X_{n, U^{\prime}, \Delta^{\prime}}$ (resp. $\left.g: \mathcal{X}_{n, U^{p}\left(N_{1}, N_{2}\right), \Delta}^{\text {ord }} \rightarrow \mathcal{X}_{n,\left(U^{p}\right)^{\prime}\left(N_{1}^{\prime}, N_{2}^{\prime}\right), \Delta^{\prime}}^{\text {ord }}\right)$ then

$$
g^{*} \mathcal{E}_{U^{\prime}, \Delta^{\prime}, \rho}^{\mathrm{can}} \stackrel{\sim}{\longrightarrow} \mathcal{E}_{U, \Delta, \rho}^{\mathrm{can}}
$$

(resp.

$$
\left.g^{*} \mathcal{E}_{\left(U^{p}\right)^{\prime}\left(N_{1}^{\prime}, N_{2}^{\prime}\right), \Delta^{\prime}, \rho}^{\mathrm{ord}, \mathrm{can}} \stackrel{\sim}{\longrightarrow} \mathcal{E}_{U^{p}\left(N_{1}, N_{2}\right), \Delta, \rho}^{\text {ord,can }}\right) .
$$

(2) If $i>0$ then

$$
R^{i} \pi_{(U, \Delta),\left(U^{\prime}, \Delta^{\prime}\right), *} \mathcal{E}_{U, \Delta, \rho}^{\mathrm{can}}=(0)
$$

and

$$
R^{i} \pi_{(U, \Delta),\left(U^{\prime}, \Delta^{\prime}\right), *} \mathcal{E}_{U, \Delta, \rho}^{\mathrm{sub}}=(0) .
$$

Similarly, for $i>0$ we have

$$
R^{i} \pi_{\left(U^{p}\left(N_{1}, N_{2}\right), \Delta\right),\left(\left(U^{p}\right)^{\prime}\left(N_{1}^{\prime}, N_{2}^{\prime}\right), \Delta^{\prime}\right), *} \mathcal{E}_{U^{p}\left(N_{1}, N_{2}\right), \Delta, \rho}^{\mathrm{ord} \text { can }}=(0)
$$

and

$$
R^{i} \pi_{\left(U^{p}\left(N_{1}, N_{2}\right), \Delta\right),\left(\left(U^{p}\right)^{\prime}\left(N_{1}^{\prime}, N_{2}^{\prime}\right), \Delta^{\prime}\right), *} \mathcal{E}_{U^{p}\left(N_{1}, N_{2}\right), \Delta, \rho}^{\mathrm{ord}, \mathrm{sub}}=(0) .
$$


(3)

$$
\left(\lim _{\rightarrow(U, \Delta)} \pi_{(U, \Delta),\left(U^{\prime}, \Delta^{\prime}\right), *} \mathcal{E}_{U, \Delta, \rho}^{\mathrm{can}}\right)^{U^{\prime}}=\mathcal{E}_{U^{\prime}, \Delta^{\prime}, \rho}
$$

and

$$
\left(\lim _{\rightarrow(U, \Delta)} \pi(U, \Delta),\left(U^{\prime}, \Delta^{\prime}\right), \mathcal{E}_{U, \Delta, \rho}^{\mathrm{sub}}\right)^{U^{\prime}}=\mathcal{E}_{U^{\prime}, \Delta^{\prime}, \rho}^{\mathrm{sub}}
$$

and

$$
\begin{aligned}
& \mathcal{E}_{\left(U^{p}\right)^{\prime}\left(N_{1}^{\prime}, N_{2}\right), \Delta^{\prime}, \rho}^{\mathrm{ord}, \mathrm{can}} \\
& =\left(\lim _{\rightarrow\left(U^{p}\left(N_{1}, N_{2}\right), \Delta\right)} \pi_{\left(U^{p}\left(N_{1}, N_{2}\right), \Delta\right),\left(\left(U^{p}\right)^{\prime}\left(N_{1}^{\prime}, N_{2}\right), \Delta^{\prime}\right), *} \mathcal{E}_{U^{p}\left(N_{1}, N_{2}\right), \Delta, \rho}^{\mathrm{ord}, \mathrm{can}}\right)^{\left(U^{p}\right)^{\prime}\left(N_{1}^{\prime}\right)}
\end{aligned}
$$

and

$$
\begin{aligned}
& \mathcal{E}_{\left(U^{p}\right)^{\prime}\left(N_{1}^{\prime}, N_{2}\right), \Delta^{\prime}, \rho}^{\text {ordsub }} \\
& \quad=\left(\lim _{\rightarrow\left(U^{p}\left(N_{1}, N_{2}\right), \Delta\right)} \pi_{\left(U^{p}\left(N_{1}, N_{2}\right), \Delta\right),\left(\left(U^{p}\right)^{\prime}\left(N_{1}^{\prime}, N_{2}\right), \Delta^{\prime}\right), *} \mathcal{E}_{U^{p}\left(N_{1}, N_{2}\right), \Delta, \rho}^{\text {ord,sub }}\right)^{\left(U^{p}\right)^{\prime}\left(N_{1}^{\prime}\right)} .
\end{aligned}
$$

Proof The first part follows easily from the corresponding facts for $\Omega_{n, U, \Delta}$ and $\Xi_{n, U, \Delta}$ (resp. $\Omega_{n, U^{p}\left(N_{1}, N_{2}\right), \Delta}^{\text {ord }}$ and $\left.\Xi_{n, U^{p}\left(N_{1}, N_{2}\right), \Delta}^{\text {ord }}\right)$. The second and third parts follow from the first part and parts 1 and 2 of Lemma 5.1 (resp. Lemma 5.2).

We next deduce our first main observation.

Theorem 5.4 If $i>0$ and $U$ is neat then $R^{i} \pi_{X^{\mathrm{tor}} / X^{\min }, *} \mathcal{E}_{U, \Delta, \rho}^{\mathrm{sub}}=(0)$.

Similarly if $i>0$ and $U^{p}$ is neat then $R^{i} \pi_{\mathcal{X}}^{\text {ord,tor } / \mathcal{X}^{\text {ord,min }, *}} \mathcal{E}_{U^{p}\left(N_{1}, N_{2}\right), \Delta, \rho}^{\text {ord sub }}=(0)$.

Proof The argument is the same in both cases, so we explain the argument only in the first case. Write $X_{n, U, \Delta, i, h}^{\wedge}$ (resp. $X_{n, U, i, h}^{\min , \wedge}$ ) for the open and closed subset of $X_{n, U, \Delta, i}^{\wedge}$ (resp. $\left.X_{n, U, i}^{\min , \wedge}\right)$ corresponding to $T_{n,(i), h U h^{-1} \cap P_{n,(i)}^{+}\left(\mathbb{A}^{\infty}\right), \Delta(h)}\left(\right.$ resp. $\left.X_{n,(i), h U h^{-1} \cap P_{n,(i)}^{+}\left(\mathbb{A}^{\infty}\right)}^{\natural}\right)$. (Recall that $X_{n, U, \Delta, i}^{\wedge}$ is the completion of a smooth toroidal compactification of the Shimura variety $X_{n, U}$ along the locally closed subspace of the boundary corresponding to the parabolic subgroup $P_{n,(i)}^{+} \subset G_{n}$. The formal scheme $X_{n, U, i}^{\min , \wedge}$ is the completion of the minimal (BailyBorel) compactification of the same Shimura variety along the locally closed subspace of the boundary corresponding to the same parabolic. Each of these formal schemes is a disjoint union of subformal schemes indexed by certain elements $h \in G_{n}\left(\mathbb{A}^{\infty}\right)$.)

We have maps of locally ringed spaces

$$
\begin{array}{ccc}
T_{n,(i), h U h^{-1} \cap P_{n,(i)}^{+}\left(\mathbb{A}^{\infty}\right), \Delta(h)}^{\natural, \wedge} & \stackrel{\sim}{\longrightarrow} X_{n, U, \Delta, i, h}^{\wedge} \\
\downarrow & & \downarrow \\
X_{n,(i), h U h^{-1} \cap P_{n,(i)}^{+}\left(\mathbb{A}^{\infty}\right)}^{\natural} & \hookrightarrow & X_{n, U, i, h}^{\min , \wedge}
\end{array}
$$

(Recall that $T_{n,(i), h U h^{-1} \cap P_{n,(i)}^{+}\left(\mathbb{A}^{\infty}\right), \Delta(h)}$ is a formal local model for the boundary of the toroidal compactification. It is the quotient by a discrete group of the formal completion of a toroidal embedding over a principal homogeneous space for an abelian scheme over a 
disjoint union of smaller Shimura varieties. The scheme $X_{n,(i), h U h^{-1} \cap P_{n,(i)}^{+}(\mathbb{A} \infty)}$ is a disjoint union of smaller Shimura varieties, and also a locally closed subscheme of the boundary of the minimal compactification of $X_{n, U}$.)

This diagram is commutative as a diagram of topological spaces (but not of locally ringed spaces) and the lower horizontal map is an isomorphism on the underlying topological spaces. It suffices to show that the higher direct images from the topological space $T_{n,(i), h U h^{-1} \cap P_{n,(i)}^{+}\left(\mathbb{A}^{\infty}\right), \Delta(h)}^{\natural}$ to the topological space $X_{n,(i), h U h^{-1} \cap P_{n,(i)}^{+}\left(\mathbb{A}^{\infty}\right)}^{\natural}$ of the pull-back of $\mathcal{E}_{U, \Delta, \rho}^{\text {sub }}$ vanishes. The theorem follows from Corollary 4.14.

We set

$$
\mathcal{E}_{U, \rho}^{\mathrm{sub}}=\pi_{X^{\mathrm{tor}} / X^{\mathrm{min}}, *} \mathcal{E}_{U, \Delta, \rho}^{\mathrm{sub}}
$$

(resp.

$$
\left.\mathcal{E}_{U^{p}\left(N_{1}, N_{2}\right), \rho}^{\text {ord,sub }}=\pi_{\mathcal{X}^{\text {ord,tor }} / \mathcal{X}^{\text {ord,min }}, *} \mathcal{E}_{U^{p}\left(N_{1}, N_{2}\right), \Delta, \rho}^{\text {ord,sub }}\right)
$$

a coherent sheaf on $X_{n, U}^{\min } \times \operatorname{Spec} R_{0}$ (resp. $\left.\mathcal{X}_{n, U^{p}\left(N_{1}, N_{2}\right)}^{\text {ord,min }} \times \operatorname{Spec} R_{0}\right)$. (Note that we do not expect these sheaves to be locally free in general.) These definitions are independent of $\Delta$. Note that

$$
\mathcal{E}_{U, \rho}^{\mathrm{sub}} \otimes \omega_{U}^{\otimes N} \cong \mathcal{E}_{U, \rho \otimes\left(\wedge^{n[F: \mathbb{Q}]} \operatorname{Std}^{\vee}\right)^{\otimes N}}^{\text {sub }}
$$

and

$$
\mathcal{E}_{U^{p}\left(N_{1}, N_{2}\right), \rho}^{\text {ord,sub }} \otimes\left(\omega_{U^{p}\left(N_{1}, N_{2}\right)}\right)^{\otimes N} \cong \mathcal{E}_{U^{p}\left(N_{1}, N_{2}\right), \rho \otimes\left(\wedge^{n[F: \mathbb{Q}]} \operatorname{Std}^{\vee}\right)^{\otimes N}}^{\text {ord,sub }}
$$

We will let $\mathcal{E}_{U^{p}(N), \Delta^{\text {ord }, \rho}}^{\text {ord,can }}$ (resp. $\mathcal{E}_{U^{p}(N), \Delta^{\text {ord }, \rho}}^{\text {ord,sub }}$, resp. $\mathcal{E}_{U^{p}(N), \rho}^{\text {ord,sub }}$ ) denote the pull-back of $\mathcal{E}_{U^{p}\left(N, N^{\prime}\right), \Delta, \rho}^{\text {ord,can }}\left(\right.$ resp. $\mathcal{E}_{U^{p}\left(N, N^{\prime}\right), \Delta, \rho}^{\text {ord,sub }}$, resp. $\left.\mathcal{E}_{U^{p}\left(N, N^{\prime}\right), \rho}^{\text {ord,sub }}\right)$ to $\mathfrak{X}_{U^{p}(N), \Delta^{\text {ord }}}^{\text {ord }}$ (resp. $\mathfrak{X}_{U^{p}(N), \Delta^{\text {ord }}}^{\text {ord }}$, resp. $\left.\mathfrak{X}_{U^{p}(N)}^{\text {ord,min }}\right)$. It is independent of the choice of $N^{\prime}$ and $\Delta$.

If $\rho$ is a representation of $L_{n,(n)}$ on a finite $\mathbb{Q}$-vector space, we will set

$$
\begin{aligned}
H^{i}\left(X_{n}^{\min }, \mathcal{E}_{\rho}^{\mathrm{sub}}\right) & =\lim \underset{U^{\prime}}{\longrightarrow} H^{i}\left(X_{n, U^{\prime}}^{\min }, \mathcal{E}_{U^{\prime}, \rho}^{\mathrm{sub}}\right) \\
& =\lim \underset{U^{\prime}, \Delta}{\longrightarrow} H^{i}\left(X_{n, U^{\prime}, \Delta}, \mathcal{E}_{U^{\prime}, \Delta, \rho}^{\mathrm{sub}}\right) .
\end{aligned}
$$

It is an admissible $G_{n}\left(\mathbb{A}^{\infty}\right)$-module with

$$
H^{i}\left(X_{n}^{\min }, \mathcal{E}_{\rho}^{\mathrm{sub}}\right)^{U^{\prime}}=H^{i}\left(X_{n, U^{\prime}}^{\min }, \mathcal{E}_{U^{\prime}, \rho}^{\mathrm{sub}}\right)
$$

Similarly, if $\rho$ is a representation of $L_{n,(n)}$ on a finite free $\mathbb{Z}_{(p)}$-module, we will set

$$
\begin{aligned}
H^{0} & \left(\mathcal{X}_{n}^{\text {ord,min }}, \mathcal{E}_{\rho}^{\text {ord,sub }} \otimes \mathbb{Z} / p^{r} \mathbb{Z}\right) \\
& =\lim _{U^{p}, N_{1}, N_{2}} H^{0}\left(\mathcal{X}_{n, U^{p}\left(N_{1}, N_{2}\right)}^{\text {ord,min }}, \mathcal{E}_{U^{p}\left(N_{1}, N_{2}\right), \rho}^{\text {ord,sub }} \otimes \mathbb{Z} / p^{r} \mathbb{Z}\right) \\
& =\lim _{U^{p}, N_{1}, N_{2}, \Delta}^{\longrightarrow} H^{0}\left(\mathcal{X}_{n, U^{p}\left(N_{1}, N_{2}\right), \Delta^{\prime}}^{\text {ord }}, \mathcal{E}_{U^{p}\left(N_{1}, N_{2}\right), \Delta, \rho}^{\text {ord,sub }} \otimes \mathbb{Z} / p^{r} \mathbb{Z}\right)
\end{aligned}
$$


and

$$
\begin{aligned}
& H^{0}\left(\mathfrak{X}_{n}^{\text {ord,min }}, \mathcal{E}_{\rho}^{\text {ord,sub }}\right) \\
& \quad=\lim _{U^{p}, N} H^{0}\left(\mathfrak{X}_{n, U^{p}(N)}^{\text {ord,min }}, \mathcal{E}_{U^{p}(N), \rho}^{\text {ord,sub }}\right) \\
& =\lim _{U^{\vec{p}, N, \Delta}} H^{0}\left(\mathfrak{X}_{n, U^{p}(N), \Delta^{\prime}}^{\text {ord }}, \mathcal{E}_{U^{p}(N), \Delta, \rho}^{\text {ordsub }}\right)
\end{aligned}
$$

They are smooth $G_{n}\left(\mathbb{A}^{\infty}\right)^{\text {ord, } \times}$-modules with

$$
H^{0}\left(\mathcal{X}_{n}^{\text {ord,min }}, \mathcal{E}_{\rho}^{\text {ord,sub }} \otimes \mathbb{Z} / p^{r} \mathbb{Z}\right)^{U^{p}\left(N_{1}\right)}=H^{0}\left(\mathcal{X}_{n, U^{p}\left(N_{1}, N_{2}\right)}^{\text {ord,min }}, \mathcal{E}_{U^{p}\left(N_{1}, N_{2}\right), \rho}^{\text {ord,sub }} \otimes \mathbb{Z} / p^{r} \mathbb{Z}\right)
$$

and

$$
H^{0}\left(\mathfrak{X}_{n}^{\text {ord,min }}, \mathcal{E}_{\rho}^{\text {ord,sub }}\right)^{U^{p}(N)}=H^{0}\left(\mathfrak{X}_{n, U^{p}(N)}^{\text {ord, min }}, \mathcal{E}_{U^{p}(N), \rho}^{\text {ord,sub }}\right) .
$$

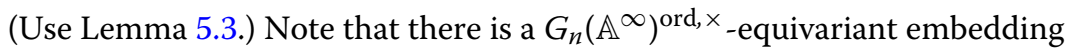

$$
H^{0}\left(\mathfrak{X}_{n}^{\text {ord,min }}, \mathcal{E}_{\rho}^{\text {ord,sub }}\right) \otimes_{\mathbb{Z}_{p}} \mathbb{Z} / p^{r} \mathbb{Z} \hookrightarrow H^{0}\left(\mathcal{X}_{n}^{\text {ord,min }}, \mathcal{E}_{\rho}^{\text {ord,sub }} \otimes \mathbb{Z} / p^{r} \mathbb{Z}\right) .
$$

Finally set

$$
H^{0}\left(\mathfrak{X}^{\text {ord,min }}, \mathcal{E}_{\rho}^{\text {ord,sub }}\right)_{\overline{\mathbb{Q}}_{p}}=H^{0}\left(\mathfrak{X}^{\text {ord,min }}, \mathcal{E}_{\rho}^{\text {ord,sub }}\right) \otimes_{\mathbb{Z}_{p}} \mathbb{Q}_{p},
$$

a smooth representation of $G_{n}\left(\mathbb{A}^{\infty}\right)^{\text {ord }, \times}$.

We record the following result from [44].

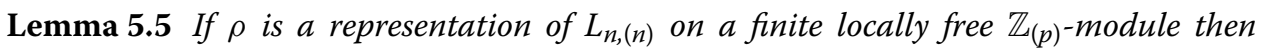
there is a unique system $\left\{\mathcal{E}_{U^{p}\left(N_{1}, N_{2}\right), \rho}^{\text {sub }}\right\}$ of $\mathcal{O}_{\mathcal{X}_{n, U^{p}\left(N_{1}, N_{2}\right)}^{\min }}$-torsion free coherent sheaves with $G_{n}\left(\mathbb{A}^{\infty}\right)^{\text {ord, }} \times$-action over $\left\{\mathcal{X}_{n, U^{p}\left(N_{1}, N_{2}\right)}\right\}$ with the following properties.

(1) $\left\{\mathcal{E}_{U^{p}\left(N_{1}, N_{2}\right), \rho}^{\mathrm{sub}}\right\}$ pulls back to $\left\{\mathcal{E}_{U^{p}\left(N_{1}, N_{2}\right), \rho \otimes_{\mathbb{Z}_{(p)}}}^{\mathrm{sub}}\right\}$ on $\left\{X_{n, U^{p}\left(N_{1}, N_{2}\right)}^{\min }\right\}$;

(2) $\left\{\mathcal{E}_{U^{p}\left(N_{1}, N_{2}\right), \rho}^{\text {sub }}\right\}$ pulls back to $\left\{\mathcal{E}_{U^{p}\left(N_{1}, N_{2}\right), \rho}^{\text {ord,sub }}\right\}$ on $\left\{\mathcal{X}_{n, U^{p}\left(N_{1}, N_{2}\right)}^{\text {ord min }}\right\}$;

(3) if $U^{p}$ is a normal subgroup of $\left(U^{p}\right)^{\prime}$ and if $g \in\left(U^{p}\right)^{\prime}\left(N_{1}^{\prime}, N_{2}\right)$ then

$$
g: g^{*} \mathcal{E}_{U^{p}\left(N_{1}, N_{2}\right), \rho}^{\text {uub }} \stackrel{\sim}{\rightarrow} \mathcal{E}_{U^{p}\left(N_{1}, N_{2}\right), \rho}^{\text {sub }}
$$

(4) if $U^{p}$ is a normal subgroup of $\left(U^{p}\right)^{\prime}$ then

$$
\mathcal{E}_{\left(U^{p}\right)^{\prime}\left(N_{1}, N_{2}\right), \rho}^{\mathrm{sub}} \stackrel{\sim}{\rightarrow}\left(\pi_{U^{p}\left(N_{1}^{\prime}, N_{2}\right),\left(U^{p}\right)^{\prime}\left(N_{1}, N_{2}\right), *} \mathcal{E}_{U^{p}\left(N_{1}^{\prime}, N_{2}\right), \rho}^{\text {sub }}\right)^{\left(U^{p}\right)^{\prime}\left(N_{1}, N_{2}\right)} ;
$$

(5) $\left\{\mathcal{E}_{U^{p}\left(N_{1}, N_{2}\right), \rho \otimes \wedge^{n[F: \mathbb{Q}]} \operatorname{Std}^{\mathrm{sub}}}\right\} \cong\left\{\omega_{U^{p}\left(N_{1}, N_{2}\right)} \otimes \mathcal{E}_{U^{p}\left(N_{1}, N_{2}\right), \rho}^{\mathrm{sub}}\right\}$.

Proof For the definition of $\mathcal{E}_{U^{p}\left(N_{1}, N_{2}\right), \rho}^{\text {sub }}$ see definition 8.3.5.1 of [44]. For the $\mathcal{O}_{\mathcal{X}_{n, U^{p}\left(N_{1}, N_{2}\right)}}$ torsion freeness see corollary 8.3.5.8 of [44]. For the $G_{n}\left(\mathbb{A}^{\infty}\right)^{\text {ord, } \times}$-action see corollary 8.3.6.5 of [44]. For part one of the lemma see lemma 8.3.5.2 of [44]. For the second part see corollary 8.3.5.4 of [44]. The third part is clear. For the fourth part see proposition 8.3.6.9 of [44], and for the final part see lemma 8.3.5.10 of [44]. 
We will write $\Omega_{A_{n, U, \Sigma}^{(m)}}^{i}(\log \infty)$ (resp. $\Omega_{A_{n, U, \Sigma}^{(m)} / X_{n, U^{\prime}, \Delta}}^{i}(\log \infty)$ ) as shorthand for the sheaf $\Omega_{A_{n, U, \Sigma}^{(m)} i \operatorname{Spec} \mathbb{Q}}^{i}\left(\log \mathcal{M}_{\Sigma}\right)$ (resp. $\left.\Omega_{A_{n, U, \Sigma}^{(m)} / X_{n, U^{\prime}, \Delta}}^{i}\left(\log \mathcal{M}_{\Sigma} / \mathcal{M}_{\Delta}\right)\right)$. Then the collection $\left\{\Omega_{A_{n, U, \Sigma}^{(m)}}^{1}(\log \infty)\right\}\left(\right.$ resp. $\left.\left\{\Omega_{A_{n, U, \Sigma}^{(m)} / X_{n, U^{\prime}, L}}^{1}(\log \infty)\right\}\right)$ is a system of locally free sheaves (for the Zariski topology) with $G_{n}^{(m)}\left(\mathbb{A}^{\infty}\right)$-action.

There are natural differentials

$$
d: \Omega_{A_{n, U, \Sigma}^{(m)}}^{i}(\log \infty) \longrightarrow \Omega_{A_{n, U, \Sigma}^{(m)}}^{i+1}(\log \infty)
$$

(resp.

$$
\left.d: \Omega_{A_{n, U, \Sigma}^{(m)} / X_{n, U^{\prime}, \Delta}}^{i}(\log \infty) \longrightarrow \Omega_{A_{n, U, \Sigma}^{(m)} / X_{n, U^{\prime}, \Delta}}^{i+1}(\log \infty)\right)
$$

making $\Omega_{A_{n, U, \Sigma}^{(m)}}^{\bullet}(\log \infty)\left(\right.$ resp. $\left.\Omega_{A_{n, U, \Sigma}^{(m)} / X_{n, U^{\prime}, \Delta}}^{\bullet}(\log \infty)\right)$ a complex. The tensor product $\Omega_{A_{n, U, \Sigma}^{(m)}}^{\bullet}(\log \infty) \otimes \mathcal{I}_{\partial A_{n, U, \Sigma}^{(m)}}^{(m)}\left(\right.$ resp. $\left.\Omega_{A_{n, U, \Sigma}^{(m)} / X_{n, U^{\prime}, \Delta}}^{\bullet, U, \Sigma}(\log \infty) \otimes \mathcal{I}_{\partial A_{n, U, \Sigma}^{(m)}}\right)$ is a subcomplex.

Lemma 5.6 (1) If $(U, \Sigma) \geq\left(U^{\prime}, \Delta\right) \geq\left(U^{\prime \prime}, \Delta^{\prime}\right)$ then the natural morphism

$$
\Omega_{A_{n, U, \Sigma}^{(m)} / X_{n, U^{\prime \prime}, \Delta^{\prime}}}^{1}(\log \infty) \stackrel{\sim}{\rightarrow} \Omega_{A_{n, U, \Sigma}^{(m)} / X_{n, U^{\prime}, \Delta}}^{1}(\log \infty)
$$

is an isomorphism, so we will simply write $\Omega_{A_{n, U, \Sigma}^{(m)} / X}^{1}(\log \infty)$ for this sheaf.

(2) If $\left(U^{\prime}, \Sigma^{\prime}\right) \geq(U, \Sigma)$ then

$$
\pi_{\left(U^{\prime}, \Sigma^{\prime}\right),(U, \Sigma)}^{*} \Omega_{A_{n, U, \Sigma}^{(m)}}^{1}(\log \infty) \stackrel{\sim}{\rightarrow} \Omega_{A_{n, U^{\prime}, \Sigma^{\prime}}^{(m)}}^{1}(\log \infty)
$$

and

$$
\pi_{\left(U^{\prime}, \Sigma^{\prime}\right),(U, \Sigma)}^{*} \Omega_{A_{n, U, \Sigma}^{(m)} / X}^{1}(\log \infty) \stackrel{\sim}{\rightarrow} \Omega_{A_{n, U^{\prime}, \Sigma^{\prime}}^{(m)} / X}^{1}(\log \infty) .
$$

(3) If $(U, \Sigma) \geq\left(U^{\prime}, \Delta\right)$ then there is an exact sequence

$$
(0) \rightarrow \pi_{(U, \Sigma),\left(U^{\prime}, \Delta\right)}^{*} \Omega_{X_{n, U^{\prime}, \Delta}}^{1}(\log \infty) \rightarrow \Omega_{A_{n, U, \Sigma}^{(m)}}^{1}(\log \infty) \rightarrow \Omega_{A_{n, U, \Sigma}^{(m)} / X}^{1}(\log \infty) \rightarrow(0) .
$$

(4) Suppose that $\left(U_{1}, \Sigma_{1}\right) \geq\left(U_{2}, \Sigma_{2}\right) \geq\left(U^{\prime}, \Delta\right)$ and that $U^{\prime}$ is the image of both $U_{1}$ and $U_{2}$ in $G_{n}\left(\mathbb{A}^{\infty}\right)$. Then the natural maps

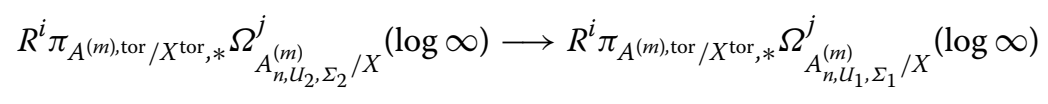

and

$$
\begin{aligned}
& R^{i} \pi_{A^{(m), \text { tor }} / X^{\text {tor }, *}}\left(\Omega_{A_{n, U_{2}, \Sigma_{2}}^{(m)} / X}^{j}(\log \infty) \otimes \mathcal{I}_{\partial A_{n, U_{2}, \Sigma_{2}}^{(m)}}\right) \\
& \longrightarrow R^{i} \pi_{A^{(m), \text { tor }} / X^{\mathrm{tor}, *}}\left(\Omega_{A_{n, U_{1}, \Sigma_{1}}^{j} / X}^{j}(\log \infty) \otimes \mathcal{I}_{\partial A_{n, U_{1}, \Sigma_{1}}^{(m)}}\right)
\end{aligned}
$$


on $X_{n, U^{\prime}, \Delta}$ are isomorphisms. We will write simply

$$
\left(R^{i} \pi_{*} \Omega_{A^{(m)} / X}^{j}(\log \infty)\right)_{\left(U^{\prime}, \Delta\right)}
$$

and

$$
\left(R^{i} \pi_{*}\left(\Omega_{A^{(m)} / X}^{j}(\log \infty) \otimes \mathcal{I}_{\left.\partial A^{(m)}\right)}\right)\right)_{\left(U^{\prime}, \Delta\right)}
$$

for these sheaves.

(5) $\left\{\left(R^{i} \pi_{*} \Omega_{A^{(m)} / X}^{j}(\log \infty)\right)_{\left(U^{\prime}, \Delta\right)}\right\}$ and $\left\{\left(R^{i} \pi_{*}\left(\Omega_{A^{(m)} / X}^{j}(\log \infty) \otimes \mathcal{I}_{\left.\partial A^{(m)}\right)}\right)\right)_{\left(U^{\prime}, \Delta\right)}\right\}$ are systems of coherent sheaves with $G_{n}^{(m)}\left(\mathbb{A}^{\infty}\right)$-action over $\left\{X_{n, U^{\prime}, \Delta}\right\}$. Moreover the maps

$$
g: g^{*}\left(R^{i} \pi_{*} \Omega_{A^{(m)} / X}^{j}(\log \infty)\right)_{\left(U^{\prime}, \Delta\right)} \longrightarrow\left(R^{i} \pi_{*} \Omega_{A^{(m)} / X}^{j}(\log \infty)\right)_{\left(U^{\prime \prime}, \Delta^{\prime}\right)}
$$

are isomorphisms.

(6) The $G_{n}^{(m)}\left(\mathbb{A}^{\infty}\right)$-actions on both the systems

$$
\left\{\left(R^{i} \pi_{*} \Omega_{A^{(m)} / X}^{j}(\log \infty)\right)_{\left(U^{\prime}, \Delta\right)}\right\}
$$

and

$$
\left\{\left(R^{i} \pi_{*}\left(\Omega_{A^{(m)} / X}^{j}(\log \infty) \otimes \mathcal{I}_{\partial A^{(m)}}\right)\right)_{\left(U^{\prime}, \Delta\right)}\right\}
$$

factor through $G_{n}\left(\mathbb{A}^{\infty}\right)$.

(7) The pull-back of $\left(\pi_{*} \Omega_{A^{(m)} / X}^{1}(\log \infty)\right)_{(U, \Delta)}$ to $T_{n,(i), h U h^{-1} \cap P_{n,(i)}^{+}\left(\mathbb{A}^{\infty}\right), \Delta(h)_{0}}$ is isomorphic to

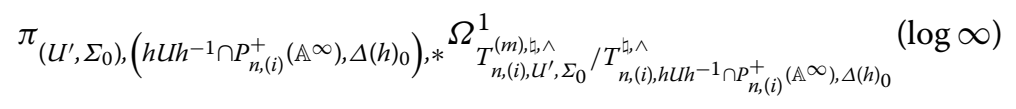

for some $U^{\prime}$ and $\Sigma_{0}$.

Proof This follows from the properties of log differentials for log smooth maps (see Sect. 2.2). For part 4 we also use Lemma 5.1. For part 6 we also use the discussion of Sect. 3.4 and a density argument.

The next lemma follows from Lemma 4.10.

Lemma 5.7 (1) The natural maps

$$
\left(\pi_{*} \Omega_{A^{(m)} / X}^{1}(\log \infty)\right)_{\left(U^{\prime}, \Delta\right)} \otimes_{\mathcal{O}_{X_{n, U}, \Delta}} \mathcal{O}_{A_{n, U, \Sigma}^{(m)}} \longrightarrow \Omega_{A_{n, U, \Sigma}^{(m)} / X}^{1}(\log \infty)
$$

are $G_{n}^{(m)}\left(\mathbb{A}^{\infty}\right)$-equivariant isomorphisms. 
(2) The natural maps

$$
\left(\wedge^{j}\left(\pi_{*} \Omega_{A^{(m)} / X}^{1}(\log \infty)\right)_{\left(U^{\prime}, \Delta\right)}\right) \otimes\left(R^{i} \pi_{*} \mathcal{O}_{A^{(m)}}\right)_{\left(U^{\prime}, \Delta\right)} \longrightarrow\left(R^{i} \pi_{*} \Omega_{A^{(m)} / X}^{j}(\log \infty)\right)_{\left(U^{\prime}, \Delta\right)}
$$

and

$$
\begin{aligned}
& \left(\wedge^{j}\left(\pi_{*} \Omega_{A^{(m)} / X}^{1}(\log \infty)\right)_{\left(U^{\prime}, \Delta\right)}\right) \otimes\left(R^{i} \pi_{*} \mathcal{O}_{A^{(m)}}\right)_{\left(U^{\prime}, \Delta\right)} \otimes \mathcal{I}_{\partial X_{n, U^{\prime}, \Delta}} \\
& \longrightarrow\left(R^{i} \pi_{*}\left(\Omega_{A^{(m)} / X}^{j}(\log \infty) \otimes \mathcal{I}_{\partial A^{(m)}}\right)\right)_{\left(U^{\prime}, \Delta\right)}
\end{aligned}
$$

are $G_{n}\left(\mathbb{A}^{\infty}\right)$-equivariant isomorphisms.

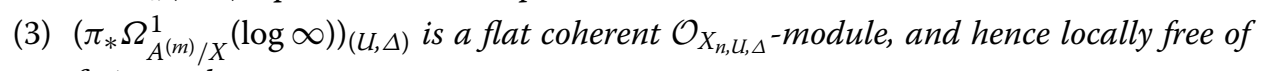
finite rank.

Next we record some results from $[42,44]$.

Lemma 5.8 (1) There are natural $G_{n}\left(\mathbb{A}^{\infty}\right)$-equivariant isomorphisms

$$
\operatorname{Hom}_{F}\left(F^{m}, \Omega_{n, U^{\prime}, \Delta}\right) \stackrel{\sim}{\longrightarrow}\left(\pi_{*} \Omega_{A_{n}^{(m)} / X_{n}}^{1}(\log \infty)\right)_{\left(U^{\prime}, \Delta\right)} .
$$

(2) The cup product maps

$$
\wedge^{i}\left(R^{1} \pi_{*} \mathcal{O}_{\left.A^{(m)}\right)}\right)_{\left(U^{\prime}, \Delta\right)} \longrightarrow\left(R^{i} \pi_{*} \mathcal{O}_{A^{(m)}}\right)_{\left(U^{\prime}, \Delta\right)}
$$

are $G_{n}\left(\mathbb{A}^{\infty}\right)$-equivariant isomorphisms.

(3) There is a unique embedding

$$
\Xi_{n, U^{\prime}, \Delta} \hookrightarrow\left(R^{1} \pi_{*} \Omega_{A^{(m)} / X}^{1}(\log \infty)\right)_{\left(U^{\prime}, \Delta\right)}
$$

extending

$$
\Xi_{n, U^{\prime}} \hookrightarrow\left(R^{1} \pi_{*} \Omega_{A^{(m)} / X}^{1}\right)_{U^{\prime}}
$$

It is $G_{n}\left(\mathbb{A}^{\infty}\right)$-equivariant.

(4) The composite maps

$$
\begin{aligned}
& \operatorname{Hom}\left(\left(\pi_{*} \Omega_{A_{n}^{(m)} / X_{n}}^{1}(\log \infty)\right)_{\left(U^{\prime}, \Delta\right)}, \Xi_{n, U^{\prime}, \Delta}\right) \\
& \longrightarrow \operatorname{Hom}\left(\left(\pi_{*} \Omega_{A_{n}^{(m)} / X_{n}}^{1}(\log \infty)\right)_{\left(U^{\prime}, \Delta\right)}\right. \\
& \left.\quad\left(\pi_{*} \Omega_{A_{n}^{(m)} / X_{n}}^{1}(\log \infty)\right)_{\left(U^{\prime}, \Delta\right)} \otimes\left(R^{1} \pi_{*} \mathcal{O}_{\left.A^{(m)}\right)}\right)_{\left(U^{\prime}, \Delta\right)}\right) \\
& \stackrel{\operatorname{tr}}{\longrightarrow}\left(R^{1} \pi_{*} \mathcal{O}_{\left.A^{(m)}\right)}\right)_{\left(U^{\prime}, \Delta\right)}
\end{aligned}
$$

are $G_{n}\left(\mathbb{A}^{\infty}\right)$-equivariant isomorphisms. 
(5) The boundary maps

$$
\begin{aligned}
\Omega_{n, U^{\prime}, \Delta} & \longrightarrow R^{1} \pi_{A^{(1)} / X, *}\left(\pi_{A^{(1)} / X}^{*} \Omega_{X_{n, U^{\prime}, \Delta}}^{1}(\log \infty)\right) \\
& \cong \Omega_{X_{n, U^{\prime}, \Delta}}^{1}(\log \infty) \otimes \operatorname{Hom}\left(\Omega_{n, U^{\prime}, \Delta}, \Xi_{n, U^{\prime}, \Delta}\right)
\end{aligned}
$$

associated to the short exact sequence of part 3 of Lemma 5.6, give rise to isomorphisms

$$
S\left(\Omega_{n, U^{\prime}, \Delta}\right) \stackrel{\sim}{\longrightarrow} \Omega_{X_{n, U^{\prime}, \Delta}}^{1}(\log \infty) \otimes \Xi_{n, U^{\prime}, \Delta} .
$$

(6) There are $G_{n}\left(\mathbb{A}^{\infty, p} \times \mathbb{Z}_{p}\right)$-equivariant identifications between the pull-back of $\omega_{U}$ from $\mathcal{X}_{n, U}^{\min }$ to $X_{n, U, \Delta}$ and $\omega_{U, \Delta}$.

Proof For the first four parts see theorem 2.15 and proposition 6.9 of [42] and theorem 1.3.3.15 of [44]. For the fifth part see theorem 1.3.1.3(4) of [44]. For the sixth part see propositions 2.2.1.2 and 2.2.3.1 of [44].

Corollary 5.9 There are equivariant isomorphisms $\mathcal{E}_{U, \Delta, \mathrm{KS}}^{\mathrm{can}} \cong \Omega_{X_{n, U, \Delta}}^{1}(\log \infty)$. (See Sect. 1.2 for the definition of the representation $\mathrm{KS}$.)

Lemma 5.10 Suppose that $U$ is a neat open compact subgroup of $G_{n}^{(m)}\left(\mathbb{A}^{\infty}\right)$ with image $U^{\prime}$ in $G_{n}\left(\mathbb{A}^{\infty}\right)$. The coherent sheaf $\Omega_{A_{n, U, \Sigma}^{(m)}}^{r}(\log \infty)$ admits a decreasing filtration by local direct summands $\mathrm{Fil}^{j} \Omega_{A_{n, U, \Sigma}^{(m)}}^{r}(\log \infty)$ with

- $\mathrm{Fil}^{0} \Omega_{A_{n, U, \Sigma}^{(m)}}^{r}(\log \infty)=\Omega_{A_{n, U, \Sigma}^{(m)}}^{r}(\log \infty)$,

- $\mathrm{Fil}^{r+1} \Omega_{A_{n, U, \Sigma}^{r}}^{r(m)}(\log \infty)=(0)$,

- and $\operatorname{gr}^{j} \Omega_{A_{n, U, \Sigma}^{r}}^{r}(\log \infty) \cong\left(\pi_{A^{(m), \text { tor }} / X^{\mathrm{tor}}}^{*} \Omega_{X_{n, U^{\prime}, \perp}^{j}}^{j}(\log \infty)\right) \otimes \Omega_{A_{n, U, \Sigma}^{(m)} / X}^{r-j}(\log \infty)$.

This filtration is $G_{n}\left(\mathbb{A}^{\infty}\right)$-equivariant.

Moreover there are representations $\rho_{m, r}^{i, j}$ of $L_{n,(n)}$ such that there are $G_{n}\left(\mathbb{A}^{\infty}\right)$-equivariant isomorphisms

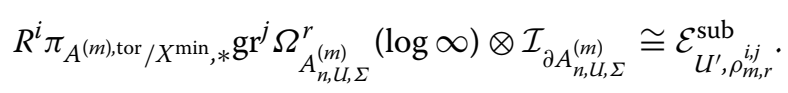

Thus there is a spectral sequence with first page

$$
E_{1}^{i, j}=\mathcal{E}_{U^{\prime}, \rho_{m, r}^{i, j}}^{\mathrm{sub}} \Rightarrow R^{i+j} \pi_{A^{(m), \operatorname{tor}} / X^{\min , *}}\left(\Omega_{A_{n, U, \Sigma}^{(m)}}^{r}(\log \infty) \otimes \mathcal{I}_{\partial A_{n, U, \Sigma}^{(m)}}\right)
$$

This spectral sequence is $G_{n}\left(\mathbb{A}^{\infty}\right)$-equivariant.

Proof Using part 2 of Corollary 5.6 and parts 1 and 2 of Lemma 5.1, we may reduce to the case that there is a cone decomposition $\Delta$ compatible with $\Sigma$. The first assertion now follows from part 3 of Lemma 5.6. 
For the second assertion, note that by Lemma 5.7 we have that

$$
\begin{aligned}
& \left(\wedge^{j} \Omega_{X_{n, U^{\prime}, \Delta}}^{1}(\log \infty)\right) \otimes\left(\wedge^{r-j}\left(\pi_{*} \Omega_{A^{(m)} / X}^{1}(\log \infty)\right)_{\left(U^{\prime}, \Delta\right)}\right) \\
& \otimes\left(R^{i} \pi_{A^{(m), \text { tor }} / X^{\mathrm{tor}, *}} \mathcal{O}_{A^{(m)}}\right)_{\left(U^{\prime}, \Delta\right)} \otimes \mathcal{I}_{\partial X_{n, U^{\prime}, \Delta}} \\
& \stackrel{\sim}{\longrightarrow} R^{i} \pi_{A^{(m), \text { tor }} / X^{\mathrm{tor}, *}}\left(\pi_{A^{(m), \text { tor } / X^{\mathrm{tor}}}}^{*} \Omega_{X_{n, U^{\prime}, \Delta}^{j}}^{j}(\log \infty) \otimes \Omega_{A_{n, U, \Sigma}^{(m)} / X}^{r-j}(\log \infty)\right. \\
& \left.\otimes \mathcal{I}_{\partial A_{n, U, \Sigma}^{(m)}}\right)
\end{aligned}
$$

Combining this with parts $1,2,4$ and 5 of Lemma 5.8 we find representations $\rho_{m, r}^{i, j}$ such that there are $G_{n}\left(\mathbb{A}^{\infty}\right)$-equivariant isomorphisms

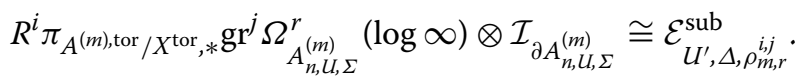

The second assertion now follows from Theorem 5.4.

The third assertion follows from the first two.

\subsection{Connection to the complex theory}

\section{Lemma 5.11 Suppose that}

$$
b=\left(b_{0},\left(b_{\tau, i}\right)_{\tau \in \operatorname{Hom}(F, \mathbb{C})}\right) \in X^{*}\left(T_{n} / \mathbb{C}\right)_{(n)}^{+}
$$

satisfies

$$
-2 n \geq b_{\tau, 1}+b_{\tau c, 1}
$$

for all $\tau \in \operatorname{Hom}(F, \mathbb{C})$. Then $H^{0}\left(X^{\min }, \mathcal{E}_{\rho_{(n), b}}^{\text {sub }}\right)$ is a semi-simple $G_{n}\left(\mathbb{A}^{\infty}\right)$-module. If $\pi$ is an irreducible subquotient of $H^{0}\left(X^{\mathrm{min}}, \mathcal{E}_{\rho_{(n), b}}^{\mathrm{sub}}\right)$, then $\pi$ is the finite part of a cohomological, cuspidal automorphic representation of $G_{n}(\mathbb{A})$.

Proof According to proposition 5.4.2 and lemma 5.2.3 of [28] and theorems 4.1.1, 5.1.1 and 5.2.12 of [43] we have an isomorphism

$$
H^{0}\left(X_{n}^{\min }, \mathcal{E}_{\rho_{(n), b}}^{\mathrm{sub}}\right) \cong \bigoplus_{\Pi} \Pi^{\infty} \otimes H^{0}\left(\mathfrak{q}_{n}, U_{n, \infty}^{0} A_{n}(\mathbb{R})^{0}, \Pi_{\infty} \otimes \rho_{(n), b}\right)
$$

where $\Pi$ runs over cuspidal automorphic representations of $G_{n}(\mathbb{A})$ taken with their multiplicity in the space of cuspidal automorphic forms.

Thus $\pi \cong \Pi^{\infty}$ for some cuspidal automorphic representation $\Pi$ of $G_{n}(\mathbb{A})$ with

$$
H^{0}\left(\mathfrak{q}_{n}, U_{n, \infty}^{0} A_{n}(\mathbb{R})^{0}, \Pi_{\infty} \otimes \rho_{(n), b}\right) \neq(0)
$$

It follows from theorem 2.6 of [21] that the Harish-Chandra parameter of the infinitesimal character of $\Pi_{\infty}$ equals

$$
\varrho_{n}-2 \varrho_{n,(n)}-b
$$

As we have assumed that

$$
b-2\left(\varrho_{n}-\varrho_{n,(n)}\right) \in X^{*}\left(T_{n} / \mathbb{C}\right)^{+},
$$


we see that $\Pi_{\infty}$ has the same infinitesimal character as $\rho_{b-2\left(\varrho_{n}-\varrho_{n,(n)}\right)}^{\vee}$. Moreover proposition 4.5 of [28] tells us that

$$
\operatorname{Hom}_{U_{n, \infty}^{0} A_{n}(\mathbb{R})^{0}}\left(\rho_{(n), b}^{\vee}, \Pi_{\infty}\right) \neq(0) .
$$

We deduce that

$$
\operatorname{Hom}_{U_{n, \infty}^{0} A_{n}(\mathbb{R})^{0}}\left(\rho_{(n),-2\left(\varrho_{n}-\varrho_{n,(n)}\right)}, \Pi_{\infty} \otimes \rho_{b-2\left(\varrho_{n}-\varrho_{n,(n)}\right)}\right) \neq(0) .
$$

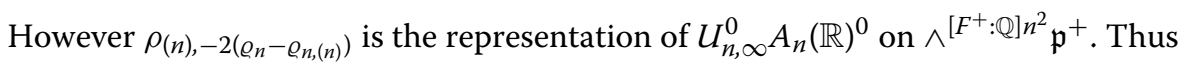

$$
\operatorname{Hom}_{U_{n, \infty}^{0} A_{n}(\mathbb{R})^{0}}\left(\wedge^{\left[F^{+}: \mathbb{Q}\right] n^{2}} \mathfrak{p} \otimes_{\mathbb{R}} \mathbb{C}, \Pi_{\infty} \otimes \rho_{b-2\left(\varrho_{n}-\varrho_{n,(n)}\right)}\right) \neq(0) .
$$

Proposition II.3.1 of [15] then tells us that

$$
H^{\left[F^{+}: \mathbb{Q}\right] n^{2}}\left(\left(\operatorname{Lie} G_{n}(\mathbb{R})\right) \otimes_{\mathbb{R}} \mathbb{C}, U_{n, \infty}^{0} A_{n}(\mathbb{R})^{0}, \Pi_{\infty} \otimes \rho_{b-2\left(\varrho_{n}-\varrho_{n,(n)}\right)}\right) \neq(0),
$$

and the lemma follows.

Corollary 5.12 Suppose that

$$
b=\left(b_{0},\left(b_{\tau, i}\right)_{\tau \in \operatorname{Hom}\left(F, \overline{\mathbb{Q}}_{p}\right)}\right) \in X^{*}\left(T_{n} / \overline{\mathbb{Q}}_{p}\right)_{(n)}^{+}
$$

satisfies

$$
-2 n \geq b_{\tau, 1}+b_{\tau c, 1}
$$

for all $\tau \in \operatorname{Hom}\left(F, \overline{\mathbb{Q}}_{p}\right)$. If $\Pi$ is an irreducible subquotient of $H^{0}\left(X_{n}^{\min }, \mathcal{E}_{\rho_{(n), b}}^{\mathrm{sub}}\right)$, then there is a continuous representation

$$
R_{p}(\Pi): G_{F} \longrightarrow G L_{2 n}\left(\overline{\mathbb{Q}}_{p}\right)
$$

which is de Rham above $p$ and has the following property: Suppose that $v$ is a prime of $F$ above a rational prime $q \neq p$ such that

- either $q$ splits in $F_{0}$,

- or $F$ and $\Pi$ are unramified above q;

then

$$
\mathrm{WD}\left(\left.R_{p}(\Pi)\right|_{G_{F_{v}}}\right)^{\mathrm{F}-\mathrm{ss}} \cong \operatorname{rec}_{F_{v}}\left(\mathrm{BC}\left(\Pi_{q}\right)_{v}|\operatorname{det}|_{v}^{(1-2 n) / 2}\right),
$$

where $q$ is the rational prime below $v$.

Proof By the lemma $\imath \Pi$ is the finite part of a cohomological, square integrable, automorphic representation of $G_{n}(\mathbb{A})$. The result now follows from Corollary 1.3.

\section{The ordinary locus}

We will now fairly systematically drop the subscript $n$, as it will be fixed throughout this section. 


\subsection{P-adic automorphic forms}

Let $U$ be a neat open compact subgroup of $G_{n}\left(\mathbb{A}^{\infty, p} \times \mathbb{Z}_{p}\right)$. Zariski locally on $\mathcal{X}_{U}^{\text {min }}$ we may lift Hasse $U$ to a (non-canonical) section $\widetilde{\mathrm{Hasse}_{U}}$ of $\omega^{\otimes(p-1)}$ over (an open subset of) $\mathcal{X}_{U}^{\mathrm{min}}$. Although $\widetilde{\operatorname{Hasse}_{U}}$ is non-canonical,

$$
\widetilde{\operatorname{Hass}} \mathrm{p}_{U}^{M-1} \bmod p^{M}
$$

is canonical, and so these glue to give a canonical element

$$
\operatorname{Hasse}_{M, U} \in H^{0}\left(\mathcal{X}_{U}^{\min } \times \operatorname{Spec} \mathbb{Z} / p^{M} \mathbb{Z}, \omega_{U}^{\otimes(p-1) p^{M-1}}\right)
$$

Again if $g \in G_{n}\left(\mathbb{A}^{\infty, p} \times \mathbb{Z}_{p}\right)$ and $U^{\prime} \supset g^{-1} U g$ then

$$
g \operatorname{Hasse}_{M, U^{\prime}}=\operatorname{Hasse}_{M, U}
$$

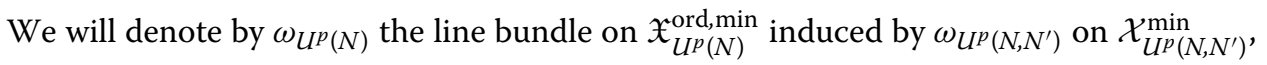
and by $\operatorname{Hasse}_{M, U^{p}(N)}$ the restriction of $\operatorname{Hasse}_{M, U^{p}\left(N, N^{\prime}\right)}$ to

$$
H^{0}\left(\mathfrak{X}_{U^{p}(N)}^{\text {ord,min }} \times \operatorname{Spec} \mathbb{Z} / p^{M} \mathbb{Z},\left(\omega_{U^{p}(N)}^{\text {ord }}\right)^{\otimes(p-1) p^{M-1}}\right)
$$

This is independent of $N^{\prime}$.

If $\rho$ is a representation of $L_{n,(n)}$ on a finite free $\mathbb{Z}_{p}$-module then, for any integer $i$, there is a natural map

$$
\begin{aligned}
H^{0}\left(\mathcal{X}_{U^{p}\left(N_{1}, N_{2}\right)}^{\min }, \mathcal{E}_{\rho \otimes(\wedge n[F: \mathbb{Q}]}^{\text {sub } \left.\operatorname{std}^{\vee}\right)^{i p^{M-1}(p-1)}}\right) & \cong H^{0}\left(\mathcal{X}_{U^{p}\left(N_{1}, N_{2}\right)}^{\min }, \mathcal{E}_{\rho}^{\text {sub }} \otimes \omega_{U}^{\otimes i(p-1) p^{M-1}}\right) \\
& \longrightarrow H^{0}\left(\mathcal{X}_{U^{p}\left(N_{1}, N_{2}\right)}^{\text {ord,min }}, \mathcal{E}_{\rho}^{\text {ord,sub }} \otimes \mathbb{Z} / p^{M} \mathbb{Z}\right)
\end{aligned}
$$

which sends $f$ to

$$
\left(\left.f\right|_{\mathcal{X}_{U^{p}\left(N_{1}, N_{2}\right)}^{\text {ord,min }}}\right) / \operatorname{Hasse}_{M, U^{p}\left(N_{1}, N_{2}\right)}^{i} .
$$

These maps are $G_{n}\left(\mathbb{A}^{\infty}\right)^{\text {ord, } \times}$-equivariant.

Lemma 6.1 For any $r$ the induced map

$$
\begin{gathered}
\bigoplus_{j=r}^{\infty} H^{0}\left(\mathcal{X}_{U^{p}\left(N_{1}, N_{2}\right)}^{\min }, \mathcal{E}_{\left.U^{p}\left(N_{1}, N_{2}\right), \rho \otimes\left(\wedge^{n[F: \mathbb{Q}]} \operatorname{Std}^{\vee}\right)^{j p^{M-1}(p-1)}\right)}^{\text {sub }}\right) \\
\longrightarrow H^{0}\left(\mathcal{X}_{U^{p}\left(N_{1}, N_{2}\right)}^{\text {ord,min }}, \mathcal{E}_{U^{p}\left(N_{1}, N_{2}\right), \rho}^{\text {ord,sub }} \otimes \mathbb{Z} / p^{M} \mathbb{Z}^{\mathbb{Z}}\right)
\end{gathered}
$$

is surjective.

Proof The proof here follows standard lines. As far as we know the argument originated in [35]. For the properties of $\mathcal{X}_{U}^{\min }$ see Sect. 5.1.

To simplify the formulae in this proof, for the duration of the proof we will write $U$ for $U^{p}\left(N_{1}, N_{2}\right)$.

Multiplying by a power of $\operatorname{Hasse}_{M, U}$ we may replace $\rho$ by

$$
\rho \otimes\left(\wedge^{n[F: \mathbb{Q}]} \operatorname{Std}^{\vee}\right)^{t p^{M-1}(p-1)}
$$


and $r$ by $r-t$ for any $t$. Thus, using the ampleness of $\omega_{U}$ over $\mathcal{X}_{U}^{\min }$, we may suppose that

$$
H^{i}\left(\mathcal{X}_{U}^{\min }, \mathcal{E}_{U, \rho}^{\mathrm{sub}} \otimes \omega_{U}^{\otimes j}\right)=(0)
$$

for all $i>0$ and $j \geq 0$. We may also suppose that $r \leq 0$. Then we may replace $r$ by 0 .

Because $\mathcal{X}_{U}^{\text {ord,min }} \times$ Spec $\mathbb{Z} / p^{M} \mathbb{Z}$ is a union of connected components of

$$
\mathcal{Y}=\mathcal{X}_{U}^{\min } \times \operatorname{Spec} \mathbb{Z} / p^{M} \mathbb{Z}-\bar{X}_{U}^{\min , \mathrm{n}-\text { ord }}
$$

it suffices to replace $\mathcal{X}_{U}^{\text {ord,min }} \times \operatorname{Spec} \mathbb{Z} / p^{M} \mathbb{Z}$ by $\mathcal{Y}$.

Now we need to show that

$$
\bigoplus_{j=0}^{\infty} H^{0}\left(\mathcal{X}_{U}^{\mathrm{min}}, \mathcal{E}_{U, \rho}^{\mathrm{sub}} \otimes \omega_{U}^{\otimes j(p-1) p^{M-1}}\right) \rightarrow H^{0}\left(\mathcal{Y}, \mathcal{E}_{U, \rho}^{\mathrm{ord}, \mathrm{sub}} \otimes \mathbb{Z} / p^{M} \mathbb{Z}\right)
$$

under the assumption that

$$
H^{i}\left(\mathcal{X}_{U}^{\min }, \mathcal{E}_{U, \rho}^{\mathrm{sub}} \otimes \omega_{U}^{\otimes j}\right)=(0)
$$

for all $i>0$ and $j \geq 0$.

The scheme $\mathcal{Y}$ is relatively affine over $\mathcal{X}_{U}^{\min }$ corresponding to the sheaf of algebras

$$
\left(\bigoplus_{j=0}^{\infty} \omega_{U}^{\otimes j p^{M-1}(p-1)}\right) /\left(\operatorname{Hasse}_{M, U}-1, p^{M}\right)
$$

Hence

$$
H^{0}\left(\mathcal{Y}, \mathcal{E}_{U, \rho}^{\mathrm{sub}}\right) \cong H^{0}\left(\mathcal{X}_{U}^{\min },\left(\bigoplus_{j=0}^{\infty} \mathcal{E}_{U, \rho}^{\mathrm{sub}} \otimes \omega_{U}^{\otimes p^{M-1}(p-1) j}\right) /\left(\operatorname{Hasse}_{M, U}-1, p^{M}\right)\right)
$$

and the map

$$
\bigoplus_{j=0}^{\infty} H^{0}\left(\mathcal{X}_{U}^{\mathrm{min}}, \mathcal{E}_{U, \rho}^{\mathrm{sub}} \otimes \omega_{U}^{\otimes j(p-1) p^{M-1}}\right) \longrightarrow H^{0}\left(\mathcal{Y}, \mathcal{E}_{U, \rho}^{\mathrm{ord}, \mathrm{sub}} \otimes \mathbb{Z} / p^{M} \mathbb{Z}\right)
$$

is induced by the map

$$
\bigoplus_{j=0}^{\infty} \mathcal{E}_{U, \rho}^{\text {sub }} \otimes \omega_{U}^{\otimes j(p-1) p^{M-1}} \rightarrow\left(\bigoplus_{j=0}^{\infty} \mathcal{E}_{U, \rho}^{\text {sub }} \otimes \omega_{U}^{\otimes p^{M-1}(p-1) j}\right) /\left(\text { Hasse }_{M, U}-1, p^{M}\right)
$$

of sheaves over $\mathcal{X}_{U}^{\min }$.

Because

$$
H^{i}\left(\mathcal{X}_{U}^{\min }, \mathcal{E}_{U, \rho}^{\mathrm{sub}} \otimes \omega_{U}^{\otimes j}\right)=(0)
$$

for all $i>0$ and $j \geq 0$, we see that

$$
H^{0}\left(\mathcal{X}_{U}^{\min }, \mathcal{E}_{U^{p}, \rho}^{\text {sub }} \otimes \omega_{U}^{\otimes j}\right) \otimes \mathbb{Z} / p^{M} \mathbb{Z} \stackrel{\sim}{\longrightarrow} H^{0}\left(\mathcal{X}_{U}^{\min }, \mathcal{E}_{U, \rho}^{\text {sub }} \otimes \omega_{U}^{\otimes j} \otimes \mathbb{Z} / p^{M} \mathbb{Z}\right)
$$


for all $j \geq 0$, and

$$
H^{i}\left(\mathcal{X}_{U}^{\min }, \mathcal{E}_{U, \rho}^{\mathrm{sub}} \otimes \omega_{U}^{\otimes j} \otimes \mathbb{Z} / p^{M} \mathbb{Z}\right)=(0)
$$

for all $i>0$ and $j \geq 0$. Thus it suffices to check that

$$
\begin{gathered}
H^{0}\left(\mathcal{X}_{U}^{\min }, \bigoplus_{j=0}^{\infty} \mathcal{E}_{U, \rho}^{\text {sub }} \otimes \omega_{U}^{\otimes p^{M-1}(p-1) j} \otimes \mathbb{Z} / p^{M} \mathbb{Z}\right) /\left(\text { Hasse }_{M, U}-1\right) \\
H^{0}\left(\mathcal{X}_{U}^{\min },\left(\bigoplus_{j=0}^{\infty} \mathcal{E}_{U, \rho}^{\text {sub }} \otimes \omega_{U}^{\otimes p^{M-1}(p-1) j} \otimes \mathbb{Z} / p^{M} \mathbb{Z}\right) /\left(\text { Hasse }_{M, U}-1\right)\right)
\end{gathered}
$$

is surjective. This follows using the long exact sequence in cohomology associated to the short exact sequence

$$
\begin{aligned}
& (0) \underset{\text { Hasse }_{M, U}-1}{\longrightarrow} \bigoplus_{j, \rho}^{\infty} \mathcal{E}_{U=0}^{\text {sub }} \otimes \omega_{U}^{\otimes p^{M-1}(p-1) j} \otimes \mathbb{Z} / p^{M} \mathbb{Z} \\
& \stackrel{\text { sub }^{M}}{\longrightarrow} \omega_{U}^{\otimes p^{M-1}(p-1) j} \otimes \mathbb{Z} / p^{M} \mathbb{Z} \\
& \longrightarrow\left(\bigoplus_{j=0}^{\infty} \mathcal{E}_{U, \rho}^{\text {sub }} \otimes \omega_{U}^{\otimes p^{M-1}(p-1) j} \otimes \mathbb{Z} / p^{M} \mathbb{Z}\right) /\left(\text { Hasse }_{M, U}-1\right) \longrightarrow(0)
\end{aligned}
$$

and the vanishing

$$
H^{1}\left(\mathcal{X}_{U}^{\min }, \bigoplus_{j=0}^{\infty} \mathcal{E}_{U, \rho}^{\mathrm{sub}} \otimes \omega_{U}^{\otimes p^{M-1}(p-1) j} \otimes \mathbb{Z} / p^{M} \mathbb{Z}\right)=(0) .
$$

Let $S$ denote a finite set of rational primes containing $p$ and all rational primes $q$ which are both non-split in $F_{0}$ and ramified in $F$. Also choose a neat open compact subgroup

$$
U^{p}=G_{n}\left(\widehat{\mathbb{Z}}^{S}\right) \times U_{S}^{p} \subset G_{n}\left(\mathbb{A}^{\infty, p}\right) .
$$

Suppose that $v$ is a place of $F$ above a rational prime $q \notin S$ and let $i \in \mathbb{Z}$. There is a unique element $\mathfrak{t}_{v}^{(i)}$ in the Bernstein centre of $G_{n}\left(\mathbb{Q}_{q}\right)$ such that

- $\mathfrak{t}_{v}^{(i)}$ acts as 0 on any irreducible smooth representation of $G_{n}\left(\mathbb{Q}_{q}\right)$ over $\mathbb{C}$ which is not a subquotient of an unramified principal series;

- on an unramified representation $\Pi_{q}$ of $G_{n}\left(\mathbb{Q}_{q}\right)$ the eigenvalue of $\mathfrak{t}_{v}^{(i)}$ on $\Pi_{q}$ equals $\operatorname{tr} \operatorname{rec}_{F_{v}}\left(\mathrm{BC}\left(\Pi_{q}\right)_{v}|\operatorname{det}|_{v}^{(1-2 n) / 2}\right)\left(\operatorname{Frob}_{v}^{i}\right)$.

(See [4].) Multiplying $\mathfrak{t}_{v}^{(i)}$ by the characteristic function of $G_{n}\left(\mathbb{Z}_{q}\right)$ we obtain a unique element $T_{\nu}^{(i)} \in \mathbb{C}\left[G_{n}\left(\mathbb{Z}_{q}\right) \backslash G_{n}\left(\mathbb{Q}_{q}\right) / G_{n}\left(\mathbb{Z}_{q}\right)\right]$ such that if $\Pi_{q}$ is an unramified representation of $G_{n}\left(\mathbb{Q}_{q}\right)$ and if $T_{v}^{(i)}$ has eigenvalue $t_{v}^{(i)}\left(\Pi_{q}\right)$ on $\Pi_{q}^{G_{n}\left(\mathbb{Z}_{q}\right)}$ then

$$
\operatorname{tr} \operatorname{rec}_{F_{v}}\left(\mathrm{BC}\left(\Pi_{q}\right)_{v}|\operatorname{det}|_{v}^{(1-2 n) / 2}\right)\left(\operatorname{Frob}_{v}^{i}\right)=t_{v}^{(i)}\left(\Pi_{q}\right) .
$$

(See [29].) If $\sigma \in \operatorname{Aut}(\mathbb{C})$ we see that ${ }^{\sigma} T_{\nu}^{(i)}=T_{\nu}^{(i)}$. (Use the fact that

$$
\left.{ }^{\sigma} \operatorname{rec}_{F_{v}}\left(\mathrm{BC}\left(\Pi_{q}\right)_{v}|\operatorname{det}|_{v}^{(1-2 n) / 2}\right) \cong \operatorname{rec}_{F_{v}}\left(\mathrm{BC}\left({ }^{\sigma} \Pi_{q}\right)_{v}|\operatorname{det}|_{v}^{(1-2 n) / 2}\right) .\right)
$$


Thus

$$
T_{v}^{(i)} \in \mathbb{Q}\left[G_{n}\left(\mathbb{Z}_{q}\right) \backslash G_{n}\left(\mathbb{Q}_{q}\right) / G_{n}\left(\mathbb{Z}_{q}\right)\right]
$$

Choose $d_{\nu}^{(i)} \in \mathbb{Q}^{\times}$such that

$$
d_{v}^{(i)} T_{v}^{(i)} \in \mathbb{Z}\left[G_{n}\left(\mathbb{Z}_{q}\right) \backslash G_{n}\left(\mathbb{Q}_{q}\right) / G_{n}\left(\mathbb{Z}_{q}\right)\right]
$$

Suppose that $q \notin S$ is a rational prime. Let $u_{1}, \ldots, u_{r}$ denote the primes of $F^{+}$above $\mathbb{Q}$ which split $u_{i}=w_{i}{ }^{c} w_{i}$ in $F$, and let $v_{1}, \ldots, v_{s}$ denote the primes of $F^{+}$above $q$ which do not split in $F$. Then under the identification

$$
G_{n}\left(\mathbb{Q}_{q}\right) \cong \prod_{i=1}^{r} G L_{2 n}\left(F_{w_{i}}\right) \times H
$$

of Sect. 1.3, the Hecke operator $T_{w_{i}}^{(1)}$ is identified with the double coset

$$
G_{n}\left(\mathbb{Z}_{q}\right) a_{i} G_{n}\left(\mathbb{Z}_{q}\right)
$$

where $a_{i} \in G L_{n}\left(F_{w_{i}}\right)$ is the diagonal matrix $\operatorname{diag}\left(1, \ldots, 1, \varpi_{w_{i}}\right)$, and we may take $d_{w_{i}}^{(1)}=1$.

We will call a topological $\mathbb{Z}_{p}\left[G_{n}\left(\widehat{\mathbb{Z}}^{S}\right) \backslash G_{n}\left(\mathbb{A}^{S}\right) / G_{n}\left(\widehat{\mathbb{Z}}^{S}\right)\right]$-algebra $\mathbb{T}$ of Galois type if there is a continuous pseudo-representation (see [55])

$$
T: G_{F}^{S} \longrightarrow \mathbb{T}
$$

such that

$$
d_{v}^{(i)} T\left(\operatorname{Frob}_{v}^{i}\right)=\theta\left(d_{v}^{(i)} T_{v}^{(i)}\right)
$$

for all $v \mid q \notin S$ and all $i \in \mathbb{Z}$.

Let $\mathbb{T}_{U^{p}\left(N_{1}, N_{2}\right), \rho}$ denote the image of $\mathbb{Z}_{p}\left[G_{n}\left(\widehat{\mathbb{Z}}^{S}\right) \backslash G_{n}\left(\mathbb{A}^{S}\right) / G_{n}\left(\widehat{\mathbb{Z}}^{S}\right)\right]$ in the endomorphism algebra $\operatorname{End}\left(H^{0}\left(\mathcal{X}_{U^{p}\left(N_{1}, N_{2}\right.}^{\min }, \mathcal{E}_{\rho}^{\text {sub }}\right)\right)$, which is also the image in the endomorphism algebra $\operatorname{End}\left(H^{0}\left(X_{U^{p}\left(N_{1}, N_{2}\right)}^{\min }, \mathcal{E}_{\rho}^{\text {sub }}\right)\right)$.

Lemma 6.2 For t sufficiently large $\mathbb{T}_{U^{p}\left(N_{1}, N_{2}\right), \rho \otimes\left(\wedge^{n[F: \mathbb{Q}]} \operatorname{Std}^{\vee}\right)^{\otimes t}}^{S}$ is of Galois type.

Proof Write

$$
\rho_{t}=\rho \otimes\left(\wedge^{n[F: \mathbb{Q}]} \operatorname{Std}^{\vee}\right)^{\otimes t} .
$$

It suffices to show that there is a continuous pseudo-representation

$$
T: G_{F}^{S} \longrightarrow \mathbb{T}_{U^{p}\left(N_{1}, N_{2}\right), \rho_{t}}^{S} \otimes \overline{\mathbb{Q}}_{p}
$$

which is unramified outside $S$ and satisfies

$$
T\left(\operatorname{Frob}_{v}^{i}\right)=T_{v}^{(i)}
$$


for all $v \mid q \notin S$ and all $i \in \mathbb{Z}$. (Because $T$ will then automatically be valued in $\mathbb{T}_{U^{p}\left(N_{1}, N_{2}\right), \rho_{t}}^{S}$, by the Cebotarev density theorem. Note that if $v$ is a prime of $F$ split over $F^{+}$and lying above a rational prime $q \notin S$, then

$$
\left.T\left(\operatorname{Frob}_{v}\right)=T_{v}^{(1)} \in \mathbb{T}_{U^{p}\left(N_{1}, N_{2}\right), \rho_{t}}^{\cdot}\right)
$$

We may then reduce to the case that $\rho \otimes \overline{\mathbb{Q}}_{p}$ is irreducible. Let

$$
\left(b_{0},\left(b_{\tau, i}\right)\right) \in X^{*}\left(T_{n} / \overline{\mathbb{Q}}_{p}\right)_{(n)}^{+}
$$

denote the highest weight of $\rho \otimes \overline{\mathbb{Q}}_{p}$.

Suppose that $t$ satisfies the inequality

$$
-2 n \geq\left(b_{\tau, 1}-t\right)+\left(b_{\tau c, 1}-t\right) .
$$

By Lemma 5.11,

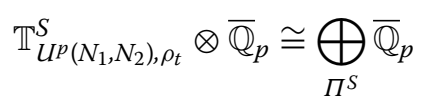

where the sum runs over irreducible admissible representations of $G_{n}\left(\mathbb{A}^{S, \infty}\right)$ for which there exists an irreducible admissible representation $\Pi_{S}$ of $\prod_{v \in S} G_{n}\left(\mathbb{Q}_{v}\right)$ such that $\Pi^{S} \otimes \Pi_{S}$ occurs in $H^{0}\left(X^{\min } \times \operatorname{Spec} \overline{\mathbb{Q}}_{p}, \mathcal{E}_{\rho_{t}}^{\text {sub }}\right)$ and $\left(\Pi^{S} \otimes \Pi_{S}\right)^{U^{p}\left(N_{1}, N_{2}\right)} \neq(0)$. Further, from Corollary 5.12 , we deduce that there is a continuous representation

$$
r: G_{F}^{S} \longrightarrow G L_{2 n}\left(\mathbb{T}_{U^{p}\left(N_{1}, N_{2}\right), \rho_{t}}^{S} \otimes \overline{\mathbb{Q}}_{p}\right)
$$

such that if $v \mid q \notin S$ then $r$ is unramified at $v$ and

$$
\operatorname{tr} r\left(\operatorname{Frob}_{v}^{i}\right)=T_{v}^{(i)}
$$

for all $i \in \mathbb{Z}$. Taking $T=\operatorname{tr} r$ completes the proof of the lemma.

If

$$
W \subset H^{0}\left(\mathfrak{X}_{U^{p}(N)}^{\mathrm{ord}, \min }, \mathcal{E}_{\rho}^{\mathrm{ord}, \mathrm{sub}}\right)
$$

(resp.

$$
\left.W \subset H^{0}\left(\mathcal{X}_{U^{p}\left(N_{1}, N_{2}\right)}^{\text {ord,min }}, \mathcal{E}_{\rho}^{\text {ord,sub }} \otimes \mathbb{Z} / p^{M} \mathbb{Z}\right)\right)
$$

is a finitely generated $\mathbb{Z}_{p}$-submodule invariant under the action of the Hecke algebra

$$
\mathbb{Z}_{p}\left[G_{n}\left(\widehat{\mathbb{Z}}^{S}\right) \backslash G_{n}\left(\mathbb{A}^{S}\right) / G_{n}\left(\widehat{\mathbb{Z}}^{S}\right)\right]
$$

then we will let $\mathbb{T}_{U^{p}(N), \rho}^{\text {ord }, S}(W)$ (resp. $\mathbb{T}_{U^{p}\left(N_{1}, N_{2}\right), \rho}^{\text {ord },}(W)$ ) denote the image of the abstract Hecke algebra $\mathbb{Z}_{p}\left[G_{n}\left(\widehat{\mathbb{Z}}^{S}\right) \backslash G_{n}\left(\mathbb{A}^{S}\right) / G_{n}\left(\widehat{\mathbb{Z}}^{S}\right)\right]$ in $\operatorname{End}_{\mathbb{Z}_{p}}(W)$. The next corollary follows from Lemmas 6.1 and 6.2 . 


\section{Corollary 6.3 If}

$$
W \subset H^{0}\left(\mathcal{X}_{U^{p}\left(N_{1}, N_{2}\right)}^{\text {ord, min }}, \mathcal{E}_{\rho}^{\text {ord,sub }} \otimes \mathbb{Z} / p^{M} \mathbb{Z}\right)
$$

is a finitely generated $\mathbb{Z}_{p}$-submodule invariant under the action of the Hecke algebra

$$
\mathbb{Z}_{p}\left[G_{n}\left(\widehat{\mathbb{Z}}^{S}\right) \backslash G_{n}\left(\mathbb{A}^{S}\right) / G_{n}\left(\widehat{\mathbb{Z}}^{S}\right)\right]
$$

then $\mathbb{T}_{U^{p}\left(N_{1}, N_{2}\right), \rho}^{\mathrm{ord},}(W)$ is of Galois type.

We deduce from this the next corollary.

\section{Corollary 6.4 If}

$$
W \subset H^{0}\left(\mathfrak{X}_{U^{p}(N)}^{\mathrm{ord}, \min }, \mathcal{E}_{\rho}^{\mathrm{ord}, \mathrm{sub}}\right)
$$

is a finitely generated $\mathbb{Z}_{p}$-submodule invariant under the action of the Hecke algebra

$$
\mathbb{Z}_{p}\left[G_{n}\left(\widehat{\mathbb{Z}}^{S}\right) \backslash G_{n}\left(\mathbb{A}^{S}\right) / G_{n}\left(\widehat{\mathbb{Z}}^{S}\right)\right],
$$

then $\mathbb{T}_{U^{p}(N), \rho}^{\mathrm{ord}, S}(W)$ is of Galois type.

Finally we deduce the following proposition.

Proposition 6.5 Suppose that $\rho$ is a representation of $L_{n,(n)}$ over $\mathbb{Z}_{(p)}$. Suppose also that $\Pi$ is an irreducible quotient of an admissible $G_{n}\left(\mathbb{A}^{\infty}\right)^{\text {ord, }} \times$-submodule $\Pi^{\prime}$ of $H^{0}\left(\mathfrak{X}^{\text {ord,min }}, \mathcal{E}_{\rho}^{\text {ord,sub }}\right)_{\overline{\mathbb{Q}}_{p}}$. Then there is a continuous semi-simple representation

$$
R_{p}(\Pi): G_{F} \longrightarrow G L_{2 n}\left(\overline{\mathbb{Q}}_{p}\right)
$$

with the following property: If $\Pi$ is unramified at a rational prime $q \neq p$ (in the sense of Sect. 1.3) and if $v \mid q$ is a prime of $F$, then

$$
\mathrm{WD}\left(\left.R_{p}(\Pi)\right|_{G_{F_{v}}}\right)^{\mathrm{F}-\mathrm{ss}} \cong \operatorname{rec}_{F_{v}}\left(\mathrm{BC}\left(\Pi_{q}\right)_{v}|\operatorname{det}|_{v}^{(1-2 n) / 2}\right) .
$$

Proof Let $S$ denote the set of rational primes consisting of $p$ and the primes where $F$ or $\Pi$ ramifies. Also choose a neat open compact subgroup

$$
U^{p}=G_{n}\left(\widehat{\mathbb{Z}}^{S}\right) \times U_{S}^{p}
$$

and integer $N$ such that

$$
\Pi^{U^{p}(N)} \neq(0)
$$

As $\left(\Pi^{\prime}\right)^{U^{p}(N)}$ is a finite dimensional, and hence closed, subspace of the topological vector space $H^{0}\left(\mathfrak{X}^{\text {ord,min }}, \mathcal{E}_{\rho}^{\text {ord,sub }}\right)_{\overline{\mathbb{Q}}_{p}}$ preserved by $\mathbb{Z}_{p}\left[G_{n}\left(\widehat{\mathbb{Z}}^{S}\right) \backslash G_{n}\left(\mathbb{A}^{S}\right) / G_{n}\left(\widehat{\mathbb{Z}}^{S}\right)\right]$ and, as there is a $\mathbb{Z}_{p}\left[G_{n}\left(\widehat{\mathbb{Z}}^{S}\right) \backslash G_{n}\left(\mathbb{A}^{S}\right) / G_{n}\left(\widehat{\mathbb{Z}}^{S}\right)\right]$-equivariant map $\left(\Pi^{\prime}\right)^{U^{p}(N)} \rightarrow \Pi^{U^{p}(N)}$, there is a continuous homomorphism

$$
\theta: \mathbb{T}_{U^{p}(N), \rho}^{\mathrm{ord}, S}\left(\left(\Pi^{\prime}\right)^{U^{p}(N)}\right) \longrightarrow \overline{\mathbb{Q}}_{p}
$$


which for $v \mid q \notin S$ sends $T_{v}^{(i)}$ to its eigenvalue on $\Pi^{G_{n}\left(\mathbb{Z}_{q}\right)}$. Proposition 6.5 now follows from the above corollary and the main theorem on pseudo-representations (see [55]).

We remark that we don't know how to prove this proposition for a general irreducible subquotient of $H^{0}\left(\mathfrak{X}^{\text {ord,min }}, \mathcal{E}_{\rho}^{\text {ord,sub }}\right)_{\overline{\mathbb{Q}}_{p}}$ (or indeed whether the corresponding statement remains true).

\subsection{Interlude concerning linear algebra}

Suppose that $K$ is an algebraic extension of $\mathbb{Q}_{p}$. For $a \in \mathbb{Q}$, we say that a polynomial $P(X) \in K(X)$ has slopes $\leq a$ if $P(X) \neq 0$ and every root of $P(X)$ in $\bar{K}$ has $p$-adic valuation $\leq a$. (We normalize the $p$-adic valuation so that $p$ has valuation 1.) If $V$ is a $K$-vector space and $T$ is an endomorphism of $V$, then we say that $V$ admits slope decompositions for $T$, if for each $a \in \mathbb{Q}$ there is a decomposition

$$
V=V_{\leq a} \oplus V_{>a}
$$

with the following properties:

- $T$ preserves $V_{\leq a}$ and $V_{>a}$;

- $V_{\leq a}$ is finite dimensional;

- if $P(X) \in K[X]$ has slopes $\leq a$ then the endomorphism $P(T)$ restricts to an automorphism of $V_{>a}$;

- there is a nonzero polynomial $P(X) \in K[X]$ with slopes $\leq a$ such that the endomorphism $P(T)$ restricts to 0 on $V_{\leq a}$.

In this case $V_{\leq a}$ and $V_{>a}$ are unique, and we refer to them as the slope a decomposition of $V$ with respect to $T$.

Lemma 6.6 (1) If $V$ is finite dimensional then it always admits slope decompositions.

(2) If $K$ is a finite extension of $\mathbb{Q}_{p}$, if $V$ is a $K$-Banach space and if $T$ is a completely continuous (see [50]) endomorphism of $V$ then $V$ admits slope decompositions for $T$.

(3) Suppose that $L / K$ is an algebraic extension and that $V$ is a $K$ vector space which admits slope decompositions with respect to an endomorphism $T$. Then $V \otimes_{K} L$ also admits slope decompositions with respect to $T$.

(4) Suppose that $V_{1}$ admits slope decompositions with respect to $T_{1}$; that $V_{2}$ admits a slope decomposition with respect to $T_{2}$; and that $d: V_{1} \rightarrow V_{2}$ is a linear map such that

$$
d \circ T_{1}=T_{2} \circ d
$$

Then for all $a \in \mathbb{Q}$ we have

$$
d V_{1, \leq a} \subset V_{2, \leq a}
$$

and

$$
d V_{1,>a} \subset V_{2,>a}
$$


Moreover ker $d$ admits slope decompositions for $T_{1}$, while $\operatorname{Im} d$ and coker $d$ admit slope decompositions for $T_{2}$. More specifically

$(\operatorname{ker} d)_{\leq a}=(\operatorname{ker} d) \cap V_{1, \leq a}$

and

$(\operatorname{ker} d)_{>a}=(\operatorname{ker} d) \cap V_{1,>a}$

and

$$
(\operatorname{Im} d)_{\leq a}=V_{1, \leq a} /(\operatorname{ker} d)_{\leq a}
$$

and

$$
(\operatorname{Im} d)_{>a}=V_{1,>a} /(\operatorname{ker} d)_{>a}
$$

and

$$
(\operatorname{coker} d)_{\leq a}=V_{2, \leq a} /(\operatorname{Im} d)_{\leq a}
$$

and

$$
(\operatorname{coker} d)_{>a}=V_{2,>a} /(\operatorname{Im} d)_{>a} .
$$

(5) Suppose that

$$
V_{1} \subset V_{2} \subset V_{3} \subset \cdots \subset V_{\infty}
$$

are vector spaces with

$$
V_{\infty}=\bigcup_{i=1}^{\infty} V_{i} .
$$

Suppose also that $T$ is an endomorphism of $V_{\infty}$ such that for all $i>1$

$$
T V_{i} \subset V_{i-1} .
$$

If for each $i$ the space $V_{i}$ admits slope decompositions for $i$, then $V_{\infty}$ admits slope decompositions for $T$.

(6) Suppose that

$$
(0) \longrightarrow V_{1} \longrightarrow V \longrightarrow V_{2} \longrightarrow(0)
$$

is an exact sequence of $K$-vector spaces and that $T$ is an endomorphism of $V$ that preserves $V_{1}$. If $V_{1}$ and $V_{2}$ both admit slope decompositions with respect to $T$, then so does $V$. Moreover we have short exact sequences

$$
(0) \longrightarrow V_{1, \leq a} \longrightarrow V_{\leq a} \longrightarrow V_{2, \leq a} \longrightarrow(0)
$$

and

$$
(0) \longrightarrow V_{1,>a} \longrightarrow V_{>a} \longrightarrow V_{2,>a} \longrightarrow(0)
$$


Proof The first and third and fourth parts are straightforward. The second part follows from [50].

For the fifth part one checks that $V_{i, \leq a}$ is independent of $i$. If we set

$$
V_{\infty, \leq a}=V_{i, \leq a}
$$

for any $i$, and

$$
V_{\infty,>a}=\bigcup_{i=1}^{\infty} V_{i,>a}
$$

then these provide the slope $a$ decomposition of $V_{\infty}$ with respect to $T$.

Finally we turn to the sixth part. Choose nonzero polynomials $P_{i}(X) \in K[X]$ with slopes $\leq a$ such that $P_{i}(T) V_{i, \leq a}=(0)$, for $i=1,2$. Set $P(X)=P_{1}(X) P_{2}(X)$. Also set $V_{\leq a}=\operatorname{ker} P(T)$ and $V_{>a}=\operatorname{Im} P(T)$. We have complexes

$$
(0) \longrightarrow V_{1,>a} \longrightarrow V_{>a} \longrightarrow V_{2,>a} \longrightarrow(0)
$$

and

$$
(0) \longrightarrow V_{1, \leq a} \longrightarrow V_{\leq a} \longrightarrow V_{2, \leq a} \longrightarrow(0)
$$

It suffices to show that these complexes are both short exact sequences. For then we see that, if $Q(X) \in K[X]$ has slopes $\leq a$, then the restriction of $Q(T)$ to $V_{>a}$ is an automorphism of $V_{>a}$. Applying this to $P(T)$, we see that $V_{\leq a} \cap V_{>a}=(0)$. Moreover $V_{\leq a}+V_{>a}$ contains $V_{1}$ and maps onto $V_{2}$, so that $V=V_{\leq a}+V_{>a}$.

To show the first complex is short exact we need only check that $V_{1,>a}=V_{>a} \cap V_{1}$, i.e. that $V_{1, \leq a} \cap V_{>a}=(0)$. So suppose that $v \in V_{1, \leq a} \cap V_{>a}$. Then $v=P(T) v^{\prime}$ and $P_{1}(T) v=0$. Thus $P_{1}(T)^{2} P_{2}(T) v^{\prime}=0$ so the image of $v^{\prime}$ in $V_{2}$ lies in $V_{2, \leq a}$ and so $P_{2}(T) v^{\prime} \in V_{1}$, and in fact $P_{2}(T) v^{\prime} \in V_{1, \leq a}$. Finally we see that $v=P_{1}(T) P_{2}(T) v^{\prime}=0$, as desired.

To show the second complex is short exact we have only to show that $V_{\leq a} \rightarrow V_{2, \leq a}$ is surjective. So suppose that $\bar{v} \in V_{2, \leq a}$ and suppose that $v \in V$ lifts $\bar{v}$. Then $P(T) v \in V_{1,>a}$. Set

$$
v^{\prime}=v-\left(\left.P(T)\right|_{V_{1,>a}} ^{-1}\right) P(T) v \in v+V_{1,>a}
$$

Then $v^{\prime}$ maps to $\bar{v} \in V_{2}$, while

$$
P(T) v^{\prime}=P(T) v-P(T) v=0,
$$

so that $v^{\prime} \in V_{\leq a}$.

We warn the reader that to the best of our knowledge it is not in general true that, if $V$ admits a slope decomposition for $T$ and $V_{1} \subset V$ is $T$-invariant, then either $V_{1}$ or $V / V_{1}$ admits slope decompositions for $T$. 


\subsection{The ordinary locus of a toroidal compactification as a dagger space}

\subsubsection{Review of dagger spaces}

We first review some general facts about dagger spaces. We refer to [27] for the basic facts.

Suppose that $K / \mathbb{Q}_{p}$ is a finite extension with ring of integers $\mathcal{O}_{K}$ and residue field $k$. Suppose also that $\mathcal{Y} / \mathcal{O}_{K}$ is quasi-projective. Let $Y$ denote the generic fibre $\mathcal{Y} \times \operatorname{Spec} K$, let $\bar{Y}$ denote the special fibre $\mathcal{Y} \times \operatorname{Spec} k$, and let $\mathcal{Y}^{\wedge}$ denote the formal completion of $\mathcal{Y}$ along $\bar{Y}$. Let $Y^{\text {an }}$ (resp. $Y^{\dagger}$ ) denote the rigid analytic (resp. dagger) space associated to $Y$. (For the latter see section 3.3 of [27].) Thus $Y^{\text {an }}$ and $Y^{\dagger}$ share the same underlying G-topological space, and in fact the completion $\left(Y^{\dagger}\right)^{\prime}$ (see theorem 2.19 of [27]) of $Y^{\dagger}$ equals $Y^{\text {an }}$. Let $\mathcal{Y}_{\eta}^{\wedge}$ denote the rigid analytic space associated to $\mathcal{Y}^{\wedge}$, its 'generic fibre'. Then $\mathcal{Y}_{\eta}^{\wedge}$ is identified with an admissible open subset $] \bar{Y}\left[\subset Y^{\text {an }}\right.$. We will denote by $\mathcal{Y}^{\dagger}$ the admissible open dagger subspace of $Y^{\dagger}$ with the same underlying topological space as ] $\bar{Y}[$.

To a coherent sheaf $\mathcal{F} / Y$ one can associate a coherent sheaf $\mathcal{F}^{\dagger} / Y^{\dagger}$ and hence $\mathcal{F}^{\dagger} / \mathcal{Y}^{\dagger}$. The functor $\mathcal{F} \mapsto \mathcal{F}^{\dagger}$ from coherent sheaves on $Y$ to coherent sheaves on $\mathcal{Y}^{\dagger}$ is exact.

Lemma 6.7 If $\mathcal{Y}$ and $\mathcal{Y}^{\prime}$ are two quasi-projective $\mathcal{O}_{K}$-schemes as described in the previous paragraphand iff $: \mathcal{Y} \rightarrow \mathcal{Y}^{\prime}$ is a morphism, then there is an induced mapf $f^{\dagger}: \mathcal{Y}^{\dagger} \rightarrow\left(\mathcal{Y}^{\prime}\right)^{\dagger}$. If further $f: \bar{Y} \stackrel{\sim}{\rightarrow} \bar{Y}^{\prime}$ and $f$ is etale in a neighbourhood of $\bar{Y}$ then $f^{\dagger}$ is an isomorphism.

Proof The first part of the lemma is clear.

For the second part, let $\mathcal{Y} \hookrightarrow \mathbb{P}_{\mathcal{O}_{K}}^{M}$ and $\mathcal{Y}^{\prime} \hookrightarrow \mathbb{P}_{\mathcal{O}_{K}}^{M^{\prime}}$ be closed embeddings. Let $\mathcal{P}^{\prime}$ denote the closure of $\mathcal{Y}^{\prime}$ in $\mathbb{P}_{\mathcal{O}_{K}}^{M^{\prime}}$. Also let $\mathcal{P}$ denote the closure of $\mathcal{Y}$ in $\mathbb{P}_{\mathcal{O}_{K}}^{M} \times \mathbb{P}_{\mathcal{O}_{K}}^{M^{\prime}}$. Then $f$ extends to a map $\mathcal{P} \rightarrow \mathcal{P}^{\prime}$. The second part of the lemma follows from theorem 1.3.5 of [6] applied to $\bar{Y} \subset \mathcal{P}$ and $\bar{Y}^{\prime} \subset \mathcal{P}^{\prime}$.

We will let $H_{\text {rig }}^{i}(\bar{Y})$ denote the rigid cohomology of $\bar{Y}$ in the sense of Berthelot-see, for instance, [46].

Lemma 6.8 (1) If $\mathcal{Y} / \mathcal{O}_{K}$ is a smooth and quasi-projective scheme, then there is a canonical isomorphism

$$
H_{\text {rig }}^{i}(\bar{Y}) \cong \mathbb{H}^{i}\left(\mathcal{Y}^{\dagger}, \Omega_{\mathcal{Y}^{\dagger}}^{\bullet}\right)
$$

(2) If $f: \mathcal{Y} \rightarrow \mathcal{Z}$ is a morphism of smooth quasi-projective schemes over $\mathcal{O}_{K}$ then the following diagram is commutative:

$$
\begin{array}{ccc}
H_{\mathrm{rig}}^{i}(\bar{Z}) & \stackrel{f^{*}}{\longrightarrow} & H_{\mathrm{rig}}^{i}(\bar{Y}) \\
\mathbb{H}^{i}\left(\mathcal{Z}^{\dagger}, \Omega_{\mathcal{Z}^{\dagger}}^{\bullet}\right) \stackrel{f^{*}}{\longrightarrow} \mathbb{H}^{i}\left(\mathcal{Y}^{\dagger}, \Omega_{\mathcal{Y}^{\dagger}}^{\bullet}\right) .
\end{array}
$$

Proof For the first part apply theorem 5.1 of [27] to the closure of $\mathcal{Y}$ in some projective space over $\mathcal{O}_{K}$. For the second part choose embeddings $i: \mathcal{Y} \hookrightarrow \mathbb{P}_{\mathcal{O}_{K}}^{M}$ and $i^{\prime}: \mathcal{Z} \hookrightarrow \mathbb{P}_{\mathcal{O}_{K}}^{M^{\prime}}$. Let $\mathcal{P}^{\prime}$ denote the closure of $\mathcal{Z}$ in $\mathbb{P}_{\mathcal{O}_{K}}^{M^{\prime}}$ and $\mathcal{P}$ the closure of $\mathcal{Y}$ in $\mathbb{P}_{\mathcal{O}_{K}}^{M} \times \mathcal{P}^{\prime}$, so that $f$ extends to a map $\mathcal{P} \rightarrow \mathcal{P}^{\prime}$. The desired result again follows from theorem 5.1 of [27], because the isomorphisms of theorem 5.1 of [27] are functorial under morphisms of the setup in that theorem. 
[It is unclear to us whether this functoriality is supposed to be implied by the word 'canonical' in the statement of theorem 5.1 of [27]. For safety's sake we sketch the argument for this functoriality. More precisely if $f: \mathcal{X}_{1} \rightarrow \mathcal{X}_{2}$ is a morphism of proper admissible formal Spf $R$-schemes which takes $Y_{1} \subset \mathcal{X}_{1, s}$ to $Y_{2} \subset \mathcal{X}_{2, s}$, then we will show that the isomorphisms of theorem 5.1 of [27] are compatible with the maps in cohomology induced by $f$. For part (a) we also suppose that we are given a map $f^{*}: f^{*} \mathcal{F}_{2} \rightarrow \mathcal{F}_{1}$.

Using the notation of part (a) of theorem 5.1 of [27], it suffices to show that the diagram

$$
\begin{array}{ccc}
H^{q}\left(X_{2}, \mathcal{F}_{2, X_{2}}\right) & \stackrel{f^{*}}{\longrightarrow} & H^{q}\left(X_{1}, \mathcal{F}_{1, X_{1}}\right) \\
\downarrow & \downarrow & \downarrow \\
H^{q}\left(\bar{Y}_{2}\left[\mathcal{X}_{2}, j_{2}^{\dagger} \mathcal{F}_{2}^{\prime}\right)\right. & \stackrel{f^{*}}{\longrightarrow} & H^{q}(] \bar{Y}_{1}\left[\mathcal{X}_{2}, j_{2}^{\dagger} \mathcal{F}_{2}^{\prime}\right)
\end{array}
$$

commutes. (The functoriality of parts (b) and (c) follow easily from the functoriality of part (a).) The vertical morphisms arise from maps $L_{k}^{\bullet} \rightarrow K_{k}^{\bullet}$ of resolutions of the sheaves $R i_{*} \mathcal{F}_{k, X_{k}}$ and $j_{k}^{\dagger} \mathcal{F}_{k}^{\prime}$, respectively. To define these resolutions one needs to choose affine covers $\left\{Y_{k, i}\right\}$ of $Y_{k}$. We may suppose these are chosen so that $f$ carries $Y_{1, i}$ to $Y_{2, i}$ for all $i$. Then $L_{k}^{\bullet}$ and $K_{k}^{\bullet}$ are the Cech complexes with

$$
L_{k}^{q}=\bigoplus_{\# J=q} i_{J *} \mathcal{F}_{k,] Y_{k, J}\left[\mathcal{X}_{k}\right.}
$$

and

$$
K_{k}^{q}=\bigoplus_{\# J=q} j_{k, J}^{\dagger} \mathcal{F}_{k}^{\prime}
$$

The maps $L_{k}^{\bullet} \rightarrow K_{k}^{\bullet}$ arise from maps

$$
\left(i_{J *} \mathcal{F}_{k,] Y_{k, J}\left[\mathcal{X}_{k}\right.}\right)(U) \cong \lim _{\rightarrow V} \mathcal{F}_{k}^{\prime}(V) \longrightarrow \lim _{\rightarrow V^{\prime}} \mathcal{F}_{k}^{\prime}\left(V^{\prime} \cap U\right)=\left(j_{k, J}^{\dagger} \mathcal{F}_{k}^{\prime}\right)(U)
$$

Here $V$ runs over strict neighbourhoods of $U \cap] Y_{k, J}\left[\mathcal{X}_{k}\right.$ in $] \bar{Y}_{k}\left[\mathcal{X}_{k}\right.$ and $V^{\prime}$ runs over strict neighbourhoods of $] Y_{k, J}\left[\mathcal{X}_{k}\right.$ in $] \bar{Y}_{k}\left[\mathcal{X}_{k}\right.$. The first isomorphism is justified in section 2.23 of [27]. The second morphism arises because, for every $V$, we can find a $V^{\prime}$ so that

$$
V^{\prime} \cap U \subset V .
$$

It suffices to show that if $f U_{1} \subset U_{2}$, then the diagrams

$$
\begin{array}{ccc}
\left(i_{J *} \mathcal{F}_{2,] Y_{2, J}\left[\mathcal{X}_{2}\right.}\right)(U)_{2} & \stackrel{f^{*}}{\longrightarrow}\left(i_{J *} \mathcal{F}_{1,] Y_{1, J}\left[\mathcal{X}_{1}\right.}\right)\left(U_{1}\right) \\
\downarrow & & \downarrow \\
\left(j_{2, J}^{\dagger} \mathcal{F}_{2}^{\prime}\right)\left(U_{2}\right) & \stackrel{f^{*}}{\longrightarrow} & \left(j_{1, J}^{\dagger} \mathcal{F}_{1}^{\prime}\right)\left(U_{1}\right)
\end{array}
$$

are commutative. But this is now clear.]

Lemma 6.9 Suppose that $f: X \rightarrow Y$ is a proper morphism between $\mathbb{Q}_{p}$-schemes of finite type and that $\mathcal{F} / X$ is a coherent sheaf. Denote by $f^{\dagger}: X^{\dagger} \rightarrow Y^{\dagger}$ the corresponding map 
of dagger spaces and by $\mathcal{F}^{\dagger}$ the coherent sheaf on $X^{\dagger}$ corresponding to $\mathcal{F} / X$. Suppose also that $V$ is an admissible open subset of $Y^{\dagger}$ and that $U$ is its pre-image in $X^{\dagger}$. Then

$$
\left.R^{i}\left(\left.f^{\dagger}\right|_{U}\right)_{*}\left(\left.\mathcal{F}^{\dagger}\right|_{U}\right) \cong\left(R^{i} f_{*} \mathcal{F}\right)^{\dagger}\right|_{V}
$$

where $\left(R^{i} f_{*} \mathcal{F}\right)^{\dagger}$ denotes the coherent sheaf on $Y^{\dagger}$ corresponding to $\left(R^{i} f_{*} \mathcal{F}\right) / Y$.

Proof It suffices to check this in the case $V=Y^{\dagger}$. There is a chain of isomorphisms

$$
\left[\left(R^{i} f_{*} \mathcal{F}\right)^{\dagger}\right]^{\text {an }} \rightarrow\left(R^{i} f_{*} \mathcal{F}\right)^{\text {an }} \rightarrow R^{i} f_{*}^{\text {an }} \mathcal{F}^{\text {an }} \rightarrow\left(R^{i} f_{*}^{\dagger} \mathcal{F}^{\dagger}\right)^{\text {an }}
$$

The first arrow is the transitivity of dagger and rigid analytification. The second arrow is theorem 6.5 of [38]. The third arrow is theorem 3.5 of [27]. Since $Y^{\dagger}$ is partially proper, theorem 2.26 of [27] implies that there is a unique isomorphism $\left(R^{i} f_{*} \mathcal{F}\right)^{\dagger} \cong R^{i} f_{*}^{\dagger} \mathcal{F}^{\dagger}$ which recovers the above map after passage to rigid spaces.

\subsubsection{The ordinary locus as a dagger space}

Now we return to our Shimura and Kuga-Sato varieties.

If $U^{p}$ is a neat open compact subgroup of $G_{n}^{(m)}\left(\mathbb{A}^{\infty, p}\right)$, if $N_{2} \geq N_{1} \geq 0$ and if $\left(U^{p}\left(N_{1}, N_{2}\right), \Sigma\right) \in \mathcal{J}^{(m) \text {,tor }}$, we will write

$$
\mathcal{A}_{U^{p}\left(N_{1}, N_{2}\right), \Sigma}^{(m), \text { ord } \dagger}
$$

(resp.

$$
\partial \mathcal{A}_{U^{p}\left(N_{1}, N_{2}\right), \Sigma}^{(m), \text { ord }}
$$

resp.

$$
\partial_{[\sigma]} \mathcal{A}_{U^{p}\left(N_{1}, N_{2}\right), \Sigma}^{(m), \text { or },}
$$

for $\left.[\sigma] \in \mathcal{S}\left(U^{p}\left(N_{1}, N_{2}\right), \Sigma\right)\right)$ for the dagger space associated to $\mathcal{A}_{U^{p}\left(N_{1}, N_{2}\right), \Sigma}^{(m) \text { ord }}$ (resp. $\partial \mathcal{A}_{U^{p}\left(N_{1}, N_{2}\right), \Sigma}^{(m), \text { rd }}$, resp. $\left.\partial_{[\sigma]} \mathcal{A}_{U^{p}\left(N_{1}, N_{2}\right), \Sigma}^{(m), \text { ord }}\right)$ as described in the paragraph before Lemma 6.7.

For $s>0$ also write

$$
\partial^{(s)} \mathcal{A}_{U^{p}\left(N_{1}, N_{2}\right), \Sigma}^{(m), \text { ord } \dagger}=\coprod_{\substack{[\sigma] \in \mathcal{S}\left(U^{p}\left(N_{1}, N_{2}\right), \Sigma\right) \\ \operatorname{dim}[\sigma]=s-1}} \partial_{[\sigma]} \mathcal{A}_{U^{p}\left(N_{1}, N_{2}\right), \Sigma}^{(m), \text { ord },}
$$

and $i^{(s)}$ for the finite map

$$
\partial^{(s)} \mathcal{A}_{U^{p}\left(N_{1}, N_{2}\right), \Sigma}^{(m), \text { ord } \dagger} \longrightarrow \partial \mathcal{A}_{U^{p}\left(N_{1}, N_{2}\right), \Sigma}^{(m), \text { ord },} \hookrightarrow \mathcal{A}_{U^{p}\left(N_{1}, N_{2}\right), \Sigma}^{(m), \text { rerd }}
$$

We set

$$
\partial^{(0)} \mathcal{A}_{U^{p}\left(N_{1}, N_{2}\right), \Sigma}^{(m), \mathrm{rd} \dagger}=\mathcal{A}_{U^{p}\left(N_{1}, N_{2}\right), \Sigma}^{(m), \mathrm{rrd},}
$$


and

$$
i^{(0)}=1_{\mathcal{A}_{u^{p}\left(N_{1}, N_{2}\right), \Sigma}^{(m), \mathrm{ord}}, \dagger} .
$$

Then the various systems of dagger spaces $\left\{\mathcal{A}_{U^{p}\left(N_{1}, N_{2}\right), \Sigma}^{(m), \text { ord },}\right\}$ and $\left\{\partial \mathcal{A}_{U^{p}\left(N_{1}, N_{2}\right), \Sigma}^{(m), \text { ord },}\right\}$ and $\left\{\partial^{(s)} \mathcal{A}_{U^{p}\left(N_{1}, N_{2}\right), \Sigma}^{(m), \Sigma^{\prime},}\right\}$ have compatible actions of $G_{n}^{(m)}\left(\mathbb{A}^{\infty}\right)^{\text {ord }}$.

If $N_{2}^{\prime} \geq N_{2}$ and if $\Sigma^{\prime}$ is a refinement of $\Sigma$ with $\Sigma^{\text {ord }}=\left(\Sigma^{\prime}\right)^{\text {ord }}$ then the natural map

$$
\mathcal{A}_{U^{p}\left(N_{1}, N_{2}^{\prime}\right), \Sigma^{\prime}}^{(m), \mathrm{rd}} \longrightarrow \mathcal{A}_{U^{p}\left(N_{1}, N_{2}\right), \Sigma}^{(m), \text { ord }}
$$

restricts to an isomorphism

$$
\bar{A}_{U^{p}\left(N_{1}, N_{2}^{\prime}\right), \Sigma^{\prime}}^{(m), \text { ord }} \stackrel{\sim}{\longrightarrow} \bar{A}_{U^{p}\left(N_{1}, N_{2}\right), \Sigma}^{(m), \text { ord }}
$$

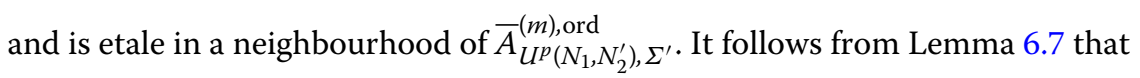

$$
\mathcal{A}_{U^{p}\left(N_{1}, N_{2}^{\prime}\right), \Sigma^{\prime}}^{(m), \text { ord } \dagger} \longrightarrow \mathcal{A}_{U^{p}\left(N_{1}, N_{2}\right), \Sigma}^{(m), \text { ord },}
$$

is an isomorphism. We will denote this dagger space simply

$$
\mathcal{A}_{U^{p}\left(N_{1}\right), \Sigma^{\text {ord }}}^{(m) \text { ord }}
$$

Similarly $\partial \mathcal{A}_{U^{p}\left(N_{1}, N_{2}\right), \Sigma}^{(m), \text { ord },}$ and $\partial_{[\sigma]} \mathcal{A}_{U^{p}\left(N_{1}, N_{2}\right), \Sigma}^{(m), \text { ord }, \dagger}$ and $\partial^{(s)} \mathcal{A}_{U^{p}\left(N_{1}, N_{2}\right), \Sigma}^{(m), \text { ord },}$ depend only on the group $U^{p}\left(N_{1}\right)$ and $\Sigma^{\text {ord }}$ and we will denote them $\partial \mathcal{A}_{U^{p}\left(N_{1}\right), \Sigma^{\text {ord }}}^{(m) \text { ond }, \uparrow} \partial_{[\sigma]} \mathcal{A}_{U^{p}\left(N_{1}\right), \Sigma^{\text {ord }}}^{(m) \text {,ord } \dagger}$ and $\partial^{(s)} \mathcal{A}_{U^{p}\left(N_{1}\right), \Sigma^{\text {ord }}}^{(m) \text {,ord }}$, respectively. If $[\sigma] \notin \mathcal{S}\left(U^{p}\left(N_{1}\right), \Sigma^{\text {ord }}\right)^{\text {ord }}$ then

$$
\partial_{[\sigma]} \mathcal{A}_{U^{p}\left(N_{1}\right), \Sigma^{\text {ord }}}^{(m), \text { od }}=\emptyset .
$$

Thus for $s>0$

$$
\partial^{(s)} \mathcal{A}_{U^{p}(N), \Sigma^{\text {ord }}}^{(m), \text { ord },}=\coprod_{\substack{[\sigma] \in \mathcal{S}\left(U^{p}(N), \Sigma^{\text {ord }}\right)^{\text {ord }} \\ \operatorname{dim}[\sigma]=s-1}} \partial_{[\sigma]} \mathcal{A}_{U^{p}(N), \Sigma^{\text {ord }}}^{(m) \text { ord }}
$$

The three projective systems of dagger spaces $\left\{\mathcal{A}_{U^{p}(N), \Sigma^{\text {ord }}}^{(m), \text { ord } \dagger}\right\}$ and $\left\{\partial \mathcal{A}_{U^{p}(N), \Sigma^{\text {ord }}}^{(m), \text { ord } \dagger}\right\}$ and $\left\{\partial^{(s)} \mathcal{A}_{U^{p}(N), \Sigma^{\text {ord }}}^{(m), \text { ord }}\right\}$ have actions of $G_{n}^{(m)}\left(\mathbb{A}^{\infty}\right)^{\text {ord }}$.

We will write $\mathfrak{X}_{\left(U^{p}\right)^{\prime}(N), \Delta}^{\mathrm{ord}, \dagger}$ for $\mathfrak{A}_{\left(U^{p}\right)^{\prime}(N), \Delta}^{(0), \text { ord }}$. If $\left(U^{p}\right)^{\prime}$ contains the projection of $U^{p}$ and if $\Delta^{\text {ord }}$ and $\Sigma^{\text {ord }}$ are compatible, then there are maps

$$
\mathcal{A}_{U_{p}(N), \Sigma^{\text {ord }}}^{(m), \text { ord } \dagger} \longrightarrow \mathcal{X}_{\left(U^{p}\right)^{\prime}(N), \Delta^{\text {ord }}}^{\text {ord } \dagger}
$$

These maps are $G_{n}^{(m)}\left(\mathbb{A}^{\infty}\right)^{\text {ord }}$-equivariant (as $U^{p},\left(U^{p}\right)^{\prime}$ and $N$ vary).

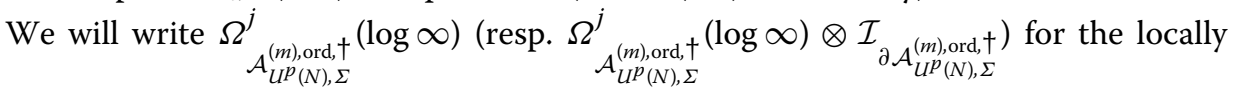
free sheaf on $\mathcal{A}_{U^{p}(N), \Sigma}^{(m), \text { ord },+}$ induced by $\Omega_{\mathcal{A}_{U^{p}\left(N, N^{\prime}\right), \Sigma^{\prime}}^{(m), \text { ord }}}^{j}(\log \infty)\left(\right.$ resp. $\Omega_{\mathcal{A}_{U p^{p}\left(N, N^{\prime}\right), \Sigma^{\prime}}^{(m), \text { ord }}}^{j}(\log \infty) \otimes$ 
$\left.\mathcal{I}_{\partial \mathcal{A}_{U p\left(N, N^{\prime}\right), \Sigma^{\prime}}^{(m), \text { ord }}}\right)$ for any $N^{\prime} \geq N$ and $\Sigma^{\prime} \in \mathcal{J}_{n}^{(m) \text {,tor }}$ with $\left(\Sigma^{\prime}\right)^{\text {ord }}=\Sigma$. This is canonically independent of the choices of $N^{\prime}$ and $\Sigma^{\prime}$. The systems of sheaves $\left\{\Omega_{\mathcal{A}_{U p(N), \Sigma}^{j}}^{(m), \text { ord } \dagger}\right.$ (log $\left.\left.\infty\right)\right\}$ and $\left\{\Omega_{\mathcal{A}_{U^{p}(N), \Sigma}^{(m), \text { ord },}}^{j}(\log \infty) \otimes \mathcal{I}_{\partial \mathcal{A}_{U^{p}(N), \Sigma}^{(m), \text { ord }, \dagger}}\right\}$ over $\left\{\mathcal{A}_{U^{p}(N), \Sigma}^{(m), \text { ord, } \dagger}\right\}$ have actions of $G_{n}^{(m)}\left(\mathbb{A}^{\infty}\right)^{\text {ord }}$. For $g \in G_{n}^{(m)}\left(\mathbb{A}^{\infty}\right)^{\text {ord }}$ the map

$$
g: g^{*} \Omega_{\mathcal{A}_{\left(U^{p}\right)^{\prime}\left(N^{\prime}\right), \Sigma^{\prime}}^{(m), \text { ord }}}^{j}(\log \infty) \longrightarrow \Omega_{\mathcal{A}_{U^{p}(N), \Sigma}^{(m), \mathrm{ord}, \dagger}}^{j}(\log \infty)
$$

is an isomorphism.

We will also write $\Omega_{\partial^{(s)} \mathcal{A}_{U^{p}(N), \Sigma}^{(m), \text { ord },}}^{j}$ for the sheaf of $j$-forms on $\partial^{(s)} \mathcal{A}_{U^{p}(N), \Sigma}^{(m), \text { ord, } \dagger}$. The system $\left\{\Omega_{\partial^{(s)} \mathcal{A}_{U^{p}(N), \Sigma}^{(m), \text { ord },}}^{j}\right\}$ over $\left\{\partial^{(s)} \mathcal{A}_{U^{p}(N), \Sigma}^{(m), \text { ord, },}\right\}$ has an action of $G_{n}^{(m)}\left(\mathbb{A}^{\infty}\right)^{\text {ord }}$

Furthermore if $\rho$ is a representation of $L_{n,(n)}$ on a finite dimensional $\mathbb{Q}_{p}$-vector space, there is a locally free sheaf $\mathcal{E}_{U^{p}(N), \Delta, \rho}^{\text {can } \dagger}\left(\right.$ resp. $\left.\mathcal{E}_{U^{p}(N), \Delta, \rho}^{\text {sub, } \dagger}\right)$ on $\mathcal{X}_{U^{p}(N), \Delta}^{\text {ord, } \dagger}$ induced by $\mathcal{E}_{U^{p}\left(N, N^{\prime}\right), \Delta^{\prime}, \rho}^{\text {can }}\left(\operatorname{resp} . \mathcal{E}_{U^{p}\left(N, N^{\prime}\right), \Delta^{\prime}, \rho}^{\text {sub }}\right)$ for any $N^{\prime} \geq N$ and $\Delta^{\prime} \in \mathcal{J}_{n}^{\text {tor }}$ with $\left(\Delta^{\prime}\right)^{\text {ord }}=\Delta$. This is canonically independent of the choices of $N^{\prime}$ and $\Delta^{\prime}$. The systems of sheaves $\left\{\mathcal{E}_{U^{p}(N), \Delta, \rho}^{\mathrm{can}, \dagger}\right\}$ and $\left\{\mathcal{E}_{U^{p}(N), \Delta, \rho}^{\text {sub } \dagger}\right\}$ over $\left\{\mathcal{X}_{U^{p}(N), \Delta}^{\text {ord, } \dagger}\right\}$ have actions of $G_{n}\left(\mathbb{A}^{\infty}\right)^{\text {ord }}$. There are equivariant identifications

$$
\mathcal{E}_{U^{p}(N), \Delta, \rho}^{\mathrm{sub}, \dagger} \cong \mathcal{E}_{U^{p}(N), \Delta, \rho}^{\mathrm{can}, \dagger} \otimes \mathcal{I}_{\partial \mathcal{X}_{U^{p}(N), \Delta}^{\mathrm{ord}, \dagger}}
$$

where $\mathcal{I}_{\partial \mathcal{X}_{U^{p}(N), \Delta}^{\text {ord, }} \dagger}$ denotes the sheaf of ideals in $\mathcal{O}_{\mathcal{X}_{U^{p}(N), \Delta}^{\text {ord, }} \dagger}$ defining $\partial \mathcal{X}_{U^{p}(N), \Delta}^{\text {ord, } \dagger}$. For $g \in$ $G_{n}\left(\mathbb{A}^{\infty}\right)^{\text {ord }}$ the map

$$
g: g^{*} \mathcal{E}_{\left(U^{p}\right)^{\prime}\left(N^{\prime}\right), \Delta^{\prime}, \rho}^{\mathrm{can}, \dagger} \longrightarrow \mathcal{E}_{U^{p}(N), \Delta, \rho}^{\mathrm{can}, \dagger}
$$

is an isomorphism. (Because the same is true over $X_{U^{p}\left(N, N^{\prime}\right), \Delta^{\prime}}$ and hence over $\left.X_{U^{p}\left(N, N^{\prime}\right), \Delta^{\prime}}^{\dagger}.\right)$ We define $H^{i}\left(\mathcal{A}^{(m) \text {,ord, } \dagger}, \Omega^{j}(\log \infty) \otimes \mathcal{I}\right)$ to be

$$
\underset{U^{p, N, \Sigma}}{\lim _{\vec{p}}} H^{i}\left(\mathcal{A}_{U^{p}(N), \Sigma}^{(m), \text { ord } \uparrow}, \Omega_{\mathcal{A}_{U^{p}(N), \Sigma}^{(m), \text { ord }, \dagger}}^{j}(\log \infty) \otimes \mathcal{I}_{\partial \mathcal{A}_{U^{p}(N), \Sigma}^{(m), \text { ord }, \dagger}}\right)
$$

and $H^{i}\left(\partial^{(s)} \mathcal{A}^{(m), \text { ord } \dagger}, \Omega^{j}\right)$ to be

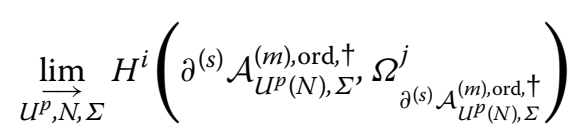

and $H^{0}\left(\mathcal{X}^{\text {ord, } \dagger}, \mathcal{E}_{\rho}^{\text {sub }}\right)$ to be

$$
\underset{U^{p, N, \Delta}}{\lim _{\longrightarrow}} H^{0}\left(\mathcal{X}_{U^{p}(N), \Delta}^{\text {ord, }}, \mathcal{E}_{U^{p}(N), \Delta, \rho}^{\text {sub,† }}\right)
$$

They are all smooth $G_{n}\left(\mathbb{A}^{\infty}\right)^{\text {ord }}$-modules. 


\section{Lemma 6.10 There are natural isomorphisms}

$$
\begin{aligned}
H^{i} & \left(\mathcal{A}_{U^{p}(N), \Sigma}^{(m), \text { ord },}, \Omega_{\mathcal{A}_{u^{\prime}(N), \Sigma}^{(m), \text { ord },}}^{j}(\log \infty) \otimes \mathcal{I}_{\partial \mathcal{A}_{U^{p}(N), \Sigma}^{(m), \text { ord },}}\right) \\
& \stackrel{\sim}{\longrightarrow} H^{i}\left(\mathcal{A}^{(m), \text { ord }, \dagger}, \Omega^{j}(\log \infty) \otimes \mathcal{I}\right)^{U^{p}(N)}
\end{aligned}
$$

and

$$
H^{0}\left(\mathcal{X}_{U^{p}(N), \Delta^{\mathrm{ord},},}^{\text {E }} \mathcal{U}^{\mathrm{sub}(N), \downarrow}, \Delta, \rho\right) \stackrel{\sim}{\longrightarrow} H^{0}\left(\mathcal{X}^{\mathrm{ord}, \dagger}, \mathcal{E}_{\rho}^{\mathrm{sub}}\right)^{U^{p}(N)}
$$

Proof Use Lemmas 5.1, 5.6, 5.7, 5.3 and 6.9.

\subsubsection{The Frobenius lift $\varsigma_{p}$ and trF $_{F}$}

The inverse of $\varsigma_{p}^{*}$ gives maps

$$
\varsigma_{p, *} \Omega_{\mathcal{A}_{U^{p}(N), \Sigma}^{(m), \text { ord }, \dagger}}^{j}(\log \infty) \stackrel{\sim}{\longrightarrow} \Omega_{\mathcal{A}_{U^{p}(N), \Sigma}^{(m), \text { ord }, ~}}^{j}(\log \infty) \otimes_{\mathcal{O}_{\mathcal{A}_{U p}^{(m), \text { ord },,}, \zeta_{p}^{*}}^{*}} \mathcal{O}_{\mathcal{A}_{U^{p}(N), \Sigma}^{(m), \text { ord }, \dagger}}
$$

and

$$
\begin{aligned}
& \varsigma_{p, *}\left(\Omega_{\mathcal{A}_{\mu p(N), \Sigma}^{(m), \text { ord },}}^{j}(\log \infty) \otimes \mathcal{I}_{\partial \mathcal{A}_{U p(N), \Sigma}^{(m), \text { ord },},}\right)
\end{aligned}
$$

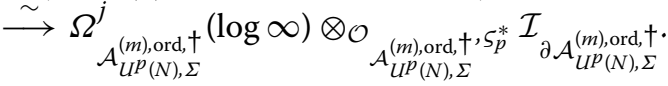

The maps

- $\varsigma_{p}: \mathcal{A}_{U^{p}(N), \Sigma^{\text {ord }}}^{(m), \text { ord },} \rightarrow \mathcal{A}_{U^{p}(N), \Sigma^{\text {ord }}}^{(m), \text { ord } \dagger}$

- and $\varsigma_{p}: \partial^{(s)} \mathcal{A}_{U^{p}(N), \Sigma^{\text {ord }}}^{(m), \text { ord },} \rightarrow \partial^{(s)} \mathcal{A}_{U^{p}(N), \Sigma^{\text {ord }}}^{(m), \text { ord },}$

are finite, flat of degrees $p^{(2 m+n) n\left[F^{+}: \mathbb{Q}\right]}$ and $p^{(2 m+n) n\left[F^{+}: \mathbb{Q}\right]-s}$, respectively. (Use the finite flatness of

$$
\varsigma_{p}: \mathfrak{A}_{U^{p}(N), \Sigma^{\text {ord }}}^{(m) \text { ord }} \rightarrow \mathfrak{A}_{U^{p}(N), \Sigma^{\text {ord }}}^{(m) \text { ord }}
$$

and

$$
\varsigma_{p}: \partial^{(s)} \mathfrak{A}_{U^{p}(N), \Sigma^{\text {ord }}}^{(m), \text { ord }} \rightarrow \partial^{(s)} \mathfrak{A}_{U^{p}(N), \Sigma^{\text {ord }}}^{(m) \text {,ord }}
$$

(see Sect. 5.3), together with theorems 1.7(1) and 1.12 of [27].)

As $\varsigma_{p}: \mathcal{A}_{U^{p}(N), \Sigma^{\text {ord }}}^{(m), \text { ord }, \dagger} \rightarrow \mathcal{A}_{U^{p}(N), \Sigma^{\text {ord }}}^{(m), \text { ord },}$ is finite and flat we get a trace map

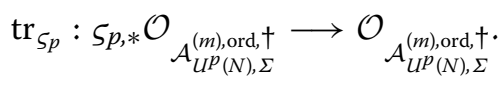

Because $\partial \mathcal{A}_{U^{p}(N), \Sigma}^{(m), \text { ord },}$ has the same support as

$$
\mathcal{A}_{U^{p}(N), \Sigma}^{(m), \text { ord }, \dagger} \times{ }_{{ }_{{ }_{p},}, \mathcal{A}_{U^{p}(N), \Sigma}^{(m), \mathrm{rd}, \dagger}} \partial \mathcal{A}_{U^{p}(N), \Sigma}^{(m), \text { ord }, \dagger}
$$


this trace map restricts to a map

$$
\operatorname{tr}_{\varsigma_{p}}: \varsigma_{p, *} \mathcal{I}_{\partial \mathcal{A}_{U p(N), \Sigma}^{(m), \mathrm{ord}, \dagger}} \longrightarrow \mathcal{I}_{\partial \mathcal{A}_{U p(N), \Sigma}^{(m), \mathrm{ord},} \dagger}
$$

(This is a consequence of the following fact: If $R$ is a noetherian ring, if $S$ is an $R$-algebra, finite and free as an $R$-module, and if $I$ and $J$ are ideals of $R$ and $S$, respectively, with

$$
\sqrt{J}=\sqrt{I S}
$$

then the trace map $\operatorname{tr}_{S / R}$ maps $J$ to $I$. To see this we may reduce to the case $I=0$. In this case every element of $J$ is nilpotent and so has trace 0 .)

Composing $\left(\varsigma_{p}^{*}\right)^{-1}$ with $\operatorname{tr}_{\varsigma_{p}}$ we get $G_{n}^{(m)}\left(\mathbb{A}^{\infty}\right)^{\text {ord } \times} \times$-equivariant maps

$$
\operatorname{tr}_{F}: \varsigma_{p, *} \Omega_{\mathcal{A}_{U^{p}(N), \Sigma}^{(m), \text { ord, }}}^{j}(\log \infty) \longrightarrow \Omega_{\mathcal{A}_{U^{p}(N), \Sigma}^{(m), \text { ord },}}^{j}(\log \infty)
$$

and

$$
\operatorname{tr}_{F}: \varsigma_{p, *}\left(\Omega_{\mathcal{A}_{U^{p}(N), \Sigma}^{(m), \text { ord },}}^{j}(\log \infty) \otimes \mathcal{I}_{\partial \mathcal{A}_{U^{p}(N), \Sigma}^{(m), \mathrm{ord}, \dagger}}\right) \longrightarrow \Omega_{\mathcal{A}_{U^{p}(N), \Sigma}^{(m), \text { ord }, \dagger}}^{j}(\log \infty) \otimes \mathcal{I}_{\partial \mathcal{A}_{U^{p}(N), \Sigma}^{(m), \text { ord }, \dagger}}
$$

We have

$$
\operatorname{tr}_{F} \circ \varsigma_{p}^{*}=p^{(n+2 m) n\left[F^{+}: \mathbb{Q}\right]}
$$

This induces endomorphisms

$$
\operatorname{tr}_{F} \in \operatorname{End}\left(H^{i}\left(\mathcal{A}_{U^{p}(N), \Sigma}^{(m), \text { ord, } \dagger}, \Omega_{\mathcal{A}_{U^{p}(N), \Sigma}^{(m), \text { ord },}}^{j}(\log \infty) \otimes \mathcal{I}_{\partial \mathcal{A}_{U^{p}(N), \Sigma}^{(m), \text { ord },}}\right)\right)
$$

which commute with the action of $G_{n}\left(\mathbb{A}^{\infty}\right)^{\text {ord, } \times}$ and satisfy

$$
\operatorname{tr}_{F} \circ \varsigma_{p}=p^{(n+2 m) n\left[F^{+}: \mathbb{Q}\right]}
$$

We obtain an element

$$
\operatorname{tr}_{F} \in \operatorname{End}\left(H^{i}\left(\mathcal{A}^{(m), \text { ord }, \dagger}, \Omega^{j}(\log \infty) \otimes \mathcal{I}\right)\right)
$$

which commutes with the $G_{n}\left(\mathbb{A}^{\infty}\right)^{\text {ord, } \times}$-action and satisfies

$$
\operatorname{tr}_{F} \circ \varsigma_{p}=p^{(n+2 m) n\left[F^{+}: \mathbb{Q}\right]}
$$

Similarly the inverse of $\varsigma_{p}^{*}$ gives maps

$$
\varsigma_{p, *} \mathcal{E}_{U^{p}(N), \Delta, \rho}^{\mathrm{can}, \dagger} \stackrel{\sim}{\longrightarrow} \mathcal{E}_{U^{p}(N), \Delta, \rho}^{\mathrm{can}, \dagger} \otimes_{\mathcal{O}_{\mathcal{X}_{U^{p}(N), \Delta}^{\mathrm{ord},} \dagger}, \varsigma_{p}^{*}} \mathcal{O}_{\mathcal{X}_{U^{p}(N), \Delta}^{\mathrm{ord},} \dagger}
$$

and

$$
\varsigma_{p, *} \mathcal{E}_{U^{p}(N), \Delta, \rho}^{\mathrm{sub}, \dagger} \stackrel{\sim}{\longrightarrow} \mathcal{E}_{U^{p}(N), \Delta, \rho}^{\mathrm{can}, \dagger} \otimes_{\mathcal{O}}{ }_{\mathcal{X}_{U^{p}(N), \Delta}^{\mathrm{ord}, \dagger}, \varsigma_{p}^{*}} \mathcal{I}_{\partial \mathcal{X}_{U^{p}(N), \Delta}^{\mathrm{ord},} \dagger} .
$$


Composing $\left(\varsigma_{p}^{*}\right)^{-1}$ with $\operatorname{tr}_{S_{p}}$ we get $G_{n}^{(m)}\left(\mathbb{A}^{\infty}\right)^{\text {ord, } \times}$-equivariant maps

$$
\operatorname{tr}_{F}: \varsigma_{p, *} \mathcal{E}_{U^{p}(N), \Delta, \rho}^{\mathrm{can}, \dagger} \longrightarrow \mathcal{E}_{U^{p}(N), \Delta, \rho}^{\mathrm{can}, \dagger}
$$

and

$$
\operatorname{tr}_{F}: \varsigma_{p, *} \mathcal{E}_{U^{p}(N), \Delta, \rho}^{\mathrm{sub}, \dagger} \longrightarrow \mathcal{E}_{U^{p}(N), \Delta, \rho}^{\mathrm{sub}, \dagger}
$$

We have

$$
\operatorname{tr}_{F} \circ \varsigma_{p}^{*}=p^{n^{2}\left[F^{+}: \mathbb{Q}\right]}
$$

This induces compatible endomorphisms

$$
\operatorname{tr}_{F} \in \operatorname{End}\left(H^{0}\left(\mathcal{X}_{U^{p}(N), \Delta}^{\mathrm{ord}, \dagger}, \mathcal{E}_{U^{p}(N), \Delta, \rho}^{\mathrm{can}, \dagger}\right)\right)
$$

and

$$
\operatorname{tr}_{F} \in \operatorname{End}\left(H^{0}\left(\mathcal{X}_{U^{p}(N), \Delta^{\mathrm{ord}} \dagger}^{\mathrm{o}}, \mathcal{E}_{U^{p}(N), \Delta, \rho}^{\mathrm{sub}, \dagger}\right)\right)
$$

which commute with the action of $G_{n}\left(\mathbb{A}^{\infty}\right)^{\text {ord, } \times}$ and satisfy

$$
\operatorname{tr}_{F} \circ \varsigma_{p}=p^{n^{2}\left[F^{+}: \mathbb{Q}\right]}
$$

We obtain an element

$$
\operatorname{tr}_{F} \in \operatorname{End}\left(H^{0}\left(\mathcal{X}^{\text {ord }, \dagger}, \mathcal{E}_{\rho}^{\text {sub }}\right)\right)
$$

which commutes with the $G_{n}\left(\mathbb{A}^{\infty}\right)^{\text {ord, } \times}$-action and satisfies

$$
\operatorname{tr}_{F} \circ \varsigma_{p}=p^{n^{2}\left[F^{+}: \mathbb{Q}\right]}
$$

We remark that $\operatorname{tr}_{F}$ is closely related to the operator often denoted $U_{p}$ : probably they differ simply by a scalar multiple.

\subsection{The ordinary locus of the minimal compactification as a dagger space}

\subsubsection{The ordinary locus as a dagger space}

Suppose that $U^{p}$ is a neat open compact subgroup of $G_{n}\left(\mathbb{A}^{\infty, p}\right)$ and that $N_{2} \geq N_{1} \geq 0$. We will write

$$
\mathcal{X}_{U^{p}\left(N_{1}, N_{2}\right)}^{\text {ord min, }}
$$

for the dagger space associated to $\mathcal{X}_{U^{p}\left(N_{1}, N_{2}\right)}^{\text {ord,min }}$ as described in the paragraph before Lemma 6.7. Then the system of dagger spaces $\left\{\mathcal{X}_{U^{p}\left(N_{1}, N_{2}\right)}^{\text {ord,min }}\right\}$ has an action of $G_{n}\left(\mathbb{A}^{\infty}\right)^{\text {ord }}$.

If $U^{p}$ denotes the image in $G_{n}\left(\mathbb{A}^{\infty, p}\right)$ of $\left(U^{p}\right)^{\prime} \subset G_{n}^{(m)}\left(\mathbb{A}^{\infty, p}\right)$ then there is a natural map

$$
\mathcal{A}_{U^{p}\left(N_{1}, N_{2}\right), \Sigma}^{(m), \text { ord } \dagger} \longrightarrow \mathcal{X}_{\left(U^{p}\right)^{\prime}\left(N_{1}, N_{2}\right)}^{\text {ord,min, }}
$$


These maps are $G_{n}^{(m)}\left(\mathbb{A}^{\infty}\right)^{\text {ord }}$-equivariant (as $\left(U^{p}\right)^{\prime}, N_{1}$ and $N_{2}$ vary).

Recall from Sect. 5.1 that, if $N_{2}^{\prime} \geq N_{2}$, then the natural map

$$
\mathcal{X}_{U^{p}\left(N_{1}, N_{2}^{\prime}\right)}^{\text {ord,min }} \longrightarrow \mathcal{X}_{U^{p}\left(N_{1}, N_{2}\right)}^{\text {ord,min }}
$$

restricts to an isomorphism

$$
\bar{X}_{U^{p}\left(N_{1}, N_{2}^{\prime}\right)}^{\text {ord,min }} \stackrel{\sim}{\longrightarrow} \bar{X}_{U^{p}\left(N_{1}, N_{2}\right)}^{\text {ord,min }}
$$

and is etale in a neighbourhood of $\bar{X}_{U^{p}\left(N_{1}, N_{2}^{\prime}\right)}^{\text {ordmin }}$. It follows from Lemma 6.7 that

$$
\mathcal{X}_{U^{p}\left(N_{1}, N_{2}^{\prime}\right)}^{\text {ord,min } \dagger} \longrightarrow \mathcal{X}_{U^{p}\left(N_{1}, N_{2}\right)}^{\text {ord,min, } \dagger}
$$

is an isomorphism. We will denote this dagger space simply

$$
\mathcal{X}_{U^{p}\left(N_{1}\right)}^{\text {ord } \min , \dagger}
$$

The system of dagger spaces $\left\{\mathcal{X}_{U^{p}(N)}^{\text {ord,min }}{ }^{\dagger}\right\}$ has an action of $G_{n}\left(\mathbb{A}^{\infty}\right)^{\text {ord }}$.

Let $\bar{e}_{U^{p}\left(N_{1}, N_{2}\right)}$ denote the idempotent in

$$
\left(\bigoplus_{i=0}^{\infty} H^{0}\left(\bar{X}_{U^{p}\left(N_{1}, N_{2}\right)}^{\min }, \omega^{\otimes(p-1) i}\right)\right) /\left(\operatorname{Hasse}_{U^{p}\left(N_{1}, N_{2}\right)}-1\right)
$$

which is 1 on $\bar{X}_{U^{p}\left(N_{1}, N_{2}\right)}^{\text {ordmin }}$ and 0 on

$$
\bar{X}_{U^{p}\left(N_{1}, N_{2}\right)}^{\min }-\bar{X}_{U^{p}\left(N_{1}, N_{2}\right)}^{\min , \mathrm{n} \text { ord }}-\bar{X}_{U^{p}\left(N_{1}, N_{2}\right)}^{\min , \text { ord }}
$$

(The existence of $\bar{e}_{U^{p}\left(N_{1}, N_{2}\right)}$ follows from the results recalled in Sect. 5.1.) Multiplying the terms of $\bar{e}_{U^{p}\left(N_{1}, N_{2}\right)}$ by suitable powers of Hasse $U_{U^{p}\left(N_{1}, N_{2}\right)}$, we may suppose that $\bar{e}_{U^{p}\left(N_{1}, N_{2}\right)}$ lies in $H^{0}\left(\bar{X}_{U^{p}\left(N_{1}, N_{2}\right)}^{\min }, \omega^{\otimes(p-1) a}\right)$ for any sufficiently large $a$, and that

$$
\bar{e}_{U^{p}\left(N_{1}, N_{2}\right)} / \operatorname{Hasse}_{U^{p}\left(N_{1}, N_{2}\right)} \in H^{0}\left(\bar{X}_{U^{p}\left(N_{1}, N_{2}\right)}^{\min }, \omega^{\otimes(p-1)(a-1)}\right) .
$$

Then

$$
\bar{X}_{U^{p}\left(N_{1}, N_{2}\right)}^{\text {ord }}=\operatorname{Spec}\left(\bigoplus_{i=0}^{\infty} H^{0}\left(\bar{X}_{U^{p}\left(N_{1}, N_{2}\right)}^{\min }, \omega^{\otimes(p-1) a i}\right)\right) /\left(\bar{e}_{U^{p}\left(N_{1}, N_{2}\right)}-1\right)
$$

For $a$ sufficiently large we have $H^{1}\left(\mathcal{X}_{U^{p}\left(N_{1}, N_{2}\right)}^{\min }, \omega^{\otimes(p-1) a}\right)=(0)$. In that case we can lift $\bar{e}_{U^{p}\left(N_{1}, N_{2}\right)}$ to a non-canonical element

$$
e_{U^{p}\left(N_{1}, N_{2}\right)} \in H^{0}\left(\mathcal{X}_{U^{p}\left(N_{1}, N_{2}\right)}^{\min }, \omega^{\otimes(p-1) a}\right)
$$

Let $\mathcal{X}_{U^{p}\left(N_{1}, N_{2}\right)}^{\min }\left[1 / e_{U^{p}\left(N_{1}, N_{2}\right)}\right]$ denote the locus in $\mathcal{X}_{U^{p}\left(N_{1}, N_{2}\right)}^{\min }$ where $e_{U^{p}\left(N_{1}, N_{2}\right)} \neq 0$. As $\omega^{\otimes(p-1) a}$ is ample, $\mathcal{X}_{U^{p}\left(N_{1}, N_{2}\right)}^{\min }\left[1 / e_{U^{p}\left(N_{1}, N_{2}\right)}\right]$ is affine and so has the form

$$
\operatorname{Spec} \mathbb{Z}_{(p)}\left[T_{1}, \ldots, T_{s}\right] / I
$$


for some $s$ and $I$. It is normal and flat over $\mathbb{Z}_{(p)}$.

For $r \in p^{\mathbb{Q} \geq 0}$ let \|\|$_{r}$ denote the norm on $\mathbb{Z}_{(p)}\left[T_{1}, \ldots, T_{s}\right]$ defined by

$$
\left\|\sum_{\vec{i}} a_{\vec{i}} T^{\vec{i}}\right\|_{r}=\sup _{\vec{i}}\left|a_{\vec{i}}\right|_{p} r^{|\vec{i}|},
$$

where $\vec{i}$ runs over $\mathbb{Z}_{\geq 0}^{s}$ and $\left|\left(i_{1}, \ldots, i_{s}\right)\right|=i_{1}+\cdots+i_{s}$. We will write $\mathbb{Z}_{p}\left\langle T_{1}, \ldots, T_{s}\right\rangle_{r}$ for the completion of $\mathbb{Z}_{(p)}\left[T_{1}, \ldots, T_{s}\right]$ with respect to \|\|$_{r}$. Thus $\mathbb{Z}_{p}\left\langle T_{1}, \ldots, T_{s}\right\rangle_{1}$ is the $p$ adic completion of $\mathbb{Z}_{(p)}\left[T_{1}, \ldots, T_{s}\right]$ and also the $p$-adic completion of $\mathbb{Z}_{p}\left\langle T_{1}, \ldots, T_{s}\right\rangle_{r}$ for any $r \geq 1$. Set $\mathbb{Q}_{p}\left\langle T_{1}, \ldots, T_{s}\right\rangle_{r}=\mathbb{Z}_{p}\left\langle T_{1}, \ldots, T_{s}\right\rangle_{r}[1 / p]$, the completion of $\mathbb{Q}\left[T_{1}, \ldots, T_{s}\right]$ with respect to \|\|$_{r}$. In the case $r=1$ we will drop it from the notation. We will write $\mathbb{Z}_{p}\left\langle T_{1} / r, \ldots, T_{s} / r\right\rangle_{1}$ for the \|\|$_{r}$ unit-ball in $\mathbb{Q}_{p}\left\langle T_{1}, \ldots, T_{s}\right\rangle_{r}$, i.e. for the set of power series

$$
\sum_{\vec{i} \in \mathbb{Z}_{\geq 0}^{s}} a_{\vec{i}} \vec{T}^{i}
$$

where $a_{\vec{i}} \in \mathbb{Q}_{p}$, and $\left|a_{\vec{i}}\right|_{p} \leq r^{-|\vec{i}|}$ for all $\vec{i}$, and $\left|a_{\vec{i}}\right|_{p} r^{|\vec{i}|} \rightarrow 0$ as $|\vec{i}| \rightarrow \infty$. We will also write

$$
\mathbb{Q}_{p}\left\langle T_{1}, \ldots, T_{s}\right\rangle^{\dagger}=\bigcup_{r>1} \mathbb{Q}_{p}\left\langle T_{1}, \ldots, T_{s}\right\rangle_{r}
$$

Let $\langle I\rangle_{r}$ denote the ideal of $\mathbb{Z}_{p}\left\langle T_{1}, \ldots, T_{s}\right\rangle_{r}$ generated by $I$ and let $\langle I\rangle_{r}^{\prime}$ denote the intersection of $\langle I\rangle_{1}$ with $\mathbb{Z}_{p}\left\langle T_{1}, \ldots, T_{s}\right\rangle_{r}$. Then $\langle I\rangle_{1}$ is the $p$-adic completion of $I$. Moreover

$$
\mathbb{Z}_{p}\left\langle T_{1}, \ldots, T_{s}\right\rangle_{1} /\langle I\rangle_{1}
$$

is normal and flat over $\mathbb{Z}_{p}$, and

$$
\mathfrak{X}_{U^{p}\left(N_{1}\right)}^{\text {ord,min }}=\operatorname{Spf} \mathbb{Z}_{p}\left\langle T_{1}, \ldots, T_{s}\right\rangle_{1} /\langle I\rangle_{1} .
$$

Note that

$$
\mathbb{Z}_{(p)}\left[T_{1}, \ldots, T_{s}\right] /(I, p) \stackrel{\sim}{\longrightarrow} \mathbb{Z}_{p}\left\langle T_{1}, \ldots, T_{s}\right\rangle_{r} /\left(\langle I\rangle_{r}, p\right)
$$

for all $r \geq 1$. Thus $\left(\langle I\rangle_{r}, p\right)=\left(\langle I\rangle_{r}^{\prime}, p\right)$.

We will also write $\langle I\rangle_{r, \mathbb{Q}_{p}}$ (resp. $\langle I\rangle_{r, \mathbb{Q}_{p}}^{\prime}$ ) for the $\mathbb{Q}_{p}$ span of $\langle I\rangle_{r}$ (resp. $\langle I\rangle_{r}^{\prime}$ ) in $\mathbb{Q}_{p}\left\langle T_{1}, \ldots, T_{s}\right\rangle_{r}$. Then

$$
\operatorname{Sp} \mathbb{Q}_{p}\left\langle T_{1}, \ldots, T_{s}\right\rangle_{1} /\langle I\rangle_{1, \mathbb{Q}_{p}} \subset \operatorname{Sp} \mathbb{Q}_{p}\left\langle T_{1}, \ldots, T_{s}\right\rangle_{r} /\langle I\rangle_{r, \mathbb{Q}_{p}}^{\prime} \subset \operatorname{Sp} \mathbb{Q}_{p}\left\langle T_{1}, \ldots, T_{s}\right\rangle_{r} /\langle I\rangle_{r, \mathbb{Q}_{p}}
$$

are all affinoid subdomains of $X_{U^{p}\left(N_{1}, N_{2}\right)}^{\min , a n}$, the rigid analytic space associated to the scheme $X_{U^{p}\left(N_{1}, N_{2}\right)}^{\min } \times \operatorname{Spec} \mathbb{Q}_{p}$. Thus they are normal. Also Sp $\mathbb{Q}_{p}\left\langle T_{1}, \ldots, T_{s}\right\rangle_{r} /\langle I\rangle_{r, \mathbb{Q}_{p}}^{\prime}$ and

$$
\operatorname{Sp} \mathbb{Q}_{p}\left\langle T_{1}, \ldots, T_{s}\right\rangle_{r} /\langle I\rangle_{r, \mathbb{Q}_{p}}-\operatorname{Sp} \mathbb{Q}_{p}\left\langle T_{1}, \ldots, T_{s}\right\rangle_{r} /\langle I\rangle_{r, \mathbb{Q}_{p}}^{\prime}
$$

form an admissible open cover of Sp $\mathbb{Q}_{p}\left\langle T_{1}, \ldots, T_{s}\right\rangle_{r} /\langle I\rangle_{r, \mathbb{Q}_{p}}$. (Sp $\mathbb{Q}_{p}\left\langle T_{1}, \ldots, T_{s}\right\rangle_{r} /\langle I\rangle_{r, \mathbb{Q}_{p}}^{\prime}$ is the union of the connected components of $\operatorname{Sp} \mathbb{Q}_{p}\left\langle T_{1}, \ldots, T_{s}\right\rangle_{r} /\langle I\rangle_{r, \mathbb{Q}_{p}}$ which contain 
a component of $\operatorname{Sp} \mathbb{Q}_{p}\left\langle T_{1}, \ldots, T_{s}\right\rangle_{1} /\langle I\rangle_{1, \mathbb{Q}_{p}}$. See proposition 8 of section 9.1.4 of [8].) Moreover Sp $\mathbb{Q}_{p}\left\langle T_{1}, \ldots, T_{s}\right\rangle_{1} /\langle I\rangle_{1, \mathbb{Q}_{p}}$ is Zariski dense in $\operatorname{Sp} \mathbb{Q}_{p}\left\langle T_{1}, \ldots, T_{s}\right\rangle_{r} /\langle I\rangle_{r, \mathbb{Q}_{p}}$. Indeed

$$
X_{U^{p}\left(N_{1}, N_{2}\right)}^{\mathrm{an}} \cap \operatorname{Sp} \mathbb{Q}_{p}\left\langle T_{1}, \ldots, T_{s}\right\rangle_{1} /\langle I\rangle_{1}
$$

is Zariski dense in $\operatorname{Sp} \mathbb{Q}_{p}\left\langle T_{1}, \ldots, T_{s}\right\rangle_{r} /\langle I\rangle_{r, \mathbb{Q}_{p}}$, where $X_{U^{p}\left(N_{1}, N_{2}\right)}^{\text {an }}$, the rigid analytic space associated to $X_{U^{p}\left(N_{1}, N_{2}\right)} \times \operatorname{Spec} \mathbb{Q}_{p}$.

If $1 \leq r^{\prime}<r$ then

$$
\operatorname{Sp} \mathbb{Q}_{p}\left\langle T_{1}, \ldots, T_{s}\right\rangle_{r^{\prime}} /\langle I\rangle_{r^{\prime}, \mathbb{Q}_{p}}^{\prime} \subset \operatorname{Sp} \mathbb{Q}_{p}\left\langle T_{1}, \ldots, T_{s}\right\rangle_{r} /\langle I\rangle_{r, \mathbb{Q}_{p}}^{\prime}
$$

and

$$
\operatorname{Sp} \mathbb{Q}_{p}\left\langle T_{1}, \ldots, T_{s}\right\rangle_{r^{\prime}} /\langle I\rangle_{r^{\prime}, \mathbb{Q}_{p}} \subset \operatorname{Sp} \mathbb{Q}_{p}\left\langle T_{1}, \ldots, T_{s}\right\rangle_{r} /\langle I\rangle_{r, \mathbb{Q}_{p}}
$$

and these are strict neighbourhoods. The natural maps

$$
i_{r, r^{\prime}}: \mathbb{Q}_{p}\left\langle T_{1}, \ldots, T_{s}\right\rangle_{r} /\langle I\rangle_{r, \mathbb{Q}_{p}} \longrightarrow \mathbb{Q}_{p}\left\langle T_{1}, \ldots, T_{s}\right\rangle_{r^{\prime}} /\langle I\rangle_{r^{\prime}, \mathbb{Q}_{p}}
$$

and

$$
i_{r, r^{\prime}}^{\prime}: \mathbb{Q}_{p}\left\langle T_{1}, \ldots, T_{s}\right\rangle_{r} /\langle I\rangle_{r, \mathbb{Q}_{p}}^{\prime} \hookrightarrow \mathbb{Q}_{p}\left\langle T_{1}, \ldots, T_{s}\right\rangle_{r^{\prime}} /\langle I\rangle_{r^{\prime}, \mathbb{Q}_{p}}^{\prime}
$$

are completely continuous. The latter is an inclusion. Moreover

$$
\left(i_{r, 1}^{\prime}\right)^{-1} \mathbb{Z}_{p}\left\langle T_{1}, \ldots, T_{s}\right\rangle_{r} /\langle I\rangle_{1}=\mathbb{Z}_{p}\left\langle T_{1}, \ldots, T_{s}\right\rangle_{r} /\langle I\rangle_{r}^{\prime}
$$

Also write $\langle I\rangle^{\dagger}$ for the ideal of $\mathbb{Q}_{p}\left\langle T_{1}, \ldots, T_{s}\right\rangle^{\dagger}$ generated by $I$. Thus

$$
\langle I\rangle^{\dagger}=\bigcup_{r>1}\langle I\rangle_{r, \mathbb{Q}_{p}}=\bigcup_{r>1}\langle I\rangle_{r, \mathbb{Q}_{p}}^{\prime}
$$

Moreover

$$
\mathbb{Q}_{p}\left\langle T_{1}, \ldots, T_{s}\right\rangle^{\dagger} /\langle I\rangle^{\dagger}=\lim _{r>1} \mathbb{Q}_{p}\left\langle T_{1}, \ldots, T_{s}\right\rangle_{r} /\langle I\rangle_{r, \mathbb{Q}_{p}}=\underset{r>1}{\lim _{r>1}} \mathbb{Q}_{p}\left\langle T_{1}, \ldots, T_{s}\right\rangle_{r} /\langle I\rangle_{r, \mathbb{Q}_{p}}^{\prime},
$$

and

$$
\mathcal{X}_{U^{p}\left(N_{1}\right)}^{\mathrm{ord}, \boldsymbol{m i n}, \dagger}=\operatorname{Sp} \mathbb{Q}_{p}\left\langle T_{1}, \ldots, T_{s}\right\rangle^{\dagger} /\langle I\rangle^{\dagger}
$$

(See, for instance, proposition 3.3.7 of [46]. For the meaning of Sp in the context of dagger algebras see section 2.11 of [27].) Thus we have the following lemma.

Lemma 6.11 $\mathcal{X}_{U^{p}(N)}^{\mathrm{ord}, \mathrm{min},}{ }^{\dagger}$ is affinoid. 
Suppose that $\rho_{0}$ is a representation of $L_{n,(n)}$ on a finite free $\mathbb{Z}_{p}$-module and let $\rho$ denote $\rho_{0}$ base changed to $\mathbb{Q}_{p}$. There are $G_{n}\left(\mathbb{A}^{\infty}\right)^{\text {ord }}$ equivariant isomorphisms

$$
H^{0}\left(\mathcal{X}_{U^{p}(N)}^{\mathrm{ord}, \mathrm{min}, \dagger}, \mathcal{E}_{U^{p}(N), \rho}^{\mathrm{sub}, \dagger}\right) \stackrel{\sim}{\longrightarrow} H^{0}\left(\mathcal{X}_{U^{p}(N), \Delta^{\mathrm{ord}}, \dagger}^{\dagger}, \mathcal{E}_{U^{p}(N), \Delta, \rho}^{\mathrm{sub}, \dagger}\right) .
$$

There are also natural $G_{n}\left(\mathbb{A}^{\infty}\right)^{\text {ord, } \times}$-equivariant embeddings

$$
H^{0}\left(\mathcal{X}_{U^{p}(N)}^{\mathrm{ord}, \min , \dagger}, \mathcal{E}_{U^{p}(N), \rho}^{\mathrm{sub}, \dagger}\right) \hookrightarrow H^{0}\left(\mathfrak{X}_{U^{p}(N)}^{\mathrm{ord}, \min }, \mathcal{E}_{U^{p}(N), \rho_{0}}^{\text {ord,sub }}\right) \otimes_{\mathbb{Z}_{p}} \mathbb{Q}_{p}
$$

We will set

$$
H^{0}\left(\mathcal{X}^{\text {ord,min }, \dagger}, \mathcal{E}_{\rho}^{\text {sub }}\right)_{\overline{\mathbb{Q}}_{p}}=\left(\lim _{\rightarrow U^{p}, N} H^{0}\left(\mathcal{X}_{U^{p}(N)}^{\text {ord,min }, \dagger}, \mathcal{E}_{U^{p}(N), \rho}^{\text {sub, } \dagger}\right)\right) \otimes_{\mathbb{Q}_{p}} \overline{\mathbb{Q}}_{p},
$$

a smooth $G_{n}\left(\mathbb{A}^{\infty}\right)^{\text {ord }}$-module. From Lemma 6.10 and the first observation of the last paragraph, we see that

$$
H^{0}\left(\mathcal{X}^{\mathrm{ord}, \mathrm{min}, \dagger}, \mathcal{E}_{\rho}^{\mathrm{sub}}\right)_{\overline{\mathbb{Q}}_{p}}^{U^{p}(N)}=H^{0}\left(\mathcal{X}_{U^{p}(N)}^{\mathrm{ord}, \mathrm{min}, \dagger}, \mathcal{E}_{U^{p}(N), \rho}^{\mathrm{sub}, \dagger}\right)_{\overline{\mathbb{Q}}_{p}} .
$$

There is a $G_{n}\left(\mathbb{A}^{\infty}\right)^{\text {ord, } \times}$-equivariant embedding

$$
H^{0}\left(\mathcal{X}^{\text {ord,min, },}, \mathcal{E}_{\rho}^{\text {sub }}\right)_{\overline{\mathbb{Q}}_{p}} \hookrightarrow H^{0}\left(\mathfrak{X}^{\text {ord,min }}, \mathcal{E}_{\rho_{0}}^{\text {ord,sub }}\right)_{\overline{\mathbb{Q}}_{p}} .
$$

The coherent sheaf $\mathcal{E}_{U^{p}\left(N_{1}, N_{2}\right), \rho}^{\text {sub }}$ gives rise to a coherent sheaf $\mathcal{E}_{U^{p}\left(N_{1}, N_{2}\right), \rho}^{\text {sub,an }}$ on the rigid space $X_{U^{p}\left(N_{1}, N_{2}\right)}^{\min , \text { an }}$. The inverse system $\left\{\mathcal{E}_{U^{p}\left(N_{1}, N_{2}\right), \rho}^{\text {sub,an }}\right\}$ is a system of coherent sheaves with $G_{n}\left(\mathbb{A}^{\infty}\right)^{\text {ord }}$-action on $\left\{X_{U^{p}\left(N_{1}, N_{2}\right)}^{\min , \text { an }}\right\}$.

\subsubsection{The Frobenius lift $S_{p}$ and tr $_{F}$}

We have a map

$$
\varsigma_{p}^{*}: \mathbb{Z}_{p}\left\langle T_{1}, \ldots, T_{s}\right\rangle_{1} /\langle I\rangle_{1} \longrightarrow \mathbb{Z}_{p}\left\langle T_{1}, \ldots, T_{s}\right\rangle_{1} /\langle I\rangle_{1}
$$

such that

- $\varsigma_{p}^{*}\left(T_{j}\right) \equiv\left(T_{j}\right)^{p} \bmod p$

- and there exists an $r_{1} \in p^{\mathbb{Q}>0}$ such that for all $j=1, \ldots, s$ the element $\varsigma_{p}^{*}\left(T_{j}\right)$ is in the image of $\mathbb{Q}_{p}\left\langle T_{1}, \ldots, T_{s}\right\rangle_{r_{1}} /\langle I\rangle_{r_{1}}$.

Thus $\left(\varsigma_{p}^{*}\left(T_{j}\right)-T_{j}^{p}\right) / p \in \mathbb{Z}_{p}\left\langle T_{1}, \ldots, T_{s}\right\rangle_{r} /\langle I\rangle_{r_{1}}^{\prime}$, and so is the image of some element $G_{j}(\vec{T}) \in \mathbb{Z}_{p}\left\langle T_{1}, \ldots, T_{s}\right\rangle_{r_{1}}$. We have

$$
S_{p}^{*}\left(T_{j}\right) \equiv\left(T_{j}\right)^{p}+p G_{j}\left(T_{1}, \ldots, T_{s}\right) \bmod \langle I\rangle_{1} .
$$

This formula then defines a map $\varsigma_{p}^{*}: \mathbb{Z}_{p}\left[T_{1}, \ldots, T_{s}\right] \rightarrow \mathbb{Z}_{p}\left\langle T_{1}, \ldots, T_{s}\right\rangle_{r_{1}}$ such that

$$
\begin{array}{ccc}
\mathbb{Z}_{p}\left[T_{1}, \ldots, T_{s}\right] & \stackrel{\varsigma_{p}^{*}}{\longrightarrow} & \mathbb{Z}_{p}\left\langle T_{1}, \ldots, T_{s}\right\rangle_{r_{1}} \\
\downarrow & \downarrow & \downarrow \\
\mathbb{Z}_{p}\left\langle T_{1}, \ldots, T_{s}\right\rangle_{1} /\langle I\rangle_{1} \stackrel{\varsigma_{p}^{*}}{\longrightarrow} & \mathbb{Z}_{p}\left\langle T_{1}, \ldots, T_{s}\right\rangle_{1} /\langle I\rangle_{1}
\end{array}
$$


commutes. Write $G_{j}(\vec{T})=\sum_{\vec{i}} g_{j, i} \vec{T}^{i}$. Choose $I_{0} \in \mathbb{Z}_{>0}$ such that

$$
p^{-1}|| G_{j} \|_{r_{1}}<\left(\sqrt{r_{1}}\right)^{I_{0}}
$$

for all $j=1, \ldots, s$ and then choose $r_{2} \in\left(1, \sqrt{r_{1}}\right) \cap p^{\mathbb{Q}}$ with

$$
r_{2}^{I_{0}}<p
$$

If $r \in\left[1, r_{2}\right] \cap p^{\mathbb{Q}}$ we have

$$
\left\|\varsigma_{p}^{*}\left(T_{j}\right)-\left(T_{j}\right)^{p}\right\|_{r}<1 .
$$

(Because if $|\vec{i}| \geq I_{0}$ then $\left\|p g_{j, i} \vec{T} \vec{i}^{i}\right\|_{r} \leq(1 / p)\left\|G_{j}\right\|_{r_{1}}\left(r / r_{1}\right)^{I_{0}}<1$, while for $|\vec{i}| \leq I_{0}$ we have $\left\|p g_{j, i} \vec{T}^{i}\right\|_{r} \leq(1 / p) r^{I_{0}}<1$.) If $r \in\left(1, r_{2}\right] \cap p^{\mathbb{Q}}$ and $H \in \mathbb{Z}_{p}\left[T_{1}, \ldots, T_{s}\right]$ we deduce that

$$
\left\|S_{p}^{*} H-H\left(\vec{T}^{p}\right)\right\|_{r} \leq r^{-p}\|H\|_{r^{p}} .
$$

(We only need check this on monomials. Hence we only need check that if it is true for $H_{1}$ and $H_{2}$ then it is also true for $H_{1} H_{2}$. For this one uses the formula

$$
\begin{array}{r}
\varsigma_{p}^{*}\left(H_{1} H_{2}\right)-\left(H_{1} H_{2}\right)\left(\vec{T}^{p}\right)=\left(\varsigma_{p}^{*} H_{1}-H_{1}\left(\vec{T}^{p}\right)\right)\left(\varsigma_{p}^{*} H_{2}-H_{2}\left(\vec{T}^{p}\right)\right) \\
\left.+\left(\varsigma_{p}^{*} H_{1}-H_{1}\left(\vec{T}^{p}\right)\right) H_{2}\left(\vec{T}^{p}\right)+\left(\varsigma_{p}^{*} H_{2}-H_{2}\left(\vec{T}^{p}\right)\right) H_{1}\left(\vec{T}^{p}\right) .\right)
\end{array}
$$

Hence, if $r \in\left(1, r_{2}\right] \cap p^{\mathbb{Q}}$ and $H \in \mathbb{Z}_{p}\left[T_{1}, \ldots, T_{s}\right]$ we deduce that

$$
\left\|s_{p}^{*} H\right\|_{r}=\|H\|_{r p}
$$

and so $S_{p}^{*}$ extends to an isometric homomorphism

$$
\varsigma_{p}^{*}: \mathbb{Z}_{p}\left\langle T_{1} / r^{p}, \ldots, T_{s} / r^{p}\right\rangle_{1} \longrightarrow \mathbb{Z}_{p}\left\langle T_{1} / r, \ldots, T_{s} / r\right\rangle_{1} .
$$

Modulo $p$ this map reduces to the Frobenius, which is finite and so

$$
\varsigma_{p}^{*}: \mathbb{Z}_{p}\left\langle T_{1} / r^{p}, \ldots, T_{s} / r^{p}\right\rangle_{1} \longrightarrow \mathbb{Z}_{p}\left\langle T_{1} / r, \ldots, T_{s} / r\right\rangle_{1}
$$

is finite. (See section 6.3.2 of [8].) Thus we get an isometric, finite homomorphism between normal rings

$$
\varsigma_{p}^{*}: \mathbb{Q}_{p}\left\langle T_{1}, \ldots, T_{s}\right\rangle_{r^{p}} /\langle I\rangle_{r p}^{\prime}, \mathbb{Q}_{p} \longrightarrow \mathbb{Q}_{p}\left\langle T_{1}, \ldots, T_{s}\right\rangle_{r} /\langle I\rangle_{r, \mathbb{Q}_{p}}^{\prime}
$$

such that the diagram

$$
\begin{array}{ccc}
\mathbb{Q}_{p}\left\langle T_{1}, \ldots, T_{s}\right\rangle_{r^{p}} /\langle I\rangle_{r p}^{\prime}, \mathbb{Q}_{p} & \stackrel{s_{p}^{*}}{\longrightarrow} & \mathbb{Q}_{p}\left\langle T_{1}, \ldots, T_{s}\right\rangle_{r} /\langle I\rangle_{r, \mathbb{Q}_{p}}^{\prime} \\
\downarrow & & \downarrow \\
\mathbb{Q}_{p}\left\langle T_{1}, \ldots, T_{s}\right\rangle^{\dagger} /\langle I\rangle^{\dagger} & \stackrel{s_{p}^{*}}{\longrightarrow} & \mathbb{Q}_{p}\left\langle T_{1}, \ldots, T_{s}\right\rangle^{\dagger} /\langle I\rangle^{\dagger} \\
\downarrow & \downarrow \\
\mathbb{Q}_{p}\left\langle T_{1}, \ldots, T_{s}\right\rangle_{1} /\langle I\rangle_{1, \mathbb{Q}_{p}} & \stackrel{s_{p}^{*}}{\longrightarrow} & \mathbb{Q}_{p}\left\langle T_{1}, \ldots, T_{s}\right\rangle_{1} /\langle I\rangle_{1, \mathbb{Q}_{p}}
\end{array}
$$

commutes. 
The map

$$
\varsigma_{p}: \operatorname{Sp} \mathbb{Q}_{p}\left\langle T_{1}, \ldots, T_{s}\right\rangle_{r} /\langle I\rangle_{r, \mathbb{Q}_{p}}^{\prime} \longrightarrow \operatorname{Sp} \mathbb{Q}_{p}\left\langle T_{1}, \ldots, T_{s}\right\rangle_{r^{p}} /\langle I\rangle_{r p}^{\prime}, \mathbb{Q}_{p}
$$

is compatible with the map

$$
\varsigma_{p}: X_{U^{p}\left(N_{1}, N_{2}\right)}^{\min , \mathrm{an}} \longrightarrow X_{U^{p}\left(N_{1}, N_{2}-1\right)}^{\min , \mathrm{an}}
$$

This latter map is finite, and away from the boundary is flat of degree $p^{n^{2}\left[F^{+}: \mathbb{Q}\right]}$. Thus the pre-image of $\operatorname{Sp} \mathbb{Q}_{p}\left\langle T_{1}, \ldots, T_{s}\right\rangle_{r^{p}} /\langle I\rangle_{r^{p}, \mathbb{Q}_{p}}^{\prime}$ has the form $\operatorname{Sp} B$ where $B$ is a normal, finite $\mathbb{Q}_{p}\left\langle T_{1}, \ldots, T_{s}\right\rangle_{r^{p}} /\langle I\rangle_{r^{p}, \mathbb{Q}_{p}}^{\prime}$ algebra, and we have a factorization

$$
\varsigma_{p}^{*}: \mathbb{Q}_{p}\left\langle T_{1}, \ldots, T_{s}\right\rangle_{r^{p}} /\langle I\rangle_{r^{p}, \mathbb{Q}_{p}}^{\prime} \longrightarrow B \longrightarrow \mathbb{Q}_{p}\left\langle T_{1}, \ldots, T_{s}\right\rangle_{r} /\langle I\rangle_{r, \mathbb{Q}_{p}}^{\prime}
$$

For $\mathfrak{m}$ a maximal ideal of $\mathbb{Q}_{p}\left\langle T_{1}, \ldots, T_{s}\right\rangle_{r^{p}} /\langle I\rangle_{r^{p}, \mathbb{Q}_{p}}^{\prime}$ corresponding to a point of the intersection $X_{U^{p}\left(N_{1}, N_{2}\right)}^{\mathrm{an}} \cap \operatorname{Sp} \mathbb{Q}_{p}\left\langle T_{1}, \ldots, T_{s}\right\rangle_{1} /\langle I\rangle_{1}$ we see that

$$
B / \mathfrak{m}=\left(\mathbb{Q}_{p}\left\langle T_{1}, \ldots, T_{s}\right\rangle_{1} /\langle I\rangle_{1}\right) / \varsigma_{p}^{*} \mathfrak{m}=\left(\mathbb{Q}_{p}\left\langle T_{1}, \ldots, T_{s}\right\rangle_{r} /\langle I\rangle_{r, \mathbb{Q}_{p}}^{\prime}\right) / \varsigma_{p}^{*} \mathfrak{m}
$$

Thus for a Zariski dense set of maximal ideals $\mathfrak{m} \in \operatorname{Sp} \mathbb{Q}_{p}\left\langle T_{1}, \ldots, T_{s}\right\rangle_{r^{p}} /\langle I\rangle_{r p}^{\prime}, \mathbb{Q}_{p}$ the map

$$
B \longrightarrow \mathbb{Q}_{p}\left\langle T_{1}, \ldots, T_{s}\right\rangle_{r} /\langle I\rangle_{r, \mathbb{Q}_{p}}^{\prime}
$$

becomes an isomorphism modulo $\mathfrak{m}$. Hence for any minimal prime $\wp$ of the ring $\mathbb{Q}_{p}\left\langle T_{1}, \ldots, T_{s}\right\rangle_{r^{p}} /\langle I\rangle_{r p}^{\prime}, \mathbb{Q}_{p}$ we have

$$
B_{\wp} / \wp=\left(\mathbb{Q}_{p}\left\langle T_{1}, \ldots, T_{s}\right\rangle_{r} /\langle I\rangle_{r, \mathbb{Q}_{p}}^{\prime}\right)_{\wp} / \wp
$$

(Choose bases over $A_{\wp} / \wp$. Then this map being an isomorphism is equivalent to some matrix having full rank. For $\mathfrak{m}$ in a dense Zariski open set these bases reduce to bases modulo $\mathfrak{m}$. So modulo a Zariski dense set of $\mathfrak{m}$ this matrix has full rank, so it has full rank.) As $B$ is normal and $\mathbb{Q}_{p}\left\langle T_{1}, \ldots, T_{s}\right\rangle_{r} /\langle I\rangle_{r, \mathbb{Q}_{p}}^{\prime}$ is finite over $B$, we see that

$$
B=\mathbb{Q}_{p}\left\langle T_{1}, \ldots, T_{s}\right\rangle_{r} /\langle I\rangle_{r, \mathbb{Q}_{p}}^{\prime}
$$

i.e.

$$
S_{p}^{-1} \operatorname{Sp} \mathbb{Q}_{p}\left\langle T_{1}, \ldots, T_{s}\right\rangle_{r^{p}} /\langle I\rangle_{r p}^{\prime}, \mathbb{Q}_{p}=\operatorname{Sp} \mathbb{Q}_{p}\left\langle T_{1}, \ldots, T_{s}\right\rangle_{r} /\langle I\rangle_{r, \mathbb{Q}_{p}}^{\prime}
$$

The sheaf $\mathcal{E}_{U^{p}\left(N_{1}, N_{2}\right), \rho}^{\text {sub }}$ induces a coherent sheaf $\mathcal{E}_{U^{p}\left(N_{1}\right), \rho}^{\text {sub, } \dagger}$ on $\mathcal{X}_{U^{p}\left(N_{1}\right)}^{\text {ord,min, } \dagger}$, which does not depend on $N_{2}$. It equals the push-forward from any $\mathcal{X}_{U^{p}\left(N_{1}\right), \Delta}^{\text {ord, } \dagger}$ of the sheaf $\mathcal{E}_{U^{p}\left(N_{1}\right), \rho}^{\text {sub },}$. The inverse system $\left\{\mathcal{E}_{U^{p}(N), \rho}^{\text {sub, },}\right\}$ is a system of coherent sheaves with $G_{n}\left(\mathbb{A}^{\infty}\right)^{\text {ord }}$-action on $\left\{\mathcal{X}_{U^{p}\left(N_{1}\right)}^{\mathrm{ord}, \mathrm{min},}\right\}$. The map

$$
\operatorname{tr}_{F}: \varsigma_{p, *} \mathcal{E}_{U^{p}(N), \Delta, \rho}^{\mathrm{sub},} \longrightarrow \mathcal{E}_{U^{p}(N), \Delta, \rho}^{\mathrm{sub}, \dagger}
$$


over $\mathcal{X}_{U^{p}\left(N_{1}\right), \Delta}^{\text {ord, }}$ induces a map

$$
\operatorname{tr}_{F}: \varsigma_{p, *} \mathcal{E}_{U^{p}(N), \rho}^{\mathrm{sub}, \dagger} \longrightarrow \mathcal{E}_{U^{p}(N), \rho}^{\mathrm{sub}, \dagger}
$$

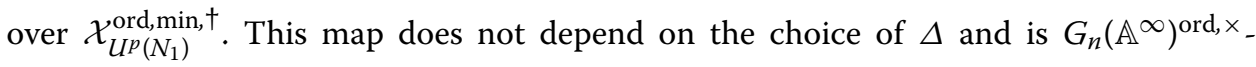
equivariant. It satisfies

$$
\operatorname{tr}_{F} \circ \varsigma_{p}=p^{n^{2}\left[F^{+}: \mathbb{Q}\right]}
$$

It induces a map

$$
\operatorname{tr}_{F} \in \operatorname{End}\left(H^{0}\left(\mathcal{X}_{U^{p}(N)}^{\text {ord,min, } \dagger}, \mathcal{E}_{U^{p}(N), \rho}^{\text {sub, } \dagger}\right)\right)
$$

also satisfying

$$
\operatorname{tr}_{F} \circ \varsigma_{p}=p^{n^{2}\left[F^{+}: \mathbb{Q}\right]} \text {. }
$$

We again remark that $\operatorname{tr}_{F}$ is closely related to the operator often denoted $U_{p}$-probably they differ simply by a scalar multiple.

The isomorphisms

$$
H^{0}\left(\mathcal{X}_{U^{p}(N)}^{\text {ord,min }, \dagger}, \mathcal{E}_{U^{p}(N), \rho}^{\text {sub, }}\right) \stackrel{\sim}{\longrightarrow} H^{0}\left(\mathcal{X}_{U^{p}(N), \Delta}^{\text {ord }, \dagger}, \mathcal{E}_{U^{p}(N), \Delta, \rho}^{\text {sub, }}\right)
$$

are $\operatorname{tr}_{F}$-equivariant. Moreover the space $H^{0}\left(\mathcal{X}^{\text {ord,min, }}{ }^{\dagger}, \mathcal{E}_{\rho}^{\text {sub }}\right)_{\overline{\mathbb{Q}}_{p}}$ inherits an endomorphism $\operatorname{tr}_{F}$, which commutes with $G_{n}\left(\mathbb{A}^{\infty}\right)^{\text {ord, } \times}$ and satisfies $\operatorname{tr}_{F} \circ \varsigma_{p}=p^{n^{2}\left[F^{+}: \mathbb{Q}\right]}$.

The sheaf $\mathcal{E}_{U^{p}\left(N_{1}, N_{2}\right), \rho}^{\text {sub,an }}$ restricted to the space $\operatorname{Sp} \mathbb{Q}_{p}\left\langle T_{1}, \ldots, T_{s}\right\rangle_{r} /\langle I\rangle_{r, \mathbb{Q}_{p}}^{\prime}$ corresponds to a finitely generated module $E_{r}$ over the ring $\mathbb{Q}_{p}\left\langle T_{1}, \ldots, T_{s}\right\rangle_{r} /\langle I\rangle_{p, \mathbb{Q}_{p}}^{\prime}$, which is naturally a Banach module. If $r^{\prime}<r$ then

$$
E_{r^{\prime}}=E_{r} \otimes_{\mathbb{Q}_{p}\left\langle T_{1}, \ldots, T_{s}\right\rangle_{r} /\langle I\rangle_{r, \mathbb{Q}}^{\prime}, i_{r, r^{\prime}}^{\prime}}\left(\mathbb{Q}_{p}\left\langle T_{1}, \ldots, T_{s}\right\rangle_{r^{\prime}} /\langle I\rangle_{r^{\prime}, \mathbb{Q}_{p}}^{\prime}\right)
$$

Then the map $E_{r} \rightarrow E_{r^{\prime}}$, which we will also denote $i_{r, r^{\prime}}^{\prime}$, is completely continuous. The map $\operatorname{tr}_{F}$ extends to a continuous $\mathbb{Q}_{p}\left\langle T_{1}, \ldots, T_{s}\right\rangle_{r^{p}} /\langle I\rangle_{r p}^{\prime}, \mathbb{Q}_{p}$ linear map

$$
t_{r}: E_{r} \longrightarrow E_{r p}
$$

for $r \in\left[1, r_{2}\right] \cap p^{\mathbb{Q}}$. We set

$$
E^{\dagger}=\bigcup_{r>1} E_{r},
$$

so that

$$
E^{\dagger}=H^{0}\left(\mathcal{X}_{U^{p}\left(N_{1}\right)}^{\text {ord,min }, \dagger}, \mathcal{E}_{U^{p}\left(N_{1}\right), \rho}^{\text {sub } \dagger}\right)
$$

We have that $\left.\operatorname{tr}_{F}\right|_{E_{r}}=t_{r}$. As $t_{r}$ is continuous and $i_{r p, r}^{\prime}$ is completely continuous we see that

$$
\operatorname{tr}_{F}: E_{r} \longrightarrow E_{r}
$$


and that this map is completely continuous. Thus each $E_{r}$ admits slope decompositions for $\operatorname{tr}_{F}$ and hence by Lemma 6.6 so does $E^{\dagger}$ and $E^{\dagger} \otimes \overline{\mathbb{Q}}_{p}$.

If $a \in \mathbb{Q}$ we thus have a well-defined, finite dimensional subspace

$$
H^{0}\left(\mathcal{X}_{U^{p}(N)}^{\mathrm{ord}, \mathrm{min}, \dagger}, \mathcal{E}_{U^{p}(N), \rho}^{\mathrm{sub}, \dagger}\right)_{\overline{\mathbb{Q}}_{p}, \leq a} \subset H^{0}\left(\mathcal{X}_{U^{p}(N)}^{\mathrm{ord}, \mathrm{min}, \dagger}, \mathcal{E}_{U^{p}(N), \rho}^{\mathrm{sub}, \dagger}\right) \otimes_{\mathbb{Q}_{p}} \overline{\mathbb{Q}}_{p}
$$

(Defined with respect to $\operatorname{tr}_{F}$.) We set

$$
H^{0}\left(\mathcal{X}^{\text {ord,min }, \dagger}, \mathcal{E}_{\rho}^{\text {sub }}\right)_{\overline{\mathbb{Q}}_{p}, \leq a}=\lim _{\rightarrow U^{p}, N} H^{0}\left(\mathcal{X}_{U^{p}(N)}^{\mathrm{ord}, \min , \dagger}, \mathcal{E}_{U^{p}(N), \rho}^{\mathrm{sub}, \dagger}\right)_{\overline{\mathbb{Q}}_{p}, \leq a},
$$

so that there are $G_{n}\left(\mathbb{A}^{\infty}\right)^{\text {ord, } \times}$-equivariant embeddings

$$
H^{0}\left(\mathcal{X}^{\text {ord,min, },}, \mathcal{E}_{\rho}^{\text {sub }}\right)_{\overline{\mathbb{Q}}_{p}, \leq a} \subset H^{0}\left(\mathcal{X}^{\text {ord,min, },}, \mathcal{E}_{\rho}^{\text {sub }}\right)_{\overline{\mathbb{Q}}_{p}} \hookrightarrow H^{0}\left(\mathfrak{X}^{\text {ord,min }}, \mathcal{E}_{\rho_{0}}^{\text {ord,sub }}\right)_{\overline{\mathbb{Q}}_{p}} .
$$

We have proved the following lemma. (The referee suggests, in politer terms, that we have made a mountain out of a mole hill in proving this lemma and Lemma 6.11. The referee is probably correct. We are not very practiced at these sorts of arguments. Neither lemma will come as any surprise to experts.)

Lemma 6.12 $H^{0}\left(\mathcal{X}^{\mathrm{ord}, \mathrm{min},{ }^{\dagger}}, \mathcal{E}_{\rho}^{\mathrm{sub}}\right)_{\overline{\mathbb{Q}}_{p}, \leq a}$ is an admissible $G_{n}\left(\mathbb{A}^{\infty}\right)^{\text {ord, } \times}$-module.

Combining this with Corollary 6.5 we obtain the following result.

Corollary 6.13 Suppose that $\rho$ is a representation of $L_{n,(n)}$ over $\mathbb{Q}$, that $a \in \mathbb{Q}$ and that $\Pi$ is an irreducible $G_{n}\left(\mathbb{A}^{\infty}\right)^{\text {ord, }} \times$-subquotient of

$$
H^{0}\left(\mathcal{X}^{\mathrm{ord}, \mathrm{min}, \dagger}, \mathcal{E}_{\rho}^{\mathrm{sub}}\right)_{\overline{\mathbb{Q}}_{p}, \leq a} .
$$

Then there is a continuous semi-simple representation

$$
R_{p}(\Pi): G_{F} \longrightarrow G L_{2 n}\left(\overline{\mathbb{Q}}_{p}\right)
$$

with the following property: If $\Pi$ is unramified at a rational prime $q \neq p$ (in the sense of Sect. 1.3) and if $v \mid q$ is a prime of $F$, then

$$
\mathrm{WD}\left(\left.R_{p}(\Pi)\right|_{G_{F_{v}}}\right)^{\mathrm{F}-\mathrm{ss}} \cong \operatorname{rec}_{F_{v}}\left(\mathrm{BC}\left(\Pi_{q}\right)_{v}|\operatorname{det}|_{v}^{(1-2 n) / 2}\right) .
$$

We will next explain the consequences of these results for sheaves of differentials on $A_{U^{p}\left(N_{1}, N_{2}\right), \Sigma}^{(m), \text { ord },}$. But we first need to record a piece of commutative algebra.

Lemma 6.14 Suppose that $A \rightarrow B \rightarrow C$ are reduced noetherian rings, with $B$ a finite flat $A$ module of rank $r_{B}$ and $C$ a finite flat $A$-module of rank $r_{C}$. Suppose also that the total ring of fractions of $C$ is finite flat over the total ring of fractions of $B$. Then $r_{B} \mid r_{C}$ and

$$
\left(r_{C} / r_{B}\right) \operatorname{tr}_{B / A}=\operatorname{tr}_{C / A}: B \longrightarrow A .
$$

Proof It suffices to check this after passing to total rings of fractions (i.e. localizations at the set of nonzero divisors). In this case $B$ is free over $A$ and $C$ is free over $B$, so the lemma is clear. 
Proposition 6.15 There are representations $\rho_{m, s}^{i, j}$ of $L_{n,(n)}$ over $\mathbb{Q}$ with the following property. If $\left(U^{p}(N), \Sigma\right) \in \mathcal{J}_{n}^{(m), \text { tor,ord }}$ and if $\left(U^{p}\right)^{\prime}$ denotes the image of $U^{p}$ in $G_{n}\left(\mathbb{A}^{\infty, p}\right)$, then there is a spectral sequence with first page

$$
\begin{aligned}
& E_{1}^{i, j}=H^{0}\left(\mathcal{X}_{\left(U^{p}\right)^{\prime}(N)}^{\text {ordmin }, \dagger}, \mathcal{E}_{\left(U^{p}\right)^{\prime}(N), \rho_{m, S}^{i, j}}^{\mathrm{sub}, \dagger}\right) \\
& \Rightarrow H^{i+j}\left(\mathcal{A}_{U^{p}(N), \Sigma}^{(m), \text { ord }, \dagger}, \Omega_{\mathcal{A}_{U^{p}(N), \Sigma}^{(m), \text { ord },}+}(\log \infty) \otimes \mathcal{I}_{\partial \mathcal{A}_{U^{p}(N), \Sigma}^{(m), \text { ord },},}\right) .
\end{aligned}
$$

These spectral sequences are equivariant for the action of $G_{n}\left(\mathbb{A}^{\infty}\right)^{\text {ord }}$. The map $\operatorname{tr}_{F}$ on the $H^{i+j}\left(\mathcal{A}_{U^{p}(N), \Sigma}^{(m), \text { ord, } \dagger}, \Omega_{A_{U^{p}(N), \Sigma}^{(m), \text { ord },}}(\log \infty) \otimes \mathcal{I}_{\partial \mathcal{A}_{U^{p}(N), \Sigma}^{(m), \text { ord },},}\right)$ is compatible with the map $p^{n m[F: \mathbb{Q}]} \operatorname{tr}_{F}$ on the $H^{0}\left(\mathcal{X}_{\left(U^{p}\right)^{\prime}(N)}^{\text {ord,min, }}, \mathcal{E}_{\left(U^{p}\right)^{\prime}(N), \rho_{m, s}^{i, j}}^{\text {sub, }}\right)$.

Proof Let $\pi$ denote the map $\mathcal{A}_{U^{p}(N), \Sigma}^{(m), \text { ord } \dagger} \rightarrow \mathcal{X}_{\left(U^{p}\right)^{\prime}(N)}^{\text {ord,min, }}$. Lemmas 5.10 and 6.9 tell us that there is a spectral sequence of coherent sheaves on $\mathcal{X}_{U^{p}(N)}^{\text {ord,min, }}{ }^{\dagger}$ with first page

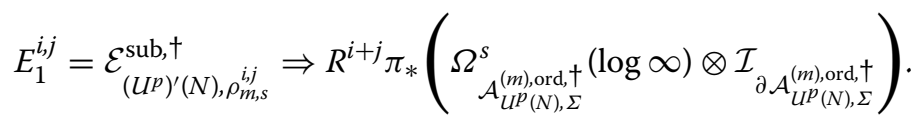

The first assertion follows from Lemma 6.11 of this paper and from proposition 3.1 of [27] (which tell us that

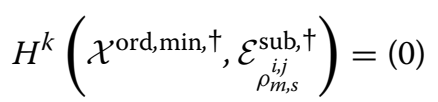

for $k>0$ ).

For the last assertion we may replace $\Sigma$ by a refinement and so reduce to the case that there is a $\Delta$ with $\left(\left(U^{p}\right)^{\prime}, \Delta\right) \in \mathcal{J}_{n}^{\text {tor,ord }}$ and $\left(\left(U^{p}\right)^{\prime}(N), \Delta\right) \leq\left(U^{p}(N), \Sigma\right)$. (Use Lemma 5.6.) To avoid confusion we will write $\varsigma_{p, \mathcal{A}}$ or $\varsigma_{p, \mathcal{X}}$ depending on whether $\varsigma_{p}$ is acting on $\mathcal{A}_{U^{p}(N), \Sigma^{\text {ord }}}^{(m) \text { or }} \mathcal{X}_{\left(U^{p}\right)^{\prime}(N), \Delta^{\text {ord }}}^{\text {ord }}$. We will also factorize $\varsigma_{p, \mathcal{A}}$ as

$$
\mathcal{A}_{U^{p}(N), \Sigma^{\text {ord }}}^{(m), \text { ord },} \stackrel{\Phi}{\longrightarrow} \varsigma_{p, \mathcal{X}}^{*} \mathcal{A}_{U^{p}(N), \Sigma^{\text {ord }}}^{(m), \text { ord } \dagger} \stackrel{\Psi}{\longrightarrow} \mathcal{A}_{U^{p}(N), \Sigma^{\text {ord }}}^{(m), \text { ord } \dagger}
$$

Write $\pi^{\prime}$ for the map

$$
\pi^{\prime}: \mathcal{A}_{U^{p}(N), \Sigma^{\text {ord }}}^{(m) \text { ord },} \rightarrow \mathcal{X}_{\left(U^{p}\right)^{\prime}(N), \Delta^{\text {ord }}}^{\text {ord } \dagger}
$$

and $\pi^{\prime \prime}$ for the map

$$
\pi^{\prime \prime}: \varsigma_{p, \mathcal{X}}^{*} \mathcal{A}_{U^{p}(N), \Sigma^{\text {ord }}}^{(m), \text { ord } \dagger} \rightarrow \mathcal{X}_{\left(U^{p}\right)^{\prime}(N), \Delta^{\text {ord }}}^{\text {ord } \dagger}
$$

The sheaf $\mathcal{E}_{\substack{i, j \\ \rho_{m, s}}}^{\text {can } \dagger}$ on $\mathcal{X}_{\left(U^{p}\right)^{\prime}(N), \Delta^{\text {ord }}}^{\text {ord } \dagger}$ is $R^{i} \pi_{*}^{\prime} \mathcal{F}_{j}$, where

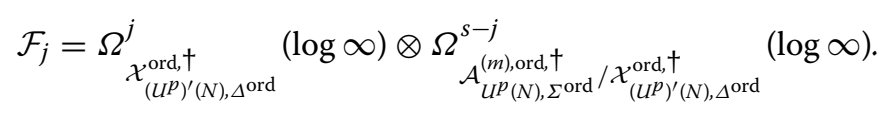


To prove the last sentence of the lemma it suffices to show that the diagrams

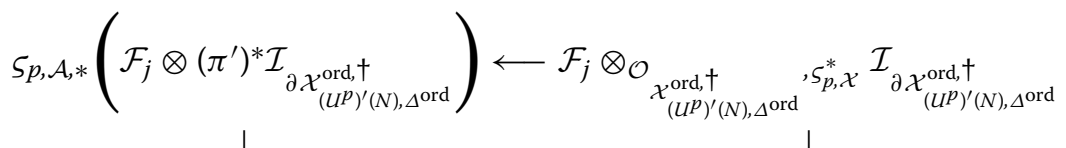

$$
\begin{aligned}
& \varsigma_{p, \mathcal{A}, *}\left(\mathcal{F}_{j} \otimes \mathcal{I}_{\partial \mathcal{A}_{U^{p}(N), \Sigma^{\text {ord }}}^{(m), \text { rd },}}\right) \longleftarrow \mathcal{F}_{j} \otimes_{\mathcal{O}_{\mathcal{A}_{U p}^{(m), \text { ord }, ~}, \text { ord }}, \varsigma_{p, \mathcal{A}}^{*}} \mathcal{I}_{\partial \mathcal{A}_{U p(N), \Sigma^{\text {ord }}}^{(m), \text { ord }}, \dagger}
\end{aligned}
$$

and

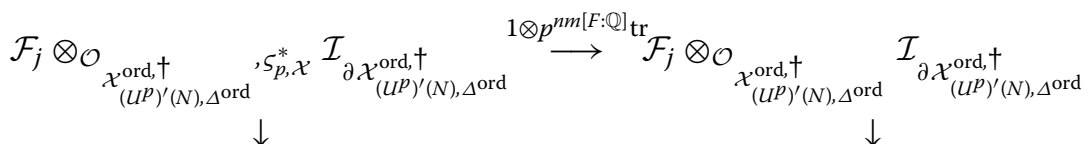

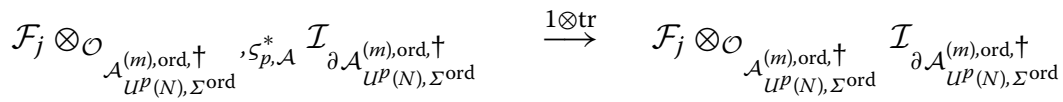

commute. In the first diagram the upper horizontal map is the composite

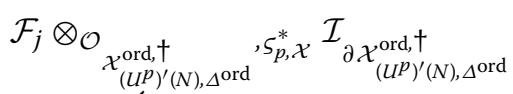

$$
\begin{aligned}
& =\Psi_{*}\left(\left(\Psi^{*} \mathcal{F}_{j}\right) \otimes\left(\pi^{\prime \prime}\right)^{*} \mathcal{I}_{\partial \mathcal{X}_{\left(U^{\prime}\right)^{\prime}(N), \Delta^{\text {ord }}}^{\text {ord }}, \dagger}\right) \\
& \longrightarrow \Psi_{*} \Phi_{*}\left(\left(\Phi^{*} \Psi^{*} \mathcal{F}_{j}\right) \otimes\left(\Phi^{*}\left(\pi^{\prime \prime}\right)^{*} \mathcal{I}_{\partial \mathcal{X}_{(U P)^{\prime}(N), \Delta}^{\mathrm{ord},},}\right)\right) \\
& =\varsigma_{p, \mathcal{A}, *}\left(\left(\varsigma_{p, \mathcal{A}}^{*} \mathcal{F}_{j}\right) \otimes\left(\pi^{\prime}\right)^{*} \mathcal{I}_{\partial \mathcal{X}_{\left(U p^{\prime}\right)^{\prime}(N), \Delta \text { ord }}^{\text {ord }} \dagger}\right) \\
& \stackrel{\varsigma_{p, \mathcal{A}}^{*}}{\longrightarrow} \varsigma_{p, \mathcal{A}, *}\left(\mathcal{F}_{j} \otimes\left(\pi^{\prime}\right)^{*} \mathcal{I}_{\partial \mathcal{X}_{\left(U^{p}\right)^{\prime}(N), \Delta^{\text {ord }}}^{\text {ord }} \dagger}\right),
\end{aligned}
$$

and the lower horizontal map is

$$
\begin{aligned}
& \mathcal{F}_{j} \otimes_{\mathcal{O}_{\mathcal{A}^{(m), \text { ord }, ~}, \text { ord }}, \varsigma_{p, \mathcal{A}}^{*}} \mathcal{I}_{\partial \mathcal{A}_{U^{p}(N), \text { ord },}^{(m), \Sigma^{\text {ord }}}} \cong \varsigma_{p, \mathcal{A}, *}\left(\left(\varsigma_{p, \mathcal{A}}^{*} \mathcal{F}_{j}\right) \otimes \mathcal{I}_{\partial \mathcal{A}_{U^{p}(N), \Sigma^{\text {ord }}}^{(m), \text { ord }},}\right) \\
& \stackrel{\varsigma_{p, \mathcal{A}}^{*}}{\longrightarrow} \varsigma_{p, \mathcal{A}, *}\left(\mathcal{F}_{j} \otimes \mathcal{I}_{\partial \mathcal{A}_{U^{p}(\mathrm{~N}), \Sigma^{\text {ord }}}^{(m), \text { ord }} \dagger}\right) .
\end{aligned}
$$

We see that the first square tautologically commutes. The second square commutes because the two maps

$$
p^{n m[F: \mathbb{Q}]} \operatorname{tr}: \Psi_{*} \mathcal{O}_{S_{p, \mathcal{X}}^{*} \mathcal{A}_{U^{p}(N), \Sigma^{\text {ord }}}^{(m), \text { ord },}} \longrightarrow \mathcal{O}_{\mathcal{A}_{U^{p}(N), \Sigma^{\text {ord }}}^{(m), \text { ord }},}
$$

and

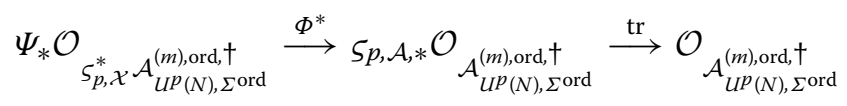

are equal. This in turn follows from Lemma 6.14. 
Corollary 6.16 For all $i$ and $s$ the vector space $H^{i}\left(\mathcal{A}_{U^{p}(N), \Sigma}^{(m), \text { ord }, \dagger}, \Omega_{\mathcal{A}_{U^{(}(N), \Sigma}^{s}}^{(m), \text { ord },}(\log \infty) \otimes\right.$ $\mathcal{I}_{\partial \mathcal{A}_{U^{p}(N), \Sigma}^{(m), o r d}, \dagger}$ admits slope decompositions for $\operatorname{tr}_{F}$.

We write

$$
\begin{aligned}
& H^{i}\left(\mathcal{A}^{(m), \mathrm{ord}, \dagger}, \Omega^{s}(\log \infty) \otimes \mathcal{I}_{\partial \mathcal{A}^{(m), \text { ord }, \dagger}}\right)_{\leq a} \\
& \quad=\lim _{\rightarrow U^{p}, N, \Sigma} H^{i}\left(\mathcal{A}_{U^{p}(N), \Sigma}^{(m), \mathrm{ord}, \dagger}, \Omega_{\mathcal{A}_{U^{p}(N), \Sigma}^{(m), \text { ord },}}^{s}(\log \infty) \otimes \mathcal{I}_{\partial \mathcal{A}_{U^{p}(N), \Sigma}^{(m), \text { ord } \dagger}}\right)_{\leq a} .
\end{aligned}
$$

The next corollary now follows from the proposition and Lemma 6.6.

Corollary 6.17 For any $a \in \mathbb{Q}$ there is $a G_{n}\left(\mathbb{A}^{\infty}\right)^{\text {ord, } \times}$-equivariant spectral sequence with first page $E_{1}^{i, j}$ :

$$
H^{0}\left(\mathcal{X}^{\text {ord,min, },}, \mathcal{E}_{\rho_{i, j}^{i, s}}^{\mathrm{sub}}\right)_{\leq a} \Rightarrow H^{i+j}\left(\mathcal{A}^{(m), \text { ord }, \dagger}, \Omega_{\mathcal{A}^{(m), \text { ord },},}^{s}(\log \infty) \otimes \mathcal{I}_{\left.\partial \mathcal{A}^{(m), \text { ord },},\right)_{\leq a+m n[F: \mathbb{Q}]}}\right.
$$

Combining this with Corollary 6.13 we obtain the following corollary.

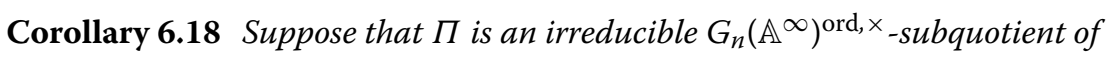

$$
H^{i}\left(\mathcal{A}^{(m), \text { ord }, \dagger}, \Omega_{\mathcal{A}^{(m), \text { ord },}+}(\log \infty) \otimes \mathcal{I}_{\left.\partial \mathcal{A}^{(m), \text { ord },},\right)_{\leq a}} \otimes_{\mathbb{Q}_{p}} \overline{\mathbb{Q}}_{p}\right.
$$

for some $a \in \mathbb{Q}$. Then there is a continuous semi-simple representation

$$
R_{p}(\Pi): G_{F} \longrightarrow G L_{2 n}\left(\overline{\mathbb{Q}}_{p}\right)
$$

with the following property: If $\Pi$ is unramified at a rational prime $q \neq p$ (in the sense of Sect. 1.3) and if $v \mid q$ is a prime of $F$, then

$$
\mathrm{WD}\left(\left.R_{p}(\Pi)\right|_{G_{F_{v}}}\right)^{\mathrm{F}-\mathrm{ss}} \cong \operatorname{rec}_{F_{v}}\left(\mathrm{BC}\left(\Pi_{q}\right)_{v}|\operatorname{det}|_{\nu}^{(1-2 n) / 2}\right)
$$

\subsection{Rigid cohomology}

Our main object of study will be the groups

$$
H_{c-\partial}^{i}\left(\bar{A}_{U^{p}(N), \Sigma}^{(m), \text { ord }}\right)=\mathbb{H}^{i}\left(\mathcal{A}_{U^{p}(N), \Sigma}^{(m), \text { ord, } \dagger}, \Omega_{\mathcal{A}_{U^{p}(N), \Sigma}^{(m), \text { ord },}}^{\bullet}(\log \infty) \otimes \mathcal{I}_{\partial \mathcal{A}_{U^{p}(N), \Sigma}^{(m), \text { ord },}}\right),
$$

where $\left(U^{p}(N), \Sigma\right) \in \mathcal{J}_{n}^{(m) \text {,tor,ord }}$. This can be thought of as a sort of rigid cohomology of $\bar{A}_{U^{p}(N), \Sigma}^{(m), \text { ord }}$ with compact supports towards the toroidal boundary, but not towards the non-ordinary locus. It seems plausible to us that this can be intrinsically attached to the pair $\bar{A}_{U^{p}(N)}^{(m) \text {,ord }} \supset \partial \bar{A}_{U^{p}(N)}^{(m) \text {,ord }}$. Hence our notation. However we will not prove this, so the reader is cautioned that our notation is nothing more than a short-hand, and the group $H_{c-\partial}^{i}\left(\bar{A}_{U^{p}(N), \Sigma}^{(m), \text { ord }}\right)$ must be assumed to depend on the pair $\mathcal{A}_{U^{p}(N), \Sigma}^{(m), \text { ord } \dagger} \supset \partial \mathcal{A}_{U^{p}(N), \Sigma}^{(m), \text { ord }, \dagger}$. We will also set

$$
H_{c-\partial}^{i}\left(\bar{A}^{(m), \text { ord }}\right)=\underset{U^{p, N}, \Sigma}{\lim } H_{c-\partial}^{i}\left(\bar{A}_{U^{p}(N), \Sigma}^{(m), \text { ord }}\right)
$$


It has a smooth action of $G_{n}\left(\mathbb{A}^{\infty}\right)^{\text {ord }}$. The maps

$$
\operatorname{tr}_{F}: \varsigma_{p, *} \Omega_{\mathcal{A}_{U p(N), \Sigma}^{(m), \text { ord },}}^{j}(\log \infty) \longrightarrow \Omega_{\mathcal{A}_{U p(N), \Sigma}^{(m), \text { ord },}}^{j}(\log \infty)
$$

induce endomorphisms

$$
\operatorname{tr}_{F} \in \operatorname{End}\left(H_{c-\partial}^{i}\left(\bar{A}_{U^{p}(N), \Sigma}^{(m), \text { ord }}\right)\right)
$$

which commute with the action of $G_{n}\left(\mathbb{A}^{\infty}\right)^{\text {ord, } \times}$ and satisfy

$$
\operatorname{tr}_{F} \circ \varsigma_{p}=p^{(n+2 m) n\left[F^{+}: \mathbb{Q}\right]} .
$$

\section{Lemma 6.19 There are natural isomorphisms}

$$
H_{c-\partial}^{i}\left(\bar{A}_{U^{p}(N), \Sigma}^{(m), \text { ord }}\right) \stackrel{\sim}{\longrightarrow} H_{c-\partial}^{i}\left(\bar{A}^{(m), \text { ord }}\right)^{U^{p}(N)} .
$$

Proof Use Lemmas 5.1, 5.6, 5.7 and 6.9.

We will compute the group $H_{c-\partial}^{i}\left(\bar{A}_{U^{p}(N), \Sigma}^{(m), \text { ord }}\right)$ in two ways. The first way will be in terms of $p$-adic cusp forms and will allow us to attach Galois representations to irreducible $G_{n}\left(\mathbb{A}^{\infty}\right)^{\text {ord, } \times}$-subquotients of $H_{c-\partial}^{i}\left(\bar{A}^{(m), \text { ord }}\right) \otimes_{\mathbb{Q}_{p}} \overline{\mathbb{Q}}_{p}$. The second way will be geometrical, in terms of the stratification of the boundary. In this second approach the cohomology of the locally symmetric spaces associated to $L_{n,(n) \text { lin }}^{(m)}$ will appear.

Here is our first calculation.

Lemma 6.20 The vector spaces $H_{c-\partial}^{i}\left(\bar{A}_{U^{p}(N), \Sigma}^{(m), \text { ord }}\right)$ admit slope decompositions for $\operatorname{tr}_{F}$. If moreover we set

$$
H_{c-\partial}^{i}\left(\bar{A}^{(m), \text { ord }}\right)_{\leq a}=\underset{U^{\vec{p}, N, \Sigma}}{\lim } H_{c-\partial}^{i}\left(\bar{A}_{U^{p}(N), \Sigma}^{(m), \text { ord }}\right)_{\leq a},
$$

then there is a $G_{n}\left(\mathbb{A}^{\infty}\right)^{\text {ord, } \times}$-spectral sequence with first page

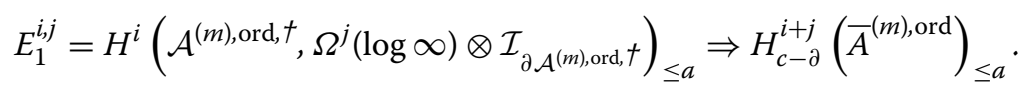

Proof This follows from Lemma 6.6, Corollary 6.16 and the spectral sequence

$$
E_{1}^{i, j}=H^{i}\left(\mathcal{A}_{U^{p}(N), \Sigma}^{(m), \text { ord }, \dagger}, \Omega_{\mathcal{A}_{U p(N), \Sigma}^{(m), \text { ord },}, j}^{j}(\log \infty) \otimes \mathcal{I}_{\partial \mathcal{A}_{U^{p}(N), \Sigma}^{(m), \text { ord },},}\right) \Rightarrow H_{c-\partial}^{i+j}\left(\bar{A}_{U^{p}(N), \Sigma}^{(m), \text { ord }}\right) .
$$

And here is our second calculation.

Lemma 6.21 There are $G_{n}\left(\mathbb{A}^{\infty}\right)^{\text {ord, } \times}$-equivariant spectral sequences with first page

$$
E_{1}^{i, j}=H_{\mathrm{rig}}^{i}\left(\partial^{(j)} \bar{A}_{U^{p}(N), \Sigma}^{(m), \mathrm{ord}}\right) \Rightarrow H_{c-\partial}^{i+j}\left(\bar{A}_{U^{p}(N), \Sigma}^{(m), \mathrm{ord}}\right)
$$

Moreover the action of Frobenius on the left hand side is compatible with the action of $s_{p}$ on the right hand side. 
Proof By Lemmas 2.3 and 6.9 the group $H_{c-\partial}^{i}\left(\bar{A}_{U^{p}(N), \Sigma}^{(m), \text { ord }}\right)$ is isomorphic to the hypercohomology of the double complex

$$
\mathbb{H}^{i}\left(\mathcal{A}_{U^{p}(N), \Sigma}^{(m), \text { ord },}, i_{*}^{(s)} \Omega_{\partial^{(s)}}^{r} \mathcal{A}_{U^{p}(N), \Sigma}^{(m), \text { ord },}\right),
$$

and so there is a spectral sequence with first page

$$
E_{1}^{i, j}=\mathbb{H}^{i}\left(\partial^{(j)} \mathcal{A}_{U^{p}(N), \Sigma}^{(m), \text { ord }, \dagger}, \Omega_{\partial^{(j)} \mathcal{A}_{U p(N), \Sigma}^{(m), \text { ord },}}^{\bullet}\right) \Rightarrow H_{c-\partial}^{i}\left(\bar{A}_{U^{p}(N), \Sigma}^{(m), \text { ord }}\right) .
$$

However, by Lemma 6.8 and the quasi-projectivity of $\partial^{(j)} \mathcal{A}_{U^{p}(N), \Sigma}^{(m), \text { ord }}$, we see that there are $G_{n}^{(m)}\left(\mathbb{A}^{\infty}\right)^{\text {ord }}$-equivariant isomorphisms

$$
\mathbb{H}^{i}\left(\partial^{(j)} \mathcal{A}_{U^{p}(N), \Sigma}^{(m), \mathrm{ord}, \dagger}, \Omega_{\partial^{(j)} \mathcal{A}_{U p(N), \Sigma}^{(m), \mathrm{ord},},}^{\bullet}\right) \cong H_{\mathrm{rig}}^{i}\left(\partial^{(j)} \bar{A}_{U^{p}(N), \Sigma}^{(m), \text { ord }}\right),
$$

and that under this identification $\varsigma_{p}$ corresponds to Frobenius (because $\varsigma_{p}$ equals Frobenius on the special fibre).

Corollary 6.22 $H_{c-\partial}^{i}\left(\bar{A}_{U^{p}(N), \Sigma}^{(m), \text { ord }}\right)$ is finite dimensional. Moreover

$$
H_{c-\partial}^{i}\left(\bar{A}_{U^{p}(N), \Sigma}^{(m), \text { ord }}\right)=H_{c-\partial}^{i}\left(\bar{A}_{\left.U^{p}(N), \Sigma\right), \text { ord }}^{(m)}\right)_{\leq a},
$$

for some a, and so

$$
H_{c-\partial}^{i}\left(\bar{A}^{(m), \text { ord }}\right)=\bigcup_{a \in \mathbb{Q}} H_{c-\partial}^{i}\left(\bar{A}^{(m), \text { ord }}\right)_{\leq a} .
$$

Proof The first assertion follows from the lemma and theorem 3.1 of [7]. The second assertion follows because $\operatorname{tr}_{F} \circ \zeta_{p}=p^{n(n+2 m)\left[F^{+}: \mathbb{Q}\right]}$ and so by the first part $\operatorname{tr}_{F}$ must be an automorphism of $H_{c-\partial}^{i}\left(\bar{A}_{U^{p}(N), \Sigma}^{(m), \text { ord }}\right)$.

Combining this with Corollary 6.18 and Lemma 6.20 we obtain the following corollary.

Corollary 6.23 Suppose that $\Pi$ is an irreducible $G_{n}\left(\mathbb{A}^{\infty}\right)^{\text {ord, } \times}$-subquotient of

$$
H_{c-\partial}^{i}\left(\bar{A}^{(m), \text { ord }}\right) \otimes_{\mathbb{Q}_{p}} \overline{\mathbb{Q}}_{p}
$$

Then there is a continuous semi-simple representation

$$
R_{p}(\Pi): G_{F} \longrightarrow G L_{2 n}\left(\overline{\mathbb{Q}}_{p}\right)
$$

with the following property: If $\Pi$ is unramified at a rational prime $q \neq p$ (in the sense of Sect. 1.3) and if $v \mid q$ is a prime of $F$. Then

$$
\mathrm{WD}\left(\left.R_{p}(\Pi)\right|_{G_{F_{v}}}\right)^{\mathrm{F}-\mathrm{ss}} \cong \operatorname{rec}_{F_{v}}\left(\mathrm{BC}\left(\Pi_{q}\right)_{v}|\operatorname{det}|_{\nu}^{(1-2 n) / 2}\right) .
$$


Corollary 6.24 The eigenvalues of $\varsigma_{p}$ on $H_{c-\partial}^{i}\left(\bar{A}^{(m), \text { ord }}\right)_{\overline{\mathbb{Q}}_{p}}$ are Weil $p^{w}$-numbers for some $w \in \mathbb{Z}_{\geq 0}$ (depending on the eigenvalue). We will write

$$
W_{0} H_{c-\partial}^{i}\left(\bar{A}^{(m), \text { ord }}\right)_{\overline{\mathbb{Q}}_{p}}
$$

for the subspace of $H_{c-\partial}^{i}\left(\bar{A}^{(m), \text { ord }}\right)_{\overline{\mathbb{Q}}_{p}}$ spanned by generalized eigenspaces of $\varsigma_{p}$ with eigenvalue a $p^{0}$-Weil number.

For $i>0$ there is a $G_{n}\left(\mathbb{A}^{\infty}\right)^{\text {ord }}$-equivariant isomorphism

$$
\underset{U^{p, N, \Sigma}}{\lim _{\longrightarrow}} H^{i}\left(\left|\mathcal{S}\left(\partial \bar{A}_{U^{p}(N), \Sigma}^{(m), \text { ord }}\right)\right|, \overline{\mathbb{Q}}_{p}\right) \stackrel{\sim}{\longrightarrow} W_{0} H_{c-\partial}^{i+1}\left(\bar{A}^{(m), \text { ord }}\right)_{\overline{\mathbb{Q}}_{p}} \cdot
$$

(For $i=0$ there is still a surjection.)

Proof By theorem 2.2 of [19], the eigenvalues of the Frobenius endomorphism on $H_{\text {rig }}^{i}\left(\partial^{(j)} \bar{A}_{U^{p}(N), \Sigma}^{(m), \text { ord }}\right)$ are all Weil $p^{w}$-numbers for some $w \in \mathbb{Z}_{\geq i}$ (depending on the eigenvalue). The first part of the corollary follows.

It follows moreover that $W_{0} H_{c-\partial}^{i}\left(\bar{A}_{U^{p}(N), \Sigma}^{(m), \text { ord }}\right)_{\overline{\mathbb{Q}}_{p}}$ is the cohomology of the complex

$$
\cdots \longrightarrow H_{\text {rig }}^{0}\left(\partial^{(i)} \bar{A}_{U^{p}(N), \Sigma}^{(m), \text { ord }}, \overline{\mathbb{Q}}_{p}\right) \longrightarrow H_{\text {rig }}^{0}\left(\partial^{(i+1)} \bar{A}_{U^{p}(N), \Sigma}^{(m), \text { ord }}, \overline{\mathbb{Q}}_{p}\right) \longrightarrow \cdots
$$

However by proposition 8.2 .15 of [46]

$$
H_{\text {rig }}^{0}\left(\partial^{(i)} \bar{A}_{U^{p}(N), \Sigma}^{(m), \text { ord }}, \overline{\mathbb{Q}}_{p}\right) \cong \overline{\mathbb{Q}}_{p}^{\pi_{0}\left(\partial^{(i)} \bar{A}_{U^{p}(N), \Sigma}^{(m), \text { ord }} \times \operatorname{Spec} \overline{\mathbb{F}}_{p}\right)},
$$

and so the cohomology of the above complex becomes

$$
\begin{array}{ll}
\operatorname{ker}\left(H_{\text {rig }}^{0}\left(\bar{A}_{U^{p}(N), \Sigma}^{(m), \text { ord }}, \overline{\mathbb{Q}}_{p}\right) \longrightarrow H^{0}\left(\left|\mathcal{S}\left(\partial \bar{A}_{U^{p}(N), \Sigma}^{(m), \text { ord }}\right)\right|, \overline{\mathbb{Q}}_{p}\right)\right) & \text { in degree } 0 \\
H^{0}\left(\left|\mathcal{S}\left(\partial \bar{A}_{U^{p}(N), \Sigma}^{(m), \text { ord }}\right)\right|, \overline{\mathbb{Q}}_{p}\right) / \operatorname{Im} H_{\text {rig }}^{0}\left(\bar{A}_{U^{p}(N), \Sigma}^{(m), \text { ord }}, \overline{\mathbb{Q}}_{p}\right) & \text { in degree } 1 \\
H^{i-1}\left(\left|\mathcal{S}\left(\partial \bar{A}_{U^{p}(N), \Sigma}^{(m), \text { ord }}\right)\right|, \overline{\mathbb{Q}}_{p}\right) & \text { in degree } i>1 .
\end{array}
$$

The last part of the corollary follows.

The discussion at the end of Sect. 5.3 shows that there are $G_{n}\left(\mathbb{A}^{\infty}\right)^{\text {ord }}$-equivariant open embeddings

$$
\mathfrak{T}_{U^{p}(N),=n}^{(m), \text { ord }} \hookrightarrow\left|\mathcal{S}\left(\partial \bar{A}_{U^{p}(N), \Sigma}^{(m), \text { ord }}\right)\right| .
$$

Thus the following corollary follows by applying Lemma 1.7 and Corollary 1.6.

Corollary 6.25 For $i>0$,

$$
H_{\text {Int }}^{i}\left(\mathfrak{T}_{=n}^{(m), \text { ord }}, \overline{\mathbb{Q}}_{p}\right) \cong \operatorname{Ind}_{P_{n,(n)}^{(m),+}\left(\mathbb{A}^{\infty, p}\right)}^{G_{(m)}^{(m)}\left(\mathbb{I}^{\infty, p}\right)} H_{\text {Int }}^{i}\left(\mathfrak{T}_{(n)}^{(m)}, \overline{\mathbb{Q}}_{p}\right)^{\mathbb{Z}_{p}^{\times}}
$$

is a $G_{n}\left(\mathbb{A}^{\infty}\right)^{\text {ord }}$ subquotient of $W_{0} H_{c-\partial}^{i+1}\left(\bar{A}_{n}^{(m), \text { ord }}\right)_{\overline{\mathbb{Q}}_{p}}$.

Combining this proposition with Corollary 6.23 (and using Lemma 1.1) we deduce the following consequence. 
Corollary 6.26 Suppose that $i>0$ and that $\pi$ is an irreducible $L_{n,(n), \operatorname{lin}}\left(\mathbb{A}^{\infty}\right)$-subquotient of $H_{\text {Int }}^{i}\left(\mathfrak{T}_{(n)}^{(m)}, \overline{\mathbb{Q}}_{p}\right)$. Then there is a continuous semi-simple representation

$$
R_{p}(\pi): G_{F} \longrightarrow G L_{2 n}\left(\overline{\mathbb{Q}}_{p}\right)
$$

with the following property: Suppose that $q \neq p$ is a rational prime which either splits in $F_{0}$ or is unramified in $F$. Suppose further that $\pi$ is unramified at all primes of $F$ above $q$. If $v \mid q$ is a prime of $F$, then

$$
\left.R_{p}(\pi)\right|_{W_{F_{v}}} ^{\mathrm{F}-\mathrm{ss}} \cong \operatorname{rec}_{F_{\nu}}\left(\pi_{v}|\operatorname{det}|_{\nu}^{(1-n) / 2}\right) \oplus \operatorname{rec}_{F_{c_{\nu}}}\left(\pi c_{\nu}|\operatorname{det}|_{c_{\nu}}^{(1-n) / 2}\right)^{\vee, c} \epsilon_{p}^{1-2 n}
$$

Combining this with Corollary 1.9 we obtain the following result.

Corollary 6.27 Suppose that $n>1$, that $\rho$ is an irreducible algebraic representation of $L_{n,(n), \text { lin }}$ on a finite dimensional $\mathbb{C}$-vector space and that $\pi$ is a cuspidal automorphic representation of $L_{n,(n), \text { lin }}(\mathbb{A})$ so that $\pi_{\infty}$ has the same infinitesimal character as $\rho^{\vee}$. Then, for all sufficiently large integers $N$, there is a continuous, semi-simple representation

$$
R_{p, l}(\pi, N): G_{F} \longrightarrow G L_{2 n}\left(\overline{\mathbb{Q}}_{p}\right)
$$

with the following property: Suppose that $q \neq p$ is a rational prime which either splits in $F_{0}$ or is unramified in F. Suppose further that $\pi$ is unramified at all primes of $F$ above $q$. If $v \mid q$ is a prime of $F$, then

$$
\left.R_{p, l}(\pi, N)\right|_{W_{F_{\nu}}} ^{\mathrm{F}-\mathrm{ss}}=l^{-1} \operatorname{rec}_{F_{\nu}}\left(\pi_{\nu}|\operatorname{det}|_{\nu}^{(1-n) / 2}\right) \oplus\left(l^{-1} \operatorname{rec}_{F_{c_{\nu}}}\left(\pi c_{v}|\operatorname{det}|_{c_{\nu}}^{(1-n) / 2}\right)\right)^{\vee, c} \epsilon_{p}^{1-2 n-2 N} .
$$

Proof Take

$$
R_{p, l}(\pi, N)=R_{p}\left(l^{-1}\left(\pi^{\infty}\|\operatorname{det}\|^{N}\right)\right) \otimes \epsilon_{p}^{-N} .
$$

\section{Galois representations}

In order to improve upon Corollary 6.27 it is necessary to apply some simple group theory. To this end, let $\Gamma$ be a topological group and let $\mathfrak{F}$ be a dense set of elements of $\Gamma$. Let $k$ be an algebraically closed, topological field of characteristic 0 and let $d \in \mathbb{Z}_{>0}$.

Let

$$
\mu: \Gamma \longrightarrow k^{\times}
$$

be a continuous homomorphism such that $\mu(f)$ has infinite order for all $f \in \mathfrak{F}$. For $f \in \mathfrak{F}$ let $\mathcal{E}_{f}^{1}$ and $\mathcal{E}_{f}^{2}$ be two $d$-element multisets of elements of $k^{\times}$. Let $\mathcal{M}$ be an infinite subset of $\mathbb{Z}$. For $m \in \mathcal{M}$ let

$$
\rho_{m}: \Gamma \longrightarrow G L_{2 d}(k)
$$

be a continuous semi-simple representation such that for every $f \in \mathfrak{F}$ the multiset of roots of the characteristic polynomial of $\rho_{m}(f)$ equals

$$
\mathcal{E}_{f}^{1} \amalg \mathcal{E}_{f}^{2} \mu(f)^{m} .
$$


Suppose that $\mathcal{M}^{\prime}$ is a finite subset of $\mathcal{M}$. Let $G_{\mathcal{M}^{\prime}}$ denote the Zariski closure in $\mathbb{G}_{m} \times$ $G L_{2 d}^{\mathcal{M}^{\prime}}$ of the image of

$$
\mu \oplus \bigoplus_{m \in \mathcal{M}^{\prime}} \rho_{m}
$$

It is a, possibly disconnected, reductive group. There is a natural continuous homomorphism

$$
\rho_{\mathcal{M}^{\prime}}=\mu \times \prod_{m \in \mathcal{M}^{\prime}} \rho_{m}: \Gamma \longrightarrow G_{\mathcal{M}^{\prime}}(k)
$$

Note that $\rho_{\mathcal{M}^{\prime}}(\mathfrak{F})$ is Zariski dense in $G_{\mathcal{M}^{\prime}}$. We will use $\mu$ for the character of $G_{\mathcal{M}^{\prime}}$ which is projection to $\mathbb{G}_{m}$. For $m \in \mathcal{M}^{\prime}$ we will let

$$
R_{m}: G_{\mathcal{M}^{\prime}} \longrightarrow G L_{2 d}
$$

denote the projection to the factor indexed by $m$.

Lemma 7.1 For every $g \in G_{\mathcal{M}^{\prime}}(k)$ there are two d-element multisets $\Sigma_{g}^{1}$ and $\Sigma_{g}^{2}$ of elements of $k^{\times}$such that for every $m \in \mathcal{M}^{\prime}$ the multiset of roots of the characteristic polynomial of $R_{m}(g)$ equals

$$
\Sigma_{g}^{1} \amalg \Sigma_{g}^{2} \mu(g)^{m} .
$$

Proof It suffices to show that the subset of $k^{\times} \times G L_{2 d}^{\mathcal{M}^{\prime}}(k)$ consisting of elements $\left(t,\left(g_{m}\right)_{m \in \mathcal{M}^{\prime}}\right)$ such that there are $d$-element multisets $\Sigma^{1}$ and $\Sigma^{2}$ of elements of $k^{\times}$such that for all $m \in \mathcal{M}^{\prime}$ the multiset of roots of the characteristic polynomial of $g_{m}$ equals $\Sigma^{1} U \Sigma^{2} t^{m}$, is Zariski closed. Let $\operatorname{Pol}_{2 d}$ denote the space of monic polynomials of degree $2 d$. It even suffices to show that the subset $X$ of $k^{\times} \times \operatorname{Pol}_{2 d}^{\mathcal{M}^{\prime}}(k)$ consisting of elements $\left(t,\left(P_{m}\right)_{m \in \mathcal{M}^{\prime}}\right)$ such that there are $d$-element multisets $\Sigma^{1}$ and $\Sigma^{2}$ of elements of $k$ such that for all $m \in \mathcal{M}^{\prime}$ the multiset of roots of $P_{m}$ equals $\Sigma^{1} U \Sigma^{2} t^{m}$, is Zariski closed.

There is a natural finite map

$$
\begin{aligned}
\pi: \mathrm{Aff}^{2 d} & \longrightarrow \mathrm{Pol}_{2 d} \\
\left(\alpha_{i}\right) & \longmapsto \prod_{i}\left(T-\alpha_{i}\right) .
\end{aligned}
$$

If

$$
\left(\sigma_{m}\right) \in S_{2 d}^{\mathcal{M}^{\prime}}
$$

where $S_{2 d}$ denotes the symmetric group on $2 d$ letters, define $V_{\left(\sigma_{m}\right)}$ to be the set of

$$
\left(t,\left(a_{m, i}\right)\right) \in \mathbb{G}_{m} \times\left(\mathrm{Aff}^{2 d}\right)^{\mathcal{M}^{\prime}}
$$

such that, for all $m, m^{\prime} \in \mathcal{M}^{\prime}$ we have

$$
a_{m, \sigma_{m} i}=a_{m^{\prime}, \sigma_{m^{\prime}} i}
$$




$$
\begin{aligned}
& \text { if } i=1, \ldots, d \text { and } \\
& \quad a_{m, \sigma_{m} i}=a_{m^{\prime}, \sigma_{m^{\prime}} i} t^{m^{\prime}-m} \\
& \text { if } i=d+1, \ldots, 2 d \text {. Then } V_{\left(\sigma_{m}\right)} \text { is closed in } \mathbb{G}_{m} \times\left(\mathrm{Aff}^{2 d}\right)^{\mathcal{M}^{\prime}} \text {. Moreover } \\
& \quad X=\bigcup_{\left(\sigma_{m}\right) \in S_{2 d}^{\mathcal{M}^{\prime}}}\left(1 \times \pi^{\mathcal{M}^{\prime}}\right) V_{\left(\sigma_{m}\right)} .
\end{aligned}
$$

The lemma now follows from the finiteness of $1 \times \pi^{\mathcal{M}^{\prime}}$.

Corollary 7.2 If $\emptyset \neq \mathcal{M}^{\prime} \subset \mathcal{M}^{\prime \prime}$ are finite subsets of $\mathcal{M}$ then $G_{\mathcal{M}^{\prime \prime}} \stackrel{\sim}{\rightarrow} G_{\mathcal{M}^{\prime}}$.

Proof Suppose that $g$ is in the kernel of the natural map

$$
G_{\mathcal{M}^{\prime \prime}} \rightarrow G_{\mathcal{M}^{\prime}}
$$

Then for all $m \in \mathcal{M}^{\prime \prime}$ the only eigenvalue of $R_{m}(g)$ is 1 . Thus $g$ must be unipotent. However $\operatorname{ker}\left(G_{\mathcal{M}^{\prime \prime}} \rightarrow G_{\mathcal{M}^{\prime}}\right)$ is reductive and so must be trivial.

Thus we can write $G$ for $G_{\mathcal{M}^{\prime}}$ without danger of confusion.

Corollary 7.3 For everyg $\in G(k)$ there are two d-element multisets $\Sigma_{g}^{1}$ and $\Sigma_{g}^{2}$ of elements of $k^{\times}$such that for every $m \in \mathcal{M}$ the multiset of roots of the characteristic polynomial of $R_{m}(g)$ equals

$$
\Sigma_{g}^{1} \amalg \Sigma_{g}^{2} \mu(g)^{m} .
$$

Moreover if $\mu(g)$ has infinite order then the multisets $\Sigma_{g}^{1}$ and $\Sigma_{g}^{2}$ are unique.

Proof Choose non-empty finite subsets

$$
\mathcal{M}_{1}^{\prime} \subset \mathcal{M}_{2}^{\prime} \subset \cdots \subset \mathcal{M}
$$

with

$$
\mathcal{M}=\bigcup_{i=1}^{\infty} \mathcal{M}_{i}^{\prime} .
$$

For each $i$ we can find two $d$-element multisets $\Sigma_{g, i}^{1}$ and $\Sigma_{g, i}^{2}$ of elements of $k^{\times}$such that for every $m \in \mathcal{M}_{i}^{\prime}$ the multiset of roots of the characteristic polynomial of $R_{m}(g)$ equals

$$
\Sigma_{g, i}^{1} \amalg \Sigma_{g, i}^{2} \mu(g)^{m} .
$$

Let $m_{1} \in \mathcal{M}_{1}^{\prime}$ and let $\Sigma$ denote the set of eigenvalues of $R_{m_{1}}(g)$. Then, for every $i$, the multiset $\Sigma_{g, i}^{1}$ consists of elements of $\Sigma$ and the multiset $\Sigma_{g, i}^{2}$ consists of elements of $\Sigma \mu(g)^{-m_{1}}$. Thus there are only finitely many possibilities for the pair of multisets $\left(\Sigma_{g, i}^{1}, \Sigma_{g, i}^{2}\right)$ as $i$ varies. Hence some such pair $\left(\Sigma_{g}^{1}, \Sigma_{g}^{2}\right)$ occurs infinitely often. This pair satisfies the requirements of the corollary. 
For uniqueness suppose that $\Sigma_{g}^{1, \prime}$ and $\Sigma_{g}^{2, \prime}$ is another such pair of multisets. Choose $m \in \mathcal{M}$ with $\mu(g)^{m} \neq \alpha / \beta$ for any $\alpha, \beta \in \Sigma_{g}^{1} \amalg \Sigma_{g}^{2} \amalg \Sigma_{g}^{1, \prime} \amalg \Sigma_{g}^{2, \prime}$. Then the equality

$$
\Sigma_{g}^{1} \amalg \Sigma_{g}^{2} \mu(g)^{m}=\Sigma_{g}^{1, \prime} \amalg \Sigma_{g}^{2, \prime} \mu(g)^{m}
$$

implies that $\Sigma_{g}^{1, \prime}=\Sigma_{g}^{1}$ and $\Sigma_{g}^{2, \prime}=\Sigma_{g}^{2}$.

The connected component $Z(G)^{0}$ of the centre of $G$ is a torus.

Lemma 7.4 The character $\mu$ is non-trivial on $Z(G)^{0}$.

Proof If $\mu$ were trivial on $Z\left(G^{0}\right)^{0}$ then it would be trivial on $G^{0}$ (because $G^{0} / Z\left(G^{0}\right)^{0}$ is semi-simple), and so $\mu$ would have finite order, a contradiction. Thus $\left.\mu\right|_{Z\left(G^{0}\right)^{0}}$ is nontrivial.

The space

$$
X^{*}\left(Z\left(G^{0}\right)^{0}\right) \otimes_{\mathbb{Z}} \mathbb{Q}
$$

is a representation of the finite group $G / G^{0}$ and we can decompose

$$
X^{*}\left(Z\left(G^{0}\right)^{0}\right) \otimes_{\mathbb{Z}} \mathbb{Q}=\left(X^{*}\left(Z(G)^{0}\right) \otimes_{\mathbb{Z}} \mathbb{Q}\right) \oplus Y
$$

where $Y$ is a $\mathbb{Q}\left[G / G^{0}\right]$-module with

$$
Y^{G / G^{0}}=(0)
$$

But

$$
\left.\mu\right|_{Z\left(G^{0}\right)^{0}} \in X^{*}\left(Z\left(G^{0}\right)^{0}\right)^{G / G^{0}} \subset X^{*}\left(Z(G)^{0}\right) \otimes_{\mathbb{Z}} \mathbb{Q}
$$

is non-trivial, and so $\left.\mu\right|_{Z(G)^{0}}$ is non-trivial.

For $m \in \mathcal{M}$ let $\mathfrak{X}_{m}$ denote the $2 d$-element multiset of characters of $Z(G)^{0}$ which occur in $R_{m}$ (taken with their multiplicity). If $g \in G$ then we will write $\mathfrak{Y}(g)_{m}$ for the $2 d$-element multiset of pairs $(\chi, a)$, where $\chi$ is a character of $Z(G)^{0}$ and $a$ is a root of the characteristic polynomial of $g$ acting on the $\chi$ eigenspace of $Z(G)^{0}$ in $R_{m}$. (The pair $(\chi, a)$ occurs with the same multiplicity as $a$ has as a root of the characteristic polynomial of $g$ acting on the $\chi$-eigenspace of $R_{m}$.)

If $\mathfrak{Y} \subset \mathfrak{Y}(g)_{m}$ and if $\psi \in X^{*}(G)$ then we will set

$$
\mathfrak{Y} \psi=\{(\chi \psi, a \psi(g)):(\chi, a) \in \mathfrak{Y}\}
$$

We warn the reader that this depends on $g$ and not just on the set $\mathfrak{Y}$.

Lemma 7.5 Suppose that $T / k$ is a torus and that $\mathfrak{X}$ is a finite set of non-trivial characters of $T$. Let $A$ be a finite subset of $k^{\times}$. Then we can find $t \in T(k)$ such that $\chi(t) \neq$ a for all $\chi \in \mathfrak{X}$ and $a \in A$. 
Proof Let (, ) denote the usual perfect pairing

$$
X^{*}(T) \times X_{*}(T) \longrightarrow \mathbb{Z}
$$

We can find $v \in X_{*}(T)$ such that $(\chi, v) \neq 0$ for all $\chi \in \mathfrak{X}$. Thus we are reduced to the case $T=\mathbb{G}_{m}$, in which case we may take $t$ to be any element of $k^{\times}$that does not lie in the divisible hull of the subgroup $H$ of $k^{\times}$generated by $A$. (For example, we can take $t$ to be a rational prime such that all elements of a finite set of generators of $H \cap \mathbb{Q}^{\times}$are units at t.)

Corollary 7.6 Suppose that $T / k$ is a torus and that $\mathfrak{X}$ is a finite set of characters of $T$. Then we can find $t \in T(k)$ such that if $\chi \neq \chi^{\prime}$ lie in $\mathfrak{X}$ then

$$
\chi(t) \neq \chi^{\prime}(t)
$$

Lemma 7.7 If $m, m^{\prime}, m^{\prime \prime} \in \mathcal{M}$, then we can decompose

$$
\mathfrak{Y}(g)_{m}=\mathfrak{Y}(g)_{m, m^{\prime}, m^{\prime \prime}}^{1} \amalg \mathfrak{Y}(g)_{m, m^{\prime}, m^{\prime \prime}}^{2}
$$

into two d-element multisets, such that

$$
\mathfrak{Y}(g)_{m^{\prime}}=\mathfrak{Y}(g)_{m, m^{\prime}, m^{\prime \prime}}^{1} \amalg \mathfrak{Y}(g)_{m, m^{\prime}, m^{\prime \prime}}^{2} \mu^{m^{\prime}-m}
$$

and

$$
\mathfrak{Y}(g)_{m^{\prime \prime}}=\mathfrak{Y}(g)_{m, m^{\prime}, m^{\prime \prime}}^{1} \amalg \mathfrak{Y}(g)_{m, m^{\prime}, m^{\prime \prime}}^{2} \mu^{m^{\prime \prime}-m} .
$$

If $\mu^{m-m^{\prime}} \neq \chi / \chi^{\prime}$ for all $\chi, \chi^{\prime} \in \mathfrak{X}_{m}$ then the equation

$$
\mathfrak{Y}(g)_{m^{\prime}}=\mathfrak{Y}(g)_{m, m^{\prime}, m^{\prime \prime}}^{1} \amalg \mathfrak{Y}(g)_{m, m^{\prime}, m^{\prime \prime}}^{2} \mu^{m^{\prime}-m}
$$

uniquely determines this decomposition.

Proof Choose $t \in Z(G)^{0}(k)$ such that $a \chi(t) \neq a^{\prime} \chi^{\prime}(t)$ for $(\chi, a) \neq\left(\chi^{\prime}, a^{\prime}\right)$ with

$$
(\chi, a),\left(\chi^{\prime}, a^{\prime}\right) \in \mathfrak{Y}(g)_{m} \cup \mathfrak{Y}(g)_{m} \mu^{m^{\prime}-m} \cup \mathfrak{Y}(g)_{m} \mu^{m^{\prime \prime}-m} \cup \mathfrak{Y}(g)_{m^{\prime}} \cup \mathfrak{Y}(g)_{m^{\prime \prime}}
$$

(Note that it suffices to choose $t \in Z(G)^{0}(k)$ such that for

$$
(\chi, a),\left(\chi^{\prime}, a^{\prime}\right) \in \mathfrak{Y}(g)_{m} \cup \mathfrak{Y}(g)_{m} \mu^{m^{\prime}-m} \cup \mathfrak{Y}(g)_{m} \mu^{m^{\prime \prime}-m} \cup \mathfrak{Y}(g)_{m^{\prime}} \cup \mathfrak{Y}(g)_{m^{\prime \prime}},
$$

with $\chi \neq \chi^{\prime}$ we have $\left(\chi / \chi^{\prime}\right)(t) \neq a^{\prime} / a$.) We can decompose

$$
\mathfrak{Y}(g)_{m}=\mathfrak{Y}(g)_{m, m^{\prime}, m^{\prime \prime}}^{1} \amalg \mathfrak{Y}(g)_{m, m^{\prime}, m^{\prime \prime}}^{2}
$$

into two $d$-element multisets, such that

$$
\left\{a \chi(t):(\chi, a) \in \mathfrak{Y}(g)_{m, m^{\prime}, m^{\prime \prime}}^{1}\right\}=\Sigma_{g t}^{1}
$$


and

$$
\left\{a \chi(t):(\chi, a) \in \mathfrak{Y}(g)_{m, m^{\prime}, m^{\prime \prime}}^{2} \mu^{-m}\right\}=\Sigma_{g t}^{2}
$$

Then

$$
\begin{aligned}
& \left\{a \chi(t):(\chi, a) \in \mathfrak{Y}(g)_{m^{\prime}}\right\} \\
& \quad=\left\{a \chi(t):(\chi, a) \in \mathfrak{Y}(g)_{m, m^{\prime}, m^{\prime \prime}}^{1}\right\} \amalg\left\{a \chi(t):(\chi, a) \in \mathfrak{Y}(g)_{m, m^{\prime}, m^{\prime \prime}}^{2} \mu^{m^{\prime}-m}\right\}
\end{aligned}
$$

and

$$
\begin{aligned}
& \left\{a \chi(t):(\chi, a) \in \mathfrak{Y}(g)_{m^{\prime \prime}}\right\} \\
& \quad=\left\{a \chi(t):(\chi, a) \in \mathfrak{Y}(g)_{m, m^{\prime}, m^{\prime \prime}}^{1}\right\} \amalg\left\{a \chi(t):(\chi, a) \in \mathfrak{Y}(g)_{m, m^{\prime}, m^{\prime \prime}}^{2} \mu^{m^{\prime \prime}-m}\right\} .
\end{aligned}
$$

It follows that

$$
\mathfrak{Y}(g)_{m^{\prime}}=\mathfrak{Y}(g)_{m, m^{\prime}, m^{\prime \prime}}^{1} \amalg \mathfrak{Y}(g)_{m, m^{\prime}, m^{\prime \prime}}^{2} \mu^{m^{\prime}-m}
$$

and

$$
\mathfrak{Y}(g)_{m^{\prime \prime}}=\mathfrak{Y}(g)_{m, m^{\prime}, m^{\prime \prime}}^{1} \amalg \mathfrak{Y}(g)_{m, m^{\prime}, m^{\prime \prime}}^{2} \mu^{m^{\prime \prime}-m} .
$$

If $\mu^{m-m^{\prime}} \neq \chi / \chi^{\prime}$ for all $\chi, \chi^{\prime} \in \mathfrak{X}_{m}$ then

$$
\mathfrak{Y}(g)_{m, m^{\prime}, m^{\prime \prime}}^{1}=\mathfrak{Y}(g)_{m} \cap \mathfrak{Y}(g)_{m^{\prime}}
$$

so the uniqueness assertion is clear.

Corollary 7.8 If $m \in \mathcal{M}$, then we can uniquely decompose

$$
\mathfrak{Y}(g)_{m}=\mathfrak{Y}(g)_{m}^{1} \amalg \mathfrak{Y}(g)_{m}^{2}
$$

into two d-element multisets, such that for all $m^{\prime} \in \mathcal{M}$ we have

$$
\mathfrak{Y}(g)_{m^{\prime}}=\mathfrak{Y}(g)_{m}^{1} \amalg \mathfrak{Y}(g)_{m}^{2} \mu^{m^{\prime}-m} .
$$

Proof Choose $m^{\prime}$ such that $\mu^{m-m^{\prime}} \neq \chi / \chi^{\prime}$ for all $\chi, \chi^{\prime} \in \mathfrak{X}_{m}$. Then we see that for all $m^{\prime \prime}, m^{\prime \prime \prime} \in \mathcal{M}$ we have

$$
\mathfrak{Y}(g)_{m, m^{\prime}, m^{\prime \prime}}^{1}=\mathfrak{Y}(g)_{m, m^{\prime}, m^{\prime \prime \prime}}^{1}
$$

and

$$
\mathfrak{Y}(g)_{m, m^{\prime}, m^{\prime \prime}}^{2}=\mathfrak{Y}(g)_{m, m^{\prime}, m^{\prime \prime \prime}}^{2}
$$

Then we can simply take $\mathfrak{Y}(g)_{m}^{i}=\mathfrak{Y}(g)_{m, m^{\prime}, m^{\prime \prime}}^{i}$. 
Corollary 7.9 For all $m, m^{\prime} \in \mathcal{M}$ we have

$$
\mathfrak{Y}(g)_{m^{\prime}}^{1}=\mathfrak{Y}(g)_{m}^{1}
$$

and

$$
\mathfrak{Y}(g)_{m^{\prime}}^{2}=\mathfrak{Y}(g)_{m}^{2} \mu^{m^{\prime}-m}
$$

Proof It is immediate from the previous corollary that $\mathfrak{Y}(g)_{m}^{1}$ and $\mathfrak{Y}(g)_{m}^{2} \mu^{m^{\prime}-m}$ have the properties that uniquely characterize $\mathfrak{Y}(g)_{m^{\prime}}^{1}$ and $\mathfrak{Y}(g)_{m^{\prime}}^{2}$.

Corollary 7.10 For all $g \in G$ and $m \in \mathcal{M}$ and for $i=1,2$ we have

$$
\mathfrak{Y}(1)_{m}^{i}=\left\{(\chi, 1): \exists a,(\chi, a) \in \mathfrak{Y}(g)_{m}^{i}\right\} .
$$

Proof It is again immediate that $\left\{(\chi, 1): \exists a,(\chi, a) \in \mathfrak{Y}(g)_{m}^{1}\right\}$ and $\{(\chi, 1): \exists a,(\chi, a) \in$ $\left.\mathfrak{Y}(g)_{m}^{2}\right\}$ have the properties that uniquely characterize $\mathfrak{Y}(1)_{m}^{1}$ and $\mathfrak{Y}(1)_{m}^{2}$.

We set

$$
\mathfrak{X}_{m}^{i}=\left\{\chi:(\chi, 1) \in \mathfrak{Y}(1)_{m}^{i}\right\} .
$$

Note that

$$
\mathfrak{X}_{m^{\prime}}^{1}=\mathfrak{X}_{m}^{1}
$$

and that

$$
\mathfrak{X}_{m^{\prime}}^{2}=\mathfrak{X}_{m}^{2} \mu^{m^{\prime}-m}
$$

Corollary 7.11 For all but finitely many $m \in \mathcal{M}$ the multisets $\mathfrak{X}_{m}^{1}$ and $\mathfrak{X}_{m}^{2}$ are disjoint.

Let $\mathcal{M}^{\prime}$ denote the set of $m \in \mathcal{M}$ such that $\mathfrak{X}_{m}^{1}$ and $\mathfrak{X}_{m}^{2}$ are disjoint. Then we see that for $m \in \mathcal{M}^{\prime}$ we have

$$
\mathfrak{Y}(g)_{m}^{i}=\left\{(\chi, a) \in \mathfrak{Y}(g)_{m}: \chi \in \mathfrak{X}_{m}^{i}\right\}
$$

Moreover for $m \in \mathcal{M}^{\prime}$ we may decompose

$$
R_{m}=R_{m}^{1} \oplus R_{m}^{2}
$$

where $R_{m}^{i}$ is the sum of the $\chi$-eigenspaces of $Z(G)^{0}$ for $\chi \in \mathfrak{X}_{m}^{i}$. We see that the multiset of roots of the characteristic polynomial of $R_{m}^{i}(g)$ equals

$$
\left\{a:(\chi, a) \in \mathfrak{Y}(g)_{m}^{i}\right\}
$$

Thus $R_{m}^{1}$ is independent of $m \in \mathcal{M}^{\prime}$, as is $R_{m}^{2} \mu^{-m}$. Denote these representations of $G$ by $r_{1}$ and $r_{2}$, so that

$$
R_{m} \cong r_{1} \oplus r_{2} \mu^{m}
$$


for all $m \in \mathcal{M}^{\prime}$. From Corollary 7.3 (applied to $\mathcal{M}^{\prime}$ ) we see that if $g \in G$ and $\mu(g)$ has infinite order then $\Sigma_{g}^{i}$ is the multiset of roots of the characteristic polynomial of $r^{i}(g)$. Thus we have proved the following result.

Proposition 7.12 Keep the notation and assumptions of the first two paragraphs of this section. Then there are continuous semi-simple representations

$$
\rho^{i}: \Gamma \longrightarrow G L_{d}(k)
$$

for $i=1,2$ such that for all $f \in \mathfrak{F}$ the multiset of roots of the characteristic polynomial of $\rho^{i}(f)$ equals $\mathcal{E}_{f}^{i}$.

This proposition allows us to deduce our main theorem from Corollary 6.27.

Theorem 7.13 Suppose that $\pi$ is a cuspidal automorphic representation of $G L_{n}\left(\mathbb{A}_{F}\right)$ such that $\pi_{\infty}$ has the same infinitesimal character as an algebraic representation of $\mathrm{RS}_{\mathbb{Q}}^{F} G L_{n}$. Then there is a continuous semi-simple representation

$$
r_{p, l}(\pi): G_{F} \longrightarrow G L_{n}\left(\overline{\mathbb{Q}}_{p}\right)
$$

with the following property: Suppose that $q \neq p$ is a rational prime which either splits in $F_{0}$ or is unramified in F. Suppose further that $\pi$ is unramified at all primes of $F$ above $q$. If $v \mid q$ is a prime of $F$, then

$$
\left.r_{p, l}(\pi)\right|_{W_{F_{v}}} ^{\mathrm{F}-\mathrm{sS}}=l^{-1} \operatorname{rec}_{F_{v}}\left(\pi_{v}|\operatorname{det}|_{\nu}^{(1-n) / 2}\right) .
$$

Proof We may suppose that $n>1$, as in the case $n=1$ the result is well known. Let $S$ denote the set of rational primes above which $F$ or $\pi$ ramifies together with $p$; and let $G_{F, S}$ denote the Galois group over $F$ of the maximal extension of $F$ unramified outside $S$. Apply Proposition 7.12 to $\Gamma=G_{F, S}$, and $k=\overline{\mathbb{Q}}_{p}$, and $\mu=\epsilon_{p}^{-2}$, and $\mathcal{M}$ consisting of all sufficiently large integers, and $\rho_{m}=R_{p, l}(\pi, m)$ (as in Theorem 6.27), and $\mathcal{F}$ the set of Frobenius elements at primes not above $S$, and $\mathcal{E}_{\text {Frob }_{v}}^{1}$ equal to the multiset of roots of the characteristic polynomial of $\imath^{-1} \operatorname{rec}_{F_{v}}\left(\pi_{v}|\operatorname{det}|_{v}^{(1-n) / 2}\right)\left(\operatorname{Frob}_{v}\right)$, and $\mathcal{E}_{\mathrm{Frob}_{v}}^{2}$ equal to the multiset of roots of the characteristic polynomial of $l^{-1} \operatorname{rec}_{F_{c_{v}}}\left(\pi c_{v}|\operatorname{det}|_{c_{v}}^{(-1+3 n) / 2}\right)\left(\operatorname{Frob}_{c_{v}}^{-1}\right)$.

Corollary 7.14 Suppose that E is a totally real or CM field and that $\pi$ is a cuspidal automorphic representation such that $\pi_{\infty}$ has the same infinitesimal character as an algebraic representation of $\mathrm{RS}_{\mathbb{Q}}^{E} G L_{n}$. Then there is a continuous semi-simple representation

$$
r_{p, l}(\pi): G_{E} \longrightarrow G L_{n}\left(\overline{\mathbb{Q}}_{p}\right)
$$

such that, if $q \neq p$ is a rational prime above which $\pi$ is unramified and if $v \mid q$ is a prime of $E$, then $r_{p, l}(\pi)$ is unramified at $v$ and

$$
\left.r_{p, l}(\pi)\right|_{W_{E_{v}}} ^{\mathrm{F}-\mathrm{ss}}=l^{-1} \operatorname{rec}_{E_{v}}\left(\pi_{v}|\operatorname{det}|_{\nu}^{(1-n) / 2}\right) .
$$

Proof This can be deduced from Theorem 7.13 by using lemma 1 of [54]. (This is the same argument used in the proof of theorem VII.1.9 of [29].) 


\section{Author details}

${ }^{1}$ Institut de Mathématiques de Jussieu, Paris, France, ${ }^{2}$ School of Mathematics, University of Minnesota, Minneapolis, MN, USA, ${ }^{3}$ School of Mathematics, IAS, Princeton, NJ, USA, ${ }^{4}$ Department of Pure Mathematics and Mathematical Statistics,

University of Cambridge, Cambridge, UK.

\section{Acknowledgements}

We are pleased to dedicate this paper to the memory of our friend Robert Coleman, who was both a personal and professional inspiration. This paper owes a lot to his ideas. The origin of this paper was the conviction that one should be able to relate all the cohomology of a Shimura variety to overconvergent $p$-adic automorphic forms. The source of this conviction was [22].

We would all like to thank the Institute for Advanced Study for its support and hospitality. This project was begun, and the key steps completed, while we were all attending the special IAS special year on 'Galois representations and automorphic forms'. M.H.'s research received funding from the European Research Council under the European Community's Seventh Framework Programme (FP7/2007-2013)/ERC Grant Agreement Number 290766 (AAMOT). K.-W.L.'s research was partially supported by NSF Grants DMS-1069154, DMS-1258962 and DMS-1352216 and by an Alfred P. Sloan Research Fellowship. R.T.'s research was partially supported by NSF Grants DMS-0600716,

DMS-1062759 and DMS-1252158 and by the IAS Oswald Veblen and Simonyi Funds. During some of the period when this research was being written up J.T. served as a Clay Research Fellow.

We would like to thank the referee for their helpful comments. We would also like to thank Elly Gustafsson and Anthony Pulido for help in compiling the index of notations.

\section{Appendix A. Guide to notation}

As the paper contains a lot of different notations, we will informally summarize some of the principal notations in this section. We hope that this will help the reader. Formal definitions will be given in the main body of the paper.

\section{A.1 Shimura varieties}

We fix a totally real field $F^{+}$, an imaginary quadratic field $F_{0}$ and let $F$ denote their composite. We also fix a prime $p$ which splits in $F_{0}$.

We will consider a quasi-split unitary similitude group $G_{n} / \mathbb{Q}$ defined with respect to (the trace of) the skew-hermitian form on $V_{n}=F^{2 n}$

$$
\langle x, y\rangle_{n}=\operatorname{tr}_{F / \mathbb{Q}}\left({ }^{t} x J_{n}{ }^{c} y\right)
$$

where $J_{n}$ is an anti-diagonal matrix with 1's in the first $n$ rows and -1 's in the last $n$ rows. The similitude factor $v: G_{n} \rightarrow \mathbb{G}_{m}$. (See Sect. 1.1.) For $U \subset G_{n}\left(\mathbb{A}^{\infty}\right)$ a neat open compact subgroup we have a corresponding Shimura variety $X_{n, U}$ and over it a universal abelian scheme $A^{\text {univ }}$, which is unique up to unique quasi-isogeny. The system $\left\{X_{n, U}\right\}$ has an action of $G_{n}\left(\mathbb{A}^{\infty}\right)$. (See Sect. 3.1.)

We will also consider the group

$$
G_{n}^{(m)}=G_{n} \ltimes \operatorname{RS}_{\mathbb{Q}}^{F} \operatorname{Hom}\left(\mathbb{G}_{a}^{m}, \mathbb{G}_{a}^{2 n}\right) .
$$

It has a (left) action of $\mathrm{RS}_{\mathbb{Q}}^{F} G L_{m}$. (See Sect. 1.1.) For $U \subset G_{n}^{(m)}\left(\mathbb{A}^{\infty}\right)$ a neat open compact subgroup we have the corresponding Kuga-Sato variety $A_{n, U}^{(m)}$ which is smooth and projective over $X_{n, U^{\prime}}$, where $U^{\prime}$ denotes the projection of $U$. The system of schemes $\left\{A_{n, U}^{(m)}\right\}$ has a right action of $G_{n}^{(m)}\left(\mathbb{A}^{\infty}\right)$ and a left action of $G L_{m}(F)$, which don't commute. (See Sect. 3.2.)

We will also need to consider integral models of these varieties over $\mathbb{Z}_{(p)}$, but only for certain level structures at $p$. To define these level structures we give $G_{n}$ the integral structure coming from the (self-dual) lattice $\Lambda_{n}=\left(\mathcal{D}_{F}^{-1}\right)^{n} \oplus \mathcal{O}_{F}^{n} \subset F^{2 n}$. We give $G_{n}^{(m)}$ a compatible integral structure so that

$$
G_{n}^{(m)}(\mathbb{Z})=G_{n}(\mathbb{Z}) \ltimes\left(\left(\mathcal{D}_{F}^{-1}\right)^{n} \oplus \mathcal{O}_{F}^{n}\right)^{m} .
$$


We will only consider integral models for Shimura varieties with level $U^{p}\left(N_{1}, N_{2}\right)$, where $N_{2} \geq N_{1} \geq 0$ are integers and $U^{p}$ is a neat open compact subgroup of $G_{n}\left(\mathbb{A}^{\infty, p}\right)$ or $G_{n}^{(m)}\left(\mathbb{A}^{\infty, p}\right)$. Here $U^{p}\left(N_{1}, N_{2}\right)=U^{p} \times U_{p}\left(N_{1}, N_{2}\right)_{n}$ where $U_{p}\left(N_{1}, N_{2}\right)_{n} \subset G_{n}\left(\mathbb{Z}_{p}\right)$ is the subgroup of matrices of the form

$$
\left(\begin{array}{c|c}
\mu 1_{n} \bmod p^{N_{1}} & * \\
\hline 0 \bmod p^{N_{2}} & 1_{n} \bmod p^{N_{1}}
\end{array}\right),
$$

or $U^{p}\left(N_{1}, N_{2}\right)=U^{p} \times U_{p}\left(N_{1}, N_{2}\right)_{n}^{(m)}$ where $U_{p}\left(N_{1}, N_{2}\right)_{n}^{(m)} \subset G_{n}^{(m)}\left(\mathbb{Z}_{p}\right)$ is the subgroup of matrices of the form

$$
\left(\begin{array}{c|c}
\mu 1_{n} \bmod p^{N_{1}} & * \\
\hline 0 \bmod p^{N_{2}} & 1_{n} \bmod p^{N_{1}}
\end{array}\right)\left(\begin{array}{c}
* \\
\hline 0 \bmod p^{N_{1}}
\end{array}\right) .
$$

(See Sect. 1.1.)

We will consider a certain integral model $\mathcal{X}_{n, U^{p}\left(N_{1}, N_{2}\right)}^{\text {ord }}$ of $X_{n, U^{p}\left(N_{1}, N_{2}\right)}$. (See Sect. 3.1.) Although its generic fibre is all of $X_{n, U^{p}\left(N_{1}, N_{2}\right)}$, its special fibre consists only of points parameterizing ordinary abelian schemes with a level structure with respect to which the distinguished subgroup scheme of order $p^{N_{2}[F: \mathbb{Q}] n}$ is connected. Thus, for example, if $N_{2}^{\prime}>N_{2}$ then $\mathcal{X}_{n, U^{p}\left(N_{1}, N_{2}^{\prime}\right)}^{\text {ord }} \rightarrow \mathcal{X}_{n, U^{p}\left(N_{1}, N_{2}\right)}^{\text {ord }}$ is not finite, because we are only including some of the possible $U^{p}\left(N_{1}, N_{2}^{\prime}\right)$-level structures extending a given $U^{p}\left(N_{1}, N_{2}\right)$-level structure. We denote the universal abelian scheme over $\mathcal{X}_{n, U^{p}\left(N_{1}, N_{2}\right)}^{\text {ord }}$ by $\mathcal{A}^{\text {univ }}$. It is uniquely defined up to prime-to- $p$ quasi-isogeny. The action of the whole group $G_{n}\left(\mathbb{A}^{\infty}\right)$ on $\left\{X_{n, U}\right\}$ does not extend to an action on $\left\{\mathcal{X}_{n, U^{p}\left(N_{1}, N_{2}\right)}^{\text {ord }}\right\}$. However the action of a sub-semigroup

$$
G_{n}\left(\mathbb{A}^{\infty}\right)^{\text {ord }}=G_{n}\left(\mathbb{A}^{\infty, p}\right) \times \varsigma_{p}^{\mathbb{Z} \geq 0} P_{n,(n)}^{+}\left(\mathbb{Z}_{p}\right)
$$

does extend. Here $P_{n,(n)}^{+}$denotes the subgroup of $G_{n}$ consisting of elements of the form

$$
\left(\begin{array}{c|c}
* & * \\
\hline 0_{n \times n} & *
\end{array}\right)
$$

(over $\mathbb{Q}$ this defines a maximal parabolic subgroup) and $\varsigma_{p}$ denotes the element

$$
\left(\begin{array}{c|c}
p^{-1} 1_{n} & 0 \\
\hline 0 & 1_{n}
\end{array}\right)
$$

We write $G_{n}\left(\mathbb{A}^{\infty}\right)^{\text {ord, } \times}$ for the maximal subgroup of the semi-group $G_{n}\left(\mathbb{A}^{\infty}\right)^{\text {ord }}$. We will also write

$$
U^{p}\left(N_{1}\right)=U^{p}\left(N_{1}, N_{2}\right) \cap G_{n}\left(\mathbb{A}^{\infty}\right)^{\text {ord }, \times}
$$

which is independent of $N_{2}$. (See Sect. 1.2.)

The formal completion of $\mathcal{X}_{n, U^{p}\left(N_{1}, N_{2}\right)}^{\text {ord }}$ along its $\mathbb{F}_{p}$-fibre only depends on $U^{p}\left(N_{1}\right)=$ $U^{p}\left(N_{1}, N_{2}\right) \cap G_{n}\left(\mathbb{A}^{\infty}\right)^{\text {ord, } \times}$, and so we will denote it $\mathfrak{X}_{n, U^{p}\left(N_{1}\right)}^{\text {ord }}$. We will also denote its reduced subscheme $\bar{X}_{n, U^{p}\left(N_{1}\right)}^{\text {ord }}$. The systems $\left\{\mathfrak{X}_{n, U^{p}\left(N_{1}\right)}^{\text {ord }}\right\}$ and $\left\{\bar{X}_{n, U^{p}\left(N_{1}\right)}^{\text {ord }}\right\}$ also have actions of $G_{n}\left(\mathbb{A}^{\infty}\right)^{\text {ord }}$. (See Sect. 3.1.) 
We will also consider a certain integral model $\mathcal{A}_{n, U^{p}\left(N_{1}, N_{2}\right)}^{(m) \text { ord }}$ of $A_{n, U^{p}\left(N_{1}, N_{2}\right)}^{(m)}$. Note that $\mathcal{A}_{n, U^{p}\left(N_{1}, N_{2}\right)}^{(m) \text { ord }}$ is smooth and projective over $\mathcal{X}_{n,\left(U^{p}\right)^{\prime}\left(N_{1}, N_{2}\right)}^{\text {ord }}$, where $\left(U^{p}\right)^{\prime}$ denotes the image of $U^{p}$ in $G_{n}\left(\mathbb{A}^{\infty, p}\right)$. The system of schemes $\left\{\mathcal{A}_{n, U^{p}\left(N_{1}, N_{2}\right)}^{(m), \text { ord }}\right\}$ has an action of the semi-group

$$
G_{n}^{(m)}\left(\mathbb{A}^{\infty}\right)^{\text {ord }}=G_{n}^{(m)}\left(\mathbb{A}^{\infty, p}\right) \times \varsigma_{p}^{\mathbb{Z} \geq 0} P_{n,(n)}^{(m),+}\left(\mathbb{Z}_{p}\right) \subset G_{n}^{(m)}\left(\mathbb{A}^{\infty}\right),
$$

where $P_{n,(n)}^{(m),+}$ denotes the subgroup of $G_{n}^{(m)}$ consisting of elements of the form

$$
\left(\begin{array}{c|c}
* & * \\
\hline 0_{n \times n} & *
\end{array}\right)\left(\begin{array}{c}
* \\
\hline *
\end{array}\right) .
$$

We also write $G_{n}^{(m)}\left(\mathbb{A}^{\infty}\right)^{\text {ord, } \times}$ for the maximal subgroup of the semi-group $G_{n}^{(m)}\left(\mathbb{A}^{\infty}\right)^{\text {ord }}$ and

$$
U^{p}\left(N_{1}\right)=U^{p}\left(N_{1}, N_{2}\right) \cap G_{n}^{(m)}\left(\mathbb{A}^{\infty}\right)^{\text {ord }, \times}
$$

It also has an action of $G L_{m}\left(\mathcal{O}_{F,(p)}\right)$. The formal completion of $\mathcal{A}_{n, U^{p}\left(N_{1}, N_{2}\right)}^{(m)}$ along its $\mathbb{F}_{p^{-}}$ fibre depends only on $U^{p}\left(N_{1}\right)$, so we will denote it $\mathfrak{A}_{n, U^{p}\left(N_{1}\right)}^{(m) \text {,ord }}$. We will also denote its special fibre $\bar{A}_{n, U^{p}\left(N_{1}\right)}^{(m) \text { ord }}$ (See Sects. 1.2, 3.2.)

We will write $L_{n,(n)}$ for the subgroup of $P_{n,(n)}^{+}$consisting of matrices of the form

$$
\left(\begin{array}{c|c}
* & 0_{n \times n} \\
\hline 0_{n \times n} & *
\end{array}\right) .
$$

We let Std denote the representation of $L_{n,(n)}$ over $\mathbb{Z}$ which sends the above matrix to the lower left $n \times n$-block in $\operatorname{RS}_{\mathbb{Z}}^{\mathcal{O}_{F}} G L_{n} \subset G L_{n[F: \mathbb{Q}]}$. In fact,

$$
v \times \operatorname{Std}: L_{n,(n)} \stackrel{\sim}{\longrightarrow} \mathbb{G}_{m} \times \mathrm{RS}_{\mathbb{Z}}^{\mathcal{O}_{F}} G L_{n} .
$$

We will let $T_{n}$ denote the subgroup of $G_{n}$ consisting of diagonal matrices and $B_{n}$ the subgroup of upper triangular matrices. The isomorphism $v \times \operatorname{Std}$ allows us to identify $T_{n}$ with $\mathbb{G}_{m} \times \mathrm{RS}_{\mathbb{Z}}^{\mathcal{O}_{F}} \mathbb{G}_{m}^{n}$ and hence we get an isomorphism

$$
X^{*}\left(T_{n}\right) \stackrel{\sim}{\longrightarrow} \mathbb{Z} \oplus \bigoplus_{\tau \in \operatorname{Hom}(F, \overline{\mathbb{Q}})} \mathbb{Z}^{n}
$$

We will denote a typical element of this group $\left(b_{0},\left(b_{\tau, i}\right)\right)$. The set $X^{*}\left(T_{n}\right)_{(n)}^{+} \subset X^{*}\left(T_{n}\right)$ of positive elements of $X^{*}\left(T_{n}\right)$ with respect to the subgroup $B_{n} \cap L_{n,(n)}$ is characterized by

$$
b_{\tau, 1} \geq b_{\tau, 2} \geq \cdots \geq b_{\tau, n}
$$

for all $\tau$. The set $X^{*}\left(T_{n}\right)_{(n)}^{+} \subset X^{*}\left(T_{n}\right)$ of positive elements with respect to $B_{n}$ is further characterized by

$$
b_{\tau, 1}+b_{\tau c, 1} \leq 0
$$

for all $\tau$. Over $\overline{\mathbb{Q}}$ we can decompose

$$
\operatorname{Std}=\bigoplus_{\tau: F \hookrightarrow \overline{\mathbb{Q}}} \operatorname{Std}_{\tau}
$$


where $\operatorname{Std}_{\tau}: L_{n,(n)} \rightarrow G L_{n}$. There is a representation

$$
\mathrm{KS}: L_{n,(n)} \longrightarrow G L_{\left[F^{+}: \mathbb{Q}\right] n^{2}}
$$

over $\mathbb{Z}$, such that over $\overline{\mathbb{Q}}$

$$
\mathrm{KS} \cong v \otimes \bigoplus_{\tau \in \operatorname{Hom}(F, \overline{\mathbb{Q}}) /\{1, c\}} \operatorname{Std}_{\tau}^{\vee} \otimes \operatorname{Std}_{\tau c}^{\vee}
$$

(See Sect. 1.2.)

If $R_{0}$ is an irreducible noetherian $\mathbb{Q}$-algebra and $\rho$ is a representation of $L_{n,(n)}$ on a locally free $R_{0}$-module then we may associate a locally free sheaf $\mathcal{E}_{U, \rho} / X_{n, U} \times \operatorname{Spec} R_{0}$ (in the Zariski topology). As examples we have the following.

- $\mathcal{E}_{U, \mathrm{Std}} \cong \Omega_{n, U}$, the pull-back by the identity section of the sheaf of relative differentials $\Omega_{A^{\text {univ }} / X_{n, U}}^{1}$.

- $\mathcal{E}_{U, \wedge[F: \mathbb{Q}] \operatorname{Std}^{\vee}} \cong \wedge^{n[F: \mathbb{Q}]} \Omega_{n, U}=\omega_{U}$.

- $\mathcal{E}_{U, \mathrm{KS}} \cong \Omega_{X_{n, U} / \mathbb{Q}}^{1}$.

The system of sheaves $\left\{\mathcal{E}_{U, \rho}\right\}$ has an action of $G_{n}\left(\mathbb{A}^{\infty}\right)$. (See Sect. 3.4.)

Similarly if $R_{0}$ is an irreducible noetherian $\mathbb{Z}_{(p)}$-algebra and $\rho$ is a representation of $L_{n,(n)}$ on a locally free $R_{0}$-module then we may associate a locally free sheaf $\mathcal{E}_{U^{p}\left(N_{1}, N_{2}\right), \rho}^{\text {ord }} / \mathcal{X}_{n, U^{p}\left(N_{1}, N_{2}\right)}^{\text {ord }} \times \operatorname{Spec} R_{0}$ (in the Zariski topology). As examples we have the following.

- $\mathcal{E}_{U_{p}\left(N_{1}, N_{2}\right), \operatorname{Std}^{\vee}}^{\text {ord }} \cong \Omega_{n, U^{p}\left(N_{1}, N_{2}\right)}^{\text {ord }}$, the pull-back by the identity section of the sheaf of relative differentials $\Omega_{\mathcal{A}^{\text {univ }} / \mathcal{X}_{n, U}^{\text {ord }}\left(N_{1}, N_{2}\right)}^{\text {or }}$.

- $\mathcal{E}_{U^{p}\left(N_{1}, N_{2}\right), \wedge^{n[F: \mathbb{Q}]} \operatorname{Std}^{\vee}} \cong \wedge^{n[F: \mathbb{Q}]} \Omega_{n, U^{p}\left(N_{1}, N_{2}\right)}^{\text {ord }}=\omega_{U^{p}\left(N_{1}, N_{2}\right)}^{\text {ord }}$.

- $\mathcal{E}_{U^{p}\left(N_{1}, N_{2}\right), \mathrm{KS}} \cong \Omega_{\left.\mathcal{X}_{n, U^{p}\left(N_{1}, N_{2}\right)}\right)}^{1} \mathbb{Z}_{(p)}$.

The system of sheaves $\left\{\mathcal{E}_{U^{p}\left(N_{1}, N_{2}\right), \rho}\right\}$ has an action of $G_{n}\left(\mathbb{A}^{\infty}\right)^{\text {ord, } \times}$. (See Sect. 3.4.)

The scheme $X_{n, U}$ has a canonical compactification $X_{n, U}^{\min }$ called the minimal or BailyBorel compactification with boundary $\partial X_{n, U}^{\min }$. It is a normal projective scheme over $\mathbb{Q}$. The line bundle $\omega_{U}$ extends (uniquely, if $n>1$ or $F^{+} \neq \mathbb{Q}$ ) to an ample line bundle $\omega_{U}$ on $X_{n, U}^{\min }$. However we can not expect the vector bundles $\mathcal{E}_{U, \rho}$ to all extend to vector bundles on $X_{n, U}^{\min } \times \operatorname{Spec} R_{0}$. All these systems of spaces and the line bundles $\omega_{U}$ have compatible actions of $G_{n}\left(\mathbb{A}^{\infty}\right)$. (See Sect. 5.1.) We can describe the scheme $\partial X_{n, U}^{\min }$ more precisely, but this will require considerably more notation, so we will come back to this in the next section.

We can also define a normal quasi-projective scheme $\mathcal{X}_{n, U^{p}\left(N_{1}, N_{2}\right)}^{\text {ord,min }}$ over $\mathbb{Z}_{(p)}$ with a closed subscheme $\partial \mathcal{X}_{n, U^{p}\left(N_{1}, N_{2}\right)}^{\text {ord,min }}$ such that

$$
\mathcal{X}_{n, U^{p}\left(N_{1}, N_{2}\right)}^{\text {ord }}=\mathcal{X}_{n, U^{p}\left(N_{1}, N_{2}\right)}^{\text {ord,min }}-\partial \mathcal{X}_{n, U^{p}\left(N_{1}, N_{2}\right)}^{\text {ord,min }}
$$

is a dense open subscheme. The scheme $\mathcal{X}_{n, U^{p}\left(N_{1}, N_{2}\right)}^{\text {ord,min }}$ is not proper-informally speaking

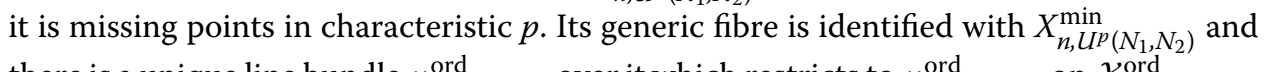
there is a unique line bundle $\omega_{U^{p}\left(N_{1}, N_{2}\right)}^{\mathrm{ord}}$ over it which restricts to $\omega_{U^{p}\left(N_{1}, N_{2}\right)}^{\mathrm{ord}}$ on $\mathcal{X}_{n, U^{p}\left(N_{1}, N_{2}\right)}^{\text {ord }}$ 
and to $\omega_{U^{p}\left(N_{1}, N_{2}\right)}$ on $X_{n, U^{p}\left(N_{1}, N_{2}\right)}^{\min }$. We will write $\mathfrak{X}_{n, U^{p}\left(N_{1}, N_{2}\right)}^{\text {ord,min }}$ for the formal completion of $\mathcal{X}_{n, U^{p}\left(N_{1}, N_{2}\right)}^{\text {ord,min }}$ along its $\mathbb{F}_{p}$-fibre $\bar{X}_{n, U^{p}\left(N_{1}, N_{2}\right)}^{\text {ord,min }}$. Schemes $\partial \bar{X}_{n, U^{p}\left(N_{1}, N_{2}\right)}^{\text {ord,min }}$ are defined similarly. All these systems of (formal) schemes have compatible actions of $G_{n}\left(\mathbb{A}^{\infty}\right)^{\text {ord }}$. The system of line bundles $\left\{\omega_{U^{p}\left(N_{1}, N_{2}\right)}^{\text {ord }}\right\}$ has an action of $G_{n}\left(\mathbb{A}^{\infty}\right)^{\text {ord, } \times}$. (See Sect. 5.1.) Again we will describe the schemes $\partial \mathcal{X}_{n, U^{p}\left(N_{1}, N_{2}\right)}^{\text {ord,min }}$ more precisely in the next section.

If $U \subset G_{n}\left(\mathbb{A}^{\infty, p} \times \mathbb{Z}_{p}\right)$ then we can define a normal scheme $\mathcal{X}_{n, U}^{\text {min }}$ which is projective and flat over $\mathbb{Z}_{(p)}$ with generic fibre $X_{n, U}^{\min }$, together with an extension of $\omega_{U}$ to an ample line bundle on $\mathcal{X}_{n, U}^{\min }$, which we will also denote $\omega_{U}$. These systems of schemes and line bundles have compatible actions of $G_{n}\left(\mathbb{A}^{\infty, p} \times \mathbb{Z}_{p}\right)$. There are $G_{n}\left(\mathbb{A}^{\infty}\right)^{\text {ord, } \times}$-equivariant open embeddings $\mathcal{X}_{n, U^{p}\left(N_{1}, N_{2}\right)}^{\text {ord,min }} \hookrightarrow \mathcal{X}_{n, U^{p}\left(N_{1}, N_{2}\right)}^{\min }$. We will write $\bar{X}_{n, U}^{\text {min }}$ for the $\mathbb{F}_{p}$-fibre of $\mathcal{X}_{U, n}^{\min }$. There is a canonical section

$$
\operatorname{Hasse}_{U} \in H^{0}\left(\bar{X}_{n, U}^{\min }, \omega_{U}^{\otimes p-1}\right)
$$

These sections are invariant under the action of $G_{n}\left(\mathbb{A}^{\infty, p} \times \mathbb{Z}_{p}\right)$. We write $\bar{X}_{n, U}^{\text {min,n-ord }}$ for the vanishing locus of Hasse $U$. Then $\bar{X}_{n, U^{p}\left(N_{1}, N_{2}\right)}^{\text {ord,min }}$ is an open and closed subscheme of $\bar{X}_{n, U^{p}\left(N_{1}, N_{2}\right)}^{\text {min }}-\bar{X}_{n, U^{p}\left(N_{1}, N_{2}\right)}^{\text {min, }}$. They are equal if $N_{1}=N_{2}=0$. (See Sect. 5.1.)

To certain additional data $\Delta$, with $(U, \Delta)$ in a certain partially ordered set $\mathcal{J}_{n}^{\text {tor }}$, which we will describe more carefully in the next section, one can associate a smooth projective scheme $X_{n, U, \Delta} / \mathbb{Q}$ and a simple normal crossings divisor

$$
\partial X_{n, U, \Delta} \subset X_{n, U, \Delta}
$$

such that

$$
X_{n, U}=X_{n, U, \Delta}-\partial X_{n, U, \Delta}
$$

We write $\left(X_{n, U, \Delta}, \mathcal{M}_{\Delta}\right)$ for the log scheme associated to $\left(X_{n, U, \Delta}, \partial X_{n, U, \Delta}\right)$. There is a natural map $X_{n, U, \Delta} \rightarrow X_{n, U}^{\mathrm{min}}$ which is the identity of $X_{n, U}$ and sends $\partial X_{n, U, \Delta}$ to $\partial X_{n, U}^{\min }$. The universal abelian scheme $A^{\text {univ }} / X_{n, U}$ extends uniquely to a semi-abelian scheme $A^{\text {univ }} / X_{n, U, \Delta}$. The system of schemes $\left\{X_{n, U, \Delta}\right\}$ has an action of $G_{n}\left(\mathbb{A}^{\infty}\right)$ via log etale maps. Similarly to certain additional data $\Sigma$, with $(U, \Sigma)$ in a certain partially ordered set $\mathcal{J}_{n}^{(m) \text {, tor }}$, which we will describe more carefully in the next section, one can associate a smooth projective scheme $A_{n, U, \Sigma}^{(m)} / \mathbb{Q}$ and a simple normal crossings divisor $\partial A_{n, U, \Sigma}^{(m)} \subset A_{n, U, \Sigma}^{(m)}$ such that

$$
A_{n, U}^{(m)}=A_{n, U, \Sigma}^{(m)}-\partial A_{n, U, \Sigma}^{(m)}
$$

We write $\left(A_{n, U, \Sigma}^{(m)}, \mathcal{M}_{\Sigma}\right)$ for the log scheme associated to $\left(A_{n, U, \Sigma}^{(m)}, \partial A_{n, U, \Sigma}^{(m)}\right)$. If $(U, \Sigma)$ and $\left(U^{\prime}, \Delta\right)$ are compatible in a suitable sense, then there is a natural $\log$ smooth map $A_{n, U, \Sigma}^{(m)} \rightarrow$ $X_{n, U^{\prime}, \Delta}$. (See Sects. 5.2, 5.3.) More details on the structure of the boundary will be given in the next section.

If $R_{0}$ is an irreducible noetherian $\mathbb{Q}$-algebra and $\rho$ is a representation of $L_{n,(n)}$ on a locally free $R_{0}$-module then we may associate a locally free sheaf $\mathcal{E}_{U, \Delta, \rho}^{\mathrm{can}} / X_{n, U, \Delta} \times \operatorname{Spec} R_{0}$ (in the Zariski topology) such that

$$
\left.\mathcal{E}_{U, \Delta, \rho}^{\mathrm{can}}\right|_{X_{n, U} \times \operatorname{Spec} R_{0}}=\mathcal{E}_{U, \rho}
$$


As examples we have the following.

- $\mathcal{E}_{U, \Delta, \mathrm{Std}^{\vee}}^{\mathrm{can}} \cong \Omega_{n, U, \Delta}$, the pull-back by the identity section of the sheaf of relative differentials $\Omega_{A^{\text {univ }} / X_{n, U, \Delta}}^{1}$.

- $\mathcal{E}_{U, \Delta, \wedge^{n[F: \mathbb{Q}]} \mathrm{Std}^{\vee}}^{\mathrm{can}} \cong \wedge^{n[F: \mathbb{Q}]} \Omega_{n, U, \Delta}=\omega_{U, \Delta}$ is naturally identified with the pull-back of $\omega_{U}$ from $X_{n, U}^{\min }$.

- $\mathcal{E}_{U, \Delta, \mathrm{KS}}^{\mathrm{can}} \cong \Omega_{X_{n, U, \Delta}}^{1}(\log \infty)$, the sheaf of differentials with log poles along the boundary.

We will write

$$
\mathcal{E}_{U, \Delta, \rho}^{\mathrm{sub}}=\mathcal{E}_{U, \Delta, \rho}^{\mathrm{can}} \otimes \mathcal{I}_{\partial X_{n, U, \Delta}}
$$

where $\mathcal{I}_{\partial X_{n}, u, \Delta}$ denotes the ideal sheaf of the boundary. It is again a locally free sheaf. We will also write $\mathcal{E}_{U, \rho}^{\text {sub }}$ for the coherent sheaf on $X_{n, U}^{\min }$ which is the push-forward of $\mathcal{E}_{U, \Delta, \rho}^{\text {sub }}$ from $X_{n, U, \Delta}$. (This is independent of the choice of $\Delta$.) The systems of sheaves $\left\{\mathcal{E}_{U, \Delta, \rho}^{\mathrm{can}}\right\}$, $\left\{\mathcal{E}_{U, \Delta, \rho}^{\text {sub }}\right\}$, and $\left\{\mathcal{E}_{U, \rho}^{\text {sub }}\right\}$ have actions of $G_{n}\left(\mathbb{A}^{\infty}\right)$. (See Sect. 5.4.)

We will write $\Omega_{A_{U, \Sigma}^{(m)}}^{1}(\log \infty)$ for the sheaf of differentials on $A_{U, \Sigma}^{(m)}$ with $\log$ poles along $\partial A_{U, \Sigma}^{(m)}$, and $\Omega_{A_{U, \Sigma}^{(m)}}^{i}(\log \infty)$ for its $i$ th exterior power.

Similarly to certain additional data $\Delta$, with $\left(U^{p}\left(N_{1}, N_{2}\right), \Delta\right) \in \mathcal{J}_{n}^{\text {tor }}$, one can associate a smooth quasi-projective scheme $\mathcal{X}_{n, U^{p}\left(N_{1}, N_{2}\right), \Delta}^{\text {ord }} / \mathbb{Q}$ and a simple relative normal crossings divisor

$$
\partial \mathcal{X}_{n, U^{p}\left(N_{1}, N_{2}\right), \Delta}^{\text {ord }} \subset \mathcal{X}_{n, U^{p}\left(N_{1}, N_{2}\right), \Delta}^{\text {ord }}
$$

such that

$$
\mathcal{X}_{n, U^{p}\left(N_{1}, N_{2}\right)}^{\text {ord }}=\mathcal{X}_{n, U^{p}\left(N_{1}, N_{2}\right), \Delta}^{\text {ord }}-\partial \mathcal{X}_{n, U^{p}\left(N_{1}, N_{2}\right), \Delta^{\circ}}^{\text {ord }}
$$

The $\mathbb{Q}$-fibre is identified with $X_{n, U^{p}\left(N_{1}, N_{2}\right), \Delta}$. We write $\left(\mathcal{X}_{n, U^{p}\left(N_{1}, N_{2}\right), \Delta}^{\text {ord }}, \mathcal{M}_{\Delta}\right)$ for the $\log$ scheme associated to $\left(\mathcal{X}_{n, U^{p}\left(N_{1}, N_{2}\right), \Delta}^{\text {ord }}, \partial \mathcal{X}_{n, U^{p}\left(N_{1}, N_{2}\right), \Delta}^{\text {ord }}\right)$. There is a natural map $\mathcal{X}_{n, U^{p}\left(N_{1}, N_{2}\right), \Delta}^{\text {ord }} \rightarrow \mathcal{X}_{n, U^{p}\left(N_{1}, N_{2}\right)}^{\text {ordmin }}$ which equals the identity of $\mathcal{X}_{n, U^{p}\left(N_{1}, N_{2}\right)}^{\text {ord }}$ and sends $\partial \mathcal{X}_{n, U^{p}\left(N_{1}, N_{2}\right), \Delta}^{\text {ord }}$ to $\partial \mathcal{X}_{n, U^{p}\left(N_{1}, N_{2}\right)}^{\text {ord, }}$. The universal abelian scheme $\mathcal{A}^{\text {univ }} / \mathcal{X}_{n, U^{p}\left(N_{1}, N_{2}\right)}^{\text {ord }}$ extends uniquely to a semi-abelian scheme $\mathcal{A}^{\text {univ }} / \mathcal{X}_{n, U^{p}\left(N_{1}, N_{2}\right), \Delta}^{\text {ord }}$. The system of schemes $\left\{\mathcal{X}_{n, U^{p}\left(N_{1}, N_{2}\right), \Delta}^{\text {ord }}\right\}$ has an action of $G_{n}\left(\mathbb{A}^{\infty}\right)^{\text {ord }}$. Also to certain additional data $\Sigma$, with $\left(U^{p}\left(N_{1}, N_{2}\right), \Sigma\right) \in \mathcal{J}_{n}^{(m), \text { tor }}$, one can associate a smooth quasi-projective scheme $\mathcal{A}_{n, U^{p}\left(N_{1}, N_{2}\right), \Sigma}^{(m), \text { ord }} / \mathbb{Z}_{(p)}$ and a simple normal crossings divisor

$$
\partial \mathcal{A}_{n, U^{p}\left(N_{1}, N_{2}\right), \Sigma}^{(m), \text { ord }} \subset \mathcal{A}_{n, U^{p}\left(N_{1}, N_{2}\right), \Sigma}^{(m), \text { ord }}
$$

such that

$$
\mathcal{A}_{n, U^{p}\left(N_{1}, N_{2}\right)}^{(m), \text { ord }}=\mathcal{A}_{n, U^{p}\left(N_{1}, N_{2}\right), \Sigma}^{(m) \text { ord }}-\partial \mathcal{A}_{n, U^{p}\left(N_{1}, N_{2}\right), \Sigma}^{(m), \text { ord }}
$$

The $\mathbb{Q}$-fibre is identified with $A_{n, U^{p}\left(N_{1}, N_{2}\right), \Sigma}^{(m)}$. We write $\left(\mathcal{A}_{n, U^{p}\left(N_{1}, N_{2}\right), \Sigma}^{\left.(m), \mathcal{M}_{\Sigma}\right)}\right.$ for the log

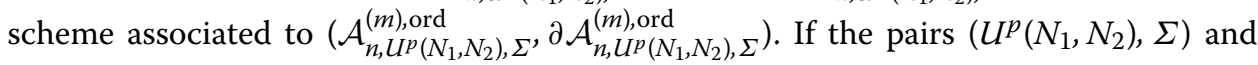
$\left(\left(U^{p}\right)^{\prime}\left(N_{1}^{\prime}, N_{2}^{\prime}\right), \Delta\right)$ are compatible in a suitable sense, then there is a natural log smooth 
$\operatorname{map} \mathcal{A}_{n, U^{p}\left(N_{1}, N_{2}\right), \Sigma}^{(m), \text { ord }} \rightarrow \mathcal{X}_{n,\left(U^{p}\right)^{\prime}\left(N_{1}^{\prime}, N_{2}^{\prime}\right), \Delta}^{\text {ord }}$. (See Sects. 5.2, 5.3.) More details on the structure of the boundary will be given in the next section.

Similarly if $R_{0}$ is an irreducible noetherian $\mathbb{Z}_{(p)}$-algebra and $\rho$ is a representation of $L_{n,(n)}$ on a locally free $R_{0}$-module then we may associate a locally free sheaf $\mathcal{E}_{U^{p}\left(N_{1}, N_{2}\right), \Delta, \rho}^{\text {ord,can }} / \mathcal{X}_{n, U^{p}\left(N_{1}, N_{2}\right), \Delta}^{\text {ord }} \times \operatorname{Spec} R_{0}$ (in the Zariski topology) such that

$$
\left.\mathcal{E}_{U^{p}\left(N_{1}, N_{2}\right), \Delta, \rho}^{\text {ord,can }}\right|_{\mathcal{X}_{n, U^{p}\left(N_{1}, N_{2}\right)}^{\text {ord }} \times \operatorname{Spec} R_{0}}=\mathcal{E}_{U^{p}\left(N_{1}, N_{2}\right), \rho}^{\text {ord }}
$$

and the pull-back to the $\mathbb{Q}$-fibre is identified with $\mathcal{E}_{U^{p}\left(N_{1}, N_{2}\right), \Delta, \rho}^{\mathrm{can}}$. As examples we have the following.

- $\mathcal{E}_{U^{p}\left(N_{1}, N_{2}\right), \Delta, \operatorname{Std}^{\vee}}^{\text {ord,can }} \cong \Omega_{n, U^{p}\left(N_{1}, N_{2}\right), \Delta}^{\text {ord }}$, the pull-back by the identity section of the sheaf of relative differentials $\Omega_{\mathcal{A}^{1}}^{1}{ }^{\text {univ }} / \mathcal{X}_{n, U^{p}\left(N_{1}, N_{2}\right), \Delta}^{\text {ord }}$.

- $\mathcal{E}_{U^{p}\left(N_{1}, N_{2}\right), \Delta, \wedge^{n[F: \mathbb{Q}]} \operatorname{Std}^{\vee}}^{\text {ord,can }} \cong \wedge^{n[F: \mathbb{Q}]} \Omega_{n, U^{p}\left(N_{1}, N_{2}\right), \Delta}^{\text {ord }}=\omega_{U^{p}\left(N_{1}, N_{2}\right), \Delta}^{\text {ord }}$ is naturally identified with the pull-back of $\omega_{U^{p}\left(N_{1}, N_{2}\right)}^{\text {ord }}$ from $\mathcal{X}_{n, U^{p}\left(N_{1}, N_{2}\right)}^{\text {ord,min }}$.

- $\mathcal{E}_{U^{p}\left(N_{1}, N_{2}\right), \Delta, \mathrm{KS}}^{\text {ord,can }} \cong \Omega_{\mathcal{X}_{n, U^{p}\left(N_{1}, N_{2}\right), \Delta}^{\text {ord }}}^{1}(\log \infty)$, the sheaf of differentials with log poles along the boundary.

We will write

$$
\mathcal{E}_{U^{p}\left(N_{1}, N_{2}\right), \Delta, \rho}^{\text {ord,sub }}=\mathcal{E}_{U^{p}\left(N_{1}, N_{2}\right), \Delta, \rho}^{\text {ord,can }} \otimes \mathcal{I}_{\partial \mathcal{X}_{n, U^{p}\left(N_{1}, N_{2}\right), \Delta}^{\text {ord }}},
$$

where $\mathcal{I}_{\partial \mathcal{X}_{n, U}^{\text {ord }}\left(N_{1}, N_{2}\right), \Delta}$ denotes the ideal sheaf of the boundary. It is again a locally free sheaf. We will also write $\mathcal{E}_{U^{p}\left(N_{1}, N_{2}\right), \rho}^{\text {ord,sub }}$ for the coherent sheaf on $\mathcal{X}_{n, U^{p}\left(N_{1}, N_{2}\right)}^{\text {ord,min }}$ which is the pushforward of $\mathcal{E}_{U^{p}\left(N_{1}, N_{2}\right), \Delta, \rho}^{\text {ord,sub }}$ from $\mathcal{X}_{n, U^{p}\left(N_{1}, N_{2}\right), \Delta}$. (This is independent of the choice of $\Delta$.) The systems of sheaves $\left\{\mathcal{E}_{U^{p}\left(N_{1}, N_{2}\right), \Delta, \rho}^{\text {ord,can }}\right\},\left\{\mathcal{E}_{U^{p}\left(N_{1}, N_{2}\right), \Delta, \rho}^{\text {ord,sub }}\right\}$, and $\left\{\mathcal{E}_{U^{p}\left(N_{1}, N_{2}\right), \rho}^{\text {ord,sub }}\right\}$ have actions of $G_{n}\left(\mathbb{A}^{\infty}\right)^{\text {ord }, \times}$. (See Sect. 5.4.)

The formal completion of $\mathcal{X}_{n, U^{p}\left(N_{1}, N_{2}\right), \Delta}^{\text {ord }}$ along its special fibre depends only on $U^{p}\left(N_{1}\right)$ and $\Delta^{\text {ord }}$, a subset of the data contained in $\Delta$, which we will describe in more detail in the next section. Thus we will denote this completion $\mathfrak{X}_{n, U^{p}\left(N_{1}\right), \Delta^{\text {ord }}}^{\text {ord }}$, and its reduced sub-

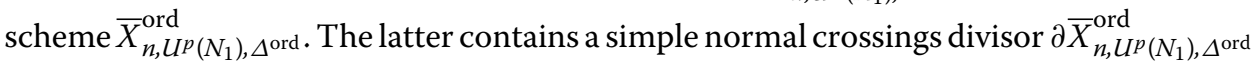
such that $\bar{X}_{n, U^{p}\left(N_{1}\right), \Delta^{\text {ord }}}^{\text {ord }}-\partial \bar{X}_{n, U^{p}\left(N_{1}\right), \Delta^{\text {ord }}}^{\text {ord }}=\bar{X}_{n, U^{p}\left(N_{1}\right)}^{\text {ord }}$. The systems of these (formal) schemes have an action of $G_{n}\left(\mathbb{A}^{\infty}\right)^{\text {ord }}$. The map

$$
\varsigma_{p}: \mathfrak{X}_{n, U^{p}\left(N_{1}\right), \Delta^{\text {ord }}}^{\text {ord }} \longrightarrow \mathfrak{X}_{n, U^{p}\left(N_{1}\right), \Delta^{\text {ord }}}^{\text {ord }}
$$

is finite flat of degree $p^{n^{2}\left[F^{+}: \mathbb{Q}\right]}$ and the induced map on reduced subschemes is absolute Frobenius. Similarly the formal completion of $\mathcal{A}_{n, U^{p}\left(N_{1}, N_{2}\right), \Sigma}^{(m) \text {,ord }}$ along its special fibre depends only on $U^{p}\left(N_{1}\right)$ and $\Sigma^{\text {ord }}$, a subset of the data contained in $\Delta$, which we will describe in more detail in the next section. Thus we will denote this completion $\mathfrak{A}_{n, U^{p}\left(N_{1}\right), \Sigma^{\text {ord }}}^{(m) \text {,ord }}$, and its

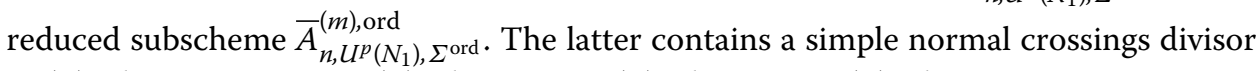
$\partial \bar{A}_{n, U^{p}\left(N_{1}\right), \Sigma^{\text {ord }}}^{(m) \text { ord }}$ such that $\bar{A}_{n, U^{p}\left(N_{1}\right), \Sigma^{\text {ord }}}^{(m)}-\partial \bar{A}_{n, U^{p}\left(N_{1}\right), \Sigma^{\text {ord }}}^{(m) \text {,ord }}=\bar{A}_{n, U^{p}\left(N_{1}\right)}^{(m) \text {,ord }}$. The systems of these (formal) schemes have an action of $G_{n}^{(m)}\left(\mathbb{A}^{\infty}\right)^{\text {ord }}$. The map

$$
\varsigma_{p}: \mathfrak{A}_{n, U^{p}\left(N_{1}\right), \Sigma^{\text {ord }}}^{(m), \text { ord }} \longrightarrow \mathfrak{A}_{n, U^{p}\left(N_{1}\right), \Sigma^{\text {ord }}}^{(m), \text { ord }}
$$


is finite flat of degree $p^{(n+2 m) n\left[F^{+}: \mathbb{Q}\right]}$ and the induced map on reduced subschemes is absolute Frobenius. (See Sect. 5.3.)

We will write $X_{U^{\prime}}^{\mathrm{min}, \dagger}$ and $X_{U^{\prime}, \Delta}^{\dagger}$ and $A_{U, \Sigma}^{(m), \dagger}$ for the dagger spaces in the sense of [27] associated to $X_{n, U^{\prime}}^{\min } / \mathbb{Q}_{p}$ and $X_{n, U^{\prime}, \Delta} / \mathbb{Q}_{p}$ and $A_{n, U, \Sigma}^{(m)} / \mathbb{Q}_{p}$. We will write $\mathcal{X}_{\left(U^{p}\right)^{\prime}\left(N_{1}\right)}^{\text {ord,min } \dagger}$ and $\mathcal{X}_{\left(U^{p}\right)^{\prime}\left(N_{1}\right), \Delta^{\text {ord }}}^{\text {ord }} \dagger$ and $\mathcal{A}_{U^{p}\left(N_{1}\right), \Sigma^{\text {ord }}}^{(m), \text { ord } \dagger}$ for the subdagger spaces of $X_{\left(U^{p}\right)^{\prime}\left(N_{1}, N_{2}\right)}^{\min \dagger}$ and $X_{\left(U^{p}\right)^{\prime}\left(N_{1}, N_{2}\right), \Delta}^{\dagger}$ and $A_{U^{p}\left(N_{1}, N_{2}\right), \Sigma}^{(m),}$ corresponding to the admissible opens in the corresponding rigid spaces which are the generic fibres of $\mathfrak{X}_{n,\left(U^{p}\right)^{\prime}\left(N_{1}\right)}^{\text {ord,min }}$ and $\mathfrak{X}_{n,\left(U^{p}\right)^{\prime}\left(N_{1}\right), \Delta^{\text {ord }}}^{\text {ord }}$ and $\mathfrak{A}_{n, U^{p}\left(N_{1}\right), \Sigma^{\text {ord }}}^{(m) \text {, respec- }}$ tively. These depend only on $\left(U^{p}\right)^{\prime}\left(N_{1}\right), \Delta^{\text {ord }}, U^{p}\left(N_{1}\right)$ and $\Sigma^{\text {ord }}$. If $U^{p}$ maps to $\left(U^{p}\right)^{\prime}$ then the spaces $\mathcal{X}_{\left(U^{p}\right)^{\prime}\left(N_{1}\right), \Delta^{\text {ord }}}^{\text {ord } \dagger}$ and $\mathcal{A}_{U^{p}\left(N_{1}\right), \Sigma^{\text {ord }}}^{(m), \text { ord }}$ are the pre-images in $X_{\left(U^{p}\right)^{\prime}\left(N_{1}, N_{2}\right), \Delta}^{\dagger}$ and $A_{U^{p}\left(N_{1}, N_{2}\right), \Sigma}^{(m),}$ of

$$
\mathcal{X}_{\left(U^{p}\right)^{\prime}\left(N_{1}\right)}^{\mathrm{ord}, \min }, \subset X_{\left(U^{p}\right)^{\prime}\left(N_{1}, N_{2}\right)}^{\mathrm{min}, \dagger}
$$

Similarly we define closed subdagger spaces $\partial \mathcal{X}_{\left(U^{p}\right)^{\prime}\left(N_{1}\right)}^{\text {ord,min } \dagger}$ and $\partial \mathcal{X}_{\left(U^{p}\right)^{\prime}\left(N_{1}\right), \Delta^{\text {ord }}}^{\text {ord } \dagger}$ and $\partial \mathcal{A}_{U^{p}\left(N_{1}\right), \Sigma^{\text {ord }}}^{(m) \text { ord } †}$ (See Sects. 6.3, 6.4.)

The systems of dagger spaces $\left\{\mathcal{X}_{V^{p}\left(N_{1}\right)}^{\text {ord,min }} \dagger^{\prime}\right\}$ and $\left\{\mathcal{X}_{V^{p}\left(N_{1}\right), \Delta^{\text {ord }}}^{\text {ord } \dagger}\right\}$ and $\left\{\mathcal{A}_{U^{p}\left(N_{1}\right), \Sigma^{\text {ord }}}^{(m) \text {,ord } \dagger}\right\}$ have actions of $G_{n}\left(\mathbb{A}^{\infty}\right)^{\text {ord }}$ and $G_{n}\left(\mathbb{A}^{\infty}\right)^{\text {ord }}$ and $G_{n}^{(m)}\left(\mathbb{A}^{\infty}\right)^{\text {ord }}$, respectively, which respect the boundaries. If $g \in G_{n}\left(\mathbb{A}^{\infty}\right)^{\text {ord }}$ or $G_{n}\left(\mathbb{A}^{\infty}\right)^{\text {ord }}$ or $G_{n}^{(m)}\left(\mathbb{A}^{\infty}\right)^{\text {ord }}$, respectively, then $\mathcal{X}_{V^{p}\left(N_{1}\right)}^{\text {ord,min }}$ and $\mathcal{X}_{V^{p}\left(N_{1}\right), \Delta^{\text {ord }}}^{\text {ord, }}$ and $\mathcal{A}_{U^{p}\left(N_{1}\right), \Sigma^{\text {ord }}}^{(m), \text { ord, } \dagger}$ are the pre-images of $\mathcal{X}_{\left(V^{p}\right)^{\prime}\left(N_{1}^{\prime}\right)}^{\text {ord,min }}$ and $\mathcal{X}_{\left(V^{p}\right)^{\prime}\left(N_{1}^{\prime}\right), \Delta^{\prime, \text { ord }}}^{\text {ord, }}$ and $\mathcal{A}_{\left(U^{p}\right)^{\prime}\left(N_{1}^{\prime}\right), \Sigma^{\prime \text {,ord }}}^{(m), \text { ord }}$ under

$$
g: X_{V^{p}\left(N_{1}, N_{2}\right)}^{\min , \dagger} \longrightarrow X_{\left(V^{p}\right)^{\prime}\left(N_{1}^{\prime}, N_{2}+\operatorname{val}_{p}(v(g))\right)}^{\min \dagger}
$$

and

$$
g: X_{V^{p}\left(N_{1}, N_{2}\right), \Delta}^{\dagger} \longrightarrow X_{\left(V^{p}\right)^{\prime}\left(N_{1}^{\prime}, N_{2}+\operatorname{val}_{p}(v(g))\right), \Delta^{\prime}}^{\dagger}
$$

and

$$
g: A_{U^{p}\left(N_{1}, N_{2}\right), \Sigma}^{(m), \dagger} \longrightarrow A_{\left(U^{p}\right)^{\prime}\left(N_{1}^{\prime}, N_{2}+\operatorname{val}_{p}(v(g))\right), \Sigma^{\prime \prime}}^{(m), \dagger}
$$

provided that either $\operatorname{val}_{p}(v(g))=0$ or $N_{2}+\operatorname{val}_{p}(v(g))>0$. (See Sects. 6.3, 6.4.)

We will write $\mathcal{E}_{U^{p}\left(N_{1}\right), \Delta^{\text {ord }, \rho}}^{\text {can } \dagger}$ for the restriction to $\mathcal{X}_{U^{p}\left(N_{1}\right), \Delta^{\text {ord }}}^{\text {ord }} \dagger$ of the locally free sheaf on $X_{U^{p}\left(N_{1}, N_{2}\right), \Delta}^{\dagger}$ associated to $\mathcal{E}_{U^{p}\left(N_{1}, N_{2}\right), \Delta, \rho}^{\mathrm{can}}$. It does not depend on $N_{2}$ or $\Delta$. We define $\mathcal{E}_{U^{p}\left(N_{1}\right), \Delta^{\text {ord }, \rho}}^{\text {sub, }}$ similarly. The systems of sheaves $\left\{\mathcal{E}_{U^{p}\left(N_{1}\right), \Delta^{\text {ord }, \rho}}^{\text {can } \dagger}\right\}$ and $\left\{\mathcal{E}_{U^{p}\left(N_{1}\right), \Delta^{\text {ord }, \rho}}^{\text {sub } \dagger}\right\}$ have actions of $G_{n}\left(\mathbb{A}^{\infty}\right)^{\text {ord }}$. If $g \in G_{n}\left(\mathbb{A}^{\infty}\right)^{\text {ord }}$ then the map

$$
g: g^{*} \mathcal{E}_{\left(U^{p}\right)^{\prime}\left(N_{1}^{\prime}\right), \Delta^{\prime, \text { ord }, \rho}}^{\mathrm{can},} \longrightarrow \mathcal{E}_{U^{p}\left(N_{1}\right), \Delta^{\text {ord }, \rho}}^{\mathrm{can},}
$$

is an isomorphism. We have maps, which we will denote $\operatorname{tr}_{F}$ :

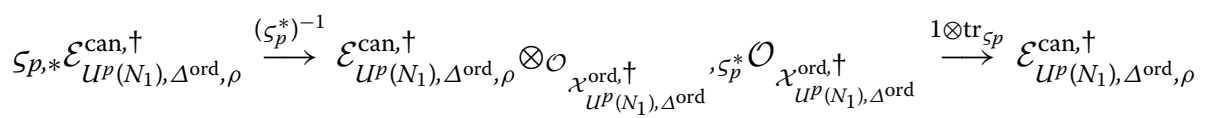


and

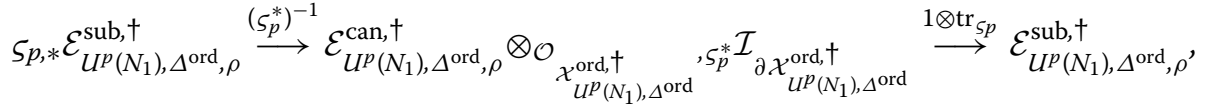

where $\mathcal{I}_{\partial \mathcal{X}_{U^{p}\left(N_{1}\right), \Delta^{\text {ord }}}^{\text {ord }}} \subset \mathcal{O}_{\mathcal{X}_{U^{p}\left(N_{1}\right), \Delta^{\text {ord }}}^{\text {ord }} \dagger}$ denotes the ideal sheaf of the boundary. (See Sect. 6.3.)

We will also write $\mathcal{E}_{U^{p}\left(N_{1}\right), \rho}^{\text {sub }, ~ f o r ~ t h e ~ r e s t r i c t i o n ~ t o ~} \mathcal{X}_{U^{p}\left(N_{1}\right)}^{\text {ord,min }} \dagger$ of the locally free sheaf on $X_{U^{p}\left(N_{1}, N_{2}\right)}^{\min \dagger}$ associated to $\mathcal{E}_{U^{p}\left(N_{1}, N_{2}\right), \rho}^{\text {sub }}$. It does not depend on $N_{2}$. It can be identified with the push-forward from $\mathcal{X}_{U^{p}\left(N_{1}\right), \Delta^{\text {ord }}}^{\text {ord }, \dagger}$ to $\mathcal{X}_{U^{p}\left(N_{1}\right)}^{\text {ord,min, } \dagger}$ of $\mathcal{E}_{U^{p}\left(N_{1}\right), \Delta^{\text {ord }, \rho}}^{\text {can }, \dagger}$. The system of sheaves $\left\{\mathcal{E}_{U^{p}\left(N_{1}\right), \rho}^{\text {sub, }}\right\}$ has an action of $G_{n}\left(\mathbb{A}^{\infty}\right)^{\text {ord }}$. Moreover the map

$$
\operatorname{tr}_{F}: \varsigma_{p, *} \mathcal{E}_{U^{p}\left(N_{1}\right), \Delta^{\text {ord }, \rho}}^{\text {sub }} \longrightarrow \mathcal{E}_{U^{p}\left(N_{1}\right), \Delta^{\text {ord }, \rho}}^{\text {sub } \dagger}
$$

pushes forward to a map

$$
\operatorname{tr}_{F}: \varsigma_{p, *} \mathcal{E}_{U^{p}\left(N_{1}\right), \rho}^{\mathrm{sub}, \dagger} \longrightarrow \mathcal{E}_{U^{p}\left(N_{1}\right), \rho}^{\mathrm{sub}, \dagger}
$$

which does not depend on $\Delta^{\text {ord }}$. (See Sect. 6.4.)

We will denote by $\Omega_{\mathcal{A}_{U^{p}\left(N_{1}\right), \Sigma}^{(m), \text { ord }}}^{i}(\log \infty)$ the sheaf on $\mathcal{A}_{U^{p}\left(N_{1}\right), \Sigma^{\text {ord }}}^{(m), \text { ord } \dagger}$ associated to the sheaf $\Omega_{A_{n, U^{p}\left(N_{1}, N_{2}\right), \Sigma}^{(m)}}^{i}(\log \infty)$. It is independent of $N_{2}$ and $\Sigma$. Also let $\mathcal{I}_{\partial \mathcal{A}_{U^{p}\left(N_{1}\right), \Sigma^{\text {ord }}}^{(m), \text { ord }} \dagger}$ denote the ideal

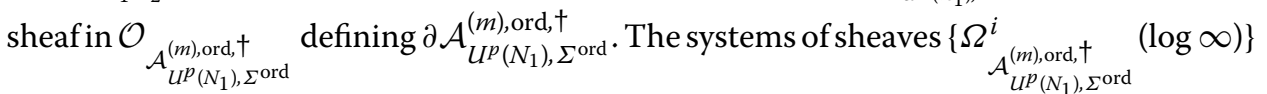
and $\left\{\Omega_{\mathcal{A}_{U^{p}\left(N_{1}\right), \Sigma^{\text {ord }}}^{(m) \text { ord } \dagger}}^{i}(\log \infty) \otimes \mathcal{I}_{\partial \mathcal{A}_{U^{p}\left(N_{1}\right), \Sigma^{\text {ord }}}^{(m), \text { ord }} \dagger}\right\}$ both have actions of $G_{n}^{(m)}\left(\mathbb{A}^{\infty}\right)^{\text {ord }}$. If $g \in$ $G_{n}^{(m)}\left(\mathbb{A}^{\infty}\right)^{\text {ord }}$ then the map

$$
g: g^{*} \Omega_{\mathcal{A}_{\left(U^{p}\right)^{\prime}\left(N_{1}^{\prime}\right), \Sigma^{\prime} \text {,ord }}^{(m) \text { ord } \dagger}}^{i}(\log \infty) \longrightarrow \Omega_{\mathcal{A}_{U^{p}\left(N_{1}\right), \Sigma^{\text {ord }}}^{(m), \text { ord }} \dagger}^{i}(\log \infty)
$$

is an isomorphism. We have maps, which we will denote $\operatorname{tr}_{F}$ :

$$
\begin{aligned}
& \varsigma_{p, *} \Omega_{\mathcal{A}_{U^{p}\left(N_{1}\right), \Sigma}^{(m), \text { ord }}}^{i}(\log \infty) \\
& \stackrel{\left(\varsigma_{p}^{*}\right)^{-1}}{\longrightarrow} \Omega_{\mathcal{A}_{U^{p}\left(N_{1}\right), \Sigma^{\text {ord }}}^{i(m), \text { ord }} \dagger}^{i}(\log \infty) \otimes_{\mathcal{O}} \underbrace{}_{\mathcal{A}_{U^{p}\left(N_{1}\right), \Sigma^{\text {ord }}}^{(m), \text { ord }} \dagger}, \varsigma_{p}^{*} \mathcal{O}_{\mathcal{A}_{U^{p}\left(N_{1}\right), \Sigma^{\text {ord }}}^{(m), \text { ord }}, \dagger} \\
& \stackrel{1 \otimes \operatorname{tr}_{S p}}{\longrightarrow} \Omega_{\mathcal{A}_{U^{p}\left(N_{1}\right), \Sigma^{\text {ord }}}^{(m) \text { ord }} \dagger}^{i}(\log \infty)
\end{aligned}
$$

and

$$
\begin{aligned}
& \varsigma_{p, *}\left(\begin{array}{ll}
\Omega_{\mathcal{A}_{U^{p}\left(N_{1}\right), \Sigma}^{(m), \text { ord }} \dagger}^{i} & (\log \infty) \otimes \mathcal{I}_{\partial \mathcal{A}_{U^{p}\left(N_{1}\right), \Sigma}^{(m), \text { ord }}, \dagger}
\end{array}\right) \\
& \stackrel{\left(\varsigma_{p}^{*}\right)^{-1}}{\longrightarrow} \Omega_{\mathcal{A}_{U^{p}\left(N_{1}\right), \Sigma^{\text {ord }}}^{(m), \text { ord }},}^{i}(\log \infty) \otimes_{\mathcal{O}_{\mathcal{A}^{p}\left(N_{1}\right), \Sigma^{\text {ord }}}^{(m), \text { ord }},}, \varsigma_{p}^{*} \mathcal{I}_{\partial \mathcal{A}_{U^{p}\left(N_{1}\right), \Sigma^{\text {ord }}}^{(m), \text { ord }} \dagger} \\
& \stackrel{1 \otimes \operatorname{tr}_{S p}}{\longrightarrow} \Omega_{\mathcal{A}_{U^{p}\left(N_{1}\right), \Sigma^{\text {ord }}}^{(m) \text {,rd }, \dagger}}^{i}(\log \infty) \otimes \mathcal{I}_{\partial \mathcal{A}_{U^{p}\left(N_{1}\right), \Sigma}^{(m), \text { ord }}, \dagger} .
\end{aligned}
$$

(See Sect. 6.3.) 


\section{A.2 The boundary}

Unfortunately to describe the boundaries of our various compactifications requires significantly more notation. We remind the reader of our convention that, if $U \subset G$ are groups and $G \rightarrow H$ is a homomorphism, we will sometimes also use $U$ to denote the image of $U$ in $H$, where from the context it is clear that we need a subgroup of $H$.

We will first consider the boundary of $X_{n, U}^{\min }$. For $i=0, \ldots, n$ let $P_{n,(i)}^{+}$denote the subgroup of $G_{n}$ consisting of matrices of the form

$$
\left(\begin{array}{c|c|c}
* & * & * \\
\hline 0_{2(n-i) \times i} & * & * \\
\hline 0_{i \times i} & 0_{i \times 2(n-i)} & *
\end{array}\right)
$$

and let $L_{n,(i)}$ denote the subgroup consisting of block diagonal matrices. Then $L_{n,(i)}=$ $L_{n,(i) \text {,lin }} \times L_{n,(i), \text { herm }}$, where $L_{n,(i) \text {, lin }} \cong \mathrm{RS}_{\mathbb{Z}}^{\mathcal{O}_{F}} G L_{i}$ is the set of matrices of the form

$$
\left(\begin{array}{c|c|c}
* & 0 & 0 \\
\hline 0 & 1_{2(n-i)} & 0 \\
\hline 0 & 0 & h
\end{array}\right)
$$

with $h \in \mathrm{RS}_{\mathbb{Z}}^{\mathcal{O}_{F}} G L_{i}$, and where $L_{n,(i) \text { herm }} \cong G_{n-i}$ is the set of matrices of the form

$$
\left(\begin{array}{c|c|c}
\nu(g) 1_{i} & 0 & 0 \\
\hline 0 & g & 0 \\
\hline 0 & 0 & 1_{i}
\end{array}\right)
$$

with $g \in G_{n-i}$. (See Sect. 1.2.)

For $U \subset L_{n,(i)}\left(\mathbb{A}^{\infty}\right)$ a neat open compact subgroup we set

$$
X_{n,(i), U}^{+}=\coprod_{h \in L_{n,(i), \text { lin }}\left(\mathbb{A}^{\infty}\right) / U} X_{n-i, h U h^{-1} \cap L_{n,(i), \text { herm }}\left(\mathbb{A}^{\infty}\right)}
$$

This is locally of finite type, but not of finite type over $\mathbb{Q}$. We refer to it as a generalized Shimura variety. It has a left action of $L_{n,(i), \text { lin }}(\mathbb{Q})=G L_{i}(F)$ such that $\delta \in L_{n,(i), \text { lin }}(\mathbb{Q})$ acts via the coproduct of the identity maps

$$
X_{n-i, h U h^{-1} \cap L_{n, i}(i) \operatorname{herm}\left(\mathbb{A}^{\infty}\right)} \longrightarrow X_{n-i, \delta h U h^{-1} \delta^{-1} \cap L_{n,(i), \operatorname{herm}}\left(\mathbb{A}^{\infty}\right)} .
$$

The inverse system $\left\{X_{n,(i), U}^{+}\right\}$has a commuting right action of $L_{n,(i)}\left(\mathbb{A}^{\infty}\right)$ such that $g=$ $\left(g_{\text {lin }}, g_{\text {herm }}\right) \in L_{n,(i)}\left(\mathbb{A}^{\infty}\right)$ acts via the coproduct of the maps

$$
g_{\text {herm }}: X_{n-i, h U h^{-1} \cap L_{n,(i), \text { herm }}\left(\mathbb{A}^{\infty}\right)} \longrightarrow X_{n-i, h g_{\text {lin }}} V g_{\text {lin }}^{-1} h^{-1} \cap L_{n,(i) \text { herm }}\left(\mathbb{A}^{\infty}\right)
$$

if $V \supset g^{-1} U g$. We further define

$$
X_{n,(i), U}^{\natural}=L_{n,(i), \operatorname{lin}}(\mathbb{Q}) \backslash X_{n,(i), U}^{+}
$$

The inverse system $\left\{X_{n,(i), U}^{\natural}\right\}$ has a right action of $L_{n,(i)}\left(\mathbb{A}^{\infty}\right)$. (See Sect. 4.1.) 
With these definitions we can describe the boundary of $X_{n, U}^{\min }$. There is a family of closed subschemes

$$
X_{n, U}^{\min }=\partial_{0} X_{n, U}^{\min } \supset \partial_{1} X_{n, U}^{\min }=\partial X_{n, U}^{\min } \supset \partial_{2} X_{n, U}^{\min } \supset \cdots \supset \partial_{n+1} X_{n, U}^{\min }=\emptyset
$$

which are preserved by the action of $G_{n}\left(\mathbb{A}^{\infty}\right)$. We set

$$
\partial_{i}^{0} X_{n, U}^{\min }=\partial_{i} X_{n, U}^{\min }-\partial_{i+1} X_{n, U}^{\min }
$$

which is smooth over $\mathbb{Q}$ of dimension $(n-i)^{2}\left[F^{+}: \mathbb{Q}\right]$ and write $X_{n, U, i}^{\min , \wedge}$ for the formal completion of $X_{n, U}^{\min }$ along $\partial_{i}^{0} X_{n, U}^{\min }$. We can describe $\partial_{i}^{0} X_{n, U}^{\min }$ as

$$
\coprod_{h \in P_{n, i(i)}^{+}\left(\mathbb{A}^{\infty}\right) \backslash G_{n}\left(\mathbb{A}^{\infty}\right) / U} X_{n,(i), h U h^{-1} \cap P_{n,(i)}^{+}\left(\mathbb{A}^{\infty}\right)^{.}}^{\natural}
$$

If $g \in G_{n}\left(\mathbb{A}^{\infty}\right)$ and if $g^{-1} U g \subset V$ then the map

$$
g: \partial_{i}^{0} X_{n, U}^{\min } \longrightarrow \partial_{i}^{0} X_{n, V}^{\min }
$$

is the coproduct of the maps

$$
g^{\prime}: X_{n,(i), h U h^{-1} \cap P_{n,(i)}^{+}\left(\mathbb{A}^{\infty}\right)}^{\natural} \longrightarrow X_{n,(i), h^{\prime} V h^{\prime}-1 \cap P_{n,(i)}^{+}\left(\mathbb{A}^{\infty}\right)}^{\natural}
$$

where $h g=g^{\prime} h^{\prime}$ with $g^{\prime} \in P_{h,(i)}\left(\mathbb{A}^{\infty}\right)$. (See Sect. 5.1.)

We write

$$
U_{p}\left(N_{1}\right)_{n,(i), \operatorname{lin}}=\operatorname{ker}\left(L_{n,(i), \operatorname{lin}}\left(\mathbb{Z}_{p}\right) \rightarrow L_{n,(i), \operatorname{lin}}\left(\mathbb{Z} / p^{N_{1}} \mathbb{Z}\right)\right) \subset L_{n,(i), \operatorname{lin}}\left(\mathbb{A}^{\infty}\right)
$$

and

$$
U_{p}\left(N_{1}, N_{2}\right)_{n,(i)}=U_{p}\left(N_{1}\right)_{n,(i), \text { lin }} \times U_{p}\left(N_{1}, N_{2}\right)_{n-i} \subset L_{n,(i)}\left(\mathbb{Q}_{p}\right),
$$

and if $U^{p}$ is a neat open compact subgroup of $L_{n,(i), \operatorname{lin}}\left(\mathbb{A}^{\infty, p}\right)$ or $L_{n,(i)}\left(\mathbb{A}^{\infty, p}\right)$ we will write

$$
U^{p}\left(N_{1}\right)=U^{p} \times U_{p}\left(N_{1}\right)_{n,(i), \text { lin }}
$$

or

$$
U^{p}\left(N_{1}, N_{2}\right)=U^{p} \times U_{p}\left(N_{1}, N_{2}\right)_{n,(i)} .
$$

Moreover we write

$$
L_{n,(i), \operatorname{lin}}\left(\mathbb{A}^{\infty}\right)^{\text {ord }}=L_{n,(i), \operatorname{lin}}\left(\mathbb{A}^{\infty, p} \times \mathbb{Z}_{p}\right)
$$

and

$$
L_{n,(i)}\left(\mathbb{A}^{\infty}\right)^{\text {ord }}=L_{n,(i), \text { lin }}\left(\mathbb{A}^{\infty}\right)^{\text {ord }} \times G_{n-i}\left(\mathbb{A}^{\infty}\right)^{\text {ord }}
$$


and

$$
L_{n,(i)}\left(\mathbb{A}^{\infty}\right)^{\text {ord }, \times}=L_{n,(i), \text { lin }}\left(\mathbb{A}^{\infty}\right)^{\text {ord }} \times G_{n-i}\left(\mathbb{A}^{\infty}\right)^{\text {ord } \times} .
$$

(See Sect. 1.2.)

We set

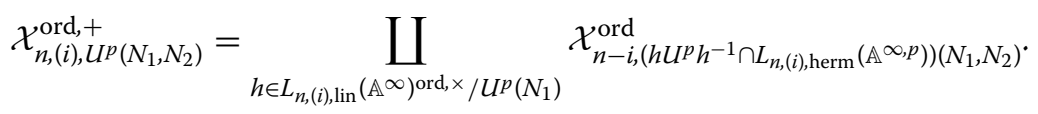

It has a left action of $L_{n,(i), \operatorname{lin}}\left(\mathbb{Z}_{(p)}\right)=G L_{i}\left(\mathcal{O}_{F,(p)}\right)$ such that $\delta \in L_{n,(i), \text { lin }}\left(\mathbb{Z}_{(p)}\right)$ acts via the coproduct of the identity maps

$$
\mathcal{X}_{n-i,\left(h U^{p} h^{-1} \cap L_{n,(i), \text { herm }}\left(\mathbb{A}^{\infty, p}\right)\right)\left(N_{1}, N_{2}\right)}^{\text {ord }} \longrightarrow \mathcal{X}_{n-i,\left(\delta h U^{p} h^{-1} \delta^{-1} \cap L_{n,(i), \text { herm }}\left(\mathbb{A}^{\infty, p}\right)\right)\left(N_{1}, N_{2}\right)}^{\text {ord }}
$$

The inverse system $\left\{\mathcal{X}_{n,(i), U^{p}\left(N_{1}, N_{2}\right)}^{\text {ord, }}\right\}$ has a commuting right action of $L_{n,(i)}\left(\mathbb{A}^{\infty}\right)^{\text {ord }}$ such that $g=\left(g_{\text {lin }}, g_{\text {herm }}\right) \in L_{n,(i)}\left(\mathbb{A}^{\infty}\right)^{\text {ord }}$ acts via the coproduct of the maps

$$
\left.g_{\text {herm }}: \mathcal{X}_{n-i,\left(h U^{p} h^{-1} \cap L_{n,(i), \text { herm }}\left(\mathbb{A}^{\infty, p}\right)\right)\left(N_{1}, N_{2}\right)}^{\longrightarrow} \mathcal{X}_{n-i,\left(h g_{\operatorname{lin}} V^{p} g_{\operatorname{lin}}^{-1} h^{-1} \cap L_{n, i}\right. \text { ord herm }}\left(\mathbb{A}^{\infty, p}\right)\right)\left(N_{1}^{\prime}, N_{2}^{\prime}\right)
$$

if $V^{p}\left(N_{1}^{\prime}, N_{2}^{\prime}\right) \supset g^{-1} U^{p}\left(N_{1}, N_{2}\right) g$. We further define

$$
\mathcal{X}_{n,(i), U^{p}\left(N_{1}, N_{2}\right)}^{\mathrm{ord}, \natural}=L_{n,(i), \operatorname{lin}}\left(\mathbb{Z}_{(p)}\right) \backslash \mathcal{X}_{n,(i), U^{p}\left(N_{1}, N_{2}\right)}^{\mathrm{ord}} .
$$

The inverse system $\left\{\mathcal{X}_{n,(i), U^{p}\left(N_{1}, N_{2}\right)}^{\text {ord }\}}\right\}$ has a right action of $L_{n,(i)}\left(\mathbb{A}^{\infty}\right)^{\text {ord }}$. (See Sect. 4.1.)

There is a family of closed subschemes

$$
\begin{aligned}
\mathcal{X}_{n, U^{p}\left(N_{1}, N_{2}\right)}^{\text {ord,min }}= & \partial_{0} \mathcal{X}_{n, U^{p}\left(N_{1}, N_{2}\right)}^{\text {ord,min }} \supset \partial_{1} \mathcal{X}_{n, U^{p}\left(N_{1}, N_{2}\right)}^{\text {ord,min }} \\
= & \partial \mathcal{X}_{n, U^{p}\left(N_{1}, N_{2}\right)}^{\text {ord, }} \supset \partial_{2} \mathcal{X}_{n, U^{p}\left(N_{1}, N_{2}\right)}^{\text {ord, }} \\
& \supset \cdots \supset \partial_{n+1} \mathcal{X}_{n, U^{p}\left(N_{1}, N_{2}\right)}^{\text {ord,mi }}=\emptyset
\end{aligned}
$$

which are preserved by the action of $G_{n}\left(\mathbb{A}^{\infty}\right)^{\text {ord }}$. We set

$$
\partial_{i}^{0} \mathcal{X}_{n, U^{p}\left(N_{1}, N_{2}\right)}^{\text {ord,min }}=\partial_{i} \mathcal{X}_{n, U^{p}\left(N_{1}, N_{2}\right)}^{\text {ord,min }}-\partial_{i+1} \mathcal{X}_{n, U^{p}\left(N_{1}, N_{2}\right)}^{\text {ord,min }}
$$

which is smooth over $\mathbb{Z}_{(p)}$ of relative dimension $(n-i)^{2}\left[F^{+}: \mathbb{Q}\right]$. We can describe it as

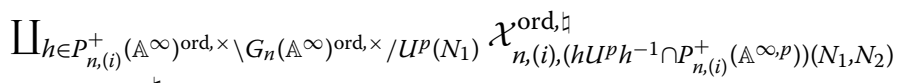

$$
\begin{aligned}
& \amalg \coprod_{h} X_{n,(i), h U^{p}\left(N_{1}, N_{2}\right) h^{-1} \cap P_{n,(i)}^{+}\left(\mathbb{A}^{\infty}\right)^{\prime}}
\end{aligned}
$$

where the second coproduct runs over

$$
h \in\left(P_{n,(i)}^{+}\left(\mathbb{A}^{\infty}\right) \backslash G_{n}\left(\mathbb{A}^{\infty}\right) / U^{p}\left(N_{1}, N_{2}\right)-P_{n,(i)}^{+}\left(\mathbb{A}^{\infty}\right)^{\text {ord }, \times} \backslash G_{n}\left(\mathbb{A}^{\infty}\right)^{\text {ord }, \times} / U^{p}\left(N_{1}\right)\right) .
$$

The action of $G_{n}\left(\mathbb{A}^{\infty}\right)^{\text {ord }}$ can be described as in the case of $\partial_{i}^{0} X_{n, U}^{\min }$. (See Sect. 5.1.)

We will now turn to the boundary of $X_{n, U, \Delta}$ and $A_{n, U, \Sigma}^{(m)}$. The former is the special case of the latter in which $m=0$, so we will discuss only the case of $A_{n, U, \Sigma}^{(m)}$ and allow the reader to specialize to the case $m=0$. We will first describe more precisely what is the data encoded in $\Sigma$. 
If $X$ is an $F$-vector space we let $\operatorname{Herm}_{X}$ denote the space of symmetric $\mathbb{Q}$-bilinear forms

$$
(, \quad): X \times X \longrightarrow \mathbb{Q}
$$

such that

$$
(a x, y)=\left(x,{ }^{c} a y\right)
$$

for $x, y \in X$ and $a \in F$. If $W \subset V_{n}$ is an isotropic $F$-subspace we set

$$
\mathfrak{C}^{(m)}(W)=\left(\operatorname{Herm}_{V_{n} / W^{\perp}} \oplus \operatorname{Hom}_{F}\left(F^{m}, W\right)\right) \otimes_{\mathbb{Q}} \mathbb{R}
$$

If $V_{n,(i)}$ denotes the subspace of $V_{n}$ with the last $2 n-i$ entries zero then $\mathfrak{C}^{(m)}\left(V_{n,(i)}\right)$ can be identified with $Z\left(N_{n,(i)}^{(m)}\right)(\mathbb{R})$, where $Z\left(N_{n,(i)}^{(m)}\right)$ is the subgroup of $G_{n}^{(m)}$ consisting of matrices of the following shape:

$$
\left(\begin{array}{c|c|c}
1_{i} & 0 & * \\
\hline 0 & 1_{2(n-i)} & 0 \\
\hline 0 & 0 & 1_{i}
\end{array}\right)\left(\begin{array}{c}
* \\
\hline 0 \\
\hline 0
\end{array}\right) .
$$

If $W^{\prime} \subset W$ then $V_{n} / W^{\perp} \rightarrow V_{n} /\left(W^{\prime}\right)^{\perp}$ and so

$$
\mathfrak{C}^{(m)}\left(W^{\prime}\right) \hookrightarrow \mathfrak{C}^{(m)}(W) .
$$

If $\gamma \in G_{n}^{(m)}(\mathbb{Q})$ then there is a natural map

$$
\gamma: \mathfrak{C}^{(m)}(W) \stackrel{\sim}{\longrightarrow} \mathfrak{C}^{(m)}(\gamma W) .
$$

We define $\mathfrak{C}^{(m),>0}(W)\left(\right.$ resp. $\left.\mathfrak{C}^{(m), \geq 0}(W)\right)$ to be the set of pairs $((, \quad), f)$ such that $(, \quad)$ is positive definite (positive semi-definite) on $\left(V_{n} / W^{\perp}\right) \otimes_{\mathbb{Q}} \mathbb{R}$, and set

$$
\mathfrak{C}^{(m),>0}(W)=\bigcup_{W^{\prime} \subset W} \mathfrak{C}^{(m),>0}\left(W^{\prime}\right) \subset \mathfrak{C}^{(m), \geq 0}(W) .
$$

Alternatively $\mathfrak{C}^{(m), \succ 0}(W)$ can be described as the set of $((, \quad), f) \in \mathfrak{C}^{(m), \geq 0}(W)$ such that the kernel (i.e. radical) of $\left(\right.$, ) is defined over $\mathbb{Q}$. We then define a topological space $\mathfrak{C}^{(m)}$ by

$$
\mathfrak{C}^{(m)}=\left(\bigcup_{W} \mathfrak{C}^{(m), \succ 0}(W)\right) / \sim,
$$

where $\sim$ is the equivalence relation generated by the identification of $\mathfrak{C}^{(m), \succ 0}\left(W^{\prime}\right)$ with its image in $\mathfrak{C}^{(m), \succ 0}(W)$ whenever $W^{\prime} \subset W$. Thus as a set

$$
\mathfrak{C}^{(m)}=\coprod_{W} \mathfrak{C}^{(m),>0}(W) .
$$

The space $\mathfrak{C}^{(m)}$ has a continuous action of $G_{n}^{(m)}(\mathbb{Q})$ and of $\mathbb{R}_{>0}^{\times}$, the latter acting by scalar multiples on each $\mathfrak{C}^{(m), \succ 0}(W)$. The natural projections $\mathfrak{C}^{(m), \succ 0}(W) \rightarrow \mathfrak{C}^{(0), \succ 0}(W)$ give rise to a projection $\mathfrak{C}^{(m)} \rightarrow \mathfrak{C}=\mathfrak{C}^{(0)}$. (See Sect. 1.4.)

By a $U$-admissible cone decomposition $\Sigma$ of $G_{n}^{(m)}\left(\mathbb{A}^{\infty}\right) \times \pi_{0}\left(G_{n}(\mathbb{R})\right) \times \mathfrak{C}^{(m)}$ we shall mean a set of closed subsets $\sigma \subset G_{n}^{(m)}\left(\mathbb{A}^{\infty}\right) \times \pi_{0}\left(G_{n}(\mathbb{R})\right) \times \mathfrak{C}^{(m)}$ such that 
(1) each $\sigma$ is contained in $\{(g, \delta)\} \times \mathfrak{C}^{(m), \succ 0}(W)$ for some isotropic subspace $W \subset V_{n}$ and some $(g, \delta) \in G_{n}^{(m)}\left(\mathbb{A}^{\infty}\right) \times \pi_{0}\left(G_{n}(\mathbb{R})\right)$ and is the set of $\mathbb{R}_{\geq 0}$-linear combinations of a finite set of elements of $\operatorname{Herm}_{V / W^{\perp}} \times W^{m}$;

(2) no $\sigma \in \Sigma$ contains a complete line through the origin in any $(g, \delta) \times \mathfrak{C}^{(m)}(W)$;

(3) if $\sigma \in \Sigma$ then any face of $\sigma$ also lies in $\Sigma$;

(4) if $\sigma, \sigma^{\prime} \in \Sigma$ then either $\sigma \cap \sigma^{\prime}=\emptyset$ or $\sigma \cap \sigma^{\prime}$ is a face of $\sigma$ and $\sigma^{\prime}$;

(5) $\quad G_{n}^{(m)}\left(\mathbb{A}^{\infty}\right) \times \pi_{0}\left(G_{n}(\mathbb{R})\right) \times \mathfrak{C}^{(m)}=\bigcup_{\sigma \in \Sigma} \sigma$;

(6) $\quad \Sigma$ is invariant by the diagonal action of $G_{n}^{(m)}(\mathbb{Q})$ on $G_{n}^{(m)}\left(\mathbb{A}^{\infty}\right) \times \pi_{0}\left(G_{n}(\mathbb{R})\right) \times \mathfrak{C}^{(m)}$;

(7) $\quad \Sigma$ is invariant by the right action of $U$ on $G_{n}^{(m)}\left(\mathbb{A}^{\infty}\right) \times \pi_{0}\left(G_{n}(\mathbb{R})\right) \times \mathfrak{C}^{(m)}$ (acting only on the first factor);

(8) $G_{n}^{(m)}(\mathbb{Q}) \backslash \Sigma / U$ is a finite set;

(9) if $\sigma \in \Sigma$ lies in $G_{n}^{(m)}\left(\mathbb{A}^{\infty}\right) \times \pi_{0}\left(G_{n}(\mathbb{R})\right) \times \mathfrak{C}^{(m), \succ 0}\left(V_{n,(i)}\right)$ and if $h \in P_{n,(i)}^{(m)}(\mathbb{A})$, then $h \sigma \in \Sigma$;

if $\sigma \in \Sigma$ lies in $G_{n}^{(m)}\left(\mathbb{A}^{\infty}\right) \times \pi_{0}\left(G_{n}(\mathbb{R})\right) \times \mathfrak{C}^{(m), \succ 0}\left(V_{n,(i)}\right)$, if $\gamma \in G_{n}^{(m)}(\mathbb{Q})$, if $u \in U$ and if $h \in P_{n,(i)}^{(m)}(\mathbb{A})$ satisfy

$$
\sigma \cap \gamma h \sigma u \cap\left(G_{n}^{(m)}\left(\mathbb{A}^{\infty}\right) \times \pi_{0}\left(G_{n}(\mathbb{R})\right) \times \mathfrak{C}^{(m),>0}\left(V_{n,(i)}\right)\right) \neq \emptyset
$$

then $\gamma \in P_{n,(i)}^{(m)}(\mathbb{Q})$.

(Here we let $G_{n}^{(m)}(\mathbb{A})$ act on $G_{n}^{(m)}\left(\mathbb{A}^{\infty}\right) \times \pi_{0}\left(G_{n}(\mathbb{R})\right) \times \mathfrak{C}^{(m)}$ via multiplication on the first two factors. The restriction of this action to $G_{n}^{(m)}(\mathbb{Q})$ does not coincide with the standard action of $G_{n}^{(m)}(\mathbb{Q})$, which we are using.) We call $\Sigma$ an admissible cone decomposition if it is $U$-admissible for some $U$. The group $\mathbb{G}_{n}^{(m)}\left(\mathbb{A}^{\infty}\right)$ acts on admissible cone decompositions. We call $\Sigma^{\prime}$ a refinement of $\Sigma$ if every element of $\Sigma$ is a union of elements of $\Sigma^{\prime}$. We write $\left(U^{\prime}, \Sigma^{\prime}\right) \geq(U, \Sigma)$ if $U^{\prime} \subset U$ and $\Sigma^{\prime}$ is a refinement of $\Sigma$. We say that $\Sigma$ is compatible with $\Delta$, an admissible cone decomposition of $G_{n}\left(\mathbb{A}^{\infty}\right) \times \pi_{0}\left(G_{n}(\mathbb{R})\right) \times \mathfrak{C}$, if the projection of each element of $\Sigma$ is contained in an element of $\Delta$. We write $(U, \Sigma) \geq(V, \Delta)$ if $U$ maps to $V$ and $\Sigma$ is compatible with $\Delta$. For each $m$ there is a cofinal collection $\mathcal{J}_{n}^{(m) \text {,tor }}$ of pairs $(U, \Sigma)$ of a neat open compact subgroup $U \subset G_{n}^{(m)}\left(\mathbb{A}^{\infty}\right)$ and a $U$-admissible cone decomposition $\Sigma$ of $G_{n}^{(m)}\left(\mathbb{A}^{\infty}\right) \times \pi_{0}\left(G_{n}(\mathbb{R})\right) \times \mathfrak{C}^{(m)}$ with various natural properties, some of which are listed in Sect. 5.2. In particular it is preserved by the action of $G_{n}^{(m)}\left(\mathbb{A}^{\infty}\right)$. (See Sect. 5.2.)

We define $\left(G_{n}^{(m)}\left(\mathbb{A}^{\infty}\right) \times \pi_{0}\left(G_{n}(\mathbb{R})\right) \times \mathfrak{C}^{(m)}\right)^{\text {ord }}$ to be the subset of $(g, \delta, x)$ such that for some $W$ we have $x \in \mathfrak{C}^{(m), \succ 0}(W)$ and $W \otimes \mathbb{Q}_{p}=g_{p}\left(V_{n,(n)} \otimes \mathbb{Q}_{p}\right)$. It is invariant under the left action of $G_{n}^{(m)}(\mathbb{Q})$, under the right action of $G_{n}^{(m)}\left(\mathbb{A}^{\infty}\right)^{\text {ord }}$ and under the action of $\mathbb{R}_{>0}^{\times}$. (See Sect. 1.4.) For $U^{p}(N) \subset G_{n}^{(m)}\left(\mathbb{A}^{\infty}\right)^{\text {ord, } \times}$, by a $U^{p}(N)$-admissible cone decomposition of $\left(G_{n}^{(m)}\left(\mathbb{A}^{\infty}\right) \times \pi_{0}\left(G_{n}(\mathbb{R})\right) \times \mathfrak{C}^{(m)}\right)^{\text {ord }}$ we mean a collection $\Sigma$ of closed subsets $\sigma \subset\left(G_{n}^{(m)}\left(\mathbb{A}^{\infty}\right) \times \pi_{0}\left(G_{n}(\mathbb{R})\right) \times \mathfrak{C}^{(m)}\right)^{\text {ord }}$ satisfying analogous properties to those listed in the previous paragraph. Notions of 'refinement' and 'compatibility' are defined just as in the previous paragraph. In the same manner we also define a partial ordering on pairs $\left(U^{p}(N), \Sigma\right)$. If $\Sigma$ is a $U^{p}\left(N_{1}, N_{2}\right)$-admissible cone decomposition of $G_{n}^{(m)}\left(\mathbb{A}^{\infty}\right) \times \pi_{0}\left(G_{n}(\mathbb{R})\right) \times \mathfrak{C}^{(m)}$, then the collection $\Sigma^{\text {ord }}$ of elements of $\Sigma$ which are contained in $\left(G_{n}^{(m)}\left(\mathbb{A}^{\infty}\right) \times \pi_{0}\left(G_{n}(\mathbb{R})\right) \times \mathfrak{C}^{(m)}\right)^{\text {ord }}$ is a $U^{p}\left(N_{1}\right)$-admissible cone decomposition of $\left(G_{n}^{(m)}\left(\mathbb{A}^{\infty}\right) \times \pi_{0}\left(G_{n}(\mathbb{R})\right) \times \mathfrak{C}^{(m)}\right)^{\text {ord }}$. For each $m$ we define $\mathcal{J}_{n}^{(m) \text {,tor,ord }}$ to be the set of $\left(U^{p}\left(N_{1}\right), \Sigma^{\text {ord }}\right)$, where $\left(U^{p}\left(N_{1}, N_{2}\right), \Sigma\right) \in \mathcal{J}_{n}^{(m) \text {,tor }}$ for some $N_{2}$ and $\Sigma$. It has various nat- 
ural properties listed in Sect. 5.2. In particular it is preserved by the action of $G_{n}^{(m)}\left(\mathbb{A}^{\infty}\right)^{\text {ord }}$. (See Sect. 5.2.)

As was already mentioned, to each $(U, \Sigma) \in \mathcal{J}_{n}^{(m) \text {.tor }}$ we can associate a smooth projective variety $A_{n, U, \Sigma}^{(m)} / X_{n, U}^{\min }$ together with a simple normal crossings divisor $\partial A_{n, U, \Sigma}^{(m)} \subset A_{n, U, \Sigma}^{(m)}$ such that

$$
A_{n, U, \Sigma}^{(m)}-\partial A_{n, U, \Sigma}^{(m)}=A_{n, U}^{(m)}
$$

and $\partial A_{n, U, \Sigma}^{(m)}$ is the reduced pre-image of $\partial X_{n, U}^{\min }$. We write $\partial_{i} A_{n, U, \Sigma}^{(m)}$ for the reduced preimage of $\partial_{i} X_{n, U}^{\min }$;

$$
\partial_{i}^{0} A_{n, U, \Sigma}^{(m)}=\partial_{i} A_{n, U, \Sigma}^{(m)}-\partial_{i+1} A_{n, U, \Sigma}^{(m)} .
$$

The irreducible components of $\partial A_{n, U, \Sigma}^{(m)}$ are in bijection with the one-dimensional cones in $G_{n}^{(m)}(\mathbb{Q}) \backslash \Sigma / U$. A collection of such irreducible components have a non-empty intersection if and only if there is a cone $\sigma \in \Sigma$ such that the given irreducible components correspond to the one-dimensional faces of $\sigma$. In that case we write $\partial_{[\sigma]} A_{n, U, \Sigma}^{(m)}$ for this intersection. We introduce the simplicial complex $\mathcal{S}\left(\partial A_{n, U, \Sigma}^{(m)}\right)$ whose vertices are the irreducible components of $\partial A_{n, U, \Sigma}^{(m)}$ and whose simplices correspond to collections of such irreducible components with nonzero intersection. Then the topological realization $\left|\mathcal{S}\left(\partial A_{n, U, \Sigma}^{(m)}\right)\right|$ of $\mathcal{S}\left(\partial A_{n, U, \Sigma}^{(m)}\right)$ can be identified with

$$
G_{n}^{(m)}(\mathbb{Q}) \backslash\left(G_{n}^{(m)}\left(\mathbb{A}^{\infty}\right) / U \times \pi_{0}\left(G_{n}(\mathbb{R})\right) \times\left(\mathfrak{C}^{(m)}-\{0\}\right) / \mathbb{R}_{>0}^{\times}\right) .
$$

Moreover

$$
\left|\mathcal{S}\left(\partial A_{n, U, \Sigma}^{(m)}\right)\right|-\left|\mathcal{S}\left(\partial A_{n, U, \Sigma}^{(m)}-\partial_{n} A_{n, U, \Sigma}^{(m)}\right)\right|
$$

is identified with

$$
G_{n}^{(m)}(\mathbb{Q}) \backslash\left(G_{n}^{(m)}\left(\mathbb{A}^{\infty}\right) / U \times \pi_{0}\left(G_{n}(\mathbb{R})\right) \times \mathfrak{C}_{=n}^{(m)} / \mathbb{R}_{>0}^{\times}\right),
$$

where

$$
\mathfrak{C}_{=n}^{(m)}=\bigcup_{\operatorname{dim} W=n} \mathfrak{C}^{(m),>0}(W) \subset \mathfrak{C}^{(m)}
$$

is a dense open subset. Moreover $G_{n}^{(m)}(\mathbb{Q}) \backslash\left(G_{n}^{(m)}\left(\mathbb{A}^{\infty}\right) / U \times \pi_{0}\left(G_{n}(\mathbb{R})\right) \times \mathfrak{C}_{=n}^{(m)} / R_{>0}^{\times}\right)$can be identified with

$$
\begin{aligned}
& \coprod_{h \in P_{n,(n)}^{(m),+}\left(\mathbb{A}^{\infty}\right) \backslash G_{n}^{(m)}\left(\mathbb{A}^{\infty}\right) / U} L_{n,(n)}^{(m)}(\mathbb{Q}) \backslash L_{n,(n)}^{(m)}(\mathbb{A}) /\left(h U h^{-1} \cap P_{n,(n)}^{(m),+}\left(\mathbb{A}^{\infty}\right)\right) \\
& L_{n,(n), \text { herm }}(\mathbb{R})^{+}\left(L_{n,(n), \operatorname{lin}}^{(m)}(\mathbb{R}) \cap U_{n, \infty}^{0}\right) A_{n,(n)}(\mathbb{R})^{0},
\end{aligned}
$$

where $U_{n, \infty}^{0}$ is a certain maximal connected compact subgroup of $G_{n}(\mathbb{R})$ defined in Sect. 1.1, and $A_{n,(n)}$ denotes the maximal split torus in the centre of $L_{n,(n)}$. (See Sect. 5.3.) 
Similarly to each $\left(U^{p}\left(N_{1}, N_{2}\right), \Sigma\right) \in \mathcal{J}_{n}^{(m) \text {.tor }}$ we can associate a smooth quasi-projective variety $\mathcal{A}_{n, U^{p}\left(N_{1}, N_{2}\right), \Sigma}^{(m), \text { ord }} / \mathcal{X}_{n, U^{p}\left(N_{1}, N_{2}\right)}^{\text {ordmin }}$ together with a simple normal crossings divisor

$$
\partial \mathcal{A}_{n, U^{p}\left(N_{1}, N_{2}\right), \Sigma}^{(m), \text { ord }} \subset \mathcal{A}_{n, U^{p}\left(N_{1}, N_{2}\right), \Sigma}^{(m), \text { ord }}
$$

such that

$$
\mathcal{A}_{n, U^{p}\left(N_{1}, N_{2}\right), \Sigma}^{(m) \text { ord }}-\partial \mathcal{A}_{n, U^{p}\left(N_{1}, N_{2}\right), \Sigma}^{(m), \text { rd }}=\mathcal{A}_{n, U^{p}\left(N_{1}, N_{2}\right)}^{(m) \text { ord }}
$$

and $\partial \mathcal{A}_{n, U^{p}\left(N_{1}, N_{2}\right), \Sigma}^{(m), \text { ord }}$ is the reduced pre-image of $\partial \mathcal{X}_{n, U^{p}\left(N_{1}, N_{2}\right)}^{\text {ord,min }}$. We will also write $\partial_{i} \mathcal{A}_{n, U^{p}\left(N_{1}, N_{2}\right), \Sigma}^{(m) \text {,ord }}$ for the reduced pre-image of $\partial_{i} \mathcal{X}_{n, U^{p}\left(N_{1}, N_{2}\right)}^{\text {ord,min }}$ and set

$$
\partial_{i}^{0} \mathcal{A}_{n, U^{p}\left(N_{1}, N_{2}\right), \Sigma}^{(m) \text { ord }}=\partial_{i} \mathcal{A}_{n, U^{p}\left(N_{1}, N_{2}\right), \Sigma}^{(m), \text { ord }}-\partial_{i+1} \mathcal{A}_{n, U^{p}\left(N_{1}, N_{2}\right), \Sigma}^{(m) \text { ord }}
$$

The irreducible components of $\partial \mathcal{A}_{n, U^{p}\left(N_{1}, N_{2}\right), \Sigma}^{(m), \text { ord }}$ are in bijection with the one-dimensional cones in $G_{n}^{(m)}(\mathbb{Q}) \backslash \Sigma / U^{p}\left(N_{1}, N_{2}\right)$. A collection of such irreducible components have a non-empty intersection if and only if there is a cone $\sigma \in \Sigma$ such that the given irreducible components correspond to the one-dimensional faces of $\sigma$. In that case we write $\partial_{[\sigma]} \mathcal{A}_{n, U^{p}\left(N_{1}, N_{2}\right), \Sigma}^{(m) \text {,ord }}$ for this intersection. The $\mathbb{F}_{p}$-fibre of $\partial_{[\sigma]} \mathcal{A}_{n, U^{p}\left(N_{1}, N_{2}\right), \Sigma}^{(m), \text { ord }}$ is non-empty if and only if $G_{n}^{(m)}(\mathbb{Q}) \sigma U$ contains an element of $\Sigma^{\text {ord }}$. (See Sect. 5.3.)

We let $\bar{A}_{n, U^{p}\left(N_{1}\right), \Sigma^{\text {ord }}}^{(m) \text {,ord }}$ denote the $\mathbb{F}_{p}$-fibre of $\mathcal{A}_{n, U^{p}\left(N_{1}, N_{2}\right), \Sigma}^{(m), \text { It }}$ is independent of $N_{2} \geq N_{1}$ and $\Sigma$ inducing $\Sigma^{\text {ord }}$. Similarly we define $\partial \bar{A}_{n, U^{p}\left(N_{1}\right), \Sigma^{\text {ord }}}^{(m), \text { ord }}$ and $\partial_{i} \bar{A}_{n, U^{p}\left(N_{1}\right), \Sigma^{\text {ord }}}^{(m) \text { ord }}$ and, if $\sigma \in$

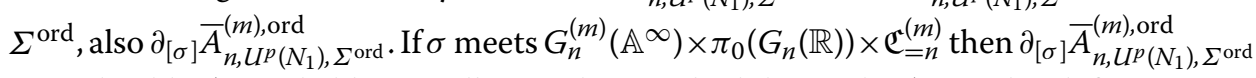
is irreducible. (It probably is in all cases, but we don't know that.) We also define

$$
\partial^{(s)} \bar{A}_{n, U^{p}\left(N_{1}\right), \Sigma^{\text {ord }}}^{(m), \text { ord }}=\coprod_{\operatorname{dim} \sigma=s} \partial_{[\sigma]} \bar{A}_{n, U^{p}\left(N_{1}\right), \Sigma^{\text {ord }}}^{(m), \text { ord }}
$$

where the disjoint union is over $s$-dimensional cones $\sigma \in \Sigma^{\text {ord }}$ taken up to equivalence, where two cones are considered equivalent if they have the same image in $G_{n}^{(m)}(\mathbb{Q}) \backslash \Sigma / U^{p}\left(N_{1}, N_{2}\right)$. We have an identification of the topological spaces associated to the simplicial complex recording the intersections of the irreducible components of the boundary:

$$
\left|\mathcal{S}\left(\partial \bar{A}_{n, U^{p}\left(N_{1}\right), \Sigma^{\text {ord }}}^{(m), \text { ord }}\right)\right|-\left|\mathcal{S}\left(\partial \bar{A}_{n, U^{p}\left(N_{1}\right), \Sigma^{\text {ord }}}^{(m), \text { ord }}-\partial_{n} \bar{A}_{n, U^{p}\left(N_{1}\right), \Sigma^{\text {ord }}}^{(m) \text {,ord }}\right)\right|
$$

with

$$
\begin{aligned}
& \coprod_{h \in P_{n,(n)}^{(m),+}\left(\mathbb{A}^{\infty, p} \times \mathbb{Z}_{p}\right) \backslash G_{n}^{(m)}\left(\mathbb{A}^{\infty}\right)^{\operatorname{ord}, \times} / U^{p}(N)} L_{n,(n)}^{(m)}(\mathbb{Q}) \backslash L_{n,(n)}^{(m)}(\mathbb{A}) / \\
& \quad\left(h U^{p}\left(N_{1}\right) h^{-1} \cap P_{n,(n)}^{(m),+}\left(\mathbb{A}^{\infty, p} \times \mathbb{Z}_{p}\right)\right) L_{n,(n), \text { herm }}(\mathbb{R})^{+} \\
& \quad\left(L_{n,(n), \operatorname{lin}}^{(m)}(\mathbb{R}) \cap U_{n, \infty}^{0}\right) A_{n,(n)}(\mathbb{R})^{0} .
\end{aligned}
$$

(See Sect. 5.3.) We write

$$
\mathfrak{T}_{(n), V}^{(m)}=L_{n,(n)}^{(m)}(\mathbb{Q}) \backslash L_{n,(n)}^{(m)}(\mathbb{A}) / V L_{n,(n), \operatorname{herm}}(\mathbb{R})^{+}\left(L_{n,(n), \operatorname{lin}}^{(m)}(\mathbb{R}) \cap U_{n, \infty}^{0}\right) A_{n,(n)}(\mathbb{R})^{0},
$$


an $\left(S^{1}\right)^{n m[F: \mathbb{Q}]}$-bundle over a locally symmetric space associated to $L_{n,(n) \text {,lin. With this }}$ notation

$$
\begin{aligned}
& \left|\mathcal{S}\left(\partial \bar{A}_{n, U^{p}\left(N_{1}\right), \Sigma^{\text {ord }}}^{(m), \text { ord }}\right)\right|-\mid \mathcal{S}\left(\partial \bar{A}_{n, U^{p}\left(N_{1}\right), \Sigma^{\text {ord }}}^{\left.(m), \partial_{n} \bar{A}_{n, U^{p}\left(N_{1}\right), \Sigma^{\text {ord }}}^{(m), \text { ord }}\right) \mid}\right. \\
& \quad \cong \coprod_{h \in P_{n,(n)}^{(m),+}\left(\mathbb{A}^{\infty, p} \times \mathbb{Z}_{p}\right) \backslash G_{n}^{(m)}\left(\mathbb{A}^{\infty}\right)^{\text {ord }, \times} / U^{p}(N)}^{\mathfrak{T}_{(n),(h)}^{(m)}\left(h U^{p}\left(N_{1}\right) h^{-1} \cap P_{n,(n)}^{(m),+}\left(\mathbb{A}^{\infty, p} \times \mathbb{Z}_{p}\right)\right)}
\end{aligned}
$$

(See Sect. 1.4.)

We also define $\mathfrak{A}_{n, U^{p}\left(N_{1}\right), \Sigma^{\text {ord }}}^{(m) \text {,ord }}$ to be the formal completion of $\mathcal{A}_{n, U^{p}\left(N_{1}, N_{2}\right), \Sigma}^{(m) \text { along }}$ its $\mathbb{F}_{p}$-fibre. It is independent of $N_{2} \geq N_{1}$ and $\Sigma$ inducing $\Sigma^{\text {ord }}$. We similarly define $\partial \mathfrak{A}_{n, U^{p}\left(N_{1}\right), \Sigma^{\text {ord }}}^{(m), \text { ord }}$ and $\partial_{i} \mathfrak{A}_{n, U^{p}\left(N_{1}\right), \Sigma^{\text {ord }}}^{(m) \text {,ord }}$ and $\partial_{[\sigma]} \mathfrak{A}_{n, U^{p}\left(N_{1}\right), \Sigma^{\text {ord }}}^{(m) \text {,ord }}$ and $\partial^{(s)} \mathfrak{A}_{n, U^{p}\left(N_{1}\right), \Sigma^{\text {ord }}}^{(m) \text {. }}$. (See Sect. 5.3.) We will write $\partial_{[\sigma]} \mathcal{A}_{n, U^{p}\left(N_{1}\right), \Sigma^{\text {ord }}}^{(m) \text { ord } \dagger}$ and $\partial^{(s)} \mathcal{A}_{n, U^{p}\left(N_{1}\right), \Sigma^{\text {ord }}}^{(m), \text { ord } \dagger}$ for the tube over the corresponding schemes in characteristic $p$ inside the dagger spaces associated to $\partial_{[\sigma]} A_{n, U^{p}\left(N_{1}, N_{2}\right), \Sigma}^{(m)}$ and $\partial^{(s)} A_{n, U^{p}\left(N_{1}, N_{2}\right), \Sigma}^{(m)}$. Again this is independent of $N_{2}$ and $\Sigma$. (See Sect. 6.3.)

We write $A_{n, U, \Sigma, i}^{(m), \wedge}$ for the formal completion of $A_{n, U, \Sigma}^{(m)}$ along $\partial_{i}^{0} A_{n, U, \Sigma}^{(m)}$. There is an explicit description of $A_{n, U, \Sigma, i}^{(m), \wedge}$, but it will require considerable extra notation, which we now explain. (See Sect. 5.3.)

The group $P_{n,(i)}^{(m),+}$ is the subgroup of $G_{n}^{(m)}$ consisting of elements of the form

$$
\left(\begin{array}{c|c|c}
* & * & * \\
\hline 0_{2(n-i) \times i} & * & * \\
\hline 0_{i \times i} & 0_{i \times 2(n-i)} & *
\end{array}\right)\left(\begin{array}{c}
* \\
\hline * \\
\hline *
\end{array}\right) .
$$

It is the semi-direct product of the subgroup $N_{n,(i)}^{(m)}$ consisting of elements of the form

$$
\left(\begin{array}{c|c|c}
1_{i} & * & * \\
\hline 0 & 1_{2(n-i)} & * \\
\hline 0 & 0 & 1_{i}
\end{array}\right)\left(\begin{array}{c}
* \\
\hline * \\
\hline 0
\end{array}\right)
$$

by $L_{n,(i)}^{(m)}=L_{n,(i) \text {,herm }} \times L_{n,(i), \text { lin }}^{(m)}$, where $L_{n,(i), \text { lin }}^{(m)}$ is the subgroup consisting of elements of the form

$$
\left(\begin{array}{c|c|c}
* & 0 & 0 \\
\hline 0 & 1_{2(n-i)} & 0 \\
\hline 0 & 0 & *
\end{array}\right)\left(\begin{array}{c}
\frac{0}{0} \\
\hline *
\end{array}\right)
$$

Thus

$$
L_{n,(i), \operatorname{lin}}^{(m)} \cong R S_{\mathbb{Z}}^{\mathcal{O}_{F}}\left(G L_{i} \ltimes \operatorname{Hom}\left(\mathbb{G}_{a}^{m}, \mathbb{G}_{a}^{i}\right)\right) \hookrightarrow \mathrm{RS}_{\mathbb{Z}}^{\mathcal{O}_{F}} G L_{i+m}
$$

We also write $P_{n,(i)}^{(m)}$ for the subgroup of $P_{n,(i)}^{(m),+}$ consisting of matrices of the form

$$
\left(\begin{array}{c|c|c}
1_{i} & * & * \\
\hline 0 & * & * \\
\hline 0 & 0 & 1_{i}
\end{array}\right)\left(\begin{array}{c}
* \\
\hline * \\
\hline 0
\end{array}\right)
$$


Then

$$
P_{n,(i)}^{(m)} / Z\left(N_{n,(i)}^{(m)}\right) \cong G_{n-i}^{(i+m)}
$$

and

$$
P_{n,(i)}^{(m),+}=L_{n,(i), \text { lin }}^{(m)} \ltimes P_{n,(i)}^{(m)}
$$

(See Sect. 1.2.)

For $U \subset L_{n,(i)}^{(m)}\left(\mathbb{A}^{\infty}\right)$ a neat open compact subgroup we set

$$
X_{n,(i), U}^{(m),+}=\coprod_{h \in L_{h,(i), \operatorname{lin}}^{(m)}\left(\mathbb{A}^{\infty}\right) / U} X_{n-i, h U h^{-1} \cap L_{n,(i), \mathrm{herm}}\left(\mathbb{A}^{\infty}\right)} .
$$

It has a left action of $L_{n,(i), \text { lin }}^{(m)}(\mathbb{Q})=G L_{i}(F) \ltimes \operatorname{Hom}_{F}\left(F^{m}, F^{i}\right)$ and the inverse system $\left\{X_{n,(i), U}^{(m),+}\right\}$ has a commuting right action of $L_{n,(i)}^{(m)}\left(\mathbb{A}^{\infty}\right)$. These actions are defined exactly similarly to those on $X_{n,(i), U}^{+}$. We have

$$
X_{n,(i), U}^{\natural}=L_{n,(i), \operatorname{lin}}^{(m)}(\mathbb{Q}) \backslash X_{n,(i), U}^{(m),} .
$$

(See Sect. 4.1.)

If $U$ is a neat open compact subgroup of $\left(P_{n,(i)}^{(m),+} / Z\left(N_{n,(i)}^{(m)}\right)\right)\left(\mathbb{A}^{\infty}\right)$ we set

$$
A_{n,(i), U}^{(m),+}=\coprod_{h \in L_{h, i) \operatorname{lin}}^{(m)}\left(\mathbb{A}^{\infty}\right) / U} A_{n-i, h U h^{-1} \cap G_{n-i}^{(i+m)}\left(\mathbb{A}^{\infty}\right)^{.}}^{(i+m)}
$$

The scheme $A_{n,(i), U}^{(m),+}$ has a left action of $L_{n,(i), \text { lin }}^{(m)}(\mathbb{Q})$ such that $\delta \in L_{n,(i), \text { lin }}^{(m)}(\mathbb{Q})$ acts via the coproduct of the maps

$$
\delta: A_{n-i, h U h^{-1} \cap G_{n-i}^{(i+m)}\left(\mathbb{A}^{\infty}\right)}^{(i+m)} \longrightarrow A_{n-i, \delta h U h^{-1} \delta^{-1} \cap G_{n-i}^{(i+m)}\left(\mathbb{A}^{\infty}\right)}^{(i+m)}=A_{n-i, \delta\left(h U h^{-1} \cap G_{n-i}^{(i+m)}\left(\mathbb{A}^{\infty}\right)\right)}^{(i+m)}
$$

The system of schemes $\left\{A_{n,(i), U}^{(m),+}\right\}$ has a commuting right action of the quotient group

$$
\left(P_{n,(i)}^{(m),+} / Z\left(N_{n,(i)}^{(m)}\right)\right)\left(\mathbb{A}^{\infty}\right)
$$

such that if $g \in\left(P_{n,(i)}^{(m),+} / Z\left(N_{n,(i)}^{(m)}\right)\right)\left(\mathbb{A}^{\infty}\right)$ maps to $g_{\text {lin }} \in L_{n,(i), \text { lin }}^{(m)}\left(\mathbb{A}^{\infty}\right)$ then $g$ acts by the coproduct of the maps

$$
h g g_{\operatorname{lin}}^{-1} h^{-1}: A_{n-i, h U h^{-1} \cap G_{n-i}^{(i+m)}\left(\mathbb{A}^{\infty}\right)}^{(i+m)} \longrightarrow A_{n-i, h g_{\operatorname{lin}} V g_{\operatorname{lin}}^{-1} h^{-1} \cap G_{n-i}^{(i+m)}\left(\mathbb{A}^{\infty}\right)}^{(i+m)}
$$

(See Sect. 4.2.)

If $U$ is a neat open compact subgroup of $P_{n,(i)}^{(m),+}\left(\mathbb{A}^{\infty}\right)$ we define $S_{n,(i), U}^{(m),+}$ to be the torus over $X_{n,(i), U}^{(m),+}$ with cocharacter group constant over

$$
X_{n-i, h \bar{U} h^{-1} \cap L_{n,(i), \text { herm }}\left(\mathbb{A}^{\infty}\right)} \subset X_{n,(i), U}^{+}
$$


(where $\bar{U}$ denotes the image of $U$ in $L_{n,(i)}^{(m)}\left(\mathbb{A}^{\infty}\right)$ ) and identified with

$$
h U h^{-1} \cap Z\left(N_{n,(i)}^{(m)}\right)(\mathbb{Q})
$$

In fact, we define

$$
Y_{n,(i), U}^{+}=\coprod_{h \in L_{n,(i), \operatorname{lin}}\left(\mathbb{A}^{\infty}\right) / U} \operatorname{Spec} \mathbb{Q}
$$

so that $S_{n,(i), U}^{(m),+}$ is already defined over $Y_{n,(i), U}^{+}$. The torus $S_{n,(i), U}^{(m),+}$ has a left action of $L_{n,(i), \text { lin }}^{(m)}(\mathbb{Q})$ such that $\delta \in L_{n,(i), \text { lin }}^{(m)}$ sends

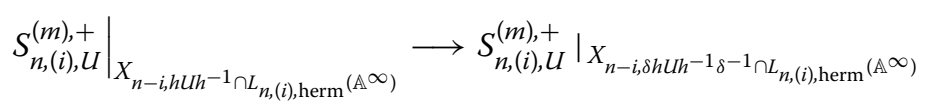

via the morphism induced by the map on cocharacter groups

$$
h U h^{-1} \cap Z\left(N_{n,(i)}^{(m)}\right)(\mathbb{Q}) \longrightarrow \delta h U h^{-1} \delta^{-1} \cap Z\left(N_{n,(i)}^{(m)}\right)(\mathbb{Q})
$$

given by conjugation by $\delta$. The system of tori $\left\{S_{n,(i), U}^{(m),+}\right\}$ also has a right action of $P_{n,(i)}^{(m),+}\left(\mathbb{A}^{\infty}\right)$ such that $g \in P_{n,(i)}^{(m),+}\left(\mathbb{A}^{\infty}\right)$ mapping to $g_{\text {lin }} \in L_{n,(i), \text { lin }}^{(m)}\left(\mathbb{A}^{\infty}\right)$ sends

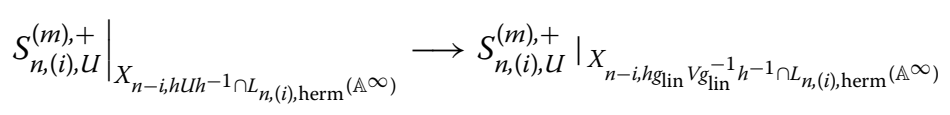

via the morphism induced by the map on cocharacter groups

$$
h U h^{-1} \cap Z\left(N_{n,(i)}^{(m)}\right)(\mathbb{Q}) \longrightarrow h g_{\operatorname{lin}} V g_{\operatorname{lin}}^{-1} h^{-1} \cap Z\left(N_{n,(i)}^{(m)}\right)(\mathbb{Q})
$$

by conjugation by $h g_{\text {lin }} g^{-1} h^{-1}$. (See Sect. 4.3.)

If $(U, \Sigma) \in \mathcal{J}_{n}^{(m), \text { tor }}$ then we define a partial fan (in the sense of Sect. 2.5) $\Sigma(h)_{0}$ in $X_{*}\left(S_{n,(i), h U h^{-1} \cap P_{n,(i)}^{(m),+}\left(\mathbb{A}^{\infty}\right)}^{(m)+}\right)$ as follows. Over

$$
X_{n-i, h^{\prime}}\left(h U h^{-1} \cap P_{n,(i)}^{(m),+}\left(\mathbb{A}^{\infty}\right)\right) h^{\prime,-1} \cap L_{n,(i), \operatorname{herm}}\left(\mathbb{A}^{\infty}\right)
$$

we take the cones

$$
\sigma \subset\left(h^{\prime}\left(h U h^{-1} \cap P_{n,(i)}^{(m),+}\left(\mathbb{A}^{\infty}\right)\right) h^{\prime,-1} \cap Z\left(N_{n,(i)}^{(m)}\right)(\mathbb{Q})\right) \otimes_{\mathbb{Z}} \mathbb{R}=\mathfrak{C}^{(m)}\left(V_{n,(i)}\right)
$$

such that

$$
\left\{\left(h^{\prime} h, 1\right)\right\} \times \sigma \in \Sigma
$$

and $\sigma \cap \mathfrak{C}^{(m),>0}\left(V_{n,(i)}\right)$ is non-empty. It is preserved by the action of $L_{n,(i), \text { lin }}^{(m)}(\mathbb{Q})$. (See Sect. 5.2.) This, in the sense of Sect. 4.4, is an 'admissible cone decomposition' of $X_{*}\left(S_{n,(i), h U h^{-1} \cap P_{n,(i)}^{(m),+}\left(\mathbb{A}^{\infty}\right)}\right)^{\succ 0}$. We write $\widetilde{\Sigma(h)_{0}}$ for the fan consisting of all faces of elements of $\Sigma(h)_{0}$. 
If $U$ is a neat open compact subgroup of $P_{n,(i)}^{(m),+}\left(\mathbb{A}^{\infty}\right)$ we define an $S_{n,(i), U^{-}}^{(m),+}$ torsor $T_{n,(i), U}^{(m),+} / A_{n,(i), U}^{(m),+}$. It has an action of $L_{n,(i), \text { lin }}^{(m)}(\mathbb{Q})$. The system $\left\{T_{n,(i), U}^{(m),+}\right\}$ has a commuting action of $P_{n,(i)}^{(m),+}\left(\mathbb{A}^{\infty}\right)$. (See Sect. 4.3.) There is a torus embedding

$$
T_{n,(i), U}^{(m),+} \hookrightarrow T_{n,(i), U, \widetilde{\Sigma(h)}}^{(m),+}
$$

over $A_{n,(i), U}^{(m),+}$ corresponding to $\widetilde{\Sigma(h)_{0}}$. We write

$$
\partial_{\Sigma(h)_{0}} T_{n,(i), U}^{(m),+}=\partial_{\Sigma(h)_{0}} T_{n,(i), U, \widetilde{\Sigma(h)}}^{(m),+}
$$

for the closed subset of the boundary corresponding to $\Sigma(h)_{0} \subset \widetilde{\Sigma(h)_{0}}$, and let

$$
T_{n,(i), U, \Sigma(h)_{0}}^{(m),+\wedge}
$$

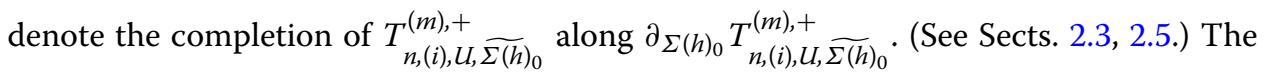
$L_{n,(i), \text { lin }}^{(m)}(\mathbb{Q})$ action extends to $T_{n,(i), U, \widetilde{\Sigma(h)_{0}}}^{(m),+}$ and $\partial_{\Sigma(h)_{0}} T_{n,(i), U}^{(m),+}$ and $T_{n,(i), U, \Sigma(h))_{0}}^{(m),+, \wedge}$. The quotients

$$
\partial_{\Sigma(h)_{0}} T_{n,(i), U}^{(m), \natural}=L_{n,(i)}^{(m)}(\mathbb{Q}) \backslash \partial_{\Sigma(h)_{0}} T_{n,(i), U}^{(m),+}
$$

and

$$
T_{n,(i), U, \Sigma(h)_{0}}^{(m), \iota, \wedge}=L_{n,(i)}^{(m)}(\mathbb{Q}) \backslash T_{n,(i), U, \Sigma(h)_{0}}^{(m),+\wedge}
$$

make sense. Sometimes it will be convenient to take the quotient in two stages. Thus we set

$$
\partial_{\Sigma(h)_{0}} T_{n,(i), U}^{(m), \sharp+}=\operatorname{Hom}_{F}\left(F^{m}, F^{i}\right) \backslash \partial_{\Sigma(h)_{0}} T_{n,(i), U}^{(m),+}
$$

and

$$
T_{n,(i), U, \Sigma(h)_{0}}^{(m), \iota+, \wedge}=\operatorname{Hom}_{F}\left(F^{m}, F^{i}\right) \backslash T_{n,(i), U, \Sigma(h)_{0}}^{(m),+\wedge}
$$

These still carry an action of $L_{n,(i)}(\mathbb{Q})$. If $(U, \Sigma) \geq(V, \Delta)$, then there is also a natural $L_{n,(i)}(\mathbb{Q})$-equivariant map

$$
T_{n,(i), U, \Sigma(h)_{0}}^{(m), \natural+\wedge} \longrightarrow T_{n,(i), V, \Delta(h)_{0}}^{(m),+,}
$$

The inverse systems $\left\{T_{n,(i), U, \Sigma(h)_{0}}^{(m), \downarrow, \wedge}\right\}$ and $\left\{\partial_{\Sigma(h)_{0}} T_{n,(i), U}^{(m), \natural}\right\}$ as $U$ and $\Sigma$ vary has an action of $P_{n,(i)}^{(m),+}\left(\mathbb{A}^{\infty}\right)$. (See Sect. 4.5.)

Then we have an identification

$$
A_{n, U, \Sigma, i}^{(m), \wedge}=\coprod_{h \in P_{n,(i)}^{(m),+}\left(\mathbb{A}^{\infty}\right) \backslash G_{n}^{(m)}\left(\mathbb{A}^{\infty}\right) / U} T_{n,(i), h U h^{-1} \cap P_{n,(i)}^{(m),+}\left(\mathbb{A}^{\infty}\right), \Sigma(h)_{0}}^{(m), \natural, \wedge}
$$


where the term indexed by $h$ is exactly the open and closed subformal scheme whose underlying topological space is the pre-image of $X_{n,(i), h U h^{-1} \cap P_{n,(i)}^{(m),+}{ }_{\mathbb{A} \infty)}^{\natural}} \subset \partial_{i}^{0} X_{n, U}^{\min }$. These identifications are compatible with the action of $G_{n}^{(m)}\left(\mathbb{A}^{\infty}\right)$ and the maps

$$
A_{n, U, \Sigma, i}^{(m), \wedge} \longrightarrow A_{n, V, \Delta, i}^{(0), \wedge}=X_{n, V, \Delta, i}^{\wedge} \longrightarrow X_{n, V, i}^{\min , \wedge}
$$

(See Sect. 5.3.)

There is a similar description for the formal completion of $\mathcal{A}_{n, U^{p}\left(N_{1}, N_{2}\right), \Sigma}^{(m) \text {,ord }}$ along $\partial_{i}^{0} \mathcal{A}_{n, U^{p}\left(N_{1}, N_{2}\right), \Sigma}^{(m) \text {, rord }}$, which we will denote $\mathcal{A}_{n, U^{p}\left(N_{1}, N_{2}\right), \Sigma, i^{\prime}}^{(m) \text {, r d } \wedge}$

We let $L_{n,(i), \text { lin }}^{(m)}\left(\mathbb{A}^{\infty}\right)^{\text {ord }}=L_{n,(i), \text { lin }}^{(m)}\left(\mathbb{A}^{\infty}\right)^{\text {ord }, \times} \operatorname{denote} L_{n,(i), \text { lin }}^{(m)}\left(\mathbb{A}^{\infty, p} \times \mathbb{Z}_{p}\right)$. We write

$$
U_{p}(N)_{n,(i), \operatorname{lin}}^{(m)}=\operatorname{ker}\left(L_{n,(i), \operatorname{lin}}^{(m)}\left(\mathbb{Z}_{p}\right) \rightarrow L_{n,(i), \operatorname{lin}}^{(m)}\left(\mathbb{Z} / p^{N} \mathbb{Z}\right)\right) .
$$

If $U^{p} \subset L_{n,(i), \text { lin }}^{(m)}\left(\mathbb{A}^{\infty, p}\right)$ we write $U^{p}(N)=U^{p} \times U_{p}(N)_{n,(i), \text { lin }}^{(m)}$. We also define

$$
\mathcal{Y}_{n,(i), U^{p}(N)}^{(m), \text { ord },+}=\coprod_{L_{n,(i), \text { lin }}^{(m)}\left(\mathbb{A}^{\infty}\right)^{\text {ord }, \times} / U^{p}(N)} \operatorname{Spec} \mathbb{Z}_{(p)}
$$

which has a left action of $L_{n,(i), \text { lin }}^{(m)}\left(\mathbb{Z}_{(p)}\right)$. The inverse system $\left\{\mathcal{Y}_{n,(i), U^{p}(N)}^{(m), \text { ord, }}\right\}$ has a commuting right action of $L_{n,(i), \text { lin }}^{(m)}\left(\mathbb{A}^{\infty}\right)^{\text {ord }}$. We set

$$
\mathcal{Y}_{n,(i), U^{p}(N)}^{(m), \text { ord }, \sharp}=L_{n,(i), \text { lin }}^{(m)}\left(\mathbb{Z}_{(p)}\right) \backslash \mathcal{Y}_{n,(i), U^{p}(N)}^{(m), \text { ord },+}
$$

(See Sect. 4.1.)

We set

$$
L_{n,(i)}^{(m)}\left(\mathbb{A}^{\infty}\right)^{\text {ord }}=L_{n,(i), \text { herm }}\left(\mathbb{A}^{\infty}\right)^{\text {ord }} \times L_{n,(i), \text { lin }}^{(m)}\left(\mathbb{A}^{\infty}\right)^{\text {ord }}
$$

and

$$
L_{n,(i)}^{(m)}\left(\mathbb{A}^{\infty}\right)^{\text {ord }, \times}=L_{n,(i), \text { herm }}\left(\mathbb{A}^{\infty}\right)^{\text {ord }, \times} \times L_{n,(i), \text { lin }}^{(m)}\left(\mathbb{A}^{\infty}\right)^{\text {ord }, \times} .
$$

We set

$$
U_{p}\left(N_{1}, N_{2}\right)_{n,(i)}^{(m)}=U_{p}\left(N_{1}, N_{2}\right)_{n-i} \times U_{p}\left(N_{1}\right)_{n,(i), \text { lin }}^{(m)},
$$

and, if $U^{p} \subset L_{n,(i)}^{(m)}\left(\mathbb{A}^{\infty, p}\right)$, then we set $U^{p}\left(N_{1}, N_{2}\right)=U^{p} \times U_{p}\left(N_{1}, N_{2}\right)_{n,(i)}^{(m)}$. We define

$$
\mathcal{X}_{n,(i), U^{p}\left(N_{1}, N_{2}\right)}^{(m), \text { ord, }}=\coprod_{h \in L_{n, i}^{(m), \operatorname{lin}}\left(\mathbb{A}^{\infty}\right)^{\text {ord }, \times} / U^{p}\left(N_{1}\right)} \mathcal{X}_{n-i,\left(h U^{p} h^{-1} \cap L_{n,(i), \text { herm }}\left(\mathbb{A}^{\infty, p}\right)\right)\left(N_{1}, N_{2}\right)^{\prime}}^{\text {ord }}
$$

which has a left action of $L_{n,(i), \text { lin }}^{(m)}\left(\mathbb{Z}_{(p)}\right)$. The inverse system $\left\{\mathcal{X}_{n,(i), U^{p}\left(N_{1}, N_{2}\right)}^{(m), \text { hrd, }}\right\}$ has a commuting right action of $L_{n,(i)}^{(m)}\left(\mathbb{A}^{\infty}\right)^{\text {ord }}$. We set

$$
\mathcal{X}_{n,(i), U^{p}(N)}^{(m), \text { ord, }}=L_{n,(i), \text { lin }}^{(m)}\left(\mathbb{Z}_{(p)}\right) \backslash \mathcal{X}_{n,(i), U^{p}(N)}^{(m), \text { ord },+}
$$

(See Sect. 4.1.) 
Further we set $P_{n,(i)}^{(m),+}\left(\mathbb{A}^{\infty}\right)^{\text {ord }}$ to be

$$
P_{n,(i)}^{(m),+}\left(\mathbb{A}^{\infty, p}\right) \times L_{n,(i)}^{(m)}\left(\mathbb{A}^{\infty}\right)^{\text {ord }} N_{n,(i)}^{(m)}\left(\mathbb{Z}_{p}\right)
$$

and $P_{n,(i)}^{(m),+}\left(\mathbb{A}^{\infty}\right)^{\text {ord } \times}$ to be

$$
P_{n,(i)}^{(m),+}\left(\mathbb{A}^{\infty, p}\right) \times L_{n,(i)}^{(m)}\left(\mathbb{A}^{\infty}\right)^{\mathrm{ord}, \times} N_{n,(i)}^{(m)}\left(\mathbb{Z}_{p}\right) .
$$

Moreover we define $U_{p}\left(N_{1}, N_{2}\right)_{n,(i)}^{(m),+}$ to be the subgroup of $P_{n,(i)}^{(m),+}\left(\mathbb{Z}_{p}\right)$ consisting of matrices of the form

$$
\left(\begin{array}{c|c|c|c}
* & * & * & * \\
\hline 0 & * & * & * \\
\hline 0 & \equiv 0 \bmod p^{N_{2}} & \equiv 1_{n-i} \bmod p^{N_{1}} & \equiv 0 \bmod p^{N_{1}} \\
\hline 0 & 0 & 0 & \equiv 1_{i} \bmod p^{N_{1}}
\end{array}\right)\left(\begin{array}{c}
\frac{*}{*} \equiv 0 \bmod p^{N_{1}} \\
\frac{\equiv \bmod p^{N_{1}}}{\equiv 0}
\end{array}\right) .
$$

If $U^{p} \subset P_{n,(i)}^{(m),+}\left(\mathbb{A}^{\infty, p}\right)$ (resp. $\left.\left(P_{n,(i)}^{(m),+} / Z\left(N_{n,(i)}^{(m)}\right)\right)\left(\mathbb{A}^{\infty, p}\right)\right)$ we define $U^{p}\left(N_{1}, N_{2}\right)$ to be $U^{p} \times$ $U_{p}\left(N_{1}, N_{2}\right)_{n,(i)}^{(m),+}\left(\right.$ resp. $\left.U^{p} \times U_{p}\left(N_{1}, N_{2}\right)_{n,(i)}^{(m),+} /\left(Z\left(N_{n,(i)}^{(m)}\right)\left(\mathbb{Z}_{p}\right)\right)\right)$. If $U^{p}$ is a neat open compact subgroup of $\left(P_{n,(i)}^{(m),+} / Z\left(N_{n,(i)}^{(m)}\right)\right)\left(\mathbb{A}^{\infty, p}\right)$ we define

$$
\mathcal{A}_{n,(i), U^{p}\left(N_{1}, N_{2}\right)}^{(m), \text { ord }+}=\underset{h \in L_{n,(i), \text { lin }}^{(m)}\left(\mathbb{A}^{\infty}\right)^{\text {ord }, \times} / U^{p}\left(N_{1}\right)}{\mathcal{A}_{n-i,\left(h U^{p} h^{-1} \cap G_{n-i}^{(i+m)}\left(\mathbb{A}^{\infty, p}\right)\right)\left(N_{1}, N_{2}\right)^{\prime}}^{(i+m), \text { ord }}}
$$

which has a left action of $L_{n,(i), \text { lin }}^{(m)}\left(\mathbb{Z}_{(p)}\right)$. The system of schemes $\left\{\mathcal{A}_{n,(i), U^{p}\left(N_{1}, N_{2}\right)}^{(m), \text { has a }}\right.$ commuting right action of $P_{n,(i)}^{(m),+}\left(\mathbb{A}^{\infty}\right)^{\text {ord }} /\left(Z\left(N_{n,(i)}^{(m)}\right)\left(\mathbb{A}^{\infty, p} \times \mathbb{Z}_{p}\right)\right)$. (See Sect. 4.2.)

If $U^{p}$ is a neat open compact subgroup of $P_{n,(i)}^{(m),+}\left(\mathbb{A}^{\infty, p}\right)$ we define $\mathcal{S}_{n,(i), U^{p}(N)}^{(m),+r d,+}$ to be the torus over $\mathcal{Y}_{n,(i), U^{p}(N)}^{(m), \text { ord,+ }}$ with cocharacter group over the Spec $\mathbb{Z}_{(p)}$ indexed by $h \in$ $L_{n,(i), \text { lin }}^{(m)}\left(\mathbb{A}^{\infty}\right)^{\text {ord }, \times} / U^{p}(N)$ identified with

$$
h U^{p} h^{-1} \cap Z\left(N_{n,(i)}^{(m)}\right)\left(\mathbb{Z}_{(p)}\right)
$$

The torus $\mathcal{S}_{n,(i), U^{p}(N)}^{(m), \text { ord, }+}$ has a left action of $L_{n,(i), \text { lin }}^{(m)}\left(\mathbb{Z}_{(p)}\right)$. The system of tori $\left\{\mathcal{S}_{n,(i), U^{p}(N)}^{(m), \text { ord, }}\right\}$ also has a right action of $P_{n,(i)}^{(m),+}\left(\mathbb{A}^{\infty}\right)^{\text {ord }}$. (See Sect. 4.3.)

If $\left(U^{p}(N), \Sigma\right) \in \mathcal{J}_{n}^{(m) \text {,tor,ord }}$ and $h \in G_{n}^{(m)}\left(\mathbb{A}^{\infty}\right)^{\text {ord, } \times}$ then we define a partial fan $\Sigma(h)_{0}$ in $X_{*}\left(\mathcal{S}_{n,(i),\left(h U^{p} h^{-1} \cap P_{n,(i)}^{(m),+}\left(\mathbb{A}^{\infty, p}\right)\right)(N)}\right)$ as follows. Over the Spec $\mathbb{Z}_{(p)}$ corresponding to $h^{\prime} \in$ $L_{n,(i), \text { lin }}^{(m)}\left(\mathbb{A}^{\infty}\right)^{\text {ord }, \times} / U^{p}(N)$ we take the cones

$$
\sigma \subset\left(h^{\prime}\left(h U^{p} h^{-1} \cap P_{n,(i)}^{(m),+}\left(\mathbb{A}^{\infty, p}\right)\right) h^{\prime,-1} \cap Z\left(N_{n,(i)}^{(m)}\right)\left(\mathbb{Z}_{(p)}\right)\right) \otimes_{\mathbb{Z}} \mathbb{R}=\mathfrak{C}^{(m)}\left(V_{n,(i)}\right)
$$

such that

$$
\left\{\left(h^{\prime} h, 1\right)\right\} \times \sigma \in \Sigma
$$

and $\sigma \cap \mathfrak{C}^{(m),>0}\left(V_{n,(i)}\right)$ is non-empty. It is preserved by the action of $L_{n,(i), \text { lin }}^{(m)}\left(\mathbb{Z}_{(p)}\right)$. (See Sect. 5.2.) This, in the sense of Sect. 4.4, is an 'admissible cone decomposition' of 
$X_{*}\left(\mathcal{S}_{n,(i),\left(h U^{p} h^{-1} \cap P_{n,(i)}^{(m),+}\left(\mathbb{A}^{\infty, p}\right)\right)(N)}\right)^{\succ 0}$. We write $\left.\widetilde{\Sigma(h}\right)_{0}$ for the fan consisting of all faces of elements of $\Sigma(h)_{0}$.

If $U^{p}$ is a neat open compact subgroup of $P_{n,(i)}^{(m),+}\left(\mathbb{A}^{\infty, p}\right)$ we define an $\mathcal{S}_{n,(i), U^{p}\left(N_{1}\right)^{- \text {torsor }}}^{(m), \text { ord, }}$ $\mathcal{T}_{n,(i), U^{p}\left(N_{1}, N_{2}\right)}^{(m) \text {,ord, }} / \mathcal{A}_{n,(i), U^{p}\left(N_{1}, N_{2}\right)}^{(m) \text {,ord }+}$. It has an action of $L_{n,(i), \text { lin }}^{(m)}\left(\mathbb{Z}_{(p)}\right)$. The system $\left\{\mathcal{T}_{n,(i), U^{p}\left(N_{1}, N_{2}\right)}^{(m), \text { ord }+}\right.$ has a commuting action of $P_{n,(i)}^{(m),+}\left(\mathbb{A}^{\infty}\right)^{\text {ord }}$. (See Sect. 4.3.) There is a torus embedding

$$
\mathcal{T}_{n,(i), U^{p}\left(N_{1}, N_{2}\right)}^{(m), \text { ord }+} \hookrightarrow \mathcal{T}_{n,(i), U^{p}\left(N_{1}, N_{2}\right), \widetilde{\Sigma(h)}}^{(m), \text { ord }+}
$$

over $\mathcal{A}_{n,(i), U^{p}\left(N_{1}, N_{2}\right)}^{(m), \text { cord }+}$ correonding to $\widetilde{\Sigma(h)_{0}}$. We write

$$
\partial_{\Sigma(h)_{0}} \mathcal{T}_{n,(i), U^{p}\left(N_{1}, N_{2}\right)}^{(m), \text { ord }+}=\partial_{\Sigma(h)_{0}} \mathcal{T}_{n,(i), U^{p}\left(N_{1}, N_{2}\right), \widetilde{\Sigma(h)}}^{(m),+}
$$

for the closed subset of the boundary corresponding to $\Sigma(h)_{0} \subset \widetilde{\Sigma(h)_{0}}$, and let

$$
\mathcal{T}_{n,(i), U^{p}\left(N_{1}, N_{2}\right), \Sigma(h)_{0}}^{(m), \text { ord }+, \wedge}
$$

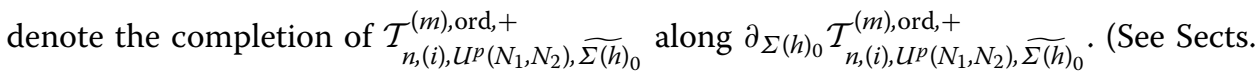
2.3, 2.5.) The $L_{n,(i), \text { lin }}^{(m)}\left(\mathbb{Z}_{(p)}\right)$ action extends to $\mathcal{T}_{\left.n,(i), U^{p}\left(N_{1}, N_{2}\right), \widetilde{\Sigma(h)}\right)_{0}}^{(m), \text { and } \partial_{\Sigma(h)_{0}}} \mathcal{T}_{n,(i), U^{p}\left(N_{1}, N_{2}\right)}^{(m),+}$ and $\mathcal{T}_{n,(i), U^{p}\left(N_{1}, N_{2}\right), \Sigma(h)_{0}}^{(m), \text { The quotients }}$

$$
\partial_{\Sigma(h)_{0}} \mathcal{T}_{n,(i), U^{p}\left(N_{1}, N_{2}\right)}^{(m), \text { ord } \sharp}=L_{n,(i)}^{(m)}\left(\mathbb{Z}_{(p)}\right) \backslash \partial_{\Sigma(h)_{0}} \mathcal{T}_{n,(i), U^{p}\left(N_{1}, N_{2}\right)}^{(m), \text { rd }+}
$$

and

$$
\mathcal{T}_{n,(i), U^{p}\left(N_{1}, N_{2}\right), \Sigma(h)_{0}}^{(m), \text { ard } \sharp \wedge}=L_{n,(i)}^{(m)}\left(\mathbb{Z}_{(p)}\right) \backslash \mathcal{T}_{n,(i), U^{p}\left(N_{1}, N_{2}\right), \Sigma(h)_{0}}^{(m), \text { ord }+, \wedge}
$$

make sense. Sometimes it will be convenient to take the quotient in two stages. Thus we set

$$
\partial_{\Sigma(h)_{0}} \mathcal{T}_{n,(i), U^{p}\left(N_{1}, N_{2}\right)}^{(m), \text { ord },+}=\operatorname{Hom}_{\mathcal{O}_{F}}\left(\mathcal{O}_{F,(p)}^{m}, \mathcal{O}_{F,(p)}^{i}\right) \backslash \partial_{\Sigma(h)_{0}} \mathcal{T}_{n,(i), U^{p}\left(N_{1}, N_{2}\right)}^{(m), o r d}
$$

and

$$
\mathcal{T}_{n,(i), U^{p}\left(N_{1}, N_{2}\right), \Sigma(h)_{0}}^{(m), \text { ord } \sharp+, \wedge}=\operatorname{Hom}_{\mathcal{O}_{F}}\left(\mathcal{O}_{F,(p)}^{m}, \mathcal{O}_{F,(p)}^{i}\right) \backslash \mathcal{T}_{n,(i), U^{p}\left(N_{1}, N_{2}\right), \Sigma(h)_{0}}^{(m), \text { ord },+\wedge}
$$

These still carry an action of $L_{n,(i)}\left(\mathbb{Z}_{(p)}\right)$. The inverse systems $\left\{\mathcal{T}_{n,(i), U^{p}\left(N_{1}, N_{2}\right), \Sigma(h)}^{(m), \text { ord, }, \wedge}\right\}$ and $\left\{\partial_{\Sigma(h)_{0}} \mathcal{T}_{n,(i), U^{p}\left(N_{1}, N_{2}\right)}^{(m), \text { ord },}\right\}$ as $U^{p}\left(N_{1}, N_{2}\right)$ and $\Sigma$ vary have actions of $P_{n,(i)}^{(m),+}\left(\mathbb{A}^{\infty}\right)^{\text {ord }}$. (See Sect. 4.5.)

Then we have an identification

$$
\begin{aligned}
& \mathcal{A}_{n, U^{p}\left(N_{1}, N_{2}\right), \Sigma, i}^{(m), \text { ord } \wedge} \\
& \quad=\coprod_{h \in P_{n,(i)}^{(m),+}\left(\mathbb{A}^{\infty}\right)^{\text {ord }, \times} \backslash G_{n}^{(m)}\left(\mathbb{A}^{\infty}\right)^{\text {ord }, \times} / U^{p}\left(N_{1}\right)} \mathcal{T}_{n,(i),\left(h U^{p} h^{-1} \cap P_{n,(i)}^{(m),+}\left(\mathbb{A}^{\infty, p}\right)\right)\left(N_{1}, N_{2}\right), \Sigma^{\text {ord }}(h)_{0}}^{(m), \text { ord }, \llbracket \wedge} \\
& \amalg \coprod_{h \in\left(P_{n,(i)}^{(m),+}\left(\mathbb{A}^{\infty}\right) \backslash G_{n}^{(m)}\left(\mathbb{A}^{\infty}\right) / U^{p}\left(N_{1}, N_{2}\right)\right)-\left(P_{n,(i)}^{(m),+}\left(\mathbb{A}^{\infty}\right)^{\text {ord }, \times} \backslash G_{n}^{(m)}\left(\mathbb{A}^{\infty}\right)^{\text {ord }, \times} / U^{p}\left(N_{1}\right)\right)} \\
& \quad T_{n,(i), h U^{p}\left(N_{1}, N_{2}\right) h^{-1} \cap P_{n,(i)}^{(m),+}\left(\mathbb{A}^{\infty}\right), \Sigma(h)_{0}}^{(m),}
\end{aligned}
$$


where the term indexed by $h$ is exactly the open and closed subformal scheme whose underlying topological space is the pre-image of

$$
\mathcal{X}_{n,(i),\left(h U^{p} h^{-1} \cap P_{n,(i)}^{(m),+}\left(\mathbb{A}^{\infty, p}\right)\right)\left(N_{1}, N_{2}\right)}^{\text {ord } \natural}
$$

or

$$
X_{n,(i), h U^{p}\left(N_{1}, N_{2}\right) h^{-1} \cap P_{n,(i)}^{(m),+}\left(\mathbb{A}^{\infty}\right)}^{\natural} \subset \partial_{i}^{0} \mathcal{X}_{n, U^{p}\left(N_{1}, N_{2}\right)^{\circ}}^{\mathrm{ord}, \min }
$$

These identifications are compatible with the action of $G_{n}^{(m)}\left(\mathbb{A}^{\infty}\right)^{\text {ord }}$ and the maps

$$
\mathcal{A}_{n, U^{p}\left(N_{1}, N_{2}\right), \Sigma, i}^{(m), \text { ord } \wedge} \longrightarrow \mathcal{A}_{n, V^{p}\left(N_{1}, N_{2}\right), \Delta, i}^{(0), \text { rd }, \wedge}=\mathcal{X}_{n, V^{p}\left(N_{1}, N_{2}\right), \Delta, i}^{\text {ord }, \wedge} \longrightarrow \mathcal{X}_{n, V^{p}\left(N_{1}, N_{2}\right), i^{*}}^{\text {ord, min }, \wedge}
$$

(See Sect. 5.3.)

\section{Appendix B. Comparison with [44]}

This appendix is meant to be a guide to the notation system in [44] (and in the earlier works [41-43]). While we will not cover everything, we will highlight the key definitions and results used in this paper so that, it is hoped, the reader can understand the references more easily. We will adopt the notation and conventions introduced in [41, Notation and Conventions], which might be quite different from the ones introduced in this paper. (We will explain the differences when necessary.)

\section{B.1 Shimura varieties as PEL moduli in characteristic zero}

In $[41,44]$ the symbols $\mathbb{Z}(1)$ are used to denote $\operatorname{ker}\left(\exp : \mathbb{C} \rightarrow \mathbb{C}^{\times}\right)$. In those papers, unlike this paper, Tate twists just mean tensor products with powers of $\mathbb{Z}(1)$ as a $\mathbb{Z}$-module, with no attached Galois actions at all. Let us fix the choice of a square-root $\sqrt{-1}$ in $\mathbb{C}$, which defines an isomorphism $2 \pi \sqrt{-1}: \mathbb{Z} \stackrel{\sim}{\rightarrow} \mathbb{Z}(1)=\operatorname{ker}\left(\exp : \mathbb{C} \rightarrow \mathbb{C}^{\times}\right)$.

Consider the integral PEL datum

$$
\left(\mathcal{O}, \star, L,\langle\cdot, \cdot\rangle, h_{0}\right)=\left(\mathcal{O}_{F}, c, \Lambda_{n}, 2 \pi \sqrt{-1}\langle,\rangle_{n}, h_{0}\right)
$$

in the sense of definition 1.1.1.1 of [44], where $h_{0}$ is the $\mathbb{R}$-algebra homomorphism

$$
\begin{aligned}
& h_{0}: \mathbb{C} \rightarrow \operatorname{End}_{\mathcal{O} \otimes_{\mathbb{Z}} \mathbb{R}}\left(L \otimes_{\mathbb{Z}} \mathbb{R}\right) \\
& z=z_{1}+\sqrt{-1} z_{2} \mapsto\left(\begin{array}{cc}
z_{1} \mathrm{Id}_{n} & -z_{2} \Psi_{n} \\
z_{2} \Psi_{n} & z_{1} \operatorname{Id}_{n}
\end{array}\right)
\end{aligned}
$$

with $\Psi_{n}$ being the $n \times n$-matrix with 1's on the anti-diagonal and 0's elsewhere (see Sect. 1.1). Note that the technical condition 1.4.3.10 in [41] or condition 1.2.1.1 in [44] are satisfied.

The group functor $G$ of definition 1.1.1.3 of [44] is just our $G_{n}$, and $v$ has the same meaning in this paper and in [44]. The reflex field $F_{0}$ defined by $\left(L \otimes_{\mathbb{Z}} \mathbb{R},\langle\cdot, \cdot\rangle, h_{0}\right)$ (see section 1.2.5 of [41]) is just $\mathbb{Q}$, and $\mathrm{S}_{0}=\operatorname{Spec} F_{0}=\operatorname{Spec} \mathbb{Q}$.

For each open compact subgroup $\mathcal{H}$ of $\mathrm{G}(\hat{\mathbb{Z}})$, [44] defines a moduli problem 
$\mathrm{M}_{\mathcal{H}}$

over $\mathrm{S}_{0}=$ Spec $\mathbb{Q}$ (parameterizing isomorphism classes of abelian schemes with additional PEL structures) as in the beginning of section 1.1.2 of [44], which is representable by a smooth quasi-projective scheme over $\operatorname{Spec} \mathbb{Q}$ when $\mathcal{H}$ is neat. As explained in remark 1.1.2.1 in [44], the definition of $\mathrm{M}_{\mathcal{H}}$ only depends on the rational PEL datum $\left(\mathcal{O} \otimes_{\mathbb{Z}}\right.$ $\left.\mathbb{Q} \star \star, L \otimes_{\mathbb{Z}} \mathbb{Q}\langle\cdot, \cdot\rangle \otimes_{\mathbb{Z}} \mathbb{Q}, h_{0}\right)$, and hence can be extended to the cases of all open compact subgroups $\mathcal{H}$ of $G\left(\mathbb{A}^{\infty}\right)$, up to replacing $L$ with any lattice in $L \otimes_{\mathbb{Z}} \mathbb{Q}$ stabilized by $\mathcal{H}$ (and replacing $\langle\cdot, \cdot\rangle$ with a suitable $\mathbb{Q}_{>0}^{\times}$-multiple of the induced pairing). By proposition 1.4.3.4 in [41], this moduli problem is canonically isomorphic to the

$$
X_{n, U}
$$

in this paper when $U=\mathcal{H}$ is contained in $\mathrm{G}(\hat{\mathbb{Z}})$ (see Sect. 3.1.1). (The condition on Lie algebras in this paper is equivalent to the determinantal condition in $[39,41]$.) The collection $\left\{M_{\mathcal{H}}\right\}_{\mathcal{H}}$ indexed by neat open compact subgroups $\mathcal{H}$ of $G\left(\mathbb{A}^{\infty}\right)$ can be $G\left(\mathbb{A}^{\infty}\right)$ equivariantly identified with $\left\{X_{n, U}\right\}_{U}$ (with $U=\mathcal{H}$ ), because the (Hecke) actions of $G\left(\mathbb{A}^{\infty}\right)$ on them are defined by twisting level structures on quasi-isogeny classes in the same way (see remark 1.4.3.11 of [41]). (Later we will not repeat the explanation on such canonical isomorphisms between moduli of isomorphism classes and of quasi-isogeny classes. Also, we will tacitly assume that all Hecke actions to be introduced are compatible with previously introduced ones under canonical morphisms.)

\section{B.2 Filtrations}

Consider any filtration $\mathrm{V}=\left\{\mathrm{V}_{-j}\right\}_{j}$ on $L \otimes_{\mathbb{Z}} \mathbb{Q} \cong F^{2 n}$ such that

$$
\text { (0) }=\mathrm{V}_{-3} \subset \mathrm{V}_{-2} \subset \mathrm{V}_{-1}=\mathrm{V}_{-2}^{\perp} \subset \mathrm{V}_{0}=L \otimes_{\mathbb{Z}} \mathbb{Q}
$$

which is symplectic in the sense of definition 1.2.6.8 in [41] (or definition 1.2.1.2(4) in $[44])$. By lemma A.4.3 in [45], up to the action of $G(\mathbb{Q})$, we may assume that there exists an integer $0 \leq i \leq n$ such that

$$
(0)=\mathrm{V}_{-3} \subset \mathrm{V}_{-2}=V_{n,(i)} \subset \mathrm{V}_{-1}=V_{n,(i)}^{\perp} \subset \mathrm{V}_{0}=L \otimes_{\mathbb{Z}} \mathbb{Q}
$$

which we call the $i$-th standard symplectic filtration, and write $\mathrm{V}=\mathrm{V}^{(i)}$. The stabilizer of $\mathrm{V}^{(i)}$ is then the parabolic subgroup $P_{n,(i)}^{+}(\mathbb{Q})$ of $G_{n}(\mathbb{Q})$.

We will need to consider filtrations $Z=\left\{Z_{-j}\right\}_{j}$ on $L \otimes_{\mathbb{Z}} \hat{\mathbb{Z}}$ such that

$$
\text { (0) }=\mathrm{Z}_{-3} \subset \mathrm{Z}_{-2} \subset \mathrm{Z}_{-1}=\mathrm{Z}_{-2}^{\perp} \subset \mathrm{Z}_{0}=L \otimes_{\mathbb{Z}} \hat{\mathbb{Z}}
$$

which is fully symplectic admissible as in definition 5.2.7.1 in [41] or definition 1.2.1.4 in [44]. If $0 \leq i \leq n$ and $g \in \mathrm{G}\left(\mathbb{A}^{\infty}\right)$, then we can define such a filtration $\mathrm{z}^{(i, g)}$ by

$$
\mathrm{Z}_{-j}^{(i, g)}=\left(g^{-1}\left(\mathrm{~V}_{-j}^{(i)} \otimes_{\mathbb{Q}} \mathbb{A}^{\infty}\right)\right) \cap\left(L \otimes_{\mathbb{Z}} \hat{\mathbb{Z}}\right),
$$

for each $j$. Since we are not in the so-called type D case, by the proof of proposition A.5.9 of [45], any fully symplectic admissible filtration arises as $\mathrm{Z}^{(i, g)}$ for some $i$ and some $g$. In fact, using the Iwasawa decomposition, we may suppose that $g \in \mathrm{G}(\hat{\mathbb{Z}})$. For each $j$, let $\mathrm{Gr}_{-j}^{\mathrm{Z}}:=\mathrm{Z}_{-j} / \mathrm{Z}_{-j-1}$ as usual. Then $\langle\cdot, \cdot\rangle$ canonically induces a perfect pairing

$$
\langle\cdot, \cdot\rangle_{-1}: \mathrm{Gr}_{-1}^{\mathrm{Z}} \times \mathrm{Gr}_{-1}^{\mathrm{Z}} \rightarrow \hat{\mathbb{Z}}(1) .
$$


Let us temporarily fix such a $\mathrm{Z}=\mathrm{Z}^{(i, g)}$ with $g \in \mathrm{G}(\hat{\mathbb{Z}})$ and fix an open compact subgroup $\mathcal{H} \subset \mathrm{G}(\hat{\mathbb{Z}})$. In [44], the following groups are defined for $\hat{\mathbb{Z}}$-algebras $R$ along with open compact subgroups (see definitions 1.2.1.9, 1.2.1.10, and 1.2.1.11 in [44]):

(1) $\quad \mathrm{P}_{\mathrm{Z}}(R):=\{g \in \mathrm{G}(R): g(\mathrm{Z})=\mathrm{Z}\}=g^{-1} P_{n,(i)}^{+}(R) g$. We set $\mathcal{H}_{\mathrm{P}_{\mathrm{Z}}}:=\mathcal{H} \cap \mathrm{P}_{\mathrm{Z}}(\hat{\mathbb{Z}})$.

(2) $\mathrm{G}_{l, \mathrm{Z}}^{\prime}(R)$ is set equal to the subgroup of $\mathrm{GL}_{\mathcal{O}}\left(\mathrm{Gr}_{-2}^{\mathrm{Z}} \otimes_{\hat{\mathbb{Z}}} R\right) \times \mathrm{GL}_{\mathcal{O}}\left(\mathrm{Gr}_{0}^{Z} \otimes_{\hat{\mathbb{Z}}} R\right)$ consisting of elements respecting the pairing $\mathrm{Gr}_{-2}^{\mathrm{Z}} \times \mathrm{Gr}_{0}^{\mathrm{Z}} \rightarrow \hat{\mathbb{Z}}(1)$ induced by $\langle\cdot, \cdot\rangle$. This group comes isomorphically from $g^{-1} L_{n,(i), \operatorname{lin}}(R) g$. There is a natural surjective map $\left(v^{-1} \mathrm{Gr}_{-2}^{\mathrm{Z}}, \mathrm{Gr}_{0}^{\mathrm{Z}}\right): \mathrm{P}_{\mathrm{Z}}(R) \rightarrow \mathrm{G}_{l, \mathrm{Z}}^{\prime}(R)$ corresponding to $P_{n,(i)}^{+}(R) \rightarrow L_{n,(i), \operatorname{lin}}(R)$. We denote the kernel by $\mathrm{P}_{\mathrm{Z}}^{\prime}(R)=g^{-1} P_{n,(i)}(R) g$. We set $\mathcal{H}_{\mathrm{P}_{\mathrm{Z}}^{\prime}}:=\mathcal{H} \cap \mathrm{P}_{\mathrm{Z}}^{\prime}(\hat{\mathbb{Z}})$, $\mathcal{H}_{\mathrm{G}_{h, \mathrm{Z}}^{\prime}}:=\mathcal{H}_{\mathrm{P}_{\mathrm{Z}}^{\prime}} / \mathcal{H}_{\mathrm{U}_{\mathrm{z}}}$, and $\mathcal{H}_{\mathrm{G}_{l, \mathrm{Z}}^{\prime}}:=\mathcal{H}_{\mathrm{P}_{\mathrm{z}}} / \mathcal{H}_{\mathrm{P}_{\mathrm{Z}}^{\prime}}$.

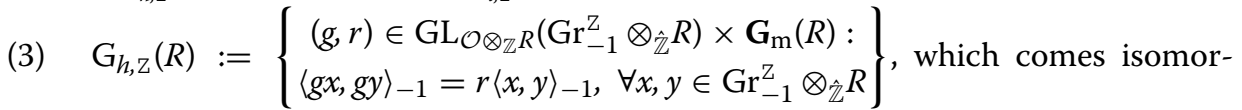
phically from $g^{-1} L_{n,(i) \text { herm }}(R) g$. Then there is a natural surjective map $\mathrm{Gr}_{-1}^{\mathrm{Z}}$ : $\mathrm{P}_{\mathrm{Z}}(R) \rightarrow \mathrm{G}_{h, \mathrm{Z}}(R)$ corresponding to $P_{n,(i)}^{+}(R) \rightarrow L_{n,(i) \text {,herm }}(R)$. We denote the kernel $\mathrm{Z}_{\mathrm{Z}}(R)=\left(g^{-1} L_{n,(i), \text { lin }}(R) g\right) \ltimes\left(g^{-1} N_{n,(i)}(R) g\right)$. We set $\mathcal{H}_{\mathrm{Z}_{\mathrm{Z}}}:=\mathcal{H} \cap \mathrm{Z}_{\mathrm{Z}}(\hat{\mathbb{Z}})$ and $\mathcal{H}_{\mathrm{G}_{h, \mathrm{Z}}}:=\mathcal{H}_{\mathrm{P}_{\mathrm{z}}} / \mathcal{H}_{\mathrm{Z}_{\mathrm{z}}}$

(4) $\mathrm{U}_{\mathrm{Z}}(R)$ is the subgroup of $\mathrm{P}_{\mathrm{Z}}(R)$ consisting of elements $g$ such that $\mathrm{Gr}^{\mathrm{Z}}(g)=$

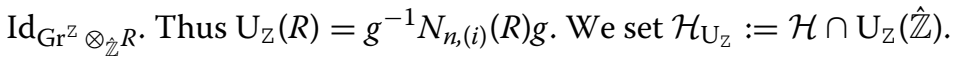

(5) $\mathrm{U}_{2, \mathrm{Z}}(R)$ is the subgroup of $\mathrm{P}_{\mathrm{Z}}(R)$ consisting of elements $g$ which induce $\operatorname{Id}_{\mathrm{z}_{-1} \otimes_{\hat{\mathbb{Z}}} R}$ and $\operatorname{Id}_{\left(\mathrm{Z}_{0} \otimes_{\hat{\mathbb{Z}}} R\right) /\left(\mathrm{z}_{-2} \otimes_{\hat{\mathbb{Z}}} R\right)}$ on $\mathrm{Z}_{-1} \otimes_{\hat{\mathbb{Z}}} R$ and $\left(\mathrm{z}_{0} \otimes_{\hat{\mathbb{Z}}} R\right) /\left(\mathrm{z}_{-2} \otimes_{\hat{\mathbb{Z}}} R\right)$, respectively. Thus we have $\mathrm{U}_{2, \mathrm{Z}}(R)=g^{-1} Z\left(N_{n,(i)}\right)(R) g \cong \operatorname{Herm}^{(i)}(R) \hookrightarrow$ $\operatorname{Hom}_{\mathcal{O} \otimes_{\mathbb{Z}} R}\left(\mathrm{Gr}_{0}^{\mathrm{Z}} \otimes_{\hat{\mathbb{Z}}} R, \mathrm{Gr}_{-2}^{\mathrm{Z}} \otimes_{\hat{\mathbb{Z}}} R\right)$. We set $\mathcal{H}_{\mathrm{U}_{2, \mathrm{Z}}}:=\mathcal{H} \cap \mathrm{U}_{2, \mathrm{Z}}(\hat{\mathbb{Z}})$.

(6) $\quad \mathrm{U}_{1, \mathrm{Z}}(R):=\mathrm{U}_{\mathrm{Z}}(R) / \mathrm{U}_{2, \mathrm{Z}}(R)=\left(g^{-1} N_{n,(i)}(R) g\right) /\left(g^{-1} Z\left(N_{n,(i)}\right)(R) g\right)$, which is isomorphic to $\operatorname{Hom}_{n-i}^{(i)}(R)$. We set $\mathcal{H}_{\mathrm{U}_{1, \mathrm{z}}}:=\mathcal{H}_{\mathrm{U}_{\mathrm{z}}} / \mathcal{H}_{\mathrm{U}_{2, \mathrm{z}}}$.

(7) $\mathrm{G}_{l, \mathrm{Z}}(R):=\mathrm{Z}_{\mathrm{Z}}(R) / \mathrm{U}_{\mathrm{Z}}(R)$. This maps isomorphically to $\mathrm{G}_{l, \mathrm{Z}}^{\prime}(R)$, but [44] chooses to distinguish it as a subgroup rather than a quotient of $\mathrm{P}_{\mathrm{Z}}(R) / \mathrm{U}_{\mathrm{Z}}(R)$. We set $\mathcal{H}_{\mathrm{G}_{l, \mathrm{z}}}:=\mathcal{H}_{\mathrm{Z}_{\mathrm{z}}} / \mathcal{H}_{\mathrm{U}_{\mathrm{z}}}$, which may differ from $\mathcal{H}_{\mathrm{G}_{L \mathrm{z}}^{\prime}}$.

(8) $\mathrm{G}_{h, \mathrm{Z}}^{\prime}(R):=\mathrm{P}_{\mathrm{Z}}^{\prime}(R) / \mathrm{U}_{\mathrm{Z}}(R)$. This maps isomorphically to $\mathrm{G}_{h, \mathrm{Z}}(R)$, but [44] chooses to distinguish it as a subgroup rather than a quotient of $\mathrm{P}_{\mathrm{Z}}(R) / \mathrm{U}_{\mathrm{Z}}(R)$. We set $\mathcal{H}_{\mathrm{G}_{h, \mathrm{z}}^{\prime}}:=\mathcal{H}_{\mathrm{P}_{\mathrm{z}}^{\prime}} / \mathcal{H}_{\mathrm{U}_{\mathrm{z}}}$, which may differ from $\mathcal{H}_{\mathrm{G}_{h, \mathrm{z}}}$.

(9) $\quad \mathrm{G}_{1, \mathrm{Z}}(R):=\mathrm{P}_{\mathrm{Z}}^{\prime}(R) / \mathrm{U}_{2, \mathrm{Z}}(R)$. It is isomorphic to $G_{n-i}^{(i)}(R)$. We set $\mathcal{H}_{\mathrm{G}_{1, \mathrm{Z}}^{\prime}}:=\mathcal{H}_{\mathrm{P}_{\mathrm{Z}}^{\prime}} / \mathcal{H}_{\mathrm{U}_{2, \mathrm{Z}}}$.

For each open compact subgroup $\mathcal{H}=U$ of $\mathrm{G}(\hat{\mathbb{Z}})$, as $g$ varies in $\mathrm{G}\left(\mathbb{A}^{\infty}\right)$, the $\mathcal{H}$-orbits $\mathrm{z}_{\mathcal{H}}^{(i, g)}$ of $\mathrm{Z}^{(i, g)}$ are parameterized by the double coset space

$$
\mathrm{P}_{Z^{(i, 1)}}\left(\mathbb{A}^{\infty}\right) \backslash G\left(\mathbb{A}^{\infty}\right) / \mathcal{H}=P_{n,(i)}^{+}\left(\mathbb{A}^{\infty}\right) \backslash G_{n}\left(\mathbb{A}^{\infty}\right) / U
$$

(See, for example, Sect. 5.1.)

\section{B.3 Cusp labels}

Given any fully symplectic admissible filtration Z, we define a torus argument to be a rigidification $\Phi=\left(X, Y, \phi, \varphi_{-2}, \varphi_{0}\right)$ on the top and bottom graded pieces (see definition 5.4.1.3 in [41] or definition 1.2.1.5 in [44]):

(1) $X$ and $Y$ are locally free $\mathcal{O}$-modules, and $\phi: Y \rightarrow X$ is an isomorphism of $\mathcal{O}$ modules. 
(2) $\varphi_{-2}: \mathrm{Gr}_{-2}^{\mathrm{Z}} \stackrel{\sim}{\rightarrow} \operatorname{Hom}_{\mathbb{Z}}(X, \hat{\mathbb{Z}}(1))$ and $\varphi_{0}: \mathrm{Gr}_{0}^{\mathrm{Z}} \stackrel{\sim}{\rightarrow} Y \otimes_{\mathbb{Z}} \hat{\mathbb{Z}}$ are isomorphisms of $\mathcal{O} \otimes_{\mathbb{Z}} \hat{\mathbb{Z}}$-modules such that the pairing $\langle\cdot, \cdot\rangle_{20}: \mathrm{Gr}_{-2}^{\mathrm{Z}} \times \mathrm{Gr}_{0}^{\mathrm{Z}} \rightarrow \hat{\mathbb{Z}}(1)$ induced by $\langle\cdot, \cdot\rangle$ is the pull-back of the pairing

$$
\langle\cdot, \cdot\rangle_{\phi}: \operatorname{Hom}_{\hat{\mathbb{Z}}}\left(X \otimes_{\mathbb{Z}} \hat{\mathbb{Z}}, \hat{\mathbb{Z}}(1)\right) \times\left(Y \otimes_{\mathbb{Z}} \hat{\mathbb{Z}}\right) \rightarrow \hat{\mathbb{Z}}(1)
$$

defined by $\langle x, y\rangle_{\phi}=x(\phi(y))$.

(For a general integral PEL datum, the first condition should be weakened to $\phi$ being injective with finite cokernel, but in our setting the second condition then forces it to be an isomorphism.) We say that two torus arguments $\Phi=\left(X, Y, \phi, \varphi_{-2}, \varphi_{0}\right)$ and $\Phi^{\prime}=$ $\left(X^{\prime}, Y^{\prime}, \phi^{\prime}, \varphi_{-2}^{\prime}, \varphi_{0}^{\prime}\right)$ are equivalent if there exists a pair of isomorphisms $\left(\gamma_{X}: X^{\prime} \stackrel{\sim}{\rightarrow} X, \gamma_{Y}\right.$ : $Y \stackrel{\sim}{\rightarrow} Y^{\prime}$ ) of $\mathcal{O}$-lattices matching all other data. For $0 \leq i \leq n$ we have a torus argument

$$
\Phi^{(i)}:=\left(\mathcal{O}_{F}^{i}, \mathcal{O}_{F}^{i}, \mathrm{Id},(2 \pi \sqrt{-1}) \Psi_{i} \mathrm{Id}^{\vee}, \mathrm{Id}\right)
$$

for $\mathrm{Z}^{(i, 1)}$, where $\Psi_{i}$ is (as before) the $i \times i$-matrix with 1's on the anti-diagonal and 0 's elsewhere (see Sect. 1.1).

If $\Phi=\left(X, Y, \phi, \varphi_{-2}, \varphi_{0}\right)$ is a torus argument for $\mathrm{Z}$ and $g \in \mathrm{P}_{\mathrm{Z}}\left(\mathbb{A}^{\infty}\right)$, then we obtain a torus argument $\Phi^{(g)}=\left(X^{(g)}, Y^{(g)}, \phi^{(g)}, \varphi_{-2}^{(g)}, \varphi_{0}^{(g)}\right)$ for $\mathrm{Z}^{(g)}$ induced by $g: \mathrm{Gr}^{g^{-1} \mathrm{z}} \rightarrow \mathrm{Gr}^{\mathrm{Z}}$ (see section 1.2.3 of [44] for more details). We shall write $\Phi^{(i, g)}=\left(\Phi^{(i)}\right)^{(g)}$.

Consider triples of the form $(\mathrm{Z}, \Phi, \delta)$, where $\mathrm{Z}$ is a filtration as in the previous section, where $\Phi$ is a torus argument as above, and where $\delta: \mathrm{Gr}^{\mathrm{Z}}=\mathrm{Gr}_{-2}^{\mathrm{z}} \oplus \mathrm{Gr}_{-1}^{\mathrm{z}} \oplus \mathrm{Gr}_{0}^{\mathrm{Z}} \stackrel{\sim}{\rightarrow} L \otimes_{\mathbb{Z}} \hat{\mathbb{Z}}$ is an $\mathcal{O} \otimes_{\mathbb{Z}} \hat{\mathbb{Z}}$-equivariant splitting. The group $\mathrm{G}(\hat{\mathbb{Z}})$ acts naturally on such triples, and hence we can consider their $\mathcal{H}$-orbits of the form $\left(\mathrm{Z}_{\mathcal{H}}, \Phi_{\mathcal{H}}, \delta_{\mathcal{H}}\right)$, for each open compact subgroup $\mathcal{H}$ of $\mathrm{G}(\hat{\mathbb{Z}})$. We consider two such orbits $\left(\mathrm{Z}_{\mathcal{H}}, \Phi_{\mathcal{H}}, \delta_{\mathcal{H}}\right)$ and $\left(\mathrm{Z}_{\mathcal{H}}^{\prime}, \Phi_{\mathcal{H}}^{\prime}, \delta_{\mathcal{H}}^{\prime}\right)$ equivalent if $\mathrm{z}_{\mathcal{H}}=\mathrm{z}_{\mathcal{H}}^{\prime}$ and if there exist representatives $\Phi$ and $\Phi^{\prime}$ of $\Phi_{\mathcal{H}}$ and $\Phi_{\mathcal{H}}^{\prime}$, respectively, which are equivalent. (Note that no compatibility condition is imposed on $\delta_{\mathcal{H}}$ and $\delta_{\mathcal{H}}^{\prime}$.) We call the equivalence classes $\left[\left(\mathrm{Z}_{\mathcal{H}}, \Phi_{\mathcal{H}}, \delta_{\mathcal{H}}\right)\right]$, or simply $\left[\left(\Phi_{\mathcal{H}}, \delta_{\mathcal{H}}\right)\right]$, the cusp labels for $\mathrm{M}_{\mathcal{H}}$.

For each $(\mathrm{Z}, \Phi, \delta)$ as above, and any $\mathbb{Z}$-algebra $R$, we define $\mathrm{G}_{l, \Phi}^{\prime}(R)=\mathrm{GL}_{\mathcal{O} \otimes_{\mathbb{Z}} R}\left(Y \otimes_{\mathbb{Z}} R\right)$, which admits a canonical map to $\mathrm{G}_{l, \mathbb{Z}}^{\prime}\left(R \otimes_{\mathbb{Z}} \hat{\mathbb{Z}}\right)$ induced by $\Phi$. When $R$ is a $\hat{\mathbb{Z}}$-algebra, we have $\mathrm{G}_{l, \Phi}^{\prime}(R) \cong \mathrm{G}_{l, \mathrm{Z}}^{\prime}(R)$. When $R=\mathbb{Q}$, we have $\mathrm{G}_{l, \Phi}^{\prime}(\mathbb{Q}) \hookrightarrow \mathrm{G}_{l, \mathrm{Z}}^{\prime}\left(\mathbb{A}^{\infty}\right)$, and we define

$$
\Gamma_{\Phi_{\mathcal{H}}}:=\mathcal{H}_{\mathrm{G}_{l, \mathrm{Z}}^{\prime}} \cap \mathrm{G}_{l, \Phi}^{\prime}(\mathbb{Q})
$$

a congruence subgroup of $G_{l, \Phi}^{\prime}(\mathbb{Z})=G_{l, \mathbb{Z}}^{\prime}(\hat{\mathbb{Z}}) \cap G_{l, \Phi}^{\prime}(\mathbb{Q})$ depending only on $\Phi_{\mathcal{H}}$ (see definition 1.2.2.3 of [44] for an equivalent definition).

The map that sends $g \in \mathrm{G}\left(\mathbb{A}^{\infty}\right)$ to $\left[\left(\mathrm{z}_{\mathcal{H}}^{(i, g)}, \Phi_{\mathcal{H}}^{(i, g)}, \delta_{\mathcal{H}}\right)\right]$ (for any $\delta$ ) sets up a bijection between the double coset space

$$
\left(\mathrm{G}_{l, \Phi^{(i, 1)}}^{\prime}(\mathbb{Q}) \ltimes \mathrm{P}_{\mathrm{Z}^{(i, 1)}}^{\prime}\left(\mathbb{A}^{\infty}\right)\right) \backslash \mathrm{G}\left(\mathbb{A}^{\infty}\right) / \mathcal{H}
$$

and cusp labels $\left[\left(\mathrm{Z}_{\mathcal{H}}, \Phi_{\mathcal{H}}, \delta_{\mathcal{H}}\right)\right]$ such that $\mathrm{Gr}_{0}^{\mathrm{Z}}$ has rank $i$ over $\mathcal{O} \otimes_{\mathbb{Z}} \hat{\mathbb{Z}}$. This identifies the set of cusp labels of the form $\left[\left(\mathrm{z}_{\mathcal{H}}^{(i, g)}, \Phi_{\mathcal{H}}, \delta_{\mathcal{H}}\right)\right]$ with

$$
\mathrm{G}_{l, \Phi^{(i, 1)}}^{\prime}(\mathbb{Q}) \backslash \mathrm{G}_{l, \mathrm{Z}^{(i, 1)}}^{\prime}\left(\mathbb{A}^{\infty}\right) /\left(g \mathcal{H} g^{-1}\right)_{\mathrm{G}_{l, \mathbb{Z}^{(i, 1)}}^{\prime}}
$$


This last double coset space is the index set of

$$
Y_{n,(i), g U g^{-1} \cap P_{n,(i)}^{+}\left(\mathbb{A}^{\infty}\right)}^{\natural}=\coprod_{L_{n,(i), \operatorname{lin}}(\mathbb{Q}) \backslash L_{n,(i), \operatorname{lin}}\left(\mathbb{A}^{\infty}\right) /\left(\operatorname{gUg} g^{-1} \cap P_{n,(i)}^{+}\left(\mathbb{A}^{\infty}\right)\right)} \operatorname{Spec} \mathbb{Q}
$$

in this paper (see Sect. 4.1), with $U=\mathcal{H}$.

\section{B.4 Minimal compactifications in characteristic zero}

For each neat open compact subgroup $\mathcal{H}=U$, there is a normal projective scheme

$$
\mathrm{M}_{\mathcal{H}}^{\min }=X_{n, U}^{\min }
$$

containing $\mathrm{M}_{\mathcal{H}}=X_{n, U}$ as an open dense subscheme (see theorem 1.3.1.5 of [44]).

The minimal compactification $M_{\mathcal{H}}^{\min }$ admits a stratification by locally closed subschemes

$$
\mathrm{M}_{\mathcal{H}}^{\min }=\coprod_{\left[\left(\Phi_{\mathcal{H}}, \delta_{\mathcal{H}}\right)\right]} \mathrm{Z}_{\left[\left(\Phi_{\mathcal{H}}, \delta_{\mathcal{H}}\right)\right]}=\coprod_{i=0}^{n} \coprod_{g} \mathrm{Z}_{\left[\left(\Phi_{\mathcal{H}}^{(i, g)}, \delta_{\mathcal{H}}\right)\right]}
$$

where $\left[\left(\Phi_{\mathcal{H}}, \delta_{\mathcal{H}}\right)\right]$ runs over cusp labels for $M_{\mathcal{H}}$ and $g$ runs over the double quotient $\left(\mathrm{G}_{l, \Phi^{(i, 1)}}^{\prime}(\mathbb{Q}) \ltimes \mathrm{P}_{\mathrm{Z}^{(i, 1)}}^{\prime}\left(\mathbb{A}^{\infty}\right)\right) \backslash \mathrm{G}\left(\mathbb{A}^{\infty}\right) / \mathcal{H}$ (see theorem 1.3.1.5(4) of $\left.[44]\right)$. If $\mathcal{H}=U$ this induces

$$
\partial_{i}^{0} X_{n, U}^{\min }=\coprod_{g} \mathrm{Z}_{\left[\left(\Phi_{\mathcal{H}}^{(i, g)}, \delta_{\mathcal{H}}\right)\right]^{\cdot}}
$$

The decomposition

$$
\partial_{i}^{0} X_{n, U}^{\min }=\coprod_{g \in P_{n, i}^{+}\left(\mathbb{A}^{\infty}\right) \backslash G_{n}\left(\mathbb{A}^{\infty}\right) / U} X_{n,(i), g U g^{-1} \cap P_{n,(i)}^{+}\left(\mathbb{A}^{\infty}\right)}^{\natural}
$$

(see Sect. 5.1) corresponds to

$$
X_{n,(i), g U g^{-1} \cap P_{n,(i)}^{+}\left(\mathbb{A}^{\infty}\right)}^{\natural}=\coprod_{h \in L_{n,(i), \operatorname{lin}}(\mathbb{Q}) \backslash L_{n,(i), \operatorname{lin}}\left(\mathbb{A}^{\infty}\right) /\left(g U g^{-1} \cap P_{n,(i)}^{+}\left(\mathbb{A}^{\infty}\right)\right)} \mathrm{Z}_{\left[\left(\Phi_{\mathcal{H}}^{(i, h g)}, \delta \mathcal{H}\right)\right]^{.}}
$$

Each $\mathrm{Z}_{\left[\left(\Phi_{\mathcal{H}}, \delta_{\mathcal{H}}\right)\right]}$ is the quotient $\mathrm{M}_{\mathcal{H}}^{\mathrm{Z} \mathcal{H}}$ of

$$
\mathrm{M}_{\mathcal{H}}^{\Phi_{\mathcal{H}}} \cong \mathrm{M}_{\mathcal{H}_{\mathrm{G}, \mathrm{Z}}^{\prime}}
$$

by $\Gamma_{\Phi_{\mathcal{H}}}$, which is isomorphic to $\mathrm{M}_{\mathcal{H}_{\mathrm{G}, \mathrm{Z}}, \Phi}$, where $\mathcal{H}_{\mathrm{G}_{h, \mathrm{Z}}, \Phi}$ is the image of $\mathcal{H}_{\mathrm{P}_{\mathrm{Z}}} \cap\left(\mathrm{G}_{l, \Phi}^{\prime}(\mathbb{Q}) \ltimes\right.$ $\mathrm{P}_{\mathrm{Z}}^{\prime}\left(\mathbb{A}^{\infty}\right)$ ) in $\mathrm{G}_{h, \mathrm{Z}}\left(\mathbb{A}^{\infty}\right)$ (see lemmas 1.3.2.1 and 1.3.2.5 of [44]). Here $\mathrm{M}_{\mathcal{H}_{\mathrm{G}_{h, \mathrm{Z}}^{\prime}}}$ and $\mathrm{M}_{\mathcal{H}_{\mathrm{G}}, \mathrm{Z}^{,}}$are moduli problems analogously defined by some integral PEL datum $\left(\mathcal{O}, \star, L^{\mathrm{Z}},\langle\cdot, \cdot\rangle^{\mathrm{Z}}, h_{0}^{\mathrm{Z}}\right)$ associated with a representative $\mathrm{Z}$ of $\mathrm{Z}_{\mathcal{H}}$ (see definition 1.2.1.15 and lemmas 1.3.2.1 and 1.3.2.5 of [44]). If we let $U_{g}^{\prime}$ (resp. $\left.U_{g, \text { lin }}^{\prime}\right)$ denote the image of $g U g^{-1} \cap P_{n,(i)}^{+}\left(\mathbb{A}^{\infty}\right)$ in $L_{n,(i)}\left(\mathbb{A}^{\infty}\right)$ (resp. $L_{n,(i), \text { lin }}\left(\mathbb{A}^{\infty}\right)$ ), then under the identifications

$$
\begin{aligned}
X_{n,(i), U_{g}^{\prime}}^{\natural} & =L_{n,(i), \operatorname{lin}}(\mathbb{Q}) \backslash X_{n,(i), U_{g}^{\prime}}^{+} \\
& =L_{n,(i), \operatorname{lin}}(\mathbb{Q}) \backslash\left(\coprod_{h \in L_{n,(i), \operatorname{lin}}\left(\mathbb{A}^{\infty}\right) /\left(U_{g}^{\prime} \cap L_{n,(i), \operatorname{lin}}\left(\mathbb{A}^{\infty}\right)\right)} X_{n,(i), U_{g}^{\prime} \cap G_{n-i}\left(\mathbb{A}^{\infty}\right)}\right) / U_{g}^{\prime} \\
& =\coprod_{h \in L_{n,(i), \operatorname{lin}}(\mathbb{Q}) \backslash L_{n,(i), \operatorname{lin}}\left(\mathbb{A}^{\infty}\right) / U_{g, \operatorname{lin}}^{\prime}}\left(\left(L_{n,(i), \operatorname{lin}}(\mathbb{Q}) \cap h U_{g, \operatorname{lin}}^{\prime} h^{-1}\right) \backslash X_{\left.n,(i), U_{g}^{\prime} \cap G_{n-i}\left(\mathbb{A}^{\infty}\right)\right),}\right.
\end{aligned}
$$

of Sect. 4.1, 
- the term $X_{n,(i), U_{g}^{\prime} \cap G_{n-i}\left(\mathbb{A}^{\infty}\right)}$ indexed by $h$ is identified with $\mathrm{M}_{\mathcal{H}}^{\Phi_{\mathcal{H}}^{(i, h)}}$

- the group $L_{n,(i), \operatorname{lin}}(\mathbb{Q}) \cap h U_{g, \operatorname{lin}}^{\prime} h^{-1}$ is identified with $\Gamma_{\Phi_{\mathcal{H}}^{(i h g)}}$;

- and the term

$$
\left(L_{n,(i), \operatorname{lin}}(\mathbb{Q}) \cap h U_{g, \operatorname{lin}}^{\prime} h^{-1}\right) \backslash X_{n,(i), U_{g}^{\prime} \cap G_{n-i}\left(\mathbb{A}^{\infty}\right)}
$$

is identified with $\mathrm{M}_{\mathcal{H}}^{\mathrm{z}_{\mathcal{H}}^{(i, h g)}}$.

Similarly, under the identification

$$
X_{n,(i), U_{g}^{\prime}}^{+}=\coprod_{h \in L_{n,(i), \operatorname{lin}}\left(\mathbb{A}^{\infty}\right) / U_{g}^{\prime}} X_{n,(i), U_{g}^{\prime} \cap G_{n-i}\left(\mathbb{A}^{\infty}\right)}
$$

the term $X_{n,(i), U_{g}^{\prime} \cap G_{n-i}\left(\mathbb{A}^{\infty}\right)}$ indexed by $h$ is identified with $\mathrm{M}_{\mathcal{H}}^{\Phi_{\mathcal{H}}^{(i, h)}}$. These identifications are all Hecke equivariant.

\section{B.5 Toroidal compactifications of Shimura varieties in characteristic zero}

For each representative $\left(\mathrm{Z}_{\mathcal{H}}, \Phi_{\mathcal{H}}, \delta_{\mathcal{H}}\right)$ of a cusp label $\left[\left(\mathrm{z}_{\mathcal{H}}, \Phi_{\mathcal{H}}, \delta_{\mathcal{H}}\right)\right]$ for $\mathrm{M}_{\mathcal{H}}$, there is a torsor

$$
C_{\Phi_{\mathcal{H}}, \delta_{\mathcal{H}}} \rightarrow \mathrm{M}_{\mathcal{H}}^{\Phi_{\mathcal{H}}}
$$

of an abelian scheme

$$
C_{\Phi_{\mathcal{H}}, \delta_{\mathcal{H}}}^{\mathrm{grp}} \rightarrow \mathrm{M}_{\mathcal{H}}^{\Phi_{\mathcal{H}}}
$$

(see lemma 1.3.2.7 and propositions 1.3.2.12 and 1.3.2.14 of [44]). The abelian scheme $C_{\Phi_{\mathcal{H}}, \delta_{\mathcal{H}}}^{\text {grp }}$ over $\mathrm{M}_{\mathcal{H}}^{\Phi_{\mathcal{H}}}$ is $\mathbb{Q}^{\times}$-isogenous (i.e. quasi-isogenous) to $\underline{\operatorname{Hom}}_{\mathcal{O}}(X, B)$ (where $B$ is the pull-back of the universal abelian scheme over $\mathrm{M}_{\mathcal{H}}^{Z \mathcal{H}}$ ). We obtain an isomorphic abelian scheme torsor if we replace $\left(\mathrm{Z}_{\mathcal{H}}, \Phi_{\mathcal{H}}, \delta_{\mathcal{H}}\right)$ with another representative (but its universal property, which we have not described here, depends on this choice of representative).

If $U_{g}^{\prime}$ again denotes $g U g^{-1} \cap P_{n,(i)}^{+}\left(\mathbb{A}^{\infty}\right)$, then the map

$$
\begin{array}{ccc}
A_{n,(i), U_{g}^{\prime}}^{+} & =\bigsqcup_{h \in L_{n,(i), \operatorname{lin}}\left(\mathbb{A}^{\infty}\right) / U_{g}^{\prime}} A_{n-i, h g U g^{-1} h^{-1} \cap P_{n,(i)}\left(\mathbb{A}^{\infty}\right)}^{(i)} \\
\downarrow & \downarrow \\
X_{n,(i), U_{g}^{\prime}}^{+} & \bigsqcup_{h \in L_{n,(i), \operatorname{lin}}\left(\mathbb{A}^{\infty}\right) / U_{g}^{\prime}} X_{n,(i), U_{g}^{\prime} \cap P_{n,(i)}\left(\mathbb{A}^{\infty}\right)}
\end{array}
$$

in this paper (see Sects. 4.1,4.2) with $U=\mathcal{H}$ is identified with

$$
\begin{gathered}
\bigsqcup_{h \in L_{n,(i), \operatorname{lin}}\left(\mathbb{A}^{\infty}\right) / U_{g}^{\prime}} C_{\Phi_{\mathcal{H}}^{(i, h g)}, \delta_{\mathcal{H}}} \\
\downarrow \\
\bigsqcup_{h \in L_{n,(i), \operatorname{lin}}\left(\mathbb{A}^{\infty}\right) / U_{g}^{\prime}} \mathrm{M}_{\mathcal{H}}^{\Phi_{\mathcal{H}}^{(i, h g)}} .
\end{gathered}
$$

These maps are $\mathrm{P}_{\mathrm{Z}^{(i, g)}}\left(\mathbb{A}^{\infty}\right) / \mathrm{U}_{2, \mathrm{Z}^{(i, g)}}\left(\mathbb{A}^{\infty}\right)$-equivariant. (See proposition 1.3.2.24 of [44].) (Since our pairing $\langle\cdot, \cdot\rangle$ is perfect, the universal property of $C_{\Phi_{\mathcal{H}}, \delta_{\mathcal{H}}}$ in proposition 1.3.2.14 of [44] can be simplified by suppressing the dual objects. This universal property then agrees with that of $A_{n-i, h g U g^{-1} h^{-1} \cap P_{n,(i)}\left(\mathbb{A}^{\infty}\right)}^{(i)}$ in Sect. 3.2 of this paper.) 
For each representative $\left(\mathrm{Z}_{\mathcal{H}}, \Phi_{\mathcal{H}}, \delta_{\mathcal{H}}\right)$ of a cusp label $\left[\left(\mathrm{Z}_{\mathcal{H}}, \Phi_{\mathcal{H}}, \delta_{\mathcal{H}}\right)\right]$ for $\mathrm{M}_{\mathcal{H}}$, there is a torsor

$$
\Xi_{\Phi_{\mathcal{H}}, \delta \mathcal{H}} \rightarrow C_{\Phi_{\mathcal{H}}, \delta \mathcal{H}}
$$

under the pull-back of a split torus

$$
E_{\Phi_{\mathcal{H}}}
$$

over Spec $\mathbb{Z}$ with character group

$$
\mathbf{S}_{\Phi_{\mathcal{H}}}
$$

(see lemma 1.3.2.25 and proposition 1.3.2.31 of [44]). We obtain an isomorphic torus torsor if we replace $\left(\mathrm{Z}_{\mathcal{H}}, \Phi_{\mathcal{H}}, \delta_{\mathcal{H}}\right)$ with another representative, but its universal property depends on this choice of representative. For a fixed $(Z, \Phi, \delta)$, the morphisms

$$
\Xi_{\Phi_{\mathcal{H}}, \delta_{\mathcal{H}}} \rightarrow C_{\Phi_{\mathcal{H}}, \delta_{\mathcal{H}}}
$$

are $\mathrm{P}_{\mathrm{Z}}^{\prime}\left(\mathbb{A}^{\infty}\right)$-equivariant. (See proposition 1.3.2.45 of [44].)

When $\mathcal{H}_{\mathrm{U}_{2, \mathrm{Z}}}=\mathrm{G}(\hat{\mathbb{Z}})_{\mathrm{U}_{2, \mathrm{Z}}}=\mathrm{U}_{2, \mathrm{Z}}(\hat{\mathbb{Z}})$ and when $\Phi_{\mathcal{H}}$ is represented by some $\Phi=$ $\left(X, Y, \phi, \varphi_{-2}, \varphi_{0}\right)$ (where $\phi: Y \hookrightarrow X$ must be an isomorphism, as explained above), the group $\mathbf{S}_{\Phi_{\mathcal{H}}}$ is the group $S(Y)^{\mathrm{TF}}$ in the notation of this paper (see Sect. 1.1). For more general $\mathcal{H}$, we set $\mathbf{S}_{\Phi_{1}}=S(Y)^{\mathrm{TF}}$ (see section 1.2.2 of [44]), and then $\mathbf{S}_{\Phi_{\mathcal{H}}}$ is the unique lattice in $\mathbf{S}_{\Phi_{1}} \otimes_{\mathbb{Z}} \mathbb{Q}$ such that $\mathbf{S}_{\Phi_{\mathcal{H}}} / \mathbf{S}_{\Phi_{1}} \cong \mathbf{S}_{\Phi_{1}}^{\vee} / \mathbf{S}_{\Phi_{\mathcal{H}}}^{\vee} \cong \mathrm{U}_{2, \mathrm{Z}}(\hat{\mathbb{Z}}) / \mathcal{H}_{\mathrm{U}_{2, \mathrm{Z}}}$ (see proposition 1.3.2.31 of [44]), where the superscript ${ }^{\vee}$ denotes the dual of $\mathbb{Z}$-modules. Then $\left(\mathbf{S}_{\Phi_{\mathcal{H}}}\right)_{\mathbb{R}}^{\vee}$ can be identified with the space of hermitian forms over $Y \otimes_{\mathbb{Z}} \mathbb{R}$, and we define $\mathbf{P}_{\Phi_{\mathcal{H}}}$ (resp. $\left.\mathbf{P}_{\Phi_{\mathcal{H}}}^{+}\right)$to be the subset of $\left(\mathbf{S}_{\Phi_{\mathcal{H}}}\right)_{\mathbb{R}}^{\vee}$ corresponding to positive semi-definite hermitian forms with rational radicals (resp. positive definite hermitian forms). When $\mathrm{Z}_{\mathcal{H}}=\mathrm{Z}_{\mathcal{H}}^{(i, g)}$ for some $g \in \mathrm{G}\left(\mathbb{A}^{\infty}\right)$, we have $Y \otimes_{\mathbb{Z}} \mathbb{Q} \cong F^{i}$; and the sets $\mathbf{S}_{\Phi_{\mathcal{H}}} \otimes_{\mathbb{Z}} \mathbb{Q}$ and $\mathbf{P}_{\Phi_{\mathcal{H}}}^{+}$are the sets $S\left(F^{i}\right)$ and Herm $_{F^{i}}^{>0}$, respectively, in this paper (see Sects. 1.1, 1.4).

The torus

$$
S_{n,(i), U_{g}^{\prime}}^{+} \rightarrow Y_{n,(i), U_{g}^{\prime}}^{+}
$$

in this paper (see Sect. 4.3) with $U=\mathcal{H}$ is identified with

$$
\coprod_{h \in L_{h,(i), \operatorname{lin}}\left(\mathbb{A}^{\infty}\right) / U_{g}^{\prime}} E_{\Phi_{\mathcal{H}}^{(i, h g)}, \delta_{\mathcal{H}}} \rightarrow \coprod_{h \in L_{n,(i), \operatorname{lin}}\left(\mathbb{A}^{\infty}\right) / U_{g}^{\prime}} \operatorname{Spec} \mathbb{Q}
$$

Moreover, the sheaves $X^{*}\left(S_{n,(i), U_{g}^{\prime}}^{+}\right), X_{*}\left(S_{n,(i), U_{g}^{\prime}}^{+}\right)_{\mathbb{R}}^{\succ 0}$, and $X_{*}\left(S_{n,(i), U_{g}^{\prime}}^{+}\right)_{\mathbb{R}}^{>0}$ are identified with

$$
\coprod_{h \in L_{n,(i), \operatorname{lin}}\left(\mathbb{A}^{\infty}\right) / U_{g}^{\prime}} \mathbf{S}_{\Phi_{\mathcal{H}}^{(i, h g)}}, \quad \coprod_{h \in L_{n,(i), \operatorname{lin}}\left(\mathbb{A}^{\infty}\right) / U_{g}^{\prime}} \mathbf{P}_{\Phi_{\mathcal{H}}^{(i, h g)}} \quad \text { and } \quad \coprod_{h \in L_{n,(i), \operatorname{lin}}\left(\mathbb{A}^{\infty}\right) / U_{g}^{\prime}} \mathbf{P}_{\Phi_{\mathcal{H}}^{(i, h g)}}^{+}
$$


respectively. The $S_{n,(i), U_{g}^{\prime}}^{+}$-torsor

$$
\begin{array}{ccc}
T_{n,(i), U_{g}^{\prime}}^{+} & =\coprod_{\left.h \in L_{n, i}\right) \operatorname{lin}\left(\mathbb{A}^{\infty}\right) / U_{g}^{\prime}} T_{n-i, h g U g^{-1} h^{-1} \cap P_{n,(i)}\left(\mathbb{A}^{\infty}\right)}^{(i)} \\
\quad \downarrow & \downarrow \\
A_{n,(i), U_{g}^{\prime}}^{+} & \coprod_{h \in L_{n, i}, \operatorname{lin}\left(\mathbb{A}^{\infty}\right) / U_{g}^{\prime}} A_{n-i, h g U g^{-1} h^{-1} \cap P_{n,(i)}\left(\mathbb{A}^{\infty}\right)}^{(i)}
\end{array}
$$

is identified with

$$
\begin{gathered}
\bigsqcup_{h \in L_{h,(i), \operatorname{lin}\left(\mathbb{A}^{\infty}\right) / U_{g}^{\prime}}} \Xi_{\Phi_{\mathcal{H}}^{(i, h g)}, \delta_{\mathcal{H}}} \\
\downarrow \\
\amalg_{h \in L_{h,(i), \text { lin }}\left(\mathbb{A}^{\infty}\right) / U_{g}^{\prime}} C_{\Phi_{\mathcal{H}}^{(i, h g)}, \delta_{\mathcal{H}}}
\end{gathered}
$$

These maps are $\mathrm{P}_{\mathrm{Z}}^{(i, g)}\left(\mathbb{A}^{\infty}\right)$-equivariant.

Consider any compatible collection

$$
\Sigma=\left\{\Sigma_{\Phi_{\mathcal{H}}}\right\}_{\left[\left(\Phi_{\mathcal{H}}, \delta_{\mathcal{H}}\right)\right]}
$$

of admissible projective smooth rational polyhedral cone decompositions for $\mathrm{M}_{\mathcal{H}}$, where each $\Sigma_{\Phi_{\mathcal{H}}}$ is a $\Gamma_{\Phi_{\mathcal{H}}}$-admissible projective smooth rational polyhedral cone decomposition of $\mathbf{P}_{\Phi_{\mathcal{H}}}$, as in definitions 1.2.2.13 and 1.2.2.14 of [44]. (Note that in [44] rational polyhedral cones are open cones, whereas in this paper they are closed cones.) Each such $\Sigma$ considered in [44] induces a pair

in $\mathcal{J}_{n}^{\text {tor }}$ (with $U=\mathcal{H}$ ) in Sect. 5.2 of this paper, because, in order to define $(U, \Delta)$ as in Sect. 5.2, it suffices to define the admissible cone decomposition $\Delta(g)_{0}$ for $X_{*}\left(S_{n,(i) g U g^{-1} \cap P_{n,(i)}^{+}\left(\mathbb{A}^{\infty}\right)}^{+}\right)_{\mathbb{R}}^{\succ 0}$, for each $g \in \mathrm{G}\left(\mathbb{A}^{\infty}\right)$, which can be taken to be the pull-back of the subcollection $\left\{\Sigma_{\Phi_{\mathcal{H}}}\right\}_{\left[\left(\Phi_{\mathcal{H}}, \delta_{\mathcal{H}}\right)\right]}$ of $\Sigma$ indexed by the cusp labels $\left[\left(\Phi_{\mathcal{H}}, \delta_{\mathcal{H}}\right)\right]$ with underlying $\mathrm{z}_{\mathcal{H}}$ equal to $\mathrm{z}_{\mathcal{H}}^{(i, g)}$. In fact, $\mathcal{J}_{n}^{\text {tor }}$ is exactly the set of such induced pairs (as $U=\mathcal{H}$ varies).

Each $\Sigma_{\Phi_{\mathcal{H}}}$ defines an affine toroidal embedding

$$
\Xi_{\Phi_{\mathcal{H}}, \delta_{\mathcal{H}}} \hookrightarrow \bar{\Xi}_{\Phi_{\mathcal{H}}, \delta_{\mathcal{H}}}=\bar{\Xi}_{\Phi_{\mathcal{H}}, \delta_{\mathcal{H}}, \Sigma_{\Phi_{\mathcal{H}}}}=\bigcup_{\sigma \in \Sigma_{\Phi_{\mathcal{H}}}} \Xi_{\Phi_{\mathcal{H}}, \delta_{\mathcal{H}}}(\sigma)=\coprod_{\sigma \in \Sigma_{\Phi_{\mathcal{H}}}} \Xi_{\Phi_{\mathcal{H}}, \delta_{\mathcal{H}}, \sigma}
$$

over $C_{\Phi_{\mathcal{H}}, \delta_{\mathcal{H}}}$. Rather confusingly $\Xi_{\Phi_{\mathcal{H}}, \delta_{\mathcal{H}}}(\sigma)$ (in the notation of [44]) is what in this paper we would have denoted $\Xi_{\Phi_{\mathcal{H}}, \delta_{\mathcal{H}}, \sigma}$; and $\Xi_{\Phi_{\mathcal{H}}, \delta_{\mathcal{H}}, \sigma}$ is what in this paper we would have denoted $\partial_{\sigma} \Xi_{\Phi_{\mathcal{H}}, \delta_{\mathcal{H}}, \Sigma_{\Phi_{\mathcal{H}}}}$. The formal completion of $\bar{\Xi}_{\Phi_{\mathcal{H}}, \delta_{\mathcal{H}}, \Sigma_{\Phi_{\mathcal{H}}}}$ along the union of the $\sigma$-strata $\Xi_{\Phi_{\mathcal{H}}, \delta_{\mathcal{H}}, \sigma}$ for all $\sigma \in \Sigma_{\Phi_{\mathcal{H}}}$ such that $\sigma \cap \mathbf{P}_{\Phi_{\mathcal{H}}}^{+} \neq \emptyset$ is denoted

$$
\mathfrak{X}_{\Phi_{\mathcal{H}}, \delta_{\mathcal{H}}}=\mathfrak{X}_{\Phi_{\mathcal{H}}, \delta_{\mathcal{H}}, \Sigma_{\Phi_{\mathcal{H}}}} .
$$

(See (1.3.2.34), (1.3.2.35), (1.3.2.36), and lemma 1.3.2.41 of [44].) The schemes

$$
T_{n,(i), U_{g}^{\prime}, \Delta(g)_{0}}^{+} \rightarrow A_{n,(i), U_{g}^{\prime}}^{+}
$$


of this paper (see Sect. 4.4) are identified with

$$
\coprod_{h \in L_{n,(i), \operatorname{lin}}\left(\mathbb{A}^{\infty}\right) / U_{g}^{\prime}} \bar{\Xi}_{\Phi_{\mathcal{H}}^{(i, h g)}, \delta_{\mathcal{H}}} \rightarrow \coprod_{h \in L_{n,(i), \text { lin }}\left(\mathbb{A}^{\infty}\right) / U_{g}^{\prime}} C_{\Phi_{\mathcal{H}}^{(i, h g)}, \delta_{\mathcal{H}}} .
$$

Moreover, $T_{n,(i), U_{g}^{\prime}, \Delta(g)_{0}}^{+, \wedge}$ is identified with

$$
\coprod_{h \in L_{n,(i), \operatorname{lin}}\left(\mathbb{A}^{\infty}\right) / U_{g}^{\prime}} \mathfrak{X}_{\Phi_{\mathcal{H}}^{(i, h)}, \delta_{\mathcal{H}}}
$$

and $T_{n,(i), U_{g}^{\prime}, \Delta(g)_{0}}^{\natural, \wedge}$ is identified with

$$
\begin{aligned}
& \quad \coprod_{h \in L_{n,}(i), \operatorname{lin}(\mathbb{Q}) \backslash L_{n,(i), \operatorname{lin}}\left(\mathbb{A}^{\infty}\right) / U_{g}^{\prime}}\left(\mathfrak{X}_{\Phi_{\mathcal{H}}^{(i, h g)}, \delta_{\mathcal{H}}} / \Gamma_{\Phi_{\mathcal{H}}^{(i, h)}}\right) \\
& =\coprod_{\left[\left(\Phi_{\mathcal{H}}, \delta_{\mathcal{H}}\right)\right]}\left(\mathfrak{X}_{\Phi_{\mathcal{H}}, \delta_{\mathcal{H}}} / \Gamma_{\Phi_{\mathcal{H}}}\right),
\end{aligned}
$$

where the second disjoint union runs over all cusp labels $\left[\left(\Phi_{\mathcal{H}}, \delta_{\mathcal{H}}\right)\right]$ with underlying $\mathrm{Z}_{\mathcal{H}}$ equal to $\mathrm{z}_{\mathcal{H}}^{(i, g)}$. (Again $U=\mathcal{H}$.)

For each pair $(\mathcal{H}, \Sigma)$ as above, we have a smooth projective scheme

$$
\mathrm{M}_{\mathcal{H}, \Sigma}^{\text {tor }}
$$

containing $\mathrm{M}_{\mathcal{H}}$ as an open dense subscheme, called a toroidal compactification of $\mathrm{M}_{\mathcal{H}}$ (see theorems 1.3.1.3 and 1.3.1.10 of [44]). This is the

$$
X_{n, U, \Delta}
$$

in this paper (see Sect. 5.3), with $U=\mathcal{H}$ and with $(U, \Delta) \in \mathcal{J}_{n}^{\text {tor }}$ induced by $\Sigma$ as above. This identification is compatible with the actions of $\mathrm{G}\left(\mathbb{A}^{\infty}\right)$ on the collections $\left\{\mathrm{M}_{\mathcal{H}, \Sigma}^{\text {tor }}\right\}_{(\mathcal{H}, \Sigma)}$ (see proposition 1.3.1.15 of [44]) and $\left\{X_{n, U, \Delta}\right\}_{(U, \Delta)}$.

The toroidal compactification $\mathrm{M}_{\mathcal{H}, \Sigma}^{\text {tor }}$ admits a stratification by locally closed subschemes

$$
\mathrm{M}_{\mathcal{H}, \Sigma}^{\text {tor }}=\coprod_{\left[\left(\Phi_{\mathcal{H}}, \delta_{\mathcal{H}}, \sigma\right)\right]} \mathrm{Z}_{\left[\left(\Phi_{\mathcal{H}}, \delta_{\mathcal{H}}, \sigma\right)\right]}
$$

(see theorem 1.3.1.3(2) of [44]) indexed by equivalence classes $\left[\left(\Phi_{\mathcal{H}}, \delta_{\mathcal{H}}, \sigma\right)\right]$ as in definition 1.2.2.10 of [44]. Without repeating the definition in detail, let us just note that the equivalence classes $\left[\left(\Phi_{\mathcal{H}}, \delta_{\mathcal{H}}, \sigma\right)\right]$ with the same underlying cusp label $\left[\left(\Phi_{\mathcal{H}}, \delta_{\mathcal{H}}\right)\right]$ can be identified with the $\Gamma_{\Phi_{\mathcal{H}}}$-orbits of the cones $\sigma \in \Sigma_{\Phi_{\mathcal{H}}}$ such that $\sigma \cap \mathbf{P}_{\Phi_{\mathcal{H}}}^{+} \neq \emptyset$. Each stratum $\mathrm{Z}_{\left[\left(\Phi_{\mathcal{H}}, \delta_{\mathcal{H}}, \sigma\right)\right]}$ is canonically isomorphic to $\Xi_{\Phi_{\mathcal{H}}, \delta_{\mathcal{H}}, \sigma}$, and the formal completion of $\mathrm{M}_{\mathcal{H}, \Sigma}^{\text {tor }}$ along the union of the strata $Z_{\left[\left(\Phi_{\mathcal{H}}, \delta_{\mathcal{H}}, \sigma\right)\right]}$ labelled by equivalence classes $\left[\left(\Phi_{\mathcal{H}}, \delta_{\mathcal{H}}, \sigma\right)\right]$ with the same underlying cusp label $\left[\left(\Phi_{\mathcal{H}}, \delta_{\mathcal{H}}\right)\right]$ is canonically isomorphic to $\mathfrak{X}_{\Phi_{\mathcal{H}}, \delta_{\mathcal{H}}} / \Gamma_{\Phi_{\mathcal{H}}}$.

When $U=\mathcal{H}$, and when $(U, \Delta) \in \mathcal{J}_{n}^{\text {tor }}$ is induced by $\Sigma$ as above, the formal completion of $\mathrm{M}_{\mathcal{H}, \Sigma}^{\text {tor }}$ along the union of all strata $\mathrm{Z}_{\left[\left(\Phi_{\mathcal{H}}, \delta_{\mathcal{H}}, \sigma\right)\right]}$ labelled by equivalence classes $\left[\left(\Phi_{\mathcal{H}}, \delta_{\mathcal{H}}, \sigma\right)\right]$ with underlying $\mathrm{z}_{\mathcal{H}}$ equal to $\mathrm{z}_{\mathcal{H}}^{(i, g)}$ for some $g \in \mathrm{G}\left(\mathbb{A}^{\infty}\right)$ is

$$
X_{n, U, \Delta, i}^{\wedge} \cong \coprod_{g} T_{n,(i), g U g^{-1} \cap P_{n,(i)}^{+}\left(\mathbb{A}^{\infty}\right), \Delta(g)_{0}}=\coprod_{g} \coprod_{h}\left(\mathfrak{X}_{\Phi_{\mathcal{H}}^{(i, h), \delta \mathcal{H}}} / \Gamma_{\Phi_{\mathcal{H}}^{(i, h g)}}\right)
$$


where the indices $g$ and $h$ run over $P_{n,(i)}^{+}\left(\mathbb{A}^{\infty}\right) \backslash G_{n}\left(\mathbb{A}^{\infty}\right) / U$ and

$$
L_{n,(i), \operatorname{lin}}(\mathbb{Q}) \backslash L_{n,(i), \operatorname{lin}}\left(\mathbb{A}^{\infty}\right) /\left(g U g^{-1} \cap P_{n,(i)}^{+}\left(\mathbb{A}^{\infty}\right)\right),
$$

respectively, in this paper (see Sect. 5.3).

These identifications are all Hecke equivariant (see proposition 1.3.2.45 of [44]).

\section{B.6 Kuga families in characteristic zero}

Consider any $\mathcal{O}$-lattice $Q$. Define $\widehat{G}$ to be the subgroup of automorphisms of $L \oplus Q$ which preserve $L$, act trivially on the quotient $(L \oplus Q) / L$ and preserve, up to scalar multiples, the pairing $\langle\cdot, \cdot\rangle$ on $L$. Restriction to $L$ gives a surjective homomorphism $\widehat{G} \rightarrow G$, and we denote the kernel $\widehat{U}$. This homomorphism is naturally split. (Compare with definition 1.2.4.3 of [44].) If $Q=\mathcal{O}_{F}^{m}$, then $\widehat{\mathrm{G}}=G_{n}^{(m)}$ and $\widehat{\mathrm{U}}=\operatorname{Hom}_{n}^{(m)}$. If $\widehat{\mathcal{H}}$ is any open compact subgroup of $\widehat{\mathrm{G}}(\hat{\mathbb{Z}})$, then $\widehat{\mathcal{H}}_{\widehat{\mathrm{U}}}$ denotes $\widehat{\mathcal{H}} \cap \widehat{\mathrm{U}}(\hat{\mathbb{Z}})$, and $\widehat{\mathcal{H}}_{\mathrm{G}}$ denotes $\widehat{\mathcal{H}} / \widehat{\mathcal{H}}_{\widehat{\mathrm{U}}}$.

To each neat open compact subgroup $\widehat{\mathcal{H}}$ of $\widehat{\mathrm{G}}(\hat{\mathbb{Z}})$ with image $\mathcal{H}=\widehat{\mathcal{H}}_{\mathrm{G}}$ in $\mathrm{G}(\hat{\mathbb{Z}})$, [44] attached a generalized Kuga family $\mathrm{N} \rightarrow \mathrm{M}_{\mathcal{H}}$. (See definition 1.3.3.4 of [44].) If $Q=\mathcal{O}_{F}^{m}$ and $U=\widehat{\mathcal{H}}$, then it is the scheme denoted $A_{n, U}^{(m)}$ in this paper. The generalized Kuga family attached to $\widehat{\mathcal{H}}_{\mathrm{G}} \ltimes \widehat{\mathcal{H}}_{\widehat{\mathrm{U}}}$ is denoted $\mathrm{N}^{\text {grp }} \rightarrow \mathrm{M}_{\mathcal{H}}$, and is a Kuga family as in definition 1.3.3.3 of [44]. It is an abelian scheme $\mathbb{Q}^{\times}$-isogenous to the $m$-fold fibre product of the universal abelian scheme over $\mathrm{M}_{\mathcal{H}}$ when $Q \cong \mathcal{O}_{F}^{m}$. The generalized Kuga family $\mathrm{N} \rightarrow \mathrm{M}_{\mathcal{H}}$ is a torsor for $\mathrm{N}^{\text {grp }} \rightarrow \mathrm{M}_{\mathcal{H}}$.

To study these schemes and their compactifications [44] realizes them, in a noncanonical way, inside the boundary of a larger Shimura variety. Concretely, as in section 1.2.4 of [44], to define such generalized Kuga families, we start with an $\mathcal{O}$-lattice $Q$; consider

$$
\left.Q_{-2}:=\operatorname{Hom}_{\mathcal{O}}\left(Q, \operatorname{Diff}_{\mathcal{O} / \mathbb{Z}}^{-1}(1)\right)\right) \text { and } Q_{0}:=Q
$$

(where $\operatorname{Diff}_{\mathcal{O} / \mathbb{Z}}^{-1}$ denotes the inverse different), with the natural perfect pairing

$$
\langle\cdot, \cdot\rangle_{Q}: Q_{-2} \times Q_{0} \rightarrow \mathbb{Z}(1)
$$

induced by the trace pairing; and set

$$
\widetilde{L}:=Q_{-2} \oplus L \oplus Q_{0},
$$

with the (self-dual) pairing

$$
\langle\cdot, \cdot\rangle: \widetilde{L} \times \widetilde{L} \rightarrow \mathbb{Z}(1)
$$

defined by

$$
\left\langle\left(x_{-2}, x_{-1}, x_{0}\right),\left(y_{-2}, y_{-1}, y_{0}\right)\right\rangle^{\sim}=\left\langle x_{-2}, y_{0}\right\rangle_{Q}+\left\langle x_{-1}, y_{-2}\right\rangle-\left\langle y_{-2}, x_{0}\right\rangle_{Q}
$$

for $x_{-2}, y_{-2} \in Q_{-2}, x_{-1}, y_{-1} \in L$, and $x_{0}, y_{0} \in Q_{0}$. We shall fix the choice of

$$
Q=\mathcal{O}_{F}^{m}
$$


in what follows, since this is all we need in this paper. Then the above $\widetilde{L}$ and $\langle\cdot, \cdot\rangle$ can be identified with the $\Lambda_{m+n}$ and $\langle,\rangle_{m+n}: \Lambda_{m+n} \times \Lambda_{m+n} \rightarrow \mathbb{Z}$ in this paper (see Sects. 1.1, B.1), up to reversing the ordering of the coordinates of $Q_{-2}=\left(\operatorname{Diff}_{\mathcal{O}_{F} / \mathbb{Z}}^{-1}(1)\right)^{m}$ and dividing by $2 \pi \sqrt{-1}$. Then there is a natural choice of $\widetilde{h}_{0}: \mathbb{C} \rightarrow \operatorname{End}_{\mathcal{O} \otimes_{\mathbb{Z}} \mathbb{R}}\left(\widetilde{L} \otimes_{\mathbb{Z}} \mathbb{R}\right)$ extending $h_{0}: \mathbb{C} \rightarrow \operatorname{End}_{\mathcal{O} \otimes_{\mathbb{Z}} \mathbb{R}}\left(L \otimes_{\mathbb{Z}} \mathbb{R}\right)$ (see section 1.2.4 of [44]) which makes

$$
\left(\mathcal{O}, \star, \widetilde{L},\left\langle\cdot, \cdot \widetilde{\gamma}, \tilde{h}_{0}\right)\right.
$$

an integral PEL datum as in definition 1.1.1.1 of [44], which defines a group functor $\widetilde{\mathrm{G}}$ and a PEL moduli problem $\widetilde{\mathrm{M}}_{\widetilde{\mathcal{H}}}$ for each open compact subgroup $\widetilde{\mathcal{H}}$ of $\widetilde{\mathrm{G}}(\hat{\mathbb{Z}})$ as in the case of $\mathrm{G}$ and $\mathrm{M}_{\mathcal{H}}$ in Sect. B.1. We shall always denote the analogues of objects for $\left(\mathcal{O}, \star \widetilde{L},\left\langle\cdot, \cdot \widetilde{\gamma}, \widetilde{h}_{0}\right)\right.$ with a tilde , without explicitly introducing them.

By the definition of $\left(\widetilde{L},\langle\cdot, \cdot \gamma)\right.$, there is a fully symplectic admissible filtration $\widetilde{Z}$ on $\widetilde{L} \otimes_{\mathbb{Z}} \hat{\mathbb{Z}}$ induced by

$$
0 \subset Q_{-2} \subset Q_{-2} \oplus L \subset Q_{-2} \oplus L \oplus Q_{0}=\widetilde{L} .
$$

Let $\widetilde{X}:=\operatorname{Hom}_{\mathcal{O}}\left(Q_{-2}, \operatorname{Diff}_{\mathcal{O} / \mathbb{Z}}^{-1}(1)\right)$ and $\widetilde{Y}:=Q_{0}$. The pairing $\langle\cdot, \cdot\rangle_{Q}: Q_{-2} \times Q_{0} \rightarrow \mathbb{Z}(1)$ induces a canonical isomorphism $\widetilde{\phi}: \widetilde{Y} \stackrel{\sim}{\rightarrow} \widetilde{X}$, and there are canonical isomorphisms $\widetilde{\varphi}_{-2}: \mathrm{Gr}_{-2}^{\widetilde{Z}} \stackrel{\sim}{\rightarrow} \operatorname{Hom}_{\hat{\mathbb{Z}}}\left(\widetilde{X} \otimes_{\mathbb{Z}} \hat{\mathbb{Z}}, \hat{\mathbb{Z}}(1)\right)$ and $\widetilde{\varphi}_{0}: \mathrm{Gr}_{0}^{\widetilde{z}} \stackrel{\sim}{\rightarrow} \widetilde{Y} \otimes_{\mathbb{Z}} \hat{\mathbb{Z}}$ (of $\mathcal{O} \otimes_{\mathbb{Z}} \hat{\mathbb{Z}}$-modules). These data define a torus argument

$$
\widetilde{\Phi}:=\left(\widetilde{X}, \widetilde{Y}, \widetilde{\phi}, \widetilde{\varphi}_{-2}, \widetilde{\varphi}_{0}\right)
$$

for $\widetilde{Z}$ (see definition 5.4.1.3 in [41] or definition 1.2.1.5 in [44]). Let $\widetilde{\delta}$ be the obvious splitting of $\widetilde{z}$ induced by the equality $Q_{-2} \oplus L \oplus Q_{0}=\widetilde{L}$.

Then we can define algebraic groups $\widetilde{\mathrm{P}}_{\widetilde{\mathrm{Z}}}, \widetilde{\mathrm{U}}_{\widetilde{\mathrm{Z}}}, \widetilde{\mathrm{U}}_{2, \widetilde{\mathrm{Z}}}, \widetilde{\mathrm{U}}_{1, \widetilde{\mathrm{Z}}}, \widetilde{\mathrm{G}}_{l, \widetilde{\mathrm{Z}}}, \widetilde{\mathrm{G}}_{l, \widetilde{\mathrm{z}}}^{\prime}, \widetilde{\mathrm{P}}_{\widetilde{\mathrm{Z}}}^{\prime}, \widetilde{\mathrm{G}}_{1, \widetilde{\mathrm{Z}}}, \widetilde{\mathrm{G}}_{h, \widetilde{\mathrm{z}}}$ and $\widetilde{\mathrm{G}}_{h, \widetilde{\mathrm{z}}}^{\prime}$. By definition, we have canonical isomorphisms

$$
\mathrm{G}_{h, \widetilde{\mathrm{Z}}} \cong \mathrm{G}_{h, \widetilde{\mathrm{Z}}}^{\prime} \cong \mathrm{G} \otimes_{\mathbb{Z}} \hat{\mathbb{Z}}
$$

and

$$
\widehat{\mathrm{G}}=\widetilde{\mathrm{G}}_{1, \widetilde{\mathrm{Z}}} \text { and } \widehat{\mathrm{U}}=\widetilde{\mathrm{U}}_{1, \widetilde{\mathrm{Z}}} \text {. }
$$

For each open compact subgroup $\widetilde{\mathcal{H}}$ of $\widetilde{\mathrm{G}}(\hat{\mathbb{Z}})$, we define

(1) $\widehat{\mathcal{H}}:=\widetilde{\mathcal{H}}_{\widehat{\mathrm{G}}}:=\widetilde{\mathcal{H}}_{\widetilde{\mathrm{G}}_{1, \tilde{z}}}$.

(2) $\widehat{\mathcal{H}}_{\widehat{\mathrm{U}}}:=\widetilde{\mathcal{H}}_{\widehat{\mathrm{U}}}:=\widetilde{\mathcal{H}}_{\widetilde{\mathrm{U}}_{1, \tilde{\mathrm{z}}}}$.

(3) $\widehat{\mathcal{H}}_{\mathrm{G}}:=\widehat{\mathcal{H}} / \widehat{\mathcal{H}}_{\widehat{\mathrm{U}}} \cong \widetilde{\mathcal{H}}_{\widetilde{\mathrm{G}}_{h, \tilde{\mathrm{z}}}^{\prime}}$.

(See definition 1.2.4.4 of [44].)

Given any neat open compact $\widehat{\mathcal{H}} \subset \widehat{\mathrm{G}}(\hat{\mathbb{Z}})$, we can always find some neat $\widetilde{\mathcal{H}} \subset \widetilde{\mathrm{G}}(\hat{\mathbb{Z}})$ such that $\widehat{\mathcal{H}}=\widetilde{\mathcal{H}}_{\widehat{\mathrm{G}}}$ as above and

$$
\widetilde{\mathcal{H}}_{\widetilde{\mathrm{P}}_{\widetilde{\mathrm{Z}}}} / \widetilde{\mathcal{H}}_{\widetilde{\mathrm{U}}_{\tilde{\mathrm{z}}}} \cong \widetilde{\mathcal{H}}_{\widetilde{\mathrm{G}}_{l, \tilde{\mathrm{z}}}} \times \widehat{\mathcal{H}}_{\mathrm{G}}
$$

(cf. condition 1.2.4.7 of [44]). In this case the abelian scheme torsor

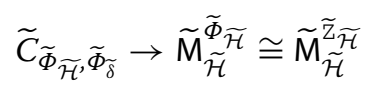


depends only on $\widehat{\mathcal{H}}$ (but not on the auxiliary choice of $\widetilde{\mathcal{H}}$ ) and equals the above generalized Kuga family $\mathrm{N} \rightarrow \mathrm{M}_{\mathcal{H}}$ attached to $\widehat{\mathcal{H}}$.

We can explicitly compare some of the above groups with the related groups defined in our paper (see Sects. 1.1, 1.2), as follows:

(1) $\quad \widetilde{\mathrm{G}}_{h, \widetilde{\mathrm{z}}}^{\prime}(R) \cong \widetilde{\mathrm{G}}_{h, \widetilde{\mathrm{z}}}(R)=\mathrm{G}(R)=G_{n}(R)$.

(2) $\quad \widetilde{\mathrm{G}}_{l, \widetilde{\mathrm{Z}}}(R) \cong \widetilde{\mathrm{G}}_{l, \widetilde{\mathrm{Z}}}^{\prime}(R) \cong \mathrm{GL}_{m}\left(\mathcal{O}_{F} \otimes_{\mathbb{Z}} R\right)$.

(3) $\quad \widetilde{\mathrm{P}}_{\widetilde{\mathrm{Z}}}(R) \cong \mathrm{GL}_{m}\left(\mathcal{O}_{F} \otimes_{\mathbb{Z}} R\right) \ltimes \widetilde{G}_{n}^{(m)}(R) \cong\left(\mathrm{GL}_{m}\left(\mathcal{O}_{F} \otimes_{\mathbb{Z}} R\right) \times G_{n}(R)\right) \ltimes N_{n}^{(m)}(R)$.

(4) $\widetilde{\mathrm{U}}_{\widetilde{\mathrm{Z}}}(R)=N_{n}^{(m)}(R)$.

(5) $\quad \widetilde{\mathrm{U}}_{2, \widetilde{\mathrm{Z}}}(R)=Z\left(N_{n}^{(m)}\right)(R)=\operatorname{Herm}^{(m)}(R)$.

(6) $\widehat{\mathrm{U}}(R)=\widetilde{\mathrm{U}}_{1, \widetilde{\mathrm{Z}}}(R)=\operatorname{Hom}_{n}^{(m)}(R)$.

(7) $\widetilde{\mathrm{P}}_{\widetilde{\mathrm{z}}}^{\prime}(R)=\widetilde{G}_{n}^{(m)}(R)=G_{n}(R) \ltimes N_{n}^{(m)}(R)$.

(8) $\quad \widehat{\mathrm{G}}(R)=\widetilde{\mathrm{G}}_{1, \widetilde{\mathrm{Z}}}(R) \cong G_{n}^{(m)}(R)=G_{n}(R) \ltimes \operatorname{Hom}_{n}^{(m)}(R)$.

(9) $\quad \widetilde{\mathrm{P}}_{\widetilde{\mathrm{Z}}}(R) / \widetilde{\mathrm{U}}_{\widetilde{\mathrm{Z}}}(R) \cong \mathrm{GL}_{m}\left(\mathcal{O}_{F} \otimes_{\mathbb{Z}} R\right) \times G_{n}(R)$.

\section{B.7 Toroidal compactifications of Kuga families in characteristic zero}

Let us fix the choice of some $(\widetilde{Z}, \widetilde{\Phi}, \widetilde{\delta})$ as above. Then there is a bijection between the fully symplectic admissible filtrations $\check{Z}$ of $\widetilde{L} \otimes_{\mathbb{Z}} \hat{\mathbb{Z}}$ such that

$$
0 \subset \widetilde{\mathrm{Z}}_{-2} \subset \check{\mathrm{Z}}_{-2} \subset \breve{\mathrm{Z}}_{-1} \subset \widetilde{\mathrm{Z}}_{-1} \subset \widetilde{L} \otimes_{\mathbb{Z}} \hat{\mathbb{Z}}
$$

and the fully symplectic admissible filtrations $\mathrm{Z}$ of $L \otimes_{\mathbb{Z}} \hat{\mathbb{Z}}$ such that $\mathrm{Z}_{-2}=\check{Z}_{-2} / \widetilde{Z}_{-2}$. (The notation ` will always mean some objects related to such a filtration $\check{z}$.) When $z=z^{(i, 1)}$, we have $\check{\mathrm{Z}}=\widetilde{\mathrm{Z}}^{(i+m, 1)}$.

For each $\breve{Z}$ as above, and for each $\hat{\mathbb{Z}}$-algebra $R$, we define the following quotients of subgroups of $\widehat{\mathrm{G}}(R)$ (see definition 1.2.4.53 of [44]):

(1) $\quad \widehat{\mathrm{P}}_{\check{\mathrm{Z}}}(R):=\left(\widetilde{\mathrm{P}}_{\check{\mathrm{Z}}}(R) \cap \widetilde{\mathrm{P}}_{\widetilde{\mathrm{Z}}}^{\prime}(R)\right) / \widetilde{\mathrm{U}}_{2, \widetilde{\mathrm{Z}}}(R)$, so that $\widehat{\mathrm{P}}_{\widetilde{\mathrm{Z}}^{(i+m, 1)}}(R)=P_{n,(i)}^{(m),+}(R)$.

(2) $\quad \widehat{\mathrm{P}}_{\check{z}}^{\prime}(R):=\widetilde{\mathrm{P}}_{\check{z}}^{\prime}(R) / \widetilde{\mathrm{U}}_{2, \widetilde{\mathrm{z}}}(R)$, so that $\widehat{\mathrm{P}}_{\widetilde{\mathrm{Z}}^{(i+m, 1)}}^{\prime}(R)=P_{n,(i)}^{(m)}(R)$.

(3) $\widehat{\mathrm{U}}_{\check{\mathrm{Z}}}(R):=\widetilde{\mathrm{U}}_{\check{\mathrm{Z}}}(R) / \widetilde{\mathrm{U}}_{2, \widetilde{\mathrm{Z}}}(R)$, so that $\widehat{\mathrm{U}}_{\widetilde{\mathrm{Z}}^{(i+m, 1)}}(R)=N_{n,(i)}^{(m)}(R)$.

(4) $\widehat{\mathrm{U}}_{2, \check{z}}(R):=\widetilde{\mathrm{U}}_{2, \check{z}}(R) / \widetilde{\mathrm{U}}_{2, \tilde{\mathrm{z}}}(R)$, so that

$$
\widehat{\mathrm{U}}_{2, \widetilde{\mathrm{Z}}^{(i+m, 1)}}(R)=Z\left(N_{n,(i)}^{(m)}\right)(R) \cong \operatorname{Herm}^{(i+m)}(R) / \operatorname{Herm}^{(m)}(R) .
$$

(5) $\widehat{\mathrm{U}}_{1, \check{z}}(R):=\widehat{\mathrm{U}}_{\check{\mathrm{Z}}}(R) / \widehat{\mathrm{U}}_{2, \check{\mathrm{z}}}(R) \cong \widetilde{\mathrm{U}}_{1, \check{\mathrm{z}}}(R)$, so that

$$
\widehat{\mathrm{U}}_{1, \widetilde{\mathrm{Z}}^{(i+m, 1)}}(R)=N_{n,(i)}^{(m)}(R) / Z\left(N_{n,(i)}^{(m)}\right)(R) \cong \operatorname{Hom}_{n-i}^{(i+m)}(R) .
$$

(6) $\widehat{\mathrm{G}}_{h, \check{\mathrm{z}}}(R):=\widetilde{\mathrm{G}}_{h, \breve{\mathrm{z}}}(R)$ and $\widehat{\mathrm{G}}_{h, \breve{\mathrm{z}}}^{\prime}(R):=\widetilde{\mathrm{G}}_{h, \breve{z}}^{\prime}(R)$, so that $\widehat{\mathrm{G}}_{h, \check{\mathrm{z}}}(R) \cong \widehat{\mathrm{G}}_{h, \breve{\mathrm{z}}}^{\prime}(R) \cong \mathrm{G}_{h, \mathrm{z}}(R)$, and $\widehat{\mathrm{G}}_{h, \widetilde{\mathrm{Z}}^{(i+m, 1)}}(R)=L_{n,(i), \text { herm }}(R)$.

(7) $\widehat{\mathrm{G}}_{l, \check{\mathrm{Z}}}(R):=\left(\widetilde{\mathrm{Z}}_{\check{\mathrm{Z}}}(R) \cap \widetilde{\mathrm{P}}_{\widetilde{\mathrm{Z}}}^{\prime}(R)\right) / \widetilde{\mathrm{U}}_{\check{\mathrm{z}}}(R)$ and $\widehat{\mathrm{G}}_{l, \grave{\mathrm{z}}}^{\prime}(R):=\widehat{\mathrm{P}}_{\check{\mathrm{z}}}(R) / \widehat{\mathrm{P}}_{\check{\mathrm{z}}}^{\prime}(R)$, so that $\widehat{\mathrm{G}}_{l, \check{\mathrm{z}}}(R) \stackrel{\sim}{\rightarrow}$ $\widehat{\mathrm{G}}_{l, \breve{\mathrm{z}}}^{\prime}(R)$ and $\widehat{\mathrm{G}}_{l, \widetilde{\mathrm{z}}^{(i+m, 1)}}^{\prime}(R) \cong L_{n,(i), \text { lin }}^{(m)}(R)$.

(8) $\quad \widehat{\mathrm{G}}_{1, \check{\mathrm{Z}}}(R):=\widehat{\mathrm{P}}_{\check{\mathrm{z}}}^{\prime}(R) / \widehat{\mathrm{U}}_{2, \check{\mathrm{z}}}(R) \cong \widetilde{\mathrm{G}}_{1, \check{\mathrm{z}}}(R)$, so that $\widehat{\mathrm{G}}_{1, \widetilde{\mathrm{Z}}^{(i+m, 1)}}(R) \cong G_{n-i}^{(i+m)}(R)$.

Hence it makes sense to define $\widehat{\mathcal{H}}_{\widehat{\mathrm{P}}_{\check{Z}}}:=\left(\widetilde{\mathcal{H}}_{\widetilde{\mathrm{P}}_{\check{z}}} \cap \widetilde{\mathcal{H}}_{\widetilde{\mathrm{P}}_{\widehat{z}}}\right) / \widetilde{\mathcal{H}}_{\widetilde{\mathrm{U}}_{2, \tilde{z}}}$, etc., when $\widehat{\mathcal{H}}=\widetilde{\mathcal{H}}_{\widehat{\mathrm{G}}}$, so that we have $\widehat{\mathcal{H}}_{\widehat{\mathrm{P}}_{\check{\mathrm{Z}}}} \rightarrow \mathcal{H}_{\mathrm{P}_{\mathrm{Z}}}$, etc. 
If $\widetilde{g} \in \widetilde{\mathrm{P}}_{\widetilde{Z}}^{\prime}\left(\mathbb{A}^{\infty}\right)$, then $\widetilde{Z}^{(i+m, \widetilde{g})}$ depends only on the image $\widehat{g}$ of $\widetilde{g}$ in $\widehat{\mathrm{G}}(\hat{\mathbb{Z}})$, so we will denote it $\check{Z}^{(i, \widehat{g})}$. This sets up a bijection between the $\widehat{\mathcal{H}}$-orbits $\widetilde{\mathrm{Z}}_{\widehat{\mathcal{H}}}^{(i+m, \widetilde{g})}=\check{z}_{\widehat{\mathcal{H}}}^{(i, \widehat{g})}$ of $\widetilde{\mathrm{Z}}^{(i+m, \widetilde{g})}=\check{\mathrm{Z}}^{(i, \widehat{g})}$ and the double coset space

$$
\widehat{\mathrm{P}}_{\check{\mathrm{Z}}^{(i, 1)}}\left(\mathbb{A}^{\infty}\right) \backslash \widehat{\mathrm{G}}\left(\mathbb{A}^{\infty}\right) / \widehat{\mathcal{H}}
$$

which equals

$$
P_{n,(i)}^{(m),+}\left(\mathbb{A}^{\infty}\right) \backslash G_{n}^{(m)}\left(\mathbb{A}^{\infty}\right) / U
$$

in this paper (see, for example, Sect. 1.4), with $U=\widehat{\mathcal{H}}$.

By taking graded pieces with respect to filtrations induced by $0 \subset \widetilde{\mathrm{Z}}_{-2} \subset \breve{\mathrm{Z}}_{-2} \subset \breve{\mathrm{Z}}_{-1} \subset$ $\widetilde{Z}_{-1} \subset \widetilde{L} \otimes_{\mathbb{Z}} \hat{\mathbb{Z}}$, there is also a bijection between the torus arguments $\breve{\Phi}=\left(\check{X}, \breve{Y}, \breve{\phi}, \breve{\varphi}_{-2}, \breve{\varphi}_{0}\right)$ for $\check{Z}$ which induce $\widetilde{\Phi}$ and the torus arguments $\Phi=\left(X, Y, \phi, \varphi_{-2}, \varphi_{0}\right)$ for the filtration Z corresponding to $\breve{Z}$. If we set $\mathcal{H}=\widehat{\mathcal{H}}_{\mathrm{G}}$, then this bijection is compatible with the formation of the $\widehat{\mathcal{H}}$-orbits of $\breve{\Phi}$ and the $\mathcal{H}$-orbits of the corresponding $\Phi$, which induces bijections among the following three sets (see lemmas 1.2.4.15 and 1.2.4.16 of [44]):

(1) The cusp labels $\left[\left(\check{Z}_{\widetilde{\mathcal{H}}}, \breve{\Phi}_{\widetilde{\mathcal{H}}}, \breve{\delta}_{\widetilde{\mathcal{H}}}\right)\right]$ for $\widetilde{\mathrm{M}}_{\widetilde{\mathcal{H}}}$ such that the stratum $\widetilde{Z}_{\left[\left(\breve{\Phi}_{\widetilde{\mathcal{H}}}, \breve{\mathcal{X}}_{\widetilde{\mathcal{H}}}\right)\right]}$ of $\widetilde{\mathrm{M}}_{\widetilde{\mathcal{H}}}^{\min }$ is contained in the closure of $\widetilde{Z}_{\left[\left(\widetilde{\Phi}_{\widetilde{\mathcal{H}}}, \widetilde{\mathcal{H}}_{\widetilde{\mathcal{H}}}\right)\right]}$.

(2) The $\widehat{\mathcal{H}}$-orbits of equivalence classes of $(\check{Z}, \breve{\Phi}, \breve{\delta})$, where $\check{Z}$ and $\check{\Phi}$ are compatible with $\widetilde{\mathrm{Z}}$ and $\widetilde{\Phi}$ in the sense that $0 \subset \widetilde{\mathrm{Z}}_{-2} \subset \breve{\mathrm{Z}}_{-2} \subset \breve{\mathrm{Z}}_{-1} \subset \widetilde{\mathrm{Z}}_{-1} \subset \widetilde{L} \otimes_{\mathbb{Z}} \hat{\mathbb{Z}}$ and that $\breve{\Phi}$ induces $\widetilde{\Phi}$ as above, which we denote by $\left[\left(\breve{Z}_{\widehat{\mathcal{H}}}, \breve{\Phi}_{\widehat{\mathcal{H}}}, \breve{\delta}_{\widehat{\mathcal{H}}}\right)\right]$ and call it a cusp label (at level $\widehat{\mathcal{H}}$ ) for $\left(\widetilde{L},\left\langle\cdot, \cdot \gamma, \widetilde{h}_{0}, \widetilde{z}\right)\right.$ (see definition 1.2.4.17 of [44]).

(3) The cusp labels $\left[\left(\mathrm{Z}_{\mathcal{H}}, \Phi_{\mathcal{H}}, \delta_{\mathcal{H}}\right)\right]$ for $\mathrm{M}_{\mathcal{H}}$.

The stratum $\widetilde{Z}_{\left[\left(\check{\Phi}_{\widetilde{\mathcal{H}}}, \check{\delta}_{\widetilde{\mathcal{H}}}\right)\right]} \cong \widetilde{\mathrm{M}}_{\tilde{\mathcal{H}}}^{\tilde{z}_{\widetilde{\mathcal{H}}}}$, the finite étale cover $\widetilde{\mathrm{M}}_{\widetilde{\mathcal{H}}_{\widetilde{\mathcal{H}}}}^{\widetilde{\mathrm{M}}_{\widetilde{\mathcal{M}}}} \widetilde{\mathrm{M}}_{\widetilde{\mathcal{H}}}^{\tilde{z}_{\widetilde{\mathcal{H}}}}$, the abelian scheme

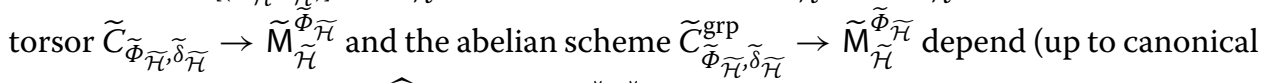
isomorphism) only on the $\widehat{\mathcal{H}}$-orbit of ( $\breve{Z}, \breve{\Phi}, \breve{\delta}$ ) (see lemma 1.3.2.50 of [44]), and hence we shall denote them by

$$
\widehat{M}_{\widehat{\mathcal{H}}}^{\check{z}}, \quad \widehat{M}_{\widehat{\mathcal{H}}}^{\check{\Phi}_{\widehat{\mathcal{H}}}} \rightarrow \widehat{M}_{\widehat{\mathcal{H}}}^{\check{z}_{\widehat{\mathcal{H}}}}, \quad \widehat{C}_{\check{\Phi}_{\widehat{\mathcal{H}}}, \check{\delta}_{\widehat{\mathcal{H}}}} \rightarrow \widehat{M}_{\widehat{\mathcal{H}}}^{\check{\Phi}_{\widehat{\mathcal{H}}}}, \quad \text { and } \quad \widehat{C}_{\check{\Phi}_{\widehat{\mathcal{H}}}, \check{\delta}_{\widehat{\mathcal{H}}}}^{\text {grp }} \rightarrow \widehat{M}_{\widehat{\mathcal{H}}}^{\check{\Phi}_{\widehat{\mathcal{H}}}},
$$

respectively. For a fixed $(\breve{Z}, \breve{\Phi}, \breve{\delta})$, the morphisms

$$
\widehat{C}_{\check{\Phi}_{\widehat{\mathcal{H}}}, \check{\delta}_{\widehat{\mathcal{H}}}} \rightarrow \widehat{\mathrm{M}}_{\widehat{\mathcal{H}}}^{\check{\Phi}_{\widehat{\mathcal{H}}}} \rightarrow \widehat{\mathrm{M}}_{\widehat{\mathcal{H}}}^{\check{Z}_{\widehat{\mathcal{H}}}}
$$

are equivariant with

$$
\widehat{\mathrm{G}}_{1, \check{\mathrm{Z}}}\left(\mathbb{A}^{\infty}\right) \rightarrow \widehat{\mathrm{G}}_{h, \check{\mathrm{z}}}^{\prime}\left(\mathbb{A}^{\infty}\right) \cong \widehat{\mathrm{G}}_{h, \check{\mathrm{z}}}\left(\mathbb{A}^{\infty}\right)
$$

(see propositions 1.3.2.24 and 1.3.2.55 of [44]).

For each $[(\breve{Z}, \breve{\Phi}, \breve{\delta})]$ as above, and any $\mathbb{Z}$-algebra $R$, we define $\widehat{\mathrm{G}}_{l, \breve{\Phi}}^{\prime}(R)$ to be the subgroup of $\mathrm{GL}_{\mathcal{O} \otimes_{\mathbb{Z}} R}\left(\breve{Y} \otimes_{\mathbb{Z}} R\right)$ of elements stabilizing the kernel of $\breve{Y} \rightarrow \widetilde{Y}$ and inducing $\operatorname{Id}_{\tilde{Y}}$, which admits a canonical map to $\widehat{\mathrm{G}}_{l, \breve{\mathrm{Z}}}^{\prime}\left(R \otimes_{\mathbb{Z}} \hat{\mathbb{Z}}\right)$ induced by $\breve{\Phi}$. When $R$ is a $\hat{\mathbb{Z}}$-algebra, we have $\widehat{\mathrm{G}}_{l, \breve{\Phi}}^{\prime}(R) \cong \widehat{\mathrm{G}}_{l, \breve{\mathrm{z}}}^{\prime}(R)$. When $R=\mathbb{Q}$, we have $\widehat{\mathrm{G}}_{l, \breve{\Phi}}^{\prime}(\mathbb{Q}) \hookrightarrow \widehat{\mathrm{G}}_{l, \breve{\mathrm{L}}}^{\prime}\left(\mathbb{A}^{\infty}\right)$, and we define

$$
\Gamma_{\check{\Phi}_{\widehat{\mathcal{H}}}}:=\widehat{\mathcal{H}}_{\widehat{\mathrm{G}}_{l, \check{L}}^{\prime}} \cap \widehat{\mathrm{G}}_{l, \breve{\Phi}}^{\prime}(\mathbb{Q}),
$$


a congruence subgroup of $\widehat{\mathrm{G}}_{l, \breve{\Phi}}^{\prime}(\mathbb{Z})=\widehat{\mathrm{G}}_{l, \breve{\mathrm{Z}}}^{\prime}(\hat{\mathbb{Z}}) \cap \widehat{\mathrm{G}}_{l, \breve{\Phi}}^{\prime}(\mathbb{Q})$ depending only on $\breve{\Phi}_{\widehat{\mathcal{H}}}$ (see definition 1.2.4.21 of [44] for an equivalent definition).

If $\widetilde{g} \in \widetilde{\mathrm{P}}_{\widetilde{\mathrm{Z}}}^{\prime}\left(\mathbb{A}^{\infty}\right)$, then the equivalence class of $\left(\widetilde{\mathrm{Z}}^{(i+m, \widetilde{g})}, \widetilde{\Phi}^{(i+m, \widetilde{g})}, \breve{\delta}\right)$ depends only on the image $\widehat{g}$ of $\widetilde{g}$ in $\widehat{\mathrm{G}}\left(\mathbb{A}^{\infty}\right)$. We will denote it $\left(\check{\mathrm{Z}}^{(i, \widehat{g})}, \breve{\Phi}^{(i, \widehat{g})}, \breve{\delta}\right)$. The map

$$
\widehat{g} \mapsto\left[\left(\check{Z}_{\widehat{\mathcal{H}}}^{(i, \widehat{g})}, \breve{\Phi}_{\widehat{\mathcal{H}}}^{(i, \widehat{g})}, \breve{\delta}_{\widehat{\mathcal{H}}}\right)\right]
$$

sets up a bijection between the double coset space

$$
\left(\widehat{\mathrm{G}}_{l, \check{\Phi}^{(i, 1)}}^{\prime}(\mathbb{Q}) \ltimes \widehat{\mathrm{P}}_{\check{\mathrm{Z}}^{(i, 1)}}^{\prime}\left(\mathbb{A}^{\infty}\right)\right) \backslash \widehat{\mathrm{G}}\left(\mathbb{A}^{\infty}\right) / \widehat{\mathcal{H}}
$$

and the set of cusp labels $\left[\left(\check{Z}_{\widehat{\mathcal{H}}}, \breve{\Phi}_{\widehat{\mathcal{H}}}, \breve{\delta}_{\widehat{\mathcal{H}}}\right)\right]$ such that $\check{Z}_{\widehat{\mathcal{H}}}=\check{\mathrm{Z}}_{\widehat{\mathcal{H}}}^{(i, \widehat{g})}$ for some $\widehat{g} \in \widehat{\mathrm{G}}\left(\mathbb{A}^{\infty}\right)$. The forgetful map sending $\left[\left(\check{Z}_{\widehat{\mathcal{H}}}, \breve{\Phi}_{\widehat{\mathcal{H}}}, \breve{\delta}_{\widehat{\mathcal{H}}}\right)\right]$ to $\breve{Z}_{\widehat{\mathcal{H}}}$ can be identified with the canonical map

$$
\left(\widehat{\mathrm{G}}_{l, \breve{\Phi}^{(i, 1)}}^{\prime}(\mathbb{Q}) \ltimes \widehat{\mathrm{P}}_{\check{\mathrm{Z}}^{(i, 1)}}^{\prime}\left(\mathbb{A}^{\infty}\right)\right) \backslash \widehat{\mathrm{G}}\left(\mathbb{A}^{\infty}\right) / \widehat{\mathcal{H}} \rightarrow \widehat{\mathrm{P}}_{\check{\mathrm{Z}}^{(i, 1)}}\left(\mathbb{A}^{\infty}\right) \backslash \widehat{\mathrm{G}}\left(\mathbb{A}^{\infty}\right) / \widehat{\mathcal{H}}
$$

whose fibre above the double coset of $\widehat{g} \in \widehat{\mathrm{G}}\left(\mathbb{A}^{\infty}\right)$ can be identified with

$$
\widehat{\mathrm{G}}_{l, \breve{\Phi}_{(i, 1)}^{\prime}}^{\prime}(\mathbb{Q}) \backslash \widehat{\mathrm{G}}_{l, \check{\mathrm{Z}}^{(i, 1)}}^{\prime}\left(\mathbb{A}^{\infty}\right) /\left(\widehat{\mathrm{g}} \widehat{\mathcal{H}}^{-1}\right)_{\widehat{\mathrm{G}}_{l, \mathrm{Z}(i, 1)}^{\prime}}
$$

This last double coset space is the index set of

$$
Y_{n,(i), g U g^{-1} \cap P_{n,(i)}^{(m),+}\left(\mathbb{A}^{\infty}\right)}^{(m), \llbracket}=\coprod_{L_{n,(i), \operatorname{lin}}^{(m)}(\mathbb{Q}) \backslash L_{n,(i), \operatorname{lin}}^{(m)}\left(\mathbb{A}^{\infty}\right) /\left(g U g^{-1} \cap P_{n,(i)}^{(m),+}\left(\mathbb{A}^{\infty}\right)\right)} \operatorname{Spec} \mathbb{Q}
$$

in this paper (see Sect. 4.1), with $g=\widehat{g}$ and $U=\widehat{\mathcal{H}}$.

If $U=\widehat{\mathcal{H}}$ is any neat open compact subgroup of $G_{n}^{(m)}\left(\mathbb{A}^{\infty}\right)=\widehat{\mathrm{G}}\left(\mathbb{A}^{\infty}\right)$, if $g=\widehat{g} \in$ $G_{n}^{(m)}\left(\mathbb{A}^{\infty}\right)=\widehat{G}\left(\mathbb{A}^{\infty}\right)$ and if $U_{g}^{\prime}=g U g^{-1} \cap P_{n,(i)}^{(m),+}\left(\mathbb{A}^{\infty}\right)$, then the maps

$$
\begin{aligned}
& A_{n,(i), U_{g}^{\prime}}^{(m),+}=\coprod_{h \in L_{n,(i), \operatorname{lin}}^{(m)}\left(\mathbb{A}^{\infty}\right) / U_{g}^{\prime}} A_{n-i, h U_{g}^{\prime} h^{-1} \cap P_{n,(i)}^{(m)}\left(\mathbb{A}^{\infty}\right)}^{(i+m)}
\end{aligned}
$$

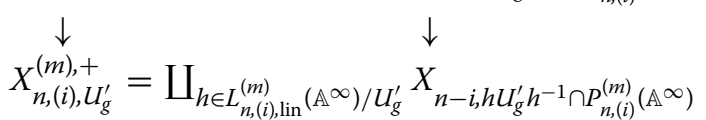

$$
\begin{aligned}
& \downarrow \\
& X_{n,(i), U_{g}^{\prime}}^{(m), \mathfrak{a}}
\end{aligned}
$$

in this paper (see Sects. 4.1, 4.2) are identified with

$$
\begin{aligned}
& \coprod_{h \in L_{n,(i), \operatorname{lin}}^{(m)}\left(\mathbb{A}^{\infty}\right) / U_{g}^{\prime}} \widehat{C}_{\breve{\Phi}_{\mathcal{\mathcal { H }}}^{(i, h g)}, \breve{\delta}_{\widehat{\mathcal{H}}}} \\
& \coprod_{h \in L_{n,(i), \operatorname{lin}}^{(m)}\left(\mathbb{A}^{\infty}\right) / U_{g}^{\prime}} \widehat{M}_{\widehat{\mathcal{H}}_{\mathcal{\mathcal { H }}}^{(i, h)}} \\
& \bigsqcup_{h \in L_{n,(i), \operatorname{lin}}^{(m)}(\mathbb{Q}) \backslash L_{n,(i), \operatorname{lin}}^{(m)}\left(\mathbb{A}^{\infty}\right) / U_{g}^{\prime}} \widehat{\mathrm{M}}_{\tilde{\mathrm{Z}}_{\mathcal{\mathcal { H }}}^{(i, h g)}} .
\end{aligned}
$$


For each representative $\left(\breve{Z}_{\widehat{\mathcal{H}}}, \breve{\Phi}_{\widehat{\mathcal{H}}}, \breve{\delta}_{\widehat{\mathcal{H}}}\right)$ of a cusp label $\left[\left(\breve{Z}_{\widehat{\mathcal{H}}}, \breve{\Phi}_{\widehat{\mathcal{H}}}, \breve{\delta}_{\widehat{\mathcal{H}}}\right)\right]$ as above, there is a torsor

$$
\widehat{\Xi}_{\check{\Phi}_{\widehat{\mathcal{H}}}, \check{\delta}_{\widehat{\mathcal{H}}}} \rightarrow \widehat{C}_{\check{\Phi}_{\widehat{\mathcal{H}}}, \check{\delta}_{\widehat{\mathcal{H}}}}
$$

under the pull-back of a split torus

$$
\widehat{E}_{\breve{\Phi}_{\widehat{\mathcal{H}}}}
$$

over Spec $\mathbb{Z}$ with character group

$$
\widehat{\mathbf{S}}_{\check{\Phi}_{\widehat{\mathcal{H}}}}:=\operatorname{ker}\left(\mathbf{S}_{\check{\Phi}_{\widetilde{\mathcal{H}}}} \rightarrow \mathbf{S}_{\widetilde{\Phi}_{\widetilde{\mathcal{H}}}}\right)
$$

(which only depends on $\widehat{\mathcal{H}}_{\widehat{\mathrm{P}}_{\check{z}}}$; see definition 1.2.4.29 and proposition 1.3.2.56 of [44]). We obtain an isomorphic torus torsor if we replace $\left(\breve{Z}_{\widehat{\mathcal{H}}}, \breve{\Phi}_{\widehat{\mathcal{H}}}, \breve{\delta}_{\widehat{\mathcal{H}}}\right)$ with another representative, but its universal property depends on this choice of representative.

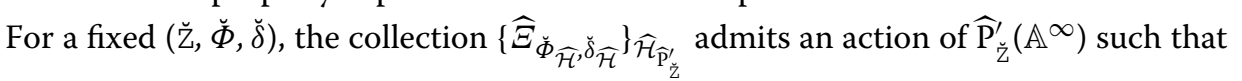
the morphisms

$$
\widehat{\Xi}_{\check{\Phi}_{\widehat{\mathcal{H}}}, \check{\delta}_{\widehat{\mathcal{H}}}} \rightarrow \widehat{C}_{\check{\Phi}_{\widehat{\mathcal{H}}}, \check{\delta}_{\widehat{\mathcal{H}}}}
$$

are equivariant with

$$
\widehat{\mathrm{P}}_{\check{\mathrm{Z}}}^{\prime}\left(\mathbb{A}^{\infty}\right) \rightarrow \widehat{\mathrm{G}}_{1, \check{\mathrm{Z}}}\left(\mathbb{A}^{\infty}\right)
$$

(see proposition 1.3.2.67 of [44]).

When $\mathcal{H}_{\widehat{\mathrm{U}}_{2, \check{z}}}=\widehat{\mathrm{G}}(\hat{\mathbb{Z}})_{\widehat{\mathrm{U}}_{2, \check{z}}}=\widehat{\mathrm{U}}_{2, \check{\mathrm{Z}}}(\hat{\mathbb{Z}})$ and when $\breve{\Phi}_{\widehat{\mathcal{H}}}$ is represented by some $\check{\Phi}=$ $\left(\breve{X}, \breve{Y}, \breve{\phi}, \breve{\varphi}_{-2}, \breve{\varphi}_{0}\right)$ (where $\breve{\phi}: \breve{Y} \hookrightarrow \breve{X}$ must be an isomorphism, as explained above), which admits a surjection $\breve{Y} \rightarrow \widetilde{Y}$ with kernel $Y$ an $\mathcal{O}$-lattice, the group $\widehat{\mathbf{S}}_{\mathscr{\Phi}_{\widehat{\mathcal{H}}}}$ is the group $\operatorname{ker}\left(S(\breve{Y})^{\mathrm{TF}} \rightarrow S(\tilde{Y})^{\mathrm{TF}}\right.$ ) in the notation of this paper (see Sect. 1.1). For more general $\widehat{\mathcal{H}}$, we write $\widehat{\mathbf{S}}_{\check{\Phi}_{1}}=\operatorname{ker}\left(S(\breve{Y})^{\mathrm{TF}} \rightarrow S(\tilde{Y})^{\mathrm{TF}}\right)$, and $\widehat{\mathbf{S}}_{\breve{\Phi}_{\widehat{\mathcal{H}}}}$ is the unique lattice in $\widehat{\mathbf{S}}_{\check{\Phi}_{1}} \otimes_{\mathbb{Z}} \mathbb{Q}$ such that

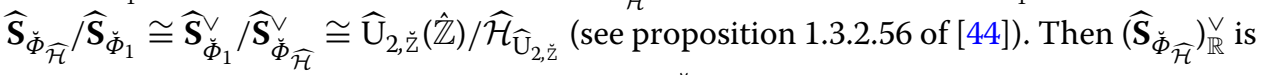
a quotient of the space of hermitian forms over $\breve{Y} \otimes_{\mathbb{Z}} \mathbb{R}$, which also admits a projection to the space of hermitian forms over $Y \otimes_{\mathbb{Z}} \mathbb{R}$. We define $\widehat{\mathbf{P}}_{\breve{\Phi}}$ (resp. $\widehat{\mathbf{P}}_{\breve{\Phi}_{\widehat{\mathcal{H}}}}^{+}$) to be the subset of $\left(\widehat{\mathbf{S}}_{\breve{\Phi}}\right)_{\mathbb{R}}^{\vee}$ consisting of images of positive semi-definite hermitian forms with rational radicals (resp. positive definite hermitian forms) over $\breve{Y} \otimes_{\mathbb{Z}} \mathbb{R}$ (see (1.2.4.33) and (1.2.4.34) of [44]), which can be identified with the subset consisting of pre-images of positive semidefinite hermitian forms with rational radicals (resp. positive definite hermitian forms) over $Y \otimes_{\mathbb{Z}} \mathbb{R}$.

The torus

$$
S_{n,(i), U_{g}^{\prime}}^{(m),+} \rightarrow Y_{n,(i), U_{g}^{\prime}}^{(m),+}
$$

in this paper (see Sect. 4.3) with $U=\mathcal{H}$ is identified with

$$
\coprod_{h \in L_{n,(i), \operatorname{lin}}^{(m)}\left(\mathbb{A}^{\infty}\right) / U_{g}^{\prime}} \widehat{E}_{\breve{\Phi}_{\overline{\mathcal{H}}}^{(i, h)}} \rightarrow \coprod_{h \in L_{h,(i), \operatorname{lin}}^{(m)}\left(\mathbb{A}^{\infty}\right) / U_{g}^{\prime}} \operatorname{Spec} \mathbb{Q} .
$$


Moreover, the sheaves $X^{*}\left(S_{n,(i), U_{g}^{\prime}}^{(m),+}\right), X_{*}\left(S_{n,(i), U_{g}^{\prime}}^{(m),+} \succ_{\mathbb{R}}^{\succ 0}\right.$, and $X_{*}\left(S_{n,(i), U_{g}^{\prime}}^{(m),+}\right)_{\mathbb{R}}^{>0}$ are identified with

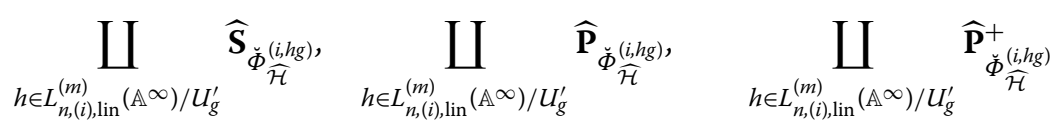

respectively. The $S_{n,(i), U_{g}^{\prime}}^{(m),+}$ torsor

$$
\begin{gathered}
T_{n,(i), U_{g}^{\prime}}^{(m),+}=\bigsqcup_{h \in L_{n,(i), \operatorname{lin}}^{(m)}\left(\mathbb{A}^{\infty}\right) / U_{g}^{\prime}} T_{n-i, h g U g^{-1} h^{-1} \cap P_{n,(i)}^{(m)}\left(\mathbb{A}^{\infty}\right)}^{(i+m)} \\
\downarrow \\
\downarrow \\
A_{n,(i), U_{g}^{\prime}}^{(m),+}= \\
\bigsqcup_{h \in L_{n,(i), \operatorname{lin}}^{(m)}\left(\mathbb{A}^{\infty}\right) / U_{g}^{\prime}} A_{n-i, h g U g^{-1} h^{-1} \cap P_{n,(i)}^{(m)}\left(\mathbb{A}^{\infty}\right)}^{(i+m)}
\end{gathered}
$$

is identified with

$$
\begin{gathered}
\bigsqcup_{h \in L_{n,(i), \operatorname{lin}}^{(m)}\left(\mathbb{A}^{\infty}\right) / U_{g}^{\prime}} \widehat{\Xi}_{\breve{\Phi}_{\mathcal{\mathcal { H }}^{(i, h g)}, \check{\delta}_{\widehat{\mathcal{H}}}}} \\
\downarrow \\
\coprod_{h \in L_{n,(i), \operatorname{lin}}^{(m)}\left(\mathbb{A}^{\infty}\right) / U_{g}^{\prime}} \widehat{C}_{\breve{\Phi}_{\overline{\mathcal{H}}}^{(i, h g)}, \check{\delta}_{\widehat{\mathcal{H}}}} .
\end{gathered}
$$

These maps are $\widehat{\mathrm{P}}_{\check{Z}^{(i, g)}}\left(\mathbb{A}^{\infty}\right)$-equivariant (see proposition 1.3.2.67 of [44]).

Consider any compatible collection

$$
\widehat{\Sigma}=\left\{\widehat{\Sigma}_{\check{\Phi}_{\widehat{\mathcal{H}}}}\right\}_{\left[\left(\check{\Phi}_{\widehat{\mathcal{H}}}, \check{\delta}_{\widehat{\mathcal{H}}}\right)\right]}
$$

of admissible projective smooth rational polyhedral cone decompositions, where each $\widehat{\Sigma}_{\breve{\Phi}_{\widehat{\mathcal{H}}}}$ is a $\Gamma_{\check{\Phi}_{\widehat{\mathcal{H}}}}$-admissible projective smooth rational polyhedral cone decomposition of $\widehat{\mathbf{P}}_{\breve{\Phi}_{\widehat{\mathcal{H}}}}$, as in lemma 1.2.4.42 of [44]. (We caution the reader that the definition there is rather ad hoc.) The set of pairs $\kappa=(\widehat{\mathcal{H}}, \widehat{\Sigma})$ with $\widehat{\mathcal{H}}_{\mathrm{G}} \subset \mathcal{H}$ is denoted $\mathbf{K}_{Q, \mathcal{H}}^{++}$; the subset of $\mathbf{K}_{Q, \mathcal{H}}^{++}$consisting of $\kappa=(\widehat{\mathcal{H}}, \widehat{\Sigma})$ with $\widehat{\mathcal{H}}_{\mathrm{G}}=\mathcal{H}$ is denoted $\mathbf{K}_{Q, \mathcal{H}}^{+}$; and the subset of $\mathbf{K}_{Q, \mathcal{H}}^{+}$consisting of $\kappa=(\widehat{\mathcal{H}}, \widehat{\Sigma})$ with $\widehat{\mathcal{H}}_{\mathrm{G}}=\mathcal{H}$ and $\widehat{\mathcal{H}}=\mathcal{H} \ltimes \widehat{\mathcal{H}}_{\widehat{\mathrm{U}}}$ is denoted $\mathbf{K}_{Q, \mathcal{H}}$ (see definitions 1.2.4.11 and 1.2.4.44 of [44]). For any given compatible collection $\Sigma$ of admissible projective smooth rational polyhedral cone decompositions for $M_{\mathcal{H}}$, and for $?=\emptyset,+$ or ++ , the subset of $\mathbf{K}_{Q, \mathcal{H}}^{\text {? }}$ consisting of $\kappa=(\widehat{\mathcal{H}}, \widehat{\Sigma})$ such that $\widehat{\Sigma}$ is compatible with $\Sigma$ in the sense that each $\widehat{\rho} \in \widehat{\Sigma}_{\breve{\Phi}_{\widehat{\mathcal{H}}}}$ is mapped into some $\sigma \in \Sigma_{\Phi_{\mathcal{H}}}$ (see condition 1.2.4.49 and definition 1.2.4.50 of [44]) is denoted $\mathbf{K}_{Q, \mathcal{H}, \Sigma}^{\text {? }}$. This notion of compatibility agrees with the one in this paper (see Sect. 5.2).

Each such $\kappa=(\widehat{\mathcal{H}}, \widehat{\Sigma})$ in $\mathbf{K}_{Q, \mathcal{H}}^{++}$induces a pair

in $\mathcal{J}_{n}^{(m) \text {,tor }}$ (with $U=\widehat{\mathcal{H}}$ ) in Sect. 5.2 of this paper, because, in order to define $(U, \Sigma)$ as in Sect. 5.2, it suffices to define the admissible cone decomposition $\Sigma(g)_{0}$ for $X_{*}\left(S_{n,(i), g U g^{-1} \cap P_{n,(i)}^{(m),+}\left(\mathbb{A}^{\infty}\right)}\right)_{\mathbb{R}}^{\succ 0}$, for each $g \in G_{n}^{(m)}\left(\mathbb{A}^{\infty}\right)$, which can be taken to be the pull-back of the subcollection $\left\{\widehat{\Sigma}_{\breve{\Phi}_{\widehat{\mathcal{H}}}}\right\}_{\left[\left(\breve{\Phi}_{\widehat{\mathcal{H}}}, \breve{\delta}_{\widehat{\mathcal{H}}}\right)\right]}$ of $\widehat{\Sigma}$ indexed by the cusp labels $\left[\left(\breve{\Phi}_{\widehat{\mathcal{H}}}, \breve{\delta}_{\widehat{\mathcal{H}}}\right)\right]$ with underlying $\breve{Z}_{\widehat{\mathcal{H}}}$ equal to $\breve{Z}_{\widehat{\mathcal{H}}}^{(i, \widehat{g})}$ for some $\widehat{g}$ in $\widehat{\mathrm{G}}\left(\mathbb{A}^{\infty}\right)$ corresponding to $g \in G_{n}^{(m)}\left(\mathbb{A}^{\infty}\right) \cong \widehat{\mathrm{G}}\left(\mathbb{A}^{\infty}\right)$. In fact, $\mathcal{J}_{n}^{(m) \text {,tor }}$ is exactly the set of such induced pairs (as $U=\widehat{\mathcal{H}}$ varies). (It is hard to 
explicitly describe the set $\mathcal{J}_{n}^{(m) \text {,tor }}$ when $m>0$, because they are induced by auxiliary choices of compatible collections $\widetilde{\Sigma}$ for $\widetilde{M}_{\tilde{\mathcal{H}}}$. Nevertheless, this is unnecessary for our purposes.)

Each $\widehat{\Sigma}_{\breve{\Phi}}$ defines an affine toroidal embedding

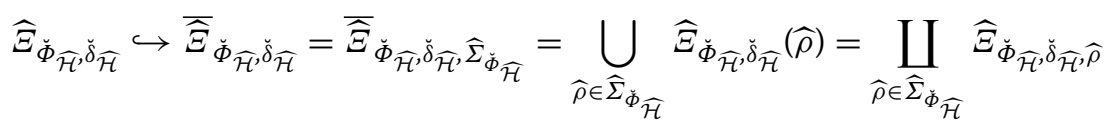

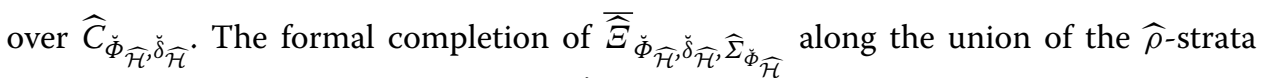
$\widehat{\Xi}_{\check{\Phi}_{\widehat{\mathcal{H}}}, \check{\delta}_{\widehat{\mathcal{H}}}, \widehat{\rho}}$ for all $\widehat{\rho} \in \widehat{\Sigma}_{\check{\Phi}_{\widehat{\mathcal{H}}}}$ such that $\widehat{\rho} \cap \widehat{\mathbf{P}}_{\dot{\Phi}_{\widehat{\mathcal{H}}}}^{+} \neq \emptyset$ is denoted

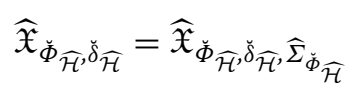

(see (1.3.2.62), (1.3.2.63), (1.3.2.64) and (1.3.2.66) of [44]). The schemes

$$
T_{n,(i), U_{g}^{\prime}, \Sigma(g)_{0}}^{(m),+} \rightarrow A_{n,(i), U_{g}^{\prime}}^{(m),+}
$$

of this paper (see Sect. 4.4) are identified with

$$
\coprod_{h \in L_{h,(i), \operatorname{lin}}^{(m)}\left(\mathbb{A}^{\infty}\right) / U_{g}^{\prime}} \widehat{\widehat{\Xi}}_{\breve{\Phi}_{\mathcal{H}}^{(i, h g)}, \check{\delta}_{\widehat{\mathcal{H}}}} \rightarrow \coprod_{h \in L_{n,(i)) \operatorname{lin}}^{(m)}\left(\mathbb{A}^{\infty}\right) / U_{g}^{\prime}} \widehat{C}_{\breve{\Phi}_{\mathcal{H}}^{(i, h g)}, \check{\delta}_{\widehat{\mathcal{H}}}} \cdot
$$

Moreover, $T_{n,(i), U_{g}^{\prime}, \Sigma(g)_{0}}^{(m),+\wedge}$ is identified with

$$
\coprod_{h \in L_{h,(i), \operatorname{lin}}^{(m)}\left(\mathbb{A}^{\infty}\right) / U_{g}^{\prime}} \widehat{\mathfrak{X}}_{\breve{\Phi}_{\mathcal{\mathcal { H }}}^{(i, h)}, \check{\delta}_{\breve{\mathcal{H}}}}
$$

and $T_{n,(i), U_{g}^{\prime}, \Sigma(g)_{0}}^{(m), \downarrow \wedge}$ is identified with

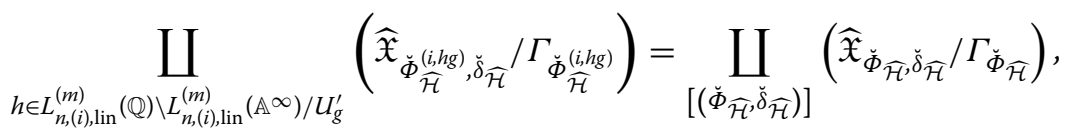

where the second disjoint union is over cusp labels with underlying $\breve{Z}_{\widehat{\mathcal{H}}}$ equal to $\breve{Z}_{\widehat{\mathcal{H}}}^{(i, \widehat{g})}$ for a fixed $\widehat{g}$ in $\widehat{\mathrm{G}}\left(\mathbb{A}^{\infty}\right)$ corresponding to $g \in G_{n}^{(m)}\left(\mathbb{A}^{\infty}\right) \cong \widehat{\mathrm{G}}\left(\mathbb{A}^{\infty}\right)$. (Again $\widehat{\mathcal{H}}=U$.)

For each $\kappa=(\widehat{\mathcal{H}}, \widehat{\Sigma}) \in \mathbf{K}_{Q, \mathcal{H}}^{++}$, we have a smooth projective scheme

$$
\mathrm{N}_{\kappa}^{\text {tor }}
$$

containing N (of Sect. B.6, which we will henceforth write as $\mathrm{N}_{\kappa}$ to emphasize the dependence on $\widehat{\mathcal{H}}$ ) as an open dense subscheme, called a toroidal compactification of $\mathrm{N}_{\kappa}$ (see theorem 1.3.3.15 of [44]). When $U=\widehat{\mathcal{H}}$, and when $(U, \Sigma) \in \mathcal{J}_{n}^{(m) \text {,tor }}$ is induced by $\kappa=(\widehat{\mathcal{H}}, \widehat{\Sigma})$ as above, the toroidal compactification

$$
\mathrm{N}_{\kappa} \hookrightarrow \mathrm{N}_{\kappa}^{\text {tor }}
$$


is the

$$
A_{n, U}^{(m)} \hookrightarrow A_{n, U, \Sigma}^{(m)}
$$

in this paper (see Sect. 5.3). Such toroidal compactifications and the identifications between them are compatible with the actions of $\widehat{G}\left(\mathbb{A}^{\infty}\right)=G_{n}^{(m)}\left(\mathbb{A}^{\infty}\right)$ (see theorem 1.3.3.15(4) of [44]).

The toroidal compactification $\mathrm{N}_{\kappa}^{\text {tor }}$ admits a stratification by locally closed subschemes

$$
\mathrm{N}_{\kappa}^{\text {tor }}=\coprod_{\left[\left(\breve{\Phi}_{\widehat{\mathcal{H}}}, \breve{\delta}_{\widehat{\mathcal{H}}}, \widehat{\rho}\right)\right]} \widehat{\mathrm{Z}}_{\left[\left(\check{\Phi}_{\widehat{\mathcal{H}}}, \breve{\delta}_{\widehat{\mathcal{H}}}, \widehat{\rho}\right)\right]}
$$

(see theorem 1.3.3.15(1) of [44]) indexed by equivalence classes $\left[\left(\breve{\Phi}_{\widehat{\mathcal{H}}}, \breve{\delta}_{\widehat{\mathcal{H}}}, \widehat{\rho}\right)\right]$ as in lemma 1.2.4.42 of [44]. Without repeating the definition in detail, let us just note that the equivalence classes $\left[\left(\breve{\Phi}_{\widehat{\mathcal{H}}}, \breve{\delta}_{\widehat{\mathcal{H}}}, \widehat{\rho}\right)\right]$ with the same underlying cusp label $\left[\left(\breve{\Phi}_{\widehat{\mathcal{H}}}, \breve{\delta}_{\widehat{\mathcal{H}}}\right)\right]$ can be identified with the $\Gamma_{\breve{\Phi}_{\widehat{\mathcal{H}}}}$-orbits of the cones $\widehat{\rho} \in \widehat{\Sigma}_{\breve{\Phi}_{\widehat{\mathcal{H}}}}$ such that $\widehat{\rho} \cap \widehat{\mathbf{P}}_{\breve{\Phi}_{\widehat{\mathcal{H}}}^{+}}^{+} \neq \emptyset$. Each stratum $\widehat{Z}_{\left[\left(\breve{\Phi}_{\widehat{\mathcal{H}}}, \breve{\delta}_{\widehat{\mathcal{H}}}, \widehat{\rho}\right)\right]}$ is canonically isomorphic to $\widehat{\Xi}_{\breve{\Phi}_{\widehat{\mathcal{H}}}, \breve{\delta}_{\widehat{\mathcal{H}}}, \widehat{\rho}}$, and the formal completion of $\mathrm{N}_{\kappa}^{\text {tor }}$ along the union of the strata $\widehat{Z}_{\left[\left(\breve{\Phi}_{\widehat{\mathcal{H}}}, \breve{\delta}_{\widehat{\mathcal{H}}}, \widehat{\rho}\right)\right]}$ labelled by equivalence classes $\left[\left(\breve{\Phi}_{\widehat{\mathcal{H}}}, \breve{\delta}_{\widehat{\mathcal{H}}}, \widehat{\rho}\right)\right]$ with the same underlying cusp label $\left[\left(\breve{\Phi}_{\widehat{\mathcal{H}}}, \breve{\delta}_{\widehat{\mathcal{H}}}\right)\right]$ is canonically isomorphic to $\widehat{\mathfrak{X}}_{\breve{\Phi}_{\widehat{\mathcal{H}}}} \breve{\delta}_{\widehat{\mathcal{H}}} / \Gamma_{\check{\Phi}_{\widehat{\mathcal{H}}}}$ (see theorem 1.3.3.15(1) of [44]).

When $U=\widehat{\mathcal{H}}$, and when $(U, \Sigma) \in \mathcal{J}_{n}^{(m) \text {,tor }}$ is induced by $\kappa=(\widehat{\mathcal{H}}, \widehat{\Sigma})$ as above, the formal completion of $\mathrm{N}_{\kappa}^{\text {tor }}$ along the union of all strata $\widehat{Z}_{\left[\left(\check{\Phi}_{\widehat{\mathcal{H}}}, \check{\delta}_{\widehat{\mathcal{H}}}, \widehat{\rho}\right)\right]}$ labelled by equivalence classes $\left[\left(\breve{\Phi}_{\widehat{\mathcal{H}}}, \breve{\delta}_{\widehat{\mathcal{H}}}, \widehat{\rho}\right)\right]$ with underlying $\breve{z}_{\widehat{\mathcal{H}}}$ equal to $\breve{\mathrm{Z}}_{\widehat{\mathcal{H}}}^{(i, \widehat{g})}$ for some $\widehat{g} \in \widehat{G}\left(\mathbb{A}{ }^{\infty}\right)$ is

$$
A_{n, U, \Sigma, i}^{(m), \wedge} \cong \coprod_{g} T_{n,(i), g U g^{-1} \cap P_{n,(i)}^{(m),+}\left(\mathbb{A}^{\infty}\right), \Sigma(g)_{0}}^{(m), 4, \wedge}=\coprod_{g} \coprod_{h}\left(\widehat{\mathfrak{X}}_{\breve{\Phi}_{\widehat{\mathcal{H}}}^{(i, h g)}, \breve{\delta}_{\widehat{\mathcal{H}}}} / \Gamma_{\breve{\Phi}_{\widehat{\mathcal{H}}}^{(i, h g)}}\right),
$$

where the indices $g$ and $h$ run over $P_{n,(i)}^{(m),+}\left(\mathbb{A}^{\infty}\right) \backslash G_{n}^{(m)}\left(\mathbb{A}^{\infty}\right) / U$ and

$$
L_{n,(i), \operatorname{lin}}^{(m)}(\mathbb{Q}) \backslash L_{n,(i), \operatorname{lin}}^{(m)}\left(\mathbb{A}^{\infty}\right) /\left(g U g^{-1} \cap P_{n,(i)}^{(m),+}\left(\mathbb{A}^{\infty}\right)\right),
$$

respectively, in this paper (see Sect. 5.3).

These identifications are all Hecke equivariant (see theorem 1.3.3.15(4) of [44]).

If $\kappa \in \mathbf{K}_{Q, \mathcal{H}, \Sigma}^{++}$, then the canonical morphism

$$
\mathrm{N}_{\kappa} \rightarrow \mathrm{M}_{\mathcal{H}}
$$

extends to a canonical log smooth morphism

$$
\mathrm{N}_{\kappa}^{\text {tor }} \rightarrow \mathrm{M}_{\mathcal{H}, \Sigma}^{\text {tor }}
$$

(see theorem 1.3.3.15(2) of [44]). When $U=\widehat{\mathcal{H}}$ and $U^{\prime}=\mathcal{H}$, and when $(U, \Sigma) \in \mathcal{J}_{n}^{(m) \text {,tor }}$ and $\left(U^{\prime}, \Delta\right) \in \mathcal{J}_{n}^{\text {tor }}$ are induced by $\kappa=(\widehat{\mathcal{H}}, \widehat{\Sigma})$ and $(\mathcal{H}, \Sigma)$, respectively, we have

$$
(U, \Sigma) \geq\left(U^{\prime}, \Delta\right)
$$


(see Sect. 5.2 in this paper) and the above morphism is the log smooth morphism

$$
A_{n, U, \Sigma}^{(m)} \rightarrow X_{n, U^{\prime}, \Delta}
$$

in this paper (see Sect. 5.3). These morphisms and identifications are equivariant with $\widehat{\mathrm{G}}\left(\mathbb{A}^{\infty}\right) \rightarrow \mathrm{G}\left(\mathbb{A}^{\infty}\right)$ (see proposition 1.3.3.15(4) of [44]).

\section{B.8 Automorphic bundles in characteristic zero}

Since the maximal isotropic submodule $V_{0}$ of $L \otimes_{\mathbb{Z}} \mathbb{C}$ on which $h_{0}(z)$ acts by $1 \otimes z$ is isomorphic to $\left(F \otimes_{\mathbb{Q}} \mathbb{C}\right)^{n}$ as an $F \otimes_{\mathbb{Q}} \mathbb{C}$-module, we can take $F_{0}^{\prime}=F_{0}=\mathbb{Q}$ and $L_{0}=F^{n}$ in the beginning of section 1.4.1 of [44], and define

$$
\mathrm{M}_{0}(R):=\mathrm{GL}_{\mathcal{O} \otimes_{\mathbb{Z}} R}\left(L_{0}^{\vee}(1) \otimes_{\mathbb{Q}} R\right) \times \mathbf{G}_{\mathrm{m}}(R)
$$

for each $\mathbb{Q}$-algebra $R$. (See definition 6.2 of [42], definition 1.4.1.1 of [44]. We will not need the other groups $\mathrm{G}_{0}(R)$ and $\mathrm{P}_{0}(R)$ in this paper.) This can be canonically identified with the group $L_{n,(n)}(R)$ in this paper (see Sect. 1.2), by matching $\mathrm{GL}_{\mathcal{O} \otimes_{\mathbb{Z}} R}\left(L_{0}^{\vee}(1) \otimes_{\mathbb{Q}} R\right.$ ) with $L_{n,(n), \text { lin }}(R)$, and by matching $\mathbf{G}_{\mathrm{m}}(R)$ with $L_{n,(n), \text { herm }}(R)$.

Let $\mathcal{H}$ be any neat open compact subgroup of $G(\hat{\mathbb{Z}})$, so that $M_{\mathcal{H}}$ is defined over Spec $\mathbb{Q}$ as in Sect. B.1. Then the tautological abelian scheme $A$ over $M_{\mathcal{H}}$ defines a locally free sheaf

$$
\underline{\mathrm{Lie}}_{A / \mathrm{M}_{\mathcal{H}}}^{\vee}:=e_{A}^{*} \Omega_{A / \mathrm{M}_{\mathcal{H}}}^{1}
$$

(where $e_{A}$ denotes the identity section), which is the

$$
\Omega_{n, U}
$$

in this paper (see Sect. 3.4.1), with $U=\mathcal{H}$. We can similarly define $\underline{\operatorname{Lie}}_{A^{\vee} / \mathrm{M}_{\mathcal{H}}}$. The action of $\mathrm{G}\left(\mathbb{A}^{\infty}\right)$ on $\left\{\mathrm{M}_{\mathcal{H}}\right\}_{\mathcal{H}}$ is defined by respecting their tautological abelian schemes up to canonical $\mathbb{Q}^{\times}$-isogenies, which induces actions of $\mathrm{G}\left(\mathbb{A}^{\infty}\right)$ on $\left\{\underline{\mathrm{Lie}}_{A / \mathrm{M}_{\mathcal{H}}^{\vee}}^{\vee}\right\}_{\mathcal{H}}$ and $\left\{\underline{\mathrm{Lie}}_{A^{\vee} / \mathrm{M}_{\mathcal{H}}}\right\}_{\mathcal{H}}$ covering the one on $\left\{M_{\mathcal{H}}\right\}_{\mathcal{H}}$, which are compatible with the isomorphisms

$$
\lambda^{*}: \underline{\operatorname{Lie}}_{A^{\vee} / M_{\mathcal{H}}}^{\vee}(1) \stackrel{\sim}{\rightarrow} \underline{\operatorname{Lie}}_{A / M_{\mathcal{H}}}^{\vee}
$$

induced by the tautological polarizations $\lambda: A \rightarrow A^{\vee}$. Here the formal Tate twist is induced by the one on de Rham homology, realized by tensor products with $\mathbb{Z}(1)$ over $\mathbb{Z}$. Therefore, the corresponding Hecke action must be twisted by the similitude character $v$, which corresponds to the tensor product with

$$
\Xi_{n, U}=\mathcal{O}_{X_{n, U}}(\|v\|)
$$

in this paper (see Sect. 3.4.1).

Let $\Sigma$ be a compatible collection of admissible projective smooth rational polyhedral cone decompositions for $M_{\mathcal{H}}$, so that $M_{\mathcal{H}, \Sigma}^{\text {tor }}$ is defined over Spec $\mathbb{Q}$ as in Sect. B.5. Then the tautological semi-abelian scheme $G$ over $\mathrm{M}_{\mathcal{H}, \Sigma}^{\text {tor }}$ defines a locally free sheaf

$$
\underline{\operatorname{Lie}}_{G / \mathrm{M}_{\mathcal{H}, \Sigma}^{\mathrm{tor}}}^{\vee}:=e_{G}^{*} \Omega_{G / \mathrm{M}_{\mathcal{H}, \Sigma}^{\mathrm{tor}}}^{1}
$$


(where $e_{G}$ denotes the identity section), which is the

$$
\Omega_{n, U, \Delta}
$$

in this paper (see Sect. 5.4), with $U=\mathcal{H}$, and with $\Delta$ induced by $\Sigma$ as in Sect. B.5. We can similarly define $\underline{\mathrm{Lie}}_{G^{\vee} / \mathrm{M}_{\mathcal{H}, \Sigma}^{\text {tor }}}^{\vee}$, where $G^{\vee}$ denotes the tautological 'dual semi-abelian scheme' over $\mathrm{M}_{\mathcal{H}, \Sigma}^{\text {tor }}$ extending $A^{\vee}$. (Note that dual semi-abelian schemes only make sense as such extensions.) The action of $G\left(\mathbb{A}^{\infty}\right)$ on $\left\{\mathrm{M}_{\mathcal{H}, \Sigma}^{\text {tor }}\right\}_{(\mathcal{H}, \Sigma)}$ is defined by respecting their tautological semi-abelian schemes up to canonical $\mathbb{Q}^{\times}$-isogenies, which induces actions of $\mathrm{G}\left(\mathbb{A}^{\infty}\right)$ on $\left\{\underline{\mathrm{Lie}}_{G / \mathrm{M}_{\mathcal{H}, \Sigma}^{\mathrm{tor}}}\right\}_{(\mathcal{H}, \Sigma)}$ and $\left\{\underline{\mathrm{Lie}}_{G^{\vee} / \mathrm{M}_{\mathcal{H}, \Sigma}^{\text {tor }}}^{\vee}\right\}_{(\mathcal{H}, \Sigma)}$ covering the one on $\left\{\mathrm{M}_{\mathcal{H}, \Sigma}^{\text {tor }}\right\}_{(\mathcal{H}, \Sigma)}$, which are compatible with the isomorphisms

$$
\lambda^{*}: \underline{\mathrm{Lie}}_{G}^{\vee} / \mathrm{M}_{\mathcal{H}, \Sigma}^{\mathrm{tor}}(1) \stackrel{\sim}{\rightarrow} \underline{\mathrm{Lie}}_{G / \mathrm{M}_{\mathcal{H}, \Sigma}^{\mathrm{tor}}}^{\vee}
$$

induced by the tautological polarizations $\lambda: G \rightarrow G^{\vee}$. Here the formal Tate twist requires (as before) the Hecke action to be twisted by the similitude character $v$, which corresponds to the tensor product with

$$
\Xi_{n, U, \Sigma}=\mathcal{O}_{X_{n, U, \Sigma}}(\|v\|)
$$

in this paper (see Sect. 5.4).

Then we have the principal $\mathrm{M}_{0}$-bundle

$$
\mathcal{E}_{\mathrm{M}_{0}}:=\underline{\operatorname{Isom}}_{\mathcal{O} \otimes_{\mathbb{Z}} \mathscr{O}_{\mathrm{M}_{\mathcal{H}}}}\left(\left(\underline{\operatorname{Lie}}_{A^{\vee} / \mathrm{M}_{\mathcal{H}}}^{\vee}(1), \mathscr{O}_{\mathrm{M}_{\mathcal{H}}}(1)\right),\left(L_{0}^{\vee}(1) \otimes_{\mathbb{Q}} \mathscr{O}_{\mathrm{M}_{\mathcal{H}}}, \mathscr{O}_{\mathrm{M}_{\mathcal{H}}}(1)\right)\right)
$$

which is an $M_{0}$-torsor over $M_{\mathcal{H}}$ (see definition 1.4.1.5 and lemma 1.4.1.7 of [44]), which canonically extends (as an $\mathrm{M}_{0}$-torsor) to a principal $\mathrm{M}_{0}$-bundle

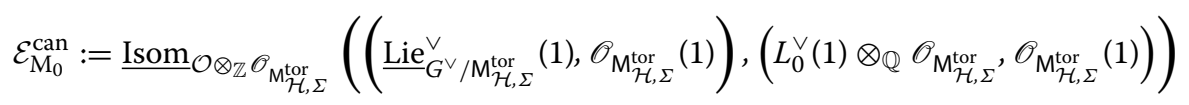

over $\mathrm{M}_{\mathcal{H}, \Sigma}^{\text {tor }}$ (see (1.4.2.7) and lemma 1.4.2.8 of [44]). These are the

$$
\mathcal{E}_{U} \text { and } \mathcal{E}_{U, \Delta}^{\mathrm{can}}
$$

in this paper (see Sects. 3.4.1, 5.4), with $U=\mathcal{H}$, and with $\Delta$ induced by $\Sigma$ as in Sect. B.5.

For each $\mathbb{Q}$-algebra $R$, we denote by $\operatorname{Rep}_{R}\left(\mathrm{M}_{0}\right)$ the category of $R$-modules with algebraic actions of $\mathrm{M}_{0} \otimes_{\mathbb{Q}} R$ (see definition 1.4.1.8 of [44]). Then we also define, for each $W \in$ $\operatorname{Rep}_{R}\left(M_{0}\right)$ that is locally free of finite rank as an $R$-module, the automorphic bundle

$$
\mathcal{E}_{\mathrm{M}_{0}, R}(W):=\left(\mathcal{E}_{\mathrm{M}_{0}} \otimes_{\mathbb{Q}} R\right) \times{ }^{\left(\mathrm{M}_{0} \otimes_{\mathbb{Q}} R\right)} W
$$

over $M_{\mathcal{H}} \otimes_{\mathbb{Q}} R$ (see definition 1.4.1.9 of [44]), which extends to the canonical extension

$$
\mathcal{E}_{\mathrm{M}_{0}, R}^{\mathrm{can}}(W):=\left(\mathcal{E}_{\mathrm{M}_{0}}^{\mathrm{can}} \otimes_{\mathbb{Q}} R\right) \times{ }^{\left(\mathrm{M}_{0} \otimes_{\mathbb{Q}} R\right)} W
$$

and the subcanonical extension

$$
\mathcal{E}_{\mathrm{M}_{0}, R}^{\mathrm{sub}}(W):=\mathcal{E}_{\mathrm{M}_{0}, R}^{\mathrm{can}}(W) \otimes_{\mathscr{O}_{\mathrm{M}_{\mathcal{H}, \Sigma}^{\text {tor }}}} \mathscr{I}_{\mathrm{D}_{\infty, \mathcal{H}, \Sigma}}
$$


over $\mathrm{M}_{\mathcal{H}}^{\text {tor }} \otimes_{\mathbb{Q}} R$ (see definition 1.4.2.9 of [44]), where $\mathscr{I}_{\mathrm{D}_{\infty, \mathcal{H}, \Sigma}}$ is the $\mathscr{O}_{\mathrm{M}_{\mathcal{H}, \Sigma}}$-ideal defining the boundary divisor $D_{\infty, \mathcal{H}, \Sigma}:=\mathrm{M}_{\mathcal{H}, \Sigma}^{\text {tor }}-\mathrm{M}_{\mathcal{H}}$ (with its canonical reduced subscheme structure). These are the vector bundles

$$
\mathcal{E}_{U, \rho}, \quad \mathcal{E}_{U, \Delta, \rho}^{\mathrm{can}}, \quad \text { and } \quad \mathcal{E}_{U, \Delta, \rho}^{\text {sub }}
$$

in this paper (see Sects. 3.4.1, 5.4), with $U=\mathcal{H}$ and $W_{\rho}=W$. The bundles $\mathcal{E}_{\mathrm{M}_{0}, R}(W)$ and $\mathcal{E}_{\mathrm{M}_{0}, R}^{\mathrm{can}}(W)$ admit compatible actions of $\mathrm{G}\left(\mathbb{A}^{\infty}\right)$ (see proposition 1.4.3.1 of [44]), which are compatible with the compatible actions of $G_{n}\left(\mathbb{A}^{\infty}\right)$ on $\mathcal{E}_{U, \rho}$ and $\mathcal{E}_{U, \Delta, \rho}^{\mathrm{can}}$, covering the ones on their respective base schemes.

\section{B.9 Total objects in mixed characteristics}

For each open compact subgroup $\mathcal{H}$ of $\mathrm{G}(\hat{\mathbb{Z}})$ whose image $\mathcal{H}^{p}$ under the canonical homomorphism $\mathrm{G}(\hat{\mathbb{Z}}) \rightarrow \mathrm{G}\left(\hat{\mathbb{Z}}^{p}\right)$ is neat, which implies, a fortiori, that $\mathcal{H}$ is also neat, we have a normal scheme

$$
\overrightarrow{\mathrm{M}}_{\mathcal{H}}
$$

which is quasi-projective and flat over $\vec{S}_{0}=\operatorname{Spec} \mathcal{O}_{F_{0},(p)}=\operatorname{Spec} \mathbb{Z}_{(p)}$ and satisfies $\vec{M}_{\mathcal{H}} \otimes_{\mathbb{Z}}$ $\mathbb{Q} \cong \mathrm{M}_{\mathcal{H}}$ (see proposition 2.2.1.1 in [44]). This is simply the normalization of $\mathrm{M}_{\mathcal{H}}$ over the auxiliary (Siegel) moduli $\mathbf{M}_{\mathcal{H}_{\text {aux }}}$ over $\operatorname{Spec} \mathcal{O}_{F_{0, \text { aux }}(p)}=\operatorname{Spec} \mathbb{Z}_{(p)}$ defined by the auxiliary integral PEL datum

$$
\left(\mathcal{O}_{\text {aux }}, \star_{\text {aux }}, L_{\text {aux }},\langle\cdot, \cdot\rangle_{\text {aux }}, h_{0, \text { aux }}\right)=\left(\mathbb{Z}, \text { Id }, L,\langle\cdot, \cdot\rangle, h_{0}\right),
$$

forgetting the actions of $\mathcal{O}=\mathcal{O}_{F}$, for any neat open compact subgroup $\mathcal{H}_{\text {aux }}$ of $\mathrm{G}_{\text {aux }}(\hat{\mathbb{Z}})$ (defined by the above auxiliary integral PEL datum) containing the image of $\mathcal{H}$ under the canonical homomorphism $\mathrm{G}(\hat{\mathbb{Z}}) \rightarrow \mathrm{G}_{\text {aux }}(\hat{\mathbb{Z}})$ (see lemma 2.1.1.18 of [44] for the existence of $\mathcal{H}_{\text {aux }}$ ).

Similarly, we have a normal scheme

$$
\overrightarrow{\mathrm{M}}_{\mathcal{H}}^{\min }
$$

which is projective and flat over Spec $\mathbb{Z}_{(p)}$ and satisfies $\vec{M}_{\mathcal{H}}^{\min } \otimes_{\mathbb{Z}} \mathbb{Q} \cong M_{\mathcal{H}}^{\min }$, and contains $\vec{M}_{\mathcal{H}}$ as an open fibrewise dense subscheme (see propositions 2.2.1.2 and 2.2.1.7, and corollary 2.2.1.15, in [44]). The scheme $\vec{M}_{\mathcal{H}}^{\min }$ is the

$$
\mathcal{X}_{n, U}^{\min }
$$

in this paper with $U=\mathcal{H}$, and the special fibre

$$
\overrightarrow{\mathrm{M}}_{\mathcal{H}}^{\min } \otimes_{\mathbb{Z}} \mathbb{F}_{p}
$$

is the

$$
\bar{X}_{n, U}^{\min },
$$

in this paper (see Sect. 5.1). 
The projective scheme $\overrightarrow{\mathrm{M}}_{\mathcal{H}}^{\min }$ is equipped with an ample invertible sheaf

$$
\omega_{\overrightarrow{\mathrm{M}}}^{\min }
$$

(see proposition 2.2.1.2 in [44]). (Since $\langle\cdot, \cdot\rangle$ is self-dual, we can take $a_{1}=1, a_{2}=0$, $a_{0}=1$ and $a=1$ in lemmas 2.1.1.1 and 2.1.2.35 of [44].) This is the

$\omega_{U}$

in this paper (see Sect. 5.1). We have a section

$$
\operatorname{Hasse}_{\mathcal{H}} \in H^{0}\left(\overrightarrow{\mathrm{M}}^{\min } \otimes_{\mathbb{Z}} \mathbb{F}_{p}, \omega_{\overrightarrow{\mathrm{M}}_{\mathcal{H}}^{\min }}^{\otimes(p-1)} \otimes_{\mathbb{Z}} \mathbb{F}_{p}\right)
$$

(see corollary 6.3.1.7 of [44]), which is the

$$
\text { Hasse }_{U} \in H^{0}\left(\bar{X}_{n, U}^{\min }, \omega_{U}^{\otimes(p-1)}\right)
$$

in this paper (see Sect. 5.1), whose vanishing and non-vanishing loci are

$$
\left(\overrightarrow{\mathrm{M}}_{\mathcal{H}}^{\min } \otimes_{\mathbb{Z}} \mathbb{F}_{p}\right)^{\text {non-ord }} \text { and }\left(\overrightarrow{\mathrm{M}}_{\mathcal{H}}^{\min } \otimes_{\mathbb{Z}} \mathbb{F}_{p}\right)^{\text {full-ord }}
$$

(see definition 6.3.2.1 of [44]), which are the

$$
\bar{X}_{n, U}^{\min , \mathrm{n}-\text { ord }} \text { and } \bar{X}_{n, U}^{\min }-\bar{X}_{n, U}^{\min , \mathrm{n}-\text { ord }},
$$

respectively, in this paper (see Sect. 5.1).

The collections $\left.\left\{\overrightarrow{\mathrm{M}}_{\mathcal{H}}\right\}_{\mathcal{H}},\left\{\overrightarrow{\mathrm{M}}_{\mathcal{H}}^{\min }\right\}_{\mathcal{H}},\left\{\omega_{\left.\overrightarrow{\mathrm{M}}_{\mathcal{H}}^{\min }\right\}_{\mathcal{H}} \text { and }\{\text { Hasse }}\right\}_{\mathcal{H}}\right\}_{\mathcal{H}}$ admit compatible actions of $\mathrm{G}\left(\mathbb{A}^{\infty, p}\right) \times \mathrm{G}\left(\mathbb{Z}_{p}\right)$ (see proposition 2.2.3.1 and corollary 6.3.1.8 of [44]).

While the reader might be interested in knowing more about $\vec{M}_{\mathcal{H}}$ and $\vec{M}_{\mathcal{H}}^{\min }$, we emphasize that we need to know almost nothing about them in this paper. What we really need to know in detail are their ordinary loci (or more precisely just their multiplicative-type ordinary loci, rather than the whole $\left(\vec{M}_{\mathcal{H}}^{\min } \otimes_{\mathbb{Z}} \mathbb{F}_{p}\right)^{\text {full-ord }}$ as above), which we will explain below.

\section{B.10 Ordinary loci of Shimura varieties}

To define the ordinary loci $\vec{M}_{\mathcal{H}}^{\text {ord }}$ in [44], which will be compared with the $\mathcal{X}_{n, U^{p}\left(N_{1}, N_{2}\right)}^{\text {ord }}$ for suitable choices of $\mathcal{H}$ and $U^{p}\left(N_{1}, N_{2}\right)$, we consider the maximal totally isotropic filtration

$$
0=\mathrm{D}^{1} \subset \mathrm{D}^{0} \subset \mathrm{D}^{-1}=L \otimes_{\mathbb{Z}} \mathbb{Z}_{p}
$$

(see lemma 3.2.2.1 of [44]) given by

$$
\mathrm{D}^{0}=\mathrm{V}^{(n)} \cap\left(L \otimes_{\mathbb{Z}} \mathbb{Z}_{p}\right)
$$

Since $\langle\cdot, \cdot\rangle$ is self-dual, the dual filtration $\mathrm{D}^{\#}$ in lemma 3.2.2.4 of [44] can be identified with $\mathrm{D}$, and the induced inclusions $\phi_{\mathrm{D}}^{0}: \mathrm{Gr}_{\mathrm{D}}^{0} \hookrightarrow \mathrm{Gr}_{\mathrm{D}^{*}}^{0}$ and $\phi_{\mathrm{D}}^{-1}: \mathrm{Gr}_{\mathrm{D}}^{-1} \hookrightarrow \mathrm{Gr}_{\mathrm{D}^{\ddagger}}^{-1}$ (again, see lemma 3.2.2.4 of [44]) are isomorphisms. Moreover, the group $\overrightarrow{\boldsymbol{S}}_{\mathrm{D}}$ in theorem 3.4.1.9 of [44] is torsion free because it can be identified with $S\left(\mathcal{O}_{F, p}^{n}\right)$ in this paper (see Sect. 1.1), 
and hence the invariant $r_{\mathrm{D}}$ in definition 3.4.2.1 of [44] is just zero under the assumptions of this paper.

Then we define the following groups, for each $\mathbb{Z}_{p}$-algebra $R$ (see definition 3.2.2.7 of [44]):

$$
\begin{aligned}
& \mathrm{P}_{\mathrm{D}}^{\text {ord }}(R):=\left\{\begin{array}{c}
(g, r) \in \mathrm{GL}_{\mathcal{O} \otimes_{\mathbb{Z}} R}\left(L \otimes_{\mathbb{Z}} R\right) \times \mathbf{G}_{\mathrm{m}}(R): \\
(g, r) \in \mathrm{G}(R), g\left(\mathrm{D} \otimes_{\mathbb{Z}_{p}} R\right)=\mathrm{D} \otimes_{\mathbb{Z}_{p}} R
\end{array}\right\}=P_{n,(n)}^{+}(R), \\
& \mathrm{M}_{\mathrm{D}}^{\text {ord }}(R):=\left\{\begin{array}{c}
(g, r) \in \mathrm{GL}_{\mathcal{O} \otimes_{\mathbb{Z}} R}\left(\mathrm{Gr}_{\mathrm{D}} \otimes_{\mathbb{Z}_{p}} R\right) \times \mathbf{G}_{\mathrm{m}}(R): \\
\langle g x, g y\rangle=r\langle x, y\rangle, \forall x, y \in \mathrm{Gr}_{\mathrm{D}} \otimes_{\mathbb{Z}_{p}} R
\end{array}\right\}=L_{n,(n)}(R), \\
& \mathrm{U}_{\mathrm{D}}^{\text {ord }}(R):=\operatorname{ker}\left(\mathrm{Gr}_{\mathrm{D}}: \mathrm{P}_{\mathrm{D}}^{\text {ord }}(R) \rightarrow \mathrm{M}_{\mathrm{D}}^{\text {ord }}(R)\right)=N_{n,(n)}(R),
\end{aligned}
$$

and

$$
\mathrm{U}_{\mathrm{D}}^{\mathrm{ord},-1}(R):=\operatorname{ker}\left(\mathrm{Gr}_{\mathrm{D}}^{-1}: \mathrm{P}_{\mathrm{D}}^{\mathrm{ord}}(R) \rightarrow \mathrm{GL}_{\mathcal{O} \otimes_{\mathbb{Z}} R}\left(\mathrm{Gr}_{\mathrm{D}}^{-1} \otimes_{\mathbb{Z}_{p}} R\right)\right)=P_{n,(n)}(R) .
$$

Then $G\left(\mathbb{A}^{\infty, p}\right) \times \mathrm{P}_{\mathrm{D}}^{\text {ord }}\left(\mathbb{Z}_{p}\right)=G_{n}\left(\mathbb{A}^{\infty}\right)^{\text {ord, } \times}$, in the notation of this paper.

For all integers $0 \leq r$ and $0 \leq r_{1} \leq r_{0}$, we set (see definition 3.2.2.8 of [44]):

(2) $\quad \mathcal{U}_{p, 1}\left(p^{r}\right):=\left(\mathrm{G}\left(\mathbb{Z}_{p}\right) \rightarrow \mathrm{G}\left(\mathbb{Z} / p^{r} \mathbb{Z}\right)\right)^{-1}\left(\mathrm{U}_{\mathrm{D}}^{\text {ord, }-1}\left(\mathbb{Z} / p^{r} \mathbb{Z}\right)\right)=U_{p}(r, r)$.

(3) $\mathcal{U}_{p, 1}^{\text {bal }}\left(p^{r}\right):=\left(\mathrm{G}\left(\mathbb{Z}_{p}\right) \rightarrow \mathrm{G}\left(\mathbb{Z} / p^{r} \mathbb{Z}\right)\right)^{-1}\left(\mathrm{U}_{\mathrm{D}}^{\text {ord }}\left(\mathbb{Z} / p^{r} \mathbb{Z}\right)\right)$.

(4) $\mathcal{U}_{p, 1,0}\left(p^{r_{1}}, p^{r_{0}}\right):=\mathcal{U}_{p, 1}\left(p^{r_{1}}\right) \cap \mathcal{U}_{p, 0}\left(p^{r_{0}}\right)=U_{p}\left(r_{1}, r_{0}\right)$.

(5) $\quad \mathcal{U}^{\text {ord }}\left(p^{r}\right):=\operatorname{ker}\left(\mathrm{M}_{\mathrm{D}}^{\text {ord }}\left(\mathbb{Z}_{p}\right) \rightarrow \mathrm{M}_{\mathrm{D}}^{\text {ord }}\left(\mathbb{Z} / p^{r} \mathbb{Z}\right)\right)=U_{p}(r)_{n,(n)} \times\left(1+p^{r} \mathbb{Z}_{p}\right)^{\times}$.

An open compact subgroup $\mathcal{H}_{p}$ of $\mathrm{G}\left(\mathbb{Q}_{p}\right)$ is said to be of standard form and of depth $r$ in the sense of definition 3.2.2.9 of [44] if

$$
\mathcal{U}_{p, 1}^{\mathrm{bal}}\left(p^{r}\right) \subset \mathcal{H}_{p} \subset \mathcal{U}_{p, 0}\left(p^{r}\right)
$$

In this case we define $\mathcal{H}_{p}^{\text {ord }}$ to be the unique open compact subgroup of $\mathrm{M}_{\mathrm{D}}^{\text {ord }}\left(\mathbb{Z}_{p}\right)$ such that $\mathcal{H}_{p}^{\text {ord }} / \mathcal{U}^{\text {ord }}\left(p^{r}\right) \cong \mathcal{H}_{p} / \mathcal{U}_{p, 1}^{\text {bal }}\left(p^{r}\right)$ (see definition 3.3.3.4 of [44]).

The theory in [44] is developed for open compact subgroups $\mathcal{H}$ of $\mathrm{G}(\hat{\mathbb{Z}})$ of the form $\mathcal{H}=\mathcal{H}^{p} \mathcal{H}_{p}$, where $\mathcal{H}^{p}$ is a neat open compact subgroup of $\mathrm{G}\left(\hat{\mathbb{Z}}^{p}\right)$, and where $\mathcal{H}_{p}$ is an open compact subgroup of $\mathrm{G}\left(\mathbb{Z}_{p}\right)$ of standard form. In this paper, we will only need $\mathcal{H}$ of the form $U^{p}\left(N_{1}, N_{2}\right)$, which satisfies the above requirement with $\mathcal{H}^{p}=U^{p}$ and $\mathcal{H}_{p}=\mathcal{U}_{p, 1,0}\left(p^{N_{1}}, p^{N_{2}}\right)$. In this case, since $v\left(\mathcal{H}_{p}\right)=\mathbb{Z}_{p}^{\times}$and $r_{\mathrm{D}}=0$, the invariant $r_{\mathcal{H}}$ in definition 3.4.2.1 of [44] is just zero. Then we have a smooth quasi-projective scheme

$$
\overrightarrow{\mathrm{M}}_{\mathcal{H}}^{\text {ord }}
$$

over $\overrightarrow{\mathrm{S}}_{0}=\operatorname{Spec} \mathcal{O}_{F_{0},(p)}=\operatorname{Spec} \mathbb{Z}_{(p)}$, which satisfies $\overrightarrow{\mathrm{M}}_{\mathcal{H}}^{\text {ord }} \otimes_{\mathbb{Z}} \mathbb{Q} \cong \mathrm{M}_{\mathcal{H}}$ and can be canonically embedded as an open subscheme of $\vec{M}_{\mathcal{H}}$ (see theorem 3.4.2.5 and proposition 3.4.6.3 of [44]). Note that, in theorem 3.4.2.5 of [44], $\overrightarrow{\mathrm{M}}_{\mathcal{H}}^{\text {ord }}$ is defined as a normalization of the base change from $\operatorname{Spec} \mathbb{Z}_{(p)}$ to $\operatorname{Spec}\left(\mathcal{O}_{F_{0},(p)}\left[\zeta_{p^{r} \mathcal{H}}\right]\right)$ of a naive moduli $\dddot{M}_{\mathcal{H}}^{\text {ord }}$ over $\operatorname{Spec} \mathbb{Z}_{(p)}$ (see definition 3.4.1.1 and theorem 3.4.1.9 of [44]), but since $F_{0}=\mathbb{Q}, r_{\mathrm{D}}=0$, and $r_{\mathcal{H}}=0$, the base change has no effect, and the normalization merely singles out the correct components satisfying the condition on Lie algebra. Hence, $\vec{M}_{\mathcal{H}}^{\text {ord }}$ coincides with the moduli problem

$$
\mathcal{X}_{n, U^{p}\left(N_{1}, N_{2}\right)}^{\text {ord }}
$$


in this paper (see Sect. 3.1.1), with $U^{p}\left(N_{1}, N_{2}\right)=\mathcal{H}$ (that is, with $U^{p}=\mathcal{H}^{p}, N_{1}=r_{1}$, and $N_{2}=r_{0}$ ). (See remark 3.4.2.8 of [44] for the comparison between the definition using isomorphism classes of abelian schemes with additional structures in [44], and the definition of $\mathcal{X}_{n, U^{p}\left(N_{1}, N_{2}\right)}^{\text {ord }}$ using prime-to- $p$ quasi-isogeny classes in this paper, and for the extension of the definition of $\vec{M}_{\mathcal{H}}^{\text {ord }}$ to allow $\mathcal{H}=\mathcal{H}^{p} \mathcal{H}_{p}$ for all open compact subgroups $\mathcal{H}^{p}$ of $\mathrm{G}\left(\hat{\mathbb{Z}}^{p}\right)$. Again, since the pairing $\langle\cdot, \cdot\rangle$ is self-dual, the consideration of dual objects in [44] can be suppressed, although they were clarifying when developing the general theory.)

The formal completion of $\overrightarrow{\mathrm{M}}_{\mathcal{H}}^{\text {ord }} \cong \mathcal{X}_{n, U^{p}\left(N_{1}, N_{2}\right)}^{\text {ord }}\left(\right.$ with $\left.U^{p}\left(N_{1}, N_{2}\right)=\mathcal{H}\right)$ along

$$
\overrightarrow{\mathrm{M}}_{\mathcal{H}}^{\text {ord }} \otimes_{\mathbb{Z}} \mathbb{F}_{p} \cong \bar{X}_{n, U^{p}\left(N_{1}\right)}^{\text {ord }}
$$

is denoted

$$
\overrightarrow{\mathfrak{M}}_{\mathcal{H}}^{\text {ord }} \cong \mathfrak{X}_{n, U^{p}\left(N_{1}\right)}^{\text {ord }}
$$

(see definition 3.4.4.2 of [44]). Their independence of $N_{2}=r_{0}$ is explained in corollary 3.4.4.4 of [44].

The collection $\left\{\vec{M}_{\mathcal{H}}^{\text {ord }}\right\}_{\mathcal{H}}$ indexed by neat open compact subgroups $\mathcal{H}$ of $G\left(\mathbb{A}^{\infty}\right)$ of the form considered above admits compatible actions (see proposition 3.4.4.1 of [44]) of $\mathrm{G}\left(\mathbb{A}^{\infty, p}\right) \times \mathrm{P}_{\mathrm{D}}^{\text {ord }}\left(\mathbb{Z}_{p}\right)$ and of the element of $\mathrm{P}_{\mathrm{D}}^{\text {ord }}\left(\mathbb{Q}_{p}\right) \subset \mathrm{G}\left(\mathbb{Q}_{p}\right)$ corresponding to $\varsigma_{p} \in$ $L_{n,(n), \text { herm }}\left(\mathbb{Q}_{p}\right)$ in this paper (see Sect. 1.2) under the identification between $G\left(\mathbb{Q}_{p}\right)$ and $G_{n}\left(\mathbb{Q}_{p}\right)$, which are compatible with the action of $G_{n}\left(\mathbb{A}^{\infty}\right)^{\text {ord }}$ on $\left\{\mathcal{X}_{n, U^{p}\left(N_{1}, N_{2}\right)}^{\text {ord }}\right\}_{U^{p}\left(N_{1}, N_{2}\right)}$. (In fact, proposition 3.4.4.1 of [44] gives the actions of many more elements, but we omit them because they are not needed in this paper.) While these actions are given by quasi-finite morphisms which are often not finite, the induced morphisms on the $p$-adic completions are always finite (see corollary 3.4.4.3 of [44]). The element of $\mathrm{P}_{\mathrm{D}}^{\text {ord }}\left(\mathbb{Q}_{p}\right)$ corresponding to $\varsigma_{p}$ is a typical example of an element of $\mathrm{U}_{p}$ type in definition 3.3.6.1 of [44], which induces the composite of absolute Frobenius and forgetful morphisms in characteristic $p$ (see corollary 3.4.4.6 of [44]).

\section{B.11 Ordinary cusp labels}

Let $\mathcal{H}$ be any open compact subgroup of $\mathrm{G}(\hat{\mathbb{Z}})$ as above. We say that a cusp label $\left(\mathrm{Z}_{\mathcal{H}}, \Phi_{\mathcal{H}}, \delta_{\mathcal{H}}\right)$ is ordinary (see definition 3.2.3.8 of [44]) if $\mathrm{Z}_{\mathcal{H}}$ contains an element $\mathrm{Z}$ that is compatible with the filtration $\mathrm{D}$ in the sense that

$$
0 \subset \mathrm{Z}_{-2} \otimes_{\hat{\mathbb{Z}}} \mathbb{Z}_{p} \subset \mathrm{D} \subset \mathrm{Z}_{-1} \otimes_{\hat{\mathbb{Z}}} \mathbb{Z}_{p} \subset L \otimes_{\mathbb{Z}} \mathbb{Z}_{p}
$$

(see definition 3.2.3.1 of [44]). Then we have an induced filtration $\mathrm{D}_{-1}$ on $\mathrm{Gr}_{-1}^{\mathrm{Z}} \otimes_{\hat{\mathbb{Z}}} \mathbb{Z}_{p}$ given by 


$$
0=\mathrm{D}_{-1}^{1} \subset \mathrm{D}_{-1}^{0}:=\mathrm{D}^{0} /\left(\mathrm{Z}_{-2} \otimes_{\hat{\mathbb{Z}}} \mathbb{Z}_{p}\right) \subset \mathrm{D}_{-1}^{-1}=\mathrm{Gr}_{-1}^{\mathrm{Z}} \otimes_{\hat{\mathbb{Z}}} \mathbb{Z}_{p}
$$

For any such $\mathrm{Z}$, and for each $\mathbb{Z}_{p}$-algebra $R$, we define the following quotients of subgroups of $\mathrm{P}_{\mathrm{Z}}(R)$ (see definition 3.2.3.9 of [44]):

(1) $\quad \mathrm{P}_{\mathrm{Z}, \mathrm{D}}^{\text {ord }}(R):=\mathrm{P}_{\mathrm{Z}}(R) \cap \mathrm{P}_{\mathrm{D}}^{\text {ord }}(R)$.

(2) $\quad \mathrm{P}_{\mathrm{Z}, \mathrm{D}}^{\mathrm{ord},}(R):=\mathrm{P}_{\mathrm{Z}}^{\prime}(R) \cap \mathrm{P}_{\mathrm{D}}^{\text {ord }}(R)$.

(3) $\quad \mathrm{P}_{1, \mathrm{Z}, \mathrm{D}}^{\text {ord }}(R):=\mathrm{P}_{\mathrm{Z}, \mathrm{D}}^{\text {ord, }}(R) / \mathrm{U}_{2, \mathrm{Z}}(R)$.

(4) $\mathrm{P}_{h, Z, \mathrm{D}}^{\text {ord }}(R)$ is the subgroup of elements of $\mathrm{G}_{h, \mathrm{Z}}(R)$ preserving the filtration $\mathrm{D}_{-1}$ induced by $\mathrm{D}$ on $\mathrm{Gr}_{-1}^{\mathrm{Z}} \otimes_{\hat{\mathbb{Z}}} \mathbb{Z}_{p}$.

(5) $\quad \mathrm{P}_{h, \mathrm{Z}, \mathrm{D}}^{\text {ord, }}(R):=\mathrm{P}_{\mathrm{Z}, \mathrm{D}}^{\text {ord, }}(R) / \mathrm{U}_{\mathrm{Z}}(R) \stackrel{\sim}{\rightarrow} \mathrm{P}_{h, \mathrm{Z}, \mathrm{D}}^{\text {ord }}(R)$.

We have, for example,

$$
\mathrm{P}_{\mathrm{Z}^{(i, 1)}}\left(\mathbb{A}^{\infty, p}\right) \times \mathrm{P}_{\mathrm{Z}^{(i, 1), \mathrm{D}}}^{\mathrm{ord}}\left(\mathbb{Z}_{p}\right)=P_{n,(i)}^{+}\left(\mathbb{A}^{\infty}\right)^{\text {ord }, \times}
$$

in the notation of this paper (see Sect. 1.2). (In this paper, all intersections with $\mathrm{P}_{\mathrm{D}}^{\text {ord }}\left(\mathbb{Z}_{p}\right)=$ $P_{n,(n)}^{+}\left(\mathbb{Z}_{p}\right)$ at the factors at $p$ are denoted by the superscript ${ }^{\text {ord, } \times}$.) By definition, as $g$ varies in $G\left(\mathbb{A}^{\infty}\right)$, the filtration $\mathrm{Z}^{(i, g)}$ is compatible with $\mathrm{D}$ if and only if $g \in G\left(\mathbb{A}^{\infty, p}\right) \times$ $\mathrm{P}_{\mathrm{Z}^{(i, 1)}}\left(\mathbb{Q}_{p}\right) \mathrm{P}_{\mathrm{D}}^{\text {ord }}\left(\mathbb{Q}_{p}\right)$.

Now suppose $\mathcal{H}=\mathcal{H}^{p} \mathcal{H}_{p}$, where $\mathcal{H}^{p}$ is an open compact subgroup of $\mathrm{G}\left(\mathbb{A}^{\infty, p}\right)$ and $\mathcal{H}_{p}=\mathcal{U}_{p, 1,0}\left(p^{r_{1}}, p^{r_{0}}\right)$ for some integers $0 \leq r_{1} \leq r_{0}$. The $\mathcal{H}$-orbits $\mathrm{Z}_{\mathcal{H}}^{(i, g)}$ of $\mathrm{z}^{(i, g)}$ that contains a filtration compatible with $D$ are parameterized by the image of $G\left(\mathbb{A}^{\infty, p}\right) \times$ $\mathrm{P}_{\mathrm{Z}^{(i, 1)}}\left(\mathbb{Q}_{p}\right) \mathrm{P}_{\mathrm{D}}^{\text {ord }}\left(\mathbb{Q}_{p}\right)$ in $\mathrm{P}_{\mathrm{Z}^{(i, 1)}}\left(\mathbb{A}^{\infty}\right) \backslash \mathrm{G}\left(\mathbb{A}^{\infty}\right) / \mathcal{H}$. As in Sect. 5.1, this image is in bijection with the double coset space

$$
\left(\mathrm{P}_{\mathrm{Z}^{(i, 1)}}\left(\mathbb{A}^{\infty, p}\right) \times \mathrm{P}_{\mathrm{Z}^{(i, 1), \mathrm{D}}}^{\text {ord }}\left(\mathbb{Z}_{p}\right)\right) \backslash\left(\mathrm{G}\left(\mathbb{A}^{\infty, p}\right) \times \mathrm{P}_{\mathrm{D}}^{\text {ord }}\left(\mathbb{Z}_{p}\right)\right) /\left(\mathcal{H}^{p} \times\left(\mathcal{H}_{p} \cap \mathrm{P}_{\mathrm{D}}^{\text {ord }}\left(\mathbb{Z}_{p}\right)\right)\right),
$$

which is the double coset space

$$
P_{n,(i)}^{+}\left(\mathbb{A}^{\infty}\right)^{\text {ord }, \times} \backslash G_{n}\left(\mathbb{A}^{\infty}\right)^{\text {ord }, \times} / U^{p}\left(N_{1}\right)
$$

in this paper (see, e.g., Sect. 5.1), with $U^{p}\left(N_{1}, N_{2}\right)=\mathcal{H}$ and with $U^{p}\left(N_{1}\right)$ denoting the intersection of $U^{p}\left(N_{1}, N_{2}\right)$ with $G_{n}\left(\mathbb{A}^{\infty}\right)^{\text {ord, } \times}$. The ordinary cusp labels $\left[\left(\mathrm{Z}_{\mathcal{H}}, \Phi_{\mathcal{H}}, \delta_{\mathcal{H}}\right)\right]$ for $\mathrm{M}_{\mathcal{H}}$ with underlying $\mathrm{Z}_{\mathcal{H}}$ equal to $\mathrm{Z}_{\mathcal{H}}^{(i, g)}$ for some $g \in \mathrm{G}\left(\mathbb{A}^{\infty, p}\right) \times \mathrm{P}_{\mathrm{D}}^{\text {ord }}\left(\mathbb{Z}_{p}\right)$ are parameterized by the double coset space

$$
\begin{aligned}
& \left(\mathrm{G}_{l, \Phi^{(i, 1)}}^{\prime}\left(\mathbb{Z}_{(p)}\right) \ltimes\left(\mathrm{P}_{\mathrm{Z}^{(i, 1)}}^{\prime}\left(\mathbb{A}^{\infty, p}\right) \times \mathrm{P}_{\mathrm{Z}^{(i, 1)}, \mathrm{D}}^{\text {ord },}\left(\mathbb{Z}_{p}\right)\right)\right) \backslash\left(\mathrm{G}\left(\mathbb{A}^{\infty}\right) \times \mathrm{P}_{\mathrm{D}}^{\text {ord }}\left(\mathbb{Z}_{p}\right)\right) \\
& \quad /\left(\mathcal{H}^{p} \times\left(\mathcal{H}_{p} \cap \mathrm{P}_{\mathrm{D}}^{\text {ord }}\left(\mathbb{Z}_{p}\right)\right)\right),
\end{aligned}
$$

and the forgetful map sending $\left[\left(\mathrm{z}_{\mathcal{H}}, \Phi_{\mathcal{H}}, \delta_{\mathcal{H}}\right)\right]$ to $\mathrm{Z}_{\mathcal{H}}$ can be identified with the canonical map from this double coset space to

$$
\left(\mathrm{P}_{\mathrm{Z}^{(i, 1)}}\left(\mathbb{A}^{\infty, p}\right) \times \mathrm{P}_{\mathrm{Z}^{(i, 1)}, \mathrm{D}}^{\text {ord }}\left(\mathbb{Z}_{p}\right)\right) \backslash\left(\mathrm{G}\left(\mathbb{A}^{\infty, p}\right) \times \mathrm{P}_{\mathrm{D}}^{\text {ord }}\left(\mathbb{Z}_{p}\right)\right) /\left(\mathcal{H}^{p} \times\left(\mathcal{H}_{p} \cap \mathrm{P}_{\mathrm{D}}^{\text {ord }}\left(\mathbb{Z}_{p}\right)\right)\right),
$$

whose fibre above the double coset of $g \in \mathrm{G}\left(\mathbb{A}^{\infty, p}\right) \times \mathrm{P}_{\mathrm{D}}^{\text {ord }}\left(\mathbb{Z}_{p}\right)$ can be identified with

$$
\mathrm{G}_{l, \Phi^{(i, 1)}}^{\prime}\left(\mathbb{Z}_{(p)}\right) \backslash \mathrm{G}_{l, \mathrm{Z}^{(i, 1)}}^{\prime}\left(\mathbb{A}^{\infty, p} \times \mathbb{Z}_{p}\right) /\left(g \mathcal{H} g^{-1}\right)_{\mathrm{G}_{h \mathbb{Z}^{(i, 1)}}^{\prime}}
$$


This last double coset space is the index set of

$$
\begin{aligned}
& \mathcal{Y}_{n,(i),\left(g U^{p} g^{-1} \cap P_{n,(i)}^{+}\right.}^{\text {ord, } \left.\left(\mathbb{A}^{\infty, p}\right)\right)\left(N_{1}\right)} \\
& =\coprod_{L_{n,(i), \operatorname{lin}}\left(\mathbb{Z}_{(p)}\right) \backslash L_{n,(i), \operatorname{lin}}\left(\mathbb{A}^{\infty}\right)^{\text {ord, } \times} /\left(g U^{p} g^{-1} \cap P_{n,(i)}^{+}\left(\mathbb{A}^{\infty, p}\right)\right)\left(N_{1}\right)} \operatorname{Spec} \mathbb{Z}_{(p)}
\end{aligned}
$$

in this paper (see Sect. 4.1), with $U^{p}\left(N_{1}, N_{2}\right)=\mathcal{H}$.

\section{B.12 Partial minimal compactifications of ordinary PEL moduli}

For each $\mathcal{H}=\mathcal{H}^{p} \mathcal{H}_{p}$, where $\mathcal{H}^{p}$ is a neat open compact subgroup of $\mathrm{G}\left(\mathbb{A}^{\infty, p}\right)$, and where $\mathcal{H}_{p}=\mathcal{U}_{p, 1,0}\left(p^{r_{1}}, p^{r_{0}}\right)$ for some integers $0 \leq r_{1} \leq r_{0}$, there is a normal scheme

$$
\overrightarrow{\mathrm{M}}_{\mathcal{H}}^{\text {ord,min }}
$$

quasi-projective and flat over $\operatorname{Spec} \mathbb{Z}_{(p)}$, with geometrically normal fibres, which contains $\overrightarrow{\mathrm{M}}_{\mathcal{H}}^{\text {ord }}$ as an open fibrewise dense subscheme and can be canonically embedded as an open subscheme of $\vec{M}_{\mathcal{H}}^{\min }$, called the partial minimal compactification of $\vec{M}_{\mathcal{H}}^{\text {ord }}$, whose characteristic zero pull-back $\vec{M}_{\mathcal{H}}^{\text {ord,min }} \otimes_{\mathbb{Z}} \mathbb{Q}$ is an open subscheme of $\mathrm{M}_{\mathcal{H}}^{\min }$ which can be identified with the union of the strata $\mathrm{Z}_{\left[\left(\Phi_{\mathcal{H}}, \delta_{\mathcal{H}}\right)\right]}$ indexed by ordinary cusp labels (see theorem 6.2.1.1 and proposition 6.2.1.6 of [44]). The union

$$
\overrightarrow{\mathrm{M}}_{\mathcal{H}}^{\text {ord,min }} \cup \mathrm{M}_{\mathcal{H}}^{\min }
$$

(by gluing along their common open subscheme described above) is the

$$
\mathcal{X}_{n, U^{p}\left(N_{1}, N_{2}\right)}
$$

in this paper (see Sect. 5.1), with $U^{p}\left(N_{1}, N_{2}\right)=\mathcal{H}$. The collection $\left\{\overrightarrow{\mathrm{M}}_{\mathcal{H}}^{\text {ord,min }}\right\}_{\mathcal{H}}$ admits compatible actions of $G\left(\mathbb{A}^{\infty, p}\right) \times \mathrm{P}_{\mathrm{D}}^{\text {ord }}\left(\mathbb{Z}_{p}\right)$ and of the element of $\mathrm{P}_{\mathrm{D}}^{\text {ord }}\left(\mathbb{Q}_{p}\right)$ corresponding to $\varsigma_{p}$ (see proposition 6.2.2.1 of [44]), which are compatible with the action of $G_{n}\left(\mathbb{A}^{\infty}\right)^{\text {ord }}$ on $\left\{\mathcal{X}_{n, U^{p}\left(N_{1}, N_{2}\right)}\right\}_{U^{p}\left(N_{1}, N_{2}\right)}$.

The partial minimal compactification $\overrightarrow{\mathrm{M}}_{\mathcal{H}}^{\text {ord,min }}$ admits a stratification by locally closed subschemes

$$
\overrightarrow{\mathrm{M}}_{\mathcal{H}}^{\text {ord,min }}=\coprod_{\left[\left(\Phi_{\mathcal{H}}, \delta_{\mathcal{H}}\right)\right]} \overrightarrow{\mathrm{Z}}_{\left[\left(\Phi_{\mathcal{H}}, \delta_{\mathcal{H}}\right)\right]}^{\text {ord }}=\coprod_{i=0}^{n} \coprod_{g} \vec{Z}_{\left[\left(\Phi_{\mathcal{H}}^{\text {ord }}, \delta_{\mathcal{H}}\right)\right]}^{\text {ord }}
$$

where $\left[\left(\Phi_{\mathcal{H}}, \delta_{\mathcal{H}}\right)\right]$ runs over ordinary cusp labels for $\mathrm{M}_{\mathcal{H}}$ and $g$ runs over

$$
\begin{aligned}
& \left(\mathrm{G}_{l, \Phi^{(i, 1)}}^{\prime}\left(\mathbb{Z}_{(p)}\right) \ltimes\left(\mathrm{P}_{\mathrm{Z}^{(i, 1)}}^{\prime}\left(\mathbb{A}^{\infty, p}\right) \times \mathrm{P}_{\mathrm{Z}^{(i, 1)}, \mathrm{D}}^{\text {ord },}\left(\mathbb{Z}_{p}\right)\right)\right) \backslash\left(\mathrm{G}\left(\mathbb{A}^{\infty}\right) \times \mathrm{P}_{\mathrm{D}}^{\text {ord }}\left(\mathbb{Z}_{p}\right)\right) \\
& \quad /\left(\mathcal{H}^{p} \times\left(\mathcal{H}_{p} \cap \mathrm{P}_{\mathrm{D}}^{\text {ord }}\left(\mathbb{Z}_{p}\right)\right)\right)
\end{aligned}
$$

(see theorem 6.2.1.1(4) of [44]). If $\mathcal{H}=U^{p}\left(N_{1}, N_{2}\right)$, this matches

$$
\coprod_{g} \vec{Z}_{\left[\left(\Phi_{\mathcal{H}}^{(i, g)}, \delta_{\mathcal{H}}\right)\right]}^{\text {ord }}
$$


(where the indices $g$ are as above) with the subscheme

$$
\coprod_{g \in P_{n,(i)}^{+}\left(\mathbb{A}^{\infty}\right)^{\text {ord } \times} \backslash G_{n}\left(\mathbb{A}^{\infty}\right)^{\text {ord } \times} / U^{p}\left(N_{1}\right)} \mathcal{X}_{n,(i),\left(g U^{p} g^{-1} \cap P_{n,(i)}^{+}\left(\mathbb{A}^{\infty, p}\right)\right)\left(N_{1}, N_{2}\right)}^{\text {ord },}
$$

of $\partial_{i}^{0} \mathcal{X}_{n, U^{p}\left(N_{1}, N_{2}\right)}^{\text {ord,min }}($ see Sect. 5.1), where

$$
\begin{aligned}
& \mathcal{X}_{n,(i),\left(g U^{p} g^{-1} \cap P_{n,(i)}^{+}\left(\mathbb{A}^{\infty, p}\right)\right)\left(N_{1}, N_{2}\right)}^{\text {ord, }} \\
& =\coprod_{h \in L_{n,(i), \operatorname{lin}}\left(\mathbb{Z}_{(p)}\right) \backslash L_{n,(i), \operatorname{lin}}\left(\mathbb{A}^{\infty}\right)^{\text {ord, } \times} /\left(g U^{p} g^{-1} \cap P_{n,(i)}^{+}\left(\mathbb{A}^{\infty, p}\right)\right)\left(N_{1}\right)} \vec{Z}_{\left[\left(\Phi_{\mathcal{H}}^{(i, h g)}, \delta \mathcal{H}\right)\right]^{\text {ord }}} \text {. }
\end{aligned}
$$

Each $\vec{Z}_{\left[\left(\Phi_{\mathcal{H}}, \delta \mathcal{H}\right)\right]}^{\text {ord }}$ is the quotient $\overrightarrow{\mathrm{M}}_{\mathcal{H}}^{\text {ord, } \mathrm{Z}_{\mathcal{H}}}$ of

$$
\overrightarrow{\mathrm{M}}_{\mathcal{H}}^{\text {ord }, \Phi_{\mathcal{H}}} \cong \overrightarrow{\mathrm{M}}_{\mathcal{H}_{\mathrm{G}, \mathrm{z}}^{\prime}}^{\text {ord }}
$$

by $\Gamma_{\Phi_{\mathcal{H}}}$, which is isomorphic to $\overrightarrow{\mathrm{M}}_{\mathcal{H}_{\mathrm{G}_{h, Z}, \Phi}}^{\text {ord }}$, where $\mathcal{H}_{\mathrm{G}_{h, \mathrm{Z}}, \Phi}$ is as in Sect. B.4 (see lemmas

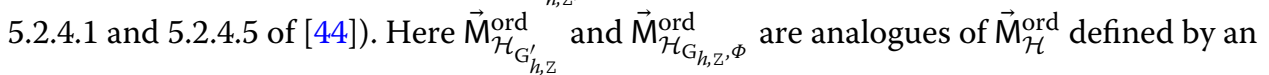
integral PEL datum $\left(\mathcal{O}, \star L^{\mathrm{Z}},\langle\cdot, \cdot\rangle^{\mathrm{z}}, h_{0}^{\mathrm{Z}}\right)$ defining $\mathrm{M}_{\mathcal{H}_{\mathrm{G}_{h, \mathrm{Z}}^{\prime}}}$ and $\mathrm{M}_{\mathcal{H}_{\mathrm{G}_{h, \mathrm{Z}}, \Phi}}$, which is associated with a representative $\mathrm{Z}$ of $\mathrm{Z}_{\mathcal{H}}$ that is compatible with $\mathrm{D}$, and by the filtration $\mathrm{D}_{-1}$ on $L^{\mathrm{Z}} \otimes_{\mathbb{Z}} \mathbb{Z}_{p} \cong \mathrm{Gr}_{-1}^{\mathrm{Z}} \otimes_{\hat{\mathbb{Z}}} \mathbb{Z}_{p}$ determined by D as above (see definition 1.2.1.15 and lemma 5.2.4.1 of [44]). If we let $U_{g}^{p, \prime}$ (resp. $U_{g, \operatorname{lin}}^{p, \prime}$ ) denote the image of $g U^{p} g^{-1} \cap P_{n,(i)}^{+}\left(\mathbb{A}^{\infty, p}\right)$ in $L_{n,(i)}\left(\mathbb{A}^{\infty, p}\right)\left(\right.$ resp. $\left.L_{n,(i), \text { lin }}\left(\mathbb{A}^{\infty, p}\right)\right)$, then under the identifications

$$
\begin{aligned}
& \mathcal{X}_{n,(i), U_{g}^{p, \prime}\left(N_{1}, N_{2}\right)}^{\text {ord } \natural}=L_{n,(i), \operatorname{lin}}\left(\mathbb{Z}_{(p)}\right) \backslash \mathcal{X}_{n,(i), U_{g}^{p, \prime}\left(N_{1}, N_{2}\right)}^{\text {ord, }} \\
& =L_{n,(i), \operatorname{lin}}\left(\mathbb{Z}_{(p)}\right) \\
& \backslash\left(\coprod_{h \in L_{n,(i), \operatorname{lin}}\left(\mathbb{A}^{\infty}\right)^{\text {ord }, \times} /\left(U_{g}^{p, \prime} \cap L_{n,(i), \operatorname{lin}}\left(\mathbb{A}^{\infty, p}\right)\right)\left(N_{1}\right)} \mathcal{X}_{n,(i),\left(U_{g}^{p, \prime} \cap G_{n-i}\left(\mathbb{A}^{\infty, p}\right)\right)\left(N_{1}, N_{2}\right)}^{\text {ord }}\right) / U_{g}^{p, \prime} \\
& =\coprod_{h \in L_{n,(i), \operatorname{lin}}\left(\mathbb{Z}_{(p)}\right) \backslash L_{n,(i), \operatorname{lin}}\left(\mathbb{A}^{\infty}\right)^{\text {ord }, \times} / U_{g, \operatorname{lin}}^{p, \prime}\left(N_{1}\right)} \\
& \left(\left(L_{n,(i), \operatorname{lin}}\left(\mathbb{Z}_{(p)}\right) \cap\left(h U_{g, \operatorname{lin}}^{p, \prime} h^{-1}\right)\left(N_{1}\right)\right) \backslash \mathcal{X}_{n,(i),\left(U_{g}^{p, \prime} \cap G_{n-i}\left(\mathbb{A}^{\infty, p}\right)\right)\left(N_{1}, N_{2}\right)}^{\text {ord }}\right),
\end{aligned}
$$

of Sect. 4.1,

- the term $\mathcal{X}_{n,(i),\left(U_{g}^{p, \prime} \cap G_{n-i}\left(\mathbb{A}^{\infty, p} p\right)\right)\left(N_{1}, N_{2}\right)}^{\text {ord }}$ indexed by $h$ is identified with $\vec{M}_{\mathcal{H}}^{\text {ord }, \Phi_{\mathcal{H}}^{(i, h g)}}$;

- the group $L_{n,(i), \operatorname{lin}}\left(\mathbb{Z}_{(p)}\right) \cap\left(h U_{g, \operatorname{lin}}^{p, \prime} h^{-1}\right)\left(N_{1}\right)$ is identified with $\Gamma_{\Phi_{\mathcal{H}}^{(i, h g}}$;

- and the term

$$
\left(L_{n,(i), \operatorname{lin}}\left(\mathbb{Z}_{(p)}\right) \cap\left(h U_{g, \operatorname{lin}}^{p, \prime} h^{-1}\right)\left(N_{1}\right)\right) \backslash \mathcal{X}_{n,(i),(}^{\text {ord }}\left(U_{g}^{p, \prime} \cap G_{n-i}\left(\mathbb{A}^{\infty, p}\right)\right)\left(N_{1}, N_{2}\right)
$$

is identified with $\overrightarrow{\mathrm{M}}_{\mathcal{H}}^{\text {ord, }} \mathrm{z}_{\mathcal{H}}^{(\text {i.h })}$. 
Similarly, under the identification

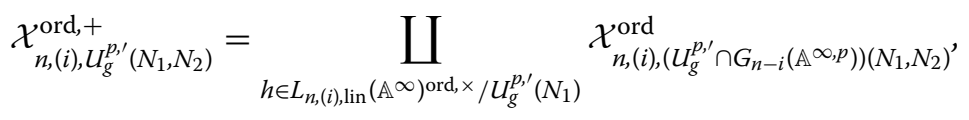

the term $\mathcal{X}_{n,(i),\left(U_{g}^{p, '} \cap G_{n-i}\left(\mathbb{A}^{\infty, p}\right)\right)\left(N_{1}, N_{2}\right)}^{\text {ord }}$ indexed by $h$ is identified with $\overrightarrow{\mathrm{M}}_{\mathcal{H}}^{\text {ord } \Phi_{\mathcal{H}}^{(i, h)}}$. These identifications are Hecke equivariant.

If $\mathcal{H}=U^{p}\left(N_{1}, N_{2}\right)$, then the formal completion of $\overrightarrow{\mathrm{M}}_{\mathcal{H}}^{\text {ord,min }} \cong \mathcal{X}_{n, U^{p}\left(N_{1}, N_{2}\right)}^{\text {ord,min }}$ along

$$
\overrightarrow{\mathrm{M}}_{\mathcal{H}}^{\text {ord,min }} \otimes_{\mathbb{Z}} \mathbb{F}_{p} \cong \bar{X}_{n, U^{p}\left(N_{1}\right)}^{\text {ord,min }}
$$

is denoted

$$
\overrightarrow{\mathfrak{M}}_{\mathcal{H}}^{\text {ord,min }} \cong \mathfrak{X}_{n, U^{p}\left(N_{1}\right)}^{\text {ord,min }}
$$

(see definition 3.4.4.2 of [44]). Their independence of $N_{2}=r_{0}$ is explained in corollary 6.2.2.8 and example 3.4.4.5 of [44]. For the Hecke actions on these formal schemes, see corollaries 6.2.2.7, 6.2.2.8 and 6.2.2.9 of [44].

\section{B.13 Partial toroidal compactifications of ordinary PEL moduli}

For each representative $\left(\mathrm{Z}_{\mathcal{H}}, \Phi_{\mathcal{H}}, \delta_{\mathcal{H}}\right)$ of an ordinary cusp label $\left[\left(\mathrm{Z}_{\mathcal{H}}, \Phi_{\mathcal{H}}, \delta_{\mathcal{H}}\right)\right]$ for $\mathrm{M}_{\mathcal{H}}$, there is a torsor

$$
\vec{C}_{\Phi_{\mathcal{H}}, \delta \mathcal{H}}^{\text {ord }} \rightarrow \overrightarrow{\mathrm{M}}_{\mathcal{H}}^{\text {ord }, \Phi_{\mathcal{H}}}
$$

of an abelian scheme

$$
\vec{C}_{\Phi_{\mathcal{H}}, \delta_{\mathcal{H}}}^{\text {ord,grp }} \rightarrow \overrightarrow{\mathrm{M}}_{\mathcal{H}}^{\text {ord }, \Phi_{\mathcal{H}}}
$$

this abelian scheme is $\mathbb{Q}^{\times}$-isogenous to $\underline{\operatorname{Hom}}_{\mathcal{O}}(X, B)$, and in fact its $\mathbb{Z}_{(p)}^{\times}$-isogeny class (i.e. prime-to- $p$ quasi-isogeny class) can be described explicitly (see lemma 5.2.4.7 and propositions 5.2.4.11 and 5.2.4.13 of [44]). We obtain an isomorphic abelian scheme torsor if we replace $\left(\mathrm{Z}_{\mathcal{H}}, \Phi_{\mathcal{H}}, \delta_{\mathcal{H}}\right)$ with another representative, but its universal property depends on this choice of representative.

If $U_{g}^{p, \prime}$ again denotes $g U^{p} g^{-1} \cap P_{n,(i)}^{+}\left(\mathbb{A}^{\infty, p}\right)$ then the map

$$
\begin{array}{ccc}
\mathcal{A}_{n,(i), U_{g}^{p, \prime}\left(N_{1}, N_{2}\right)}^{\text {ord }++} & =\coprod_{h \in L_{n,(i), \text { lin }}\left(\mathbb{A}^{\infty}\right)^{\text {ord }, \times} / U_{g}^{p, \prime}\left(N_{1}\right)} \mathcal{A}_{n-i,\left(h g U^{p} g^{-1} h^{-1} \cap P_{n,(i)}\left(\mathbb{A}^{\infty, p}\right)\right)\left(N_{1}, N_{2}\right)}^{(i), \text { ord }} \\
\downarrow & \downarrow \\
\mathcal{X}_{n,(i), U_{g}^{p, \prime}\left(N_{1}, N_{2}\right)}^{\text {ord }++} & = & \coprod_{h \in L_{n,(i), \text { lin }}\left(\mathbb{A}^{\infty}\right)^{\text {ord }, \times} / U_{g}^{p, \prime}\left(N_{1}\right)} \mathcal{X}_{n,(i),\left(U_{g}^{p, \prime} \cap P_{n,(i)}\left(\mathbb{A}^{\infty, p}\right)\right)\left(N_{1}, N_{2}\right)}^{\text {ord }}
\end{array}
$$

in this paper (see Sects. 4.1, 4.2) with $U^{p}\left(N_{1}, N_{2}\right)=\mathcal{H}$ is identified with

$$
\begin{aligned}
& \coprod_{h \in L_{n,(i), \operatorname{lin}}\left(\mathbb{A}^{\infty}\right)^{\text {ord }, \times} / U_{g}^{p,{ }^{p}\left(N_{1}\right)}} \vec{C}_{\Phi_{\mathcal{H}}^{(i, h g)}, \delta_{\mathcal{H}}}^{\text {ord }} \\
& \downarrow \\
& \bigsqcup_{\left.h \in L_{n, i}\right) \operatorname{lin}\left(\mathbb{A}^{\infty}\right)^{\text {ord }, \times} / U_{g}^{p,}\left(N_{1}\right)} \vec{M}_{\mathcal{H}}^{\text {ord }, \Phi_{\mathcal{H}}^{(i, h)}} .
\end{aligned}
$$


These maps are equivariant with compatible actions of $\mathrm{P}_{\mathrm{Z}^{(i, g)}}\left(\mathbb{A}^{\infty, p}\right) \times \mathrm{P}_{\mathrm{Z}^{(i, g)}, \mathrm{D}}^{\text {ord }}\left(\mathbb{Z}_{p}\right)$ and of the element of $\mathrm{P}_{z^{(i, g)}, \mathrm{D}}^{\text {ord }}\left(\mathbb{Q}_{p}\right)$ corresponding to $\varsigma_{p}$. (See proposition 5.2.4.25 of [44].) (Since our pairing $\langle\cdot, \cdot\rangle$ is perfect, the universal property of $\vec{C}_{\Phi_{\mathcal{H}}, \delta_{\mathcal{H}}}^{\text {ord }}$ in proposition 5.2 .4 .13 of [44] can be simplified by suppressing the dual objects. This universal property then agrees

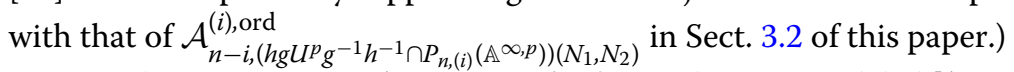

For each representative $\left(\mathrm{Z}_{\mathcal{H}}, \Phi_{\mathcal{H}}, \delta_{\mathcal{H}}\right)$ of an ordinary cusp label $\left[\left(\mathrm{Z}_{\mathcal{H}}, \Phi_{\mathcal{H}}, \delta_{\mathcal{H}}\right)\right]$ for $\mathrm{M}_{\mathcal{H}}$, there is a torsor

$$
\overrightarrow{\boldsymbol{\Xi}}_{\Phi_{\mathcal{H}}, \delta_{\mathcal{H}}}^{\text {ord }} \rightarrow \vec{C}_{\Phi_{\mathcal{H}}, \delta_{\mathcal{H}}}^{\text {ord }}
$$

under the pull-back of the same split torus $E_{\Phi_{\mathcal{H}}}$ over Spec $\mathbb{Z}$ as before (see Sect. B.5; and see lemma 5.2.4.26 and proposition 5.2.4.30 of [44]). We obtain an isomorphic torus torsor if we replace $\left(Z_{\mathcal{H}}, \Phi_{\mathcal{H}}, \delta_{\mathcal{H}}\right)$ with another representative, but its universal property depends on this choice of representative. For a fixed $(\mathrm{Z}, \Phi, \delta)$, the collection $\left\{\vec{\Xi}_{\Phi_{\mathcal{H}}, \delta_{\mathcal{H}}}^{\text {ord }}\right\}_{\mathcal{H}_{\mathrm{P}_{\mathrm{Z}}^{\prime}}}$ admits compatible actions of $\mathrm{P}_{\mathrm{Z}}^{\prime}\left(\mathbb{A}^{\infty, p}\right) \times \mathrm{P}_{\mathrm{Z}, \mathrm{D}}^{\text {ord, }}\left(\mathbb{Z}_{p}\right)$ and of the element of $\mathrm{P}_{\mathrm{Z}, \mathrm{D}}^{\text {ord, }}\left(\mathbb{Q}_{p}\right)$ corresponding to $\varsigma_{p}$ such that the morphisms

$$
\vec{\Xi}_{\Phi_{\mathcal{H}}, \delta_{\mathcal{H}}}^{\text {ord }} \rightarrow \vec{C}_{\Phi_{\mathcal{H}}, \delta_{\mathcal{H}}}^{\text {ord }}
$$

are equivariant with

$$
\mathrm{P}_{\mathrm{Z}}^{\prime}\left(\mathbb{A}^{\infty, p}\right) \times \mathrm{P}_{\mathrm{Z}, \mathrm{D}}^{\text {ord, }}\left(\mathbb{Z}_{p}\right) \rightarrow \mathrm{G}_{1, \mathrm{Z}}\left(\mathbb{A}^{\infty, p}\right) \times \mathrm{P}_{1, \mathrm{Z}, \mathrm{D}}\left(\mathbb{Z}_{p}\right)
$$

and with the compatible actions of the elements corresponding to $\varsigma_{p}$ (see proposition 5.2.4.41 of [44]).

The torus

$$
\mathcal{S}_{n,(i), U_{g}^{p, \prime}\left(N_{1}\right)}^{\text {ord }+} \rightarrow \mathcal{Y}_{n,(i), U_{g}^{p,{ }^{\prime}}\left(N_{1}\right)}^{\text {ord }+}
$$

in this paper (see Sect. 4.3) with $U^{p}\left(N_{1}, N_{2}\right)=\mathcal{H}$ is identified with

$$
\coprod_{h \in L_{n,(i), \operatorname{lin}}\left(\mathbb{A}^{\infty}\right)^{\text {ord }, \times} / U_{g}^{p, \prime}\left(N_{1}\right)} \vec{E}_{\Phi_{\mathcal{H}}^{(i, h g)}, \delta \mathcal{H}}^{\text {ord }} \rightarrow \coprod_{h \in L_{n,(i), \operatorname{lin}}\left(\mathbb{A}^{\infty}\right)^{\text {ord }, \times} / U_{g}^{p,{ }^{\prime}}\left(N_{1}\right)} \operatorname{Spec} \mathbb{Z}_{(p)}
$$

Moreover, the sheaves $X^{*}\left(\mathcal{S}_{n,(i), U_{g}^{p,{ }^{\prime}}\left(N_{1}\right)}^{\text {ord, }}\right), X_{*}\left(\mathcal{S}_{n,(i), U_{g}^{p,{ }^{\prime}}\left(N_{1}\right)}^{\text {ord }+}\right)_{\mathbb{R}}^{\succ 0}$, and $X_{*}\left(\mathcal{S}_{n,(i), U_{g}^{p,,}}^{\text {ord, }}\right)_{\mathbb{R}}^{>0}$ are identified with

$$
\coprod_{h \in L_{n,(i), \operatorname{lin}}\left(\mathbb{A}^{\infty}\right)^{\text {ord }, \times} / U_{g}^{p,{ }^{\prime \prime}}\left(N_{1}\right)} \mathbf{S}_{\Phi_{\mathcal{H}}^{(i, h g)}}, \quad \coprod_{h \in L_{n,(i), \operatorname{lin}}\left(\mathbb{A}^{\infty}\right)^{\text {ord }, \times} / U_{g}^{p,{ }^{\prime}}\left(N_{1}\right)} \mathbf{P}_{\Phi_{\mathcal{H}}^{(i, h g)}}
$$

and

$$
\coprod_{h \in L_{n,(i), \operatorname{lin}}\left(\mathbb{A}^{\infty}\right)^{\text {ord }, \times} / U_{g}^{p,{ }^{\prime}}\left(N_{1}\right)} \mathbf{P}_{\Phi_{\mathcal{H}}^{(i, h g)}}^{+}
$$


respectively. The $\mathcal{S}_{n,(i), U_{g}^{p, \prime}\left(N_{1}\right)}^{\text {ord, }}$-torsor

$$
\begin{aligned}
& \mathcal{T}_{n,(i), U_{g}^{p, \prime}\left(N_{1}, N_{2}\right)}^{\text {ord }}=\coprod_{h \in L_{n,(i), \text { lin }}\left(\mathbb{A}^{\infty}\right) \text { ord, } \times / U_{g}^{p, \prime}\left(N_{1}\right)} \mathcal{T}_{\left.n-i, h g U^{p} g^{-1} h^{-1} \cap P_{n,(i)}\left(\mathbb{A}^{\infty}, p\right)\right)\left(N_{1}, N_{2}\right)}^{(i) \text { ord }}
\end{aligned}
$$

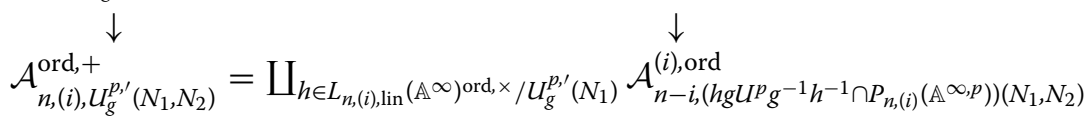

is identified with

$$
\begin{aligned}
& \bigsqcup_{h \in L_{n,(i), \operatorname{lin}}\left(\mathbb{A}^{\infty}\right)^{\text {ord }, \times} / U_{g}^{p, '}\left(N_{1}\right)} \vec{\Xi}_{\Phi_{\mathcal{H}}^{(i, h g)}, \delta_{\mathcal{H}}}^{\text {ord }} \\
& \downarrow \\
& \coprod_{\left.h \in L_{n, i}\right) \operatorname{lin}\left(\mathbb{A}^{\infty}\right)^{\text {ord }, \times} / U_{g}^{p, \prime}\left(N_{1}\right)} \vec{C}_{\Phi_{\mathcal{H}}^{(i, h g)}, \delta \mathcal{H}}^{\text {ord }}
\end{aligned}
$$

These maps are equivariant with the compatible actions of $\mathrm{P}_{\mathrm{Z}^{(i, g)}(}\left(\mathbb{A}^{\infty, p}\right) \times \mathrm{P}_{\mathrm{Z}^{(i, g), \mathrm{D}}}^{\text {ord }}\left(\mathbb{Z}_{p}\right)$ and of the elements corresponding to $\varsigma_{p}$. (See proposition 5.2.4.41 of [44].)

Consider any compatible collection

$$
\Sigma^{\text {ord }}=\left\{\Sigma_{\Phi_{\mathcal{H}}}\right\}_{\left[\left(\Phi_{\mathcal{H}}, \delta_{\mathcal{H}}\right)\right]}
$$

of admissible projective smooth rational polyhedral cone decompositions for $\overrightarrow{\mathrm{M}}_{\mathcal{H}}^{\text {ord }}$, where each $\Sigma_{\Phi_{\mathcal{H}}}$ is a $\Gamma_{\Phi_{\mathcal{H}}}$-admissible projective smooth rational polyhedral cone decomposition of $\mathbf{P}_{\Phi_{\mathcal{H}}}$, and where the indices $\left[\left(\Phi_{\mathcal{H}}, \delta_{\mathcal{H}}\right)\right]$ are ordinary cusp labels for $\mathrm{M}_{\mathcal{H}}$, as in definitions 5.1.3.1 and 5.1.3.3 of [44]. Any compatible collection $\Sigma$ for $M_{\mathcal{H}}$ induces a compatible collection $\Sigma^{\text {ord }}$ for $\overrightarrow{\mathrm{M}}_{\mathcal{H}}^{\text {ord }}$ by restricting to the indices given by ordinary cusp labels, and conversely any compatible collection $\Sigma^{\text {ord }}$ for $\overrightarrow{\mathrm{M}}_{\mathcal{H}}^{\text {ord }}$ extends to a compatible collection $\Sigma$ for $M_{\mathcal{H}}$ (see proposition 5.1.3.4 of [44]). Each such $\Sigma^{\text {ord }}$ considered in [44] induces a pair

$$
\left(U^{p}\left(N_{1}\right), \Delta\right)
$$

in $\mathcal{J}_{n}^{\text {tor,ord }}$ (with $U^{p}\left(N_{1}, N_{2}\right)=\mathcal{H}$ ) in Sect. 5.2 of this paper, because, in order to define $\left(U^{p}\left(N_{1}\right), \Delta\right)$ as in Sect. 5.2, it suffices to define the admissible cone decomposition $\Delta(g)_{0}$ for $X_{*}\left(\mathcal{S}_{n,(i),\left(g U^{p} g^{-1} \cap P_{n, i}^{+}(i)\left(\mathbb{A}^{\infty, p}\right)\right)\left(N_{1}\right)}\right)_{\mathbb{R}}^{\succ 0}$, for each $g \in G_{n}\left(\mathbb{A}^{\infty}\right)^{\text {ord, } \times}=\mathrm{G}\left(\mathbb{A}^{\infty, p}\right) \times \mathrm{P}_{\mathrm{D}}^{\text {ord }}\left(\mathbb{Z}_{p}\right)$, which can be taken to be the pull-back of the subcollection $\left\{\Sigma_{\Phi_{\mathcal{H}}}\right\}_{\left[\left(\Phi_{\mathcal{H}}, \delta_{\mathcal{H}}\right)\right]}$ of $\Sigma^{\text {ord }}$ indexed by the cusp labels $\left[\left(\Phi_{\mathcal{H}}, \delta_{\mathcal{H}}\right)\right]$ with underlying $\mathrm{z}_{\mathcal{H}}$ equal to $\mathrm{z}_{\mathcal{H}}^{(i, g)}$. In fact, $\mathcal{J}_{n}^{\text {tor,ord }}$ is exactly the set of such induced pairs (as $U^{p}\left(N_{1}, N_{2}\right)=\mathcal{H}$ varies).

Each $\Sigma_{\Phi_{\mathcal{H}}}$ defines an affine toroidal embedding

$$
\vec{\Xi}_{\Phi_{\mathcal{H}}, \delta_{\mathcal{H}}}^{\text {ord }} \hookrightarrow \overline{\vec{\Xi}}_{\Phi_{\mathcal{H}}, \delta_{\mathcal{H}}}^{\text {ord }}=\overline{\vec{\Xi}}_{\Phi_{\mathcal{H}}, \delta_{\mathcal{H}}, \Sigma_{\Phi_{\mathcal{H}}}}^{\text {ord }}=\bigcup_{\sigma \in \Sigma_{\Phi_{\mathcal{H}}}} \vec{\Xi}_{\Phi_{\mathcal{H}}, \delta_{\mathcal{H}}}^{\text {ord }}(\sigma)=\coprod_{\sigma \in \Sigma_{\Phi_{\mathcal{H}}}} \vec{\Xi}_{\Phi_{\mathcal{H}}, \delta_{\mathcal{H}}, \sigma}^{\text {ord }}
$$

over $\vec{C}_{\Phi_{\mathcal{H}}, \delta \mathcal{H}}^{\text {ord }}$. Rather confusingly $\vec{\Xi}_{\Phi_{\mathcal{H}}, \delta \mathcal{H}}^{\text {ord }}(\sigma)$ (in the notation of [44]) is what in this paper we would have denoted $\vec{\Xi}_{\Phi_{\mathcal{H}}, \delta_{\mathcal{H}}, \sigma}^{\text {ord }}$; and $\vec{\Xi}_{\Phi_{\mathcal{H}}, \delta_{\mathcal{H}}, \sigma}^{\text {ord }}$ is what in this paper we would have denoted $\partial_{\sigma} \vec{\Xi}_{\Phi_{\mathcal{H}}, \delta_{\mathcal{H}}, \Sigma_{\Phi_{\mathcal{H}}}}^{\text {ord }}$. The formal completion of $\overrightarrow{\vec{\Xi}}_{\Phi_{\mathcal{H}}, \delta_{\mathcal{H}}, \Sigma_{\Phi_{\mathcal{H}}}}^{\text {ord }}$ along the union of the $\sigma$-strata $\vec{\Xi}_{\Phi_{\mathcal{H}}, \delta_{\mathcal{H}}, \sigma}^{\text {ord }}$ for all $\sigma \in \Sigma_{\Phi_{\mathcal{H}}}$ such that $\sigma \cap \mathbf{P}_{\Phi_{\mathcal{H}}}^{+} \neq \emptyset$ is denoted

$$
\overrightarrow{\mathfrak{X}}_{\Phi_{\mathcal{H}}, \delta_{\mathcal{H}}}^{\text {ord }}=\overrightarrow{\mathfrak{X}}_{\Phi_{\mathcal{H}}, \delta_{\mathcal{H}}, \Sigma_{\Phi_{\mathcal{H}}}}^{\mathrm{ord}}
$$


(see (4.2.2.4), (5.2.4.32), (5.2.4.33), and lemma 5.2.4.38 of [44]).

For a fixed $g \in \mathrm{G}\left(\mathbb{A}^{\infty, p}\right) \times \mathrm{P}_{\mathrm{D}}^{\text {ord }}\left(\mathbb{Z}_{p}\right)$, the schemes

$$
\mathcal{T}_{n,(i), U_{g}^{p, \prime}\left(N_{1}, N_{2}\right), \Delta(g)_{0}}^{\text {ord }+} \rightarrow \mathcal{A}_{n,(i), U_{g}^{p, \prime}\left(N_{1}, N_{2}\right)}^{\text {ord }+}
$$

of this paper (see Sect. 4.4) are identified with

$$
\coprod_{h \in L_{n,(i), \operatorname{lin}}\left(\mathbb{A}^{\infty}\right)^{\text {ord } \times} \times / U_{g}^{p, \prime}\left(N_{1}\right)} \overrightarrow{\vec{\Xi}}_{\Phi_{\mathcal{H}}^{(i, h)}, \delta_{\mathcal{H}}}^{\text {ord }} \rightarrow \coprod_{h \in L_{n,(i), \operatorname{lin}}\left(\mathbb{A}^{\infty}\right)^{\text {ord } \times} \times / U_{g}^{p, '}\left(N_{1}\right)}{\overrightarrow{\Phi_{\mathcal{H}}}}_{\Phi^{\text {ord }}, \delta_{\mathcal{H}}}^{\text {(i, }}
$$

Moreover, $\mathcal{T}_{n,(i), U_{g}^{p, \prime}\left(N_{1}, N_{2}\right), \Delta(g)_{0}}^{\text {ord },, \wedge}$ is identified with

$$
\coprod_{h \in L_{n, i(i) \operatorname{lin}}\left(\mathbb{A}^{\infty}\right)^{\text {ord }, \times} / U_{g}^{p, \prime}\left(N_{1}\right)} \overrightarrow{\mathfrak{X}}_{\Phi_{\mathcal{H}}^{\text {ord }} \text { (ihg) } \delta \mathcal{H}}
$$

and $\mathcal{T}_{n,(i), U_{g}^{p, j}\left(N_{1}, N_{2}\right), \Delta(g)_{0}}^{\text {ord }, \wedge}$ is identified with

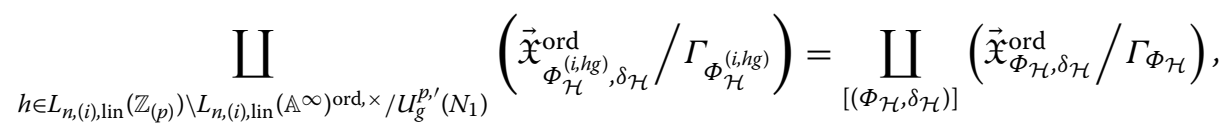

where the second disjoint union runs over all ordinary cusp labels $\left[\left(\Phi_{\mathcal{H}}, \delta_{\mathcal{H}}\right)\right]$ with underlying $\mathrm{z}_{\mathcal{H}}$ equal to $\mathrm{z}_{\mathcal{H}}^{(i, g)}$. (Again $U=\mathcal{H}$.)

For each $\Sigma^{\text {ord }}$ as above (and each $\mathcal{H}$ as above; see the beginning of Sect. B.12), we have a smooth quasi-projective scheme

$$
\overrightarrow{\mathrm{M}}_{\mathcal{H}, \Sigma \text { ord }}^{\text {ord tor }}
$$

over Spec $\mathbb{Z}_{(p)}$, which contains $\overrightarrow{\mathrm{M}}_{\mathcal{H}}^{\text {ord }}$ as an open fibrewise dense subscheme, and is called a partial toroidal compactification of $\vec{M}_{\mathcal{H}}^{\text {ord }}$. Its characteristic zero pull-back $\vec{M}_{\mathcal{H}, \Sigma^{\text {ord }}}^{\text {ord,tor }} \otimes_{\mathbb{Z}} \mathbb{Q}$ is an open subscheme of $\mathrm{M}_{\mathcal{H}, \Sigma}^{\text {tor }}$, for any $\Sigma$ extending $\Sigma^{\text {ord }}$, which can be identified with the union of the strata $Z_{\left[\left(\Phi_{\mathcal{H}}, \delta_{\mathcal{H}}, \sigma\right)\right]}$ indexed by equivalence classes whose underlying cusp labels $\left[\left(\Phi_{\mathcal{H}}, \delta_{\mathcal{H}}\right)\right]$ are ordinary (see theorems 5.2.1.1 and 6.2.3.1 and remark 5.2.1.5 of [44]). The union

$$
\overrightarrow{\mathrm{M}}_{\mathcal{H}, \Sigma \text { ord }}^{\text {ord,tor }} \cup \mathrm{M}_{\mathcal{H}, \Sigma}^{\text {tor }}
$$

(by gluing along their common open subscheme described above) is the

$$
\mathcal{X}_{n, U^{p}\left(N_{1}, N_{2}\right), \Delta}^{\text {ord }}
$$

in this paper (see Sect. 5.3), with $U^{p}\left(N_{1}, N_{2}\right)=\mathcal{H}$ and with $\left(U^{p}\left(N_{1}, N_{2}\right), \Delta\right) \in \mathcal{J}_{n}^{\text {tor }}$ induced by $\Sigma$ as in Sect. B.5. In this case, the $\left(U^{p}\left(N_{1}\right), \Delta^{\text {ord }}\right) \in \mathcal{J}_{n}^{\text {tor,ord }}$ induced by $\left(U^{p}\left(N_{1}, N_{2}\right), \Delta\right)$ is induced by the $\Sigma^{\text {ord }}$ induced by $\Sigma$. The collection $\left\{\overrightarrow{\mathrm{M}}_{\mathcal{H}, \Sigma^{\text {ord }}}^{\text {ord tor }}\right\}_{\left(\mathcal{H}, \Sigma^{\text {ord }}\right)}$ admits compatible actions of $G\left(\mathbb{A}^{\infty, p}\right) \times \mathrm{P}_{D}^{\text {ord }}\left(\mathbb{Z}_{p}\right)$ and of the element of $\mathrm{P}_{D}^{\text {ord }}\left(\mathbb{Q}_{p}\right)$ corresponding to $s_{p}$ (see proposition 5.2.2.2 of [44]), which are compatible with the action of $G_{n}\left(\mathbb{A}^{\infty}\right)^{\text {ord }}$ on $\left\{\mathcal{X}_{n, U^{p}\left(N_{1}, N_{2}\right), \Delta}\right\}_{\left(U^{p}\left(N_{1}, N_{2}\right), \Delta\right)}$. 
The partial toroidal compactification $\overrightarrow{\mathrm{M}}_{\mathcal{H}, \Sigma^{\text {ord }}}^{\text {ord,tor }}$ admits a stratification by locally closed subschemes

$$
\overrightarrow{\mathrm{M}}_{\mathcal{H}, \Sigma^{\text {ord }}}^{\text {ord,tor }}=\coprod_{\left[\left(\Phi_{\mathcal{H}}, \delta_{\mathcal{H}}, \sigma\right)\right]} \vec{Z}_{\left[\left(\Phi_{\mathcal{H}}, \delta_{\mathcal{H}}, \sigma\right)\right]}^{\text {ord }}
$$

(see theorem 5.2.1.1(2) of [44]) indexed by equivalence classes $\left[\left(\Phi_{\mathcal{H}}, \delta_{\mathcal{H}}, \sigma\right)\right]$ as in definition 1.2.2.10 of [44] whose underlying cusp labels $\left[\left(\Phi_{\mathcal{H}}, \delta_{\mathcal{H}}\right)\right]$ are ordinary. Each stratum $\vec{Z}_{\left[\left(\Phi_{\mathcal{H}}, \delta_{\mathcal{H}}, \sigma\right)\right]}^{\text {ord }}$ is canonically isomorphic to $\vec{\Xi}_{\Phi_{\mathcal{H}}, \delta_{\mathcal{H}}, \sigma}^{\text {ord }}$, and the formal completion of $\vec{M}_{\mathcal{H}, \Sigma}^{\text {ord,tor }}$ along the union of the strata $\vec{Z}_{\left[\left(\Phi_{\mathcal{H}}, \delta_{\mathcal{H}}, \sigma\right)\right]}^{\text {ord }}$ labelled by equivalence classes $\left[\left(\Phi_{\mathcal{H}}, \delta_{\mathcal{H}}, \sigma\right)\right]$ with the same underlying ordinary cusp label $\left[\left(\Phi_{\mathcal{H}}, \delta_{\mathcal{H}}\right)\right]$ is canonically isomorphic to $\overrightarrow{\mathfrak{X}}_{\Phi_{\mathcal{H}}, \delta_{\mathcal{H}}}^{\text {ord }} / \Gamma_{\Phi_{\mathcal{H}}}$ (see lemma 5.2.4.38 of [44]).

When $U^{p}\left(N_{1}, N_{2}\right)=\mathcal{H}$, and when $\left(U^{p}\left(N_{1}\right), \Delta^{\text {ord }}\right) \in \mathcal{J}_{n}^{\text {tor,ord }}$ is induced by $\Sigma^{\text {ord }}$ as above, the formal completion of $\vec{M}_{\mathcal{H}, \Sigma}^{\text {ord,tor }}$ along the union of all strata $\vec{Z}_{\left[\left(\Phi_{\mathcal{H}}, \delta_{\mathcal{H}}, \sigma\right)\right]}^{\text {ord }}$ labelled by equivalence classes $\left[\left(\Phi_{\mathcal{H}}, \delta_{\mathcal{H}}, \sigma\right)\right]$ with underlying $\mathrm{Z}_{\mathcal{H}}$ equal to $\mathrm{Z}_{\mathcal{H}}^{(i, g)}$ for some $g \in$ $\mathrm{G}\left(\mathbb{A}^{\infty, p}\right) \times \mathrm{P}_{\mathrm{D}}^{\text {ord }}\left(\mathbb{Z}_{p}\right)$ is

$$
\coprod_{g \in P_{n,(i)}^{+}\left(\mathbb{A}^{\infty}\right)^{\text {ord }, \times} \backslash G_{n}\left(\mathbb{A}^{\infty}\right)^{\text {ord }, \times} / U^{p}\left(N_{1}\right)} \mathcal{T}_{n,(i),\left(g U^{p} g^{-1} \cap P_{n,(i)}^{+}\left(\mathbb{A}^{\infty, p}\right)\right)\left(N_{1}, N_{2}\right), \Delta(g)_{0}}^{\text {ord } \downarrow, \wedge} \subset \mathcal{X}_{n, U^{p}\left(N_{1}, N_{2}\right), \Delta, i}^{\text {ord, } \wedge}
$$

in this paper, whose union with $X_{n, U^{p}\left(N_{1}, N_{2}\right), \Delta, i}^{\wedge}$ is

$$
\mathcal{X}_{n, U^{p}\left(N_{1}, N_{2}\right), \Delta, i}^{\text {ord } \wedge}
$$

(see Sect. 5.3). It can be identified with

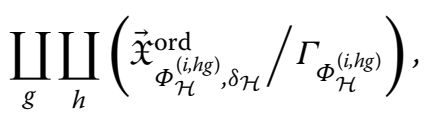

where $g$ runs over $P_{n,(i)}^{+}\left(\mathbb{A}^{\infty}\right)^{\text {ord }, \times} \backslash G_{n}\left(\mathbb{A}^{\infty}\right)^{\text {ord }, \times} / U^{p}\left(N_{1}\right)$ and $h$ runs over

$$
L_{n,(i), \operatorname{lin}}\left(\mathbb{Z}_{(p)}\right) \backslash L_{n,(i), \operatorname{lin}}\left(\mathbb{A}^{\infty}\right)^{\text {ord }, \times} /\left(g U^{p} g^{-1} \cap P_{n,(i)}^{+}\left(\mathbb{A}^{\infty, p}\right)\right)\left(N_{1}\right) .
$$

These identifications are all Hecke equivariant (see proposition 5.2.4.41 of [44]).

The formal completion of $\vec{M}_{\mathcal{H}, \Sigma}^{\text {ord,tor }}$ along

$$
\overrightarrow{\mathrm{M}}_{\mathcal{H}, \Sigma^{\text {ord }}}^{\text {ord,tor }} \otimes_{\mathbb{Z}} \mathbb{F}_{p}
$$

is denoted

$$
\overrightarrow{\mathfrak{M}}_{\mathcal{H}, \Sigma^{\text {ord }}}^{\text {ord,tor }}
$$

in [44] (see definition 3.4.4.2). When $U^{p}\left(N_{1}, N_{2}\right)=\mathcal{H}$, and when $\left(U^{p}\left(N_{1}\right), \Delta\right) \in \mathcal{J}_{n}^{\text {tor,ord }}$ induced by $\Sigma^{\text {ord }}$ as above, these are denoted $\bar{X}_{n, U^{p}\left(N_{1}\right), \Delta}^{\text {ord }}$ and $\mathfrak{X}_{n, U^{p}\left(N_{1}\right), \Delta}^{\text {ord }}$, respectively, in this paper (see Sect. 5.3). (Their independence of $N_{2}=r_{0}$ is explained in corollary 5.2.2.4 of [44].) For the Hecke actions on these formal schemes, see corollaries 5.2.2.3, 5.2.2.4 and 5.2.2.5 of [44]. 


\section{B.14 Ordinary loci of Kuga families}

Recall the choice of $Q=\mathcal{O}_{F}^{m}$ in Sect. B.6, and the associated algebraic groups. For a $\mathbb{Z}_{p}$-algebra $R$, we set

$$
\widehat{\mathrm{P}}_{\mathrm{D}}^{\text {ord }}(R)
$$

to be the pre-image of $\mathrm{P}_{\mathrm{D}}^{\text {ord }}(R)$ under the homomorphism $\widehat{\mathrm{G}}(R) \rightarrow \mathrm{G}(R)$. This is $P_{n,(n)}^{(m),+}(R)$ in the notation of this paper. Thus,

$$
\widehat{\mathrm{G}}\left(\mathbb{A}^{\infty, p}\right) \times \widehat{\mathrm{P}}_{\mathrm{D}}^{\text {ord }}\left(\mathbb{Z}_{p}\right)=G_{n}^{(m)}\left(\mathbb{A}^{\infty}\right)^{\text {ord }, \times}
$$

For all integers $0 \leq r$ and $0 \leq r_{1} \leq r_{0}$, we set:

(1) $\widehat{\mathcal{U}}_{p, 0}\left(p^{r}\right):=\left(\widehat{\mathrm{G}}\left(\mathbb{Z}_{p}\right) \rightarrow \widehat{\mathrm{G}}\left(\mathbb{Z} / p^{r} \mathbb{Z}\right)\right)^{-1}\left(\widehat{\mathrm{P}}_{\mathrm{D}}^{\mathrm{ord}}\left(\mathbb{Z} / p^{r} \mathbb{Z}\right)\right)=U_{p}(0, r)_{n}^{(m)}$.

(2) $\widehat{\mathcal{U}}_{p, 1}\left(p^{r}\right):=U_{p}(r, r)_{n}^{(m)}$.

(3) $\widehat{\mathcal{U}}_{p, 1}^{\mathrm{bal}}\left(p^{r}\right):=\operatorname{ker}\left(v: \widehat{\mathcal{U}}_{p, 1}\left(p^{r}\right) \rightarrow\left(\mathbb{Z} / p^{r} \mathbb{Z}\right)^{\times}\right)$.

(4) $\widehat{\mathcal{U}}_{p, 1,0}\left(p^{r_{1}}, p^{r_{0}}\right):=\widehat{\mathcal{U}}_{p, 1}\left(p^{r_{1}}\right) \cap \widehat{\mathcal{U}}_{p, 0}\left(p^{r_{0}}\right)=U_{p}\left(r_{1}, r_{0}\right)_{n}^{(m)}$.

(The first and third of these definitions are consistent with definition 7.1.1.2 of [44].) An open compact subgroup $\widehat{\mathcal{H}}_{p} \subset \widehat{G}\left(\mathbb{Q}_{p}\right)$ is said to be of standard form and of depth $r$ if

$$
\widehat{\mathcal{U}}_{p, 1}^{\mathrm{bal}}\left(p^{r}\right) \subset \widehat{\mathcal{H}}_{p} \subset \widehat{\mathcal{U}}_{p, 0}\left(p^{r}\right)
$$

(see definition 7.1.1.2 of [44]).

The theory in [44] is developed for open compact subgroups $\widehat{\mathcal{H}}$ of $\widehat{G}(\hat{\mathbb{Z}})$ of the form $\widehat{\mathcal{H}}=\widehat{\mathcal{H}}^{p} \widehat{\mathcal{H}}_{p}$, where $\widehat{\mathcal{H}}^{p}$ is a neat open compact subgroup of $\widehat{\mathrm{G}}\left(\hat{\mathbb{Z}}^{p}\right)$, and where $\widehat{\mathcal{H}}_{p}$ is an open compact subgroup of $\widehat{\mathrm{G}}\left(\mathbb{Z}_{p}\right)$ of standard form. In this paper, we will only need $\widehat{\mathcal{H}}$ of the form $U^{p}\left(N_{1}, N_{2}\right)$, which satisfies the above requirement with $\widehat{\mathcal{H}}^{p}=U^{p}$ and $\widehat{\mathcal{H}}_{p}=\widehat{\mathcal{U}}_{p, 1,0}\left(p^{N_{1}}, p^{N_{2}}\right)$. Then $r_{\widehat{\mathcal{H}}_{\mathrm{G}}}=0$ and $\widehat{\mathcal{H}}$ satisfies conditions 7.1.1.4 and 7.1.1.5 of [44]. To each $\widehat{\mathcal{H}}$ as above with image $\mathcal{H}=\widehat{\mathcal{H}}_{\mathrm{G}}$ in $\mathrm{G}(\hat{\mathbb{Z}})$, [44] attached a generalized ordinary Kuga family

$$
\overrightarrow{\mathrm{N}}^{\text {ord }} \rightarrow \overrightarrow{\mathrm{M}}_{\mathcal{H}}^{\text {ord }}
$$

(see definition 7.1.3.2 of [44]). Its characteristic zero pull-back is a generalized Kuga family $\mathrm{N} \rightarrow \mathrm{M}_{\mathcal{H}}$ as in Sect. B.6. Since $Q=\mathcal{O}_{F}^{m}$, it is the scheme denoted $\mathcal{A}_{n, U^{p}\left(N_{1}, N_{2}\right)}^{(m) \text {,ord }}$ in this paper. The generalized ordinary Kuga family attached to $\widehat{\mathcal{H}}_{\widehat{\mathrm{G}}} \ltimes \widehat{\mathcal{H}}_{\widehat{\mathrm{U}}}$ is denoted

$$
\overrightarrow{\mathrm{N}}^{\text {ord,grp }} \rightarrow \overrightarrow{\mathrm{M}}_{\mathcal{H}}^{\text {ord }}
$$

and is a Kuga family as in definition 7.1.3.1 of [44]. It is an abelian scheme and, since $Q=\mathcal{O}_{F}^{m}$, it is $\mathbb{Q}^{\times}$-isogenous to the $m$-fold fibre product of the universal abelian scheme over $\vec{M}_{\widehat{\mathcal{H}}_{G}}^{\text {ord }}$. Its characteristic zero pull-back is a Kuga family N $\mathrm{N}^{\text {grp }} \rightarrow \mathrm{M}_{\mathcal{H}}$ as in Sect. B.6. The generalized ordinary Kuga family $\vec{N}^{\text {ord }} \rightarrow \vec{M}_{\mathcal{H}}^{\text {ord }}$ is a torsor for $\vec{N}^{\text {ord,grp }} \rightarrow \vec{M}_{\mathcal{H}}^{\text {ord }}$.

To study these schemes and their compactification [44] again realizes them in a noncanonical way inside the boundary of a larger Shimura variety. We will continue to use the notation of Sect. B.6. We consider the maximal totally isotropic filtration

$$
0=\widetilde{\mathrm{D}}^{1} \subset \widetilde{\mathrm{D}}^{0}=\left(\left(\widetilde{\mathrm{Z}}_{-2} \otimes_{\hat{\mathbb{Z}}} \mathbb{Z}_{p}\right) \oplus \mathrm{D}\right) \subset \widetilde{\mathrm{D}}^{-1}=\widetilde{L} \otimes_{\mathbb{Z}} \mathbb{Z}_{p}
$$


of $\widetilde{L} \otimes_{\mathbb{Z}} \mathbb{Z}_{p}$ (see the beginning of section 7.1.1 of [44]). We have

$$
\widetilde{\mathrm{D}}^{0}=\widetilde{\mathrm{V}}^{(m+n)} \cap\left(\widetilde{L} \otimes_{\mathbb{Z}} \mathbb{Z}_{p}\right) .
$$

We define (see definition 7.1.1.22 of [44]):

(1) $\quad \widetilde{\mathrm{P}}_{\widetilde{\mathrm{Z}}, \widetilde{\mathrm{D}}}^{\text {ord }}(R):=\widetilde{\mathrm{P}}_{\widetilde{\mathrm{Z}}}(R) \cap \widetilde{\mathrm{P}}_{\widetilde{\mathrm{D}}}^{\text {ord }}(R)$.

(2) $\quad \widetilde{\mathrm{P}}_{\widetilde{\mathrm{Z}}, \widetilde{\mathrm{D}}}^{\text {ord, }}(R):=\widetilde{\mathrm{P}}_{\widetilde{\mathrm{Z}}}^{\prime}(R) \cap \widetilde{\mathrm{P}}_{\widetilde{\mathrm{D}}}^{\text {ord }}(R)=\widetilde{\mathrm{P}}_{n,(n)}^{(m),+}$.

For any such $\widehat{\mathcal{H}}$, we can always find some

$$
\widetilde{\mathcal{H}}=\widetilde{\mathcal{H}}^{p} \times \widetilde{\mathcal{U}}_{p, 1,0}\left(p^{N_{1}}, p^{N_{2}}\right) \subset \widetilde{\mathrm{G}}(\hat{\mathbb{Z}})
$$

with $\widetilde{\mathcal{H}}^{p}$ neat such that $\widehat{\mathcal{H}}=\widetilde{\mathcal{H}}_{\widehat{\mathrm{G}}}$ (see Sect. B.6) and

$$
\widetilde{\mathcal{H}}_{\widetilde{\mathrm{P}}_{\tilde{\mathrm{Z}}}} / \widetilde{\mathcal{H}}_{\widetilde{\mathrm{U}}_{\tilde{\mathrm{z}}}} \cong \widetilde{\mathcal{H}}_{\widetilde{\mathrm{G}}_{l, \tilde{\mathrm{z}}}} \times \widehat{\mathcal{H}}_{\mathrm{G}} .
$$

(Then $r_{\tilde{\mathcal{H}}}=0$.) In this case, the abelian scheme torsor

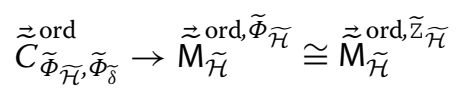

depends only on $\widehat{\mathcal{H}}$ (but not on the auxiliary choice of $\widetilde{\mathcal{H}}$ ) and equals the above generalized ordinary Kuga family $\overrightarrow{\mathrm{N}}^{\text {ord }} \rightarrow \overrightarrow{\mathrm{M}}_{\mathcal{H}}^{\text {ord }}$ attached to $\widehat{\mathcal{H}}$.

\section{B.15 Partial toroidal compactifications of ordinary loci of Kuga families}

Let us fix the choice of some $(\widetilde{Z}, \widetilde{\Phi}, \widetilde{\delta})$ as in Sect. B.6. Then the bijection described at the start of Sect. B.7 restricts to a bijection between the following two sets:

(1) The fully symplectic admissible filtrations $\breve{Z}$ of $\widetilde{L} \otimes_{\mathbb{Z}} \hat{\mathbb{Z}}$ compatible with $\widetilde{D}$ such that $0 \subset \widetilde{Z}_{-2} \subset \breve{Z}_{-2} \subset \breve{Z}_{-1} \subset \widetilde{Z}_{-1} \subset \widetilde{L} \otimes_{\mathbb{Z}} \hat{\mathbb{Z}}$.

(2) The fully symplectic admissible filtrations $\mathrm{Z}$ of $L \otimes_{\mathbb{Z}} \hat{\mathbb{Z}}$ compatible with D such that $\mathrm{Z}_{-2}=\breve{\mathrm{Z}}_{-2} / \widetilde{\mathrm{Z}}_{-2}$.

(Recall that the notation “ will always mean objects related to such a filtration ž.)

For each $\check{Z}$ as above, and for each $\mathbb{Z}_{p}$-algebra $R$, we define the following quotients of subgroups of $\widehat{\mathrm{P}}_{\check{Z}}(R)$ (see definition 7.1.1.27 of [44]):

(1) $\widehat{\mathrm{P}}_{\tilde{z}, \mathrm{D}}^{\text {ord }}(R):=\left(\widetilde{\mathrm{P}}_{\check{z}, \widetilde{\mathrm{D}}}^{\text {ord }}(R) \cap \widetilde{\mathrm{P}}_{\widetilde{\mathrm{Z}}}^{\prime}(R)\right) / \widetilde{\mathrm{U}}_{2, \widetilde{\mathrm{Z}}}(R)$.

(2) $\widehat{\mathrm{P}}_{\tilde{\mathrm{Z}}, \mathrm{D}}^{\mathrm{ord},}(R):=\widetilde{\mathrm{P}}_{\check{\mathrm{Z}}, \widetilde{\mathrm{D}}}^{\mathrm{ord},}(R) / \widetilde{\mathrm{U}}_{2, \widetilde{\mathrm{Z}}}(R)$.

(3) $\widehat{\mathrm{P}}_{1, \mathrm{z}, \mathrm{D}}^{\text {ord }}(R):=\widehat{\mathrm{P}}_{\check{z}, \mathrm{D}}^{\text {ord, }}(R) / \widehat{\mathrm{U}}_{2, \check{z}}(R)$.

(4) $\widehat{\mathrm{P}}_{h, \check{\mathrm{z}}, \mathrm{D}}^{\text {ord, }}(R):=\widehat{\mathrm{P}}_{1, \mathrm{z}, \mathrm{D}}^{\text {ord }}(R) / \widehat{\mathrm{U}}_{1, \check{\mathrm{z}}}(R) \cong \widehat{\mathrm{P}}_{h, \mathrm{z}, \mathrm{D}}^{\text {ord }}(R)$.

(5) $\widehat{\mathrm{P}}_{h, \mathrm{z}, \mathrm{D}}^{\text {ord }}(R)$ denotes the subgroup of elements of $\widehat{\mathrm{G}}_{h, \check{\mathrm{Z}}}(R) \cong \mathrm{G}_{h, \mathrm{Z}}(R)$ which preserve $\widetilde{\mathrm{D}}_{-1}=\mathrm{D} \subset \mathrm{Gr}_{-1}^{\check{z}} \otimes_{\mathbb{Z}^{\mathbb{Z}}} \mathbb{Z}_{p} \cong \mathrm{Gr}_{-1}^{\mathrm{Z}} \otimes_{\mathbb{\mathbb { Z }}} \mathbb{Z}_{p}$. Thus $\widehat{\mathrm{P}}_{h, \check{\mathrm{z}}, \mathrm{D}}^{\text {ord, }}(R) \stackrel{\sim}{\rightarrow} \widehat{\mathrm{P}}_{h, \check{z}, \mathrm{D}}^{\text {ord }}(R)$.

When $\mathrm{Z}=\mathrm{Z}^{(i, 1)}$, in which case $\breve{\mathrm{z}}=\widetilde{\mathrm{Z}}^{(i+m, 1)}=\check{\mathrm{z}}^{(i, 1)}$, we have, for example,

$$
\widehat{\mathrm{P}}_{\check{\mathrm{Z}}^{(i, 1)}}\left(\mathbb{A}^{\infty, p}\right) \times \widehat{\mathrm{P}}_{\check{\mathrm{Z}}^{(i, 1)}, \mathrm{D}}^{\mathrm{ord}}\left(\mathbb{Z}_{p}\right)=P_{n,(i)}^{(m),+}\left(\mathbb{A}^{\infty}\right)^{\text {ord }, \times}
$$

in the notation of this paper (see Sect. 1.2). 
The filtration $\check{Z}^{(i, \widehat{g})}$ is compatible with $\widetilde{\mathrm{D}}$ if and only if

$$
\widehat{g} \in \widehat{\mathrm{G}}\left(\mathbb{A}^{\infty, p}\right) \times \widehat{\mathrm{P}}_{\check{\mathrm{z}}^{(i, 1)}}^{\mathrm{ord}}\left(\mathbb{Q}_{p}\right) \widehat{\mathrm{P}}_{\mathrm{D}}^{\mathrm{ord}}\left(\mathbb{Q}_{p}\right),
$$

where $\widehat{P}_{D}^{\text {ord }}\left(\mathbb{Q}_{p}\right)=\widetilde{\mathrm{P}}_{1, \widetilde{\mathrm{Z}}, \widetilde{\mathrm{D}}}^{\text {ord }}\left(\mathbb{Q}_{p}\right)$.

Now suppose $\widehat{\mathcal{H}}$ is an open compact subgroup $\widehat{\mathcal{H}}$ of $\widehat{\mathrm{G}}(\hat{\mathbb{Z}})$ of the form $\widehat{\mathcal{H}}=\widehat{\mathcal{H}}^{p} \widehat{\mathcal{H}}_{p}$, where $\widehat{\mathcal{H}}^{p}$ is a neat open compact subgroup of $\widehat{\mathrm{G}}\left(\hat{\mathbb{Z}}^{p}\right)$, and where $\widehat{\mathcal{H}}_{p}=\widehat{\mathcal{U}}_{p, 1,0}\left(p^{r_{1}}, p^{r_{0}}\right)$ for some integers $0 \leq r_{1} \leq r_{0}$. The $\widehat{\mathcal{H}}$-orbits $\breve{Z}_{\widehat{\mathcal{H}}}^{(i, \widehat{g})}$ containing a filtration compatible with $\widetilde{\mathrm{D}}$ are

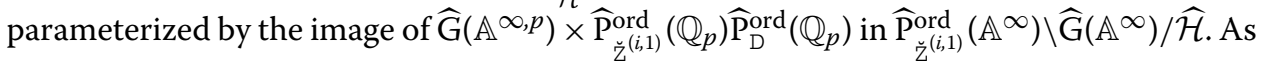
in Sect. 5.1, this set is in bijection with the double coset space

$$
\left(\widehat{\mathrm{P}}_{\check{\mathrm{Z}}^{(i, 1)}}\left(\mathbb{A}^{\infty, p}\right) \times \widehat{\mathrm{P}}_{\check{\mathrm{Z}}^{(i, 1)}, \mathrm{D}}^{\text {ord }}\left(\mathbb{Z}_{p}\right)\right) \backslash\left(\widehat{\mathrm{G}}\left(\mathbb{A}^{\infty, p}\right) \times \widehat{\mathrm{P}}_{\mathrm{D}}^{\text {ord }}\left(\mathbb{Z}_{p}\right)\right) /\left(\widehat{\mathcal{H}}^{p} \times\left(\widehat{\mathcal{H}}_{p} \cap \widehat{\mathrm{P}}_{\mathrm{D}}^{\text {ord }}\left(\mathbb{Z}_{p}\right)\right)\right),
$$

which is the double coset space

$$
P_{n,(i)}^{(m),+}\left(\mathbb{A}^{\infty}\right)^{\text {ord }, \times} \backslash G_{n}^{(m)}\left(\mathbb{A}^{\infty}\right)^{\text {ord }, \times} / U^{p}\left(N_{1}\right)
$$

in this paper (see, e.g., Sect. 1.4), with $U^{p}\left(N_{1}, N_{2}\right)=\widehat{\mathcal{H}}$. It maps isomorphically to

$$
P_{n,(i)}^{+}\left(\mathbb{A}^{\infty}\right)^{\text {ord }, \times} \backslash G_{n}\left(\mathbb{A}^{\infty}\right)^{\text {ord }, \times} / U^{p}\left(N_{1}\right) .
$$

The correspondence of cusp labels from Sect. B.7 sets up bijections between the following sets (see lemmas 1.2.4.15 and 1.2.4.16, definitions 1.2.4.17 and 3.2.3.8, and lemma 7.1.1.8 of [44]):

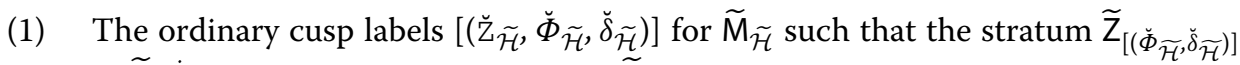

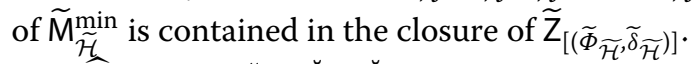

(2) The $\widehat{\mathcal{H}}$-orbits, $\left[\left(\breve{Z}_{\widehat{\mathcal{H}}}, \breve{\Phi}_{\widehat{\mathcal{H}}}, \breve{\delta}_{\widehat{\mathcal{H}}}\right)\right]$, of equivalence classes of $(\check{Z}, \breve{\Phi}, \breve{\delta})$, where $\check{\mathrm{Z}}$ and $\check{\Phi}$ are compatible with $\widetilde{\mathrm{Z}}$ and $\widetilde{\Phi}$, as described in Sect. B.7, and with $\widetilde{\mathrm{D}}$. We call such an orbit an ordinary cusp label for $(\widetilde{L},\langle\cdot, \cdot \gamma, \widetilde{h} 0, \widetilde{Z}, \widetilde{D})$.

(3) The ordinary cusp labels $\left[\left(\mathrm{Z}_{\mathcal{H}}, \Phi_{\mathcal{H}}, \delta_{\mathcal{H}}\right)\right]$ for $\mathrm{M}_{\mathcal{H}}$.

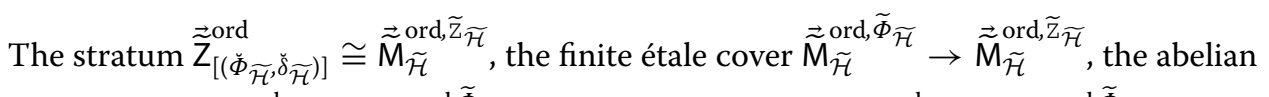
scheme torsor $\overrightarrow{\widetilde{C}}_{\widetilde{\Phi}_{\widetilde{\mathcal{H}}}, \widetilde{\delta}_{\widetilde{\mathcal{H}}}} \rightarrow \overrightarrow{\widetilde{M}}_{\widetilde{\mathcal{H}}}^{\text {ord }}, \widetilde{\Phi}_{\widetilde{\mathcal{H}}}$, and the abelian scheme $\overrightarrow{\widetilde{C}}_{\widetilde{\Phi}_{\widetilde{\mathcal{H}}} \widetilde{\delta}_{\widetilde{\mathcal{H}}}}^{\text {ord, grp }} \rightarrow \overrightarrow{\widetilde{M}}_{\widetilde{\mathcal{H}}}^{\text {ord }} \widetilde{\Phi}_{\widetilde{\mathcal{H}}}$ depend (up to canonical isomorphism) only on the $\widehat{\mathcal{H}}$-orbit of ( $(\breve{Z}, \breve{\Phi}, \breve{\delta}$ ) (see lemma 7.1.2.1 of [44]), and hence we shall denote them by

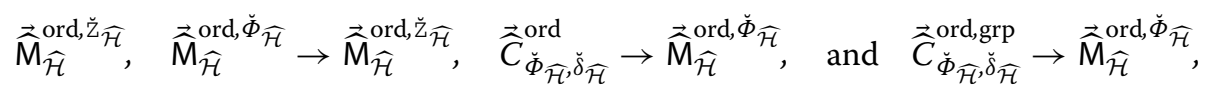

respectively. For a fixed $(\breve{Z}, \breve{\Phi}, \breve{\delta})$, the morphisms

$$
\overrightarrow{\widehat{C}}_{\check{\Phi}_{\widehat{\mathcal{H}}}, \check{\delta}_{\widehat{\mathcal{H}}}}^{\text {ord }} \rightarrow \overrightarrow{\widehat{\mathrm{M}}}_{\widehat{\mathcal{H}}}^{\text {ord, } \check{\Phi}_{\widehat{\mathcal{H}}}} \rightarrow \overrightarrow{\widehat{\mathrm{M}}}_{\widehat{\mathcal{H}}}^{\text {ord, } \check{\mathcal{H}}_{\widehat{\mathcal{H}}}}
$$

are equivariant with

$$
\widehat{\mathrm{G}}_{1, \check{\mathrm{z}}}\left(\mathbb{A}^{\infty, p}\right) \times \widehat{\mathrm{P}}_{1, \check{\mathrm{z}}, \mathrm{D}}^{\mathrm{ord}}\left(\mathbb{Z}_{p}\right) \rightarrow \widehat{\mathrm{G}}_{h, \check{\mathrm{z}}}^{\prime}\left(\mathbb{A}^{\infty, p}\right) \times \widehat{\mathrm{P}}_{h, \breve{\mathrm{Z}}, \mathrm{D}}^{\mathrm{ord},}\left(\mathbb{Z}_{p}\right) \cong \widehat{\mathrm{G}}_{h, \check{\mathrm{z}}}\left(\mathbb{A}^{\infty, p}\right) \times \widehat{\mathrm{P}}_{h, \breve{\mathrm{z}}, \mathrm{D}}^{\mathrm{ord}}\left(\mathbb{Z}_{p}\right)
$$


and with the compatible actions of the elements corresponding to $s_{p}$ (see propositions 5.2.4.25 and 7.1.2.5 of [44]).

The cusp labels $\left[\left(\breve{Z}_{\widehat{\mathcal{H}}}, \breve{\Phi}_{\widehat{\mathcal{H}}}, \breve{\delta}_{\widehat{\mathcal{H}}}\right)\right]$ such that $\breve{Z}_{\widehat{\mathcal{H}}}=\breve{Z}_{\widehat{\mathcal{H}}}^{(i, \widehat{g})}$ for some $\widehat{g} \in \widehat{\mathrm{G}}\left(\mathbb{A}^{\infty, p}\right) \times \widehat{\mathrm{P}}_{\mathrm{D}}^{\text {ord }}\left(\mathbb{Z}_{p}\right)$ are parameterized by the double coset space

$$
\begin{aligned}
& \left(\widehat{\mathrm{G}}_{l, \breve{\Phi}^{(i, 1)}}^{\prime}\left(\mathbb{Z}_{(p)}\right) \ltimes\left(\widehat{\mathrm{P}}_{\check{\check{C}}^{(i, 1)}}^{\prime}\left(\mathbb{A}^{\infty, p}\right) \times \widehat{\mathrm{P}}_{\check{\mathrm{Z}}^{(i, 1)}, \mathrm{D}}^{\text {ord },}\left(\mathbb{Z}_{p}\right)\right)\right) \backslash\left(\widehat{\mathrm{G}}\left(\mathbb{A}^{\infty, p}\right) \times \widehat{\mathrm{P}}_{\mathrm{D}}^{\text {ord }}\left(\mathbb{Z}_{p}\right)\right) \\
& \quad /\left(\widehat{\mathcal{H}}^{p} \times\left(\widehat{\mathcal{H}}_{p} \cap \widehat{\mathrm{P}}_{\overline{\mathrm{D}}}^{\text {ord }}\left(\mathbb{Z}_{p}\right)\right)\right),
\end{aligned}
$$

and the forgetful map sending $\left[\left(\breve{Z}_{\widehat{\mathcal{H}}}, \breve{\Phi}_{\widehat{\mathcal{H}}}, \breve{\delta}_{\widehat{\mathcal{H}}}\right)\right]$ to $\breve{Z}_{\widehat{\mathcal{H}}}$ can be identified with the canonical map from this double coset space to

$$
\left(\widehat{\mathrm{P}}_{\check{\mathrm{Z}}^{(i, 1)}}\left(\mathbb{A}^{\infty, p}\right) \times \widehat{\mathrm{P}}_{\check{\mathrm{Z}}^{(i, 1)}, \mathrm{D}}^{\text {ord }}\left(\mathbb{Z}_{p}\right)\right) \backslash\left(\widehat{\mathrm{G}}\left(\mathbb{A}^{\infty, p}\right) \times \widehat{\mathrm{P}}_{\mathrm{D}}^{\text {ord }}\left(\mathbb{Z}_{p}\right)\right) /\left(\widehat{\mathcal{H}}^{p} \times\left(\widehat{\mathcal{H}}_{p} \cap \widehat{\mathrm{P}}_{\mathrm{D}}^{\text {ord }}\left(\mathbb{Z}_{p}\right)\right)\right)
$$

whose fibre above the double coset of $\widehat{g} \in \widehat{\mathrm{G}}\left(\mathbb{A}^{\infty, p}\right) \times \widehat{\mathrm{P}}_{\mathrm{D}}^{\text {ord }}\left(\mathbb{Z}_{p}\right)$ can be identified with

$$
\widehat{\mathrm{G}}_{l, \breve{\Phi}^{(i, 1)}}^{\prime}\left(\mathbb{Z}_{(p)}\right) \backslash \widehat{\mathrm{G}}_{l, \breve{\mathrm{Z}}^{(i, 1)}}^{\prime}\left(\mathbb{A}^{\infty, p} \times \mathbb{Z}_{p}\right) /\left(\widehat{\mathrm{g}} \widehat{\mathcal{H}}^{-1}\right)_{\widehat{\mathrm{G}}_{l, \breve{\mathbb{Z}}^{(i, 1)}}^{\prime}}
$$

(See Sect. B.7 for the definition of $\widehat{\mathrm{G}}_{l, \breve{\Phi}\left({ }^{(i 1)}\right)}^{\prime}$.) This last double coset space is the index set of

$$
\begin{aligned}
& \mathcal{Y}_{n,(i),\left(g U^{p} g^{-1} \cap P_{n, i}^{(m),+}\left(\mathbb{A}^{\infty, p}\right)\right)\left(N_{1}\right)}^{(m), \text { rd },}
\end{aligned}
$$

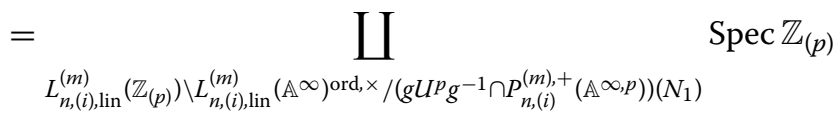

in this paper (see Sect. 4.1), with $g=\widehat{g}$ and $U^{p}\left(N_{1}, N_{2}\right)=\widehat{\mathcal{H}}$.

If $U^{p}\left(N_{1}, N_{2}\right)=\widehat{\mathcal{H}}$ is a neat open compact subgroup of $G_{n}^{(m)}\left(\mathbb{A}^{\infty}\right)=\widehat{\mathrm{G}}\left(\mathbb{A}^{\infty}\right)$ as above, if $g=\widehat{g} \in G_{n}^{(m)}\left(\mathbb{A}^{\infty}\right)^{\text {ord, } \times}=\widehat{\mathrm{G}}\left(\mathbb{A}^{\infty, p}\right) \times \widehat{\mathrm{P}}_{\mathrm{D}}^{\text {ord }}\left(\mathbb{Z}_{p}\right)$ and if $U_{g}^{p, \prime}=g U^{p} g^{-1} \cap P_{n,(i)}^{(m),+}\left(\mathbb{A}^{\infty}\right)$, then the maps

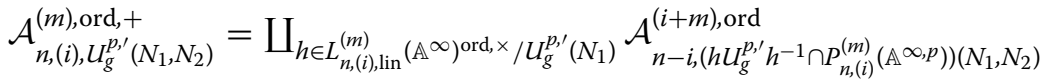

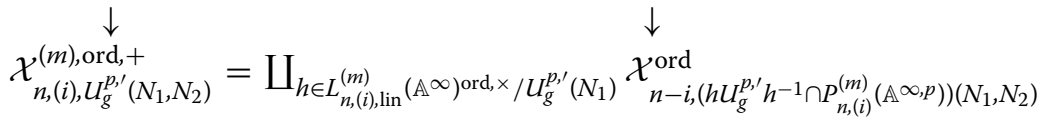

$$
\begin{aligned}
& \underset{n,(i), U_{g}^{p, \prime}\left(N_{1}, N_{2}\right)}{\downarrow}
\end{aligned}
$$

in this paper (see Sects. 4.1, 4.2) are identified with

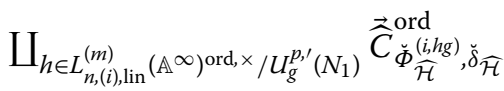

$$
\begin{aligned}
& \amalg_{h \in L_{n,(i), \operatorname{lin}}^{(m)}\left(\mathbb{A}^{\infty}\right)^{\text {ord } \times} \times / U_{g}^{p, \prime^{\prime}}\left(N_{1}\right)} \overrightarrow{\widehat{M}}_{\widehat{\mathcal{H}}}^{\text {ord, } \breve{\Phi}_{\mathcal{\mathcal { H }}}^{(i h h)}} \\
& \downarrow \\
& \bigsqcup_{h \in L_{n,(i), \operatorname{lin}}^{(m)}\left(\mathbb{Z}_{(p)}\right) \backslash L_{n,(i), \operatorname{lin}}^{(m)}\left(\mathbb{A}^{\infty}\right)^{\text {ord }, \times} / U_{g}^{p, \prime}\left(N_{1}\right)} \overrightarrow{\widehat{M}}_{\widehat{\mathcal{H}}}^{\text {ord, } \tilde{\bar{Z}}^{(i, h g)}} .
\end{aligned}
$$


For each representative $\left(\breve{Z}_{\widehat{\mathcal{H}}}, \breve{\Phi}_{\widehat{\mathcal{H}}}, \breve{\delta}_{\widehat{\mathcal{H}}}\right)$ of an ordinary cusp label [( $\left.\left.\breve{\mathrm{Z}}_{\widehat{\mathcal{H}}}, \breve{\Phi}_{\widehat{\mathcal{H}}}, \breve{\delta}_{\widehat{\mathcal{H}}}\right)\right]$ as above, there is a torsor

$$
\overrightarrow{\widehat{\Xi}}_{\check{\Phi}_{\widehat{\mathcal{H}}}, \check{\delta}_{\widehat{\mathcal{H}}}}^{\text {ord }} \rightarrow \overrightarrow{\widehat{C}}_{\mathscr{\Phi}_{\widehat{\mathcal{H}}}, \check{\delta}_{\widehat{\mathcal{H}}}}^{\text {ord }}
$$

under the pull-back of the same split torus $\widehat{E}_{\breve{\Phi}_{\widehat{\mathcal{H}}}}$ over Spec $\mathbb{Z}$ with character group $\widehat{\mathbf{S}}_{\breve{\Phi}_{\widehat{\mathcal{H}}}}$ as before (see Sect. B.7; and see proposition 7.1.2.6 of [44]). We obtain an isomorphic torus torsor if we replace $\left(\breve{Z}_{\widehat{\mathcal{H}}}, \breve{\Phi}_{\widehat{\mathcal{H}}}, \breve{\delta}_{\widehat{\mathcal{H}}}\right)$ with another representative, but its universal property depends on this choice of representative.

The torus

$$
\mathcal{S}_{n,(i), U_{g}^{p,}\left(N_{1}\right)}^{(m), \text { ord }+} \rightarrow \mathcal{Y}_{n,(i), U_{g}^{p, \prime}\left(N_{1}\right)}^{(m), \text { ord }+}
$$

in this paper (see Sect. 4.3) with $U^{p}\left(N_{1}, N_{2}\right)=\mathcal{H}$ is identified with

$$
\coprod_{h \in L_{n,(i), \operatorname{lin}}^{(m)}\left(\mathbb{A}^{\infty}\right)^{\text {ord, } \times} / U_{g}^{p, \prime}\left(N_{1}\right)} \widehat{E}_{\check{\Phi}_{\mathcal{H}}^{(i, h g)}} \rightarrow \coprod_{h \in L_{n,(i), \text { lin }}^{(m)}\left(\mathbb{A}^{\infty}\right)^{\text {ord } \times} / U_{g}^{p, \prime}\left(N_{1}\right)} \operatorname{Spec} \mathbb{Z}_{(p)} .
$$

Moreover, the sheaves $X^{*}\left(\mathcal{S}_{n,(i), U_{g}^{p,}\left(N_{1}\right)}^{(m), \text { ord, }}\right), X_{*}\left(\mathcal{S}_{n,(i), U_{g}^{p, \prime}\left(N_{1}\right)}^{(m), \text { ord },+}\right)_{\mathbb{R}}^{\succ 0}$, and $X_{*}\left(\mathcal{S}_{n,(i), U_{g}^{p, \prime}\left(N_{1}\right)}^{(m) \text { ord, }}\right)_{\mathbb{R}}^{>0}$ are identified with

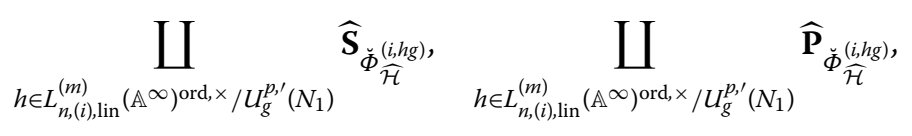

and

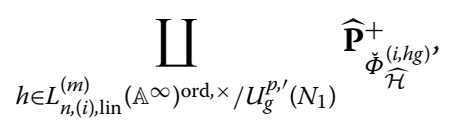

respectively. The $\mathcal{S}_{n,(i), U_{g}^{(m),}\left(N_{1}\right)}^{(m) \text { ord, }}$-torsor

$$
\begin{aligned}
& \mathcal{T}_{n,(i), U_{g}^{p, \prime}\left(N_{1}, N_{2}\right)}^{(m),+ \text { ord }}=\coprod_{h \in L_{n,(i), \text { lin }}^{(m)}\left(\mathbb{A}^{\infty}\right) \text { ord, } \times / U_{g}^{p, \prime}\left(N_{1}\right)} \mathcal{T}_{\left.n-i, h g U^{p} g^{-1} h^{-1} \cap P_{n, i}^{(m)}\left(\mathbb{A}^{\infty, p}\right)\right)\left(N_{1}, N_{2}\right)}^{(i+m), \text { ord }}
\end{aligned}
$$

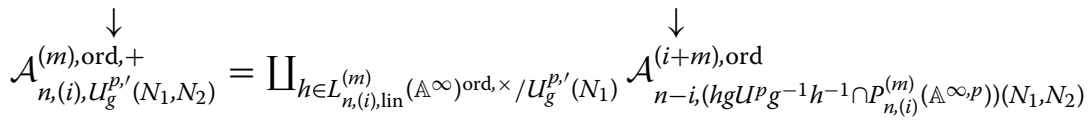

is identified with

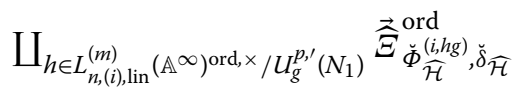

$$
\begin{aligned}
& \downarrow
\end{aligned}
$$

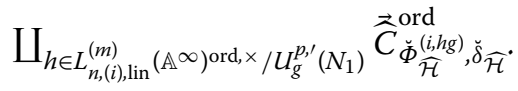

These maps are equivariant with $\widehat{\mathrm{P}}_{\check{Z}^{(i, g)}}\left(\mathbb{A}^{\infty, p}\right) \times \widehat{\mathrm{P}}_{\check{\mathrm{Z}}^{(i, g)}, \mathrm{D}}^{\mathrm{ord}}\left(\mathbb{Z}_{p}\right)$ and with the compatible actions of the elements corresponding to $\zeta_{p}$ (see proposition 1.3.2.67 of [44]). 
In lemma 7.1.1.9 of [44], we have introduced an ad hoc definition of a compatible collection

$$
\widehat{\Sigma}^{\text {ord }}=\left\{\widehat{\Sigma}_{\breve{\Phi}_{\widehat{\mathcal{H}}}}\right\}_{\left[\left(\breve{\Phi}_{\widehat{\mathcal{H}}}, \check{\delta}_{\widehat{\mathcal{H}}}\right)\right]}
$$

of admissible projective smooth rational polyhedral cone decompositions, where each $\widehat{\Sigma}_{\breve{\Phi}_{\widehat{\mathcal{H}}}}$ is a $\Gamma_{\check{\Phi}_{\widehat{\mathcal{H}}}}$-admissible projective smooth rational polyhedral cone decomposition of $\widehat{\mathbf{P}}_{\check{\Phi}_{\widehat{\mathcal{H}}}}$, and where the indices are ordinary cusp labels [( $\left.\left.\breve{\Phi}_{\widehat{\mathcal{H}}}, \breve{\delta}_{\widehat{\mathcal{H}}}\right)\right]$. In [44], we allow $\widehat{\mathcal{H}}_{p}$ to be all subgroups of $\widehat{\mathrm{G}}\left(\mathbb{Z}_{p}\right)$ of standard form (which is more general than just of the form $\widehat{\mathcal{U}}_{p, 1,0}\left(p^{r_{1}}, p^{r_{0}}\right)$ for some integers $\left.0 \leq r_{1} \leq r_{0}\right)$, and the set of pairs $\kappa=\left(\widehat{\mathcal{H}}, \widehat{\Sigma}^{\text {ord }}\right)$ with $\widehat{\mathcal{H}}_{\mathrm{G}} \subset \mathcal{H}$ is denoted $\mathbf{K}_{Q, \mathcal{H}}^{\text {ord,++}}$; the subset of $\mathbf{K}_{Q, \mathcal{H}}^{\text {ord,++ }}$ consisting of $\kappa=\left(\widehat{\mathcal{H}}, \widehat{\Sigma}^{\text {ord }}\right)$ with $\widehat{\mathcal{H}}_{\mathrm{G}}=\mathcal{H}$ is denoted $\mathbf{K}_{Q, \mathcal{H}}^{\mathrm{ord}+}$; and the subset of $\mathbf{K}_{Q, \mathcal{H}}^{\text {ord, }}$ consisting of $\kappa=\left(\widehat{\mathcal{H}}, \widehat{\Sigma}^{\text {ord }}\right)$ with $\widehat{\mathcal{H}}_{\mathrm{G}}=\mathcal{H}$ and $\widehat{\mathcal{H}}=\mathcal{H} \ltimes \widehat{\mathcal{H}}_{\widehat{\mathrm{U}}}$ is denoted $\mathbf{K}_{Q, \mathcal{H}}^{\text {ord }}$ (see definitions 7.1.1.7 and 7.1.1.11 of [44]). For $?=\emptyset,+$ or ++ , any pair $\kappa=(\widehat{\mathcal{H}}, \widehat{\Sigma})$ in $\mathbf{K}_{Q, \mathcal{H}}^{?}$ introduced earlier in Sect. B.7 such that $\widehat{\mathcal{H}}$ is of the form allowed here induces a pair $\left(\widehat{\mathcal{H}}, \widehat{\Sigma}^{\text {ord }}\right)$ in $\mathbf{K}_{Q, \mathcal{H}}^{\text {ord? }}$, and conversely any pair in $\mathbf{K}_{Q, \mathcal{H}}^{\text {ord? }}$ extends to a pair in $\mathbf{K}_{Q, \mathcal{H}}^{\text {? }}$ (see proposition 7.1.1.21 of [44]). For any compatible collection $\Sigma^{\text {ord }}$ of admissible projective smooth rational polyhedral cone decompositions for $\vec{M}_{\mathcal{H}}^{\text {ord }}$, and for ? $=\emptyset,+,++$, the subset of $\mathbf{K}_{Q, \mathcal{H}}^{\text {ord? }}$ consisting of $\kappa=\left(\widehat{\mathcal{H}}, \widehat{\Sigma}^{\text {ord }}\right)$ such that $\widehat{\Sigma}^{\text {ord }}$ is compatible with $\Sigma^{\text {ord }}$ in the sense that each $\widehat{\rho} \in \widehat{\Sigma}_{\check{\Phi}_{\widehat{\mathcal{H}}}}$ is mapped into some $\sigma \in \Sigma_{\Phi_{\mathcal{H}}}$ (see condition 7.1.1.17 and definition 7.1.1.19 of [44]) is denoted $\mathbf{K}_{Q, \mathcal{H}, \Sigma}^{\text {ord } \Sigma^{\text {ord }}}$. This notion of compatibility agrees with the one in this paper (see Sect. 5.2).

Each such $\kappa=\left(\widehat{\mathcal{H}}, \widehat{\Sigma}^{\text {ord }}\right)$ in $\mathbf{K}_{Q, \mathcal{H}}^{\text {ord,++ }}$ induces a pair

$$
\left(U^{p}\left(N_{1}\right), \Sigma\right)
$$

in $\mathcal{J}_{n}^{(m) \text {,tor,ord }}$ (with $U^{p}\left(N_{1}, N_{2}\right)=\widehat{\mathcal{H}}$ ) in Sect. 5.2 of this paper, because, in order to define $\left(U^{p}\left(N_{1}\right), \Sigma\right)$ as in Sect. 5.2, it suffices to define the admissible cone decomposition $\Sigma(g)_{0}$ for $X_{*}\left(\mathcal{S}_{n,(i),\left(g U^{p} g^{-1} \cap P_{n,(i)}^{(m),+}\left(\mathbb{A}^{\infty, p}\right)\right)\left(N_{1}\right)}\right)_{\mathbb{R}}^{\succ 0}$, for each $g \in G_{n}^{(m)}\left(\mathbb{A}^{\infty}\right)^{\text {ord, } \times}=\widehat{\mathrm{G}}\left(\mathbb{A}^{\infty, p}\right) \times \widehat{\mathrm{P}}_{\mathrm{D}}^{\text {ord }}\left(\mathbb{Z}_{p}\right)$, which can be taken to be the pull-back of the subcollection $\left\{\widehat{\Sigma}_{\breve{\Phi}_{\widehat{\mathcal{H}}}}\right\}_{\left[\left(\breve{\Phi}_{\widehat{\mathcal{H}}}, \check{\delta}_{\widehat{\mathcal{H}}}\right)\right]}$ of $\widehat{\Sigma}^{\text {ord }}$ indexed by the ordinary cusp labels $\left[\left(\breve{\Phi}_{\widehat{\mathcal{H}}}, \breve{\delta}_{\widehat{\mathcal{H}}}\right)\right]$ with underlying $\breve{Z}_{\widehat{\mathcal{H}}}$ equal to $\breve{Z}_{\widehat{\mathcal{H}}}^{(i, g)}$. In fact, $\mathcal{J}_{n}^{(m) \text {,tor,ord }}$ is exactly the set of such induced pairs (as $U^{p}\left(N_{1}, N_{2}\right)=\widehat{\mathcal{H}}$ varies). (As before, it is hard to explicitly describe the set $\mathcal{J}_{n}^{(m) \text {,tor,ord }}$ when $m>0$, because they are induced by auxiliary choices of compatible collections $\widetilde{\Sigma}^{\text {ord }}$ for $\vec{M}_{\widetilde{\mathcal{H}}}^{\text {ord }}$. Nevertheless, this is unnecessary for our purpose.)

Each $\widehat{\Sigma}_{\breve{\Phi}_{\widehat{\mathcal{H}}}}$ defines an affine toroidal embedding

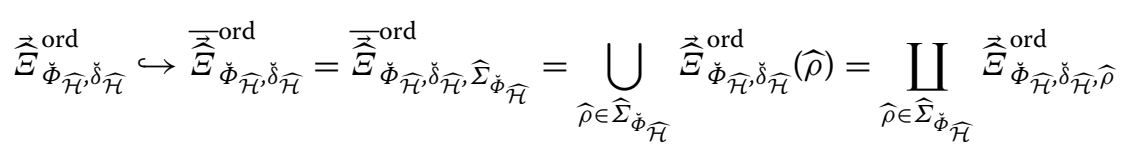

over $\overrightarrow{\widehat{C}}_{\check{\Phi}_{\widehat{\mathcal{H}}}, \check{\delta}_{\widehat{\mathcal{H}}}}^{\text {ord }}$. The formal completion of $\overline{\vec{\Xi}}_{\check{\Phi}_{\widehat{\mathcal{H}}}, \check{\delta}_{\widehat{\mathcal{H}}}, \widehat{\Sigma}_{\check{\Phi}_{\widehat{\mathcal{H}}}}}^{\text {ord }}$ along the union of the $\widehat{\rho}$-strata $\overrightarrow{\widehat{\Xi}}_{\mathscr{\Phi}_{\widehat{\mathcal{H}}}, \breve{\delta}_{\widehat{\mathcal{H}}}, \widehat{\rho}}^{\text {ord }}$ for all $\widehat{\rho} \in \widehat{\Sigma}_{\check{\Phi}_{\widehat{\mathcal{H}}}}$ such that $\widehat{\rho} \cap \widehat{\mathbf{P}}_{\breve{\Phi}_{\widehat{\mathcal{H}}}}^{+} \neq \emptyset$ is denoted

$$
\overrightarrow{\hat{\mathfrak{X}}}_{\check{\Phi}_{\widehat{\mathcal{H}}}, \check{\delta}_{\widehat{\mathcal{H}}}}^{\text {ord }}=\overrightarrow{\widehat{\mathfrak{X}}}_{\check{\Phi}_{\widehat{\mathcal{H}}}, \check{\delta}_{\widehat{\mathcal{H}}}, \widehat{\Sigma}_{\check{\Phi}_{\widehat{\mathcal{H}}}}^{\text {ord }}}
$$


(see (7.1.2.12), (7.1.2.13), (7.1.2.14) and (7.1.2.16) of [44]). The schemes

$$
\underset{\mathcal{T}_{n,(i), U_{g}^{p, \prime}\left(N_{1}, N_{2}\right), \Sigma(g)_{0}}^{(m), \text { rd }+}}{\rightarrow} \mathcal{A}_{n,(i), U_{g}^{p, '}\left(N_{1}, N_{2}\right)}^{(m), \text { ord }+}
$$

of this paper (see Sect. 4.4) are identified with

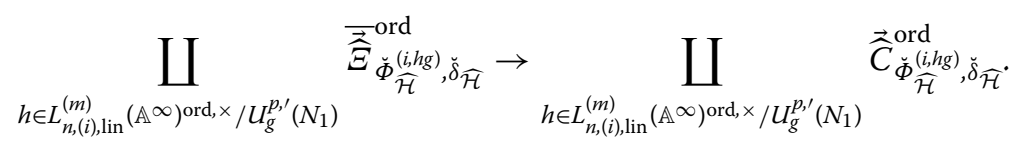

Moreover, $\mathcal{T}_{n,(i), U_{g}^{p, \prime}\left(N_{1}, N_{2}\right), \Sigma(g)_{0}}^{(m),+ \text { id }+, \wedge}$ identified with

$$
\coprod_{h \in L_{n,(i), \operatorname{lin}}^{(m)}\left(\mathbb{A}^{\infty}\right)^{\text {ord }, \times} / U_{g}^{p, \prime}\left(N_{1}\right)}{\overrightarrow{\widehat{\mathfrak{X}}_{\breve{\Phi}}^{\text {ord }}}}_{\breve{\mathcal{H}}^{(i, h g)}, \breve{\delta}_{\widehat{\mathcal{H}}}}
$$

and $\mathcal{T}_{n,(i), U_{g}^{p,{ }^{\prime, ~}}\left(N_{1}\right), \Sigma(g)_{0}}^{(m), \text { is }}$ identified with

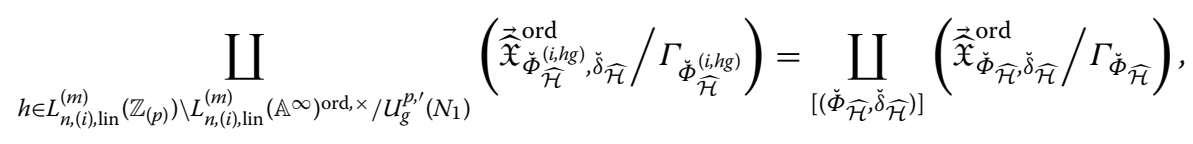

where the second disjoint union is over cusp labels with underlying $\check{Z}_{\widehat{\mathcal{H}}}$ equal to $\mathrm{Z}_{\widehat{\mathcal{H}}}^{(i, \widehat{g})}$ for a fixed $\widehat{g}$ in $\widehat{\mathrm{G}}\left(\mathbb{A}^{\infty, p}\right) \times \widehat{\mathrm{P}}_{\mathrm{D}}^{\text {ord }}\left(\mathbb{Z}_{p}\right)$ corresponding to $g \in G_{n}^{(m)}\left(\mathbb{A}^{\infty}\right)^{\text {ord }, \times}$. (Again $\widehat{\mathcal{H}}=$ $U^{p}\left(N_{1}, N_{2}\right)$.)

For each $\kappa=\left(\widehat{\mathcal{H}}, \widehat{\Sigma}^{\text {ord }}\right) \in \mathbf{K}_{Q, \mathcal{H}}^{\text {ord,++}}$, we have a smooth quasi-projective scheme

$$
\overrightarrow{\mathrm{N}}_{\kappa}^{\text {ord,tor }}
$$

containing $\overrightarrow{\mathrm{N}}^{\text {ord }}$ (of Sect. B.14, which we will henceforth write as $\overrightarrow{\mathrm{N}}_{\kappa}^{\text {ord }}$ to emphasize the dependence on $\widehat{\mathcal{H}}$ ) as an open fibrewise dense subscheme. The scheme $\overrightarrow{\mathrm{N}}_{\kappa}^{\text {ord,tor }}$ is called a partial toroidal compactification of $\overrightarrow{\mathrm{N}}_{\kappa}^{\text {ord }}$ (see theorem 7.1.4.1 of [44]). Its characteristic zero fibre $\overrightarrow{\mathrm{N}}_{\kappa}^{\text {ord,tor }} \otimes_{\mathbb{Z}} \mathbb{Q}$ is an open subscheme of $\overrightarrow{\mathrm{N}}_{\kappa^{\prime}}^{\text {tor }}$ for any $\kappa^{\prime}=(\widehat{\mathcal{H}}, \widehat{\Sigma}) \in \mathbf{K}_{Q, \mathcal{H}}^{++}$ extending $\kappa=\left(\widehat{\mathcal{H}}, \widehat{\Sigma}^{\text {ord }}\right) \in \mathbf{K}_{Q, \mathcal{H}}^{\text {ord,++}}$, which is identified with the union of the strata $\widehat{Z}_{\left[\left(\check{\Phi}_{\widehat{\mathcal{H}}}, \check{\delta}_{\widehat{\mathcal{H}}}, \widehat{\rho}\right)\right]}$ indexed by equivalence classes $\left[\left(\check{\Phi}_{\widehat{\mathcal{H}}}, \breve{\delta}_{\widehat{\mathcal{H}}}, \widehat{\rho}\right)\right]$ whose underlying cusp labels $\left[\left(\breve{\Phi}_{\widehat{\mathcal{H}}}, \breve{\delta}_{\widehat{\mathcal{H}}}\right)\right]$ are ordinary (see theorem 7.1.4.1(6) of [44]). When $U^{p}\left(N_{1}, N_{2}\right)=\widehat{\mathcal{H}}$, and when $\left(U^{p}\left(N_{1}, N_{2}\right), \Sigma\right) \in \mathcal{J}_{n}^{(m), \text { tor }}$ is induced by $\kappa^{\prime}=(\widehat{\mathcal{H}}, \widehat{\Sigma})$ as in Sect. B.7, the partial toroidal compactification

$$
\overrightarrow{\mathrm{N}}_{\kappa}^{\text {ord }} \hookrightarrow \overrightarrow{\mathrm{N}}_{\kappa}^{\text {ord,tor }} \cup \mathrm{N}_{\kappa^{\prime}}^{\text {tor }}
$$

(by gluing along their common open subscheme described above) is the

$$
\mathcal{A}_{n, U^{p}\left(N_{1}, N_{2}\right)}^{(m), \text { ord }} \hookrightarrow \mathcal{A}_{n, U^{p}\left(N_{1}, N_{2}\right), \Sigma}^{(m), \text { ord }}
$$

in this paper (see Sect. 5.3). In this case, the $\left(U^{p}\left(N_{1}\right), \Sigma^{\text {ord }}\right) \in \mathcal{J}_{n}^{(m) \text {,tor,ord }}$ induced by $\left(U^{p}\left(N_{1}, N_{2}\right), \Sigma\right)$ is induced by the $\kappa=\left(\widehat{\mathcal{H}}, \widehat{\Sigma}^{\text {ord }}\right)$ induced by $\kappa^{\prime}=(\widehat{\mathcal{H}}, \widehat{\Sigma})$. The partial 
toroidal compactifications $\overrightarrow{\mathrm{N}}_{\kappa}^{\text {ord }} \hookrightarrow \overrightarrow{\mathrm{N}}_{\kappa}^{\text {ord,tor }} \cup \mathrm{N}_{\kappa^{\prime}}^{\text {tor }}$ are compatible with the actions of $\widehat{\mathrm{G}}\left(\mathbb{A}^{\infty, p}\right) \times \widehat{\mathrm{P}}_{\mathrm{D}}^{\text {ord }}\left(\mathbb{Z}_{p}\right)$ and of the element of $\widehat{\mathrm{P}}_{\mathrm{D}}^{\text {ord }}\left(\mathbb{Q}_{p}\right)$ corresponding to $\varsigma_{p}$ (see theorem 7.1.4.1, (4) and (6), of [44]), and they are compatible with the actions of $G_{n}^{(m)}\left(\mathbb{A}^{\infty}\right)^{\text {ord }}$ on the partial toroidal compactifications $\mathcal{A}_{n, U^{p}\left(N_{1}, N_{2}\right)}^{(m), \text { ord }} \hookrightarrow \mathcal{A}_{n, U^{p}\left(N_{1}, N_{2}\right), \Sigma}^{(m) \text {, }}$.

The partial toroidal compactification $\overrightarrow{\mathrm{N}}_{\kappa}^{\text {ord,tor }}$ admits a stratification by locally closed subschemes

$$
\overrightarrow{\mathrm{N}}_{\kappa}^{\text {ord,tor }}=\coprod_{\left[\left(\check{\Phi}_{\widehat{\mathcal{H}}}, \check{\delta}_{\widehat{\mathcal{H}}}, \widehat{\rho}\right)\right]} \overrightarrow{\mathrm{Z}}_{\left[\left(\check{\Phi}_{\widehat{\mathcal{H}}}, \check{\delta}_{\widehat{\mathcal{H}}}, \widehat{\rho}\right)\right]}^{\text {ord }}
$$

(see theorem 7.1.4.1(1) of [44]) indexed by equivalence classes $\left[\left(\check{\Phi}_{\widehat{\mathcal{H}}}, \breve{\delta}_{\widehat{\mathcal{H}}}, \widehat{\rho}\right)\right]$ as in lemma 1.2.4.42 of [44] whose underlying cusp labels $\left[\left(\breve{\Phi}_{\widehat{\mathcal{H}}}, \breve{\delta}_{\widehat{\mathcal{H}}}\right)\right]$ are ordinary. Each stratum $\overrightarrow{\mathrm{Z}}_{\left[\left(\breve{\Phi}_{\widehat{\mathcal{H}}}, \check{\mathcal{X}}_{\widehat{\mathcal{H}}}, \widehat{\rho}\right)\right]}^{\text {ord }}$ is canonically isomorphic to $\overrightarrow{\widehat{\Xi}}_{\mathscr{\Phi}_{\widehat{\mathcal{H}}}, \breve{\delta}_{\widehat{\mathcal{H}}}, \widehat{\rho}}^{\text {ord }}$, and the formal completion of $\overrightarrow{\mathrm{N}}_{\kappa}^{\text {ord,tor }}$ along the union of the strata $\overrightarrow{\mathrm{Z}}_{\left[\left(\breve{\Phi}_{\widehat{\mathcal{H}}}, \check{\delta}_{\widehat{\mathcal{H}}}, \widehat{\rho}\right)\right]}^{\text {ord }}$ labelled by equivalence classes $\left[\left(\breve{\Phi}_{\widehat{\mathcal{H}}}, \breve{\delta}_{\widehat{\mathcal{H}}}, \widehat{\rho}\right)\right]$ with the same underlying ordinary cusp label $\left[\left(\breve{\Phi}_{\widehat{\mathcal{H}}}, \breve{\delta}_{\widehat{\mathcal{H}}}\right)\right]$ is canonically isomorphic to $\overrightarrow{\hat{\mathfrak{X}}}_{\bar{\Phi}_{\widehat{\mathcal{H}}}, \breve{\delta}_{\widehat{\mathcal{H}}}}^{\text {ord }} / \Gamma_{\check{\Phi}_{\widehat{\mathcal{H}}}}$ (see theorem 7.1.4.1(1) of [44]).

When $U^{p}\left(N_{1}, N_{2}\right)=\widehat{\mathcal{H}}$, and when $\left(U^{p}\left(N_{1}\right), \Sigma\right) \in \mathcal{J}_{n}^{(m) \text {,tor,ord }}$ is induced by $\kappa=$ $\left(\widehat{\mathcal{H}}, \widehat{\Sigma}^{\text {ord }}\right)$ as above, the formal completion of $\overrightarrow{\mathrm{N}}_{\kappa}^{\text {ord,tor }}$ along the union of all strata $\overrightarrow{\mathrm{Z}}_{\left[\left(\breve{\Phi}_{\widehat{\mathcal{H}}}, \check{\delta}_{\widehat{\mathcal{H}}}, \widehat{\rho}\right)\right]}^{\text {ord }}$ labelled by equivalence classes $\left[\left(\breve{\Phi}_{\widehat{\mathcal{H}}}, \breve{\delta}_{\widehat{\mathcal{H}}}, \widehat{\rho}\right)\right]$ with underlying $\check{Z}_{\widehat{\mathcal{H}}}$ equal to $\check{Z}_{\widehat{\mathcal{H}}}^{(i, \widehat{g})}$ for some $\widehat{g} \in \widehat{\mathrm{G}}\left(\mathbb{A}^{\infty, p}\right) \times \widehat{\mathrm{P}}_{\mathrm{D}}^{\text {ord }}\left(\mathbb{Z}_{p}\right)$ is

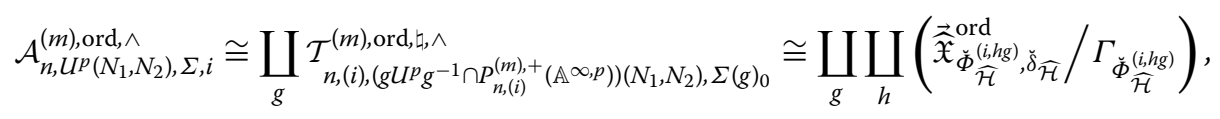

where the indices $g$ and $h$ run over $P_{n,(i)}^{(m),+}\left(\mathbb{A}^{\infty}\right)^{\text {ord, } \times} \backslash G_{n}^{(m)}\left(\mathbb{A}^{\infty}\right)^{\text {ord, } \times} / U^{p}\left(N_{1}, N_{2}\right)$ and $L_{n,(i), \text { lin }}^{(m)}\left(\mathbb{Z}_{(p)}\right) \backslash L_{n,(i), \text { lin }}^{(m)}\left(\mathbb{A}^{\infty}\right)^{\text {ord, } \times} / U_{g}^{p, \prime}\left(N_{1}\right)$, respectively, in this paper (see Sect. 5.3).

If $\kappa \in \mathbf{K}_{Q, \mathcal{H}, \Sigma^{\text {ord }}}^{\mathrm{ord},+}$, then the canonical morphism

$$
\overrightarrow{\mathrm{N}}_{\kappa}^{\text {ord }} \rightarrow \overrightarrow{\mathrm{M}}_{\mathcal{H}}^{\text {ord }}
$$

extends to a canonical log smooth morphism

$$
\overrightarrow{\mathrm{N}}_{\kappa}^{\text {ord,tor }} \rightarrow \overrightarrow{\mathrm{M}}_{\mathcal{H}, \Sigma^{\text {ord }}}^{\text {ord,tor }}
$$

(see theorem 7.1.4.1(2) of [44]). When $U^{p}\left(N_{1}, N_{2}\right)=\widehat{\mathcal{H}}$ and $\left(U^{\prime}\right)^{p}\left(N_{1}^{\prime}, N_{2}^{\prime}\right)=\mathcal{H}$, and when $\left(U^{p}\left(N_{1}, N_{2}\right), \Sigma\right) \in \mathcal{J}_{n}^{(m), \text { tor }}$ and $\left(\left(U^{\prime}\right)^{p}\left(N_{1}^{\prime}, N_{2}^{\prime}\right), \Delta\right) \in \mathcal{J}_{n}^{\text {tor }}$ are induced by some $\kappa^{\prime}=(\widehat{\mathcal{H}}, \widehat{\Sigma})$ and $(\mathcal{H}, \Sigma)$ extending $\kappa=\left(\widehat{\mathcal{H}}, \widehat{\Sigma}^{\text {ord }}\right)$ and $\left(\mathcal{H}, \Sigma^{\text {ord }}\right)$, respectively, we have

$$
\left(U^{p}\left(N_{1}, N_{2}\right), \Sigma\right) \geq\left(\left(U^{\prime}\right)^{p}\left(N_{1}^{\prime}, N_{2}^{\prime}\right), \Delta\right)
$$

(see Sect. 5.2 in this paper) and the union of the above morphism with

$$
\mathrm{N}_{\kappa^{\prime}}^{\text {tor }} \rightarrow \mathrm{M}_{\mathcal{H}, \Sigma}^{\text {tor }}
$$

(see Sect. B.7) is the log smooth morphism 


$$
\mathcal{A}_{n, U^{p}\left(N_{1}, N_{2}\right), \Sigma}^{(m), \text { ord }} \rightarrow \mathcal{X}_{n,\left(U^{\prime}\right)^{p}\left(N_{1}^{\prime}, N_{2}^{\prime}\right), \Delta}^{\text {ord }}
$$

in this paper (see Sect. 5.3).

These identifications are all Hecke equivariant (see theorem 7.1.4.1(4) of [44]).

The formal completion of $\overrightarrow{\mathrm{N}}_{\kappa}^{\text {ord,tor }}$ along

$$
\overrightarrow{\mathrm{N}}_{\kappa}^{\text {ord,tor }} \otimes_{\mathbb{Z}} \mathbb{F}_{p}
$$

is denoted

$$
\overrightarrow{\mathfrak{N}}_{\kappa}^{\text {ord,tor }}
$$

in [44] (see definition 3.4.4.2). When $U^{p}\left(N_{1}, N_{2}\right)=\widehat{\mathcal{H}}$, and when $\left(U^{p}\left(N_{1}\right), \Sigma\right) \in$ $\mathcal{J}_{n}^{(m), \text { tor,ord }}$ is induced by $\kappa=\left(\widehat{\mathcal{H}}, \widehat{\Sigma}^{\text {ord }}\right)$, these are the

$$
\bar{A}_{n, U^{p}\left(N_{1}\right), \Sigma}^{(m), \text { ord }}
$$

and

$$
\mathfrak{A}_{n, U^{p}\left(N_{1}\right), \Sigma}^{\text {ord }}
$$

respectively, in this paper (see Sect. 5.3). (Their independence of $N_{2}=r_{0}$ is explained in theorem 7.1.4.1(4h) of [44].) For the Hecke actions on these formal schemes, see theorem 7.1.4.1, (4g)-(4j), of [44].

\section{B.16 Automorphic bundles in mixed characteristics}

We first recall how some of the notation of [44] specializes in our case. In the beginning of section 8.1.1 of [44], we can take:

(1) $\quad R_{0}=\mathbb{Z}_{(p)}$ and $\tilde{R}_{0}=\mathbb{Z}_{p}$.

(2) $\mathrm{Gr}_{\mathrm{D}, 0}^{-1}=\mathrm{Gr}_{\mathrm{D}^{\#}, 0}^{-1}=\mathcal{O}_{F,(p)}^{n}$ with its canonical $\mathcal{O} \otimes_{\mathbb{Z}} \mathbb{Z}_{(p)}$-module structure, with $\phi_{\mathrm{D}, 0}^{-1}$ : $\mathrm{Gr}_{\mathrm{D}, 0}^{-1} \stackrel{\sim}{\rightarrow} \mathrm{Gr}_{\mathrm{D}^{*}, 0}^{-1}$ given by the identity morphism.

Then we have

$$
\operatorname{Gr}_{\mathrm{D}, 0}^{0}:=\operatorname{Hom}_{R_{0}}\left(\mathrm{Gr}_{\mathrm{D}^{\sharp}, 0}^{-1}, R_{0}\right) \cong\left(\operatorname{Diff}_{\mathcal{O}_{F,(p)} / \mathbb{Z}_{(p)}}^{-1}\right)^{n}
$$

and, for each $\mathbb{Z}_{(p)}$-algebra $R$,

$$
\mathrm{M}_{\mathrm{D}, 0}^{\mathrm{ord}}(R):=\mathrm{GL}_{\mathcal{O} \otimes_{\mathbb{Z}} R}\left(\mathrm{Gr}_{\mathrm{D}, 0}^{-1} \otimes_{\mathbb{Z}_{(p)}} R\right) \times \mathbf{G}_{\mathrm{m}}(R) \cong \mathrm{GL}_{\mathcal{O} \otimes_{\mathbb{Z}} R}\left(\operatorname{Gr}_{\mathrm{D}, 0}^{0} \otimes_{\mathbb{Z}_{(p)}} R\right) \times \mathbf{G}_{\mathrm{m}}(R)
$$

which is canonically isomorphic to the one in definition 8.1.1.1 of [44] because of the simpler setting here. (We will not need the other groups $G_{\mathrm{D}, 0}^{\text {ord }}(R)$ and $\mathrm{P}_{\mathrm{D}, 0}^{\text {ord }}(R)$ in this paper.) This can be canonically identified with the group $L_{n,(n)}(R)$ in this paper (see Sect. 1.2). If $R$ is a $\mathbb{Q}$-algebra, then $\mathrm{M}_{\mathrm{D}, 0}^{\mathrm{ord}}(R) \cong \mathrm{M}_{0}(R)$ (see Sect. B.8). 
Let $\mathcal{H}$ be any open compact subgroup of $\mathrm{G}(\hat{\mathbb{Z}})$ of the form $\mathcal{H}=\mathcal{H}^{p} \mathcal{H}_{p}$, where $\mathcal{H}^{p}$ is a neat open compact subgroup of $G\left(\hat{\mathbb{Z}}^{p}\right)$, and where $\mathcal{H}_{p}=\mathcal{U}_{p, 1,0}\left(p^{r_{1}}, p^{r_{0}}\right)$ for some integers $0 \leq r_{1} \leq r_{0}$, so that $\overrightarrow{\mathrm{M}}_{\mathcal{H}}^{\text {ord }}$ and $\overrightarrow{\mathrm{M}}_{\mathcal{H}}^{\text {ord,min }}$ are defined over Spec $\mathbb{Z}_{(p)}$ as in Sects. B.10 and B.12. Then the tautological abelian scheme $A$ over $\vec{M}_{\mathcal{H}}^{\text {ord }}$ defines a locally free sheaf

$$
\underline{\mathrm{Lie}}_{A / \overrightarrow{\mathrm{M}}_{\mathcal{H}}^{\mathrm{ord}}}^{\vee}:=e_{A}^{*} \Omega_{A / \overrightarrow{\mathrm{M}}_{\mathcal{H}}^{\text {ord }}}^{1}
$$

(where $e_{A}$ denotes the identity section), which is the

$$
\Omega_{n, U^{p}\left(N_{1}, N_{2}\right)}^{\text {ord }}
$$

in this paper (see Sect. 3.4.3), with $U^{p}\left(N_{1}, N_{2}\right)=\mathcal{H}$. We can similarly define $\underline{\mathrm{Lie}}_{A^{\vee} / \overrightarrow{\mathrm{M}}_{\mathcal{H}}^{\mathrm{ord}}}$. The action of $G\left(\mathbb{A}^{\infty, p}\right) \times \mathrm{P}_{\mathrm{D}}^{\text {ord }}\left(\mathbb{Z}_{p}\right)$ (resp. of the element of $\mathrm{P}_{\mathrm{D}}^{\text {ord }}\left(\mathbb{Q}_{p}\right)$ corresponding to $\varsigma_{p}$ ) on $\left\{\vec{M}_{\mathcal{H}}^{\text {ord }}\right\}_{\mathcal{H}}$ is defined by respecting their tautological abelian schemes up to canonical $\mathbb{Z}_{(p)}^{\times}$-isogenies (resp. $\mathbb{Q}^{\times}$-isogenies). Therefore, such an action induces actions on $\left\{\underline{\mathrm{Lie}}_{A / \overrightarrow{\mathrm{M}}_{\mathcal{H}}^{\vee \text { ord }}}^{\vee}\right\}_{\mathcal{H}}$ and $\left\{\underline{\mathrm{Lie}}_{A^{\vee} / \mathrm{M}_{\mathcal{H}}}^{\vee}\right\}_{\mathcal{H}}$ covering the one on $\left\{\overrightarrow{\mathrm{M}}_{\mathcal{H}}^{\text {ord }}\right\}_{\mathcal{H}}$, which are compatible with the isomorphisms

$$
\lambda^{*}: \underline{\mathrm{Lie}}_{A^{\vee} / \overrightarrow{\mathrm{M}}_{\mathcal{H}}^{\text {ord }}}(1) \stackrel{\sim}{\rightarrow} \underline{\mathrm{Lie}}_{A / \overrightarrow{\mathrm{M}}_{\mathcal{H}}^{\mathrm{ord}}}
$$

induced by the tautological polarizations $\lambda: A \rightarrow A^{\vee}$. Here the formal Tate twist requires (as before) the Hecke action to be twisted by the similitude character $v$, which corresponds to the tensor product with

$$
\Xi_{n, U}^{\text {ord }}=\mathcal{O}_{\mathcal{X}_{n, U}^{\text {ord }}}(\|v\|)
$$

in this paper (see Sect. 3.4.3).

Let $\Sigma^{\text {ord }}$ be a compatible collection of admissible projective smooth rational polyhedral cone decompositions for $\vec{M}_{\mathcal{H}}^{\text {ord }}$, so that $\vec{M}_{\mathcal{H}, \Sigma^{\text {ord }}}^{\text {ord tor }}$ is defined over Spec $\mathbb{Z}_{(p)}$ as in Sect. B.13. Then the tautological semi-abelian scheme $G$ over $\vec{M}_{\mathcal{H}, \Sigma}^{\text {ord,tor }}$ defines a locally free sheaf

$$
\underline{\mathrm{Lie}}_{G / \overrightarrow{\mathrm{M}}_{\mathcal{H}, \Sigma}^{\text {ord tor }}}^{\vee}:=e_{G}^{*} \Omega_{G / \overrightarrow{\mathrm{M}}_{\mathcal{H}, \Sigma^{\text {ord }}}^{\text {ord,tor }}}^{1}
$$

(where $e_{G}$ denotes the identity section), which is

$$
\left.\Omega_{n, U, \Delta}^{\text {ord }}\right|_{\vec{M}_{\mathcal{H}, \Sigma}^{\text {ord tor }}}
$$

in this paper (see Sect. 5.4), with $U^{p}\left(N_{1}, N_{2}\right)=\mathcal{H}$, and with $\Delta$ induced by any extension $\Sigma$ of $\Sigma^{\text {ord }}$ as in Sect. B.13. We can similarly define $\underline{L i e}_{G^{\vee} / \overrightarrow{\mathrm{M}}_{\mathcal{H}, \Sigma}^{\text {ord tor }}}^{\text {ord where }}$ $G^{\vee}$ denotes the tautological dual semi-abelian scheme over $\overrightarrow{\mathrm{M}}_{\mathcal{H}, \Sigma^{\text {ord }}}^{\text {ord }}$ extending $A^{\vee}$. The action of $\mathrm{G}\left(\mathbb{A}^{\infty, p}\right) \times \mathrm{P}_{\mathrm{D}}^{\text {ord }}\left(\mathbb{Z}_{p}\right)$ (resp. of the element of $\mathrm{P}_{\mathrm{D}}^{\text {ord }}\left(\mathbb{Q}_{p}\right)$ corresponding to $\varsigma_{p}$ ) on $\left\{\vec{M}_{\mathcal{H}, \Sigma^{\text {ord }}}^{\text {ord,tor }}\right\}_{\left(\mathcal{H}, \Sigma^{\text {ord }}\right)}$ is defined by respecting their tautological semi-abelian schemes up to canonical $\mathbb{Z}_{(p)}^{\times}$-isogenies (resp. $\mathbb{Q}^{\times}$-isogenies). Therefore, such an action induces actions on $\left\{\underline{\mathrm{Lie}}_{G / \overrightarrow{\mathrm{M}}_{\mathcal{H}, \Sigma^{\text {ord }}}^{\mathrm{ord}}}^{\text {ord,or }}\right\}_{\left(\mathcal{H}, \Sigma^{\text {ord }}\right)}$ and $\left\{\underline{\mathrm{Lie}}_{G^{\vee} / \overrightarrow{\mathrm{M}}_{\mathcal{H}, \Sigma^{\text {ord }}}^{\text {ord tor }}}\right\}_{\left(\mathcal{H}, \Sigma^{\text {ord }}\right)}$ covering the one on $\left\{\overrightarrow{\mathrm{M}}_{\mathcal{H}, \Sigma^{\text {ord }}}^{\text {ord,tor }}\right\}_{\left(\mathcal{H}, \Sigma^{\text {ord }}\right)}$, which are compatible with the isomorphisms 


$$
\lambda^{*}: \underline{\mathrm{Lie}}_{G^{\vee}}^{\vee} / \overrightarrow{\mathrm{M}}_{\mathcal{H}, \Sigma^{\text {ord }}}^{\text {ordtor }}(1) \stackrel{\sim}{\rightarrow} \underline{\mathrm{Lie}}_{G / \overrightarrow{\mathrm{M}}_{\mathcal{H}, \Sigma^{\text {ord }}}^{\vee \text { ord tor }}}^{\vee}
$$

induced by the tautological polarizations $\lambda: G \rightarrow G^{\vee}$. Here the formal Tate twist requires (as before) the Hecke action to be twisted by the similitude character $v$, which corresponds to the tensor product with

$$
\Xi_{n, U, \Delta}^{\text {ord }}=\mathcal{O}_{\mathcal{X}_{n, U, \Delta}^{\text {ord }}}(\|v\|)
$$

in this paper (see Sect. 5.4).

Then we have the principal $\mathrm{M}_{\mathrm{D}, 0}^{\text {ord }}$-bundle

$$
\overrightarrow{\mathcal{E}}_{\mathrm{M}_{\mathrm{D}, 0}^{\text {ord }}}^{\text {ord }}:=\underline{\operatorname{Isom}}_{\mathcal{O} \otimes_{\mathbb{Z}} \mathscr{O}_{\overrightarrow{\mathcal{M}}}^{\text {ord }}}\left(\left(\underline{\operatorname{Lie}}_{A^{\vee} / \overrightarrow{\mathrm{M}}_{\mathcal{H}}^{\vee \text { ord }}}^{\vee}(1), \mathscr{O}_{\overrightarrow{\mathrm{M}}_{\mathcal{H}}^{\text {ord }}}^{\text {ord }}(1)\right),\left(\operatorname{Gr}_{\mathrm{D}, 0}^{0} \otimes_{\mathbb{Z}_{(p)}} \mathscr{O}_{\overrightarrow{\mathrm{M}}_{\mathcal{H}}}^{\text {ord }}, \mathscr{O}_{\overrightarrow{\mathrm{M}}_{\mathcal{H}}^{\text {ord }}}(1)\right)\right),
$$

which is an $\mathrm{M}_{\mathrm{D}, 0}^{\text {ord }}$-torsor over $\overrightarrow{\mathrm{M}}_{\mathcal{H}}^{\text {ord }}$ (see definition 8.1.2.4 and lemma 8.1.2.6 of [44]), which canonically extends (as an $\mathrm{M}_{\mathrm{D}, 0}^{\text {ord }}$-torsor) to a principal $\mathrm{M}_{\mathrm{D}, 0}^{\text {ord }}$-bundle

$$
\begin{aligned}
& \overrightarrow{\mathcal{E}}_{\mathrm{M}_{0}}^{\text {ord,can }}:=\underline{\operatorname{Isom}}_{\mathcal{O} \otimes_{\mathbb{Z}} \mathscr{O}_{\overrightarrow{\mathrm{M}}_{\mathcal{H}, \Sigma^{\text {ord }}}^{\text {ord,tor }}}}\left(\left({\underline{\mathrm{Lie}^{\vee}}}_{G^{\vee} / \overrightarrow{\mathrm{M}}_{\mathcal{H}, \Sigma^{\text {ord }}}^{\text {ord,tor }}}(1), \mathscr{O}_{\overrightarrow{\mathrm{M}}_{\mathcal{H}, \Sigma^{\text {ord }}}^{\text {ord,tor }}}(1)\right),\right. \\
& \left.\left(\operatorname{Gr}_{\mathrm{D}, 0}^{0} \otimes_{\mathbb{Z}_{(p)}} \mathscr{O}_{\overline{\mathrm{M}}_{\mathcal{H}, \Sigma}^{\text {ord,tor }}}, \mathscr{O}_{\overrightarrow{\mathrm{M}}_{\mathcal{H}, \Sigma^{\text {ord }}}^{\text {ord,tor }}}(1)\right)\right)
\end{aligned}
$$

over $\vec{M}_{\mathcal{H}, \Sigma^{\text {ord }}}^{\text {ord, }}$ (see (8.1.3.11) and lemma 8.1.3.12 of [44]). These are the restrictions (to $\vec{M}_{\mathcal{H}}^{\text {ord }}$ and $\vec{M}_{\mathcal{H}, \Sigma^{\text {ord }}}^{\text {ord,tor }}$, respectively) of the

$$
\mathcal{E}_{U^{p}\left(N_{1}, N_{2}\right)}^{\text {ord }} \quad \text { and } \quad \mathcal{E}_{U^{p}\left(N_{1}, N_{2}\right), \Delta}^{\text {ord,can }}
$$

in this paper (see Sects. 3.4.3, 5.4), with $U^{p}\left(N_{1}, N_{2}\right)=\mathcal{H}$, and with $\Delta$ induced by some extension $\Sigma$ of $\Sigma^{\text {ord }}$ as in Sect. B.13.

For each $\mathbb{Z}_{(p)}$-algebra $R$, we denote by $\operatorname{Rep}_{R}\left(\mathrm{M}_{\mathrm{D}, 0}^{\text {ord }}\right)$ the category of $R$-modules with algebraic actions of $\mathrm{M}_{\mathrm{D}, 0}^{\text {ord }} \otimes_{\mathbb{Z}_{(p)}} R$ (see definition 8.1.2.7 of [44]). Then we also define, for each $W \in \operatorname{Rep}_{R}\left(\mathrm{M}_{\mathrm{D}, 0}^{\text {ord }}\right)$ that is locally free of finite rank as an $R$-module, the automorphic bundle

$$
\overrightarrow{\mathcal{E}}_{\mathrm{M}_{\mathrm{D}, 0}^{\text {ord }}, R}^{\text {ord }}(W):=\left(\overrightarrow{\mathcal{E}}_{\mathrm{M}_{\mathrm{D}, 0}^{\text {ord }}}^{\text {ord }} \otimes_{\mathbb{Z}_{(p)}} R\right) \times{ }^{\left(\mathrm{M}_{\mathrm{D}, 0}^{\text {ord }} \otimes_{\mathbb{Z}_{(p)}} R\right)} W
$$

over $\vec{M}_{\mathcal{H}}^{\text {ord }} \otimes_{\mathbb{Z}_{(p)}} R$ (see definition 8.1.2.8 of [44]), which extends to the canonical extension

$$
\overrightarrow{\mathcal{E}}_{\mathrm{M}_{\mathrm{D}, 0}^{\text {ord }}, R}^{\text {ord, can }}(W):=\left(\overrightarrow{\mathcal{E}}_{\mathrm{M}_{\mathrm{D}, 0}^{\text {ord }}}^{\text {ord,can }} \otimes_{\mathbb{Z}_{(p)}} R\right) \times{ }^{\left(\mathrm{M}_{\mathrm{D}, 0}^{\text {ord }} \otimes_{\mathbb{Z}_{(p)}} R\right)} W
$$

and the subcanonical extension

$$
\overrightarrow{\mathcal{E}}_{\mathrm{M}_{\mathrm{D}, 0}^{\text {ord }}, R}^{\text {ord,sub }}(W):=\overrightarrow{\mathcal{E}}_{\mathrm{M}_{\mathrm{D}, 0}^{\text {ord }, R}}^{\text {ord,can }}(W) \otimes_{\mathcal{M}_{\mathrm{M}_{\mathcal{H}} \text { ord,tor }}^{\text {ord }}} \mathscr{I}_{\overrightarrow{\mathrm{D}}_{\infty}^{\text {ord }}}
$$


over $\overrightarrow{\mathrm{M}}_{\mathcal{H}, \Sigma \text { ord }}^{\text {ord,tor }} \otimes_{\mathbb{Z}_{(p)}} R$ (see definition 8.1.3.13 of [44]), where $\mathscr{I}_{\overrightarrow{\mathrm{D}}_{\infty}^{\text {ord }}}$ is the $\mathscr{O}_{\overrightarrow{\mathrm{M}}_{\mathcal{H}, \Sigma^{\text {ord }}}^{\text {ord,tor }}}$-ideal defining the boundary divisor $\vec{D}_{\infty}^{\text {ord }}:=\vec{M}_{\mathcal{H}, \Sigma^{\text {ord }}}^{\text {ord }}-\vec{M}_{\mathcal{H}}^{\text {ord }}$ (with its canonical reduced subscheme structure). These are restrictions of the vector bundles

$$
\mathcal{E}_{U^{p}\left(N_{1}, N_{2}\right), \rho}^{\text {ord }}, \quad \mathcal{E}_{U^{p}\left(N_{1}, N_{2}\right), \Delta, \rho}^{\text {ord,can }}, \quad \text { and } \quad \mathcal{E}_{U^{p}\left(N_{1}, N_{2}\right), \Delta, \rho}^{\text {ord,sub }}
$$

in this paper (see Sects. 3.4.3, 5.4), with $U^{p}\left(N_{1}, N_{2}\right)=\mathcal{H}$, and with $\Delta$ induced by some extension $\Sigma$ of $\Sigma^{\text {ord }}$ as in Sect. B.13. The bundles $\overrightarrow{\mathcal{E}}_{\mathrm{M}_{\mathrm{D}, 0}^{\text {ord }}, R}^{\text {ord }}(W)$ and $\overrightarrow{\mathcal{E}}_{\mathrm{M}_{\mathrm{D}, 0}^{\text {ord }}, R}^{\text {ord,can }}(W)$ admit compatible actions of $\mathrm{G}\left(\mathbb{A}^{\infty, p}\right) \times \mathrm{P}_{\mathrm{D}}^{\text {ord }}\left(\mathbb{Z}_{p}\right)$ (see proposition 8.1.4.1 of [44]), which are compatible with the compatible actions of $G_{n}\left(\mathbb{A}^{\infty}\right)^{\text {ord, } \times}$ on $\mathcal{E}_{U^{p}\left(N_{1}, N_{2}\right), \rho}^{\text {ord }}$ and $\mathcal{E}_{U^{p}\left(N_{1}, N_{2}\right), \Delta, \rho}^{\text {ord,can }}$, covering the ones on their respective base schemes. The base extensions of these bundles from $\mathbb{Z}_{(p)}$ to $\mathbb{Q}$ are canonically isomorphic to restrictions of the corresponding bundles introduced in Sect. B.8.

Beyond the ordinary loci, we still have the tautological abelian scheme $\vec{A}$ and the principal polarization $\vec{\lambda}: \vec{A} \stackrel{\sim}{\rightarrow} \vec{A}^{\vee}$ over $\vec{M}_{\mathcal{H}}$ (see proposition 2.2.1.1 of [44]). Hence, we can still define the principal bundle

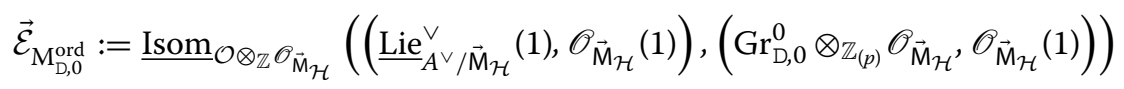

(see (8.3.1.2) and lemma 8.3.1.4 of [44]), for any $\mathcal{H}$, and accordingly the automorphic bundle

$$
\overrightarrow{\mathcal{E}}_{\mathrm{M}_{\mathrm{D}, 0}^{\text {ord }}, R}(W):=\left(\overrightarrow{\mathcal{E}}_{\mathrm{M}_{\mathrm{D}, 0}^{\text {ord }}} \otimes_{\mathbb{Z}_{(p)}} R\right) \times{ }^{\left(\mathrm{M}_{\mathrm{D}, 0}^{\text {ord }} \otimes_{\mathbb{Z}_{(p)}} R\right)} W
$$

over $\vec{M}_{\mathcal{H}}$ (see definition 8.3.2.1 of [44]), for any $W \in \operatorname{Rep}_{R}\left(\mathrm{M}_{\mathrm{D}, 0}^{\text {ord }}\right)$ that is locally free of finite rank as an $R$-module.

For simplicity, assume that $R$ is just $\mathbb{Z}_{(p)}$. Then we can still define some canonical and subcanonical extensions

$$
\overrightarrow{\mathcal{E}}_{\mathrm{M}_{\mathrm{D}, 0}^{\text {ord }}, R}^{\mathrm{can}, \min }(W) \quad \text { and } \quad \overrightarrow{\mathcal{E}}_{\mathrm{M}_{\mathrm{D}, 0}^{\text {ord }}, R}^{\mathrm{sub}, \min }(W)
$$

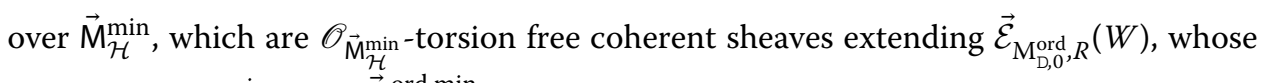
pull-backs to $\mathrm{M}_{\mathcal{H}}^{\min }$ (resp. $\overrightarrow{\mathrm{M}}_{\mathcal{H}}^{\text {ord,min }}$ ) are canonically isomorphic to the push-forwards from $\mathrm{M}_{\mathcal{H}, \Sigma}^{\text {tor }}$ (resp. $\overrightarrow{\mathrm{M}}_{\mathcal{H}, \Sigma^{\text {ord }}}^{\text {ord,tor }}$ ) (for any $\Sigma$ inducing $\Sigma^{\text {ord }}$ ) of the corresponding canonical and subcanonical extensions for the automorphic bundle associated with $W \otimes_{\mathbb{Z}} \mathbb{Q}$ (resp. $W$ ). (See definition 8.3.5.1, lemma 8.3.5.2, corollary 8.3.5.4, lemma 8.3.5.7, and corollary 8.3.5.8 of [44].) The above $\overrightarrow{\mathcal{E}}_{\mathrm{M}_{\mathrm{D}, 0}^{\text {sub, }} \text {,rin }}(W)$ is the

$$
\mathcal{E}_{U^{p}\left(N_{1}, N_{2}\right), \rho}^{\mathrm{sub}}
$$

in lemma 5.5 of this paper, with $U^{p}\left(N_{1}, N_{2}\right)=\mathcal{H}$, with $R=\mathbb{Z}_{(p)}$, and with $W_{\rho}=W$. Such coherent sheaves admit compatible actions of $\mathrm{G}\left(\mathbb{A}^{\infty, p}\right) \times \mathrm{G}\left(\mathbb{Z}_{p}\right)$ (see proposition 8.3.6.5 of [44]), and the identifications in this paragraph are all Hecke equivariant. 
Index of Notations

$\mathcal{A}_{\Delta}^{\text {univ }}, 176$

$\left(\mathcal{A}_{n, U^{p}\left(N_{1}, N_{2}\right), \Sigma}^{(m) \text { ord }}\right)^{0}, 177$

$\mathcal{A}_{U^{p}\left(N_{1}, N_{2}\right), \Sigma}^{(m), 0 r, \dagger}, 206$

$\mathcal{A}_{n,(i), U^{p}\left(N_{1}, N_{2}\right)}^{\text {ord }}, 130$

$\left\{\mathcal{A}_{n, U^{p}\left(N_{1}, N_{2}\right)}^{(m) \text { ord }}\right\}, 101$

$\left[\left(\mathcal{A}^{\text {univ }}, i^{\text {univ }}, \lambda^{\text {univ }},\left[\eta^{\text {univ }}\right]\right)\right], 91$

$\mathcal{A}_{n,(i), U^{p}\left(N_{1}, N_{2}\right)}^{(m), \text { ord }+}, 130$

$\mathcal{A}_{n, U^{p}\left(N_{1}, N_{2}\right), \Sigma}^{(m), \text { ord }}, 175$

$\mathcal{A}_{U^{p}\left(N_{1}\right), \Sigma^{\text {ord }}}^{(m), 207}$

$\mathfrak{A}^{(m), \text { ord }}$

$\mathfrak{A}_{n, U^{p}\left(N_{1}\right), \Sigma^{\text {ord }}}, 178$

$\mathfrak{A}_{U^{p}\left(N_{1}\right)}^{(m), \text { ord }}, 106$

$\alpha_{*} T, 46$

$A_{n}, 16$

$\bar{A}_{n, U^{p}\left(N_{1}\right), \Sigma^{\text {ord }}}^{(m), 178}$

(A, $i), 85$

$A_{n,(i)}, 25$

$A_{n,(i), \text { herm }}, 25$

$(A, i, \lambda), 85$

$\left(A, i, \lambda,\left[\left(\eta_{0}, \eta_{1}\right)\right]\right) g, 90$

$\left(A, i, \lambda,\left[\left(\eta_{0}^{p}, \eta_{1}^{p}, C, \eta_{p}\right)\right]\right) g, 90$

$A_{n,(i), \text { lin }}, 25$

$A^{\perp}, 54$

$A_{n,(i), U}^{+}, 127$

$A_{n, U, \Sigma}^{(m)}, 173$

$\bar{A}_{n, U^{p}(N)}^{(m), \text { ord }}, 106$

$\left(A^{\vee}, i^{\vee}\right), 85$

$A^{\text {univ }}, 173$

$A_{n,(i), U}^{(m),+}, 127$

$A_{n, U, \Sigma, i}^{(m), \wedge}, 174$

$A_{n, U}^{(m)}, 95$

$\left[\left(A^{\text {univ }}, i^{\text {univ }}, \lambda^{\text {univ }},\left[\eta^{\text {univ }}\right]\right)\right], 91$

$A^{\vee}, 53$

$A^{\vee, 0}, 54$

\|\|$_{F}, 10$

||$_{v}, 10$
$B_{n}, 16$

$B_{n}^{1}, 28$

$\mathrm{BC}(\Pi)_{w}, 29$

$B_{G L_{m}}, 28$

$\mathfrak{C}, 34$

$\mathfrak{C}_{=i}, 34$

$\mathfrak{C}^{(m)}, 33$

$\mathfrak{C}_{=i}^{(m)}, 34$

$\mathfrak{C}^{(m)}(W), 32$

$\mathfrak{C}(W), 32$

$\mathfrak{C}^{>0}(W), 33$

$\mathfrak{C}^{\geq 0}(W), 33$

$\mathfrak{C}^{(m),>0}(W), 33$

$\mathfrak{C}^{(m), \geq 0}(W), 33$

$\mathfrak{C}^{(m), \succ 0}(W), 33$

$\mathfrak{C}^{\succ 0}(W), 33$

$C_{n}, 12$

$\mathcal{D}_{F}^{-1}, 10$

$\Delta_{n}, 16$

$\Delta_{(n)}, 25$

$\delta_{P}, 11$

$D^{(s)}, 51$

$D_{S}, 51$

$d_{v}^{(i)}, 198$

$\partial A_{n, U, \Sigma}^{(m)}, 173$

$\partial \bar{X}_{n, U^{p}\left(N_{1}\right)}^{\text {ord, } \min }, 164$

$\partial \mathcal{A}_{U^{p}\left(N_{1}\right), \Sigma^{\text {ord }}}^{(m), \text { ord }}, 207$

$\partial \mathcal{A}_{U^{p}\left(N_{1}, N_{2}\right), \Sigma}^{(m), 206}$

$\partial \mathcal{A}_{n, U^{p}\left(N_{1}, N_{2}\right), \Sigma}^{(m), \text { ord }}, 175$

$\partial \mathcal{X}_{n, U^{p}\left(N_{1}, N_{2}\right)}^{\text {ord,min }}, 162$

$\partial_{i} X_{n, U}^{\min }, 161$

$\partial^{(s)} \mathcal{A}_{U^{p}\left(N_{1}, N_{2}\right), \Sigma}^{(m), \text { ord },}, 206$

$\partial^{(s)} \mathcal{A}_{U^{p}\left(N_{1}\right), \Sigma^{\text {ord }}}^{(m), \text { ord }}, 207$

$\partial T_{\sigma}, 54$

$\partial T_{\Sigma}, 57,80$

$\partial_{\{0\}}^{0} T_{\Sigma}, 58$

$\partial_{[\sigma]} A_{n, U, \Sigma}^{(m)}, 175$ 


$$
\begin{aligned}
& \partial_{[\sigma]} \bar{A}_{n, U^{p}\left(N_{1}\right), \Sigma^{\text {ord }}}^{(m), 179} \\
& \partial_{[\sigma]} \mathcal{A}_{U^{p}\left(N_{1}\right), \Sigma^{\text {ord }}}^{(m), \text { ord },} 207 \\
& \partial_{[\sigma]} \mathcal{A}_{U^{p}\left(N_{1}, N_{2}\right), \Sigma}^{(m), 2006} \\
& \partial_{[\sigma]} \mathfrak{A}_{n, U^{p}\left(N_{1}\right), \Sigma^{\text {ord }}}^{(m), 179} \\
& \partial_{[\sigma]} \mathcal{A}_{n, U^{p}\left(N_{1}, N_{2}\right), \Sigma}^{(m), 177} \\
& \partial_{i} A_{n, U, \Sigma}^{(m)}, 173 \\
& \partial_{i} \bar{A}_{n, U^{p}(N), \Sigma^{\text {ord }}}^{(m), 178} \\
& \partial_{i} \bar{X}_{n, U^{p}(N)}^{\text {ord,min }}, 164 \\
& \partial_{i} \mathcal{A}_{n, U^{p}\left(N_{1}, N_{2}\right), \Sigma}^{(m), \text { ord }}, 176 \\
& \partial_{i} \mathcal{X}_{n, U^{p}\left(N_{1}, N_{2}\right)}^{\text {ord,min }}, 162 \\
& \partial_{i}^{0} A_{n, U, \Sigma}^{(m)}, 174 \\
& \partial_{i}^{0} \bar{A}_{n, U^{p}(N), \Sigma^{\text {ord }}}^{(m), 178} \\
& \partial_{i}^{0} \bar{X}_{n, U^{p}(N)}^{\text {ord, min }}, 164 \\
& \partial_{i}^{0} X_{n, U}^{\min }, 161 \\
& \partial_{i}^{0} \mathcal{A}_{n, U^{p}\left(N_{1}, N_{2}\right), \Sigma}^{(m), \text { ord }}, 176 \\
& \partial_{i}^{0} \mathcal{X}_{n, U^{p}\left(N_{1}, N_{2}\right)}^{\text {ord,min }}, 162 \\
& \partial_{i} T_{\Sigma}, 59,80 \\
& \partial_{\Sigma_{0}} T_{\Sigma}, 56 \\
& \partial_{\Sigma_{0}} T_{n,(i), U}^{(m), t}, 147 \\
& \partial_{\Sigma_{0}} \bar{T}_{n,(i), U^{p}(N)}^{(m), \text { ord },}, 149 \\
& \partial_{\Sigma_{0}} \mathcal{T}_{n,(i), U^{p}\left(N_{1}, N_{2}\right)}^{(m), \text { ord },}, 147 \\
& \partial_{\Sigma_{0}} T_{\sigma}, 54 \\
& \partial_{\Sigma_{0}} T_{n,(i), U}^{(m),+}, 145 \\
& \partial_{\sigma} T_{\Sigma}, 58,80 \\
& \partial_{\Sigma_{0}} T, 81 \\
& \partial_{\Sigma_{0}} T, 65 \\
& \partial_{\Sigma_{0}} T_{\Sigma}, 80 \\
& \partial_{\Sigma_{0}} \mathcal{T}_{n,(i), U^{p}\left(N_{1}, N_{2}\right)}^{(m), \text { ord }+}, 145 \\
& \partial_{\sigma}^{0} T_{\Sigma}, 58 \\
& \partial X_{n, U}^{\min }, 161 \\
& \mathcal{E}_{(i), U^{p}\left(N_{1}, N_{2}\right)}^{\text {ord, }}, 126 \\
& \mathcal{E}_{(i), U, \rho}^{+}(W), 124 \\
& \mathcal{E}_{U, \Delta, \rho}^{\mathrm{can}}, 184 \\
& \mathcal{E}_{U, \Delta}^{\mathrm{can}}, 183 \\
& \mathcal{E}_{U^{p}(N), \Delta, \rho}^{\mathrm{can}, \dagger}, 208
\end{aligned}
$$

$\mathcal{E}^{\text {ord, }+}$

$\mathcal{E}_{(i), U^{p}\left(N_{1}, N_{2}\right), \rho}, 126,131$

$\mathcal{E}_{U^{p}(N), \rho}^{\text {ord,sub }}, 187$

$\mathcal{E}_{U^{p}(N), \Delta^{\text {ord }}, \rho}^{\text {ord,sub }}, 187$

$\mathcal{E}_{U^{p}(N), \Delta^{\text {ord }, \rho}}^{\text {ord,can }}, 187$

$\mathcal{E}^{(m) \text {,ord }}$

$\mathcal{E}_{U, \rho}^{(m)}, 113$

$\mathcal{E}_{U^{p}(N), \Delta, \rho}^{\mathrm{sub}, \dagger}, 208$

$\widetilde{\mathcal{E}}_{(i), U}^{+}, 129$

$\mathcal{E}_{(i), U}^{+}, 124$

$\mathcal{E}_{(i), U, \Delta_{0}, \rho}^{\natural}, 152$

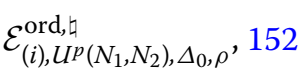

$\mathcal{E}^{\text {ord }}$

$\mathcal{E}^{p}\left(N_{1}, N_{2}\right), 115$

$\mathcal{E}_{U}, 111$

$\mathcal{E}_{U, \Delta, \rho}^{\mathrm{sub}}, 185$

$\mathcal{E}_{U, \rho}, 112$

$\mathcal{E}_{U, \rho}^{\text {sub }}, 187$

$\mathcal{E}^{\text {ord,sub }}$

$\mathcal{E}_{U^{p}\left(N_{1}, N_{2}\right), \rho}^{\text {ord,sub }}, 187$

$\mathcal{E}_{U^{p}\left(N_{1}, N_{2}\right), \Delta, \rho}^{\text {ord,can }}, 184$

$\mathcal{E}_{U^{p}\left(N_{1}, N_{2}\right), \Delta}^{\text {ord,can }}, 183$

$\mathcal{E}_{U^{p}\left(N_{1}, N_{2}\right), \Delta, \rho}^{\text {ord,sub }}, 185$

$\mathcal{E}_{U}^{(m)}, 113$

$\mathcal{E}_{U^{p}\left(N_{1}, N_{2}\right), \rho}^{\text {ord }}, 115$

$\mathcal{E}_{U^{p}\left(N_{1}, N_{2}\right)}^{(m), \text { ord }}, 116$

$\widetilde{\mathcal{E}}_{(i), U^{p}\left(N_{1}, N_{2}\right)}^{\text {ord, }+}, 131$

$\mathfrak{E}_{\rho}, 18$

$\mathfrak{E}_{U, \rho}, 112$

$\mathfrak{E}_{\rho}, 25$

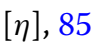

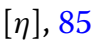

$\left(\eta_{0}^{p}, \eta_{1}^{p}, C, \eta_{p}\right), 85$

$\left\|\eta_{0}^{p}\right\|, 86$

$\left\|\eta_{0}\right\|, 85$

e, 15

$\mathcal{F}^{\dagger}, 204$

$f^{\dagger}, 206$

$\widetilde{\mathcal{G}}^{\text {univ }}, 131$ 
$\left(\mathcal{G}^{\text {univ }}, i^{\text {univ }}, j^{\text {univ }}, \lambda^{\text {univ }},\left[\eta^{\text {univ }}\right]\right), 102$

$\gamma\left(G, i, j, \lambda,\left[\left(\eta_{0}^{p}, \eta_{1}^{p}, C, D, \eta_{p}\right)\right]\right), 101$

$\gamma\left(G, i, j, \lambda,\left[\left(\eta_{0}, \eta_{1}\right)\right]\right), 95$

$G_{n}, 12$

$G_{n}^{1}, 28$

$G_{F}, 10$

$G_{F}^{S}, 10$

$(G, i, j), 93$

$(G, i, j), 98$

$\left(G, i, j, \lambda,\left[\left(\eta_{0}^{p}, \eta_{1}^{p}, C, D, \eta_{p}\right)\right]\right) g, 100$

$\left(G, i, j, \lambda,\left[\left(\eta_{0}^{p}, \eta_{1}^{p}, C, D, \eta_{p}\right)\right]\right) \varsigma_{p}, 101$

$\left(G, i, j, \lambda,\left[\left(\eta_{0}, \eta_{1}\right)\right]\right) g, 95$

$G_{n}^{(m)}, 13$

$\left(G_{n}^{(m)}\left(\mathbb{A}^{\infty}\right) \times \pi_{0}\left(G_{n}(\mathbb{R})\right) \times \mathfrak{C}_{=i}^{(m)}\right)^{\text {ord }}, 35$

$\left(G_{n}^{(m)}\left(\mathbb{A}^{\infty}\right) \times \pi_{0}\left(G_{n}(\mathbb{R})\right) \times \mathfrak{C}^{(m)}\right)^{\text {ord }}, 35$

$G_{n}^{(m)}\left(\mathbb{A}^{\infty}\right)^{\text {ord } \times}, 28$

$\widetilde{G}_{n}^{(m)}\left(\mathbb{A}^{\infty}\right)^{\text {ord }, \times}, 28$

$\widetilde{G}_{n}^{(m)}\left(\mathbb{A}^{\infty}\right)^{\text {ord }}, 28$

$\widetilde{G}_{n}^{(m)}, 16$

$G_{n}\left(\mathbb{A}^{\infty}\right)^{\text {ord }, \times}, 28$

$\left[\left(G^{\text {univ }}, i^{\text {univ }}, j^{\text {univ }}, \lambda^{\text {univ }},\left[\eta^{\text {univ }}\right]\right)\right], 96$

$\widetilde{G}^{\text {univ }} / A_{n,(i), U}^{+}, 129$

$\geq, 167$

$\geq, 168$

$\geq, 169$

Hom, 9

$\mathfrak{H}_{n}^{ \pm}, 18$

$H(R)^{+}, 17$

Hasse $_{M, U}, 195$

Hasse $_{M, U^{p}(N)}, 195$

Hasse $_{U}, 165$

$\operatorname{Herm}_{X}^{>0}, 31$

$\operatorname{Herm}_{X}^{\geq 0}, 31$

$\operatorname{Herm}^{(m)}, 14$

$\operatorname{Herm}_{X}, 13$

$H_{\text {Int }}^{i}, 36$

$H_{\text {Int }}^{i}\left(\mathfrak{T}_{(n)}^{(m)}, \overline{\mathbb{Q}}_{p}\right), 36$
$H_{\text {Int }}^{i}\left(\mathfrak{T}_{=n}^{(m), \text { ord }}, \overline{\mathbb{Q}}_{p}\right), 36$

$H_{\text {Int }}^{i}\left(\mathfrak{T}_{(n)}, \mathcal{L}_{\rho}\right), 41$

$\operatorname{Hom}_{n}^{(m)}, 12$

$H^{0}\left(\mathcal{X}_{n}^{\text {ord,min }}, \mathcal{E}_{\rho}^{\text {ord,sub }} \otimes \mathbb{Z} / p^{r} \mathbb{Z}\right), 188$

$H^{0}\left(\mathcal{X}_{U^{p}(N)}^{\text {ord,min, } \dagger}, \mathcal{E}_{U^{p}(N), \rho}^{\text {sub, }}\right)_{\overline{\mathbb{Q}}_{p}, \leq a}, 219$

$H^{0}\left(\mathcal{X}^{\text {ord }, \dagger}, \mathcal{E}_{\rho}^{\text {sub }}\right), 208$

$H^{0}\left(\mathcal{X}^{\text {ord,min }, \dagger}, \mathcal{E}_{\rho}^{\text {sub }}\right)_{\overline{\mathbb{Q}}_{p}, \leq a}, 219$

$H^{0}\left(\mathfrak{X}^{\text {ord,min }}, \mathcal{E}_{\rho}^{\text {ord,sub }}\right)_{\overline{\mathbb{Q}}_{p}}, 188$

$H^{0}\left(\mathfrak{X}_{n}^{\text {ord,min }}, \mathcal{E}_{\rho}^{\text {ord,sub }}\right), 188$

$H^{i}\left(\mathcal{A}^{(m), \text { ord } \dagger}, \Omega^{j}(\log \infty) \otimes \mathcal{I}\right), 208$

$H^{i}\left(\partial^{(s)} \mathcal{A}^{(m), \text { ord } \dagger}, \Omega^{j}\right), 208$

$H_{c-\partial}^{i}\left(\bar{A}^{(m), \text { ord }}\right), 223$

$H_{c-\partial}^{i}\left(\bar{A}^{(m), \text { ord }}\right)_{\leq a}, 223$

$H_{c-\partial}^{i}\left(\bar{A}_{U^{p}(N), \Sigma}^{(m), \text { ord }}\right), 222$

$H_{\text {rig }}^{i}(\bar{Y}), 204$

$H^{i}\left(X_{n}^{\min }, \mathcal{E}_{\rho}^{\mathrm{sub}}\right), 187$

$H^{0}\left(\mathcal{X}^{\text {ord,min }, \dagger}, \mathcal{E}_{\rho}^{\text {sub }}\right)_{\overline{\mathbb{Q}}_{p}}, 215$

$H^{i}\left(\mathcal{A}^{(m), \text { ord }, \dagger}, \Omega^{s}(\log \infty) \otimes \mathcal{I}_{\partial \mathcal{A}^{(m), \text { ord }, \dagger}}\right)_{\leq a}$, 222

$\mathcal{I}_{\partial_{\Sigma_{0}} T_{\Sigma}}, 56$

$\mathcal{I}_{\partial T_{\Sigma}}, 57$

$\mathcal{I}_{\partial, n,(i), U, \Sigma_{0}}^{(m), \mathfrak{}+, \wedge}, 150$

$\mathcal{I}_{\partial, n,(i), U, \Sigma_{0}}^{(m), 150}$

$\mathcal{I}_{\partial, n,(i), U^{p}\left(N_{1}, N_{2}\right), \Sigma_{0}}^{(m), o r d, \natural+, \wedge}, 150$

$\mathcal{I}^{(m), \text { ord, }+, \wedge}$

$\mathcal{I}_{\partial, n,(i), U^{p}\left(N_{1}, N_{2}\right), \Sigma_{0}}^{(m), 150}$

$\mathcal{I}_{\partial A_{U, \Sigma}^{(m)} \times \operatorname{Spec} R_{0}}, 180$

$\mathcal{I}_{\partial, \Sigma_{0}}^{\wedge}, 66,81$

$\mathcal{I}_{\partial T_{\Sigma}}, 80$

$\mathcal{I}_{\partial X_{n, U, \Delta}}, 180$

$\mathcal{I}_{\partial A_{U, \Sigma}^{(m)}}, 180$

$\mathcal{I}_{\partial X_{n, U}^{\min } \times \operatorname{Spec} R_{0}}, 180$

$\mathcal{I}_{\partial X_{n, U, \Delta} \times \operatorname{Spec} R_{0}}, 180$

$\mathcal{I}_{T_{\Sigma_{0}}^{\wedge}}, 66,81$

$\mathcal{I}_{\partial X_{n, U}^{\min }}, 180$

Ind, 10

$i^{(s)}, 206$ 


\begin{tabular}{|c|c|}
\hline$i_{A^{\text {univ }}}^{(m)}, 97$ & $\mathcal{M}_{\Sigma_{0}}^{\wedge}, 65$ \\
\hline$i_{\mathcal{A}^{\text {univ }}}^{(m)}, 103$ & $\mathcal{M}_{\Sigma}, 57,80$ \\
\hline$i_{\lambda}^{(m)}, 98,105$ & $\mathcal{M}_{\Sigma, i}^{\wedge}, 174$ \\
\hline$[\infty], 9$ & $\left(\mathcal{M}^{\wedge}, \alpha^{\wedge}\right), 48$ \\
\hline$\left[\infty^{p}\right], 9$ & $\mathcal{M}_{\Sigma_{0}}^{\wedge}, 81$ \\
\hline $\mathcal{J}_{n}^{(m), \text { tor,ord }}, 170$ & $v, 12$ \\
\hline $\mathcal{J}_{n}^{(m), \text { tor }}, 170$ & $\mathbf{N}, 29$ \\
\hline$J_{n}, 11$ & $N_{n}, 16$ \\
\hline$j_{U}^{\min }, 161$ & $N_{n,(i)}, 20$ \\
\hline$j_{U, \Sigma}^{(m)}, 173$ & $N_{n,(i)}^{+}, 19$ \\
\hline$j_{U^{p}\left(N_{1}, N_{2}\right), \Sigma}^{(m), \text { ord }}, 175$ & $N_{n,(i)}^{(m)}, 20$ \\
\hline$j_{U^{p}\left(N_{1}, N_{2}\right)}, 162$ & $N_{n,(i)}^{(m),+}, 19$ \\
\hline KS, 26 & $\tilde{N}_{n,(i)}^{(m)}, 20$ \\
\hline$k(v), 10$ & $\tilde{N}_{n,(i)}^{(m),+}, 19$ \\
\hline $\mathcal{L}_{\rho, U}, 41$ & n-Ind, 11 \\
\hline $\mathcal{L}_{n, \widetilde{U}}^{(m)}(\chi), 107$ & $N\left(L_{n,(i), \operatorname{lin}}^{(m)}\right), 22$ \\
\hline $\mathcal{L}_{n, \widetilde{U}^{p}\left(N_{1}, N_{2}\right)}^{(m), \text { ord }}(\chi), 108$ & $N_{n}^{(m)}, 15$ \\
\hline $\mathcal{L}_{T}(\chi), 46$ & $N\left(R_{n,(n),(i)}\right), 24$ \\
\hline $\mathcal{I}_{\partial \mathcal{X}_{U^{p}(N), \Delta}^{\text {ord }}, \dagger}, 208$ & $\mathcal{O}_{T_{\Sigma_{0}}^{\wedge}}, 66$ \\
\hline $\mathcal{L}_{\psi}, 59$ & $\Omega^{j} \quad, 208$ \\
\hline $\mathcal{L}_{\psi}^{\wedge}, 66$ & $\partial^{(s)} \mathcal{A}_{U^{p}(N), \Sigma}^{(m), \text { ord }}$ \\
\hline $\mathcal{L}_{U}^{+}(a), 139$ & $\Omega_{\mathcal{A}_{U^{p}(N), \Sigma}^{(m), \text { ord }, \dagger}}^{J}(\log \infty) \otimes \mathcal{I}_{\partial \mathcal{A}_{U^{p}(N), \Sigma}^{(m), \text { ord }, \dagger}}, 207$ \\
\hline $\begin{array}{l}\mathcal{L}_{U^{p}\left(N_{1}, N_{2}\right)}^{+}(a), 140 \\
\Lambda_{n}, 11\end{array}$ & $\Omega_{\mathcal{A}_{U^{p}(N), \Sigma}^{(m), \text { ord } \dagger}}^{j}(\log \infty), 207$ \\
\hline$\Lambda_{n,(i)}, 12$ & $\Omega_{n, U^{p}\left(N_{1}, N_{2}\right), \Delta^{\prime}}^{\text {ord }}, 182$ \\
\hline$\Lambda_{n,(i)}^{\prime}, 20$ & $\Omega_{n,(i), U^{p}\left(N_{1}, N_{2}\right)}^{\text {ord }, 1}, 127$ \\
\hline$\Lambda_{n}^{(m)}, 12$ & $\Omega_{n,(i), U^{p}\left(N_{1}, N_{2}\right)}^{\mathrm{ord},}, 126$ \\
\hline$L_{n,(i), \text { herm }}, 23$ & $\Omega_{A^{\text {univ }} / X_{n, U}, 110}^{1}$ \\
\hline$L_{n,(i), \text { herm }}^{-}, 24$ & $\Omega_{\mathcal{A}^{\text {univ }} / \mathcal{X}_{n, U^{p}\left(N_{1}, N_{2}\right)}^{\text {ord }}}^{1}, 114$ \\
\hline $\begin{array}{l}L_{n,(i)}, 24 \\
L_{n,(i)}^{(m)}, 24\end{array}$ & $\Omega_{\mathcal{G}^{\text {univ }} / \mathcal{A}_{n, U^{p}\left(N_{1}, N_{2}\right)}^{(m) \text { ord }}}^{1}, 116$ \\
\hline$\widetilde{L}_{n,(i)}^{(m)}, 24$ & $\Omega_{T_{\Sigma_{0}}^{\wedge} / \operatorname{Spf} R_{0}}^{1}(\log \infty), 81$ \\
\hline$L_{n,(i), \operatorname{lin}}, 22$ & $\Omega_{T_{\Sigma} / \operatorname{Spec} R_{0}}^{1}(\log \infty), 80$ \\
\hline$L_{n,(i), \operatorname{lin}}^{(m)}, 22$ & $\Omega_{T_{\Sigma_{0}}^{\wedge} / \operatorname{Spf} R_{0}}^{1}(\log \infty), 66$ \\
\hline$\widetilde{L}_{n,(i), \operatorname{lin}}^{(m)}, 22$ & $\Omega_{\mathfrak{X} / \mathfrak{Y}}^{i}(\log \mathcal{M} / \mathcal{N}), 50$ \\
\hline $\mathcal{M}(D), 48$ & $\Omega_{\mathfrak{X} / \mathrm{Spf} R_{0}}^{i}, 50$ \\
\hline $\mathcal{M}_{\Sigma, i}^{\wedge}, 176$ & $\Omega_{\mathfrak{X} / \operatorname{Spf} R_{0}}^{i}(\log \mathcal{M}), 50$ \\
\hline
\end{tabular}


$\Omega_{X}^{i}(\log \mathcal{M}), 48$

$\Omega_{X / Y}^{i}(\log \mathcal{M} / \mathcal{N}), 48$

$\omega_{n, U^{p}\left(N_{1}, N_{2}\right)}, 114$

$\widetilde{\Omega}^{\text {ord, }+}$

$\widetilde{\Omega}_{n,(i), U^{p}\left(N_{1}, N_{2}\right)}^{\mathrm{ord}, 131}$

$\widetilde{\Omega}_{n,(i), U}^{+}, 129$

$\Omega_{n, U, \Delta}, 182$

$\Omega_{n, U}, 110$

$\omega_{n, U}, 110$

$\Omega_{n, U}^{(m)}, 113$

$\Omega^{(m), \text { ord }}$

$\Omega_{n, U^{p}\left(N_{1}, N_{2}\right)}, 116$

$\Omega_{n, U^{p}\left(N_{1}, N_{2}\right)}^{\text {ord }}, 114$

$\Omega_{T_{\Sigma} /\left(\alpha_{*} T\right)_{\Sigma^{\prime}}}^{1}(\log \infty), 82$

$\Omega_{T_{\Sigma} /\left(\alpha_{*} T\right)_{\Sigma^{\prime}}}^{1}(\log \infty), 60$

$\Omega_{T_{\Sigma_{0}}^{\wedge} /\left(\alpha_{*} T\right)_{\Sigma_{0}^{\prime}}^{\wedge}}^{1}(\log \infty), 82$

$\omega_{U}, 165$

$\omega_{U}, 110$

$\omega_{U, \Delta}, 182$

$\omega_{U^{p}(N)}, 195$

$\omega_{U^{p}\left(N_{1}, N_{2}\right), \Delta}^{\text {ord }}, 183$

$\omega_{U^{p}\left(N_{1}, N_{2}\right)}, 114$

$\Omega_{n,(i), U}^{\natural}, 125$

$\Omega_{n,(i), U}^{+}, 124$

$\widetilde{\Omega}_{n,(i), U, \Delta_{0}}^{\natural}, 152$

$\widetilde{\Omega}_{n,(i), U^{p}\left(N_{1}, N_{2}\right), \Delta_{0}}^{\mathrm{ord}, \sharp}, 152$

ord, 28

ord, $\times, 28$

$\mathfrak{p}_{n}, 18$

$\mathfrak{p}_{n}^{ \pm}, 18$

$\Phi_{n}, 16$

$\Phi_{n}^{+}, 16$

$\Phi_{n,(n)}, 25$

$\Phi_{n,(n)}^{+}, 25$

$\varpi_{v}, 10$

$\pi_{m, m^{\prime}}, 95$

$\left(\pi_{A^{+} / X^{\natural}, *} \mathcal{L} \otimes \mathcal{F}\right)_{U}^{+}\left(a^{\natural}\right), 139$

$\left(\pi_{A^{+} / X^{+}, *} \mathcal{L}\right)_{U}^{+}(a), 139$

$\pi_{A^{(m) \text { tor }}} / X^{\text {tor }}, 173$
$\pi_{A^{(m), \text { tor }} / X^{\min }}, 173$

$\pi_{A_{n}^{(m)} / A_{n}^{\left(m^{\prime}\right)}}, 96$

$\pi_{A_{n}^{(m)} / X_{n}}, 96$

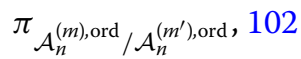

$\pi_{\mathcal{A}^{(m), \text { ord,tor }} / \mathcal{X}^{\text {ord, } \min }, 175}$

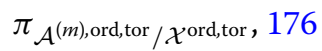

$\left(\pi_{\mathcal{A}^{\text {ord },+} / \mathcal{X}^{\text {ord, }, \text {, } *}} \mathcal{L} \otimes \mathcal{F}\right)_{U^{p}\left(N_{1}, N_{2}\right)}^{+}\left(a^{\natural}\right), 141$

$\pi_{m, m^{\prime}}\left(G, i, j, \lambda,\left[\left(\eta_{0}^{p}, \eta_{1}^{p}, C, D, \eta_{p}\right)\right]\right), 101$

$\pi_{(U, \Sigma),\left(U^{\prime}, \Sigma^{\prime}\right)}, 173$

$\Psi_{n}, 11$

$\psi_{\Sigma}, 60$

$P_{n,(i)}, 19$

$P_{n,(i)}^{+}, 19$

$P_{n,(i)}^{(m)}, 19$

$P_{n,(i)}^{(m),+}, 19$

$P_{n,(i)}^{(m),-}, 24$

$\widetilde{P}_{n,(i)}^{(m)}, 19$

$\widetilde{P}_{n,(i)}^{(m),+}, 19$

$\mathfrak{Q}_{n}, 18$

$\mathfrak{q}_{n}, 18$

$Q_{m,\left(m^{\prime}\right)}, 24$

$Q_{m,\left(m^{\prime}\right)}^{\prime}, 24$

$\varrho_{n}, 16$

$\rho_{\lambda}, 16$

$\varrho_{n,(n)}, 25$

$\rho_{(n), \lambda}, 25$

$\rho_{m, s}^{i, j}, 220$

rec, 10

$R_{n,(n),(i)}, 24$

$R_{n,(n)}^{(m)}, 24$

$r_{p, l}(\Pi), 31$

RS, 9

$R_{p, l}(\pi, N), 226$

$R_{p}(\Pi), 219,222$

$R_{p}(\pi), 226$

$R_{p}(\Pi), 200,224$

$|\mathcal{S}|, 10$ 
$|\mathcal{S}(D)|, 51$

$|\mathcal{S}(D, \geq)|, 51$

$\left|\mathcal{S}\left(\partial A_{n, U, \Sigma}^{(m)}\right)\right|_{=i}, 174$

$|\mathcal{S}(U, \Sigma)|_{=i}, 171$

$\left|\mathcal{S}\left(U^{p}(N), \Sigma\right)^{\text {ord }}\right|_{=i}, 172$

$\widehat{S}^{(m), \text { ord, }+}$

$\mathcal{S}_{n,(i), \widetilde{U}^{p}(N)}, 135$

$\mathcal{S}(D), 51$

$\mathcal{S}(D, \geq), 51$

$\mathcal{S}_{n, \widetilde{U}^{p}}^{(m), \text { ord }}, 106$

$\mathcal{S}_{n,(i), U^{p}(N)}^{+}, 135$

$\mathcal{S}_{n,(i), U^{p}(N)}^{(m), \text { ord, }}, 135$

$\mathcal{S}(U, \Sigma), 171$

$\mathcal{S}(U, \Sigma)_{\leq i}, 171$

$\mathcal{S}\left(U^{p}(N), \Sigma\right)_{\leq i}^{\text {ord }}, 172$

$\widetilde{\mathcal{S}}^{(m), \text { ord, }+}$

$\mathcal{S}_{n,(i), \widetilde{U}^{p}(N)}, 133$

$|\Sigma|, 55,79$

$|\Sigma|^{*}, 79$

$|\Sigma|^{\vee}, 79$

$|\Sigma|^{\vee, 0}, 79$

$\left|\Sigma_{0}\right|, 57,65,81$

$\left|\Sigma_{0}\right|^{0}, 54$

$\left|\Sigma_{0}\right|^{0}, 65,81$

$\left|\Sigma_{0}\right|^{0}, 57$

$\left|\Sigma_{0}\right|^{*}, 81$

$\left|\Sigma_{0}\right|^{\vee}, 81$

$\left|\Sigma_{0}\right|^{\vee, 0}, 81$

$\bar{\Sigma}_{0}(\sigma), 59$

$\bar{\Sigma}(\sigma), 59$

$\Sigma(\sigma), 58$

$\Sigma^{\text {ord }}, 168$

$\widetilde{\Sigma}_{0}, 65,81$

$\varsigma_{p}, 27$

$\widehat{S}_{n,(i), \widetilde{U}}^{(m),+}, 134$

$S\left(F^{m}\right)^{>0}, 31$

$S_{n}, 9$

$S_{n, \widetilde{U}^{\prime}}^{(m)}, 106$

$S_{n,(i), U}^{+}, 134$
$S(\sigma), 59$

$S_{n,(i), U}^{(m),+}, 134$

Std, 26

$\operatorname{Std}_{\tau}, 26$

sw, 14

$S(X), 14$

$\widetilde{S}_{n,(i), \widetilde{U}}^{(m),+}, 132$

$\mathbb{T}_{U^{p}(N), \rho}^{\mathrm{ord}, S}(W), 199$

$\mathbb{T}_{U^{p}\left(N_{1}, N_{2}\right), \rho}^{S}, 198$

$\mathbb{T}_{U^{p}\left(N_{1}, N_{2}\right), \rho}^{\text {ord },}(W), 199$

$\widetilde{\mathcal{T}}_{n,(i), \widetilde{U}^{p}\left(N_{1}, N_{2}\right)}^{(m), \text { rd }+}, 138$

$\left(\mathcal{T}_{n,(i), U^{p}\left(N_{1}, N_{2}\right), \widetilde{\Sigma}_{0}}^{(m), \text { ord }+}, \mathcal{M}_{\widetilde{\Sigma}_{0}}\right), 144$

$\left(\mathcal{T}_{n,(i), U^{p}\left(N_{1}, N_{2}\right), \Sigma_{0}}^{(m), \text { ord },+,}, \mathcal{M}_{\Sigma_{0}}^{\wedge}\right), 147$

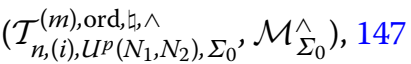

$\left(\mathcal{T}_{n,(i), U^{p}\left(N_{1}, N_{2}\right), \Sigma_{0}}^{(m), \text { ord }+, \wedge}, \mathcal{M}_{\Sigma_{0}}^{\wedge}\right), 145$

$\mathcal{T}_{n,(i), U^{p}\left(N_{1}, N_{2}\right)}^{(m), \text { ord }+}, 138$

$\mathcal{T}_{n,(i), U^{p}\left(N_{1}, N_{2}\right)}^{\text {ord, }}, 138$

$\mathcal{T}_{n, \widetilde{U}^{p}\left(N_{1}, N_{2}\right)}^{(m) \text {,ord }}, 108$

$\mathfrak{T}_{(i), U}^{(m)}, 36$

$\mathfrak{T}_{U^{p}\left(N_{1}\right),=n}^{(m), \text { ord }}, 36$

$\mathfrak{T}_{(n)}, 41$

$\mathfrak{t}_{v}^{(i)}, 197$

$T, 9$

$T_{n}, 16$

$T_{n}^{1}, 28$

$T_{1} \otimes_{S} T_{2}, 47$

TF, 9

$T_{G L_{m}}, 28$

$\widetilde{T}_{n,(i), \widetilde{U}}^{(m),+}, 137$

$\left(T_{n,(i), U, \Sigma_{0}}^{(m), 4+, \wedge}, \mathcal{M}_{\Sigma_{0}}^{\wedge}\right), 147$

$T^{p}, 9$

$T^{p} S_{\bar{y}}, 45$

$\operatorname{tr}_{F}, 210$

$\operatorname{tr}_{F}, 218$

$\operatorname{tr}_{F}, 223$

$T_{n,(i), U}^{+}, 137$ 
$T S_{\bar{y}}, 45$

$\left(T_{n,(i), U, \widetilde{\Sigma}_{0}}^{(m),+}, \mathcal{M}_{\widetilde{\Sigma}_{0}}\right), 144$

$\left(T_{n,(i), U, \Sigma_{0}}^{(m),+, \wedge}, \mathcal{M}_{\Sigma_{0}}^{\wedge}\right), 145$

$\left(T_{n,(i), U, \Sigma_{0}}^{(m), \natural}, \mathcal{M}_{\Sigma_{0}}^{\wedge}\right), 147$

$T_{n, \widetilde{U}}^{(m)}, 107$

$T_{\Sigma_{0}}^{\wedge}, 81$

$\operatorname{tr}_{5 p}, 209$

$T_{n,(i), U}^{(m),}, 137$

$T_{\Sigma}, 56,79$

$T_{\sigma}, 54$

$T_{\Sigma_{0}}^{\wedge}, 65$

$\left(T_{\Sigma_{0}}^{\wedge}\right)_{\sigma}, 66$

$T_{v}^{(i)}, 197$

$U_{n, \infty}, 17$

$U(n), 9$

$U^{p}(N), 27,28$

$U^{p}\left(N_{1}, N_{2}\right), 19,27,28$

$U_{K, \infty}, 30$

$U_{p}\left(N_{1}, N_{2}\right)_{n}, 19$

$U_{p}\left(N_{1}, N_{2}\right)_{n,(i)}^{(m)}, 27$

$U_{p}\left(N_{1}, N_{2}\right)_{n,(i)}^{(m),+}, 27$

$\widetilde{U}_{p}\left(N_{1}, N_{2}\right)_{n,(i)}^{(m),+}, 27$

$U_{p}\left(N_{1}, N_{2}\right)_{n}^{(m)}, 19$

$\widetilde{U}_{p}\left(N_{1}, N_{2}\right)_{n}, 19$

$U_{p}(N)_{n,(i)}, 27$

$U_{p}(N)_{n,(i)}^{(m)}, 27$

$V_{n}, 12$

$V, 9$

$V_{n,(i)}, 12$

$V_{n}^{(m)}, 12$

$V^{p}, 9$

$V S_{\bar{y}}, 45$

$V_{\leq a}, 201$

$V_{>a}, 201$

WD, 10

$W_{0} H_{c-\partial}^{i}\left(\bar{A}^{(m), \text { ord }}\right)_{\overline{\mathbb{Q}}_{p}}, 225$
$\mathcal{X}_{n,(i), U^{p}\left(N_{1}, N_{2}\right)}^{(m), \text { ord } \natural}, 125$

$\mathcal{X}^{\text {ord, }, ~}$

$\mathcal{X}_{n,(i), U^{p}\left(N_{1}, N_{2}\right)}, 125$

$\mathcal{X}^{(m), \text { ord, }+}$

$\mathcal{X}_{n,(i), U^{p}\left(N_{1}, N_{2}\right)}^{(m),} 125$

$\mathcal{X}_{n, U^{p}\left(N_{1}, N_{2}\right)}^{\text {ord,min }}, 161$

$\mathcal{X}_{U^{p}\left(N_{1}\right)}^{\text {ord,min, }}, 212$

$\mathcal{X}_{U^{p}\left(N_{1}, N_{2}\right)}^{\text {ord,min, }}, 211$

$\mathcal{X}_{n, U}^{\min }, 164$

$\mathcal{X}_{n, U^{p}\left(N_{1}, N_{2}\right)}^{\text {ord }}, 91$

$\mathfrak{X}_{n, U^{p}\left(N_{1}\right), \Sigma^{\text {ord }}}^{\text {ord }}, 178$

$\mathfrak{X}_{\left(U^{p}\right)^{\prime}(N), \Delta}^{\text {ord, }}, 207$

$\mathfrak{X}_{n, U^{p}\left(N_{1}\right)}^{\text {ord,min }}, 164$

$\mathfrak{X}_{n, U^{p}\left(N_{1}\right)}^{\text {ord }}, 93$

$\widetilde{\Xi}_{n,(i), U^{p}\left(N_{1}, N_{2}\right)}^{\text {ord, }}, 131$

$\widetilde{\Xi}_{n,(i), U}^{+}, 129$

$\Xi_{n,(i), U}^{+}, 124$

$\Xi_{n,(i), U}^{\natural}, 125$

$\Xi_{n, U^{p}\left(N_{1}, N_{2}\right), \Delta}^{\text {ord }}, 183$

ord,

$\Xi_{n,(i), U^{p}\left(N_{1}, N_{2}\right)}^{\mathrm{ord}, \llbracket} 127$

$\Xi_{n,(i), U^{p}\left(N_{1}, N_{2}\right)}^{\text {ord, }}, 126$

$\Xi_{n, U}, 110$

$\Xi_{n, U^{p}\left(N_{1}, N_{2}\right)}, 114$

$\Xi_{n, U, \Delta}, 183$

$\bar{X}_{n, U}^{\min }, 165$

$\bar{X}_{n, U^{p}\left(N_{1}\right)}^{\text {ord, min }}, 164$

$\bar{x}_{n, U^{p}\left(N_{1}\right), \Sigma}^{\text {ord }}, 178$

$\bar{X}_{n, U}^{\min , \mathrm{n} \text {-ord }}, 165$

$X^{*}\left(T_{n}\right), 16$

$X^{*}\left(T_{n}\right)^{+}, 16$

$X^{*}\left(T_{n}\right)_{(n)}^{+}, 25$

$X_{n,(i), U}^{+}, 123$

$X_{n,(i), U}^{(m), \llbracket}, 123$

$X_{n, U}^{\min }, 161$

$\bar{X}_{n, U^{p}(N)}^{\text {ord }}, 93$

$X^{*}(G), 93$

$X^{*}, 45$

$X^{*}\left(\mathcal{S}_{n,(i), U^{p}(N)}^{(m), \text { ord }+}\right)^{\natural}, 137$ 


$$
\begin{aligned}
& X^{*}\left(\mathcal{S}_{n,(i), U^{p}(N)}^{(m), \text { ord, }}\right)^{>0, \natural}, 137 \\
& X^{*}\left(\mathcal{S}_{n,(i), U^{p}(N)}^{(m), \text { ord, }}\right)^{>0}, 136 \\
& X^{*}\left(\mathcal{S}_{n,(i), U^{p}(N)}^{(m), \text { ord, }}\right) \geq 0, \text {, } 137 \\
& X^{*}\left(\mathcal{S}_{n,(i), U^{p}(N)}^{(m), \text { ord, }}\right) \geq 0,136 \\
& X^{*}\left(\mathcal{S}_{n,(i), U^{p}(N)}^{(m), \text { ord, }}\right)_{\mathbb{R}}^{>0}, 136 \\
& X^{*}\left(\mathcal{S}_{n,(i), U^{p}(N)}^{(m), \text { ord, }}\right)_{\mathbb{R}}^{\geq 0}, 136 \\
& X^{*}\left(S_{n,(i), U}^{(m),+}\right)^{\natural}, 136 \\
& X^{*}\left(S_{n,(i), U}^{(m),+}\right)^{>0, \natural}, 136 \\
& X^{*}\left(S_{n,(i), U}^{(m),+}\right)^{\geq 0, \natural, 136} \\
& X^{*}\left(S_{n,(i), U}^{(m),+}\right)^{\geq 0}, 136 \\
& X^{*}\left(S_{n,(i), U}^{(m),+}\right)_{\mathbb{R}}^{>0}, 136 \\
& X^{*}\left(S_{n,(i), U}^{(m),+}\right)_{\mathbb{R}}^{\geq 0}, 136 \\
& X_{n,(i), U}^{(m), 1}, 123 \\
& X_{n, U}, 91 \\
& X_{*}, 45 \\
& X_{*}\left(S_{n,(i), U}^{(m),+}\right)_{\mathbb{R}}^{\succ 0}, 136
\end{aligned}
$$

$X_{*}\left(\mathcal{S}_{n,(i), U^{p}(N)}^{(m), \text { ord }+}\right)_{\mathbb{R}}^{>0}, 136$
$X_{*}\left(\mathcal{S}_{n,(i), U^{p}(N)}^{(m), \text { ord, }+}\right)_{\mathbb{R}}^{\geq 0}, 136$
$X_{*}\left(\mathcal{S}_{n,(i), U^{p}(N)}^{(m), \text { ord, }+}\right)_{\mathbb{R}}^{\geq 0}, 136$
$X_{*}\left(S_{n,(i), U}^{(m),+}\right)_{\mathbb{R}}^{\geq 0}, 136$
$X_{*}\left(S_{n,(i), U}^{(m),+}\right)_{\mathbb{R}}^{>0}, 136$
$\mathcal{Y}^{\dagger}, 204$
$\mathcal{Y}_{\eta}^{\wedge}, 204$
$\mathcal{Y}_{n,(i), U U^{p}(N)}^{(m), 122}$
$\mathcal{Y}_{n,(i), U^{p}(N)}^{\text {ord, }}, 122$
]$_{Y}[, 204$
$Y^{\text {an }}, 204$
$Y^{\dagger}, 204$
$Y_{n,(i), U^{\prime}}^{(m), 122}$
$Y_{n,(i), U^{+}}^{+}, 121$
$Y_{n,(i), U^{\prime}}^{(m),+}, 121$
$Z(\quad), 9$

Received: 30 June 2015 Accepted: 20 June 2016 Published online: 26 October 2016

\section{References}

1. Andreatta, F., lovita, A., Pilloni, V.: p-Adic families of Siegel modular cuspforms Ann. Math. 181(2), 623-697 (2015)

2. Andreatta, F., lovita, A., Pilloni, V.: On overconvergent Hilbert modular cusp forms (to appear in Astérisque)

3. Ash, A., Mumford, D., Rapoport, M., Tai, Y.-S.: Smooth Compactifications of Locally Symmetric Varieties, 2nd edn. Cambridge University Press, Cambridge (2010)

4. Bernstein, J., Deligne, P.: Le "centre" de Bernstein. In: Deligne, P. (ed.) Representations des groupes reductifs sur un corps local: Travaux en cours. Hermann, Paris (1984)

5. Berkovich, V.:Complex analytic vanishing cycles for formal schemes (2015) (preprint). http://www.wisdom.weizmann ac.il/ vova/

6. Berthelot, P.: Cohomologie rigide et cohomologie rigide à supports propres. Prépublication IRMAR 96-03. http:// perso.univ-rennes1.fr/pierre.berthelot/ (1996)

7. Berthelot, P.: Finitude et pureté cohomologique en cohomologie rigide. Invent. Math. 128(2), 329-377 (1997)

8. Bosch, S., Günzer, U., Remmert, R.: Non-Archimedean Analysis. Springer, Berlin (1984)

9. Barnet-Lamb, T., Gee, T., Geraghty, D., Taylor, R.: Potential automorphy and change of weight. Ann. Math 179(2), 501-609 (2014)

10. Barnet-Lamb, T., Gee, T., Geraghty, D., Taylor, R.: Local-global compatibility for $/=p$ II. Ann. Sci. Éc. Norm. Supér. (4) 47(1), 165-179 (2014)

11. Barnet-Lamb, T., Geraghty, D., Harris, M., Taylor, R.: A family of Calabi-Yau varieties and potential automorphy II. Publ. Res. Inst. Math. Sci. 47(1), 29-98 (2011)

12. Bosch, S., Lütkebohmert, W., Raynaud, M.: Néron Models. Springer, Berlin (1980)

13. Borel, A.: Stable real cohomology of arithmetic groups II. In: Hano, J., Morimoto, A., Murakami, S., Okamoto, K., Ozeki, H. (eds.) Manifolds and Lie Groups, Papers in Honor of Y. Matsushima", Progress in Mathematics, vol. 14. Birkhaüser, Basel (1981)

14. Bredon, G.: Sheaf Theory, 2nd edn. Springer, Berlin (1997)

15. Borel, A., Wallach, N.: Continuous Cohomology, Discrete Subgroups, and Representations of Reductive, Annals of Mathematics Studies, vol. 94. Princeton University Press, Princeton (1980)

16. Caraiani, A.: Local-global compatibility and the action of monodromy on nearby cycles. Duke Math. J. 161(12), 2311-2413 (2012)

17. Chai, C.-L., Faltings, G.: Degeneration of Abelian Varieties. Springer, Berlin (1990)

18. Chenevier, G., Harris, M.: Construction of automorphic Galois representations II. Camb. J. Math. 1(1), 53-73 (2013)

19. Chiarellotto, B.: Weights in rigid cohomology applications to unipotent F-isocrystals. Ann. Sci. Éc. Norm. Supér. (4) 31(5), 683-715 (1998) 
20. Clozel, L.: Motifs et formes automorphes: applications du principe de fonctorialité. In: Clozel, L., Milne, J.S. (eds.) Automorphic Forms, Shimura Varieties, and L-Functions I, Perspectives in Mathematics, vol. 10. Academic Press, London (1990)

21. Casselman, W., Osborne, M.S.: The $n$-cohomology of representations with an infinitesimal character. Compos. Math. 31(2), 219-227 (1975)

22. Coleman, R.: Classical and overconvergent modular forms. Invent. Math. 124(1-3), 215-41 (1996)

23. Dieudonné, J., Grothendieck, A.: Eléments de géométrie algébrique III. Publ. Math. IHES 11, 5-167 (1961)

24. Dieudonné, J., Grothendieck, A.: Eléments de géométrie algébrique IV. Publ. Math. IHES 28, 5-255 (1966)

25. Friedman, G.: Survey article: an elementary illustrated introduction to simplicial sets. Rocky Mountain J. Math. 42 (2), 353-423 (2012)

26. Fulton, W.: Introduction to Toric Varieties, Annals of Mathematics Studies, vol. 131. Princeton University Press, Princeton (1993)

27. Grosse-Klönne, E.: Rigid analytic spaces with overconvergent structure sheaf. J. Reine Angew. Math. 519, 73-95 (2000)

28. Harris, M.: Automorphic forms of $\bar{\partial}$-cohomology type as coherent cohomology classes. J. Differ. Geom. 32(1), 1-63 (1990)

29. Harris, M., Taylor, R.: The Geometry and Cohomology of Some Simple Shimura Varieties, Annals of Mathematics Studies, vol. 151. Princeton University Press, Princeton (2001)

30. Harris, M., Zucker, S.: Boundary cohomology of Shimura varieties, I: Coherent cohomology on the toroidal boundary. Ann. Sci. Éc. Norm. Supér. (4) 27(3), 249-344 (1994)

31. Harris, M., Zucker, S.: Boundary cohomology of Shimura varieties, II: Mixed Hodge structures. Invent. Math. 116(1-3), 243-308 (1994) erratum, Invent. Math. 121(2), 437 (1995)

32. Harris, M., Zucker, S.: Boundary cohomology of Shimura varieties, III: Coherent cohomology on higher-rank boundary strata and applications to Hodge theory. Mémoire Société mathématique de France, nouv. sér., No. 85. Société Mathématique de France, Paris, pp. vi+116 (2001)

33. Hida, H.: p-Adic Automorphic Forms on Shimura Varieties. Springer, Berlin (2004)

34. Kato, K.: Logarithmic structures of Fontaine-Illusie. In: Igusa, J.-I. (ed.) Algebraic Analysis, Geometry, and Number Theory. Johns Hopkins University Press, Baltimore (1989)

35. Katz, N.: P-Adic properties of modular schemes and modular forms. In: Modular Functions of One Variable, III, LNM 350. Springer, Berlin (1973)

36. Katz, N.: Serre-Tate local moduli. In: Algebraic Surfaces (Orsay, 1976-78), LNM 868. Springer, Berlin (1981)

37. Katz, N., Mazur, B.: Arithmetic Moduli of Elliptic Curves, Annals of Mathematics Studies, vol. 108. Princeton University Press, Princeton (1985)

38. Köpf, U.: Über eigentliche Familien algebraischer Varietäten über affinoiden Räumen, Schriftenreihe des Mathematischen Instituts der Universität Münster (2). Heft 7 (1974)

39. Kottwitz, R.: Points on some Shimura varieties over finite fields. J. Am. Math. Soc. 5(2), 373-444 (1992)

40. Kunz, E.: Kähler Differentials. Viehweg, Brunswick (1986)

41. Lan, K.-W.: Arithmetic Compactifications of PEL-Type Shimura Varieties, London Mathematical Society Monographs, vol. 36. Princeton University Press, Princeton (2013)

42. Lan, K.-W.: Toroidal compactifications of PEL-type Kuga families. Algebra Number Theory 6(5), 885-966 (2012)

43. Lan, K.-W.: Comparison between analytic and algebraic constructions of toroidal compactifications of PEL-type Shimura varieties. J. Reine Angew. Math. 664, 163-228 (2012)

44. Lan, K.-W.: Compactifications of PEL-type Shimura varieties and Kuga families with ordinary loci (2013) (preprint). http://www.math.umn.edu/ kwlan/academic.html

45. Lan, K.-W.: Boundary strata of connected components in positive characteristics (an appendix to the article Families of nearly ordinary Eisenstein series on unitary groups by Xin Wan). Algebra Number Theory 9(9), 1955-2054 (2015)

46. Le Stum, B.: Rigid Cohomology, Cambridge Tracts in Mathematics, vol. 172. Cambridge University Press, Cambridge (2007)

47. Mumford, D.: Abelian Varieties, Tata Institute of Fundamental Research Studies in Mathematics, vol. 5. Oxford University Press, Oxford (1970)

48. Mœglin, C., Waldspurger, J.-L.: Le spectre résiduel de GL(n). Ann. Sci. Éc. Norm. Supér. (4) 22(4), 605-674 (1989)

49. Pink, R.: Arithmetical Compactification of Mixed Shimura Varieties, Bonner Mathematische Schriften, vol. 209. Universität Bonn, Bonn (1990)

50. Serre, J.-P.: Endomorphismes complètements continues des espaces de Banach p-adiques. Publ. Math. IHES 12,69-85 (1962)

51. Shin, S.-W.: Galois representations arising from some compact Shimura varieties. Ann. Math. (2) 173(3), 1645-1741 (2011)

52. Shin, S.-W.: On the cohomological base change for unitary similitude groups (an appendix to the article Galois representations associated to holomorphic limits of discrete series by W. Goldring). Compos. Math. 150(2), 191-228 (2014)

53. Demazure, M.: Structures algébriques. Cohomologie des groupes. In: Demazure, M., Grothendieck, A. (eds.) Séminaire de Géométrie Algébrique du Bois Marie 3: Schémas en groupes I, LNM 151. Springer, Berlin (1970)

54. Sorensen, C.: A patching lemma (2008) (preprint). http://fa.institut.math.jussieu.fr/node/45

55. Taylor, R.: Galois representations associated to Siegel modular forms of low weight. Duke Math. J. 63(2), 281-332 (1991)

56. Taylor, R., Yoshida, T.: Compatibility of local and global Langlands correspondences. J. Am. Math. Soc. 20(2), 467-493 (2007)

57. Weibel, C.: An Introduction to Homological Algebra, Cambridge Studies in Advanced Mathematics 38. Cambridge University Press, Cambridge (1994) 